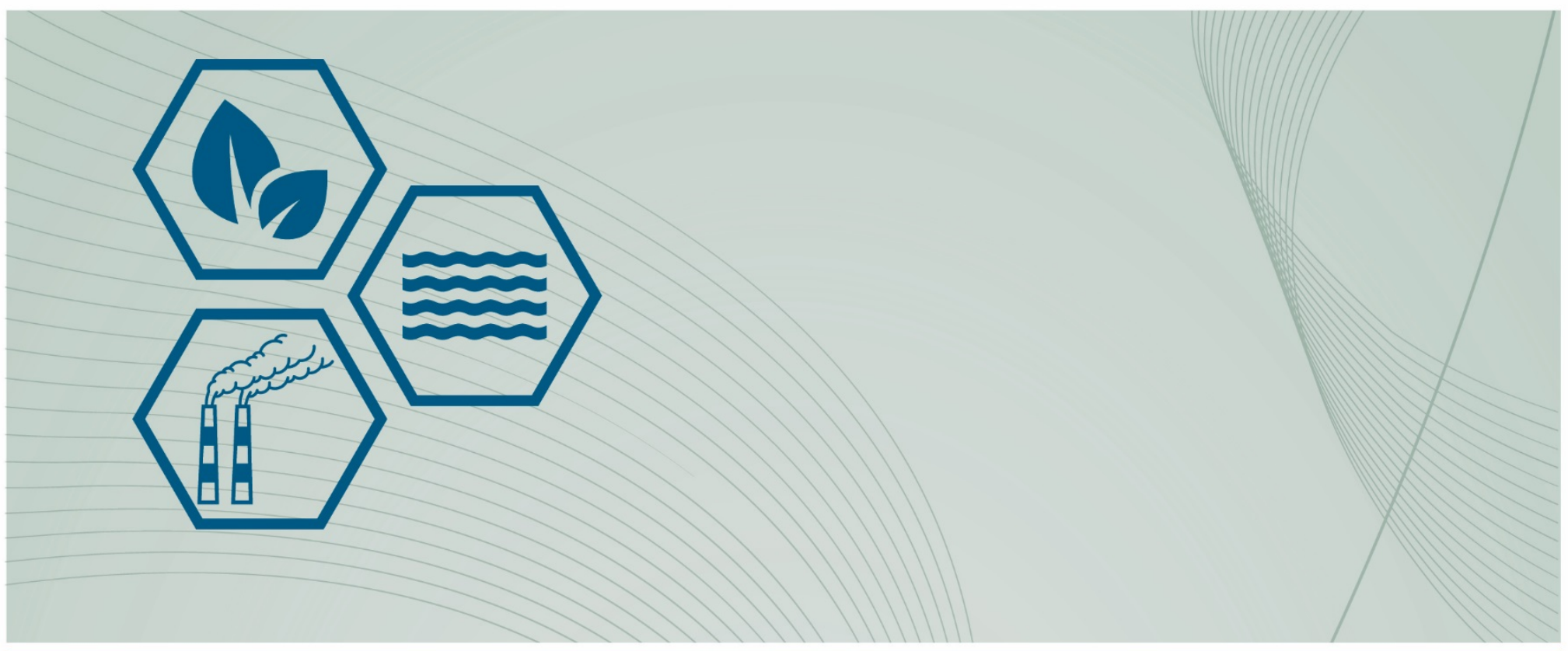

\title{
Toxicological Profile for
}

\section{Lead}

August 2020

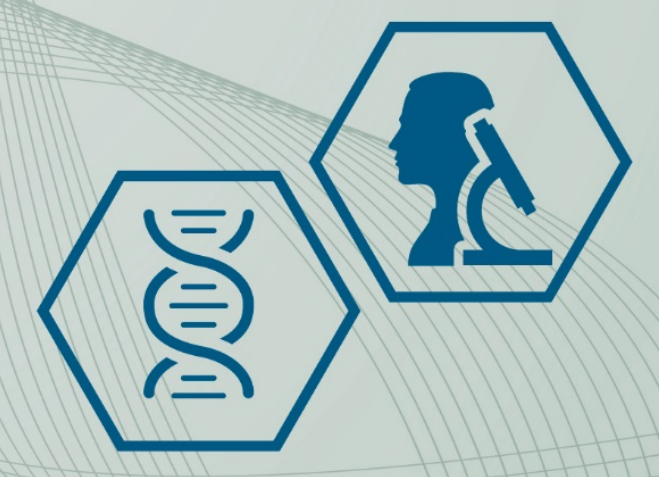




\section{DISCLAIMER}

Use of trade names is for identification only and does not imply endorsement by the Agency for Toxic Substances and Disease Registry, the Public Health Service, or the U.S. Department of Health and Human Services. 


\section{FOREWORD}

This toxicological profile is prepared in accordance with guidelines* developed by the Agency for Toxic Substances and Disease Registry (ATSDR) and the Environmental Protection Agency (EPA). The original guidelines were published in the Federal Register on April 17, 1987. Each profile will be revised and republished as necessary.

The ATSDR toxicological profile succinctly characterizes the toxicologic and adverse health effects information for these toxic substances described therein. Each peer-reviewed profile identifies and reviews the key literature that describes a substance's toxicologic properties. Other pertinent literature is also presented, but is described in less detail than the key studies. The profile is not intended to be an exhaustive document; however, more comprehensive sources of specialty information are referenced.

The focus of the profiles is on health and toxicologic information; therefore, each toxicological profile begins with a relevance to public health discussion which would allow a public health professional to make a real-time determination of whether the presence of a particular substance in the environment poses a potential threat to human health. The adequacy of information to determine a substance's health effects is described in a health effects summary. Data needs that are of significance to the protection of public health are identified by ATSDR.

Each profile includes the following:

(A) The examination, summary, and interpretation of available toxicologic information and epidemiologic evaluations on a toxic substance to ascertain the levels of significant human exposure for the substance due to associated acute, intermediate, and chronic exposures;

(B) A determination of whether adequate information on the health effects of each substance is available or in the process of development to determine levels of exposure that present a significant risk to human health of acute, intermediate, and chronic health effects; and

(C) Where appropriate, identification of toxicologic testing needed to identify the types or levels of exposure that may present significant risk of adverse health effects in humans.

The principal audiences for the toxicological profiles are health professionals at the Federal, State, and local levels; interested private sector organizations and groups; and members of the public.

This profile reflects ATSDR's assessment of all relevant toxicologic testing and information that has been peer-reviewed. Staffs of the Centers for Disease Control and Prevention and other Federal scientists have also reviewed the profile. In addition, this profile has been peer-reviewed by a nongovernmental panel and was made available for public review. Final responsibility for the contents and views expressed in this toxicological profile resides with ATSDR.

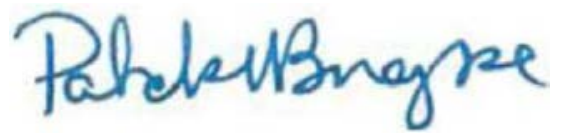

Patrick N. Breysse, Ph.D., CIH

Director, National Center for Environmental Health and

Agency for Toxic Substances and Disease Registry

Centers for Disease Control and Prevention 


\section{*Legislative Background}

The toxicological profiles are developed under the Comprehensive Environmental Response, Compensation, and Liability Act of 1980, as amended (CERCLA or Superfund). CERCLA section 104(i)(1) directs the Administrator of ATSDR to “...effectuate and implement the health related authorities" of the statute. This includes the preparation of toxicological profiles for hazardous substances most commonly found at facilities on the CERCLA National Priorities List (NPL) and that pose the most significant potential threat to human health, as determined by ATSDR and the EPA. Section 104(i)(3) of CERCLA, as amended, directs the Administrator of ATSDR to prepare a toxicological profile for each substance on the list. In addition, ATSDR has the authority to prepare toxicological profiles for substances not found at sites on the NPL, in an effort to “...establish and maintain inventory of literature, research, and studies on the health effects of toxic substances" under CERCLA Section 104(i)(1)(B), to respond to requests for consultation under section 104(i)(4), and as otherwise necessary to support the site-specific response actions conducted by ATSDR. 


\section{VERSION HISTORY}

\begin{tabular}{ll}
\hline Date & Description \\
\hline August 2020 & Final toxicological profile released \\
May 2019 & Draft for public comment toxicological profile released \\
August 2007 & Final toxicological profile released \\
April 1993 & Final toxicological profile released
\end{tabular}




\section{CONTRIBUTORS \& REVIEWERS}

\section{CHEMICAL MANAGER TEAM}

Henry Abadin, M.S.P.H. (Lead)

Jessilynn Taylor, M.S., CDR USPHS

Melanie Buser, M.P.H.

Franco Scinicariello, M.D., M.P.H.

Jennifer Przybyla, Ph.D.

ATSDR, Division of Toxicology and Human Health

Sciences, Atlanta, GA
Julie M. Klotzbach, Ph.D.

Gary L. Diamond, Ph.D.

Mario Citra, Ph.D.

Lara L. Chappell, Ph.D.

Laura A. Mcllroy, B.A.

SRC, Inc., North Syracuse, NY

\section{REVIEWERS}

\section{Interagency Minimal Risk Level Workgroup:}

Includes ATSDR; National Center for Environmental Health (NCEH); National Institute for Occupational Safety and Health (NIOSH); U.S. Environmental Protection Agency (EPA); National Toxicology Program (NTP).

\section{Additional reviews for science and/or policy:}

ATSDR, Division of Community Health Investigations; ATSDR, Office of Science; NCEH, Division of Laboratory Science; NCEH, Division of Environmental Health Science and Practice; Occupational Safety and Health Administration (OSHA); Department of Defense (DoD); EPA; NIOSH.

\section{PEER REVIEWERS}

1. Howard Hu, M.D., M.P.H., Sc.D., Department of Environmental Health Sciences, University of Michigan School of Public Health, Ann Arbor, Michigan

2. Anthony Knafla, M.Sc., DABT, P. Biol., Founder/Senior Scientist \& Manager, Equilibrium Environmental Inc., Calgary, Canada

3. Nelly Mañay, Ph.D., Professor, Department of Toxicology and Environmental Hygiene, Faculty of Chemistry, University of the Republic of Uruguay, Montevideo, Uruguay

These experts collectively have knowledge of toxicology, chemistry, and/or health effects. All reviewers were selected in conformity with Section 104(I)(13) of the Comprehensive Environmental Response, Compensation, and Liability Act, as amended.

ATSDR scientists review peer reviewers' comments and determine whether changes will be made to the profile based on comments. The peer reviewers' comments and responses to these comments are part of the administrative record for this compound.

The listing of peer reviewers should not be understood to imply their approval of the profile's final content. The responsibility for the content of this profile lies with ATSDR. 


\section{CONTENTS}

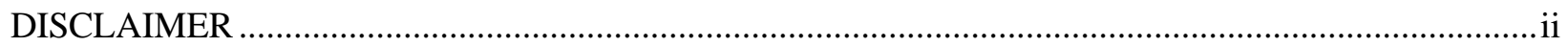

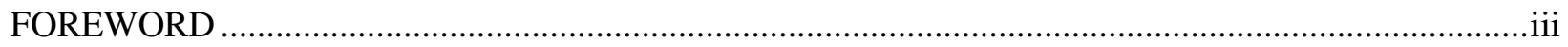

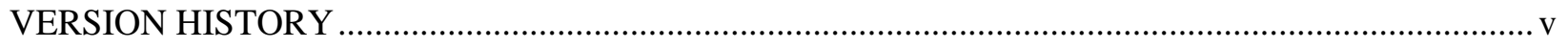

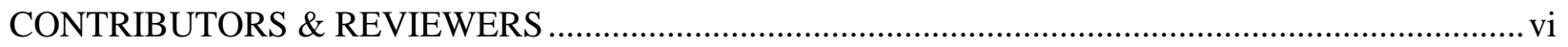

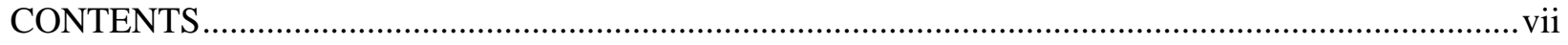

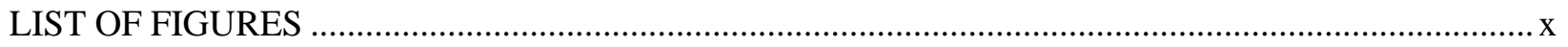

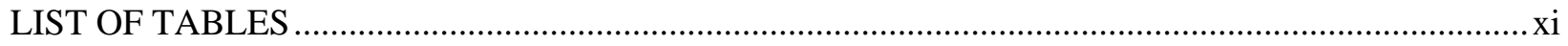

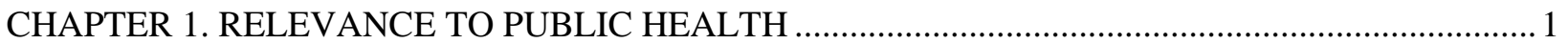

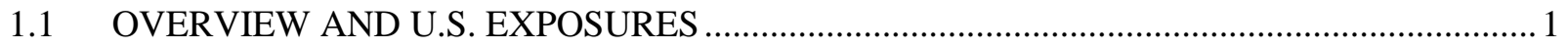

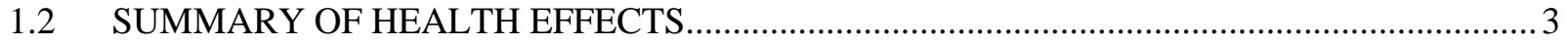

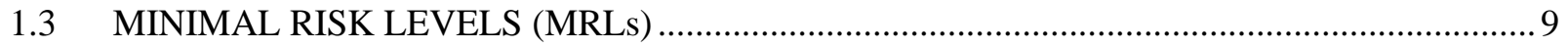

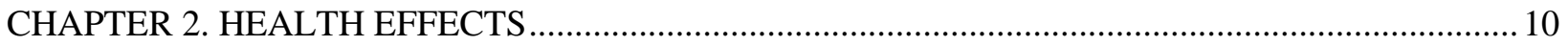

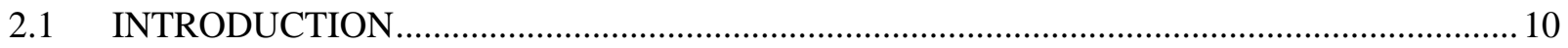

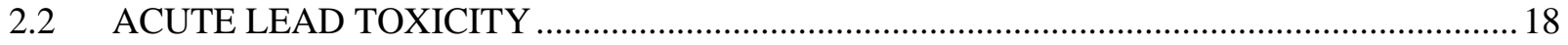

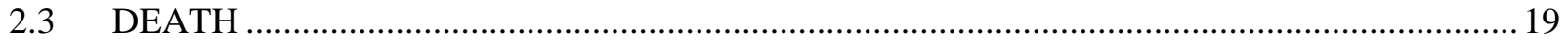

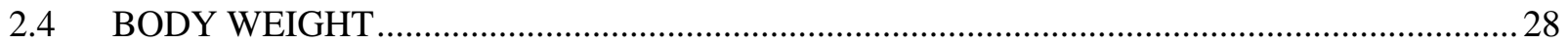

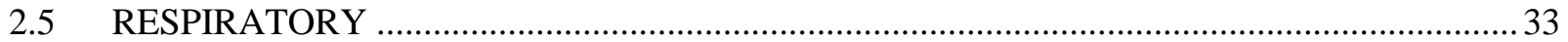

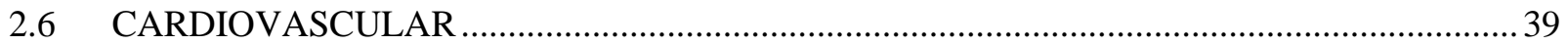

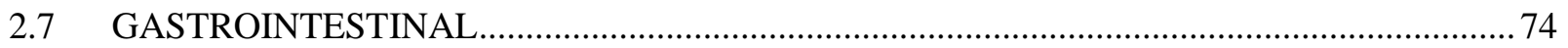

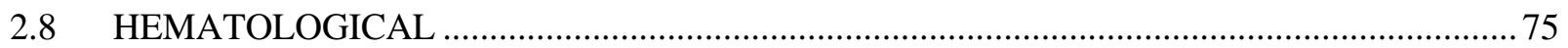

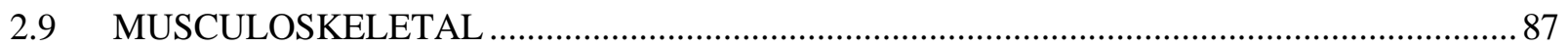

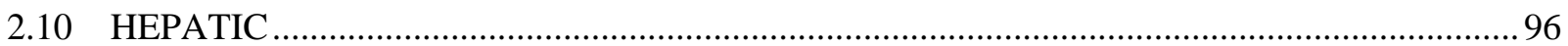

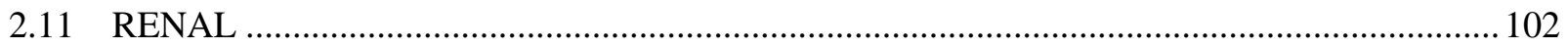

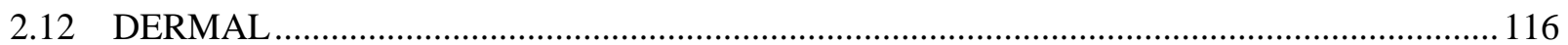

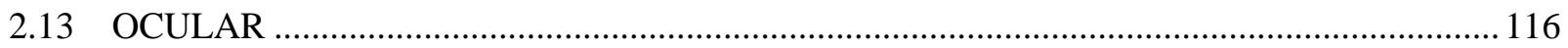

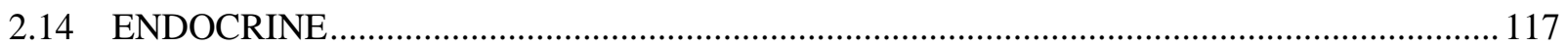

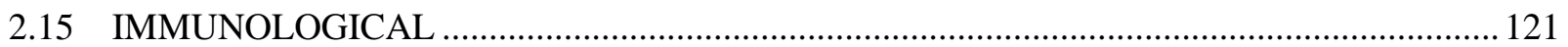

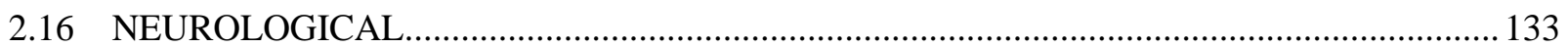

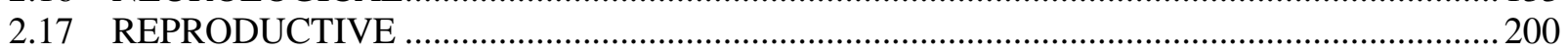

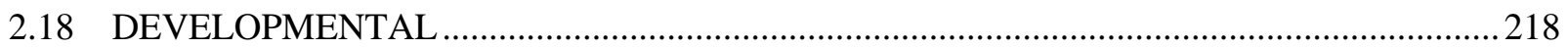

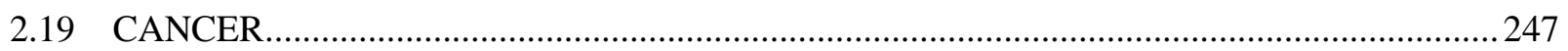

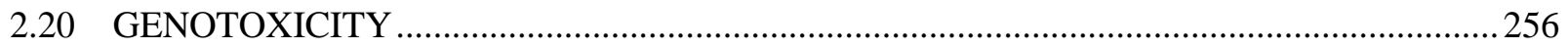

2.21 GENERAL CELLULAR MECHANISMS OF ACTION ..................................................262

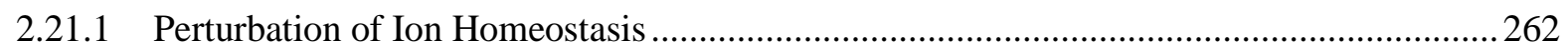

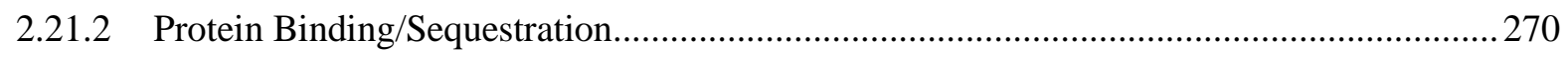

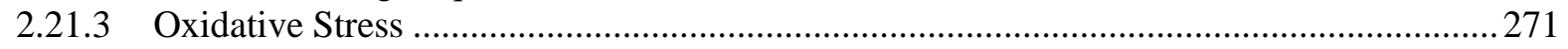

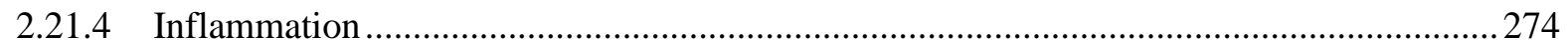

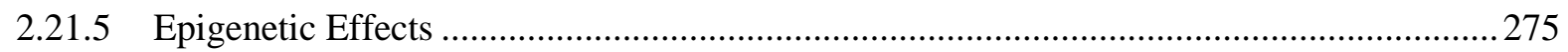

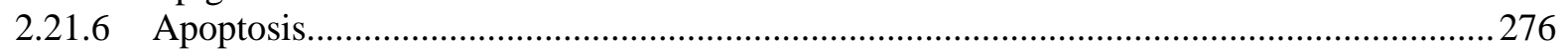

CHAPTER 3. TOXICOKINETICS, SUSCEPTIBLE POPULATIONS, BIOMARKERS, CHEMICAL

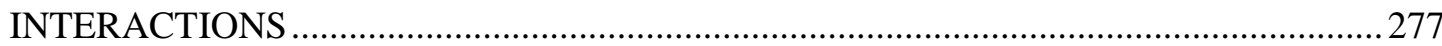

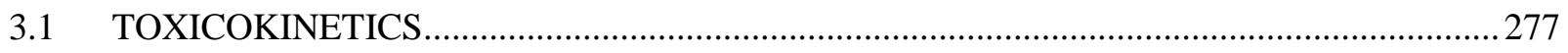

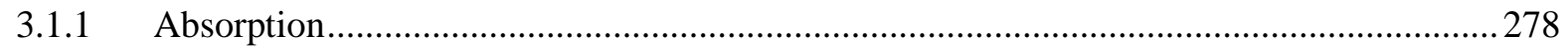

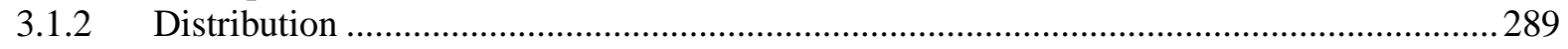




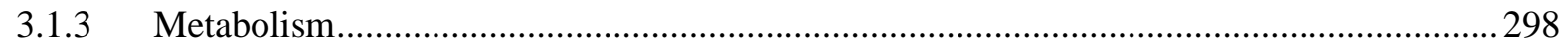

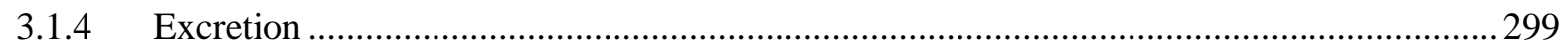

3.1.5 Physiologically Based Pharmacokinetic (PBPK)/Pharmacodynamic (PD) Models ........... 302

3.2 CHILDREN AND OTHER POPULATIONS THAT ARE UNUSUALLY SUSCEPTIBLE... 321

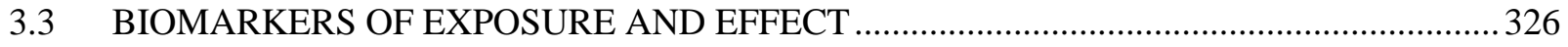

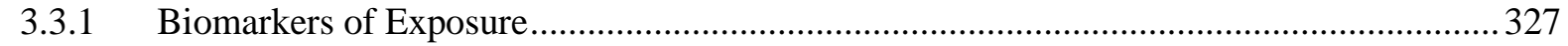

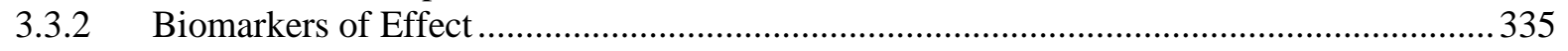

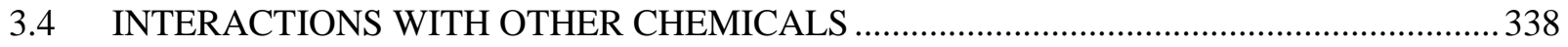

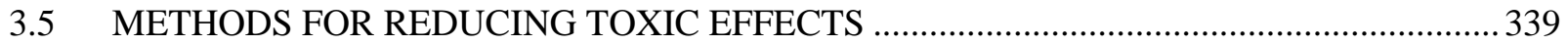

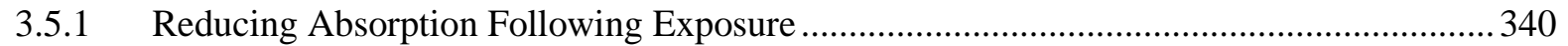

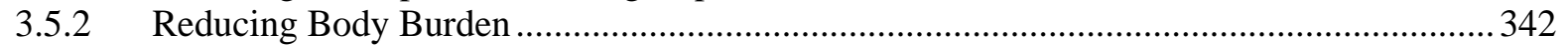

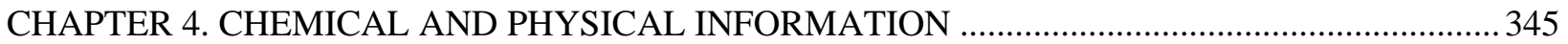

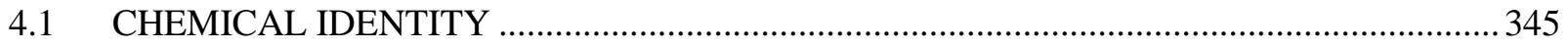

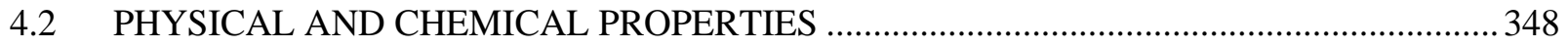



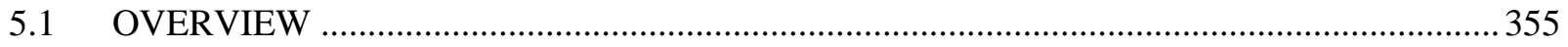

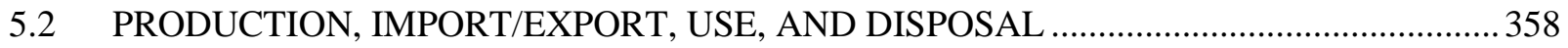

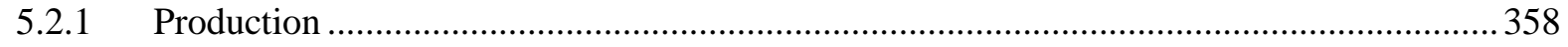

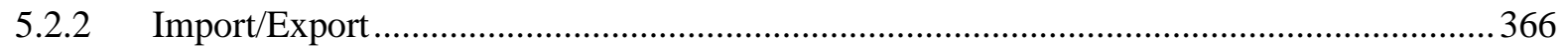

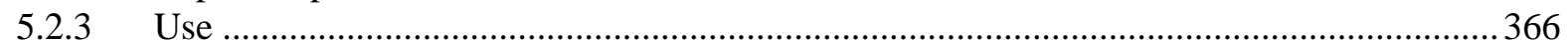

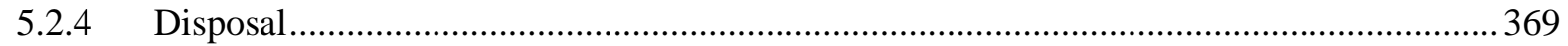

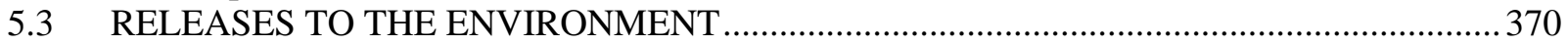

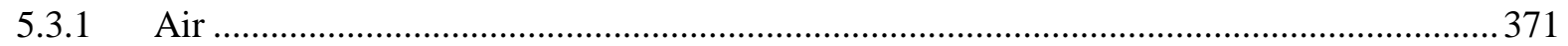

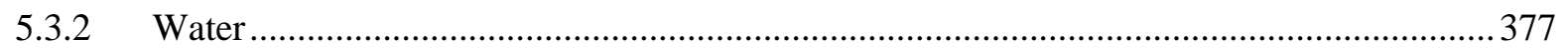

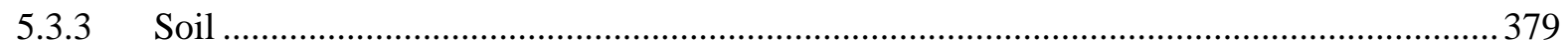

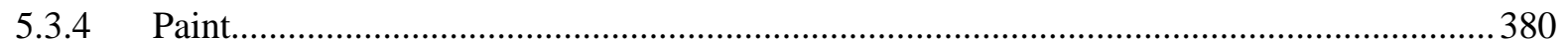

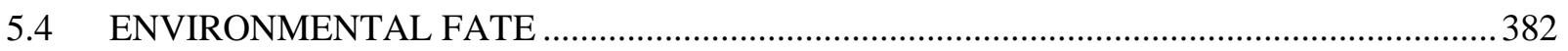

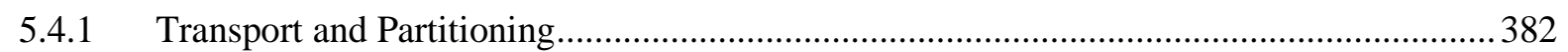

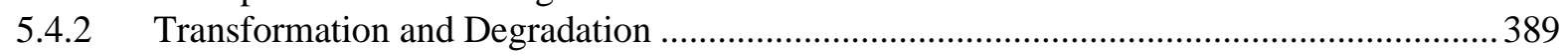

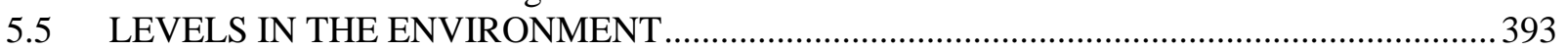

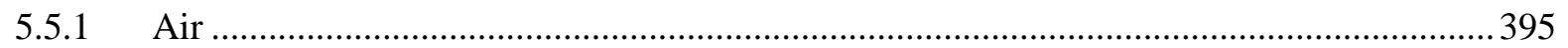

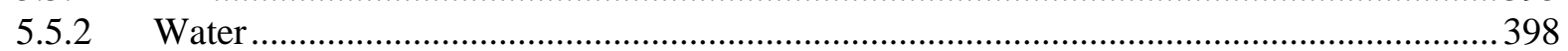

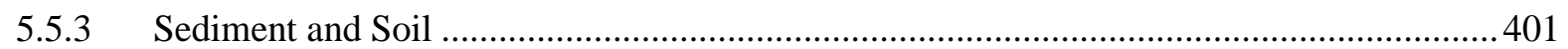

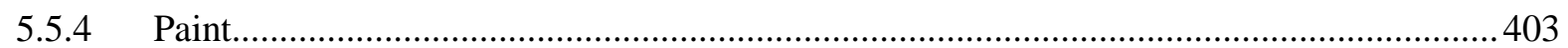

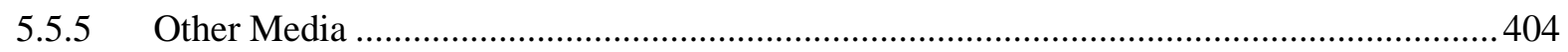

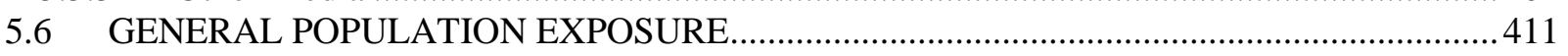

5.7 POPULATIONS WITH POTENTIALLY HIGH EXPOSURES ........................................ 428

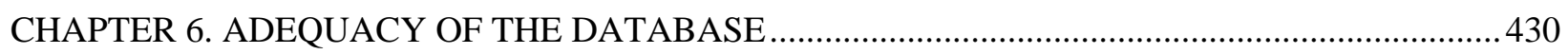

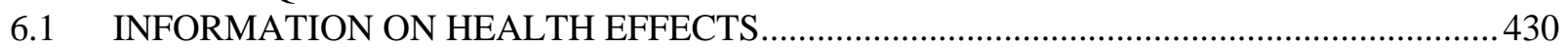

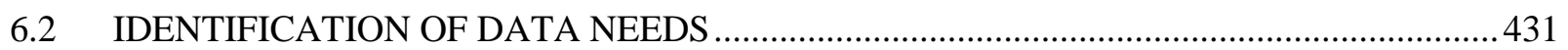

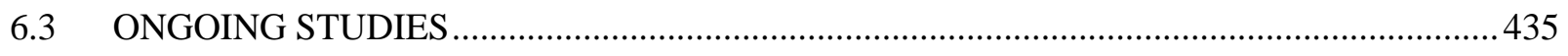

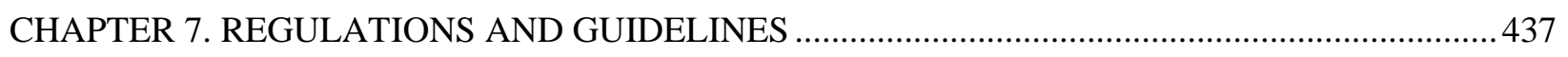

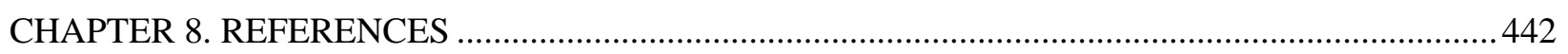




\section{APPENDICES}

APPENDIX A. ATSDR MINIMAL RISK LEVEL WORKSHEETS A-1

APPENDIX B. LITERATURE SEARCH FRAMEWORK FOR LEAD .............................................. B-1

APPENDIX C. INGESTION OF LEAD DEBRIS …............................................................................ C-1

APPENDIX D. QUICK REFERENCE FOR HEALTH CARE PROVIDERS …................................. D-1

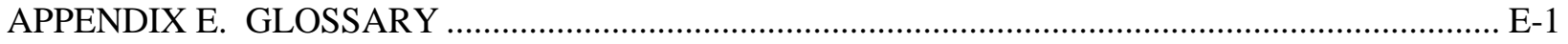

APPENDIX F. ACRONYMS, ABBREVIATIONS, AND SYMBOLS ……......................................... 


\section{LIST OF FIGURES}

2-1. Overview of the Number of Studies Examining Associations Between PbB and Health Effects...... 17

2-2. Change in the Systolic Pressure Associated with a Doubling of the Blood Lead Concentration $(\mathrm{PbB})$... 46

2-3. Change in the Diastolic Pressure Associated with a Doubling of the Blood Lead Concentration $(\mathrm{PbB})$ 47

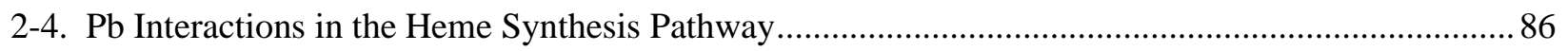

2-5. Multiorgan Impact of Reduction of Heme Body Pool by Lead...................................................... 87

2-6. Immunological Pathways by which Pb Exposure Potentially may Increase Risk of ImmuneRelated Diseases

2-7. Relationship Between Blood Lead Concentration (PbB) and Birth Weight at $\mathrm{PbB} \leq 10 \mu \mathrm{g} / \mathrm{dL} \ldots . . . .231$

3-1. Compartments and Pathways of Lead (Pb) Exchange in the O’Flaherty Model.............................305

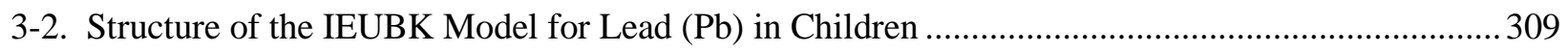

3-3. Compartments and Pathways of Lead $(\mathrm{Pb})$ Exchange in the Leggett Model .................................. 313

3-4. Blood Lead Concentrations (PbBs) in Children Predicted by the IEUBK, Leggett, and O’Flaherty Models and AALM.....

3-5. Blood Lead Concentrations (PbBs) in Adults Predicted by the Leggett and O’Flaherty Models and AALM.

5-1. Number of NPL Sites with Lead Contamination. 355

5-2. Number of NPL Sites with Lead Compound Contamination..... 356

5-3. Annual Maximum 3-Month Average Representing the National Trend 396 


\section{LIST OF TABLES}

2-1. Summary of Epidemiological Studies Evaluating Death 22

2-2. Summary of Epidemiological Studies Evaluating Effects on Body Weight at Mean Blood Lead Concentrations $(\mathrm{PbB}) \leq 10 \mu \mathrm{g} / \mathrm{dL}$ 30

2-3. Effects on Body Weight Associated with Mean Blood Lead Concentrations $(\mathrm{PbBs}) \leq 10 \mu \mathrm{g}$. 33

2-4. Overview of Respiratory Effects in Adults and Children Chronically Exposed to Lead (Pb) ... 35

2-5. Summary of Epidemiological Studies Evaluating Respiratory Effects at Mean Blood Lead Concentration $(\mathrm{PbB}) \leq 10 \mu \mathrm{g} / \mathrm{dL}$ 36

2-6. Overview of Cardiovascular Effects in Adults and Children Associated with Chronic Exposure to Lead $(\mathrm{Pb})$.

2-7. Characteristics of the Study Population in Meta-Analyses of Effects of Lead (Pb) on Blood Pressure.

2-8. Summary of Epidemiological Studies Evaluating Effects on Blood Pressure at Mean Blood Lead Concentration $(\mathrm{PbB}) \leq 10 \mu \mathrm{g} / \mathrm{dL}$

2-9. Summary of Epidemiological Studies Evaluating Atherosclerosis at Mean Blood Lead Concentration $(\mathrm{PbB}) \leq 10 \mu \mathrm{g} / \mathrm{dL}$

2-10. Summary of Epidemiological Studies Evaluating Heart Disease at Mean Blood Lead Concentration $(\mathrm{PbB}) \leq 10 \mu \mathrm{g} / \mathrm{dL}$

2-11. Summary of Epidemiological Studies Evaluating Mortality due to Cardiovascular Disease at Mean Blood Lead Concentrations $(\mathrm{PbB}) \leq 10 \mu \mathrm{g} / \mathrm{dL}$ 70

2-12. Associations Between Bone $\mathrm{Pb}$ and Blood Pressure Outcomes 71

2-13. Associations Between Bone Pb and Cardiac Function, Disease, and Mortality. 73

2-14. Summary of Studies Evaluating Gastrointestinal Symptoms Associated with Chronic Exposure to Lead $(\mathrm{Pb})$

2-15. Overview of Hematological Effects Associated with Chronic Exposure to Lead (Pb).....

2-16. Summary of Epidemiological Studies Evaluating Hematological Effects at Mean Blood Lead Concentration $(\mathrm{PbB}) \leq 10 \mu \mathrm{g} / \mathrm{dL}$

2-17. Overview of Musculoskeletal Effects Associated with Chronic Exposure to Lead (Pb)

2-18. Summary of Epidemiological Studies Evaluating Musculoskeletal Effects at Mean Blood Lead Concentration $(\mathrm{PbB}) \leq 10 \mu \mathrm{g} / \mathrm{dL}$

2-19. Summary of Epidemiological Studies Evaluating Hepatic Effects Associated with Blood Lead Concentration (PbB)

2-20. Effects on Liver Function Tests Associated with Chronic Exposure to Lead (Pb) 101 
2-21. Overview of Renal Effect Associated with Chronic Exposure to Lead (Pb)..... .104

2-22. Summary of Epidemiological Studies Evaluating Renal Effects at Mean Blood Lead Concentration $(\mathrm{PbB}) \leq 10 \mu \mathrm{g} / \mathrm{dL}$ 106

2-23. Associations Between Bone Pb and Renal Function. 115

2-24. Overview of Endocrine Effects Associated with Chronic Exposure to Lead (Pb).....

2-25. Effects on Thyroid Hormones Associated with Blood Lead Concentration (PbB) 119

2-26. Overview of Immunological Effects Associated with Chronic Exposure to Lead (Pb)

2-27. Summary of Epidemiological Studies Evaluating Immunological Effects at Mean Blood Lead Concentration $(\mathrm{PbB}) \leq 10 \mu \mathrm{g} / \mathrm{dL}$

2-28. Overview of Neurological Effects in Children Associated with Chronic Exposure to Lead $(\mathrm{Pb})$ 136

2-29. Overview of Neurological Effects in Adults Associated with Chronic Exposure to Lead $(\mathrm{Pb})$

2-30. Summary of Epidemiological Studies Evaluating Neurological Effects in Children at Mean Blood Lead Concentration $(\mathrm{PbB}) \leq 10 \mu \mathrm{g} / \mathrm{dL}$

2-31. Associations Between Bone $\mathrm{Pb}$ and Neurological Outcomes in Children 175

2-32. Summary of Epidemiology Studies Evaluating Neurodevelopmental Effects in Adults at Mean Blood Lead Concentration $(\mathrm{PbB}) \leq 10 \mu \mathrm{g} / \mathrm{dL}$

2-33. Associations Between Bone Pb and Neurological Outcomes in Adults. 191

2-34. Overview of Effects on the Male Reproductive System Associated with Chronic Exposure to Lead $(\mathrm{Pb})$

2-35. Effects on Reproductive Hormones Associated with Chronic Exposure to Lead $(\mathrm{Pb})$ in Males 204

2-36. Summary of Epidemiological Studies Evaluating Effects on the Male Reproductive System at Mean Blood Lead Concentration $(\mathrm{PbB}) \leq 10 \mu \mathrm{g} / \mathrm{dL}$

2-37. Overview of Effects on the Female Reproductive System and Pregnancy Outcomes Associated with Chronic Exposure to Lead $(\mathrm{Pb})$.

2-38. Summary of Epidemiological Studies Evaluating Effects on the Female Reproductive System at Mean Blood Lead Concentration $(\mathrm{PbB}) \leq 10 \mu \mathrm{g} / \mathrm{dL}$

2-39. Overview of Developmental Effects Associated with Chronic Exposure to Lead (Pb). 220

2-40. Effects on Birth Outcomes at Blood Lead Concentration $(\mathrm{PbB}) \leq 10 \mu \mathrm{g} / \mathrm{dL}$

2-41. Summary of Epidemiological Studies Evaluating Birth Outcomes Effects of Mean Blood Lead Concentration $(\mathrm{PbB}) \leq 10 \mu \mathrm{g} / \mathrm{dL}$ 
2-42. Overview of Decreased Anthropometric Measures in Children at Blood Lead Concentration $(\mathrm{PbB}) \leq 10 \mu \mathrm{g} / \mathrm{dL}$

2-43. Summary of Epidemiological Studies Evaluating Anthropometric Measurements in Infants and Children with Mean Blood Lead Concentration $(\mathrm{PbB}) \leq 10 \mu \mathrm{g} / \mathrm{dL}$

2-44. Summary of Epidemiological Studies Evaluating the Onset of Puberty at Children with Mean Blood Lead Concentration $(\mathrm{PbB}) \leq 10 \mu \mathrm{g} / \mathrm{dL}$ 242

2-45. Associations Between Maternal Bone $\mathrm{Pb}$ and Birth Outcome and Postnatal Growth. 246

2-46. Summary of Epidemiological Studies Evaluating Cancer Endpoints and Blood Lead Concentration $(\mathrm{PbB})$ .250

2-47. Overview of Epidemiology Studies Evaluating Genotoxicity Associated with Chronic Exposure to Lead $(\mathrm{Pb})$. 258

2-48. Results of Genotoxicity Studies at Blood Lead Concentration $(\mathrm{PbB}) \leq 10 \mu \mathrm{g} / \mathrm{dL}$ 260

2-49. Effects of Lead $(\mathrm{Pb})$ on Function of Various Proteins. 266

3-1. Ranking of Relative Bioavailability of Lead (Pb) Mineral Phases in Soil 286

3-2. Comparison of Slope Factors in Selected Slope Factor Models 320

3-3. Influence of Other Metals and Metalloids on Lead (Pb) Toxicity. 338

3-4. Recommended Actions Based on Child Blood Lead Level (PbB). 341

3-5. Recommended Actions for Workers Based on Blood Lead Level (PbB) 342

4-1. Chemical Identity of Lead and Compounds 345

4-2. Physical and Chemical Properties of Lead and Compounds 350

5-1. U.S. Manufacturers of Lead Metal and Selected Lead Compounds. 359

5-2. U.S. Lead Production 2015-2018 362

5-3. Facilities that Produce, Process, or Use Lead. 362

5-4. Facilities that Produce, Process, or Use Lead Compounds.............................................................. 364

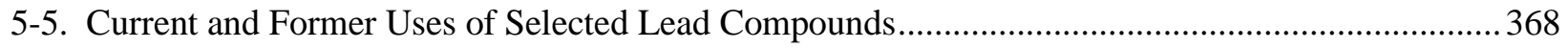

5-6. Releases to the Environment from Facilities that Produce, Process, or Use Lead ........................... 372

5-7. Releases to the Environment from Facilities that Produce, Process, or Use Lead Compounds ........ 374

5-8. Historic Levels of Lead Emissions to the Atmosphere in the United States (in Thousand Metric Tons) 376

5-9. U.S. Surface Water Discharges of Lead and Lead Compounds (Pounds/Year) 378 
5-10. Canada Surface Water Discharges of Lead and Lead Compounds (Tonnes) 379

5-11. Lowest Limit of Detection Based on Standards 394

5-12. Lead Levels in Water, Soil, and Air of National Priorities List (NPL) Sites 395

5-13. Summary Data for Lead Monitors Across the United States, 2008-2010 ( $\left.\mu \mathrm{g} / \mathrm{m}^{3}\right)$ 396

5-14. Percentile Distribution of Mean Lead (TSP) Concentrations $\left(\mu \mathrm{g} / \mathrm{m}^{3}\right)$ Measured in Ambient Air at Locations Across the United States. 397

5-15. Lead Levels in Foods Commonly Eaten by Toddlers and Infants. 404

5-16. Selected Mean Lead Concentrations in Food from the FDA Total Diet Study 405

5-17. Estimated Median and Maximum Lead Exposures 407

5-18. Lead Content in Ayurvedic Medications and Other Health Remedies. 408

5-19. Lowest Limit of Detection Based on Standards

5-20. Geometric Mean Blood Lead Levels $(\mu \mathrm{g} / \mathrm{dL})$ and the $95^{\text {th }}$ Percentile Confidence Interval, by Race/Ethnicity, Sex, and Age for the Years for 2011-2016.

5-21. Geometric Mean Urine Lead Levels ( $\mu \mathrm{g} / \mathrm{dL})$ and the $95^{\text {th }}$ Percentile Confidence Interval, by Race/Ethnicity, Sex, and Age

5-22. Industries by Sector with Most Workers having Blood Lead Concentrations (PbBs) $\geq 25 \mu \mathrm{g} / \mathrm{dL}, 2010-2016$

5-23. Number and Rate per 100,000 Children Aged < 5 Years with Blood Lead Levels 5-9 $\mu \mathrm{g} / \mathrm{dL}$ in the Childhood Blood Lead Surveillance System, United States, 2010-2014.

5-24. Geometric Mean Urine Lead Levels $(\mu \mathrm{g} / \mathrm{dL})$ and the 95th Percentile Confidence Interval by Smoking Status

5-25. Measurements of Lead in Indoor Dust in the United States from 2006 to 2011

6-1. Ongoing Studies on Lead (Pb)

7-1. Regulations and Guidelines Applicable to Lead (Pb) 


\section{CHAPTER 1. RELEVANCE TO PUBLIC HEALTH}

\subsection{OVERVIEW AND U.S. EXPOSURES}

Lead $(\mathrm{Pb})$ is an element that is found in concentrated and easily accessible $\mathrm{Pb}$ ore deposits that are widely distributed throughout the world. A major source of $\mathrm{Pb}$ in the U.S. environment has historically been anthropogenic emissions to the atmosphere from combustion of leaded gasoline, which was phased out of use after 1973 and then banned in 1995 (with the exception of fuels for piston-driven aircraft) (EPA 1996a). Pb continued to be used as an anti-knock agent in National Association for Stock Car Auto Racing (NASCAR) fuels until it was phased out beginning in 2008. Deteriorating Pb-based paints from weathered surfaces (which produce highly concentrated $\mathrm{Pb}$ debris and dusts) in older housing stock (pre-1978) continues to be a source of childhood Pb poisoning in the United States (CDC 1991, 2012d). The combination of corrosive water and $\mathrm{Pb}$ pipes or $\mathrm{Pb}$-soldered joints in either the distribution system or individual houses can create localized zones of high Pb water concentrations (EPA 1989b, 2007a; HannaAttisha et al. 2016). Other anthropogenic sources of $\mathrm{Pb}$ have included mining and smelting of ore; manufacture of and use of Pb-containing products (e.g., Pb-based paints, pigments, and glazes; electrical shielding; plumbing; storage batteries; solder; and welding fluxes); manufacture and application of $\mathrm{Pb}$-containing pesticides; combustion of coal and oil; and waste incineration.

$\mathrm{Pb}$ does not degrade in the environment, although it can exist in various chemical forms (see Section 5.4 for a more detailed discussion of the environmental fate of $\mathrm{Pb}$ ). Particulate matter containing $\mathrm{Pb}$ can be transported through air, water, and soil. In general, atmospheric deposition is the largest source of $\mathrm{Pb}$ found in soils not impacted by other local non-air sources (e.g., dust from deteriorating leaded paint). $\mathrm{Pb}$ is transferred continuously between air, water, and soil by natural chemical and physical processes such as weathering, runoff, precipitation, dry deposition of dust, and stream/river flow; however, soil and sediments appear to be important sinks for $\mathrm{Pb}$. $\mathrm{Pb}$ adsorbs strongly to most soils, which limits the rate of leaching. Soil acidity $(\mathrm{pH})$ and composition are the most important factors affecting solubility, mobility, and phytoavailability of $\mathrm{Pb}$ in soil. Other conditions that increase $\mathrm{Pb}$ mobility in soil are reducing conditions and high chloride content.

The general population may be exposed to $\mathrm{Pb}$ in ambient air, foods, drinking water, soil, and dust. $\mathrm{Pb}$ has also been found in a variety of other consumer products including storage batteries, solders, pottery glazes, leaded crystal glassware, cosmetics, hair dyes, jewelry, gun shot and ammunition, relic fishing sinkers, tire weights, and imported children's toys, traditional or folk remedies, and candy/food 
packaging. For adults, exposure to levels of $\mathrm{Pb}$ beyond background is usually associated with occupational exposures. For children, exposure to high levels of $\mathrm{Pb}$ is associated with living in areas contaminated by $\mathrm{Pb}$ (e.g., soil or indoor dust in older homes with Pb-based paint). The primary source of $\mathrm{Pb}$ exposure to children is from surface dusts (on the ground or entrained) that contain $\mathrm{Pb}$ from a variety of sources including deteriorated Pb-based paint (CDC 2009; Lanphear et al. 1998a; Succop et al. 1998). Environmental $\mathrm{Pb}$ is particularly accessible to children because of their more intensive hand-to-mouth activity and the proximity of the child breathing zone to $\mathrm{Pb}$ entrained from surface dusts. Because $\mathrm{Pb}$ is transported from soil very slowly, historic sources of deposition of $\mathrm{Pb}$ to soil continue to contribute to current exposures (Laidlaw and Filipelli 2008; Laidlaw et al. 2012). Based on a multimedia Pb exposure modeling analysis for children 1-5 years old at upper percentiles of blood $\mathrm{Pb}(\mathrm{PbB})$ levels in the U.S. population, soil and dust ingestion are dominant exposure pathways, but for lower percentiles, other age groups (e.g., younger children), or specific local U.S. locations, the main other exposure sources/ pathways could be important, such as drinking water and food (Zartarian et al. 2017).

$\mathrm{PbB}$ has been used as a biomarker of $\mathrm{Pb}$ exposure, and periodic surveys of $\mathrm{PbB}$ of the U.S. population are conducted by the Centers for Disease Control and Prevention (CDC). Based on data from the National Health and Nutrition Examination Survey (NHANES) (2015-2016, CDC 2018a, 2019), the geometric mean $\mathrm{PbB}$ in a representative sample of U.S. adults, $\geq 20$ years old, was $0.920 \mu \mathrm{g} / \mathrm{dL}$ ( $95 \%$ confidence interval [CI] 0.862, 0.982). The geometric mean blood $\mathrm{PbB}$ of a representative sample of U.S. children, 1-5 years old, was $0.758 \mu \mathrm{g} / \mathrm{dL}$ (95\% CI 0.675, 0.850). PbBs in the U.S. have decreased considerably in the last several decades as a result of removal of $\mathrm{Pb}$ from gasoline and restrictions placed on the use of $\mathrm{Pb}$ in residential paints (Brody et al. 1994; CDC 2011, 2018a; Pirkle et al. 1994, 1998; Schwartz and Pitcher 1989).

Seasonal variations in blood lead concentration ( $\mathrm{PbB}$ ) levels in children have been observed, with a general trend of increasing PbB during late summer and early fall (Gulson et al. 2008; Johnson and Bretsch 2002; Laidlaw et al. 2005). Seasonal patterns in behavior (e.g., outdoor activities) and weather that promotes re-entrainment and transport of dust $\mathrm{Pb}$ (humidity and wind velocity) may contribute to the observed seasonal patterns in PbB (Laidlaw et al. 2005, 2012) and provide additional evidence for surface dusts being a major contributor to child $\mathrm{Pb}$ exposure and $\mathrm{PbB}$. 


\subsection{SUMMARY OF HEALTH EFFECTS}

The toxicity of $\mathrm{Pb}$ to humans has been known for over 2,000 years, and is not disputed. Early epidemiological studies focused on overt toxicity associated with high occupational exposures. However, during the past few decades, there has been a growing awareness that low-level environmental exposure resulting in $\mathrm{PbB}<10 \mu \mathrm{g} / \mathrm{dL}$ is associated with adverse effects, particularly in children. PbB levels associated with adverse effects vary by endpoint. Adverse effects occur at $\mathrm{PbB}<5 \mu \mathrm{g} / \mathrm{dL}$ and for the most studied endpoints (neurological, renal, cardiovascular, hematological, immunological, reproductive, and developmental), effects occur at the lowest PbBs studied ( $\leq 5 \mu \mathrm{g} / \mathrm{dL})$. CDC (2018b) states that "no safe blood lead level in children has been identified.” As a result, U.S. public health policy has changed to focus on eliminating lead poisoning as a public health problem. CDC considers $\mathrm{PbB}$ to be elevated in children when it exceeds a reference value defined as the $97.5^{\text {th }}$ percentile for the U.S. population. The current CDC reference value, based on data from the NHANES 2007-2008 and 2009-2010, is $5 \mu \mathrm{g} / \mathrm{dL}$. Therefore, the primary objective of current research is on health effects associated with $\mathrm{PbB} \leq 5 \mu \mathrm{g} / \mathrm{dL}$.

The literature evaluating the health effects of $\mathrm{Pb}$ is enormous, and includes an extensive database in humans, including children and infants. Information on health effects reviewed below is taken from epidemiological studies that identify the major lines of evidence regarding health effects in humans. Although the literature on adverse effects of $\mathrm{Pb}$ in laboratory animals also is extensive, due to the large number of available epidemiological studies, results of animal studies were not considered for the identification of health effects associated with $\mathrm{Pb}$. This potentially leaves out discussion of effects that may have been observed in animal models that have not been studied in humans and that may be future targets of human epidemiology and clinical toxicology studies. Animal studies were included in discussion of mechanisms of toxicity of $\mathrm{Pb}$ and toxicokinetics.

To quantify exposure, epidemiological studies on the toxicity of Pb rely on internal exposure metrics, rather than measurements of external exposures (e.g., concentration of $\mathrm{Pb}$ in water or air) or ingested dose. The most common internal dose metric for $\mathrm{Pb}$ is the concentration of $\mathrm{Pb}$ in blood $(\mathrm{PbB}$, typically expressed in terms of $\mu \mathrm{g} / \mathrm{dL})$. Blood $\mathrm{Pb}$ concentration reflects both ongoing exposure and $\mathrm{Pb}$ stores in bone, which can be transferred to blood. Because of the relatively rapid elimination of $\mathrm{Pb}$ from blood compared to bone, blood $\mathrm{Pb}$ will reflect mainly the exposure history of the previous few months and not necessarily the larger burden of $\mathrm{Pb}$ in bone (see Section 3.1). As a result, a single $\mathrm{PbB}$ measurement may not be a reliable metric for $\mathrm{Pb}$ body burden or cumulative exposure. Longitudinal measurements of $\mathrm{PbB}$ can be used to construct a cumulative blood $\mathrm{Pb}$ index (CBLI), which may be a better reflection of 
exposure history; however, the CBLI will not capture shorter-term variation in exposure that may occur between measurements. Direct, noninvasive measurements of bone $\mathrm{Pb}$ concentrations have been used as a metric of long-term exposure on the basis that most of the absorbed $\mathrm{Pb}$ retained in the body will reside in bone (see Section 3.1). The health effects of $\mathrm{Pb}$ are the same, regardless of the route of exposure (e.g., inhalation or ingestion). Given that exposure is quantified by internal exposure metrics (e.g., $\mathrm{PbB}$, bone $\mathrm{Pb}$ ), epidemiological studies do not attempt to define the route of exposure. Environmental exposure to $\mathrm{Pb}$ occurs continuously over a lifetime and $\mathrm{Pb}$ is retained in the body for decades. Because internal dose metrics cannot define the complete history of exposure, the exposure duration and timing that correlates most strongly with the observed health effect are typically unknown or highly uncertain.

Toxic effects of $\mathrm{Pb}$ have been observed in every organ system that has been rigorously studied. Clinical significance of some of the organ system effects at low levels of exposure and blood $\mathrm{Pb}$ is more substantial than for others (e.g., neurological, renal, cardiovascular, hematological, immunological, reproductive, and developmental effects). This is not surprising because the mechanisms that induce toxicity are common to all cell types and because $\mathrm{Pb}$ is widely distributed throughout the body. Adverse health effects have been observed in these systems at $\mathrm{PbB} \leq 10 \mu \mathrm{g} / \mathrm{dL}$. Exposure thresholds for effects on specific organ systems have not been identified (i.e., no safe level has been identified). Cognitive deficits in children occurring at the lowest PbBs $(\leq 5 \mu \mathrm{g} / \mathrm{dL})$ are the best substantiated effects. However, data for some organ systems results are inconsistent, and insufficient data are available to provide information on dose-response relationships. It is also important to note that effects observed in adults, especially older adults, may be due to higher environmental or occupational exposures in the past; therefore, exposure history is an important consideration in epidemiological studies on the health effects of $\mathrm{Pb}$.

The most extensively studied health outcomes, as described below, are neurological, renal, cardiovascular, hematological, immunological, reproductive, and developmental effects. Neurological effects of $\mathrm{Pb}$ are of greatest concern because effects are observed in infants and children and may result in life-long decrements in neurological function. Infants are born with a $\mathrm{Pb}$ burden derived from maternal transfer in utero and subsequently can continue to absorb maternal $\mathrm{Pb}$ from ingestion of breast milk. Children are also more vulnerable because of behaviors that increase ingestion of $\mathrm{Pb}$ surface dusts (e.g., hand-to-mouth activity) and because gastrointestinal absorption of ingested $\mathrm{Pb}$ is higher in children compared to adults, possibly due to a combination of physiological differences and differences in diet and nutrition. The following briefly summarizes health effects of chronic exposure to Pb observed in humans. More detailed information, including reference citations, is provided in Chapter 2. 
Neurological Effects in Children. Numerous prospective and large cross-section studies in children provide consistent evidence of decrements in neurological function, including decrements in cognitive function (learning and memory), altered behavior and mood (attention, hyperactivity, impulsivity, irritably, delinquency), and altered neuromotor and neurosensory function (visual-motor integration, dexterity, postural sway, changes in hearing and visual thresholds). These effects have been associated with a $\mathrm{PbB}$ range from $\leq 5$ to $>50 \mu \mathrm{g} / \mathrm{dL}$, with numerous studies providing evidence for effects at $\mathrm{PbB} \leq 5 \mu \mathrm{g} / \mathrm{dL}$. Taken together, studies support the concept that $\mathrm{Pb}$ affects cognitive function in children prenatally and/or environmentally exposed to low levels of $\mathrm{Pb}$. No threshold for these effects has been identified (i.e., no safe level has been identified). Decrements in cognitive function increase with $\mathrm{PbB}$, and several $\mathrm{PbB}$-effect models predict that larger decrements in cognitive function would occur when PbB increases from 1 to $10 \mu \mathrm{g} / \mathrm{dL}$, compared to when PbB increases from levels $>10 \mu \mathrm{g} / \mathrm{dL}$. Supralinear dose-response relationships for neurological outcomes are discussed in greater detail in Section 2.16 (Neurological). At higher PbB (>30 $\mu \mathrm{g} / \mathrm{dL}$ ), other neurotoxic effects have been observed, including alterations in nerve function (decrements in fine and gross motor skills, peripheral neuropathy) and encephalopathy.

Neurological Effects in Adults. Epidemiological studies in adults demonstrate decrements in neurological function associated with $\mathrm{PbB}$. All of the cognitive and neurobehavioral effects of $\mathrm{Pb}$ observed in children also have been observed in adults associated with $\mathrm{PbB}$ ranging from $\leq 10$ to $>50 \mu \mathrm{g} / \mathrm{dL}$, with evidence of effects occurring at $\mathrm{PbB} \leq 5 \mu \mathrm{g} / \mathrm{dL}$. At higher $\mathrm{PbB}(>30 \mu \mathrm{g} / \mathrm{dL})$, other observed neurotoxic effects include peripheral neuropathy, psychiatric symptoms (depression, panic disorders, anxiety, hostility, confusion, anger, and schizophrenia), and changes in regional brain volumes and neurochemistry. It is not clear if cognitive decrements are related to exposures that occurred during adulthood or during periods of nervous system development (e.g., prenatal and childhood exposures) or if effects are due to cumulative exposure. Results of a few studies that have followed children to early adulthood show an association between child $\mathrm{PbB}$ and behavioral and neuroanatomical changes in adults, suggesting a possible impact of exposures on childhood to adult outcomes.

Renal Effects. Adverse renal effects of $\mathrm{Pb}$ are well-established in numerous epidemiological studies. Studies show consistent evidence of renal damage and reduced renal function associated with a wide range of $\mathrm{PbB}(\leq 10-50 \mu \mathrm{g} / \mathrm{dL})$, with several studies providing evidence for effects at $\mathrm{PbB} \leq 5 \mu \mathrm{g} / \mathrm{dL}$. Deficits in renal function include enzymuria, proteinuria, impaired transport of organic anions and glucose, and depressed glomerular filtration rate (GFR). At higher PbB ( $>30 \mu \mathrm{g} / \mathrm{dL}$ ), Pb-induced nephrotoxicity is characterized by proximal tubular nephropathy, glomerular sclerosis, interstitial fibrosis, 
and tubular necrosis. Note that $\mathrm{Pb}$-induced decrements in renal function can lead to higher $\mathrm{Pb}$ body burden due to decreased excretion of Pb (i.e., reverse causality). In addition, other causes of decreased renal function could result in an increased body burden of $\mathrm{Pb}$.

Cardiovascular Effects. A large number of epidemiological studies in adults show adverse cardiovascular effects associated with a $\mathrm{PbB}$ range from $\leq 5$ to $>50 \mu \mathrm{g} / \mathrm{dL}$. Effects on blood pressure is the most-studied cardiovascular outcome, with studies showing increased systolic and diastolic blood pressure, with some evidence of effects occurring at $\mathrm{PbB} \leq 5 \mu \mathrm{g} / \mathrm{dL}$. A few studies show increased blood pressure in children and pregnant women. Nawrot et al. (2002) estimated that with doubling of PbB (for example, from 5 to $10 \mu \mathrm{g} / \mathrm{dL}$ ), systolic and diastolic blood pressure would increase by 1 and 0.6 millimeters of mercury, respectively. Other cardiovascular effects include increased risk of hypertension and heart disease, atherosclerosis, altered cardiac conduction, cardiac disease, and increased mortality due to cardiovascular disease. A recent study concluded that low-level environmental $\mathrm{Pb}$ exposure is an important risk factor for cardiovascular disease mortality (Lanphear et al. 2018).

Hematological Effects. The toxicity of $\mathrm{Pb}$ to the hematological system of humans has been established in numerous studies in adults and children. Exposure to $\mathrm{Pb}$ causes dose-dependent decreases in heme synthesis through inhibition of the enzyme delta-aminolevulinic acid dehydratase ( $\delta$-ALAD). At $\mathrm{PbB} \leq 10 \mu \mathrm{g} / \mathrm{dL}$, decreased blood hemoglobin is observed; however, it should be noted that the magnitude of this decrease is typically small and may not represent a biologically significant change. As $\mathrm{PbB}$ increases, further decreases in blood hemoglobin and loss of erythrocytes due to a Pb-induced increased membrane fragility results in the development of anemia (NAS 2013). Other effects of Pb on the hematological system include decreased activity of other erythrocyte enzymes (pyrimidine 5'-nucleotidase or red blood cell membrane $\mathrm{Ca}^{2+} / \mathrm{Mg}^{2+} \mathrm{ATPase}$ ) and altered levels of plasma erythropoietin (a hormone that stimulates red blood cell formation); however, fewer studies on these endpoints have been published and study results are mixed.

Immunological Effects. Epidemiological studies provide evidence that $\mathrm{Pb}$ exposure can perturb the immune systems of children and adults. Evidence for this derives from changes in various indicators of humoral and cell-mediated immunity in association with increasing PbB. Effects have been observed in populations that had average $\mathrm{PbB}<10 \mu \mathrm{g} / \mathrm{dL}$. These effects are consistent with more extensive studies conducted in animal models and isolated immune cells that have shown that $\mathrm{Pb}$ can perturb the humoral and cell-mediated immune systems, leading to sensitization, autoimmunity, and inflammation (EPA 2014c; NAS 2013). 
Reproductive Effects in Males. Health effects of $\mathrm{Pb}$ on the male reproductive system have been evaluated in numerous epidemiological studies. Effects include damage to sperm (decreased sperm count, concentration, motility, and viability, and increased immature sperm concentration and percentage of morphologically abnormal sperm), possible alterations in serum levels of reproductive hormones (testosterone, estradiol, luteinizing hormone [LH], and follicle-stimulating hormone [FSH]), decreased fertility, and histopathological changes to the testes. Severity of these effects increases with PbB. Studies conducted in populations with mean $\mathrm{PbB} \leq 10 \mu \mathrm{g} / \mathrm{dL}$ provide evidence of damage to sperm, although effects are more consistently observed at $\mathrm{PbB}>10 \mu \mathrm{g} / \mathrm{dL}$. Regarding effects on serum levels of reproductive hormones, results of available studies for $\mathrm{PbB}$ ranging from $\leq 10$ to $>50 \mu \mathrm{g} / \mathrm{dL}$ are inconsistent; thus, Pb-induced effects on circulating reproductive hormones are not firmly established. At higher $\mathrm{PbB}$ (>10 $\mu \mathrm{g} / \mathrm{dL})$, a few studies provide evidence of more severe effects, including decreased fertility and histopathological damage to testes.

Reproductive Effects in Females. Compared to studies of male reproductive effects, the epidemiologic literature database for effects of $\mathrm{Pb}$ on the female reproductive system is smaller, with most epidemiological studies conducted in populations with mean $\mathrm{PbB} \leq 10 \mu \mathrm{g} / \mathrm{dL}$. Studies provide some evidence of alterations in serum reproductive hormone levels (estradiol, LH, and FSH), decreased fertility, increased spontaneous abortion, increased preterm birth, and earlier age at onset of menopause. However, results are inconsistent, with several studies reporting no association between $\mathrm{PbB}$ and female reproductive effects.

Developmental Effects (Excluding Neurodevelopmental). Numerous epidemiological studies have evaluated developmental outcomes, with most studies conducted in populations with maternal and/or umbilical cord $\mathrm{PbB} \leq 10 \mu \mathrm{g} / \mathrm{dL}$. Some studies provide evidence of decreased birth size (weight, length, head circumference), decreased child growth (weight, height, head circumference, trunk length, leg length, arm length, body mass index [BMI]), and delayed onset of puberty in males and females. Although it is difficult to assess dose-dependence for developmental effects within the relatively narrow range of $\mathrm{PbB}(\leq 10 \mu \mathrm{g} / \mathrm{dL})$ in most studies, dose-related decreases in birth weight have been observed in populations with $\mathrm{PbB} \leq 10 \mu \mathrm{g} / \mathrm{dL}$. Although studies provide evidence of associations between $\mathrm{PbB}$ and developmental outcomes, results are inconsistent and several studies, including prospective studies, show no associations with non-neurodevelopmental outcomes. 
Other Health Effects Associated with Pb. In addition to the effects summarized above, health effects to other organ systems have been reported. The epidemiological databases for these effects are much less extensive than for the effects reviewed above. Effects described below occur over a wide range of $\mathrm{PbBs}$, including $\mathrm{PbB} \leq 10 \mu \mathrm{g} / \mathrm{dL}$. However, results for most endpoints are inconsistent and insufficient data are available to provide information on dose-response relationships.

- Respiratory Effects. Associations have been observed between $\mathrm{PbB}$ and decreased lung function, increased bronchial hyperreactivity, symptoms of respiratory disease, and increased risk of respiratory diseases (e.g., asthma and obstructive lung disease).

- Endocrine Effects (Excluding Reproductive Hormones). Studies in adults, adolescents, and children show effects on thyroid function, cortisol levels, vitamin D levels, and serum levels of growth factors. Effects on thyroid function are the most studied effect, although results do not demonstrate a consistent pattern of effect.

- Hepatic Effects. Most studies were conducted in workers with $\mathrm{PbB}>10 \mu \mathrm{g} / \mathrm{dL}$. Several studies show altered plasma levels of liver enzymes, although no consistent pattern of effects has been observed. Liver enlargement and increased gall bladder wall thickness have been associated with $\mathrm{PbB}$.

- Musculoskeletal Effects. Studies provide evidence of bone loss, increased markers of bone metabolism/turn over, and adverse periodontal and dental effects (periodontal bone loss, tooth loss, periodontal disease, dental caries) in adults and children.

- Gastrointestinal Effects. Gastrointestinal colic is a predominant clinical symptom of acute $\mathrm{Pb}$ poisoning. Epidemiological studies provide evidence of gastrointestinal symptoms (abdominal colic/pain, nausea, vomiting, diarrhea, and/or constipation) associated with PbB ranging from $8 \mu \mathrm{g} / \mathrm{dL}$ to approximately $100 \mu \mathrm{g} / \mathrm{dL}$. However, most studies are survey or cross-sectional studies of small populations of workers.

- Body Weight Effects. A few studies evaluating effects of $\mathrm{PbB} \leq 10 \mu \mathrm{g} / \mathrm{dL}$ on body weight provide some evidence of decreased body weight in children and adults, although inconsistent results have been reported.

- Ocular Effects (Excluding Neurological Effects). Limited data provide some evidence that exposure to $\mathrm{Pb}$ is associated with macular degeneration in adults and increased risk of cataracts.

Cancer. Numerous epidemiological studies have evaluated associations between $\mathrm{Pb}$ exposure and cancer. Although studies provide limited evidence of carcinogenicity of $\mathrm{Pb}$ in humans, results are inconsistent, with several negative studies, and interpretation of data may be limited due to confounding 
factors (e.g., smoking status, family history of cancer, co-exposure to other carcinogens). At $\mathrm{PbB}$ $\leq 10 \mu \mathrm{g} / \mathrm{dL}$, increased risks were reported for all cancers and lung cancer. At $\mathrm{PbB}>10 \mu \mathrm{g} / \mathrm{dL}$, increased risks were observed for all cancer, respiratory tract cancer, stomach cancer, intestinal cancer, cancer of the larynx, and glioma.

The Department of Health and Human Services classified $\mathrm{Pb}$ and $\mathrm{Pb}$ compounds as reasonably anticipated to be human carcinogens (NTP 2016). In 1988, EPA classified Pb as a probable human carcinogen based on sufficient evidence in animals; evidence in humans was considered inadequate (IRIS 2004). The International Agency for Research on Cancer (IARC) has classified inorganic Pb compounds as probably carcinogenic to humans (Group 2A) based on sufficient evidence in animals and limited evidence in humans; evidence for organic $\mathrm{Pb}$ compounds was considered to be inadequate in humans and animals (IARC 2006).

\subsection{MINIMAL RISK LEVELS (MRLS)}

As reviewed in Section 1.2, epidemiological studies have evaluated the health effects of $\mathrm{Pb}$ in all organ systems. For the most studied endpoints (neurological, renal, cardiovascular, hematological, immunological, reproductive, and developmental), effects occur at the lowest PbBs studied ( $\leq 5 \mu \mathrm{g} / \mathrm{dL})$. Because the lowest PbBs are associated with serious adverse effects (e.g., declining cognitive function in children), MRLs for Pb have not been derived. 


\section{CHAPTER 2. HEALTH EFFECTS}

\subsection{INTRODUCTION}

The primary purpose of this chapter is to provide public health officials, physicians, toxicologists, and other interested individuals and groups with an overall perspective on the toxicology of lead $(\mathrm{Pb})$. It contains descriptions and evaluations of epidemiological investigations and provides conclusions, where possible, on the relevance of toxicity and toxicokinetic data to public health.

A glossary and list of acronyms, abbreviations, and symbols can be found at the end of this profile.

To help public health professionals and others identify potential health effects in persons with elevated PbB, the information in this section is organized by health effect.

As discussed in Appendix B, a literature search was conducted to identify relevant studies examining health effect endpoints. Figure 2-1 provides an overview of the database of epidemiology studies included in this chapter of the profile.

Since development of the 2007 Toxicological Profile on Lead (ATSDR 2007), results of numerous epidemiological studies have prompted growing attention to the adverse health effects of $\mathrm{Pb}$ exposures that result in blood $\mathrm{Pb}$ concentrations (PbB) of $<10 \mu \mathrm{g} / \mathrm{dL}$ (EPA 2014c). Awareness of the potential adverse consequences of such exposures has led to changes in U.S. public health policy, with a focus on eliminating lead poisoning as a public health problem (CDC 2012d; EPA 2016b). In 2012, CDC accepted their Advisory Committee on Childhood Lead Poisoning Prevention (ACCLPP) recommendation to establish a $\mathrm{PbB}$ reference value for $\mathrm{Pb}$, replacing the $10 \mu \mathrm{g} / \mathrm{dL}$ level of concern. The reference value is based on the $97.5^{\text {th }}$ percentile of the $\mathrm{PbB}$ distribution among children 1-5 years of age in the United States, using data generated by NHANES (CDC 2012d). At that time, the PbB reference was approximately 5 fg/dL (NHANES 2007-2010) (CDC 2018a). ACCLPP recommended that the reference value be updated every 4 years using the two most recent NHANES cycles and would be used in recommendations for follow-up evaluations and identification of high-risk childhood populations (CDC 2012d). It is likely that PbB values among children will continue to decline; therefore, the primary focus of this toxicological profile is on health effects associated with low $\mathrm{Pb}$ exposure (i.e., those observed at $\mathrm{PbB} \leq 5 \mu \mathrm{g} / \mathrm{dL}$ ). Detailed information on effects at $\mathrm{PbB} \leq 10 \mu \mathrm{g} / \mathrm{dL}$ is also presented to examine potential 
exposure-response relationships. Information on health effects observed at higher $\mathrm{PbB}$ levels ( $>10 \mu \mathrm{g} / \mathrm{dL})$ is also included to provide a comprehensive overview of the adverse effects of $\mathrm{Pb}$.

Literature Search Strategy. The literature on health effects of $\mathrm{Pb}$ in humans is enormous, with countless epidemiological studies in workers and the general population, including children. Due to the extent of the $\mathrm{Pb}$ database in humans, it is impossible to cite all, or even most, of the studies on health effects of $\mathrm{Pb}$; thus, this profile does not attempt to provide a comprehensive review of all literature; instead, the profile summarizes the major lines of epidemiological evidence regarding health effects in humans. Although the literature database on adverse effects of $\mathrm{Pb}$ in laboratory animals is also extensive, given the large number of studies available in humans, animal studies are not included in this toxicological profile. For a recent review of studies in animal models, the reader should consult the EPA's Integrated Science Assessment for Lead (EPA 2014c).

The following were used as primary sources to identify literature on health effects of $\mathrm{Pb}$ :

- The previous Toxicological Profile for Lead (ATSDR 2007) was used to identify literature published through 2007.

- The EPA (2014c) Integrated Science Assessment for Lead was used to identify literature published from 2006 to 2013.

- Literature searches were conducted from 2013 to 2019 to identify studies published after EPA (2014c).

In addition, recent reviews by NTP (2012) and NAS (2013) were consulted. As anticipated, the literature search revealed an extensive epidemiological database of literature published since 2013. To narrow the evaluation to those studies of greatest utility identifying health effects of low exposures to $\mathrm{Pb}$, a series of inclusion criteria were defined; only studies meeting the criteria were considered for inclusion in the toxicological profile. These criteria are described further in Appendix B. Data from selected studies were tabulated and discussed in subsequent sections of this chapter.

Duration of Exposure. Typically, toxicological profiles organize the discussion of health effects according to exposure duration categories. However, this is not a particularly informative approach to the discussion of $\mathrm{Pb}$ epidemiology. The epidemiologic study of $\mathrm{Pb}$ toxicity in human populations has relied on internal dose metrics (e.g., $\mathrm{PbB}$, bone $\mathrm{Pb}$ ) for evaluating associations between health outcomes. These metrics are considered to represent relatively recent exposure history, in the case of $\mathrm{PbB}$, and longer-term 
cumulative exposure, in the case of CBLI or bone $\mathrm{Pb}$. However, neither metric offers a confident estimate of exposure duration or of changes in $\mathrm{Pb}$ exposure over time (including peak exposure periods that may have occurred in the past), and, in general, the complete exposure history is not known. Health outcomes associated with acute exposures is available from clinical case studies of $\mathrm{Pb}$ poisoning (see Section 2.2). However, even in these cases, the exposure duration that proceeded the identification of the case is rarely known with certainty.

Routes of Exposure. For the general population, exposure to $\mathrm{Pb}$ occurs primarily via the oral route, with some contribution from the inhalation route, whereas inhalation exposures can be more important in occupational settings, depending on particle size. In addition, occupational exposure to organic $\mathrm{Pb}$ compounds may involve dermal absorption as a significant exposure route. This profile does not attempt to separate health effects by route of exposure. As noted previously, epidemiology studies have relied on internal dose metrics (e.g., $\mathrm{PbB}$, bone $\mathrm{Pb}$ ), which reflect $\mathrm{Pb}$ body burden (to varying degrees), irrespective of the route of exposure. The primary systemic toxic effects of $\mathrm{Pb}$ are the same regardless of the route of entry into the body,

Exposure Metric. To quantify exposure in humans, data are expressed in terms of absorbed $\mathrm{Pb}$, and not in terms of external exposure levels (e.g., concentration in water) or dose (e.g., $\mathrm{mg} / \mathrm{kg} / \mathrm{day}$ ). The most common metric of absorbed dose for $\mathrm{Pb}$ is the concentration of lead in blood ( $\mathrm{PbB})$, although other measures of exposure (e.g., concentration of $\mathrm{Pb}$ in bone, hair, teeth, or urine) are used; however, measurements of $\mathrm{Pb}$ in urine, teeth, and hair are not as reliable as measurements in blood or bone. $\mathrm{PbB}$ mainly reflects exposure history of the previous few months and does not necessarily reflect the larger burden and much slower elimination kinetics of $\mathrm{Pb}$ in bone (see Section 3.1). $\mathrm{Pb}$ in bone is considered a biomarker of cumulative or long-term exposure because $\mathrm{Pb}$ accumulates in bone over the lifetime and most of the $\mathrm{Pb}$ body burden resides in bone. Most of the body burden of $\mathrm{Pb}$ (the total amount of $\mathrm{Pb}$ in the body) is distributed to the bone, with approximately 94 and 76\% of the body burden found in bone in adults and children, respectively. The remainder is distributed to blood and soft tissues. However, the concentration of $\mathrm{Pb}$ in blood can vary considerably with age and physiology/lifestage (e.g., pregnancy, lactation, menopause). For this reason, measurement of $\mathrm{Pb}$ in bone has seen wider application in epidemiological studies of adults in which measures of cumulative lifetime exposures are of interest. However, bone $\mathrm{Pb}$ measurements require specialized radiologic equipment (e.g., K-shell x-ray fluorescence; XRF) and, as a result, are used less commonly than PbB in human epidemiology. Since most of the epidemiology has relied on $\mathrm{PbB}$ as the dose metric, this profile has focused on describing dose-response relationships based on $\mathrm{PbB}$ to facilitate comparisons across studies and endpoints. This 
approach also aligns with public health practices, which rely on $\mathrm{PbB}$ for evaluating elevated exposures to $\mathrm{Pb}$ (CDC 2012d; EPA 2016b). However, it is recognized that some health outcomes may be correlated with cumulative exposure, in which case, bone $\mathrm{Pb}$ may be a better dose metric than $\mathrm{PbB}$. For these outcomes, short-term variation in $\mathrm{PbB}$ may contribute to exposure classification error (i.e., the same $\mathrm{PbB}$ could be observed in individuals who have different bone $\mathrm{Pb}$ ). The exposure history of the subjects may also be an important factor in determining associations observed between outcomes and blood or bone $\mathrm{Pb}$. Some studies of historically exposed occupational populations (e.g., former workers) have found stronger associations between bone $\mathrm{Pb}$ and health outcomes than with $\mathrm{PbB}$, while some studies of concurrently exposed populations have found stronger associations with PbB (Shih et al. 2007).

Confounding Factors and Effect Modifiers. Bias can occur in epidemiological studies when the background risk of the outcome being measured is not the same in the exposed and reference groups. Confounders are variables that affect the measured outcome and are also associated with the $\mathrm{Pb}$ exposure metric (e.g., $\mathrm{PbB}$, bone $\mathrm{Pb}$ ). For example, $\mathrm{Pb}$ body burden increases with age; therefore, age can be a confounding factor if it is also a risk factor for the outcome (e.g., renal or cardiovascular disease). Not adjusting for confounders may attenuate or strengthen the apparent associations between $\mathrm{Pb}$ exposure and the outcome, depending on whether it is a negative or positive confounding variable. Effect modifiers are variables that affect the measured outcome independently of the $\mathrm{Pb}$ exposure metric. For example, renal disease from any cause can affect blood pressure and, thereby, could interact with $\mathrm{Pb}$ to change blood pressure. Effect modifiers can also be confounders, if they are associated with the Pb exposure metric (e.g., socio-economic status [SES] and cognitive development). Recall bias may also contribute to uncertainties and should be considered as a confounding factor. For example, interviews of parents are a standard method for estimating potential co-variables that might affect child development in prospective studies, as there are no alternatives for studies in children. Thus, inaccurate recall may potentially influence study outcomes. Failure to account for important effect modifiers can result in underestimation or overestimation of the apparent strength of the association, depending on the direction of the effect of the modifying variable. Confounding factors and effect modifiers are discussed in greater detail in sections that describe specific categories of health effects. Epidemiological studies provide information about the strengths of statistical associations between exposure metrics (e.g., blood $\mathrm{Pb}$ ) and health outcomes. However, statistical associations do not necessarily reflect causal associations. Evidence for causal associations can include demonstration of exposure-response relationships, occurrence of the outcome or its precursors in controlled studies conducted in experimental models (in vivo and in vitro), and consistency of observed statistical associations with known modes of action of $\mathrm{Pb}$. 
Overview of Health Effects of Pb. The health effects of $\mathrm{Pb}$ are diverse, and exposure to $\mathrm{Pb}$ is associated with toxicity to every organ system. This is not surprising because the mechanisms of action associated with $\mathrm{Pb}$-induced toxicity, including perturbations of ion homeostasis and transport, protein binding, oxidative stress, and inflammation, are common to all cell types. In addition, $\mathrm{Pb}$ is widely distributed throughout the body, and has been measured in all tissues evaluated (see Section 3.1.2). For all organ systems, toxicity has been observed at $\mathrm{PbB} \leq 10 \mu \mathrm{g} / \mathrm{dL}$. Neurological effects of $\mathrm{Pb}$ are of greatest concern because effects are observed in infants and children; furthermore, these effects may result in life-long decrements in neurological function. Children are also more vulnerable because of behaviors that increase ingestion of $\mathrm{Pb}$ surface dusts (e.g., hand-to-mouth activity) and because gastrointestinal absorption of ingested $\mathrm{Pb}$ is higher in children compared to adults, possibly due to a combination of physiological differences and differences in diet and nutrition. The weight-of-evidence for all adverse health effects is strongly supported by studies in animal models and in vitro systems; see EPA (2014c) for a review of this literature.

Effects observed in association with $\mathrm{PbB}$ are briefly described below. Note that for some of the effects listed below, study results are not consistent, which limits interpretation of observations; this is reviewed in more detail in subsequent sections for each organ system in Chapter 2. The most extensive epidemiological databases examining $\mathrm{Pb}$ are for neurological, renal, cardiovascular, hematological, immunological, reproductive, and developmental effects.

\section{- Neurological Effects:}

o Children. Decreased cognitive function; altered mood and behaviors that may contribute to learning deficits, altered neuromotor and neurosensory function, peripheral neuropathy, and encephalopathy.

o Adults. Decreased cognitive function including attention, memory, and learning; altered neuromotor and neurosensory function; altered mood and behavior; and decreased peripheral nerve conduction velocity.

- Renal Effects. Decreased GFR, proteinuria, enzymuria, impaired tubular transport, and histopathological damage.

- Cardiovascular Effects. Increased systolic and diastolic blood pressure, increased risk of hypertension, atherosclerosis, altered cardiac conduction, increased risk of heart disease, and increased mortality due to cardiovascular disease. 
- Hematological Effects. Inhibition of $\delta$-ALAD leading to decreased blood hemoglobin and anemia, decreased activity of other erythrocyte enzymes, and altered plasma erythropoietin (EPO) levels.

- Immunological Effects. Perturbation of humoral and cell-mediated immune systems, decreased resistance to disease, sensitization, autoimmunity, and inflammation.

- Reproductive Effects:

o Males. Effects on sperm, alterations in semen quality, decreased fertility, histopathological damage to the testes, and possible altered serum concentrations of reproductive hormones.

o Females. Possible alterations in serum concentrations of reproductive hormones, decreased fertility, spontaneous abortion, preterm birth, and earlier age at the onset of menopause.

- Developmental Effects. Decreased birth weight and size, decreased anthropometric measures in children, and delayed onset of puberty in males and females.

Other health outcomes associated with PbB include the following:

- Respiratory Effects. Decreased lung function, increased bronchial hyperreactivity, increased risk of asthma, and obstructive lung disease.

- Hepatic Effects. Possible increases in plasma liver enzymes and cholesterol, enlarged liver, and increased thickness of gall bladder wall.

- Endocrine Effects. Possible alterations in serum of thyroid hormones, altered cortisol responses, alteration in serum growth factors, and decreased serum vitamin D levels.

- Gastrointestinal Effects. Abdominal pain/colic, nausea, vomiting, and diarrhea and/or constipation.

- Musculoskeletal Effects. Bone loss, osteoporosis, dental caries, tooth loss, and periodontitis. 
- Ocular Effects. Possible macular degeneration and cataracts.

- Cancer. Increased risk of cancer, including all cancers, cancer of the respiratory tract, intestinal tract, and larynx, and glioma.

\section{Many specific health effect endpoints have been evaluated in numerous studies. To provide the reader with a weight-of-evidence for these endpoints, the profile indicates if results are consistent and corroborated in numerous studies or if results are inconsistent (or mixed).}

Figure 2-1 shows the numbers of epidemiological studies included in this chapter of the toxicological profile, based on health outcome studied. The number of studies evaluating effects at $\mathrm{PbB} \leq 10 \mu \mathrm{g} / \mathrm{dL}$ also is indicated. The $\mathrm{PbB} \leq 10 \mu \mathrm{g} / \mathrm{dL}$ was selected to evaluate effects at the lowest $\mathrm{PbB}$ (e.g., $\leq 5 \mu \mathrm{g} / \mathrm{dL}$ ) and to evaluate potential exposure-response relationships for $\mathrm{PbB} \leq 10 \mu \mathrm{g} / \mathrm{dL}$. As noted above, due to the enormous number of epidemiological studies published, the profile does not attempt to provide a comprehensive review of all literature. Therefore, this figure should not be interpreted as depicting all epidemiological studies that have been published on $\mathrm{Pb}$ toxicity. 
Figure 2-1. Overview of the Number of Studies Examining Associations Between PbB and Health Effects ${ }^{\mathrm{a}}$

Most studies examined the potential cardiovascular, renal, and neurological effects of lead

A subset of studies evaluating health effects for $\mathrm{PbB} \leq 10 \mu \mathrm{g} / \mathrm{dL}$ compared to all $\mathrm{PbB}$ studies (counts represent studies examining endpoint)

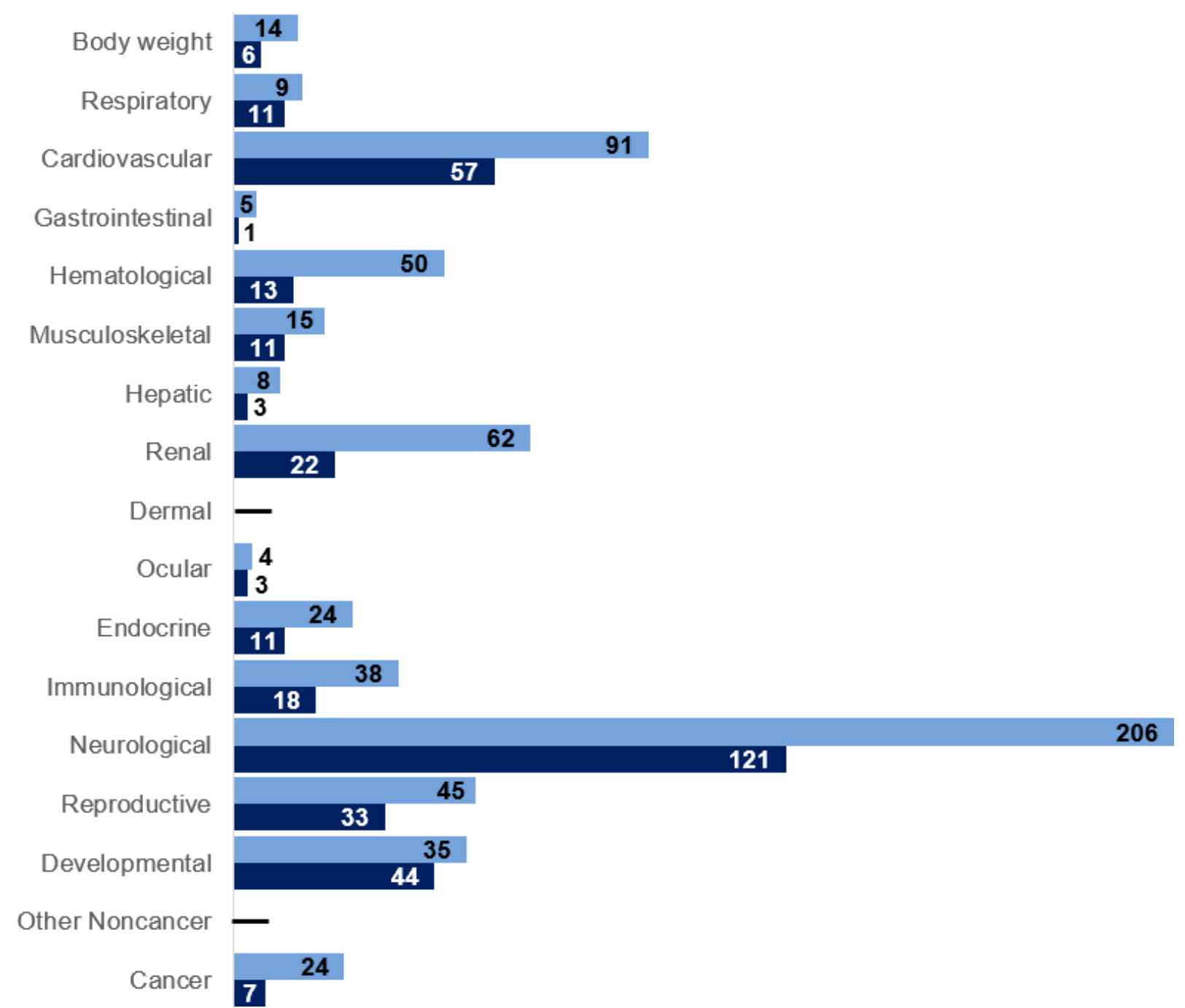

ancludes studies discussed in Chapter 2. A total of 694 epidemiological studies (including those finding no effect) have examined toxicity; some studies examined multiple endpoints. 


\subsection{ACUTE LEAD TOXICITY}

Overview. No controlled studies in humans have evaluated the acute toxicity of $\mathrm{Pb}$ (acute $\mathrm{Pb}$ poisoning). Available information is anecdotal, obtained from numerous case reports. Thus, data are not sufficient to establish a dose-response relationship for acute toxicity relative to $\mathrm{PbB}$. Acute $\mathrm{Pb}$ toxicity is characterized by symptoms of abdominal pain/colic, vomiting, constipation, peripheral neuropathy, and cerebral edema and encephalopathy, which can lead to seizures, coma, and death. Children are more susceptible than adults to acute $\mathrm{Pb}$ poisoning. Additional information on toxicity of ingested $\mathrm{Pb}$ debris (e.g., Pb shot) is provided in Appendix C.

Rather than reviewing numerous case reports, the information presented below was taken from the following reviews: Beers et al. (1999); Chisolm (1977); Klaassen (2001); Landrigan (1995); NAS (1972); Needleman (2004); and Skerfving and Bergdahl (2015). Citations are only specifically noted below if quantitative information is discussed.

Confounding Factors, Effect Modifiers, and Uncertainties. There are several uncertainties from case reports on acute toxicity of $\mathrm{Pb}$. Therefore, it is difficult to establish dose-response relationships for acute toxicity relative to $\mathrm{PbB}$. Uncertainties include:

- $\quad$ Baseline PbB data are rarely available.

- There is a lack of quantitative data on the dose of $\mathrm{Pb}$ ingested.

- No information on the fractional absorption of ingested Pb.

- Time from ingestion of $\mathrm{Pb}$ to development of symptoms of acute $\mathrm{Pb}$ toxicity is often unknown.

- Time from ingestion of $\mathrm{Pb}$ to first clinical evaluation and $\mathrm{PbB}$ assessment is often unknown.

- Gastrointestinal symptoms and general malaise are typically the first symptoms of acute $\mathrm{Pb}$ toxicity to appear; these general symptoms are often attributed to other causes, leading to an initial misdiagnosis or delay in diagnosis.

- Data to develop PbB time-concentration curves are incomplete.

- Numerous factors may contribute to individual susceptibility to acute Pb exposure, including age, intercurrent illness, underlying developmental issues, dietary and nutritional status, concurrent medication use, and exposure to other chemicals.

Clinical Presentation of Acute Pb Toxicity. The onset of acute toxicity is rapid, usually occurring within 1-5 days of exposure. The main organ systems involved are the gastrointestinal, hematological, and 
neurological systems. Signs and symptoms increase in severity with increasing $\mathrm{PbB}$, ranging from mild to severe. Gastrointestinal effects include abdominal colic/pain, nausea, vomiting, diarrhea, and constipation. Massive loss of gastrointestinal fluids can lead to dehydration. Hematological effects include decreased hemoglobin synthesis, anemia, and acute hemolytic crisis characterized by anemia and hemoglobinuria. Numerous neurological symptoms are associated with acute $\mathrm{Pb}$ toxicity, including headache, hyperirritability, decreased activity, paresthesia, muscle pain and weakness, ataxic gait, decreased consciousness, cerebral edema leading to seizures and coma, encephalopathy, and death. Other reported symptoms include astringency of the mouth, metallic taste in the mouth, and thirst.

Susceptibility of Children. Children are more susceptible than adults to $\mathrm{Pb}$ poisoning because the fractional absorption of ingested $\mathrm{Pb}$ is higher than in adults and the developing central nervous system is more vulnerable to toxicity compared to a fully developed nervous system (Needleman 2004). In addition to being more sensitive than adults, acute toxicity in children may have long-lasting effects. For example, children who recover from acute encephalopathy can have long-term decreases in cognitive abilities, attention deficits, and impaired behavior. Children are also susceptible due to increased exposure.

Dose-Response Relationship for Acute Toxicity Relative to PbB. As noted above, data from case reports are not sufficient to establish a dose-response relationship for acute toxicity relative to $\mathrm{PbB}$. Some general observations can be made from available reports; however, dose-response relationships are highly uncertain and may not apply to individuals acutely exposed to $\mathrm{Pb}$. At $\mathrm{PbB}<30 \mu \mathrm{g} / \mathrm{dL}$, signs and symptoms of acute toxicity typically are not observed. This should not be interpreted to mean that no Pb-induced adverse effects (e.g., decreased hemoglobin synthesis) occur at $\mathrm{PbB}<30 \mu \mathrm{g} / \mathrm{dL}$, but that symptoms causing individuals to seek medical intervention (e.g., abdominal colic and vomiting) typically are not observed at $\mathrm{PbB}<30 \mu \mathrm{g} / \mathrm{dL}$. As PbBs increase to $>30 \mu \mathrm{g} / \mathrm{dL}$, signs and symptoms of gastrointestinal and neurological toxicity are observed, with severity increasing with $\mathrm{PbB}$. Pb-induced encephalopathy has been reported at $\mathrm{PbB}<100 \mu \mathrm{g} / \mathrm{dL}$, but is more commonly associated with $\mathrm{PbB}$ $>100 \mu \mathrm{g} / \mathrm{dL}$ (NAS 1972). In a review of 96 cases of death due to acute $\mathrm{Pb}$ poisoning in children, death occurred at $\mathrm{PbB}>100 \mu \mathrm{g} / \mathrm{dL}$ (NAS 1972).

\subsection{DEATH}

Overview. Numerous epidemiological studies have investigated associations between $\mathrm{Pb}$ exposure and death. Studies include exposure of workers and general populations, and report a wide range of $\mathrm{PbB}$ levels. In the general population, studies have shown significant associations between $\mathrm{PbB}$ and mortality 
due to disease of blood and blood-forming organs. In occupationally exposed individuals, mortality due to infection, endocrine diseases, and digestive diseases were associated with $\mathrm{PbB}$ in male workers, but not female workers, while mortality due to respiratory disease was associated with $\mathrm{PbB}$ in a cohort of male workers. In addition, studies of the general population and $\mathrm{Pb}$ occupations show an association between $\mathrm{PbB}$ and cumulative "all-cause” mortality (including cancer). However, results are inconsistent and interpretation may be limited due to confounding factors. Studies assessing associations between PbB and mortality due to cardiovascular diseases and cancer are discussed in Sections 2.5 and 2.19, respectively, and are not reviewed here.

The following causes of death have been associated with PbB:

- $\leq 10 \mu \mathrm{g} / \mathrm{dL}$ :

o Increased risk of death from all causes (including cancer and cardiovascular disease); evaluated in a few studies with generally consistent results.

- $>10 \mu \mathrm{g} / \mathrm{dL}$ :

o Increased risk of death from all causes (including cancer and cardiovascular disease); evaluated in several studies with positive associations in some studies.

o Increased risk of death from chronic or unspecified nephritis or non-malignant kidney disease; evaluated in several studies with positive associations in some studies.

o Increase risk of death from infection; demonstrated in one study.

o Increased risk of death from endocrine disease; demonstrated in one study.

o Increased risk of death from digestive disease; evaluated in several studies with positive associations in some studies.

o Increased risk of death from diseases of the blood and blood forming organs; demonstrated in one study.

o Increased risk of death from respiratory diseases (emphysema, pneumonia, and other respiratory diseases); evaluated in several studies with positive associations in some studies.

Confounding Factors and Effect Modifiers. Numerous factors can influence results of epidemiological studies evaluating associations between $\mathrm{Pb}$ exposure and mortality, including age, sex, BMI, ethnicity, poverty level, education, alcohol consumption, smoking status, hypertension, diabetes, family history of diseases, activity level, total cholesterol, postmenopausal status, nutritional status, and co-exposure with other metals (i.e., arsenic or cadmium). Failure to account for these factors may attenuate or strengthen 
the apparent associations between $\mathrm{Pb}$ exposure and the outcome, depending on the direction of the effect of the variable on the outcome.

Measures of Exposure. Studies examining the association between $\mathrm{Pb}$ exposure and mortality evaluate exposure by measurement of $\mathrm{PbB}$.

Characterization of Effects. Numerous epidemiological studies have assessed associations between PbB and mortality. Studies of general populations and workers are briefly summarized in Table 2-1. In the general population, at $\mathrm{PbB} \leq 10 \mu \mathrm{g} / \mathrm{dL}$, a positive dose-response relationship was suggested for all-cause mortality and mortality due to coronary heart disease (Khalil 2009, 2010; Menke et al. 2006; Schober et al. 2006), although Weisskopf et al. (2009) did not show an increased risk for all-cause mortality. At $>10 \mu \mathrm{g} / \mathrm{dL}$, results of occupational exposure and general population studies are mixed and do not establish a pattern of effects or exposure-response relationships. In the general population, findings of the Lustberg and Silbergeld (2002) study suggested dose-response for PbB and all-cause mortality. In Pb workers, a dose-effect relationship was observed for all-cause mortality and mortality due to endocrine disease, infection, and digestive disease (Chowdhury et al. 2014; Kim et al. 2015), although Malcolm and Barnett (1982) did not observe a dose-effect relationship between $\mathrm{Pb}$ and all-cause mortality in $\mathrm{Pb}$ battery workers. 
Table 2-1. Summary of Epidemiological Studies Evaluating Death ${ }^{\mathrm{a}}$

\begin{tabular}{|c|c|c|c|}
\hline Reference and study population & $\mathrm{PbB}(\mu \mathrm{g} / \mathrm{dL})$ & Mortality outcome & Effects $^{b}$ \\
\hline \multicolumn{4}{|l|}{$\mathrm{PbB} \leq 10 \mu \mathrm{g} / \mathrm{dL}$} \\
\hline \multicolumn{4}{|l|}{$\begin{array}{l}\text { Cross-sectional study; } n=3,482 \\
\text { (NHANES III) }\end{array}$} \\
\hline $\begin{array}{l}\text { Khalil 2010; Khalil et al. } 2009 \\
\text { Prospective cohort study; } n=533 \text { women } \\
\text { (age 65-87 years) }\end{array}$ & 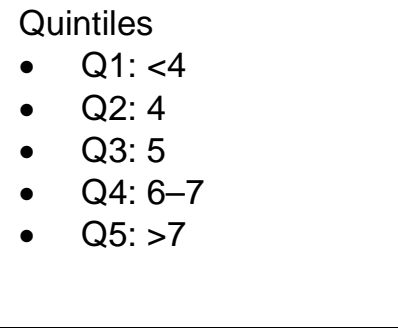 & All-cause mortalityc & $\begin{array}{l}\text { HR Q1 (reference) } \\
\text { HR Q2: } 0.80(0.45,1.42) \\
\text { HR Q3: } 0.70(0.39,1.24) \\
\text { HR Q4: } 0.60(0.34,1.06) \\
\text { HR Q5: } 1.20(0.69,2.09) \\
\text { p-trend=0.905 } \\
\text { Spline for } 5^{\text {th }} \text { knot: } \mathbf{p}=\mathbf{0 . 0 0 9 *} \\
\text { Wald test: } p=0.0843\end{array}$ \\
\hline Khalil et al. 2009 & \multirow{2}{*}{$\begin{array}{l}\text { Mean: } 5.3 \\
<8(n=453) \\
\geq 8(n=79)\end{array}$} & All-cause mortalityc & $\begin{array}{l}\text { Adjusted HR } \geq 8 \mu \mathrm{g} / \mathrm{dL}: 1.59(1.02, \\
2.49) ; p=0.041^{*}\end{array}$ \\
\hline $\begin{array}{l}\text { Prospective cohort study; } n=533 \text { women } \\
\text { (age 65-87 years) }\end{array}$ & & $\begin{array}{l}\text { All-cause mortality } \\
\text { excluding deaths due to } \\
\text { cancer and cardiovascular } \\
\text { disease }\end{array}$ & $\begin{array}{l}\text { Adjusted HR } \geq 8 \mu \mathrm{g} / \mathrm{dL}: 1.22(0.48,3.10) ; \\
\mathrm{p}=0.673\end{array}$ \\
\hline & $\begin{array}{l}\text { Mean: } 2.58 \\
\text { Tertiles: }\end{array}$ & All-cause mortalityc & $\begin{array}{l}\text { Adjusted HR } \\
\text { T1 (reference) }\end{array}$ \\
\hline $\begin{array}{l}\text { Longitudinal study; } n=13,946 \text { (NHANES } \\
\text { 1988-1994; mean age } 44.4 \text { years) }\end{array}$ & $\begin{array}{ll}\text { - } & \text { T1: }<1.93 \\
\text { - } & \text { T2: } 1.94-3.62 \\
\text { - } & \text { T3: } \geq 3.63\end{array}$ & & $\begin{array}{l}\text { T2: } 0.91(0.72,1.15) \\
\text { T3: } 1.25(1.04, \mathbf{1 . 5 1})^{*} \\
\text { p-trend=0.002* }\end{array}$ \\
\hline Neuberger et al. 2009 & 5.8 & Tuberculosis & SMR: $0.0(0.0,10.80)$ \\
\hline \multirow{2}{*}{$\begin{array}{l}\text { Retrospective cohort study; mortality data } \\
\text { from Oklahoma State Department of Health; } \\
\text { 1999-2001 }\end{array}$} & & $\begin{array}{l}\text { Bronchitis, emphysema, } \\
\text { asthma }\end{array}$ & SMR: $1.10(0.863,13.84)$ \\
\hline & & Kidney disease & SMR: $0.984(0.573,1.576)$ \\
\hline $\begin{array}{l}\text { Schober et al. } 2006 \\
\text { Longitudinal study; } n=9,757 \text { (NHANES III; } \\
\text { age } \geq 40 \text { years) }\end{array}$ & 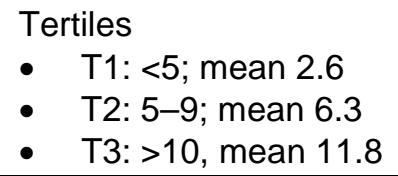 & All-cause mortalityc & $\begin{array}{l}\text { RR T2: } 1.24(1.05,1.48)^{*} \\
\text { RR T3: } 1.59(1.28,1.98)^{*} \\
\text { p-trend<0.001 }\end{array}$ \\
\hline
\end{tabular}


Table 2-1. Summary of Epidemiological Studies Evaluating Death ${ }^{\mathrm{a}}$

\begin{tabular}{|c|c|c|c|}
\hline Reference and study population & $\mathrm{PbB}(\mu \mathrm{g} / \mathrm{dL})$ & Mortality outcome & Effects $^{b}$ \\
\hline $\begin{array}{l}\text { Weisskopf et al. } 2009 \\
\text { Longitudinal study; } n=868 \text { men (Normative } \\
\text { Aging Study; age } 21-80 \text { years) }\end{array}$ & $\begin{array}{l}\text { Mean (SD): } 5.6(3.4) \\
\text { Tertiles: } \\
\text { - } \quad \text { T1: }<4 \\
\text { - } \quad \text { T2: } 4-6 \\
\text { - } \quad \text { T3: }>6\end{array}$ & All-cause mortalityc & $\begin{array}{l}\text { Adjusted HR } \\
\text { - } \quad \text { T1: } 1 \text { (reference) } \\
\text { - } \quad \text { T2: } 0.99(0.71,1.37) \\
\text { - } \quad \text { T3: } 1.01(0.71,1.44) \\
\text { - } \quad \text { p-trend=0.92 } \\
\end{array}$ \\
\hline \multicolumn{4}{|l|}{$\mathrm{PbB}>10 \mu \mathrm{g} / \mathrm{dL}$} \\
\hline \multirow{5}{*}{$\begin{array}{l}\text { Barry and Steenland } 2019 \\
\text { Retrospective study; } n=58,368 \text { male } \\
\text { workers (10-year follow-up of Chowdhury et } \\
\text { al. 2014) }\end{array}$} & \multirow{5}{*}{$\begin{array}{l}\text { Q1: } 0-<5 \\
\text { Q2: } 5-<25 \\
\text { Q3: } 25-<40 \\
\text { Q4: } \geq 40 \\
\text { T1: } 0-<25 \\
\text { T2: } 25-<40 \\
\text { T3: } \geq 40\end{array}$} & All-cause mortalityc & HR Q4: 1.38 (1.24, 1.53)* \\
\hline & & $\begin{array}{l}\text { Chronic obstructive } \\
\text { pulmonary disease }\end{array}$ & HR Q4: $1.46(0.94,2.28)$ \\
\hline & & Chronic renal disease & HR T3: $1.81(0.91,3.57)$ \\
\hline & & $\begin{array}{l}\text { Cerebrovascular disease } \\
\text { (stroke) }\end{array}$ & SMR Q4: $0.73(0.58,0.91)$ \\
\hline & & Ischemic heart disease & SMR Q4: $0.70(0.63,0.77)$ \\
\hline \multirow{3}{*}{$\begin{array}{l}\text { Chowdhury et al. } 2014 \\
\text { Survey study; n=58,368 male workers } \\
\text { (mean age } 38.9 \text { years) }\end{array}$} & \multirow{3}{*}{$\begin{array}{l}\text { Quartiles } \\
\begin{array}{l}\text { - } \\
\text { - }\end{array} \text { Q2: } 0-<5-<25 \\
\text { - } \quad \text { Q3: } 25-<40 \\
\text { - } \quad \text { Q4: } \geq 40\end{array}$} & All-cause mortalityc & $\begin{array}{l}\text { SMR Q4: } \mathbf{0 . 8 0}(\mathbf{0 . 7 5}, \mathbf{0 . 8 4})^{*} \\
\text { SMR overall: } 0.69(0.66,0.71)\end{array}$ \\
\hline & & $\begin{array}{l}\text { Chronic obstructive } \\
\text { pulmonary disease }\end{array}$ & $\begin{array}{l}\text { SMR Q4: } 0.86(0.64,1.12) \\
\text { SMR overall: } 0.65(0.54,0.78)\end{array}$ \\
\hline & & Chronic renal disease & $\begin{array}{l}\text { SMR Q4: } 1.01(0.58,1.64) \\
\text { SMR overall: } 0.65(0.44,0.93)\end{array}$ \\
\hline \multirow{4}{*}{$\begin{array}{l}\text { Cooper 1988; Cooper et al. } 1985 \\
\text { Cohort study; } n=4,519 \text { battery workers; } \\
\text { 2,300 smelters }\end{array}$} & \multirow{4}{*}{$\begin{array}{l}\text { Mean } \\
\text { - } \quad \text { Battery }(n=1326): 62.7 \\
\text { - } \quad \text { Smelters }(n=537): 79.7\end{array}$} & $\begin{array}{l}\text { Nonmalignant respiratory } \\
\text { disease }\end{array}$ & $\begin{array}{l}\text { Battery PMR: } 0.90(0.74,1.10) \\
\text { Smelter PMR: } 0.76(0.53,1.11)\end{array}$ \\
\hline & & Cirrhosis of the liver & $\begin{array}{l}\text { Battery PMR:1.29 }(0.96,1.73) \\
\text { Smelter PMR: } 0.63(0.35,1.15)\end{array}$ \\
\hline & & $\begin{array}{l}\text { Chronic or unspecified } \\
\text { nephritis }\end{array}$ & $\begin{array}{l}\text { Battery PMR: } 2.06(\mathbf{1 . 2 6}, \mathbf{3 . 1 8}) * ; \\
\text { p<0.01 } \\
\text { Smelter PMR: } 1.86(0.80,3.66)\end{array}$ \\
\hline & & Chronic nephritis & $\begin{array}{l}\text { Battery PMR: } 1.48(0.88,2.49) \\
\text { Smelter PMR: } 1.20(0.50,2.86)\end{array}$ \\
\hline
\end{tabular}


Table 2-1. Summary of Epidemiological Studies Evaluating Death ${ }^{\mathrm{a}}$

\begin{tabular}{|c|c|}
\hline Reference and study population & $\mathrm{PbB}(\mu \mathrm{g} / \mathrm{dL})$ \\
\hline Kim et al. 2015 & $\begin{array}{l}\text { Mean } \\
\text { - } \quad \text { Males: } 8.8\end{array}$ \\
\hline $\begin{array}{l}\text { Cross-sectional study; } n=81,067 \text { inorganic } \\
\mathrm{Pb} \text { workers ( } 54,788 \text { males; } 26,279 \text { females; } \\
\text { age } 20-\leq 50 \text { years) }\end{array}$ & 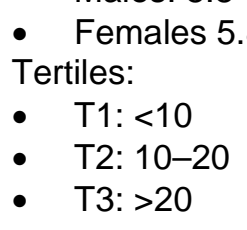 \\
\hline
\end{tabular}

\begin{tabular}{|c|c|}
\hline Mortality outcome & Effects $^{b}$ \\
\hline All-cause mortalityc & $\begin{array}{l}\text { Males: RR T3: } 1.36(1.03,1.79)^{*} ; \\
\text { p<0.05 } \\
\text { Females: RR T3: } 1.30(0.41,4.16)\end{array}$ \\
\hline Non-malignant death & $\begin{array}{l}\text { Males: RR T3: } 0.95(0.56,1.51) \\
\text { Females RR T3: } 0.99(0.13,7.19)\end{array}$ \\
\hline Infection & $\begin{array}{l}\text { Males: RR T2: } 3.73(1.06,13.06) * ; \\
\text { p<0.05 } \\
\text { Females: not reported }\end{array}$ \\
\hline Endocrine disease & $\begin{array}{l}\text { Males: RR T3: } 4.25(0.90,20.04) * ; \\
\text { p<0.1 } \\
\text { Females: not reported }\end{array}$ \\
\hline Respiratory disease & $\begin{array}{l}\text { Males: RR T2: } 1.46(0.28,7.49) \\
\text { Females: RR T2: } 3.49(0.31,39.05)\end{array}$ \\
\hline Digestive disease & $\begin{array}{l}\text { Males: RR T3: } \mathbf{3 . 2 3}(\mathbf{1 . 3 3}, \mathbf{7 . 8 6})^{*} \\
\text { p<0.05 } \\
\text { Females: RR T2: } 3.66(0.33,40.70)\end{array}$ \\
\hline All-cause mortalityc & Total cohort SMR: $0.9(0.8,1.0)$ \\
\hline Respiratory disease & Total cohort SMR: $0.4(0.2,0.8)$ \\
\hline Digestive organs & Total cohort SMR: $0.6(0.3,1.1)$ \\
\hline All-cause mortalityc & $\begin{array}{l}\text { RR T2: } 1.17(0.90,1.52) \\
\text { RR T3: } \mathbf{1 . 4 6}(\mathbf{1 . 1 4}, \mathbf{1 . 8 6})^{*}\end{array}$ \\
\hline All-cause mortalityc ${ }^{c}$ & Group 3 SMR: 1.07; $p=0.134$ \\
\hline
\end{tabular}


Table 2-1. Summary of Epidemiological Studies Evaluating Death ${ }^{\mathrm{a}}$

\begin{tabular}{l} 
Reference and study population \\
\hline McDonald and Potter 1996 \\
Prospective cohort study; $\mathrm{n}=454$ pediatric \\
patients diagnosed with $\mathrm{Pb}$ poisoning, \\
Massachusetts, 1923-1966, followed
\end{tabular}

through 1991; age of diagnosis $<1-9$ years

$\mathrm{PbB}(\mu \mathrm{g} / \mathrm{dL})$
Mean 113

McElvenny et al. 2015

Cohort study; $n=9,122$ workers; mean age 29.2 years

\begin{tabular}{|c|c|}
\hline Mortality outcome & Effects $^{b}$ \\
\hline $\begin{array}{l}\text { Diseases of the blood and } \\
\text { blood forming organs }\end{array}$ & SMR: $9.68(1.95,28.28)^{*}$ \\
\hline $\begin{array}{l}\text { Nervous-system and } \\
\text { sense-organ diseases }\end{array}$ & SMR: $2.86(0.57,8.35)$ \\
\hline Respiratory diseases & SMR: $1.95(0.78,4.02)$ \\
\hline Pneumonia & SMR: $2.10(0.68,4.90)$ \\
\hline Digestive system diseases & SMR: $1.37(0.44,3.21)$ \\
\hline $\begin{array}{l}\text { Genitourinary system } \\
\text { diseases }\end{array}$ & SMR: $1.69(0.02,9.43)$ \\
\hline Chronic nephritis & SMR: $5.00(0.06,27.82)$ \\
\hline All-cause mortalityc & SMR: $1.74(1.40,2.15)^{*}$ \\
\hline All-cause mortalityc & $\begin{array}{l}\text { Males: SMR } 1.10(1.06,1.14)^{*} \\
\text { Females: SMR } 1.00(0.91,1.09) \\
\text { Total SMR: } 1.09(1.05,1.12)^{*}\end{array}$ \\
\hline $\begin{array}{l}\text { Respiratory system } \\
\text { diseases }\end{array}$ & $\begin{array}{l}\text { Males: SMR: } 1.17(1.06,1.30)^{*} \\
\text { Females: SMR: } 1.24(0.98,1.57) \\
\text { Total SMR: } 1.18(1.08,1.30)^{*}\end{array}$ \\
\hline Digestive system diseases & $\begin{array}{l}\text { Males: SMR: } 1.22(\mathbf{1 . 0 3}, \mathbf{1 . 4 5})^{*} \\
\text { Females: SMR: } 0.84(0.52,1.35) \\
\text { Total SMR: } 1.16(0.99,1.36)\end{array}$ \\
\hline Genitourinary diseases & $\begin{array}{l}\text { Males: SMR: } 1.02(0.72,1.44) \\
\text { Females: SMR: } 0.67(0.28,1.60) \\
\text { Total SMR: } 0.95(0.69,1.31)\end{array}$ \\
\hline $\begin{array}{l}\text { Non-malignant kidney } \\
\text { disease }\end{array}$ & $\begin{array}{l}\text { Males: SMR: } 1.30(0.76,2.24) \\
\text { Total SMR: } 1.29(0.79 .2 .11\end{array}$ \\
\hline
\end{tabular}


2. HEALTH EFFECTS

Table 2-1. Summary of Epidemiological Studies Evaluating Death ${ }^{\mathrm{a}}$

\begin{tabular}{l} 
Reference and study population \\
\hline Selevan et al. 1985 \\
Retrospective cohort study; $n=1,987$ male \\
workers
\end{tabular}
workers

\section{$\operatorname{PbB}(\mu \mathrm{g} / \mathrm{dL})$ \\ Mean: 56.3}

Steenland et al. 1992
Cohort study (same cohort as Selevan et al.
1985); $n=1,990$ male smelter workers
Cohort study; $\mathrm{n}=88,187 \mathrm{~Pb}$ workers (United States $n=58,313$, United Kingdom $n=9,122$,

Finland $n=20,752$ )
Median: 26

Tertiles:

Mean: 56.3

- T1: $20-<30$

- $T 2: 30-<409$

- T3: $>40$

\begin{tabular}{|c|c|}
\hline Mortality outcome & Effects $^{b}$ \\
\hline All tuberculosis & SMR: $1.39(0.69,2.49)$ \\
\hline $\begin{array}{l}\text { Diseases of the central } \\
\text { nervous system }\end{array}$ & SMR: $0.84(0.61,1.12)$ \\
\hline \multicolumn{2}{|c|}{$\begin{array}{l}\text { Diseases of the respiratory SMR: } 1.25(0.92,1.66) \\
\text { system }\end{array}$} \\
\hline Other respiratory diseases & SMR: $1.87(1.28,2.64)^{*}$ \\
\hline $\begin{array}{l}\text { Diseases of the digestive } \\
\text { system }\end{array}$ & SMR: $0.51(0.26,0.89)$ \\
\hline $\begin{array}{l}\text { Diseases of the } \\
\text { genitourinary system }\end{array}$ & SMR: $0.93(0.42,1.77)$ \\
\hline $\begin{array}{l}\text { Chronic and unspecified } \\
\text { nephritis and other renal } \\
\text { sclerosis }\end{array}$ & SMR: $1.92(0.88,3.64)$ \\
\hline All other & SMR: $0.88(0.67,1.14)$ \\
\hline All-cause mortality ${ }^{c}$ & SMR: $1.07(1.00,1.14)^{*}$ \\
\hline $\begin{array}{l}\text { Non-malignant respiratory } \\
\text { disease }\end{array}$ & SMR: $1.44(1.16,1.77)^{*}$ \\
\hline Emphysema & SMR: $2.20(1.45,3.20)^{*}$ \\
\hline $\begin{array}{l}\text { Pneumonia and other } \\
\text { respiratory disease }\end{array}$ & SMR: $1.88(1.34,2.56)^{\star}$ \\
\hline Acute kidney disease & SMR: $0.91(0.02,5.07)$ \\
\hline Chronic kidney disease & SMR: $1.26(0.54,2.49)$ \\
\hline All-cause mortalityc & HR T1: $1.15(1.10,1.21)^{*}$ \\
\hline Stroke & HR T1: $1.24(1.03,1.50)^{*}$ \\
\hline Ischemic heart disease & HR T1: $1.14(1.04,1.26)^{*}$ \\
\hline $\begin{array}{l}\text { Chronic obstructive } \\
\text { pulmonary disease }\end{array}$ & HR T1: $1.43(1.10,1.86)^{*}$ \\
\hline Chronic kidney disease & HR T3: $1.54(0.77,3.08)$ \\
\hline
\end{tabular}


2. HEALTH EFFECTS

Table 2-1. Summary of Epidemiological Studies Evaluating Death ${ }^{a}$

\begin{tabular}{llll}
\hline Reference and study population & PbB $(\mu \mathrm{g} / \mathrm{dL})$ & Mortality outcome & Effects $^{\mathrm{b}}$ \\
\hline Wong and Harris et al. $\mathbf{2 0 0 0}$ & Mean: & All-cause mortalityc & \\
& $\bullet \quad$ All workers: 64.0 & \\
Cohort study; $\mathrm{n}=4,519$ battery workers; & $\bullet \quad$ Battery workers: 62.7 & \\
$\begin{array}{l}\text { 2,300 smelters (same cohort as Cooper et } \\
\text { al. } 1985)\end{array}$ & $\bullet \quad$ Smelters: 79.7 & \\
\hline
\end{tabular}

a Studies assessing death due to cardiovascular disease and cancer are discussed in Sections 2.5 and 2.19 , respectively.

${ }^{b}$ Asterisk and bold indicate association with $\mathrm{Pb}$; unless otherwise specified, values in parenthesis are $95 \%$ Cls; $p$-values $<0.05$ unless otherwise noted in the table.

Includes cancer and/or cardiovascular deaths.

$\mathrm{Cl}=$ confidence interval; $\mathrm{HR}=$ hazard ratio; NHANES = National Health and Nutrition Examination Survey; OR = odds ratio; $\mathrm{Pb}=$ lead; $\mathrm{PbB}=\mathrm{blood}$ lead concentration; $\mathrm{PMR}=$ proportionate mortality ratio; $\mathrm{RR}=$ rate ratio or relative risk; $\mathrm{SD}=$ standard deviation; $\mathrm{SMR}=\mathrm{standard}$ mortality ratio 


\subsection{BODY WEIGHT}

Overview. Compared to other health effect endpoints, there is little information on $\mathrm{Pb}$ exposure and body weight measures. However, a few epidemiological studies have evaluated effects of $\mathrm{Pb}$ exposure on body weight in children, adolescents, and adults. The studies reviewed below focused on effects at $\mathrm{PbB}$ $\leq 10 \mu \mathrm{g} / \mathrm{dL}$. Inverse associations have been observed between PbB and BMI, and decreased risks of being overweight or obese have been reported. However, some studies did not observe associations and one study reported a positive association between $\mathrm{PbB}$ and the risk of obesity in women.

Note that studies evaluating the effects of exposure to $\mathrm{Pb}$ on birth weight are reviewed in Section 2.18 (Developmental).

The following effects on body weight have been associated with $\mathrm{PbB} \leq 10 \mu \mathrm{g} / \mathrm{dL}$ :

- Decreased BMI and risk of being overweight or obese in children and adolescents; observed in a few studies.

- Decreased BMI and risk of being overweight or obese in adults; not corroborated.

- Increased risk of obesity in women; not corroborated.

Measures of Exposure. Most studies evaluating effects of chronic Pb exposure on body weight evaluate exposure by measurement of $\mathrm{PbB}$. A few other studies examining associations between $\mathrm{Pb}$ exposure and body weight used $\mathrm{Pb}$ concentration in urine, bone, and/or dentin as biomarkers of exposure; however, these studies did not report PbB (Kim et al. 1995; Liu et al. 2019a; Padilla et al. 2010; Shao et al. 2017).

Confounding Factors and Effect Modifiers. Numerous factors contribute to body weight (or BMI), including age, sex, race, nutrition, diet, daily activity level, intercurrent illness, genetic pre-disposition for body type, income level, education, and alcohol and tobacco use. Failure to account for these factors may attenuate or strengthen the apparent associations between $\mathrm{Pb}$ exposure and the outcome, depending on the direction of the effect of the variable on the outcome. 
Effects at Blood Pb Levels $\leq 10 \mu \boldsymbol{g} / \mathbf{d L}$. Results of studies evaluating effects of $\mathrm{PbB} \leq 10 \mu \mathrm{g} / \mathrm{dL}$ on body weight are briefly summarized in Table 2-2 and an overview of results is provided in Table 2-3; study details are provided in the Supporting Document for Epidemiological Studies for Lead, Table 1. Studies have been conducted in children and adolescents (Burns et al. 2017; Cassidy-Bushrow et al. 2016; Hauser et al. 2008; Scinicariello et al. 2013) and adults (Scinicariello et al. 2013; Wang et al. 2015). The largest study evaluating associations between $\mathrm{PbB}$ and body weight is a study of children, adolescents, and adults participating in NHANES, 1999-2006; this study included adjustments for numerous confounding factors (see the Supporting Document for Epidemiological Studies for Lead, Table 1) (Scinicariello et al. 2013). In children and adolescents $(n=10,693)$, results show an inverse association between PbB and BMI-Z score and risk of being overweight or obese. In a smaller study in children ( $n=131)$, inverse associations were observed between PbB and BMI and BMI-Z score (Cassidy-Bushrow et al. 2016). Other studies in small populations of boys showed no associations between weight, BMI and/or BMI-Z score (Burns et al. 2017; Hauser et al. 2008). Results of studies in adults are mixed. The largest study in adults $(n=15,899)$ shows inverse associations between $\mathrm{PbB}$ and $\mathrm{BMI}$ and risk of being overweight and obese, with a negative trend (p-trend: $\leq 0.01$ ) over quartiles (Scinicariello et al. 2013). No association was observed between PbB and BMI in a small study on women ( $\mathrm{n}=107)$ (Ronco et al. 2010) or a larger study in men $(n=2235)$ (Wang et al. 2015). In contrast, the risk of being obese was increased in a large population ( $n=3323$ ) of women (Wang et al. 2015). Thus, except for the Wang et al. (2015) study, available studies show either no association or an inverse association between $\mathrm{PbB} \leq 10 \mu \mathrm{g} / \mathrm{dL}$ and body weight and/or BMI.

Mechanisms of Action. The mechanisms involved in the development of Pb-induced changes in body weight have not been established. However, alterations of the hypothalamic-pituitary-adrenal axis, stressinduced elevations in glucocorticoid levels, oxidative stress, and altered lipid metabolism have been proposed (reviewed by Scinicariello et al. 2013; Shao et al. 2017; Wang et al. 2015). 
2. HEALTH EFFECTS

\section{Table 2-2. Summary of Epidemiological Studies Evaluating Effects on Body Weight at Mean Blood Lead Concentrations $(\mathrm{PbB}) \leq 10 \mu \mathrm{g} / \mathrm{dL}^{\mathrm{a}}$}

\begin{tabular}{|c|c|c|c|}
\hline Reference and study population & $\mathrm{PbB}(\mu \mathrm{g} / \mathrm{dL})$ & Outcome evaluated & Result $^{b}$ \\
\hline Burns et al. 2017 & Median 3.0 & HT-Z score & $\begin{array}{l}\text { Adjusted } \beta(95 \% \mathrm{Cl}), \mathrm{HT}-\mathrm{Z} \text { score per unit } \\
\text { InPbB: }-0.26(-0.40,-0.13) ; p<0.001^{*}\end{array}$ \\
\hline $\begin{array}{l}\text { Prospective cohort of } 481 \text { Russian } \\
\text { boys enrolled at age } 8-9 \text { years and } \\
\text { followed until age } 18 \text { years }\end{array}$ & & BMI-Z score & $\begin{array}{l}\text { Adjusted } \beta(95 \% \mathrm{Cl}), \mathrm{BMI}-\mathrm{Z} \text { score per unit } \\
\text { InPbB: }-0.14(-0.31,0.04) ; p=0.12\end{array}$ \\
\hline Cassidy-Bushrow et al. 2016 & Mean (SD): 2.45 (2.53) & BMI & $\begin{array}{l}\text { Adjusted RR }(95 \% \mathrm{CI}) \text { for } \mathrm{BMI} \geq 85^{\text {th }} \\
\text { percentile } 0.57(0.33,0.98) ; p=0.041^{*}\end{array}$ \\
\hline $\begin{array}{l}\text { Birth cohort of } 131 \text { children, } 2- \\
3 \text { years of age }\end{array}$ & & BMI-Z score & $\begin{array}{l}\text { Adjusted } \beta(95 \% \mathrm{CI}) \text { for BMI Z-score: }-0.35 \\
(-0.60,-0.10) ; p=0.012^{*}\end{array}$ \\
\hline Hauser et al. 2008 & Mean: 3 & Weight & $\begin{array}{l}\text { Adjusted } \beta(95 \% \mathrm{Cl}) \text {, per unit log-PbB: }-0.761 \\
(-1.54,0.02) ; p=0.067\end{array}$ \\
\hline $\begin{array}{l}\text { Cross-sectional study of } 489 \text { boys, } 8- \\
9 \text { years of age }\end{array}$ & & BMI & $\begin{array}{l}\text { Adjusted } \beta(95 \% \mathrm{Cl}) \text {, per unit log-PbB: }-0.107 \\
(-0.44,0.23) ; p=0.53\end{array}$ \\
\hline $\begin{array}{l}\text { Ronco } 2010 \\
\text { Cross-sectional study of } 107 \text { women } \\
\text { of childbearing age (median age: } \\
27 \text { years) from Chile; data collection } \\
\text { period not reported }\end{array}$ & $\begin{array}{l}\text { Median } \\
\text { - } \quad \text { All: } 1.0 \\
\text { - } \quad \text { Low weight: } 1.7 \\
\text { - } \quad \text { Normal weight: } 2.3 \\
\text { - } \quad \text { Overweight: } 1.0\end{array}$ & BMI & $\begin{array}{l}\text { No differences in PbB were observed between } \\
\text { BMI categories }\end{array}$ \\
\hline
\end{tabular}


2. HEALTH EFFECTS

Table 2-2. Summary of Epidemiological Studies Evaluating Effects on Body Weight at Mean Blood Lead Concentrations $(\mathrm{PbB}) \leq 10 \mu \mathrm{g} / \mathrm{dL}^{\mathrm{a}}$

\begin{tabular}{|c|c|c|c|}
\hline Reference and study population & $\mathrm{PbB}(\mu \mathrm{g} / \mathrm{dL})$ & Outcome evaluated & Result $^{\mathrm{b}}$ \\
\hline \multirow{6}{*}{$\begin{array}{l}\text { Scinicariello et al. } 2013 \\
\text { Cross-sectional study of children and } \\
\text { adolescents ( } n=10,693 ; \text { age } 3- \\
19 \text { years) adults ( } n=15,899, \text { age } \\
\geq 20 \text { years) using NHANES data } \\
(1999-2006)\end{array}$} & \multirow{6}{*}{$\begin{array}{ll}\text { Gmean (SE) } \\
\text { - } \quad \text { Children/adolescents: } \\
\text { - } 1.12(0.02) \\
\text { - } \quad \text { Adults: } 1.59(0.02) \\
\text { o } \quad \text { Q1: } \leq 0.70 \\
\text { o Q2: } 0.71-1.09 \\
\text { o Q3: } 1.10-1.60 \\
\text { o Q4: } \geq 1.61\end{array}$} & $\begin{array}{l}\text { BMI-Z score } \\
\text { (children and adolescents) }\end{array}$ & 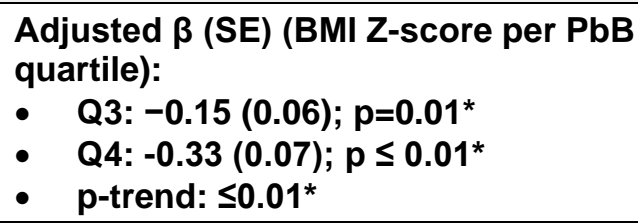 \\
\hline & & $\begin{array}{l}\text { Overweight } \\
\text { (children and adolescents) }\end{array}$ & Adjusted OR for Q4: $0.67(0.52,0.88)^{*}$ \\
\hline & & $\begin{array}{l}\text { Obesity } \\
\text { (children and adolescents) }\end{array}$ & $\begin{array}{l}\text { Adjusted OR } \\
\text { - } \quad \text { Q3: } 0.70(0.54,0.90)^{*} \\
\text { - } \quad \text { Q4: } 0.42(0.30,0.59)^{*}\end{array}$ \\
\hline & & BMI (adults) & $\begin{array}{l}\text { Adjusted } \beta \text { (SE) (BMI per quartile): } \\
-\quad \text { Q2: }-0.90(0.20) ; p \leq 0.01^{*} \\
-\quad \text { Q3: }-1.41(0.22) ; p \leq 0.01^{*} \\
-\quad \text { Q4: }-2.58(0.25) ; p \leq 0.01^{*} \\
-\quad \text { p-trend: } \leq 0.01^{*}\end{array}$ \\
\hline & & Overweight (adults) & Adjusted OR for Q4: $0.79(0.65-0.95)^{*}$ \\
\hline & & Obesity (adults) & $\begin{array}{l}\text { Adjusted OR } \\
\text { - } \quad \text { Q2: } 0.76(0.66-0.87)^{*} \\
\text { - } \quad \text { Q3: } 0.66(0.56-0.77)^{*} \\
-\quad \text { Q4: } 0.42(0.35-0.50)^{*}\end{array}$ \\
\hline
\end{tabular}


2. HEALTH EFFECTS

\section{Table 2-2. Summary of Epidemiological Studies Evaluating Effects on Body Weight at Mean Blood Lead Concentrations $(\mathrm{PbB}) \leq 10 \mu \mathrm{g} / \mathrm{dL}^{\mathrm{a}}$}

\begin{tabular}{|c|c|c|c|}
\hline Reference and study population & $\mathrm{PbB}(\mu \mathrm{g} / \mathrm{dL})$ & Outcome evaluated & Result $^{b}$ \\
\hline \multirow[t]{3}{*}{$\begin{array}{l}\text { Cross-sectional study of } 5,558 \text { adults } \\
\text { (men: } 2,235 \text {, ages 39-65 years; } \\
\text { women: } 3,323 \text {, ages } 40-65 \text { years) } \\
\text { from } 16 \text { locations in China }\end{array}$} & $\begin{array}{l}\text { PbB: } \\
\text { Men } \\
\text { - } \quad \text { Median: } 4.40 \\
\text { - } \quad \text { Quartiles: } \\
\text { o Q1: } \leq 29.00 \\
\text { o Q2: } 29.01-44.00 \\
\text { o Q3: } 44.01-62.16\end{array}$ & BMI & $\begin{array}{l}\beta \text { (SE) per PbB quartile } \\
\text { - Men } \\
\text { o Q4: } 0.01(0.20) \\
\text { o p-trend: } 0.82 \\
\text { - Women } \\
\text { o Q4: } 0.59(0.17) ; \text { p<0.05* } \\
\text { o p-trend: }<0.001^{*}\end{array}$ \\
\hline & $\begin{array}{l}\text { o Q4: } \geq 62.17 \\
\text { Women: } \\
\text { - Median: } 3.78 \\
\text { - Quartiles: } \\
\text { o Q1: } \leq 25.13 \\
\text { o Q2: } 25.14-37.79 \\
\text { o Q3: } 37.80-54.35\end{array}$ & Overweight & $\begin{array}{l}\text { Adjusted OR } \\
\text { - Men } \\
\text { o Q4: } 0.95(0.72,1.26) \\
\text { o p-trend: } 0.74 \\
\text { Women } \\
\text { o Q4: } 1.16(0.92,1.46) \\
\text { o p-trend: } 0.07\end{array}$ \\
\hline & o Q4: $\geq 54.36$ & Obesity & $\begin{array}{l}\text { Adjusted OR } \\
\text { - Men } \\
\text { o Q4: } 0.88(0.48,1.61) \\
\text { o p-trend: } 0.99 \\
\text { Women } \\
\text { o Q4: } 1.86(\mathbf{1 . 1 6 , 2 . 9 8 ) *} \\
\text { o p-trend: }<0.01^{*}\end{array}$ \\
\hline
\end{tabular}

aSee the Supporting Document for Epidemiological Studies for Lead, Table 1 for more detailed descriptions of studies.

${ }^{b}$ Asterisk and bold indicate association with $\mathrm{Pb}$; unless otherwise specified, values in parenthesis are $95 \% \mathrm{Cls}$; $\mathrm{p}$-values $<0.05$ unless otherwise noted in the table.

$\mathrm{BMI}=$ body mass index; BMI-Z = BMI z-scores; $\mathrm{Cl}=$ confidence interval; Gmean = geometric mean; HT-Z = height Z-Scores; NHANES = National Health and Nutrition Examination Survey; $\mathrm{OR}=$ odds ratio; $\mathrm{Pb}=$ lead; $\mathrm{RR}=$ risk ratio; $\mathrm{SD}=$ standard deviation; $\mathrm{SE}=\mathrm{standard}$ error 


\section{Table 2-3. Effects on Body Weight Associated with Mean Blood Lead Concentrations (PbBs) $\leq 10 \mu g^{\mathrm{a}}$}

\begin{tabular}{|c|c|c|c|c|c|c|c|}
\hline $\begin{array}{l}\text { Mean PbB } \\
(\mu \mathrm{g} / \mathrm{dL})\end{array}$ & Population (n) & Weight & $\mathrm{BMI}$ & $\begin{array}{l}\text { BMI-Z } \\
\text { score }\end{array}$ & Overweight & Obese & Reference \\
\hline 3.0 & C (481 boys) & - & - & 0 & - & - & Burns et al. 2017 \\
\hline 2.45 & $C(131)$ & - & $\downarrow$ & $\downarrow$ & - & - & $\begin{array}{l}\text { Cassidy-Bushrow } \\
\text { et al. } 2016\end{array}$ \\
\hline 3 & C (489 boys) & 0 & 0 & - & - & - & Hauser et al. 2008 \\
\hline 1.0 & A (107 women) & - & 0 & - & - & - & Ronco et al. 2010 \\
\hline 1.12 & $\mathrm{C}, \mathrm{Ad}(10,693)^{\mathrm{c}}$ & - & - & $\downarrow$ & $\downarrow$ & $\downarrow$ & $\begin{array}{l}\text { Scinicariello et al. } \\
2013\end{array}$ \\
\hline 1.59 & $A(15,899)^{c}$ & - & $\downarrow$ & - & $\downarrow$ & $\downarrow$ & $\begin{array}{l}\text { Scinicariello et al. } \\
2013\end{array}$ \\
\hline 4.40 & A $(2,235$, men $)$ & - & 0 & - & 0 & 0 & Wang et al. 2015 \\
\hline 3.78 & A $(3,323$, women $)$ & - & 0 & - & 0 & $\uparrow$ & Wang et al. 2015 \\
\hline
\end{tabular}

$\mathrm{a} \uparrow=$ increased; $\downarrow=$ decreased; $0=$ no change; $-=$ not assessed.

bUnless otherwise specified, study was conducted in males and females.

cParticipants from the National Health and Nutrition Examination Survey 1999-2006.

$\mathrm{A}=$ adults; $\mathrm{Ad}=$ adolescents; $\mathrm{BMI}=$ body mass index; $\mathrm{BMI}-\mathrm{Z}=\mathrm{BMI} \mathrm{z}$-scores; $\mathrm{C}=$ children

\subsection{RESPIRATORY}

Overview. Few epidemiological studies have evaluated respiratory effects associated with exposure to $\mathrm{Pb}$; those that are available include cross-sectional studies in adults and prospective and cross-sectional studies in children. Associations have been observed between $\mathrm{PbB}$ and decreased lung function, increased bronchial hyperreactivity, increased number and severity of symptoms of respiratory disease, and increased risk of respiratory diseases (e.g., asthma and obstructive lung disease). Although most studies found associations between respiratory effects and $\mathrm{PbB}$, other studies did not observe associations.

The following respiratory effects have been associated with PbB:

- $\leq 10 \mu \mathrm{g} / \mathrm{dL}$ :

o Decreased lung function; corroborated in a few studies, including studies in children.

o Increased bronchial hyperreactivity.

o Increased risk of asthma and obstructive lung disease; evaluated in a few studies with mixed results. 
- $\quad>10 \mu \mathrm{g} / \mathrm{dL}:$

o Decreased lung function.

o Symptoms of respiratory disease (e.g., shortness of breath).

o Increased risk/prevalence of asthma; evaluated in a few studies with mixed results.

Measures of Exposure. Studies evaluating the association between respiratory effects and $\mathrm{Pb}$ exposure evaluate exposure by measurement of PbB.

Confounding Factors and Effect Modifiers. The etiology for most respiratory diseases is multifactorial; therefore, several factors may contribute to clinical findings. Factors that may contribute to the development of respiratory diseases include poor housing conditions, exposure to allergens (e.g., pet dander, seasonal allergies), exposure to tobacco smoke and other respiratory irritants, and asthma compounded by obesity (Ali and Ulirk 2013). In addition, Aligne et al. (2000) reported that children living in urban settings have an increased risk of asthma. Failure to account for these factors may attenuate or strengthen the apparent associations between $\mathrm{Pb}$ exposure and the outcome, depending on the direction of the effect of the variable on the outcome.

Characterization of Effects. General trends for studies showing a relationship between $\mathrm{PbB}$ and respiratory effects are shown in Table 2-4. Compared to other toxicological endpoints (e.g., neurological or cardiovascular effects), few studies have evaluated adverse respiratory effects associated with $\mathrm{PbB}$. Data are from cross-sectional studies in adults (Bagci et al. 2004; Bener et al. 2001; Chung et al. 2015; Min et al. 2008a; Pugh Smith and Nriagu 2011; Rokadia and Agarwal 2013), and prospective (Joseph et al. 2005; Rabinowitz et al. 1990) and cross-sectional (Wells et al. 2014) studies in children. Over a range of $\mathrm{PbBs}$ that includes $\mathrm{PbB} \leq 10 \mu \mathrm{g} / \mathrm{dL}$ and $\mathrm{PbB}>50 \mu \mathrm{g} / \mathrm{dL}$, studies provide evidence for effects in $\mathrm{Pb}$ workers compared to controls or associations between $\mathrm{PbB}$ and decreased pulmonary function tests indicative of obstructive pulmonary disease (forced expiratory volume in 1 second [FEV 1 ], FEV 1 /forced vital capacity [FVC] ratio, forced expiratory flow at 25-75\% of $\left.\mathrm{FVC}_{\text {[FEF }} 25-75\right]$ ), increased bronchial hyperreactivity (indicative of asthma), symptoms of respiratory disease (cough, shortness of breath), and increased risk of respiratory diseases (e.g., asthma and obstructive lung disease). With the exception of a prospective study in children, which showed no increased risk of asthma at umbilical cord $\mathrm{PbB} \geq 10 \mu \mathrm{g} / \mathrm{dL}$ compared to $<10 \mu \mathrm{g} / \mathrm{dL}$ (Rabinowitz et al. 1990), studies showed positive associations between $\mathrm{PbB}$ and respiratory effects. 
Table 2-4. Overview of Respiratory Effects in Adults and Children Chronically Exposed to Lead (Pb)

\begin{tabular}{|c|c|c|}
\hline $\begin{array}{l}\text { Mean blood lead } \\
\text { concentration } \\
(\mathrm{PbB})(\mu \mathrm{g} / \mathrm{dL})\end{array}$ & Effects associated with $\mathrm{Pb}$ exposure & References \\
\hline \multirow[t]{3}{*}{$\leq 10$} & Decreased lung function & $\begin{array}{l}\text { Chung et al. 2015; Leem et al. 2015; } \\
\text { Little et al. 2017; Zeng et al. } 2017\end{array}$ \\
\hline & Increased bronchial responsiveness & Min et al. $2008 a$ \\
\hline & $\begin{array}{l}\text { Lung disease (asthma and obstructive lung } \\
\text { disease) }\end{array}$ & $\begin{array}{l}\text { Joseph et al. 2005; Rokadia and } \\
\text { Agarwal 2013; Wang et al. 2017a; Wells } \\
\text { et al. 2014; Zeng et al. } 2016\end{array}$ \\
\hline$>10-30$ & Lung disease (asthma) & Pugh Smith and Nriagu 2011 \\
\hline$>30-50$ & Decreased lung function & Bagci et al. 2004 \\
\hline \multirow[t]{2}{*}{$>50$} & Symptoms of lung disease (phlegm) & Bener et al. 2001 \\
\hline & Lung disease (asthma) & Bener et al. 2001 \\
\hline
\end{tabular}

Effect at Blood Pb Levels $\leq 10 \mu g / d L$. Results of studies evaluating respiratory effects of $\mathrm{PbB} \leq 10 \mu \mathrm{g} / \mathrm{dL}$ are summarized in Table 2-5, with study details provided in the Supporting Document for Epidemiological Studies for Lead, Table 2. Studies show associations between $\mathrm{PbB} \leq 10 \mu \mathrm{g} / \mathrm{dL}$ and decreased lung function, increased bronchial hyperreactivity, and increased risk of asthma; findings are consistent with obstructive lung disease. In a cross-sectional study in adults from China with mean $\mathrm{PbB}$ of $2.50 \mu \mathrm{g} / \mathrm{dL}$, an inverse association was observed for the $\mathrm{FEV}_{1} / \mathrm{FVC}$ ratio in a population; results are consistent with obstructive airway disease (Chung et al. 2015). In a large pooled cross-sectional study, Korean adults showed a decrease in the $\mathrm{FEV}_{1} / \mathrm{FVC}$ ratio in the highest exposure quartile (Leem et al. 2015). A small study in children with a mean PbBs of $5.53 \mu \mathrm{g} / \mathrm{dL}$ show inverse associations between $\mathrm{PbB}$ and pulmonary functions tests, including $\mathrm{FEV}_{1}$ and FVC (Little et al. 2017). Increased bronchial reactivity in response to methacholine challenge, consistent with a diagnosis of asthma, was observed in adults with mean $\mathrm{PbB}$ of $2.96 \mu \mathrm{g} / \mathrm{dL}$ (Min et al. 2008a). In addition, risk of obstructive lung disease was observed in a large NHANES population of adults with a mean PbB of $1.73 \mu \mathrm{g} / \mathrm{dL}$ (Rokadia and Agarwal 2013). Studies in children examining associations between PbB and risk of asthma do not provide consistent results. A large prospective study showed an increased risk of asthma in black children with $\mathrm{PbB}<5$ and $\geq 5 \mu \mathrm{g} / \mathrm{dL}$ compared to white children with $\mathrm{PbB}<5 \mu \mathrm{g} / \mathrm{dL}$; however, no increased risk was observed for white children with $\mathrm{PbB} \geq 5 \mu \mathrm{g} / \mathrm{dL}$ compared to white children with $\mathrm{PbB}<5 \mu \mathrm{g} / \mathrm{dL}$ (Joseph et al. 2005). The underlying causes for the racial disparity of results have not been established. However, the study authors noted the following as possible contributors: socio-economic factors; racial differences in IgE; differences in housing conditions and indoor $\mathrm{Pb}$ sources (e.g., $\mathrm{Pb}$ paint); and genetic variability in susceptibility to $\mathrm{Pb}$ toxicity (e.g., vitamin $\mathrm{D}$ receptor gene). In cross-sectional studies, asthma risk was 


\section{Table 2-5. Summary of Epidemiological Studies Evaluating Respiratory Effects at Mean Blood Lead Concentration $(\mathrm{PbB}) \leq 10 \mu \mathrm{g} / \mathrm{dL}^{\mathrm{a}}$}

\begin{tabular}{|c|c|c|c|}
\hline Reference and study population & $\mathrm{PbB}(\mu \mathrm{g} / \mathrm{dL})$ & Outcome evaluated & Result ${ }^{b}$ \\
\hline \multicolumn{4}{|l|}{ Decreased lung function } \\
\hline \multirow{5}{*}{$\begin{array}{l}\text { Chung et al. } 2015 \\
\text { Cross-sectional study; n=870 adults }\end{array}$} & \multirow{5}{*}{$\begin{array}{l}\text { Mean: } 2.50 \\
\text { Tertiles: } \\
\text { - } \quad \text { T1: }<2.03 \\
\text { - } \quad \text { T2: } 2.03-2.81 \\
\text { - } \quad \text { T3: }>2.81\end{array}$} & FVC\% & Correlation coefficient: 0.070 \\
\hline & & $\mathrm{FEV}_{1} \%$ & Correlation coefficient: 0.00 \\
\hline & & \multirow[t]{3}{*}{$\mathrm{FEV}_{1} / \mathrm{FVC}$ ratio } & Correlation coefficient: $-0.115 ; p<0.01^{*}$ \\
\hline & & & OR T3: $0.006(0,0.286)^{*}$ \\
\hline & & & p-trend: $0.03^{*}$ \\
\hline Leem et al. 2015 & $\begin{array}{l}\text { Mean: } \\
-\quad \text { Men: } 2.92\end{array}$ & $\mathrm{FEV}_{1} / \mathrm{FVC}$ ratio & $\begin{array}{l}\text { Difference (SE) between reference and Q4: } \\
-0.6(0.3) ; p=0,025^{*}\end{array}$ \\
\hline $\begin{array}{l}\text { Pooled cross-sectional study; } \\
n=5,972 \text { adults }\end{array}$ & $\begin{array}{l}\text { - } \quad \text { Women: } 2.33 \\
\text { Quartiles (men and women) } \\
\text { - } \quad \text { Q1: } \leq 1.85 \text { (reference) } \\
\text { - } \quad \text { Q2: } 1.86-2.43 \\
\text { - } \quad \text { Q3: } 2.44-3.16 \\
\text { - } \quad \text { Q4: } \geq 3.17\end{array}$ & & \\
\hline Little et al. 2017 & \multirow{2}{*}{$\begin{array}{l}\text { Mean: } \\
\text { - } \quad \text { Boys: } 5.27 \\
\text { - } \quad \text { Girls: } 3.82\end{array}$} & \multirow[t]{2}{*}{ FVC } & $\begin{array}{l}\text { Boys, } \beta \text { (SE), per log10 increase in } \mathrm{PbB} \text { : } \\
-5.11(4.47) ; p=0.25\end{array}$ \\
\hline $\begin{array}{l}\text { Cross-sectional study; } n=184 \text { boys and } \\
189 \text { girls (age } \geq 10-\leq 15.9 \text { years) }\end{array}$ & & & $\begin{array}{l}\text { Girls, } \beta \text { (SE), per log10 increase in PbB: } \\
-12.90 \text { (5.25); } p=0.02^{*}\end{array}$ \\
\hline Zeng et al. 2017 & \multirow{2}{*}{$\begin{array}{l}\text { PbB: } \\
\text { Median } \\
\text { - } \quad \text { Control: } 3.57 \\
\text { - } \quad \text { Exposed: } 5.53\end{array}$} & $\mathrm{FEV}_{1}$ & $\begin{array}{l}\text { Regression coefficient for exposed: - } \\
0.02(-0.100,0.043)\end{array}$ \\
\hline $\begin{array}{l}\text { Cross-sectional study; } n=200 \text { children } \\
\text { (ages } 5-7 \text { years) }\end{array}$ & & FVC & $\begin{array}{l}\text { Regression coefficient for exposed: } \\
\text { FVC: }-0.015(-0.093,0.063)\end{array}$ \\
\hline \multicolumn{4}{|l|}{ Increased bronchial responsiveness } \\
\hline Min et al. 2008a & Mean (SD): 2.96 (1.59) & BR & $\begin{array}{l}\text { A } 1 \mu \mathrm{g} / \mathrm{dL} \text { increase in } \mathrm{PbB} \text { was associated } \\
\text { with a higher BR; } \beta \text { (SE): } 0.018(0.007)^{\star}\end{array}$ \\
\hline
\end{tabular}


2. HEALTH EFFECTS

\section{Table 2-5. Summary of Epidemiological Studies Evaluating Respiratory Effects at Mean Blood Lead Concentration $(\mathrm{PbB}) \leq 10 \mu \mathrm{g} / \mathrm{dL}^{\mathrm{a}}$}

\begin{tabular}{|c|c|c|c|}
\hline Reference and study population & $\mathrm{PbB}(\mu \mathrm{g} / \mathrm{dL})$ & Outcome evaluated & Result $^{b}$ \\
\hline \multicolumn{4}{|l|}{ Asthma } \\
\hline \multirow{5}{*}{$\begin{array}{l}\text { Joseph et al. } 2005 \\
\text { Prospective study; } n=4,634 \text { children } \\
\text { (ages } 3 \text { months to } 3 \text { years) }\end{array}$} & \multirow{5}{*}{$\begin{array}{l}\text { Mean } \\
\text { - } \quad \text { White: } 3.2 \\
\text { - } \quad \text { Black: } 5.5\end{array}$} & \multirow[t]{5}{*}{ Asthma } & All compared to $\mathrm{PbB}<5 \mu \mathrm{g} / \mathrm{dL}$ in white children \\
\hline & & & HR white $(\mathrm{PbB} \geq 5): 2.3(0.8,6.7) ; p=0.12$ \\
\hline & & & HR black (PbB <5): $1.8(1.3,2.4) ; p<0.01^{*}$ \\
\hline & & & HR black (PbB $\geq 5): 1.5(1.2,1.8) ; p<0.01^{*}$ \\
\hline & & & HR black (PbB $\geq 10): 3.0(1.2,7.1) ; p=0.01^{*}$ \\
\hline \multirow{3}{*}{$\begin{array}{l}\text { Rokadia and Agarwal 2013c } \\
\text { Pooled cross-sectional study; } \\
n=9,575 \text { adults }(8,411 \text { without OLD; } \\
1,164 \text { with OLD) }\end{array}$} & \multirow{3}{*}{$\begin{array}{l}\text { Mean } \\
\text { - Non-OLD: } 1.18 \\
\text { - OLD: } 1.73\end{array}$} & \multirow[t]{3}{*}{ OLD } & OR for all OLD: $1.94(1.10,3.42)^{\star}$ \\
\hline & & & OR for mild OLD: $1.21(0.55,2.65)$ \\
\hline & & & $\begin{array}{l}\text { OR for moderate-severe OLD: } 3.49 \text { (1.70, } \\
7.15)^{*}\end{array}$ \\
\hline \multirow{3}{*}{$\begin{array}{l}\text { Wang et al. } 2017 \text { a } \\
\text { Cross-sectional study; } n=930 \text { children } \\
\text { (mean age: } 5.74 \text { years) }\end{array}$} & \multirow{3}{*}{$\begin{array}{l}\text { Gmean (GSD) } \\
\text { - } \quad \text { All: } 1.86(1.21) \\
\text { - } \quad \text { Boys: } 1.89(1.22) \\
\text { - } \quad \text { Girls: } 1.83(1.20)\end{array}$} & \multirow[t]{3}{*}{ Asthma } & $\begin{array}{l}\text { OR (all participants), <5 versus } \geq 5 \mu \mathrm{g} / \mathrm{dL}: \\
5.50(1.69,17.94) ; p=0.005^{*}\end{array}$ \\
\hline & & & 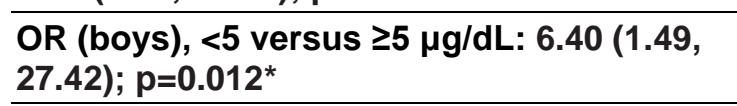 \\
\hline & & & 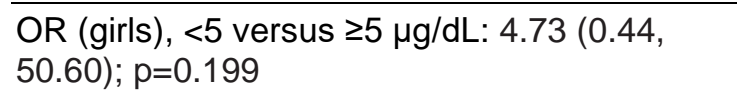 \\
\hline Wells et al. $2014^{c}$ & \multirow[t]{2}{*}{ Gmean: 1.07} & \multirow[t]{2}{*}{ Asthma } & OR for asthma with atopy: $0.97(0.61,1.55)$ \\
\hline $\begin{array}{l}\text { Cross-sectional study; NHANES 2005- } \\
2006 ; n=1,430 \text { children (ages 4- } \\
12 \text { years) }\end{array}$ & & & OR for asthma with no atopy: $1.07(0.86,1.33)$ \\
\hline
\end{tabular}


2. HEALTH EFFECTS

\section{Table 2-5. Summary of Epidemiological Studies Evaluating Respiratory Effects at Mean Blood Lead Concentration $(\mathrm{PbB}) \leq 10 \mu \mathrm{g} / \mathrm{dL}^{\mathrm{a}}$}

\begin{tabular}{|c|c|c|c|}
\hline Reference and study population & $\mathrm{PbB}(\mu \mathrm{g} / \mathrm{dL})$ & Outcome evaluated & Result $^{\mathrm{b}}$ \\
\hline Zeng et al. 2016 & $\begin{array}{l}\text { Median } \\
\text { - Haojiang area: } 4.75\end{array}$ & Asthma & $\begin{array}{l}\text { OR for asthma at } \mathrm{PbB} \geq 5 \mu \mathrm{g} / \mathrm{dL}: 9.50 \text { (1.16, } \\
\text { 77.49); } \mathrm{p}<0.01^{*}\end{array}$ \\
\hline $\begin{array}{l}\text { Cross-sectional study; } n=470 \text { children } \\
\text { (ages 3-8 years) }\end{array}$ & - $\quad$ Guiyu area: 6.24 & & \\
\hline
\end{tabular}

aSee the Supporting Document for Epidemiological Studies for Lead, Table 2 for more detailed descriptions of studies.

${ }^{b}$ Asterisk and bold indicate association with $\mathrm{Pb}$; unless otherwise specified, values in parenthesis are $95 \%$ Cls; $p$-values $<0.05$ unless otherwise noted in the table.

cStudy population was from NHANES.

$\mathrm{BR}=$ bronchial responsiveness; $\mathrm{Cl}=$ confidence interval; $F E V_{1}=$ forced expiratory volume in 1 second $(\mathrm{L} / \mathrm{s}) ; \mathrm{FEV} \mathrm{V}_{1} \%=$ percent of predicted $\mathrm{FEV}$; FVC = forced vital capacity (L); FVC\% = percent of predicted FVC; Gmean = geometric mean; GSD = geometric standard deviation; HR = hazard ratio; NHANES = National Health and Nutrition Examination Survey; OLD = obstructive lung disease; OR = odds ratio; $\mathrm{Pb}=$ lead; SD = standard deviation; SE = standard error 
increased in Taiwanese children, with elevated risks in the total population and for boys, but not for girls (Wang et al. 2017a) and in Chinese children with $\mathrm{PbB} \geq 5 \mu \mathrm{g} / \mathrm{dL}$ (Zeng et al. 2016). In contrast, a large cross-sectional study of children participating in NHANES did not observe an association between PbB (mean $1.07 \mu \mathrm{g} / \mathrm{dL}$ ) and asthma, with or without atopy (Wells et al. 2014).

Mechanisms of Action. General mechanisms of toxicity of $\mathrm{Pb}$ (reviewed in Section 2.21) are likely involved in the development of toxicity to the respiratory system. EPA (2014c) specifically noted that oxidative stress through reactive oxygen species (ROS), resulting in tissue damage and inflammation and immune effects, is a plausible mechanism for the underlying cause of respiratory damage. Increased ROS, along with depletion of antioxidants, results in inflammation and production and release of metabolites and cytokines. Immune-mediated inflammation is observed with asthma and bronchial hyperreactivity.

\subsection{CARDIOVASCULAR}

Overview. A large number of epidemiological studies showing adverse effects on the cardiovascular system associated with $\mathrm{Pb}$ exposure have been published. Most studies evaluated effects in adults, although a few studies in children have been conducted. The effect of $\mathrm{Pb}$ exposure on blood pressure is the most studied cardiovascular outcome, with results providing consistent evidence of positive associations between $\mathrm{Pb}$ exposure and blood pressure. Other cardiovascular endpoints (atherosclerosis, cardiac conduction, cardiovascular disease, and mortality due to cardiovascular disease) also show positive and inverse associations with $\mathrm{PbB}$, although the majority of studies had positive associations. In some cases, although no associations between $\mathrm{PbB}$ and cardiovascular outcomes were observed, associations were observed for bone $\mathrm{Pb}$, a biomarker of cumulative $\mathrm{Pb}$ exposure that, among individuals with high historical $\mathrm{Pb}$ exposures, typically remains elevated for many years after the $\mathrm{PbB}$ declines to $\leq 10 \mu \mathrm{g} / \mathrm{dL}$; these cases are noted in the discussions below.

The following cardiovascular effects have been associated with PbB:

- $\quad \leq 10 \mu \mathrm{g} / \mathrm{dL}$ :

o Greater systolic and diastolic blood pressure:

- In adults; corroborated in multiple studies.

- In children; evaluated in a few studies.

- During pregnancy; evaluated in a few studies. 
o Greater risk of hypertension:

- In adults, including during pregnancy; evaluated in numerous studies.

o Greater risk of atherosclerosis; evaluated in a few studies.

o Altered cardiac conduction; evaluated in a few studies.

o Greater risk of mortality due to cardiovascular diseases; evaluated in a few studies with mixed results.

- $\quad>10 \mu \mathrm{g} / \mathrm{dL}$ :

o Increased systolic and diastolic blood pressure:

- In adults; corroborated in multiple studies and meta-analyses.

- In children; evaluated in a few studies.

o Increased risk of hypertension; corroborated in multiple studies.

o Atherosclerosis; evaluated in a few studies.

o Increased risk or prevalence of heart disease; evaluated in a few studies.

o Increased mortality due to cardiovascular diseases; corroborated in multiple studies.

Measures of Exposure. $\mathrm{PbB}$ and bone $\mathrm{Pb}$ concentrations have been used as biomarkers to evaluate cardiovascular effects of $\mathrm{Pb}$ exposure. However, $\mathrm{PbB}$ may not provide the ideal biomarker for long-term exposure to target tissues that contribute a hypertensive effect of $\mathrm{Pb}$. Because the development of cardiovascular effects has a long latency period, associations between $\mathrm{PbB}$ and cardiovascular disease at concurrent $\mathrm{PbB} \leq 10 \mu \mathrm{g} / \mathrm{dL}$ may be related to higher past $\mathrm{Pb}$ exposures. Bone $\mathrm{Pb}$, a metric of cumulative or long-term exposure to $\mathrm{Pb}$, appears to be a better predictor of $\mathrm{Pb}$-induced elevations in blood pressure and alterations in cardiac conduction than PbB.

Confounding Factors and Effect Modifiers. Numerous factors affect blood pressure, including age, body mass, race, smoking, alcohol consumption, ongoing or family history of cardiovascular/renal disease, LDL cholesterol levels, and various dietary factors (e.g., dietary calcium). In addition, renal disease, as well as Pb-induced renal damage, can lead to cardiovascular effects, including increased blood pressure (EPA 2014c; NTP 2012); thus, interpretation of studies examining cardiovascular outcomes is complicated by the link between cardiovascular and renal function. Failure to account for these factors may attenuate or strengthen the apparent associations between $\mathrm{Pb}$ exposure and the outcome, depending on the direction of the effect of the variable on the outcome (e.g., Møller and Kristensen 1992). For example, adjusting for alcohol consumption will decrease the apparent association between $\mathrm{PbB}$ and blood pressure, if alcohol consumption contributes to Pb intake and, thereby, PbB (Bost et al. 1999; Hense et al. 1993; Hertz-Picciotto and Croft 1993; Wolf et al. 1995). Varying approaches and breadth of 
inclusion of these may account for the disparity of results that have been reported. Measurement error may also be an important factor. Blood pressure estimates based on multiple measurements or, preferably, 24-hour ambulatory measurements, are more reproducible than single measurements (Staessen et al. 2000). Ambulatory measurements also can decrease bias in estimates related to increases in blood pressure that can accompany clinic visits (Yang et al. 2018).

Characterization of Effects. General trends between studies showing a relationship between $\mathrm{PbB}$ and cardiovascular effects are shown in Table 2-6. Over the $\mathrm{PbB}$ range of $\leq 10->50 \mu \mathrm{g} / \mathrm{dL}$, results of epidemiological studies provide evidence for increased blood pressure and hypertension, atherosclerosis (increased intimal medial thickening and peripheral artery disease), heart disease (myocardial infarction, ischemic heart disease, left ventricular hypertrophy, cardiac arrhythmias, and angina), and increased risk of mortality due to cardiovascular diseases. The effect of $\mathrm{Pb}$ exposure on blood pressure is the most studied cardiovascular outcome. A review by Navas-Acien et al. (2007) concluded that available literature provides evidence that "is sufficient to infer a causal relationship of $\mathrm{Pb}$ exposure and hypertension" and evidence that "is suggestive but not sufficient to infer a causal relationship of $\mathrm{Pb}$ exposure with clinical cardiovascular outcomes” (cardiovascular, coronary heart disease, and stroke mortality; and peripheral arterial disease). Well-controlled studies in laboratory animals provide additional support regarding effects of Pb on blood pressure; see EPA (2014c) for additional information.

\section{Table 2-6. Overview of Cardiovascular Effects in Adults and Children Associated with Chronic Exposure to Lead (Pb)}

\begin{tabular}{ll}
$\begin{array}{l}\text { Mean blood lead } \\
\text { concentration }\end{array}$ & Effects associated with $\mathrm{Pb}$ \\
$(\mathrm{PbB})(\mu \mathrm{g} / \mathrm{dL})$ & exposure \\
\hline$\leq 10$ & $\begin{array}{l}\text { Increased blood pressure and } \\
\text { hypertension }\end{array}$
\end{tabular}

References

Almeida Lopes et al. 2017; Al-Saleh et al. 2005; Barry et al. 2019; Bost et al. 1999; Bushnik et al. 2014; Cheng et al. 2001; Chu et al. 1999; Den Hond et al. 2002; Disha et al. 2019; Elmarsafawy et al. 2006; Faramawi et al. 2015; Gambelunghe et al. 2016; Gerr et al. 2002; Glenn et al. 2003; Gump et al. 2005, 2011; Hense et al. 1993; Hu et al. 1996a; Korrick et al. 1999; Lee et al. 2016a, 2016b; Martin et al. 2006; Muntner et al. 2005; Nash et al. 2003; Obeng-Gyasi and Obeng-Gyasi 2018; Park et al. 2009b; Perlstein et al. 2007; Proctor et al. 1996; Rothenberg et al. 2002; Schwartz 1995; Scinicariello et al. 2010, 2011; Vupputuri et al. 2003; Wells et al. 2011; Yang et al. 2017, 2018; Yazbeck et al. 2009; Zhang et al. 2011; Zota et al. 2013 


\section{Table 2-6. Overview of Cardiovascular Effects in Adults and Children Associated with Chronic Exposure to Lead (Pb)}

\begin{tabular}{|c|c|c|}
\hline $\begin{array}{l}\text { Mean blood lead } \\
\text { concentration } \\
(\mathrm{PbB})(\mu \mathrm{g} / \mathrm{dL})\end{array}$ & $\begin{array}{l}\text { Effects associated with } \mathrm{Pb} \\
\text { exposure }\end{array}$ & References \\
\hline & Atherosclerosis $^{a}$ & $\begin{array}{l}\text { Ari et al. 2011; Muntner et al. 2005; Navas-Acien } \\
\text { et al. 2004; }\end{array}$ \\
\hline & $\begin{array}{l}\text { Heart disease }{ }^{b} \text { and cardiac } \\
\text { function }\end{array}$ & $\begin{array}{l}\text { Chen et al. 2017; Cheng et al. 1998; Eum et al. } \\
\text { 2011; Jain et al. 2007; Jing et al. 2019; Park et } \\
\text { al. 2009a }\end{array}$ \\
\hline & $\begin{array}{l}\text { Mortality due to cardiovascular } \\
\text { disease }\end{array}$ & $\begin{array}{l}\text { Aoki et al. 2016; Khalil et al. 2009; Lanphear et } \\
\text { al. 2018; Menke et al. 2006; Schober et al. 2006; } \\
\text { Weisskopf et al. } 2009\end{array}$ \\
\hline \multirow[t]{4}{*}{$>10-30$} & $\begin{array}{l}\text { Increased blood pressure and } \\
\text { hypertension }\end{array}$ & $\begin{array}{l}\text { Coate and Fowles 1989; Factor-Litvak et al. } \\
\text { 1999; Grandjean et al. 1989; Han et al. 2018; } \\
\text { Harlan et al. 1985; Møller and Kristensen 1992; } \\
\text { Pirkle et al. 1985; Rabinowitz et al. } 1987\end{array}$ \\
\hline & Atherosclerosis ${ }^{a}$ & Pocock et al. 1988; Poreba et al. 2011, 2012 \\
\hline & $\begin{array}{l}\text { Heart disease }{ }^{b} \text { and cardiac } \\
\text { function }\end{array}$ & Karakulak et al. 2019; Poreba et al. 2013 \\
\hline & $\begin{array}{l}\text { Mortality due to cardiovascular } \\
\text { disease }\end{array}$ & $\begin{array}{l}\text { Barry and Steenland 2019; Lustberg and } \\
\text { Silbergeld 2002; Min et al. 2017; Schober et al. } \\
\text { 2006; Steenland et al. } 2017\end{array}$ \\
\hline \multirow[t]{4}{*}{$>30-50$} & $\begin{array}{l}\text { Increased blood pressure and } \\
\text { hypertension }\end{array}$ & $\begin{array}{l}\text { Aiba et al. 1999; Al-Saleh et al. 2005; Factor- } \\
\text { Litvak et al. 1996, 1999; Ghiasvand et al. 2013; } \\
\text { Glenn et al. 2006; Rapisarda et al. 2016; } \\
\text { Weaver et al. 2008; Weiss et al. 1986, } 1988\end{array}$ \\
\hline & Atherosclerosis ${ }^{a}$ & Karakulak et al. 2017 \\
\hline & Heart disease ${ }^{b}$ & $\begin{array}{l}\text { Bockelmann et al. 2002; Jain et al. 2007; } \\
\text { Kieltucki et al. } 2017\end{array}$ \\
\hline & $\begin{array}{l}\text { Mortality due to cardiovascular } \\
\text { disease }\end{array}$ & $\begin{array}{l}\text { Barry and Steenland 2019; Gerhardsson et al. } \\
\text { 1995a; Steenland et al. } 2017\end{array}$ \\
\hline \multirow[t]{3}{*}{$>50$} & $\begin{array}{l}\text { Increased blood pressure and } \\
\text { hypertension }\end{array}$ & Kirby and Gyntelberg 1985; Were et al. 2014 \\
\hline & Atherosclerosis ${ }^{a}$ & Kirby and Gyntelberg 1985 \\
\hline & $\begin{array}{l}\text { Mortality due to cardiovascular } \\
\text { disease }\end{array}$ & $\begin{array}{l}\text { Cooper 1988; Cooper et al. 1985; Fanning 1988; } \\
\text { Gerhardsson et al. 1995a; McDonald and Potter } \\
1996\end{array}$ \\
\hline
\end{tabular}

\footnotetext{
aAtherosclerosis includes increased intimal medial thickening and peripheral artery disease.

${ }^{b}$ Heart disease includes myocardial infarction, ischemic heart disease, left ventricular hypertrophy, cardiac arrhythmias, and angina.
}

Numerous studies provide a weight of evidence for associations between $\mathrm{PbB}$ and increased blood pressure over a wide $\mathrm{PbB}$ range in adults (Table 2-6). Results of meta-analyses estimate small but consistent increases in blood pressure per doubling of $\mathrm{PbB}$. The largest meta-analysis of 31 studies published between 1980 and 2001 included a total of 58,518 subjects (Nawrot et al. 2002); blood pressure 
data from studies included in the analysis are shown in Table 2-7 and Figures 2-2 and 2-3. Nawrot et al. (2002), in an update of an earlier meta-analysis by Staessen et al. (1994), estimated the increase in systolic pressure per doubling of $\mathrm{PbB}$ to be $1 \mathrm{mmHg}(95 \% \mathrm{CI} 0.5,1.4)$ and the increase in diastolic pressure to be $0.6 \mathrm{mmHg}$ (95\% CI 0.4, 0.8). The range of mean (or median) PbBs for studies included in the analysis was 2.28-63.82 $\mu \mathrm{g} / \mathrm{dL}$. Although a PbB mean was not estimated for the entire study population, only nine studies had a mean $\mathrm{PbB}<10 \mu \mathrm{g} / \mathrm{dL}$; therefore, it is likely that the overall $\mathrm{PbB}$ mean for the entire study population was $>10 \mu \mathrm{g} / \mathrm{dL}$. Similar outcomes were observed in two other metaanalyses (Schwartz 1995; Staessen et al. 1994). A meta-analysis reported by Staessen et al. (1994) included 23 studies (published between 1984 and 1993; 33,141 subjects) and found a 1 mmHg (95\% CI $0.4,1.6)$ increase in systolic blood pressure and $0.6 \mathrm{mmHg}(95 \%$ CI 0.2, 1.0) increase in diastolic pressure per doubling of PbB. Schwartz (1995) conducted a meta-analysis that encompassed a similar time frame (15 studies published between 1985 and 1993) and found a $1.25 \mathrm{mmHg}$ (95\% CI 0.87, 1.63) increase in systolic blood pressure per doubling of $\mathrm{PbB}$ (diastolic not reported). The latter analysis included only those studies that reported a standard error (SE) on effect measurement (e.g., increase in blood pressure per doubling of PbB). Of the 15 studies included in the Schwartz (1995) analysis, 8 were also included in the Staessen et al. (1994) analysis. The estimated increase in blood pressure per doubling of $\mathrm{PbB}$ in these meta-analyses is small; however, on a population basis, the consequences of increased blood pressure includes increased risks of serious and potentially fatal effects, including atherosclerosis, stroke, and myocardial infarction. Increased blood pressure during pregnancy has been associated with $\mathrm{PbB}$ and bone $\mathrm{Pb}$ (Rothenberg et al. 2002; Wells et al. 2011; Yazbeck et al. 2009); these studies are discussed in more detail below (Effect at Blood $\mathrm{Pb}$ Levels $\leq 10 \mu \mathrm{g} / \mathrm{dL}$ ).

\section{Table 2-7. Characteristics of the Study Population in Meta-Analyses of Effects of Lead $(\mathrm{Pb})$ on Blood Pressure}

\begin{tabular}{|c|c|c|c|c|c|c|c|c|c|}
\hline & Reference & Number ${ }^{a}$ & Population $^{\mathrm{b}}$ & $\begin{array}{l}\text { Men } \\
(\%)^{\mathrm{c}}\end{array}$ & $\mathrm{HT}^{\mathrm{d}}$ & $\begin{array}{l}\text { Age } \\
\text { (years) }^{\mathrm{e}}\end{array}$ & $\mathrm{SBP}^{f}$ & $\mathrm{DBP}^{f}$ & $\begin{array}{l}\text { Lead } \\
(\mu \mathrm{g} / \mathrm{dL})^{\mathrm{g}}\end{array}$ \\
\hline $1^{\mathrm{h}}$ & $\begin{array}{l}\text { Pocock et al. 1984i,j; } \\
\text { Shaper et al. } 1981\end{array}$ & 7,379 & GP & 100 & $\mathrm{Y}$ & $\begin{array}{l}49 \\
(40-59) \\
\end{array}$ & 145 & 82 & $\begin{array}{l}15.13 \\
(2.07-66.3)^{\mathrm{a}, \mathrm{e}}\end{array}$ \\
\hline 2 & $\begin{array}{l}\text { Kromhout } 1988^{\mathrm{i}, \mathrm{j}} \\
\text { Kromhout et al. } 1985^{\mathrm{i}}\end{array}$ & 152 & GP & 100 & $\mathrm{Y}$ & $\begin{array}{l}67 \\
(57-76)\end{array}$ & 154 & 92 & $\begin{array}{l}18.23 \\
(10.77- \\
27.97)^{a, c}\end{array}$ \\
\hline 3 & $\begin{array}{l}\text { Moreau et al. 1982j, } \\
\text { 1988; Orssaud et al. } \\
1985^{\mathrm{i}, \mathrm{j}}\end{array}$ & 431 & WC & 100 & $\mathrm{Y}$ & $\begin{array}{l}41 \\
(24-55)\end{array}$ & 131 & 75 & $\begin{array}{l}18.23 \\
(8.91-49.94)^{a, e}\end{array}$ \\
\hline 4 & Weiss et al. 1986', $1988^{i}$ & 89 & WC & 100 & $\mathrm{Y}$ & $\begin{array}{l}47 \\
(30-64)\end{array}$ & 122 & 83 & $\begin{array}{l}24.45 \\
(18.65- \\
29.01)^{m, x}\end{array}$ \\
\hline
\end{tabular}


Table 2-7. Characteristics of the Study Population in Meta-Analyses of Effects of Lead (Pb) on Blood Pressure

\begin{tabular}{|c|c|c|c|c|c|c|c|c|c|}
\hline & Reference & Number ${ }^{a}$ & Population $^{b}$ & $\begin{array}{l}\text { Men } \\
(\%)^{\mathrm{c}}\end{array}$ & $\mathrm{HT}^{\mathrm{d}}$ & $\begin{array}{l}\text { Age } \\
\text { (years) }^{\mathrm{e}}\end{array}$ & $\mathrm{SBP}^{\mathrm{f}}$ & $\mathrm{DBP}^{f}$ & $\begin{array}{l}\text { Lead } \\
(\mu \mathrm{g} / \mathrm{dL})^{\mathrm{g}}\end{array}$ \\
\hline 5 & $\begin{array}{l}\text { de Kort and Zwennis } \\
1988^{\mathrm{i}, j} ; \text { de Kort et al. } \\
1987^{i}\end{array}$ & 105 & $\mathrm{BC}$ & 100 & $\mathrm{~N}$ & $\begin{array}{l}40 \\
(25-80)\end{array}$ & 136 & 83 & $\begin{array}{l}29.22 \\
(4.35-83.29)^{a, e}\end{array}$ \\
\hline 6 & $\begin{array}{l}\text { Lockett and Arbuckle } \\
1987^{i}\end{array}$ & 116 & $\mathrm{BC}$ & 100 & $\mathrm{Y}$ & $\begin{array}{l}32 \\
(?-?)\end{array}$ & 119 & 80 & $\begin{array}{l}37.5 \\
(14.92- \\
95.52)^{\mathrm{a}, \mathrm{e}}\end{array}$ \\
\hline 7 & Parkinson et al. $1987^{i}$ & 428 & $\mathrm{BC}$ & 100 & $\mathrm{Y}$ & $\begin{array}{l}36 \\
(18-60) \\
\end{array}$ & 127 & 80 & $\begin{array}{l}27.97 \\
(6.01-49.52)^{\mathrm{a}, \mathrm{c}}\end{array}$ \\
\hline 8 & Rabinowitz et al. $1987^{i}$ & 3,851 & GP & 0 & Y & $\begin{array}{l}28 \\
(18-38)\end{array}$ & 121 & 76 & $\begin{array}{l}7.04 \\
(3.73-10.15)^{a, c}\end{array}$ \\
\hline 9 & $\begin{array}{l}\text { Elwood et al. 1988ai,j, } \\
\text { 1988bk }\end{array}$ & 1,136 & GP & 100 & $\mathrm{Y}$ & $\begin{array}{l}56 \\
(49-65)\end{array}$ & 146 & 87 & $\begin{array}{l}12.64 \\
(6.01-26.11)^{\mathrm{g}, \mathrm{c}}\end{array}$ \\
\hline 10 & $\begin{array}{l}\text { Elwood et al. 1988a, } \\
\text { 1988bi,j,l }\end{array}$ & 1,721 & GP & 50 & $\mathrm{Y}$ & $\begin{array}{l}41 \\
(18-64)\end{array}$ & 127 & 78 & $\begin{array}{l}10.15 \\
(4.56-23.21)^{g, c}\end{array}$ \\
\hline 11 & $\begin{array}{l}\text { Gartside et al. 1988; } \\
\text { Harlan 1988; Harlan et } \\
\text { al. 1985; Pirkle et al. } \\
\text { 1985; Ravnskov } 1992^{\mathrm{m}}\end{array}$ & 6,289 & GP & 53 & $\mathrm{Y}$ & $\begin{array}{l}30 \\
(10-74)\end{array}$ & 127 & 80 & $\begin{array}{l}13.47 \\
(2.07-95.93)^{\mathrm{g}, \mathrm{e}}\end{array}$ \\
\hline 12 & Neri et al. $1988^{\mathrm{i}, \mathrm{j}, \mathrm{n}}$ & 288 & $\mathrm{BC}$ & 100 & $?$ & $\begin{array}{l}? \\
(?-?)\end{array}$ & $?$ & $?$ & $\begin{array}{l}45.17 \\
(6.01-65.06)^{\mathrm{a}, \mathrm{e}}\end{array}$ \\
\hline 13 & Neri et al. $1988^{\mathrm{i}, \mathrm{o}}$ & 2,193 & GP & $?$ & $\mathrm{Y}$ & $\begin{array}{l}45 \\
(25-65) \\
\end{array}$ & $?$ & $?$ & $\begin{array}{l}23.41 \\
(0-47.03)^{\mathrm{m}, \mathrm{e}} \\
\end{array}$ \\
\hline 14 & $\begin{array}{l}\text { Grandjean et al. } 1989, \\
1991^{i, p}\end{array}$ & 1,050 & GP & 48 & $\mathrm{Y}$ & $\begin{array}{l}40 \\
(40-40)\end{array}$ & $?$ & $?$ & $\begin{array}{l}11.6 \\
(3.94-60.09)^{\mathrm{a}, \mathrm{e}}\end{array}$ \\
\hline 15 & $\begin{array}{l}\text { Reimer and Tittelbach } \\
1989^{i}\end{array}$ & 58 & $\mathrm{BC}$ & 100 & $?$ & $\begin{array}{l}32 \\
(?-?)\end{array}$ & 134 & 81 & $\begin{array}{l}39.99 \\
(12.85- \\
70.24)^{\mathrm{a}, \mathrm{c}} \\
\end{array}$ \\
\hline 16 & Apostoli et al. $1990^{i}$ & 525 & GP & 48 & $\mathrm{Y}$ & $\begin{array}{l}45 \\
(21-60) \\
\end{array}$ & 132 & 84 & $\begin{array}{l}13.05 \\
(2.07-28.18)^{\mathrm{a}, \mathrm{e}} \\
\end{array}$ \\
\hline 17 & Morris et al. $1990^{i, j}$ & 251 & GP & 58 & $\mathrm{Y}$ & $\begin{array}{l}? \\
(23-79)\end{array}$ & $?$ & $?$ & $\begin{array}{l}7.46 \\
(4.97-38.95)^{\mathrm{a}, \mathrm{e}}\end{array}$ \\
\hline 18 & $\begin{array}{l}\text { Sharp et al. 1988i,j, } \\
1989^{i}, 1990^{i}\end{array}$ & 249 & WC & 100 & $\mathrm{~N}$ & $\begin{array}{l}43 \\
(31-65) \\
\end{array}$ & 128 & 83 & $\begin{array}{l}6.63 \\
(2.07-14.92)^{\mathrm{p}, \mathrm{e}} \\
\end{array}$ \\
\hline 19 & Staessen et al. 1984i,q & 531 & WC & 75 & $\mathrm{Y}$ & $\begin{array}{l}48 \\
(37-58)\end{array}$ & 126 & 78 & $\begin{array}{l}11.4 \\
(4.14-35.22)^{\mathrm{g}, \mathrm{e}}\end{array}$ \\
\hline 20 & $\begin{array}{l}\text { Møller and Kristensen } \\
1992^{\mathrm{i}, \mathrm{j}, \mathrm{r}}\end{array}$ & 439 & GP & 100 & $\mathrm{Y}$ & $\begin{array}{l}40 \\
(40-40) \\
\end{array}$ & $?$ & $?$ & $\begin{array}{l}13.68 \\
(4.97-60.09)^{a, e}\end{array}$ \\
\hline 21 & Hense et al. $1993^{i, j}$ & 3,364 & GP & 51 & $\mathrm{Y}$ & $\begin{array}{l}48 \\
(28-67) \\
\end{array}$ & 129 & 80 & $\begin{array}{l}7.87 \\
(1.24-37.09)^{\mathrm{a}, \mathrm{e}} \\
\end{array}$ \\
\hline 22 & Maheswaran et al. $1993^{i}$ & 809 & $\mathrm{BC}$ & 100 & $\bar{Y}$ & $\begin{array}{l}43 \\
(20-65)\end{array}$ & 129 & 84 & $\begin{array}{l}31.7 \\
(0-98.01)^{\mathrm{a}, \mathrm{e}}\end{array}$ \\
\hline 23 & Menditto et al. 1994 & 1,319 & GP & 100 & $\mathrm{Y}$ & $\begin{array}{l}63 \\
(55-75)\end{array}$ & 140 & 84 & $\begin{array}{l}11.19 \\
(6.22-24.66)\end{array}$ \\
\hline
\end{tabular}



Table 2-7. Characteristics of the Study Population in Meta-Analyses of Effects of
Lead (Pb) on Blood Pressure

\begin{tabular}{|c|c|c|c|c|c|c|c|c|c|}
\hline & Reference & Number $^{a}$ & Population $^{b}$ & $\begin{array}{l}\text { Men } \\
(\%)^{\mathrm{c}}\end{array}$ & $\mathrm{HT}^{\mathrm{d}}$ & $\begin{array}{l}\text { Age } \\
\text { (years) }^{\mathrm{e}}\end{array}$ & SBP & $\mathrm{DBP}^{f}$ & $\begin{array}{l}\text { Lead } \\
(\mu \mathrm{g} / \mathrm{dL})^{\mathrm{g}}\end{array}$ \\
\hline 24 & $\begin{array}{l}\text { Hu et al. 1996a; Proctor } \\
\text { et al. } 1996^{\mathrm{s}}\end{array}$ & 798 & GP & 100 & $\mathrm{Y}$ & $\begin{array}{l}66 \\
(43-93) \\
\end{array}$ & 134 & 80 & $\begin{array}{l}5.59 \\
(0.41-35.02)^{\mathrm{p}, \mathrm{e}} \\
\end{array}$ \\
\hline 25 & $\begin{array}{l}\text { Staessen et al. 1996a', } \\
\text { 1996bi,t }\end{array}$ & 728 & GP & 49.3 & $\mathrm{Y}$ & $\begin{array}{l}46 \\
(20-82)\end{array}$ & 130 & 77 & $\begin{array}{l}9.12 \\
(1.66-72.52)^{\mathrm{g}, \mathrm{e}}\end{array}$ \\
\hline 26 & Sokas et al. $1997^{u}$ & 186 & $\mathrm{BC}$ & 99 & Y & $\begin{array}{l}43 \\
(18-79)\end{array}$ & 130 & 85 & $\begin{array}{l}7.46 \\
(2.07-30.04)^{\mathrm{p}, \mathrm{e}}\end{array}$ \\
\hline 27 & Bost et al. 1999 & 5,326 & GP & 48 & Y & $\begin{array}{l}48 \\
(16-?) \\
\end{array}$ & 135 & 75 & $\begin{array}{l}63.82 \\
(?-?)^{9} \\
\end{array}$ \\
\hline 28 & Chu et al. 1999 & 2,800 & GP & 53 & Y & $\begin{array}{l}44 \\
(15-85) \\
\end{array}$ & 123 & 78 & $\begin{array}{l}6.42 \\
(0.41-69)^{\mathrm{a}, \mathrm{e}}\end{array}$ \\
\hline 29 & $\begin{array}{l}\text { Rothenberg et al. } \\
\text { 1999a, 1999b }\end{array}$ & 1,627 & GP & 0 & Y & $\begin{array}{l}27 \\
(?-?) \\
\end{array}$ & 110 & 59 & $\begin{array}{l}2.28 \\
(?-?)^{\mathrm{g}} \\
\end{array}$ \\
\hline 30 & Schwartz et al. 2000c & 543 & $\mathrm{BC}$ & 100 & Y & $\begin{array}{l}58 \\
(41-73) \\
\end{array}$ & 128 & 77 & $\begin{array}{l}4.56 \\
(1.04-20.1)^{\mathrm{a}, \mathrm{e}}\end{array}$ \\
\hline 31 & Den Hond et al. $2001^{v}$ & 13,781 & GP & 53.2 & $\mathrm{Y}$ & $\begin{array}{l}48 \\
(20-90)\end{array}$ & 125 & 73 & $\begin{array}{l}3.11 \\
(0.62-55.94)^{\mathrm{g}, \mathrm{e}}\end{array}$ \\
\hline
\end{tabular}

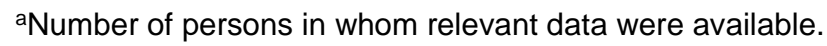

bStudy population: $\mathrm{BC}=$ blue collar workers; $\mathrm{GP}=$ sample from general population; $\mathrm{WC}=$ white collar employees.

'Men: Percentage of men.

${ }^{\mathrm{d}} \mathrm{HT}$ : Indicates whether the sample included $(\mathrm{Y}=$ yes $)$ or did not include $(\mathrm{N}=\mathrm{no})$ hypertensive patients.

${ }^{\mathrm{e}} \mathrm{Age}$ : Mean age or midpoint of age span (range or approximate range given between parentheses).

fSBP, DBP: Mean systolic and diastolic blood pressures.

gLead: Measure of central tendency: $A=$ arithmetic mean; $G=$ geometric mean; $M=$ midpoint of range;

$\mathrm{P}=\mathrm{P}_{50}$ (median). The spread of blood lead is given between parentheses: $c=P_{5}-P_{05}$ interval; $P_{10}-P_{90}$ interval, or interval equal to 4 times the standard deviation; $\mathrm{e}=$ extremes; $\mathrm{x}=$ approximate limits of distribution.

hNumber refers to reference in Figures 2-2 and 2-3.

IIncluded in the Staessen et al. (1994) meta-analysis.

inncluded in the Schwartz (1995) meta-analysis.

${ }^{\mathrm{k}}$ Caerphilly Study.

'Welsh Heart Program.

mNHANES (National Health and Nutrition Examination Survey).

nFoundry workers.

${ }^{\circ}$ Canadian Health Survey.

PGlostrup Population Study, cross-sectional analysis (1976).

qLondon Civil Servants.

'Glostrup Population Study, longitudinal analysis (1976-1987).

sNormative Aging Study.

tPheeCad (Public Health and Environmental Exposure to Cadmium) Study.

uBecause of missing information, only the effect in whites is included.

vNHANES III.

Source: Nawrot et al. 2002 


\section{Figure 2-2. Change in the Systolic Pressure Associated with a Doubling of the Blood Lead Concentration (PbB)*}

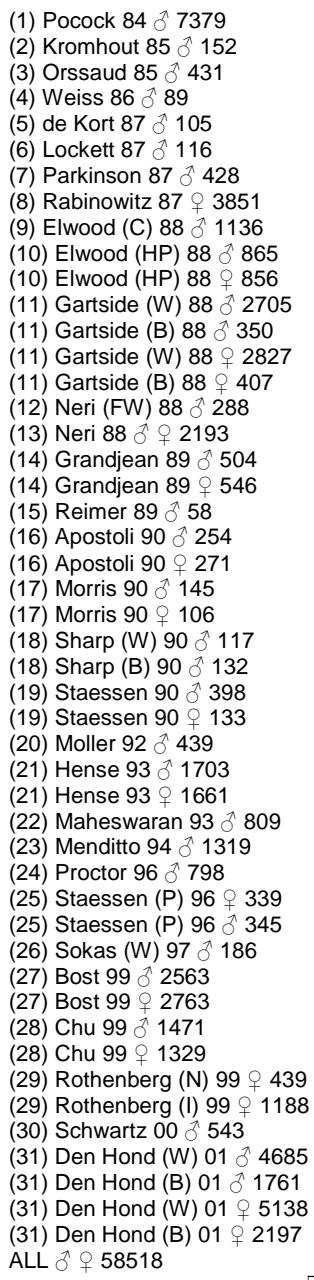

(1) Pocock 847379

(2) Kromhout $85 \AA 152$

(3) Orssaud 85431

(4) Weiss 8689

(7) Parkinson 87,428

(9) Elwood (C) 88 1136

(10) Elwood (HP) 88 ते 865

(10) Elwood (HP) 88 우 856

(11) Gartside (W) 88 त 2705

(11) Gartside (B) 883350

(11) Gartside (B) $88 \circ 407$

(12) Neri (FW) 88 288

(13) Neri 88 우 2193

(14) Grandjean 89504

(14) Grandjean $89+546$

(19) Staessen 90398

(19) Staessen 90 ㅇ 133

(20) Moller 92 o 439

(21) Hense $93 \circ 1661$

(22) Maheswaran 93809

(23) Menditto $94 \quad 1319$

(24) Proctor 96798

(25) Staessen (P) $96+339$

(26) Sokas (W) 97186

(27) Bost 99 त 2563

(27) Bost 99 ㅇ 2763

(28) Chu 99 1471

(29) Rothenberg (N) 99 우 439

(29) Rothenberg (I) 99 우 1188

(30) Schwartz $00 \quad 543$

(31) Den Hond (W) 014685

(31) Den Hond (B) 011761

(31) Den Hond (B) $01+2197$

ALL $\gtrsim+q 58518$

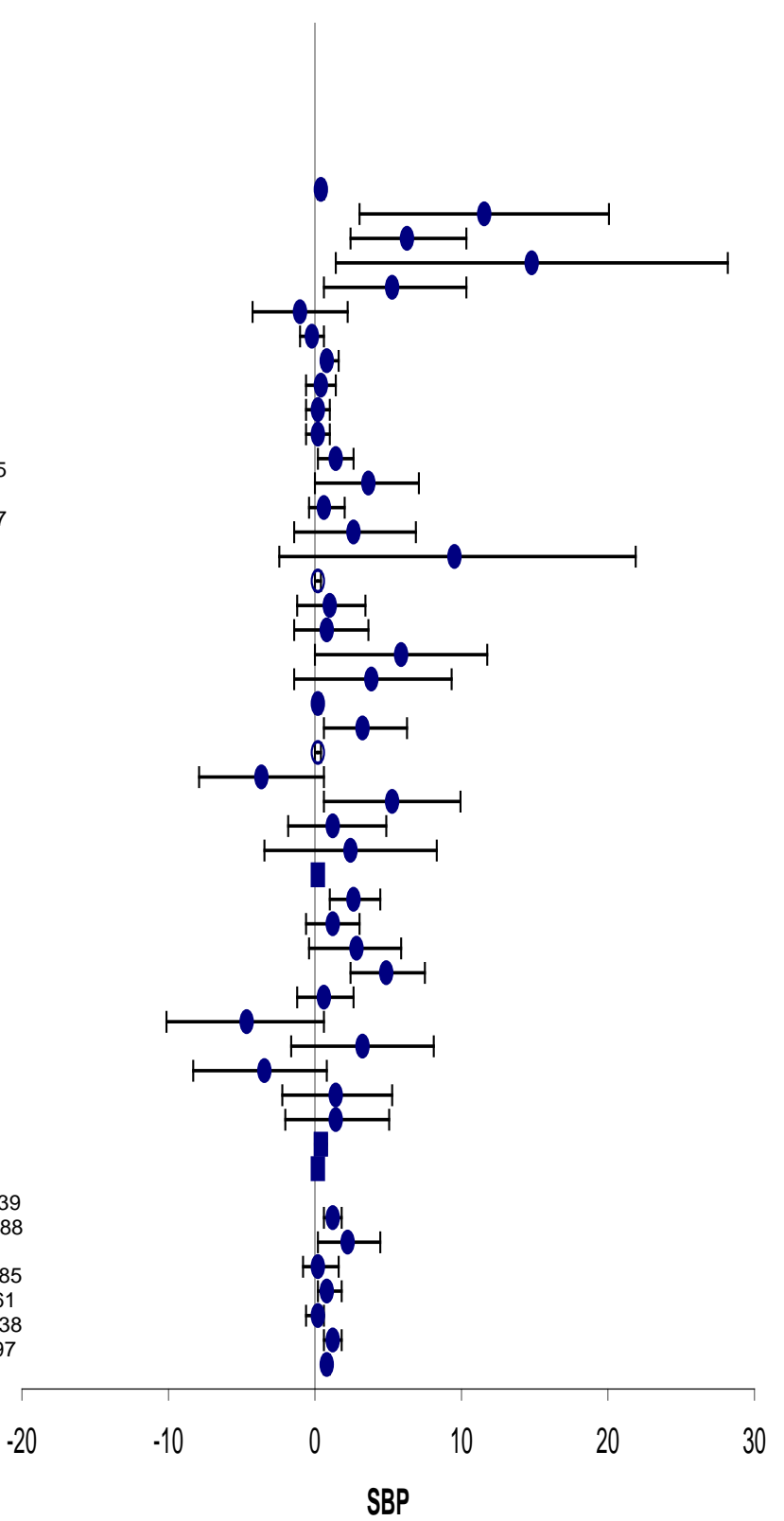

*Data were digitized from Nawrot et al. 2002. Circles represent means (mmHg) of individual groups; squares represent combined groups; and open circles represent nonsignificant associations (plotted as zero). Bars represent $95 \%$ confidence limits. See Table $2-7$ for more details on study groups.

$\mathrm{B}=$ blacks; $\mathrm{C}=$ Caerphilly Study; $\mathrm{CS}=$ civil servants; FW = foundry workers; HP = Welsh Heart Program;

$\mathrm{I}=$ immigrants; $\mathrm{NI}=$ non-immigrants; $\mathrm{P}=$ Public Health and Environmental Exposure to Cadmium Study; $\mathrm{W}=$ whites 


\section{Figure 2-3. Change in the Diastolic Pressure Associated with a Doubling of the Blood Lead Concentration (PbB)*}

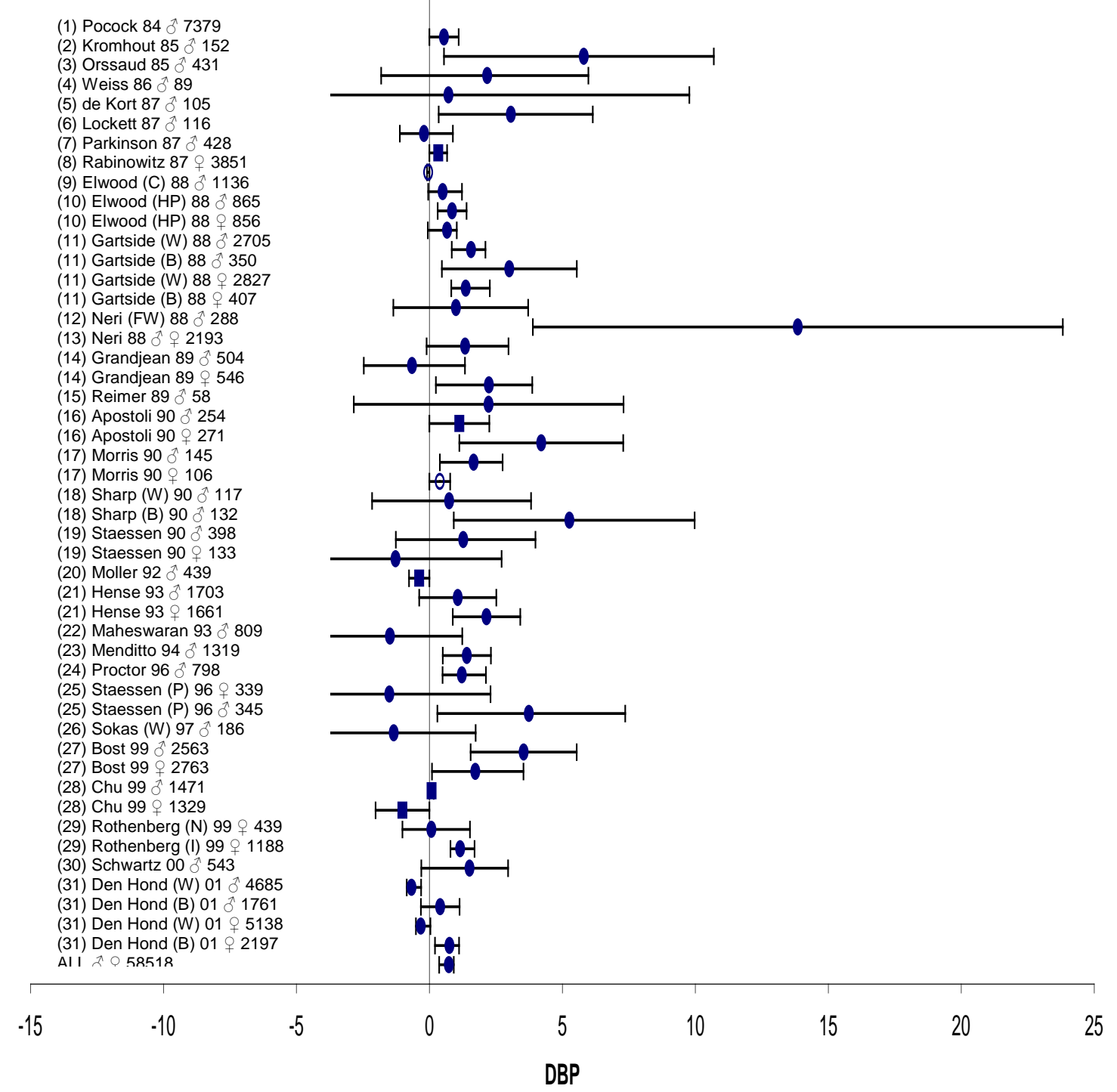

*Data were digitized from Nawrot et al. 2002. Circles represent means (mmHg) of individual groups; squares represent combined groups; and open circles represent nonsignificant associations (plotted as zero). Bars represent 95\% confidence limits. See Table 2-7 for more details on study groups.

B = blacks; $C=$ Caerphilly Study; CS = civil servants; FW = foundry workers; HP = Welsh Heart Program;

$\mathrm{I}=$ immigrants; $\mathrm{N}=$ non-immigrants; $\mathrm{P}=$ Public Health and Environmental Exposure to Cadmium Study; $\mathrm{W}=$ whites 
Within individual studies, dose-effect relationships are evident at $\mathrm{PbB} \leq 10 \mu \mathrm{g} / \mathrm{dL}$. A positive dose-effect was observed for PbB and diastolic blood pressure (Zota et al. 2013). An observed positive dose-effect was observed for tibia $\mathrm{Pb}$ concentration and hypertension (Hu et al. 1996a). No dose-effect was observed for $\mathrm{PbB}$ and pulse pressure (PP), although a positive dose-effect was observed for tibia $\mathrm{Pb}$ and $\mathrm{PP}$ (Perlstein et al. 2007). In a cross-sectional study of women, diastolic hypertension was observed to have a positive dose-effect when pre- and postmenopausal women were analyzed together and when postmenopausal women were analyzed alone. In contrast, a dose-effect relationship was not observed for $\mathrm{PbB}$ and hypertension in a cross-sectional study of men and women (Muntner et al. 2005). A positive dose-effect relationship was observed for PbB and peripheral artery disease (PAD) (Muntner et al. 2005). In men, tibia blood levels had a positive dose-effect relationship with QT interval, but a negative doseeffect relationship with atrioventricular conduction defect (Eum et al. 2011). Studies have also found positive dose-effect relationships between mortality due to cardiovascular disease, myocardial infarction, and stroke and PbB (Menke et al. 2006; Schober et al. 2006).

Several studies have evaluated associations between PbB and cardiovascular function in children (Ahn et al. 2018; Factor-Litvak et al. 1999, 1996; Gump et al. 2005, 2011; Kapuku et al. 2006; Khalil et al. 2009, 2010; Lustberg and Silbergeld 2002; Menke et al. 2006; Schober et al. 2006; Zhang et al. 2011). Results show alterations in cardiovascular function, including increases in blood pressure and altered cardiovascular function under stress (decreased stroke volume and cardiac output) over a PbB range from $<10$ to approximately $40 \mu \mathrm{g} / \mathrm{dL}$.

Effect at Blood Pb Levels $\leq 10 \mu \boldsymbol{\mu g} / \mathbf{d L}$. Studies investigating relationships between $\mathrm{PbB} \leq 10 \mu \mathrm{g} / \mathrm{dL}$ and cardiovascular effects have evaluated effects on blood pressure (including hypertension), atherosclerosis, heart disease (alterations in cardiac conduction and ischemic heart disease), and death due to cardiovascular disease.

Increased blood pressure and hypertension. Numerous studies of large populations show associations between $\mathrm{PbB} \leq 10 \mu \mathrm{g} / \mathrm{dL}$ and increased systolic and/or diastolic blood pressure and increased risk of hypertension and prehypertension (see Table 2-8). The lowest mean PbB associated with increased systolic and diastolic is $1.33 \mu \mathrm{g} / \mathrm{dL}$ (Obeng-Gyasi and Obeng-Gyasi 2018). A few studies did not show associations between $\mathrm{PbB}$ and blood pressure parameters; however, positive associations between bone $\mathrm{Pb}$ concentrations and blood pressure at concomitant $\mathrm{PbB} \leq 10 \mu \mathrm{g} / \mathrm{dL}$ were observed (Barry et al. 2019; Gerr et al. 2002; Hu et al. 1996a; Korrick et al. 1999; Zhang et al. 2011). Studies are briefly summarized 
Table 2-8. Summary of Epidemiological Studies Evaluating Effects on Blood Pressure at Mean Blood Lead Concentration $(\mathrm{PbB}) \leq 10 \mu \mathrm{g} / \mathrm{dL}$

\begin{tabular}{|c|c|c|c|}
\hline Reference and study population & $\mathrm{PbB}(\mu \mathrm{g} / \mathrm{dL})$ & Outcome evaluated & Result ${ }^{a, b}$ \\
\hline \multicolumn{4}{|c|}{ Women and men combined (not stratified by sex)c } \\
\hline \multirow{3}{*}{$\begin{array}{l}\text { Almeida Lopes et al. } 2017 \\
\text { Population-based study; } n=948 \text { adults } \\
\text { ( } \geq 40 \text { years of age) }\end{array}$} & \multirow{3}{*}{$\begin{array}{l}\text { Gmean: } 1.97 \\
\text { Quartiles: } \\
\text { - } \quad \text { Q1: } \leq 1.32 \\
\text { - } \quad \text { Q2: } 1.32-1.93 \\
\text { - } \quad \text { Q3: } 1.93-2.76 \\
\text { - } \quad \text { Q4: }>2.76\end{array}$} & SBP & $\begin{array}{l}\text { Change in SBP, Q4: }-0.00(-0.00,-0.00) \text {; } \\
\text { p-trend: } 0.002^{*}\end{array}$ \\
\hline & & DBP & $\begin{array}{l}\text { Change in DBP, Q4: } 0.06(0.04,0.09) \\
\text { p-trend: }<0.001^{*}\end{array}$ \\
\hline & & Hypertension & OR, Q4: $2.54(1.17,5.53)^{*}$ \\
\hline \multirow{2}{*}{$\begin{array}{l}\text { Faramawi et al. } \mathbf{2 0 1 5}^{\mathbf{d}} \\
\text { Cross-sectional study; } n=13,757\end{array}$} & \multirow[t]{2}{*}{ Mean: 3.44} & SBP & $\begin{array}{l}\beta \text { (SE), } \mathrm{mmHg} \text { for change in blood pressure } \\
\text { SD per } \mu \mathrm{g} / \mathrm{dL}: 0.07(0.02) ; p<0.01^{*}\end{array}$ \\
\hline & & DBP & $\begin{array}{l}\beta(\mathrm{SE}), \text { for change in blood pressure SD per } \\
\mu \mathrm{g} / \mathrm{dL}: 0.04(0.03) ; p=0.08\end{array}$ \\
\hline \multirow{3}{*}{$\begin{array}{l}\text { Gambelunghe et al. } 2016 \\
\text { Cross-section study; } n=4,452 \text { adults }\end{array}$} & \multirow{3}{*}{$\begin{array}{l}\text { Mean: } 2.8 \\
\text { Quartiles: } \\
\text { - } \quad \text { Q1: } 0.15-1.9 \\
\text { - } \quad \text { Q2: } 1.9-2.5 \\
\text { - } \quad \text { Q3: } 2.5-3.3 \\
\text { - } \quad \text { Q4: 3.3-25.8 }\end{array}$} & SBP & $\begin{array}{l}\text { Regression coefficient, } \beta, Q 4 \text { versus } Q 1-Q 3 \\
(\mathrm{mmHg}): 1.7 ; p=0.01^{*}\end{array}$ \\
\hline & & DBP & $\begin{array}{l}\text { Regression coefficient, } \beta, Q 4 \text { versus } Q 1-Q 3 \\
(\mathrm{mmHg}): 1.3 ; p<0.001^{*}\end{array}$ \\
\hline & & Hypertension & $\begin{array}{l}\text { OR, Q4 versus Q1-Q3: } 1.3(1.1-1.5) \\
p=0.004^{*}\end{array}$ \\
\hline Lee et al. $2016 b$ & $\begin{array}{l}\text { Study population mean not } \\
\text { reported } \\
\text { Quartiles: }\end{array}$ & Prehypertension & \multirow{2}{*}{$\begin{array}{ll}\text { OR, versus Q1: } \\
\text { - } \quad \text { Q2: } 1.24(1.04,1.48)^{*} \\
-\quad \text { Q3: } 1.27(1.06,1.52)^{\star} \\
-\quad \text { Q4: } 1.30(1.07,1.60)^{*} \\
-\quad \text { p-trend: } 0.0152^{*}\end{array}$} \\
\hline Cross-sectional study; $n=8,493$ adults & $\begin{array}{l}\text { - } \quad \text { Q1: } 0.206-1.539 \\
\text { - } \quad \text { Q2: } 1.540-2.056 \\
\text { - } \quad \text { Q3: } 2.057-2.716 \\
\text { - } \quad \text { Q4: } 2.717-24.532\end{array}$ & & \\
\hline Martin et al. 2006 & Mean: 3.5 & SBP & $\begin{array}{l}\beta, \mathrm{mmHg} \text { per } 1 \mu \mathrm{g} / \mathrm{dL}: 0.99(0.47,1.51) ; \\
p<0.01^{*}\end{array}$ \\
\hline \multicolumn{2}{|l|}{$\begin{array}{l}\text { Cross-sectional study; } n=964 \text { (ages } 50- \\
70 \text { years) }\end{array}$} & DBP & $\begin{array}{l}\beta, \mathrm{mmHg} \text { per } 1 \mu \mathrm{g} / \mathrm{dL}: 0.51(0.24,0.79) ; \\
p<0.01^{*}\end{array}$ \\
\hline
\end{tabular}


Table 2-8. Summary of Epidemiological Studies Evaluating Effects on Blood Pressure at Mean Blood Lead Concentration $(\mathrm{PbB}) \leq 10 \mu \mathrm{g} / \mathrm{dL}$

\begin{tabular}{ll}
\hline Reference and study population & $\mathrm{PbB}(\mu \mathrm{g} / \mathrm{dL})$ \\
\hline Zota et al. 2013 $^{\text {d }}$ & Mean: 1.69 \\
& Quintiles: \\
Cross-sectional study; $\mathrm{n}=8,194$ (ages & $\bullet$ Q1: $\leq 1.05$ \\
$40-65$ years) & Q Q2: $1.06-1.44$ \\
& Q $3: 1.45-1.90$ \\
& Q4: $1.91-2.69$ \\
& Q Q5: $>2.70$ \\
\hline Obeng-Gyasi and Obeng-Gyasi 2018 & Mean: 1.33
\end{tabular}

Cross-sectional study; $n=22,747$ adults

\section{Women and men (stratified by sex)}

Bost et al. 1999

Cross-sectional study; $n=2,563$ males and 2,763 females

\begin{tabular}{ll} 
Outcome evaluated & Result $^{\mathrm{a}, \mathrm{b}}$ \\
$\begin{array}{l}\text { Elevated SBP } \\
(\geq 140 \mathrm{mmHg})\end{array}$ & OR (Q5): $1.23(0.92,1.65) ; \mathrm{p}-$ \\
\hline Elevated DBP & OR (Q3): 1.56 (1.11, 2.19)* \\
$(\geq 90 \mathrm{mmHg})$ & OR (Q4): $\mathbf{1 . 8 0}(\mathbf{1 . 2 4}, \mathbf{2 . 6 0})^{*}$ \\
& $\begin{array}{l}\text { OR (Q5): } \mathbf{1 . 7 7}(\mathbf{1 . 2 5}, \mathbf{2 . 5 0})^{*} \\
\text { p-trend } \mathbf{0 . 0 0 0 2}\end{array}$
\end{tabular}

SBP

$\beta$, increase in blood pressure $(\mathrm{mmHg})$ per unit increased in In PbB: 0.238 (0.122, 0.355); $\mathrm{p}=0.0001^{*}$

DBP $\quad \beta$, increase in blood pressure $(\mathrm{mmHg})$ per unit increased in In PbB: 0.132 (0.049, 0.215); $p=0.002^{*}$

\begin{tabular}{|c|c|c|}
\hline \multirow{4}{*}{$\begin{array}{l}\text { Mean } \\
\text { - } \quad \mathrm{M}: 3.7 \\
\text { - } \quad \mathrm{F}: 2.6\end{array}$} & \multirow[t]{2}{*}{ SBP } & $\begin{array}{l}\text { M: no association with PbB (regression } \\
\text { coefficient not reported) }\end{array}$ \\
\hline & & $\begin{array}{l}\text { F: no association with } \mathrm{PbB} \text { (regression } \\
\text { coefficient not reported) }\end{array}$ \\
\hline & \multirow[t]{2}{*}{ DBP } & $\mathrm{M}: \beta$, per doubling of $\mathrm{PbB}: 0.78(0.01,1.55)^{*}$ \\
\hline & & F: regression coefficients not reported \\
\hline \multirow{5}{*}{$\begin{array}{l}\text { Mean } \\
\text { - } \quad \text { All: } 1.64 \\
\text { - } \quad \text { Non-hypertensive: } 1.59 \\
\text { - } \quad \text { Hypertensive: } 1.74\end{array}$} & \multirow[t]{3}{*}{ SBP } & $\begin{array}{l}\text { All } \beta, \mathrm{mmHg} \text { per } 1 \mu \mathrm{g} / \mathrm{dL}: 1.85(-0.20,3.90) \text {; } \\
\mathrm{p}=0.075\end{array}$ \\
\hline & & $\begin{array}{l}M \beta, m m H g \text { per } 1 \mu \mathrm{g} / \mathrm{dL}: 2.17(-0.08,4.42) \\
p=0.058\end{array}$ \\
\hline & & $\begin{array}{l}F \beta, m m H g \text { per } 1 \mu \mathrm{g} / \mathrm{dL}: 0.76(-2.72,4.24) \\
p=0.656\end{array}$ \\
\hline & \multirow[t]{2}{*}{ DBP } & $\begin{array}{l}\text { All } \beta, \mathrm{mmHg} \text { per } 1 \mu \mathrm{g} / \mathrm{dL}: 1.91(0.75,3.08) ; \\
p=0.002^{*}\end{array}$ \\
\hline & & $\begin{array}{l}M \beta, m m H g \text { per } 1 \mu g / d L: 2.36(0.94,3.79) ; \\
p=0.002^{*}\end{array}$ \\
\hline
\end{tabular}


Table 2-8. Summary of Epidemiological Studies Evaluating Effects on Blood Pressure at Mean Blood Lead Concentration $(\mathrm{PbB}) \leq 10 \mu \mathrm{g} / \mathrm{dL}$

\begin{tabular}{|c|c|c|c|}
\hline Reference and study population & $\mathrm{PbB}(\mu \mathrm{g} / \mathrm{dL})$ & Outcome evaluated & Result ${ }^{a, b}$ \\
\hline & & & $\begin{array}{l}F \beta, m m H g \text { per } 1 \mu g / d L: 1.43(-0.51,3.38) \\
p=0.142\end{array}$ \\
\hline & & Hypertension & $\begin{array}{l}\text { All } \beta, \mathrm{mmHg} \text { per } 1 \mu \mathrm{g} / \mathrm{dL}:-3.87(-7.46,-0.29) \text {; } \\
\mathrm{p}=0.035^{*}\end{array}$ \\
\hline & & & $\begin{array}{l}\mathrm{M} \beta, \mathrm{mmHg} \text { per } 1 \mu \mathrm{g} / \mathrm{dL}:-6.37(-15.02,2.29) ; \\
\mathrm{p}=0.142\end{array}$ \\
\hline & & & $\begin{array}{l}\mathrm{F} \beta, \mathrm{mmHg} \text { per } 1 \mu \mathrm{g} / \mathrm{dL}:-4.18(-8.78,0.42) \\
p=0.073\end{array}$ \\
\hline & $\begin{array}{l}\text { Mean } \\
-\quad \text { M: } 7.3\end{array}$ & SBP & $\begin{array}{l}M \beta(S E), m m H g \text { per } 1 \log _{10} \mu g / d L: \\
0.185(0.076) ; p=0.015^{*}\end{array}$ \\
\hline \multirow[t]{3}{*}{$\begin{array}{l}\text { Population-based survey study; } \\
n=1,471 \text { males and } 1,329 \text { females }\end{array}$} & - $\quad F: 5.7$ & & $\begin{array}{l}F \beta(S E), m m H g \text { per } 1 \log _{10} \\
\mu g / d L:-0.057 \text { (0.109); } p=0.603\end{array}$ \\
\hline & & DBP & $\begin{array}{l}\text { M } \beta \text { (SE), mmHg per } 1 \log _{10} \mu g / d L: \\
0.075(0.053) ; p=0.159\end{array}$ \\
\hline & & & $\begin{array}{l}\mathrm{F} \beta(\mathrm{SE}), \mathrm{mmHg} \text { per } 1 \log _{10} \\
\mu \mathrm{g} / \mathrm{dL}:-0.083(0.072) ; p=0.250\end{array}$ \\
\hline \multirow{4}{*}{$\begin{array}{l}\text { Hense et al. } 1993 \\
\text { Population-based survey study; } \\
n=1,703 \text { males and } 1,661 \text { females }\end{array}$} & \multirow{4}{*}{$\begin{array}{l}\text { Mean } \\
\text { - } \quad \mathrm{M}: 8.3 \\
\text { - } \mathrm{F}: 6.0\end{array}$} & SBP & $\mathrm{M} \beta, \mathrm{mmHg}$ per $1 \mu \mathrm{g} / \mathrm{dL}: 0.29(0.08,0.49)^{*}$ \\
\hline & & & $\mathrm{F} \beta, \mathrm{mmHg}$ per $1 \mu \mathrm{g} / \mathrm{dL}: 0.17(-0.14,0.48)$ \\
\hline & & DBP & $\mathrm{M} \beta, \mathrm{mmHg}$ per $1 \mu \mathrm{g} / \mathrm{dL}: 0.08(-0.06,0.23)$ \\
\hline & & & F $\beta, m m H g$ per $1 \mu \mathrm{g} / \mathrm{dL}: 0.29(0.09,0.49)^{*}$ \\
\hline
\end{tabular}


2. HEALTH EFFECTS

Table 2-8. Summary of Epidemiological Studies Evaluating Effects on Blood Pressure at Mean Blood Lead Concentration $(\mathrm{PbB}) \leq 10 \mu \mathrm{g} / \mathrm{dL}$

\begin{tabular}{|c|c|c|c|}
\hline Reference and study population & $\mathrm{PbB}(\mu \mathrm{g} / \mathrm{dL})$ & Outcome evaluated & Result ${ }^{a, b}$ \\
\hline \multirow{9}{*}{$\begin{array}{l}\text { Lee et al. 2016a } \\
\text { Cross-sectional study; } n=5,920 \text { men } \\
\text { and } 6,059 \text { women }\end{array}$} & \multirow{2}{*}{$\begin{array}{l}\text { Gmean }(95 \% \mathrm{Cl}) \\
\text { - } \quad \text { Men: } 2.396\end{array}$} & \multirow[t]{2}{*}{ SBP } & M difference, T3 versus T1: $0.25(-0.90,1.41)$ \\
\hline & & & F difference, T3 versus T1: $1.48(0.29,2.67)$ \\
\hline & \multirow{2}{*}{$\begin{array}{l}\text { - Women: } 1.919 \\
\text { Tertiles: } \\
\text { - } \quad \text { Men }\end{array}$} & \multirow[t]{2}{*}{ DBP } & M difference, T3 versus T1 : $0.73(-0.12,1.60)$ \\
\hline & & & $\begin{array}{l}\text { F difference, T3 versus T1: } 1.059 \text { (0.308, } \\
1.811)\end{array}$ \\
\hline & o T2: $2.096-2.886$ & \multirow[t]{2}{*}{ Hypertension } & M OR, T3: $0.88(0.72,1.07)$ \\
\hline & \multirow{4}{*}{$\begin{array}{l}\text { o T3: }>2.886 \\
\text { Women } \\
\text { o T1: }<1.516 \\
\text { o T2: } 1.516-2.147 \\
\text { o } \quad \text { T3: }>2.14\end{array}$} & & F OR, T3: $1.26(0.999,1.58)$ \\
\hline & & \multirow[t]{3}{*}{ Prehypertension } & M OR, T3: $0.95(0.79,1.16)$ \\
\hline & & & F OR, T3: $1.22(1.01,1.48)^{*}$ \\
\hline & & & \\
\hline \multicolumn{4}{|l|}{ Men onlyc } \\
\hline \multirow{2}{*}{$\begin{array}{l}\text { An et al. } 2017 \\
\text { Cross-sectional study; } n=310 \text { male } \\
\text { smelters ( } 21-61 \text { years of age) }\end{array}$} & \multirow[t]{2}{*}{ Gmean: 5.839} & SBP & $\begin{array}{l}\beta, \text { per doubling of } \mathrm{PbB}:-0.636(-2.661,1.389) \text {; } \\
p=0.537\end{array}$ \\
\hline & & DBP & $\begin{array}{l}\beta, \text { per doubling of } \mathrm{PbB}:-1.182(-2.763,0.399) ; \\
p=0.142\end{array}$ \\
\hline $\begin{array}{l}\text { Barry et al. } 2019 \\
\text { Cross-sectional study; } n=211 \text { male } \mathrm{Pb} \\
\text { workers }\end{array}$ & 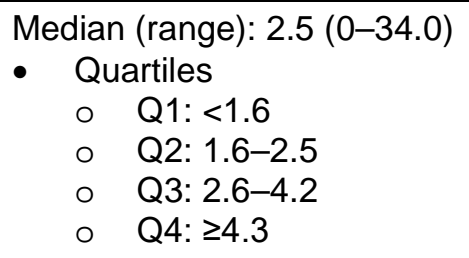 & SBP & $\begin{array}{l}\text { Regression coefficient (SE) for } \\
\text { PbB Q4: } 7.33(4.40) ; p=0.10 \\
\text { PbB continuous: } 0.19(0.30) 0.52 \\
\text { Bone Pb Q4: } 5.32(5.26) ; p=0.31 \\
\text { Bone Pb continuous: } \mathbf{0 . 3 6}(\mathbf{0 . 1 5 )} ; \mathbf{p}=\mathbf{0 . 0 2 *}\end{array}$ \\
\hline & $\begin{array}{l}\text { Bone } \mathrm{Pb} \text { (tibia) median, } \mu \mathrm{g} / \mathrm{g} \\
\text { (range): } 13.8 \text { (0-127.3) } \\
\begin{array}{ll}\text { - } & \text { Bone Pb quartiles: } \\
\text { o } & \text { Q1: < } \\
\text { o } & \text { Q2: } 9.6-13.7 \\
\text { o } & \text { Q3: } 13.8-19.5 \\
\text { o } & \text { Q4: } \geq 19.6\end{array}\end{array}$ & & \\
\hline
\end{tabular}




\section{Table 2-8. Summary of Epidemiological Studies Evaluating Effects on Blood Pressure at Mean Blood Lead Concentration $(\mathrm{PbB}) \leq 10 \mu \mathrm{g} / \mathrm{dL}$}

\begin{tabular}{l} 
Reference and study population \\
\hline Cheng et al. 2001 \\
Longitudinal study; $n=833$ men \\
Analysis for hypertension limited to \\
474 participants who had no history of \\
definite hypertension; analysis for SBP \\
limited to 519 participants who were \\
free from definite hypertension at \\
baseline
\end{tabular}

baseline

\section{$\mathrm{PbB}(\mu \mathrm{g} / \mathrm{dL})$}

PbB mean (all): 6.09

Tibia $\mathrm{Pb}(\mu \mathrm{g} / \mathrm{g})$

- Definite: 22.69

Patella $\mathrm{Pb}(\mu \mathrm{g} / \mathrm{g})$

- Borderline: 33.73

- Definite: 32.72
- Borderline: 23.46

\section{Outcome evaluated Resulta,b}

Hypertension (borderline RR, per 1 SD increase in PbB: $1.00(0.76,1.33)$ and definite)

$\mathrm{RR}$, per $1 \mathrm{SD}$ increase in tibia $\mathrm{Pb}: 1.22(0.95$, 1.57)

RR, per 1 SD increased in patella Pb: 1.29 $(1.04,1.61) ; p<0.05^{*}$

SBP RR, per 1 SD increase in PbB: -0.13 (-1.35, 1.09)

RR, per 1 SD increase in tibia Pb: 1.37 (0.02, 2.73); $p<0.05^{\star}$

$\mathrm{RR}$, per $1 \mathrm{SD}$ increased in patella $\mathrm{Pb}: 0.57$ $(-0.71,1.84)$

Elmarsafawy et al. $2006^{\mathbf{e}}$
Cross-sectional study; $\mathrm{n}=471$

Mean

Hypertension

- Low $\mathrm{Ca}^{2+}$ intake: 6.6

- High $\mathrm{Ca}^{2+}$ intake: 6.6

Glenn et al. 2003 Mean: 4.6

Occupational longitudinal study; $n=496$

Hu et al. $1996 a^{e}$

Mean

- Cases: 6.9

Case-control study of men $(n=146)$ with $\quad$ Controls: 6.1

hypertension and controls $(n=444)$

Low $\mathrm{Ca}^{2+}$ : OR: $1.07(1.00,1.15)^{*}$

High Ca ${ }^{2+}$ : OR: $1.03(0.97,1.11)$

$\beta$ (SE; 95\% Cl), per 1 SD increased in PbB: $0.64(0.25 ; 0.14,1.14)^{*}$

$\beta$ (SE; 95\% Cl); per 1 SD increased in PbB: $0.09(0.17 ;-0.24,0.43)$

Risk of hypertension based on tibia $\mathrm{Pb}$ : logistic $\beta$ (SE): 0.19 (0.0078); $p=0.01^{*}$ $\mathrm{PbB}$ was not associated with hypertension OR for $1 \mu \mathrm{g} / \mathrm{g}$ change in tibia $\mathrm{Pb}: 1.019$ $(1.004,1.035)^{*}$

OR for quintile range (8-37 $\mu \mathrm{g} / \mathrm{g})$ : 1.5 (1.1, 1.8)*

Perlstein et al. 2007

Mean: 6.12

PP

$\mathrm{PbB}:$ no trend over quintiles $(\mathrm{p}=0.82)$

Bone $\mathrm{Pb}$ : $\mathrm{p}$-trend $=0.02 *$ 
2. HEALTH EFFECTS

Table 2-8. Summary of Epidemiological Studies Evaluating Effects on Blood Pressure at Mean Blood Lead Concentration $(\mathrm{PbB}) \leq 10 \mu \mathrm{g} / \mathrm{dL}$

\begin{tabular}{|c|c|c|c|}
\hline Reference and study population & $\mathrm{PbB}(\mu \mathrm{g} / \mathrm{dL})$ & Outcome evaluated & Result $\mathrm{a,b}$ \\
\hline Proctor et al. $1996^{e}$ & $\begin{array}{l}\text { Mean: } \\
-\quad \text { All: } 6.5\end{array}$ & SBP & $\begin{array}{l}\text { All } \beta, \mathrm{mmHg} \text { per } 1 \mathrm{ln} \mu \mathrm{g} / \mathrm{dL} \mathrm{PbB}: 0.85 \text { (-1.1, } \\
2.7) ; p>0.05\end{array}$ \\
\hline \multirow[t]{3}{*}{$\begin{array}{l}\text { Cross-sectional study; } \leq 74 \text { years } \\
(n=681) ;>74 \text { years }(n=117)\end{array}$} & $\begin{array}{l}-\quad \leq 74 \text { years: } 6.5 \\
-\quad>74 \text { years: } 6.3\end{array}$ & & $\begin{array}{l}\leq 74 \beta, \mathrm{mmHg} \text { per } 1 \mathrm{ln} \mu \mathrm{g} / \mathrm{dL} \text { PbB: } 1.2(-0.86, \\
3.2) ; p>0.05\end{array}$ \\
\hline & & DBP & $\begin{array}{l}\text { All } \beta, \mathrm{mmHg} \text { per } 1 \mathrm{ln} \mu \mathrm{g} / \mathrm{dL} \text { PbB: } 1.2(0.11, \\
2.2) ; p \leq 0.05^{*}\end{array}$ \\
\hline & & & $\begin{array}{l}\leq 74 \beta, \mathrm{mmHg} \text { per } 1 \mathrm{ln} \mu \mathrm{g} / \mathrm{dL} \text { PbB: } 1.6(0.42 \\
2.7) ; p \leq 0.01^{*}\end{array}$ \\
\hline \multirow[t]{3}{*}{$\begin{array}{l}\text { Yang et al. } 2018 \\
\text { Cross-sectional study; n=236 Pb } \\
\text { workers }\end{array}$} & $\begin{array}{l}\text { Gmean (IQR): } 4.50(2.60- \\
9.15)\end{array}$ & SBP & 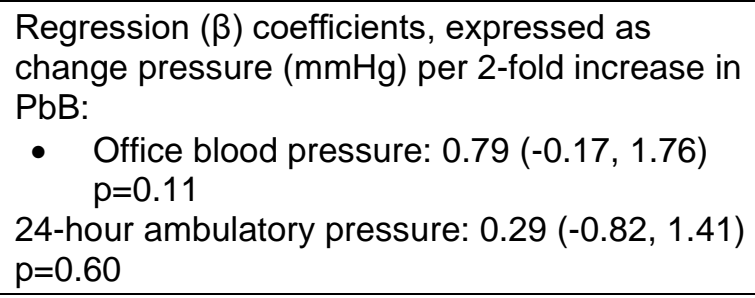 \\
\hline & & DBP & 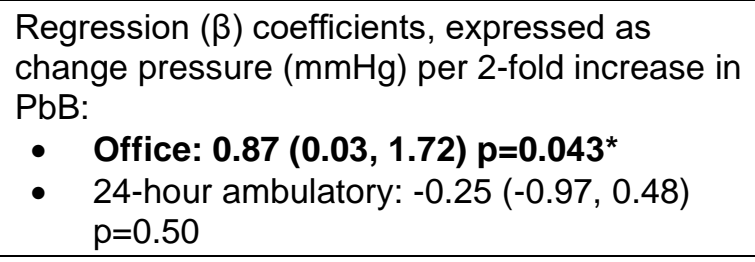 \\
\hline & & Hypertension & $\begin{array}{l}\text { OR: } \\
\bullet \quad \text { Office: } 0.89(0.62-1.28) ; p=0.052 \\
\bullet \quad 24-h o u r \text { ambulatory: } 1.21(0.94-1.57) \\
\quad p=0.14\end{array}$ \\
\hline \multicolumn{4}{|l|}{ Women onlyc } \\
\hline $\begin{array}{l}\text { Al-Saleh et al. } 2005 \\
\text { Case-control study of women with } \\
\text { hypertension }(n=100) \text { and control } \\
\text { subjects }(n=85)\end{array}$ & $\begin{array}{l}\text { Mean } \\
-\quad \text { Hypertension: } 4.75 \\
\text { - } \quad \text { Controls: } 4.56\end{array}$ & Hypertension & $\begin{array}{l}\text { OR for } \mathrm{PbB} \geq 3.85 \text { compared to } \mathrm{PbB}<3.85 \text { : } \\
5.27(0.93,29.86) ; \mathrm{p}=0.06\end{array}$ \\
\hline
\end{tabular}


Table 2-8. Summary of Epidemiological Studies Evaluating Effects on Blood Pressure at Mean Blood Lead Concentration $(\mathrm{PbB}) \leq 10 \mu \mathrm{g} / \mathrm{dL}$

\begin{tabular}{|c|c|c|c|}
\hline Reference and study population & $\mathrm{PbB}(\mu \mathrm{g} / \mathrm{dL})$ & Outcome evaluated & Result ${ }^{a, b}$ \\
\hline Korrick et al. 1999 & Mean (all): 3 & Hypertension & PbB: no increased risk (ORs not reported) \\
\hline Case-control study of women with & & & $\begin{array}{l}\text { Patella Pb OR per } 1 \mu \mathrm{g} / \mathrm{g} \text { increase in PbB: } \\
1.03(1.00,1.05) ; p=0.02^{*}\end{array}$ \\
\hline
\end{tabular}

hypertension $(n=89)$ and control

subjects $(n=195)$
Nash et al. 2003

Cross-sectional study; $n=2,165$ all; 1,084 premenopausal, and 663 postmenopausal
Mean (all): 2.9

Quartiles; mean (range)

- Q1: $1.0(0.5-1.6)$

- Q2: $2.1(1.7-2.5)$

- Q3: $3.2(2.6-3.9)$

- Q4: $6.4(4.0-31.1)$

\begin{tabular}{|c|c|}
\hline \multirow[t]{3}{*}{ SBP } & $\begin{array}{l}\text { All } \beta \text { (SE), mmHg per } 1 \mathrm{In} \mu \mathrm{g} / \mathrm{dL} \text { PbB: } \\
0.32(0.16) ; p=0.03^{*}\end{array}$ \\
\hline & $\begin{array}{l}\text { Premenopausal } \beta \text { (SE), mmHg per } 1 \mathrm{ln} \mu \mathrm{g} / \mathrm{dL} \\
\text { PbB: } 0.14(0.26) ; p=0.59\end{array}$ \\
\hline & $\begin{array}{l}\text { Postmenopausal } \beta \text { (SE), mmHg per } 1 \mathrm{ln} \mu \mathrm{g} / \mathrm{dL} \\
\text { PbB: } 0.42(0.21) ; p=0.29\end{array}$ \\
\hline \multirow[t]{3}{*}{ DBP } & $\begin{array}{l}\text { All } \beta \text { (SE): } 0.25 \text { (0.09), mmHg per } 1 \mathrm{ln} \mu \mathrm{g} / \mathrm{dL} \\
\text { PbB; } p=0.009^{*}\end{array}$ \\
\hline & $\begin{array}{l}\text { Premenopausal } \beta \text { (SE), mmHg per } 1 \mathrm{ln} \mu \mathrm{g} / \mathrm{dL} \\
\text { PbB: } 0.38(0.25) ; p=0.12\end{array}$ \\
\hline & $\begin{array}{l}\text { Postmenopausal } \beta \text { (SE) mmHg per } 1 \text { In } \\
\mu \mathrm{g} / \mathrm{dL} \text { PbB: } 0.14 \text { (0.13); } p=0.04^{*}\end{array}$ \\
\hline Hypertension & $\begin{array}{l}\text { Percent of total population with } \\
\text { hypertension: p-trend<0.001* (Q1: } 19.4 ; \text { Q2: } \\
\text { 20.6; Q3: } 25.5 \text { Q4: } 28.3)\end{array}$ \\
\hline \multirow[t]{4}{*}{ SBP } & $\begin{array}{l}\text { MW } \beta \text {, per doubling of PbB: } 0.3(-0.2,0.7) \text {; } \\
p=0.29\end{array}$ \\
\hline & $\begin{array}{l}\text { FW } \beta \text {, per doubling of } \mathrm{PbB}: 0.1(-0.4,0.5) \\
p=0.80\end{array}$ \\
\hline & $\begin{array}{l}\text { MB } \beta \text {, per doubling of PbB: } 0.9(0.04,1.8) \\
p=0.04^{*}\end{array}$ \\
\hline & $\begin{array}{l}\text { FB } \beta \text {, per doubling of } \mathrm{PbB}: 1.2(0.4,2.0) \\
\mathrm{p}=0.004^{*}\end{array}$ \\
\hline DBP & $\begin{array}{l}\text { MW } \beta \text {, per doubling of PbB: }-0.6(-0.9,-0.3) \text {; } \\
p=0.0003^{*}\end{array}$ \\
\hline
\end{tabular}

Mean

- $\quad \mathrm{MW}: 3.6$

- FW: 2.1

- $\mathrm{MB}: 4.2$

- FB: 2.3
Cross-sectional study $n=4,685 \mathrm{MW}$; 5,138 FW; 1,761 MB; and 2,197 FB

\subsection{3 (1.00, 1.05); $p=0.02^{*}$}

\section{All $\beta$ (SE), mmHg per $1 \mathrm{ln} \mu \mathrm{g} / \mathrm{dL}$ PbB}

PbB: 0.14 (0.26); $p=0.59$

Postmenopausal $\beta$ (SE), mmHg per $1 \mathrm{ln} \mu \mathrm{g} / \mathrm{dL}$

$\mathrm{PbB} ; \mathrm{p}=0.009^{*}$

PbB: 0.38 (0.25); $p=0.12$

Percent of total population with

hypertension: p-trend<0.001* (Q1: 19.4; Q2: 20.6; Q3: 25.5 Q4: 28.3)

Women and men stratified by race ${ }^{\mathrm{c}}$

Den Hond et al. 2002d

(1) 
Table 2-8. Summary of Epidemiological Studies Evaluating Effects on Blood Pressure at Mean Blood Lead Concentration $(\mathrm{PbB}) \leq 10 \mu \mathrm{g} / \mathrm{dL}$

\begin{tabular}{|c|c|c|c|}
\hline \multirow[t]{4}{*}{ Reference and study population } & \multirow[t]{4}{*}{$\mathrm{PbB}(\mu \mathrm{g} / \mathrm{dL})$} & \multirow[t]{4}{*}{ Outcome evaluated } & \multirow{2}{*}{$\begin{array}{l}\text { Resulta,b } \\
\text { FW } \beta \text {, per doubling of } \mathrm{PbB}:-0.2(-0.5,0.1) \\
\mathrm{p}=0.13\end{array}$} \\
\hline & & & \\
\hline & & & $\begin{array}{l}\text { MB } \beta \text {, per doubling of } \mathrm{PbB}: 0.3(-0.3,1.0) \\
p=0.28\end{array}$ \\
\hline & & & $\begin{array}{l}\text { FB } \beta \text {, per doubling of PbB: } 0.5(0.01,1.1) \\
p=0.047^{*}\end{array}$ \\
\hline Muntner et al. $2005^{d}$ & \multirow{3}{*}{$\begin{array}{l}\text { Mean: } 1.64 \\
\text { Quartiles: } \\
\text { - } \quad \text { Q1: }<1.06 \\
\text { - } \quad \text { Q2: } 1.06-1.63 \\
\text { - } \quad \text { Q3: } 1.63-2.47 \\
\text { - } \quad \text { 44: } \geq 2.47\end{array}$} & \multirow[t]{3}{*}{ Hypertension } & W (Q4) OR: 1.10 (0.87, 1.41); p-trend=0.61 \\
\hline \multirow[b]{2}{*}{$\begin{array}{l}\text { Cross-sectional study; } n=9,961 \text { (men } \\
\text { and women), stratified by race } \\
(W, B, M A)\end{array}$} & & & B (Q4) OR: $1.44(0.89,2.32) ; p$-trend=0.06 \\
\hline & & & MA (Q4) OR: $1.54(0.99,2.39) ; p$-trend $=0.04^{\star}$ \\
\hline \multirow[t]{2}{*}{ Park et al. 2009b } & \multirow{8}{*}{$\begin{array}{ll}\text { Mean } \\
\text { - } & \mathrm{MW}(<50 \text { years old }) 4.02 \\
\text { - } & \mathrm{MW}(\geq 50 \text { years old }) 4.92 \\
\text { - } & \mathrm{MB}(<50 \text { years old }) 4.55 \\
\text { - } & \mathrm{MB}(\geq 50 \text { years old }) 7.57 \\
\text { - } & \mathrm{FW}(<50 \text { years old }) 2.09 \\
\text { - } & \mathrm{FW}(\geq 50 \text { years old }) 3.53 \\
\text { - } & \mathrm{FB}(<50 \text { years old }) 2.52 \\
\text { - } & \mathrm{FB}(\geq 50 \text { years old }) 4.49\end{array}$} & \multirow[t]{8}{*}{ Hypertension } & MW OR: $1.06(0.92,1.22)$ \\
\hline & & & FW OR: $1.16(1.04,1.29)^{*}$ \\
\hline \multirow{6}{*}{$\begin{array}{l}\text { Cross-sectional study; } n=12,500 \text { all, } \\
2,130 \mathrm{MW} \text { ( }<50 \text { years old); } 2,152 \mathrm{MW} \\
\text { ( } \geq 50 \text { years old); } 1,048 \mathrm{MB}(<50 \text { years } \\
\text { old); } 540 \mathrm{MB} \text { ( } \geq 50 \text { years old); } 2,429 \mathrm{FW} \\
\text { ( }<50 \text { years old); } 2,180 \mathrm{FW}(\geq 50 \text { years } \\
\text { old); 1,409 FB ( }<50 \text { years old); and } \\
612 \text { FB ( } \geq 50 \text { years old) }\end{array}$} & & & MB OR: $1.17(0.98,1.38)$ \\
\hline & & & FB OR: $1.19(1.04,1.38)^{*}$ \\
\hline & & & $\mathrm{M}(<50$ years old) OR: $0.98(0.80,1.22)$ \\
\hline & & & M (>50 years old) OR: $1.20(1.02,1.41)^{*}$ \\
\hline & & & F (<50 years old) OR: $1.23(1.04,1.46)^{*}$ \\
\hline & & & F (>50 years old) OR: $1.09(0.94,1.26)$ \\
\hline Scinicariello et al. $2010^{d}$ & \multirow{4}{*}{\begin{tabular}{ll}
\multicolumn{2}{l}{ Mean } \\
$-\quad$ W 2.87 \\
$-\quad$ B 3.59 \\
$-\quad$ MA 3.33
\end{tabular}} & SBP & $\begin{array}{l}W \beta(S E), m m H g \text { per In } \mu g / d L \text { PbB: } 1.05 \\
(0.37) ; p=0.01^{*}\end{array}$ \\
\hline \multirow[t]{3}{*}{$\begin{array}{l}\text { Cross-sectional study; } \\
n=6,016 \text { (stratified by race) }\end{array}$} & & & $\begin{array}{l}\text { B } \beta(S E), m m H g \text { per In } \mu g / d L \text { PbB: } 2.55 \\
(0.49) ; p=0.001^{*}\end{array}$ \\
\hline & & & $\begin{array}{l}\text { MA } \beta(S E), m m H g \text { per } \ln \mu g / d L \text { PbB: } 0.84 \\
(0.46) ; p=0.08\end{array}$ \\
\hline & & DBP & $\begin{array}{l}W \beta(S E), m m H g \text { per } \ln \mu g / d L ~ P b B:-0.14 \\
(0.49) ; p=0.77\end{array}$ \\
\hline
\end{tabular}


Table 2-8. Summary of Epidemiological Studies Evaluating Effects on Blood Pressure at Mean Blood Lead Concentration $(\mathrm{PbB}) \leq 10 \mu \mathrm{g} / \mathrm{dL}$

\begin{tabular}{|c|c|c|c|}
\hline Reference and study population & $\mathrm{PbB}(\mu \mathrm{g} / \mathrm{dL})$ & Outcome evaluated & Result ${ }^{a, b}$ \\
\hline & & & $\begin{array}{l}\text { B } \beta \text { (SE), } m m H g \text { per In } \mu g / d L \text { PbB: } 1.99 \\
(0.44) ; p=0.0002^{*}\end{array}$ \\
\hline & & & $\begin{array}{l}\text { MA } \beta \text { (SE), mmHg per In } \mu g / d L \text { PbB: } 0.74 \\
(0.74) ; p=0.06\end{array}$ \\
\hline \multirow{14}{*}{ 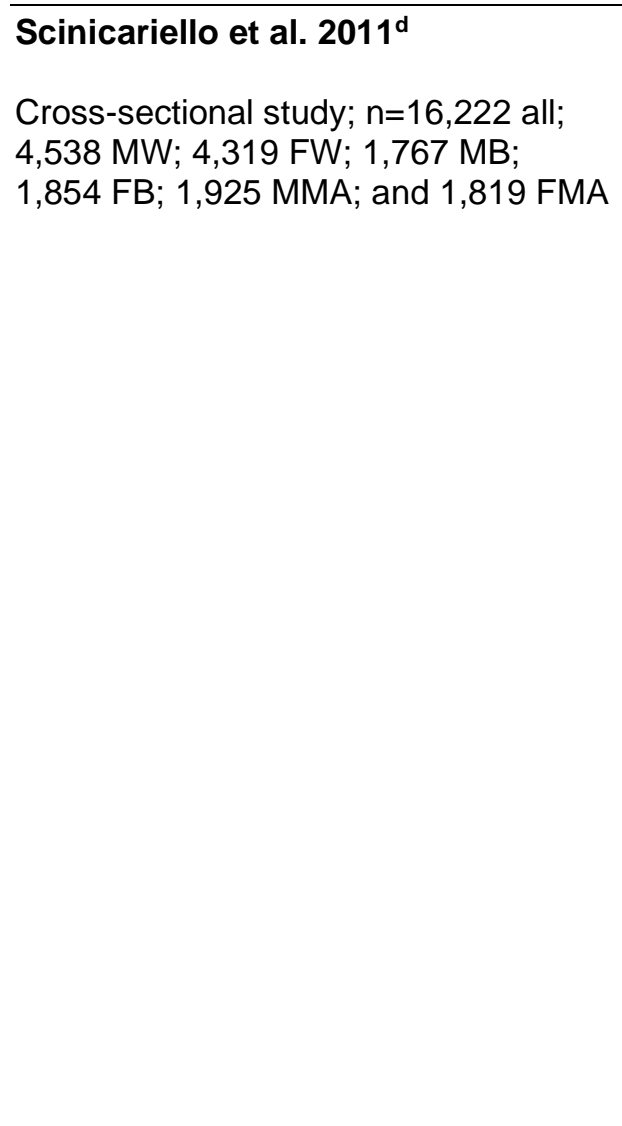 } & \multirow{14}{*}{\begin{tabular}{ll}
\multicolumn{2}{l}{ Mean } \\
- & All 1.41 \\
- & MW 2.20 \\
- & FW 1.55 \\
- & MB 2.44 \\
- & FB 1.81 \\
- & MMA 2.47 \\
- & FMA 1.56
\end{tabular}} & \multirow[t]{7}{*}{ SBP } & $\begin{array}{l}\text { All } \beta \text { (SE), per In } \mu g / d L \text { PbB: } 1.07 \text { (0.35); } \\
p<0.05^{*}\end{array}$ \\
\hline & & & $\begin{array}{l}\text { MW } \beta \text { (SE), per In } \mu \mathrm{g} / \mathrm{dL} \text { PbB: } 0.87 \text { (0.53); } \\
\mathrm{p}>0.05\end{array}$ \\
\hline & & & $\begin{array}{l}\text { FW } \beta \text { (SE), per In } \mu g / d L \text { PbB: } 0.89 \text { (0.55); } \\
p>0.05\end{array}$ \\
\hline & & & $\begin{array}{l}\text { MB } \beta(S E) \text {, per In } \mu g / d L \text { PbB: } 2.30(0.71) \\
p<0.05^{*}\end{array}$ \\
\hline & & & $\begin{array}{l}\text { FB } \beta(S E) \text {, per In } \mu g / d L \text { PbB: } 2.40 \text { (1.14); } \\
\text { p<0.05* }\end{array}$ \\
\hline & & & $\begin{array}{l}\text { MMA } \beta \text { (SE), per In } \mu g / d L \text { PbB: } 0.10 \text { (0.70); } \\
p>0.05\end{array}$ \\
\hline & & & $\begin{array}{l}\text { FMA } \beta \text { (SE), per In } \mu g / d L \text { PbB: -0.03 (0.64); } \\
p>0.05\end{array}$ \\
\hline & & \multirow[t]{7}{*}{ DBP } & All $\beta$ (SE): 0.71 (0.27): $p<0.05^{*}$ \\
\hline & & & MW $\beta$ (SE): 0.90 (0.45): $p<0.05^{*}$ \\
\hline & & & FW $\beta$ (SE): 0.95 (0.38): $p<0.05^{*}$ \\
\hline & & & MB $\beta$ (SE): 2.75 (0.82); p<0.05* \\
\hline & & & $\begin{array}{l}\text { FB } \beta(S E) \text {, per In } \mu g / d L \text { PbB: } 0.30 \text { (0.81); } \\
p>0.05\end{array}$ \\
\hline & & & $\begin{array}{l}\text { MMA } \beta(\text { (SE), per In } \mu g / d L \text { PbB: -1.34 (0.66); } \\
p<0.05^{*}\end{array}$ \\
\hline & & & $\begin{array}{l}\text { FMA } \beta \text { (SE), per In } \mu g / d L \text { PbB: }-0.74 \text { (0.44); } \\
p>0.05\end{array}$ \\
\hline
\end{tabular}


2. HEALTH EFFECTS

Table 2-8. Summary of Epidemiological Studies Evaluating Effects on Blood Pressure at Mean Blood Lead Concentration $(\mathrm{PbB}) \leq 10 \mu \mathrm{g} / \mathrm{dL}$

\begin{tabular}{|c|c|c|c|}
\hline Reference and study population & $\mathrm{PbB}(\mu \mathrm{g} / \mathrm{dL})$ & Outcome evaluated & Resulta,b \\
\hline Vupputuri et al. $2003^{d}$ & $\begin{array}{l}\text { Mean } \\
-\quad \text { MW } 4.4\end{array}$ & SBP & $\begin{array}{l}\text { MW } \beta \text {, per } 1 \mathrm{SD}(3.3 \mu \mathrm{g} / \mathrm{dL}) \text { increase of } \mathrm{PbB} \text { : } \\
0.29(-0.24,0.83)\end{array}$ \\
\hline \multirow{11}{*}{$\begin{array}{l}\text { Cross-sectional study; } n=14,952 \text { total; } \\
n=5,360 \mathrm{MW} ; 5,188 \mathrm{FW} ; 2,104 \mathrm{MB} \text {; and } \\
2,300 \mathrm{FB}\end{array}$} & 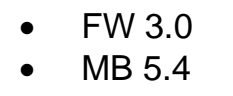 & & $\begin{array}{l}\text { FW } \beta \text {, per } 1 \mathrm{SD}(3.3 \mu \mathrm{g} / \mathrm{dL}) \text { increase of } \mathrm{PbB}: \\
0.34(-0.49,1.17)\end{array}$ \\
\hline & - $\quad$ FB 3.4 & & $\begin{array}{l}\text { MB } \beta \text {, per } 1 \text { SD }(3.3 \mu \mathrm{g} / \mathrm{dL}) \text { increase of PbB: } \\
0.82(0.19,1.44) ; p<0.05^{*}\end{array}$ \\
\hline & & & $\begin{array}{l}\text { FB } \beta \text {, per } 1 \text { SD }(3.3 \mu g / d L) \text { increase of PbB: } \\
1.55(0.47,2.64) ; p<0.01^{*}\end{array}$ \\
\hline & & DBP & $\begin{array}{l}\text { MW } \beta \text {, per } 1 \text { SD }(3.3 \mu \mathrm{g} / \mathrm{dL}) \text { increase of } \mathrm{PbB}: \\
0.01(-0.38,0.40) ; p \geq 0.05\end{array}$ \\
\hline & & & $\begin{array}{l}\text { FW } \beta \text {, per } 1 \mathrm{SD}(3.3 \mu \mathrm{g} / \mathrm{dL}) \text { increase of } \\
\text { PbB: }-0.04(-0.56,0.47) p \geq 0.05\end{array}$ \\
\hline & & & $\begin{array}{l}\text { MB } \beta \text {, per } 1 \text { SD }(3.3 \mu \mathrm{g} / \mathrm{dL}) \text { increase of PbB: } \\
0.64(0.08,1.20) ; p<0.05^{*}\end{array}$ \\
\hline & & & $\begin{array}{l}\text { FB } \beta \text {, per 1 SD }(3.3 \mu \mathrm{g} / \mathrm{dL}) \text { increase of PbB: } \\
1.07(0.37,1.77) ; p<0.01^{*}\end{array}$ \\
\hline & & Hypertension & MW OR: $1.04(0.93,1.16) ; p=0.47$ \\
\hline & & & FW OR: $1.32(1.14,1.52) p<0.001^{*}$ \\
\hline & & & MB OR: $1.08(0.99,1.19) ; p=0.08$ \\
\hline & & & FB OR: $1.39(1.21,1.61) ; p<0.001^{*}$ \\
\hline
\end{tabular}


Table 2-8. Summary of Epidemiological Studies Evaluating Effects on Blood Pressure at Mean Blood Lead Concentration $(\mathrm{PbB}) \leq 10 \mu \mathrm{g} / \mathrm{dL}$

\begin{tabular}{|c|c|c|c|}
\hline Reference and study population & $\mathrm{PbB}(\mu \mathrm{g} / \mathrm{dL})$ & Outcome evaluated & Result $^{\mathrm{a}, \mathrm{b}}$ \\
\hline \multicolumn{4}{|l|}{ Children and young adults ${ }^{c}$} \\
\hline Ahn et al. 2018 & $\begin{array}{l}\text { Mean }(95 \% \mathrm{Cl}): 1.192(1.165, \\
1.219)\end{array}$ & DBP & $\begin{array}{l}\text { Mean difference with doubling of } \mathrm{PbB} \\
\text { continuous variable: }-0.680(-1.581,0.221)\end{array}$ \\
\hline \multirow[t]{2}{*}{$\begin{array}{l}\text { Cross-sectional study; } n=1,776 \\
\text { adolescents (ages 10-18 years) }\end{array}$} & $\begin{array}{l}\text { Quartiles } \\
\bullet \quad \text { Males }\end{array}$ & SBP & $\begin{array}{l}\text { Mean difference with doubling of } \mathrm{PbB} \text {, } \\
\text { continuous variable: }-0.099(-1.098,0.898)\end{array}$ \\
\hline & 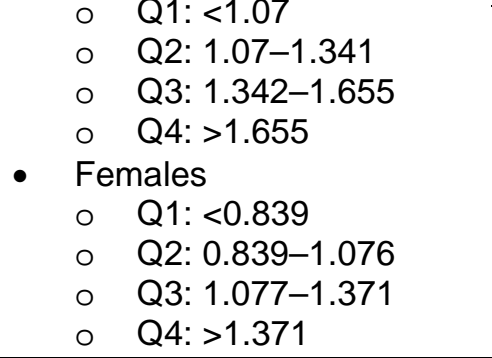 & Prehypertension & OR, continuous variable: $0.906(0.629,1.305)$ \\
\hline \multirow{2}{*}{$\begin{array}{l}\text { Gerr et al. } 2002 \\
\text { Cross-sectional study; } n=508 \text { young } \\
\text { adults (ages 19-29 years) }\end{array}$} & \multirow{2}{*}{$\begin{array}{l}\text { PbB mean associated with the } \\
\text { following bone } \mathrm{Pb} \\
\text { concentrations: } \\
\text { - } \quad<1 \mu \mathrm{g} / \mathrm{g}: 1.91(1.58) \\
\text { - } \quad 1-5 \mu \mathrm{g} / \mathrm{g}: 2.31(2.06) \\
\text { - } \quad 6-10 \mu \mathrm{g} / \mathrm{g}: 2.43(2.36) \\
\text { - } \quad>10 \mu \mathrm{g} / \mathrm{g}: 3.15(2.28) \\
\end{array}$} & SBP & $\begin{array}{l}\text { Increase (mmHg) associated with bone } \mathrm{Pb} \\
>10 \mu \mathrm{g} / \mathrm{g}(\mathrm{SE}): 4.26(1.48) ; p=0.004^{*}\end{array}$ \\
\hline & & DBP & $\begin{array}{l}\text { Increase }(\mathrm{mmHg}) \text { associated with bone } \mathrm{Pb} \\
>10 \mu \mathrm{g} / \mathrm{g}(\mathrm{SE}): 2.80(1.25) ; p=0.03^{*}\end{array}$ \\
\hline \multirow{2}{*}{$\begin{array}{l}\text { Gump et al. } 2005 \\
\text { Prospective study; } n=122 \text { children } \\
\text { assessed at } 9 \text { years of age }\end{array}$} & \multirow[t]{2}{*}{ Cord PbB mean: 2.97} & SBP & 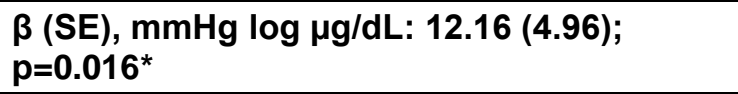 \\
\hline & & DBP & 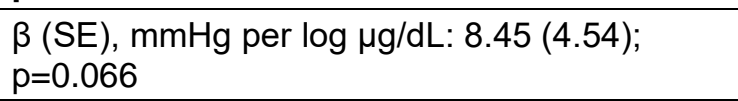 \\
\hline \multirow{3}{*}{$\begin{array}{l}\text { Gump et al. } 2011 \\
\text { Cross-sectional study; n=140 children } \\
\text { (ages 9-11 years) }\end{array}$} & \multirow{3}{*}{$\begin{array}{l}\text { Mean: } 1.01 \\
\text { Quartiles: } \\
\text { - } \quad \text { Q1: 0.14-0.68 } \\
\text { - } \quad \text { Q2: 0.69-0.93 } \\
\text { - } \quad \text { Q3: 0.94-1.20 } \\
\text { - } \quad \text { Q4: 1.21-3.76 }\end{array}$} & SBP & Under acute stress, p-trend over quartiles: 0.31 \\
\hline & & DBP & Under acute stress, p-trend over quartiles: 0.29 \\
\hline & & TPR & $\begin{array}{l}\text { Under acute stress, p-trend over quartiles: } \\
0.03^{*}\end{array}$ \\
\hline
\end{tabular}




\section{Table 2-8. Summary of Epidemiological Studies Evaluating Effects on Blood Pressure at Mean Blood Lead Concentration $(\mathrm{PbB}) \leq 10 \mu \mathrm{g} / \mathrm{dL}$}

\begin{tabular}{l} 
Reference and study population \\
\hline Zhang et al. 2011 \\
Prospective longitudinal study; \\
$\mathrm{n}=457$ mother-child pairs; children \\
evaluated at ages $7-15$ years
\end{tabular}

$\mathrm{n}=457$ mother-child pairs; childre

evaluated at ages 7-15 years

$\mathrm{PbB}(\mu \mathrm{g} / \mathrm{dL})$

- Mean umbilical cord: 5.51 SBP

- Mean child concurrent:

$$
2.96
$$

- Median maternal postnatal tibia $\mathrm{Pb}(\mu \mathrm{g} / \mathrm{g}): 9.3$
Outcome evaluated

Result ${ }^{a, b}$

Maternal tibia $\mathrm{Pb}$, boys, $\beta, \mathrm{mmHg}$ increased per maternal tibia $\mathrm{Pb}(13 \mu \mathrm{g} / \mathrm{g}):-0.34(-1.98,1.30)$

Maternal tibia $\mathrm{Pb}$, girls, $\beta \mathrm{mmHg}$ increased per maternal tibia $\mathrm{Pb}(13 \mu \mathrm{g} / \mathrm{g}): 2.11(0.69$, 3.52); $\mathrm{p}=0.025^{*}$

DBP

Maternal tibia $\mathrm{Pb}$, boys, $\beta, \mathrm{mmHg}$ increased per maternal tibia $\mathrm{Pb}(13 \mu \mathrm{g} / \mathrm{g}):-0.83(-2.05,0.38)$

Maternal tibia $\mathrm{Pb}$, girls, $\beta, \mathrm{mmHg}$ increased per maternal tibia $\mathrm{Pb}(13 \mu \mathrm{g} / \mathrm{g})$ : $1.60(0.28$, 2.91); $\mathrm{p}=0.007^{*}$

Blood pressure during pregnancy

Disha et al. 2019 PbB:

Cross-sectional study; $n=44$ healthy pregnant women; $n=23$ pre-eclamptic women

\section{Rothenberg et al. 2002}

Longitudinal study; $n=667$ pregnant women

\section{Wells et al. 2011}

Cross-sectional study; $n=285$ pregnan women during labor

\section{Mean}

- Control: 2.38

- Pre-eclampsia: 3.42

Mean: 1.9
Bone (calcaneus) $\mathrm{Pb}(\mu \mathrm{g} / \mathrm{g})$
mean:10.7

Umbilical cord PbB

- mean: 0.66

- Quartiles:

$$
\begin{array}{ll}
\text { O } & \text { Q1: }<0.46 \\
\text { o } & \text { Q2: } 0.47-0.65 \\
\text { o } & \text { Q3: } 0.66-0.95 \\
\text { o } & \text { Q4: } 0.96-6.47
\end{array}
$$

Pearson correlation (mmHg): 0.71; $\mathrm{p}<0.0001$ *

Pearson correlation $(\mathrm{mmHg})$ : $0.57 ; \mathrm{p}=0.004^{*}$

Ln-PbB, $\beta:-0.04(-1.26,1.18)$ Bone Pb, $\beta$ : $0.70(0.04,1.36)^{*}$

Ln-PbB, $\beta: 0.20(-0.78,1.18)$

Bone Pb, $\beta$ : $0.54(0.01,1.08)^{*}$

SBP

Q4 versus Q1 increase in SBP in $\mathrm{mmHg}$ at admission: 6.87 (1.51, 12.21); $p<0.05^{*}$

Q4 versus Q4 maximum increase in SBP in mmHg: 7.72 (1.83, 13.60); $p<0.05^{*}$

DBP admission: 4.40 (0.21, 8.59); $p<0.05^{*}$

Q4 versus Q4 maximum increase in DBP in mmHg: Q4: 8.33 (1.14, 15.53); $p<0.05 *$ 
Table 2-8. Summary of Epidemiological Studies Evaluating Effects on Blood Pressure at Mean Blood Lead Concentration $(\mathrm{PbB}) \leq 10 \mu \mathrm{g} / \mathrm{dL}$

\begin{tabular}{|c|c|c|c|}
\hline Reference and study population & $\mathrm{PbB}(\mu \mathrm{g} / \mathrm{dL})$ & Outcome evaluated & Resulta,b \\
\hline & $\begin{array}{l}\text { Mean } \\
\text { - } \quad \text { Participants with PIH: } 2.2\end{array}$ & $\mathrm{PIH}$ & $\begin{array}{l}\text { OR for PIH for an increase of } 1 \log _{10} \mu \mathrm{g} / \mathrm{dL} \\
\text { in PbB; } 3.29(1.11,9.74) ; p=0.03^{*}\end{array}$ \\
\hline $\begin{array}{l}\text { Cross-sectional study; } n=971 \text { pregnant } \\
\text { women }\end{array}$ & $\begin{array}{l}\text { - Participants without PIH: } \\
1.9\end{array}$ & & \\
\hline
\end{tabular}

${ }^{a}$ Asterisk and bold indicate association with $\mathrm{Pb}$; unless otherwise specified, values in parenthesis are $95 \% \mathrm{Cls}$; $\mathrm{p}$-values $<0.05$ unless otherwise noted in the table. 'blf bone $\mathrm{Pb}$ is noted under results, study did not show associations between $\mathrm{PbB}$ and blood pressure parameters; however, results showed associations between bone $\mathrm{Pb}$ concentrations and increased blood pressure at concomitant $\mathrm{PbB} \leq 10 \mu \mathrm{g} / \mathrm{dL}$.

'See the Supporting Document for Epidemiological Studies for Lead, Table 3 for more detailed descriptions of studies.

dStudy population was from NHANES.

eStudy population was from the Normative Aging Study.

$\mathrm{B}=$ black $\mathrm{Cl}=$ confidence interval; $\mathrm{CL}=$ confidence limit; $\mathrm{DBP}=$ diastolic blood pressure; $\mathrm{F}=$ female(s); Gmean = geometric mean; $\mathrm{M}=$ male(s); $\mathrm{MA}=\mathrm{Mexican}$ American; NHANES = National Health and Nutrition Examination Survey; OR = odds ratio; $\mathrm{Pb}=$ lead; $\mathrm{PIH}=$ pregnancy-induced hypertension; $\mathrm{PP}=$ pulse pressure; $\mathrm{RR}=$ rate ratio; $\mathrm{SBP}=$ systolic blood pressure; $\mathrm{SD}=$ standard deviation; $\mathrm{SE}=$ standard error; $\mathrm{TPR}=$ total peripheral resistance; $\mathrm{W}=$ white 
in Table 2-8, with additional details provided in the Supporting Document for Epidemiological Studies for Lead, Table 3.

The magnitude of effect on blood pressure observed in individual large-scale, cross-sectional studies is consistent with results of meta-analyses (see discussion above on Characterization of Effects). For example, Martin et al. (2006) reported that systolic and diastolic blood pressure increased by 0.99 (95\% CI 0.47, 1.51; $\mathrm{p}<0.01) \mathrm{mmHg}$ and 0.51 (95\% CI 0.24, 0.79; $\mathrm{p}<0.01) \mathrm{mmHg}$, respectively, per $1 \mu \mathrm{g} / \mathrm{dL}$ increase in $\mathrm{PbB}$.

Several studies have examined the relationship between $\mathrm{PbB}$ and blood pressure with study populations stratified according to gender, race, and/or age. For example, within study populations, positive associations were observed between $\mathrm{PbB}$ and systolic and diastolic blood pressure in men but not in women (Bushnik et al. 2014; Chu et al. 1999; Hense et al. 1993). A cross-sectional study reported an increased risk of prehypertension (defined as a diastolic blood pressure of at least $80 \mathrm{mmHg}$ but below $90 \mathrm{mmHg}$ or a systolic blood pressure of at least $120 \mathrm{mmHg}$ but below $140 \mathrm{mmHg}$ ) in women but not in men, although PbB was lower $(\mathrm{p}<0.05)$ in women $(1.9 \mu \mathrm{g} / \mathrm{dL})$ than men $(2.4 \mu \mathrm{g} / \mathrm{dL})$ (Lee et al. 2016a). However, other studies did not find differences between men and women (Bost et al. 1999; Scinicariello et al. 2011). Stratification by sex and age indicates additional differences between men and women. For example, Park et al. (2009b) reported a greater risk of hypertension in men $>50$ years of age (odds ratio [OR] 1.20; 95\% CI 1.02, 1.41), but not in men <50 years of age (OR 0.98; 95\% CI 0.80, 1.22), whereas in women, the opposite effect of age was observed, with a greater risk of hypertension in women $<50$ years of age (OR 1.23; 95\% CI 1.04, 1.46) but not >50 years of age (OR 1.09; 95\% CI 0.94, 1.26). Studies that stratify populations by race have found race differences in effect sizes on blood pressure. Large-scale cross-sectional studies based on data from NHANES have found larger effect sizes in non-Hispanic blacks and Mexican-Americans than in whites (Den Hond et al. 2002; Muntner et al. 2005; Scinicariello et al. 2011; Vupputuri et al. 2003). Cross-sectional studies based on data from NHANES have consistently shown elevations of systolic blood pressure in association with increasing PbB among black males and females, with less consistency in findings for other demographic groups or for diastolic blood pressure (Den Hond et al. 2002; Nash et al. 2003; Scinicariello et al. 2010, 2011; Vupputuri et al. 2003). Scinicariello et al. (2011) estimated increases in systolic blood pressure ranging from 1.07 to 2.4 per 1 ln increase in $\mathrm{PbB}$ (equivalent to approximately 0.7-1.66 per doubling of $\mathrm{PbB}$ ). The largest effects sizes were observed in black males (2.3; SE 0.71 per ln PbB) and black females (2.4; SE 1.14). Den Hond et al. (2002) estimated the effect size for systolic blood pressure in black males and females to be $0.9 \mathrm{mmHg}$ $(95 \%$ CI 0.04, 1.8) and $1.2 \mathrm{mmHg}(95 \%$ CI 0.4, 2.0) per doubling of PbB, respectively. Vupputuri et al. 
(2003) estimated the effect size for systolic blood pressure in black males and females to be $0.82 \mathrm{mmHg}$ (95\% CI 0.19, 1.44) and 1.55 mmHg (95\% CI 0.47, 2.64) per 1 standard deviation (SD) increase $(3.3 \mu \mathrm{g} / \mathrm{dL})$ of $\mathrm{PbB}$, respectively. As discussed above (see Confounding Factors and Effect Modifiers), numerous co-variables and confounders affect studies of associations between $\mathrm{PbB}$ and blood pressure, complicating comparisons between studies.

Few studies have evaluated effects of chronic $\mathrm{Pb}$ exposure in children or young adults on blood pressure parameters at $\mathrm{PbB}$ at $\leq 10 \mu \mathrm{g} / \mathrm{dL}$ (Ahn et al. 2018; Gerr et al. 2002; Gump et al. 2005, 2011; Zhang et al. 2011). Studies are briefly summarized in Table 2-8, with additional details provided in the Supporting Document for Epidemiological Studies for Lead, Table 3. Population sizes in these studies are small $(n=122-1,776)$ compared to studies in adults. Positive associations were observed between concurrent $\mathrm{PbB}$ and increased systolic and diastolic blood pressure in young adults (Gerr et al. 2002). Two prospective studies suggest that prenatal exposure to $\mathrm{Pb}$ is associated with increased blood pressure in childhood (Gump et al. 2005; Zhang et al. 2011). Umbilical cord PbB was positively associated with increased systolic, but not diastolic, blood pressure in children (Gump et al. 2005). Maternal postnatal bone $\mathrm{Pb}$ concentration was associated with increased systolic and diastolic blood pressure in girls, but not boys; however, no association was observed between umbilical cord $\mathrm{PbB}$ or patella $\mathrm{Pb}$ concentration and increased blood pressure (Zhang et al. 2011). No association between PbB and diastolic or systolic blood pressure or risk of prehypertension in a larger population of adolescents $(n=1,776)$ with a mean $\mathrm{PbB}$ of $1.19 \mu \mathrm{g} / \mathrm{dL}$ (Ahn et al. 2018).

Effects of $\mathrm{Pb}$ on blood pressure and hypertension at $\mathrm{PbB}$ at $\leq 10 \mu \mathrm{g} / \mathrm{dL}$ have also been evaluated during pregnancy (Disha et al. 2019; Rothenberg et al. 2002; Wells et al. 2011; Yazbeck et al. 2009). Studies are briefly summarized in Table 2-8, with additional details provided in the Supporting Document for Epidemiological Studies for Lead, Table 3. Increases in systolic and diastolic blood pressure during pregnancy and labor were associated with $\mathrm{PbB} \leq 10 \mu \mathrm{g} / \mathrm{dL}$ umbilical cord $\mathrm{PbB}$, or bone $\mathrm{Pb}$ concentrations with concomitant $\mathrm{PbB} \leq 10 \mu \mathrm{g} / \mathrm{dL}$ (Rothenberg et al. 2002; Wells et al. 2011; Yazbeck et al. 2009). Pregnancy-induced hypertension has been positively associated with $\mathrm{PbB} \leq 10 \mu \mathrm{g} / \mathrm{dL}$ (Yazbeck et al. 2009). A small cross-sectional study reported a positive association between $\mathrm{PbB}$ and increased systolic and diastolic blood pressure in women with pre-eclampsia (Disha et al. 2019).

Atherosclerosis. Few studies have evaluated associations between $\mathrm{PbB} \leq 10 \mu \mathrm{g} / \mathrm{dL}$ and atherosclerosis (Ari et al. 2011; Muntner et al. 2005; Navas-Acien et al. 2004). Studies are briefly summarized in Table 2-9, with additional details provided in the Supporting Document for Epidemiological Studies for 
Lead, Table 3. Ari et al. (2011) reported a positive correlation between PbB and intimal medial thickening of the greater carotid artery in non-diabetic hemodialysis patients at a concurrent $\mathrm{PbB}$ of $0.41 \mu \mathrm{g} / \mathrm{dL}$. Peripheral artery disease was positively associated with PbB levels $\geq 2.47 \mu \mathrm{g} / \mathrm{dL}$, with a positive trend across quartiles, in a study of a large NHANES 1999-2002 (age 18 years or older) population (Muntner et al. 2005), whereas analyses restricted to adult ( $\geq 40$ years old) participants of NHANES 1999-2000 reported a positive trend for the risk of peripheral artery disease, although ORs for $\mathrm{PbB}$ quartiles (highest $\mathrm{PbB}$ quartile $>2.90 \mu \mathrm{g} / \mathrm{dL}$ ) were not associated with peripheral artery disease (Navas-Acien et al. 2004).

Cardiac function and heart disease. Several studies have investigated cardiac function and heart disease, including a series of studies conducted in men from the Normative Aging Study in the greater Boston, Massachusetts area that evaluated associations between $\mathrm{PbB} \leq 10 \mu \mathrm{g} / \mathrm{dL}$ and alterations in cardiac conduction and ischemic heart disease (Cheng et al. 1998; Eum et al. 2011; Jain et al. 2007; Park et al. 2009a). Studies are briefly summarized in Table 2-10, with additional details provided in the Supporting Document for Epidemiological Studies for Lead, Table 2. Studies on the Normative Aging Study population show positive associations between bone $\mathrm{Pb}$ concentrations (at concomitant $\mathrm{PbB} \leq 10 \mu \mathrm{g} / \mathrm{dL}$ ) and changes to electrocardiograms (prolonged QT and QRS intervals) and atrioventricular conduction defect; however, no associations were observed between PbB and conduction abnormalities (Cheng et al. 1998; Eum et al. 2011; Park et al. 2009a). For ischemic heart disease, increased risks were associated with PbB and with tibia and patella Pb concentrations (Jain et al. 2007). A 1 SD increase in PbB was associated with a 1.27-fold increase in risk for ischemic heart disease (Jain et al. 2007). In addition to the evaluations of the Normative Aging Study population, a large cross-sectional study of 2,163 men and 3,185 women found an increased risk of cardiovascular disease (including coronary artery disease, myocardial infarction, and stroke) for women in the two highest exposure PbB quartiles (Q3: 3.77$5.460 \mu \mathrm{g} / \mathrm{dL} ; \mathrm{Q} 4: \geq 5.461 \mu \mathrm{g} / \mathrm{dL}$ ), although risk was not increased for men in any PbB quartile (Q4: $\geq 6.25 \mu \mathrm{g} / \mathrm{dL}$ ) (Chen et al. 2017). Other studies have evaluated left ventricular function and structure, heart rate variability, and QRS-T wave angle (Jing et al. 2019l Yang et al. 2017; Yu et al. 2019a). A small $(\mathrm{n}=179)$ prospective study in adults with a mean $\mathrm{PbB}$ of $4.18 \mu \mathrm{g} / \mathrm{dL}$ showed an inverse association between $\mathrm{PbB}$ and left ventricular systolic function, but not left ventricular diastolic function or left ventricular structure (Yang et al. 2017). Results of a small ( $n=328)$ cross-sectional study in newly hired male $\mathrm{Pb}$ workers did not observe an association between $\mathrm{PbB}$ (mean $4.54 \mu \mathrm{g} / \mathrm{dL}$ ) and heart rate variability (Yu et al. 2019a). A large $(n=7,179)$ study of NHANES III participants showed that PbB was associated 
Table 2-9. Summary of Epidemiological Studies Evaluating Atherosclerosis at Mean Blood Lead Concentration $(\mathrm{PbB}) \leq 10 \mu \mathrm{g} / \mathrm{dL}^{\mathrm{a}}$

\begin{tabular}{|c|c|c|c|}
\hline Reference and study population & $\mathrm{PbB}(\mu \mathrm{g} / \mathrm{dL})$ & Outcome evaluated & Result $^{b}$ \\
\hline $\begin{array}{l}\text { Ari et al. } 2011 \\
\text { Clinical study; } n=50 \text { adult male and } \\
\text { female hemodialysis patients and } \\
48 \text { age- and sex-matched controls }\end{array}$ & $\begin{array}{l}\text { Mean } \\
\text { - } \quad \text { Hemodialysis patients: } \\
\quad 0.41 \\
\text { - Controls: } 0.10\end{array}$ & $\begin{array}{l}\text { Greater carotid artery } \\
\text { intima-media thickness }\end{array}$ & $\begin{array}{l}\beta \text { (SE), mm per } \mu g / d L \text { PbB: } 0.101(0.040) \\
p=0.013^{*}\end{array}$ \\
\hline $\begin{array}{l}\text { Muntner et al. 2005' } \\
\text { Cross-sectional study; } \\
\text { n=9,961participants }\end{array}$ & $\begin{array}{l}\text { Mean: } 1.64 \\
\text { Quartiles: } \\
\text { - } \quad \text { Q1: }<1.06 \\
\text { - } \quad \text { 2: } 1.06-1.63 \\
\text { - } \quad \text { Q3: } 1.63-2.47 \\
\text { - } \quad \text { Q4: } \geq 2.47 \\
\end{array}$ & PAD & $\begin{array}{l}\text { OR for prevalence in Q4: } 1.92(1.02-3.61)^{\star} \\
\text { p-trend (across quartiles): }<0.001^{*}\end{array}$ \\
\hline $\begin{array}{l}\text { Navas-Acien et al. } \mathbf{2 0 0 4}^{\mathrm{c}} \\
\text { Cross-sectional study; } \\
\mathrm{n}=2,125 \text { participants }\end{array}$ & $\begin{array}{l}\text { Mean: } 2.07 \\
\text { Quartiles: } \\
\begin{array}{l}\text { - } \\
\text { - }\end{array} \text { Q2: } 1.45-2.07 \\
\text { - } \quad \text { Q3: } 2.07-2.90 \\
\text { - } \quad \text { 4: }>2.90\end{array}$ & PAD & $\begin{array}{l}\text { OR for prevalence in Q4: } 2.88(0.87,9.47) \\
\text { p-trend (across quartiles) for risk: } \mathbf{0 . 0 2 *}\end{array}$ \\
\hline
\end{tabular}

aSee the Supporting Document for Epidemiological Studies for Lead, Table 3 for more detailed descriptions of studies.

${ }^{\mathrm{b}}$ Asterisk and bold indicate association with $\mathrm{Pb}$; unless otherwise specified, values in parenthesis are $95 \% \mathrm{Cls}$

'Study population was from NHANES.

$\mathrm{Cl}=$ confidence interval; NHANES = National Health and Nutrition Examination Survey; OR = odds ratio; PAD = peripheral artery disease; pb = lead;

$\mathrm{SE}=$ standard error 
2. HEALTH EFFECTS

Table 2-10. Summary of Epidemiological Studies Evaluating Heart Disease at Mean Blood Lead Concentration $(\mathrm{PbB}) \leq 10 \mu \mathrm{g} / \mathrm{dL}^{\mathrm{a}}$

\begin{tabular}{|c|c|c|c|}
\hline Reference and study population & $\mathrm{PbB}(\mu \mathrm{g} / \mathrm{dL})$ & Outcome evaluated & Result $^{\text {b,c }}$ \\
\hline Chen et al. 2017 & $\begin{array}{l}\text { Quartiles: } \\
\text { - Men }\end{array}$ & $\begin{array}{l}\text { Cardiovascular } \\
\text { disease }\end{array}$ & $\begin{array}{l}\text { ORs: } \\
\text { Men, Q4: } 1.01(0.58,1.78) ; p \text {-trend: } 0.59\end{array}$ \\
\hline $\begin{array}{l}\text { Cross-sectional study; } \\
n=5,348 \text { adults (men: } 2,163 \text {; women: }\end{array}$ & $\begin{array}{ll}\text { o } & \text { Q1: } \leq 2.900 \\
\text { o } & \text { O2: } 2.901-4.400\end{array}$ & & $\begin{array}{l}\text { Women, Q3: } 1.65(1.03,2.66)^{*} \\
\text { Women, Q4, } 1.93(1.22,3.04) \text {; p-trend: }<0.01^{*}\end{array}$ \\
\hline 3,185 ) aged $\geq 18$ years & o O3: $4.401-6.248$ & & 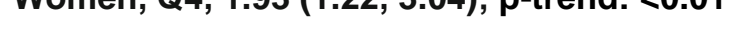 \\
\hline
\end{tabular}

3,185 ) aged $\geq 18$ years $\quad 0$ O3: $4.401-6.248$

o Q4: $\geq 6.249$

- Women

o $\mathrm{Q1}: \leq 2.50$

0 Q2: $2.501-3.770$

o Q3:3.771-5.460

o Q4: $\geq 5.461$

Cheng et al. 1998

Longitudinal study; $\mathrm{n}=775$ men $(\mathrm{n}=277$ for men $<65$ years of age)
PbB mean: 5.8 Bone $\mathrm{Pb}, \mu \mathrm{g} / \mathrm{g}$, mean (SD)

- Tibia: $22.2(13.4)$

- Patella: 30.8 (19.2)

\begin{tabular}{|c|c|}
\hline \multirow[t]{3}{*}{ QT interval } & $\begin{array}{l}\beta, \text { msec per } 10 \text {-fold increase in } \mathrm{PbB}:-0.65 \\
(-10.40,9.10) ; p=0.90\end{array}$ \\
\hline & $\begin{array}{l}\beta, \text { msec per } 10 \text {-fold increase in tibia } \mathrm{Pb} \text { : } \\
5.03(0.83,9.22) ; p=0.02^{*}\end{array}$ \\
\hline & $\begin{array}{l}\beta, \text { msec per } 10 \text {-fold increase in patella } \mathrm{Pb}: 3.00 \\
(0.16,5.84) ; p=0.04^{*}\end{array}$ \\
\hline \multirow[t]{3}{*}{ QRS interval } & $\begin{array}{l}\beta, \text { msec per } 1 \text { unit increase in PbB: }-3.49(-10.72 \text {, } \\
3.75) ; p=0.35\end{array}$ \\
\hline & $\begin{array}{l}\beta, \text { msec per 1-fold increase in tibia } \mathrm{Pb}: \\
4.83(1.83,7.83) ; p<0.01^{*}\end{array}$ \\
\hline & $\begin{array}{l}\beta, \text { msec per 1-fold increase in patella } \mathrm{Pb} \text { : } \\
2.23(0.10,4.36) ; p=0.04^{*}\end{array}$ \\
\hline IVCD & $\begin{array}{l}\text { OR for a } 10 \text {-fold increase in tibia } \mathrm{Pb}: 2.23(1.28 \\
3.90) ; p<0.01^{*}\end{array}$ \\
\hline
\end{tabular}


2. HEALTH EFFECTS

\section{Table 2-10. Summary of Epidemiological Studies Evaluating Heart Disease at Mean Blood Lead Concentration $(\mathrm{PbB}) \leq 10 \mu \mathrm{g} / \mathrm{dL}^{\mathrm{a}}$}

\begin{tabular}{|c|c|c|c|}
\hline Reference and study population & $\mathrm{PbB}(\mu \mathrm{g} / \mathrm{dL})$ & Outcome evaluated & Result $^{\text {b,c }}$ \\
\hline \multirow{4}{*}{$\begin{array}{l}\text { Eum et al. } \mathbf{2 0 1 1}^{\mathrm{d}} \\
\text { Prospective longitudinal study; } \\
\mathrm{n}=600 \text { men }\end{array}$} & \multirow{4}{*}{$\begin{array}{l}\text { PbB baseline mean: } 5.8 \\
\text { PbB Tertiles: } \\
\text { - T1: }<4 \\
\text { - T2: } 4-6 \\
\text { - T3: }>6 \\
\text { Tibia } \mathrm{Pb}(\mu \mathrm{g} / \mathrm{g}) \text { baseline mean: } \\
21.6 \\
\text { Tertiles: } \\
\text { - T1: }<16 \\
\text { - T2: } 16-23 \\
\text { - T3: }>23 \\
\end{array}$} & \multirow[t]{2}{*}{ QT interval } & PbB OR for T3: $1.31(0.69,2.48) ; p$-trend: 0.41 \\
\hline & & & $\begin{array}{l}\text { Tibia OR for T3: } 2.53(1.22,5.25)^{*} \text {; p-trend: } \\
\text { 0.003* }^{*}\end{array}$ \\
\hline & & \multirow{2}{*}{$\begin{array}{l}\text { Atrioventricular } \\
\text { conduction defect }\end{array}$} & PbB OR for T3: $0.52(0.19,1.45) ; p$-trend: 0.16 \\
\hline & & & Tibia OR for T3: $0.23(0.06,0.87) ; p$-trend: 0.03 \\
\hline \multirow{3}{*}{$\begin{array}{l}\text { Jain et al. } \mathbf{2 0 0 7}^{\mathbf{d}} \\
\text { Longitudinal prospective study; } \\
n=837 \text { men }\end{array}$} & \multirow{3}{*}{$\begin{array}{l}\text { PbB baseline mean } \\
\text { - } \quad \text { Non-cases } 6.2 \\
\text { - } \quad \text { Cases } 7.0 \\
\text { Patella } \mathrm{Pb}(\mu \mathrm{g} / \mathrm{g}) \text { baseline mean } \\
\text { - } \quad \text { Non-cases } 30.6 \\
\text { - } \quad \text { Cases } 36.8\end{array}$} & \multirow[t]{3}{*}{$\begin{array}{l}\text { Ischemic heart } \\
\text { disease }\end{array}$} & $\begin{array}{l}\text { PbB } \beta \text { per } 1 \text { SD increase in PbB: } 1.27 \text { (1.01, } \\
1.59)^{\star}\end{array}$ \\
\hline & & & $\begin{array}{l}\text { PbB HR per } 1 \text { log increased in PbB: } 1.45 \text { (1.01, } \\
2.06) ; p=0.05^{*}\end{array}$ \\
\hline & & & $\begin{array}{l}\text { Patella Pb HR per } 1 \text { log increased in bone } \mathrm{Pb} \text { : } \\
2.64(1.09,6.37) ; p=0.05^{*}\end{array}$ \\
\hline \multirow{3}{*}{$\begin{array}{l}\text { Park et al. } \mathbf{2 0 0 9}^{\mathbf{d}} \\
\text { Longitudinal prospective study; } \\
n=613 \text { men }\end{array}$} & \multirow{3}{*}{$\begin{array}{ll}- & \text { PbB median (IQR): } 5(4-7) \\
- & \text { Patella } \mathrm{Pb}(\mu \mathrm{g} / \mathrm{dL}), \text { median } \\
& \text { (IQR): } 26(18-37) \\
\text { - } & \text { Tibia Pb }(\mu \mathrm{g} / \mathrm{dL}), \text { median } \\
& \text { (IQR): } 19(14-27)\end{array}$} & \multirow[t]{3}{*}{ QT interval } & $\begin{array}{l}\mathrm{PbB} \beta \text { for msec increase per IQR: } 1.3(-0.76 \\
3.36)\end{array}$ \\
\hline & & & $\begin{array}{l}\text { Patella } \beta \text { for msec increase per IQR: } 2.64 \text { (0.13, } \\
5.15)^{*}\end{array}$ \\
\hline & & & $\begin{array}{l}\text { Tibia } \beta \text { for msec increase per IQR: } 2.85(0.29 \text {, } \\
5.40)^{*}\end{array}$ \\
\hline \multirow{3}{*}{$\begin{array}{l}\text { Yang et al. } 2017 \\
\text { Prospective study; } n=179 \text { adults } \\
\text { (50.3\% women); follow-up period } \\
11.9 \text { years }\end{array}$} & \multirow[t]{3}{*}{ PbB baseline Gmean: 4.19} & \multirow[t]{3}{*}{$\begin{array}{l}\text { Left ventricular } \\
\text { systolic function }\end{array}$} & $\begin{array}{l}\beta, \text { per doubling of } \mathrm{PbB} \text { for ejection fraction }(\%) \text { : } \\
0.150(-1.019,1.320) ; p=0.800\end{array}$ \\
\hline & & & $\begin{array}{l}\beta, \text { per doubling of PbB for global longitudinal } \\
\text { strain (\%): }-0.392(-0.753,-0.030) ; p=0.034^{*}\end{array}$ \\
\hline & & & $\begin{array}{l}\beta, \text { per doubling of } \mathrm{PbB} \text { for regional } \\
\text { longitudinal strain }(\%):-0.618(-1.167,-0.068) \\
p=0.028^{*}\end{array}$ \\
\hline
\end{tabular}


Table 2-10. Summary of Epidemiological Studies Evaluating Heart Disease at Mean Blood Lead Concentration $(\mathrm{PbB}) \leq 10 \mu \mathrm{g} / \mathrm{dL} \mathrm{L}^{\mathrm{a}}$

\begin{tabular}{|c|c|c|c|}
\hline \multirow[t]{7}{*}{ Reference and study population } & $\mathrm{PbB}(\mu \mathrm{g} / \mathrm{dL})$ & Outcome evaluated & Result $^{\mathrm{b}, \mathrm{c}}$ \\
\hline & & & $\begin{array}{l}\beta, \text { per doubling of } \mathrm{PbB} \text { for regional } \\
\text { longitudinal strain rate (per second): }-0.056 \\
(-0.097,-0.015) ; p=0.008^{*}\end{array}$ \\
\hline & & & $\begin{array}{l}\beta, \text { per doubling of } \mathrm{PbB} \text { for regional radial strain } \\
(\%):-1.825(-3.740,0.090) ; p=0.062\end{array}$ \\
\hline & & & $\begin{array}{l}\beta, \text { per doubling of } \mathrm{PbB} \text { for regional radial } \\
\text { strain rate (per second): }-0.113 \\
(-0.226,-0.0002) ; p=0.050^{*}\end{array}$ \\
\hline & & $\begin{array}{l}\text { Left ventricular } \\
\text { structure }\end{array}$ & $\begin{array}{l}\beta, \text { per doubling of } \mathrm{PbB} \text { for left ventricular mass } \\
\left(\mathrm{g} / \mathrm{m}^{2}\right):-1.399(-4.504,1.707) ; p=0.375\end{array}$ \\
\hline & & & $\begin{array}{l}\beta, \text { per doubling of } \mathrm{PbB} \text { for end-diastolic diameter } \\
(\mathrm{cm}):-0.064(-0.134,0.006) ; p=0.072\end{array}$ \\
\hline & & & $\begin{array}{l}\beta \text {, per doubling of } \mathrm{PbB} \text { for relative wall thickness: } \\
0.0065(-0.0031,0.0162) ; p=0.185\end{array}$ \\
\hline $\begin{array}{l}\text { Yu et al. 2019a } \\
\text { Cross-sectional study; } \mathrm{n}=328 \text { newly } \\
\text { hired male } \mathrm{Pb} \text { workers }\end{array}$ & Mean: 4.54 & Heart rate variability & $\begin{array}{l}\text { Regression }(\beta) \text { coefficients }(95 \% \mathrm{Cl}) \text { per } 10 \text {-fold } \\
\text { increase in PbB: } \\
\text { - Supine position: } 3.0(-20.4,33.0) ; p=0.82 \\
\text { - } \quad \text { Standing position: }-6.0(-26.2,19.7) ; p=0.61 \\
\text { - } \quad \text { Orthostatic change: }-8.8(-31.8,17.5) ; p=0.47\end{array}$ \\
\hline
\end{tabular}

aSee the Supporting Document for Epidemiological Studies for Lead, Table 3 for more detailed descriptions of studies.

${ }^{b}$ Asterisk and bold indicate association with $\mathrm{Pb}$; unless otherwise specified, values in parenthesis are $95 \% \mathrm{Cls}$; $\mathrm{p}$-values $<0.05$ unless otherwise noted in the table.

If bone $\mathrm{Pb}$ is noted under results, study did not show associations between $\mathrm{PbB}$ and blood pressure parameters; however, results showed associations between bone $\mathrm{Pb}$ concentrations and increased blood pressure at concomitant $\mathrm{PbB} \leq 10 \mu \mathrm{g} / \mathrm{dL}$.

${ }^{d}$ Study population was from the Normative Aging Study.

$\mathrm{Cl}=$ confidence interval; Gmean = geometric mean; HR = hazard ratio; IQR = intraquartile range; IVCD = intraventricular conduction defect; OR = odds ratio; $\mathrm{Pb}=$ lead; $\mathrm{SD}=$ standard deviation 
with an abnormal QRS-T wave angle in men (mean PbB: $4.10 \mu \mathrm{g} / \mathrm{dL}$ ), but not in women (mean PbB: $2.93 \mu \mathrm{g} / \mathrm{dL}$ ) (Jing et al. 2019).

Mortality due to cardiovascular disease. Mortality due to cardiovascular disease at $\mathrm{PbB} \leq 10 \mu \mathrm{g} / \mathrm{dL}$ has been examined in large prospective and longitudinal studies, which provide mixed results. Studies are briefly summarized in Table 2-11, with additional details provided in the Supporting Document for Epidemiological Studies for Lead, Table 3. Three of these were conducted in large studies of men and women participating in NHANES (Aoki et al. 2016; Lanphear et al. 2018; Menke et al. 2006; Schober et al. 2006). Aoki et al. (2016), Lanphear et al. (2018), and Menke et al. (2006) observed positive associations of mortality due to cardiovascular disease, including ischemic heart disease, myocardial infarction, and stroke and at $\mathrm{PbB} \leq 10 \mu \mathrm{g} / \mathrm{dL}$, including positive trends for mortality with increasing $\mathrm{PbB}$.

In contrast, Schober et al. (2006) did not find increased cardiovascular mortality risk at $\mathrm{PbB}<10 \mu \mathrm{g} / \mathrm{dL}$, although risk was increased at $\mathrm{PbB} \geq 10 \mu \mathrm{g} / \mathrm{dL}$ and a positive trend for mortality was observed with increasing $\mathrm{PbB}$. For $\mathrm{PbB}$, no increased risk or positive trend for morality due to cardiovascular was observed in men from the Normative Aging Study (Weisskopf et al. 2009). In women, the risk of mortality due to coronary heart disease was increased at $\mathrm{PbB} \geq 8 \mu \mathrm{g} / \mathrm{dL}$ compared to $\mathrm{PbB}<8 \mu \mathrm{g} / \mathrm{dL}$ (Khalil et al. 2009).

Associations Between Bone Pb and Cardiovascular Effects. Several studies have evaluated associations between bone $\mathrm{Pb}$ concentration and blood pressure and cardiac outcomes. Results provide evidence that long-term exposure to $\mathrm{Pb}$ produces adverse effects on the cardiovascular system.

Increased blood pressure and hypertension. Numerous studies show associations between bone $\mathrm{Pb}$ concentration and increased blood pressure and increased risk of hypertension (see Table 2-12). The most studied population is older men participating in the Normative Aging Study. Results consistently show positive associations between tibia $\mathrm{Pb}$ and systolic blood pressure (Cheng et al. 2001), pulse pressure (Jhun et al. 2015; Perlstein et al. 2007; Zhang et al. 2010), and risk of hypertension (Cheng et al. 2001; Elmarsafawy et al. 2006; Hu et al. 1996a; Peters et al. 2007). The association between bone $\mathrm{Pb}$ and elevated pulse pressure suggests that $\mathrm{Pb}$ may alter cardiovascular function through loss of arterial elasticity (Jhun et al. 2015; Perlstein et al. 2007; Zhang et al. 2010). Associations between patella Pb and blood pressure outcomes have been somewhat less consistent, with some studies showing positive associations (Hu et al. 1997; Jhun et al. 2015; Perlstein et al. 2007; Peters et al. 2007; Zhang et al. 2010) and other studies showing no associations (Cheng et al. 2001; Elmarsafawy et al. 2006). Other study 
2. HEALTH EFFECTS

\section{Table 2-11. Summary of Epidemiological Studies Evaluating Mortality due to Cardiovascular Disease at Mean Blood Lead Concentrations (PbB) $\leq 10 \mu \mathrm{g} / \mathrm{dL}^{\mathrm{a}}$}

\begin{tabular}{|c|c|c|c|}
\hline Reference and study population & $\mathrm{PbB}(\mu \mathrm{g} / \mathrm{dL})$ & Outcome evaluated & Result $^{b}$ \\
\hline Aoki et al. $2016^{c}$ & Mean: 1.73 & $\begin{array}{l}\text { Mortality due to } \\
\text { cardiovascular disease }\end{array}$ & $\begin{array}{l}\text { RR, per 10-fold increase in PbB: } 1.44 \text { (1.05, } \\
1.98)^{*}\end{array}$ \\
\hline \multicolumn{4}{|l|}{ Prospective study; $n=18,602$} \\
\hline Khalil et al. 2009 & Mean: 5.3 & $\begin{array}{l}\text { Mortality due to coronary heart } \\
\text { disease }\end{array}$ & $\begin{array}{l}\text { PbB } \geq 8.0 \text { compared to women with } \mathrm{PbB}<8.0 \text {. } \\
\text { HR: } 3.08(1.23,7.70) ; p=0.016^{*}\end{array}$ \\
\hline \multicolumn{4}{|l|}{ Prospective study; n=533 women } \\
\hline \multirow{4}{*}{$\begin{array}{l}\text { Menke et al. 2006c } \\
\text { Longitudinal study; } n=13,946\end{array}$} & \multirow{4}{*}{$\begin{array}{l}\text { Baseline mean: } 2.58 \\
\text { Tertiles: } \\
\text { - } \quad \text { T1: }<1.93 \\
\text { - } \quad \text { T2: } 1.94-3.62 \\
\text { - } \quad \text { T3: } \geq 3.63\end{array}$} & $\begin{array}{l}\text { Mortality due to cardiovascular } \\
\text { disease }\end{array}$ & $\begin{array}{l}\text { HR for T3 versus T1: } 1.55(1.08,2.24)^{*} ; \\
\text { p-trend: } 0.003^{*}\end{array}$ \\
\hline & & $\begin{array}{l}\text { Mortality due to myocardial } \\
\text { infarction }\end{array}$ & $\begin{array}{l}\text { HR for T3 versus T1: } 1.89(1.04,3.43)^{*} \text {; } \\
\text { p-trend: } 0.007^{*}\end{array}$ \\
\hline & & Mortality due to stroke & $\begin{array}{l}\text { HR for T3 versus T1: } 2.51(1.20,5.26)^{*} \text {; } \\
\text { p-trend: } 0.017^{*}\end{array}$ \\
\hline & & & $\begin{array}{l}\text { RR for T3 versus T1: } 1.55(1.16,2.07)^{*} ; \\
\text { p-trend: }<0.01^{*}\end{array}$ \\
\hline \multirow{2}{*}{$\begin{array}{l}\text { Lanphear et al. } \mathbf{2 0 1 8}^{\mathrm{c}} \\
\text { Longitudinal study; } n=14,289\end{array}$} & \multirow[t]{2}{*}{ Mean: 2.71} & $\begin{array}{l}\text { Mortality due to cardiovascular } \\
\text { disease }\end{array}$ & $\begin{array}{l}\text { HR for PbB increase from } 1.0 \text { to } 6.7 \mu \mathrm{g} / \mathrm{dL} \text { : } \\
1.70(1.30,2.22)^{*}\end{array}$ \\
\hline & & $\begin{array}{l}\text { Mortality due to ischemic heart } \\
\text { disease }\end{array}$ & $\begin{array}{l}\text { HR for } \mathrm{PbB} \text { increase from } 1.0 \text { to } 6.7 \mu \mathrm{g} / \mathrm{dL} \text { : } \\
2.08(1.52,2.85)^{\star}\end{array}$ \\
\hline Weisskopf et al. 2009d & $\begin{array}{l}\text { Mean: } 5.6 \\
\text { Tertiles }\end{array}$ & $\begin{array}{l}\text { Mortality due to cardiovascular } \\
\text { disease }\end{array}$ & $\begin{array}{l}\text { HR for T3 versus T1: } 1.10(0.67,1.80) \text {; } p \text {-trend: } \\
0.72\end{array}$ \\
\hline Longitudinal study; $n=868$ men & $\begin{array}{ll}\text { - } & \mathrm{T} 1:<4 \\
\text { - } & \mathrm{T} 2: 4-6 \\
\text { - } & \mathrm{T} 3:>6\end{array}$ & & \\
\hline
\end{tabular}

asee the Supporting Document for Epidemiological Studies for Lead, Table 3 for more detailed descriptions of studies.

${ }^{b}$ Asterisk and bold indicate association with $\mathrm{Pb}$; unless otherwise specified, values in parenthesis are $95 \% \mathrm{Cls}$; $\mathrm{p}$-values <0.05 unless otherwise noted in the table.

'Study population was from NHANES.

dStudy population was from the Normative Aging Study.

$\mathrm{Cl}=$ confidence interval; $\mathrm{HR}=$ hazard ratio; NHANES = National Health and Nutrition Examination Survey; $\mathrm{Pb}=$ lead; $\mathrm{RR}=$ risk ratio 
populations examined include adults (Martin et al. 2006), young adults (Gerr et al. 2002), current and former Pb workers (Glenn et al. 2003; Lee et al. 2001), women (Korrick et al. 1999), pregnant women (Rothenberg et al. 2002), and mother-child pairs (Zhang et al. 2011). Although study results are not consistent, positive associations between bone $\mathrm{Pb}$ and blood pressure and risk of hypertension have been reported. Navas-Acien et al. (2008) conducted a meta-analysis of 10 studies (see Table 2-12 for studies included in the analysis) to evaluate associations between tibia and patella $\mathrm{Pb}$ and blood pressure outcomes. Positive associations were observed between tibia $\mathrm{Pb}$ and systolic blood pressure and hypertension risk, but no associations were observed between tibia $\mathrm{Pb}$ and diastolic blood pressure or between patella $\mathrm{Pb}$ and systolic blood pressure, diastolic blood pressure, or hypertension risk.

\section{Table 2-12. Associations Between Bone $\mathrm{Pb}$ and Blood Pressure Outcomes}

\begin{tabular}{|c|c|c|c|c|c|}
\hline \multirow[b]{2}{*}{ Reference } & \multirow[b]{2}{*}{ Population } & \multicolumn{4}{|c|}{ Blood pressure outcome } \\
\hline & & $\begin{array}{l}\text { Systolic } \\
\text { blood } \\
\text { pressure }\end{array}$ & $\begin{array}{l}\text { Diastolic } \\
\text { blood } \\
\text { pressure }\end{array}$ & $\begin{array}{l}\text { Pulse } \\
\text { pressure }\end{array}$ & Hypertension \\
\hline $\begin{array}{l}\text { Cheng et al. } \\
2001^{\mathrm{a}}\end{array}$ & 833 men $^{b}$ & $\begin{array}{l}\uparrow \mathrm{T} \\
0 \mathrm{P}\end{array}$ & - & - & $\begin{array}{l}\uparrow \mathrm{T} \\
0 \mathrm{P}\end{array}$ \\
\hline $\begin{array}{l}\text { Elmarsafawy } \\
\text { et al. } 2006\end{array}$ & 471 men $^{\mathrm{b}}$ & - & - & - & $\begin{array}{c}\uparrow \mathrm{T} \text { (at low dietary } \\
\text { calcium) } \\
\text { O P (at high dietary } \\
\text { calcium) }\end{array}$ \\
\hline $\begin{array}{l}\text { Gerr et al. } \\
2002^{a}\end{array}$ & 508 young adults ${ }^{c}$ & $\uparrow \mathrm{T}$ & $\uparrow \top$ & - & - \\
\hline $\begin{array}{l}\text { Glenn et al. } \\
2003^{a}\end{array}$ & 496 male $\mathrm{Pb}$ workers ${ }^{\mathrm{d}}$ & $\begin{array}{l}\uparrow T \\
\uparrow P\end{array}$ & $\begin{array}{l}0 \mathrm{~T} \\
0 \mathrm{P}\end{array}$ & - & - \\
\hline $\begin{array}{l}\text { Glenn et al. } \\
2006^{a}\end{array}$ & 575 adult $\mathrm{Pb}$ workers ${ }^{\mathrm{e}}$ & $\downarrow T$ & $0 \mathrm{~T}$ & - & - \\
\hline $\begin{array}{l}\text { Hu et al. } \\
1996 a^{a}\end{array}$ & 590 & - & - & - & $\begin{array}{l}\uparrow T \\
\uparrow P\end{array}$ \\
\hline $\begin{array}{l}\text { Jhun et al. } \\
2015\end{array}$ & 727 men $^{b}$ & - & - & $\begin{array}{l}\uparrow T \\
\uparrow P\end{array}$ & - \\
\hline $\begin{array}{l}\text { Korrick et al. } \\
1999^{a}\end{array}$ & $\begin{array}{l}689 \text { women }(214 \\
\text { cases; } 475 \text { controls })^{f}\end{array}$ & - & - & - & $\begin{array}{l}0 T \\
\uparrow P\end{array}$ \\
\hline $\begin{array}{l}\text { Lee et al. } \\
2001^{a}\end{array}$ & $\begin{array}{l}924 \text { adult } \mathrm{Pb} \text { workers } \\
\text { ( } 789 \text { cases; } \\
135 \text { controls }^{\mathrm{e}}\end{array}$ & $\uparrow \mathrm{T}$ & $0 \mathrm{~T}$ & - & $\uparrow \mathrm{T}$ \\
\hline $\begin{array}{l}\text { Martin et al. } \\
2006^{a}\end{array}$ & 964 adults & $0 \mathrm{~T}$ & $0 \mathrm{~T}$ & - & $\uparrow \mathrm{T}$ \\
\hline $\begin{array}{l}\text { Perlstein et } \\
\text { al. } 2007\end{array}$ & 593 men $^{b}$ & - & - & $\begin{array}{l}\uparrow T \\
\uparrow P\end{array}$ & - \\
\hline
\end{tabular}


Table 2-12. Associations Between Bone $\mathrm{Pb}$ and Blood Pressure Outcomes

\begin{tabular}{|c|c|c|c|c|c|}
\hline \multirow[b]{2}{*}{ Reference } & \multirow[b]{2}{*}{ Population } & \multicolumn{4}{|c|}{ Blood pressure outcome } \\
\hline & & $\begin{array}{l}\text { Systolic } \\
\text { blood } \\
\text { pressure }\end{array}$ & $\begin{array}{l}\text { Diastolic } \\
\text { blood } \\
\text { pressure }\end{array}$ & $\begin{array}{l}\text { Pulse } \\
\text { pressure }\end{array}$ & Hypertension \\
\hline $\begin{array}{l}\text { Peters et al. } \\
2007\end{array}$ & 512 men $^{b}$ & - & - & - & $\begin{array}{l}\uparrow \mathrm{T} \text { (with high stress) } \\
\uparrow \mathrm{P} \text { (with high stress) }\end{array}$ \\
\hline $\begin{array}{l}\text { Rothenberg } \\
\text { et al. } 2002^{a}\end{array}$ & 1,006 pregnant women & - & - & - & $\begin{array}{l}\uparrow \mathrm{C}\left(3^{\text {rd }} \text { trimester }\right) \\
0 \mathrm{~T}\left(3^{\text {rd }} \text { trimester }\right)\end{array}$ \\
\hline $\begin{array}{l}\text { Schwartz et } \\
\text { al. } 2000 c^{a}\end{array}$ & 543 male $\mathrm{Pb}$ workers ${ }^{\mathrm{d}}$ & $0 \mathrm{~T}$ & $0 \mathrm{~T}$ & - & $0 \mathrm{~T}$ \\
\hline $\begin{array}{l}\text { Weaver et al. } \\
2008\end{array}$ & $652 \mathrm{~Pb}$ workers & $0 P$ & $0 P$ & - & $0 P$ \\
\hline $\begin{array}{l}\text { Zhang et al. } \\
2010\end{array}$ & 612 men $^{b}$ & - & - & $\begin{array}{l}\uparrow T \\
\uparrow P\end{array}$ & - \\
\hline $\begin{array}{l}\text { Zhang et al. } \\
2011\end{array}$ & 457 mother-child pairs ${ }^{g}$ & $\begin{array}{l}\uparrow \mathrm{T} \text { (girls) } \\
0 \mathrm{~T} \text { (boys) }\end{array}$ & $\begin{array}{l}\uparrow \mathrm{T} \text { (girls) } \\
0 \mathrm{~T} \text { (boys) }\end{array}$ & - & - \\
\hline
\end{tabular}

ancluded in the Navas-Acien et al. (2008) meta-analysis.

${ }^{b}$ Participants in the Normative Aging Study.

c19-29 years of age.

dCurrent and former $\mathrm{Pb}$ workers in the United States.

eCurrent and former $\mathrm{Pb}$ workers in South Korea.

${ }^{f}$ Nurses Health Study.

gBased on maternal bone $\mathrm{Pb}$ measurement.

$\uparrow=$ positive association; $\downarrow=$ inverse association; $0=$ no association; $-=$ not reported; $\mathrm{C}=$ calcaneus bone;

$\mathrm{P}=$ patella; $\mathrm{Pb}=$ lead; $\mathrm{T}=$ tibia

Cardiac function. Several studies evaluating associations between bone $\mathrm{Pb}$ and cardiac function, disease, and mortality were conducted in participants of the Normative Aging Study (see Table 2-13). For tibia $\mathrm{Pb}$, positive associations have been observed for QT and QRS intervals (Cheng et al. 1998; Eum et al. 2011; Park et al. 2009a), atrioventricular and intraventricular block (Cheng et al. 1998), and ischemic heart disease (Jain et al. 2007). For patella Pb, positive associations were observed for QT and QRS intervals (Cheng et al. 1998; Park et al. 2009a). Both tibia Pb and patella Pb were positively associated with ischemic heart disease (Jain et al. 2007), and patella and tibia Pb were associated with an increased risk of coronary heart disease (Ding et al. 2016, 2019). However, no association was observed between tibia or patella $\mathrm{Pb}$ and all cardiovascular mortality or mortality due to ischemic heart disease (Weisskopf et al. 2009). 


\begin{tabular}{|c|c|c|c|c|}
\hline \multirow[b]{2}{*}{ Reference } & \multirow[b]{2}{*}{ Population } & \multicolumn{3}{|c|}{ Outcome } \\
\hline & & Function & Disease & Mortality \\
\hline $\begin{array}{l}\text { Cheng et al. } \\
1998\end{array}$ & 775 men $^{\mathrm{a}}$ & $\begin{array}{l}\uparrow \mathrm{T}(\mathrm{QT} \text { and QRS } \\
\text { intervals; } \\
\text { AV block; IV block) } \\
\uparrow \mathrm{P} \text { (QT and QRS } \\
\text { intervals) } \\
\text { O P (AV block; IV block) }\end{array}$ & - & - \\
\hline Ding et al. 2016 & 589 men $^{a}$ & - & $\uparrow \mathrm{P}(\mathrm{CHD})$ & - \\
\hline Ding et al. 2019 & 594 men $^{\mathrm{a}}$ & - & $\begin{array}{l}\uparrow \mathrm{T}(\mathrm{CHD}) \\
\uparrow \mathrm{P}(\mathrm{CHD})\end{array}$ & - \\
\hline Eum et al. 2011 & 600 men $^{a}$ & $\begin{array}{l}\uparrow T(Q T \text { and } Q R S \\
\text { intervals) } \\
0 \mathrm{P} \text { (QT and QRS } \\
\text { intervals) }\end{array}$ & - & - \\
\hline Jain et al. 2007 & 837 men $^{a}$ & - & $\begin{array}{l}\uparrow \mathrm{T}(\mathrm{IHD}) \\
\uparrow \mathrm{P}(\mathrm{IHD}) \\
\end{array}$ & - \\
\hline Park et al. 2006 & 413 men $^{a}$ & $\begin{array}{l}0 \mathrm{~T} \text { (HRV with MetS) } \\
0 \mathrm{~T} \text { (HRV without MetS) } \\
\uparrow \mathrm{P} \text { (HRV with MetS) } \\
0 \text { P (HRV without MetS) }\end{array}$ & - & - \\
\hline $\begin{array}{l}\text { Park et al. } \\
2009 a\end{array}$ & 613 men $^{a}$ & $\begin{array}{l}\uparrow \mathrm{T} \text { (QT interval) } \\
\uparrow \mathrm{P}(\mathrm{QT} \text { interval) } \\
\end{array}$ & - & - \\
\hline $\begin{array}{l}\text { Weisskopf et al. } \\
2009\end{array}$ & 868 men $^{a}$ & - & - & $\begin{array}{l}0 \mathrm{~T} \text { (all cardiovascular or IHD } \\
\text { deaths) } \\
0 \mathrm{P} \text { (all cardiovascular or IHD } \\
\text { deaths) }\end{array}$ \\
\hline
\end{tabular}

aParticipants in the Normative Aging Study.

$\uparrow=$ positive association; $\downarrow=$ inverse association; $0=$ no association; $-=$ not reported; AV $=$ atrioventricular; $\mathrm{CHD}=$ coronary heart disease; HRV = heart rate variability; IHD = ischemic heart disease (defined as myocardial infarction or angina pectoris); IV = intraventricular; MetS = metabolic syndrome (three or more of the following: obesity, diabetes, hypertension, and dyslipidemia); $\mathrm{P}=$ patella; $\mathrm{Pb}=$ lead; $\mathrm{T}=$ tibia

Mechanisms of Action. Several studies and recent reviews include discussions of mechanisms that may be involved in Pb-induced effects on cardiovascular function (Faramawai et al. 2015; Ghiasvand et al. 2013; Mitra et al. 2017; Nawrot et al. 2002; Shiue et al. 2014; Weisskopf et al. 2009; Xu et al. 2015; Zota et al. 2013). Control of cardiovascular function is multi-factorial; therefore, numerous mechanisms are likely involved in $\mathrm{Pb}$-induced cardiovascular effects. Specific mechanisms for cardiovascular effects include: impairment of renal function; effects on vascular smooth muscle, including constrictive effects and disruption of NO-induced vasodilatory actions; increase of sympathetic nervous system activity; 
altered chemoreceptor activity; and altered regulation of the renin-angiotensin-aldosterone axis and the renal kallikrein system. In addition, general mechanisms of toxicity of $\mathrm{Pb}$, including oxidative stress, inflammation, and altered transport of ions across cellular membranes, also are likely to be involved (see Section 2.21).

\subsection{GASTROINTESTINAL}

Overview. Few epidemiological studies have evaluated gastrointestinal effects associated with chronic exposure to $\mathrm{Pb}$. Almost all available studies were conducted in small numbers of workers with $\mathrm{PbB}$ $>10 \mu \mathrm{g} / \mathrm{dL}$, although one study included a group of workers with $\mathrm{PbB} \leq 10 \mu \mathrm{g} / \mathrm{dL}$. Study results consistently show gastrointestinal symptoms (abdominal colic/pain, nausea, vomiting, diarrhea, and/or constipation) associated with PbB ranging from $8.04 \mu \mathrm{g} / \mathrm{dL}$ to approximately $100 \mu \mathrm{g} / \mathrm{dL}$. As reviewed in Section 2.2 (Acute Lead Toxicity), acute exposure to $\mathrm{Pb}$ is associated with gastrointestinal symptoms and intestinal paralysis.

The following gastrointestinal effects have been associated with $\mathrm{PbB}$ :

- $\leq 10 \mu \mathrm{g} / \mathrm{dL}$ :

o Gastrointestinal symptoms (abdominal colic/discomfort).

- $\quad>10 \mu \mathrm{g} / \mathrm{dL}$ :

o Gastrointestinal symptoms (abdominal colic/pain, nausea, vomiting, diarrhea and/or constipation); corroborated in a few studies.

Measures of Exposure. Studies examining the association between gastrointestinal effects of $\mathrm{Pb}$ exposure evaluate exposure by measurement of PbB.

Confounding Factors and Effect Modifiers. Most epidemiological studies on gastrointestinal effects of $\mathrm{Pb}$ are survey or cross-sectional studies of small populations of workers. In general, studies did not consider factors, such as age, diet, nutritional factors, alcohol use, and potential exposure to other occupational chemicals or limitations such as study design (cross-sectional and survey). Failure to account for these factors may attenuate or strengthen the apparent associations between $\mathrm{Pb}$ exposure and the outcome, depending on the direction of the effect of the variable on the outcome. 
Characterization of Effects. In contrast to the large number of epidemiological studies evaluating effects of $\mathrm{Pb}$ on other organ systems (e.g., neurological and cardiovascular outcomes), few epidemiological studies have investigated the gastrointestinal effects of chronic exposure to $\mathrm{Pb}$ (see Table 2-14). With the exception of a survey study conducted in 497 workers (Rosenman et al. 2003), studies were conducted in small worker populations ( $\mathrm{n}=69-155)$. Increased gastrointestinal symptoms (abdominal colic/pain, nausea, vomiting, diarrhea, and/or constipation) were observed in all studies. The lowest $\mathrm{PbB}$ associated with increased gastrointestinal symptoms showed an increased percentage of workers reporting abdominal colic and discomfort at a mean PbB of $8.04 \mu \mathrm{g} / \mathrm{dL}$, compared to controls (PbB 5.76 $\mu \mathrm{g} / \mathrm{dL}$ ) (Kuruvilla et al. 2006). For example, 18.9\% of painters reported abdominal colic compared to 0 in the control group.

Effect at Blood Pb Levels $\leq 10 \mu \boldsymbol{g} / \mathbf{d L}$. See discussion above on Kuruvilla et al. (2006).

Mechanisms of Action. General mechanisms of toxicity of $\mathrm{Pb}$ (reviewed in Section 2.21) are likely involved in the development of gastrointestinal toxicity. EPA (2014c) specifically noted that oxidative stress through ROS could result in gastrointestinal toxicity; as a result, damage to the intestinal mucosa epithelium is possible.

\subsection{HEMATOLOGICAL}

Overview. Pb-induced toxicity to the hematological system has long been established. $\mathrm{Pb}$ inhibits heme synthesis, leading to the development of microcytic, hypochromic anemia. Numerous epidemiological studies have evaluated hematological effects associated with exposure to $\mathrm{Pb}$ in adults and children. Most studies were cross-sectional in design and evaluated effects on heme synthesis and subsequent changes in erythrocyte hemoglobin parameters and anemia. Studies in adults (general populations and workers) and children consistently show inhibition of heme synthesis enzymes, particularly $\delta$-ALAD, and subsequent decreases in blood hemoglobin, red blood cell parameters (e.g., mean cell hemoglobin, mean cell volume), and development of anemia. Other hematological effects observed in epidemiological studies include alterations in erythrocyte function (decreased activities of pyrimidine 5'-nucleotidase and membrane $\left.\mathrm{Ca}^{2+} / \mathrm{Mg}^{2+} \mathrm{ATPase}\right)$, changes in serum EPO concentration, and decreased platelet count. 
2. HEALTH EFFECTS

Table 2-14. Summary of Studies Evaluating Gastrointestinal Symptoms Associated with Chronic Exposure to Lead (Pb)

Reference and study population

Awad el Karin et al. 1986

Cross-sectional study; $\mathrm{n}=92$

exposed; 40 controls

\section{Baker et al. 1979}

Survey study; $\mathrm{n}=160 \mathrm{~Pb}$ workers

Kuruvilla et al. 2006

Cross-sectional study; $\mathrm{n}=155$; exposed workers: $n=105$ (52 battery workers; 53 painters); controls: $\mathrm{n}=50$

\begin{tabular}{ll}
$\mathrm{PbB}(\mu \mathrm{g} / \mathrm{dL})$ & Outcomes evaluated $^{\mathrm{a}}$ \\
$\begin{array}{l}\text { Range of means (by job } \\
\text { category): } 48.1-80.7\end{array}$ & Abdominal colic $^{\text {Controls mean: } 21.2}$ \\
\cline { 2 - 2 }
\end{tabular}

Constipation Range of means (by job category): 41.8-87.2

\section{Effects $^{b}$}

- $\quad$ Exposed (\% reporting symptom) 41.3; exposed versus control $\mathrm{p}=0.01^{*}$

- Control (\% reporting symptom): 7.5

- Exposed (\% reporting symptom) 41.4; exposed versus control $\mathrm{p}=0.01^{*}$

- Control (\% reporting symptom): 10.0 $101.24 \mu \mathrm{g} / \mathrm{dL}(\mathrm{p}<0.01)^{*}$

- $\quad$ PbB, symptom absent: $65.98 \mu \mathrm{g} / \mathrm{dL}$

- $\mathrm{PbB}$, symptoms present: $100.77 \mu \mathrm{g} / \mathrm{dL}$ $(p<0.01)^{*}$

- PbB, symptom absent: $68.25 \mu \mathrm{g} / \mathrm{dL}$

- Battery workers (\% reporting symptom): 17.3; $p<0.01^{*}$

- Painters (\% reporting symptom):18.9; $\mathrm{p}<0.01^{*}$

- Controls (\% reporting symptom): 0

Abdominal discomfort • Battery workers (\% reporting symptom): 19.2; $p<0.01^{*}$

- Painters (\% reporting symptom): 26.4; p $<0.001^{*}$

- Controls (\% reporting symptom): 2

Vomiting • Battery workers (\% reporting symptom): 1.9

- Painters (\% reporting symptom): 1.9

- Controls (\% reporting symptom): 0

Constipation $\quad$ - Battery workers (\% reporting symptom): 0

- Painters (\% reporting symptom): 1.9

- Controls (\% reporting symptom): 2 
2. HEALTH EFFECTS

Table 2-14. Summary of Studies Evaluating Gastrointestinal Symptoms Associated with Chronic Exposure to Lead $(\mathrm{Pb})$

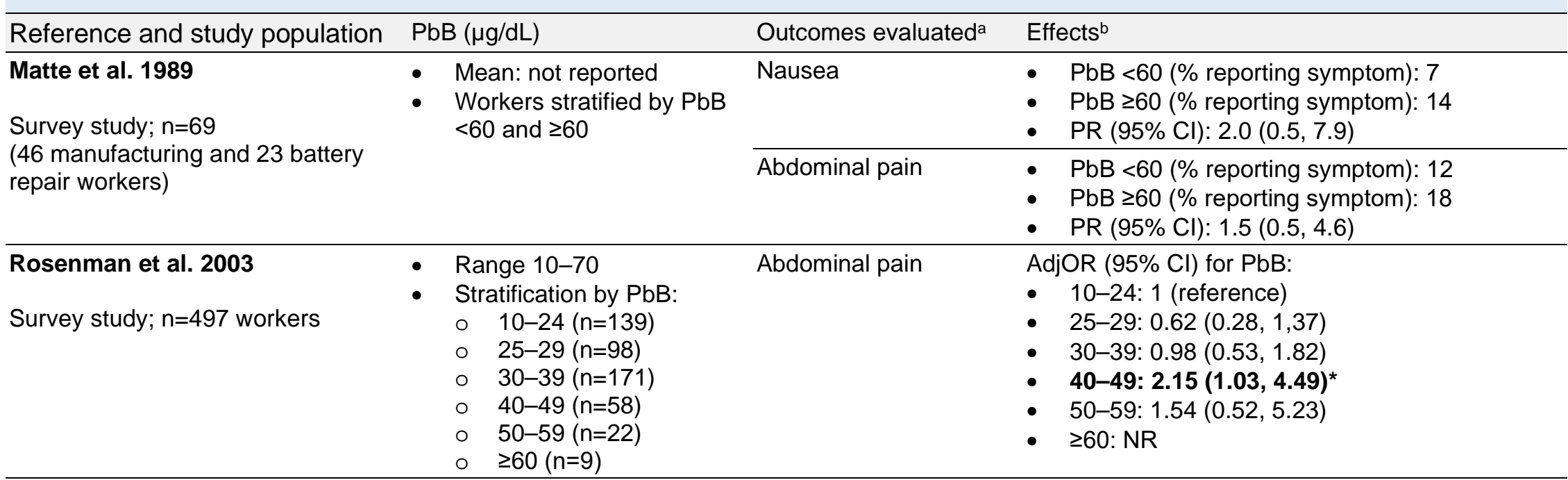

${ }^{a} G$ astrointestinal symptoms include abdominal colic, nausea, vomiting, diarrhea, and/or constipation.

${ }^{\mathrm{b}}$ Asterisk and bold indicate association with $\mathrm{Pb}$; unless otherwise specified, values in parenthesis are $95 \% \mathrm{Cls}$; $\mathrm{p}$-values <0.05 unless otherwise noted in the table.

AdjOR = adjusted odds ratio (adjusted by age, ethnicity group, company screening, and smoking status); $\mathrm{Cl}=\mathrm{confidence}$ interval; $\mathrm{NR}=$ not reported; PbB = blood lead concentration; PR: prevalence ratio 
The following hematological effects have been associated with PbB:

- $\quad \leq 10 \mu \mathrm{g} / \mathrm{dL}$ :

o Inhibition of $\delta$-ALAD; demonstrated in a few studies.

o Decreased blood hemoglobin; evaluated in several studies with mixed results.

o Decreased platelet count.

o Decreased plasma EPO in adult males.

- $\quad>10 \mu \mathrm{g} / \mathrm{dL}$ :

o Dose-dependent decreased heme synthesis due to inhibition of $\delta$-ALAD and other heme metabolism enzymes; demonstrated in numerous studies.

o Anemia and decreased blood hemoglobin; demonstrated in numerous studies.

o Decreased activity of other erythrocyte enzymes (pyrimidine 5'-nucleotidase or red blood cell membrane $\left.\mathrm{Ca}^{2+} / \mathrm{Mg}^{2+} \mathrm{ATPase}\right)$; demonstrated in a few studies.

o Altered plasma EPO concentration:

- Decreased in adult males; evaluated in a few studies with mixed results.

- Decreased in pregnant females; demonstrated in one study, but findings not corroborated.

- Mixed results (both increases and decreases observed) in children; evaluated in a few studies.

Measures of Exposure. Studies evaluating the association between hematological effects and $\mathrm{Pb}$ exposure most commonly evaluate exposure by measurement of $\mathrm{PbB}$.

Confounding Factors and Effect Modifiers. In general, available epidemiological studies on hematological effects do not control for factors, including concomitant exposure to other chemicals, that may affect the hematological system. In addition, dietary insufficiency of iron is the primary cause of microcytic, hypochromic anemia; however, few studies evaluated this as an effect modifier. Age and renal function are also confounding factors, as impairment of renal function can affect renal EPO synthesis and $\mathrm{PbB}$. Failure to account for these factors may attenuate or strengthen the apparent associations between $\mathrm{Pb}$ exposure and the outcome, depending on the direction of the effect of the variable on the outcome.

Characterization of Effects. General trends for studies showing a relationship between $\mathrm{PbB}$ and hematological effects are shown in Table 2-15. Most epidemiological studies of hematological effects have examined effects on heme metabolism and its consequences, with fewer studies examining other 
hematological endpoints (altered serum levels of EPO, altered erythrocyte function, and decreased platelet count). As noted above, Pb-induced toxicity to the hematological system, specifically inhibition of heme synthesis enzymes and resulting anemia and decreased erythrocyte hemoglobin, have long been established. Numerous epidemiological studies in adults and children provide consistent evidence that $\delta$-ALAD activity is inversely correlated with $\mathrm{PbB}$ over a $\mathrm{PbB}$ range of $<10->50 \mu \mathrm{g} / \mathrm{dL}$ (see Table 2-15) with $\delta$-ALAD inhibition and subsequent effects of inhibition showing concentration-dependence for $\mathrm{PbB}$ (Murata et al. 2009; Schwartz et al. 1990). A few studies have reported other hematological effects, including decreased platelet count in Pb workers at PbB of $5.4 \mu \mathrm{g} / \mathrm{dL}$ (Conterato et al. 2013) and $>41 \mu \mathrm{g} / \mathrm{dL}$ (Barman et al. 2014). Inhibition of non-heme metabolism enzymes in erythrocytes was also associated with $\mathrm{PbB}$. In $\mathrm{Pb}$ workers, membrane $\mathrm{Ca}^{2+} / \mathrm{Mg}^{2+} \mathrm{ATPase}$ was inhibited at a $\mathrm{PbB}$ range of approximately 29-42 $\mu \mathrm{g} / \mathrm{dL}$ (Abam et al. 2008), and pyrimidine 5'-nucleotidase was inhibited at a PbB of $>50$ g/dL (Buc and Kaplan 1978). Pyrimidine 5'-nucleotidase also was inhibited in children (aged 15 years) with a PbB range of 30-72 $\mu \mathrm{g} / \mathrm{dL}$ (Angle et al. 1982).

\section{Table 2-15. Overview of Hematological Effects Associated with Chronic Exposure to Lead (Pb)}

\begin{tabular}{|c|c|c|}
\hline $\begin{array}{l}\text { Mean blood lead } \\
\text { concentration }(\mathrm{PbB}) \\
(\mu \mathrm{g} / \mathrm{dL})\end{array}$ & $\begin{array}{l}\text { Effects associated } \\
\text { with } \mathrm{Pb} \text { exposure }\end{array}$ & References \\
\hline \multirow[t]{5}{*}{$\leq 10$} & Altered heme synthesis ${ }^{a}$ & $\begin{array}{l}\text { Ahamed et al. 2006; Ergurhan-Ilhan et al. 2008; } \\
\text { Wang et al. } 2010\end{array}$ \\
\hline & $\begin{array}{l}\text { Anemia and/or } \\
\text { decreased measures of } \\
\text { RBC hemoglobin }{ }^{\mathrm{b}}\end{array}$ & $\begin{array}{l}\text { Ahamed et al. 2006; Conterato et al. 2013; Olivero- } \\
\text { Verbel et al. 2007; Queirolo et al. 2010; Riddell et al. } \\
\text { 2007; Ukaejiofo et al. } 2009\end{array}$ \\
\hline & Increased hemoglobin & Chen et al. 2019 \\
\hline & $\begin{array}{l}\text { Decreased platelet } \\
\text { count }\end{array}$ & Conterato et al. 2013 \\
\hline & Decreased EPO & Sakata et al. 2007 \\
\hline \multirow[t]{5}{*}{$>10-30$} & Altered heme synthesis $^{a}$ & $\begin{array}{l}\text { Ahamed et al. 2005, 2006; Counter et al. 2008, } \\
\text { 2009; Grandjean and Lintrup 1978; La-Llave-Leon et } \\
\text { al. 2017; Lauwerys et al. 1978; Mohammad et al. } \\
\text { 2008; Murata et al. 2009; Piomelli et al. 1982 } \\
\text { Rabinowitz et al. 1985; Roels et al. 1975, 1976; } \\
\text { Roels and Lauwerys 1987; Schumacher et al. 1997; } \\
\text { Stuik } 1974\end{array}$ \\
\hline & $\begin{array}{l}\text { Anemia and/or } \\
\text { decreased measures of } \\
\text { RBC hemoglobin }{ }^{b}\end{array}$ & $\begin{array}{l}\text { Adebonojo 1974; Ahamed et al. 2007; Karita et al. } \\
\text { 2005; Li et al. 2018; Schwartz et al. 1990; Shah et al. } \\
2010\end{array}$ \\
\hline & Altered RBC function ${ }^{c}$ & Abam et al. 2008; Huel et al. 2008 \\
\hline & $\begin{array}{l}\text { Decreased platelet } \\
\text { count }\end{array}$ & Barman et al. 2014 \\
\hline & Decreased EPO & Graziano et al. 1991, Liebelt et al. 1999 \\
\hline
\end{tabular}




\section{Table 2-15. Overview of Hematological Effects Associated with Chronic Exposure to Lead (Pb)}

\begin{tabular}{|c|c|c|}
\hline $\begin{array}{l}\text { Mean blood lead } \\
\text { concentration }(\mathrm{PbB}) \\
(\mu \mathrm{g} / \mathrm{dL})\end{array}$ & $\begin{array}{l}\text { Effects associated } \\
\text { with } \mathrm{Pb} \text { exposure }\end{array}$ & References \\
\hline & Increased EPO & Factor-Litvak et al. 1999; \\
\hline \multirow[t]{6}{*}{$>30-50$} & Altered heme synthesis ${ }^{a}$ & $\begin{array}{l}\text { Ademuyiwa et al. 2005; Alessio et al. 1976; } \\
\text { Conterato et al. 2013; Fukumoto et al. 1983; Griffin } \\
\text { et al. 1975; Murata et al. 2009; Roels et al. 1976; } \\
\text { Secchi et al. 1974; Solliway et al. } 1996\end{array}$ \\
\hline & $\begin{array}{l}\text { Anemia and/or } \\
\text { decreased measures of } \\
\text { RBC hemoglobin }{ }^{b}\end{array}$ & $\begin{array}{l}\text { Chwalba et al. 2018; Conterato et al. 2013; } \\
\text { Dobrakowski et al. 2016; Schwartz et al. 1990; } \\
\text { Solliway et al. } 1996\end{array}$ \\
\hline & Altered RBC function & $\begin{array}{l}\text { Abam et al. 2008; Angle et al. 1982; Buc and Kaplan } \\
1978\end{array}$ \\
\hline & Increased reticulocytes & Kalahasthi and Barman 2016 \\
\hline & Decreased EPO & Romeo et al. 1996 \\
\hline & Increased EPO & Factor-Litvak et al. 1998; Graziano et al. 2004; \\
\hline \multirow[t]{4}{*}{$>50$} & Altered heme synthesis ${ }^{a}$ & $\begin{array}{l}\text { Cools et al. 1976; Gurer-Orhan et al. 2004; Jin et al. } \\
\text { 2006; Meredith et al. 1978; Murata et al. 2009; } \\
\text { Pagliuca et al. 1990; Schwartz et al. } 1990\end{array}$ \\
\hline & $\begin{array}{l}\text { Anemia and/or } \\
\text { decreased measures of } \\
\text { RBC hemoglobin }{ }^{b}\end{array}$ & $\begin{array}{l}\text { Baker et al. 1979; Lilis et al. 1978; Malekirad et al. } \\
\text { 2013; Grandjean1979; Patil et al. 2006; Roels et al. } \\
\text { 1979 }\end{array}$ \\
\hline & Decreased EPO & Romeo et al. 1996 \\
\hline & Altered RBC function ${ }^{c}$ & Buc and Kaplan 1978 \\
\hline
\end{tabular}

anhibition of heme synthesis measured by decreased $\delta$-ALAD activity, elevated RBC levels or urinary levels of heme precursors (e.g., protoporphyrin, erythrocyte protoporphyrin, free erythrocyte protoporphyrin), and/or increased RBC zinc protoporphyrin/heme ratio.

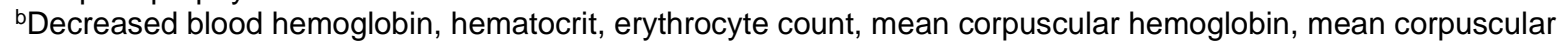
hemoglobin concentration, and/or mean cell volume.

'Altered erythrocyte function includes inhibition of pyrimidine 5'-nucleotidase or decreased RBC membrane $\mathrm{Ca}^{2+} / \mathrm{Mg}^{2+}$ ATPase.

ALAD = aminolevulinic acid dehydratase $;$ EPO = serum erythropoietin $; \mathrm{RBC}=$ red blood cell

Several studies have evaluated the relationship between PbB and serum EPO levels in adults (Graziano et al. 1991; Osterode et al. 1999; Romeo 1996; Sakata et al. 2007) and children (Factor-Litvak 1998, 1999; Graziano et al. 2004; Liebelt et al. 1999). Erythropoietin is a glycoprotein hormone produced in renal proximal tubules that regulates steady-state and accelerated erythrocyte production. As a compensatory response to conditions producing low blood oxygen (e.g., anemia), proximal tubular cells release EPO, resulting in stimulated erythrocyte production. However, if renal function is compromised due to disease or toxicity (e.g., Pb-induced renal damage), the compensatory increases in serum EPO may be diminished or absent. Results of three cross-sectional studies in adult male workers are inconsistent, showing 
decreased serum EPO levels at PbB 6.4-65.1 $\mu \mathrm{g} / \mathrm{dL}$ (Romeo et al. 1996; Sakata et al. 2007), but no effect on EPO at a PbB of $45.5 \mu \mathrm{g} / \mathrm{dL}$ (Osterode et al. 1999). Study populations in these cross-sectional studies were small ( $\mathrm{n}$ for exposed groups=10-27). In a subgroup of 48 pregnant women (selected from a larger cohort of 1,502 pregnant women), serum EPO was decreased; the range of PbB means based on hemoglobin stratifications was 23.1-36.2 $\mu \mathrm{g} / \mathrm{dL}$ (Graziano et al. 1991). Studies in children have yielded mixed results on associations between $\mathrm{PbB}$ and serum EPO. Results of a series of prospective studies of children ( $\mathrm{n}=280$ ) in former Yugoslavia indicate that serum EPO levels in Pb-exposed children exhibit age-dependence (Factor-Litvak et al. 1998, 1999; Graziano et al. 2004). Serum EPO was increased in children 4.5 (mean PbB: $39.3 \mu \mathrm{g} / \mathrm{dL}$ ) and 6.5 years of age (mean PbB: $36.2 \mu \mathrm{g} / \mathrm{dL}$ ), but not in children 9.5 (mean PbB: $28.1 \mu \mathrm{g} / \mathrm{dL}$ ) or 12 years of age (mean PbB: 30.6 g/dL) (Factor-Litvak et al. 1998, 1999; Graziano et al. 2004). The study authors suggested that the capacity for compensatory increases in EPO in response to $\mathrm{Pb}$-induced anemia declines over time, possibly due to $\mathrm{Pb}$-induced damage to the renal proximal tubule. In contrast to increases in EPO levels observed in the Yugoslavian cohort, Liebelt et al. (1999) showed decreased EPO levels in a group of children ages 1-6 years $(n=95)$ who had a mean PbB of $18 \mu \mathrm{g} / \mathrm{dL}$.

Effect at Blood Pb Levels $\leq 10 \mu \boldsymbol{\mu g} / \mathbf{d L}$. Epidemiological studies evaluating hematological effects of $\mathrm{PbB}$ $\leq 10 \mu \mathrm{g} / \mathrm{dL}$ are summarized in Table 2-16, with additional details provided in the Supporting Document for Epidemiological Studies for Lead, Table 4. Studies were conducted in small populations (n for exposed groups=25-391), except for two larger $(n=855-2,861)$ cross-sectional studies in children (Liu et al. 2015a; Riddell et al. 2007). In general, studies show inverse associations between $\mathrm{PbB} \leq 10 \mu \mathrm{g} / \mathrm{dL}$ and $\delta$-ALAD activity and blood hemoglobin in adults and children, although results are mixed. Negative correlations between $\mathrm{PbB}$ and $\delta$-ALAD activity (measured by plasma $\delta$-ALAD activity or zinc protoporphyrin:heme ratio) have been observed in children (Wang et al. 2010), adolescent males (Ahamed et al. 2006), and adults (Wang et al. 2010) at mean PbB of 5.95-9.96 $\mu \mathrm{g} / \mathrm{dL}$; however, no effect on $\delta$-ALAD activity was observed in children with a mean PbB of $7.11 \mu \mathrm{g} / \mathrm{dL}$ (Ahamed et al. 2005). Differences in $\delta$-ALAD activity were observed for male automotive repair workers (mean PbB: $7.9 \mu \mathrm{g} / \mathrm{dL}$ ) and male controls (mean PbB: $2.6 \mu \mathrm{g} / \mathrm{dL}$ ). Additionally, two studies in adults showed that blood hemoglobin concentration was lower in Pb workers (mean PbB: 5.4-7.0 $\mu \mathrm{g} / \mathrm{dL}$ ) compared to controls (mean PbB: 1.5-3.0 g/dL) (Conterato et al. 2013; Ukaejiofo et al. 2009). In contrast, blood hemoglobin and erythrocyte count were increased in adults living near an electronic waste site (median PbB $8.7 \mu \mathrm{g} / \mathrm{dL}$ ), compared to controls (median PbB $8.7 \mu \mathrm{g} / \mathrm{dL}$ ) (Chen et al. 2019). In children with mean 
2. HEALTH EFFECTS

Table 2-16. Summary of Epidemiological Studies Evaluating Hematological Effects at Mean Blood Lead Concentration $(\mathrm{PbB}) \leq 10 \mu \mathrm{g} / \mathrm{dL}^{\mathrm{a}}$

\begin{tabular}{|c|c|c|c|}
\hline Reference and study population & $\mathrm{PbB}(\mu \mathrm{g} / \mathrm{dL})$ & Outcome evaluated & Result $^{\mathrm{b}}$ \\
\hline \multicolumn{4}{|l|}{ Heme metabolism } \\
\hline $\begin{array}{l}\text { Ahamed et al. } 2005 \\
\text { Cross-sectional study; } n=62 \text { children } \\
\text { (ages } 4-12 \text { years) }\end{array}$ & $\begin{array}{l}\text { Mean (SD) } \\
-\quad \text { Group 1: } 3.93(0.61) \\
-\quad \text { Group 2: } 7.11(1.25)\end{array}$ & $\delta$-ALAD activity & $\begin{array}{l}\text { No difference between groups: } \\
\text { - Group 1: } 4.82(1.25) \\
\text { - Group 2: } 4.56(1.20)\end{array}$ \\
\hline $\begin{array}{l}\text { Ahamed et al. } 2006 \\
\text { Cross-sectional study; } \\
\mathrm{n}=39 \text { adolescent males (ages 15- } \\
18 \text { years) }\end{array}$ & $\begin{array}{l}\text { Mean (SD): } 9.96(3.63) \\
\text { Range: } 4.62-18.64\end{array}$ & $\delta$-ALAD activity & $\begin{array}{l}\text { A negative correlation between } \mathrm{PbB} \text { and blood } \\
\delta \text {-ALAD activity: } r=-0.592 ; p<0.001^{*}\end{array}$ \\
\hline Ergurhan-Ilhan et al. 2008 & \multirow{2}{*}{$\begin{array}{l}\text { Mean (SD) } \\
-\quad \text { Controls: } 2.6(2.0) \\
\text { - } \quad \text { Workers: } 7.9(5.2)\end{array}$} & ALAD index & $\begin{array}{l}\text { - Controls: } 0.40(0.34) \\
\text { - Workers: } 0.73\left(\mathbf{0 . 4 7 )} ; \mathbf{p}=\mathbf{0 . 0 4 8 ^ { * }}\right.\end{array}$ \\
\hline $\begin{array}{l}\text { Cross-sectional study; } \mathrm{n}=25 \text { male } \\
\text { automotive repair workers (mean age } \\
16.8 \text { years): } 24 \text { male controls (mean } \\
\text { age } 16.3 \text { years) }\end{array}$ & & ZPP:heme ratio & $\begin{array}{l}\text { - } \quad \text { Controls: } 26.4(7) \\
\text { - Workers: } 37.2 \text { (15.9); } \mathbf{p}=\mathbf{0 . 0 4 5 ^ { * }}\end{array}$ \\
\hline \multirow{2}{*}{$\begin{array}{l}\text { Wang et al. } 2010 \\
\text { Cross-sectional study; } n=307 \text { children } \\
\text { (ages } 4-13 \text { years) and } 391 \text { adults } \\
\text { (ages } 16-77 \text { years) from China }\end{array}$} & \multirow[t]{2}{*}{$\begin{array}{l}\text { Median } \\
\text { - } \quad \text { Children: } 6.83 \\
\text { - } \quad \text { Adults: } 5.95\end{array}$} & $\delta$-ALAD activity & $\begin{array}{l}\text { Pearson correlation coefficients: } \\
\text { - } \quad \text { Children: }-0.256 ; p<0.05^{*} \\
\text { - } \quad \text { Adults: }-0.213 ; p<0.05^{*}\end{array}$ \\
\hline & & ZPP & $\begin{array}{l}\text { Pearson correlation coefficients: } \\
\text { - Children: } 0.135 ; p<0.05^{*} \\
\text { - } \quad \text { Adults: } 0.083 ; p<0.05^{*} \\
\end{array}$ \\
\hline \multicolumn{4}{|l|}{ Blood hemoglobin/erythrocyte count } \\
\hline \multirow{2}{*}{$\begin{array}{l}\text { Chen et al. } 2019 \\
\text { Cross-sectional study; } n=158 \text { exposed } \\
\text { adults living near an electronic waste } \\
\text { area (mean age: } 44 \text { years); } \\
n=109 \text { controls (mean age: } 47 \text { years) }\end{array}$} & \multirow{2}{*}{$\begin{array}{l}\text { Median }\left(\mathrm{P}_{25}, \mathrm{P}_{75}\right) \\
\text { - } \quad \text { Control: } 5.1(3.9,8.4) \\
\text { Exposed: } 8.7(6.2,12.2)\end{array}$} & $\mathrm{Hb}$ & $\begin{array}{l}\text { Median }\left(\mathrm{P}_{25}, \mathrm{P}_{75}\right), \mathrm{g} / \mathrm{dL} \\
\text { - } \quad \text { Control: } 123.0(107.0,143.0) \\
\text { - } \quad \text { Exposed: } \mathbf{1 3 7 . 0}(\mathbf{1 1 9 . 5}, \mathbf{1 5 0 . 0}), \mathbf{p}=\mathbf{0 . 0 0 1}^{*}\end{array}$ \\
\hline & & Erythrocyte count & $\begin{array}{l}\text { RBC count }\left(\times 10^{3}\right), \text { median }\left(\mathrm{P}_{25}, \mathrm{P}_{75}\right) \\
\text { - } \quad \text { Control: } 4.2(3.5,4.6) \\
\text { - } \quad \text { Exposed: } \mathbf{4 . 5}(\mathbf{4 . 1}, \mathbf{4 . 8}), \mathbf{p}=\mathbf{0 . 0 0 1 *}\end{array}$ \\
\hline
\end{tabular}


2. HEALTH EFFECTS

Table 2-16. Summary of Epidemiological Studies Evaluating Hematological Effects at Mean Blood Lead Concentration $(\mathrm{PbB}) \leq 10 \mu \mathrm{g} / \mathrm{dL}^{\mathrm{a}}$

\begin{tabular}{|c|c|c|c|}
\hline Reference and study population & $\mathrm{PbB}(\mu \mathrm{g} / \mathrm{dL})$ & Outcome evaluated & Result $^{b}$ \\
\hline $\begin{array}{l}\text { Conterato et al. } 2013 \\
\text { Cross-sectional study; n=50 painters; } \\
36 \text { controls }\end{array}$ & $\begin{array}{l}\text { Mean (SE) } \\
\text { - } \quad \text { Control: } 1.5(0.1) \\
\text { - } \quad \text { Painters: } 5.4(0.4)\end{array}$ & $\mathrm{Hb}$ & $\begin{array}{l}\text { Mean (SE), } \mu \mathrm{g} / \mathrm{dL} \\
\text { - } \quad \text { Control: } 15.4(0.2) \\
\text { - } \quad \text { Painters: } 15.0(\mathbf{0 . 1}) ; \mathbf{p}<\mathbf{0 . 0 5 *}\end{array}$ \\
\hline $\begin{array}{l}\text { Liu et al. } 2015 a \\
\text { Cross-sectional study; } n=855 \text { children } \\
\text { (age range: } 3-7 \text { years) }\end{array}$ & \begin{tabular}{ll}
\multicolumn{2}{l}{ PbB quartiles: } \\
- $\quad$ Q1: $2.20-5.16$ \\
- $\quad$ Q2: $5.16-7.33$ \\
- $\quad$ Q3: $7.33-10.62$ \\
- $\quad$ Q4: $10.62-37.78$ \\
Erythrocyte Pb quartiles: \\
- $\quad$ Q1: 5.98-13.52 \\
- $\quad$ Q2: 13.52-19.35 \\
- $\quad$ Q3: $19.35-28.42$ \\
- $\quad$ Q4: $28.42-101.01$
\end{tabular} & $\mathrm{Hb}$ & $\begin{array}{l}\text { Change in Hb compared to Q1: } \\
\text { - PbB Q3: } 1.45(-0.28,3.18) \\
\text { - Erythrocyte Pb } \\
\text { o Q3: }-3.01(-4.71,1.31) ; p<0.05^{\star, c} \\
\text { o Q4: -3.97 (-5.68, -2.27); } \mathbf{p}<0.05^{*}\end{array}$ \\
\hline $\begin{array}{l}\text { Olivero-Verbel et al. } 2007 \\
\text { Cross-sectional study, } n=189 \text { children } \\
\text { (age range 5-9 years) }\end{array}$ & Mean (SE): $5.49(0.23)$ & $\mathrm{Hb}$ & Spearman correlation coefficient: $0.069 ; p=0.348$ \\
\hline $\begin{array}{l}\text { Queirolo et al. } 2010 \\
\text { Cross-sectional study; } n=222 \text { children } \\
\text { (age: } 5-45 \text { months) }\end{array}$ & Mean (SD): $9.0(6.0)$ & $\mathrm{Hb}$ & $\begin{array}{l}\text { Blood } \mathrm{Hb}<10.5 \mathrm{~g} / \mathrm{L} \text { was a predictor of } \mathrm{PbB} ; \\
\beta(95 \% \mathrm{Cl}): 2.40(0.77,4.03) ; p<0.01^{*}\end{array}$ \\
\hline $\begin{array}{l}\text { Riddell et al. } 2007 \\
\text { Cross-sectional study; } \\
n=2,861 \text { children (age } 6 \text { months- } \\
5 \text { years) }\end{array}$ & Mean: 6.9 & $\mathrm{Hb}$ & $\begin{array}{l}\text { A } 1 \mathrm{~g} / \mathrm{dL} \text { increase in } \mathrm{Hb} \text { was associated with a } 3 \% \\
\text { decrease in } \mathrm{PbB}(p=0.043)^{*}\end{array}$ \\
\hline $\begin{array}{l}\text { Ukaejiofo et al. } 2009 \\
\text { Cross-sectional study; n=81 Pb } \\
\text { workers; } 30 \text { controls }\end{array}$ & $\begin{array}{l}\text { Mean (SD) } \\
-\quad \text { Controls: } 3.00(0.19) \\
-\quad \text { Workers: } 7.00(0.07)\end{array}$ & $\mathrm{Hb}$ & $\begin{array}{l}\text { Mean (SE), g/dL } \\
\text { - } \quad \text { Controls: } 12.96(0.089) \\
\text { - Workers: } 12.05\left(\mathbf{1 . 6 2 )} ; \mathbf{p}<0.001^{*}\right.\end{array}$ \\
\hline
\end{tabular}


Table 2-16. Summary of Epidemiological Studies Evaluating Hematological Effects at Mean Blood Lead Concentration $(\mathrm{PbB}) \leq 10 \mu \mathrm{g} / \mathrm{dL}^{\mathrm{a}}$

\begin{tabular}{|c|c|c|c|}
\hline Reference and study population & $\mathrm{PbB}(\mu \mathrm{g} / \mathrm{dL})$ & Outcome evaluated & Result $^{b}$ \\
\hline Zentner et al. 2006 & $\begin{array}{l}\text { Umbilical mean (SD): } 3.9 \\
(3.6)\end{array}$ & $\mathrm{Hb}$ & Pearson correlation coefficient: $-0.04 ; p=0.721$ \\
\hline \multicolumn{4}{|c|}{ Cross-sectional study; $n=55$ newborns } \\
\hline \multicolumn{4}{|l|}{ Other hematological effects } \\
\hline $\begin{array}{l}\text { Conterato et al. } 2013 \\
\text { Cross-sectional study; } n=50 \text { painters; } \\
36 \text { controls }\end{array}$ & $\begin{array}{l}\text { Mean (SE) } \\
\text { - } \quad \text { Control: } 1.5(0.1) \\
\text { - } \quad \text { Painters: } 5.4(0.4)\end{array}$ & Platelet count & $\begin{array}{l}\text { Mean (SE), \% } \\
\text { - } \quad \text { Control: } 244.3(8.3) \\
\text { - } \quad \text { Painters: } 203.7(6.5) ; p<0.05^{\star}\end{array}$ \\
\hline $\begin{array}{l}\text { Sakata et al. } 2007 \\
\text { Cross-sectional studies: } n=27 \text { exposed } \\
\text { workers; } 9 \text { controls }\end{array}$ & $\begin{array}{l}\text { Mean (SD); range } \\
\text { - } \quad \text { Controls: } 2.4(1.1) \\
\text { - } \quad \text { Workers: } 6.4(2.2)\end{array}$ & EPO & $\begin{array}{l}\text { Mean (SD), } \mathrm{mU} / \mathrm{mL}: \\
\text { - } \quad \text { Controls:18.8 (4.6) } \\
\text { - } \quad \text { Workers: } 12.7(3.5) ; \text { p<0.01* }\end{array}$ \\
\hline
\end{tabular}

aSee the Supporting Document for Epidemiological Studies for Lead, Table 4 for more detailed descriptions of studies.

${ }^{\mathrm{b}}$ Asterisk and bold indicate association with $\mathrm{Pb}$; unless otherwise specified, values in parenthesis are $95 \% \mathrm{Cls}$.

'The discrepancy between the $95 \%$ confidence limits and the p-value appears to be caused by an error in the reporting of the upper confidence limit (i.e., - 1.31 , rather than 1.31).

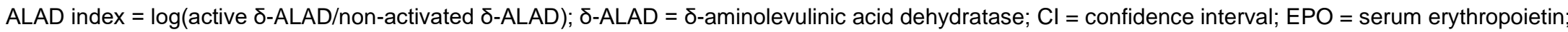
$\mathrm{Hb}=$ hemoglobin; $\mathrm{Pb}=$ lead; $\mathrm{SD}=$ standard deviation; $\mathrm{SE}=$ standard error; $\mathrm{ZPP}=$ zinc-protoporphyrin 
$\mathrm{PbB}$ of 6.9-9.0 $\mu \mathrm{g} / \mathrm{dL}$, there was an inverse association between blood hemoglobin concentrations and PbB (Queirolo et al. 2010; Riddell et al. 2007) and erythrocyte Pb concentration (Liu et al. 2015a). At lower PbB in newborns (PbB $3.9 \mu \mathrm{g} / \mathrm{dL}$ ) and children (PbB $5.5 \mu \mathrm{g} / \mathrm{dL}$ ), no correlation was found; however, these study populations were small ( $n=50-189)$ (Olivero-Verbel et al. 2007; Zentner et al. 2006). Thus, data are not adequate to establish an exposure-response relationship for decreased hemoglobin at $\mathrm{PbB} \leq 10 \mu \mathrm{g} / \mathrm{dL}$. Studies in small groups of workers ( $\mathrm{n}=27-50)$ showed lower platelet count (PbB $5.4 \mu \mathrm{g} / \mathrm{dL}$ ) and serum EPO concentrations (PbB $6.4 \mu \mathrm{g} / \mathrm{dL}$ ) compared to controls (Conterato et al. 2013; Sakata et al. 2007). Although these findings have not been evaluated in other studies with $\mathrm{PbB} \leq 10 \mu \mathrm{g} / \mathrm{dL}$, similar effects have been observed at $\mathrm{PbB}>10 \mu \mathrm{g} / \mathrm{dL}$.

Mechanisms of Action. $\mathrm{Pb}$ inhibits heme synthesis by inhibiting $\delta$-ALAD and ferrochelatase (see Figure 2-4). As a consequence, the activity of the rate-limiting enzyme of the pathway, $\delta$-aminolevulinic synthetase ( $\delta$-ALAS), which is feedback inhibited by heme, is subsequently increased. The end results of these changes in enzyme activities are increased urinary porphyrins, coproporphyrin, and $\delta$-aminolevulinic acid ( $\delta$-ALA), increased blood and plasma $\delta$-ALA, increased erythrocyte protoporphyrin (EP), and decreased hemoglobin. The impairment of heme synthesis by $\mathrm{Pb}$ may have a far-ranging impact not limited to the hematopoietic system. EPA (1986) provided an overview of the known and potential consequences of the reduction of heme synthesis as shown in Figure 2-5. Solid arrows indicate welldocumented effects, whereas dashed arrows indicate effects considered to be plausible further consequences of the impairment of heme synthesis.

In addition to decreased hemoglobin synthesis, general mechanisms of toxicity of $\mathrm{Pb}$ (reviewed in Section 2.21) are likely involved in the development of adverse effects to the hematological system. EPA (2014c) specifically noted effects of oxidative stress (altered antioxidant enzymes, decreased cellular glutathione, and lipid peroxidation) as an important mechanism for hematological effects. As reviewed in Section 3.2.3 (Toxicokinetics, Distribution), 99\% of $\mathrm{Pb}$ in blood is distributed to erythrocytes, providing a toxicokinetic mechanism for hematological effects (Bergdahl et al. 1997a, 1998, 1999; Hernandez-Avila et al. 1998; Manton et al. 2001; Schutz et al. 1996; Smith et al. 2002). 
Figure 2-4. Pb Interactions in the Heme Synthesis Pathway

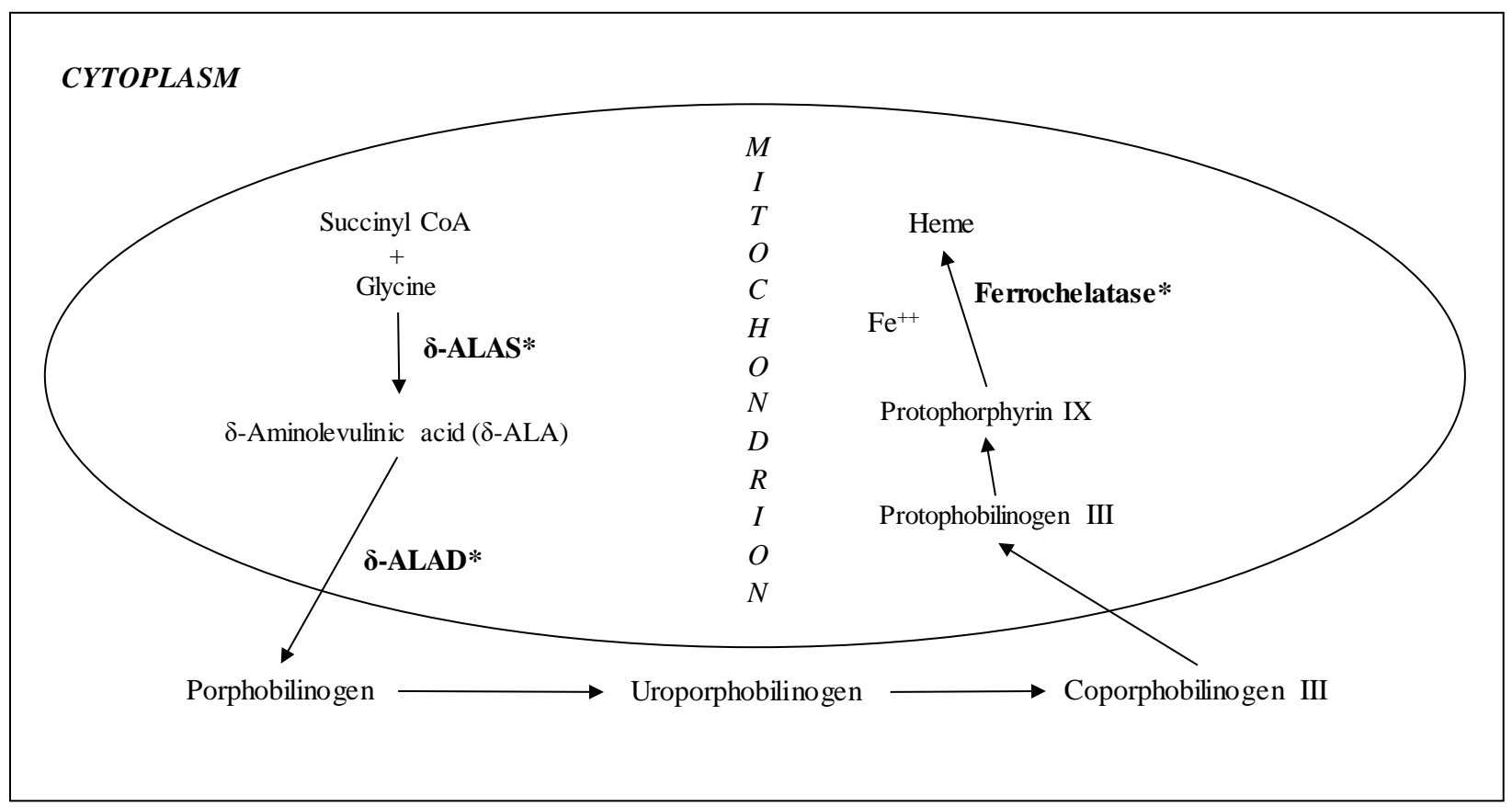

Abbreviations as noted in Ahamed and Siddiqui (2007): $\delta$-ALAS = delta-aminolevulinic acid synthetase; $\delta$-ALAD = delta-aminolevulinic dehydratase $; \mathrm{COA}=$ coenzyme $A$

${ }^{*}$ Activity of enzymes inhibited by lead.

Source: Reprinted from Ahamed and Siddiqui (2007) with permission from Elsevier. 


\section{Figure 2-5. Multiorgan Impact of Reduction of Heme Body Pool by Lead}

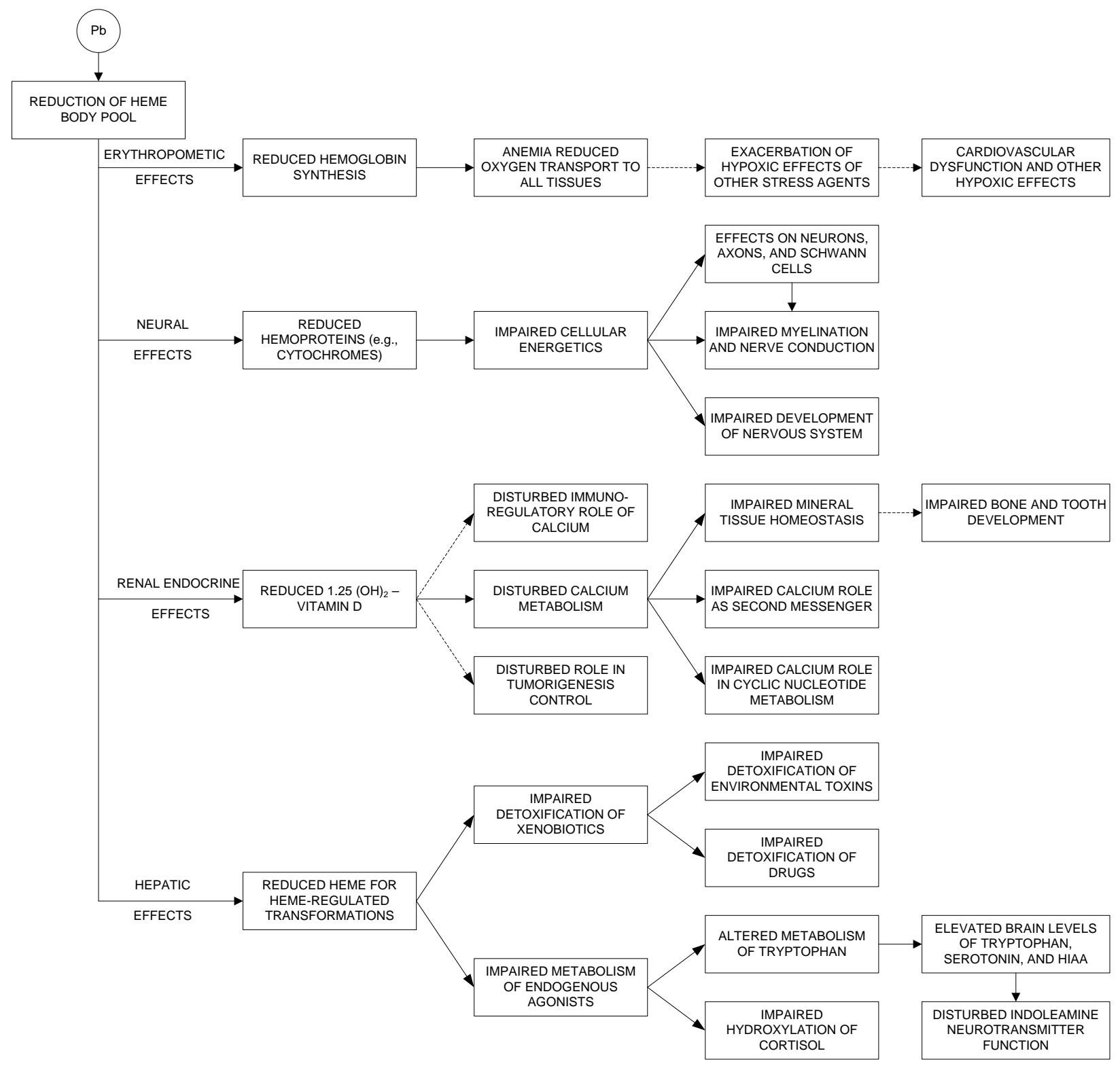

Source: EPA 1986a

\subsection{MUSCULOSKELETAL}

Overview. Few epidemiological studies have evaluated musculoskeletal effects associated with $\mathrm{Pb}$ exposure; thus, limited data are available to fully describe the exposure-response relationship or evaluate the weight-of-evidence for certain effects. Studies provide evidence of bone loss, increased markers of bone metabolism/turnover, and adverse periodontal and dental effects (periodontal bone loss, tooth loss, periodontal disease, dental caries). However, within dose ranges $(\leq 10,10-30,30-50$, and $>50 \mu \mathrm{g} / \mathrm{dL})$, 
few studies examined the same endpoints. Available studies include a prospective study in women and cross-sectional studies in adults and children, with some studies in large populations.

The following musculoskeletal effects have been associated with PbB:

- $\leq 10 \mu \mathrm{g} / \mathrm{dL}$ :

o Bone loss or markers of increased bone or joint tissue metabolism.

o Periodontal bone loss.

o Tooth loss.

o Dental caries.

o Periodontitis.

- $>10 \mu \mathrm{g} / \mathrm{dL}$ :

o Muscle soreness/weakness.

o Osteoporosis/decreased bone mineral density (BMD) in adults.

o Increased BMD in children.

o Periodontal disease.

o Dental caries.

Measures of Exposure. Most studies examining the association between musculoskeletal effects and $\mathrm{Pb}$ exposure have evaluated exposure by measurement of $\mathrm{PbB}$, although some studies also evaluated exposure by bone $\mathrm{Pb}$ concentration.

Confounding Factors and Effect Modifiers. A complicating factor in the interpretation of studies examining associations between $\mathrm{PbB}$ and bone loss or measures of bone metabolism is that increased bone metabolism (bone turnover or loss) can result in higher $\mathrm{PbB}$ due to $\mathrm{Pb}$ released from bone into the blood (reverse causality). This contributes to confounding from other factors that are associated with bone loss, including nutrition, age, pregnancy and menopause, and activity. Results of studies examining $\mathrm{Pb}$-induced periodontal or dental effects need to account for dental hygiene, diet/nutrition, and previous dental interventions. For example, interpretation of results on associations between dental caries and $\mathrm{PbB}$ would be uncertain if daily fluoride intake or prophylactic dental treatments (e.g., fluoride treatments or coating of molars during childhood) were not considered as confounding factors. Studies that rely on in vivo estimates of bone $\mathrm{Pb}$ (e.g., XRF) as the exposure metric for changes in BMD should also consider the potential for changes in BMD affecting the measurement of the concentration of $\mathrm{Pb}$ in bone mineral (Hu et al. 2007). 
Characterization of Effects. Studies evaluating musculoskeletal effects associated with $\mathrm{PbB}$ provide evidence of bone loss, altered bone or joint tissue metabolism, and adverse periodontal and dental effects (periodontal bone loss, tooth loss, periodontal disease, dental caries). Due to the small number of studies, it is difficult to establish exposure-response relationships; in addition, within specific dose-ranges ( $\leq 10$, 10-30, 30-50, and $>50 \mu \mathrm{g} / \mathrm{dL}$ ), few studies examined the same endpoints. Effects associated with chronic $\mathrm{Pb}$ exposure are shown in Table 2-17. In adults, decreased BMD has been observed over a PbB range of $\leq 10->50 \mu \mathrm{g} / \mathrm{dL}$ (Campbell and Auinger 2007; Dongre et al. 2013; Khalil et al. 2008; Lee and Park 2018), although BMD was not decreased in women at $\mathrm{PbB} \leq 10 \mu \mathrm{g} / \mathrm{dL}$ (Pollack et al. 2013). BMD was increased in a single study in children with a mean PbB of $23.6 \mu \mathrm{g} / \mathrm{dL}$ (Campbell et al. 2004). The study authors suggested that the effect may represent accelerated bone maturation due to $\mathrm{Pb}$-induced inhibition of parathyroid hormone-related peptide and transforming growth factor $\beta-1$. The study authors also noted that the accelerated bone maturation may be a predisposing factor for osteoporosis later in life. Sun et al. (2008a, 2008b) showed that PbB was associated with increased prevalence of osteoporosis (mean PbB men: $20.22 \mu \mathrm{g} / \mathrm{dL}$; women $15.50 \mu \mathrm{g} / \mathrm{dL}$ ). Periodontal disease (including periodontitis), periodontal bone loss, tooth loss, and dental caries have been reported over a $\mathrm{PbB}$ range of $\leq 10-30 \mu \mathrm{g} / \mathrm{dL}$ (Arora et al. 2009; Campbell et al. 2000a; Dye et al. 2002; Gemmel et al. 2002; Kim and Lee 2013; Kim et al. 2017a; Moss et al. 1999; Youravong and Teanpaisan 2015). Most studies examining periodontal and dental effects of $\mathrm{Pb}$ are conducted in populations with $\mathrm{PbB} \leq 10 \mu \mathrm{g} / \mathrm{dL}$. Muscle soreness and weakness has also been reported, although at higher PbB (40-49 $\mu \mathrm{g} / \mathrm{dL})$ (Rosenman et al. 2003).

\begin{tabular}{|c|c|c|}
\hline $\begin{array}{l}\text { Mean blood lead } \\
\text { concentration }(\mathrm{PbB})(\mu \mathrm{g} / \mathrm{dL})\end{array}$ & $\begin{array}{l}\text { Effects associated with } \mathrm{Pb} \\
\text { exposure }\end{array}$ & References \\
\hline \multirow[t]{5}{*}{$\leq 10$} & $\begin{array}{l}\text { Bone loss/increased bone } \\
\text { metabolism }\end{array}$ & $\begin{array}{l}\text { Khalil et al. 2008; Lee and Park 2018; } \\
\text { Machida et al. 2009; Nelson et al. } \\
2009\end{array}$ \\
\hline & Tooth loss & Arora et al. 2009 \\
\hline & Periodontal bone loss & Dye et al. 2002 \\
\hline & Periodontitis & Kim and Lee 2013 \\
\hline & Dental caries & $\begin{array}{l}\text { Gemmel et al. } 2002 ; \text { Kim et al. } 2017 \text { a } \\
\text { Moss et al. } 1999\end{array}$ \\
\hline
\end{tabular}




\section{Table 2-17. Overview of Musculoskeletal Effects Associated with Chronic Exposure to Lead (Pb)}

\begin{tabular}{|c|c|c|}
\hline $\begin{array}{l}\text { Mean blood lead } \\
\text { concentration }(\mathrm{PbB})(\mu \mathrm{g} / \mathrm{dL})\end{array}$ & $\begin{array}{l}\text { Effects associated with } \mathrm{Pb} \\
\text { exposure }\end{array}$ & References \\
\hline \multirow[t]{5}{*}{$>10-30$} & Osteoporosis & Sun et al. $2008 a, 2008 b$ \\
\hline & $\begin{array}{l}\text { Decreased bone mineral density } \\
\text { (adults) }\end{array}$ & Campbell and Auinger 2007 \\
\hline & $\begin{array}{l}\text { Increased bone mineral density } \\
\text { (children) }\end{array}$ & Campbell et al. 2004 \\
\hline & Periodontal disease & Youravong and Teanpaisan 2015 \\
\hline & Dental caries & Campbell et al. 2000a \\
\hline \multirow[t]{2}{*}{$>30-50$} & Muscle soreness/weakness & Rosenman et al. 2003 \\
\hline & Decreased bone mineral density & Campbell and Auinger 2007 \\
\hline$>50$ & Decreased bone mineral density & Dongre et al. 2013 \\
\hline
\end{tabular}

Effects at Blood Pb Levels $\leq 10 \mu \mathrm{g} / \mathrm{dL}$. Epidemiological studies of musculoskeletal effects associated with $\mathrm{PbB} \leq 10 \mu \mathrm{g} / \mathrm{dL}$ have examined effects on bone and periodontal and dental health; studies are briefly summarized in Table 2-18, with additional details provided in the Supporting Document for Epidemiological Studies for Lead, Table 5. A prospective study in women reported an increased rate of bone loss at $\mathrm{PbB}$ ranges of 4-7 and 8-21 $\mu \mathrm{g} / \mathrm{dL}$ and an increased risk of non-spine fractures at a $\mathrm{PbB}$ range of 8-21 $\mu \mathrm{g} / \mathrm{dL}$ (Khalil et al. 2008). In cross-sectional studies, markers of bone metabolism were positively associated with $\mathrm{PbB}$ in women at mean $\mathrm{PbBs}$ of $<2$ and $2.9 \mu \mathrm{g} / \mathrm{dL}$, although no relationship was observed for these markers and PbB in men (mean PbB: $1.2 \mu \mathrm{g} / \mathrm{dL}$ ) (Machida et al. 2009; Nelson et al. 2011). In non-occupationally exposed men and women ( $\mathrm{n}=443)$, $\mathrm{PbB}$ (mean $4.44 \mu \mathrm{g} / \mathrm{dL}$ ) was negatively associated with BMD (Lee and Park 2018). However, no associations between PbB and BMD have been observed in cross-sectional studies in women at slightly lower PbB median PbB (1.8$2.2 \mu \mathrm{g} / \mathrm{dL}$ ) (Machida et al. 2009; Pollack et al. 2013). Studies examining periodontal and dental effects include large ( $n=2,805-10,033)$ cross-sectional studies in adults and children (Dye et al. 2002; Kim and Lee 2013; Kim et al. 2017a; Moss et al. 1999). Positive associations have been observed between PbB and presence of dental furcations in male and female adults (mean PbB: 1.9-3.3 $\mu \mathrm{g} / \mathrm{dL}$ ) (Dye et al. 2002), periodontitis in adult males (PbB mean $3.1 \mu \mathrm{g} / \mathrm{dL}$ ), but not females (mean PbB: 2.2) (Kim and Lee 2013), and dental 
Table 2-18. Summary of Epidemiological Studies Evaluating Musculoskeletal Effects at Mean Blood Lead Concentration $(\mathrm{PbB}) \leq 10 \mu \mathrm{g} / \mathrm{dL}^{\mathrm{a}}$

\begin{tabular}{|c|c|c|c|}
\hline Reference and study population & $\mathrm{PbB}(\mu \mathrm{g} / \mathrm{dL})$ & Outcome evaluated & Result $^{b, c}$ \\
\hline \multicolumn{4}{|l|}{ Bone metabolism } \\
\hline Khalil et al. 2008 & $\begin{array}{l}\text { PbB: } \\
\text { Mean (SD): } 5.3(2.3)\end{array}$ & Bone loss & $\begin{array}{l}\text { Percentage rate of calcaneus bone loss } \\
\text { - T1: }-\mathbf{1 . 0 1}(-\mathbf{1 . 2 7},-\mathbf{0 . 7 4})^{*}\end{array}$ \\
\hline \multirow[t]{2}{*}{$\begin{array}{l}\text { Prospective cohort study; } n=533 \\
\text { women (age range: } 65-87 \text { years). }\end{array}$} & \multirow{2}{*}{$\begin{array}{l}\text { Tertiles: } \\
\text { - } \quad \text { T1 }(n=122): \leq 3 \\
\text { (reference) } \\
\text { - } \quad \text { T2 }(n=332): 4-7 \\
\text { - } \quad \text { T3 }(n=79): 8-21\end{array}$} & & $\begin{array}{l}-\quad \text { T2: }-1.41(-1.57,-1.24)^{*} \\
-\quad \text { T3: }-1.49(-1.86,-1.10)^{*} ; p=t r e n d: 0.03\end{array}$ \\
\hline & & Non-spine fractures & HR T3: $2.50(1.25,5.03) * ; p$-trend: 0.016 \\
\hline \multicolumn{3}{|l|}{$\begin{array}{l}\text { Cross-sectional study; } n=443 \text { adults } \\
\text { (age range: } 39-69 \text { years) }\end{array}$} & \\
\hline \multirow{4}{*}{$\begin{array}{l}\text { Machida et al. } 2009 \\
\text { Cross-sectional study; } n=1,225 \text { female } \\
\text { Japanese farmers (age range: } 35- \\
75 \text { years) }\end{array}$} & \multirow{4}{*}{ 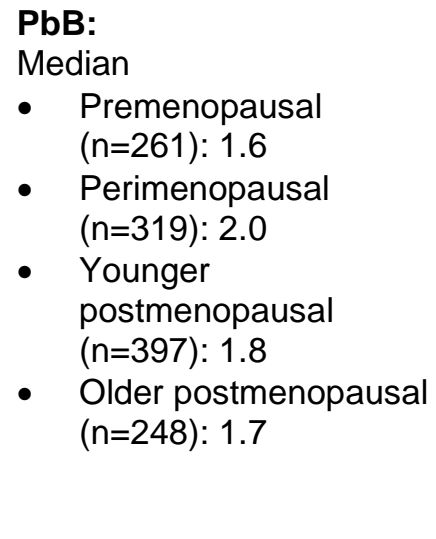 } & BALP & $\begin{array}{l}\text { Spearman's correlation coefficients } \\
-\quad \text { All women: } 0.143 ; p=0.000^{*} \\
-\quad \text { Perimenopausal women: } 0.234 ; p=0.000^{*}\end{array}$ \\
\hline & & $\mathrm{OC}$ & $\begin{array}{l}\text { Spearman's correlation coefficients } \\
-\quad \text { All women: } 0.191 ; p=0.000^{*} \\
-\quad \text { Perimenopausal women: } 0.391 ; p=0.000^{*}\end{array}$ \\
\hline & & NTX & $\begin{array}{l}\text { Spearman's correlation coefficients } \\
-\quad \text { All women: } 0.181 ; p=0.000^{*} \\
-\quad \text { Perimenopausal women: } 0.261 ; p=0.000^{*}\end{array}$ \\
\hline & & BMD & $\begin{array}{l}\text { Spearman's correlation coefficients } \\
\text { - } \quad \text { All women: }-0.016 ; p=0.570 \\
\text { - } \quad \text { Perimenopausal women: }-0.101 ; p=0.071\end{array}$ \\
\hline
\end{tabular}


2. HEALTH EFFECTS

Table 2-18. Summary of Epidemiological Studies Evaluating Musculoskeletal Effects at Mean Blood Lead Concentration $(\mathrm{PbB}) \leq 10 \mu \mathrm{g} / \mathrm{dL}^{\mathrm{a}}$

\begin{tabular}{l} 
Reference and study population \\
\hline Nelson et al. 2011 \\
Cross-sectional study; $\mathrm{n}=329$ males \\
(mean age: 65 years) and
\end{tabular}

$\mathrm{n}=342$ females (mean age: 62 years)

\author{
$\operatorname{PbB}(\mu \mathrm{g} / \mathrm{dL})$ \\ Median (range) \\ - Males: $2.2(0.5-25.1)$ \\ - Females: $1.9(0.5-25.4)$
}

Outcome evaluated Result ${ }^{\mathrm{b}, \mathrm{c}}$

$\beta$ change in biomarker per $5 \mu \mathrm{g} / \mathrm{dL}$ increase in

In-PbB

- Males: $1.06(0.95,1.18)$

- Females: $1.45(1.21,1.74)^{*}$

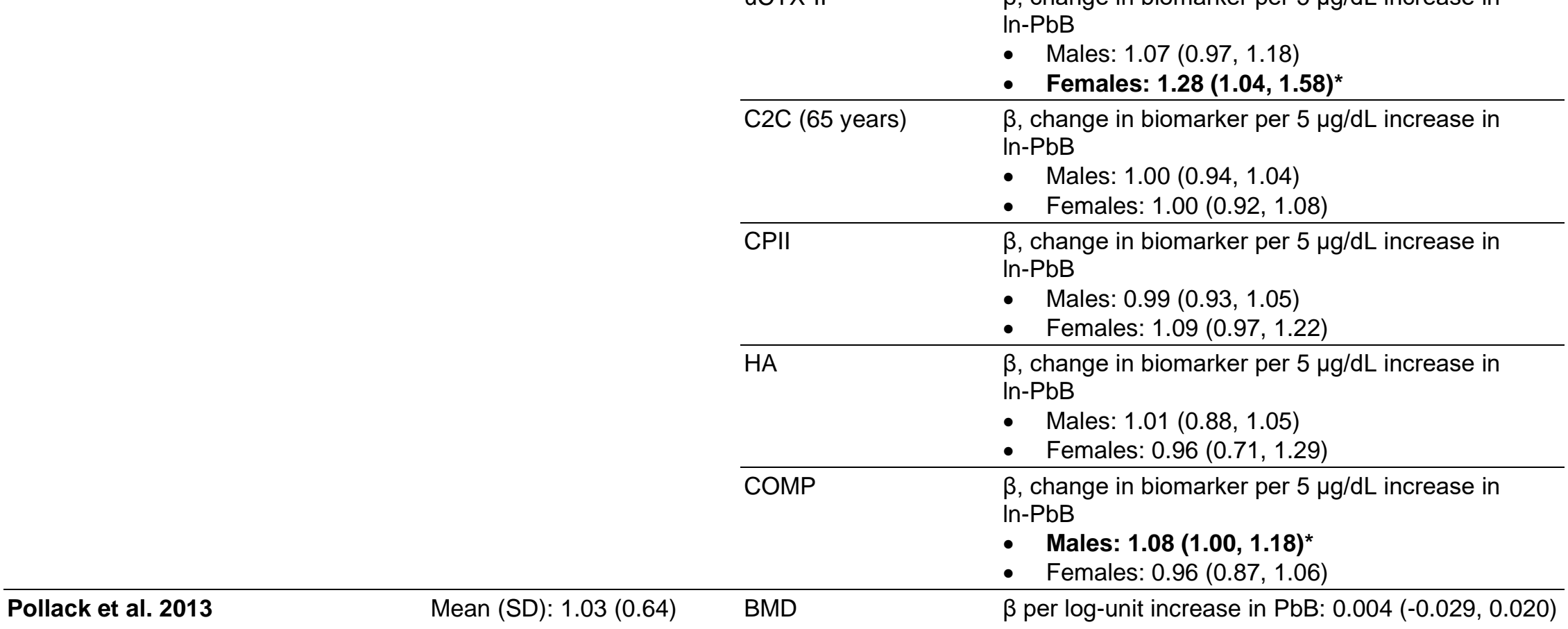

Cross-sectional study;

$\mathrm{n}=249$ premenopausal women (ages

18-44 years) 
2. HEALTH EFFECTS

Table 2-18. Summary of Epidemiological Studies Evaluating Musculoskeletal Effects at Mean Blood Lead Concentration $(\mathrm{PbB}) \leq 10 \mu \mathrm{g} / \mathrm{dL}^{\mathrm{a}}$

\begin{tabular}{|c|c|c|c|}
\hline Reference and study population & $\mathrm{PbB}(\mu \mathrm{g} / \mathrm{dL})$ & Outcome evaluated & Result $^{b, c}$ \\
\hline \multicolumn{4}{|l|}{ Periodontal and dental effects } \\
\hline \multirow{3}{*}{$\begin{array}{l}\text { Arora et al. } 2009 \\
\text { Cross-sectional study; } n=333 \text { men } \\
\text { (age range: 50-94 years) }\end{array}$} & \multirow{3}{*}{ 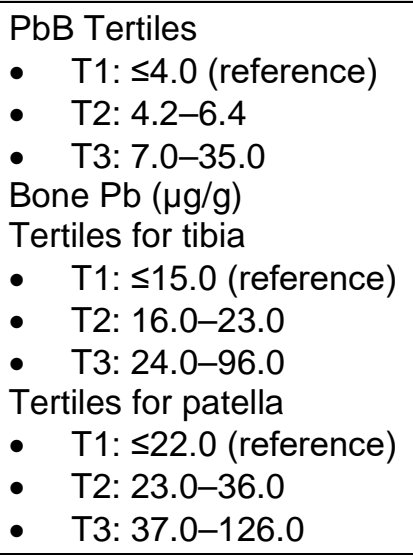 } & \multirow[t]{3}{*}{ Tooth loss } & $\begin{array}{l}\text { OR PbB (compared to T1) } \\
\qquad \quad \text { T3: } 0.88(0.52,1.50) ; p \text {-trend }=0.57\end{array}$ \\
\hline & & & $\begin{array}{l}\text { OR Tibia Pb (compared to T1) } \\
\qquad \quad \text { T2: } 1.81(1.02,3.18)^{\star} \\
\bullet \quad \text { T3: } \mathbf{3 . 0 3}(\mathbf{1 . 6 0}, \mathbf{5 . 7 6})^{\star} ; \text { p-trend=0.001* } \\
\end{array}$ \\
\hline & & & $\begin{array}{l}\text { OR Patella } \mathrm{Pb} \text { (compared to T1) } \\
\qquad \quad \text { T3: } \mathbf{2 . 4 1}(\mathbf{1 . 3 0}, \mathbf{4 . 4 9})^{\star} ; \text { p-trend } 0.005^{\star}\end{array}$ \\
\hline $\begin{array}{l}\text { Dye et al. } 2002 \\
\text { Cross-sectional study in } \\
\text { 10,033 participants in NHANES III } \\
\text { (ages 20-69 years) }\end{array}$ & $\begin{array}{l}\text { Mean (SE) } \\
\text { - } \quad \text { Males: } 3.3(0.12) \\
\quad \quad \text { Females: } 1.9(0.05)\end{array}$ & $\begin{array}{l}\text { Presence of dental } \\
\text { furcations }\end{array}$ & $\begin{array}{l}\beta \text { (SE), for presence of dental furcations } \\
\text { (combined men and women): } 0.13(0.05) \\
p=0.005^{\star}\end{array}$ \\
\hline
\end{tabular}


2. HEALTH EFFECTS

Table 2-18. Summary of Epidemiological Studies Evaluating Musculoskeletal Effects at Mean Blood Lead Concentration $(\mathrm{PbB}) \leq 10 \mu \mathrm{g} / \mathrm{dL}^{\mathrm{a}}$

\begin{tabular}{|c|c|c|c|}
\hline Reference and study population & $\mathrm{PbB}(\mu \mathrm{g} / \mathrm{dL})$ & Outcome evaluated & Result $^{b, c}$ \\
\hline $\begin{array}{l}\text { Gemmel et al. } 2002 \\
\text { Cross-sectional study in } 498 \text { children } \\
\text { (age range: } 6-10 \text { years) from rural } \\
(n=239) \text { and urban }(n=259) \text { settings. }\end{array}$ & $\begin{array}{l}\text { Mean (SD) } \\
\text { - } \quad \text { Rural: } 1.7(1.0) \\
\text { - } \quad \text { Urban: } 2.9(2.0)\end{array}$ & Dental caries & $\begin{array}{l}\text { Regression coefficient (SE): } \\
\text { - } \quad \text { Rural: }-0.15(0.09) ; p=0.09 \\
\text { - Urban: }-\mathbf{0 . 2 2}\left(\mathbf{0 . 0 8 )} ; \mathbf{p}=\mathbf{0 . 0 0 5 ^ { * }}\right.\end{array}$ \\
\hline $\begin{array}{l}\text { Kim and Lee } 2013 \\
\text { Cross-sectional study; } n=3,966 \text { adults } \\
\text { ( } \geq 20 \text { years of age) }\end{array}$ & $\begin{array}{l}\text { PbB: } \\
\text { Mean (SE): } \\
\text { - } \quad \text { Men } \\
\text { o no periodontitis: } \\
\\
\quad 2.625(0.028) \\
\text { o periodontitis: } 3.118 \\
\quad(0.057) ; p<0.001 \\
\text { - Women, } \\
\text { o no periodontitis: } \\
\quad 1.906(0.025) \\
\text { o periodontitis: } 2.222 \\
\quad(0.052) ; p<0.001\end{array}$ & Periodontitis & $\begin{array}{l}\text { OR }(95 \% \mathrm{Cl}), \text { per doubling of } \mathrm{PbB} \text { : } \\
\text { - } \quad \text { Men: } \mathbf{1 . 6 9 9}\left(\mathbf{1 . 1 5 4 , 2 . 5 0 3 ) ^ { * }}\right. \\
\text { - } \quad \text { Women: } 1.242(0.833,1.850)\end{array}$ \\
\hline $\begin{array}{l}\text { Kim et al. } 2017 \text { a } \\
\text { Cross-sectional study; } \\
n=2,805 \text { school-aged children (age } \\
\text { range: } \leq 9-\geq 12 \text { years) }\end{array}$ & $\begin{array}{l}\text { PbB: } \\
\text { Gmean: } 1.53 \\
\text { Range: } 0.11-4.89\end{array}$ & Dental caries & $\begin{array}{l}\text { PR for combined teeth with caries and filled teeth } \\
\text { - } \quad \text { Deciduous teeth: } 1.14(\mathbf{1 . 0 2}, \mathbf{1 . 2 7})^{*} \\
\text { - } \quad \text { Permanent teeth: } 0.83(0.69,0.99)\end{array}$ \\
\hline
\end{tabular}




\section{Table 2-18. Summary of Epidemiological Studies Evaluating Musculoskeletal Effects at Mean Blood Lead Concentration $(\mathrm{PbB}) \leq 10 \mu \mathrm{g} / \mathrm{dL}^{\mathrm{a}}$}

\begin{tabular}{|c|c|c|c|}
\hline Reference and study population & $\mathrm{PbB}(\mu \mathrm{g} / \mathrm{dL})$ & Outcome evaluated & Result ${ }^{b, c}$ \\
\hline $\begin{array}{l}\text { Moss et al. } 1999 \\
\text { Cross-sectional study; } \\
n=24,901 \text { participants }(2-5 \text { years old: } \\
n=3,547 ; 6-11 \text { years old: } n=2,894 ; \\
\geq 12 \text { years: } n=18,460) \text { in NHANES III }\end{array}$ & 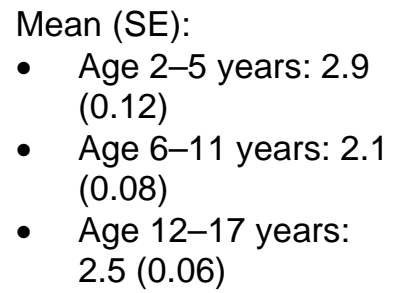 & $\begin{array}{l}\text { Dental caries in } \\
\text { children (ages 5- } \\
17 \text { years) }\end{array}$ & OR per $5 \mu \mathrm{g} / \mathrm{dL}$ increased in PbB: $1.8(1.3,2.5)^{*}$ \\
\hline
\end{tabular}

aSee the Supporting Document for Epidemiological Studies for Lead, Table 5 for more detailed descriptions of studies.

${ }^{b}$ Asterisk and bold indicate association with $\mathrm{Pb}$; unless otherwise specified, values in parenthesis are $95 \%$ Cls; $p$-values $<0.05$ unless otherwise noted in the table.

If bone $\mathrm{Pb}$ is noted under results, study did not show associations between $\mathrm{PbB}$ and musculoskeletal effects; however, results showed associations between bone $\mathrm{Pb}$ concentrations and musculoskeletal effects at concomitant $\mathrm{PbB} \leq 10 \mu \mathrm{g} / \mathrm{dL}$.

BALP = bone-specific alkaline phosphatase (marker of bone metabolism); BMD = bone mineral density; C2C = serum cleavage neoepitope of type II collagen (marker of joint tissue metabolism); $\mathrm{Cl}=$ confidence interval; COMP = serum cartilage oligomeric matrix protein (marker of joint tissue metabolism); CPII = serum type II procollagen synthesis C-propeptide (marker of joint tissue metabolism); Gmean = geometric mean; HA = serum hyaluronic acid (marker of joint tissue metabolism); HR = hazard ratio; NHANES = National Health and Nutrition Examination Survey; NTx = N-telopeptide cross-linked collagen type I (marker of bone metabolism); $\mathrm{OC}=$ osteocalcin (marker of bone metabolism); OR = odds ratio; $\mathrm{Pb}=$ lead; $\mathrm{PR}=$ prevalence ratio; $\mathrm{SD}=\mathrm{standard}$ deviation; $\mathrm{SE}=\mathrm{standard}$ error; uCTX-II = C-telopeptide urine fragments of type II collagen (marker of joint tissue metabolism); uNTX-I = urine cross-linked N telopeptide of type I collagen (marker of joint tissue metabolism) 
caries in children ages 6-17 years (PbB 2.1-2.4 $\mu \mathrm{g} / \mathrm{dL}$ ) (Moss et al. 1999). Kim et al. (2017a) reported that the prevalence of dental caries and filled teeth in children was increased for deciduous teeth, but not for permanent teeth; the mean $\mathrm{PbB}$ was $1.53 \mu \mathrm{g} / \mathrm{dL}$, with all $\mathrm{PbB}<5 \mu \mathrm{g} / \mathrm{dL}$. One study in adult males showed an association between bone $\mathrm{Pb}$ and tooth loss, but not PbB and tooth loss (Arora et al. 2009).

Mechanisms of Action. In bone and teeth, Pb substitutes for calcium (see Section 3.1.2, Toxicokinetics, Distribution). As reviewed by EPA (2014c) and Mitra et al. (2017), several mechanisms may be involved in the development of bone and periodontal/dental effects. Possible mechanisms include the following:

- Alterations in plasma growth hormones and calcitropic hormones (e.g., 1,25-[OH]2D3) leading to altered bone cell differentiation and function.

- Suppression in bone cell proliferation due to altered growth factors and hormones, including growth hormone, epidermal growth factor, transforming growth factor-beta 1 (TGF- $\beta$ ), and parathyroid hormone-related protein.

- Alterations in vitamin D-stimulated production of osteocalcin production, with inhibition of secreted bone-related proteins (e.g., osteonectin and collagen).

- Increased chondrogenesis through alterations of multiple signaling pathways, including TGF- $\beta$, bone morphogenic protein, activator protein-1, and nuclear factor kappa B.

- Inhibition of the posteruptive enamel proteinases.

- Decreased microhardness of tooth surface enamel.

\subsection{HEPATIC}

Overview. Few epidemiological studies have evaluated hepatic effects associated with exposure to $\mathrm{Pb}$, with most available studies comparing hepatic effects in small numbers of workers with $\mathrm{PbB}>10 \mu \mathrm{d} / \mathrm{dL}$ to controls with $\mathrm{PbB}$ lower than workers. Results of studies evaluating effects of $\mathrm{Pb}$ on liver function tests are inconsistent and do not demonstrate exposure-response relationships. Liver enlargement and increased gall bladder wall thickness was observed in workers with mean $\mathrm{PbB}$ of $\geq 28.66 \mu \mathrm{g} / \mathrm{dL}$. Observed effects are consistent with oxidative stress. Histopathological effects of the liver associated with $\mathrm{Pb}$ have not been established.

The following hepatic effects have been associated with $\mathrm{PbB}>10 \mu \mathrm{g} / \mathrm{dL}$ :

- Greater plasma liver enzymes; evaluated in a few studies with mixed results. 
- Greater total cholesterol.

- Enlarged liver and increased thickness of gall bladder wall.

Measures of Exposure. Studies examining the association between hepatic effects $\mathrm{Pb}$ exposure evaluate exposure by measurement of $\mathrm{PbB}$.

Confounding Factors and Effect Modifiers. Most epidemiological studies on hepatic effects of $\mathrm{Pb}$ were of small populations of workers using cross-sectional designs. In general, studies did not consider factors, such as age, diet, concurrent diseases, and potential exposure to other workplace chemicals that could affect hepatic function in association with, or independent of, $\mathrm{Pb}$ exposure. Failure to account for these factors may attenuate or strengthen the apparent associations between $\mathrm{Pb}$ exposure and the outcome, depending on the direction of the effect of the variable on the outcome.

Characterization of Effects. In contrast to the large number of epidemiological studies evaluating effects of $\mathrm{Pb}$ on other organ systems (e.g., neurological and cardiovascular outcomes), few studies have investigated the hepatic effects of $\mathrm{Pb}$. Brief study descriptions are provided in Table 2-19. Available studies were conducted in small populations ( $\mathrm{n}=23-100)$ of workers with mean PbB of 5.4-77.5 $\mu \mathrm{g} / \mathrm{dL}$. The most serious effects reported for $\mathrm{Pb}$-induced hepatic damage are liver enlargement and greater gall bladder wall thickness observed in workers with low PbB (28.66 $\mu \mathrm{g} / \mathrm{dL})$ and high $\mathrm{PbB}(40.58 \mu \mathrm{g} / \mathrm{dL})$, respectively, compared to the control group (PbB $8.34 \mu \mathrm{g} / \mathrm{dL}$ ) (Kasperczyk et al. 2013). However, these findings have not been corroborated in other studies. The study authors stated that no signs consistent with liver necrosis were observed. A cross-sectional study of a Chinese population evaluated the association between PbB and non-alcoholic fatty liver disease in China (Zhai et al. 2017). In women, a positive association between $\mathrm{PbB}$ and non-alcoholic fatty liver disease was observed in the two highest $\mathrm{PbB}$ quartiles (4.50-6.59 and >6.59 $\mu \mathrm{g} / \mathrm{dL}$; upper range not reported); no association was observed for men in the highest $\mathrm{PbB}$ quartile (>7.29 $\mu \mathrm{g} / \mathrm{dL}$; upper range not reported).

Most studies evaluated hepatic toxicity by liver function tests measuring plasma levels of liver enzymes. As shown in Table 2-20, results on effects of $\mathrm{Pb}$ on liver function tests are inconsistent and do not demonstrate exposure-response relationships. For example, Patil et al. (2007) reported greater alanine aminotransferase (ALT) and aspartate aminotransferase (AST) in spray painters with a mean $\mathrm{PbB}$ of $22.32 \mu \mathrm{g} / \mathrm{dL}$, but no change in ALT or AST in battery workers or silver jewelry workers with higher mean PbB (53.64 and $48.56 \mu \mathrm{g} / \mathrm{dL}$, respectively), compared to controls (mean PbB: $12.52 \mu \mathrm{g} / \mathrm{dL}$ ). Similarly, AST was elevated in painters with a mean PbB of $5.4 \mu \mathrm{g} / \mathrm{dL}$, but no change in AST was 
2. HEALTH EFFECTS

\section{Table 2-19. Summary of Epidemiological Studies Evaluating Hepatic Effects Associated with Blood Lead Concentration (PbB)}

\begin{tabular}{|c|c|c|c|}
\hline Reference and study population & $\mathrm{PbB}(\mu \mathrm{g} / \mathrm{dL})$ & Outcomes evaluated & Effects $^{b, c}$ \\
\hline $\begin{array}{l}\text { Al-Neamy et al. } 2001 \\
\text { Cross-sectional study; } \\
n=100 \text { workers; } 100 \text { controls }\end{array}$ & $\begin{array}{l}\text { Mean (SD) } \\
\text { - } \quad \text { Workers: } 77.5(42.8) \\
\text { - } \quad \text { Controls: } 19.8(12.3)\end{array}$ & LFTs & $\begin{array}{l}\text { - } \quad \text { Greater: LDH, AP } \\
\text { - } \quad \text { No difference: ALT, AST, GGT, bilirubin, } \\
\text { albumin }\end{array}$ \\
\hline $\begin{array}{l}\text { Can et al. } 2008 \\
\text { Cross-sectional study; } n=22 \text { battery } \\
\text { workers; } 38 \text { muffler repair workers; } \\
24 \text { controls }\end{array}$ & $\begin{array}{l}\text { Mean (SD) } \\
\text { - } \quad \text { Battery workers: } 36.83(8.13) \\
\text { - } \quad \text { Muffler workers: } 26.99(9.42) \\
\text { - } \quad \text { Controls: } 14.81(3.01)\end{array}$ & LFTs & $\begin{array}{l}\text { Battery workers: } \\
\text { - Greater LDH, AP, TC } \\
\text { Muffler workers: } \\
\text { - Greater" LDH, AP }\end{array}$ \\
\hline $\begin{array}{l}\text { Chen et al. } 2019 \\
\text { Cross-sectional study; } \\
\text { n=158 exposed adults living near an } \\
\text { electronic waste area; } 109 \text { controls }\end{array}$ & $\begin{array}{l}\text { Median }\left(\mathrm{P}_{25}, \mathrm{P}_{75}\right) \\
\text { - } \quad \text { Control: } 5.1(3.9,8.4) \\
\text { - } \quad \text { Exposed: } 8.7(6.2-12.2)\end{array}$ & LFTs & $\begin{array}{l}\text { - Greater: GGT } \\
\text { - } \quad \text { No difference: AST, ALT, LDH }\end{array}$ \\
\hline $\begin{array}{l}\text { Conterato et al. } 2013 \\
\text { Cross-sectional study; } \\
n=50 \text { painters; } 23 \text { battery workers; } \\
\text { and } 36 \text { controls }\end{array}$ & $\begin{array}{l}\text { Mean (SE) } \\
\text { - } \quad \text { Painters: } 5.4(0.4) \\
\text { - } \quad \text { Battery workers 49.8 (4.0) } \\
\text { - } \quad \text { Controls: } 1.5(0.1)\end{array}$ & LFTs & $\begin{array}{l}\text { Painters: } \\
\text { - } \quad \text { Greater: AST } \\
\text { - } \quad \text { No difference: GGT } \\
\text { Battery workers: } \\
\text { - } \quad \text { No difference: AST, GGT }\end{array}$ \\
\hline $\begin{array}{l}\text { Hsiao et al. } 2001 \\
\text { Longitudinal study (baseline 1989; } \\
\text { follow-up 1999); } n=30 \text { battery } \\
\text { workers }\end{array}$ & $\begin{array}{l}\text { Baseline: } 60 \\
\text { Follow-up: } 30\end{array}$ & LFTs & No correlation of $\mathrm{PbB}$ to $\mathrm{ALT}$ \\
\hline
\end{tabular}


2. HEALTH EFFECTS

\section{Table 2-19. Summary of Epidemiological Studies Evaluating Hepatic Effects Associated with Blood Lead Concentration (PbB)}

\begin{tabular}{|c|c|c|c|}
\hline Reference and study population & $\mathrm{PbB}(\mu \mathrm{g} / \mathrm{dL})$ & Outcomes evaluated & Effects $^{b, c}$ \\
\hline \multirow{3}{*}{$\begin{array}{l}\text { Kasperczyk et al. } 2013 \\
\text { Cross-sectional study; n (from Pb-Zn } \\
\text { processing facility): } 57 \text { low Pb } \\
\text { exposure; } 88 \text { high Pb exposure; and } \\
36 \text { controls }\end{array}$} & $\begin{array}{l}\text { Mean (SD); range } \\
\text { - Low Pb: } 28.66(6.60) ; 20-35\end{array}$ & Liver size & $\begin{array}{l}\text { - Low PbB: Greater } \\
\text { - High BPb: Greater }\end{array}$ \\
\hline & $\begin{array}{l}\text { - High Pb: } 40.58(6.74) ; 35-60 \\
\text { - } \quad \text { Control: } 8.34(2.91)\end{array}$ & $\begin{array}{l}\text { Gall bladder wall } \\
\text { thickness }\end{array}$ & $\begin{array}{l}\text { - Low PbB: Greater } \\
\text { - } \quad \text { High PbB: Greater }\end{array}$ \\
\hline & & LFTs & $\begin{array}{l}\text { - Low PbB: } \\
\text { o No difference: ALT, AST, LDH, GGT, } \\
\text { bilirubin }\end{array}$ \\
\hline
\end{tabular}

- High PbB:

o No difference: ALT, LDH, AST, bilirubin o Greater AST, GGT

\begin{tabular}{|c|c|c|c|}
\hline $\begin{array}{l}\text { Khan et al. } 2008 \\
\text { Cross-sectional study; } \\
n=87 \text { workers; } 61 \text { controls }\end{array}$ & $\begin{array}{l}\text { Median (range) } \\
\text { - } \quad \text { Workers: } 29.1(9.0-61.1) \\
\text { - } \quad \text { Controls: } 8.3(1.0-21.7)\end{array}$ & LFTs & $\begin{array}{l}\text { - } \quad \text { Greater ALT, GGT, albumin } \\
\text { - } \quad \text { No change: AP, bilirubin }\end{array}$ \\
\hline $\begin{array}{l}\text { Kristal-Boneh et al. } 1999 \\
\text { Cross-sectional study; } \\
\mathrm{n}=56 \text { exposed; } 87 \text { controls }\end{array}$ & $\begin{array}{l}\text { Mean (SD) } \\
\text { - } \quad \text { Workers: } 42.3(14.9) \\
\text { - } \quad \text { Controls: } 2.7(3.6)\end{array}$ & $\begin{array}{l}\text { Cholesterol and } \\
\text { lipoproteins }\end{array}$ & $\begin{array}{l}\text { - } \quad \text { Greater: TC, HDL } \\
\text { - } \quad \text { No change: LDL, TG, HDL:TC ratio }\end{array}$ \\
\hline $\begin{array}{l}\text { Patil et al. } 2007 \\
\text { Cross-sectional study; n=30 battery } \\
\text { workers; } 30 \text { silver jewelry workers; } \\
30 \text { spray painters }{ }^{\text {; }} 35 \text { controls }\end{array}$ & $\begin{array}{l}\text { Mean (SD) } \\
\text { - } \quad \text { Battery workers: } 53.63 \\
\text { (16.98) } \\
\text { - } \quad \text { Silver jewelry workers: } 48.56 \\
\text { (7.39) } \\
\text { - } \quad \text { Spray painters: } 22.32(8.87) \\
\text { - } \quad \text { Controls: } 12.52(4.08)\end{array}$ & LFTs & $\begin{array}{l}\text { Battery workers: } \\
\text { - } \quad \text { Greater percentage change: albumin, } \\
\text { bilirubin } \\
\text { - } \quad \text { No change: ALT, AST } \\
\text { Silver jewelry workers: } \\
\text { - } \quad \text { Lesser percentage change: albumin } \\
\text { compared to controls } \\
\text { - } \quad \text { No change: ALT, AST, bilirubin compared to } \\
\quad \text { controls } \\
\text { Spray painters: } \\
\text { - } \quad \text { Greater percentage change: ALT, AST } \\
\text { - } \quad \text { Decreased percentage change: albumin } \\
\text { - No change: bilirubin }\end{array}$ \\
\hline
\end{tabular}


Table 2-19. Summary of Epidemiological Studies Evaluating Hepatic Effects Associated with Blood Lead Concentration (PbB)

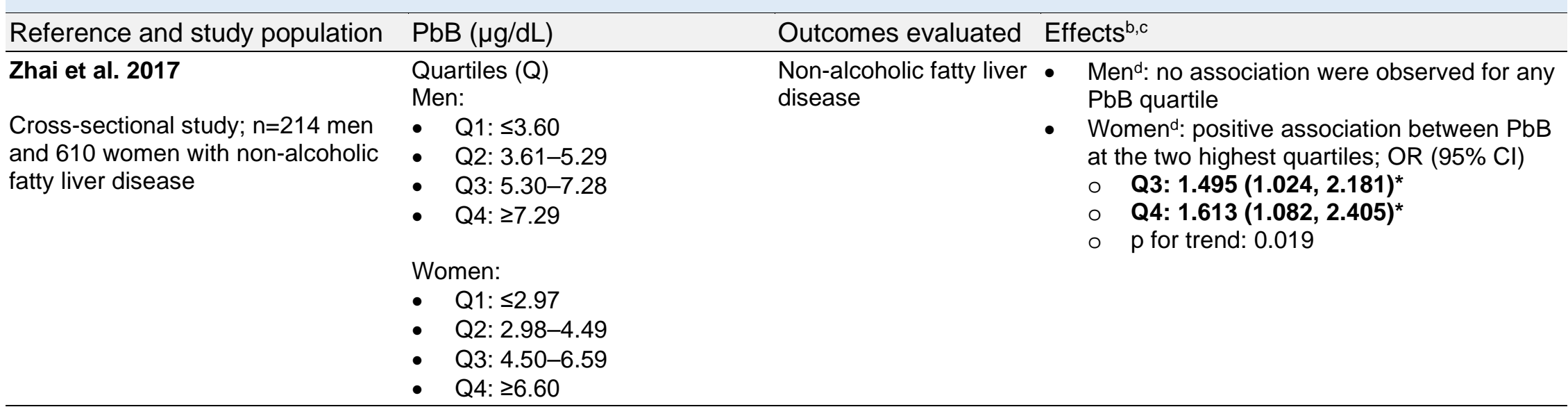

aReporting inconsistencies regarding number of spray painters evaluated; reported as 30 and 35.

${ }^{b}$ Asterisk and bold indicate association with $\mathrm{Pb}$; unless otherwise specified, values in parenthesis are $95 \%$ Cls; $\mathrm{p}$-values <0.05 unless otherwise noted in the table.

cUnless otherwise specified, comparisons are to control groups.

${ }^{\mathrm{d}}$ Comparison to lowest $\mathrm{PbB}$ quartile.

$\mathrm{ALT}=$ alanine aminotransferase; $\mathrm{AP}=$ alkaline phosphatase; $\mathrm{AST}=$ aspartate aminotransferase $; \mathrm{Cl}=$ confidence interval; $\mathrm{GGT}=$ gamma-glutamyl transpeptidase; $\mathrm{HDL}=$ high-density lipoprotein; $\mathrm{LDH}=$ lactate dehydrogenase; $\mathrm{LDL}=$ low-density lipoprotein; $L F T=$ liver function test (plasma activity of hepatic enzymes); $\mathrm{Pb}=$ lead; $\mathrm{Q}=$ quartiles; $\mathrm{SD}=$ standard deviation; $\mathrm{SE}=$ standard error; $\mathrm{TC}=$ total cholesterol; $\mathrm{TG}=$ triglycerides; $\mathrm{Zn}=$ zinc 


\begin{tabular}{|c|c|c|c|c|c|c|c|}
\hline Mean PbB $(\mu \mathrm{g} / \mathrm{dL})$ & Population $(n)^{b}$ & ALT & AST & GGT & LDH & AP & Reference \\
\hline 5.4 & $\mathrm{P}(50)$ & - & $\uparrow$ & 0 & - & - & Conterato et al. 2013 \\
\hline 8.7 & G (158) & 0 & 0 & $\uparrow$ & 0 & $\uparrow$ & Chen et al. 2019 \\
\hline 22.32 & $P(35)^{c}$ & $\uparrow$ & $\uparrow$ & - & - & - & Patil et al. 2007 \\
\hline 26.99 & $\mathrm{~Pb}-\mathrm{A}(38)$ & 0 & 0 & 0 & $\uparrow$ & $\uparrow$ & Can et al. 2008 \\
\hline 28.66 & $\mathrm{~Pb}-\mathrm{Zn}(57)$ & 0 & 0 & 0 & 0 & 0 & Kasperczyk et al. 2013 \\
\hline 29.1 & $\mathrm{~Pb}(87)$ & $\uparrow$ & - & $\uparrow$ & - & 0 & Khan et al. 2008 \\
\hline 30 & $\mathrm{~B}(30)$ & 0 & - & - & - & - & Hsiao et al. 2001 \\
\hline 36.83 & $\mathrm{~B}(22)$ & 0 & 0 & 0 & $\uparrow$ & 0 & Can et al. 2008 \\
\hline 40.58 & $\mathrm{~Pb}-\mathrm{Zn}(88)$ & 0 & $\uparrow$ & $\uparrow$ & 0 & $\uparrow$ & Kasperczyk et al. 2013 \\
\hline 48.56 & $\mathrm{~J}(30)$ & 0 & 0 & - & - & - & Patil et al. 2007 \\
\hline 9.8 & $\mathrm{~B}(23)$ & 0 & 0 & 0 & - & - & Conterato et al. 2013 \\
\hline 53.63 & $\mathrm{~B}(30)$ & 0 & 0 & - & - & - & Patil et al. 2007 \\
\hline 77.5 & $\mathrm{~Pb}(100)$ & 0 & 0 & 0 & $\uparrow$ & $\uparrow$ & Al-Neamy et al. 2001 \\
\hline
\end{tabular}

aReporting inconsistencies regarding number of spray painters evaluated; reported as 30 and 35.

$\uparrow=$ increased; 0 = no change; $-=$ not assessed; ALT = alanine aminotransferase; AP = alkaline phosphatase; AST = aspartate aminotransferase; $\mathrm{B}$ = battery workers; $\mathrm{G}=$ general population; $\mathrm{GGT}$ = gamma-glutamyl transpeptidase; $\mathrm{J}$ = silver jewelry workers; $\mathrm{LDH}=$ lactate dehydrogenase; MDA: malondialdehyde; $\mathrm{P}=$ painters; $\mathrm{Pb}=\mathrm{Pb}$-exposed industrial workers; $\mathrm{Pb}-\mathrm{A}=\mathrm{Pb}$-exposed auto workers; $\mathrm{Pb}-\mathrm{Zn}=\mathrm{Pb}$-zinc processers

observed in battery workers with a mean PbB of $49.8 \mu \mathrm{g} / \mathrm{dL}$, compared to controls with a mean PBB of $1.5 \mu \mathrm{g} / \mathrm{dL}$ (Conterato et al. 2013). Effects in painters with lower PbB compared to other workers with higher PbB may be due to co-exposure to other occupational chemicals. In a cross-sectional study of residents living close to an electronic waste site in China, $\mathrm{PbB}$ (median PbB: $8.7 \mu \mathrm{g} / \mathrm{dL}$ ) was associated with an increase in gamma-glutamyl transpeptidase (GGT) compared to controls (median PbB: $5.1 \mu \mathrm{g} / \mathrm{dL}$ ), although no effects were observed for ALT or AST (Chen et al. 2019). In addition to liver enzymes, total serum cholesterol and high-density lipoprotein (HDL)-cholesterol were greater in workers with a mean $\mathrm{PbB}$ of $26.99-42.3 \mu \mathrm{g} / \mathrm{dL}$, compared to controls with a mean PbB 2.7-14.81 $\mu \mathrm{g} / \mathrm{dL}$ (Can et al. 2008; Kristal-Boneh et al. 1999).

Effect at Blood Pb Levels $\leq 10 \mu \boldsymbol{\mu g} / \mathbf{d L}$. See discussion above on Conterato et al. (2013), Chen et al. (2019), and Zhai et al. (2017).

Mechanisms of Action. General mechanisms of toxicity of $\mathrm{Pb}$ (reviewed in Section 2.21) are likely involved in the development of hepatic toxicity. EPA (2014c) specifically noted that oxidative stress 
through ROS can result in damaged function and histopathological damage to the liver, including peroxidation of lipid membranes.

\subsection{RENAL}

Overview. Numerous epidemiologic studies in adults show that exposure to $\mathrm{Pb}$ can cause altered kidney function and contribute to the development of chronic kidney disease (CKD). A few studies in children also show decreases in renal function. Pb-induced nephrotoxicity is characterized by proximal tubular nephropathy, glomerular sclerosis, and interstitial fibrosis (Diamond 2005; Goyer 1989; LoghmanAdham 1997). Functional deficits in humans that have been associated with excessive $\mathrm{Pb}$ exposure include enzymuria, low- and high-molecular weight proteinuria, impaired transport of organic anions and glucose, and depressed GFR. A few studies have revealed histopathological features of renal injury in humans, including intranuclear inclusion bodies and cellular necrosis in the proximal tubule and interstitial fibrosis (Biagini et al. 1977; Cramer et al. 1974; Wedeen et al. 1975, 1979). Studies show consistent evidence of renal damage and reduced renal function associated over a wide range of $\mathrm{PbB}$ $(\leq 10->50 \mu \mathrm{g} / \mathrm{dL})$, with the overall dose-effect pattern suggesting an increasing severity of nephrotoxicity associated with increasing $\mathrm{PbB}$.

The following renal effects have been associated with PbB:

- $\leq 10 \mu \mathrm{g} / \mathrm{dL}$ :

o Decreased GFR; corroborated in numerous studies.

o Proteinuria; demonstrated in a few studies.

o Chronic kidney disease (CKD); demonstrated in two studies.

- $\quad>10 \mu \mathrm{g} / \mathrm{dL}$ :

o Decreased GFR; corroborated in numerous studies.

o Enzymuria; corroborated in numerous studies.

o Proteinuria; corroborated in numerous studies.

o Impaired tubular transport; demonstrated in a few studies.

o Histopathological damage; demonstrated in a few studies.

Measures of Effect. Endpoints demonstrating renal damage include various measures of glomerular and tubular dysfunction. Effects on GFR typically are assessed from measurements of creatinine clearance, serum creatinine concentration, or blood urea nitrogen (BUN). Increased excretion of albumin 
(albuminuria) is an indication of damage to the glomerular endothelium or basement membrane, resulting in increased filtration of albumin, or impaired function of the proximal tubule, resulting in decreased reabsorption of filtered albumin. Increased excretion of low molecular weight serum proteins (e.g., $2 \mu \mathrm{G}$ or retinol-binding protein) are an indication of impaired reabsorption of protein in the proximal tubule. Increased excretion of enzymes associated with the renal tubule (renal tubular enzymuria) is an indication of injury to renal tubular cells resulting in release of membrane or intracellular enzymes into the tubular fluid. Pb-induced renal tubular enzymuria is most commonly evaluated from measurements of urinary $\mathrm{N}$-acetyl-D-glucosaminidase (NAG). Increased excretion of NAG has been found in Pb-exposed workers in the absence of increased excretion of other proximal tubule enzymes (e.g., alanine aminopeptidase, alkaline phosphatase, glutamyltransferase) (Pergande et al. 1994). Indices of impaired transport include altered clearance or transport maxima for organic anions (e.g., p-aminohippurate, urate) or glucose (Biagini et al. 1977; Hong et al. 1980; Wedeen et al. 1975). Proximal tubular injury can also be confirmed through histopathological examination of renal tissue, although few studies provide this information (Biagini et al. 1977; Cramer et al. 1974; Wedeen et al. 1975, 1979).

Measures of Exposure. Most studies evaluating renal damage use $\mathrm{PbB}$ as the biomarker for exposure, although more recent epidemiological studies have explored associations between toxicity and bone $\mathrm{Pb}$ concentrations. These studies provide a basis for establishing $\mathrm{PbB}$, and, in some cases, bone $\mathrm{Pb}$ concentration ranges associated with specific nephrotoxicity outcomes.

Confounding Factors and Effect Modifiers. Inconsistencies in the reported outcomes for renal effects across studies may derive from several causes, including failure to account for confounding factors and effect modifiers. Various factors can affect kidney function, including age, underlying diseases (e.g., hypertension), and concomitant exposure to other nephrotoxicants (e.g., cadmium). Results of epidemiological studies of general populations have shown an effect of age on the relationship between GFR (assessed from creatinine clearance of serum creatinine concentration or cystatin $\mathrm{C}$ ) and $\mathrm{PbB}$ (Kim et al. 1996a; Muntner et al. 2003; Payton et al. 1994; Staessen et al. 1990, 1992). Pb-induced decrements in renal function can lead to higher $\mathrm{Pb}$ body burden due to decreased excretion of $\mathrm{Pb}$ (i.e., reverse causality) (Bellinger 2011; Diamond et al. 2019; Evans and Elinder 2011; Marsden 2003). Thus, reverse causality potentially confounds interpretation of the dose-response relationship between $\mathrm{PbB}$ and decreased renal function. Pb exposure has also been associated with increases in GFR (Hsiao et al. 2001; Roels et al. 1994). This may represent a benign outcome or a potentially adverse hyperfiltration, which may contribute to subsequent adverse renal effects. Hypertension can be both a confounder in studies of associations between $\mathrm{Pb}$ exposure and creatinine clearance (Perneger et al. 1993) and a covariable with $\mathrm{Pb}$ 
exposure (Harlan et al. 1985; Muntner et al. 2003; Payton et al. 1994; Pirkle et al. 1985; Pocock et al. 1984, 1988; Tsaih et al. 2004; Weiss et al. 1986). Renal damage can cause increased blood pressure, which in turn can result in further damage to the kidneys. In addition, varying uncertainty also exists across studies in exposure history of subjects and in the biomarkers assessed.

Characterization of Effects. A large number of studies showing decrements in renal function associated with $\mathrm{Pb}$ exposure in humans have been published (Table 2-21). Most of these studies are of adults whose exposures were of occupational origin; however, a few environmental, mixed, and/or unknown exposures are represented, and a few studies of children are also included. Although these studies demonstrate adverse renal effects across the $\mathrm{PbB}$ range, some studies did not find associations (Buchet et al. 1980; de Kort et al. 1987; Fadrowski et al. 2010; Gennart et al. 1992; Huang et al. 2002; Karimooy et al. 2010; Mujaj et al. 2019; Omae et al. 1990). However, collectively, the body of evidence demonstrates that long-term exposure to $\mathrm{Pb}$ is nephrotoxic. General trends regarding the relationship between $\mathrm{PbB}$ and qualitative aspects of the kidney response are shown in Table 2-21. Decreased GFR and proteinuria have been observed in association with $\mathrm{PbB} \leq 10 \mu \mathrm{g} / \mathrm{dL}$; the significance of these studies is discussed in greater detail below. Enzymuria and proteinuria have been observed in association with $\mathrm{PbB}>10-\leq 50 \mu \mathrm{g} / \mathrm{dL}$. Functional deficits, including enzymuria, proteinuria, impaired transport, and depressed GFR have been observed at $\mathrm{PbB}>50 \mu \mathrm{g} / \mathrm{dL}$. Histopathological findings, including tubular atrophy, focal sclerosis of glomeruli, and periglomerular and interstitial fibrosis have also been observed at $\mathrm{PbB}>50 \mu \mathrm{g} / \mathrm{dL}$. The overall dose-effect pattern suggests an increasing severity of nephrotoxicity associated with increasing $\mathrm{PbB}$, with effects on glomerular filtration evident at $\mathrm{PbBs}<10 \mu \mathrm{g} / \mathrm{dL}$, enzymuria and proteinuria becoming evident $>10 \mu \mathrm{g} / \mathrm{dL}$, and severe deficits in function and pathological changes occurring in association with $\mathrm{PbBs}>50 \mu \mathrm{g} / \mathrm{dL}$.

\section{Table 2-21. Overview of Renal Effect Associated with Chronic Exposure to Lead} (Pb)

\begin{tabular}{|c|c|c|}
\hline $\begin{array}{l}\text { Mean blood lea } \\
\text { concentration } \\
(\mathrm{PbB})(\mu \mathrm{g} / \mathrm{dL}) \\
\end{array}$ & $\begin{array}{l}\text { Effects } \\
\text { associated with } \\
\text { Pb exposure }\end{array}$ & References \\
\hline \multirow[t]{2}{*}{$\leq 10$} & Increased GFR & de Burbure et al. 2006 \\
\hline & Decreased GFR & $\begin{array}{l}\text { Åkesson et al. 2005; Fadrowski et al. 2010; Harari et al. 2018; Lin } \\
\text { et al. 2001; Khan et al. 2010a; Kim et al. 1996a; Lin et al. 2003; } \\
\text { Lin et al. 2006a, 2006b; Muntner et al. 2003; Navas-Acien et al. } \\
\text { 2009; Payton et al. 1994; Pollack et al. 2015; Spector et al. 2011; } \\
\text { Staessen et al. 1992, 2001; Yu et al. } 2004\end{array}$ \\
\hline
\end{tabular}




\begin{tabular}{|c|c|c|}
\hline \multirow[t]{2}{*}{$\begin{array}{l}\text { Mean blood leac } \\
\text { concentration } \\
(\mathrm{PbB})(\mu \mathrm{g} / \mathrm{dL})\end{array}$} & $\begin{array}{l}\text { Effects } \\
\text { associated with } \\
\text { Pb exposure }\end{array}$ & References \\
\hline & $\begin{array}{l}\text { Proteinuria } \\
\text { chronic kidney } \\
\text { disease }\end{array}$ & Navas-Acien et al. 2009; Harari et al. 2018; Pollack et al. 2015 \\
\hline \multirow[t]{3}{*}{$>10-\leq 30$} & Decreased GFR & Kim et al. 1996a; Staessen et al. 1990 \\
\hline & Enzymuria & $\begin{array}{l}\text { Bernard et al. 1995; Chia et al. 1994; Sonmez et al. 2002; Sun et } \\
\text { al. 2008b }\end{array}$ \\
\hline & Proteinuria & Bernard et al. 1995; Chia et al. 1995a, 1995b \\
\hline \multirow[t]{5}{*}{$>30-\leq 50$} & Increased GFR & Hsiao et al. 2001; Roels et al. 1994 \\
\hline & Decreased GFR & $\begin{array}{l}\text { Orisakwe et al. 2007; Weaver et al. 2003a, 2003b, 2005a; } \\
\text { Wedeen et al. } 1975\end{array}$ \\
\hline & Enzymuria & $\begin{array}{l}\text { Cardenas et al. 1993; Cardozo dos Santos et al. 1994; Fels et al. } \\
\text { 1994; Garcon et al. 2007; Gerhardsson et al. 1992; Kim et al. } \\
\text { 1996a; Kumar and Krishnaswamy 1995; Lin and Tai-yi 2007; } \\
\text { Mortada et al. 2001; Pergande et al. 1994; Roels et al. 1994; } \\
\text { Verberk et al. 1996; Verschoor et al. 1987; Weaver et al. 2003a, } \\
\text { 2003b, 2005a }\end{array}$ \\
\hline & Proteinuria & $\begin{array}{l}\text { Factor-Litvak et al. 1999; Fels et al. 1998; Garcon et al. 2007; } \\
\text { Gerhardsson et al. 1992; Kumar and Krishnaswamy 1995; } \\
\text { Mortada et al. 2001; Pergande et al. 1994; Verschoor et al. } 1987\end{array}$ \\
\hline & $\begin{array}{l}\text { Impaired tubular } \\
\text { transport }\end{array}$ & Pinto de Almeida et al. 1987 \\
\hline \multirow[t]{5}{*}{$>50$} & Decreased GFR & $\begin{array}{l}\text { Baker et al. 1979; Biagini et al. 1977; Cramer et al. 1974; Ehrlich } \\
\text { et al. 1998; Hong et al. 1980; Lilis et al. 1968, 1980; Onuegbu et } \\
\text { al. 2011; Wedeen et al. 1975, } 1979\end{array}$ \\
\hline & Enzymuria & Cabral et al. 2012; Gao et al. 2010; Garcon et al. 2007 \\
\hline & Proteinuria & Cabral et al. 2012; Gao et al. 2010; Garcon et al. 2007 \\
\hline & $\begin{array}{l}\text { Impaired tubular } \\
\text { transport }\end{array}$ & $\begin{array}{l}\text { Biagini et al. 1977; Ehrlich et al. 1998; Hong et al. 1980; Wedeen } \\
\text { et al. } 1975\end{array}$ \\
\hline & $\begin{array}{l}\text { Histopathological } \\
\text { changes }\end{array}$ & Biagini et al. 1977; Cramer et al. 1974; Wedeen et al. 1975, 1979 \\
\hline
\end{tabular}

GFR = glomerular filtration rate

Effects at Blood Pb Levels $\leq \mathbf{1 0} \boldsymbol{\mu g} / \mathbf{d L}$. Studies of renal function in populations with $\mathrm{PbB} \leq 10 \mu \mathrm{g} / \mathrm{dL}$ provide evidence for effects of Pb on GFR in children and adults. Results are summarized in Table 2-22, with study details provided in the Supporting Document for Epidemiological Studies for Lead, Table 6. Most studies found that increasing PbB was associated with decreased GFR; however, one study found evidence for increasing GFR in children (de Burbure et al. 2006). 
2. HEALTH EFFECTS

Table 2-22. Summary of Epidemiological Studies Evaluating Renal Effects at Mean Blood Lead Concentration $(\mathrm{PbB}) \leq 10 \mu \mathrm{g} / \mathrm{dL}^{\mathrm{a}}$

\begin{tabular}{|c|c|c|c|}
\hline Reference and study population ${ }^{b}$ & $\mathrm{PbB}(\mu \mathrm{g} / \mathrm{dL})$ & Outcome evaluated $^{c}$ & Result $^{d}$ \\
\hline Åkesson et al. 2005 & Median: 2.2 & $\mathrm{CCr}$ & $\begin{array}{l}\text { Linear regression } \beta \text { coefficient }(\mathrm{mL} / \text { minute per } \\
\mu \mathrm{g} / \mathrm{dL}):-0.018(95 \% \mathrm{Cl}-0.03,-0.006)^{*}\end{array}$ \\
\hline \multirow[t]{3}{*}{$\begin{array}{l}\text { Cross-sectional study; } \mathrm{n}=820 \text { adult } \\
\text { women }\end{array}$} & & GFR & $\begin{array}{l}\text { Linear regression } \beta \text { coefficient }(\mathrm{mL} / \text { minute per } \\
\mu \mathrm{g} / \mathrm{dL}):-0.02(95 \% \mathrm{Cl}-0.03,-0.009)^{*}\end{array}$ \\
\hline & & $\mathrm{UPHC}$ & $\begin{array}{l}\text { Linear regression } \beta \text { coefficient }(\mu \mathrm{g} / \mathrm{L} \text { per } \mu \mathrm{g} / \mathrm{dL}) \text { : } \\
\text { reported as NS }\end{array}$ \\
\hline & & UNAG & $\begin{array}{l}\text { Linear regression } \beta \text { coefficient }(\mathrm{U} / \mathrm{g} \text { creatinine per } \\
\mu \mathrm{g} / \mathrm{dL}) \text { : reported as NS }\end{array}$ \\
\hline $\begin{array}{l}\text { Barry et al. } 2019 \\
\text { Cross-sectional study; n=211 adult men }\end{array}$ & Median: 2.5 & GFR & $\begin{array}{l}\text { Linear regression coefficient }(\mathrm{SE}) \text { for: } \\
\mathrm{PbB} \mathrm{Q} 4:-2.71(4.16) ; \mathrm{p}=0.52 \\
\mathrm{PbB} \text { continuous: }-0.13(0.28) ; \mathrm{p}=0.65 \\
\text { Bone } \mathrm{Pb} \text { Q4: }-5.66(4.86) ; p=0.25 \\
\text { Bone } \mathrm{Pb} \text { Continuous: }-0.15(0.11) ; p=0.18\end{array}$ \\
\hline \multirow{2}{*}{$\begin{array}{l}\text { de Burbure et al. } 2006 \\
\text { Cross-sectional study; } n>800 \text { children } \\
\text { (ages } 8.5-12.3 \text { years) }\end{array}$} & \multirow{2}{*}{$\begin{array}{l}\text { Mean range (three } \\
\text { locations) } \\
\text { Control: } 2.81-3.81 \\
\text { Exposure: } 3.64-6.51\end{array}$} & $\mathrm{SCr}$ & $\begin{array}{l}\text { Decreased } 7 \%(p<0.01) \text { in } \mathrm{Q} 4(\mathrm{PbB} \\
>5.59 \mu \mathrm{g} / \mathrm{dL}), \text { compared to } \mathrm{Q} 1(\mathrm{PbB} \\
<2.85 \mu \mathrm{g} / \mathrm{dL})^{*}\end{array}$ \\
\hline & & $\mathrm{S} \beta 2 \mathrm{M}$ & $\begin{array}{l}\text { Decreased } 9 \%(p<0.01) \text { in } \mathrm{Q} 4(\mathrm{PbB} \\
>5.86 \mu \mathrm{g} / \mathrm{dL}), \text { compared to } \mathrm{Q} 1(\mathrm{PbB} \\
<3.10 \mu \mathrm{g} / \mathrm{dL})^{*}\end{array}$ \\
\hline $\begin{array}{l}\text { Fadrowski et al. } 2010 \\
\text { Cross-sectional study; } \\
n=769 \text { adolescents (ages 12-20 years) }\end{array}$ & $\begin{array}{l}\text { Median: } 1.5 \\
\text { Quartiles: } \\
\text { - } \quad \text { Q1: }<1.0 \\
\text { - } \quad \text { 2: } 1.0-1.5 \\
\text { - } \quad \text { Q3: } 1.6-2.9 \\
\text { Q4: }>2.9\end{array}$ & GFR & $\begin{array}{l}\text { Change in GFR }\left(\mathrm{mL} / \mathrm{min} u t e / 1.73 \mathrm{~m}^{2}\right) \mathrm{Q4} \\
\text { compared to Q1: } \\
-6.6(-12.6,-0.7)^{*} \\
\text { p-Trend across Q1-Q4=0.009* } \\
\text { - Mean difference in GFR associated with a } \\
\text { 2-fold increase in blood lead } \\
\text { level: }-2.9(-5.0,-0.7)^{*}\end{array}$ \\
\hline
\end{tabular}


Table 2-22. Summary of Epidemiological Studies Evaluating Renal Effects at Mean Blood Lead Concentration $(\mathrm{PbB}) \leq 10 \mu \mathrm{g} / \mathrm{dL}^{\mathrm{a}}$

\begin{tabular}{ll}
\hline Reference and study population $^{\mathrm{b}}$ & $\mathrm{PbB}(\mu \mathrm{g} / \mathrm{dL})$ \\
\hline Harari et al. 2018 & Median at baseline (range) \\
& $2.5(0.15-25.8)$ \\
Prospective cohort study; & Quartiles (range): \\
$\mathrm{n}=2,567$ adults; with a 16-year follow-up & $\bullet$ Q1: $1.5(0.15-1.85)$ \\
period & - Q2: $2.2(1.85-2.47)$ \\
& - Q3: $2.9(2.47-3.30)$ \\
& - Q4: $4.6(3.30-25.8)$
\end{tabular}

Kim et al. 1996a

Mean: 9.9 Outcome evaluated $^{\mathrm{c}}$

Result $^{\mathrm{d}}$

At follow-up, GFR for Q1 decreased from 89 to

$62 \mathrm{~mL} /$ minute from baseline

Additional decreases in GFR, $\mathrm{mL} /$ minutel

$1.73 \mathrm{~m}^{2}$, per quartile:

- Q3: -2.6 (-4.0, -1.2); $p<0.001^{*}$

- Q4: $-2.3(-3.8,-0.85) ; p=0.002^{*}$

- p-trend: $<0.001^{*}$

HR for Q4 compared to combined Q1-Q3:

1.49 (1.07-2.08); $p=0.02^{*}$

- Regression coefficient (SE) for all participants $(\mu \mathrm{mol} / \mathrm{L}$ per $\mu \mathrm{g} / \mathrm{dL})$ : 0.033 (0.012); $\mathrm{p}=0.005^{*}$

Retrospective cohort study; $\mathrm{n}=459$ men

- Regression coefficient (SE) for PbB $\leq 10$ ( $\mu \mathrm{mol} / \mathrm{L}$ per $\mu \mathrm{g} / \mathrm{dL}): 0.060$ (0.019); $\mathrm{p}=0.002^{*}$

\section{Khan et al. 2010}

Cross sectional study children (ages 1-

6 years) of $\mathrm{Pb}$ workers $(\mathrm{n}=123)$ and controls $(n=123)$

\section{Lin et al. 2001}

Prospective, longitudinal study; $\mathrm{n}=110$ patients with chronic renal insufficiency
Median

$\mathrm{SCr}$

- Control: 6.7

Exposed: 8.10 Low PbB mean: 3.9 High PbB mean: 6.6

$\mathrm{CCr}$

$\mathrm{CCr}$

Serum creatinine ( $\mu \mathrm{mol} / \mathrm{L})$ : control: 52 ; exposed: $56 ; p \leq 0.01^{*}$

- Spearman's correlation coefficient: $r=0.13$; $p \leq 0.05^{*}$

- 18 Months $\mathrm{CCr}$ (mL/second) mean $\pm S D$ : low $\mathrm{Pb}: 0.72 \pm 0.25$; high $\mathrm{Pb}: 0.59 \pm 0.22 \mu \mathrm{g} / \mathrm{dL}$ $(p=0.007)^{*}$

- 21 Months $\mathrm{CCr}$ (mL/second) mean $\pm S D$ : low $\mathrm{Pb}: 0.70 \pm 0.24$; High $\mathrm{Pb}: 0.57 \pm 0.22 \mu \mathrm{g} / \mathrm{dL}$ $(p=0.006)^{*}$

- 24 Months $\mathrm{CCr}$ (mL/second) mean $\pm \mathrm{SD}$ : low $\mathrm{Pb}: 0.70 \pm 0.24$; High Pb: $0.55 \pm 0.22 \mu \mathrm{g} / \mathrm{dL}$ $(p=0.001)^{*}$ 
Table 2-22. Summary of Epidemiological Studies Evaluating Renal Effects at Mean Blood Lead Concentration $(\mathrm{PbB}) \leq 10 \mu \mathrm{g} / \mathrm{dL} \mathrm{L}^{\mathrm{a}}$

\section{Reference and study population}

Lin et al. 2003

Prospective, longitudinal study; $\mathrm{n}=202$ patients with chronic renal insufficiency

\section{$\mathrm{PbB}(\mu \mathrm{g} / \mathrm{dL})$ \\ Baseline: 5.3}

After 24-month observation, prior to

chelation

- Placebo: 5.9

- Chelation: 6.1
After 24-month

observation, prior to

chelatione

- Placebo: 3.0

- Chelation: 2.6

Outcome evaluated ${ }^{c} \quad$ Result $^{d}$

GFR

- GFR (mL/minute/1.73 $\left.\mathrm{m}^{2}\right)$ following

treatment (mean $\pm S D$ ): placebo $25.5 \pm 12.3$; chelation $34.4 \pm 14.7(p=0.01)^{*}$

- Change in GFR (mL/minute/1.73 $\left.\mathrm{m}^{2}\right)$ following treatment (mean $\pm S D$ ): placebo $-6.0 \pm 5.8$; chelation $2.1 \pm 5.7$ $(p>0.001)^{*}$

GFR

- GFR (mL/minute/1.73 $\left.\mathrm{m}^{2}\right)$ following treatment (mean $\pm S D$ ): placebo $38.0 \pm 8.9$; chelation $47.9 \pm 17.0(p=0.0493)^{*}$

$\mathrm{n}=124$ patients with chronic rena insufficiency

- Change in GFR (mL/minute/1.73 $\left.\mathrm{m}^{2}\right)$ following treatment (mean $\pm S D$ ): placebo $-4.6 \pm 4.3$; chelation $6.6 \pm 10.7$ $(p>0.0005)^{*}$

UP (24-hour) • Urine protein (g) following chelation: placebo 1.11 \pm 1.63 ; chelation: $0.92 \pm 1.16(p=0.6236)$

\section{Lin et al. 2006b}

End of 12-month observation, prior to chelatione

Prospective, longitudinal study; $\mathrm{n}=238$ patients with type II diabetes and • Placebo: 5.9 progressive diabetic neuropathy

- Chelation: 7.5

Lin-Tan et al. 2007 Mean after 51-month chelation

Placebo-controlled clinical study; $\mathrm{n}=116$ - Placebo: 6.0 non-diabetic patients with chronic kidney disease

- Chelation: 3.5 chelation $18.0 \pm 7.3(p=0.0352)^{*}$ GFR

- GFR (mL/minute/1.73 $\left.\mathrm{m}^{2}\right)$ following treatment (mean $\pm S D$ ): placebo 13.1 \pm 4.5 ;

- Decrements in GFR (mL/minute/1.73 $\mathrm{m}^{2}$ ) following treatment (mean $\pm S D)$ : placebo 13.2 \pm 7.6 ; chelation $4.4 \pm 6.8(p>0.0045)^{*}$

- GFR (mL/minute/1.73 $\left.\mathrm{m}^{2}\right)$ following treatment (mean $\pm S D$ ): placebo $23.7 \pm 10.8$; Chelation 35.4 $\pm 17.0(p<0.0001)^{*}$

- Change in GFR (mL/minute/1.73 $\left.\mathrm{m}^{2}\right)$ following treatment (mean $\pm S D$ ): placebo $-12.7 \pm 8.4$; chelation $-1.8 \pm 8.8$ $(p>0.0001)^{*}$

UP (24-hour)
UP (mean $\pm S D)$ : placebo $0.96 \pm 1.04$; chelation: $0.81 \pm 0.86(p=0.3369)$ 
2. HEALTH EFFECTS

Table 2-22. Summary of Epidemiological Studies Evaluating Renal Effects at Mean Blood Lead Concentration $(\mathrm{PbB}) \leq 10 \mu \mathrm{g} / \mathrm{dL}^{\mathrm{a}}$

\begin{tabular}{|c|c|c|c|}
\hline Reference and study population ${ }^{b}$ & $\mathrm{PbB}(\mu \mathrm{g} / \mathrm{dL})$ & Outcome evaluated $^{c}$ & Result $^{d}$ \\
\hline \multirow{2}{*}{$\begin{array}{l}\text { Mujaj et al. } 2019 \\
\text { Cross-sectional study; } n=447 \text { newly } \\
\text { hired male workers }\end{array}$} & \multirow[t]{2}{*}{ Mean: 4.34} & GFR & $\begin{array}{l}\begin{array}{l}\beta, \text { per doubling of } \mathrm{PbB}:-0.281(-3.07,2.50) \\
p=0.84\end{array}\end{array}$ \\
\hline & & ACR & $\begin{array}{l}\beta, \text { per doubling of } \mathrm{PbB}:-0.071(-0.14,0.59) \\
p=0.06\end{array}$ \\
\hline \multirow{3}{*}{$\begin{array}{l}\text { Muntner et al. } 2003 \\
\text { Cross-sectional study; } \\
n=4,813 \text { hypertensive; } \\
n=10,398 \text { normotensive adults } 9\end{array}$} & \multirow{3}{*}{$\begin{array}{l}\text { Normotensive } \\
\text { Mean: } 3.30 \pm 0.10 \\
\text { Quartiles } \\
\text { - } \quad \text { Q1 (reference): 0.7-1.6 } \\
\text { - } \quad \text { Q2: } 1.7-2.8 \\
\text { - } \quad \text { Q3: } 2.9-4.6 \\
\text { - Q4: 4.7-52.9 } \\
\text { Hypertensive } \\
\text { Mean: 4.21 } 0.14 \\
\text { Quartiles: } \\
\text { - } \quad \text { Q1 (reference): 0.7-2.4 } \\
\text { - } \quad \text { 2: } 2.5-3.8 \\
\text { - } \quad \text { Q3: 3.9-5.9 } \\
\text { - } 4: 6.0-56.0\end{array}$} & GFR & $\begin{array}{l}\text { Estimated GFR, } \mathrm{mL} / \text { minute }(\text { mean } \pm S D) \\
\text { - } \quad \text { Normotensive: } 115 \pm 0.7 \\
\text { - Hypertensive: } \mathbf{9 5} \pm 0.7(\mathbf{p}<0.001)^{*}\end{array}$ \\
\hline & & $\overline{\mathrm{SCr}}$ & 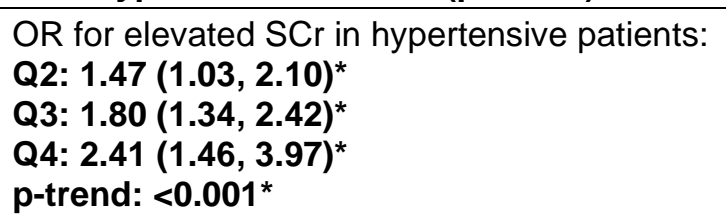 \\
\hline & & CKD & $\begin{array}{l}\text { OR for elevated CKD in hypertensive patients: } \\
\text { Q2: } 1.44(1.00,2.09) \\
\text { Q3: } \mathbf{1 . 8 5}(\mathbf{1 . 3 2}, \mathbf{2 . 5 9})^{\star} \\
\text { Q4: } \mathbf{2 . 6 0}(\mathbf{1 . 5 2 , 4 . 4 5})^{\star} \\
\text { p-trend: }<\mathbf{0 . 0 0 1 ^ { * }}\end{array}$ \\
\hline \multirow[t]{2}{*}{$\begin{array}{l}\text { Navas-Acien et al. } \mathbf{2 0 0 9 ^ { \mathbf { f } }} \\
\text { Cross-sectional study; } n=14,778 \text { adults }\end{array}$} & \multirow[t]{2}{*}{$\begin{array}{l}\text { Mean: } 1.58 \\
\text { Quartiles: } \\
\text { - } \quad \text { Q1 (reference): } \leq 1.1 \\
\text { - } \quad \text { 22: }>1.1-1.6 \\
\text { - } \quad \text { Q3: }>1.6-2.4 \\
\text { - } \quad \text { 4: }>2.4\end{array}$} & GFR & $\begin{array}{ll}\text { ORs for reduced GFR } \\
\text { - } & \text { Q2: } 1.10(0.80,1.51) \\
\text { - } & \text { Q3: } 1.36(0.99,1.85) \\
\text { - } & \text { Q4: } \mathbf{1 . 5 6}(\mathbf{1 . 1 7}, \mathbf{2 . 0 8})^{*} \\
\text { - } & \text { p-trend: }<\mathbf{0 . 0 0 1}^{*} \\
\end{array}$ \\
\hline & & Albuminuria & $\begin{array}{l}\text { ORs for albuminuria } \\
\text { - Q2: } 0.83(0.66,1.04) \\
\text { - Q3: } 0.92(0.76,1.12) \\
\text { - } \quad \text { Q4: } 1.19(0.96,1.47) \\
\text { - p-trend: }<0.001^{*}\end{array}$ \\
\hline
\end{tabular}


2. HEALTH EFFECTS

Table 2-22. Summary of Epidemiological Studies Evaluating Renal Effects at Mean Blood Lead Concentration $(\mathrm{PbB}) \leq 10 \mu \mathrm{g} / \mathrm{dL}^{\mathrm{a}}$

Reference and study population $^{\mathrm{b}}$

Payton et al. 1994

Cross-sectional study; $n=744$ men

Pollack et al. 2015

Prospective cohort study;

$\mathrm{n}=257$ premenopausal women

\section{$\mathrm{PbB}(\mu \mathrm{g} / \mathrm{dL})$}

Mean: 8.1

Outcome evaluated

Result $^{\mathrm{d}}$

Regression coefficient, $\beta$ (SE), $\mathrm{mL} /$ minute per $\mu \mathrm{g} / \mathrm{dL}$ : -0.0403 (0.0198); $p=0.0426^{*}$

- Regression $\beta$ coefficient (\% change per twofold increase in $\mathrm{PbB})$ :

Median: 0.88

- T1 (reference): $<0.72$

- T2: 0.72-1.10

- T3: $>1.10$

GFR

- Regression $\beta$ coefficient (\% change per 2-fold increase in $\mathrm{PbB}$ ) by tertile: o T2: $-8.28(-14.07,-2.5)$; $p<0.05^{\star}$

o T3: $-6.79(-13.10,-0.49) ; p<0.05^{*}$

\begin{tabular}{ll}
\hline $\mathrm{SCr}$ & $\begin{array}{l}\text { Regression } \beta \text { coefficient }(\% \text { change per 2-fold } \\
\text { increase in } \mathrm{PbB}): 3.47(0.86,6.16)\end{array}$ \\
\hline BUN & $\begin{array}{l}\text { Regression } \beta \text { coefficient }(\% \text { change per 2-fold } \\
\text { increase in } \mathrm{PbB}):-0.13(-4.97,4.96)\end{array}$ \\
\hline Blood albumin & $\begin{array}{l}\text { Regression } \beta \text { coefficient }(\% \text { change per } 2 \text {-fold } \\
\text { increase in } \mathrm{PbB}):-0.38(-1.28,0.52)\end{array}$ \\
\hline Blood glucose & $\begin{array}{l}\text { Regression } \beta \text { coefficient }(\% \text { change per } 2 \text {-fold } \\
\text { increase in } \mathrm{PbB}): 0.93(-0.28,2.15)\end{array}$ \\
\hline Blood protein & $\begin{array}{l}\text { Regression } \beta \text { coefficient }(\% \text { change per } 2 \text {-fold } \\
\text { increase in } \mathrm{PbB}):-0.76(-1.61,0.09)\end{array}$ \\
&
\end{tabular}


Table 2-22. Summary of Epidemiological Studies Evaluating Renal Effects at Mean Blood Lead Concentration $(\mathrm{PbB}) \leq 10 \mu \mathrm{g} / \mathrm{dL}^{\mathrm{a}}$

\section{Reference and study population ${ }^{\mathrm{b}}$}

Spector et al. $2011^{f}$

Cross-sectional study; $n=3,941$ adults

$\mathrm{PbB}(\mu \mathrm{g} / \mathrm{dL})$

Mean (all): 1.7

Mean ( $\geq 60$ years)

Tertiles (all):

- T1 (reference): $\leq 1.3$

- T2: $>1.3-2.2$

- T3: $>2.2$

Outcome evaluated ${ }^{c} \quad$ Result $^{d}$

GFR

- All participants: change in GFR ( $\mathrm{mL} / \mathrm{minute} / 1.73 \mathrm{~m}^{2}$ ) per 2-fold increase in PbB: -1.9 (-3.2, -0.7)*

- All participants: OR for reduced GFR by tertiles

o T2: $-1.6(-4.2,1.0)$

o T3: $-3.3(-5.3,-1.4)^{*}$

o p-trend: $0.001^{*}$

- Participants $\geq 60$ years: change in GFR (mL/minute/1.73 $\mathrm{m}^{2}$ ) per 2-fold increase in PbB: -4.5 (-5.6, -3.3)

Staessen et al. 1992
Cross-sectional study; $n=1,981$ adults
(965 men; 1,016 women)

Staessen et al. 2001

$$
\text { Mean men: } 11.4
$$

Mean women: 7.5

$\mathrm{CCr}$

Staessen et al. 2001

Cross-sectional study; $n=200$ 17-yearold adolescent girls

\section{Tsaih et al. 2004}

Prospective study; $\mathrm{n}=448$ (66-72 years

of age); $\mathrm{n}=26$ participants with diabetes, and $n=115$ participants with

hypertension
Mean control: 1.4

Mean exposed area 1: 1.8

Mean at baseline: 6.5

Mean at follow-up: 4.5

Mean exposed area $2: 2.7$

$\mathrm{SCr}$ Serum cystatin C

Urine $\beta_{2}$-microblobulin
Partial regression coefficient (SE) for $\mathrm{CCr}$

(mL/minute per log $\mu \mathrm{g} \mathrm{Pb/L):}$

- Men: -13.1 (4.0); $p \leq 0.001$ *

- Women: -30.1 (3.4); $p \leq 0.001^{*}$

Change in per 2-fold increase in PbB: $+3.6 \%$ $(1.5,5.7)^{*}$

Change per 2-fold increase in PbB: $+\mathbf{1 6 . 0 \%}$ $(2.7,31)^{*}$

Baseline regression $\beta$ coefficients ( $\mathrm{mg} / \mathrm{dL}$ per In $\mu \mathrm{g} / \mathrm{dL}$ ):

- All participants: 0.009 (SE 0.006)

- Participants with diabetes: 0.076 (SE 0.023); $p<0.05^{\star}$

- Participants with hypertension: 0.008 (0.010); Follow-up (4-8 years) regression $\beta$ coefficients (mg/dL per In $\mu \mathrm{g} / \mathrm{dL}$ ):

- Participants with diabetes: 0.223 (SE 0.183); Participants with hypertension: $\mathbf{0 . 3 5 2}$ (0.097); $p<0.05^{*}$ 
Table 2-22. Summary of Epidemiological Studies Evaluating Renal Effects at Mean Blood Lead Concentration $(\mathrm{PbB}) \leq 10 \mu \mathrm{g} / \mathrm{dL}^{\mathrm{a}}$

\begin{tabular}{llll}
\hline Reference and study population $^{\mathrm{b}}$ & $\mathrm{PbB}(\mu \mathrm{g} / \mathrm{dL})$ & Outcome evaluated $^{\mathrm{c}}$ & Result $^{\mathrm{d}}$ \\
\hline Yu et al. $\mathbf{2 0 0 4}$ & Mean: 4.2 & GFR & $\begin{array}{l}\text { Change in GFR (mL/minute/1.73 } \mathbf{m}^{\mathbf{2}} \text { per } \\
\mathbf{1} \boldsymbol{\mu g} / \mathrm{dL}):-\mathbf{- 4 . 0}(\mathbf{p}=\mathbf{0 . 0 1 4 8})^{*}\end{array}$
\end{tabular}

Prospective longitudinal study; $\mathrm{n}=121$ patients with chronic renal insufficiency; progression of renal insufficiency was evaluated for 48 months

a See the Supporting Document for Epidemiological Studies for Lead, Table 6 for more detailed descriptions of studies.

bParticipants had no known occupational exposure to $\mathrm{Pb}$.

${ }^{\mathrm{c} A}$ variety of methods are used to estimate GFR (Chao et al. 2015). Each has limitations for application to both clinical evaluations and epidemiology. The preferred method is to measure the clearance of substance from plasma that is known to be eliminated solely by glomerular filtration and is not reabsorbed in the renal tubule. Typically, in the clinical setting, this is accomplished with intravenous administration of GFR markers, such as ${ }^{125}$-iothalmate, for the radiocontrast agent (e.g., iohexol). These procedures are feasible in the clinical setting, but not in epidemiology studies in which invasive procedures and administration of such agents is not practical or possible. Clearance of endogenous creatinine is an alternative that has had wide use in epidemiology. However, it requires concurrent measurements of serum creatinine and the rate of urinary excretion of creatinine, which can be accurately determined only with a carefully timed urine sample that can represent the amount of glomerular filtrate formed over a given time interval. Achieving accurately timed urine samples requires a rigidly implemented and supervised collection protocol, which is not always feasible, particularly in large-scale epidemiology studies. Alternatives to clearance methods are measurement of endogenous metabolites in plasma whose clearance approximates GFR. Typically, this is achieved with endogenous creatinine or cystatin $\mathrm{C}$. The serum concentration of these two metabolites strongly correlates with GFR; however, the relationship between concentration and GFR is also affected by other variables, including age, sex, race, and creatinine muscle mass. Several approaches have been developed to improve estimates of GFR from serum creatinine that attempt to account for these co-variables. These methods rely on multiple variable regression models that relate GFR to serum creatinine and other significant determinants of GFR (Cockcroft and Gault 1976; Levey et al. 1999, 2009). An evaluation of two of the more commonly used methods for estimating GFR from serum creatinine, the CKD-EPI and MDRD equations, found that both achieved a median difference between calculated and measured GFR (from clearance measurements) that range from 2 to $6 \mathrm{~mL} /$ minute per $1.73 \mathrm{~m}^{2}$ (Levey et al. 2009). The interquartile range in the difference was approximately 18 $\mathrm{mL} /$ minute per $1.73 \mathrm{~m}^{2}$ in a validation dataset consisting of data for 3,986 study subjects. This suggests that approximately $25 \%$ of the GFR estimates from these methods are expected to be in error of the true GFR by $>18 \mathrm{~mL} /$ minute (or approximately $15 \%$ of the GFR in a healthy adult, $120 \mathrm{~mL} / \mathrm{minute}$ ).

${ }^{\mathrm{d}}$ Asterisk and bold indicate association with $\mathrm{Pb}$; unless otherwise specified, values in parenthesis are $95 \%$ Cls; $\mathrm{p}$-values $<0.05$ unless otherwise noted in the table.

eBlood lead estimated by EDTA mobilization.

fPopulation from NHANES.

$\mathrm{ACR}=$ albumin-to-creatinine ratio; $\mathrm{BUN}=$ blood urea nitrogen; $\mathrm{CCr}=$ creatinine clearance; $\mathrm{Cl}=$ confidence interval; $\mathrm{CKD}=\mathrm{chronic}$ kidney disease; CKD-EPI = Chronic Kidney Disease Epidemiology Collaboration; EDTA = ethylenediaminetetraacetic acid; GFR = glomerular filtration rate; HR = hazard ratio; MDRD = Modification of Diet in Renal Disease; NHANES = National Health and Nutrition Examination Survey; OR = odds ratio; Pb = lead; S $\beta 2 \mathrm{M}=$ serum $\beta_{2}$-microglobulin; SCr = serum creatinine concentration; SD = standard deviation; SE = standard error; UNAG = urine $N$-acetyl- $\beta$-D-glucosaminidase; UP = urine protein; UPHC = urine human complex-forming protein ( $\alpha 1$-microglobulin) 
A few studies have examined associations between low PbB and GFR in children and adolescents (de Burbure et al. 2006; Fadrowski et al. 2010; Khan et al. 2010a; Staessen et al. 2001). de Burbure et al. (2006) examined serum creatinine in a cross-sectional study of approximately 800 children (age range 8.5-12.3 years) who resided near nonferrous smelters. Serum creatinine and cystatin C decreased (indicating an increase in GFR) by approximately $7 \%$ in the upper quartile PbB group (mean $7.8 \mu \mathrm{g} / \mathrm{dL}$ ) compared to the lowest quartile $(<2.84 \mu \mathrm{g} / \mathrm{dL})$. Fadrowski et al. (2010) examined adolescents (1220 years, $n=769$ ). GFR (estimated from serum cystatin $\mathrm{C}$ ) decreased with increasing $\mathrm{PbB}$. In the upper quartile PbB group (>2.9 $\mu \mathrm{g} / \mathrm{dL}$ ), the decrease was $6.6 \mathrm{~mL} / \mathrm{minute} / 1.73 \mathrm{~m}^{2}$, which represented approximately a 6\% decrease in GFR. In a smaller study of younger children of Pb-exposed workers (ages 1-6 years; $\mathrm{n}=123 ; \mathrm{PbB}: 8.1 \mu \mathrm{g} / \mathrm{dL}$ ), serum creatinine was higher compared to controls (ages 16 years; n=123; PbB: 6.7 g/dL) (Khan et al. 2010), indicating decreased GFR. Several factors may have contributed to the different outcomes in these studies (decrease or increase in GFR), including a different age range of the study groups, different approaches to adjusting outcome metrics for confounders, and different exposures (e.g., co-exposure to Pb, cadmium, and mercury in the de Burbure et al. 2006 study).

A smaller study of adolescents (17 years of age, $n=200$ ) also found evidence for higher serum cystatin C (indicating lower GFR) in a group with a mean $\mathrm{PbB}$ of $2.7 \mu \mathrm{g} / \mathrm{dL}$ compared to a group with a mean $\mathrm{PbB}$ of $1.4 \mu \mathrm{g} / \mathrm{dL}$ (Staessen et al. 2001).

A larger number of studies have been conducted in adult populations (Table 2-22). These include several prospective studies (Harari et al. 2018; Lin et al. 2001, 2003, 2006a, 2006b; Lin-Tan et al. 2007; Pollack et al. 2015; Tsaih et al. 2004; Yu et al. 2004). Most of these studies have examined changes in GFR in patients who had ongoing renal disease and depressed GFR (Lin et al. 2001, 2003, 2006a, 2006b; Lin-Tan et al. 2007; Yu et al. 2004). In adult participants with a median baseline PbB of $2.5 \mu \mathrm{g} / \mathrm{dL}$, GFR decreased from 89 to $62 \mathrm{~mL} /$ minute after 16 years; GFR further decreased with increasing PbB (Harari et al. 2018). In addition, the risk of CKD was increased in participants with a median PbB of $4.6 \mu \mathrm{g} / \mathrm{dL}$ compared to participants with a $\mathrm{PbB}$ range of $0.15-3.30 \mu \mathrm{g} / \mathrm{dL}$. In adult patients who had indications of renal insufficiency (e.g., serum creatinine concentration >1.5 mg/dL), GFR increased following repeated chelation therapy with calcium disodium ethylenediaminetetraacetic acid (EDTA) (Lin et al. 2003, 2006b). Yu et al. (2004) estimated the decline in GFR in patients with renal insufficiency to be approximately $4 \mathrm{~mL} /$ minute/1.73 $\mathrm{m}^{2}$ per $1 \mu \mathrm{g} / \mathrm{dL}$ increase in PbB. A prospective study of premenopausal women estimated the decline in GFR to be approximately 3.73\% per doubling of PbB (Pollack et al. 2015). The median PbB in the cohort was $0.88 \mu \mathrm{g} / \mathrm{dL}$. A prospective study of older males found an association between increased serum creatinine (indicative in decreasing GFR) and PbB in subjects 
diagnosed with hypertension or diabetes. Mean PbBs were $6.5 \mu \mathrm{g} / \mathrm{dL}$ at baseline and $4.5 \mu \mathrm{g} / \mathrm{dL}$ at followup (Tsaih et al. 2004).

Several large cross-sectional studies have examined associations between $\mathrm{PbB}$ and GFR in adults (Table 2-22). Three large studies relied on data collected in the NHANES (Munter et al. 2003; Navas-Acien et al. 2009; Spector et al. 2011). The Munter et al. (2003) study, which included 4,813 hypertensive subjects and 10,938 normotensive subjects, found an association between increasing $\mathrm{PbB}$ and decreasing GFR in the hypertensive group. Navas-Acien et al. (2009) included 14,788 adult subjects and reported decreased GFR ( $<60 \mathrm{~mL} /$ minute $\left./ 1.73 \mathrm{~m}^{2}\right)$ among participants in the highest $\mathrm{PbB}$ quartile (mean $>2.4 \mu \mathrm{g} / \mathrm{dL}$ ). Spector et al. (2011) included 3,941 adults. In the age group $\geq 60$ years, the estimate for the decline in GFR was $4.5 \mathrm{~mL} /$ minute $/ 1.73 \mathrm{~m}^{2}$ per doubling of $\mathrm{PbB}$. The mean $\mathrm{PbB}$ in this group was $2.2 \mu \mathrm{g} / \mathrm{dL}$. Several smaller cross-sectional studies have also found associations between increasing $\mathrm{PbB}$ and decreasing GFR in adult populations in which mean or median PbBs were $<10 \mu \mathrm{g} / \mathrm{dL}$ (Åkesson et al. 2005; Payton et al. 1994; Staessen et al. 1992). Collectively, these studies indicate that Pb exposure is associated with decreasing GFR, and effects on GFR are evident in populations with PbB $<10 \mu \mathrm{g} / \mathrm{dL}$. People with on-going renal disease or hypertension may be more vulnerable to the effects of $\mathrm{Pb}$. Estimates of the decline in GFR associated with increasing PbB vary across studies, with some studies indicating declines of 3-6 mL/minute/1.73 $\mathrm{m}^{2}$ at $\mathrm{PbB}<10 \mu \mathrm{g} / \mathrm{dL}$ (Pollack et al. 2015; Spector et al. 2011; Yu et al. 2004). However, as noted above, the estimates may be inflated by reverse causality for associations between decreasing GFR and increasing Pb body burden.

Associations Between Bone Pb and Renal Effects. Studies evaluating associations between bone $\mathrm{Pb}$ and renal function are summarized in Table 2-23. Weaver et al. (2003a, 2005a, 2005b, 2006, 2009) conducted a series of studies evaluating associations between bone $\mathrm{Pb}$ and metrics of renal GFR (e.g., serum creatinine concentration, creatinine clearance calculated from serum creatinine concentration, BUN) and renal tubular injury (urinary NAG) in current and former Pb workers in South Korea. These studies provide evidence that tibia $\mathrm{Pb}$ is positively associated with serum creatinine concentration in older workers (Weaver et al. 2003a, 2005a, 2005b) and in male, but not female, workers (Weaver et al. 2009); and negatively associated with tibia $\mathrm{Pb}$ and creatinine clearance in male workers (Weaver et al. 2009) and in workers with vitamin D receptor (VDR) genotypes BB and Bb (Weaver et al. 2006). Tibia Pb was also positively associated with urinary NAG in older workers (Weaver et al. 2005a). Studies of participants of the longitudinal Normative Aging Study have found positive associations between tibia $\mathrm{Pb}$ and serum creatinine concentration in participants with diabetes (Tsaih et al. 2004) and with ALAD genotypes 1-2 and 2-2 (Wu et al. 2003a). One cross-sectional study did not find an association between tibia $\mathrm{Pb}$ and 
estimated GFR (Barry et al. 2019). A small case-control study did not find an association between tibia $\mathrm{Pb}$ and end-stage renal disease. Taken together, the results suggest that long-term exposure to $\mathrm{Pb}$ is associated with diminished renal function.

Table 2-23. Associations Between Bone $\mathrm{Pb}$ and Renal Function

\begin{tabular}{|c|c|c|c|c|c|c|c|c|}
\hline \multirow[b]{2}{*}{ Reference } & \multirow[b]{2}{*}{ Population } & \multicolumn{7}{|c|}{ Effect } \\
\hline & & GFR & $\mathrm{SCr}$ & $\mathrm{CCr}$ & NAG & $\mathrm{RBP}$ & BUN & ESRD \\
\hline $\begin{array}{l}\text { Barry et al. } \\
2019\end{array}$ & $\begin{array}{l}211 \text { adult } \\
\text { men }\end{array}$ & $0(\mathrm{~T})$ & - & - & - & - & - & - \\
\hline $\begin{array}{l}\text { Muntner et } \\
\text { al. } 2007\end{array}$ & $\begin{array}{l}55 \text { adult } \\
\text { ESRD } \\
\text { patients; } \\
53 \text { controls }\end{array}$ & - & - & - & - & - & - & $0 \mathrm{~T}$ \\
\hline $\begin{array}{l}\text { Tsaih et al. } \\
2004\end{array}$ & 448 men $^{\mathrm{a}}$ & - & $\begin{array}{l}0 \mathrm{~T} \\
\uparrow \mathrm{T} \text { (diabetics) } \\
0 \mathrm{P} \\
\text { O P (diabetics) }\end{array}$ & - & - & - & - & - \\
\hline $\begin{array}{l}\text { Weaver et } \\
\text { al. } 2003 a\end{array}$ & $\begin{array}{l}803 \text { adult } \mathrm{Pb} \\
\text { workers; } \\
135 \text { controls }\end{array}$ & - & $\begin{array}{l}0 \mathrm{~T} \text { (all workers) } \\
\uparrow \mathrm{T}\left(>46 \text { years }{ }^{c}\right)\end{array}$ & $0 \mathrm{~T}^{\mathrm{c}}$ & $0 \mathrm{~T}^{\mathrm{c}}$ & $0 \mathrm{~T}^{\mathrm{c}}$ & $0 \mathrm{~T}^{\mathrm{c}}$ & - \\
\hline $\begin{array}{l}\text { Weaver et } \\
\text { al. } 2005 a\end{array}$ & $\begin{array}{l}803 \text { adult } \mathrm{Pb} \\
\text { workers }^{\mathrm{b}}\end{array}$ & - & $\uparrow \mathrm{T}\left(>46\right.$ years $\left.^{\mathrm{c}}\right)$ & - & $\begin{array}{l}\uparrow \top \\
(>46 \text { years) }\end{array}$ & - & - & - \\
\hline $\begin{array}{l}\text { Weaver et } \\
\text { al. 2005b }\end{array}$ & $\begin{array}{l}795 \text { adult } \mathrm{Pb} \\
\text { workers }^{\mathrm{b}}\end{array}$ & - & $\uparrow \mathrm{T}(>40.6$ years $)$ & - & - & - & - & - \\
\hline $\begin{array}{l}\text { Weaver et } \\
\text { al. } 2006\end{array}$ & $\begin{array}{l}647 \text { adult } \mathrm{Pb} \\
\text { workers }^{\mathrm{b}}\end{array}$ & - & $\begin{array}{l}0 \mathrm{~T}\left(\mathrm{VDR}^{\mathrm{d}}\right) \\
0 \mathrm{~T}\left(\mathrm{VDR}^{\mathrm{e}}\right) \\
0 \mathrm{P}\left(\mathrm{VDR}^{\mathrm{d}}\right) \\
0 \mathrm{P}\left(\mathrm{VDR}^{\mathrm{e}}\right)\end{array}$ & $\begin{array}{l}0 \mathrm{~T} \\
\left(\mathrm{VDR}^{\mathrm{d}}\right) \\
\downarrow \mathrm{T} \\
\left(\mathrm{VDR}^{\mathrm{e}}\right) \\
0 \mathrm{P} \\
\left(\mathrm{VDR}^{\mathrm{d}}\right) \\
0 \mathrm{P} \\
\left(\mathrm{VDR}^{\mathrm{e}}\right)\end{array}$ & - & - & - & - \\
\hline $\begin{array}{l}\text { Weaver et } \\
\text { al. } 2009\end{array}$ & $\begin{array}{l}398 \text { adult } \\
\text { male and } \\
139 \text { female } \\
\text { Pb workers }{ }^{b}\end{array}$ & - & $\begin{array}{l}\uparrow \mathrm{T}(\mathrm{M}) \\
0 \mathrm{~T}(\mathrm{~F})\end{array}$ & $\begin{array}{l}\downarrow \mathrm{T}(\mathrm{M}) \\
0 \mathrm{~T}(\mathrm{~F})\end{array}$ & & - & $\begin{array}{l}0 \mathrm{~T}(\mathrm{M}) \\
\uparrow \mathrm{T}(\mathrm{F})\end{array}$ & - \\
\hline
\end{tabular}


Table 2-23. Associations Between Bone $\mathrm{Pb}$ and Renal Function

\begin{tabular}{llllllll}
\hline & \multicolumn{7}{c}{ Effect } \\
\cline { 2 - 7 } Reference Population & GFR & SCr & CCr & NAG & RBP & BUN & ESRD \\
\hline $\begin{array}{lllll}\text { Wu et al. } 709 \text { men }^{\mathrm{a}} \\
\text { 2003a }\end{array}$ & - & $\begin{array}{l}\uparrow \mathrm{T}\left(\mathrm{ALAD}^{\prime}\right) \\
\mathrm{OP}\end{array}$ & $\begin{array}{c}0 \mathrm{~T} \\
\downarrow \mathrm{P}\end{array}$ & - & - & - & - \\
\hline
\end{tabular}

aParticipants in the Normative Aging Study.

${ }^{\mathrm{b}} \mathrm{Current}$ and former $\mathrm{Pb}$ workers in South Korea.

'Data were analyzed for all study participants and by age tertiles (Tertile 1: $\leq 36$ years old; Tertile 2: 36.1-46 years old; Tertile 3: $>46$ years old). Any association observed in a specific age tertile are noted. If no association was observed for all participants and for all age tertiles, this is noted with a single entry of 0 .

dVitamin $\mathrm{D}$ receptor genotype bb.

eVitamin $\mathrm{D}$ receptor genotypes $\mathrm{BB}$ and $\mathrm{Bb}$.

fInteraction between ALAD genotype (ALAD 1-2/2-2 versus ALAD 1-1).

$\uparrow=$ positive association; $\downarrow=$ inverse association; $0=$ no association; $-=$ not reported; ALAD = aminolevulinic acid dehydratase; $\mathrm{BUN}=$ blood urea nitrogen; $\mathrm{CCr}=$ creatinine clearance; $\mathrm{ESRD}=$ end-stage renal disease; $\mathrm{F}=$ female; $\mathrm{M}=$ male; $\mathrm{NAG}=\mathrm{N}$-acetyl-D-glucosaminidase; $\mathrm{P}=$ patella; $\mathrm{Pb}=$ lead $; \mathrm{RBP}=$ retinol binding protein; $\mathrm{SCr}=$ serum creatinine concentration; $\mathrm{T}=$ tibia; $\mathrm{VDR}=$ vitamin $\mathrm{D}$ receptor

Mechanisms of Action. Several mechanisms have been established or proposed as mechanisms for kidney damage associated with exposure to $\mathrm{Pb}$, including general mechanisms of Pb-induced toxicity (reviewed in Section 2.21). Mechanisms of renal damage associated with $\mathrm{Pb}$ exposure were recently reviewed in detail by EPA (2014c), including oxidative stress, inflammation, apoptosis of glomerular and tubular cells, alterations in renal gangliosides (plasma membrane lipids that play a role in the control of GFR), changes in renal vascular tone, and alterations in the renin-angiotensin-aldosterone system. As discussed in $\mathrm{Pb}$ Section 3.1.2 (Toxicokinetics, Distribution), $\mathrm{Pb}$ is distributed to the kidney, providing a toxicokinetic mechanism for direct effects to the kidney.

\subsection{DERMAL}

No epidemiological studies evaluating adverse dermal effects of chronic exposure to $\mathrm{Pb}$ were identified.

\subsection{OCULAR}

Few epidemiological studies have evaluated non-neurological ocular effects of Pb exposure, with studies examining associations with macular degeneration (Erie et al. 2009; Park et al. 2015) and cataract development (Schaumberg et al. 2004). In a cross-sectional study of 3,865 participants with a mean $\mathrm{PbB}$ of $2.69 \mu \mathrm{g} / \mathrm{dL}$ participating in the Korea National Health and Nutrition Examination study (2008-2011), 
the risks of age-related early (adjusted OR 1.12; 95\% CI 1.02, 1.23; p=0.009) and late (adjusted OR 1.25; 95\% CI 1.05, 1.50; p=0.015) macular degeneration were increased (Park et al. 2015). A cross-sectional study of human donor eyes with $(n=25)$ and without $(n=36)$ age-related macular degeneration found no association between $\mathrm{Pb}$ concentration in the retinal pigment epithelium-choroid complex and subjects with age-related macular degeneration and normal subjects (Erie et al. 2009). A prospective study of 642 men participating in the Normative Aging Study found no association between PbB (range: 1.0$35.0 \mu \mathrm{g} / \mathrm{dL}$ ) and risk of cataracts, although the risk of cataracts was increased in association with tibia $\mathrm{Pb}$ levels (Schaumberg et al. 2004). A prospective cohort study of 634 male participants of the Normative Aging Study found an association between patellar bone $\mathrm{Pb}$ concentration and incidence of primary openangle glaucoma, with an HR of 5.06 (95\% CI 1.61, 15.88; p=0:005) (Wang et al. 2018).

\subsection{ENDOCRINE}

Effects of chronic exposure to $\mathrm{Pb}$ on reproductive hormones are reviewed in Section 2.17 (Reproductive).

Overview. Effects on endocrine systems have been evaluated in several epidemiological studies in adults (general populations and workers), adolescents, and children. Investigations have focused on effects on thyroid function, cortisol levels, vitamin D levels, serum levels of other growth factors, and diabetes. Associations between $\mathrm{PbB}$ and thyroid function, assessed by measurement of serum thyroid hormone levels, is the most investigated endocrine outcome, although results do not demonstrate a consistent pattern of effect or dose-response relationships. Other endocrine endpoints have been evaluated in only a few studies.

The following endocrine effects have been associated with PbB:

- $\leq 10 \mu \mathrm{g} / \mathrm{dL}:$

o Altered serum levels of thyroid hormones (thyroxine [T4], triiodothyronine [T3], thyroidstimulating hormone [TSH]); evaluated in multiple studies. Few effects were observed and results do not demonstrate consistent patterns of effects or exposure-response relationships.

o Altered salivary cortisol awakening response in pregnant women.

o Increased stress-induced salivary cortisol response in children.

o Decreased serum levels of insulin-like growth factor-1 (IGF-1) in children. 
- $\quad>10 \mu \mathrm{g} / \mathrm{dL}$ :

o Altered serum levels of thyroid hormones (T4, T3, TSH); evaluated in a few studies; results do not demonstrate consistent patterns of effects or exposure-response relationships.

o Increased thyroid peroxidase antibodies.

o Decreased serum levels of vitamin D; evaluated in a few studies in children with consistent results.

Measures of Exposure. Studies evaluating the association between endocrine effects and $\mathrm{Pb}$ exposure evaluate exposure by measurement of PbB.

Confounding Factors and Effect Modifiers. Results of epidemiological studies on endocrine effects have not been consistent. In general, statistical analyses were not rigorous and potential confounding factors and effect modifiers were not fully considered. Exposure to other metals and other chemical with endocrine effects is an important confounding factor to consider when interpreting study results. Although a few studies were of large populations (e.g., NHANES participants); most studies examined relatively small populations and used cross-sectional designs.

Characterization of Effects. General trends for studies showing a relationship between $\mathrm{PbB}$ and endocrine effects are shown in Table 2-24. Several studies have evaluated associations between $\mathrm{PbB}$ and effects on serum levels of thyroid hormones (T4, T3, and TSH) at mean $\mathrm{PbB}$ ranging from $<1$ to $71 \mu \mathrm{g} / \mathrm{dL}$; an overview of study results is presented in Table 2-25. Based on evaluation of thyroid hormones, it is unclear if $\mathrm{PbB}$ is associated with altered thyroid function. At $\mathrm{PbB} \leq 10 \mu \mathrm{g} / \mathrm{dL}$, results of epidemiological studies, including cross-sectional studies of large NHANES populations, show associations between $\mathrm{PbB}$ and some alterations in serum levels of thyroid hormones; however, results do not demonstrate apparent patterns or exposure response relationships (see discussion below on Effect at Blood $\mathrm{Pb}$ Levels $\leq 10 \mu \mathrm{g} / \mathrm{dL}$ ). Increased thyroid peroxidase (TPO) antibodies were observed at $\mathrm{PbB}$ $\leq 10 \mu \mathrm{g} / \mathrm{dL}$, although TSH was not increased. Epidemiological studies at $\mathrm{PbB}>10 \mu \mathrm{g} / \mathrm{dL}$, conducted in smaller populations ( $\mathrm{n}=25-309$ ), show more effects on thyroid hormones than observed at PbB $\leq 10 \mu \mathrm{g} / \mathrm{dL}$. However, similar to studies at lower PbB, results are inconsistent. Kahn et al. (2014) found decreased T4 ( $<<0.0001)$ and increased TPO antibodies ( $\mathrm{p}=0.0002)$ during the second trimester of pregnancy in women ( $\mathrm{n}=144)$ with mean PbB $20.00 \mu \mathrm{g} / \mathrm{dL}$ compared to women ( $\mathrm{n}=147)$ with PbB of $5.57 \mu \mathrm{g} / \mathrm{dL}$; no increase in TSH was observed. The adjusted OR (95\% CI) for testing positive for TPO antibodies was 2.41 (1.563, 3.82). Results indicate that autoimmunity is a potential mechanism for altered thyroid function. This finding has not been corroborated in other studies. 


\begin{tabular}{|c|c|c|}
\hline $\begin{array}{l}\text { Mean PbB } \\
(\mu \mathrm{g} / \mathrm{dL})\end{array}$ & $\begin{array}{l}\text { Effects associated with } \mathrm{Pb} \\
\text { exposure }\end{array}$ & References \\
\hline \multirow[t]{3}{*}{$\leq 10$} & $\begin{array}{l}\text { Altered levels of thyroid hormones } \\
\text { and increased TPO antibodies }\end{array}$ & $\begin{array}{l}\text { Abdelouahab et al. 2008; Dundar et al. 2006; Luo and } \\
\text { Hendryx 2014; Mendy et al. 2013; Nie et al. 2017; } \\
\text { Yorita Christensen } 2013\end{array}$ \\
\hline & Altered salivary cortisol levels & Braun et al. 2014; Gump et al. 2008 \\
\hline & Decreased serum IGF-1 & Fleisch et al. 2013 \\
\hline$>10-30$ & $\begin{array}{l}\text { Altered levels of thyroid hormones } \\
\text { and increased TPO antibodies }\end{array}$ & $\begin{array}{l}\text { Gustafson et al. 1989; Kahn et al. 2014; Lamb et al. } \\
\text { 2008; Lopez et al. } 2000\end{array}$ \\
\hline$>30-50$ & Decreased serum vitamin D level & $\begin{array}{l}\text { Luo and Hendryx 2014; Mahaffey et al. 1982; Rosen et } \\
\text { al. } 1980\end{array}$ \\
\hline \multirow[t]{2}{*}{$>50$} & Altered levels of thyroid hormones ${ }^{a}$ & $\begin{array}{l}\text { Lopez et al. 2000; Pekcici et al. 2010; Robins et al. } \\
\text { 1983; Singh et al. 2000; Tuppurainen et al. } 1988\end{array}$ \\
\hline & Decreased serum vitamin D level & Rosen et al. 1980 \\
\hline
\end{tabular}

aThyroid hormones: T4, T3, and/or TSH.

IGF-1 = insulin-like growth factor-1; $\mathrm{PbB}=$ blood lead concentration; $\mathrm{T} 3$ = triiodothyronine; $\mathrm{T} 4$ = thyroxine; $\mathrm{TSH}=$ thyroid-stimulating hormone; TPO = thyroid peroxidase

\section{Table 2-25. Effects on Thyroid Hormones Associated with Blood Lead Concentration (PbB)}

\begin{tabular}{|c|c|c|c|c|c|c|c|}
\hline \multirow{2}{*}{$\begin{array}{l}\text { Mean PbB } \\
(\mu \mathrm{g} / \mathrm{dL})\end{array}$} & \multirow{2}{*}{$\begin{array}{l}\text { Number of } \\
\text { participants }\end{array}$} & \multicolumn{2}{|c|}{$\mathrm{T} 4$} & \multicolumn{2}{|c|}{ T3 } & \multirow[b]{2}{*}{$\mathrm{TSH}$} & \multirow[b]{2}{*}{ Reference } \\
\hline & & Total & Free & Total & Free & & \\
\hline \multicolumn{8}{|c|}{$\mathrm{PbB} \leq 10 \mu \mathrm{g} / \mathrm{dL}$} \\
\hline 0.93 & 1,109 adolescents $^{a}$ & 0 & 0 & 0 & 0 & 0 & Chen et al. 2013 \\
\hline 1.3 & 1,587 adults $^{\mathrm{a}}$ & $\downarrow$ & 0 & 0 & 0 & 0 & Yorita Christensen 2013 \\
\hline 1.52 & 4,652 adults $^{a}$ & $\downarrow$ & 0 & 0 & 0 & 0 & Mendy et al. 2013 \\
\hline 1.74 & 87 women & 0 & - & 0 & - & $\downarrow$ & Abdelouahab et al. 2008 \\
\hline 1.75 & 4,409 adults $^{a}$ & 0 & 0 & 0 & 0 & 0 & Chen et al. 2013 \\
\hline 1.82 & 6,231 adults $^{a}$ & - & 0 & - & $\uparrow$ & 0 & Luo and Hendryx 2014 \\
\hline 3.5 & 3,350 women & - & - & - & - & 0 & Nie et al. 2017 \\
\hline 4.1 & 2,278 men & - & - & - & - & 0 & Nie et al. 2017 \\
\hline $6.3^{b}$ & 24 infants $^{b}$ & - & 0 & - & - & 0 & lijama et al. 2007 \\
\hline 7.3 & 42 adolescents & - & $\downarrow$ & - & 0 & 0 & Dundar et al. 2006 \\
\hline \multicolumn{8}{|c|}{$\mathrm{PbB}>10 \mu \mathrm{g} / \mathrm{dL}$} \\
\hline 20.00 & 291 adults & - & $\downarrow$ & - & - & 0 & Kahn et al. 2014 \\
\hline 20.56 & 309 pregnancyc $^{c}$ & - & $\downarrow$ & - & - & - & Lamb et al. 2008 \\
\hline 24.1 & 151 adults & 0 & 0 & - & - & 0 & Schumacher et al. 1998 \\
\hline 25 & 68 children & 0 & 0 & - & - & - & Siegel et al. 1989 \\
\hline
\end{tabular}




\section{Table 2-25. Effects on Thyroid Hormones Associated with Blood Lead Concentration (PbB)}

\begin{tabular}{|c|c|c|c|c|c|c|c|}
\hline \multirow{2}{*}{$\begin{array}{l}\text { Mean PbB } \\
(\mu \mathrm{g} / \mathrm{dL})\end{array}$} & \multirow{2}{*}{$\begin{array}{l}\text { Number of } \\
\text { participants }\end{array}$} & \multicolumn{2}{|c|}{$\mathrm{T} 4$} & \multicolumn{2}{|c|}{ T3 } & \multirow[b]{2}{*}{$\mathrm{TSH}$} & \multirow[b]{2}{*}{ Reference } \\
\hline & & Total & Free & Total & Free & & \\
\hline 31 & 77 adults & - & 0 & - & 0 & 0 & Erfurth et al. 2001 \\
\hline$<33.19^{c}$ & 6,231 adults $^{a}$ & 0 & - & - & $\uparrow$ & 0 & Luo and Hendryx 2014 \\
\hline 39.5 & 25 adults & $\uparrow$ & - & - & - & $\uparrow$ & Gustafson et al. 1989 \\
\hline 50.9 & 75 adults & $\uparrow$ & $\downarrow$ & 0 & - & 0 & Lopez et al. 2000 \\
\hline 51.9 & 47 adults & $\downarrow$ & $\downarrow$ & - & - & 0 & Robins et al. 1983 \\
\hline 51.9 & 58 adults & 0 & - & $\downarrow$ & - & $\uparrow$ & Singh et al. 2000 \\
\hline 56.1 & 176 adults & $\downarrow$ & $\downarrow$ & 0 & - & 0 & Tuppurainen et al. 1988 \\
\hline 71.1 & 65 adults & - & $\uparrow$ & - & $\uparrow$ & $\uparrow$ & Pekcici et al. 2010 \\
\hline
\end{tabular}

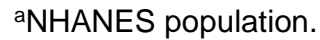

bUmbilical cord $\mathrm{PbB}$; assessments in infants.

cMean not reported.

$\uparrow=$ Increased; $\downarrow=$ decreased; $0=$ no change; $-=$ not assessed; NHANES $=$ National Health and Nutrition Examination Survey; T3 = triiodothyronine; T4 = thyroxine; $\mathrm{TSH}=$ thyroid-stimulating hormone

Studies also have investigated alterations in serum levels of vitamin D at PbB $>30 \mu \mathrm{g} / \mathrm{dL}$ (Mahaffey et al. 1982). In children and adolescents, serum levels of 1,25-dihydroxycholecalciferol were negatively associated with $\mathrm{PbB}$ over a range of 30-120 $\mu \mathrm{g} / \mathrm{dL}$ (Mahaffey et al. 1982). Similar results were observed for vitamin D in children with $\mathrm{PbB}>50 \mu \mathrm{g} / \mathrm{dL}$ (Rosen et al. 1980). However, in children with $\mathrm{PbB}$ $<10 \mu \mathrm{g} / \mathrm{dL}$, no associations between PbB and vitamin D levels were observed (Kemp et al. 2007) (see discussion below on Effect at Blood $\mathrm{Pb}$ Levels $\leq 10 \mu \mathrm{g} / \mathrm{dL}$ ). Studies investigating associations between $\mathrm{PbB}$ and other endocrine outcomes (salivary cortisol levels, serum levels of growth factors and diabetes) were conducted in populations with $\mathrm{PbB} \leq 10 \mu \mathrm{g} / \mathrm{dL}$ (see discussion below on Effect at Blood Pb Levels $\leq 10 \mu \mathrm{g} / \mathrm{dL})$.

Effect at Blood Pb Levels $\leq \mathbf{1 0} \boldsymbol{\mu g} / \mathbf{d L}$. Epidemiological studies of endocrine effects associated with PbB $\leq 10 \mu \mathrm{g} / \mathrm{dL}$ have examined thyroid function, as assessed by serum levels of thyroid hormones (Abdelouahab et al. 2008; Chen et al. 2013; Dundar et al. 2006; Iijama et al. 2007; Luo and Hendryx 2014; Mendy et al. 2013; Yorita Christensen 2013), cortisol levels and cortisol responses to stress (Braun et al. 2014; Gump et al. 2008), vitamin D levels (Kemp et al. 2007), IGF-1 levels (Fleisch et al. 2013), and diabetes (Moon 2013); study details are summarized in Supporting Document for Epidemiological Studies for Lead, Table 7. Studies examining thyroid function, including several large cross-sectional studies of NHANES populations (Chen et al. 2013; Mendy et al. 2013; Luo and Hendryx 2014; Yorita Christensen 2013), report inconsistent results; see Table 2-25. Results of NHANES studies at low PbB 
(range of means: 0.93-1.82 $\mu \mathrm{g} / \mathrm{dL}$ ) are mixed, showing decreased total T4 and no change for free T4 (Mendy et al. 2013; Yorita Christensen 2013), and no change for total or free T4 (Chen et al. 2013; Luo and Hendryx 2014). The NHANES studies did not show associations between PbB and T3 or TSH levels, except for an increase in FT3 (Luo and Hendryx 2014). In smaller studies, decreased TSH and increased free T4 were observed at $\mathrm{PbB}$ of 3.10 and $7.3 \mu \mathrm{g} / \mathrm{dL}$, respectively (Abdelouahab et al. 2008; Dundar et al. 2006). In a large, cross-sectional study, increased TPO antibodies were observed in women with PbBs $>2.9 \mu \mathrm{g} / \mathrm{dL}$, with a significant positive trend $(\mathrm{p}=0.008)$ for increased TSH; in men, there was no association (Nie et al. 2017). Thus, few effects on measures of thyroid function have been observed at $\mathrm{PbB} \leq 10 \mu \mathrm{g} / \mathrm{dL}$, and results do not demonstrate consistent patterns of effects or exposure-response relationships. Results of studies examining other endocrine effects associated with $\mathrm{PbB}$ have not been corroborated. Study findings include: associations between $\mathrm{PbB}$ and decreased cortisol awakening response during pregnancy at $\mathrm{PbB} \geq 5.1 \mu \mathrm{g} / \mathrm{dL}$ (Braun et al. 2014); enhanced salivary cortisol response to cold stress in children at PbB 1.1-6.2 $\mu \mathrm{g} / \mathrm{dL}$ (Gump et al. 2008); no association between PbB and basal cortisol levels or cortisol levels under stress (Ngueta et al. 2018); no association between PbB and serum vitamin D in children at PbB means 4.94-6.54 $\mu \mathrm{g} / \mathrm{dL}$ (Kemp et al. 2007); decreased serum IGF-1 in children at PbB 5-9 $\mu \mathrm{g} / \mathrm{dL}$ (Fleisch et al. 2013); and no association between PbB and diabetes in children at mean PbB $4.08 \mu \mathrm{g} / \mathrm{dL}$ (Moon 2013).

Mechanisms of Action. Adverse effects on the endocrine system (non-reproductive effects) associated with chronic $\mathrm{Pb}$ exposure have not been established; therefore, mechanisms of toxicity have not been identified. Thyroid function could be decreased through stimulation of autoimmunity to the thyroid gland, as shown by increased thyroid peroxidase antibodies (Kahn et al. 2014). In addition, general mechanisms of toxicity (reviewed in Section 2.21) of Pb would likely be involved in any endocrine toxicity.

\subsection{IMMUNOLOGICAL}

Overview. This section of the profile summarizes the immunological effects of $\mathrm{Pb}$, exclusive of asthma, which is summarized in Section 2.5. Studies conducted in animal models have shown that $\mathrm{Pb}$ can perturb the humoral and cell-mediated immune systems, leading to decreased resistance to disease, sensitization, autoimmunity, and inflammation (EPA 2014c). These studies support epidemiological evidence of associations between $\mathrm{Pb}$ exposures (as indexed to $\mathrm{PbB}$ ) and changes in biomarkers of humoral and cellmediated immunity. 
The following immunological effects have been associated with PbB:

- $\quad \leq 10 \mu \mathrm{g} / \mathrm{dL}$ :

o Increases in susceptibility to infections.

o Sensitization to allergens.

o Changes in indicators of humoral immunity (immunoglobulins, B-cells); demonstrated in several studies.

o Changes in indicators of cell-mediated immunity (T-cells, eosinophils, neutrophils); demonstrated in several studies.

o Changes in indicators of inflammatory response (circulating inflammation cytokines).

- $\quad>10 \mu \mathrm{g} / \mathrm{dL}$ :

o Changes in indicators of humoral immunity (immunoglobulins, B-cells).

o Changes in indicators of cell-mediated immunity (T-cells, natural killer [NK]-cells, neutrophils).

o Changes in indicators of inflammatory response (inflammatory response of activated monocytes).

o Decreases in circulating complement.

Measures of Exposure. Studies of associations between $\mathrm{Pb}$ exposure and immunological outcomes have relied on $\mathrm{PbB}$ as a biomarker of exposure. Most studies have been cross-sectional in design, which increases uncertainty in the interpretation of the results since the exposure history of the subjects is not necessarily indicated by the cross-sectional PbB measurement.

Confounding Factors and Effect Modifiers. The immune system is responsive to a multitude of environmental and physiological factors, which can be confounding factors or effect modifiers in studies of associations between $\mathrm{Pb}$ exposure and immunological outcomes. Factors that have been considered in some studies, but not consistently across studies, include age, sex, smoking, physical activity, allergen exposures, history of inflammatory disease, SES factors, recreational activities, and co-exposures to other chemicals. Immunological outcomes observed in epidemiological studies may also be secondary to other systemic effects of $\mathrm{Pb}$ (e.g., hematological, splenic gene expression) that affect the immune system.

Characterization of Effects. Table 2-26 lists epidemiological studies that have found associations between $\mathrm{PbB}$ and immunological outcomes, grouped by population $\mathrm{PbB}$ (typically mean or geometric 
mean). Several studies have found alterations in immunological endpoints in association with PbB over the range $<10->50 \mu \mathrm{g} / \mathrm{dL}$.

\section{Table 2-26. Overview of Immunological Effects Associated with Chronic Exposure to Lead (Pb)}

\begin{tabular}{|c|c|c|}
\hline $\begin{array}{l}\text { Mean PbB } \\
(\mu \mathrm{g} / \mathrm{dL})\end{array}$ & $\begin{array}{l}\text { Effects associated with } \mathrm{Pb} \\
\text { exposure }\end{array}$ & References \\
\hline \multirow[t]{5}{*}{$\leq 10$} & $\begin{array}{l}\text { Increased susceptibility to } \\
\text { infections }\end{array}$ & Krueger and Wade 2016; Park et al. 2019 \\
\hline & Sensitization to allergens & Jedrychowski et al. 2011; Pizent et al. 2008 \\
\hline & $\begin{array}{l}\text { Changes in indicators of } \\
\text { humoral immunity }\end{array}$ & $\begin{array}{l}\text { Hon et al. 2009, 2010; Karmaus et al. 2005; Min and Min } \\
\text { 2015; Pizent et al. 2008; Sarasua et al. 2000; Wells et al. } \\
\text { 2014; Xu et al. } 2015\end{array}$ \\
\hline & $\begin{array}{l}\text { Changes in indicators of cell- } \\
\text { mediated immunity }\end{array}$ & $\begin{array}{l}\text { Boscolo et al. 2000; Conterato et al. 2013; Hsiao et al. } \\
\text { 2011; Karmaus et al. 2005; Sarasua et al. 2000; Wells et } \\
\text { al. } 2014\end{array}$ \\
\hline & $\begin{array}{l}\text { Changes in indicators of } \\
\text { inflammatory response }\end{array}$ & Kim et al. 2007, Sirivarasai et al. 2013; Songdej et al. 2010 \\
\hline \multirow[t]{3}{*}{$>10-30$} & $\begin{array}{l}\text { Changes in indicators of } \\
\text { humoral immunity }\end{array}$ & $\begin{array}{l}\text { Heo et al. 2004, Lutz et al. 1999; Sun et al. 2003; Wang et } \\
\text { al. } 2017 \text { a }\end{array}$ \\
\hline & $\begin{array}{l}\text { Changes in indicators of cell- } \\
\text { mediated immunity }\end{array}$ & $\begin{array}{l}\text { Alomran and Shleamoon 1988; Bergeret et al. 1990; } \\
\text { Boscolo et al. 1999; Di Lorenzo et al. 2006; Fischbein et al. } \\
\text { 1993; Kimber et al. 1986; Mishra et al. 2003; Queiroz et al. } \\
\text { 1993, 1994; Sata et al. 1998; Valentino et al. 1991, 2007; } \\
\text { Zhao et al. } 2004\end{array}$ \\
\hline & $\begin{array}{l}\text { Changes in indicators of } \\
\text { inflammatory response }\end{array}$ & Valentino et al. 2007 \\
\hline \multirow[t]{2}{*}{$>30-50$} & $\begin{array}{l}\text { Changes in indicators of } \\
\text { humoral immunitya }\end{array}$ & Ewers et al. 1982; Heo et al. 2004; Pinkerton et al. 1998 \\
\hline & $\begin{array}{l}\text { Changes in indicators of cell- } \\
\text { mediated immunity }\end{array}$ & $\begin{array}{l}\text { Conterato et al. 2013; Fischbein et al. 1993; Garcia-Leston } \\
\text { et al. 2012; Niu et al. 2015; Pinkerton et al. } 1998\end{array}$ \\
\hline \multirow[t]{3}{*}{$>50$} & $\begin{array}{l}\text { Changes in indicators of } \\
\text { humoral immunity }\end{array}$ & Basaran and Undeger 2000 \\
\hline & $\begin{array}{l}\text { Changes in indicators of cell- } \\
\text { mediated immunity }\end{array}$ & $\begin{array}{l}\text { Basaran and Undeger 2000; Mishra et al. 2010; Undeger } \\
\text { et al. } 1996\end{array}$ \\
\hline & $\begin{array}{l}\text { Decreases in circulating } \\
\text { complement levels }\end{array}$ & Ewers et al. 1982; Undeger et al. 1996 \\
\hline
\end{tabular}

aImmunoglobulins, B-cells.

bT-cells, natural killer (NK) cells, eosinophils, neutrophils and related receptors and cytokines.

cCirculating cytokines (e.g., C-reactive protein [CRP], interleukin-6 [IL-6], tumor necrosis factor-alpha [TNFa]).

Humoral immunity. Numerous epidemiological studies have examined associations between $\mathrm{Pb}$ exposure and circulating levels of immunoglobulins. These studies provide evidence that exposure to $\mathrm{Pb}$ is associated with increases in circulating IgE in children (Hon et al. 2009, 2010; Karmaus et al. 2005; Lutz 
et al. 1999; Sun et al. 2003; Wang et al. 2017a) and in adults (Heo et al. 2004; Sarasua et al. 2000). IgE is an important mediator of hypersensitivity reactions and inflammation and Pb-induced perturbations in IgE may contribute to associations between $\mathrm{Pb}$ exposure and sensitization and inflammation. Although some studies have found changes in levels of other immunoglobins, the evidence for these effects is not as strong as for IgE (Alomran and Shleamoon 1988; Anetor and Adeniyi 1998; Ewers et al. 1982; Kimber et al. 1986; Pinkerton et al. 1998; Queiroz et al. 1994b; Ündeger et al. 1996). The association between circulating IgE levels and PbB appears to extend to PbB levels $<10 \mu \mathrm{g} / \mathrm{dL}$ (Karmaus et al. 2005; Min and Min 2015; Pizent et al. 2008; Sarasua et al. 2000; Wells et al. 2014).

T-cells. T-cells are important mediators of immunity to self-cells (e.g., cancer cells and cells infected with virus) and for activation of B-cells and humoral immunity. Epidemiological studies provide evidence that exposure to $\mathrm{Pb}$ is associated with decreases in T-cell abundance in children (Karmaus et al. 2005; Lutz et al. 1999; Sarasua et al. 2000; Zhao et al. 2004) and increases in abundance in adults (Boscolo et al. 1999, 2000; Sarasua et al. 2000). Several studies in adults found no consistent effect on T-cell abundance (Fischbein et al. 1993; Mishra et al. 2010; Pinkerton et al. 1998; Ündeger et al. 1996; Yücesoy et al. 1997b). Most of the studies on T-cell abundance did not differentiate specific classes of T-cell population affected; however, evidence is stronger for effects on CD3+ cells (Karmaus et al. 2005; Lutz et al. 1999; Sarasua et al. 2000; Zhao et al. 2004), with some studies finding effects on abundances of CD4+ (T helper) or CD8+ (T cytotoxic) cells (Boscolo et al. 1999, 2000; Karmaus et al. 2005; Sarasua et al. 2000). The association between circulating T-cell abundance and PbB appears to extend to PbB levels $\leq 10 \mu \mathrm{g} / \mathrm{dL}$ (Boscolo et al. 2000; Karmaus et al. 2005; Sarasua et al. 2000).

Neutrophils. Neutrophils are phagocytic cells that function in the immune defense against bacterial infections. Epidemiological studies have found associations between $\mathrm{Pb}$ exposure and neutrophil function. The effects on cultured human PMNs in populations that had mean $\mathrm{PbB}>10 \mu \mathrm{g} / \mathrm{dL}$ includes suppression of chemotaxis, phagocytosis, respiratory oxidative burst, and antigen killing (Alomran and Shleamon 1988; Bergeret et al. 1990; Fischbein et al. 1993; Kimber et al. 1986; Queiroz et al. 1993, 1994; Valentino et al. 1991). In a worker population having mean $\mathrm{PbB} \leq 10 \mu \mathrm{g} / \mathrm{dL}$, increasing $\mathrm{PbB}$ was associated with decreases in circulating neutrophil abundance (Conterato et al. 2013), whereas in a worker population having mean $\mathrm{PbB}>10 \mu \mathrm{g} / \mathrm{dL}, \mathrm{PbB}$ was associated with increases in neutrophil abundance (Di Lorenzo et al. 2006) and decreases in circulating complement levels (Ewers et al. 1982; Undeger et al. 1996). 
NK cells. NK cells contribute to the immune defense (cytotoxicity) against tumor cells and viral infected cells. Although a few studies have found associations between $\mathrm{PbB}$ and NK cell abundance (Boscolo et al. 1999, 2000), most studies have found no associations (Fischbein et al. 1993; Garcia-Leston et al. 2011; Karmaus et al. 2005; Kimber et al. 1986; Mishra et al. 2003; Pinkerton et al. 1998; Sarasua et al. 2000; Undeger et al. 1996; Yucesoy et al. 1997) at population mean $\mathrm{PbBs} \leq 10$ or $>10 \mu \mathrm{g} / \mathrm{dL}$.

Lymphocyte activation. A few epidemiological studies have found associations between exposure to $\mathrm{Pb}$ and increased lymphocyte activation (HLA-DR expression) and proliferation in children (Lutz et al. 1999) and adults (Alomran and Shleamon 1988; Boscolo et al. 1999; Cohen et al. 1989; Fischbein et al. 1993; Kimber et al. 1986; Mishra et al. 2003). These studies found effects in populations that had PbB $>10 \mu \mathrm{g} / \mathrm{dL}$.

Sensitization. Epidemiological studies provided evidence for associations between exposure to $\mathrm{Pb}$ and sensitization. This evidence includes increased risk of atopy to airborne allergens in children (Jedrychowski et al. 2011) and adults (Pizent et al. 2008). Consistent with findings in animal studies which found that Pb exposure suppresses delayed type hypersensitivity (DTH), Hsiao et al. (2011) found that higher $\mathrm{PbB}$ was associated with decreases in circulating levels of IFN- $\gamma$ ya T-helper cytokine known to be important in DTH). The above effects related to sensitization have been observed in populations that had mean $\mathrm{PbB} \leq 10 \mu \mathrm{g} / \mathrm{dL}$.

Inflammation. A few epidemiological studies have examined possible associations between $\mathrm{Pb}$ exposure and biomarkers of inflammation. Results for these studies suggest that Pb exposure can modify the control of inflammatory responses, including modifying macrophage NO release and ROS production in macrophages harvested from exposed children (Pineda-Zavaleta et al. 2004), and in adults, decreases in abundance of circulating monocytes (Conterato et al. 2013; Pinkerton et al. 1998), and lower circulating levels of HLA-DR+ (Fischbein et al. 1993) in adults. Three studies found evidence for effects indicative of enhancement or stimulation of inflammation in adults at mean $\mathrm{PbB} \leq 10 \mu \mathrm{g} / \mathrm{dL}$. Outcomes included increases in circulating tumor necrosis factor-alpha (TNF $\alpha)(\mathrm{Kim}$ et al. 2007) and C-reactive protein (CRP) in men (Songdej et al. 2010; Sirivarasai et al. 2013).

Effect at Blood Pb Levels $\leq 10 \mu \boldsymbol{g} / \mathbf{d L}$. Epidemiological studies that have evaluated immunological effects associated with $\mathrm{PbB} \leq 10 \mu \mathrm{g} / \mathrm{dL}$ are summarized in Table 2-27, with additional details provided in the Supporting Document for Epidemiological Studies for Lead, Table 8. Outcomes that have been observed in populations with $\mathrm{PbB} \leq 10 \mu \mathrm{g} / \mathrm{dL}$ include susceptibility to infections, sensitization in children and 
adults, humoral and cell-mediated immunity in children and adults, and inflammation in children and adults.

Susceptibility to infections. A cross-sectional study of data from NHANES (1999-2012) found a trend for increasing OR for being seropositive for H. pylori, T. gondii, and Hepatitis B virus in a population that has a geometric mean PbB of $1.5 \mu \mathrm{g} / \mathrm{dL}$ (Krueger and Wade 2016).

Humoral immunity. Several studies have found associations between circulating IgE levels and $\mathrm{PbB}$ in populations with mean or geometric mean $\mathrm{PbB}$ levels $\leq 10 \mu \mathrm{g} / \mathrm{dL}$ (Karmaus et al. 2005; Min and Min 2015; Pizent et al. 2008; Sarasua et al. 2000; Wells et al. 2014). In general, these studies found increases in serum IgE levels in association with increasing PbB in children (Karmaus et al. 2005; Sarasua et al. 2000; Wang et al. 2017a; Wells et al. 2014) and adults (Min and Min 2015; Pizent et al. 2008). A crosssectional study of children (3-7 years of age) found an association between increasing $\mathrm{PbB}$ and decreasing Hepatitis B virus antibody titers (Xu et al. 2015).

T-cells, neutrophils, and NK cells. Several studies have found associations between T-cell abundance and $\mathrm{PbB}$ in populations with mean or geometric mean $\mathrm{PbB}$ levels $\leq 10 \mu \mathrm{g} / \mathrm{dL}$. In studies of children, T-cell abundances decreased (Karmaus et al. 2005), whereas in a study of adults, T-cell abundance increased (Boscolo et al. 2000). In a study of Pb workers, neutrophil abundance was lower in Pb workers compared to controls (Contertato et al. 2013). The worker populations included a group of painters in which the mean $\mathrm{PbB}$ was $5.4 \pm 0.4$ (SE) $\mu \mathrm{g} / \mathrm{dL}$, compared to the control group (1.5 $\pm 0.1, \mathrm{SE}$ ). A study of a population of atopic adult women with median $\mathrm{PbB} 6.6 \mu \mathrm{g} / \mathrm{dL}$ (25 $-75^{\text {th }}$ percentile range: 4.9-7.9), found an association between increasing PbB and increasing abundance of NK cells (CD4+CD45RO+; Boscolo et al. 2000).

Sensitization. Exposures to $\mathrm{Pb}$ that resulted in population geometric mean $\mathrm{PbB} \leq 10 \mu \mathrm{g} / \mathrm{dL}$ was associated with increased risk of atopy to airborne allergens in children (Jedrychowski et al. 2011) and adults (Pizent et al. 2008). Higher PbB was associated with decreases in circulating levels of IFN- $\gamma$ (a T-helper cytokine known to be important in DTH) in a population of children with a mean PbB of 8.8 \pm 0.45 (SD) $\mu \mathrm{g} / \mathrm{dL}$ (Hsaio et al. 2011). 
2. HEALTH EFFECTS

Table 2-27. Summary of Epidemiological Studies Evaluating Immunological Effects at Mean Blood Lead Concentration $(\mathrm{PbB}) \leq 10 \mu \mathrm{g} / \mathrm{dL}^{\mathrm{a}}$

\begin{tabular}{|c|c|c|c|}
\hline Reference and study population & $\mathrm{PbB}(\mu \mathrm{g} / \mathrm{dL})$ & Outcome evaluated & Result $^{\mathrm{b}}$ \\
\hline \multicolumn{4}{|l|}{ Sensitization } \\
\hline $\begin{array}{l}\text { Jedrychowski et al. } 2011 \\
\text { Prospective study; n=224 children (at } \\
5 \text { years of age) of women recruited in } \\
\text { the } 2^{\text {nd }} \text { trimester of pregnancy }\end{array}$ & $\begin{array}{l}\text { Gmean }(95 \% \mathrm{Cl}) \text { : } \\
\text { Cord: } 1.16(1.12,1.22) \\
\text { Maternal: } 1.60(1.52,1.67)\end{array}$ & Atopy & $\begin{array}{l}\text { Adjusted RR: } \\
\text { - } \quad \text { Cord PbB: } 2.28(1.12,4.62)^{*} \\
\text { - } \quad \text { Maternal PbB: } 1.72(0.98,3.00)\end{array}$ \\
\hline $\begin{array}{l}\text { Pizent et al. } 2008 \\
\text { Cross-sectional study; } n=216 \text { adults } \\
\text { (age range } 19-67 \text { years) }\end{array}$ & $\begin{array}{l}\text { Gmean }(95 \% \mathrm{Cl}:) \\
\text { - } \quad \text { Male: } 3.17(0.99,7.23) \\
\text { - } \quad \text { Female: } 2.16(0.56,7.35)\end{array}$ & SPT & Adjusted OR for positive SPT: $0.92(0.86,0.98)^{*}$ \\
\hline \multicolumn{4}{|l|}{ Humoral immunity } \\
\hline \multirow{2}{*}{$\begin{array}{l}\text { Karmaus et al. } 2005 \\
\text { Cross-sectional study; } n=671 \text { children } \\
\text { (age } 7-10 \text { years) }\end{array}$} & \multirow{2}{*}{$\begin{array}{l}\text { Gmean }(95 \% \mathrm{Cl}:) \\
\text { - } \quad \text { Males: } 2.78(1.48,4.82) \\
\text { - } \quad \text { Females: } 2.54(1.10,4.38)\end{array}$} & $\lg \mathrm{E}$ & $\begin{array}{l}\text { Mean serum IgE levels were higher }(p \leq 0.05) \text { in } \\
\text { PbB strata }>2.84 \text { and }>3.41 \mu g / d^{*}\end{array}$ \\
\hline & & B-cells & $\begin{array}{l}\text { B-cell abundance was lower }(\mathrm{p} \leq 0.05) \text { in } \mathrm{PbB} \\
\text { stratum } 2.21-2.83 \text { compared to }<2.2 \mu \mathrm{g} / \mathrm{dL}^{*}\end{array}$ \\
\hline $\begin{array}{l}\text { Min and Min } 2015 \\
\text { Cross-sectional study; } n=4,287 \text { adults } \\
\text { (age } \geq 22 \text { years)c }^{c}\end{array}$ & $\begin{array}{l}\text { Gmean }(95 \% \mathrm{Cl}) \text { : } \\
-\quad 1.46(1.44,1.50)\end{array}$ & $\lg \mathrm{E}$ & $\begin{array}{l}\beta \text { for } 1 \log _{10} \text { increase in IgE per } 1 \log _{10} \text { increase in } \\
\text { PbB: } \\
\text { - } \quad \text { Q2 }(1.1-1.69 \mu \mathrm{g} / \mathrm{dL}): 0.20(0.05,0.34)^{*} \\
\text { - } \quad \text { Q3 }(1.7-2.6 \mu \mathrm{g} / \mathrm{dL}): 0.26(0.10,0.42)^{*} \\
\text { - } \quad \text { Q }(2.61-26.4 \mu \mathrm{g} / \mathrm{dL}): 0.35(0.20,0.51)^{*}\end{array}$ \\
\hline $\begin{array}{l}\text { Pizent et al. } 2008 \\
\text { Cross-sectional study; } n=216 \text { adults } \\
\text { (age range } 19-67 \text { years) }\end{array}$ & $\begin{array}{l}\text { Gmean }(95 \% \mathrm{Cl}:) \\
\text { - } \quad \text { Male: } 3.17(0.99,7.23) \\
\text { - } \quad \text { Female: } 2.16(0.56,7.35)\end{array}$ & $\lg \mathrm{E}$ & $\begin{array}{l}\beta \text { log increase in IgE per log increase in } \mathrm{PbB} \\
\mu \mathrm{g} / \mathrm{L}(\mathrm{SE}), \text { females not taking oral contraceptives } \\
\text { or hormone replacement therapy: } 0.600(0.298) ; \\
p=0.046^{*}\end{array}$ \\
\hline
\end{tabular}


2. HEALTH EFFECTS

\section{Table 2-27. Summary of Epidemiological Studies Evaluating Immunological Effects at Mean Blood Lead Concentration $(\mathrm{PbB}) \leq 10 \mu \mathrm{g} / \mathrm{dL}^{\mathrm{a}}$}

\begin{tabular}{|c|c|c|c|}
\hline Reference and study population & $\mathrm{PbB}(\mu \mathrm{g} / \mathrm{dL})$ & Outcome evaluated & Result $^{\mathrm{b}}$ \\
\hline \multirow{5}{*}{$\begin{array}{l}\text { Sarasua et al. } 2000 \\
\text { Cross-sectional study; } \\
\mathrm{n}=1,561 \text { residents of communities } \\
\text { with elevated levels of } \mathrm{Cd} \text { or } \mathrm{Pb} \text { in } \\
\text { soil (age range } 6 \text { months }-75 \text { years) } \\
\text { (1991) }\end{array}$} & \multirow{5}{*}{$\begin{array}{l}\text { Gmean }(95 \% \text { Cl: }) \\
\text { - } \quad \text { Age 6-35 months: } \\
\quad 7.0(1.7,16.1) \\
\text { - } \quad \text { Age 36-71 months: } \\
\quad 6.0(1.6,14.1) \\
\text { - } \quad \text { Age 6-15 years: } \\
\quad 4.0(1.1,9.2)\end{array}$} & $\lg \mathrm{A}$ & $\beta$ per $1 \mu \mathrm{g} / \mathrm{dL} \mathrm{PbB}$, age $6-35$ months: $0.8, \mathrm{p}<0.01^{*}$ \\
\hline & & $\lg G$ & 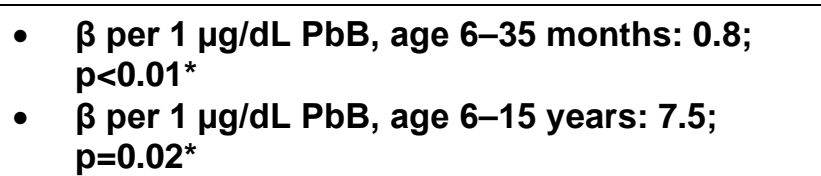 \\
\hline & & $\lg M$ & $\beta$ per $1 \mu \mathrm{g} / \mathrm{dL} \mathrm{PbB}$, age $6-35$ months: $1.0 ; p=0.03^{*}$ \\
\hline & & B-cell count & $\begin{array}{l}\beta \text { per } 1 \mu \mathrm{g} / \mathrm{dL} \mathrm{PbB} \text {, age } 6-35 \text { months: } 16.9 \\
\text { p<0.01* }\end{array}$ \\
\hline & & B-cell\% & $\begin{array}{l}\beta \text { per } 1 \mu \mathrm{g} / \mathrm{dL} \text { PbB, age } 6-35 \text { months: } 0.19 \\
p=0.02^{*}\end{array}$ \\
\hline $\begin{array}{l}\text { Wang et al. } 2017 a \\
\text { Cross-sectional study; } n=930 \text { children } \\
\text { (mean age: } 5.74 \text { years; } 469 \text { boys and } \\
461 \text { girls) }\end{array}$ & $\begin{array}{l}\text { PbB Gmean (GSD) } \\
\text { - } \quad \text { All: } 1.86(1.21) \\
\text { Boys: } 1.88(1.22) \\
\text { - Girls: } 1.83(1.20)\end{array}$ & $\lg E$ & $\begin{array}{l}\text { - All participants, } \beta \text { per In-unit increase in PbB } \\
(2.72 \mu \mathrm{g} / \mathrm{dL}): 0.26(0.009,0.50) ; p=0.042^{*} \\
\text { Boys, } \beta \text { per In-unit increase in } \mathrm{PbB} \\
(2.72 \mu \mathrm{g} / \mathrm{dL}): 0.40(0.03,0.76) ; p=0.036^{*} \\
\text { - Girl, } \beta \text { per In-unit increase in } \mathrm{PbB}(2.72 \mu \mathrm{g} / \mathrm{dL}): \\
0.02(-0.35,0.40) ; p=0.901\end{array}$ \\
\hline $\begin{array}{l}\text { Wells et al. } 2014 \\
\text { Cross-sectional study; } \\
n=1,788 \text { children (age } 2-12 \text { years) }\end{array}$ & $\begin{array}{l}\text { Gmean }(95 \% \mathrm{Cl}:) \\
-\quad 1.13(1.04,1.22)\end{array}$ & $\lg E$ & $\begin{array}{l}\beta \text { per } 1 \mu \mathrm{g} / \mathrm{dL} \text { PbB for } \% \text { increase per } 1 \mu \mathrm{g} / \mathrm{dL}: \\
10.27(3.52,17.47)^{*}\end{array}$ \\
\hline $\begin{array}{l}\text { Xu et al. } 2015 \\
\text { Cross-sectional study; } n=590 \text { children } \\
\text { (age } 3-7 \text { years) }\end{array}$ & $\begin{array}{l}\text { Gmean (SD of log } \mathrm{PbB}) \text { : } \\
\text { - } \quad \text { Male: } 6.61(0.19) \\
\text { - } \quad \text { Female: } 6.16(0.18)\end{array}$ & Hepatitis B virus & $\begin{array}{l}\text { Antibody titers decreased with increasing } \mathrm{PbB} \\
\beta \text { signal to cut-off ratio per } 1 \mu \mathrm{g} / \mathrm{dL}(\mathrm{SE}) \text { at two } \\
\text { assessment dates: } \\
\text { - 2011: }-0.4467(0.0225) ; \boldsymbol{p}<0.001^{*} \\
\text { - 2012: } 0.3661\left(\mathbf{0 . 0 1 9 3 )} ; \boldsymbol{p}<0.001^{*}\right.\end{array}$ \\
\hline
\end{tabular}




\section{Table 2-27. Summary of Epidemiological Studies Evaluating Immunological Effects at Mean Blood Lead Concentration $(\mathrm{PbB}) \leq 10 \mu \mathrm{g} / \mathrm{dL}^{\mathrm{a}}$}

\begin{tabular}{|c|c|c|c|}
\hline Reference and study population & $\mathrm{PbB}(\mu \mathrm{g} / \mathrm{dL})$ & Outcome evaluated & Result $^{\mathrm{b}}$ \\
\hline \multicolumn{4}{|l|}{ Cell-mediated immunity } \\
\hline $\begin{array}{l}\text { Boscolo et al. } 2000 \\
\text { Cross-sectional study; } n=30 \text { atopic } \\
\text { women (age range } 19-49 \text { years) and } \\
30 \text { non-atopic women }\end{array}$ & $\begin{array}{l}\text { Median: } \\
\text { - } \quad \text { Atopic: } 6.4(4.9,7.9) \\
\text { - } \quad \text { Control: } 5.5(4.4,6.7)\end{array}$ & T-cell abundance & $\begin{array}{l}\text { Positive correlation between } \mathrm{PbB} \text { and T-cell } \\
\text { abundances in non-atopic subjects ( } r \text { for cell count): } \\
\text { - } \quad \text { CD4+CD45RO-: } 0.464 ; p<0.05^{*} \\
\text { - } \quad \text { CD3+ CD8+: } 0.430 ; p<0.05^{*} \\
\text { - } \quad \text { CD3- HLA-DR+>: } 0.435 ; p<0.05^{*}\end{array}$ \\
\hline $\begin{array}{l}\text { Conterato et al. } 2013 \\
\text { Cross-section study of battery } \\
\text { manufacture workers }(n=59) \text {, and } \\
\text { automobile painters }(n=23) ; \text { ages } 15- \\
61 \text { years }\end{array}$ & $\begin{array}{l}\text { Median: } \\
\text { - } \quad \text { Battery workers: } 49.8(4.0) \\
\text { - } \quad \text { Painters: } 5.4(0.4) \\
\text { - } \quad \text { Controls: } 1.5(0.1) \\
\end{array}$ & Neutrophil abundance & $\begin{array}{l}\text { Mean (SE), } 10^{3} / \mathrm{mm}^{3}: \\
\text { - } \quad \text { Battery workers: } 2.87(0.27) ; p<0.05^{*} \\
\text { - } \quad \text { Painters: } 3.07(0.13) ; p<0.05^{\star} \\
\text { - } \quad \text { Controls: } 3.75(2.49)\end{array}$ \\
\hline \multirow{5}{*}{$\begin{array}{l}\text { Hsiao et al. } 2011 \\
\text { Cross-sectional study; } n=214 \text { children } \\
\text { (primary school grades 5-6) }\end{array}$} & \multirow{5}{*}{$\begin{array}{l}\text { Mean (SD): } \\
\text { - } \quad \text { Allergic and residing near } \\
\text { oil refinery: } \\
8.80(0.45) \\
\text { - } \quad \text { Non-allergic and residing } \\
\text { near oil refinery: } \\
5.23(0.36) \\
\text { Other rural or urban } \\
\text { groups, allergic or not: } \\
3.16-3.83 \\
\end{array}$} & & $\begin{array}{l}\text { Compared to all other groups, allergic group residing } \\
\text { near the refinery had: }\end{array}$ \\
\hline & & IFN-Y & $>96 \%$ decrease in serum IFN-Y; $p<0.05^{*}$ \\
\hline & & IL-12 & $>96 \%$ decrease; $p<0.05^{*}$ \\
\hline & & IL-4 & $>500 \%$ increase; $p<0.05^{\star}$ \\
\hline & & IL-25 & $>500 \%$ increase; $p<0.05^{\star}$ \\
\hline $\begin{array}{l}\text { Karmaus et al. } 2005 \\
\text { Cross-sectional study; } n=67 \text { children } \\
\text { (age } 7-10 \text { years) }\end{array}$ & $\begin{array}{l}\text { Gmean }(95 \% \mathrm{Cl}:) \\
\text { - } \quad \text { Males: } 2.78(1.48,4.82) \\
\text { - } \quad \text { Females: } 2.54(1.10,4.38)\end{array}$ & T-cell and $\mathrm{Tc}_{\mathrm{C}}$ & $\begin{array}{l}\text { Lower }(\mathrm{p} \leq 0.05) \text { in } \mathrm{PbB} \text { stratum } 2.21-2.83 \\
\text { compared to }<2.2 \mu \mathrm{g} / \mathrm{dL}^{*}\end{array}$ \\
\hline
\end{tabular}


2. HEALTH EFFECTS

\section{Table 2-27. Summary of Epidemiological Studies Evaluating Immunological Effects at Mean Blood Lead Concentration $(\mathrm{PbB}) \leq 10 \mu \mathrm{g} / \mathrm{dL}^{\mathrm{a}}$}

\begin{tabular}{|c|c|c|c|}
\hline Reference and study population & $\mathrm{PbB}(\mu \mathrm{g} / \mathrm{dL})$ & Outcome evaluated & Result $^{b}$ \\
\hline Sarasua et al. 2000 & \multirow{4}{*}{ 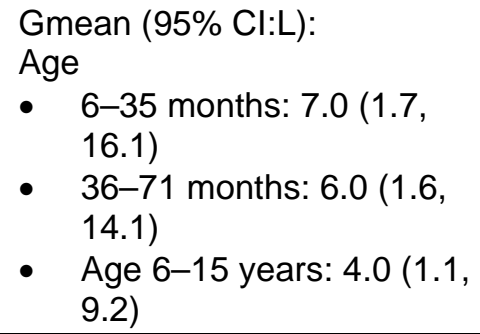 } & T-cell\% & $\beta$ per $1 \mu \mathrm{g} / \mathrm{dL}$ PbB: $-0.18 ; p=0.03^{*}$ \\
\hline \multirow{3}{*}{$\begin{array}{l}\text { Cross-sectional study; } n=1,561 ; \text { age } \\
\text { range } 6 \text { months- } 75 \text { years) }\end{array}$} & & T-cell count & $\beta$ per $1 \mu \mathrm{g} / \mathrm{dL}$ PbB: $7.2 ; p=0.59$ \\
\hline & & NK-cell\% & $\beta$ per $1 \mu \mathrm{g} / \mathrm{dL}$ PbB: $0.00 ; p=0.99$ \\
\hline & & NK-cell count & $\beta$ per $1 \mu \mathrm{g} / \mathrm{dL}$ PbB: $1.3 ; p=0.60$ \\
\hline Wells et al. 2014 & $\begin{array}{l}\text { Gmean }(95 \% \mathrm{Cl}:) \\
1.13(1.04,1.22)\end{array}$ & Eosinophils \% & $\begin{array}{l}\beta \text { for } \% \text { increase per } 1 \mu \mathrm{g} / \mathrm{dL} \text { : } \\
4.61(2.44,6.83)^{\star}\end{array}$ \\
\hline \multicolumn{4}{|l|}{$\begin{array}{l}\text { Cross-sectional study; } \\
n=1,788 \text { children (age } 2-12 \text { years) }\end{array}$} \\
\hline \multicolumn{4}{|l|}{ Inflammation } \\
\hline Kim et al. 2007 & $\begin{array}{l}\text { Mean (range): } \\
-\quad \text { Q1: } 1.46(0.337,1.885)\end{array}$ & & $\begin{array}{l}\text { In males for } \mathrm{PbB} \text { stratum }>2.51 \text { relative to lower } \mathrm{PbB} \\
\text { stratum. } \% \text { per } 1 \mu \mathrm{g} / \mathrm{dL} \text { increase in } \mathrm{PbB} \text { : }\end{array}$ \\
\hline \multirow{3}{*}{$\begin{array}{l}\text { Cross-sectional study; } n=300 \text { adults } \\
\text { (mean age } 24 \pm 2 \text { years) }\end{array}$} & \multirow{3}{*}{$\begin{array}{ll}- & \text { Q2: } 2.22(1.886,2.511) \\
- & \text { Q3: } 2.77(2.513,3.103) \\
\text { - } & \text { Q4: } 3.93(3.110,10.470)\end{array}$} & TNFa & $23 \%(4,55) ; p=0.015^{*}$ \\
\hline & & WBC & $15 \%(0,35) ; p=0.004^{*}$ \\
\hline & & IL-6 & $26 \%(0,55 \%) ; p=0.082$ \\
\hline $\begin{array}{l}\text { Sirivarasai et al. } 2013 \\
\text { Cross-sectional study; } n=924 \text { male } \\
\text { adults (mean age } 43 \text { years) }\end{array}$ & $\begin{array}{l}\text { Mean: } 5.45 \\
\text { Quartiles, mean (range): } \\
\text { - } \quad \text { Q1: } 2.44(1.23,3.47) \\
\text { - } \quad \text { Q2: } 3.95(3.48,4.55) \\
\text { - } \quad \text { Q3: } 5.77(4.56,6.47) \\
\text { - } \quad \text { Q4: } 9.21(6.48,24.62)\end{array}$ & CRP & $\begin{array}{l}\text { CRP was higher in upper quartile PbB stratum } \\
\text { compared to Q1 and Q2 (p<0.001). In Q4 stratum, } \\
\text { adjusted OR was elevated for GSTM1 and GSTT1 } \\
\text { null genotypes: } \\
\text { - } \quad \text {-GSTM1-I- and GSTT1-I-: 1.98 }(\mathbf{1 . 4 7 , ~ 2 . 5 5 ) *} \\
\text { - -GSTM1-I-: } 1.32(1.03,1.69)^{*} \\
\text { - -GSTT1-I-: } \mathbf{1 . 6 5}(\mathbf{1 . 1 7}, \mathbf{2 . 3 5})^{*}\end{array}$ \\
\hline
\end{tabular}


Table 2-27. Summary of Epidemiological Studies Evaluating Immunological Effects at Mean Blood Lead Concentration $(\mathrm{PbB}) \leq 10 \mu \mathrm{g} / \mathrm{dL}^{\mathrm{a}}$

\begin{tabular}{|c|c|c|c|}
\hline Reference and study population & $\mathrm{PbB}(\mu \mathrm{g} / \mathrm{dL})$ & Outcome evaluated & Result $^{b}$ \\
\hline Songdej et al. 2010 & Gmean: 1.89 & & OR for $<1.16$ versus $>3.09 \mu \mathrm{g} / \mathrm{dL}$ : \\
\hline \multirow{3}{*}{$\begin{array}{l}\text { Cross-sectional study; } n=9,145 \text { adults } \\
\text { (age }>40 \text { years) }^{c}\end{array}$} & & CRP & $\begin{array}{ll} & \text { Males: } 2.85(1.49,5.45)^{*} \\
\text { - } & \text { Females: } 0.57(0.43,0.76)\end{array}$ \\
\hline & & Fibrinogen & $\begin{array}{ll}- & \text { Males: } 1.15(0.61,2.16) \\
\text { - } & \text { Females: } 0.87(0.57,1.33)\end{array}$ \\
\hline & & WBC & $\begin{array}{ll}\text { - } & \text { Males: } 1.55(0.96,2.49) \\
\text { - } & \text { Females: } 0.84(0.62,1.13)\end{array}$ \\
\hline
\end{tabular}

a See the Supporting Document for Epidemiological Studies for Lead, Table 8 for more detailed descriptions of studies.

${ }^{b}$ Asterisk and bold indicate association with $\mathrm{Pb}$; unless otherwise specified, values in parenthesis are $95 \%$ Cls; $p$-values $<0.05$ unless otherwise noted in the table.

'Study of NHANES participants.

$\mathrm{Cd}=$ cadmium; $\mathrm{Cl}=$ confidence interval; $\mathrm{CL}=$ confidence limit; CRP = C-reactive protein; Gmean = geometric mean; GSTM1 = glutathione S-transferase Mu 1; GSTT1 = glutathione S-transferase theta 1; IFN-y = interferon gamma; Ig = immunoglobulin antibody; IL = interleukin; NHANES = National Health and Nutrition Examination Survey; $\mathrm{NK}=$ natural killer; OR = odds ratio; $\mathrm{Pb}=$ lead; $\mathrm{SD}=$ standard deviation; $\mathrm{SE}=$ standard error; SPT = skin prick test; TNF $\alpha=$ tumor necrosis factor-alpha; WBC = white blood cell 
Inflammation. A few studies have found evidence for increases in circulating TNF $\alpha$ (Kim et al. 2007) and CRP (Songdej et al. 2010; Sirivarasai et al. 2013) in adults at mean $\mathrm{PbB}<10 \mu \mathrm{g} / \mathrm{dL}$. These outcomes are indicative of enhancement or stimulation of inflammation.

Mechanisms of Action. Studies conducted in animal models and cell cultures have shown that $\mathrm{Pb}$ can disrupt the immune response through diverse mechanisms (EPA 2014c). Figure 2-6 shows the various potential pathways by which $\mathrm{Pb}$ may perturb the immune system and increase risk of atopy and inflammation, autoimmunity, and host resistance. In addition to its effects on T-cells, dendritic cells, and macrophages, $\mathrm{Pb}$ may also alter immune function at many other processes in the pathways shown in

Figure 2-6.

\section{Figure 2-6. Immunological Pathways by which $\mathrm{Pb}$ Exposure Potentially may Increase Risk of Immune-Related Diseases}

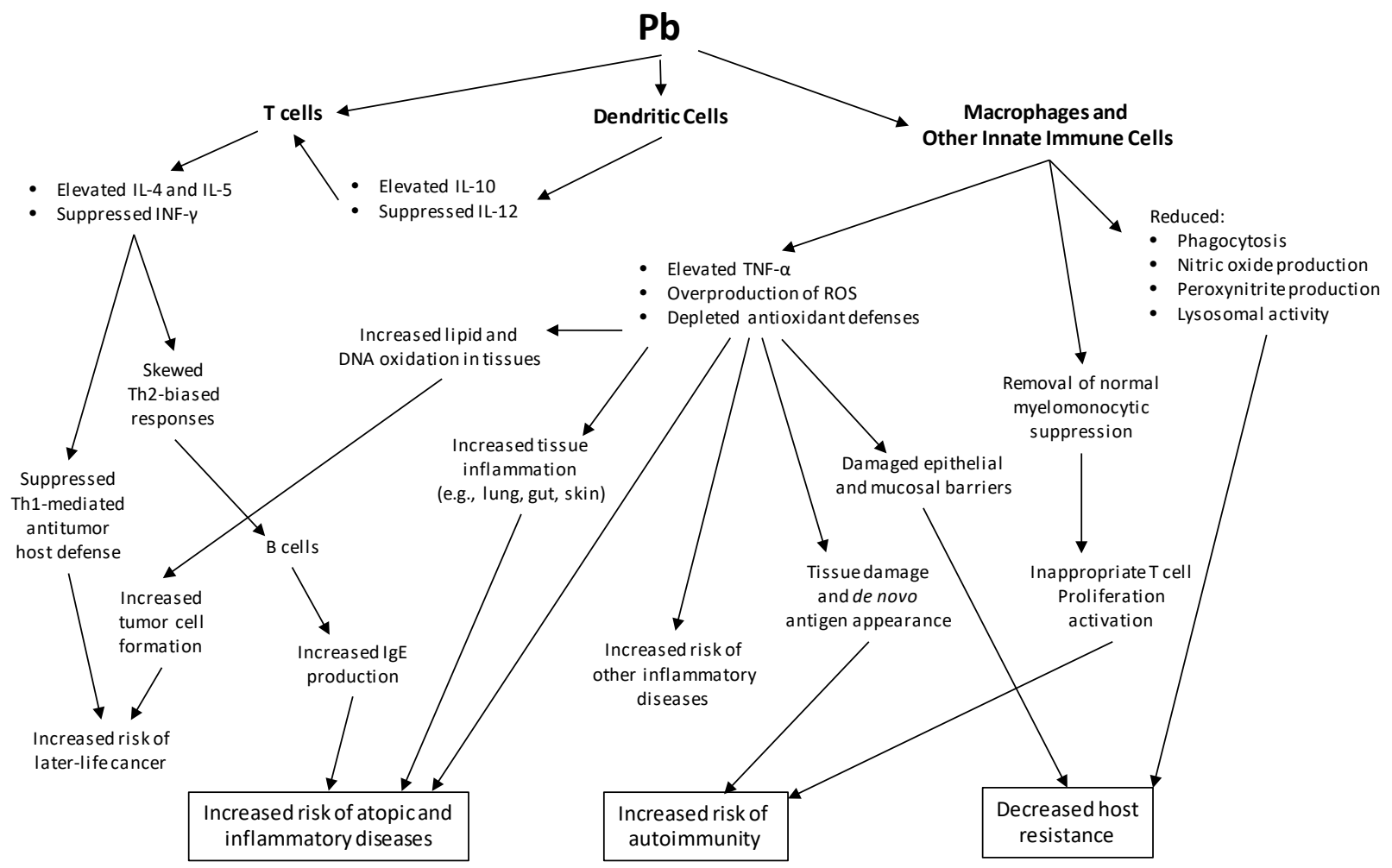

Note: As shown in the figure, immunological pathways may increase risk of diseases such as cancer and inflammatory diseases in the cardiovascular, renal, and hepatic systems.

Source: EPA 2014c 


\subsection{NEUROLOGICAL}

Overview. The literature on the neurobehavioral effects of $\mathrm{Pb}$ is extensive. With the improvement in analytical methods to detect $\mathrm{Pb}$ in the various biological media and in study designs, the concentrations of $\mathrm{Pb}$, particularly in blood, associated with alterations in neurobehavioral outcomes continue to decrease, suggesting that there may be no threshold for the effects of $\mathrm{Pb}$ on intellectual function (CDC 2012d). Due to the enormous size of the database on neurobehavioral effects of $\mathrm{Pb}$, this discussion has been limited to representative and/or major studies published on specific topics crucial to understanding dose-response relationships in the lower exposure ranges (e.g., $\mathrm{PbB} \leq 10 \mu \mathrm{g} / \mathrm{dL}$ ). For additional information, the reader is referred to a recent review of this topic (EPA 2014c).

Numerous epidemiological studies have evaluated effects of $\mathrm{Pb}$ on neurological function in children and adults. These studies show consistent evidence of associations between decrements in cognitive and neuromotor/neurosensory function with $\mathrm{PbBs}$ that range from $\leq 10$ to $>50 \mu \mathrm{g} / \mathrm{dL}$. The PbB-effect relationship for cognitive effects in children extends well below $10 \mu \mathrm{g} / \mathrm{dL}$, with no evidence for a threshold. In several PbB-effect models, the slope for decrements in cognitive function in children show greater increases at lower PbB ranges. These models predict that larger decrements in cognitive function would occur when PbB increases from 1 to $10 \mu \mathrm{g} / \mathrm{dL}$, than when PbB increases to levels $>10 \mu \mathrm{g} / \mathrm{dL}$. All of the cognitive and neurobehavioral effects of $\mathrm{Pb}$ observed in children have also been observed in adults; however, it is not certain what life-stage exposures contribute most to outcomes in adults. A few studies that have followed children to early adulthood provide evidence of associations between childhood $\mathrm{Pb}$ exposure (e.g., $\mathrm{PbB}$ ) and behavioral and neuroanatomical changes in adults, suggesting a possible role of exposures in childhood to adult outcomes. Other studies have found evidence of associations between cumulative $\mathrm{Pb}$ exposures (e.g., bone $\mathrm{Pb}$ ) and neurological outcomes in adults.

The following neurobehavioral effects in children have been associated with $\mathrm{PbB}$ :

- $\quad \leq 10 \mu \mathrm{g} / \mathrm{dL}:$

o Decreased cognitive function including full scale IQ (FSIQ).

o Altered mood and behaviors that may contribute to learning deficits, including attention deficits, hyperactivity, autistic behaviors, conduct disorders, and delinquency.

o Altered neuromotor and neurosensory function, including gross and fine motor skills, visualmotor integration, and hearing threshold. 
- $\quad>10 \mu \mathrm{g} / \mathrm{dL}$ :

o Decreased cognitive function including FSIQ.

o Altered mood and behaviors, including attention deficits, hyperactivity, autistic behaviors, conduct disorders, and delinquency.

o Altered neuromotor and neurosensory function, including gross and fine motor skills, visualmotor integration, hearing threshold, and visual evoked potentials.

o Peripheral neuropathy.

o Encephalopathy.

The following neurobehavioral effects in adults have been associated with increasing PbB:

- $\leq 10 \mu \mathrm{g} / \mathrm{dL}$ :

o Decreased cognitive function including attention, memory, and learning.

o Altered neuromotor and neurosensory function including decreased reaction time and walking speed, tremor, and increased risk of amyotrophic lateral sclerosis (ALS).

o Altered mood and behavior including risk of various psychiatric symptoms including anxiety, depression, and schizophrenia.

- $\quad>10 \mu \mathrm{g} / \mathrm{dL}$ :

o Reduced brain volume and altered brain neurochemistry.

o Decreased cognitive function.

o Altered neuromotor and neurosensory function.

o Decreased peripheral nerve conduction velocity.

Measures of Exposure. Studies conducted in children have relied heavily on $\mathrm{PbB}$ as an exposure metric. Although bone or tooth $\mathrm{Pb}$ measurements may be informative, few studies have been conducted in children (Bellinger et al. 1994; Campbell et al. 2000b; Fergusson et al. 1993; Kim et al. 1995; Needleman et al. 1979, 1990, 1996, 2002; Wasserman et al. 2003). Maternal bone Pb has been used as an exposure metric for evaluating outcomes in children (Gomaa et al. 2002; Xu et al. 2015). Bone Pb has been used as metric of cumulative exposure in a growing number of epidemiological studies of adults (see Section 3.3.1, Biomarkers of Exposure). An association between a health outcome and bone $\mathrm{Pb}$ does not necessarily infer an association between the outcome and PbB (or vice versa) as indicated by studies in which associations are not consistent for the two metrics. These differences may reflect the relative importance of cumulative exposure on the given outcome, or differences in error associated with measurements of blood and bone Pb concentrations. A review by Shih et al. (2007) concluded that 
negative associations between $\mathrm{Pb}$ and cognitive function are stronger for bone $\mathrm{Pb}$ (specifically tibia $\mathrm{Pb}$ ) for environmental exposures and for PbB for occupational exposures.

Confounding Factors and Effect Modifiers. Various factors have the potential to contribute to bias in estimates of associations between $\mathrm{PbB}$ and neurobehavioral outcomes. Failure to account for these factors may attenuate or strengthen the apparent associations between $\mathrm{Pb}$ exposure and the outcome, depending on the direction of the effect of the variable on the outcome. Neurological function can be influenced by numerous factors that may also correlate with $\mathrm{Pb}$ exposure in the population studied. A contributor to these correlations is the influence of SES-related factors on Pb exposure. Confounding factors that are typically evaluated in all high-quality studies include maternal education and IQ, SES, and HOME score (parental care). However, other factors have also been explored in some studies, including maternal substance abuse (including prenatal alcohol) and psychopathology, birth weight, exposure to tobacco smoke, nutritional status, and ALAD allele type. The relatively strong correlation between SES and $\mathrm{PbB}$ can result in overcontrol in studies of populations that have wide SES variability. Overcontrol will tend to attenuate the estimated association between $\mathrm{PbB}$ and the outcome (Bellinger 2004). However, SES may also modify the effect of Pb on neurological function (Bellinger et al. 1990; Ris et al. 2004; Tong et al. 2000). If this were to occur, then SES would also be an effect-modifier.

Characterization of Effects in Children. A large number of studies showing decrements in neurological function in children have been published (Table 2-28). Collectively, these studies support the concept that $\mathrm{Pb}$ affects cognitive function in children prenatally exposed to $\mathrm{PbB} \leq 10 \mu \mathrm{g} / \mathrm{dL}$, with numerous studies providing evidence for effects at $\mathrm{PbB} \leq 5 \mu \mathrm{g} / \mathrm{dL}$. Neurobehavioral functions that have been associated with $\mathrm{PbB} \leq 10 \mu \mathrm{g} / \mathrm{dL}$ include decrements in cognitive function (learning and memory), altered behavior and mood (e.g., attention, hyperactivity, impulsivity, irritability, delinquency), and altered neuromotor and neurosensory function (visual-motor integration, dexterity, postural sway, changes in hearing and visual thresholds). These outcomes also have been observed in association with $\mathrm{PbB}$ $>10 \mu \mathrm{g} / \mathrm{dL}$. In children who have been followed to early adulthood, mean childhood PbBs of $13 \mu \mathrm{g} / \mathrm{dL}$ were associated with altered brain volume and neurochemistry (Brubaker et al. 2010; Cecil et al. 2008, 2011). PbBs $>30 \mu \mathrm{g} / \mathrm{dL}$ are associated with a variety of decrements in cognitive function, behavior (e.g., depression, aggression), and nerve function (e.g., decrements in fine and gross motor skills, peripheral neuropathy). Encephalopathy has been observed in children who have experienced severe $\mathrm{Pb}$ poisoning typical of $\mathrm{PbB}>80 \mu \mathrm{g} / \mathrm{dL}$ (NAS 1972). 
Table 2-28. Overview of Neurological Effects in Children Associated with Chronic Exposure to Lead (Pb)

\begin{tabular}{ll}
\hline $\begin{array}{l}\text { Mean PbB } \\
(\mu \mathrm{g} / \mathrm{dL})\end{array}$ & $\begin{array}{l}\text { Effects associated with } \\
\mathrm{Pb} \text { exposure }\end{array}$ \\
\hline$\leq 10$ & Intellectual deficits $^{\mathrm{a}}$
\end{tabular}

References

Blackowicz et al. 2016; Baghurst et al. 1992; Bellinger and Needleman 2003; Bellinger et al. 1992; Boucher et al. 2014; Braun et al. 2012; Canfield et al. 2003; Chandramouli et al. 2009; Chiodo et al. 2004; Desrochers-Couture et al. 2018; Dietrich et al. 1986, 1987, 1989, 1991, 1992, 1993a; Emory et al. 2003; Evens et al. 2015; Geier et al. 2017; Gomaa et al. 2002; Hong et al. 2015; Hu et al. 2006; Jedrychowski et al. 2009; Jusko et al. 2008; Kordas et al. 2011; Krieg et al. 2010; Lanphear et al. 2000a, 2005, 2019; Lin et al. 2013; Liu et al. 2014b; Mazumdar et al. 2011; McLaine et al. 2013; Min et al. 2009; Miranda et al. 2009; Polanska et al. 2018; Rodrigues et al. 2016; Rooney et al. 2018; Ruebner et al. 2019; Schnaas et al. 2006; Shadbegian et al. 2019; Sobin et al. 2015; Tellez-Rojo et al. 2006; Vigeh et al. 2014; Wang et al. 2008; Wasserman et al. 1994, 1997, 2003; Zhang et al. 2013; Zhou et al. 2017

Altered mood and behavior $^{\mathrm{b}}$

Altered neuromotor neurosensory function ${ }^{c}$

Altered brain anatomical development and activity

$>10-30 \quad$ Intellectual deficits ${ }^{a}$

Altered mood and behavior $^{\text {b }}$

Altered neuromotor neurosensory function ${ }^{c}$
Arbuckle et al. 2016; Boucher et al. 2012; Braun et al. 2006, 2008; Choi et al. 2016; Dietrich et al. 2001; Froehlich et al. 2009; Fruh et al. 2019; Geier et al. 2018; He et al. 2019; Hong et al. 2015; Huang et al. 2016; Ji et al. 2018; Joo et al. 2017, 2018; Kim et al. 2013a, 2016; Liu et al. 2014a, 2015b; Park et al. 2016; Sioen et al. 2013; Stroustrup et al. 2016; Wang et al. 2008; Winter and Sampson 2017

Chiodo et al. 2004; Dietrich et al. 1987, 1989, 1993b; Ethier et al. 2012; Fraser et al. 2006; Kim et al. 2013b; Liu et al. 2018b; Osman et al. 1999; Silver et al. 2016; Tellez-Rojo et al. 2006

Cecil et al. 2008, 2011

Baghurst et al. 1992; Bellinger et al. 1987, 1990, 1991; Chen et al. 2005, 2007; Dietrich et al. 1992, 1993a; Factor-Litvak et al. 1999; Hornung et al. 2009; Kordas et al. 2006; Magzamen et al. 2013, 2015; Marques et al. 2014; McMichael et al. 1988; Roy et al. 2011; Schnaas et al. 2000; Shen et al. 1998; Tong et al. 1996; Wasserman et al. 1994, 1997, 2000, 2003

Amato et al. 2013; Chen et al. 2007; Dietrich et al. 1993b, 2001; Lin et al. 2019; McFarlane et al. 2013; Neugebauer et al. 2015; Nkomo et al. 2017; Rothenberg et al. 1989; Roy et al. 2009; Wu et al. 2018

Baghurst et al. 1995; Bhattacharya et al. 2006; Otto et al. 1985; Palaniappan et al. 2011; Parajuli et al. 2013, Ris et al. 2004; Robinson et al. 1985; Schwartz and Otto 1987, 1991 
Table 2-28. Overview of Neurological Effects in Children Associated with Chronic Exposure to Lead (Pb)

\begin{tabular}{|c|c|c|}
\hline $\begin{array}{l}\text { Mean PbB } \\
(\mu \mathrm{g} / \mathrm{dL})\end{array}$ & $\begin{array}{l}\text { Effects associated with } \\
\mathrm{Pb} \text { exposure }\end{array}$ & References \\
\hline$>30-50$ & Intellectual deficits ${ }^{a}$ & do Nascimento et al. 2014; Royal et al. 2013 \\
\hline \multirow[t]{4}{*}{$>50$} & Intellectual deficits ${ }^{a}$ & Hou et al. 2013 \\
\hline & $\begin{array}{l}\text { Altered mood and } \\
\text { behavior }^{\mathrm{b}}\end{array}$ & Hou et al. 2013 \\
\hline & $\begin{array}{l}\text { Altered neuromotor } \\
\text { neurosensory function }\end{array}$ & Hou et al. 2013; \\
\hline & Peripheral neuropathy ${ }^{d}$ & $\begin{array}{l}\text { Erenberg et al. 1974; Landrigan et al. 1976; Schwartz et al. } \\
\text { 1988; Seto and Freeman } 1964\end{array}$ \\
\hline$>80$ & Encephalopathy & NAS 1972 \\
\hline
\end{tabular}

aIntellectual deficits include decreased IQ, cognitive function, verbal comprehension, language development, perceptual organization, processing speed, decreased math and reading aptitude, educational attainment, school performance, and memory.

${ }^{b}$ Altered mood and behavior includes hyperactivity, ADHD, decreased adaptive skills and emotional functioning, externalizing behaviors, internalizing behaviors, social problems, delinquent behavior, impulsive behavior, irritability, autistic behavior, altered sleep, and associations between child PbB and adult behavior (see McFarlane et al. (2013).

${ }^{c}$ Altered neuromotor neurosensory function includes decreased integrated motor activities, gross motor skills; fine motor speed and dexterity, and visual-motor integration.

dPeripheral neuropathy includes decreased motor and sensory nerve conduction velocity.

$\mathrm{ADHD}=$ attention-deficit/hyperactivity disorder; $\mathrm{IQ}=$ intelligence quotient; $\mathrm{PbB}=$ blood lead concentration

Characterization of Effects in Adults. A large number of studies showing decrements in neurological function in adults have been published (Table 2-29). These studies have found neurobehavioral effects in populations whose PbBs were $\leq 10 \mu \mathrm{g} / \mathrm{dL}$. Neurobehavioral functions that have been associated with $\mathrm{PbB}$ $\leq 10 \mu \mathrm{g} / \mathrm{dL}$ include decreased cognitive function, altered behavior and mood, and altered neuromotor and neurosensory function. These outcomes also have been observed in association with $\mathrm{PbB}>10 \mu \mathrm{g} / \mathrm{dL}$. PbBs in the range of 10-20 $\mu \mathrm{g} / \mathrm{dL}$, measured either during childhood or in adulthood, have been associated with decreased brain volume and changes in brain neurochemistry (Brubaker et al. 2010; Cecil et al. 2008; 2011; Hsieh et al. 2009). PbBs $>30 \mu \mathrm{g} / \mathrm{dL}$ are associated with a variety of decrements in cognitive function, behavior and nerve function, including postural sway and stability; decreased walking speed; decreased visuospatial function and visual-motor performance; decrements in hearing; peripheral neuropathy; psychiatric symptoms (depression, panic disorders, anxiety, hostility, confusion, anger, and schizophrenia); and changes in regional brain volumes and neurochemistry. 


\section{Table 2-29. Overview of Neurological Effects in Adults Associated with Chronic Exposure to Lead (Pb)}

\begin{tabular}{ll}
$\begin{array}{l}\text { Mean } \mathrm{PbB} \\
(\mu \mathrm{g} / \mathrm{dL})\end{array}$ & Effects associated with $\mathrm{Pb}$ exposure \\
\hline$\leq 10$ & Intellectual deficits ${ }^{\mathrm{a}}$
\end{tabular}

Intellectual deficits ${ }^{a}$

Altered mood and behavior ${ }^{b}$

Altered neuromotor neurosensory function ${ }^{\mathrm{c}}$

Neurological diseases (ALS)

Intellectual deficits ${ }^{a}$

Altered mood and behavior ${ }^{\mathrm{b}}$

Altered neuromotor neurosensory function ${ }^{c}$

Altered brain architecture and metabolism

>30-50 Intellectual deficits ${ }^{a}$

Altered mood and behavior ${ }^{b}$

Altered neuromotor neurosensory function ${ }^{c}$

Altered nerve conduction
References

Muldoon et al. 1996; Payton et al. 1998;

Power et al. 2014; Seo et al. 2014; Shih et al. 2006; Weisskopf et al. 2007; Weuve et al. 2006; Wright et al. 2003b

Bouchard et al. 2009; Buser and Scinicariello 2017; Golub et al. 2010; Opler et al. 2004; Rajan et al. 2007, 2008; Rhodes et al. 2003

Hwang et al. 2009; Ji et al. 2013; Krieg et al. 2005

Fang et al. 2010

Mantere et al. 1982; Reuben et al. 2017

Beckley et al. 2018; Yoon and Ahn et al. 2016

Chuang et al. 2007; Yokoyama et al. 1997

Brubaker et al. 2010; Cecil et al. 2008, 2011; Hsieh et al. 2009

Baker et al. 1983; Barth et al. 2002; Campara et al. 1984; Fazli et al. 2014; Goodman et al. 2002; Hogstedt et al. 1983; Meyer-Baron and Seeber 2000; Schwartz et al. 2005; Vlasak et al. 2019

Baker et al. 1983; Lucchini et al. 2000; Maizlish et al. 1995; Malekirad et al. 2013; Parkinson et al. 1986

Baker et al. 1983; Barth et al. 2002; Chia et al. 1996; Choi et al. 2012; Ghiasvand et al. 2016; Haenninen et al. 1978; Iwata et al. 2005

Araki et al. 1980, 1987, 2000; Chia et al. 1996; Hirata and Kosaka et al. 1993;

Pasternak et al. 1989; Stollery et al. 1989, 1991 


\section{Table 2-29. Overview of Neurological Effects in Adults Associated with Chronic Exposure to Lead (Pb)}

\begin{tabular}{ll}
$\begin{array}{l}\text { Mean } \mathrm{PbB} \\
(\mu \mathrm{g} / \mathrm{dL})\end{array}$ & Effects associated with $\mathrm{Pb}$ exposure \\
\hline$>50$ & Intellectual deficits
\end{tabular}

Altered mood and behavior ${ }^{b}$

Altered neuromotor neurosensory function ${ }^{\mathrm{c}}$

Altered nerve conduction

Altered brain architecture
References

Arnvig et al. 1980; Campara et al. 1984;

Fenga et al. 2016; Matte et al. 1989;

Valciukas et al. 1978

Awad el Karim et al. 1986; Zimmerman-

Tansella et al. 1983

Hanninen et al. 1998

Triebig et al. 1984

Jiang et al. 2008

antellectual deficits include decreased IQ, cognitive function, learning ability, verbal reasoning, logic, memory, and concentration.

${ }^{\text {b} A l t e r e d ~ m o o d ~ a n d ~ b e h a v i o r ~ i n c l u d e ~ d e p r e s s i o n, ~ p a n i c ~ d i s o r d e r s, ~ a n x i e t y, ~ h o s t i l i t y, ~ c o n f u s i o n, ~ a n g e r, ~ a n d ~}$ schizophrenia.

cAltered neuromotor neurosensory function includes postural sway; postural stability, decreased walking speed, decreased visuospatial function and visual-motor performance, hearing loss, and altered hearing threshold.

ALS = amyotrophic lateral sclerosis; $\mathrm{PbB}=$ blood lead concentration

Effects at Blood Pb Levels $\leq 10 \mu \mathrm{g} / \mathrm{dL}$ in Children. Numerous prospective and large cross-sectional studies provide a weight of evidence for decreased cognitive function, altered mood and behavior, and altered neuromotor and neurosensory function in children in association with exposures that result in $\mathrm{PbB}$ $<10 \mu \mathrm{g} / \mathrm{dL}$, with some studies showing effects at $\mathrm{PbB} \leq 5 \mu \mathrm{g} / \mathrm{dL}$. Study details are reviewed in the Supporting Document for Epidemiological Studies for Lead, Table 9. The cognitive outcome metric that has been most extensively studied and compared across studies is FSIQ. Tests of memory, learning, and executive function have also been used to assess cognitive function. Studies that attempt to identify associations between $\mathrm{PbB}$ and cognitive function must control for major factors known to influence or correlate with cognitive development and function, including SES, parental education and IQ, quality of caregiving, nutrition, and birth weight. Many of these same factors correlate with $\mathrm{PbB}$ and can confound associations between $\mathrm{PbB}$ and outcomes. Relationships between $\mathrm{PbB}$ and outcomes appear to be nonlinear. The Lanphear et al. (2005) pooled analysis and re-analyses (Crump et al. 2013; EPA 2014e) predict a nonlinear dose-response relationship for $\mathrm{Pb}$ in which the slope for the decrement in cognitive function in children increases with decreasing PbB. The biological significance of the observed supralinear response has been the subject of several reviews and commentaries (Bowers and Beck 2006; Hornung and Lanphear 2014; Jusko et al. 2006). Decrements in cognitive function in children have been associated with increasing $\mathrm{PbB}$ measured at various life stages, including prenatal and various metrics of 
child PbB including peak, concurrent, and cumulative. No specific life stage has been conclusively identified as the critical time period for exposure.

Cognitive function in infancy. Several prospective studies have evaluated cognitive function in infancy and early child cohorts having mean $\mathrm{PbB}<10 \mu \mathrm{g} / \mathrm{dL}$ (Table 2-30). In general, these studies provide evidence for decrements in cognitive function in association with increasing $\mathrm{PbB}$. Several studies used the Mental Development Index (MDI) score from the Bayley Scales of Infant Development (BSID), allowing comparison of results across studies (Dietrich et al. 1986, 1987, 1989; Gomaa et al. 2002; Hu et al. 2006; Jedyrychowski et al. 2009; Liu et al. 2014b). Each study found decreases in MDI scores measured from 6 to 36 months in association with increasing prenatal (e.g., maternal) or neonatal PbB. Cohort mean PbB ranged from 1.2 to $7.1 \mu \mathrm{g} / \mathrm{dL}$. In a cohort that had a mean $\mathrm{PbB}$ of $1.23 \mu \mathrm{g} / \mathrm{dL}$ (range 0.44-6.9 $\mu \mathrm{g} / \mathrm{dL}$ ), the change in MDI score measured at 24 months of age was -7.6 (95\% confidence limit [CL] -14.7, -0.62) points per $1 \log _{10}$ increase in cord PbB (Jedrychowski et al. 2009). The largest effect size was reported for a cohort that had a mean $\mathrm{PbB}$ of $8 \pm 3.8$ (SD) $\mu \mathrm{g} / \mathrm{dL}$; the change in MDI score measured at age 6 months was $-15 \pm 5.1$ (SE, $\mathrm{p}<0.03$ ) points per cord $\ln \mathrm{PbB}$ (Dietrich et al. 1986). Studies that repeatedly measured MDI scores longitudinally within the same birth cohorts found that the associations observed at 6 months persisted to later ages (Dietrich et al. 1986, 1987, 1989, 1991; Jedyrychowski et al. 2009; Liu et al. 2014b). The association between $\mathrm{Pb}$ and declining cognitive behavior appears to be exacerbated by maternal prenatal psychosocial stress. A small prospective study conducted in Shanghai, China (139 mother-infant pairs) found larger effect sizes on language development in mothers who demonstrated higher prenatal stress (Zhou et al. 2017).

Cognitive function in early childhood - FSIQ. Prospective studies initiated at time of pregnancy or birth have consistently found decrements in child FSIQ in association with increasing cohort mean PbB $<10 \mu \mathrm{g} / \mathrm{dL}$ measured at various stages of development (Table 2-30). Collectively, these studies provide evidence for effect sizes ranging from -1 to -6 FSIQ points in association with a 10-fold increase in PbB and larger effect sizes in cohorts or cohort strata having a lower mean $\mathrm{PbB}$. These studies do not consistently point to a specific life stage as being more or less vulnerable, as negative associations with FSIQ have been observed with PbB measured during pregnancy, infancy, and childhood, and measured previous to or concurrently with the FSIQ evaluation. Results of an adult follow-up of a birth cohort suggest that FSIQ decrements observed in childhood may persist to adulthood (Mazumdar et al. 2011). 
Table 2-30. Summary of Epidemiological Studies Evaluating Neurological Effects in Children at Mean Blood Lead Concentration (PbB) $\leq 10 \mu \mathrm{g} / \mathrm{dL}^{\mathrm{a}}$

\begin{tabular}{l} 
Reference and study population ${ }^{\mathrm{b}}$ \\
\hline Intellectual deficits \\
\hline Baghurst et al. 1992 \\
Prospective cohort, $\mathrm{n}=494$ children \\
followed from birth to age 7 years
\end{tabular}
followed from birth to age 7 years

Quartile range:

- Birth: 4.3, 15.0

- Mean 0-2 years: 11.6, 27.1

- Mean 0-3 years: 12.2, 28.2

- Mean 0-4 years: 12.2 , 27.7

- Lifetime average (7 years):10.8, 24.8

\begin{tabular}{lll}
\hline Blackowicz et al. 2016 & Mean (SD): & ISAT
\end{tabular}

Retrospective study; $n=12,319$ thirdgrade Hispanic children

FSIQ

\section{Outcome evaluated $\quad$ Result $^{\mathrm{c}}$}

$\beta$ (SE) for $\mathrm{PbB}$ metrics per each In PbB increase:

- Prenatal: 0.6 (1.4), $p=0.68$

- Mean 0-2 years: $-4.6(2.1), p=0.03^{*}$

- Mean 0-3 years: $-4.8(2.3), p=0.04^{\star}$

- Mean 0-4 years: $-4.6(2.4), \mathrm{p}=0.05^{\star}$

- Lifetime average: -3.7 (2.5), $p=0.14$

(1)

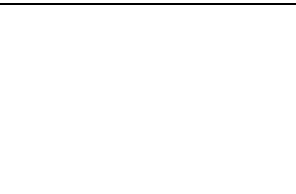

RR for failure on ISAT for 1 or $5 \mu \mathrm{g} / \mathrm{dL}$ increase in PbB:

Reading ISAT:

- $1 \mu \mathrm{g} / \mathrm{dL}$ increase: $1.07(1.05,1.10)^{*}$

- $5 \mu \mathrm{g} / \mathrm{dL}$ increase: $1.43(1.25,1.63)^{*}$ Math ISAT

- $1 \mu \mathrm{g} / \mathrm{dL}$ increase: $1.09(1.06,1.12)^{*}$

- $5 \mu \mathrm{g} / \mathrm{dL}$ increase: $1.53(1.32,1.78)^{*}$

Bellinger et al. 1992; Bellinger and Needleman 2003

Prospective cohort, $\mathrm{n}=148$ children followed from birth to age 10 years

Boucher et al. 2014

Prospective cohort, $n=93$ infants

\section{Mean (SE)}

- 6 months: 6.7 (7.0)

- 1 years: $7.7(6.5)$

- 2 years: $6.5(4.9)$
FSIQ

$\beta$ (SE) for PbB metrics per each $1 \mu \mathrm{g} / \mathrm{dL}$ increase in $\mathrm{PbB}$ :

- Prenatal: -2.55 (2.56), $p=0.57$

- 6 months: $-0.13(0.15), p=0.39$

- 2 years: $-0.58(0.21), p<0.007^{*}$

- Peak <10 $\mu \mathrm{g} / \mathrm{dL}:-1.56(\mathrm{p}=0.03)^{*}$

- Peak >10 $\mu \mathrm{g} / \mathrm{dL}$ : $-0.58(\mathrm{p}=\mathrm{NA})$

Umbilical cord $\mathrm{PbB}$ :

FTIl-fixed duration

$\beta 0.21(0.07,0.35) ; p \leq 0.01^{*}$

- Mean (SD): 4.8 (3.5)

- Range: 0.5-17.8 
2. HEALTH EFFECTS

Table 2-30. Summary of Epidemiological Studies Evaluating Neurological Effects in Children at Mean Blood Lead Concentration (PbB) $\leq 10 \mu \mathrm{g} / \mathrm{dL}^{\mathrm{a}}$

\begin{tabular}{|c|c|}
\hline Reference and study population ${ }^{b}$ & $\mathrm{PbB}(\mu \mathrm{g} / \mathrm{dL})$ \\
\hline Braun et al. 2012 & $\begin{array}{l}\text { Median }\left(5^{\text {th }}, 95^{\text {th }} \text { percentile }\right) \\
\text { at age: }\end{array}$ \\
\hline $\begin{array}{l}\text { Prospective cohort, } n=1,035 \text { mother- } \\
\text { infant pairs }\end{array}$ & $\begin{array}{ll}\text { - } & 1 \text { years: } 4.2(1.3,10.6) \\
\text { - } & 2 \text { years: } 4.6(1.5,13.4) \\
\text { - } & 3 \text { years: } 5.5(2.3,13.8) \\
\text { - } & 4 \text { years: } 5.9(2.5,12.8)\end{array}$ \\
\hline
\end{tabular}

Outcome evaluated Result ${ }^{\mathrm{c}}$

\section{Canfield et al. 2003}

Prospective cohort, $n=172$ children, followed from age 24-40 months to 5 years

\section{Mean (SD)}

Lifetime average at age 5: 7.4 (4.3)

Peak: 11.1 (7.1)

Concurrent with FSIQ: 5.8

(4.1)

\section{Coefficient} each year:

- $\quad$ PbB at 1 year: $-2.5(-5.6,0.5)$

- $\quad$ PbB at 2 years: $-3.8(-6.3,-1.4) *$

- $\quad$ PbB at 3 years: $-0.7(-3.1,1.6)$

- $\quad \mathrm{PbB}$ at 4 years: $-2.5(-5.1,0.1)$

$\beta$ per IQ for each $1 \mu \mathrm{g} / \mathrm{dL}$ increase in $\mathrm{PbB}$ at

5 years of age:

Full cohort $(n=172)$ :

- Lifetime average: $-0.57(-0.93,-0.20)$; $p=0.003^{*}$

- Peak: $-0.26(-0.47,-0.05) ; p=0.02 *$

- Concurrent: $-0.61(-0.99,-0.24) ; p<0.001$ *

Peak $\mathrm{PbB}<10(\mathrm{n}=101)$

- Lifetime average: $\mathbf{- 1 . 5 2}(-2.94,-0.09)$; $\mathrm{p}=0.04^{*}$

- Peak: -1.44 (-2.55, -0.33); $p=0.01 *$

- Concurrent: $-1.79(-3.00,-0.60) ; p=0.004^{*}$
Chandramouli et al. 2009

Prospective study; $\mathrm{n}=488$ children followed from age 4-30 months (born 1992) to age $7-8$ years
Mean (SD) at age 30 months: 4.22 (3.12)
Reading

Writing
- $\quad$ PbB 2-5 $\mu \mathrm{g} / \mathrm{dL}$ OR: $0.88(0.54,1.43) ; p=0.608$

- $\quad$ PbB 5-10 $\mu \mathrm{g} / \mathrm{dL}$ OR: $0.51(0.32,0.82)$; $p=0.006 *$

- $\quad$ PbB 2-5 $\mu \mathrm{g} / \mathrm{dL}$ OR: $1.08(0.69,1.71) ; \mathrm{p}=0.729$

- $\quad$ PbB 5-10 $\mu \mathrm{g} / \mathrm{dL}$ OR:0.49 (0.31, 0.78); $p=0.003 *$

Standard assessment test $\mathrm{A} 2$-fold increase in $\mathrm{PbB}$ was associated with a scores 
2. HEALTH EFFECTS

Table 2-30. Summary of Epidemiological Studies Evaluating Neurological Effects in Children at Mean Blood Lead Concentration (PbB) $\leq 10 \mu \mathrm{g} / \mathrm{dL}^{\mathrm{a}}$

\begin{tabular}{|c|c|c|c|}
\hline Reference and study population ${ }^{b}$ & $\mathrm{PbB}(\mu \mathrm{g} / \mathrm{dL})$ & Outcome evaluated & Result ${ }^{\mathrm{C}}$ \\
\hline $\begin{array}{l}\text { Chiodo et al. } 2004 \\
\text { Prospective study; } n=237 \text { children, age } \\
7.5 \text { years }\end{array}$ & $\begin{array}{l}\text { Mean (SD, range): } \\
5.4(3.3,1-25)\end{array}$ & FSIQ & $\begin{array}{l}\beta \text { (SE): } \\
\bullet \quad<3 \mu \mathrm{g} / \mathrm{dL}:-0.10 ; \mathrm{p} \leq 0.1^{*} \\
\bullet \quad<5 \mu \mathrm{g} / \mathrm{dL}:-0.12 ; \mathrm{p} \leq 0.1^{*} \\
\bullet \quad<7.5 \mu \mathrm{g} / \mathrm{dL}:-0.14 ; \mathrm{p} \leq 0.05^{*} \\
\bullet \quad<10 \mu \mathrm{g} / \mathrm{dL}:-0.18 ; \mathrm{p} \leq 0.01^{*} \\
\bullet \quad \text { Cohort: }-0.20 ; \mathrm{p} \leq 0.01^{*}\end{array}$ \\
\hline $\begin{array}{l}\text { Desrochers-Couture et al. } 2018 \\
\text { Prospective study; n=609 mother- } \\
\text { infant pairs with follow-up at age 3- } \\
4 \text { years }\end{array}$ & $\begin{array}{l}\text { Gmean (SD) } \\
-\quad \text { Cord: } 0.76(1.7) \\
-\quad \text { Child: } 0.70(1.7)\end{array}$ & FSIQ & $\begin{array}{l}\text { Associations with } \mathrm{PbB}(\beta \text { per } 1 \mathrm{SD} \mathrm{PbB} \text { : } \\
\text { Cord PbB } \\
\text { - } \quad \text { Male: } \mathbf{- 2 . 6 5}\left(\mathbf{- 4 . 6 6 , - 0 . 4 8 )} \mathrm{p}=\mathbf{0 . 0 4 ^ { * }}\right. \\
\text { - } \quad \text { Female: }-0.18(-1.63,1.21) \mathrm{p}=0.83 \\
\text { Child PbB } \\
\text { - } \quad \text { Male: }-0.07(-2.10,2.17), \mathrm{p}=0.96 \\
\text { - } \quad \text { Female: } 0.52(-1.23,2.40), \mathrm{p}=0.63\end{array}$ \\
\hline $\begin{array}{l}\text { Dietrich et al. } 1986 \\
\text { Prospective study; n=280 mother- } \\
\text { infant pairs }\end{array}$ & $\begin{array}{l}\text { Prenatal (maternal): } \\
\text { - } \quad \text { Mean (SD): } 8.0(3.8) \\
\text { - } \quad \text { Range: 1-27 } \\
\text { Neonatal (age } 10 \text { days): } \\
\text { - } \quad \text { Mean (SD): } 4.5(2.9) \\
\text { - } \quad \text { Range: } 1-22\end{array}$ & MDI & 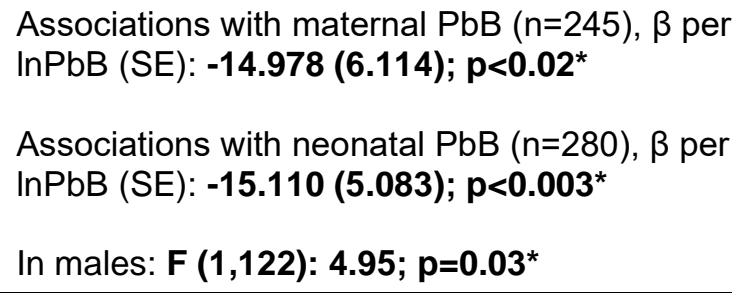 \\
\hline \multirow{3}{*}{$\begin{array}{l}\text { Dietrich et al. } 1987 \\
\text { Prospective study; n=185 mother- } \\
\text { infant pairs }\end{array}$} & \multirow{3}{*}{$\begin{array}{ll}\text { Mean }(\mathrm{SD}, \text { range): } \\
\text { - } \quad \text { Prenatal (maternal): } \\
8.3(3.8,1-27) \\
\text { - } \quad \text { Neonatal }(10 \text { days }): 4.9 \\
\quad(3.3,1-24) \\
\text { - } \quad \text { Neonatal }(3 \text { months): } \\
6.3(3.8,1-22) \\
\text { - } \quad \text { Neonatal }(6 \text { months): } \\
8.1(5.2,1-36)\end{array}$} & MDI & $\begin{array}{l}\beta \text { per InPbB (SE): } \\
\qquad \quad \text { 3-month: }-12.113(4.727) ; p=0.01^{*} \\
-\quad \text { 6-month: }-2.117(0.916) ; p=0.02^{*}\end{array}$ \\
\hline & & PDI & - $\quad \beta$ (SE): -13.248 (4.250); $p=0.002^{*}$ \\
\hline & & Motor maturity & - $\quad \beta$ (SE): $-0.570(0.260) ; p=0.03^{*}$ \\
\hline
\end{tabular}


Table 2-30. Summary of Epidemiological Studies Evaluating Neurological Effects in Children at Mean Blood Lead Concentration $(\mathrm{PbB}) \leq 10 \mu \mathrm{g} / \mathrm{dL}^{\mathrm{a}}$

\begin{tabular}{|c|c|c|c|}
\hline Reference and study population ${ }^{b}$ & $\mathrm{PbB}(\mu \mathrm{g} / \mathrm{dL})$ & Outcome evaluated & Result ${ }^{\mathrm{C}}$ \\
\hline $\begin{array}{l}\text { Dietrich et al. } 1989 \\
\text { Prospective study; n=192 mother- } \\
\text { infant pairs }\end{array}$ & 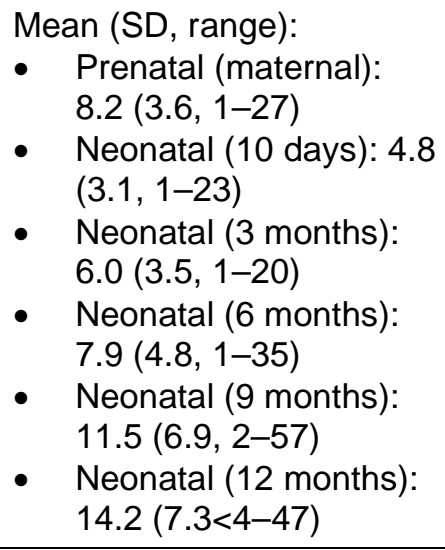 & MDI & $\begin{array}{l}\text { Structural Equation Model indicated associations } \\
(p \leq 0.05) \text { between increasing prenatal PbB and } \\
\text { 12-month MDI through decreasing birth weight. } \\
\text { Standardized regression coefficients: } \\
\text { - } \quad \text { Prenatal PbB } \rightarrow \text { birth weight: }-0.15, p \leq 0.05^{*} \\
\text { - } \quad \text { Birth weight } \rightarrow \text { 12-month MDI: } 0.18, p \leq 0.05^{\star}\end{array}$ \\
\hline $\begin{array}{l}\text { Dietrich et al. } 1991 \\
\text { Prospective study; n=258 4-year-old } \\
\text { children }\end{array}$ & $\begin{array}{l}\text { Mean (SD, range): } \\
\text { (based on Dietrich et al. } \\
\text { 1992) } \\
\text { - } \quad \text { Maternal }(6-7 \text { months): } \\
\quad 8.2(3.8,1-27) \\
\text { - Neonatal }(10 \text { days): } 4.8 \\
\quad(3.3,1-26)\end{array}$ & K-ABC scores & $\begin{array}{l}\text { Coefficients per } \mu \mathrm{g} / \mathrm{dL} \text { neonatal } \mathrm{PbB} \text { : } \\
\text { - } \quad \text { Mental processing composite: }-0.63 ; \\
\text { - }<<0.01^{*} \\
\text { - } \quad \text { Sequential processing: }-0.68, \mathrm{p}<0.01^{*} \\
\text { - } \quad \text { Nonverbal: }-0.63 ; \mathrm{p}<0.01^{*} \\
\text { - } \\
\text { Achievement: }-0.28 ; \mathrm{p}<0.05^{\star}\end{array}$ \\
\hline $\begin{array}{l}\text { Dietrich et al. } 1992 \\
\text { Prospective study; n=259 5-year-old } \\
\text { children }\end{array}$ & $\begin{array}{l}\text { Mean (SD, range): } \\
\text { - } \quad \text { Maternal }(6-7 \text { months): } \\
\quad 8.2(3.8,1-27) \\
\text { - } \quad \text { Neonatal }(10 \text { days }): 4.8 \\
\quad(3.3,1-26) \\
\text { - } \quad \text { Postnatal (5 years): } \\
\quad 11.9(6.4,3-38)\end{array}$ & FWS scores & 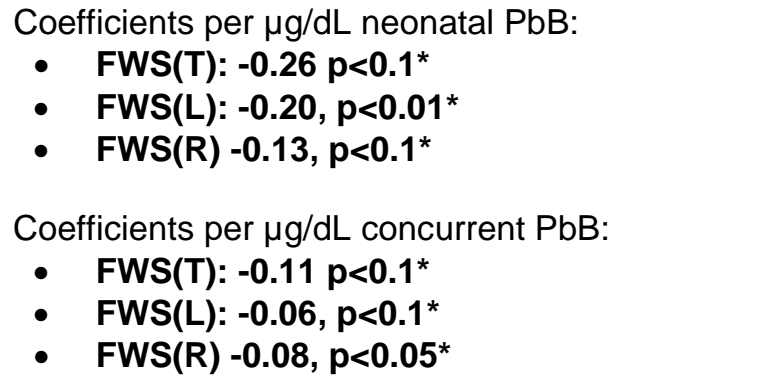 \\
\hline
\end{tabular}


Table 2-30. Summary of Epidemiological Studies Evaluating Neurological Effects in Children at Mean Blood Lead Concentration (PbB) $\leq 10 \mu \mathrm{g} / \mathrm{dL}^{\mathrm{a}}$

Reference and study population ${ }^{\text {b }}$
Dietrich et al. 1993a
Prospective study; n=253 6-7-year-old
children
Emory et al. 2003
Retrospective study; $n=79$ African-
American mother-infant pairs

American mother-infant pairs

\section{EPA 2014e}

(re-analysis of pooled cohort from Lanphear et al. 2005 with corrections to the database)

Prospective; pooled-analysis; $n=1,333$ children (4.8-6 years of age)

from seven prospective studies

Outcome evaluated
FSIQ

\section{Result $^{\mathrm{c}}$}

Adjusted $\beta(\mathrm{SE})$ in IQ per each $1 \mu \mathrm{g} / \mathrm{dL}$ :

- Prenatal: 0.15 (0.21),

- Lifetime average: $-0.13(0.11)$.

- Concurrent: $-0.33(0.14) ; p \leq 0.05^{*}$

Mean (SD, $5^{\text {th }}$ FTII, Scaled Novelty Risk Score: PbB (SD):

95 ${ }^{\text {th }}$ percentile):

(risk of mental retardation - Low risk: $0.65 \mu \mathrm{g} / \mathrm{dL}(0.80)$

- Maternal: $0.72(0.86$, later in life) $0.28-1.18)$

- Medium risk: $0.89 \mu \mathrm{g} / \mathrm{dL}(0.88)$

- High risk: $1.01 \mu \mathrm{g} / \mathrm{dL}(0.126)$

FSIQ

- Lifetime average: 12.4 $(4.1,34.8)$

- Peak: $18.0(6.2,47.0)$

- Concurrent with FSIQ: $9.7(2.5,33.2)$ $\mathrm{PbB}(95 \% \mathrm{Cl})$ :

- 6-24 months: $-2.21(-3.38,-1304)^{*}$

- Peak: $-2.86(-4.10,-1.61)^{*}$

- Concurrent: $-2.65(-3.69,-1.61)^{*}$
Mean $(95 \% \mathrm{Cl})$ :

$\beta$ in IQ for per each $\ln \mathrm{PbB}(\mu \mathrm{g} / \mathrm{dL})$ increase in

- Lifetime average: $-3.14(-4.39,-1.88)^{*}$

FSIQ change for concurrent $\mathrm{PbB}$ range:

- $2.4-10 \mu \mathrm{g} / \mathrm{dL}:-3.8$ points $(-2.3,-5.3)^{*}$

- $10-20 \mu \mathrm{g} / \mathrm{dL}:-1.8$ points $(-1.1,-2.6)^{*}$

- $20-30 \mu \mathrm{g} / \mathrm{dL}:-1.1(-0.7,-1.5)^{*}$

Evens et al. 2015

Mean (SD): 4.81 (2.22):

Participants with $\mathrm{PbB}<10$

ISAT reading scores

Regression coefficient (SE): -0.60 (0.03); $\mathrm{p}<0.0001^{*}$

Population-based retrospective cohort 100\%

study; $n=47,168$ children (third

graders)

\section{Adjusted RR:}

- $1 \mu \mathrm{g} / \mathrm{dL}: 1.06(1.05,1.07)^{\star}$

- $5 \mu \mathrm{g} / \mathrm{dL}: 1.32(1.26,1.39)^{*}$

Math
Regression coefficient (SE): $-0.50(0.03)$; $\mathrm{p}<0.0001^{*}$

Adjusted RR:

- $1 \mu \mathrm{g} / \mathrm{dL}: 1.06(1.05,1.07)^{*}$

- $5 \mu \mathrm{g} / \mathrm{dL}: 1.32(1.26,1.39)^{\star}$ 
2. HEALTH EFFECTS

Table 2-30. Summary of Epidemiological Studies Evaluating Neurological Effects in Children at Mean Blood Lead Concentration (PbB) $\leq 10 \mu \mathrm{g} / \mathrm{dL}^{\mathrm{a}}$

\begin{tabular}{|c|c|c|c|}
\hline Reference and study population ${ }^{b}$ & $\mathrm{PbB}(\mu \mathrm{g} / \mathrm{dL})$ & Outcome evaluated & Result $^{\mathrm{C}}$ \\
\hline $\begin{array}{l}\text { Geier et al. } 2017 \\
\text { Cross-sectional study; } \\
n=1,411 \text { children, age } 6-15 \text { years }\end{array}$ & $\begin{array}{l}\text { Mean (SD): } 1.32(0.95) \\
\text { <P50: 0.2-1.007 } \\
\text { P50-P75: } 1.007-1.530 \\
\text { P75-P100: } 1.530-13.50\end{array}$ & $\begin{array}{l}\text { Diagnosed with learning } \\
\text { disability }\end{array}$ & $\begin{array}{l}\text { OR per } \boldsymbol{\mu g} / \mathbf{d L}: \mathbf{1 . 1 9}(\mathbf{1 . 0 0}, \mathbf{1 . 4 0}) \mathrm{p}=\mathbf{0 . 0 4 4 ^ { * }} \\
\text { OR for quartile relative to }<50^{\text {th }} \text { percentile }(<\mathrm{P} 50) \text { : } \\
\text { - P50-75: } 1.46(1.11,1.92), \mathrm{p}=\mathbf{0 . 0 0 1 7 ^ { * }} \\
\text { - } \quad \text { P75-100: } 1.95(1.16,3.29), p=0.0033^{*}\end{array}$ \\
\hline $\begin{array}{l}\text { Gomaa et al. } 2002 \\
\text { Prospective study; } n=197 \text { children } \\
\text { followed from birth to age } 2 \text { years }\end{array}$ & $\begin{array}{l}\text { Umbilical cord mean (SD): } \\
6.7 \text { (3.4) } \\
\text { Participants with } \mathrm{PbB} \geq 10 \text { : } \\
15.7 \%\end{array}$ & MDI & $\beta$ (SE): -4.48 (2.04); $p=0.03^{*}$ \\
\hline $\begin{array}{l}\text { Hong et al. } 2015 \\
\text { Cross-sectional study; } \\
\mathrm{n}=1,001 \text { children (ages 8-11 years) }\end{array}$ & $\begin{array}{l}\text { Gmean (GSD): } 1.80(1.40) \\
5^{\text {th }}-95^{\text {th }} \text { percentile range: } \\
0.53-6.16\end{array}$ & IQ & $\begin{array}{l}\text { Regression coefficients per } 10 \text {-fold increase in } \\
\text { PbB: } \\
\begin{array}{l}\text { - Verbal IQ: }-2.64(-4.98,-0.30) ; p=0.027^{*} \\
\text { - Full-scale IQ: }-7.23(-13.39,-1.07) ; p=0.021^{*}\end{array}\end{array}$ \\
\hline $\begin{array}{l}\text { Hu et al. } 2006 \\
\text { Prospective study; n=146 mother-child } \\
\text { pairs }\end{array}$ & 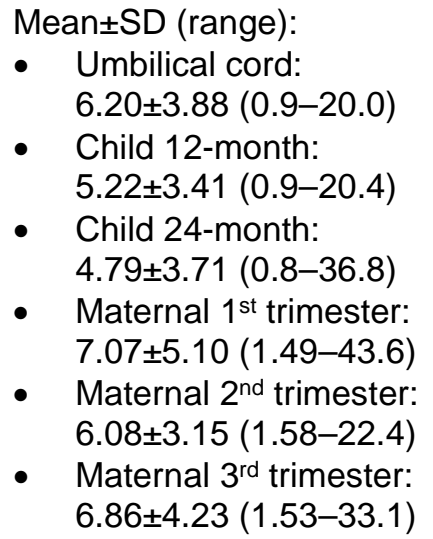 & MDI & $\begin{array}{l}\beta \text { per } 1 \text { SD change in In PbB: } \\
\text { - Umbilical cord: }-0.35(-4.72,4.03) ; p=0.88 \\
\text { - } \quad \text { Child 12-month: }-2.38(-6.24,1.49) ; p=0.23 \\
\text { - } \quad \text { Child 24-month: }-1.00(-3.93,1.94) ; p=0.50 \\
\text { - Maternal } 1^{\text {st }} \text { trimester: }-4.13(-8.10,-0.17) ; \\
\text { p=0.04* } \\
\text { Maternal } 2^{\text {nd }} \text { trimester: }-4.08(-8.29,0.12) ; \\
\text { - } \quad \text { Maternal } 3^{\text {rd }} \text { trimester: }-2.42(-6.38,1.54) ; \\
p=0.23\end{array}$ \\
\hline
\end{tabular}


Table 2-30. Summary of Epidemiological Studies Evaluating Neurological Effects in Children at Mean Blood Lead Concentration $(\mathrm{PbB}) \leq 10 \mu \mathrm{g} / \mathrm{dL}^{\mathrm{a}}$

\begin{tabular}{ll}
\hline Reference and study population $^{\mathrm{b}}$ & $\mathrm{PbB}(\mu \mathrm{g} / \mathrm{dL})$ \\
\hline Jedrychowski et al. 2009 & Umbilical cord $\mathrm{PbB}$ \\
& - Gmean: 1.29 \\
Prospective study; $\mathrm{n}=444$ children & - Median: 1.23 \\
followed prenatally to age 3 years & - Range: $0.44-6.90$
\end{tabular}

$\mathrm{MDI}$

Outcome evaluated

Result $^{\mathrm{c}}$

$\beta$ per lg cord $\mathrm{PbB} \pm \mathrm{SE}$ :

- 12 months: $-5.419 \pm 2.935$ (-11.188, 0.3495); $\mathrm{p}=0.066$

- 24 months: $-7.653 \pm 3.577(-14.684,-0.623)$; $p=0.033^{*}$

- 36 months: $-6.717 \pm 2.964(-12.546$, $-0.889) ; p=0.024^{*}$

- All participants with $\mathrm{PbB}<5$ (combination of all testing times): $-6.618 \pm 2.499(-11.517$, 1.719); $p=0.008^{*}$

\section{Jusko et al. 2008}

Prospective study; $\mathrm{n}=174$ children recruited at age $24-30$ months and evaluated for FSIQ at 6 years
Lifetime average:
- $\quad$ Mean (SD): $7.2(4.1)$
- Range: 1.4-27.1
- Participants <10: $77 \%$

FSIQ

- Associations between increasing $\mathrm{PbB}$ and decreasing FSIQ measured at age 6 years $(p=0.003) *$

- Comparison of children with PbB of 59.9 (high) to those with $\mathrm{PbB}<5$ (low) showed a 4.9-point decrease in FSIQ score (low: 91.3; high 86.4; $p=0.04$ )*

- Adjusted changes in IQ for each $1 \mu \mathrm{g} / \mathrm{dL}$ increase in peak lifetime $\mathrm{PbB}$ ( $p$ not reported):
o 2.1-10 $\mu \mathrm{g} / \mathrm{dL}:-1.2$
o 10-20 $\mu \mathrm{g} / \mathrm{dL}:-0.32$
o 20-30 $\mu \mathrm{g} / \mathrm{dL}:-0.15$

Gmean (GSD):

Prospective birth cohort; $n=884$ mother Late pregnancy: $1.3(1.5)$

MDI

infant pairs 
2. HEALTH EFFECTS

Table 2-30. Summary of Epidemiological Studies Evaluating Neurological Effects in Children at Mean Blood Lead Concentration (PbB) $\leq 10 \mu \mathrm{g} / \mathrm{dL}^{\mathrm{a}}$

\begin{tabular}{l} 
Reference and study population \\
\hline Kordas et al. 2011 \\
Prospective study; $\mathrm{n}=186$ children \\
followed prenatally (to age 4 years)
\end{tabular}

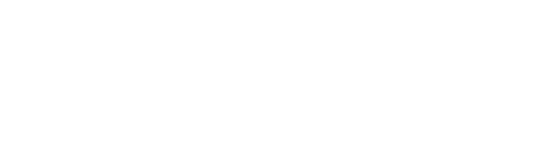

\section{Lanphear et al. 2000a}

Cross-sectional study;

$n=4,853$ children (ages $6-16$ years)

$\mathrm{PbB}(\mu \mathrm{g} / \mathrm{dL})$

Mean (SD):

- Umbilical cord: 6.6 (3.3)

- 24 months: 8.1 (4.4)

- 48 months: 8.1 (3.6)

\begin{tabular}{l} 
Outcome \\
MDI (24 mo \\
\hline GDI (24 mo \\
Memory sc \\
Arithmetic
\end{tabular}

Gmean: 1.9

Participants with $\mathrm{PbB}$

- $\quad \geq 5: 9.7 \%$

- $\geq 10: 2.1 \%$

\begin{tabular}{|c|c|}
\hline & - $\mathrm{PbB}<10:-0.89(0.32) ; \mathrm{p}=0.008^{*}$ \\
\hline Reading & 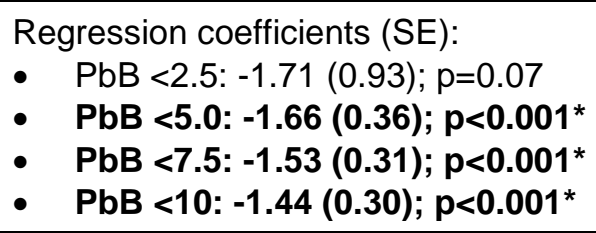 \\
\hline Block design & 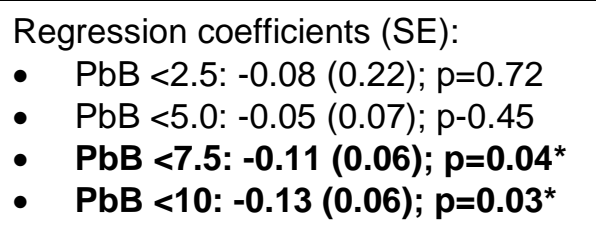 \\
\hline Digit span & $\begin{array}{l}\text { Regression coefficients (SE): } \\
\text { - } \quad \mathrm{PbB}<2.5:-0.25(0.17) ; \mathrm{p}=0.17 \\
\text { - } \quad \mathrm{PbB}<5.0:-0.09(0.07), \mathrm{p}=0.20 \\
\text { - } \quad \mathrm{PbB}<7.5:-0.09(0.05) ; \mathrm{p}=0.11 \\
\text { - } \quad \mathrm{PbB}<\mathbf{1 0}: \mathbf{- 0 . 0 8}\left(\mathbf{0 . 0 4 )} ; \boldsymbol{p}=\mathbf{0 . 0 3}^{\star}\right.\end{array}$ \\
\hline
\end{tabular}


Table 2-30. Summary of Epidemiological Studies Evaluating Neurological Effects in Children at Mean Blood Lead Concentration $(\mathrm{PbB}) \leq 10 \mu \mathrm{g} / \mathrm{dL}^{\mathrm{a}}$

\begin{tabular}{ll}
\hline Reference and study population $^{\mathrm{b}}$ & $\mathrm{PbB}(\mu \mathrm{g} / \mathrm{dL})$ \\
\hline Lanphear et al. 2005 $_{\text {(same cohorts used for Budtz- }}$ & Mean $(96 \% \mathrm{CL})$ : \\
Jorgensen et al. 2013) & - Lifetime average: 12.4 \\
& - $(4.1,34.8)$ \\
Prospective; pooled-analysis; & Peak: $18.0(6.2,47.0)$ \\
$\mathrm{n}=1,333$ children (4.8-6 years of age) & Concurrent with FSIQ: \\
from & $9.7(2.5,33.2)$
\end{tabular}

from seven prospective studies

Out

Result $^{c}$

$\beta$ in IQ for per each In $\mathrm{PbB}(\mu \mathrm{g} / \mathrm{dL})$ increase in $\mathrm{PbB}$ :

- 6-24 months: $-2.04(-3.27,-0.81)^{*}$

- Lifetime average: $-3.04(-4.33,-1.75)$

- Peak: $-2.85(-4.10,-1.60)^{*}$

- Concurrent: $-2.70(-3.74,-1.66)^{*}$

FSIQ change for lifetime average $\mathrm{PbB}$ :

- $2.4-10 \mu \mathrm{g} / \mathrm{dL}:-3.9$ points $(-2.4,-5.3)^{*}$

- $10-20 \mu \mathrm{g} / \mathrm{dL}:-1.9$ points $(-1.2,-2.6)^{*}$

- $20-30 \mu \mathrm{g} / \mathrm{dL}:-1.1(-0.7,-1.5)^{*}$

Lanphear et al. 2019
(re-analysis of data reported in
Lanphear et al. 2005; same cohorts
used for Budtz-Jorgensen et al. 2013)

Prospective; pooled-analysis;
$\mathrm{n}=1,333$ children (4.8-6 years of age)
from seven prospective studies

Median (96\% CL):

- Lifetime average: 11.9

$$
(3.6,34.5)
$$

- Peak: $18.0(6.2,47.0)$

Concurrent with FSIQ: 9.7

$(2.5,33.2)$

FSIQ

$\beta$ in IQ for per each In $\mathrm{PbB}(\mu \mathrm{g} / \mathrm{dL})$ increase in $\mathrm{PbB}$ :

- 6-24 months: $-2.21(-3.38,-1.04)^{*}$

- Peak: $-2.86(-4.10,-1.61)^{*}$

- Lifetime average: $-3.25(-4.51,-1.99)^{*}$

- Concurrent: $-2.65(-3.69,-1.61)^{*}$

FSIQ change for concurrent PbB:

- $2.4-10 \mu \mathrm{g} / \mathrm{dL}:-3.8$ points $(-2.3,-5.3)^{*}$

- $10-20 \mu \mathrm{g} / \mathrm{dL}$ : -1.8 points $(-1.1,-2.6)^{*}$

- $20-30 \mu \mathrm{g} / \mathrm{dL}:-1.1(-0.7,-1.5)^{*}$

Lin et al. 2013

\section{Umbilical cord}

- Mean (SD): 1.30 (0.75)

Prospective (Taiwan Birth Panel Study; birth dates: April 2004-January

- Range: 0.016-4.32

Cognitive score

2005) of 230 mother-infant pairs from

Taipei, Taiwan, followed until age

2 years

Regression analysis comparing $\mathrm{PbB} \geq 1.645$ ( $75^{\text {th }}$ percentile) and $\mathrm{PbB}<1.645$. Adjusted $\beta$ (SE):

- Total score: $\mathbf{- 4 . 2 3}(1.82) ; \mathrm{p}<0.05^{*}$

- Cognitive: $-5.35(2.19) ; p<0.05^{*}$

- Language: -2.53 (1.89); $\mathrm{p} \geq 0.05$ 
Table 2-30. Summary of Epidemiological Studies Evaluating Neurological Effects in Children at Mean Blood Lead Concentration (PbB) $\leq 10 \mu \mathrm{g} / \mathrm{dL}^{\mathrm{a}}$

\begin{tabular}{|c|c|c|c|}
\hline Reference and study population ${ }^{b}$ & $\mathrm{PbB}(\mu \mathrm{g} / \mathrm{dL})$ & Outcome evaluated & Result ${ }^{\mathrm{C}}$ \\
\hline $\begin{array}{l}\text { Liu et al. 2014b } \\
\text { Prospective study; } n=243 \text { infants } \\
\text { followed from birth to age } 3 \text { years }\end{array}$ & $\begin{array}{l}\text { Umbilical cord (mean } \pm S D) \text { : } \\
\text { - Low } \mathrm{PbB} \text { group: } \\
1.35 \pm 0.26 \\
\text { - High } \mathrm{PbB} \text { group: } \\
5.63 \pm 0.32\end{array}$ & MDI & 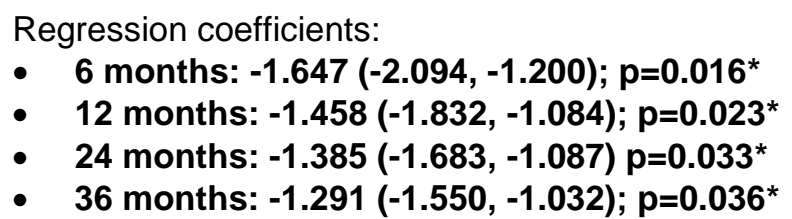 \\
\hline
\end{tabular}

Increasing $\mathrm{PbB}$ at ages 24 and 36 months was associated with decreasing MDI scores measured at 24 and 36 months, respectively; $\beta$ :

- 24 months: $-1.403 ; p=0.026^{*}$

- 36 months: $-1.298 ; p=0.036^{*}$

$\begin{array}{ll}\text { PDI } & \begin{array}{l}\text { Regression coefficients at } 36 \\ \text { months: }-1.302(-1.572,-1.031) ; p=0.041^{*}\end{array}\end{array}$

Mazumdar et al. 2011

A prospective of 43 adults followed from birth (1979-1981) to age 2830 years

McLaine et al. 2013 Mean (SD):

- Cord: $6.5(5.3)$

- 6 months: 8.0 (5.3)

- 12 months: $10.0(6.7)$

- Age 2 years: $7.7(4.0)$

- Age 4 years: $6.7(3.6)$

- Age 10 years: $3.0(2.7)$

\section{Median: 4.2}

Interquartile range: $2.6,6.0$

FSIQ months: -1.302 (-1.572, -1.031$)$; $p=0.041 *$

Change in FSIQ per $1 \mu \mathrm{g} / \mathrm{dL}$ increase in $\mathrm{PbB}$. $\beta$ for average late childhood $\mathrm{PbB}$ (mean of 4 - and 10-year PbB):

- Unadjusted: $-1.89(-3.30,-0.47), \mathrm{p}<0.01^{*}$

- Adjusted for maternal IQ: $-1.11(-2.29,0.06)$

- Other adjustments: $95 \%$ UCLs $<0$

Population-based retrospective cohort study; $n=3,406$ children

(kindergarteners)
Mean differences $(95 \% \mathrm{Cl})$ in PALS-K scores (85\% CL), compared to $\mathrm{PbB}<5$ :

- PbB 5-9: -4.51 (-6.61, -2.85); $p>0.182$

- $\mathrm{PbB} \geq 10$ : -10.13 (-13.30, -6.96$) ; \mathrm{p}>0.182$

PR for falling below the PALS-K benchmark, compared to $\mathrm{PbB}<4$ :

- PbB 5-9: 1.21 (1.19, 1.23); $p<0.001^{*}$

- $\mathrm{PbB} \geq 10$ : $1.56(1.51,1.60) ; \mathrm{p}<0.001^{*}$ 
2. HEALTH EFFECTS

Table 2-30. Summary of Epidemiological Studies Evaluating Neurological Effects in Children at Mean Blood Lead Concentration $(\mathrm{PbB}) \leq 10 \mu \mathrm{g} / \mathrm{dL}^{\mathrm{a}}$

\begin{tabular}{|c|c|c|c|}
\hline Reference and study population ${ }^{b}$ & $\mathrm{PbB}(\mu \mathrm{g} / \mathrm{dL})$ & Outcome evaluated & Result ${ }^{\mathrm{C}}$ \\
\hline $\begin{array}{l}\text { Min et al. } 2009 \\
\text { Prospective study; } n=267 \text { children } \\
\text { followed prenatally age } 11 \text { years }\end{array}$ & $\begin{array}{l}\text { Mean (SD): } \\
\text { - } 4 \text { years: } 7.0 \mu \mathrm{g} / \mathrm{dL}(4.1)\end{array}$ & FSIQ & $\begin{array}{l}\text { Regression coefficient }(\mathrm{SE}) \text { : } \\
\text { - } \quad 4 \text { years: }-0.50(0.20), p<0.05^{*} \\
\text { - } 9 \text { years: }-0.41(0.19), p<0.05^{*} \\
-\quad 11 \text { years: }-0.54(0.19) ; p<0.01^{*}\end{array}$ \\
\hline
\end{tabular}

Miranda et al. $2009 \quad$ Mean: $4.8 \quad$ EOG scores $\quad$ Multivariate regression coefficients for PbB ( $\mu$ g/dL)

Population-based retrospective cohort Range: 1-16

study; $n=57,6784^{\text {th }}$ grade children

- PbB 2: $-0.30(-0.58,-0.01) ; p<0.0001^{*}$

- $\quad$ PbB 3: $-0.46(-0.73,-0.19) ; p<0.0001^{*}$

- $\quad$ PbB 4: $-0.52(-0.79,-0.24) ; p<0.0001^{*}$

- $\quad$ PbB 5: $-0.80(-1.08,-0.51) ; p<0.0001^{*}$

- $\quad$ PbB 6: -0.99 (-1.29, -0.68); $p<0.0001^{*}$

- $\quad$ PbB 7: -1.07 (-1.40, -0.74); $p<0.0001^{*}$

- $\quad$ PbB 8: -1.35 (-1.73, -0.97$) ; p<0.0001 *$

- $\quad$ PbB 9: -1.20 (-1.64, -0.75); $p<0.0001^{*}$

- $\mathrm{PbB} \geq 10$ : -1.75 (-2.09, -1.41); $\mathrm{p}<0.0001^{*}$

\section{Polanska et al. 2018}

Prospective study; $n=538$ mother-child pairs with follow-up of 303 children at age 2 years
- $\quad 2^{\text {nd }}$ trimester: 0.99 (0.15) $(0.29,2.63)$

- Cord: 0.96 (0.16) (0.24, 5.65)
Gmean (SD) (range)

BSID III

$\beta$ score per $\mu \mathrm{g} / \mathrm{dL}$ cord In PbB:

Cognitive score:

- Females: $0.34(-1.30,1.98), p=0.68$

- Males: -2.07 (-4.07, -0.06), $\mathrm{p}=0.04^{*}$

Language score:

- Females: $-0.29(-2.23,1.65), p=0.77$

- Males: $-0.43(-2.81,1.95), p=0.72$

Rodrigues et al. 2016

Median (P24, P75,
maximum)

Prospective study; n=812 mother-child • Sirajdikhan: 7.6 (5.5, pairs with follow-up of 5251, children at age $2-3$ years $\beta$ score per $\mu g / L$ child $\mathrm{PbB}$ :

Cognitive score:

- Sirajdikhan: $-0.17(0.09), p=0.05^{*}$

- Pabna: 0.02 (0.12), $p=0.87$ 10.4)

- Pabna: <LOD (<LOD, $3.8,13.8)$ 
Table 2-30. Summary of Epidemiological Studies Evaluating Neurological Effects in Children at Mean Blood Lead Concentration (PbB) $\leq 10 \mu \mathrm{g} / \mathrm{dL}^{\mathrm{a}}$

\begin{tabular}{|c|c|}
\hline Reference and study population ${ }^{b}$ & $\mathrm{PbB}(\mu \mathrm{g} / \mathrm{dL})$ \\
\hline Rooney et al. 2018 & $\begin{array}{l}\text { Mean }(\mathrm{SD}) \text { at age } 8- \\
12 \text { years }\end{array}$ \\
\hline $\begin{array}{l}\text { Longitudinal study; } n=330 \text { children wi } \\
\text { follow-up at age } 12 \text { and } 17 \text { years }\end{array}$ & $\begin{array}{l}\text { - } \quad \text { Females: } 4.42(2.19) \\
\text { - } \quad \text { Males: } 5.26(2.73)\end{array}$ \\
\hline
\end{tabular}

Ruebner et al. 2019

Median (P24, P75): $1.2(0.8,1.8)$

Cross-sectional study; $n=412$ children (median age 15.4 years) from prospective study of CKD in children

\section{Schnaas et al. 2006}

Prospective study; $n=150$ followed from birth to age 10 years

Maternal during full pregnancy

- Gmean (range): 8.0 (1-33)

Outcome evaluated
Learning, memory, and
executive function test

\section{Result ${ }^{\mathrm{c}}$}

Genetic variants of N-methyl-D-aspartate receptors (NMDAR subunits GRIN2A and GRIN2B) were effect modifiers on associations between increasing $\mathrm{PbB}$ (at age 8-12 years) and decreasing performance on learning and memory and executive functions

$\beta$ score per $\mu \mathrm{g} / \mathrm{dL}(95 \% \mathrm{Cl})$ :

FSIQ:

- $\quad$ PbB: $-2.1(-3.9,-0.2), p=0.029 * C P T$ variability score:

- PbB: $1.8(0.2,3.5), p=0.033^{*}$

$\beta$ assessed at age $6-10$ years:

FSIQ

Maternal $\mathrm{PbB}$ during

pregnancy weeks 28-36

- $\quad$ Gmean $(95 \% \mathrm{Cl}): 7.3$ (1.5-17.4)

Child $1-5$ years

- Gmean (range): 9.8 (2.8-36.4)

Child 6-10 years

- Gmean (range): 6.2 (2.2-18.6)
- Ln maternal PbB (28 weeks pregnancy): -4.00 (-6.37, -1.65); $p=0.001 *$

- Ln child PbB (6-10 years): $-2.45(-4.09,-0.81) ; p=0.003^{*}$ 
Table 2-30. Summary of Epidemiological Studies Evaluating Neurological Effects in Children at Mean Blood Lead Concentration (PbB) $\leq 10 \mu \mathrm{g} / \mathrm{dL}^{\mathrm{a}}$

\begin{tabular}{ll}
\hline Reference and study population $^{\mathrm{b}}$ & $\mathrm{PbB}(\mu \mathrm{g} / \mathrm{dL})$ \\
\hline Shadbegian et al. 2019 & Mean $(\mathrm{SD})$ \\
& Whole cohort \\
Retrospective study; & $-<10 \mu \mathrm{g} / \mathrm{dL}: 3.66(1.90)$ \\
$\mathrm{n}=560,624$ children with $\mathrm{PbB}$ & $-\leq 5 \mu \mathrm{g} / \mathrm{dL}: 2.89(1.18)$ \\
measured at ages 0-5 years and & CEM stratum \\
cognitive assessments during school & $\leq 5 \mu \mathrm{g} / \mathrm{dL}: 2.40(1.24)$ \\
\end{tabular}

Standardized academic

achievement tests

$<10 \mu \mathrm{g} / \mathrm{dL}: 3.66(1.90)$

$\leq 5 \mu \mathrm{g} / \mathrm{dL}: 2.40(1.24)$

\section{Result $^{\mathrm{C}}$}

Percentile score change relative to $\leq 1 \mu \mathrm{g} / \mathrm{dL}$ CEM

stratum (SE) for children who had geometric mean $\mathrm{PbB}>1$ and $\leq 5 \mu \mathrm{g} / \mathrm{dL}$ :

Math percentile for $\mathrm{PbB}$ strata:

- $2 \mu \mathrm{g} / \mathrm{dL}:-0.38$ (0.19), $\mathrm{p}>0.05$

- $3 \mu \mathrm{g} / \mathrm{dL}:-0.56(0.20), \mathrm{p}<0.01^{*}$

- $4 \mu \mathrm{g} / \mathrm{dL}:-0.96(0.23), \mathrm{p}<0.001^{*}$

- $5 \mu \mathrm{g} / \mathrm{dL}:-0.51(0.30), \mathrm{p}>0.05$

Reading percentile for $\mathrm{PbB}$ strata:

- $2 \mu \mathrm{g} / \mathrm{dL}:-0.55$ (0.19), $\mathrm{p}<0.01^{*}$

- $3 \mu \mathrm{g} / \mathrm{dL}:-1.02(0.20), \mathrm{p}<0.001^{*}$

- $4 \mu \mathrm{g} / \mathrm{dL}:-1.31$ (0.23), $\mathrm{p}<0.001^{*}$

- $5 \mu \mathrm{g} / \mathrm{dL}:-0.97$ (0.30), $\mathrm{p}>0.001^{*}$

Sobin et al. 2015

Mean (SD):

Working memory

$\beta$ (SE): 0.11 (0.03), $p<0.01^{*}$

- Females: $2.7(1.5)$

Cross-sectional study; $n=252$ children (age 5.1-11.8 years)

- Males: 2.4 (1.0)

- $96 \%<5.0 \mu \mathrm{g} / \mathrm{dL}$

Taylor et al. 2017

Mean (SD):

FSIQ

- Maternal (11 weeks):

3.67 (1.46)

Prospective study; $n=14,062$ motherinfant pairs with follow-up of 404 children at age 4 years and

- $\quad$ Child (30 months): 4.22 (3.12) $\beta$ for score per $\mu \mathrm{g} / \mathrm{dL}$ at age 8 years: Females:

- Verbal: $0.71(0.11,1.32), p=0.021$ *

- Performance: $0.57(-0.11,1.24), p=0.099$

- Total: $0.73(0.13,1.33), p=0.017$

Males:

- Verbal: $-0.15(-0.90,0.60), p=0.72$

- Performance: $-0.42(-1.19,0.35), p=0.29$

- Total: $-0.29(-1.02,0.44), p=0.44$ 
2. HEALTH EFFECTS

Table 2-30. Summary of Epidemiological Studies Evaluating Neurological Effects in Children at Mean Blood Lead Concentration (PbB) $\leq 10 \mu \mathrm{g} / \mathrm{dL}^{\mathrm{a}}$

\begin{tabular}{|c|c|c|c|}
\hline Reference and study population ${ }^{b}$ & $\mathrm{PbB}(\mu \mathrm{g} / \mathrm{dL})$ & Outcome evaluated & Result $^{c}$ \\
\hline $\begin{array}{l}\text { Tellez-Rojo et al. } 2006 \\
\text { Prospective study; n=294 children } \\
\text { followed from birth to age } 2 \text { years }\end{array}$ & $\begin{array}{l}\text { Mean (SD): } \\
\text { - } \quad \text { Cord: } 4.85(3.0) \\
\text { - } \quad 12 \text { months: } 4.27(2.14) \\
\text { - } \quad 24 \text { months: } 4.28(2.25)\end{array}$ & MDI & $\begin{array}{l}\beta \text { per In PbB } 12 \text { months: } \\
-\quad<10 \mu \mathrm{g} / \mathrm{dL}:-0.15, \mathrm{p}=0.57 \\
\bullet \quad \geq 10 \mu \mathrm{g} / \mathrm{dL}:-0.71, \mathrm{p}=0.17 \\
\beta \text { per InPbB } 24 \text { months: } \\
-\quad<10 \mu \mathrm{g} / \mathrm{dL}: \mathbf{- 1 . 0 4 ,} \mathrm{p}<0.01 * \\
-\quad \geq 10 \mu \mathrm{g} / \mathrm{dL}: 0.07, \mathrm{p}=0.84\end{array}$ \\
\hline $\begin{array}{l}\text { Vigeh et al. } 2014 \\
\text { Prospective study; } n=174 \text { mother-child } \\
\text { pairs, birth to } 36 \text { months }\end{array}$ & $\begin{array}{l}\text { Mean } \pm \text { SD (range): } \\
\text { - } \quad 1^{\text {st }} \text { trimester: } 4.15 \pm 2.43 \\
(1.6-20.5) \\
\text { - } \quad 2^{\text {nd }} \text { trimester: } \\
\quad 3.44 \pm 1.28(1.1-7.5) \\
\text { - } \quad 3^{\text {rd }} \text { trimester: } 3.78 \pm 1.40 \\
\quad(1.5-8.0) \\
\text { Umbilical cord: } \\
\quad 2.86 \pm 1.09(1.2-6.9)\end{array}$ & ECDI score & OR $1^{\text {st }}$ trimester: $1.74(1.18-2.57) ; p=0.005^{*}$ \\
\hline $\begin{array}{l}\text { Wasserman et al. 1994, 1997, } 2003 \\
\text { Prospective study; n=332 children age } \\
4 \text { years, } 261 \text { children age } 7 \text { years, } 167 \\
\text { children age 10-12 years }\end{array}$ & $\begin{array}{l}\text { Mean (SD): } \\
\text { - Age } 4 \text { years: } \\
\text { 9.6, Pristina } \\
\text { 39.9, K. Mitrovica } \\
\text { - Age 10-12 years: } \\
6.1 \text { (1.9), Pristina } \\
30.9(9.6), \text { K. Mitrovica }\end{array}$ & FSIQ & 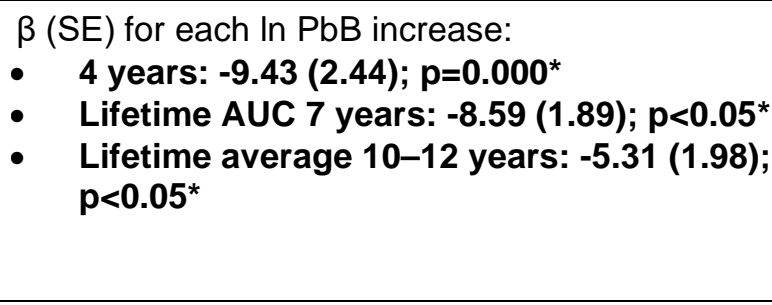 \\
\hline \multirow{3}{*}{$\begin{array}{l}\text { Zhang et al. } 2013 \\
\text { Population-based retrospective cohort } \\
\text { study; n=8,831, 7,708, and } \\
4,742 \text { students in grades } 3,5 \text {, and } 8 \text {, } \\
\text { respectively }\end{array}$} & \multirow{3}{*}{$\begin{array}{l}\text { Mean (SD): } 7.12(7.26) \\
\text { Analysis: academic } \\
\text { achievement }\end{array}$} & Math & $\begin{array}{ll}\text { - } & \text { OR 1-5 PbB }(\mu \mathrm{g} / \mathrm{dL}): 1.42(1.24,1.63)^{*} \\
\text { - } & \text { OR } 6-10 \mathrm{PbB}(\mu \mathrm{g} / \mathrm{dL}): 2.00(1.74,2.30)^{*} \\
\text { - } & \text { OR >10 PbB }(\mu \mathrm{g} / \mathrm{dL}): 2.40(2.07,2.77)^{*}\end{array}$ \\
\hline & & Science & $\begin{array}{ll}\text { - } & \text { OR 1-5 PbB ( } \mu \mathrm{g} / \mathrm{dL}): 1.33(1.10,1.62)^{*} \\
\text { - } & \text { OR 6-10 PbB }(\mu \mathrm{g} / \mathrm{dL}): 2.22(1.82,2.72)^{*} \\
\text { - } & \text { OR >10 PbB }(\mu \mathrm{g} / \mathrm{dL}): 2.26(1.84,2.78)^{*}\end{array}$ \\
\hline & & Reading & $\begin{array}{ll}\text { - } & \text { OR 1-5 PbB }(\mu \mathrm{g} / \mathrm{dL}): 1.45(1.27,1.67)^{*} \\
\text { - } & \text { OR } 6-10 \mathrm{PbB}(\mu \mathrm{g} / \mathrm{dL}): 2.21(1.92,2.55)^{\star} \\
\text { - } & \text { OR >10 PbB }(\mu \mathrm{g} / \mathrm{dL}): 2.69(2.31,3.12)^{*}\end{array}$ \\
\hline
\end{tabular}


Table 2-30. Summary of Epidemiological Studies Evaluating Neurological Effects in Children at Mean Blood Lead Concentration (PbB) $\leq 10 \mu \mathrm{g} / \mathrm{dL}^{\mathrm{a}}$

\begin{tabular}{|c|c|c|c|}
\hline Reference and study population ${ }^{b}$ & $\mathrm{PbB}(\mu \mathrm{g} / \mathrm{dL})$ & Outcome evaluated & Result ${ }^{\mathrm{C}}$ \\
\hline $\begin{array}{l}\text { Zhou et al. } 2017 \\
\text { Prospective study; } n=139 \text { mother- } \\
\text { infant pairs followed from birth to } 24 \text { - } \\
36 \text { months }\end{array}$ & $\begin{array}{l}\text { Gmean }(95 \% \mathrm{Cl}) \\
\text { Mid-late pregnancy: } 3.30 \\
(3.05,3.57)\end{array}$ & $\begin{array}{l}\text { Gesell Development } \\
\text { Scale, prenatal stress } \\
\text { Global Severity Index }\end{array}$ & $\begin{array}{l}\beta \text { for development quotient per } \mu \mathrm{g} / \mathrm{dL} \text { : } \\
\text { All children: } \\
\text { - } \quad \text { Adaptive behavior: } 3.60(-3.64,10.83) \\
\text { - Language: }-6.76(-17.29,3.77) \\
\text { - } \quad \text { Social behavior: }-6.45(-15.55,2.65)\end{array}$ \\
\hline
\end{tabular}

Children from mothers who exhibited high prenatal stress:

- Adaptive behavior: $-\mathbf{1 7 . 9 3}(-35.83,-0.03)^{*}$

- Language: $-33.82(-60.04,-7.59)^{*}$

- Social behavior: $-41.00(-63.11-18.89)^{*}$

Mood and behavior

\section{Arbuckle et al. 2016}

Cross-sectional study;

$n=2,097$ children aged $6-19$ years

\section{Boucher et al. 2012}

Prospective study; $\mathrm{n}=272$ children (mean age 11.3 years)
Gmean $(95 \% \mathrm{Cl})$

age $6-11$ years
$(0.81,0.99)$

age $12-19$ years: 0.80

$(0.74,0.85)$

Mean \pm SD (range):

- Umbilical cord:

$4.7 \pm 3.3(0.8-20.9)$

- Current: $2.7 \pm 2.2(0.4-$ 12.8)
ADD/ADHD

- ADDIADHD: $2.39(1.32,4.32)^{*}$

- Emotional symptoms: $1.08(0.68,1.71)$

- Hyperactivity/inattention: $2.33(1.59,3.43)^{*}$

- Total difficulties: $2.16(1.33,3.51)^{*}$

ADHD-inattentive type

Adjusted ORs:

- T2 (n=94): $1.06(0.42,2.66)$

- T3 (n=91): $1.01(0.38,2.64)$

ADHD-hyperactive- $\quad$ T2(n=94): $4.01(1.06,15.23)^{*}$

impulsive type • T3(n=91): $5.52(1.38,22.12)^{*}$

ODD and/or CD

- $\mathrm{T} 2(\mathrm{n}=94): 1.90(0.88,4.11)$

- $\mathrm{T3}(\mathrm{n}=91): 1.53(0.67,3.49)$

Behavior problem scores Umbilical cord $\mathrm{PbB}$ was not associated with associated with behavior problem scores (data not reported). 
2. HEALTH EFFECTS

Table 2-30. Summary of Epidemiological Studies Evaluating Neurological Effects in Children at Mean Blood Lead Concentration $(\mathrm{PbB}) \leq 10 \mu \mathrm{g} / \mathrm{dL}^{\mathrm{a}}$

\begin{tabular}{|c|c|c|c|}
\hline Reference and study population ${ }^{b}$ & $\mathrm{PbB}(\mu \mathrm{g} / \mathrm{dL})$ & Outcome evaluated & Result $^{\mathrm{c}}$ \\
\hline $\begin{array}{l}\text { Braun et al. } 2006 \\
\text { Cross-sectional study; } \\
n=4,704 \text { children (ages 4-15 years) }\end{array}$ & $\begin{array}{ll}\text { Quintiles: } \\
\text { - } \quad \text { Q1 (reference): ND- } \\
\text { - } 0.7 \\
\text { - } \quad \text { Q2: } 0.8-1.0 \\
\text { - } \quad \text { Q3: } 1.1-1.3 \\
\text { - } \quad \text { Q4: } 1.4-2.0 \\
\text { - } \quad \text { Q5: } \geq 2.0\end{array}$ & ADHD & $\begin{array}{l}\text { Adjusted ORs: } \\
\text { - } \quad \text { Q2: } 1.1(0.4,3.4) ; p=0.804 \\
\text { - } \quad \text { Q3: } 2.1(0.7,6.8) ; p=0.195 \\
\text { - } \quad \text { Q4: } 2.7(0.9,8.4) ; p=0.086 \\
\text { - } \quad \text { Q5: } 4.1(\mathbf{1 . 2}, \mathbf{1 4 . 0}) ; p=0.026^{*} \\
\text { - } \quad \text { p-trend: } 0.012^{*}\end{array}$ \\
\hline $\begin{array}{l}\text { Braun et al. } 2008 \\
\text { Cross-sectional study; } \\
n=3,082 \text { children (ages 8-15 years) }\end{array}$ & $\begin{array}{l}\text { Quartiles: } \\
\text { - } \quad \text { Q1 (reference): 0.2-0.7 } \\
\text { - } \quad \text { Q2: } 0.8-1.0 \\
\text { - } \quad \text { Q3: } 1.1-1.4 \\
\text { - } \quad \text { Q4: }>2.0\end{array}$ & Conduct disorder & $\begin{array}{l}\text { Adjusted ORs: } \\
\text { - Q2: } 7.24(1.06,49.47)^{*} \\
\text { - Q3: } 12.37\left(2.37,64.56^{*}\right) \\
\text { - } \quad \text { Q4: } 8.64(1.87,40.04)^{*}\end{array}$ \\
\hline $\begin{array}{l}\text { Choi et al. } 2016 \\
\text { Longitudinal study; } n=2,159 \text { children } \\
\text { (ages } 7-9 \text { years) }\end{array}$ & 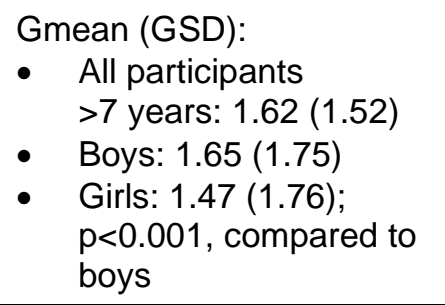 & ADHD & $\begin{array}{l}\text { - } \quad \text { RR for } \mathrm{PbB} \geq 2.17 \text { (compared to } \mathrm{PbB}<2.17 \text { ): } \\
1.552(1.002,2.403)^{*}\end{array}$ \\
\hline $\begin{array}{l}\text { Desrochers-Couture et al. } 2019 \\
\text { Longitudinal study; n=212 Inuit } \\
\text { children followed from birth and } \\
\text { evaluated at mean age } 11.4 \text { and } \\
\text { 18.5 years }\end{array}$ & $\begin{array}{l}\text { Gmean (GSD) } \\
-\quad \text { Cord: } 3.80(1.84) \\
-\quad \text { Child: } 2.34(1.86) \\
-\quad \text { Adolescent: } 1.63(2.00)\end{array}$ & ADHD & $\begin{array}{l}\beta \text { per } \log _{2} \mu \mathrm{g} / \mathrm{dL} \mathrm{PbB}: \\
\text { Child: } \\
\text { - } \quad \text { Externalizing: } 0.61(-0.63,1.96) \\
\text { - } \quad \text { Hyperactivity-impulsivity: } 0.11(-0.14,0.37) \\
\text { - Oppositional defiant/conduct disorder: } 0.02 \\
\quad(-0.20,0.21) \\
\text { Adolescent: } \\
\text { - } \quad \text { Externalizing interacting with child } \\
\text { externalizing: } \mathbf{0 . 3 2}(\mathbf{0 . 0 8 , 0 . 7 2})^{*}\end{array}$ \\
\hline
\end{tabular}


2. HEALTH EFFECTS

Table 2-30. Summary of Epidemiological Studies Evaluating Neurological Effects in Children at Mean Blood Lead Concentration (PbB) $\leq 10 \mu \mathrm{g} / \mathrm{dL}^{\mathrm{a}}$

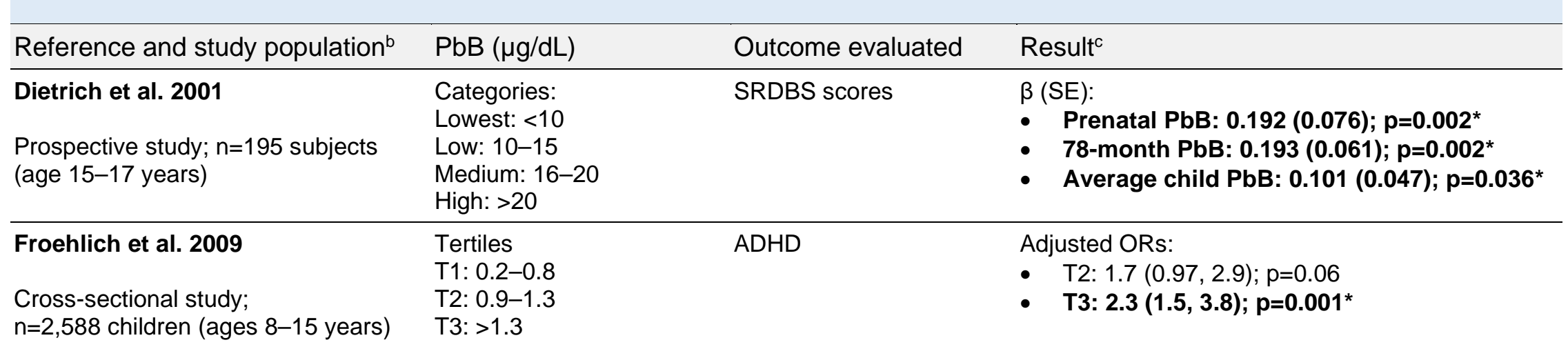

Fruh et al. $2019 \quad$ Erythrocyte $\mathbf{P b}$

Prospective study; $n=1,006$ mother- $\quad 25^{\text {th }}-75^{\text {th }} \%$ range: 0.6 child pairs with follow-up at age 8 years; Massachusetts

$\beta$ for change in score for an IQR increase in maternal $2^{\text {nd }}$ trimester erythrocyte $\mathrm{Pb}$ : Parent-rated SDQ:

- Total difficulties: $0.36(-0.04,0.77)$

- Emotional problems: $0.18(0.03,0.33)^{*}$

Parent-rated BRIEF score:

- Behavioral regulation index: $0.69(-0.13,1.51)$

- General executive composite: 0.73 (-0.06, 1.52)

- Plan organize: $0.85(0.12,1.59) *$

\section{Geier et al. 2018}

Cross-sectional study; $n=2,109$ children, age $10-19$ years
Mean (SD): 1.16 (1.27) Quartiles, range:

- $\quad 0-50^{\text {th }}: 0.2-0.88$

- $50^{\text {th }}-75^{\text {th }}: 0.88-$ 1.26

- $75^{\text {th }}-100^{\text {th }}: 1.26-$ 34.8
ADD

OR for diagnosis of ADD:

Total sample (per $\mu \mathrm{g} / \mathrm{dL}): 1.292(1.025,1.545)$ $p=0.0301^{*}$

Upper quartile $\mathrm{PbB}$ relative to $\mathbf{0}-\mathbf{5 0}^{\text {th }}$ percentile as reference:

- $\quad 50-75^{\text {th }} \%: 1.28(0.82,2.00), p=0.2466^{*}$

- $75-100^{\text {th }} \%: 1.59(1.05,2.39), p=0.0130$ * 
2. HEALTH EFFECTS

\section{Table 2-30. Summary of Epidemiological Studies Evaluating Neurological Effects in Children at Mean Blood Lead Concentration $(\mathrm{PbB}) \leq 10 \mu \mathrm{g} / \mathrm{dL}^{\mathrm{a}}$}

Reference and study population $^{\mathrm{b}}$
He et al. 2019
Meta-analysis of seven studies of
associations between PbB and risk of
ADHD diagnosis

Hong et al. 2015

A cross-sectional study;

$\mathrm{n}=1,001$ children (age 8-11 years)

\section{Huang et al. 2016}

Prospective study of mother-infant pairs with follow-up of 578 children at age 6-13 years

\section{Ji et al. 2018}

Prospective study of mother-infant pairs recruited beginning 1998 with follow-up of 1,479 children at median age 9.6 years

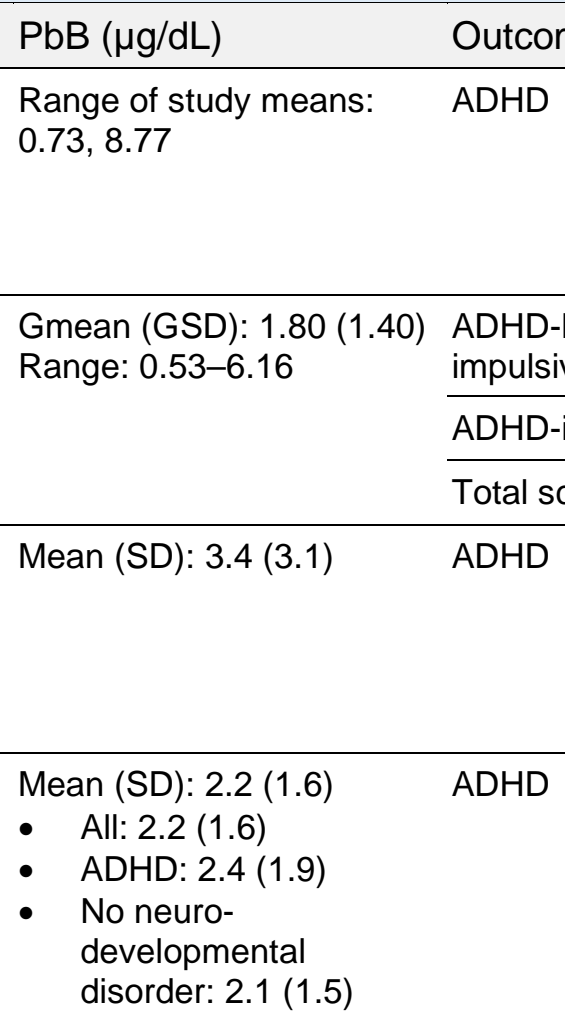

\section{Result $^{\mathrm{c}}$}

Mean risk difference $(95 \% \mathrm{Cl})$ :

- All studies (7): $0.59(0.50,0.68), p<0.0001^{*}$

- $\mathrm{PbB}<3 \mu \mathrm{g} / \mathrm{dL}: 0.47(0.39,0.56), \mathrm{p}<0.0001^{*}$

Age $5-12$ years compared to age $>12$ years:

$1.35(0.28,2.41), \mathrm{p}<0.0001^{*}$

PbB (log-transformed) OR: 3.66 (1.18, 6.13); $p=0.004^{*}$

- OR: $2.72(-0.12,5.56) ; p=0.060$

- OR: $6.38(1.36,11.40) ; p=0.013^{*}$

$\beta$ per $1 \mu \mathrm{g} / \mathrm{dL}$ :

- Hyperactivity: $1.2(0.3,2.0), p=0.01^{*}$

- Restless-impulsive: $1.2(0.3,2.0), p=0.007^{*}$

- Hyperactive-impulsive: $1.1(0.2,2.0)$, $p=0.02^{*}$

OR for ADHD diagnosis.

Males and females: OR per In PbB ( $\mu \mathrm{g} / \mathrm{dL}): 1.25$ $(1.01,1.56) p=0.045^{*}$

OR relative to $<2 \mu \mathrm{g} / \mathrm{dL}$ reference:

- $2-4 \mu \mathrm{g} / \mathrm{dL}: 1.08(0.81,1.44) \mathrm{p}=0.622$

- $5-10 \mu \mathrm{g} / \mathrm{dL}$ : $1.73(1.09,2.73) \mathrm{p}=0.019 *$

OR relative to $<5 \mu \mathrm{g} / \mathrm{dL}$ reference:

- $5-10 \mu \mathrm{g} / \mathrm{dL}$ : $1.66(1.08,2.56) \mathrm{p}=0.020$ *

Males:

OR 5-10 $\mu \mathrm{g} / \mathrm{dL}$ relative to $<5 \mu \mathrm{g} / \mathrm{dL}$ reference:

- Males: $2.49(1.46,4.26) p=0.001^{*}$

- Females: $0.68(0.27,1.69) p=0.401$ 
2. HEALTH EFFECTS

Table 2-30. Summary of Epidemiological Studies Evaluating Neurological Effects in Children at Mean Blood Lead Concentration $(\mathrm{PbB}) \leq 10 \mu \mathrm{g} / \mathrm{dL}^{\mathrm{a}}$

\begin{tabular}{ll}
\hline Reference and study population $^{\mathrm{b}}$ & $\mathrm{PbB}(\mu \mathrm{g} / \mathrm{dL})$ \\
\hline & \\
\hline Joo et al. 2017 & Gmean (SD): \\
Case-control study; $n=214$ child ADHD Controls: $1.49(1.48)$ & Cases: $1.65(1.45)$
\end{tabular}

cases and 214 controls, age 6-

10 years

Gmean (SD)

Controls: 1.49 (1.48)

Gmean (SD):

Early pregnancy: 1.28

(1.48)

Late pregnancy: 1.24

(1.57)

Cord: 0.90 (1.57)

2 years: $1.55(1.49)$

3 years: $1.43(1.44)$

5 years: $1.29(1.38$
Joo et al. 2018

Prospective study; $n=1,751$ motherinfant pairs with follow-up of

575 children at age 5 years

Behavioral problems $\quad \beta$ for score per $\mu \mathrm{g} / \mathrm{dL}$ :

(Child Behavior Checklist) PbB at age 2 years:

- Females: $3.82(1.25,3.69)^{*}$

- Males: $0.22(-1.87,2.32)$

$\mathrm{PbB}$ at age 3 years:

- Females: $2.43(-1.00,5.87)$

- Males: $0.48(-2.17,3.12)$

$\mathrm{PbB}$ at age 5 years:

- Females: $5.72(0.44,10.99)^{*}$

- Males: $1.37(-2.06,4.80)$

Kim et al. 2016

Prospective study; $\mathrm{n}=2,473$ children (age 7-8 years)

\section{Mean $(95 \% \mathrm{Cl})$ :}

- $\quad$ Ages 7-8 years: 1.64 $(1.60,1.68)$

- $\quad$ Ages 9-10 years: 1.58 $(1.55,1.61)$

- Ages 11-12 years: $1.58(1.55,1.61)$

ASSQ

$\mathrm{PbB}$ (log transformed) $\beta$ (SE):

- 7-8 years: $0.151(0.061,0.242)^{*}$

- 9-10 years: $-0.023(-0.143,0.097$

- 11-12 years: $0.054(-0.061,0.170)$

- PbB at 7-8 years: $2.489(1.378,3.600)^{*}$

- PbB at 9-10 years: $1.295(-0.235,2.825)$

- $\mathrm{PbB}$ at $11-12$ years: $\beta$ (SE): 0.724 (-0.727, 2.176) 
Table 2-30. Summary of Epidemiological Studies Evaluating Neurological Effects in Children at Mean Blood Lead Concentration (PbB) $\leq 10 \mu \mathrm{g} / \mathrm{dL}^{\mathrm{a}}$

\begin{tabular}{|c|c|c|c|}
\hline Reference and study population ${ }^{b}$ & $\mathrm{PbB}(\mu \mathrm{g} / \mathrm{dL})$ & Outcome evaluated & Result $^{\mathrm{C}}$ \\
\hline $\begin{array}{l}\text { Liu et al. } \mathbf{2 0 1 4 a} \\
\text { Prospective study; } n=332 \text { mother- } \\
\text { infant pairs }\end{array}$ & 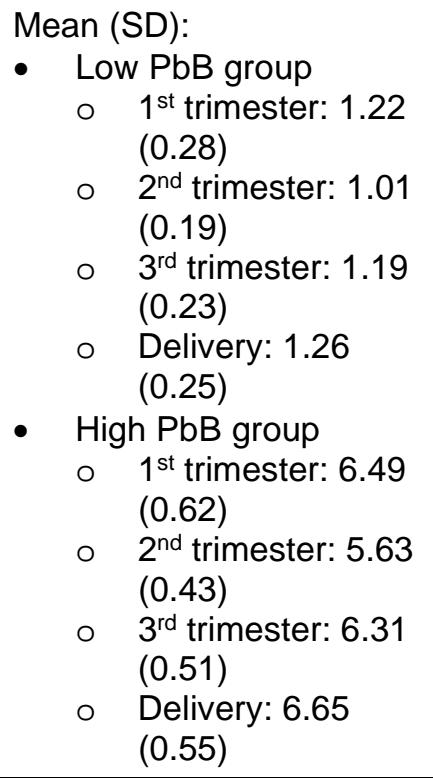 & NBNA score & $\begin{array}{ll}\beta: & \\
- & \mathbf{1}^{\text {st }} \text { trimester: }-\mathbf{4 . 8 6}(-\mathbf{8 . 8 3 1},-\mathbf{0 . 8 8 9}) ; p=0.03^{*} \\
\text { - } & 2^{\text {nd }} \text { trimester: }-3.98(-8.180,0.220) ; p=0.07^{*} \\
\text { - } & 3^{\text {rd }} \text { trimester: }-3.65(-6.609,1.309) ; p=0.21 \\
\text { - } & \text { Delivery: }-3.39(-7.531,0.751) ; p=0.11\end{array}$ \\
\hline $\begin{array}{l}\text { Liu et al. } \mathbf{2 0 1 5 b} \\
\text { Prospective study; } n=665 \text { children } \\
\text { (ages } 3-13 \text { years) }\end{array}$ & Mean (SD): 6.26 (2.54) & Sleep onset delay & $\beta: 0.033(0.009,0.056) ; p=0.006^{*}$ \\
\hline $\begin{array}{l}\text { Park et al. } 2016 \\
\text { Case-control study of child (mean age } \\
9 \text { years) ADHD cases }(n=114) \text { and } \\
\text { controls }(n=114)\end{array}$ & $\begin{array}{l}\text { Gmean } \pm \text { SD (range): } \\
\text { Cases: } 1.90 \pm 0.86(0.37 \\
\text { 5.35) } \\
\text { Controls } 1.59 \pm 0.68(0.18, \\
\text { 3.41) } \\
\text { Q1: } 0.18,1.12 \\
\text { Q2: } 1.13,1.71 \\
\text { Q2: } 1.72,2.29 \\
\text { Q4: } 2.30,5.35\end{array}$ & ADHD & $\begin{array}{l}\text { OR for ADHD diagnosis: } \\
\text { All subjects: } \mathbf{1 . 6 0}(\mathbf{1 . 0 4}, \mathbf{2 . 2 5}), \mathbf{p}=\mathbf{0 . 0 3 *} \\
\text { Relative to Q1: } \\
\text { Q2: } 1.26(0.56,2.84), p=0.39 \\
\text { Q3: } 1.26(0.55,2.87), p=0.61 \\
\text { Q4: } \mathbf{2 . 5 4}(\mathbf{1 . 0 9 , 5 . 9 4 ) , ~} \mathbf{p}=\mathbf{0 . 0 3 *}\end{array}$ \\
\hline
\end{tabular}


Table 2-30. Summary of Epidemiological Studies Evaluating Neurological Effects in Children at Mean Blood Lead Concentration (PbB) $\leq 10 \mu \mathrm{g} / \mathrm{dL}^{\mathrm{a}}$

\begin{tabular}{|c|c|c|}
\hline Reference and study population ${ }^{b}$ & $\mathrm{PbB}(\mu \mathrm{g} / \mathrm{dL})$ & Outcome evaluated \\
\hline $\begin{array}{l}\text { Sioen et al. } 2013 \\
\text { Prospective study; } n=270 \text { children, } \\
\text { followed newborn to } 8 \text { years }\end{array}$ & $\begin{array}{l}\text { Umbilical cord mean }\left(25^{\text {th }}-\right. \\
\left.75^{\text {th }} \text { percentiles }\right): 1.43 \\
(0.73-2.53)\end{array}$ & Hyperactivity \\
\hline $\begin{array}{l}\text { Stroustrup et al. } 2016 \\
\text { Prospective study, } n=948 \text { mother- } \\
\text { infant pairs with follow-up of } 500 \\
\text { children at age } 24 \text { months }\end{array}$ & $\begin{array}{l}\text { Median (IQR): } \\
2^{\text {nd }} \text { trimester: } 2.8(2.7)\end{array}$ & $\begin{array}{l}\text { Temperament } \\
\text { (TTS=easy, intermediate } \\
\text { or difficult); maternal } \\
\text { postnatal depression } \\
\text { (EPDS) }\end{array}$ \\
\hline
\end{tabular}

Result ${ }^{\mathrm{c}}$

OR: 2.940 (1.172, 7.380); $p=0.022^{*}$

$75^{\text {th }}$ percentiles): 1.43

Prospective study; $\mathrm{n}=270$ children, $\quad(0.73-2.53)$

followed newborn to 8 years

Prospective study, $n=948$ mother-

children at age 24 months

(EPDS)

OR $(95 \% \mathrm{Cl})$ corresponding to a 1 unit change in

In(maternal PbB $\mu \mathrm{g} / \mathrm{dL}$ ) for TTS score, easy score

as reference:

- Intermediate: $0.88(0.59,1.3)$

- Difficult: $1.52(1.03,2.26)^{*}$

Probability of demonstrating difficult TTS score was approximately doubled if EPDS score was high

Wang et al. 2008

Case-control study; $\mathrm{n}=630$ children

(ages 4-12 years)
Means (SE):

- ADHD cases: 8.77

(3.89)

- Controls: 5.76 (3.36)

- Cases versus control: $p<0.05$

Tertiles:

- T1 (reference): $\leq 5$

- T2: 5-10

- T3: $\geq 10$

\section{Winter and Sampson 2017}

Prospective study of birth cohort $(n=1,255)$ with follow-up from birth to
Means (SD) at age $<6$ years: $6.14(4.58)$

\section{ADHD}

OR:

- T2: $4.92(3.47,6.98) ; p<0.01^{*}$

- T3: 6.00 (4.11, 8.77); $p<0.01^{*}$ age 18 years $(n=208)$
Impulsivity, anxiety, or depression (Child Behavior Checklist) $\beta$ for score per $\mu \mathrm{g} / \mathrm{dL}$ :

- Impulsivity: $0.08(0.01,0.16)^{*}$

- Anxiety or depression: $0.11(0.01,0.21)^{*}$ 
Table 2-30. Summary of Epidemiological Studies Evaluating Neurological Effects in Children at Mean Blood Lead Concentration (PbB) $\leq 10 \mu \mathrm{g} / \mathrm{dL}^{\mathrm{a}}$

\begin{tabular}{|c|c|c|c|}
\hline Reference and study population ${ }^{b}$ & $\mathrm{PbB}(\mu \mathrm{g} / \mathrm{dL})$ & Outcome evaluated & Result ${ }^{c}$ \\
\hline \multicolumn{4}{|l|}{ Neuromotor neurosensory function } \\
\hline $\begin{array}{l}\text { Chiodo et al. } 2004 \\
\text { Prospective study; } n=237 \text { children } \\
\text { (age } 7.5 \text { years) }\end{array}$ & $\begin{array}{l}\text { Mean (SD, range): } \\
5.4(3.3,1-25)\end{array}$ & Battery test performance & 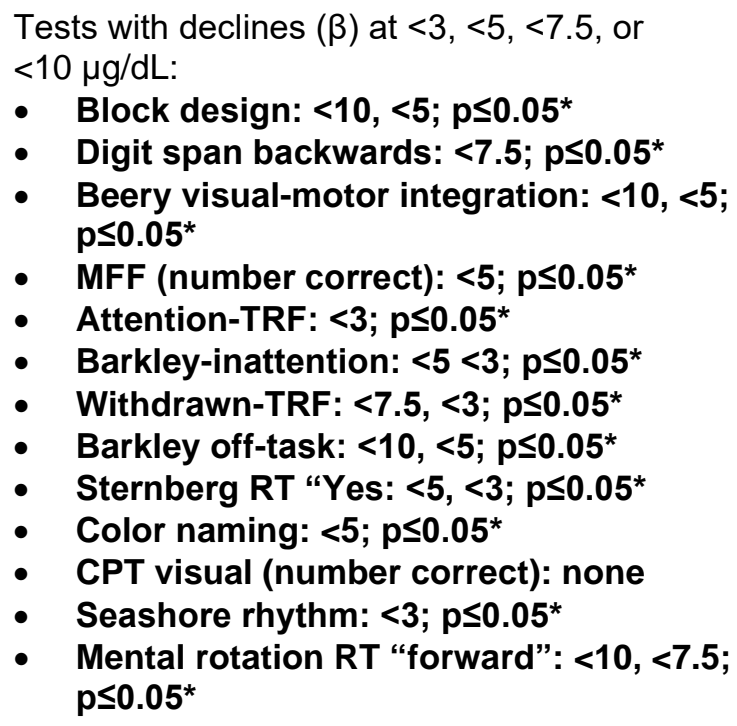 \\
\hline \multirow[t]{2}{*}{$\begin{array}{l}\text { Dietrich et al. } 1987 \\
\text { Prospective study; n=185 mother- } \\
\text { infant pairs }\end{array}$} & \multirow{2}{*}{$\begin{array}{ll}\text { Mean }(\mathrm{SD}, \text { range): } \\
\text { - } \quad \text { Prenatal (maternal): } \\
\quad 8.3(3.8,1-27) \\
\text { - } \quad \text { Neonatal }(10 \text { days): } 4.9 \\
\quad(3.3,1-24) \\
\text { - } \quad \text { Neonatal }(3 \text { months): } \\
\quad 6.3(3.8,1-22) \\
\text { - } \quad \text { Neonatal }(6 \text { months): } \\
8.1(5.2,1-36)\end{array}$} & Motor maturity & 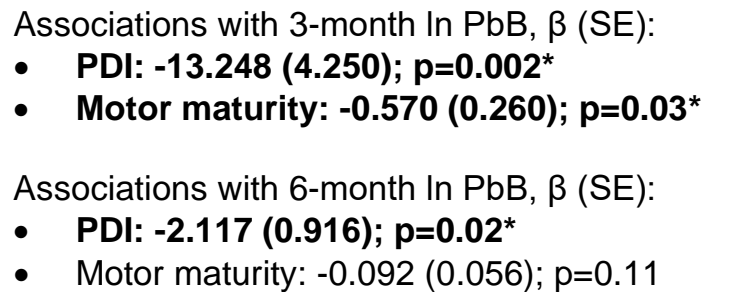 \\
\hline & & PDI & 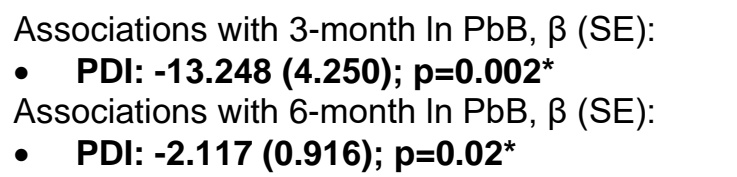 \\
\hline
\end{tabular}


Table 2-30. Summary of Epidemiological Studies Evaluating Neurological Effects in Children at Mean Blood Lead Concentration (PbB) $\leq 10 \mu \mathrm{g} / \mathrm{dL}^{\mathrm{a}}$

\begin{tabular}{|c|c|c|c|}
\hline Reference and study population ${ }^{b}$ & $\mathrm{PbB}(\mu \mathrm{g} / \mathrm{dL})$ & Outcome evaluated & Result $^{\mathrm{c}}$ \\
\hline $\begin{array}{l}\text { Dietrich et al. } 1989 \\
\text { Prospective study; n=192 mother- } \\
\text { infant pairs }\end{array}$ & $\begin{array}{ll}\text { Mean (SD, range): } \\
\text { - } \quad \text { Prenatal (maternal): } \\
8.2(3.6,1-27) \\
\text { - } \quad \text { Neonatal }(10 \text { days): } 4.8 \\
\quad(3.1,1-23) \\
\text { - } \quad \text { Neonatal (3 months): } \\
6.0(3.5,1-20) \\
\text { - } \quad \text { Neonatal (6 months): } \\
\quad 7.9 \text { (4.8, 1-35) } \\
\text { - } \\
\quad 11.5(6.9,2-57) \\
\text { - } \quad \text { Neonatal }(12 \text { months): } \\
\quad 14.2(7.3<4-47)\end{array}$ & PDI & 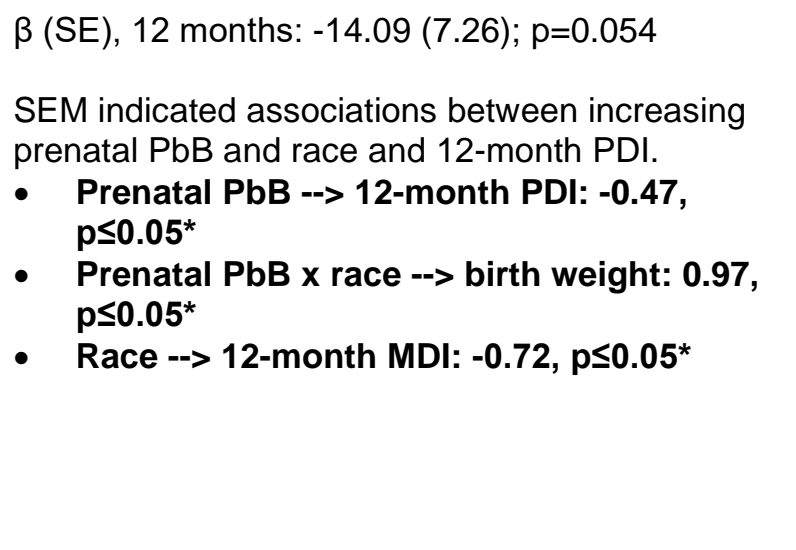 \\
\hline $\begin{array}{l}\text { Dietrich et al. } 1993 b \\
\text { Prospective study; } n=245 \text { children } \\
\text { (age } 6 \text { years) }\end{array}$ & $\begin{array}{l}\text { Mean (SD): } \\
\text { - } \quad \text { Prenatal (maternal: } 8.4 \\
\text { (3.8) } \\
\text { - } \quad \text { Neonatal: } 4.8(3.1) \\
\text { - } \quad \text { Life average } \\
\text { - } \quad \text { Lifears: } 10.1(5.6) \\
\quad \text { quartile range: } 7-22\end{array}$ & Motor performance & $\begin{array}{l}\text { Tests with }(p \leq 0.05) \text { declines }(\beta) \text { associated with } \\
\text { neonatal }(N) \text {, mean lifetime }(L) \text { or concurrent }(C) \\
\text { PbB: } \\
\text { - } \quad \text { Bilateral coordination: } N, M \\
\text { - } \quad \text { Visual motor control: } C \\
\text { - } \quad \text { Upper limb speed and dexterity: C, M, N } \\
\text { - } \quad \text { Fine motor composite: } C, M, N\end{array}$ \\
\hline $\begin{array}{l}\text { Ethier et al. } 2012 \\
\text { Prospective longitudinal, } \\
n=149 \text { children (age 10-13 years) }\end{array}$ & $\begin{array}{l}\text { Mean (SD, range): } \\
\text { - Cord: } 4.6(3.1,0.8- \\
\text { 19.5) } \\
\text { 11 years: } 2.6(2.3,0.4- \\
12.8)\end{array}$ & $\begin{array}{l}\text { Delay of N150 latency of } \\
\text { VEP }\end{array}$ & $\begin{array}{l}\text { Association between increasing cord } \mathrm{PbB} \text { and } \\
\text { delay of N150 latency of VEP at multiple contracts. } \\
\text { Mean latency (estimated from reported bar plot): } \\
\qquad \quad \geq 4.15 \mu \mathrm{g} / \mathrm{dL}: \sim 160 \mathrm{~ms}, \mathrm{p}<0.05^{*} \\
-\quad<4.15 \mu \mathrm{g} / \mathrm{dL}: \sim 153 \mathrm{~ms} \text { (reference) }\end{array}$ \\
\hline Fraser et al. 2006 & \multirow{3}{*}{$\begin{array}{l}\text { Mean (SD): } \\
\text { Cord: } 4.9(3.7) \\
\text { Child: } 5.3(4.9)\end{array}$} & Hand movements & $\beta-0.30, p \leq 0.01^{*}$ \\
\hline Prospective study; $n=101$ children & & Sway velocity & $\beta-0.28, p \leq 0.01^{*}$ \\
\hline (age 5 years) & & Transversal sway & $\beta 0.24, p \leq 0.05^{*}$ \\
\hline
\end{tabular}


2. HEALTH EFFECTS

Table 2-30. Summary of Epidemiological Studies Evaluating Neurological Effects in Children at Mean Blood Lead Concentration (PbB) $\leq 10 \mu \mathrm{g} / \mathrm{dL}^{\mathrm{a}}$

\begin{tabular}{llll}
\hline Reference and study population $^{\mathrm{b}}$ & $\mathrm{PbB}(\mu \mathrm{g} / \mathrm{dL})$ & Outcome evaluated & Result $^{\mathrm{c}}$ \\
\hline Kim et al. 2013b & Gmean (GSD): & PDI & $\beta$ per $1 \mu \mathrm{g} / \mathrm{dL}$ change in PbB: $-1.69(-3.65,-0.27) ;$ \\
& Early pregnancy: 1.4 (1.5) & & $\mathrm{p}=0.09$
\end{tabular}

Prospective birth cohort, $\mathrm{n}=884$ mother Late pregnancy: 1.3 (1.5) infant pairs

\begin{tabular}{|c|c|c|c|}
\hline $\begin{array}{l}\text { Liu et al. } \mathbf{2 0 1 8 b} \\
\text { Cross-sectional study; } n=234 \text { children, } \\
\text { age } 3-7 \text { years }\end{array}$ & $\begin{array}{l}\text { Median (SE) } \\
\text { e-waste location: } 4.94 \\
(0.20) \\
\text { reference location: } 3.85 \\
(1.81)\end{array}$ & $\begin{array}{l}\text { Hearing (pure tone } \\
\text { conduction }>25 \mathrm{~dB} \text { ) }\end{array}$ & 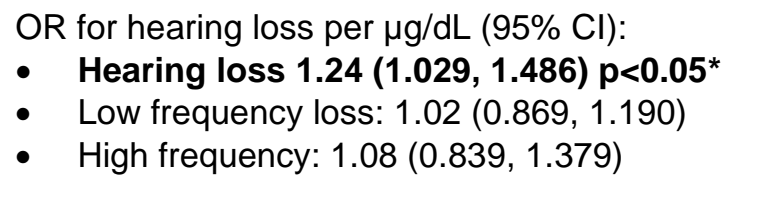 \\
\hline $\begin{array}{l}\text { Osman et al. } 1999 \\
\text { Retrospective study; } n=155 \text { children } \\
\text { (age } 4-14 \text { years) }\end{array}$ & $\begin{array}{l}\text { Median (range): } \\
\text { - } \quad 7.2(1.9-28.1)\end{array}$ & Hearing threshold & 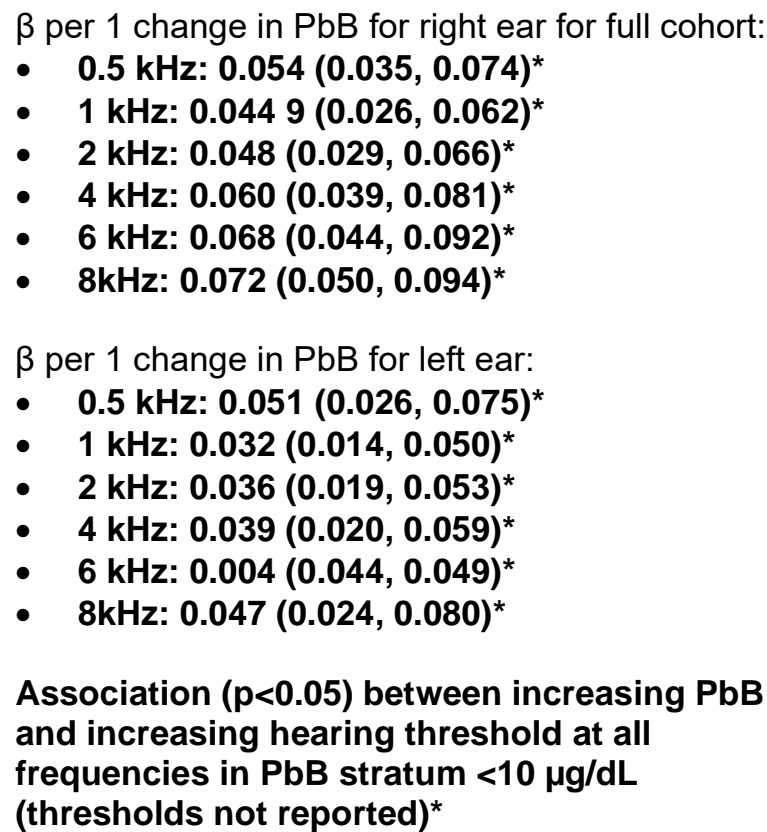 \\
\hline
\end{tabular}


Table 2-30. Summary of Epidemiological Studies Evaluating Neurological Effects in Children at Mean Blood Lead Concentration (PbB) $\leq 10 \mu \mathrm{g} / \mathrm{dL}^{\mathrm{a}}$

\begin{tabular}{l} 
Reference and study population \\
\hline Polanska et al. 2018 \\
Prospective study; $n=539$ mother-child \\
pairs with follow-up of children at age \\
2 years; 280 blood samples and 303 \\
cord blood samples were randomly \\
chosen for analysis
\end{tabular}

Rodrigues et al. 2016

Median (P25, P75,

maximum)

Prospective study with cross-sectional - Sirajdikhan: 7.6 (5.5, analysis of $\mathrm{PbB}$ and fine motor score; $\mathrm{n}=524$ children, $20-30$ months

10.4)

- Pabna: $<$ LOD $(<L O D$, $3.8,13.8)$

\section{Silver et al. 2016}

Prospective study; infants assessed for hearing at 2 days and vision at 6 weeks; maternal blood $\mathrm{Pb}$ collected at mid pregnancy and late pregnancy and in cord blood
Exposure for infants with hearing data:

Gmean (SD)

Mid-pregnancy: 2.4 (2.5)

Late-pregnancy: 2.7 (2.3)

Cord: $<\mathrm{LOQ}$

Exposure for infants with vision data:

Gmean (SD)

Mid-pregnancy: 2.4 (2.6)

$(n=1,038)$;

Late-pregnancy: 2.9 (2.2)

$(\mathrm{n}=1,058)$;

Cord: <LOQ ( $n=949)$
Hearing at age 2 days $(A B R)$; vision at age 6 weeks (GVA)

BSID III
Outcome evaluated

Result ${ }^{\mathrm{c}}$

$\beta$ score per $\mu \mathrm{g} / \mathrm{dL}$ cord $\mathrm{PbB}$ :

Motor score:

- Females: $0.48(-1.55,2.52), p=0.64$

- Males: $-0.70(-2.90,1.51), p=0.53$

$\beta$ score (SE) per child InPbB ( $\mu \mathrm{g} / \mathrm{L})$ :

Fine motor score:

- Sirajdikhan: 0.07 (0.11), $p=0.50$

- Pabna: -0.07 (0.11), $p=0.50$

Percent change in score relative to $<2 \mu \mathrm{g} / \mathrm{dL}$ (latepregnancy) reference group

GVA score for PbB strata:

- $\quad>3.8 \mu \mathrm{g} / \mathrm{dL}:-8.5(-14.7,-2.4)^{*}$

- 2 - $3.8 \mu \mathrm{g} / \mathrm{dL}:-7.2(-13.3,-1.1)^{*}$

ABR C-P ratio for $\mathrm{PbB}$ strata:

- $\quad>3.8 \mu \mathrm{g} / \mathrm{dL}: 4.6(1.8,7.4)^{*}$

- 2 - $3.8 \mu \mathrm{g} / \mathrm{dL}: 3.2(0.0,5.9)^{*}$ 
Table 2-30. Summary of Epidemiological Studies Evaluating Neurological Effects in Children at Mean Blood Lead Concentration (PbB) $\leq 10 \mu \mathrm{g} / \mathrm{dL}^{\mathrm{a}}$

\begin{tabular}{|c|c|c|c|}
\hline Reference and study population ${ }^{b}$ & $\mathrm{PbB}(\mu \mathrm{g} / \mathrm{dL})$ & Outcome evaluated & Result $^{c}$ \\
\hline $\begin{array}{l}\text { Taylor et al. } 2018 \\
\text { Prospective study; } n=14,541 \text { mother- } \\
\text { infant pairs with follow-up of } \\
1,558 \text { children at age } 7 \text { years }\end{array}$ & $\begin{array}{l}\text { Mean (SD) at gestation } \\
\text { week } 11 \\
\text { Mean (SD) } 3.66(1.55) \\
\text { Range: } 0.20,19.14\end{array}$ & $\begin{array}{l}\text { Motor skills (Movement } \\
\text { Assessment Battery) }\end{array}$ & $\begin{array}{l}\text { OR for scores per } \mu \mathrm{g} / \mathrm{dL} \text { prenatal } \mathrm{PbB} \text { : } \\
\text { - } \quad \text { Heal to toe: } 0.99(0.74,1.33), \mathrm{p}=0.93 \\
\text { - } \quad \text { Beanbag: } 0.88(0.58,1.32), \mathrm{p}=0.54 \\
\text { - } \quad \text { Threading lace: } 1.12(0.83,1.50), \mathrm{p}=0.47 \\
\text { - } \quad \text { Peg board (preferred hand): } 1.19(0.88,1.60) \text {, } \\
\text { - } \quad \text { =0.26 } \\
\text { Peg board (non-preferred hand): } 1.14(0.85, \\
\text { 1.54), } p=0.37\end{array}$ \\
\hline
\end{tabular}

\section{Tellez-Rojo et al. 2006}

Prospective study; $n=294$ children (followed from birth to age 2 years)

\section{Mean (SD):}

- $\quad$ Cord: 4.85 (3.0)

- 12 months: 4.27 (2.14)

- 24 months: 4.28 (2.25)
PDI

$\beta$ per 1 In change in $\mathrm{PbB}$ :

12 months:

- $\quad<10 \mu \mathrm{g} / \mathrm{dL}:-0.01, \mathrm{p}=0.98$

- $\geq 10 \mu \mathrm{g} / \mathrm{dL}:-1.19, \mathrm{p}=0.01^{*}$

24 months:

- $\quad<10 \mu \mathrm{g} / \mathrm{dL}:-1.18, \mathrm{p}<0.01^{*}$

- $\quad \geq 10 \mu \mathrm{g} / \mathrm{dL}: 0.04, \mathrm{p}=0.89$

\section{Zhou et al. 2017}

Prospective study; n=139 motherinfant pairs followed from birth to 24 36 months

Gmean (95\% Cl) Motor skills (Gesell

Mid-late pregnancy: 3.30

$(3.05,3.57)$ $\beta(95 \% \mathrm{Cl})$ for development quotient per $\mu \mathrm{g} / \mathrm{dL}$ : All children:

- $\quad$ Gross motor: $3.31(-6.11,12.73)$

- $\quad$ Fine motor: $0.49(-11.27,12.24)$

\begin{tabular}{|c|c|c|c|}
\hline \multicolumn{4}{|l|}{ Altered brain structure and chemistry } \\
\hline $\begin{array}{l}\text { Cecil et al. } 2008 \\
\text { Prospective study; n=157 adults, age } \\
\text { 19-24 years from a birth cohort born } \\
\text { 1979-1984 from Cincinnati, Ohio }\end{array}$ & $\begin{array}{l}\text { Mean (SD, range): } \\
\text { - } \quad 6 \text { month-6.5 years: } \\
\quad 13.3(5.9,4.6-37.2)\end{array}$ & Brain volume & $\begin{array}{l}\text { Association ( } p \leq 0.001) \text { between increasing } \\
\text { childhood mean PbB and decreasing brain volume } \\
\text { affecting } 1.2 \% \text { of the total gray matter. Effects } \\
\text { were greater in males than females. Largest } \\
\text { effects were in the anterior cingulate cortex. }\end{array}$ \\
\hline
\end{tabular}


Table 2-30. Summary of Epidemiological Studies Evaluating Neurological Effects in Children at Mean Blood Lead Concentration $(\mathrm{PbB}) \leq 10 \mu \mathrm{g} / \mathrm{dL}^{\mathrm{a}}$

\begin{tabular}{|c|c|c|c|}
\hline Reference and study population ${ }^{b}$ & $\mathrm{PbB}(\mu \mathrm{g} / \mathrm{dL})$ & Outcome evaluated & Result ${ }^{\mathrm{c}}$ \\
\hline $\begin{array}{l}\text { Cecil et al. } 2011 \\
\text { Prospective study; n=159 adults, age } \\
\text { 19-24 years from a birth cohort born } \\
\text { 1979-1984 from Cincinnati, Ohio }\end{array}$ & $\begin{array}{l}\text { Mean (SD, range): } \\
\text { - } 6 \text { months-6.5 years: } \\
\quad 13.3(6.1,4.7-37.2)\end{array}$ & Brain metabolism & $\begin{array}{l}\text { Association }(p<0.05) \text { between increasing } \\
\text { childhood mean } \mathrm{PbB} \text { and decreasing regional } \\
\text { levels of gray matter } \mathrm{N} \text {-acetyl aspartate, } \\
\text { glutamate-glutamine, creatine and } \\
\text { phosphocreatine, and white matter cholines. } \\
\text { Areas affected include the basal ganglia, } \\
\text { cerebellum vermis, parietal white matter, and } \\
\text { frontal white matter.* }\end{array}$ \\
\hline
\end{tabular}

aSee the Supporting Document for Epidemiological Studies for Lead, Table 9 for more detailed descriptions of studies.

${ }^{b}$ Participants had no known occupational exposure to $\mathrm{Pb}$.

${ }^{c}$ Asterisk and bold indicate association with $\mathrm{Pb}$; unless otherwise specified, values in parenthesis are $95 \%$ Cls; $p$-values <0.05 unless otherwise noted in the table.

$\mathrm{ABR}=$ brainstem auditory response; $\mathrm{ADD}=$ attention deficit disorder; $\mathrm{ADHD}=$ attention-deficit/hyperactivity disorder; $\mathrm{ASSQ}=\mathrm{Autism}$ Spectrum Screening Development Questionnaire; AUC = area under the curve; BRIEF = Behavior Rating Inventory of Executive Function; BSID = Bayley Scales of Infant Development $\mathrm{CD}=$ Conduct Disorder; $\mathrm{CEM}=$ Coarsened Exact Matching; $\mathrm{Cl}=$ confidence interval; $\mathrm{CKD}=\mathrm{chronic}$ kidney disease; $\mathrm{CL}=\mathrm{confidence} \mathrm{limit;}$ C-P = central-to-peripheral; CPT = Continuous Performance Test; ECDI = Early Child Development Inventory; EOG = End of Grade; EPDS = Edinburgh Postnatal Depression Scale; FSIQ = Full-Scale intelligence quotient; FTII = Fagan Test of Infant Intelligence; FWS = Filtered Word Subtest; GCI = General Cognitive Index; Gmean = geometric mean; GSD = geometric standard deviation; GVA = grating visual acuity; IQ = intelligence quotient; IQR = interquartile range; ISAT = Illinois Standard Achievement Test; K-ABC = Kaufman Assessment Battery for Children; LOD = limit of detection; LOQ = limit of quantitation; MDI = Mental Development Index; MFF = Matching Familiar Figures; MSEL = Mullen Scales of Early Learning; NA = not available; NBNA = Neonatal Behavioral Neurological Assessment; ND = not detected; ODD = Oppositional Defiant Disorder; OR = odds ratio; PALS-K = Phonological Awareness Literacy Screening-Kindergarten; Pb = lead; PDI = Psychomotor Development Index; PR = prevalence ratio; RR = relative risk; RT = reaction time; SD = standard deviation; SDQ = Strengths and Difficulties Questionnaire; SE = standard error; SRDBS = Self-Reported Delinquent Behavior Survey; SRS = Social Responsiveness Scale; TRF = Teacher Report Form from the Child Behavior Checklist; TTS = Toddler Temperament Scales; UCL = upper confidence limit; VEP = visual evoked potential 
FSIQ was assessed at age 28-30 years in 43 members of the Boston prospective study cohort (Bellinger et al. 1992). The change in FSIQ was -1.89 points (95\% CI: -3.00, -047) per $\mu \mathrm{g} / \mathrm{dL}$ increase in late child $\mathrm{PbB}$ (mean $6.7 \pm 3.6$ at age 4 years, $3.0 \pm 2.7$ at age 10 years). After adjustment for maternal IQ, the change in FSIQ was -1.11 (95\% CI: -2.29, 0.06).

The largest study was a pooled analysis from seven individual prospective studies that evaluated FSIQ (Baghurst et al. 1992; Bellinger et al. 1992; Canfield et al. 2003; Dietrich et al. 1993a; Ernhart et al. 1989; Schnaas et al. 2000; Wasserman et al. 1997). The pooled cohort consisted for 1,333 children who were evaluated for FSIQ between ages 4.8 and 6 years (Lanphear et al. 2005, 2019). Co-variates considered in the analysis included study, maternal IQ, HOME score (Home Observation for Measurement of the Environment Inventory score), maternal education, marital status, birth weight, birth order, maternal age, race, and prenatal tobacco exposure. Of these, maternal IQ, HOME, and birth weight were included in the final models. When the full cohort was considered ( $\mathrm{PbB}$ range 0.1-72 $\mu \mathrm{g} / \mathrm{dL}$ ), the adjusted change in FSIQ was loglinear, with greater changes in IQ per unit change in PbB at lower PbB levels. Several blood $\mathrm{Pb}$ metrics were explored in regression modeling, and slopes were significant for childhood, peak, lifetime average, or concurrent (with IQ testing) $\mathrm{PbB}$. The model that used concurrent $\mathrm{PbB}$ had the highest $\mathrm{r}^{2}$ (not reported). The covariate adjusted regression $\beta$ for this model was -2.65 (95\% CI: -3.69, -1.61) IQ points per $1 \ln \mathrm{PbB}$. The unadjusted $\beta$ was -4.84 (-5.98, -3.69). The concurrent $\mathrm{PbB}$ model predicts a decrease of 6.2 points in FSIQ when PbB increased from 1 to $10 \mu \mathrm{g} / \mathrm{dL}$. In a PbB stratum maximum $<7.5 \mu \mathrm{g} / \mathrm{dL}$, the mean change in FSIQ was -2.53 (95\% CI -4.48, -0.58) per $1 \mu \mathrm{g} / \mathrm{dL}$ change in $\mathrm{PbB}$, and for a $\mathrm{PbB}$ stratum maximum $\geq 7.5 \mu \mathrm{g} / \mathrm{dL}$, the mean change in FSIQ was -0.15 (95\% CI -0.23, -0.07) per $1 \mu \mathrm{g} / \mathrm{dL}$. Re-analyses of the pooled cohort reported in Lanphear et al. (2005) have been conducted (Crump et al. 2013; EPA 2014e). EPA (2014e) made several corrections to the dataset and obtained $\beta$ coefficients that were similar to those reported in Lanphear et al. (2005). The results of the EPA (2014e) reanalysis are presented in Table 2-30.

The model that used early childhood PbB (6-24 months) had the highest $\mathrm{r}^{2}$ (0.6433), although the $\mathrm{r}^{2}$ was similar to concurrent $\mathrm{PbB}$ (0.6414). A benchmark dose (BMD) analysis of the pooled data from Lanphear et al. (2005) estimated BMDLs (95\% lower one-sided confidence limit on BMD) ranging from 0.1 to $1 \mu \mathrm{g} / \mathrm{dL}$ for a $1 \%$ decrease in FSIQ for the best-fitting models (Budtz-Jorgensen et al. 2013). This BMD analysis provides supporting evidence that exposures to $\mathrm{Pb}$ may produce effects on cognitive function in populations whose PbBs are well below $5 \mu \mathrm{g} / \mathrm{dL}$, and may extend to levels below $1 \mu \mathrm{g} / \mathrm{dL}$. 
In addition to the seven prospective studies included in the Lanphear et al. $(2005,2019)$ pooled analysis, more recent prospective studies have evaluated associations between PbB and FSIQ in children (Braun et al. 2012; Chiodo et al. 2004; Jusko et al. 2008; Kordas et al. 2011; Min et al. 2009; Schnaas et al. 2006; Taylor et al. 2017; Table 2-30). Each of these studies found significant associations between increasing $\mathrm{PbB}$ and decreasing FSIQ in study populations that had mean PbBs $<10 \mu \mathrm{g} / \mathrm{dL}$. The largest of these studies combined four Mexico City birth cohorts for a total of 1,035 mother-infant pairs (Braun et al. 2012). Cognitive function assessed at age 4 years (McCarthy General Cognitive Index [GCI]) decreased with increasing $\mathrm{PbB}$ measured at age 2 years. The adjusted effect of concurrent $\mathrm{PbB}$ was estimated as 3.8 (95\% CI: -6.3, -1.4) points when PbB increased by $10 \mu \mathrm{g} / \mathrm{dL}$. Similar to the findings of the Lanphear et al. $(2005,2019)$ study, covariate adjustment decreased the regression $\beta$ by approximately $40 \%$ (from 6.4 to -3.8$)$. The cohort mean $\mathrm{PbB}$ was $4.6 \mu \mathrm{g} / \mathrm{dL}$ ( $5^{\text {th }}-95^{\text {th }}$ percentile range $\left.1.3-13.4\right)$. Studies of smaller cohorts from Mexico City found similar associations (Kordas et al. 2011; Schnaas et al. 2006). Schnaas et al. (2006) estimated the effect size to be a -4.0 (95\% CI: -6.37, -1.65) point change in FSIQ measured at ages 6-10 years in association with a natural log increase in maternal PbB; the cohort geometric mean was $7.3 \mu \mathrm{g} / \mathrm{dL}$ (95\% CI: 1.5, 17.4). Kordas et al. (2011) estimated the effect size to be -0.6 (SE 0.2) for a $1 \mu \mathrm{g} / \mathrm{dL}$ increase in concurrent $\mathrm{PbB}$ (mean $8.1 \mu \mathrm{g} / \mathrm{dL} \pm 4.4 \mathrm{SE}$ ). Prospective studies conducted in Cleveland, Ohio (Min et al. 2009) and Rochester, New York (Jusko et al. 2008) also found similar effect sizes for the associations between increasing $\mathrm{PbB}$ and decreasing IQ. In the Rochester study, the changes in FSIQ were larger at lower PbB, consistent with the outcomes of the Lanphear et al. (2005) study (Jusko et al. 2008). For the PbB range 2.1-10 $\mu \mathrm{g} / \mathrm{dL}$, the change in FSIQ measured at age 6 years was -1.2 per $1 \mu \mathrm{g} / \mathrm{dL}$ increase in PbB. This decreased to -0.32 and -0.15 for the ranges $10-20$ and $20-30 \mu \mathrm{g} / \mathrm{dL}$, respectively. In the Cleveland study, the change was $-0.50 \pm 0.20$ (SE) in FSIQ measured at age 4 years per $1 \mu \mathrm{g} / \mathrm{dL}$ increase in concurrent PbB (Min et al. 2009). A study conducted in Detroit, Michigan estimated the change in FSIQ to be -0.20 per 1 SD change in PbB (Chiodo et al. 2004). The decrement was significant $(\mathrm{p} \leq 0.05)$ in $\mathrm{PbB}$ strata $<7.5$ and $<10 \mu \mathrm{g} / \mathrm{dL}$. Not all prospective studies have found evidence for decreasing FSIQ in association with increased PbB. One of the largest birth cohorts that has been studied is the Avon Longitudinal Study of Parents and Children (ALSPC), conducted in the United Kingdom (Taylor et al. 2017). This study followed a cohort of approximately 14,000 births. In a followup of 2,127 children at age 8 years, increasing maternal PbB (mean $3^{\text {rd }}$ trimester PbB 3.67 $\pm 1.46 \mathrm{SD}$ ) was associated with an increase in FSIQ in females and no change in FSIQ in males. The changes in FSIQ were 0.73 (95\% CI: 0.13, 1.01) per $1 \mu \mathrm{g} / \mathrm{dL}$ increase in PbB in females and -0.29 (95\% CI: -1.02, 0.44) in males. A prospective study of 609 mother-infant pairs, conducted in Canada, found that increasing cord $\mathrm{PbB}$ was associated with decreasing FSIQ when assessed in male children at age 3-4 years (DesrochersCouture et al. 2018). The change in FSIQ in males was -2.61 points (95\% CI: -4.66, -0.48) per $1 \mu \mathrm{g} / \mathrm{dL}$ 
and the change in females was $-0.18(-1.63,1.21)$. The geometric mean cord $\mathrm{PbB}$ was $3.80 \pm 1.86$ (geometric standard deviation [GSD]).

Cross-sectional studies have also found associations between increasing PbB and FSIQ in children (Hong et al. 2015; Ruebner et al. 2019). A study conducted in South Korea evaluated PbB and FSIQ in 1,001 children 8-11 years of age (Hong et al. 2015). The estimated effect of PbB on FSIQ was 7.23 points (95\% CI: -13.39 , -1.07$)$ per 10 -fold increase in $\mathrm{PbB}$. The $5^{\text {th }}-95^{\text {th }}$ percentile range for the cohort PbB was 0.53-6.16 $\mu \mathrm{g} / \mathrm{dL}$. A study of 412 children (median age 15 years) who were diagnosed with $\mathrm{CKD}$ found an association between increasing child $\mathrm{PbB}$ and decreasing FSIQ, after adjustment for CKD severity (Ruebner et al. 2019). The estimated effect of PbB on FSIQ was -2.1 (95\% CI: -3.9, -0.2).

Cognitive function in early childhood-other than FSIQ. Several studies have examined outcomes other than IQ and have found associations between $\mathrm{PbB}$ and changes in cognitive function in children whose $\mathrm{PbBs}$ were $<10 \mu \mathrm{g} / \mathrm{dL}$ (Table 2-30). These include prospective studies that used the same outcome metric, the BSID MDI, allowing comparison of outcomes across studies (Dietrich et al. 1986, 1987, 1989; Kim et al. 2013b; Polanska et al. 2018; Rodrigues et al. 2016; Tellez-Rojo et al. 2006). A prospective study of 884 children conducted in South Korea found inverse associations between $\mathrm{PbB}$ in late pregnancy (geometric mean 1.3 \pm 1.5 , GSD) and MDI scores measured at age 6 months (Kim et al. 2103b). A prospective study of 294 children conducted in Mexico City found inverse associations between concurrent PbB (mean 4.27 \pm 2.14 , SD) and MDI measured at 24 months in a PbB stratum $<10 \mu \mathrm{g} / \mathrm{dL}$ (Tellez-Rojo et al. 2006). A prospective study conducted in Cincinnati, Ohio (approximately 190 infants) found declines in MDI scores at age 6 and 12 months in association with increasing maternal, neonatal, or infant PbB (Dietrich et al. 1986, 1987, 1989). A prospective study conducted in Poland (303 infants) found declines in MDI scores at age 2 years in males (but not females) in association with increasing cord $\mathrm{PbB}$ (range 0.24-5.65 $\mu \mathrm{g} / \mathrm{dL}$ ) (Polanska et al. 2018). A prospective study conducted in Bangladesh (324 infants) found declines in MDI scores at age 2-3 years in association with increasing child PbB (median $7.6 \mu \mathrm{g} / \mathrm{dL}$, maximum $10.4 \mu \mathrm{g} / \mathrm{dL}$ ) (Rodrigues et al. 2016).

Several large-scale retrospective studies linked academic performance for individual children with their corresponding blood $\mathrm{Pb}$ data recorded in state or local blood $\mathrm{Pb}$ registries (Blackowicz et al. 2016; Evens et al. 2015; Miranda et al. 2009; Shadbegian et al. 2019; Zhang et al. 2013; Table 2-30). Evens et al. (2015) linked individual $3^{\text {rd }}$ grade Illinois Standard Achievement Test (ISAT) scores and PbB data (birth72 months) for a population of 47,158 children in Chicago, Illinois. All children had $\mathrm{PbB}<10 \mu \mathrm{g} / \mathrm{dL}$ and the population mean was $4.8 \pm 2.2 \mu \mathrm{g} / \mathrm{dL}$ (SD). Increasing PbB was inversely associated with decreasing 
covariate adjusted scores in math and reading. The adjusted relative risks (RRs) for failing scores was also significant for a 1 or $5 \mu \mathrm{g} / \mathrm{dL}$ increase in PbB. A follow-up to this study of the same data from Chicago that focused on Hispanic children who had $\mathrm{PbB}<10 \mu \mathrm{g} / \mathrm{dL}$ also found that increasing $\mathrm{PbB}$ was associated with decreasing scores in math and reading and significant RRs for failing scores (Blackowicz et al. 2016). Miranda et al. (2009) linked $4^{\text {th }}$ grade reading End of Grade (EOG) scores and PbB data collected (birth-36 months) for a population of 57,678 children in North Carolina. The population mean $\mathrm{PbB}$ was $4.8 \mu \mathrm{g} / \mathrm{dL}$ (range 1-16 $\mu \mathrm{g} / \mathrm{dL}$ ); $94 \%$ of children had $\mathrm{PbB}<10 \mu \mathrm{g} / \mathrm{dL}$. Increasing $\mathrm{PbB}$ was associated with decreasing covariate adjusted scores in all PbB strata, the lowest of which was $2 \mu \mathrm{g} / \mathrm{dL}$. The effect size (change in score/ $\mu \mathrm{g} / \mathrm{dL} \mathrm{PbB}$ ) increased with increasing PbB. Another study conducted in North Carolina analyzed data on PbB and standardized achievement scores of children in grades 3-8 (Shadbegian et al. 2019). Increasing PbB was associated with decreasing score percentiles in math and reading among children who had $\mathrm{PbBs}$ within the range $>1-\leq 5 \mu \mathrm{g} / \mathrm{dL}$, relative to children who had $\mathrm{PbBs}$ $<1 \mu \mathrm{g} / \mathrm{dL}$. Zhang et al. (2013) linked Michigan Educational Assessment Program (MEAP) scores and PbB data (birth-72 months) of age for a population of approximately 21,000 children in Detroit, Michigan. Covariate adjusted ORs for failing scores in mathematics, science, and reading were significant for PbB strata 1-5, 6-10, and $>10 \mu \mathrm{g} / \mathrm{dL}$. A cross-sectional study of data from NHANES III examined associations between $\mathrm{PbB}$ and scores on tests of cognitive function (Wide Range Achievement Test-Revised [WRAT-R], Wechsler Intelligence Scales for Children-Revised [WISC-R]) in approximately 5,000 children 6-16 years of age (Lanphear et al. 2000a). Increasing PbB was significantly associated with decreasing scores in reading in blood strata $<5.0,<7.5$, and $<10 \mu \mathrm{g} / \mathrm{dL}$. McLaine et al. (2013) examined associations between PbB (9-72 months) and kindergarten readiness assessed from Phonological Awareness Literacy Screening-Kindergarten (PALS-K) scores in approximately 3,400 children in Providence, Rhode Island. The population median PbB was 4.2 $\mu \mathrm{g} / \mathrm{dL}$ (interquartile range 2.9-6.0); 93\% of children had $\mathrm{PbB}<10 \mu \mathrm{g} / \mathrm{dL}$. Mean difference in covariate adjusted scores in blood strata 5-9 and $\geq 10 \mu \mathrm{g} / \mathrm{dL}$ compared to $<4 \mu \mathrm{g} / \mathrm{dL}$ were in the inverse direction and adjusted prevalence ratios for test failure was significant in both strata. Genetic variants of N-methylD-aspartate receptors (NMDAR subunits GRIN2A and GRIN2B) were effect modifiers on associations between increasing $\mathrm{PbB}$ (at age 8-12 years) and decreasing performance tests of learning, memory, and executive function at age 17 years (Rooney et al. 2018).

Altered mood and behavior. Numerous studies have examined possible associations between neonatal and child $\mathrm{PbB}$ risk of behaviors that may contribute to learning deficits, including attention deficits, hyperactivity, autistic behaviors, conduct disorders, and delinquency (Table 2-30). 
Several studies have examined attention-deficit/hyperactivity disorder (ADHD) as an outcome, allowing comparisons of outcomes across studies (Arbuckle et al. 2016; Boucher et al. 2012; Braun et al. 2006; Choi et al. 2016; Desrochers-Couture et al. 2019; Froehlich et al. 2009; Geier et al. 2018; He et al. 2019; Hong et al. 2015; Huang et al. 2016; Ji et al. 2018; Joo et al. 2017; Park et al. 2016; Wang et al. 2008). Collectively, the ADHD studies indicate that risk of childhood ADHD increases in association with increasing $\mathrm{PbB}$ within the range of $\mathrm{PbB}<10 \mu \mathrm{g} / \mathrm{dL}$ (Table 2-30). Several case-control studies have found associations between increasing PbB and increasing OR for ADHD diagnosis in children (Joo et al. 2017; Park et al. 2016; Wang et al. 2008). In the largest case-control study (630 cases), conducted in China, covariate-adjusted ORs for ADHD in children 4-14 years of age were 4.92 (95\% CI 3.47, 6.98) for the PbB range 5-10 $\mu \mathrm{g} / \mathrm{dL}$ and $6.00(4.11,8.77)$ for $\mathrm{PbB} \geq 10 \mu \mathrm{g} / \mathrm{dL}$ compared to $<5 \mu \mathrm{g} / \mathrm{dL}$ (Wang et al. 2008). Associations between increasing $\mathrm{PbB}$ and increasing OR for ADHD diagnosis in children have also been found in several prospective studies (Boucher et al. 2012; Huang et al. 2016; Ji et al. 2008). In the largest prospective study (1,479 children, median age 9.6 years), conducted in Boston, ORs were estimated relative to $\mathrm{PbB}<2 \mu \mathrm{g} / \mathrm{dL}$ (Ji et al. 2018). The OR for the $\mathrm{PbB}$ range of $2-4 \mu \mathrm{g} / \mathrm{dL}$ was 1.08 (95\% CI 0.81, 1.44), and the OR for the PbB range of 5-10 $\mu \mathrm{g} / \mathrm{dL}$ was 1.73 (95\% CI 1.09, 2.73). The OR (5-10 $\mu \mathrm{g} / \mathrm{dL}$ relative to $<5 \mu \mathrm{g} / \mathrm{dL}$ ) for male children (OR 2.49, 95\% CI 1.46, 4.26) was larger than for female children (OR 0.68, 95\% CI 0.27, 1.69). A prospective study of 272 children (mean age 11 years) conducted in Nunavik, Canada found elevated covariate adjusted ORs of 4.01 (95\% CI 1.06, 15.23) for a PbB stratum 1.6-2.7 $\mu \mathrm{g} / \mathrm{dL}$ and 5.52 (95\% CI 1.38, 22.12) for the stratum 2.7-12.8 $\mu \mathrm{g} / \mathrm{dL}$ (Boucher et al. 2012). A longitudinal study examined ADHD outcomes of 2,159 South Korean children (ages 7-9 years) who did not exhibit ADHD symptoms at recruitment (Choi et al. 2016). Two years following baseline assessment, the covariate adjusted relative risk of ADHD was estimated to be 1.552 (95\% CI 1.002, 2.403) for children having $\mathrm{PbB}>2.17 \mu \mathrm{g} / \mathrm{dL}$ compared to $\leq 2.17 \mu \mathrm{g} / \mathrm{dL}$. The geometric mean PbB for the cohort was $1.62 \mu \mathrm{g} / \mathrm{dL} \pm 1.52$ (GSD). Several cross-sectional studies have also found associations between concurrent PbB and risk of ADHD (Braun et al. 2006; Froehlich et al. 2009; Hong et al. 2014). A study of data on approximately 4,700 children (age 4-15 years) reported in the 1999-2002 NHANES found elevated risk of ADHD in association with concurrent PbB >2 $\mu \mathrm{g} / \mathrm{dL}$ and a significant trend in risk with increasing PbB (Braun et al. 2006). Froehlich et al. (2009) examined data for children 8-15 years of age from the 2001-2004 NHANES. Covariate adjusted ORs of ADHD were elevated for the PbB stratum $>1.3 \mu \mathrm{g} / \mathrm{dL}$ (compared to $\geq 0.8 \mu \mathrm{g} / \mathrm{dL}$ ). A cross-sectional study conducted in South Korea examined associations between PbB and ADHD rating scores of 1,001 children of age 811 years (Hong et al. 2015). One $\log _{10}$ increase of $\mathrm{PbB}$ was associated with increases in teacher-rated ADHD hyperactivity (OR 3.66; 95\% CI 1.18, 6.13) and total ADHD score (OR 6.38; 95\% CI 1.36, 11.40). The cohort geometric mean PbB was $1.8 \pm 1.4 \mu \mathrm{g} / \mathrm{dL}$ (SD). 
Prospective studies have also provided evidence for associations between neonatal or early childhood $\mathrm{PbB}$ and other neurobehavioral outcomes, including neonatal behavior, emotional or temperament problems, anxiety or depression, sleep disorders, hyperactivity and impulsivity, autistic behavior, and delinquency (Dietrich et al. 2001; Fruh et al. 2019; Huang et al. 2016; Joo et al. 2018; Kim et al. 2016; Liu et al. 2014b, 2015b; Sioen et al. 2013; Stroustrup et al. 2016; Winter and Sampson 2017).

Altered neuromotor-neurosensory function. Numerous studies have examined possible associations between neonatal and child $\mathrm{PbB}$ and neuromotor or neurosensory function (Table 2-30). Several studies used the Psychomotor Development Index (PDI) score from the BSID, allowing comparison of results across studies (Dietrich et al. 1987, 1989; Kim et al. 2013b; Tellez-Rojo et al. 2006). Each study found inverse associations for PDI scores measured from 6 to 12 months in association with increasing prenatal (e.g., maternal) or neonatal PbB. Studies that repeatedly measured PDI scores longitudinally within the same birth cohorts found that associations observed at 6 months persisted to later ages (Dietrich et al. 1987, 1989, 1991; Tellez-Rojo et al. 2006). A prospective study conducted in China administered a neurobehavioral test battery to a birth cohort of 237 children at age 7 years (Chiodo at al. 2004). Significant declines in performance $(\mathrm{p} \leq 0.05)$ were observed in $\mathrm{PbB}$ strata that ranged from $<3 \mu \mathrm{g} / \mathrm{dL}$ at the lowest to $<10 \mu \mathrm{g} / \mathrm{dL}$; most tests that showed significant declines at $<10 \mu \mathrm{g} / \mathrm{dL}$, also showed declines at $<5 \mu \mathrm{g} / \mathrm{dL}(\mathrm{p} \leq 0.05)$. A prospective study conducted in Nunavik, Canada evaluated fine motor control in a birth cohort at 5 years (Fraser et al. 2006). Significant changes in motor control assessed from sway and reaction times were associated with increasing concurrent $\mathrm{PbB}(\mathrm{p} \leq 0.01)$. The cohort $\mathrm{PbB}$ mean was $5.3 \mu \mathrm{g} / \mathrm{dL} \pm 4.9$ (SD). This birth cohort also exhibited changes in visual evoked potentials that were associated in increasing cord PbB (Ethier et al. 2012). The cohort cord PbB mean was 4.6 \pm 3.1 (SD). However, not all studies have found associations between $\mathrm{PbB}$ and neuromotor performance. A followup of a prospective birth cohort of approximately 14,500 pregnancies evaluated motor skills in 1,558 children at age 7 years (Taylor et al. 2018). Prenatal (gestation week 11) PbB was not associated with performance on a movement assessment battery (e.g., heel-to-toe, threading lace, peg board).

Several studies have examined associations between $\mathrm{PbB}$ and neurosensory function in infants or children (Ethier et al. 2012; Liu et al. 2018b; Silver et al. 2016). A prospective study conducted in Nunavik, Canada found changes in visual evoked potentials at age 5 years that were associated with increasing cord $\mathrm{PbB}$ (mean 4/6 $\pm 3.1 \mu \mathrm{g} / \mathrm{dL}$ ) (Ethier et al. 2012). A prospective study of 315 mother-infant pairs conducted in China found associations between increasing prenatal $\mathrm{PbB}$ and brainstem auditory response measured at age 2 days and grating visual activity measured at age 6 weeks (Silver et al. 2016). Geometric mean 
late-pregnancy PbB was 2.7 \pm 2.3 (GSD) $\mu \mathrm{g} / \mathrm{dL}$. A cross-sectional study of 234 children (age 3-7 years), conducted in China, found that increasing PbB was associated with hearing loss (Liu et al. 2018b). The OR for hearing loss was 1.24 (95\% CI 1.029, 1.486). The median PbB was 4.94 \pm 0.20 (SE) $\mu \mathrm{g} / \mathrm{dL}$.

Altered brain structure and neurochemistry. A follow-up to the Cincinnati prospective study (Dietrich et al. 1986) estimated whole brain volumes and imaged brain metabolites in 157-159 adults at age 1924 years (Brubaker et al. 2010; Cecil et al. 2008, 2011; Table 2-30). Decreasing covariate adjusted brain volume was associated with increased childhood mean $\mathrm{PbB}$ (measured between ages 6 months and 6 years). Brain volume reductions that were associated with childhood PbB compromised approximately $1.2 \%$ of the total gray matter and were more severe in males compared to females. The largest effects were observed in the anterior cingulate cortex. This region of the brain is involved in controlling executive function, mood, and decision-making. Increasing childhood PbB was also associated with decreasing concentrations of various metabolites in the brain known to be important in the supporting metabolic structural integrity of neurons (e.g., lipid metabolism and myelin production). These included decreased N-acetyl aspartate (NAA) in the basal ganglia and cerebellar hemisphere, decreased glutamateglutamine in the vermis and parietal white matter, decreased creatine and phosphocreatine in the basal ganglia, and decreased cholines in the cerebellum, parietal white matter, and frontal white matter. These changes in association with childhood $\mathrm{PbB}$ suggest that childhood $\mathrm{Pb}$ exposure may be indicators of longer-term changes in brain glutamate-associated lipid metabolism or neuronal architecture (Cecil et al. 2011).

Associations Between Bone Pb and Neurological Effects in Children. Few studies have been conducted to assess possible associations between bone $\mathrm{Pb}$ and neurological function in children (Table 2-31). Prospective studies of outcomes in children of mother-infant pairs have found associations between maternal or child bone Pb cognitive function (Campbell et al. 2000b; Gomaa et al. 2002; Needleman et al. 1996; Wasserman et al. 2003; Xu et al. 2015). Increasing bone $\mathrm{Pb}$ measured at age 24 months was associated with decrements in cognitive development (Gomaa et al. 2002) and behaviors indicative of attention deficit hyperactivity disorder assessed at age 7-15 years (Xu et al. 2015). Increasing child bone $\mathrm{Pb}$ measured later in childhood (ages 11-14 years) was associated with decrements in language processing (Campbell et al. 2000b); full scale, verbal, and performance IQ (Wasserman et al. 2003); and delinquent, aggressive, internalizing, externalizing behaviors (Needleman et al. 1996). A case-control study of adjudicated delinquency at age 12-18 years found associations between increasing bone $\mathrm{Pb}$ and delinquency (Needleman et al. 2002). A prospective study found associations between increasing bone $\mathrm{Pb}$ and difficult temperament at age 24 months (Stroustrup et al. 2016). 


\section{Table 2-31. Associations Between Bone Pb and Neurological Outcomes in Children}

\begin{tabular}{|c|c|c|c|c|c|}
\hline \multirow[b]{2}{*}{ Reference } & \multirow[b]{2}{*}{ Population } & \multicolumn{3}{|c|}{ Neurological outcome } & \multirow[b]{2}{*}{$\begin{array}{l}\text { Outcome } \\
\text { measures }\end{array}$} \\
\hline & & $\begin{array}{l}\text { Intellectual } \\
\text { deficits }\end{array}$ & $\begin{array}{l}\text { Altered } \\
\text { neuromotor or } \\
\text { neurosensory } \\
\text { function }\end{array}$ & $\begin{array}{l}\text { Altered } \\
\text { mood or } \\
\text { behavior }\end{array}$ & \\
\hline $\begin{array}{l}\text { Campbell et al. } \\
2000 \mathrm{~b}\end{array}$ & $\begin{array}{l}156 \text { males, } \\
\text { age: } 11-14 \text { years }\end{array}$ & $\uparrow \mathrm{T}$ & - & - & $\begin{array}{l}\text { Language } \\
\text { processing }\end{array}$ \\
\hline $\begin{array}{l}\text { Gomaa et al. } \\
2002\end{array}$ & $\begin{array}{l}197 \text { mother-infant } \\
\text { pairs }\end{array}$ & $\begin{array}{l}\uparrow \mathrm{P}^{\mathrm{a}} \\
0 \mathrm{~T}^{\mathrm{a}}\end{array}$ & - & - & 24-month MDI ${ }^{\mathrm{b}}$ \\
\hline $\begin{array}{l}\text { Needleman et al. } \\
1996\end{array}$ & $\begin{array}{l}301 \text { males, } \\
\text { age: } 9-13 \text { years }\end{array}$ & - & - & $\uparrow \mathrm{T}$ & $\begin{array}{l}\text { Delinquent, } \\
\text { aggressive, } \\
\text { internalizing, } \\
\text { externalizing } \\
\text { behaviors }\end{array}$ \\
\hline $\begin{array}{l}\text { Needleman et al. } \\
2002\end{array}$ & $\begin{array}{l}194 \text { male cases, } \\
145 \text { controls, } \\
\text { age: } 12-18 \text { years }\end{array}$ & - & - & $\uparrow \mathrm{T}$ & $\begin{array}{l}\text { Adjudicated } \\
\text { delinquency }\end{array}$ \\
\hline $\begin{array}{l}\text { Stroustrup et al. } \\
2016\end{array}$ & $\begin{array}{l}948 \text { mother-infant } \\
\text { pairs, } 760 \text { children, } \\
\text { age: } 24 \text { months }\end{array}$ & - & - & $\uparrow \mathrm{T}$ & $\begin{array}{l}\text { Difficult } \\
\text { temperament }\end{array}$ \\
\hline $\begin{array}{l}\text { Wasserman et } \\
\text { al. } 2003\end{array}$ & $\begin{array}{l}167 \text { children, } \\
\text { age: } 10-12 \text { years }\end{array}$ & $\uparrow \mathrm{T}$ & - & - & $\begin{array}{l}\text { IQ (full scale, } \\
\text { verbal, } \\
\text { performance) }\end{array}$ \\
\hline Xu et al. 2015 & $\begin{array}{l}197 \text { mother-infant } \\
\text { pairs }\end{array}$ & - & - & $\uparrow P^{a}$ & $\begin{array}{l}\text { Attenuation of effect } \\
\text { of maternal self- } \\
\text { esteem on ADHD } \\
\text { assessed at age } 7- \\
15 \text { years }^{\mathrm{d}}\end{array}$ \\
\hline
\end{tabular}

aMaternal bone lead measured within 1 month of birth.

bBayley Scale.

'Wechsler Intelligence Scale for Children-III.

'Maternal self-esteem was evaluated with Coopersmith Self-Esteem Inventory. ADHD was evaluated with Conners' Parent Rating Scale-Revised and Behavior Rating Inventory of Executive Function.

$\uparrow=$ positive association; $\downarrow=$ inverse association; $0=$ no association; $-=$ not reported; ADHD $=$ Attention deficit hyperactivity disorder; $\mathrm{C}=$ calcaneus bone; $\mathrm{MDI}=$ Mental Developmental Index; $\mathrm{P}=$ patella; $\mathrm{Pb}=\mathrm{lead} ; \mathrm{T}=$ tibia; $\mathrm{O}=$ other

Effects at Blood Pb Levels $\leq 10 \mu \mathrm{g} / \mathrm{dL}$ in Adults. Numerous longitudinal and large cross-sectional studies in adults provide a weight of evidence for decreased cognitive function, altered mood and behavior, and altered neuromotor and neurosensory function in association with exposures that result in $\mathrm{PbB}<10 \mu \mathrm{g} / \mathrm{dL}$, 
with some studies showing effects in the $3-5 \mu \mathrm{g} / \mathrm{dL}$ range. Study details are reviewed in the Supporting Document for Epidemiological Studies for Lead, Table 10. Cognitive, neuromotor, and neurosensory outcomes have been evaluated with tests of memory, learning, executive function, reaction time, walking speed, and tremor. Pb exposure has been associated with risk of various psychiatric symptoms including anxiety, depression, and schizophrenia, and with risk of ALS. In some studies, associations were found between outcomes and $\mathrm{PbB}$ and/or bone $\mathrm{Pb}$. Several studies have examined cohorts of people who had mean ages within the range 50-70 years. Studies of cognitive function in elderly populations must control for factors that contribute to age-related decrements in function, including confounding from the relationship between age and bone $\mathrm{Pb}$, which increases with age. Longitudinal studies offer advantages over cross-sectional studies in that they can provide measurement changes in function of individual subjects with age.

Cognitive function. Numerous studies have examined possible associations between $\mathrm{Pb}$ exposure and cognitive function in adults (Table 2-32). Most of these studies have found associations between increasing $\mathrm{Pb}$ exposure, indicated by blood or bone $\mathrm{Pb}$, and indications of decreased cognitive function (Muldoon et al. 1996; Payton et al. 1998; Power et al. 2014; Przybyla et al. 2017; Seegal et al. 2013; Seo et al. 2014; Shih et al. 2006; Weisskopf et al. 2007; Weuve et al. 2006, 2009; Wright et al. 2003b). However, not all studies have found associations (Kreig et al. 2005; Yu et al. 2019b). One of the largest cross-sectional studies analyzed data from NHANES III (1988-1994) found no associations between PbB and performance on neurobehavioral tests (Krieg et al. 2005). This study compared scores from several tests from the Neurobehavioral Evaluation System (NBES) and concurrent PbB in approximately 5,700 adults (age 20-50 years). Implemented tests measured processing speed, attention, learning, and memory (reaction time, symbol-digit substitution, serial digit learning). The geometric mean $\mathrm{PbB}$ was $2.51 \mu \mathrm{g} / \mathrm{dL}$ (range $0.7-42$ ) and 96\% of the cohort was $<10 \mu \mathrm{g} / \mathrm{dL}$. No significant associations (defined as $\mathrm{p} \leq 0.05$ ) between $\mathrm{PbB}$ and cognitive outcomes were found. However, associations between $\mathrm{PbB}$ and cognitive performance may be stronger in elderly adults. An examination of a smaller cohort from the NHANES 1999-2000, restricted to ages $\geq 60$ years $(n=498)$, found an association between increasing $\mathrm{PbB}$ and decreasing scores on short-term memory (digit symbol test) (Przybyla et al. 2017). The geometric mean PbB in this study was $2.17 \mu \mathrm{g} / \mathrm{dL}$. Several studies have examined smaller cohorts from longitudinal studies designed to evaluate health in aging populations. Studies of male cohorts from the Normative Aging Study have found significant $(\mathrm{p} \leq 0.05)$ associations between increasing blood and/or bone $\mathrm{Pb}$ and 
2. HEALTH EFFECTS

Table 2-32. Summary of Epidemiology Studies Evaluating Neurodevelopmental Effects in Adults at Mean Blood Lead Concentration $(\mathrm{PbB}) \leq 10 \mu \mathrm{g} / \mathrm{dL}^{\mathrm{a}}$

\begin{tabular}{|c|c|c|c|}
\hline Reference and study population ${ }^{b}$ & $\mathrm{PbB}(\mu \mathrm{g} / \mathrm{dL})$ & Outcome evaluated & Result $^{\mathrm{c}}$ \\
\hline \multicolumn{4}{|l|}{ Cognitive abilities } \\
\hline Krieg et al. 2005 & $\begin{array}{l}\text { Gmean (range): } 2.51(0.7, \\
41.8)\end{array}$ & Simple visual reaction time & $\begin{array}{l}\text { No associations between } \mathrm{PbB} \text { and performance } \\
\text { scores }\end{array}$ \\
\hline \multirow{3}{*}{$\begin{array}{l}\text { Cross-sectional study; } n=5,662 \text { adults, } \\
\text { age } 20-59 \text { years }\end{array}$} & & & - Mean reaction time: $p=0.24$ \\
\hline & & Symbol-digit substitution & $\begin{array}{l}\text { - } \quad \text { Mean total latency: } p=0.27 \\
\text { - }\end{array}$ \\
\hline & & Serial digit learning & $\begin{array}{l}\text { - Trials to criterion: } p=0.26 \\
\text { - Total score: } p=0.24\end{array}$ \\
\hline \multirow[t]{3}{*}{$\begin{array}{l}\text { Muldoon et al. } 1996 \\
\text { Cross-sectional study; } n=530 \text { adult } \\
\text { women, mean age } 70 \text { years }\end{array}$} & $\begin{array}{l}\text { Mean (SD): } \\
\text { - } \quad \text { All: } 4.8(0.4) \\
\text { - } \quad \text { Rural: } 4.5(0.4) \\
\text { - } \quad \text { Urban: } 5.4(0.4) \\
\text { - } \quad \text { Low: }<4 \\
\text { - } \quad \text { Medium: } 4-7\end{array}$ & Trailmaking B & \begin{tabular}{ll} 
- & \multicolumn{2}{l}{ Urban } \\
o & Medium PbB OR: $0.97(0.40,2.40)$ \\
o & High PbB OR: $0.79(0.20,3.04)$ \\
- & Rural \\
o & Medium PbB OR: $2.05(\mathbf{1 . 0 5 , 4 . 0 2})^{*}$ \\
o & High PbB OR: $2.60\left(\mathbf{1 . 0 4 , 6 . 4 9 ) ^ { * }}\right.$ \\
\end{tabular} \\
\hline & - $\quad$ High: $>7$ & Digit symbol (correct) & $\begin{array}{ll}\text { - } & \text { Urban } \\
\text { o } & \text { Medium PbB OR: } 0.61(0.25,1.50) \\
\text { o } & \text { High PbB OR: } 0.64(0.16,2.47) \\
\text { - } & \text { Rural } \\
\text { o } & \text { Medium PbB OR: } 2.03(\mathbf{1 . 0 6 , 3 . 8 8})^{\star} \\
\text { o } & \text { High PbB OR: } \mathbf{3 . 7 3}\left(\mathbf{( 1 . 5 7 , 8 . 8 4 ) ^ { * }}\right.\end{array}$ \\
\hline & & Incidental memory & $\begin{array}{ll}\text { - Urban } \\
\text { o } \quad \text { Medium PbB OR: } 0.50(0.22,1.16) \\
\text { o } \quad \text { High PbB OR:0.99 }(0.28,1.16) \\
\text { - Rural } \\
\text { o } \quad \text { Medium PbB: OR: } 1.37(0.77,2.41) \\
\text { o } \quad \text { High PbB: OR: } 1.89(0.83,3.41)\end{array}$ \\
\hline
\end{tabular}


2. HEALTH EFFECTS

Table 2-32. Summary of Epidemiology Studies Evaluating Neurodevelopmental Effects in Adults at Mean Blood Lead Concentration (PbB) $\leq 10 \mu \mathrm{g} / \mathrm{dL}^{\mathrm{a}}$

\begin{tabular}{|c|c|c|c|}
\hline Reference and study population ${ }^{b}$ & $\mathrm{PbB}(\mu \mathrm{g} / \mathrm{dL})$ & Outcome evaluated & Result ${ }^{\mathrm{c}}$ \\
\hline \multirow{5}{*}{$\begin{array}{l}\text { Payton et al. } 1998 \\
\text { Longitudinal study; } n=141 \text { males, } \\
\text { mean age } 67 \text { years }\end{array}$} & \multirow{5}{*}{$\begin{array}{ll}\text { Mean (SD): } \\
\text { - } & 5.5(3.5) \\
\text { - } & \text { Q1: } 1.4 \\
\text { - } & \text { Q2: } 3.5 \\
\text { - } & \text { Q3: } 5.4 \\
\text { - } & \text { Q4: } 9.8\end{array}$} & Pattern recognition & - $\beta: 0.074(0.032), p=0.02^{*}$ \\
\hline & & Vocabulary & - $\beta:-0.841(0.20), p=0.0001^{*}$ \\
\hline & & Word list memory & - $\beta:-0.182(0.086), p=0.036^{*}$ \\
\hline & & Boston naming test & - $\beta:-0.036(0.016), p=0.028^{*}$ \\
\hline & & Verbal fluency & - $\quad \beta:-0.230(0.120), p=0.09$ \\
\hline \multirow{2}{*}{$\begin{array}{l}\text { Power et al. } 2014 \\
\text { Longitudinal study; } n=584 \text { adults } \\
\text { females, mean age } 61 \text { years }\end{array}$} & \multirow{2}{*}{$\begin{array}{l}\text { Mean }(\mathrm{SD}): \\
-\quad 2.9(1.9) \\
\text { Tibia } \mathrm{Pb}(\mu \mathrm{g} / \mathrm{g}): \text { Mean } \\
(\mathrm{SD}): \\
\text { Patella } \mathrm{Pb}(\mu \mathrm{g} / \mathrm{g}) \text { mean } \\
\text { (SD): } \\
-\quad 12.6(11.7)\end{array}$} & Overall cognition & $\begin{array}{l}\beta \text { for } 1 \text {-age year change in score per } 1 \text { SD PbB: } \\
-0.013(-0.044,0.017)\end{array}$ \\
\hline & & Verbal memory & $\begin{array}{l}\beta \text { for } 1 \text {-age year change in score per } 1 \mathrm{SD} \mathrm{PbB}: \\
0.006(-0.037,0.050)\end{array}$ \\
\hline $\begin{array}{l}\text { Przybyla et al. } 2017 \\
\text { Cross-sectional study; } n=498 \text { adults, } \\
\text { age } 60-84 \text { years }\end{array}$ & $\begin{array}{l}\text { Gmean (range): } 2.17(0.4, \\
16.4)\end{array}$ & Digit symbol (correct) & $\beta$ per InPbB $\mu \mathrm{g} / \mathrm{dL}:-0.10(-0.20,-0.006), p=0.04$ \\
\hline $\begin{array}{l}\text { Seo et al. } 2014 \\
\text { Cross-sectional study; } n=31 \text { retired } \\
\text { female } \mathrm{Pb} \text { workers, mean age } \\
60.4 \text { years, and } 34 \text { controls }\end{array}$ & $\begin{array}{l}\text { Gmean (range): } \\
\text { Exposed: } 4.07(0.88-13.5) \\
\text { Controls: } 2.00(1.24-6.47)\end{array}$ & Verbal memory & 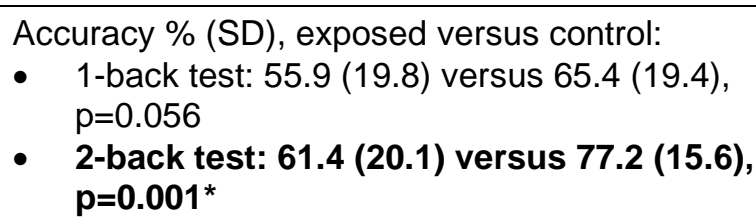 \\
\hline
\end{tabular}


Table 2-32. Summary of Epidemiology Studies Evaluating Neurodevelopmental Effects in Adults at Mean Blood Lead Concentration (PbB) $\leq 10 \mu \mathrm{g} / \mathrm{dL}^{\mathrm{a}}$

\begin{tabular}{|c|c|c|c|}
\hline Reference and study population $^{\mathrm{b}}$ & $\mathrm{PbB}(\mu \mathrm{g} / \mathrm{dL})$ & Outcome evaluated & Result $^{\mathrm{C}}$ \\
\hline \multirow{7}{*}{$\begin{array}{l}\text { Shih et al. } 2006 \\
\text { Cross-sectional study; } n=985 \text { adults, } \\
\text { mean age } 59.4 \text { years }\end{array}$} & \multirow{7}{*}{$\begin{array}{l}\text { Mean (SD): } \\
\bullet \quad 3.46(2.23) \\
\text { Tibia Pb }(\mu \mathrm{g} / \mathrm{g}) \text { mean (SD): } \\
-\quad 18.72(11.24)\end{array}$} & Language & $\begin{array}{l}\text { B per } 1 \mu \mathrm{g} / \mathrm{g} \text { tibia } \mathrm{Pb}:-0.0083(0.0023) \text {, } \\
\mathrm{p} \leq 0.01^{*}\end{array}$ \\
\hline & & Processing speed & $\begin{array}{l}\text { - } \quad \begin{array}{l}\beta \text { per } 1 \mu \mathrm{g} / \mathrm{g} \text { tibia } \mathrm{Pb}:-0.0042(0.0021) \\
\mathrm{p}<0.01^{*}\end{array} \\
\end{array}$ \\
\hline & & Eye-hand & $\begin{array}{l}\text { - } \quad \beta \text { per } 1 \mu \mathrm{g} / \mathrm{g} \text { tibia } \mathrm{Pb}:-0.0079 \text { (0.0020), } \\
\mathrm{p} \leq 0.01^{*}\end{array}$ \\
\hline & & Executive function & 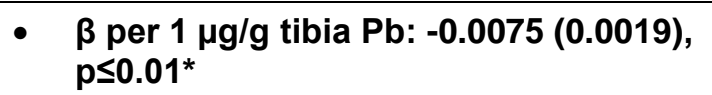 \\
\hline & & Verbal memory and learning & $\begin{array}{l}\text { - } \quad \beta \text { per } 1 \mu \mathrm{g} / \mathrm{g} \text { tibia } \mathrm{Pb}:-0.0078(0.0024) \text {, } \\
\mathrm{p} \leq 0.01^{*}\end{array}$ \\
\hline & & Visual memory & $\begin{array}{l}\text { - } \quad \beta \text { per } 1 \mu \mathrm{g} / \mathrm{g} \text { tibia } \mathrm{Pb}:-0.0067 \text { (0.0023), } \\
\mathrm{p} \leq 0.01^{*}\end{array}$ \\
\hline & & Visuoconstruction & $\begin{array}{l}\text { - } \quad \beta \text { per } 1 \mu \mathrm{g} / \mathrm{g} \text { tibia } \mathrm{Pb}:-0.0122(0.0027) \text {, } \\
\mathrm{p} \leq 0.01^{*}\end{array}$ \\
\hline \multirow{3}{*}{$\begin{array}{l}\text { Weisskopf et al. } 2007 \\
\text { Longitudinal study cohort, } \\
n=1,089 \text { males, mean age } 68.7 \text { years }\end{array}$} & \multirow{3}{*}{$\begin{array}{l}\text { Median (IQ range): } \\
\bullet \quad 5(3-6) \\
\text { Tibia } \mathrm{Pb}(\mu \mathrm{g} / \mathrm{g}) \text { median (IQ } \\
\text { range): } \\
\bullet \quad 20(13-28) \\
\text { Patella } \mathrm{Pb}(\mu \mathrm{g} / \mathrm{g}) \text { median } \\
\text { (IQ range): } \\
-\quad 25(17-37)\end{array}$} & Vocabulary & $\begin{array}{l}\beta \text { per } 3 \mu \mathrm{g} / \mathrm{dL} \text { increase in PbB: }-1.26 \\
(-2.08,-0.44), p=0.003^{*}\end{array}$ \\
\hline & & $\begin{array}{l}\text { Visuoconstruction (patella } \\
\mathrm{Pb} \text { ) }\end{array}$ & $\beta$ per IQR: $-0.067(-0.11,-0.02), p=0.0041^{*}$ \\
\hline & & $\begin{array}{l}\text { Pattern comparison latency } \\
\text { (tibia } \mathrm{Pb} \text { ) }\end{array}$ & $\beta: 0.079(0.04,0.12), p=0.0004^{*}$ \\
\hline $\begin{array}{l}\text { Weuve et al. } 2006 \\
\text { Longitudinal study cohort, } \\
\mathrm{n}=915 \text { males, mean age } 68.7 \text { years }\end{array}$ & $\begin{array}{l}\text { Median (IQ range): } \\
\qquad \quad 5.2(2.9) \\
-\quad 94 \%<10\end{array}$ & Cognitive function & $\begin{array}{l}\text { Change in MMSE score per IQR in PbB, } \\
3 \mu \mathrm{g} / \mathrm{dL} \text { : } \\
\text { - ALAD-2: IQR: }-\mathbf{0 . 2 9}(-0.56,-0.02)^{\star} \\
\text { - ALAD wildtype: IQR: }-0.05(-0.16,0.06)\end{array}$ \\
\hline $\begin{array}{l}\text { Longitudinal study cohort, } \\
n=587 \text { females, mean age } 61 \text { years }\end{array}$ & $\begin{array}{l}\text { Mean (SD): } \\
\bullet \quad 2.9(1.9) \\
\text { Tibia } \mathrm{Pb}(\mu \mathrm{g} / \mathrm{g}) \text { median } \\
(\mathrm{SD}): \\
-\quad 10.5(9.7)\end{array}$ & Cognitive function & 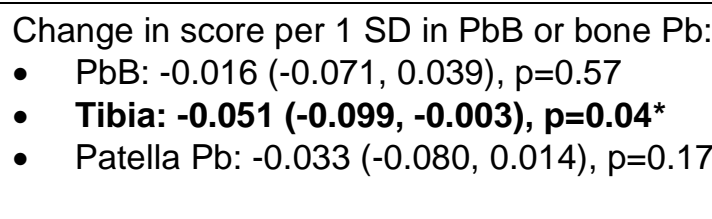 \\
\hline
\end{tabular}


Table 2-32. Summary of Epidemiology Studies Evaluating Neurodevelopmental Effects in Adults at Mean Blood Lead Concentration $(\mathrm{PbB}) \leq 10 \mu \mathrm{g} / \mathrm{dL}^{\mathrm{a}}$

\begin{tabular}{|c|c|c|c|}
\hline Reference and study population $^{\mathrm{b}}$ & $\mathrm{PbB}(\mu \mathrm{g} / \mathrm{dL})$ & Outcome evaluated & Result $^{\mathrm{C}}$ \\
\hline & $\begin{array}{l}\text { Patella } \mathrm{Pb}(\mu \mathrm{g} / \mathrm{g}) \text { median } \\
(\mathrm{SD}): \\
-\quad 12.6(11.6)\end{array}$ & & \\
\hline $\begin{array}{l}\text { Wright et al. 2003b } \\
\text { Longitudinal study cohort, } \\
n=736 \text { males, mean age } 68.2 \text { years }\end{array}$ & 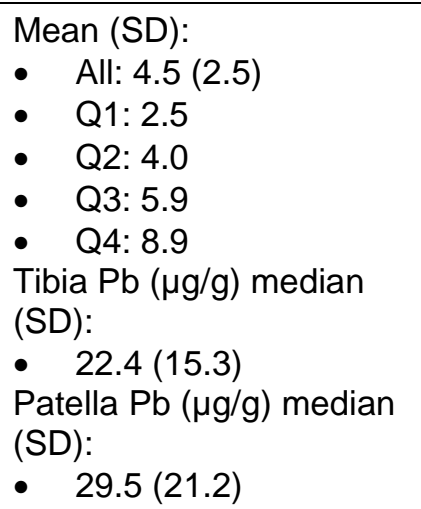 & MMSE score & $\begin{array}{l}\text { Adjusted OR with } 1 \mu \mathrm{g} / \mathrm{dL} \text { increase in } \mathrm{PbB} \text { or } \\
1 \mu \mathrm{g} / \mathrm{g} \text { increase in bone } \mathrm{Pb} \text { : } \\
\text { - PbB: } 1.21(1.07,1.36)^{*} \\
\text { - Patella Pb: } 1.02(1.00,1.03)^{*} \\
\text { - Tibia Pb: } 1.02(1.00,1.04)^{*} \\
\text { Effect of age increased with increasing PbB. } \\
\beta \text { for age with increasing Pb for PbB quartile: } \\
\text { - Q1 }-0.04(-0.07,-0.02)^{*} \\
\text { - Q2 -0.04 }(-0.08,-0.01)^{*} \\
\text { - Q3 }-0.09(-0.13,-0.06)^{*} \\
\text { - Q4 -0.12 }(-0.17,-0.02)^{*}\end{array}$ \\
\hline Yu et al. 2019b & $\begin{array}{l}\text { Gmean (IQR): } 2.47 \text { (2.00, } \\
3.00)\end{array}$ & $\begin{array}{l}\text { Digit symbol (mean total } \\
\text { latency) }\end{array}$ & $\beta$ per $\log _{10} \mathrm{PbB}: 5.4 \%(-0.4,11.5), \mathrm{p}=0.066$ \\
\hline \multirow[t]{3}{*}{$\begin{array}{l}\text { Cross-sectional study; } n=339 \text { males, } \\
\text { mean age } 28.6 \text { years }\end{array}$} & & $\begin{array}{l}\text { Stroop reaction time } \\
\text { incongruent trials }\end{array}$ & $\beta$ per $\log _{10} \mathrm{PbB}: 5.1 \%(-4.5,15.6), \mathrm{p}=0.30$ \\
\hline & & $\begin{array}{l}\text { Stroop reaction time } \\
\text { congruent trials }\end{array}$ & $\beta$ per $\log _{10} \mathrm{PbB}:-1.2 \%(-10.4,9.0), \mathrm{p}=0.81$ \\
\hline & & Stroop interference effect & $\beta$ per $\log _{10} \mathrm{PbB}: 23.0 \%(-15.4,78.9), p=0.28$ \\
\hline
\end{tabular}


Table 2-32. Summary of Epidemiology Studies Evaluating Neurodevelopmental Effects in Adults at Mean Blood Lead Concentration (PbB) $\leq 10 \mu \mathrm{g} / \mathrm{dL}^{\mathrm{a}}$

\begin{tabular}{|c|c|c|c|}
\hline Reference and study population ${ }^{b}$ & $\mathrm{PbB}(\mu \mathrm{g} / \mathrm{dL})$ & Outcome evaluated & Result $^{\mathrm{C}}$ \\
\hline \multicolumn{4}{|l|}{ Mood and behavior } \\
\hline \multirow[t]{3}{*}{$\begin{array}{l}\text { Bouchard et al. } 2009 \\
\text { Cross-sectional study; } n=1,987 \text { adults } \\
\text { (age } 20-39 \text { years) }\end{array}$} & $\begin{array}{ll}\text { Gmean } \pm \text { GSD (range): } \\
\text { - } \quad 1.24(1.96) \\
\text { - } \quad 99 \% \leq 10 \\
\text { - } \quad \text { Q1: } 0.6 \\
\text { Q2: } 0.9\end{array}$ & Major depressive disorder & $\begin{array}{l}\text { - Adjusted ORs for PbB for Q5 relative to } \\
\text { Q1: } 2.32(1.13,4.75) ; p \text {-trend=0.05* } \\
\text { Eliminating current smokers, adjusted } \\
\text { ORs for PbB for Q5 relative to Q1: } \\
2.93(1.24,6.92) ; p \text {-trend=0.03* }\end{array}$ \\
\hline & $\begin{array}{ll}- & \text { Q3: } 1.2 \\
- & \text { Q4: } 1.3 \\
- & \text { Q5: } 3.0\end{array}$ & Panic disorder & $\begin{array}{l}\text { - Adjusted ORs for } \mathrm{PbB} \text { for } \mathrm{Q} 5 \text { relative to } \\
\text { Q1: } 4.94(1.32,18.48) ; \mathrm{p} \text {-trend }=0.02^{*} \\
\text { Eliminating current smokers, adjusted } \\
\text { ORs for } \mathrm{PbB} \text { for } \mathrm{Q} 5 \text { relative to Q1: } \\
9.57(1.28,71.43) ; \mathrm{p} \text {-trend }=0.01^{*}\end{array}$ \\
\hline & & $\begin{array}{l}\text { Generalized anxiety } \\
\text { disorder }\end{array}$ & $\begin{array}{l}\text { - } \quad \text { Adjusted ORs for PbB for Q5 relative to } \\
\text { Q1: } 1.53(0.39,5.96) ; p \text {-trend=0.78 } \\
\text { Eliminating current smokers, adjusted ORs } \\
\text { for PbB for Q5 relative to Q1: } 1.59(0.19 \text {, } \\
\text { 13.31); } p \text {-trend=0.44 }\end{array}$ \\
\hline $\begin{array}{l}\text { Buser and Scinicariello } 2017 \\
\text { Cross-sectional study of 3,905 adults } \\
\text { (age } \geq 20 \text { years) from NHANES 2011- } \\
2012\end{array}$ & $\begin{array}{l}\text { Cohort stratified into PbB } \\
\text { quartiles: } \\
\text { - } \quad \text { Q1: }<0.7 \\
\text { - } \quad \text { Q2: } 0.70-1.06 \\
\text { - } \quad \text { Q3: } 1.07-1.67 \\
\text { - Q4: }>1.67\end{array}$ & Depression & $\begin{array}{l}\text { Adjusted OR for depression symptoms in adult } \\
\text { females (age 20-47 years) associated with } \\
\text { increasing PbB: } \\
\text { - Q3: } 1.86\left(\mathbf{1 . 0 1 , 3 . 4 1 ,} \mathbf{p}<0.05^{*}\right. \\
\text { - Q4: } 2.97\left(\mathbf{1 . 0 1}, \mathbf{8 . 7 4 )}, \mathbf{p}<0.05^{*}\right.\end{array}$ \\
\hline $\begin{array}{l}\text { Fan et al. } 2020 \\
\text { Cross-sectional study; } n=994 \text { adults, } \\
\text { age }>60 \text { years }\end{array}$ & $\begin{array}{l}\text { Mean (SD): } 3.229(2.357) \\
-\quad \text { Q1: }<2.027 \\
\text { Q } 2: 2.027,2.677 \\
\text { Q3: } 2.677,3.058 \\
\text { Q4: } \geq 3.058\end{array}$ & $\begin{array}{l}\text { Depression symptoms } \\
\text { (score on } 30-\text { point Geriatric } \\
\text { Depression Scale } \geq 11 \text { ) }\end{array}$ & $\begin{array}{l}\text { OR for depression for } \mathrm{PbB} \text { quartiles relative to } \\
\text { Q1: } \\
\text { - } \quad \text { Q2: } 1.28(0.79,2.08), \mathrm{p}=0.315 \\
\text { - } \quad \text { Q3: } 1.36(0.84,2.22), \mathrm{p}=0.216 \\
\text { - Q4: } \mathbf{2 . 0 3}(\mathbf{1 . 2 3}, \mathbf{3 . 3 5}), \mathbf{p}=\mathbf{0 . 0 0 6 ^ { * }} \\
-\quad \text { p-trend }=\mathbf{0 . 0 0 7 ^ { * }}\end{array}$ \\
\hline
\end{tabular}


Table 2-32. Summary of Epidemiology Studies Evaluating Neurodevelopmental Effects in Adults at Mean Blood Lead Concentration (PbB) $\leq 10 \mu \mathrm{g} / \mathrm{dL}^{\mathrm{a}}$

\begin{tabular}{|c|c|c|c|}
\hline Reference and study population ${ }^{b}$ & $\mathrm{PbB}(\mu \mathrm{g} / \mathrm{dL})$ & Outcome evaluated & Result $^{c}$ \\
\hline $\begin{array}{l}\text { Golub et al. } 2010 \\
\text { Cross-sectional study; } 4,195 \text { adults } \\
\text { (age } \geq 20 \text { years) from NHANES 2005- } \\
2006\end{array}$ & $\begin{array}{l}\text { Cohort stratified into PbB } \\
\text { quartiles: } \\
\text { - } \quad \text { Q1: } \leq 0.88 \\
\text { - } \quad \text { Q2: } 0.89-1.40 \\
\text { - } \quad \text { Q3: } 1.41-2.17 \\
\text { - } \quad \text { Q4: } 2.18-26.4\end{array}$ & & 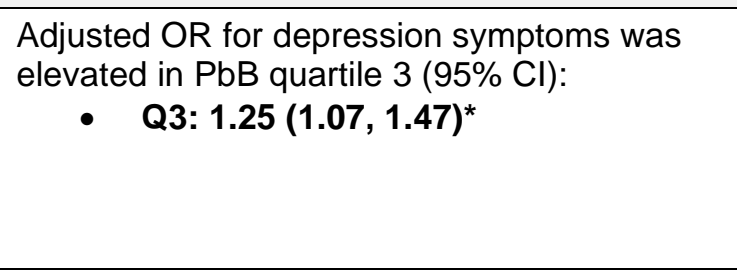 \\
\hline \multirow[t]{3}{*}{$\begin{array}{l}\text { Li et al. } 2017 \text { a } \\
\text { Cross-sectional study; } \\
n=1,931 \text { pregnancies (age } 13- \\
42 \text { years) }\end{array}$} & \multicolumn{2}{|c|}{$\begin{array}{l}\text { Gmean (range): } 3.99 \text { (0.80, Depression symptoms } \\
14.84)\end{array}$} & $\begin{array}{l}\text { per } \log _{10} \mathrm{PbB}: \\
\text { - } \quad \text { Full cohort: } 0.03(-0.05,0.10), p=0.466 \\
\text { - } \quad \mathrm{PbB} \leq 2.57: 0.34(\mathbf{0 . 1 2}, \mathbf{0 . 5 6}), \mathrm{p}=\mathbf{0 . 0 0 2} \\
\text { - } \quad \mathrm{PbB}>2.57:-0.09(-0.19,0.02), p=0.113\end{array}$ \\
\hline & & Anxiety symptoms & $\begin{array}{l}\beta \text { per } \log _{10} \mathrm{PbB}: \\
\text { - } \quad \text { Full cohort: } 0.01(-0.06,0.08), \mathrm{p}=0.770 \\
\text { - } \quad \mathrm{PbB} \leq 2.57: 0.25\left(\mathbf{0 . 0 4}, \mathbf{0 . 4 6 )}, \mathrm{p}=\mathbf{0 . 0 1 9 ^ { * }}\right. \\
\text { - } \quad \mathrm{PbB}>2.57:-0.08(-0.18,0.02), \mathrm{p}=0.136\end{array}$ \\
\hline & & $\begin{array}{l}\text { Depression or anxiety } \\
\text { symptoms (Global Severity } \\
\text { Index) }\end{array}$ & $\begin{array}{l}\beta \text { per log10 PbB: } \\
\text { - } \quad \text { Full cohort: } 0.01(-0.05,0.07), p=0.815 \\
\text { - } \quad \text { PbB } \leq 2.57: 0.22(\mathbf{0 . 0 5}, \mathbf{0 . 4 0}), p=0.013^{*} \\
\text { - } \quad \text { PbB >2.57: }-0.07(-0.16,0.01), p=0.100\end{array}$ \\
\hline $\begin{array}{l}\text { Opler et al. } 2004 \\
\text { Case-control study; } \\
\text { n=44 schizophrenia cases and } \\
75 \text { matched controls from birth cohorts }\end{array}$ & $\begin{array}{l}\text { Cohort stratified into }<15 \text { or } \\
\geq 15 \mu \mathrm{g} / \mathrm{dL} \text { based on } 2^{\text {nd }} \\
\text { trimester ALA } \\
\text { measurements }\end{array}$ & Schizophrenia & $\begin{array}{l}\text { Adjusted OR for schizophrenia associated with } \\
\text { high }(\geq 15 \mu \mathrm{g} / \mathrm{dL}) \text { prenatal } \mathrm{PbB}: 2.43(0.99, \\
5.96), \mathrm{p}=0.051\end{array}$ \\
\hline $\begin{array}{l}\text { Opler et al. } 2008 \\
\text { Case-control study; } \\
\mathrm{n}=71 \text { schizophrenia cases and } \\
129 \text { matched controls }\end{array}$ & $\begin{array}{l}\text { Cohort stratified into }<15 \text { or } \\
\geq 15 \mu \mathrm{g} / \mathrm{dL} \text { based on } 2^{\text {nd }} \\
\text { trimester ALA } \\
\text { measurements }\end{array}$ & Schizophrenia & $\begin{array}{l}\text { Adjusted OR for schizophrenia associated } \\
\text { with high }(\geq 15 \mu \mathrm{g} / \mathrm{dL}) \text { prenatal PbB: } 1.92 \\
(1.05,3.87), p=0.03^{*}\end{array}$ \\
\hline
\end{tabular}


Table 2-32. Summary of Epidemiology Studies Evaluating Neurodevelopmental Effects in Adults at Mean Blood Lead Concentration $(\mathrm{PbB}) \leq 10 \mu \mathrm{g} / \mathrm{dL}^{\mathrm{a}}$

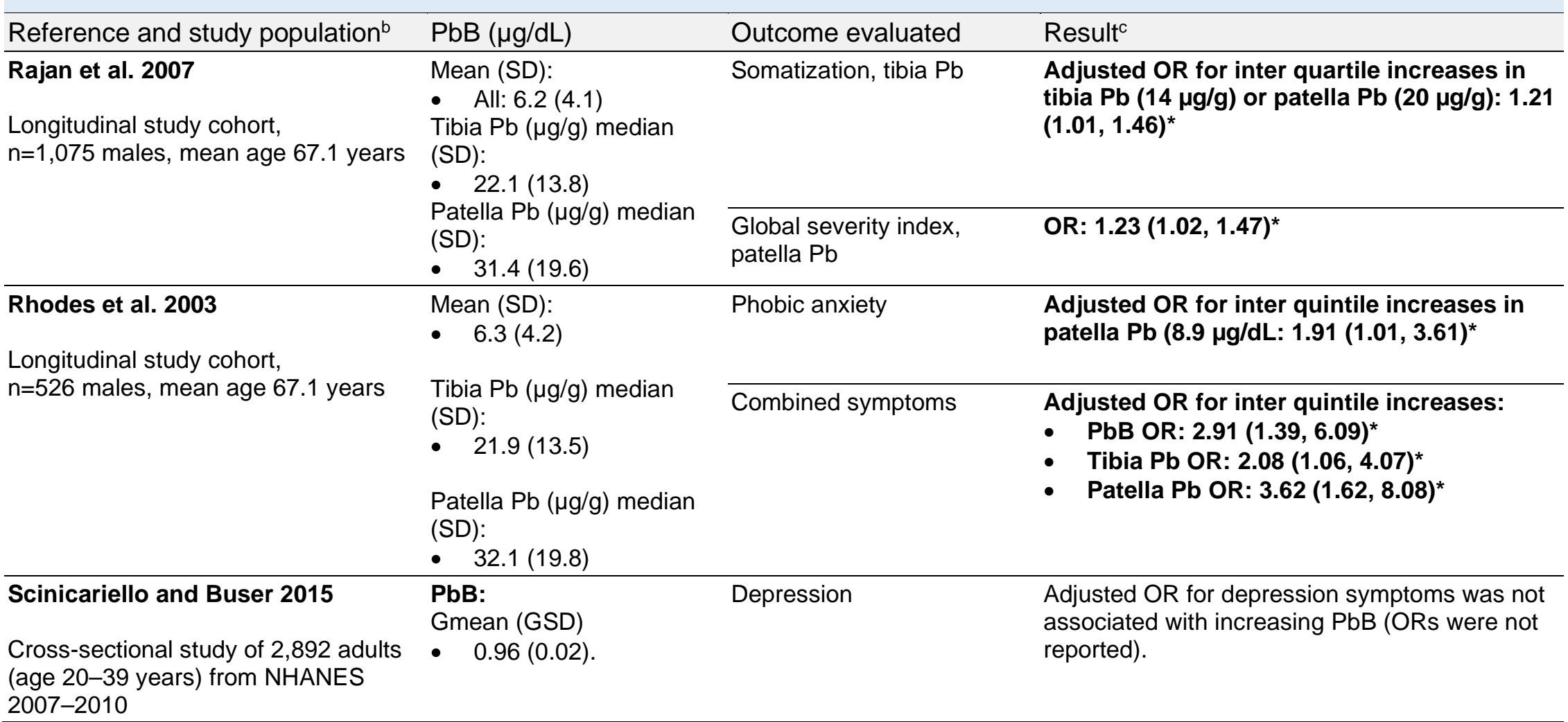


Table 2-32. Summary of Epidemiology Studies Evaluating Neurodevelopmental Effects in Adults at Mean Blood Lead Concentration $(\mathrm{PbB}) \leq 10 \mu \mathrm{g} / \mathrm{dL}^{\mathrm{a}}$

\begin{tabular}{|c|c|c|c|}
\hline Reference and study population ${ }^{b}$ & $\mathrm{PbB}(\mu \mathrm{g} / \mathrm{dL})$ & Outcome evaluated & Result $^{\mathrm{C}}$ \\
\hline \multicolumn{4}{|c|}{ Neuromotor neurosensory function } \\
\hline \multirow[t]{2}{*}{$\begin{array}{l}\text { Casjens et al. } 2018 \\
\text { Longitudinal study; } n=1,188 \text { males, } \\
\text { age 55-86 years at follow-up }\end{array}$} & $\begin{array}{l}\text { Median }(\%>9) \\
\text { - } \quad \text { Baseline: } 3.29(2.27 \%) \\
\text { - } 11-y e a r \text { follow-up: } 2.59 \\
\quad(0.84 \%)\end{array}$ & $\begin{array}{l}\text { Olfaction (score on 12-point } \\
\text { odor identification test } \leq 7 \text { ) }\end{array}$ & 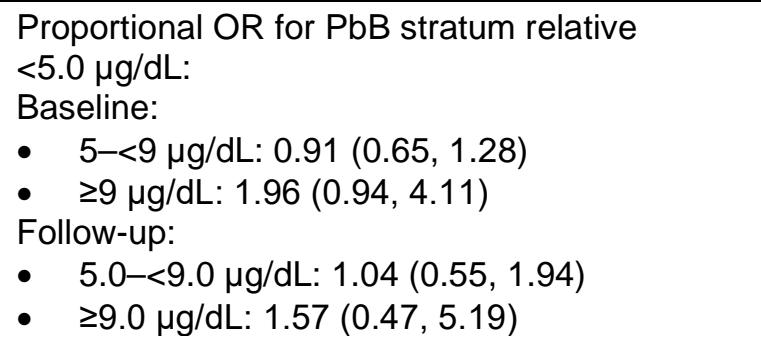 \\
\hline & & $\begin{array}{l}\text { Dexterity (finger tapping } \\
\text { errors) }\end{array}$ & 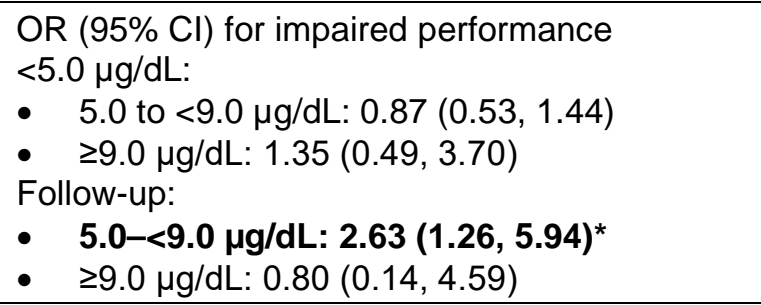 \\
\hline $\begin{array}{l}\text { Hwang et al. } 2009 \\
\text { Cross-sectional study; } n=259 \text { male } \\
\text { steel workers, mean age } 36.0 \text { years }\end{array}$ & $\begin{array}{l}\text { Mean (SD): } \\
5.43(3.46)\end{array}$ & Hearing loss & 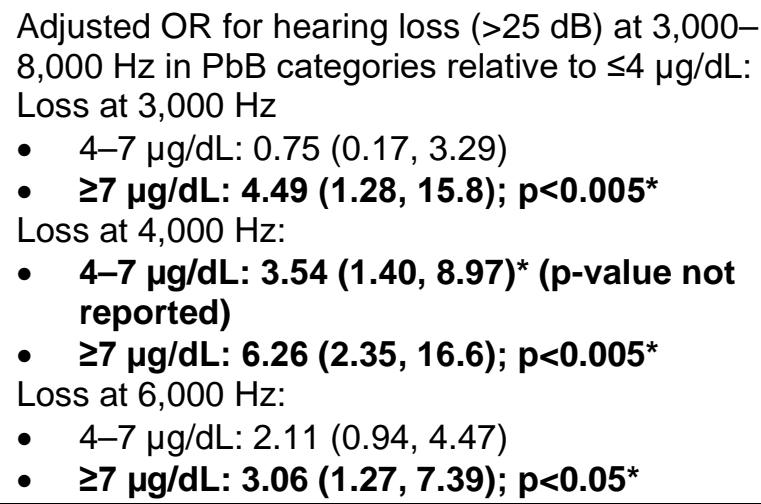 \\
\hline
\end{tabular}


Table 2-32. Summary of Epidemiology Studies Evaluating Neurodevelopmental Effects in Adults at Mean Blood Lead Concentration $(\mathrm{PbB}) \leq 10 \mu \mathrm{g} / \mathrm{dL}^{\mathrm{a}}$

\begin{tabular}{|c|c|c|c|}
\hline Reference and study population ${ }^{b}$ & $\mathrm{PbB}(\mu \mathrm{g} / \mathrm{dL})$ & Outcome evaluated & Result ${ }^{\mathrm{c}}$ \\
\hline $\begin{array}{l}\text { Huh et al. } 2018 \\
\text { Cross-sectional study; } n=2,387 \text { adults, } \\
\text { age } 19-85 \text { years }\end{array}$ & $\begin{array}{l}\text { Gmean }(95 \% \mathrm{Cl}): 2.46 \\
(2.41,2.52)\end{array}$ & $\begin{array}{l}\text { Hearing loss (pure tone } \\
\text { threshold }>25 \mathrm{~dB} \text { ) }\end{array}$ & $\begin{array}{l}\text { OR per doubling of } \mathrm{PbB}(95 \% \mathrm{Cl}): \\
-\quad \text { Low frequency: } 0.91(0.52,1.61) \\
\text { - } \quad \text { Speech frequency: } 1.21(0.72,2.04) \\
\text { - } \quad \text { High frequency: } \mathbf{1 . 8 8}(\mathbf{1 . 1 1}, \mathbf{3 . 1 7})^{*}\end{array}$ \\
\hline $\begin{array}{l}\text { Ji et al. } 2013 \\
\text { Cross-sectional study; } n=1,795 \text { males } \\
\text { and } 1,798 \text { females, age }>50 \text { years } \\
\text { (median } 61.2 \text { ) }\end{array}$ & $\begin{array}{l}\text { Mean (SD): } \\
\text { - } \quad \text { Females: } 2.17(0.06) \\
\text { - } \quad \text { Males: } 3.18(0.12)\end{array}$ & Walking speed & 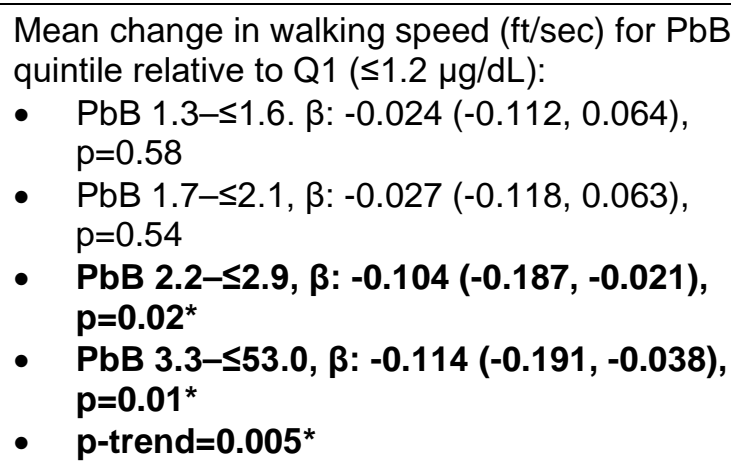 \\
\hline $\begin{array}{l}\text { Ji et al. } 2015 \\
\text { Longitudinal study cohort, } \\
n=807 \text { males, mean age } 69 \text { years }\end{array}$ & $\begin{array}{l}\text { Mean (SD): } 5.0(2.7) \\
\text { - } \%<10: 96 \% \\
\text { Bone } \mathrm{Pb}, \mu \mathrm{g} / \mathrm{g}(\mathrm{SD}) \\
\quad \quad \text { Patella: } 28.0(18.4) \\
\quad \text { Tibia: } 21.2(13.3)\end{array}$ & Tremor & $\begin{array}{l}\text { OR for tremor by PbB quintile: } \\
\text { - } \quad \mathrm{Q} 5(8-28), \mathrm{PbB}: 0.84(0.38,1.86), \mathrm{p}=0.72 \\
\text { - } \quad \mathrm{Q} 5(40-165), \text { patella } \mathrm{Pb}: 0.83(0.31,2.19) \text {, } \\
\mathrm{p}=0.41 \\
\text { - } \mathrm{Q} 5(30-126): \text { tibia } \mathrm{Pb}: 1.08(0.46,2.53), \\
\mathrm{p}=0.60\end{array}$ \\
\hline
\end{tabular}


Table 2-32. Summary of Epidemiology Studies Evaluating Neurodevelopmental Effects in Adults at Mean Blood Lead Concentration $(\mathrm{PbB}) \leq 10 \mu \mathrm{g} / \mathrm{dL}^{\mathrm{a}}$

\begin{tabular}{|c|c|c|c|}
\hline Reference and study population ${ }^{b}$ & $\mathrm{PbB}(\mu \mathrm{g} / \mathrm{dL})$ & Outcome evaluated & Result ${ }^{\mathrm{C}}$ \\
\hline \multirow{3}{*}{$\begin{array}{l}\text { Kang et al. } 2018 \\
\text { Cross-sectional study; } n=6,409 \text { adults, } \\
\text { age } 20-87 \text { years }\end{array}$} & \multirow{3}{*}{ 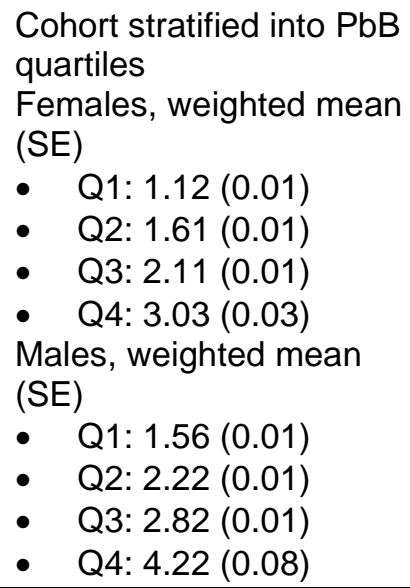 } & Hearing loss (females) & $\begin{array}{l}\text { - } \quad \text { Q2: } 0.947(0.6061 .477) \\
\text { Q3: } 1.013(0.698,1.471)\end{array}$ \\
\hline & & & - $\quad$ Q4: $1.502(1.027,2.196)^{*}$ \\
\hline & & Hearing loss (males) & $\begin{array}{ll}- & \text { Q2: } 1.368(1.006,1.859)^{*} \\
\text { - } & \text { Q3: } 1.402(1.005,1.955)^{*} \\
\text { - } & \text { Q4: } 1.629(1.161,2.287)^{*}\end{array}$ \\
\hline \multirow{4}{*}{$\begin{array}{l}\text { Muldoon et al. } 1996 \\
\text { Cross-sectional study; } n=530 \text { adult } \\
\text { women, mean age } 70 \text { years }\end{array}$} & \multirow{4}{*}{$\begin{array}{ll}\text { Mean (SD): } \\
\text { - } \quad \text { All: } 4.8(0.4) \\
\text { - } \quad \text { Rural: } 4.5(0.4) \\
\text { - } \quad \text { Urban: } 5.4(0.4) \\
\text { - } \quad \text { Low: }<4 \\
\text { - } & \text { Medium: 4-7 } \\
\text { - } & \text { High: }>7\end{array}$} & Pegboard & $\begin{array}{l}\text { OR for poor performance (low PbB reference) in } \\
\text { the rural cohort: }\end{array}$ \\
\hline & & & $\begin{array}{l}\text { ANOVA, } p=0.98 \\
\text { - } \quad \text { Medium PbB OR: } 1.37(0.71,2.65) \\
\text { - } \quad \text { High PbB OR: } 1.16(0.45,3.01)\end{array}$ \\
\hline & & Upper extremity & $\begin{array}{l}\text { ANOVA, } p<0.01 \text {, in the rural cohort } \\
\text { - } \quad \text { Medium PbB: OR: } 1.39(0.73,2.65) \\
\text { - } \quad \text { High PbB: OR: } 2.43(\mathbf{1 . 0 1 , 5 . 8 3})^{\star}\end{array}$ \\
\hline & & Lower extremity & $\begin{array}{l}\text { ANOVA, } \mathrm{p}<0.01 \text {, in the rural cohort } \\
\text { - } \quad \text { Medium PbB OR: } 1.29(0.68,2.47 \\
\text { - } \quad \text { High PbB OR: } \mathbf{2 . 8 4}(\mathbf{1 . 1 9 , 6 . 7 4 ) *}\end{array}$ \\
\hline
\end{tabular}


Table 2-32. Summary of Epidemiology Studies Evaluating Neurodevelopmental Effects in Adults at Mean Blood Lead Concentration $(\mathrm{PbB}) \leq 10 \mu \mathrm{g} / \mathrm{dL}^{\mathrm{a}}$

\begin{tabular}{|c|c|c|c|}
\hline Reference and study population $^{b}$ & $\mathrm{PbB}(\mu \mathrm{g} / \mathrm{dL})$ & Outcome evaluated & Result $^{\mathrm{C}}$ \\
\hline \multicolumn{4}{|l|}{ Neurological disease } \\
\hline $\begin{array}{l}\text { Fang et al. } 2010 \\
\text { Case-control study; } n=184 \text { male ALS } \\
\text { cases and } 194 \text { matched controls, } \\
\text { mean age } 63 \text { years }\end{array}$ & $\begin{array}{l}\text { Mean (range): } \\
\text { - Controls: } 1.76(0.32- \\
\quad 6.90) \\
\text { Cases: } 2.41(0.72- \\
\quad 7.58) \\
\end{array}$ & ALS & $\begin{array}{l}\text { Adjusted OR for ALS for doubling of PbB: } \\
\text { - All cases }(n=184): 1.9(1.3,2.7)^{*} \\
\text { - Excluding progressive muscular atrophy } \\
\text { and primary lateral sclerosis }(n=151): 1.8 \\
(1.2,2.5)^{*}\end{array}$ \\
\hline $\begin{array}{l}\text { Kamel et al. } 2002 \\
\text { Case-control study; } n=109 \text { ALS cases } \\
\text { and } 256 \text { matched controls, age } 30- \\
80 \text { years }\end{array}$ & 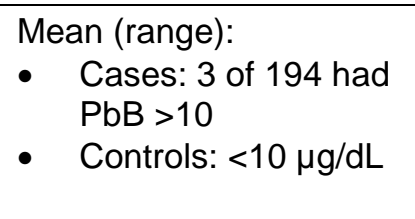 & ALS & $\begin{array}{l}\text { - Adjusted OR for ALS (for a 1- } \mu \mathrm{g} / \mathrm{dL} \\
\text { increase in PbB: } 1.9(1.4,2.6)^{*} \\
\text { - Adjusted OR for ALS relative to }<2 \mu \mathrm{g} / \mathrm{dL} \\
\text { o } 3-4 \mu \mathrm{g} / \mathrm{dL}: 14.3(3.0,69.3)^{*} \\
0 \quad 5-14 \mu \mathrm{g} / \mathrm{dL}: 24.5(4.3,139.3)^{*}\end{array}$ \\
\hline
\end{tabular}

aSee the Supporting Document for Epidemiological Studies for Lead, Table 10 for more detailed descriptions of studies.

barticipants had no known occupational exposure to $\mathrm{Pb}$.

${ }^{\mathrm{c}}$ Asterisk and bold indicate association with $\mathrm{Pb}$; unless otherwise specified, values in parenthesis are $95 \%$ Cls; $p$-values $<0.05$ unless otherwise noted in the table.

ALA = aminolevulinic acid; ALAD-2 = delta-aminolevulinic acid dehydratase allele; ALS = amyotrophic lateral sclerosis; ANOVA = analysis of variance;

$\mathrm{Cl}=$ confidence interval; $\mathrm{CL}=$ confidence limit; Gmean = geometric mean; GSD = geometric standard deviation; IQ = intelligence quotient; IQR = interquartile range; $\mathrm{MMSE}=$ Mini-Mental Status Examination; NHANES = National Health and Nutrition Examination Survey; OR = odds ratio; Pb = lead; SD = standard deviation; SE = standard error 
decreasing scores on cognitive tests, including short-term memory, verbal memory, and visuoconstruction (Payton et al. 1998; Weisskopf et al. 2007; Weuve et al. 2006). Cohort sizes in these studies ranged from approximately 600 to 1,100 and the mean $\mathrm{PbB}$ ranged from $2.9 \pm 1.9$ to $5.5 \pm 3.5 \mu \mathrm{g} / \mathrm{dL}$. Weuve et al. (2006) found that decreases in cognitive performance were associated with PbB in a cohort of ALAD-2 carriers, but not in a cohort that carried the wildtype ALAD allele. Studies of female cohorts (approximately 600 subjects) from the longitudinal Nurses’ Health Study have found mixed outcomes (Power et al. 2014; Weuve et al. 2009). Weuve et al. (2009) found significant association between increasing tibia $\mathrm{Pb}$, but not $\mathrm{PbB}$, and scores on a telephone survey of cognitive function (the Telephone Interview for Cognitive Status, TIC). The TIC has been used to assess memory and executive function and has been used to evaluate dementia. The effect size was -0.051 (95\% CI -0.099, -0.003) points per 1 SD of tibia Pb. Power et al. (2014) used the same telephone survey instrument and found no associations between blood or bone $\mathrm{Pb}$ and cognitive function; the effect size for $\mathrm{PbB}$ was -0.013 (95\% CI: $-0.044,0.017$ ) and the cohort mean PbB was $2.9 \pm 1.9$ (SD) $\mu \mathrm{g} / \mathrm{dL}$. A cross-sectional study of approximately 1,000 adults from the Boston Memory Study found inverse associations $(p \leq 0.05)$ between performance on cognitive tests and increasing tibia $\mathrm{Pb}$, but not for $\mathrm{PbB}$ (Shih et al. 2006). The cohort mean blood $\mathrm{Pb}$ was 3.46 \pm 2.2 (SD) $\mu \mathrm{g} / \mathrm{dL}$. Cognitive function evaluated included language, processing speed, executive function, verbal memory and learning, and visuoconstruction. The effect sizes were substantially attenuated by race/ethnicity and years of educational and were no longer significant $(\mathrm{p}<0.05)$ when adjusted for these covariates. A cross-sectional study of approximately 500 adult females from the Study of Osteoporotic Fractures found significant associations $(p \leq 0.05)$ between performance on cognitive tests and increasing PbB (Muldoon et al. 1996). The odds of performing worse on visual attention and short-term memory tests were significantly decreased $(\mathrm{p} \leq 0.05)$ in a PbB stratum $4-7$ and to $>7 \mu \mathrm{g} / \mathrm{dL}$ compared to stratum $<4 \mu \mathrm{g} / \mathrm{dL}$. A cross-sectional study of 339 newly hired male Pb workers did not find significant associations between $\mathrm{PbB}(\mathrm{p} \geq 0.05)$ and performance on tests that measured attention, memory, and processing speed (Stroop test, Symbol Digit Test) (Yu et al. 2019b). The geometric mean $\mathrm{PbB}$ was $2.47 \mu \mathrm{g} / \mathrm{dL}$.

Altered mood and behavior. Several studies have examined associations between Pb exposure assessed from blood or bone $\mathrm{Pb}$ and symptoms of psychiatric disorders (Table 2-32). Several studies have analyzed cross-sectional data from NHANES to explore associations between depression symptoms and PbB (Bouchard et al. 2009; Buser and Scinicariello 2017; Golub et al. 2010; Scinicariello and Buser 2015). Three studies found associations between $\mathrm{PbB}$ and depression in adult populations that had geometric mean $\mathrm{PbBs}$ that were $2-3 \mu \mathrm{g} / \mathrm{dL}$ compared to populations that have $\mathrm{PbBs}<1$ (Bouchard et al. 2009; Buser and Scinicariello 2017; Golub et al. 2010). Buser and Scinicariello (2017) found stronger 
associations in adult women than in men. Cross-sectional studies in other populations have found significant associations between $\mathrm{PbB}$ and symptoms of depression or anxiety (Fan et al. 2020; Li et al. 2017a). The Fan et al. (2020) study was restricted to adults $>60$ years ( $n=994)$ and found that increasing $\mathrm{PbB}$ was associated with increasing scores on the Geriatric Depression Scale. The OR for categorization as depressed was 2.04 (95\% CI: 1.23, 3.35) in the upper quartile PbB stratum ( $\geq 3.06 \mu \mathrm{g} / \mathrm{dL})$. The Li et al. (2017a) study examined a cross-sectional cohort of 1,931 pregnancies (age range 13-42 years) for depression, anxiety, and psychological stress. Increasing PbB was associated with increasing scores on depression and anxiety assessments; however, the association was stronger in the $\mathrm{PbB}$ stratum $\leq 2.57 \mu \mathrm{g} / \mathrm{dL}$ compared to a higher stratum $>2.57 \mu \mathrm{g} / \mathrm{dL}$. Associations between psychiatric disorders and Pb exposure metrics have also been studied in longitudinal studies (Rajan et al. 2007; Rhodes et al. 2003). Two studies of cohorts from the Normative Aging Study found significant ORs for blood or bone $\mathrm{Pb}$ and various psychiatric symptoms in males (mean age $67 \pm 7, \mathrm{SD}$ ), including somatization, phobic anxiety, and composite indices of distress. Mean PbBs in these cohorts were $6 \pm 4$ (SD) $\mu \mathrm{g} / \mathrm{dL}$. Associations between $\mathrm{PbB}$ and psychiatric disorders have also been found in case-control studies (Opler et al. 2004, 2008). The largest was a study of 71 schizophrenia cases and 129 matched controls (Opler et al. 2008). The adjusted OR for schizophrenia was $1.92(95 \%$ CI $1.05,3.87)$ for the $\mathrm{PbB}$ stratum $\geq 15 \mu \mathrm{g} / \mathrm{dL}$ compared to $15 \mu \mathrm{g} / \mathrm{dL}$. Because individual PbB data were not available, subjects were categorized into the high $(<15 \mu \mathrm{g} / \mathrm{dL})$ or low $(15 \mu \mathrm{g} / \mathrm{dL})$ PbB categories based on measurements of serum ALA and a regression model relating $\mathrm{PbB}$ and ALA derived from a different population (Graziano et al. 1990). Although the accuracy of the method for assigning subjects from Graziano et al. (1990) into low or high categories was, on average, approximately $90 \%$, uncertainty in the actual regression model is likely to have resulted in some misclassification of individuals.

Altered neuromotor neurosensory function. Several studies have examined associations between $\mathrm{Pb}$ exposure assessed from blood or bone $\mathrm{Pb}$ and performance on tests of neuromotor or neurosensory function (Table 2-32). The largest study analyzed data from NHANES III (1988-1994) and found no association ( $\mathrm{p}=0.34$ ) between concurrent $\mathrm{PbB}$ and simple visual reaction time in a cohort of 5,700 adults (age 20-50 years; Krieg et al. 2005). The geometric mean PbB was $2.51 \mu \mathrm{g} / \mathrm{dL}$ (range 0.7-42) and 96\% of the cohort was $<10 \mu \mathrm{g} / \mathrm{dL}$. A more recent analysis of data from NHANES (1999-2002) examined walking speed in cohorts of approximately 1,800 males or females and found a significant association between increasing $\mathrm{PbB}$ and decreasing walking speed in females in a PbB stratum 2.2- $\leq 2.9 \mu \mathrm{g} / \mathrm{dL}$ compared to $1.6 \mu \mathrm{g} / \mathrm{dL}$; there was a significant trend with increasing PbB (Ji et al. 2013). This outcome is consistent with a smaller cross-sectional study of women (mean age $70 \pm 4$ years) that found significant decreases in upper and lower extremity reaction times in association with increasing PbB (Muldoon et al. 
1996). A longitudinal study of a cohort from the Normative Aging Study found no significant

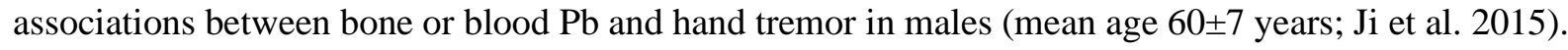

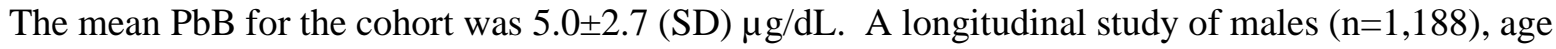
range 50-86 years, conducted in Germany, found associations between increasing $\mathrm{PbB}$ and decreasing performance scores on tests of dexterity (Casjens et al. 2018). The median PbBs were $3.29 \mu \mathrm{g} / \mathrm{dL}$ at the start of the study $(2.27 \%>9 \mu \mathrm{g} / \mathrm{dL})$ and $2.29 \mu \mathrm{g} / \mathrm{dL}(0.84 \%>9 \mu \mathrm{g} / \mathrm{dL})$ at the 11-year follow-up. This study examined several metrics of dexterity (finger tapping and aiming, line tracing, steadiness). The association with $\mathrm{Pb}$ was strongest for the finger tapping test. The OR for impaired performance on the finger tapping test at the follow-up was 2.63 (95\% CI: 1.26, 5.94) for the PbB stratum $5.0-<9 \mu \mathrm{g} / \mathrm{dL}$ and 0.80 (95\% CI: 0.14, 4.59) for the PbB stratum $>9 \mu \mathrm{g} / \mathrm{dL}$.

Several studies have examined associations between $\mathrm{PbB}$ and sensory function in adults, including olfaction (Casjens et al. 2018) and hearing (Huh et al. 2018; Hwang et al. 2009; Kang et al. 2018). Two studies examined association between $\mathrm{PbB}$ and hearing using data from the Korean National Health and Nutrition Examination Study (KNHANES) (Huh et al. 2018; Kang et al. 2018). Both studies found associations between increasing $\mathrm{PbB}$ and high-frequency hearing loss. The larger of the two studies $(n=6,409)$ estimated ORs for high-frequency hearing loss in females and males in the age range 1985 years. The ORs were 1.629 (95\% CI: 1.161, 2.287) in the highest male PbB quartile (mean PbB: $4.2 \mu \mathrm{g} / \mathrm{dL} \pm 0.04 \mathrm{SE}$ ) and 1.502 (95\% CI: 1.027, 2.196) in the highest female PbB quartile (mean PbB: $3.03 \mu \mathrm{g} / \mathrm{dL} \pm 0.03 \mathrm{SE}$ ). A smaller cross-sectional study of steel workers ( $\mathrm{n}=259)$ also found associations been increasing $\mathrm{PbB}$ and hearing loss that extended from 3,000 to $8,000 \mathrm{~Hz}$ in the $\mathrm{PbB}$ stratum $\geq 7 \mu \mathrm{g} / \mathrm{dL}$ (Hwang et al. 2009). Performance on an odor identification test was not associated with $\mathrm{PbB}$ in a longitudinal study of males ( $\mathrm{n}=1,188)$, age range 50-86 years (Casjens et al. 2018).

Neurological diseases. Possible associations between $\mathrm{Pb}$ exposure and risk of ALS have been examined in case-control studies (Fang et al. 2010; Kamel et al. 2002). A case-control study of 184 male ALS cases and 194 matched controls found a significant association between increasing PbB and ALS (Fang et al. 2010). The mean PbB for cases was $2.41 \mu \mathrm{g} / \mathrm{dL}$ (range 0.72-7.58 $\mu \mathrm{g} / \mathrm{dL}$ ). A case-control study of 109 ALS cases (43 females, 66 males) and 194 matched controls estimated the OR for ALS to be 1.9 (95\% CI: 1.4, 2.6) for a $1 \mu \mathrm{g} / \mathrm{dL}$ increase in PbB (Kamel et al. 2002).

Associations Between Bone Pb and Neurological Effects in Adults. Decrements in neurological function in adults have also been associated with bone $\mathrm{Pb}$ (Table 2-33). In general, these studies provide further support for associations between $\mathrm{Pb}$ exposure and neurobehavioral function, including decrements 
in cognitive function, altered neuromotor and neurosensory function, and altered behavior and mood. Most of these studies are of cohorts from longitudinal health studies: Boston Memory Study (BandeenRoche et al. 2009; Glass et al. 2009; Shih et al. 2006), Nurses' Health Study (Power et al. 2014; Weuve et al. 2009), or Normative Aging Study (Eum et al. 2013; Farooqui et al. 2017; Grashow et al. 2013a, 2013b, 2015; Ji et al. 2015; Park et al. 2010; Payton et al. 1998; Power et al. 2014; Rajan et al. 2007, 2008; Rhodes et al. 2003; Schwartz et al. 2005; Wang et al. 2007, 2018; Weisskopf et al. 2004, 2007; Wright et al. 2003b). These studies have provided both cross-sectional and longitudinal assessments of associations between bone $\mathrm{Pb}$ (and $\mathrm{PbB}$ ) and neurological function in adult populations. Longitudinal designs are particularly important because they allow age-related declines in cognitive function to be assessed. Longitudinal studies have found that associations between bone $\mathrm{Pb}$ and cognitive function (learning, memory) persist when adjustments are made for age (Bandeen-Roche et al. 2009; Dorsey et al. 2006; Eum et al. 2013; Grashow et al. 2013a; Khalil et al. 2009; Payton et al. 1998; Power et al. 2014; Rajan et al. 2008; Schwartz et al. 2005; Seegal et al. 2013; Shih et al. 2006; Stewart et al. 2002; van Wijngaarden et al. 2009; Weisskopf et al. 2007; Weuve et al. 2009, 2013; Wright et al. 2003b). Rates of decrement in cognitive function with age have been found to be more severe in association with increasing bone $\mathrm{Pb}$ (Farooqui et al. 2017; Power et al. 2014; Schwartz et al. 2005; Wang et al. 2007; Weisskopf et al. 2004, 2007; Wright et al. 2003b).

\section{Table 2-33. Associations Between Bone $\mathrm{Pb}$ and Neurological Outcomes in Adults}

\begin{tabular}{|c|c|c|c|c|c|}
\hline \multirow[b]{2}{*}{ Reference } & \multirow[b]{2}{*}{ Population } & \multicolumn{3}{|c|}{ Neurological outcome } & \multirow[b]{2}{*}{$\begin{array}{l}\text { Outcome } \\
\text { measures }\end{array}$} \\
\hline & & $\begin{array}{l}\text { Intellectual } \\
\text { deficits }\end{array}$ & $\begin{array}{l}\text { Altered } \\
\text { neuromotor or } \\
\text { neurosensory } \\
\text { function }\end{array}$ & $\begin{array}{l}\text { Altered } \\
\text { mood or } \\
\text { behavior }\end{array}$ & \\
\hline $\begin{array}{l}\text { Bandeen-Roche } \\
\text { et al. } 2009\end{array}$ & $\begin{array}{l}965 \text { adults, } \\
\text { age: } 50- \\
70 \text { years }^{\mathrm{a}}\end{array}$ & $\uparrow T$ & - & - & $\begin{array}{l}\text { Learning, memory, } \\
\text { executive function, } \\
\text { eye-hand } \\
\text { coordination }\end{array}$ \\
\hline Coon et al. 2006 & $\begin{array}{l}121 \text { adult cases, } \\
414 \text { controls, } \\
\text { age: } 50- \\
>80 \text { years }\end{array}$ & - & $\uparrow 0^{\mathrm{d}}$ & - & Parkinson's disease \\
\hline $\begin{array}{l}\text { Dorsey et al. } \\
2006\end{array}$ & $\begin{array}{l}652 \text { adult } \mathrm{Pb} \\
\text { workers, } \\
\text { age: } 20-70 \text { years }\end{array}$ & $\begin{array}{l}\uparrow P \\
\uparrow \mathrm{P}\end{array}$ & $\begin{array}{l}\uparrow P \\
\uparrow \mathrm{T}\end{array}$ & $\begin{array}{l}\uparrow P \\
\uparrow \mathrm{P}\end{array}$ & $\begin{array}{l}\text { Reaction time, } \\
\text { executive function, } \\
\text { manual dexterity, } \\
\text { vibration threshold, } \\
\text { depression }\end{array}$ \\
\hline
\end{tabular}




\section{Table 2-33. Associations Between Bone Pb and Neurological Outcomes in Adults}

\begin{tabular}{|c|c|c|c|c|c|}
\hline \multirow[b]{2}{*}{ Reference } & \multirow[b]{2}{*}{ Population } & \multicolumn{3}{|c|}{ Neurological outcome } & \multirow[b]{2}{*}{$\begin{array}{l}\text { Outcome } \\
\text { measures }\end{array}$} \\
\hline & & $\begin{array}{l}\text { Intellectual } \\
\text { deficits }\end{array}$ & $\begin{array}{l}\text { Altered } \\
\text { neuromotor or } \\
\text { neurosensory } \\
\text { function }\end{array}$ & $\begin{array}{l}\text { Altered } \\
\text { mood or } \\
\text { behavior }\end{array}$ & \\
\hline Eum et al. 2013 & $\begin{array}{l}789 \text { adult } \text { males }^{b} \text {, } \\
\text { age: } 68 \text { years } \\
\text { (median) }\end{array}$ & $\begin{array}{l}\uparrow P \\
\uparrow \mathrm{T}\end{array}$ & - & - & $\begin{array}{l}\text { Memory, verbal and } \\
\text { written skills, } \\
\text { executive function }\end{array}$ \\
\hline Eum et al. 2015 & $\begin{array}{l}100 \text { adult cases, } \\
194 \text { controls, } \\
\text { age: } 60 \text { years } \\
\text { (mean) }\end{array}$ & - & $\begin{array}{l}\uparrow P \\
\uparrow \mathrm{P}\end{array}$ & - & $\begin{array}{l}\text { Interaction between } \\
\mathrm{Pb} \text {, amyotrophic } \\
\text { lateral sclerosis and } \\
\text { hemochromatosis } \\
\text { gene } \\
\text { polymorphisms }\end{array}$ \\
\hline $\begin{array}{l}\text { Farooqui et al. } \\
2017\end{array}$ & $\begin{array}{l}741 \text { males, age: } \\
68 \text { years (mean) }\end{array}$ & $\begin{array}{l}\uparrow P \\
0 T\end{array}$ & - & - & $\begin{array}{l}\text { Memory, } \\
\text { visuospatial ability, } \\
\text { attention, language, } \\
\text { orientation }\end{array}$ \\
\hline Glass et al. 2009 & $\begin{array}{l}\text { 1,001 adultsa, } \\
\text { age: } 50-70 \text { years }\end{array}$ & $\uparrow \mathrm{T}$ & $\uparrow \top$ & - & $\begin{array}{l}\text { Interaction between } \\
\mathrm{Pb} \text { and } \\
\text { psychosocial hazard } \\
\text { scale for eye-hand } \\
\text { coordination, } \\
\text { executive function, } \\
\text { language }\end{array}$ \\
\hline $\begin{array}{l}\text { Grashow et al. } \\
2013 a\end{array}$ & $\begin{array}{l}51 \text { adult males }{ }^{b} \text {, } \\
\text { age: } 75 \text { years } \\
\text { (mean) }\end{array}$ & $\begin{array}{l}\uparrow P \\
0 T\end{array}$ & - & - & Fear conditioning \\
\hline $\begin{array}{l}\text { Grashow et al. } \\
2013 b\end{array}$ & $\begin{array}{l}362 \text { adult } \text { males }^{b} \text {, } \\
\text { age: } 69 \text { years } \\
\text { (mean) }\end{array}$ & - & $\begin{array}{l}\uparrow P \\
\uparrow \mathrm{T}\end{array}$ & - & Manual dexterity \\
\hline $\begin{array}{l}\text { Grashow et al. } \\
2015\end{array}$ & $\begin{array}{l}164 \text { adult males }{ }^{b} \text {, } \\
\text { age: } 80 \text { years } \\
\text { (mean) }\end{array}$ & - & $\begin{array}{l}0 P \\
\uparrow T\end{array}$ & - & Olfactory function \\
\hline Ji et al. 2015 & $\begin{array}{l}672 \text { adult males }{ }^{b} \\
\text { age: } 50-98 \text { years }\end{array}$ & - & $\begin{array}{l}0 \mathrm{P} \\
0 \mathrm{~T}\end{array}$ & - & $\begin{array}{l}\text { Tremor (no } \\
\text { association in } \\
\text { adjusted models) }\end{array}$ \\
\hline $\begin{array}{l}\text { Kamel et al. } \\
2002\end{array}$ & $\begin{array}{l}109 \text { adult cases, } \\
256 \text { controls, } \\
\text { age: } 30-80 \text { years }\end{array}$ & - & $\begin{array}{l}0 \mathrm{P} \\
0 \mathrm{~T}\end{array}$ & - & $\begin{array}{l}\text { Amyotrophic lateral } \\
\text { sclerosis (no } \\
\text { association in } \\
\text { adjusted models) }\end{array}$ \\
\hline Khalil et al. 2009 & $\begin{array}{l}83 \text { adult workers } \\
\text { and } 51 \text { controls, } \\
\text { age: }>55 \text { years }\end{array}$ & $\uparrow \top$ & - & - & Learning, memory \\
\hline
\end{tabular}




\section{Table 2-33. Associations Between Bone Pb and Neurological Outcomes in Adults}

\begin{tabular}{|c|c|c|c|c|c|}
\hline \multirow[b]{2}{*}{ Reference } & \multirow[b]{2}{*}{ Population } & \multicolumn{3}{|c|}{ Neurological outcome } & \multirow[b]{2}{*}{$\begin{array}{l}\text { Outcome } \\
\text { measures }\end{array}$} \\
\hline & & $\begin{array}{l}\text { Intellectual } \\
\text { deficits }\end{array}$ & $\begin{array}{l}\text { Altered } \\
\text { neuromotor or } \\
\text { neurosensory } \\
\text { function }\end{array}$ & $\begin{array}{l}\text { Altered } \\
\text { mood or } \\
\text { behavior }\end{array}$ & \\
\hline Park et al. 2010 & $\begin{array}{l}448 \text { adult } \text { males }^{b} \text {, } \\
\text { age: } 65 \text { years } \\
\text { (mean) }\end{array}$ & - & $\begin{array}{l}\uparrow P \\
\uparrow \mathrm{T}\end{array}$ & - & Hearing function \\
\hline $\begin{array}{l}\text { Payton et al. } \\
1998\end{array}$ & $\begin{array}{l}141 \text { adult males }{ }^{b} \text { age } 67 \text { years } \\
\text { (mean) }\end{array}$ & $\uparrow \mathrm{T}$ & - & - & $\begin{array}{l}\text { Memory, visual- } \\
\text { spatial performance }\end{array}$ \\
\hline $\begin{array}{l}\text { Power et al. } \\
2014\end{array}$ & $\begin{array}{l}584 \text { adult } \\
\text { females }^{c}, \\
\text { age: } 60-74 \text { years }\end{array}$ & $\begin{array}{l}0 \mathrm{P} \\
0 \mathrm{~T}\end{array}$ & - & - & $\begin{array}{l}\text { Learning, memory, } \\
\text { executive function }\end{array}$ \\
\hline Rajan et al. 2007 & $\begin{array}{l}1,075 \text { adult } \\
\text { males }^{\mathrm{b}}, \\
\text { age: } 48-94 \text { years }\end{array}$ & - & - & $\begin{array}{l}\uparrow P \\
\uparrow \mathrm{P}\end{array}$ & $\begin{array}{l}\text { Psychiatric } \\
\text { symptoms }\end{array}$ \\
\hline Rajan et al. 2008 & $\begin{array}{l}982 \text { adult males }{ }^{b} \text {, } \\
\text { age: } 49->72 \\
\text { years }\end{array}$ & $\begin{array}{l}0 P \\
\uparrow T\end{array}$ & - & - & $\begin{array}{l}\text { Visual-spatial } \\
\text { performance }\end{array}$ \\
\hline $\begin{array}{l}\text { Rhodes et al. } \\
2003\end{array}$ & $\begin{array}{l}536 \text { adult males } \\
\text { age: } 48-70 \text { years }\end{array}$ & - & - & $\begin{array}{l}\uparrow P \\
\uparrow T\end{array}$ & Anxiety \\
\hline $\begin{array}{l}\text { Schwartz et al. } \\
2000 b\end{array}$ & $\begin{array}{l}535 \mathrm{~Pb} \text { workers, } \\
\text { age: } 56 \text { years } \\
\text { (mean) }\end{array}$ & $\uparrow \mathrm{T}$ & $\uparrow \mathrm{T}$ & - & $\begin{array}{l}\text { Memory, executive } \\
\text { function, manual } \\
\text { dexterity }\end{array}$ \\
\hline $\begin{array}{l}\text { Schwartz et al. } \\
2001\end{array}$ & $\begin{array}{l}803 \text { exposed } \mathrm{Pb} \\
\text { workers and } \\
135 \text { controls, } \\
\text { age: } 40 \text { years } \\
\text { (mean) }\end{array}$ & $0 \mathrm{~T}$ & $0 \mathrm{~T}$ & $0 \mathrm{~T}$ & $\begin{array}{l}\text { Learning, memory, } \\
\text { executive function, } \\
\text { manual dexterity, } \\
\text { grip strength, mood } \\
\text { and depression }\end{array}$ \\
\hline $\begin{array}{l}\text { Schwartz et al. } \\
2005\end{array}$ & $\begin{array}{l}576 \text { exposed } \mathrm{Pb} \\
\text { workers, } \\
\text { age: } 41 \text { years } \\
\text { (mean) }\end{array}$ & $\uparrow \mathrm{T}$ & $\uparrow \mathrm{T}$ & $\uparrow \mathrm{T}$ & $\begin{array}{l}\text { Executive function, } \\
\text { manual dexterity, } \\
\text { vibration threshold, } \\
\text { depression }\end{array}$ \\
\hline $\begin{array}{l}\text { Seegal et al. } \\
2013\end{array}$ & $\begin{array}{l}241 \text { capacitor } \\
\text { workers, } \\
\text { age: } 64 \text { years } \\
\text { (mean) }\end{array}$ & $\uparrow \top$ & $\uparrow \top$ & - & $\begin{array}{l}\text { Learning, memory, } \\
\text { executive function, } \\
\text { manual dexterity }\end{array}$ \\
\hline Shih et al. 2006 & $\begin{array}{l}991 \text { adultsa }^{\mathrm{a}} \\
\text { age: } 50-70 \text { years }\end{array}$ & $\uparrow \mathrm{T}$ & $\uparrow \mathrm{T}$ & - & $\begin{array}{l}\text { Learning, memory, } \\
\text { executive function, } \\
\text { manual dexterity }\end{array}$ \\
\hline
\end{tabular}




\section{Table 2-33. Associations Between Bone Pb and Neurological Outcomes in Adults}

\begin{tabular}{|c|c|c|c|c|c|}
\hline \multirow[b]{2}{*}{ Reference } & \multirow[b]{2}{*}{ Population } & \multicolumn{3}{|c|}{ Neurological outcome } & \multirow[b]{2}{*}{$\begin{array}{l}\text { Outcome } \\
\text { measures }\end{array}$} \\
\hline & & $\begin{array}{l}\text { Intellectual } \\
\text { deficits }\end{array}$ & $\begin{array}{l}\text { Altered } \\
\text { neuromotor or } \\
\text { neurosensory } \\
\text { function }\end{array}$ & $\begin{array}{l}\text { Altered } \\
\text { mood or } \\
\text { behavior }\end{array}$ & \\
\hline $\begin{array}{l}\text { Stewart et al. } \\
2002\end{array}$ & $\begin{array}{l}529 \mathrm{~Pb} \text { workers, } \\
\text { age: } 40- \\
>70 \text { years }\end{array}$ & $\uparrow \mathrm{T}$ & $\uparrow \mathrm{T}$ & - & $\begin{array}{l}\text { Learning, memory, } \\
\text { executive function, } \\
\text { reaction time, } \\
\text { manual dexterity }\end{array}$ \\
\hline $\begin{array}{l}\text { van Wijngaarden } \\
\text { et al. } 2009\end{array}$ & $\begin{array}{l}47 \text { adults, } \\
\text { age: } 55-67 \text { years }\end{array}$ & $\uparrow \mathrm{C}$ & - & - & Learning, memory \\
\hline Wang et al. 2007 & $\begin{array}{l}358 \text { adult males }{ }^{b} \text {, } \\
\text { age: } 67 \text { years } \\
\text { (median) }\end{array}$ & $\uparrow \mathrm{T}$ & - & - & $\begin{array}{l}\text { Interaction between } \\
\mathrm{Pb} \text { and } \\
\text { hemochromatosis } \\
\text { gene } \\
\text { polymorphisms on } \\
\text { learning, memory, } \\
\text { executive function }\end{array}$ \\
\hline Wang et al. 2018 & $\begin{array}{l}634 \text { males, age: } \\
67 \text { years (mean) }\end{array}$ & - & $\begin{array}{l}\uparrow P \\
\uparrow T\end{array}$ & - & Glaucoma \\
\hline $\begin{array}{l}\text { Weisskopf et al. } \\
2004\end{array}$ & $\begin{array}{l}466 \text { adult males }{ }^{b} \text {, } \\
\text { age: } 68 \text { years } \\
\text { (mean) }\end{array}$ & $\uparrow P$ & - & - & $\begin{array}{l}\text { Memory, verbal and } \\
\text { written skills, } \\
\text { executive function }\end{array}$ \\
\hline $\begin{array}{l}\text { Weisskopf et al. } \\
2007\end{array}$ & $\begin{array}{l}761 \text { adult males }{ }^{b} \text {, } \\
\text { age: } 69 \text { years } \\
\text { (mean) }\end{array}$ & $\begin{array}{l}\uparrow P \\
\uparrow T\end{array}$ & - & - & $\begin{array}{l}\text { Memory, visual- } \\
\text { spatial performance }\end{array}$ \\
\hline $\begin{array}{l}\text { Weisskopf et al. } \\
2010\end{array}$ & $\begin{array}{l}330 \text { adult cases } \\
\text { and } 308 \text { controls, } \\
\text { age: } 67 \text { years } \\
\text { (mean) }\end{array}$ & - & $\uparrow \mathrm{T}$ & - & Parkinson's disease \\
\hline $\begin{array}{l}\text { Weuve et al. } \\
2009\end{array}$ & $\begin{array}{l}587 \text { adult } \\
\text { females }^{c}, \\
\text { age: } 47-74 \text { years }\end{array}$ & $\begin{array}{l}0 \mathrm{P} \\
\uparrow \mathrm{T}\end{array}$ & - & - & Learning, memory \\
\hline $\begin{array}{l}\text { Weuve et al. } \\
2013\end{array}$ & $\begin{array}{l}101 \text { cases and } \\
50 \text { controls, } \\
\text { age: } 55-80 \text { years }\end{array}$ & $\begin{array}{l}0 \mathrm{P} \\
\uparrow \mathrm{T}\end{array}$ & - & - & $\begin{array}{l}\text { Learning, memory } \\
\text { (stronger } \\
\text { association with Pb } \\
\text { among Parkinson's } \\
\text { disease cases) }\end{array}$ \\
\hline
\end{tabular}




\section{Table 2-33. Associations Between Bone Pb and Neurological Outcomes in Adults}

\begin{tabular}{|c|c|c|c|c|c|}
\hline \multirow[b]{2}{*}{ Reference } & \multicolumn{5}{|c|}{ Neurological outcome } \\
\hline & Population & $\begin{array}{l}\text { Intellectual } \\
\text { deficits }\end{array}$ & $\begin{array}{l}\text { Altered } \\
\text { neuromotor or } \\
\text { neurosensory } \\
\text { function }\end{array}$ & $\begin{array}{l}\text { Altered } \\
\text { mood or } \\
\text { behavior }\end{array}$ & $\begin{array}{l}\text { Outcome } \\
\text { measures }\end{array}$ \\
\hline $\begin{array}{l}\text { Wright et al. } \\
2003 \mathrm{~b}\end{array}$ & $\begin{array}{l}736 \text { adult males }{ }^{b} \\
\text { age: } 68 \text { years } \\
\text { (mean) }\end{array}$ & $\begin{array}{l}\uparrow P \\
\uparrow \mathrm{T}\end{array}$ & - & - & $\begin{array}{l}\text { Memory, verbal and } \\
\text { written skills, } \\
\text { executive function }\end{array}$ \\
\hline
\end{tabular}

aBoston Memory Study.

bNormative Aging Study.

cNurses Health Study.

dWhole-body $\mathrm{Pb}$ predicted from bone $\mathrm{Pb}$.

$\uparrow=$ positive association; $\downarrow=$ inverse association; $0=$ no association; $-=$ not reported; $\mathrm{C}=$ calcaneus bone;

$\mathrm{P}=$ patella; $\mathrm{Pb}=$ lead; $\mathrm{T}=$ tibia; $\mathrm{O}=$ other

Bone $\mathrm{Pb}$ has been associated with declines in neuromotor and neurosensory function. Neuromotor outcomes that have been associated with bone Pb include tremor, Parkinson's disease, and ALS (Coon et al. 2006; Eum et al. 2015; Weisskopf et al. 2010; Weuve et al. 2013). Neurosensory outcomes include decrements in olfactory and hearing function, vibration threshold, and manual dexterity (Dorsey et al. 2006; Grashow et al. 2013b, 2015; Park et al. 2010; Schwartz et al. 2000b; 2005; Shih et al. 2006; Stewart et al. 2002). Bone $\mathrm{Pb}$ has also been associated with increased risk or odds of psychiatric symptoms such as anxiety and depression (Dorsey et al. 2006; Rajan et al. 2007; Rhodes et al. 2003; Schwartz et al. 2005).

Mechanisms of Action. Numerous cellular mechanisms are likely involved in Pb-induced alterations in neurological function. $\mathrm{Pb}$ disrupts cellular function through diverse mechanisms, including displacement of metal ion co-factors from protein, enzyme inhibition, inhibition of ion transport, disruption of cell and mitochondrial membrane potentials, disruption of intracellular calcium homeostasis oxidative stress, and inflammation and endocrine disruption (see Section 2.21). All of these Pb mechanisms have been demonstrated in neuronal tissues, although there is no consensus on which mechanisms dominate. Evidence for various mechanisms that may participate in $\mathrm{Pb}$ neurotoxicity are summarized in this section. The reader is referred to references cited therein for more detailed information (Bouton and Pevsner 2000; Bressler et al. 1999; Cory-Slechta 1995, 2003; EPA 2014c; Gilbert and Lasley 2002; Lasley and Gilbert 2000; Mitra et al. 2017; Nihei and Guilarte 2002; Suszkiw 2004; Toscano and Guilarte 2005; Zawia et al. 2000; Zhang et al. 2015). 
$\mathrm{Pb}$ can affect the nervous system by multiple mechanisms, one of the most important of which is by mimicking calcium action and/or disruption of calcium homeostasis. Because calcium is involved as a cofactor in many cellular processes, it is not surprising that many cell-signaling pathways are affected by $\mathrm{Pb}$. One pathway that has been studied in more detail is the activation of protein kinase $\mathrm{C}(\mathrm{PKC})$. PKC is a serine/threonine protein kinase involved in many processes important for synaptic transmission such as the synthesis of neurotransmitters, ligand-receptor interactions, conductance of ionic channels, and dendritic branching. The PKC family is made up of 12 isozymes, each with different enzymatic cofactor requirements, tissue expression, and cellular distributions. The $\gamma$-isoform is one of several calciumdependent forms of PKC and is a likely target for $\mathrm{Pb}$ neurotoxicity; it is neuron-specific and is involved in long-term potentiation (see below), spatial learning, and memory processes. Pb has the capacity to both activate and inhibit PKCs. Studies have shown that micromolar concentrations of Pb can activate PKCdependent phosphorylation in cultured brain microvessels, whereas picomolar concentrations of $\mathrm{Pb}$ activate preparations of PKC in vitro. Interestingly, studies in rats exposed to low Pb levels have shown few significant changes in PKC activity or expression, suggesting that the whole animal may be able to compensate for $\mathrm{Pb} \mathrm{PKC}$-mediated effects compared to a system in vitro. PKC induces the formation of the AP-1 transcriptional regulatory complex, which regulates the expression of a large number of target genes via AP-1 promoter elements. A gene regulated by $\mathrm{Pb}$ via AP-1 promoters is the glial fibrillary acidic protein (GFAP), an astrocytic intermediate filament protein that is induced during periods of reactive astrocytic gliosis. Astrocytes, along with endothelial cells, make up the blood-brain barrier. Studies in rats exposed chronically to low $\mathrm{Pb}$ levels have reported alterations in the normal pattern of GFAP gene expression in the brain, and the most marked long-lasting effects occurred when the rats were exposed during the developmental period. In immature brain microvessels, most of the protein kinase $\mathrm{C}$ is in the cytosol, whereas in mature brain microvessels, this enzyme is membrane-bound. Activation of protein kinase $\mathrm{C}$ in other systems is known to result in a change in distribution from cytosol to membrane, and has been observed with exposure of immature brain microvessels to $\mathrm{Pb}$. An inhibition of microvascular formation has been observed with $\mathrm{Pb}$ concentrations that are effective in activating PKC. Thus, it appears that premature activation of PKC by Pb may impair brain microvascular formation and function, and at high levels of $\mathrm{Pb}$ exposure, may account for gross defects in the blood-brain barrier that contribute to acute $\mathrm{Pb}$ encephalopathy. The blood-brain barrier normally excludes plasma proteins and many organic molecules, and limits the passage of ions. With disruption of this barrier, molecules such as albumin freely enter the brain, and ions and water follow. Because the brain lacks a well-developed lymphatic system, clearance of plasma constituents is slow, edema occurs, and intracranial pressure rises. The particular vulnerability of the fetus and infant to the neurotoxicity of $\mathrm{Pb}$ may be due in part to 
immature brain microvessels, which affect the blood brain barrier, and to the lack of the high-affinity $\mathrm{Pb}-$ binding protein in astroglia, which sequester $\mathrm{Pb}$.

Another enzyme altered by $\mathrm{Pb}$ is calmodulin, a major intracellular receptor for calcium in eukaryotes. Normally, calcium induces a conformational change in calmodulin that converts the protein to an active form; $\mathrm{Pb}$ improperly activates the enzyme. Some studies suggest that activation of calmodulin by $\mathrm{Pb}$ results in protein phosphorylation in the rat brain and brain membrane preparations and can alter proper functioning of cAMP messenger pathways. It has been shown that calmodulin can mediate gene expression via calmodulin-dependent kinases. The effects of $\mathrm{Pb}$ on gene expression via activation of calmodulin are not as marked as those via PKC because activation of calmodulin requires 100 -fold more $\mathrm{Pb}$ than activation of PKC.

$\mathrm{Pb}$ also can substitute for zinc in some enzymes and in zinc-finger proteins, which coordinate one or more zinc cations as cofactors. The substitution of $\mathrm{Pb}$ for zinc in zinc-finger proteins can have significant effects on de novo expression of the bound proteins and in any genes transcriptionally-regulated by a particular protein. $\mathrm{Pb}$ has been found to alter the binding of zinc-finger transcriptional regulator Sp1 to its specific DNA sequences. This is accompanied by aberrant expression of Sp1 target genes such as myelin basic protein and proteolipid protein. Another gene regulated by Sp1 is the $\beta$-amyloid precursor protein (APP) gene. Recently, it was shown that Pb exposure in neonatal rats transiently induces APP mRNA, which is overexpressed with a delay of 20 months after exposure to $\mathrm{Pb}$ has ceased. In contrast, APP expression, and Sp1 activity, as well as APP and $\beta$-amyloid protein levels, were unresponsive to $\mathrm{Pb}$ during old age, suggesting that exposures occurring during brain development may predetermine the expression and regulation of APP later in life. It has been suggested that the multiple responses to $\mathrm{Pb}$ exposure are due to $\mathrm{Pb}$ specifically targeting zinc-finger proteins found in enzymes, channels, and receptors.

$\mathrm{Pb}$ affects virtually every neurotransmitter system in the brain, but most information on changes is available on the glutamatergic, dopaminergic, cholinergic, and gamma-aminobutyric acid (GABA) systems. Of these, special attention has been paid to the glutamatergic system and its role in hippocampal long-term potentiation (LTP). Hippocampal LTP is a cellular model of learning and memory characterized by a persistent increase in synaptic efficacy following delivery of brief tetanic stimulation (high-frequency stimulation). LTP provides a neurophysiological substrate for learning and storing information and is thought to utilize the same synaptic mechanisms as the learning process. LTP is established only with complex patterns of stimulation but not with single pulse stimulation. While it has 
been studied primarily in the hippocampal subregions CA1 and dentate gyrus, it can also be evoked in cortical areas. Exposure of intact animals or tissue slices to Pb diminishes LTP by a combination of three actions: increasing the threshold for induction, reducing the magnitude of potentiation, and shortening its duration by accelerating its rate of decay. This effect on LTP involves actions of $\mathrm{Pb}$ on glutamate release (presynaptic effects) and on the N-methyl-D-aspartate (NMDA) receptor function. Pb exposure inhibits release of glutamine from pre-synaptic endings, which may be mediated, in part, by altered pre-synaptic vesicle formation or activation. Studies have shown that the effects of $\mathrm{Pb}$ vary as a function of the developmental exposure period and that $\mathrm{Pb}$ exposure early in life is critical for production of impaired LTP in adult animals. LTP is more readily affected by $\mathrm{Pb}$ during early development, but exposure initiated after weaning also affects synaptic plasticity. Studies also have shown that both LTP magnitude and threshold exhibit a U-shape type response with increasing Pb doses. While LTP is primarily a glutamatergic phenomenon, it can be modulated through input from extrahippocampal sources including noradrenergic, dopaminergic, and cholinergic sources.

Studies in animals treated with $\mathrm{Pb}(\mathrm{PbB} 30-40 \mu \mathrm{g} / \mathrm{dL}$ ) have shown that induction of pair-pulse facilitation in the dentate gyrus is impaired. Since the phenomenon is mediated primarily by increased glutamate release, the reasonable assumption is that $\mathrm{Pb}$ reduces glutamate release. Support for this assumption is also derived from studies in which depolarization-induced hippocampal glutamate release was reduced in awake animals with similar PbB. This inhibition of glutamate release was shown to be due to Pb-related decrements in a calcium-dependent component. The exact mechanism for the inhibition of glutamate release by $\mathrm{Pb}$ is not known, but is consistent with $\mathrm{Pb}$ at nanomolar concentrations preventing maximal activation of PKC, rather than Pb blocking calcium influx into the presynaptic terminal through voltagegated calcium channels. Reduced glutamate release has been observed in rats exposed from conception through weaning and tested as adults, when $\mathrm{Pb}$ was no longer present, suggesting that a direct action of $\mathrm{Pb}$ is not necessary and that other mechanisms, such as reductions in synaptogenesis, also may be involved. As with LTP, depolarization-evoked hippocampal glutamate release in rats treated chronically with several dose levels of $\mathrm{Pb}$ exhibited a U-shaped response. That is, glutamate release was inhibited in rats treated with the lower $\mathrm{Pb}$ doses, but not in those exposed to the higher concentrations of $\mathrm{Pb}$. Although speculative, this was interpreted as $\mathrm{Pb}$ at the higher doses mimicking calcium in promoting transmitter release and overriding the inhibitory effects of $\mathrm{Pb}$ that occur at lower $\mathrm{Pb}$ levels.

The findings regarding the effects of $\mathrm{Pb}$ on postsynaptic glutamatergic function have been inconsistent across laboratories, but a direct inhibitory action of $\mathrm{Pb}$ on the NMDA receptor is unlikely at environmentally relevant exposure levels. Some studies have shown that continuous exposure of rats 
from gestation to adulthood results in a significant increase in NMDA receptor numbers in cortical areas, hippocampus, and forebrain. This was observed in the forebrain at PbB of $14 \mu \mathrm{g} / \mathrm{dL}$. Other studies, however, have reported changes in the opposite direction and the reason for the discrepancy in results may be due to the different exposure protocols used. From a functional point of view, it seems plausible that a $\mathrm{Pb}$-induced reduction in presynaptic transmitter release be compensated by a postsynaptic increase in number or density of receptors in order to maintain a viable function.

The dopaminergic system also has a role in aspects of cognitive function since lesions of dopaminergic neurons impair behavior in various types of learning and cognitive tasks. Also, individuals who suffer from Parkinson's disease, a disease associated with dopamine depletion in the striatum, sometimes show difficulties in cognitive functions. Most of the evidence available suggests that $\mathrm{Pb}$ may impair regulation of dopamine synthesis and release, indicating a presynaptic site of action. Studies in animals often report opposing effects of $\mathrm{Pb}$ on nigrostriatal and mesolimbic dopamine systems regarding receptor binding, dopamine synthesis, turnover, and uptake. Postweaning exposure of rats to $\mathrm{Pb}$ resulted in supersensitivity of D1 and D2 dopamine receptors, which can be interpreted as a compensatory response to decreased synthesis and/or release of dopamine. Lesions to the nucleus accumbens (a terminal dopamine projection area) and the frontal cortex resulted in perseverative deficits, suggesting that the mesolimbic system is preferentially involved in the effects of $\mathrm{Pb}$. Results of studies using dopaminergic compounds seem to indicate that changes in dopamine systems do not play a role in the effects of $\mathrm{Pb}$ on learning. Instead, it has been suggested that changes in dopaminergic systems may play a role in the altered response rates on Fixed-Interval (FI) schedules of reinforcement that have been observed in animals exposed to $\mathrm{Pb}$. This type of change has been thought to represent a failure to inhibit inappropriate responding.

It is widely accepted that the cholinergic system plays a role in learning and memory processes. Some cognitive deficits observed in patients with Alzheimer's disease have been attributed to impaired cholinergic function in the cortex and hippocampus. Exposure to $\mathrm{Pb}$ induces numerous changes in cholinergic system function, but the results, in general, have been inconsistently detected, or are of opposite direction in different studies, which may be attributed to the different exposure protocols used in the different studies. However, it is clear that $\mathrm{Pb}$ blocks evoked release of acetylcholine and diminishes cholinergic function. This has been demonstrated in central and peripheral synapses. Studies with the neuromuscular junction showed that $\mathrm{Pb}$ reduces acetylcholine release by blocking calcium entry into the terminal. At the same time, $\mathrm{Pb}$ prevents sequestration of intracellular calcium by organelles, which results in increased spontaneous release of the neurotransmitter. Studies in vitro show that Pb can block nicotinic cholinergic receptors, but it is unclear whether such effects occur in vivo or whether $\mathrm{Pb}$ alters the 
expression of nicotinic cholinergic receptors in the developing brain. Evidence for an involvement in Pb-induced behavioral deficits has been presented based on the observation that intrahippocampal transplants of cholinergic-rich septal and nucleus basalis tissue improve the deficits and that treatment with nicotinic agonists can improve learning and memory impairments following perinatal $\mathrm{Pb}$ treatment of rats. Chronic exposure of rats to $\mathrm{Pb}$ has resulted in decreased muscarinic-receptor expression in the hippocampus. Whether or not $\mathrm{Pb}$ exposure during development alters muscarinic receptor sensitivity is unclear as there are reports with opposite results. The preponderance of the binding data suggests that $\mathrm{Pb}$ does not directly affect muscarinic receptors with the exception of the visual cortex, where $\mathrm{Pb}$ may have a direct inhibitory effect on muscarinic receptors from rods and bipolar cells of the retina.

$\mathrm{Pb}$ exposure decreases spontaneous and evoked release of GABA in rats and in hippocampal cultures and brain slices. In general, GABA functions in the brain as a post-synaptic inhibitory transmitter. The role of changes in GABA release in the neurotoxicity of $\mathrm{Pb}$ has not been firmly established.

Various other mechanisms may also contribute to $\mathrm{Pb}$ neurotoxicity. Exposure to $\mathrm{Pb}$ has also been shown to stimulate inflammation in a variety of tissues, including neuronal tissue (see Section 2.21). Contributing mechanisms include alterations in levels of ROS, activation of nuclear activation factor $\mathrm{NF} \kappa \beta$, cytokine release, and alterations in prostaglandin metabolism. Pb exposure has been shown to alter neuronal nitric oxide signaling (NOS) and the hormone levels regulated by the hypothalamicpituitary-thyroid axis.

\subsection{REPRODUCTIVE}

Overview. Numerous epidemiological studies have evaluated effects of $\mathrm{Pb}$ on male and female reproductive function. In males, most exposures were occupational, with mean $\mathrm{PbB}>10 \mu \mathrm{g} / \mathrm{dL}$. In general, studies in males show consistent evidence of reproductive effects on sperm (production, motility, viability, and morphology), semen quantity and composition, serum reproductive hormone levels, and fertility, with severity of effects increasing with increasing PbB. In contrast to exposure of males, most exposures of females were non-occupational, with mean $\mathrm{PbB} \leq 10 \mu \mathrm{g} / \mathrm{dL}$. Studies investigating effects on serum reproductive hormone levels, fertility, spontaneous abortion, and preterm birth provide mixed results; thus, dose-dependence of effects in females is difficult to assess. 
The following reproductive effects in males have been associated with $\mathrm{PbB}$ :

- $\quad \leq 10 \mu \mathrm{g} / \mathrm{dL}$ :

o Increased serum testosterone; evaluated in a few studies with mixed results.

o Effects on sperm (decreased sperm count, concentration, motility, and viability, and increased immature sperm concentration and percentage of morphologically abnormal sperm); evaluated in a few studies with mixed results.

- $\quad>10 \mu \mathrm{g} / \mathrm{dL}:$

o Altered serum concentrations of reproductive hormones (testosterone, FSH, LH); evaluated in several studies with mixed results.

o Effects on sperm (decreased sperm count, concentration, motility, viability, and increased immature sperm concentration and percentage of morphologically abnormal sperm); corroborated in several studies.

o Alterations in semen quality (decreased semen volume and altered composition of seminal fluid); evaluated in a few studies.

o Decreased fertility; evaluated in a few studies.

o Histopathological changes to the testes (peritubular fibrosis, oligospermia, and vacuolization of Sertoli cells); evaluated in a few studies.

The following reproductive effects in females have been associated with PbB:

- $\quad \leq 10 \mu \mathrm{g} / \mathrm{dL}$ :

o Increased serum levels of estradiol, FSH, and LH; studies have mixed results.

o Decreased fertility; studies have mixed results.

o Increased spontaneous abortion; studies have mixed results.

o Increased preterm birth; studies have mixed results.

o Earlier age at onset of menopause; demonstrated in a few studies.

- $>10 \mu \mathrm{g} / \mathrm{dL}$ :

o Decreased fertility; studies have mixed results.

o Increased preterm birth; studies have mixed results.

Measures of Exposure. Most studies evaluating effects on male and female reproductive systems used $\mathrm{PbB}$ as the biomarker for exposure. More recent studies in men have explored the relationship between the concentration of $\mathrm{Pb}$ in semen or spermatozoa and adverse effects (Table 2-34). It has been suggested 
that semen levels of $\mathrm{Pb}$ may be a better biomarker for assessment of male reproductive effects, particularly at low $\mathrm{PbB}$, because no relationship between $\mathrm{PbB}$ and $\mathrm{Pb}$ levels in semen or spermatozoa has been observed (Hernandez-Ochoa et al. 2005; Mendiola et al. 2011). In women, other biomarkers of exposure include concentration of $\mathrm{Pb}$ in plasma (Lamadrid-Figueroa et al. 2007), red blood cells (Perkins et al. 2014), placenta (Gundacker et al. 2010), and plasma/blood ratio (Lamadrid-Figueroa et al. 2007).

Confounding Factors and Effect Modifiers. Numerous factors may add uncertainty in the interpretation of studies examining associations between $\mathrm{PbB}$ and reproductive effects, including overall health, body weight, nutrition, and SES. Exposures to other substances, including recreational drugs, alcohol, therapeutic agents, industrial chemicals, insecticides, and pesticides, also may affect fertility (Foster and Gray 2008). Failure to account for these factors may attenuate or strengthen the apparent associations between $\mathrm{Pb}$ exposure and the outcome, depending on the direction of the effect of the variable on the outcome. Some studies examining effects on sperm (discussed below) were conducted on samples obtained at fertility clinics; therefore, other causes for sperm effects could be effect modifiers (additional details are provided in the Supporting Document for Epidemiological Studies for Lead, Table 11). In addition, because sperm counts can vary by geographical location, it is important that control and exposed groups are matched for geographic location.

Characterization of Effects in Males. General trends regarding the relationship between $\mathrm{PbB}$ and male reproductive effects are shown in Table 2-34. Overall, the dose-effect pattern suggests an increasing severity of toxicity associated with increasing $\mathrm{PbB}$, with effects on sperm at $\leq 10 \mu \mathrm{g} / \mathrm{dL}$ (discussed in more detail below). At increasing PbB, effects become more severe, with decreased fertility observed at $\mathrm{PbB}>10 \mu \mathrm{g} / \mathrm{dL}$ and histopathological changes of the testes at $\mathrm{PbB}$ of approximately $30 \mu \mathrm{g} / \mathrm{dL}$. Effects on sperm, including decreased sperm count, concentration, motility, viability, and increased immature sperm concentration and percentage of morphologically abnormal sperm, have been observed at $\mathrm{PbB}$ of $\leq 10$ $>50$ g/dL (Alexander et al. 1998a; Assennato et al. 1987; Bonde et al. 2002; Cullen et al. 1984; Famurewa and Ugwuja 2017; Hernández-Ochoa et al. 2005; Kasperczyk et al. 2008; Lancranjan et al. 1975; Lerda 1992; Li et al. 2015; Meeker et al. 2008; Moran-Martinez et al. 2013; Telisman et al. 2007; Wildt et al. 1983). However, a few studies showed no association between $\mathrm{PbB}$ and adverse effects on sperm (Lancranjan et al. 1975; Mendiola et al. 2011). The significance of the observed changes to sperm on fertility is uncertain. Decreased semen volume and altered composition of seminal fluid have been observed at PbB >10 $\mu \mathrm{g} / \mathrm{dL}$ (Bonde et al. 2002; Naha and Chowdhury 2006; Telisman et al. 2000; Wildt et al. 1983). Decreased fertility has been reported in association with $\mathrm{PbB}>10->50 \mu \mathrm{g} / \mathrm{dL}$ (Sallmén et al. 2000; Shiau et al. 2004), although no effect on fertility was observed in one study of workers with PbB 
$>40 \mu \mathrm{g} / \mathrm{dL}$ (Coste et al. 1991). Histopathological assessment of biopsied testicular tissue from $\mathrm{Pb}$ workers (mean PbB: $29.0 \mu \mathrm{g} / \mathrm{dL}$ ) showed peritubular fibrosis, oligospermia, and vacuolization of Sertoli cells (Braunstein et al. 1978). Evaluations of associations between $\mathrm{PbB}$ and serum levels of reproductive hormones show inconsistent results (Table 2-35). At $\mathrm{PbB} \leq 10 \mu \mathrm{g} / \mathrm{dL}$, positive associations between $\mathrm{PbB}$ and serum testosterone levels have been observed (Kresovich et al. 2015; Lewis and Meeker 2015; Meeker et al. 2010; Telisman et al. 2007), whereas inverse associations or no effects were reported at PbB $>10 \mu \mathrm{g} / \mathrm{dL}$. No effects on FSH or $\mathrm{LH}$ were reported at $\mathrm{PbB} \leq 10 \mu \mathrm{g} / \mathrm{dL}$, and inconsistent results were observed at $\mathrm{PbB}>10 \mu \mathrm{g} / \mathrm{dL}$. Changes in serum levels of reproductive hormones may indicate disruption of the hypothalamic-pituitary-gonadal axis; however, due to inconsistent findings, an association between $\mathrm{PbB}$ and endocrine disruption in males has not been firmly established.

\section{Table 2-34. Overview of Effects on the Male Reproductive System Associated with Chronic Exposure to Lead (Pb)}

\begin{tabular}{|c|c|c|}
\hline $\begin{array}{l}\text { Mean blood lead } \\
\text { concentration } \\
(\mathrm{PbB})(\mu \mathrm{g} / \mathrm{dL})\end{array}$ & Effects associated with $\mathrm{Pb}$ exposure & References \\
\hline \multirow[t]{2}{*}{$\leq 10$} & $\begin{array}{l}\text { Effects on sperm (decreased sperm } \\
\text { concentration, motility, and viability; increased } \\
\text { morphologic abnormalities) }\end{array}$ & $\begin{array}{l}\text { Famurewa and Ugwuja 2017; } \\
\text { Hernández-Ochoa et al. 2005; Li et al. } \\
\text { 2015; Meeker et al. 2008; Telisman et } \\
\text { al. } 2007\end{array}$ \\
\hline & $\begin{array}{l}\text { Effects on hormones (increased serum levels } \\
\text { of testosterone, estradiol, LH, FSH, and } \\
\text { SHBG; decreased serum prolactin and SHBG }\end{array}$ & $\begin{array}{l}\text { Chen et al. 2016; Kresovich et al. } \\
\text { 2015; Lewis and Meeker 2015; } \\
\text { Meeker et al. 2010; Telisman et al. } \\
2007\end{array}$ \\
\hline \multirow[t]{3}{*}{$>10-30$} & $\begin{array}{l}\text { Effects on sperm (decreased sperm count, } \\
\text { concentration, density, motility, viability; } \\
\text { morphologic abnormalities) }\end{array}$ & $\begin{array}{l}\text { Alexander et al. 1998a; Bonde et al. } \\
\text { 2002; Moran-Martinez et al. } 2013\end{array}$ \\
\hline & Effects on semen (decreased volume) & Bonde et al. 2002 \\
\hline & Decreased fertility & Sallmén et al. 2000 \\
\hline \multirow[t]{5}{*}{$>30-50$} & $\begin{array}{l}\text { Effects on sperm (decreased count, } \\
\text { concentration, motility, viability; morphologic } \\
\text { abnormalities) }\end{array}$ & $\begin{array}{l}\text { Hsu et al. 2009; Lancranjan et al. } \\
\text { 1975; Lerda 1992; Telisman et al. } \\
2000\end{array}$ \\
\hline & Effects on composition of seminal fluid & Telisman et al. 2000 \\
\hline & $\begin{array}{l}\text { Effects on hormones (increased estradiol, LH, } \\
\text { FSH; decreased testosterone) }\end{array}$ & $\begin{array}{l}\text { Braunstein et al. 1978; } \mathrm{Ng} \text { et al. 1991; } \\
\text { Telisman et al. } 2000\end{array}$ \\
\hline & $\begin{array}{l}\text { Histopathological changes to testes } \\
\text { (peritubular fibrosis, oligospermia, } \\
\text { vacuolization of Sertoli cells) }\end{array}$ & Braunstein et al. 1978 \\
\hline & Decreased fertility & Sallmén et al. 2000; Shiau et al. 2004 \\
\hline
\end{tabular}


Table 2-34. Overview of Effects on the Male Reproductive System Associated with Chronic Exposure to Lead (Pb)

\begin{tabular}{|c|c|c|}
\hline $\begin{array}{l}\text { Mean blood lead } \\
\text { concentration } \\
(\mathrm{PbB})(\mu \mathrm{g} / \mathrm{dL})\end{array}$ & Effects associated with $\mathrm{Pb}$ exposure & References \\
\hline \multirow[t]{4}{*}{$>50$} & $\begin{array}{l}\text { Effects on sperm (decreased count, } \\
\text { concentration, motility, viability; morphologic } \\
\text { abnormalities) }\end{array}$ & $\begin{array}{l}\text { Assennato et al. 1987; Cullen et al. } \\
\text { 1984; Kasperczyk et al. 2008; } \\
\text { Lancranjan et al. 1975; Lerda 1992; } \\
\text { Naha and Chowdhury 2006; Wildt et } \\
\text { al. } 1983\end{array}$ \\
\hline & $\begin{array}{l}\text { Effects on semen (decreased volume; altered } \\
\text { composition) }\end{array}$ & $\begin{array}{l}\text { Naha and Chowdhury 2006; Wildt et } \\
\text { al. } 1983\end{array}$ \\
\hline & $\begin{array}{l}\text { Effects on hormones (altered serum levels of } \\
\text { testosterone, FSH, LH, prolactin) }\end{array}$ & $\begin{array}{l}\text { Assennato et al. 1987; Rodamilans et } \\
\text { al. } 1988\end{array}$ \\
\hline & Decreased fertility & Sallmén et al. 2000 \\
\hline
\end{tabular}

FSH = follicle-stimulating hormone; $\mathrm{LH}=$ luteinizing hormone; SHBG = sex hormone binding globulin

\section{Table 2-35. Effects on Reproductive Hormones Associated with Chronic Exposure to Lead $(\mathrm{Pb})$ in Males}

\begin{tabular}{|c|c|c|c|c|c|c|c|c|}
\hline \multirow{2}{*}{$\begin{array}{l}P b B \\
(\mu g / d L)\end{array}$} & \multicolumn{7}{|c|}{ Hormone } & \multirow[b]{2}{*}{ Reference } \\
\hline & $\mathrm{T}$ & $\mathrm{FSH}$ & $\mathrm{LH}$ & $E$ & $P$ & $A$ & SHBG & \\
\hline \multirow[t]{6}{*}{$\leq 10$} & $\uparrow \uparrow$ & 0 & - & - & - & 0 & 0 & Kresovich et al. 2015 \\
\hline & $\uparrow$ & 0 & 0 & - & - & - & 0 & Meeker et al. 2010 \\
\hline & $\uparrow$ & - & - & $\uparrow$ & 0 & - & - & Telisman et al. 2007 \\
\hline & $\uparrow$ & - & - & - & - & - & - & Lewis and Meeker 2015 \\
\hline & $\uparrow$ & $\uparrow$ & $\uparrow$ & 0 & - & - & $\uparrow$ & Chen et al. 2016 \\
\hline & 0 & 0 & 0 & - & - & - & - & Mendiola e al. 2011 \\
\hline \multirow[t]{2}{*}{ 10-30 } & 0 & 0 & 0 & - & - & - & - & Hsieh et al. 2009 \\
\hline & 0 & 0 & 0 & - & & - & - & Alexander et al. $1998 a$ \\
\hline \multirow[t]{8}{*}{$30-50$} & $\downarrow$ & 0 & 0 & - & 0 & - & - & Braunstein et al. 1978 \\
\hline & 0 & 0 & 0 & - & 0 & - & - & Erfurth et al. 2001 \\
\hline & 0 & $\downarrow$ & $\downarrow$ & - & - & - & - & Gustafson et al. 1989 \\
\hline & 0 & $\uparrow$ & $\uparrow$ & - & - & - & - & McGregor and Mason 1990 \\
\hline & $\downarrow$ & $\uparrow$ & $\uparrow$ & & 0 & - & - & Ng et al. 1991 \\
\hline & & & & $\uparrow$ & - & - & - & Telisman et al. 2000 \\
\hline & 0 & 0 & 0 & 0 & - & - & - & Sadeghnaiit Haghighi et al. 2013 \\
\hline & $\downarrow$ & - & - & - & - & - & - & Rodamilans et al. 1988 \\
\hline
\end{tabular}

$0=$ no effect; $\uparrow=$ increased serum level; $\downarrow=$ decreased serum level; $-=$ not evaluated; $A=$ androstenedione; $\mathrm{E}=$ estradiol; $\mathrm{FSH}=$ follicle stimulating hormone; $\mathrm{LH}=$ luteinizing hormone; $\mathrm{P}=$ prolactin; $\mathrm{SHBG}=$ sex hormone binding globulin; $\mathrm{T}=$ testosterone 
Effects in Males at Blood Pb Levels $\leq 10 \mu \mathrm{g} / \mathrm{dL}$. Cross-sectional studies evaluating adverse effects of non-occupational exposures to $\mathrm{Pb}$ on the male reproductive system show that damage to sperm, decreased semen volume, and increased serum testosterone are associated with mean $\mathrm{PbB} \leq 10 \mu \mathrm{g} / \mathrm{dL}$ or with $\mathrm{Pb}$ concentrations in semen or spermatozoa when PbBs are $\leq 10 \mu \mathrm{g} / \mathrm{dL}$. Results are summarized in Table 2-36, with study details provided in the Supporting Document for Epidemiological Studies for Lead, Table 11. None of the studies evaluated associations between $\mathrm{PbB}$ and male fertility parameters (i.e., pregnancy). Three studies assessed larger populations, including two studies using NHANES data (Kresovich et al. 2015; Lewis and Meeker 2015) and one study of a Chinese population (Chen et al. 2016). However, in general, study populations were small ( $n=61-240)$. In addition, for a few studies, participants were selected from infertility clinics and it is unclear how this may have biased study results (Meeker et al. 2008, 2010; Mendiola et al. 2011). Despite these limitations, taken together, results of non-occupational exposure studies support that adverse effects to the male reproductive system occur at $\mathrm{PbB} \leq 10 \mu \mathrm{g} / \mathrm{L}$.

Sperm and semen. A significant association between an increase in $\mathrm{PbB} \leq 10 \mu \mathrm{g} / \mathrm{dL}$ and increasing percentages of morphologically abnormal sperm, wide sperm, and round sperm was observed in a population of Croatian men (Telisman et al. 2007). The mean PbB was $4.92 \mu \mathrm{g} / \mathrm{dL}$; although the maximum $\mathrm{PbB}$ value in this study was $14.9 \mu \mathrm{g} / \mathrm{dL}$, over 90\% of participants had $\mathrm{PbB}<10 \mu \mathrm{g} / \mathrm{dL}$. Li et al. (2015) found small, but significant inverse associations between PbB and sperm count, sperm concentration, motile sperm, and morphologically normal sperm in 154 men from a reproductive clinic in Taiwan. The median PbB was $2.78 \mu \mathrm{g} / \mathrm{dL}$ (SD 1.85); range and percentiles were not reported. Sperm count was associated with $\mathrm{PbB}$ in a small population of infertile men with mean PbB 1.71-2.05 $\mu \mathrm{g} / \mathrm{dL}$ (Famurewa and Ugwuja 2017). Other studies have shown associations between Pb levels in semen and/or spermatozoa and increased percentages of morphologically abnormal sperm and decreased sperm motility and viability, although no associations were observed between $\mathrm{PbB}$ and these outcomes (HernandezOchoa et al. 2005; Mendiola et al. 2011); mean PbB levels were $9.3 \mu \mathrm{g} / \mathrm{dL}$ in the Hernandez-Ochoa et al. (2005) study and $2.8 \mu \mathrm{g} / \mathrm{dL}$ in the Mendiola et al. (2011) study. No associations were observed between $\mathrm{PbB}$ and sperm concentration, motility, or morphologic abnormalities in men at a median $\mathrm{PbB}$ of $1.5 \mu \mathrm{g} / \mathrm{dL}$ (Meeker et al. 2008). Semen volume (mL) was inversely associated with $\mathrm{PbB}$ at a mean $\mathrm{PbB}$ of $9.3 \mu \mathrm{g} / \mathrm{dL}$; however, 48\% of participants had PbB >10 $\mu \mathrm{g} / \mathrm{dL}$ (Hernandez-Ochoa et al. 2005). 
Table 2-36. Summary of Epidemiological Studies Evaluating Effects on the Male Reproductive System at Mean Blood Lead Concentration $(\mathrm{PbB}) \leq 10 \mu \mathrm{g} / \mathrm{dL}^{\mathrm{a}}$

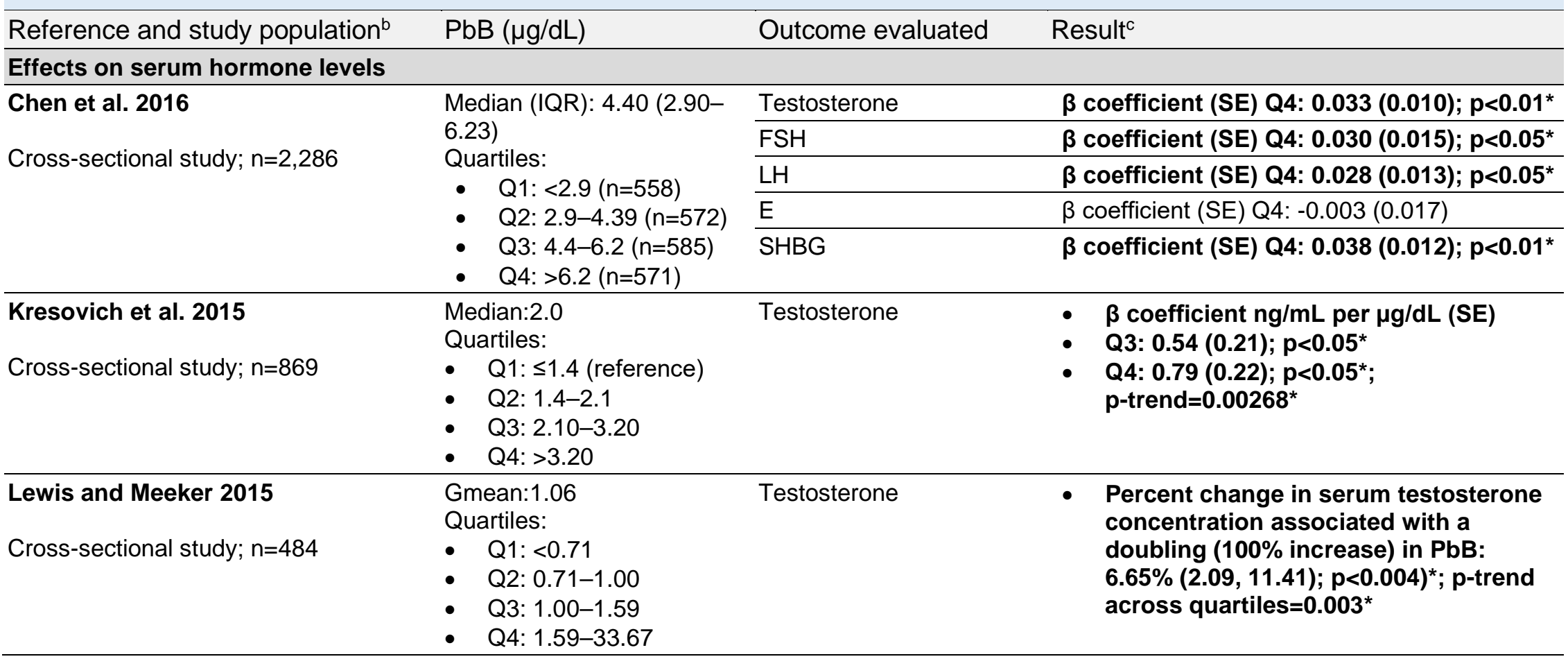


Table 2-36. Summary of Epidemiological Studies Evaluating Effects on the Male Reproductive System at Mean Blood Lead Concentration $(\mathrm{PbB}) \leq 10 \mu \mathrm{g} / \mathrm{dL}^{\mathrm{a}}$

\begin{tabular}{|c|c|c|c|}
\hline Reference and study population $^{b}$ & $\mathrm{PbB}(\mu \mathrm{g} / \mathrm{dL})$ & Outcome evaluated & Result $^{\mathrm{c}}$ \\
\hline Meeker et al. 2010 & $\begin{array}{l}\text { Median:1.5 } \\
\text { Quartiles }\end{array}$ & Testosterone & $\begin{array}{l}\text { Regression coefficient Q4 (ng/dL per } \\
\mu \mathrm{g} / \mathrm{dL}): 39.9(3.32,76.4)^{*}\end{array}$ \\
\hline \multirow[t]{5}{*}{ Cross-sectional study; $n=219$} & \multirow{5}{*}{$\begin{array}{l}-\quad \text { Q1: }<1.1 \text { (reference) } \\
\text { - } \quad \text { Q2: } 1.1-1.5 \\
\text { - } \quad \text { Q3: } 1.5-2.0 \\
\text { Q4.0-16.2 }\end{array}$} & $\mathrm{FSH}$ & $\begin{array}{l}\text { Regression coefficient Q4 }(\mathrm{mlU} / \mathrm{mL} \text { per } \mu \mathrm{g} / \mathrm{dL}) \text { : } \\
0.07(-0.18,0.31)\end{array}$ \\
\hline & & $\mathrm{LH}$ & $\begin{array}{l}\text { Regression coefficient Q4 (mIU/m per } \mu \mathrm{g} / \mathrm{dL}) \text { : } \\
0.08(-0.14,0.29)\end{array}$ \\
\hline & & Inhibin B & $\begin{array}{l}\text { Regression coefficient Q4 }(\mathrm{pg} / \mathrm{mL} \text { per } \mu \mathrm{g} / \mathrm{dL}) \text { : - } \\
7.79(-29.0,13.4)\end{array}$ \\
\hline & & SHBG & $\begin{array}{l}\text { Regression coefficient Q4 (nmol/L per } \mu \mathrm{g} / \mathrm{dL}) \text { : } \\
0.07(-0.10,0.23)\end{array}$ \\
\hline & & FAl & $\begin{array}{l}\text { Regression coefficient Q4 (per } \mu \mathrm{g} / \mathrm{dL}) \text { : } \\
0.08(-0.05,0.21)\end{array}$ \\
\hline \multirow{3}{*}{$\begin{array}{l}\text { Mendiola et al. } 2011 \\
\text { Case-control study; } n=61\end{array}$} & \multirow[t]{3}{*}{ Gmean: 2.8} & Testosterone & $\begin{array}{l}\beta \text { coefficient }(\mathrm{ng} / \mathrm{mL} \text { per } \mu \mathrm{g} / \mathrm{L}):-0.12(-0.40, \\
0.14)\end{array}$ \\
\hline & & $\mathrm{FSH}$ & $\beta$ coefficient (IU/L per $\mu \mathrm{g} / \mathrm{L}):-0.20(-0.64,0.25)$ \\
\hline & & $\mathrm{LH}$ & $\beta$ coefficient (IU/L per $\mu \mathrm{g} / \mathrm{L}):-0.07(-0.49,0.31)$ \\
\hline \multirow{3}{*}{$\begin{array}{l}\text { Telisman et al. } 2007 \\
\text { Cross-sectional study; } n=240\end{array}$} & \multirow[t]{3}{*}{ Median: 4.92} & Testosterone & $\begin{array}{l}\beta \text { coefficient }(\mathrm{nmol} / \mathrm{L} \text { per } \mu \mathrm{g} / \mathrm{L}): 0.21 \\
p<0.003^{*}\end{array}$ \\
\hline & & Estradiol & $\begin{array}{l}\beta \text { coefficient }(\mathrm{nmol} / \mathrm{L} \text { per } \mu \mathrm{g} / \mathrm{L}): 0.22 \\
p<0.0008^{*}\end{array}$ \\
\hline & & Prolactin & $\beta$ coefficient $(\mu \mathrm{g}$ per $\mu \mathrm{g} / \mathrm{L}):-0.18 ; p<0.007$ \\
\hline \multicolumn{4}{|l|}{ Sperm and semen quality } \\
\hline \multirow{4}{*}{$\begin{array}{l}\text { Famurewa and Ugwuja } 2017 \\
\text { Cross-sectional study; } n=75 \text { men with } \\
\text { infertility }\end{array}$} & \multirow{4}{*}{$\begin{array}{l}\text { PbB: } \\
\text { Mean } \\
\text { - Normospermic: } 1.49 \\
\text { - } \quad \text { Azoospermic: } 1.71 \\
\text { - Oligospermic: } 2.05\end{array}$} & Semen volume & Pearson correlation $R$ value: $-0.132 ; p=0.27$ \\
\hline & & Sperm count & Pearson correlation $\mathrm{R}$ value: $-0.280 ; p=0.02^{*}$ \\
\hline & & Sperm motility & Pearson correlation $R$ value: $-0.092 ; p=0.44$ \\
\hline & & Sperm morphology & Pearson correlation $R$ value: $-0.081 ; p=0.50$ \\
\hline
\end{tabular}


Table 2-36. Summary of Epidemiological Studies Evaluating Effects on the Male Reproductive System at Mean Blood Lead Concentration $(\mathrm{PbB}) \leq 10 \mu \mathrm{g} / \mathrm{dL}^{\mathrm{a}}$

\begin{tabular}{|c|c|c|c|}
\hline Reference and study population ${ }^{b}$ & $\mathrm{PbB}(\mu \mathrm{g} / \mathrm{dL})$ & Outcome evaluated & Result $^{\mathrm{c}}$ \\
\hline Hernandez-Ochoa et al. 2005 & \multirow{5}{*}{$\begin{array}{l}\text { Mean: } 9.3 \\
\text { SPZ Pb: } 0.047 \mathrm{ng} / 10^{6} \text { cells } \\
\text { SF Pb: } 2.02 \mu \mathrm{g} / \mathrm{L}\end{array}$} & Log sperm concentration & $\begin{array}{l}\beta \text { coefficient SPZ Pb }\left(10^{6} \text { cells } / \mathrm{mL} \text { per } \mathrm{ng} / 10^{6}\right. \\
\text { cells): }-17.17(p<0.05)^{*}\end{array}$ \\
\hline \multirow[t]{4}{*}{ Cross-sectional study; $n=68$} & & Sperm motility & $\begin{array}{l}\beta \text { coefficient } \mathrm{PbB}(\% \text { per } \mu \mathrm{g} / \mathrm{dL}):-0.006 \\
\beta \text { coefficient SPZ Pb: }\left(\% \text { per } \mathbf{n g} / 10^{6}\right. \\
\text { cells): }-\mathbf{2 . 1 2}(\boldsymbol{p}<\mathbf{0 . 0 5})^{*}\end{array}$ \\
\hline & & $\begin{array}{l}\text { Sperm morphology } \\
\text { (abnormal) }\end{array}$ & $\begin{array}{l}\beta \text { coefficient } \mathrm{PbB}(\% \text { per } \mu \mathrm{g} / \mathrm{dL}):-0.001 \\
\beta \text { coefficient SPZ Pb }\left(\% \text { per } \mathrm{ng} / 10^{6}\right. \\
\text { cells): }-\mathbf{1 . 4 2}(\boldsymbol{p}<0.05)^{*}\end{array}$ \\
\hline & & Sperm viability & $\begin{array}{l}\beta \text { coefficient } \mathrm{PbB}(\% \text { per } \mu \mathrm{g} / \mathrm{dL}):-0.095 \\
\beta \text { coefficient SPZ Pb }\left(\% \text { per } \mathbf{n g} / 10^{6}\right. \\
\text { cells): }-\mathbf{0 . 1 3 0}(\boldsymbol{p}<0.05)^{*}\end{array}$ \\
\hline & & Semen volume & $\begin{array}{l}\beta \text { coefficient } \mathrm{PbB}(\mathrm{mL} \text { per } \mu \mathrm{g} / \mathrm{dL}):-0.043 \\
\beta \text { coefficient SF Pb (mL per } \\
\boldsymbol{\mu g} / \mathrm{L}):-\mathbf{0 . 1 8 3} \mathbf{m L} ; \boldsymbol{p}<0.05^{*}\end{array}$ \\
\hline \multirow{5}{*}{$\begin{array}{l}\text { Li et al. } 2015 \\
\text { Cross-sectional study; } n=154\end{array}$} & \multirow{5}{*}{$\begin{array}{l}\text { Mean: } \\
\text { All participants: } 2.78 \\
\text { Low-quality semen group: } \\
3.43 \\
\text { High-quality semen group: } \\
2.38\end{array}$} & Low quality sperm & OR: $1.040(1.011,1.069) ; p=0.0061^{*}$ \\
\hline & & $\begin{array}{l}\text { Decreased sperm } \\
\text { concentration }\end{array}$ & OR: $1.046(1.015,1.078) ; p=0.0032^{*}$ \\
\hline & & Decreased sperm number & OR: $1.041(1.012,1.071) ; p=0.0048^{*}$ \\
\hline & & Decreased motile sperm & OR: $1.057\left(1.026,1.089 ; p=0.0003^{*}\right.$ \\
\hline & & $\begin{array}{l}\text { Decreased } \\
\text { morphologically normal } \\
\text { sperm }\end{array}$ & OR: $1.071\left(1.025,1.118 ; p=0.0021^{*}\right.$ \\
\hline \multirow{4}{*}{$\begin{array}{l}\text { Meeker et al. } 2008 \\
\text { Cross-sectional study; } n=219\end{array}$} & \multirow{4}{*}{$\begin{array}{cl}\text { Median:1.50 } \\
\begin{array}{cl}\text { - } & \text { Quartiles (Q): } \\
\text { o } & \text { Q1: }<1.10 \\
\text { o } & \text { Q2: } 1.10-1.50 \\
\text { o } & \text { Q3: } 1.50-2.00 \\
\text { o } & \text { Q4: } 2.00-16.2\end{array}\end{array}$} & Sperm concentration & $\begin{array}{l}\text { Regression coefficient }\left(10^{6} / \mathrm{mL} \text { per } \mu \mathrm{g} / \mathrm{dL}\right) \\
\text { Q4: } 0.02(-0.39,0.43)\end{array}$ \\
\hline & & Sperm motility & $\begin{array}{l}\text { Regression coefficient }(\% \text { per } \mu \mathrm{g} / \mathrm{dL}) \\
\text { Q4: } 1.10(-4.56,6.75)\end{array}$ \\
\hline & & Sperm morphology & $\begin{array}{l}\text { Regression coefficient }(\% \text { per } \mu \mathrm{g} / \mathrm{dL}) \\
\text { Q4: }-0.16(-1.58,1.26)\end{array}$ \\
\hline & & Semen volume & $\begin{array}{l}\text { Regression coefficient }(\mathrm{mL} \text { per } \mu \mathrm{g} / \mathrm{dL}) \\
\text { Q4: } 0.17(-0.41,0.74)\end{array}$ \\
\hline
\end{tabular}


Table 2-36. Summary of Epidemiological Studies Evaluating Effects on the Male Reproductive System at Mean Blood Lead Concentration (PbB) $\leq 10 \mu \mathrm{g} / \mathrm{dL}^{\mathrm{a}}$

\begin{tabular}{|c|c|c|c|}
\hline Reference and study population ${ }^{b}$ & $\mathrm{PbB}(\mu \mathrm{g} / \mathrm{dL})$ & Outcome evaluated & Resultc \\
\hline Mendiola et al. 2011 & \multirow{3}{*}{$\begin{array}{l}\text { Gmean: } 2.8 \\
\text { Median: } 2.9\end{array}$} & Sperm concentration & $\beta$ coefficient $\left(10^{6} / \mathrm{mL}\right.$ per $\left.\mu \mathrm{g} / \mathrm{L}\right): 0.08(-4.1,5.2)$ \\
\hline \multirow{2}{*}{ Case-control study; $n=61$} & & Immobile sperm & $\beta$ coefficient $(\%$ per $\mu \mathrm{g} / \mathrm{L}):-0.49(-1.8,0.62)$ \\
\hline & & $\begin{array}{l}\text { morphologically normal } \\
\text { sperm }\end{array}$ & $\beta$ coefficient(\% per $\mu \mathrm{g} / \mathrm{L}):-0.8(-3.5,3.4)$ \\
\hline Telisman et al. 2007 & \multirow[t]{4}{*}{ Median: 4.92} & Immature sperm & $\beta$ coefficient $\left(10^{6} / \mathrm{mL}\right.$ per $\left.\mu \mathrm{g} / \mathrm{L}\right): 0.13(p<0.07)$ \\
\hline \multirow{3}{*}{ Cross-sectional study; $n=240$} & & Pathologic sperm & $\beta$ coefficient ( $\%$ per $\mu g / L): 0.31(p<0.0002)^{*}$ \\
\hline & & Wide sperm & $\beta$ coefficient $(\%$ per $\mu g / L): 0.32(p<0.0001)^{*}$ \\
\hline & & Round sperm & $\beta$ coefficient $\left(\%\right.$ per $\mu: 0.16(p<0.03)^{*}$ \\
\hline
\end{tabular}

aSee the Supporting Document for Epidemiological Studies for Lead, Table 11 for more detailed descriptions of studies.

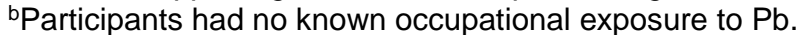

${ }^{\mathrm{c}}$ Asterisk and bold indicate association with PB; unless otherwise specified, values in parenthesis are $95 \%$ Cls; $p$-values $<0.05$ unless otherwise noted in the table.

$\mathrm{Cl}=$ confidence interval; $\mathrm{E}=$ estradiol $; \mathrm{FAI}=$ free androgen index; FSH = follicle-stimulating hormone; Gmean = geometric mean; Inhibin $\mathrm{B}=$ gonadal dimeric polypeptide hormone; IQR = interquartile range; $\mathrm{LH}=$ luteinizing hormone; OR = odds ratio; $\mathrm{Pb}=$ lead; $\mathrm{SE}=\mathrm{standard}$ error; $\mathrm{SF}=$ seminal fluid; SHBG = sex hormone-binding globulin; SPZ = spermatozoa 
Serum testosterone levels. Significant associations have also been observed between $\mathrm{PbB} \leq 10 \mu \mathrm{g} / \mathrm{dL}$ and increased serum testosterone levels (Table 2-34). Studies using NHANES data found significant positive associations between PbB and serum testosterone levels (Kresovich et al. 2015; Lewis and Meeker 2015). Examined by $\mathrm{PbB}$ quartiles, Kresovich et al. (2015) observed significant positive associations between $\mathrm{PbB}$ and serum testosterone (ng/L) for PbBs of 2.10-3.20 and $>3.2 \mu \mathrm{g} / \mathrm{dL}$; the median $\mathrm{PbB}$ of the study population was $2.0 \mu \mathrm{g} / \mathrm{dL}$. A doubling of PbB was positively associated with a $6.65 \%$ change in serum testosterone; the mean $\mathrm{PbB}$ of the study population was $1.06 \mu \mathrm{g} / \mathrm{dL}$ (Lewis and Meeker 2015). The toxicological significance of the observed associations between $\mathrm{PbB}$ and serum testosterone has not been established.

Characterization of Effects in Females. As noted above, most epidemiological studies evaluated effects at $\mathrm{PbB} \leq 10 \mu \mathrm{g} / \mathrm{dL}$, with few studies of $\mathrm{PbB}>10 \mu \mathrm{g} / \mathrm{dL}$. Studies of $\mathrm{PbB} \leq 10 \mu \mathrm{g} / \mathrm{dL}$ are discussed in detail in the section below. General trends for studies showing a relationship between $\mathrm{PbB} \leq 10-50 \mu \mathrm{g} / \mathrm{dL}$ and female reproductive effects are shown in Table 2-37. Effects associated with PbB include increased serum levels of estradiol, FSH, and LH at PbB $\leq 10 \mu \mathrm{g} / \mathrm{dL}$ (Chang et al. 2006; Krieg 2007), decreased fertility at $\mathrm{PbB} \leq 10 \mu \mathrm{g} / \mathrm{dL}$ (Chang et al. 2006), increased time to pregnancy at $\mathrm{PbB}>30-40 \mu \mathrm{g} / \mathrm{dL}$ (Sallmén et al. 1995), increased spontaneous abortion at PbB $\leq 10-30 \mu \mathrm{g} / \mathrm{dL}$ (Borja-Aburto et al. 1999; Yin et al. 2008), decreased number of gestational days at $\mathrm{PbB}>10-40 \mu \mathrm{g} / \mathrm{dL}$ (Jelliffe-Pawlowski et al. 2006), and increased preterm birth at $\mathrm{PbB} \leq 10-50 \mu \mathrm{g} / \mathrm{dL}$ (McMichael et al. 1986; Jelliffe-Pawlowski et al. 2006; Rabito et al. 2014). Although epidemiological studies demonstrate effects on reproductive function, results are inconsistent, with several studies reporting no association between $\mathrm{PbB}$ and female reproductive effects (Baghurst et al. 1987; Bloom et al. 2010, 2011, 2015; Garcia-Esquinas et al. 2014; Jackson et al. 2007; Murphy et al. 1990; Perkins et al. 2014; Pollack et al. 2011; Sallmén et al. 1995; Taylor et al. 2015; Vigeh et al. 2010). Dose-dependence has not been firmly established within the relatively narrow range of $\mathrm{PbB}(\leq 10 \mu \mathrm{g} / \mathrm{dL})$ in most studies. 


\section{Table 2-37. Overview of Effects on the Female Reproductive System and Pregnancy Outcomes Associated with Chronic Exposure to Lead (Pb)}

\begin{tabular}{|c|c|c|}
\hline $\begin{array}{l}\text { Mean blood lead } \\
\text { concentration } \\
(\mathrm{PbB})(\mu \mathrm{g} / \mathrm{dL})\end{array}$ & Effects associated with $\mathrm{Pb}$ exposure & References \\
\hline \multirow[t]{5}{*}{$\leq 10$} & Increased serum hormones (estradiol, FSH, LH) & $\begin{array}{l}\text { Chang et al. 2006; Chen et al. } \\
\text { 2016; Krieg } 2007\end{array}$ \\
\hline & Decreased fertility & Chang et al. 2006 \\
\hline & Increased spontaneous abortion & Yin et al. 2008 \\
\hline & Increased preterm birth & Li et al. 2017b; Rabito et al. 2014 \\
\hline & Earlier age at menopause & $\begin{array}{l}\text { Eum et al. 2014; Popovic et al. } \\
2005\end{array}$ \\
\hline \multirow[t]{3}{*}{$>10-30$} & Increased spontaneous abortion & Borja-Aburto et al. 1999 \\
\hline & Decreased number of gestational days & Jelliffe-Pawlowski et al. 2006 \\
\hline & Increased preterm birth & McMichael et al. 1986 \\
\hline \multirow[t]{3}{*}{$>30-40$} & Increased time to pregnancy & Sallmén et al. 1995 \\
\hline & Decreased number of gestational days & Jelliffe-Pawlowski et al. 2006 \\
\hline & Increased preterm birth & Jelliffe-Pawlowski et al. 2006 \\
\hline$>40-50$ & Increased preterm birth & Jelliffe-Pawlowski et al. 2006 \\
\hline
\end{tabular}

$\mathrm{FSH}=$ follicle-stimulating hormone; $\mathrm{LH}=$ luteinizing hormone

Effects in Females at Blood Pb Levels $\leq 10 \mu \boldsymbol{g} / \mathbf{d L}$. As discussed above, most epidemiology studies evaluating adverse effects of $\mathrm{Pb}$ on female reproductive function reported mean $\mathrm{PbB} \leq 10 \mu \mathrm{g} / \mathrm{dL}$. Although some studies provide evidence showing associations between $\mathrm{PbB} \leq 10 \mu \mathrm{g} / \mathrm{dL}$ and effects on serum reproductive hormones (Chang et al. 2006; Chen et al. 2016; Krieg 2007), fertility (Chang et al. 2006), spontaneous abortion (Lamadrid-Figueroa et al. 2007; Yin et al. 2008), and preterm birth (Li et al. 2017b; Rabito et al. 2014; Taylor et al. 2015; Vigeh et al. 2011), many studies show no associations between $\mathrm{PbB}$ and these outcomes. In general, most studies are limited by small sample sizes, although, as discussed below, some studies were of larger populations. The basis for differences in study outcomes in not readily apparent, although several factors may contribute, including low samples size, timing of evaluations in menstrual and life cycles, and inclusion of study participants identified from fertility clinics. Results are summarized in Table 2-38, with study details provided in the Supporting Document for Epidemiological Studies for Lead Table 12. 
2. HEALTH EFFECTS

Table 2-38. Summary of Epidemiological Studies Evaluating Effects on the Female Reproductive System at Mean Blood Lead Concentration (PbB) $\leq 10 \mu \mathrm{g} / \mathrm{dL}^{\mathrm{a}}$

\begin{tabular}{|c|c|c|c|}
\hline Reference and study population ${ }^{b}$ & $\mathrm{PbB}(\mu \mathrm{g} / \mathrm{dL})$ & Outcome evaluated & Result ${ }^{\mathrm{c}}$ \\
\hline \multicolumn{4}{|l|}{ Effects on serum hormone levels } \\
\hline $\begin{array}{l}\text { Chang et al. } 2006 \\
\text { Case control study; } n=147\end{array}$ & Mean: 3.55 & Estradiol & $\begin{array}{l}\beta \text { coefficient } \mathrm{pg} / \mathrm{mL} \text { per } \mu \mathrm{g} / \mathrm{dL}(\mathrm{SE}): 1.18 \\
(0.60) ; p=0.049^{\star}\end{array}$ \\
\hline \multirow{5}{*}{$\begin{array}{l}\text { Chen et al. } 2016 \\
\text { Cross-sectional study; } \\
n=1,571 \text { postmenopausal women }\end{array}$} & \multirow{5}{*}{$\begin{array}{ll}\text { Median: } 4.1 \\
-\quad \text { Q1: }<2.7(n=558) \\
-\quad \text { Q2: } 2.7-4.09 \\
& (n=572) \\
- & \text { Q3: 4.1-5.98 } \\
& (n=585) \\
- & \text { Q4: }>5.98(n=571)\end{array}$} & $\mathrm{FSH}$ & $\begin{aligned} & \beta \text { coefficients (SE) } \\
&-\quad \text { Q3: } 0.047(0.015) ; p<0.01^{*} \\
&-\quad \text { Q4: } 0.046(0.016) ; p<0.01^{*}\end{aligned}$ \\
\hline & & $\overline{\mathrm{LH}}$ & $\begin{array}{l}\beta \text { coefficients (SE), Q4: } 0.037(0.016) ; \\
p<0.05^{*}\end{array}$ \\
\hline & & Estradiol & $\beta$ coefficients (SE), Q4: $-0.021(0.020)$ \\
\hline & & Testosterone & $\beta$ coefficients (SE), Q4: $-0.016(0.020)$ \\
\hline & & Sex hormone binding & $\begin{array}{l}\beta \text { coefficients (SE), Q4: } 0.048(0.016) \\
p<0.01^{*}\end{array}$ \\
\hline \multirow{4}{*}{$\begin{array}{l}\text { Jackson et al. } 2011 \\
\text { Longitudinal cohort study; } n=252\end{array}$} & \multirow[t]{4}{*}{ Mean: 0.87} & $\mathrm{FSH}$ & $\beta$ coefficient (IU/L per $\mu \mathrm{g} / \mathrm{dL}):-2.5(-11.2,7.0)$ \\
\hline & & $\mathrm{LH}$ & $\beta$ coefficient $(\mathrm{mg} / \mathrm{L}$ per $\mu \mathrm{g} / \mathrm{dL}): 2.5(-12.3,19.9)$ \\
\hline & & Estradiol & $\beta$ coefficient $(\mathrm{pg} / \mathrm{mL}$ per $\mu \mathrm{g} / \mathrm{dL}): 4.9(-5.0,15.9)$ \\
\hline & & Progesterone & $\begin{array}{l}\beta \text { coefficient }(\mathrm{ng} / \mathrm{mL} \text { per } \mu \mathrm{g} / \mathrm{dL}): 4.6(-12.2, \\
24.6)\end{array}$ \\
\hline \multirow{3}{*}{$\begin{array}{l}\text { Krieg } 2007 \\
\text { Cross-sectional study; } n=3,375\end{array}$} & \multirow[t]{3}{*}{ Gmean: 2.2} & $\mathrm{FSH}$ & $\begin{array}{l}\text { - Slope pre-menopausal (IU/L per } \mu \mathrm{g} / \mathrm{dL} \text { ): } \\
8.3(2.2) ; 95 \% \mathrm{Cl} 3.8,12.7 ; p=0.0006^{*}\end{array}$ \\
\hline & & & $\begin{array}{l}\text { - Slope post-menopausal (IU/L per } \mu \mathrm{g} / \mathrm{dL} \text { ): } \\
22.2(4.3) ; 95 \% \mathrm{CI} 13.5,30.8 ; \mathrm{p}=0.0000^{*} \\
\text { - Slope both ovaries removed (IU/L per } \\
\mu \mathrm{g} / \mathrm{dL}): 32.6(11.2) ; 95 \% \mathrm{Cl} 10.1,55.1 ; \\
\mathrm{p}=0.0054^{*}\end{array}$ \\
\hline & & $\mathrm{LH}$ & $\begin{array}{ll} & \text { Slope pre-menopausal (IU/L per } \mu \mathrm{g} / \mathrm{dL}): 1.7 \\
& (1.2) ; 95 \% \mathrm{Cl}-0.6,4.1 ; \mathrm{p}=0.1486 \\
\text { - } & \text { Slope post-menopausal (IU/L per } \boldsymbol{\mu g} / \mathrm{dL}) \text { : } \\
\mathbf{6 . 2}(\mathbf{1 . 6}) ; \mathbf{9 5 \%} \mathbf{C}: \mathbf{3 . 0}, \mathbf{9 . 5} ; \boldsymbol{p}=\mathbf{0 . 0 0 0 3 ^ { * }}\end{array}$ \\
\hline
\end{tabular}


2. HEALTH EFFECTS

Table 2-38. Summary of Epidemiological Studies Evaluating Effects on the Female Reproductive System at Mean Blood Lead Concentration (PbB) $\leq 10 \mu \mathrm{g} / \mathrm{dL}^{\mathrm{a}}$

\begin{tabular}{|c|c|c|c|}
\hline Reference and study population ${ }^{b}$ & $\mathrm{PbB}(\mu \mathrm{g} / \mathrm{dL})$ & Outcome evaluated & Result ${ }^{\mathrm{c}}$ \\
\hline & & & $\begin{array}{l}\text { - Slope both ovaries removed (IU/L per } \\
\mu g / d L): 10.0(4.4) ; 95 \% \mathrm{CI} 1.1,18.9 ; \\
p=0.0279 *\end{array}$ \\
\hline Pollack et al. 2011 & Mean: 0.93 & Estradiol & $\begin{array}{l}\beta \text { coefficient }(\mathrm{pg} / \mathrm{mL} \text { per } \mu \mathrm{g} / \mathrm{dL}): 0.03(-0.05 \\
0.11)\end{array}$ \\
\hline \multirow[t]{3}{*}{ Longitudinal cohort study; n=252 } & & $\overline{\mathrm{FSH}}$ & $\begin{array}{l}\beta \text { coefficient ( } \mathrm{mIU} / \mathrm{mL} \text { per } \mu \mathrm{g} / \mathrm{dL}):-0.01(-0.07 \text {, } \\
0.06)\end{array}$ \\
\hline & & $\mathrm{LH}$ & $\begin{array}{l}\beta \text { coefficient }(\mathrm{ng} / \mathrm{mL} \text { per } \mu \mathrm{g} / \mathrm{dL}): 0.02(-0.06 \text {, } \\
0.10)\end{array}$ \\
\hline & & Progesterone & $\begin{array}{l}\beta \text { coefficient }(\mathrm{ng} / \mathrm{mL} \text { per } \mu \mathrm{g} / \mathrm{dL}): 0.06(-0.04, \\
0.17)\end{array}$ \\
\hline \multicolumn{4}{|l|}{ Fertility } \\
\hline $\begin{array}{l}\text { Bloom et al. } 2010 \\
\text { Longitudinal cohort study; } n=15\end{array}$ & Mean: 0.82 & Oocyte fertilization (in vitro) & RR: 1.09 (0.72, 1.65). \\
\hline $\begin{array}{l}\text { Bloom et al. } 2011 \\
\text { Longitudinal cohort study; } n=80\end{array}$ & Mean: 1.54 & $\begin{array}{l}\text { Achieving pregnancy over } \\
12 \text { menstrual cycles }\end{array}$ & $\begin{array}{l}\beta \text { coefficient (probability of pregnancy per } \\
\mu \mathrm{g} / \mathrm{dL}):-0.031(95 \% \mathrm{Cl}-1.066,1.004) ; p=0.954\end{array}$ \\
\hline $\begin{array}{l}\text { Chang et al. } 2006 \\
\text { Case control study; } n=147\end{array}$ & $\begin{array}{l}\text { Mean: } \\
\text { - } \quad \text { All: } 3.12 \\
\text { - } \quad \text { Controls: } 2.78 \\
\text { - } \quad \text { Cases: } 3.55\end{array}$ & Infertility & $\begin{array}{l}\text { OR for } \mathrm{PbB}>2.5 \text { versus } \leq 2.5 \mu \mathrm{g} / \mathrm{dL}: 2.94 \\
(95 \% \mathrm{Cl} 1.18,7.34) ; p=0.021^{*}\end{array}$ \\
\hline \multicolumn{4}{|l|}{ Pregnancy outcome } \\
\hline $\begin{array}{l}\text { Bloom et al. } 2015 \\
\text { Case control study; } n=235\end{array}$ & $\begin{array}{l}\text { Mean: } 0.71 \\
\text { Tertiles (mean): } \\
\text { - } \quad \text { T1: not reported } \\
\text { - } \quad \text { T2: } 0.55 \\
\text { - } \quad \text { T3: } 0.73\end{array}$ & Duration of gestation & $\begin{array}{l}\text { Regression coefficient gestational age per } \\
\mu \mathrm{g} / \mathrm{dL}) \mathrm{T} 3: 0.14(-0.81,1.09)\end{array}$ \\
\hline
\end{tabular}


Table 2-38. Summary of Epidemiological Studies Evaluating Effects on the Female Reproductive System at Mean Blood Lead Concentration (PbB) $\leq 10 \mu \mathrm{g} / \mathrm{dL}^{\mathrm{a}}$

\begin{tabular}{|c|c|c|c|}
\hline Reference and study population ${ }^{b}$ & $\mathrm{PbB}(\mu \mathrm{g} / \mathrm{dL})$ & Outcome evaluated & Result ${ }^{\mathrm{C}}$ \\
\hline $\begin{array}{l}\text { Garcia-Esquinas et al. } 2014 \\
\text { Birth cohort study; } n=100\end{array}$ & Gmean: 1.83 & Duration of gestation & $\begin{array}{l}\text { Mean difference in gestational age (weeks) per } \\
\text { 2-fold increase in PbB: } 0.02(95 \% \mathrm{Cl}-0.44 \text {, } \\
0.47)\end{array}$ \\
\hline $\begin{array}{l}\text { Gundacker et al. } 2010 \\
\text { Cross-sectional study; } n=30\end{array}$ & $\begin{array}{l}\text { Median PbB: } 2.5 \\
\text { Median } \mathrm{Pb} \text { (placenta): } \\
25.8 \mu \mathrm{g} / \mathrm{kg}\end{array}$ & Spontaneous abortion & $\begin{array}{l}\text { Placenta } \mathrm{Pb} \text { concentration in women with a } \\
\text { history of miscarriage was higher }(n=8 ; \\
p=0.039) \text { than in women with no history of } \\
\text { miscarriage }(n=22)^{*}\end{array}$ \\
\hline $\begin{array}{l}\text { Lamadrid-Figueroa et al. } 2007 \\
\text { Cross-sectional study; n=207 }\end{array}$ & $\begin{array}{l}\text { Mean PbB: } 6.24(4.48) \\
\text { Mean plasma } \mathrm{Pb}: 0.014 \\
\text { Mean plasma/blood } \mathrm{Pb} \\
\text { ratio: } 0.22 \% \\
\text { (tertile values not } \\
\text { reported) }\end{array}$ & Spontaneous abortion & 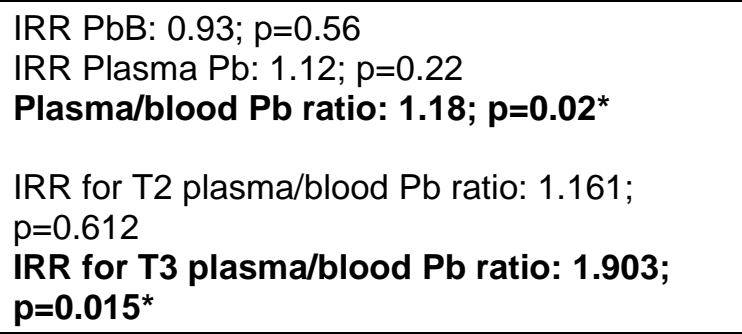 \\
\hline $\begin{array}{l}\text { Li et al. 2017b } \\
\text { Birth cohort study; } n=3,125\end{array}$ & $\begin{array}{l}\text { Mean (range): } 1.5 \text { (0.02- } \\
5.46) \\
\text { Stratified: } \\
\text { - } \quad \text { Low: }<1.18 \\
\text { - } \quad \text { Medium: } 1.18-1.70 \\
\text { - High: } \geq 1.71\end{array}$ & Preterm birth & $\begin{array}{l}\text { OR Medium PbB: } 2.33(1.49,3.65) \\
\text { p<0.001* } \\
\text { OR High PbB: } 3.09(2.01,4.76) ; p<0.001^{*}\end{array}$ \\
\hline $\begin{array}{l}\text { Perkins et al. } 2014 \\
\text { Birth cohort; } n=949\end{array}$ & $\begin{array}{l}\text { Estimated mean PbB: } 0.4 \\
\text { Mean RBC: } 1.22 \mu \mathrm{g} / \mathrm{dL} \\
\text { Quartile RBC }(\mu \mathrm{g} / \mathrm{dL}): \\
\text { - } \quad \text { Q1: } 0.65 \\
\text { - } \quad \text { Q2: } 0.96 \\
\text { - Q3: } 1.27 \\
\text { - } \quad \text { Q4: } 2.02\end{array}$ & Duration of gestation & $\begin{array}{l}\beta \text { coefficient } Q 4 \text { gestational age (weeks) per } \\
\mu \mathrm{g} / \mathrm{dL}:-0.17(-0.51,0.16)\end{array}$ \\
\hline $\begin{array}{l}\text { Rabito et al. } 2014 \\
\text { Birth cohort; } n=98\end{array}$ & $\begin{array}{l}\text { Second trimester mean: } \\
0.42 \\
\text { Third trimester mean: } \\
0.45\end{array}$ & Preterm birth & $\begin{array}{l}\text { OR second trimester: } 1.66(1.23,2.23) \\
p<0.01^{*} \\
\text { OR third trimester: } 1.24(1.01,1.52) ; p=0.04^{*}\end{array}$ \\
\hline
\end{tabular}




\section{Table 2-38. Summary of Epidemiological Studies Evaluating Effects on the Female Reproductive System at Mean Blood Lead Concentration (PbB) $\leq 10 \mu \mathrm{g} / \mathrm{dL}^{\mathrm{a}}$}

\begin{tabular}{|c|c|c|c|}
\hline Reference and study population $^{b}$ & $\mathrm{PbB}(\mu \mathrm{g} / \mathrm{dL})$ & Outcome evaluated & Result $^{\mathrm{C}}$ \\
\hline $\begin{array}{l}\text { Taylor et al. 2013, } 2015 \\
\text { Longitudinal cohort study; } n=3,870\end{array}$ & $\begin{array}{l}\text { Mean: } 3.67 \\
\text { Median: } 3.42\end{array}$ & Preterm birth & OR for $\mathrm{PbB} \geq 5.0: 2.0(1.35,3.00) ; p=0.001^{*}$ \\
\hline $\begin{array}{l}\text { Vigeh et al. } 2010 \\
\text { Longitudinal cohort study; } n=351\end{array}$ & Mean: 3.8 & Spontaneous abortion & OR (log PbB): $0.331(0.011,10.096) ; p=0.53$ \\
\hline $\begin{array}{l}\text { Vigeh et al. } 2011 \\
\text { Longitudinal cohort study; } n=44 \text { women } \\
\text { with preterm birth; } n=304 \text { women with } \\
\text { term birth }\end{array}$ & $\begin{array}{l}\text { Mean: } \\
\text { - } \quad \text { Term birth: } 3.72 \\
\text { - } \quad \text { Preterm birth: } 4.52\end{array}$ & Preterm birth & OR: $1.41(1.08,1.84)^{*}$ \\
\hline $\begin{array}{l}\text { Yin et al. } 2008 \\
\text { Case-control study; } n=80\end{array}$ & $\begin{array}{l}\text { Control (term birth): } 4.5 \\
\text { Spontaneous abortion: } \\
5.3\end{array}$ & Spontaneous abortion & $\begin{array}{l}\text { PbB was higher in cases of anembryonic } \\
\text { pregnancy during gestational weeks 8-13 } \\
\text { compared to controls with term births } \\
(p=0.03) .^{*}\end{array}$ \\
\hline $\begin{array}{l}\text { Zhu et al. } 2010 \\
\text { Retrospective cohort; } n=43,288 \text { mother- } \\
\text { infant pairs ( } n=3,519 \text { preterm birth; } \\
n=39,769 \text { term birth) }\end{array}$ & $\begin{array}{l}\text { PbB } \\
\text { Mean: } 2.1 \\
\text { Quartiles: } \\
\text { - Q1: } \leq 1.0 \\
\text { - Q2: } 1.1-2.0 \\
\text { - Q3: } 2.1-3.0 \\
\text { Q Q4: 3.1-9.9 }\end{array}$ & Preterm birth & $\begin{array}{l}\text { Adjusted ORs did not show an increased risk of } \\
\text { preterm birth for any quartile. Q4: } 1.04(0.89 \text {, } \\
1.22)\end{array}$ \\
\hline
\end{tabular}

aSee the Supporting Document for Epidemiological Studies for Lead, Table 12 for more detailed descriptions of studies.

bParticipants had no known occupational exposure to $\mathrm{Pb}$.

${ }^{\mathrm{c}}$ Asterisk and bold indicate association with PB; unless otherwise specified, values in parenthesis are $95 \%$ Cls; $p$-values < 0.05 unless otherwise noted in the table.

$\mathrm{Cl}=$ confidence interval; FSH = follicle-stimulating hormone; Gmean = geometric mean; IRR = incidence rate ratio; LH = luteinizing hormone; OR = odds ratio; $\mathrm{Pb}=$ lead; $\mathrm{PbPI}=\mathrm{Pb}$ concentration in placenta $(\mu \mathrm{g} / \mathrm{kg}) ; \mathrm{RBC}=$ red blood cell; $\mathrm{RR}=$ relative risk; $\mathrm{SE}=$ standard error 
Serum hormone levels and estrus cycle. Results of epidemiological studies on associations between PbB $\leq 10 \mu \mathrm{g} / \mathrm{dL}$ and serum hormone levels show conflicting results (Table 2-38). The strongest evidence showing that chronic $\mathrm{Pb}$ exposure alters serum hormone levels is from a large cross-sectional study (mean PbB: $2.2 \mu \mathrm{g} / \mathrm{dL}$ ) participating in the NHANES III study (Krieg 2007). Serum levels of FSH (IU/L) increased with $\mathrm{PbB}$ in both pre-menopausal and post-menopausal women. Serum levels of LH increased with PbB in post-menopausal women, but not pre-menopausal women. The lowest PbBs associated with a significant increase in FSH in pre- and post-menopausal women were $4.1 \mu \mathrm{g} / \mathrm{dL}$ and $2.4 \mu \mathrm{g} / \mathrm{dL}$, respectively. The lowest $\mathrm{PbB}$ associated with a significant increase in FSH in post-menopausal women was $2.8 \mu \mathrm{g} / \mathrm{dL}$ (slope \pm SE 8.6 \pm 3.3 ; 95\% CI 2.1, 15.2; p=0.0109). Increases in serum FSH and LH were also observed in women who had total ovariectomy, indicating that increased hormone levels may be related to effects on the hypothalamus or pituitary (Krieg 2007). A large cross-sectional study of postmenopausal Chinese women also found that elevated serum hormones levels were positively associated with $\mathrm{PbB}$. Increased FSH was observed in the two highest $\mathrm{PbB}$ quartiles (4.1-5.9 and $>5.9 \mu \mathrm{g} / \mathrm{dL}$ ), with LH increased in the highest quartile (Chen et al. 2016). SHBG was also increased in the highest quartile. No associations were observed between $\mathrm{Pb}$ and serum levels of FSH, LH, estradiol, or progesterone or menstrual cycle length in a smaller study of pre-menopausal women with a mean $\mathrm{PbB}$ of $0.87 \mu \mathrm{g} / \mathrm{dL}$ (Jackson et al. 2011). In this same study population, when PbB was examined by tertiles, increased serum progesterone levels were observed in the second $\mathrm{PbB}$ tertile $(0.73-1.10 \mu \mathrm{g} / \mathrm{dL})$ compared to the lowest tertile $(0.30-0.72 \mu \mathrm{g} / \mathrm{dL})$, but no effects were observed in the highest $\mathrm{PbB}$ tertile $(1.11-$ $6.20 \mu \mathrm{g} / \mathrm{dL}$ ) compared to the lowest (Pollack et al. 2011). In this study population, no association was observed between $\mathrm{PbB}$ and anovulation. In a case-control study of women attending a fertility clinic, a significant association was observed between PbB and serum estradiol concentrations (Chang et al. 2006).

Fertility. Little epidemiological information is available on the effects of $\mathrm{PbB} \leq 10 \mu \mathrm{g} / \mathrm{dL}$ on female fertility. A prospective cohort study with a mean $\mathrm{Pb}$ of $1.5 \mu \mathrm{g} / \mathrm{dL}$ showed no effect on achieving pregnancy over 12 menstrual cycles (Bloom et al. 2011). A case-control study of women from a fertility clinic showed a 2.9-fold risk of infertility for $\mathrm{PbB}>2.5 \mu \mathrm{g} / \mathrm{dL}$ compared to $\mathrm{PbB} \leq 2.5 \mu \mathrm{g} / \mathrm{dL}$ (Chang et al. 2006). In a study of women undergoing in vitro fertilization, no association was observed between $\mathrm{PbB}$ and oocyte fertilization; however, only 15 women were included in this study. Available epidemiological studies on the effects of $\mathrm{PbB} \leq 10 \mu \mathrm{g} / \mathrm{dL}$ on fertility are limited due to small numbers of participants and study populations of women undergoing fertility treatment; thus, data are not sufficient to determine if fertility in women is affected at $\mathrm{PbB} \leq 10 \mu \mathrm{g} / \mathrm{dL}$. 
Spontaneous abortion. Few epidemiological studies have evaluated associations between $\mathrm{PbB} \leq 10 \mu \mathrm{g} / \mathrm{dL}$ and spontaneous abortion (Table 2-38). Although studies provide some evidence suggesting associations between $\mathrm{PbB} \leq 10 \mu \mathrm{g} / \mathrm{dL}$ or plasma/blood $\mathrm{Pb}$ ratio and spontaneous abortion, results are inconsistent. In a case-control study, $\mathrm{PbB}$ was significantly higher in cases of spontaneous abortion ( $\mathrm{PbB} 5.3 \mu \mathrm{g} / \mathrm{dL}$; $\mathrm{p}=0.03$ ) during weeks 8-13, compared to women with term birth ( $\mathrm{PbB} 4.5 \mu \mathrm{g} / \mathrm{dL}$ ) (Yin et al. 2008). A cross-sectional study reported that the risk of miscarriage per $1 \mathrm{SD}$ increase of plasma/blood $\mathrm{Pb}$ ratio [mean plasma/blood $\mathrm{Pb}$ ratio $\pm \mathrm{SD}$ (\%): 0.22 \pm 0.14 ] was associated with an $18 \%$ greater incidence of spontaneous abortion, although the association between risk of spontaneous abortion and $\mathrm{PbB}$ (mean 6.24) was not significant (Lamadrid-Figueroa et al. 2007). In contrast, results of a longitudinal cohort study showed no association between $\mathrm{PbB}$ and spontaneous abortion during gestational weeks 13-19 (Vigeh et al. 2010).

Preterm birth. Several studies have evaluated associations between $\mathrm{PbB} \leq 10 \mu \mathrm{g} / \mathrm{dL}$ and preterm birth ( $<37$ weeks of gestation), including three studies of larger study populations ( $n=705-3,870$ ) (Li et al. 2017b; Perkins et al. 2014; Taylor et al. 2015). Results of these studies are mixed (Table 2-38). The strongest evidence showing that chronic $\mathrm{Pb}$ exposure is associated with preterm birth is from two large, cohort studies ( $\mathrm{Li}$ et al. 2017b; Taylor et al. 2013, 2015). Taylor et al. (2013, 2015) reported that when stratified into groups of $\mathrm{PbB}<5$ and $\geq 5.0 \mu \mathrm{g} / \mathrm{dL}$, there was a 2-fold increase in the risk of preterm birth for $\mathrm{PbB} \geq 5.0 \mu \mathrm{g} / \mathrm{dL}$ compared to $\mathrm{PbB}<5 \mu \mathrm{g} / \mathrm{dL}$. In the $\mathrm{PbB} \geq 5.0 \mu \mathrm{g} / \mathrm{dL}$ group, the maximum $\mathrm{PbB}$ was $19.14 \mu \mathrm{g} / \mathrm{dL}$, although very few $\mathrm{PbBs}$ were $>10 \mu \mathrm{g} / \mathrm{dL}$; however, the group mean $\mathrm{PbB}$ was not reported. In a large cohort study, the risk of preterm birth was increased in women with PbBs of 1.18-1.70 and 1.71-5.46 $\mu \mathrm{g} / \mathrm{dL}$, relative to women with PbBs of 0.02-1.18 $\mu \mathrm{g} / \mathrm{dL}$ (Li et al. 2017b). The risk of preterm birth also was increased in a longitudinal cohort study (Vigeh et al. 2011). Mean PbB in women with preterm birth was significantly higher than in women with term birth (preterm $\mathrm{PbB}: 4.52 \mu \mathrm{g} / \mathrm{dL}$; term birth $\mathrm{PbB}: 3.72 \mu \mathrm{g} / \mathrm{dL})$. A cohort study showed increased odds of preterm birth associated with $\mathrm{PbB}$ measured in the $2^{\text {nd }}$ (mean: $0.42 \mu \mathrm{g} / \mathrm{dL}$ ) and $3^{\text {rd }}$ (mean: $0.45 \mu \mathrm{g} / \mathrm{dL}$ ) trimesters (Rabito et al. 2014). ORs for risks of preterm birth were $1.66(\mathrm{p}<0.01)$ and $1.24(\mathrm{p}=0.04)$ for $2^{\text {nd }}$ and $3^{\text {rd }}$ trimester $\mathrm{PbB}$, respectively. Other studies reported no associations between $\mathrm{PbB}$ and preterm birth at mean $\mathrm{PbB}$ of $0.71-5.70 \mu \mathrm{g} / \mathrm{dL}$ (Bloom et al. 2015; Perkins et al. 2014; Zhu et al. 2010), including a large retrospective cohort study (Zhu et al. 2010) and a large case-control study (Perkins et al. 2014).

Age at menopause. A few studies had evaluated associations between $\mathrm{Pb}$ exposure and age at menopause (Eum et al. 2014; Popovic et al. 2005). Eum et al. (2014) found an inverse association between tibia $\mathrm{Pb}$ and age at onset of natural menopause (e.g., non-surgical) in a population of 434 participants in the 
Nurses Health Study cohort. In the highest tibia Pb tertile, the age at onset of menopause was 1.21 years earlier than controls. However, no associations were observed between $\mathrm{PbB}$ (mean $\mathrm{PbB}:<5 \mu \mathrm{g} / \mathrm{dL}$ ) or patella $\mathrm{Pb}$. In a study of 108 former smelters (mean PbB: $2.73 \mu \mathrm{g} / \mathrm{dL}$ ), the age at onset of combined natural and surgical menopause was earlier by 7 years $(\mathrm{p}=0.001)$ compared to controls $(\mathrm{n}=99$; PbB: $1.25 \mu \mathrm{g} / \mathrm{dL}$ ) (Popovic et al. 2005). No difference was observed between the age at onset and natural menopause between the exposed and control groups.

Mechanisms of Action. General mechanisms of toxicity of $\mathrm{Pb}$ (reviewed in Section 2.21) are likely involved in the development of toxicity to male and female reproductive systems. Oxidative stress through ROS is a plausible mechanism for reproductive effects, as is the disruption of calcium homeostasis. Mechanisms for alterations in circulating hormone levels have been not been established. However, EPA (2014c) and NRC (2012) noted several possible mechanisms that may be involved in alterations of serum hormones, including direct inhibition of LH secretion; reduced expression of steroidogenic acute regulatory protein (a protein required in maintaining gonadotropin-stimulated steroidogenesis); altered release of pituitary hormones due to interference with cation-dependent second messenger systems; and altered binding of hormones to receptors. $\mathrm{Pb}$ is distributed to, and has been measured in, semen, spermatozoa, the fetus, umbilical cord blood, placenta, and follicular fluid (see Section 3.1.2, Toxicokinetics, Distribution), providing a toxicokinetic mechanism for direct effects to reproductive tissues.

\subsection{DEVELOPMENTAL}

This section discusses developmental effects of $\mathrm{Pb}$ other than neurodevelopmental defects. Neurodevelopmental effects are discussed in Section 2.16 (Neurological Effects). The term "developmental” used in the discussion that follows refers to effects other than neurodevelopmental.

Overview. Numerous epidemiological studies have evaluated developmental effects (birth outcomes, birth defect, neural tube defects, decreased anthropometric measures in children, and delayed puberty) associated with $\mathrm{Pb}$ exposure, with the database for developmental effects dominated by environmental exposure studies with $\mathrm{PbB} \leq 10 \mu \mathrm{g} / \mathrm{dL}$. In general, studies provide mixed evidence for effects on birth outcomes (e.g., infant size) and anthropometric measures in children, but more consistent evidence for delayed puberty. Although studies provide evidence of associations between $\mathrm{PbB}$ and developmental outcomes, results are inconsistent, and several studies, including prospective studies, with $\mathrm{PbB} \leq 10 \mu \mathrm{g} / \mathrm{dL}$ show no associations with developmental outcomes. 
The following developmental effects have been associated with $\mathrm{PbB}$ :

- $\quad \leq 10 \mu \mathrm{g} / \mathrm{dL}$ :

o Effects on birth outcomes (decreased birth weight, head circumference, and crown-heel length); results are mixed when compared across studies.

o Decreased anthropometric measures in children (weight, height, head circumference, trunk length, leg length, arm length, BMI); results are mixed when compared across studies.

o Delayed puberty in females (breast development, pubic hair development, onset of menarche); corroborated in multiple studies.

o Delayed puberty in males (testicular volume, genitalia development, pubic hair development); a few studies with equivocal results.

- $\quad>10 \mu \mathrm{g} / \mathrm{dL}$ (based on few studies):

o Effects on birth outcomes (low birth weight).

o Decreased anthropometric measures in children (decreased weight, height, head circumference, chest circumference).

o Delayed puberty in females (breast development).

o Delayed puberty in males (decreased testicular size, delayed pubic hair development, delayed penile development).

Measures of Exposure. Most studies evaluating developmental effects used maternal $\mathrm{PbB}$ and/or cord, infant, or child $\mathrm{PbB}$ as the biomarker for exposure. In some studies, $\mathrm{Pb}$ concentrations in red blood cells (Perkins et al. 2014), maternal bone (Afeiche et al. 2011; Cantonwine et al. 2010; Hernandez-Avila et al. 2002; Kordas et al. 2009), or hair (Sanín et al. 2001; Sanna and Vallascas 2011) were used as biomarkers.

Confounding Factors and Effect Modifiers. Numerous complicating factors may add uncertainty in the interpretation of studies examining associations between $\mathrm{PbB}$ and developmental effects. These factors include nutrition during pregnancy, prenatal care, adequate nutrition during infancy and childhood, SES, intercurrent diseases, alcohol consumption, smoking status, and potential exposure to other chemicals. Failure to account for these factors may attenuate or strengthen the apparent associations between $\mathrm{Pb}$ exposure and the outcome, depending on the direction of the effect of the variable on the outcome.

Characterization of Effects. As noted above, most epidemiological studies evaluated developmental effects at $\mathrm{PbB} \leq 10 \mu \mathrm{g} / \mathrm{dL}$, with few studies of $\mathrm{PbB}>10 \mu \mathrm{g} / \mathrm{dL}$. Studies of $\mathrm{PbB} \leq 10 \mu \mathrm{g} / \mathrm{dL}$ are discussed 
in detail in the section below. General trends for studies showing a relationship between $\mathrm{PbB} \leq 10$ $50 \mu \mathrm{g} / \mathrm{dL}$ and developmental effects are shown in Table 2-39. Effects on birth outcomes, including decreased birth weight, head circumference, and crown-heel length have been observed at maternal PbBs of $\leq 10-50 \mu \mathrm{g} / \mathrm{dL}$. Decreased anthropometric measures in infants and children, including decreased weight, height, head circumference, trunk length, leg length, arm length, and BMI, have been observed over the $\mathrm{PbB}$ range of $\leq 10-30 \mu \mathrm{g} / \mathrm{dL}$. Delayed onset of puberty in males and females was observed over the $\mathrm{PbB}$ range of $\leq 10-30 \mu \mathrm{g} / \mathrm{dL}$. Very little data are available regarding in utero exposure to $\mathrm{Pb}$ and birth defects. Two studies that examined neural tube defects did not find associations with $\mathrm{Pb}$ exposure at mean blood levels over for PbB means ranging from 2.4 to $24 \mu \mathrm{g} / \mathrm{dL}$ (Brender et al. 2006; Zeyrek et al. 2009). As discussed below, although epidemiological studies demonstrate developmental effects of $\mathrm{Pb}$, results across studies are inconsistent, with several studies reporting no association between $\mathrm{PbB}$ and developmental effects. For example, results of effects on birth outcomes in study populations with maternal $\mathrm{PbB} \leq 10 \mu \mathrm{g} / \mathrm{dL}$ are equivocal (see Tables 2-40 and 2-41). For studies with maternal $\mathrm{PbB}$ $>10 \mu \mathrm{g} / \mathrm{dL}$, equivocal results also were observed for associations between $\mathrm{PbB}$ and birth weight and length (Factor-Litvak et al. 1991; Hernandez-Avila et al. 2002; McMichael et al. 1986; Murphy et al. 1990). Dose-dependence has not been firmly established within the relatively narrow range of PbB $(\leq 10 \mu \mathrm{g} / \mathrm{dL})$ in most studies.

\title{
Table 2-39. Overview of Developmental Effects Associated with Chronic Exposure to Lead (Pb)
}

\author{
Mean blood lead \\ concentration $(\mathrm{PbB}) \quad$ Effects associated with $\mathrm{Pb}$ \\ $\underline{(\mu g / d L)}$ \\ exposure \\ References \\ $\leq 10 \quad$ Effects on birth outcome (decreased \\ birth weight, crown-heel length, head \\ circumference) \\ Bornschein et al. 1989; González - \\ Cossío et al. 1997; Nishioka et al. \\ 2014; Odland et al. 1999; Taylor et al. \\ 2013, 2015; Wang et al. 2017b, 2017b; \\ Xie et al. 2013; Zhu et al. 2010 \\ Minor congenital anomalies \\ Needleman et al. 1984 \\ Decreased anthropometric measures \\ in children (decreased weight, height, \\ head circumference, waist \\ circumference, trunk length, leg \\ length, arm length, body mass index, \\ body fat) \\ Afeiche et al. 2011; Alvarez-Ortega et \\ al. 2019; Dallaire et al. 2014; Deierlein \\ et al. 2019; Hauser et al. 2008; Hong et \\ al. 2014; Ignasiak et al. 2006; Little et \\ al. 2009; Min et al. 2008b; Olivero- \\ Verbel et al. 2007; Raihan et al. 2018; \\ Schell et al. 2009; Yang et al. 2013a \\ Delayed puberty in females (breast \\ Denham et al. 2005; Den Hond et al. \\ development, pubic hair development, 2011; Gollenberg et al. 2010; Naicker \\ onset of menarche) \\ et al. 2010; Selevan et al. 2003; Wu et \\ al. $2003 \mathrm{~b}$
}




\section{Table 2-39. Overview of Developmental Effects Associated with Chronic Exposure to Lead (Pb)}

\begin{tabular}{|c|c|c|}
\hline $\begin{array}{l}\text { Mean blood lead } \\
\text { concentration }(\mathrm{PbB}) \\
(\mu \mathrm{g} / \mathrm{dL})\end{array}$ & $\begin{array}{l}\text { Effects associated with } \mathrm{Pb} \\
\text { exposure }\end{array}$ & References \\
\hline & $\begin{array}{l}\text { Delayed puberty in males (testicular } \\
\text { volume, genitalia development, pubic } \\
\text { hair development) }\end{array}$ & $\begin{array}{l}\text { Hauser et al. 2008; Williams et al. } \\
2010,2019\end{array}$ \\
\hline \multirow[t]{4}{*}{$>10-30$} & $\begin{array}{l}\text { Effects on birth outcome (decreased } \\
\text { birth weight) }\end{array}$ & $\begin{array}{l}\text { Chen et al. 2006; Hernandez-Avila et } \\
\text { al. } 2002\end{array}$ \\
\hline & $\begin{array}{l}\text { Decreased anthropometric measures } \\
\text { in children (decreased weight, height, } \\
\text { head circumference, chest } \\
\text { circumference) }\end{array}$ & $\begin{array}{l}\text { Frisancho and Ryan 1991; Kerr et al. } \\
\text { 2019; Tomoum et al. } 2010\end{array}$ \\
\hline & $\begin{array}{l}\text { Delayed puberty in females (breast } \\
\text { development) }\end{array}$ & Liu et al. 2019b; Tomoum et al. 2010 \\
\hline & $\begin{array}{l}\text { Delayed puberty in males (decreased } \\
\text { testicular size, delayed pubic hair } \\
\text { development; delayed penile } \\
\text { development) }\end{array}$ & Tomoum et al. 2010 \\
\hline$>30-50$ & $\begin{array}{l}\text { Effects on birth outcome (low birth } \\
\text { weight) }\end{array}$ & Jelliffe-Pawlowski et al. 2006 \\
\hline
\end{tabular}

\section{Table 2-40. Effects on Birth Outcomes at Blood Lead Concentration (PbB) $\leq 10 \mu \mathrm{g} / \mathrm{dL}$}

\begin{tabular}{lllll}
\hline & \multicolumn{4}{c}{ Birth outcome } \\
\cline { 2 - 5 } Reference (population size) & Birth weight & $\begin{array}{l}\text { Height or } \\
\text { C-H length }\end{array}$ & SGA & $\begin{array}{l}\text { Head } \\
\text { circumference }\end{array}$ \\
\hline Al-Saleh et al. 2014 $(\mathrm{n}=1,577)$ & $0^{\mathrm{a}}$ & 0 & 0 & 0 \\
\hline Bloom et al. 2015 $(\mathrm{n}=235)$ & $0^{\mathrm{a}}$ & 0 & - & 0 \\
\hline Bornschein et al. 1989 $(\mathrm{n}=202)$ & $\downarrow^{\mathrm{a}}$ & $\downarrow$ & - & 0 \\
\hline Garcia-Esquinas et al. 2014 (n=97) & $0^{\mathrm{a}}$ & 0 & - & - \\
\hline González-Cossín et al. 1997 $(\mathrm{n}=272)$ & $0^{\mathrm{b}}$ & - & - & - \\
\hline Kim et al. 2017b $(\mathrm{n}=280)$ & $0^{\mathrm{b}}$ & $\uparrow(\mathrm{M}), 0(\mathrm{~F})$ & - & 0 \\
\hline Nishioka et al. 2014 $(\mathrm{n}=386)$ & $\downarrow^{\mathrm{b}}$ & - & - & - \\
\hline Odland et al. 1999 $(\mathrm{n}=50)$ & $\downarrow^{\mathrm{a}, \mathrm{b}}$ & - & - & - \\
\hline Perkins et al. 2014 $(\mathrm{n}=949)$ & $0^{\mathrm{a}, \mathrm{b}}$ & 0 & - & 0 \\
\hline Rabito et al. 2014 $(\mathrm{n}=98)$ & $0^{\mathrm{a}}$ & - & - & - \\
\hline Rodosthenous et al. 2017 (n=946) & - & - & 0 & - \\
\hline Taylor et al. 2015 $(\mathrm{n}=4,285)$ & $\downarrow^{\mathrm{b}}$ & $\downarrow$ & - & $\downarrow$ \\
\hline Thomas et al. 2015 $(\mathrm{n}=1,835)$ & - & - & 0 & - \\
\hline Wang et al. 2017b & $\downarrow$ & 0 & $\downarrow$ & 0 \\
\hline Wang et al. 2017c & $\uparrow(M), 0(\mathrm{~F})$ & $0(\mathrm{~F})$ & - & 0 \\
\hline
\end{tabular}


2. HEALTH EFFECTS

Table 2-40. Effects on Birth Outcomes at Blood Lead Concentration (PbB) $\leq 10 \mu \mathrm{g} / \mathrm{dL}$

\begin{tabular}{lllll}
\hline & \multicolumn{4}{c}{ Birth outcome } \\
\cline { 2 - 5 } Reference (population size) & Birth weight & $\begin{array}{l}\text { Height or } \\
\text { C-H length }\end{array}$ & SGA & $\begin{array}{l}\text { Head } \\
\text { circumference }\end{array}$ \\
\hline Xie et al. 2013 $(n=252)$ & $\downarrow^{b}$ & 0 & - & 0 \\
\hline Zhu et al. 2010 $(n=43,288)$ & $\downarrow^{b}$ & - & 0 & - \\
\hline
\end{tabular}

airth weight not adjusted for gestational age

${ }^{\mathrm{b} B i r t h}$ weight adjusted for gestational age

$\downarrow=$ decrease in outcome measure; $\uparrow=$ increase in outcome measure; $0=$ no effect on outcome measure; $-=$ not assessed; $\mathrm{C}-\mathrm{H}=$ crown-heel; $\mathrm{F}=$ female; $\mathrm{M}=$ male; $\mathrm{SGA}=$ small for gestational age 
2. HEALTH EFFECTS

\section{Table 2-41. Summary of Epidemiological Studies Evaluating Birth Outcomes Effects of Mean Blood Lead Concentration $(\mathrm{PbB}) \leq 10 \mu \mathrm{g} / \mathrm{dL}^{\mathrm{a}}$}

\begin{tabular}{|c|c|c|c|}
\hline $\begin{array}{l}\text { Reference and study } \\
\text { population }^{\mathrm{b}}\end{array}$ & $\mathrm{PbB}(\mu \mathrm{g} / \mathrm{dL})^{\mathrm{c}}$ & Outcome evaluated & Result $^{d}$ \\
\hline Al-Saleh et al. 2014 & \multirow[t]{6}{*}{ Maternal PbB mean: 2.897} & Birth weight & OR: $1.107(0.797,1.538) ; p=0.545$ \\
\hline \multirow{5}{*}{$\begin{array}{l}\text { Cross-sectional study; } \\
\mathrm{n}=1578 \text { mother-infant pairs }\end{array}$} & & Birth height & OR: $1.299(0.945,1.786) ; p=0.107$ \\
\hline & & Crown-heel length & OR: $1.061(0.795,1.415) ; p=0.689$ \\
\hline & & SGA & OR: $1.168(0.837,1.631) ; p=0.362$ \\
\hline & & Head circumference & OR: $1.007(0.724,1.400) ; p=0.968$ \\
\hline & & Apgar & OR: $1.027(0.787,1.341) ; p=0.842$ \\
\hline \multirow{3}{*}{$\begin{array}{l}\text { Bloom et al. } 2015 \\
\text { Case-control study; } \\
\text { n=235 mother-infant pairs }\end{array}$} & \multirow{3}{*}{$\begin{array}{l}\text { Maternal PbB mean: } 0.71 \\
\text { Tertiles: } \\
\text { - T1: }<0.55 \text { (reference) } \\
\text { - T2: } 0.55-<0.73 \\
\text { - T3: } 0.73-2.23\end{array}$} & Birth weight & $\begin{array}{l}\text { Linear regression coefficient ( } \mathrm{g} \text { per } \mu \mathrm{g} / \mathrm{dL} \text { ) } \\
\text { T3: }-34.85(-97.76,128.06) ; \mathrm{p} \text {-trend }=0.202\end{array}$ \\
\hline & & Birth length & $\begin{array}{l}\text { Linear regression coefficient }(\mathrm{cm} \text { per } \mu \mathrm{g} / \mathrm{dL} \text { ) T3: } \\
0.14(-0.81,1.09) ; p \text {-trend:0.671 }\end{array}$ \\
\hline & & Head circumference & $\begin{array}{l}\text { Linear regression coefficient }(\mathrm{cm} \text { per } \mu \mathrm{g} / \mathrm{dL}) \\
\text { T3: }-0.33(-1.07,0.41) ; p \text {-trend: } 0.132\end{array}$ \\
\hline \multirow{4}{*}{$\begin{array}{l}\text { Bornschein et al. } 1989 \\
\text { Prospective study; } \\
n=202 \text { mother-infant pairs }\end{array}$} & \multirow[t]{4}{*}{$\begin{array}{l}\text { PbB: } \\
\text { Mean (SD): } 7.5\end{array}$} & Birth weight & $\begin{array}{l}\text { Regression coefficient (g per } \ln \mu g / d L) \text { for all } \\
\text { births: }-114 ; p<0.001^{*} \text {. }\end{array}$ \\
\hline & & & $\begin{array}{l}\text { Regression coefficient }(g \text { per } \mathrm{ln} \mu \mathrm{g} / \mathrm{dL} \text { ) with } \\
\text { significant interaction with maternal age } \\
(\mathrm{p}=0.0073)^{*} \text { : } \\
\text { maternal age } 18 \text { years: }-58^{*} \\
\text { maternal age } 30 \text { years: }-601^{*}\end{array}$ \\
\hline & & Birth length & $\begin{array}{l}\text { Regression coefficient (cm per In } \mu \mathrm{g} / \mathrm{dL} \text { ): }-2.5 \text {; } \\
\mathrm{p}=0.019^{*}\end{array}$ \\
\hline & & Head circumference & $\begin{array}{l}\text { Regression coefficient }(\mathrm{cm} \text { per In } \mathrm{PbB} \mu \mathrm{g} / \mathrm{dL}): 0.0 \\
\mathrm{p}=0.97\end{array}$ \\
\hline
\end{tabular}


Table 2-41. Summary of Epidemiological Studies Evaluating Birth Outcomes Effects of Mean Blood Lead Concentration $(\mathrm{PbB}) \leq 10 \mu \mathrm{g} / \mathrm{dL}^{\mathrm{a}}$

\begin{tabular}{|c|c|c|c|}
\hline $\begin{array}{l}\text { Reference and study } \\
\text { population }^{\mathrm{b}}\end{array}$ & $\mathrm{PbB}(\mu \mathrm{g} / \mathrm{dL})^{\mathrm{c}}$ & Outcome evaluated & Result $^{d}$ \\
\hline Garcia-Esquinas et al. 2014 & \multirow[t]{4}{*}{ Maternal PbB Gmean: 1.83} & Birth weight & $\begin{array}{l}\text { Adjusted mean difference in grams for a 2-fold } \\
\text { increase in } \mathrm{PbB}(\mu \mathrm{g} / \mathrm{L}): 62.4(-73.1,197.8)\end{array}$ \\
\hline \multirow[t]{3}{*}{$\begin{array}{l}\text { Birth cohort study; } \\
n=100 \text { mother-infant pairs }\end{array}$} & & Birth length & $\begin{array}{l}\text { Adjusted mean difference in } \mathrm{cm} \text { for a 2-fold } \\
\text { increase in } \mathrm{PbB}(\mu \mathrm{g} / \mathrm{L}): 0.17(-0.56,0.91)\end{array}$ \\
\hline & & Abdominal diameter & $\begin{array}{l}\text { Adjusted mean difference in } \mathrm{cm} \text { for a 2-fold } \\
\text { increase in } \mathrm{PbB}(\mu \mathrm{g} / \mathrm{d}): 0.31(-0.52,1.15)\end{array}$ \\
\hline & & Cephalic diameter & $\begin{array}{l}\text { Adjusted mean difference in } \mathrm{cm} \text { for a 2-fold } \\
\text { increase in } \mathrm{PbB}(\mu \mathrm{g} / \mathrm{L}): 0.15(-0.21,0.51)\end{array}$ \\
\hline $\begin{array}{l}\text { González-Cossío et al. } 1997 \\
\text { Birth cohort study; } \\
\text { n=272 mother-infant pairs }\end{array}$ & 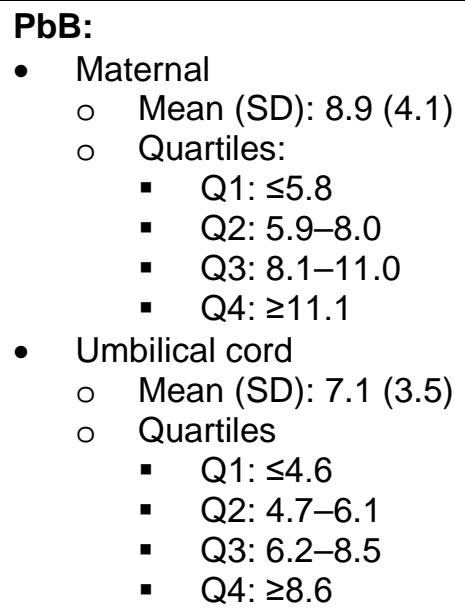 & Birth weight & $\begin{array}{l}\text { Regression coefficient: } \\
\text { - Maternal PbB for Q4: }-98.30 \text { (59.55); } p=0.100 \\
\text { - Umbilical cord PbB for Q4: }-41.74 \text { (64.04); } \\
\mathrm{p}=0.514\end{array}$ \\
\hline
\end{tabular}


2. HEALTH EFFECTS

\section{Table 2-41. Summary of Epidemiological Studies Evaluating Birth Outcomes Effects of Mean Blood Lead Concentration $(\mathrm{PbB}) \leq 10 \mu \mathrm{g} / \mathrm{dL}^{\mathrm{a}}$}

\begin{tabular}{l}
$\begin{array}{l}\text { Reference and study } \\
\text { population }^{\mathrm{b}}\end{array}$ \\
\hline Kim et al. 2017b \\
Prospective longitudinal study;
\end{tabular}

$\mathrm{n}=280$ mother-infant pairs

PbB $(\mu \mathrm{g} / \mathrm{dL})^{\mathrm{c}}$
PbB:
Umbilical cord, mean
- $\quad$ All: $1.31(0.06)$
- Boys: $1.39(0.09)$
- $\quad$ Girls: $1.21(0.07)$

- All: $1.31(0.06)$

- Girls: $1.21(0.07)$

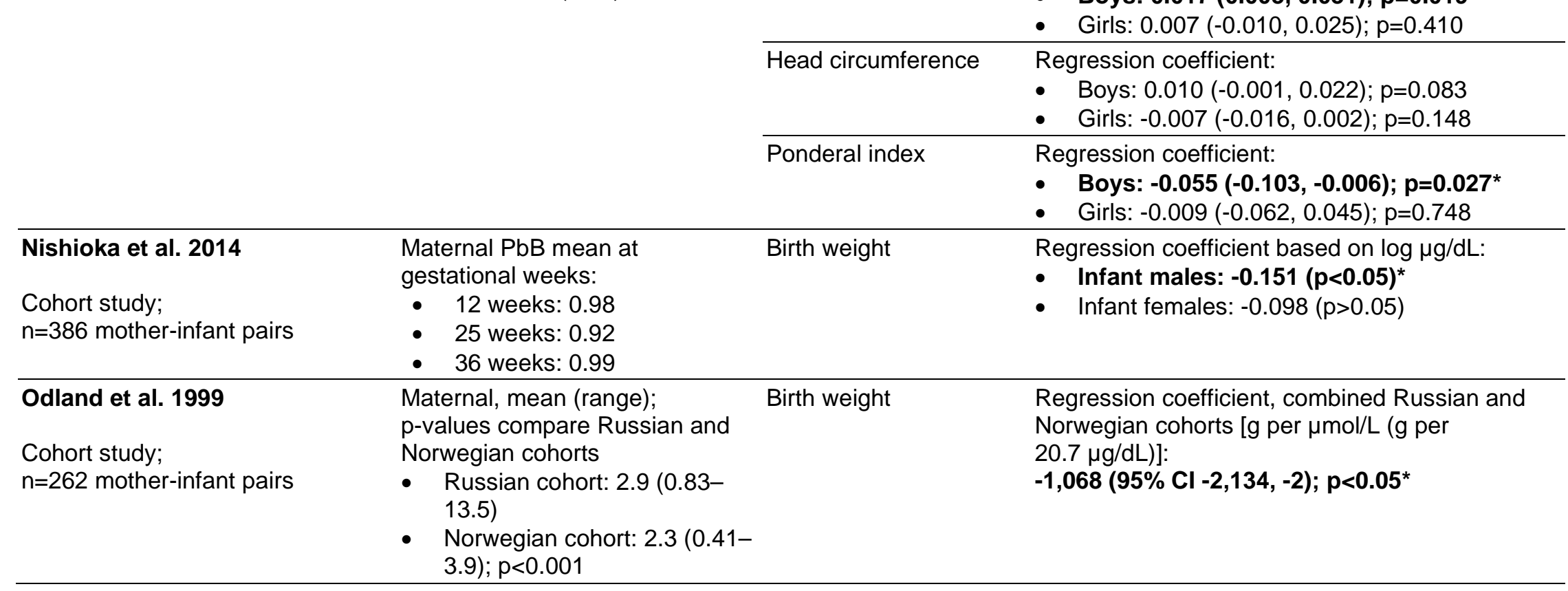


2. HEALTH EFFECTS

\section{Table 2-41. Summary of Epidemiological Studies Evaluating Birth Outcomes Effects of Mean Blood Lead Concentration $(\mathrm{PbB}) \leq 10 \mu \mathrm{g} / \mathrm{dL}^{\mathrm{a}}$}

\begin{tabular}{|c|c|c|c|}
\hline $\begin{array}{l}\text { Reference and study } \\
\text { population }^{\mathrm{b}}\end{array}$ & $\mathrm{PbB}(\mu \mathrm{g} / \mathrm{dL})^{\mathrm{c}}$ & Outcome evaluated & Result $^{d}$ \\
\hline \multirow{4}{*}{$\begin{array}{l}\text { Perkins et al. } 2014 \\
\text { Birth cohort study; } \\
n=829 \text { mother-infant pairs }\end{array}$} & \multirow{4}{*}{$\begin{array}{l}\text { Maternal RBC Pb concentration } \\
\text { ( } \mu \mathrm{g} / \mathrm{dL} \text { ) mean: } 1.22 \\
\text { Quartiles for RBC Pb; mean: } \\
\text { - } \quad \text { Q1: } 0.65 \\
\text { - } \quad \text { Q2: } 0.96 \\
\text { - } \quad \text { Q3: } 1.27 \\
\quad \text { Q4: } 2.02 \\
\text { Estimated maternal PbB mean: } \\
0.4\end{array}$} & Birth weight & $\begin{array}{l}\text { Linear regression } \beta \text { coefficient for } \mathrm{RBC}(\mu \mathrm{g} / \mathrm{dL}) \\
\text { Q4: }-47 \\
(-128,35) ; p \text {-trend: } 0.27\end{array}$ \\
\hline & & Birth length & $\begin{array}{l}\text { Linear regression } \beta \text { coefficient for } \mathrm{RBC}(\mu \mathrm{g} / \mathrm{dL}) \\
\text { Q4: }-0.15(-0.54,0.23) ; p \text {-trend: } 0.37\end{array}$ \\
\hline & & Head circumference & $\begin{array}{l}\text { Linear regression } \beta \text { coefficient for RBC }(\mu \mathrm{g} / \mathrm{dL}) \\
\text { Q4: }-0.08(-0.33,0.16) ; p \text {-trend: } 0.56\end{array}$ \\
\hline & & & \\
\hline Rabito et al. 2014 & \multirow{2}{*}{$\begin{array}{l}\text { Maternal } 2^{\text {nd }} \text { trimester } \mathrm{PbB} \text { mean: } \\
0.42 \\
\text { Maternal } 3^{\text {rd }} \text { trimester } \mathrm{PbB} \text { mean: } \\
0.45\end{array}$} & Birth weight & $\begin{array}{l}\text { Linear regression } \beta \text { coefficient, g per } \mu \mathrm{g} / \mathrm{dL} \\
\text { maternal: }\end{array}$ \\
\hline $\begin{array}{l}\text { Birth cohort study; } \\
\mathrm{n}=98 \text { mother-infant pairs }\end{array}$ & & & $\begin{array}{l}-\quad 2^{\text {nd }} \text { trimester: }-43.21(-88.6,2.18) ; p=0.06 \\
-\quad 3^{\text {rd }} \text { trimester: } \beta \text { not reported; } p=0.68 \\
\text { - } \quad \text { Delivery: } \beta \text { not reported; } p=0.83\end{array}$ \\
\hline Rodosthenous et al. 2017 & \multirow{2}{*}{ 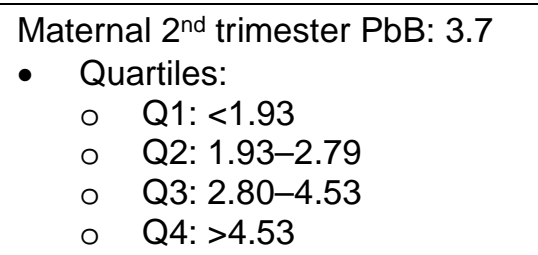 } & $\begin{array}{l}\text { Birthweight-for- } \\
\text { gestational age z-score }\end{array}$ & $\begin{array}{l}\text { Linear regression } \beta \text { for a doubling for } \\
\text { PbB: }-0.06(-0.13,0.003) ; p=0.06\end{array}$ \\
\hline $\begin{array}{l}\text { Prospective cohort study; } \\
n=944 \text { mother-infant pairs }\end{array}$ & & SGA & Logistic regression OR Q4: $1.62(0.99-2.65)$ \\
\hline Taylor et al. 2013, 2015 & Maternal PbB mean: 3.67 & Birth weight & $\begin{array}{l}\beta \text { coefficient (g per } \\
\mu \mathrm{g} / \mathrm{dL}):-13.23(-23.75,-2.70) ; p=0.014^{*}\end{array}$ \\
\hline \multirow[t]{2}{*}{$\begin{array}{l}\text { Longitudinal cohort study; } \\
n=4,285 \text { mother-infant pairs }\end{array}$} & \multirow[t]{2}{*}{$\begin{array}{l}\text { Population stratified by } \mathrm{PbB}<5.0 \\
\text { and } \geq 5.0\end{array}$} & Head circumference & $\begin{array}{l}\beta \text { coefficient }(\mathrm{cm} \text { per } \\
\mu \mathrm{g} / \mathrm{dL}):-0.04(-0.07,-0.06)^{\mathrm{e}} ; p=0.021^{*}\end{array}$ \\
\hline & & Crown-heel length & $\begin{array}{l}\beta \text { coefficient (cm per } \\
\mu \mathrm{g} / \mathrm{dL}):-0.05(-0.10,-0.00) ; p=0.034^{*}\end{array}$ \\
\hline
\end{tabular}


Table 2-41. Summary of Epidemiological Studies Evaluating Birth Outcomes Effects of Mean Blood Lead Concentration $(\mathrm{PbB}) \leq 10 \mu \mathrm{g} / \mathrm{dL}^{\mathrm{a}}$

\begin{tabular}{|c|c|c|c|}
\hline $\begin{array}{l}\text { Reference and study } \\
\text { population }^{\mathrm{b}}\end{array}$ & $\mathrm{PbB}(\mu \mathrm{g} / \mathrm{dL})^{\mathrm{c}}$ & Outcome evaluated & Result $^{d}$ \\
\hline Thomas et al. 2015 & $\begin{array}{l}\text { Maternal PbB median: } 0.59 \\
\text { Tertiles: }\end{array}$ & SGA & Adjusted RR for T3 $(95 \%$ Cl): $1.19(0.65,2.18)$ \\
\hline $\begin{array}{l}\text { Prospective cohort; } \\
n=1,835 \text { mother-infant pairs }\end{array}$ & $\begin{array}{l}-\mathrm{T} 1:<0.52 \\
-\quad \mathrm{T} 2: 0.52-1.04 \\
-\quad \mathrm{T} 3:>1.04-4.04\end{array}$ & & \\
\hline Wang et al. 2017b & \multirow{5}{*}{$\begin{array}{l}\text { Maternal serum Pb mean: } 1.50 \\
\text { Tertiles: } \\
\text { - T1: }<1.18 \\
\text { - T2: } 1.18-1.70 \\
\text { - T3: } \geq 1.71\end{array}$} & Birth weight & $\begin{array}{l}\text { Regression coefficient } \beta:-2.74(-5.17,-0.31) \\
p=0.03^{*}\end{array}$ \\
\hline \multirow[t]{4}{*}{$\begin{array}{l}\text { Prospective cohort study; } \\
n=3,125 \text { mother-infant pairs }\end{array}$} & & Birth length & $\begin{array}{l}\text { Regression coefficient } \beta:-0.013(-0.026,0.001) \\
p=0.06\end{array}$ \\
\hline & & Head circumference & $\begin{array}{l}\text { Regression coefficient } \beta:-0.008(-0.019,0.004) ; \\
p=0.18\end{array}$ \\
\hline & & Chest circumference & $\begin{array}{l}\text { Regression coefficient } \beta:-0.008(-0.018,0.002) \\
p=0.13\end{array}$ \\
\hline & & SGA & $\begin{array}{l}\text { OR T2: } 1.45(1.04,2.02) ; p=0.03^{*} \\
\text { - } \quad \text { OR T3: } 1.69(1.22,2.34) ; p=0.002^{*}\end{array}$ \\
\hline \multirow[t]{2}{*}{$\begin{array}{l}\text { Wang et al. 2017c } \\
\text { Cross-sectional study; } \\
n=1,009 \text { mother-infant pairs }\end{array}$} & \multirow[t]{2}{*}{$\begin{array}{l}\text { PbB: } \\
\text { Cord PbB, Gmean }(95 \% \mathrm{Cl}) \\
\text { All: } 4.07(3.98,4.17) \\
\text { Infant boys: } 4.07(3.89,4.17) \\
\text { Infant girls: } 4.17(3.98,4.36)\end{array}$} & Birth weight & $\begin{array}{l}\text { Regression coefficient } \beta(95 \%) \text {, per } 1 \text {-unit } \\
\text { increase in } \log _{10} \text {-transformed } \mathrm{PbB} \text { : } \\
\text { - } \quad \text { All: } 60.78(-66.30,187.85) ; p=0.35 \\
\text { - Boys: } \mathbf{1 8 2 . 3 2}\left(\mathbf{1 5 . 2 4 . 3 4 9 . 3 9 ) ; ~} \mathbf{p = 0 . 0 3 ^ { * }}\right. \\
\text { - } \quad \text { Girls: }-96.06(-289.23,97.10) ; p=0.33\end{array}$ \\
\hline & & Birth length & $\begin{array}{l}\text { Regression coefficient } \beta(95 \%) \text {, per } 1 \text {-unit } \\
\text { increase in } \log _{10} \text {-transformed PbB: } \\
\text { - } \quad \text { All: } 0.32(-0.18,0.82) ; p=0.21 \\
\text { - Boys: not reported } \\
\text { - Girls: } 0.30(-0.46,1.05) ; p=0.44\end{array}$ \\
\hline
\end{tabular}


Table 2-41. Summary of Epidemiological Studies Evaluating Birth Outcomes Effects of Mean Blood Lead Concentration $(\mathrm{PbB}) \leq 10 \mu \mathrm{g} / \mathrm{dL}^{\mathrm{a}}$

\section{Reference and study} population $^{\mathrm{b}}$
$\operatorname{PbB}(\mu \mathrm{g} / \mathrm{dL})^{\mathrm{c}}$

Outcome evaluated
Head circumference

Result $^{\mathrm{d}}$

Regression coefficient $\beta$ (95\%), per 1-unit increase in $\log _{10}$-transformed $\mathrm{PbB}$ :

- All: $-0.36(-0.78,0.06) ; p=0.09$

- Boys: $-0.50(-1.09,0.09) ; p=0.10$

- Girls: $-0.32(-0.91,0.27) ; p=0.29$

Ponderal index Regression coefficient $\beta(95 \%)$, per 1-unit increase in $\log _{10}$-transformed $\mathrm{PbB}$ :

- All: $-0.01(-0.10,0.09) ; p=0.94$

- Boys: $0.10(-0.03,0.23) ; p=0.12$

- Girls: -0.17 (-0.31, -0.02$) ; p=0.02$ *

Head circumference
Xie et al. 2013

Maternal PbB mean: 3.53

Birth weight

Birth length

$\beta$ coefficient (g per square root $\mu \mathrm{g} / \mathrm{dL}$ ): -148.99 (-286.33, -11.66); $p=0.03^{*}$

$\beta$ coefficient ( $\mathrm{cm}$ per square root $\mu \mathrm{g} / \mathrm{dL}):-0.46(-1.25,0.34) ; p=0.26$

$\mathrm{n}=252$ mother-infant pairs $\beta$ coefficients (cm per square root $\mu \mathrm{g} / \mathrm{dL})$ : $-0.37(-0.78,0.19) ; \mathrm{p}=0.24$ 
Table 2-41. Summary of Epidemiological Studies Evaluating Birth Outcomes Effects of Mean Blood Lead Concentration $(\mathrm{PbB}) \leq 10 \mu \mathrm{g} / \mathrm{dL}^{\mathrm{a}}$

\section{Reference and study}

population $^{\mathrm{b}}$

Zhu et al. 2010

Retrospective cohort study;

$\mathrm{n}=43,288$ mother-infant pairs

\begin{tabular}{ll} 
Outcome evaluated & Result $^{\mathrm{d}}$ \\
\hline Birth weight & $\beta$ coefficient g per $\mu \mathrm{g} / \mathrm{dL}(95 \% \mathrm{Cl}):$ \\
& O: reference \\
& $1:-27.4(-17.1,-37.8)^{*}$ \\
& $2:-38.8(-24.1,-53.4)^{\star}$ \\
& $3:-47.5(-29.6,-65.4)^{\star}$ \\
& $4:-54.8(-34.2,-75.5)^{*}$ \\
& $5:-61.3(-38.2,-84.4)^{*}$ \\
& $6:-67.2(-41.8,-92.5)^{*}$ \\
& $7:-72.5(-45.2,-99.9)^{*}$ \\
& $8:-77.6(-48.3,-106.8)^{*}$ \\
& $9:-82.3(-51.2,-113.3)^{*}$ \\
& $10:-86.7(-54.0,-119.4)^{*}$ \\
\hline SGA & Adjusted OR for Q4: $1.07(0.93,1.23)$
\end{tabular}

asee the Supporting Document for Epidemiological Studies for Lead, Table 13 for more detailed descriptions of studies.

${ }^{b}$ Participants had no known occupational exposure to $\mathrm{Pb}$.

cValues are for maternal PbB, unless otherwise specified.

${ }^{\mathrm{d}}$ Asterisk and bold indicate association with $\mathrm{Pb}$; unless otherwise specified, values in parenthesis are $95 \%$ Cls; $\mathrm{p}$-values $<0.05$ unless otherwise noted in the table.

eValues are reported; the value for the $\beta$ coefficient is outside of the $95 \% \mathrm{Cl}$.

$\mathrm{Cl}=$ confidence interval; Gmean = geometric mean; $\mathrm{OR}=$ odds ratio; $\mathrm{Pb}=$ lead; $\mathrm{RBC}=$ red blood cell; $\mathrm{RR}=$ relative risk; $\mathrm{SD}=\mathrm{standard}$ deviation; $\mathrm{SE}=\mathrm{standard}$ error; SGA = small for gestational age 
Effect at Blood Pb Levels $\leq 10 \mu \mathrm{g} / \mathrm{dL}$. Epidemiology studies have reported developmental effects, including birth outcomes, birth defects, anthropometric measures in children, and delayed onset of puberty, at mean $\mathrm{PbB} \leq 10 \mu \mathrm{g} / \mathrm{dL}$. Study details are provided in Supporting Document for Epidemiological Studies for Lead, Table 13. Results of studies on associations between PbB and adverse effects on birth outcomes and anthropometric measures are mixed when compared across studies. Delayed onset of puberty in females has been corroborated in several studies. Fewer studies are available regarding effects of $\mathrm{Pb}$ on onset of puberty in males, with equivocal results. Exposure to $\mathrm{Pb}$ has not been shown to cause birth defects in humans. Neural tube defects have not been associated with $\mathrm{Pb}$ exposure and findings of a single study showing minor anomalies have not been corroborated.

Birth outcomes. An overview of results of studies that evaluated associations between Pb exposure and birth outcomes (infant weight, height or crown-heel length, small for gestation age [SGA], head circumference, and ponderal index) at maternal $\mathrm{PbB} \leq 10 \mu \mathrm{g} / \mathrm{dL}$ is shown in Table 2-40, with more detailed results in Table 2-41. Studies include two prospective studies (Bornschein et al. 1989; Thomas et al. 2015), several studies of large populations ( $n=829-43,288)$ (Al-Saleh et al. 2014; Perkins et al. 2014; Rodosthenouse et al. 2017; Taylor et al. 2015; Thomas et al. 2015; Wang et al. 2017b, 2017b; Zhu et al. 2010), and cohort and case-control studies of smaller ( $n=98-386)$ populations (Bloom et al. 2015; GarciaEsquinas et al. 2014; González-Cossío et al. 1997; Kim et al. 2017b; Nishioka et al. 2014; Rabito et al. 2014). As shown in Table 2-41, results of most studies show either decreases or no change in birth outcomes. Some positive associations between $\mathrm{PbB}$ and birth outcomes have been reported. A large cross-sectional study $(\mathrm{n}=1,009)$ reported a positive association between umbilical cord $\mathrm{PbB}$ (mean: $4.07 \mathrm{~g} / \mathrm{dL}$ ) and birth weight in male infants, but no change for female infants (PbB mean: $4.17 \mu \mathrm{g} / \mathrm{dL}$ ) (Wang et al. 2017c). A longitudinal study showed a positive association between umbilical cord PbB in infant boys (mean: $1.39 \mu \mathrm{g} / \mathrm{dL}$ ) and birth length, but an inverse association for ponderal index (calculated relationship between body mass and height); no associations were observed for infant girls ( $\mathrm{PbB}$ mean: $1.21 \mu \mathrm{g} / \mathrm{dL}$ ) (Kim et al. 2017b). In a small ( $\mathrm{n}=202)$ prospective study, Bornschein et al. (1989) reported associations between maternal $\mathrm{PbB}$ (mean $7.5 \mu \mathrm{g} / \mathrm{dL}$ ) and decreased birth weight and length. The size of the effect of $\mathrm{PbB}$ varied with maternal age $(\mathrm{p}<0.007)$, with a $58 \mathrm{~g}$ per $\ln \mathrm{PbB}$ decrease for pregnancies at age 18 years and a $601 \mathrm{~g}$ decrease per $\ln \mathrm{PbB}(\mu \mathrm{g} / \mathrm{dL})$ for pregnancies at age 30 years. In the complete birth cohort from this study, which included mothers who declined participation in the infant follow-up ( $n=861$ ), the decline in birth weight was -114 g per $\ln$ PbB. Results of the largest cohort study, a retrospective study of $>43,000$ participants (mean PbB: $2.1 \mu \mathrm{g} / \mathrm{dL}$ ), showed an inverse association between PbB and birth weight (Zhu et al. 2010). The best fitting model was a linear change in birth weight with square root of PbB (Figure 2-7). The model predicts a $34 \mathrm{~g}$ decrease in birth weight for an 
increase in $\mathrm{PbB}$ from 1 to $5 \mu \mathrm{g} / \mathrm{dL}$ and a $59 \mathrm{~g}$ decrease for an increase in $\mathrm{PbB}$ from 1 to $10 \mu \mathrm{g} / \mathrm{dL}$ (adjusted for confounders).

\section{Figure 2-7. Relationship Between Blood Lead Concentration ( $\mathrm{PbB}$ ) and Birth Weight at $\mathrm{PbB} \leq 10 \mu \mathrm{g} / \mathrm{dL}$}

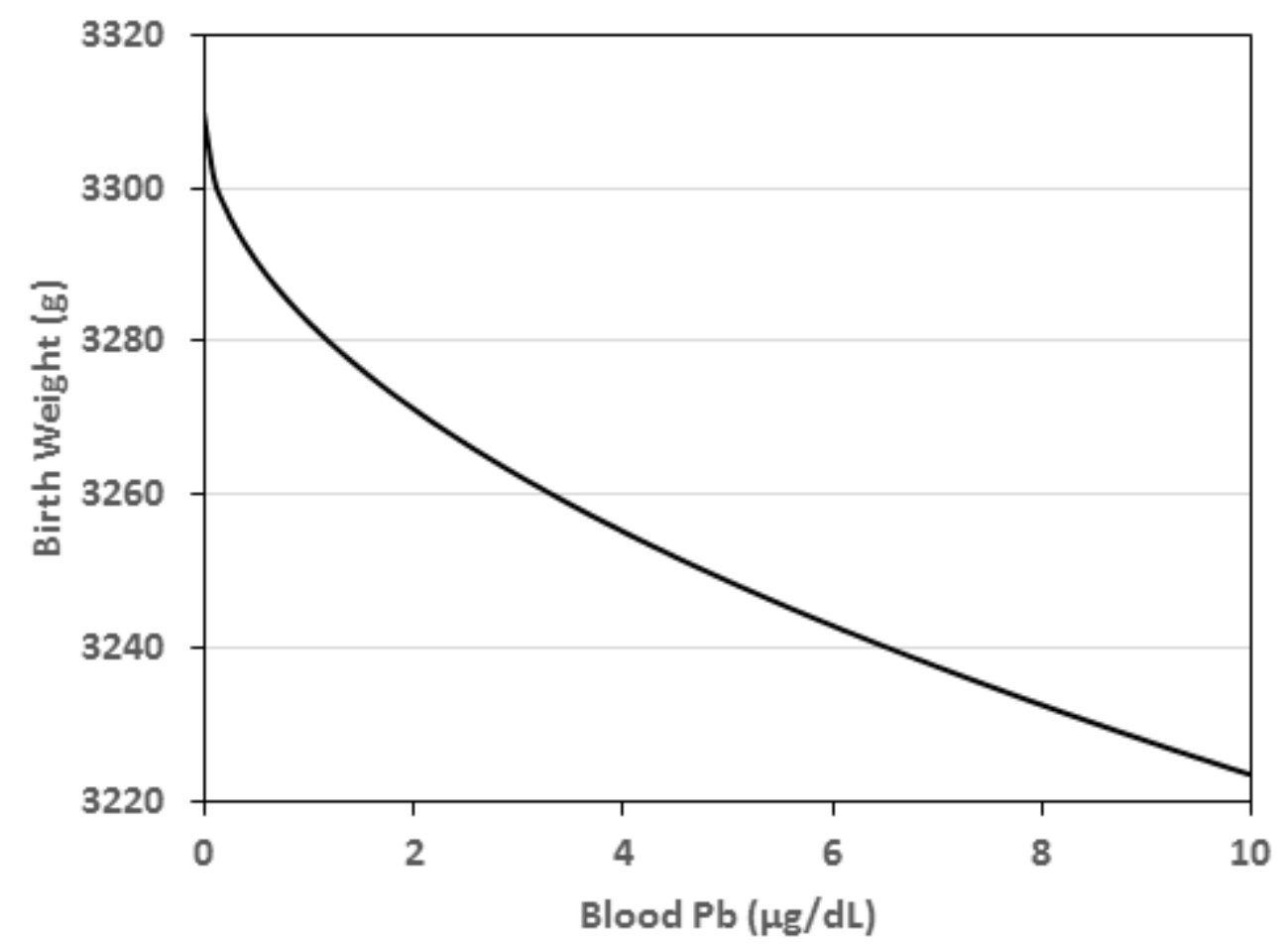

Source: Zhu et al. 2010

Results of a longitudinal cohort study of 4,285 mother-infant pairs (maternal PbB mean: $2.1 \mu \mathrm{g} / \mathrm{dL}$; range 0.42-19.14) showed inverse associations between birth weight, crown-heel length, and head circumference for participants with $\mathrm{PbB} \geq 5 \mu \mathrm{g} / \mathrm{dL}$ compared to $\mathrm{PbB}<5 \mu \mathrm{g} / \mathrm{dL}$ (Taylor et al. 2015). A prospective cohort study of 3,125 mother infant pairs observed an inverse association between maternal serum $\mathrm{Pb}$ (mean: $1.50 \mu \mathrm{g} / \mathrm{dL}$ ) and birth weight and SGA (Wang et al. 2017b). Other smaller cohort studies also showed associations between maternal $\mathrm{PbB} \leq 10 \mu \mathrm{g} / \mathrm{dL}$ and decreased birth weight (Nisioka et al. 2014; Odland et al. 1999). In contrast, other studies, including a prospective study and cohort studies of large populations, did not find associations between $\mathrm{PbB}$ and birth outcome measures. A prospective study of 1,835 mother-infant pairs did not find an association between PbB and SGA, with PbB data stratified by tertiles (range for highest tertile: 1.04-4.04 $\mu \mathrm{g} / \mathrm{dL}$ ) (Thomas et al. 2015). Similarly, no associations between maternal $\mathrm{PbB}$ and decreased birth weight, length, or head circumference were 
observed in a cohort study of 829 participants (estimated PbB mean of $0.4 \mu \mathrm{g} / \mathrm{dL}$ ) (Perkins et al. 2014), or in a cross-sectional study of 1,578 participants (Al-Saleh et al. 2014). Smaller cohort studies also report no associations between PbB and adverse birth outcome measures (Bloom et al. 2015; Garcia-Esquinas et al. 2014; González-Cossío et al. 1997; Rabito et al. 2014). Equivocal findings for birth outcomes in studies examining effects at maternal $\mathrm{PbB} \leq 10 \mu \mathrm{g} / \mathrm{dL}$ are not surprising, given that prospective studies at maternal $\mathrm{PbB}>10 \mu \mathrm{g} / \mathrm{dL}$ also have reported conflicting results for adverse effects on birth outcomes (Factor-Litvak et al. 1991; Hernandez-Avila et al. 2002; McMichael et al. 1986; Murphy et al. 1990). For example, two prospective studies found no associations between $\mathrm{PbB}$ and birth weight in birth cohorts that had mean maternal PbBs >10 $\mu \mathrm{g} / \mathrm{dL}$ (Factor-Litvak et al. 1991; McMichael et al. 1986).

Birth defects. Few studies have evaluated associations between in utero exposure to $\mathrm{Pb}$ and birth defects. Details of studies evaluating $\mathrm{PbB} \leq 10 \mu \mathrm{g} / \mathrm{dL}$ are provided in the Supporting Document for Epidemiological Studies for Lead, Table 13. No association was observed between PbB and neural tube defects in a case-control study ( $\mathrm{n}=409$ ) with mean maternal PbB of $2.5 \mu \mathrm{g} / \mathrm{dL}$ (Brender et al. 2006). Other epidemiological studies that have reported associations between $\mathrm{Pb}$ in exposure media (e.g., water, soil) and neural tube defects are limited by the lack of PbB measurement (Bound et al. 1997; Huang et al. 2011; Irgens et al. 1998). An early cross-sectional study of birth outcomes examined associations between $\mathrm{PbB}$ and congenital anomalies using hospital records on 5,183 deliveries in Boston, Massachusetts (Needleman et al. 1984). The RR of an anomaly increased with increasing cord PbB; the RR (relative to PbB $0.7 \mu \mathrm{g} / \mathrm{dL}$ ) was 1.87 (95\% CI 1.44, 2.42) for PbB of $6.3 \mu \mathrm{g} / \mathrm{dL}$ and increased to 2.39 (95\% CI 1.66, 3.43) at $15 \mu \mathrm{g} / \mathrm{dL}$ and 2.73 (95\% CI 1.80, 4.16) at $24 \mu \mathrm{g} / \mathrm{dL}$. The anomalies were considered to be minor (hemangiomas, lymphangiomas, hydrocele, minor skin anomalies, undescended testicle) and no specific anomaly was associated with $\mathrm{PbB}$. Limitations of this study are that it was a cross-sectional study of a convenience sample with outcomes obtained from hospital records.

Associations between $\mathrm{PbB}$ and congenital anomalies have not been corroborated. A case-control study of 97 cases and 201 controls did not find an increased risk for congenital heart defects (Liu et al. 2018a). For the highest umbilical cord $\mathrm{PbB}$ tertile $(\geq 0.826 \mu \mathrm{g} / \mathrm{dL})$, the OR $(95 \% \mathrm{CI})$ for congenital heart defects was $1.67(0.88,3.17)$.

Anthropometric measures in children. An overview of results of studies evaluating associations between $\mathrm{Pb}$ exposure and growth of infants and children (aged 0.5-15 years) at maternal and/or offspring $\mathrm{PbB}$ $\leq 10 \mu \mathrm{g} / \mathrm{dL}$ is shown in Table 2-42, with more detailed results in Table 2-43. Studies include five prospective studies (Dallaire et al. 2014; Deierlein et al. 2019; Lamb et al. 2008 Kim et al. 2017b; Renzetti et al. 2017), cross-sectional studies of large ( $\mathrm{n}=899-1,050)$ populations (Afeiche et al. 2011; 
Hong et al. 2014; Ignasiak et al. 2006), and several smaller ( $n=108-729)$ cohort and cross-sectional studies (Alvarez-Ortega et al. 2019; Hauser et al. 2008; Little et al. 2009; Min et al. 2008b; OliveroVerbel et al. 2007; Raihan et al. 2018; Schell et al. 2009; Yang et al. 2013a). Most studies report inverse associations between $\mathrm{Pb}$ exposure and height, with mixed results for weight and BMI (Table 2-42). In a prospective longitudinal study of girls ( $\mathrm{n}=692$; mean $\mathrm{PbB}: 1.16 \mu \mathrm{g} / \mathrm{dL}$ ), height, BMI, waist circumference, and percent body fat were decreased in participants with $\mathrm{PbB} \geq 1 \mu \mathrm{g} / \mathrm{dL}$, compared to participants with $\mathrm{PbB}<1 \mu \mathrm{g} / \mathrm{dL}$; decreases were observed at yearly assessments at ages 7-14 years (Deierlein et al. 2019). The Renzetti et al. (2017) prospective study ( $\mathrm{n}=513$ mothers) reported inverse associations between $3^{\text {rd }}$ pregnancy trimester maternal PbB (mean: $3.1 \mu \mathrm{g} / \mathrm{dL}$ ) and weight-for-age and height-for-age, but no associations for BMI or percentage body fat. No associations were observed between $2^{\text {nd }}$ trimester $\mathrm{PbB}$ or cord $\mathrm{PbB}$. In contrast, a prospective study of 280 children (18-27 months) observed positive associations between umbilical cord PbB (mean: $1.31 \mu \mathrm{g} / \mathrm{dL}$ ) and weight and BMI, but not height; no associations were observed at 18 or 27 months (Kim et al. 2017b). A small $(n=290)$ prospective study showed an association between cord $\mathrm{PbB}$ (mean $4.8 \mu \mathrm{g} / \mathrm{dL}$ ) and small decreases in height and head circumference, but not for weight or BMI (Dallaire et al. 2014). Similarly, Lamb et al. (2008) did not find an association between maternal $\mathrm{PbB}$ and height or BMI at maternal PbB means of 5.60-20.56 $\mu \mathrm{g} / \mathrm{dL}$ (means for different geographic locations). In contrast, results of large case-control studies showed inverse associations between maternal bone $\mathrm{Pb}$ and weight (Afeiche et al. 2011), maternal $\mathrm{PbB}$ and weight and height (Hong et al. 2014), and child PbB and several growth measures, including weight, height, and BMI (Ignasiak et al. 2006). The largest inverse association for decreased weight was observed for maternal bone $\mathrm{Pb}$ in females assessed at 2-5 years of age; the mean $\mathrm{PbB}$ in children was $3.8 \mu \mathrm{g} / \mathrm{dL}$ (Afeiche et al. 2011). At the 5-year assessment, body weight in females was decreased by approximately $172 \mathrm{~g}$ for each 1-SD increase in maternal bone $\mathrm{Pb}$. Smaller case-control and cohort studies reported consistent inverse associations between $\mathrm{PbB}$ and height, with equivocal findings for weight, and no associations for BMI.

Table 2-42. Overview of Decreased Anthropometric Measures in Children at Blood Lead Concentration (PbB) $\leq 10 \mu \mathrm{g} / \mathrm{dL}$

\begin{tabular}{lllll}
\hline & \multirow{2}{*}{$\begin{array}{l}\text { Age at time of } \\
\text { Reference }\end{array}$} & \multicolumn{3}{c}{ Anthropometric measurements } \\
\cline { 2 - 5 } assessment (years) & Weight & Height & BMI \\
\hline Afeiche et al. 2011 & $1-5$ & $\downarrow$ (F); 0 (M) & - & - \\
\hline Alvarez-Ortega et al. 2019 & $5-16$ & $\downarrow$ (F); 0 (M) & $\downarrow$ (F); 0 (M) & $\downarrow$ (F); 0 (M) \\
\cline { 2 - 5 } & $5-11$ & 0 & 0 & 0 \\
\cline { 2 - 5 } & $12-16$ & $\downarrow$ & $\downarrow$ & $\downarrow$ \\
\hline Dallaire et al. 2014 & $8-14$ & 0 & $\downarrow$ & $\downarrow$ \\
\hline Deierlein et al. 2019 & $7-14$ & $\downarrow$ & $\downarrow$ & \\
\hline
\end{tabular}


Table 2-42. Overview of Decreased Anthropometric Measures in Children at Blood Lead Concentration $(\mathrm{PbB}) \leq 10 \mu \mathrm{g} / \mathrm{dL}$

\begin{tabular}{|c|c|c|c|c|}
\hline \multirow[b]{2}{*}{ Reference } & \multirow{2}{*}{$\begin{array}{l}\text { Age at time of } \\
\text { assessment (years) }\end{array}$} & \multicolumn{3}{|c|}{ Anthropometric measurements } \\
\hline & & Weight & Height & $\mathrm{BMI}$ \\
\hline Hauser et al. 2008 & $8-9$ & 0 & $\downarrow$ & 0 \\
\hline Hong et al. 2014 & $0.5-2$ & $\downarrow$ & $\downarrow$ & - \\
\hline Ignasiak et al. 2006 & $7-15$ & $\downarrow(\mathrm{F}) ; 0$ (M) & $\downarrow(\mathrm{F}) ; 0(\mathrm{M})$ & $\downarrow$ \\
\hline Kim et al. 2017b & 2.25 & 0 & 0 & 0 \\
\hline Lamb et al. 2008 & $1-10$ & 0 & 0 & 0 \\
\hline Little et al. 2009 & $2-12$ & $\downarrow$ & - & - \\
\hline Min et al. 2008b & $5-13$ & 0 & $\downarrow$ & - \\
\hline Olivero-Verbel et al. 2007 & $5-9$ & 0 & $\downarrow$ & - \\
\hline Raihan et al. 2018 & $<2$ & $\downarrow^{\mathrm{a}}$ & $\downarrow^{\mathrm{a}}$ & 0 \\
\hline Renzetti et al. 2017 & $4-6$ & $\downarrow$ & $\downarrow$ & - \\
\hline Schell et al. 2009 & $0.5-1$ & 0 & $\downarrow$ & - \\
\hline Yang et al. $2013 a$ & 3-9 & $\downarrow$ & $\downarrow$ & 0 \\
\hline
\end{tabular}

aAssessments were underweight (defined as weight-for-age z-score <-2) and "stunting" (defined as length-for-age z-score <-2).

$\downarrow=$ decrease in outcome measure; $0=$ no effect on outcome measure; $-=$ not assessed; BMI $=$ body mass index; $\mathrm{F}=$ females; $\mathrm{M}=$ males

Delayed puberty. Results of studies that evaluated associations between $\mathrm{Pb}$ exposure and sexual maturation in boys and girls at child $\mathrm{PbB} \leq 10 \mu \mathrm{g} / \mathrm{dL}$ are summarized in Table 2-44. In girls, delayed onset of puberty, as measured by breast development, pubic hair development, and attainment of menarche, has been corroborated in multiple cross-sectional studies (Den Hond et al. 2011; Denham et al. 2005; Gollenberg et al. 2010; Naicker et al. 2010; Selevan et al. 2003; Wu et al. 2003b). Mean PbB in these studies ranged from 0.49 to $4.9 \mu \mathrm{g} / \mathrm{dL}$. Delays in the predicted attainment of menarche ranged from 3.6 to 10.6 months (Denham et al. 2005; Selevan et al. 2003). Fewer studies examining associations between $\mathrm{Pb}$ exposure and sexual maturation in boys at child $\mathrm{PbB} \leq 10 \mu \mathrm{g} / \mathrm{dL}$ are available. Results of these studies are equivocal. Delayed sexual maturation (time to onset to puberty and sexual maturity), measured by genitalia development, testicular volume, and pubic hair development, was observed in three cross-sectional studies of the same study population of $481-489$ boys; the median child $\mathrm{PbB}$ was $3 \mu \mathrm{g} / \mathrm{dL}$ at the time of study enrollment (Hauser et al. 2008; Williams et al. 2010, 2019). However, no association between $\mathrm{PbB}$ and the onset of puberty was observed in a cross-sectional study of 887 boys with a median $\mathrm{PbB}$ of $2.5 \mu \mathrm{g} / \mathrm{dL}$ (Den Hond et al. 2011). 
Table 2-43. Summary of Epidemiological Studies Evaluating Anthropometric Measurements in Infants and Children with Mean Blood Lead Concentration $(\mathrm{PbB}) \leq 10 \mu \mathrm{g} / \mathrm{dL}^{\mathrm{a}}$

\begin{tabular}{|c|c|c|c|}
\hline $\begin{array}{l}\text { Reference and study } \\
\text { population }^{\text {b }}\end{array}$ & $\mathrm{PbB}(\mu \mathrm{g} / \mathrm{dL})^{\mathrm{c}}$ & Outcome evaluated & Result $^{d}$ \\
\hline \multirow[t]{2}{*}{$\begin{array}{l}\text { Afeiche et al. } 2011 \\
\text { Cross-sectional study; } \\
n=999 \text { mother-child pairs }\end{array}$} & $\begin{array}{ll} & \text { Child PbB mean: } 3.8 \\
\text { - } & \text { Maternal bone } \mathrm{Pb} \text { (patella) } \\
& \text { mean }(\mu \mathrm{g} / \mathrm{g}): 10.4\end{array}$ & Weight (females) & $\begin{array}{l}\text { Associations between a 1-SD increase in } \\
\text { maternal bone } \mathrm{Pb}(\mu \mathrm{g} / \mathrm{g}) \text { and child weight }(\mathrm{g}) \text { for } \\
\text { children aged: } \\
\text { - } 12 \text { months: }-70.9(-147.9,6.0) \\
\text { - } 24 \text { months: }-96.1(-170.4,-21.8)^{*} \\
\text { - } 36 \text { months: }-121.3(-200.0,-42.6)^{*} \\
\text { - } 48 \text { months: }-146.4(-235.5,-57.4)^{*} \\
\text { - } 60 \text { months: }-171.6(-275.2,-68.0)^{*}\end{array}$ \\
\hline & & Weight (males) & $\begin{array}{l}\text { Associations between a 1-SD increase in } \\
\text { maternal bone } \mathrm{Pb}(\mu \mathrm{g} / \mathrm{g}) \text { and child weight }(\mathrm{g}) \text { for } \\
\text { children aged: } \\
\text { - } 12 \text { months: } 29.4(-42.1,100.8) \\
\text { - } 24 \text { months: } 27.8(-43.5,99.1) \\
\text { - } \quad 36 \text { months: } 7.9(-67.3,83.1) \\
\text { - } \quad 48 \text { months: }-13.6(-97.9,70.8) \\
\text { - } 60 \text { months: }-35.0(-132.4,62.3)\end{array}$ \\
\hline \multirow[t]{2}{*}{$\begin{array}{l}\text { Alvarez-Ortega et al. } 2019 \\
\text { Cross-sectional study; } \\
n=554 \text { children (ages 5-16 years) }\end{array}$} & $\begin{array}{l}\text { Mean (SE): } 3.5(0.2) \\
\text { Median: } 1.9 \\
\text { Range: } 0.1-50.1\end{array}$ & Weight & $\begin{array}{l}\text { Spearman correlations: } \\
\text { - All participants: }-0.152 ; p<0.001^{*} \\
-\quad \text { Females: }-0.226 ; p<0.001^{*} \\
\text { - Males: }-0.056 ; p=0.380 \\
\text { - Age 5-11 years: }-0.069 ; p=0.010^{*} \\
\text { - } \text { Age } 12-16 \text { years: }-0.385 ; p<0.001^{*}\end{array}$ \\
\hline & & Height & $\begin{array}{l}\text { Spearman correlations: } \\
\text { - All participants: }-\mathbf{0 . 1 0 1 ;} p=0.019^{*} \\
\text { - Females: }-\mathbf{0 . 1 5 3} ; \mathrm{p}=\mathbf{0 . 0 0 9 ^ { * }} \\
\text { - Males: }-0.037 ; p=0.567 \\
\text { - Age } 5-11 \text { years: }-0.137 ; p=0.418 \\
\text { - Age } 12-16 \text { years: }-\mathbf{0 . 2 0 6} ; p=0.009^{*}\end{array}$ \\
\hline
\end{tabular}


Table 2-43. Summary of Epidemiological Studies Evaluating Anthropometric Measurements in Infants and Children with Mean Blood Lead Concentration $(\mathrm{PbB}) \leq 10 \mu \mathrm{g} / \mathrm{dL}^{\mathrm{a}}$

Reference and study
population $^{b}$
$\operatorname{PbB}(\mu \mathrm{g} / \mathrm{dL})^{\mathrm{c}}$

Outcome evaluated
BMI

earman correlations:

- All participants: $-0.172 ; p<0.001^{*}$

- Females: $-0.273 ; p<0.001^{*}$

- Males: $-0.040 ; p=0.536$

- Age $5-11$ years: $-0.056 ; p=0.295$

- Age 12-16 years: $-0.384 ; p<0.001$ *

\section{Dallaire et al. 2014}

Prospective cohort study; $\mathrm{n}=290$ children (aged 8-14 years)
- $\quad$ Cord PbB mean: 4.8
- Child PbB mean: 2.7

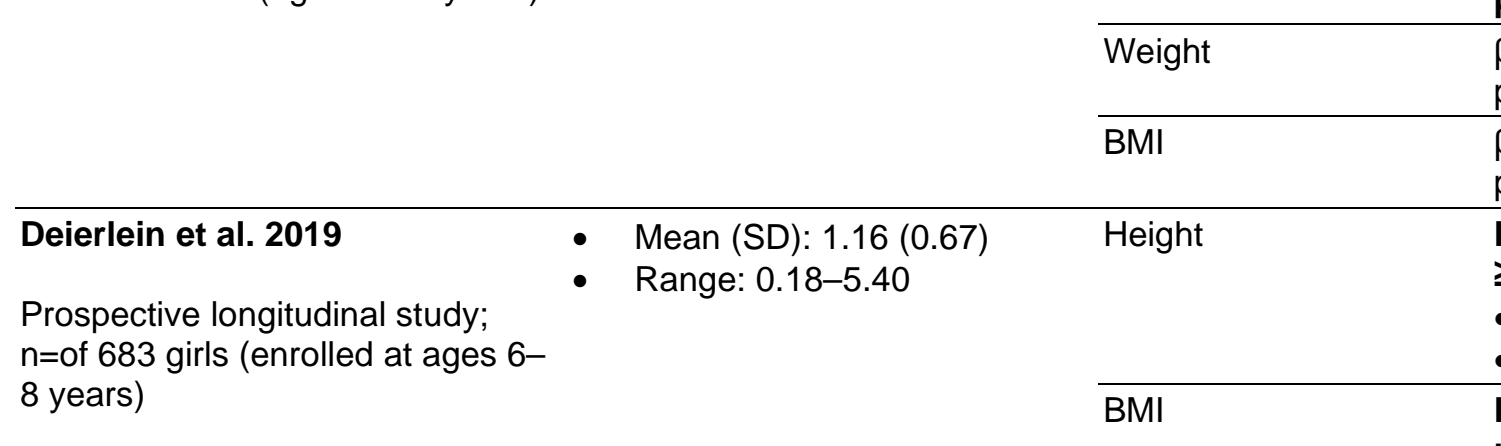

\begin{tabular}{|c|c|}
\hline & $\begin{array}{l}\mu g / d L \text { compared to }<1 \mu g / d L: \\
-\quad \text { Age } 7:-0.7(-1.2,-0.2) ; p=0.005^{\star} \\
-\quad \text { Age 14: }-0.8(-1.5,-0.02) ; p=0.05^{\star}\end{array}$ \\
\hline Waist circumference & $\begin{array}{l}\text { Predicted mean differences }(\mathrm{cm}) \text { for } \mathrm{PbB} \\
\geq 1 \mu \mathrm{g} / \mathrm{dL} \text { compared to }<1 \mu \mathrm{g} / \mathrm{dL} \text { : } \\
\text { - Age } 7:-2.2(-3.8,-0.6) ; \mathrm{p}=0.01^{*} \\
\text { - Age 14: }-2.9(-4.8,-0.9) ; \mathrm{p}=0.005^{\star}\end{array}$ \\
\hline Body fat & $\begin{array}{l}\text { Predicted mean differences (\%) for } \mathrm{PbB} \\
\geq 1 \mathrm{\mu g} / \mathrm{dL} \text { compared to }<1 \mu \mathrm{g} / \mathrm{dL} \text { : } \\
\text { - Age 7: }-1.8(-3.2,-0.4) ; \mathrm{p}=0.01^{*} \\
\text { - Age 14: }-1.7(-3.1,-0.4) ; \mathrm{p}=0.01^{*}\end{array}$ \\
\hline
\end{tabular}


Table 2-43. Summary of Epidemiological Studies Evaluating Anthropometric Measurements in Infants and Children with Mean Blood Lead Concentration $(\mathrm{PbB}) \leq 10 \mu \mathrm{g} / \mathrm{dL}^{\mathrm{a}}$

\begin{tabular}{|c|c|c|c|}
\hline $\begin{array}{l}\text { Reference and study } \\
\text { population }^{\mathrm{b}}\end{array}$ & $\mathrm{PbB}(\mu \mathrm{g} / \mathrm{dL})^{\mathrm{c}}$ & Outcome evaluated & Result $^{d}$ \\
\hline Hauser et al. 2008 & Child PbB, mean: 3 & Height & $\begin{array}{l}\text { Regression coefficient (cm per } \\
\mu \mathrm{g} / \mathrm{dL}):-1.439(-2.25,-0.63) ; p<0.001^{*}\end{array}$ \\
\hline \multirow[t]{2}{*}{$\begin{array}{l}\text { Cross-sectional study } \\
\mathrm{n}=489 \text { children (aged } 8-9 \text { years) }\end{array}$} & & Weight & $\begin{array}{l}\text { Regression coefficient }(\mathrm{kg} \text { per } \\
\mu \mathrm{g} / \mathrm{dL}):-0.761(-1.54,0.02) ; p=0.067\end{array}$ \\
\hline & & BMI & $\begin{array}{l}\text { Regression coefficient }\left(\mathrm{kg} / \mathrm{m}^{2} \text { per }\right. \\
\mu \mathrm{g} / \mathrm{dL}):-0.107(-0.44,0.23) ; p=0.53\end{array}$ \\
\hline Hong et al. 2014 & Maternal PbB mean: 1.25 & Weight & Weight z score: $-0.28(-0.48,-0.09) ; p<0.05^{\star}$ \\
\hline $\begin{array}{l}\text { Cross-sectional study; } \\
\text { n=1,150 infants (aged 6- } \\
24 \text { months) }\end{array}$ & & Height & Height z score: $-0.28(-0.49,-0.06) ; p<0.05^{*}$ \\
\hline \multirow[t]{4}{*}{$\begin{array}{l}\text { Ignasiak et al. } 2006 \\
\text { Cross-section study; } \\
\text { n=899 children (aged 7-15 years) }\end{array}$} & Child PbB mean: 7.7 & Weight & 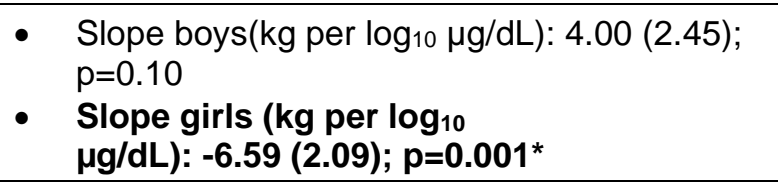 \\
\hline & & Height & 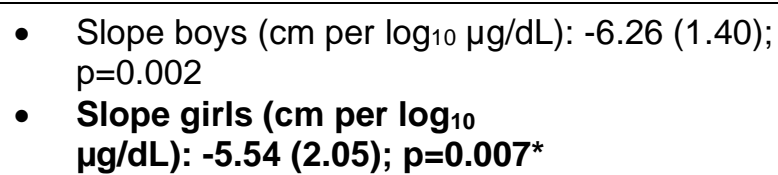 \\
\hline & & $\mathrm{BMI}$ & $\begin{array}{l}\text { - Slope boys }\left(\mathrm{kg} / \mathrm{m}^{2} \text { per } \log _{10}\right. \\
\mu \mathrm{g} / \mathrm{dL}):-0.39(0.82) ; p=N S \\
\text { Slope girls }\left(\mathbf{k g} / \mathrm{m}^{2} \text { per log }\right. \\
\text { } \mathrm{\mu g} / \mathrm{dL}):-\mathbf{1 . 8 6}(\mathbf{0 . 7 5}) ; \mathrm{p}=\mathbf{0 . 0 1 ^ { * }}\end{array}$ \\
\hline & & Trunk length & $\begin{array}{l}\text { Slope boys (cm per } \log _{10} \\
\mu \mathrm{g} / \mathrm{dL}):-2.21(0.97) ; \mathrm{p}=0.02^{*} \\
\text { Slope girls (cm per log } \mu \mathrm{g} / \mathrm{dL}):-1.47 \text { (1.00); } \\
\text { p=NS }\end{array}$ \\
\hline
\end{tabular}


Table 2-43. Summary of Epidemiological Studies Evaluating Anthropometric Measurements in Infants and Children with Mean Blood Lead Concentration $(\mathrm{PbB}) \leq 10 \mu \mathrm{g} / \mathrm{dL}^{\mathrm{a}}$

\begin{tabular}{|c|c|c|c|}
\hline $\begin{array}{l}\text { Reference and study } \\
\text { population }^{\mathrm{b}}\end{array}$ & $\mathrm{PbB}(\mu \mathrm{g} / \mathrm{dL})^{\mathrm{c}}$ & Outcome evaluated & Result $^{d}$ \\
\hline & & Leg length & $\begin{array}{l}\text { - Slope boys (cm per } \log _{10} \\
\mu g / d L):-4.05(1.27) ; p=0.002^{*} \\
\text { Slope girls (cm per } \log _{10} \\
\mu g / d L):-4.08(1.27) p=0.0001^{*}\end{array}$ \\
\hline & & Arm length & $\begin{array}{l}\text { Slope boys }(\mathrm{cm} \text { per } \mu \mathrm{g} / \mathrm{dL}):-3.20(0.97) \\
\mathrm{p}=0.0001^{*} \\
\text { Slope girls }\left(\mathrm{cm} \text { per } \log _{10}\right. \\
\mu \mathrm{g} / \mathrm{dL}):-2.61(0.98) ; p=0.008^{*}\end{array}$ \\
\hline & & Trunk-length ratio & $\begin{array}{l}\text { Slope boys }\left(\text { per } \log _{10} \mu \mathrm{g} / \mathrm{dL}\right): 0.71(0.34): \\
p=0.04^{*} \\
\text { Slope girls }\left(\text { per } \log _{10} \mu \mathrm{g} / \mathrm{dL}\right): 1.03(0.34) ; \\
p=0.003^{*}\end{array}$ \\
\hline $\begin{array}{l}\text { Lamb et al. } 2008 \\
\text { Population-based prospective } \\
\text { cohort; } n=309 \text { children (aged } 1-10 \text { ) } \\
\text { years }\end{array}$ & $\begin{array}{l}\text { Maternal PbB mean for } \\
\text { towns of: } \\
\text { - Pristina: } 5.60 \\
\text { - } \quad \text { Mitrovica: } 20.56\end{array}$ & Height/BMI & $\begin{array}{l}\text { Pristina }(\beta \text { coefficients per } \log \mu \mathrm{g} / \mathrm{dL}) \text { : } \\
\begin{array}{l}\text { - } \\
\text { - }\end{array} \text { Age } 1 \text { year: }-0.61(-2.24,1.03) \\
\text { Mitrovica }(\beta \text { coefs: }-0.09(-3.69,3.52) \\
\text { - } \quad \text { Age } 1 \text { year: }-0.30(-2.55,1.96) \\
\text { - } \quad \text { Age } 10 \text { years: }-2.87(-6.21,0.47)\end{array}$ \\
\hline Little et al. 2009 & \multirow{3}{*}{$\begin{array}{l}\text { Child PbB mean } \\
\text { - } 1980 \text { cohort: } 23.6 \\
\text { - } \quad 2002 \text { cohort: } 1.6 \\
\text { - } \quad \text { oooled cohort } \mathrm{PbB} \text { mean } \\
\quad \text { not reported }\end{array}$} & Height & $\begin{array}{l}\beta \text { coefficient (cm per10 } \mu \mathrm{g} / \mathrm{dL} \text { PbB decrease): } \\
2.1(1.9,2.3) ; p<0.0001^{*}\end{array}$ \\
\hline \multirow{2}{*}{$\begin{array}{l}\text { Cross-sectional study; } \\
\text { n=360 children (aged } 2-12 \text { years) }\end{array}$} & & Weight & $\begin{array}{l}\beta \text { coefficient (kg per } 10 \mu \mathrm{g} / \mathrm{dL} \text { PbB decrease): } \\
1.9(1.7,2.1) ; p<0.0001^{*}\end{array}$ \\
\hline & & $\mathrm{BMI}$ & $\begin{array}{l}\beta \text { coefficient }\left(\mathrm{kg} / \mathrm{m}^{2} \text { per } 10 \mu \mathrm{g} / \mathrm{dL} \text { PbB }\right. \\
\text { decrease }): 0.5(0.4,0.7) ; p<0.0001^{*}\end{array}$ \\
\hline
\end{tabular}


Table 2-43. Summary of Epidemiological Studies Evaluating Anthropometric Measurements in Infants and Children with Mean Blood Lead Concentration $(\mathrm{PbB}) \leq 10 \mu \mathrm{g} / \mathrm{dL}^{\mathrm{a}}$

\begin{tabular}{|c|c|c|c|}
\hline $\begin{array}{l}\text { Reference and study } \\
\text { population }^{\mathrm{b}}\end{array}$ & $\mathrm{PbB}(\mu \mathrm{g} / \mathrm{dL})^{\mathrm{c}}$ & Outcome evaluated & Result $^{\mathrm{d}}$ \\
\hline \multirow[t]{3}{*}{$\begin{array}{l}\text { Kim et al. 2017b } \\
\text { Prospective longitudinal study; } \\
n=280 \text { children (18-27 months) }\end{array}$} & \multirow[t]{3}{*}{$\begin{array}{l}\text { Umbilical cord, mean: } 1.31 \\
\text { - } \quad \text { All: } 1.31(0.06) \\
\text { - } \quad \text { Boys: } 1.39(0.09) \\
\text { - } \quad \text { Girls: } 1.21(0.07)\end{array}$} & Weight & $\begin{array}{l}\text { Regression coefficient } \beta \text { : } \\
\text { - } \quad 18 \text { months: } 0.897(-0.171,1.965) ; p=0.092 \\
\text { - } 24 \text { months: } 0.717(0.195,1.239) ; p=0.009^{*} \\
\text { - } \quad 27 \text { months: } 0.316(-0.345,0.977) ; p=0.333\end{array}$ \\
\hline & & Height & $\begin{array}{l}\text { Regression coefficient } \beta \text { : } \\
\text { - } \quad 18 \text { months: } 0.909(-0.222,2.040) ; p=0.101 \\
\text { - } \quad 24 \text { months: } 0.138(-0.530,0.806) ; p=0.675 \\
\text { - } \quad 27 \text { months: } 0.354(-0.497,1.205) ; p=0.394\end{array}$ \\
\hline & & BMI & $\begin{array}{l}\text { Regression coefficient } \beta \text { : } \\
\text { - } \quad 18 \text { months: } 0.157(-1.266,1.580) p=0.806 \\
\text { - } \quad 24 \text { months: } 0.695(0.077,1.313) ; p=0.029^{*} \\
\text { - } 27 \text { months: } 0.409(-0.398,1.216) ; p=0.300\end{array}$ \\
\hline \multirow{4}{*}{$\begin{array}{l}\text { Min et al. 2008b } \\
\text { Cross-sectional study; } \\
n=108 \text { children (aged 5-13 years) }\end{array}$} & \multirow[t]{4}{*}{ Child PbB mean: 2.4} & Height & $\begin{array}{l}\text { Regression coefficient } \mathrm{cm} \text { per } \mu \mathrm{g} / \mathrm{dL} \\
\text { (SE): }-1.449(0.639) ; p=0.026^{*}\end{array}$ \\
\hline & & Weight & $\begin{array}{l}\text { Regression coefficient } \mathrm{kg} \text { per } \mu \mathrm{g} / \mathrm{dL} \\
(\mathrm{SE}):-0.646(0.718) ; 0.370\end{array}$ \\
\hline & & BMI & $\begin{array}{l}\text { Regression coefficient } \mathrm{kg} / \mathrm{m}^{2} \text { per } \mu \mathrm{g} / \mathrm{dL} \\
\text { (SE): }-0.006(0.272) ; p=0.982\end{array}$ \\
\hline & & Arm length & $\begin{array}{l}\text { Regression coefficient cm per } \mu \mathrm{g} / \mathrm{dL} \\
\text { (SE): }-1.804(0.702) ; p=0.012^{*}\end{array}$ \\
\hline Olivero-Verbel et al. 2007 & \multirow[t]{2}{*}{ Child PbB mean: 5.53} & Height & Correlation coefficient: $-0.224 ; p=0.002^{*}$ \\
\hline $\begin{array}{l}\text { Cross-sectional study; } \\
\mathrm{n}=189 \text { children (aged } 5-9 \text { years) }\end{array}$ & & Weight & Correlation coefficient: $-0.126 ; p=0.087$ \\
\hline \multirow{3}{*}{$\begin{array}{l}\text { Raihan et al. } 2018 \\
\text { Cross-sectional study; } \\
n=729 \text { children ( }<2 \text { years of age) }\end{array}$} & \multirow{3}{*}{$\begin{array}{l}\text { Mean (SD): } 8.25(3.64) \\
95 \% \text { Cl: } 7.98,8.51 \\
\text { "Normal" PbB: }<5 \\
\text { "Elevated" PbB: } \geq 5\end{array}$} & Stunting ${ }^{\mathrm{e}}$ & $\begin{array}{l}\text { OR for } \mathrm{PbB} \geq 5 \mu \mathrm{g} / \mathrm{dL} \text { (compared to } \mathrm{PbB} \\
<5 \mu \mathrm{g} / \mathrm{dL}): 1.78(1.07,2.99) ; p=0.028^{*}\end{array}$ \\
\hline & & Wastinge & $\begin{array}{l}\text { OR for } \mathrm{PbB} \geq 5 \mu \mathrm{g} / \mathrm{dL} \text { (compared to } \mathrm{PbB} \\
<5 \mu \mathrm{g} / \mathrm{dL}): 1.18(0.64,2.19) ; \mathrm{p}=0.581\end{array}$ \\
\hline & & Underweight & $\begin{array}{l}\text { OR for } \mathrm{PbB} \geq 5 \mu \mathrm{g} / \mathrm{dL} \text { (compared to } \mathrm{PbB} \\
<5 \mu \mathrm{g} / \mathrm{dL}): 1.63(1.02,2.61) ; p=0.043^{*}\end{array}$ \\
\hline
\end{tabular}


Table 2-43. Summary of Epidemiological Studies Evaluating Anthropometric Measurements in Infants and Children with Mean Blood Lead Concentration $(\mathrm{PbB}) \leq 10 \mu \mathrm{g} / \mathrm{dL}^{\mathrm{a}}$

\begin{tabular}{|c|c|}
\hline $\begin{array}{l}\text { Reference and study } \\
\text { population }^{\mathrm{b}}\end{array}$ & $\mathrm{PbB}(\mu \mathrm{g} / \mathrm{dL})^{\mathrm{c}}$ \\
\hline Renzetti et al. 2017 & $\begin{array}{l}\text { Maternal PbB (Gmean) } \\
0 \quad 2^{\text {nd }} \text { trimester: } 3.0(0.8-17.8)\end{array}$ \\
\hline $\begin{array}{l}\text { Prospective study; } n=513 \text { mothers } \\
\text { (children assessed at ages } 4- \\
6 \text { years) }\end{array}$ & $\begin{array}{l}\text { - } \quad 3^{\text {rd }} \text { trimester: } 3.1(0.3-28.3) \\
\text { - } \quad \text { At delivery: } 3.5(0.7-21.9) \\
\quad \text { Umbilical cord: } 2.8(0.4-18.5)\end{array}$ \\
\hline
\end{tabular}

\begin{tabular}{|c|c|}
\hline Outcome evaluated & Result $^{d}$ \\
\hline BMI z-score & 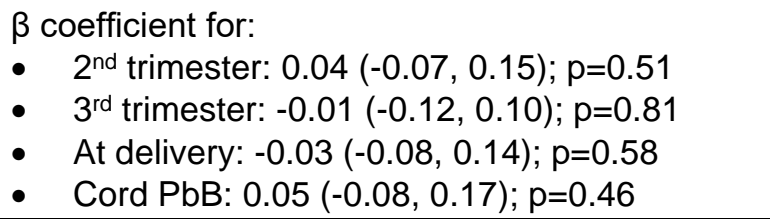 \\
\hline Percentage body fat & 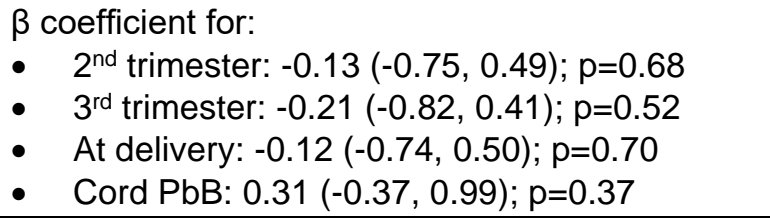 \\
\hline Weight-for-age z-score & 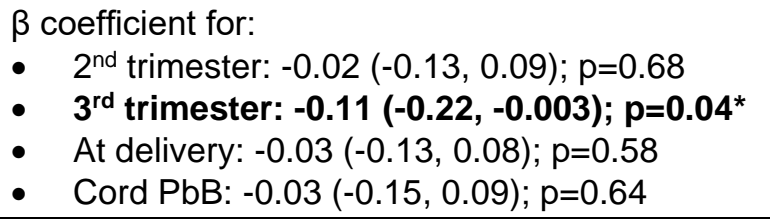 \\
\hline Height-for-age z-score & $\begin{array}{l}\beta \text { coefficient for: } \\
-\quad 2^{\text {nd }} \text { trimester: }-0.04(-0.13,0.04) ; p=0.32 \\
-\quad 3^{\text {rd }} \text { trimester: }-0.10(-0.19,-0.01) ; p=0.03^{*} \\
-\quad \text { At delivery: }-0.04(-0.13,0.05) ; p=0.39 \\
-\quad \text { Cord PbB: }-0.04(-0.14,0.06) ; p=0.39\end{array}$ \\
\hline Length & 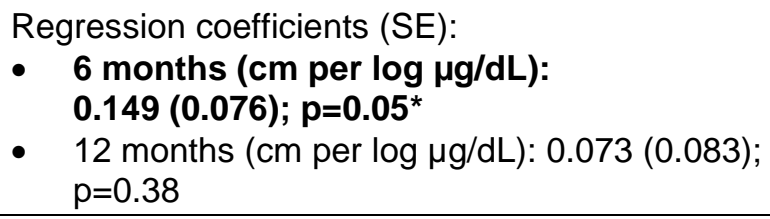 \\
\hline Weight-for-age & 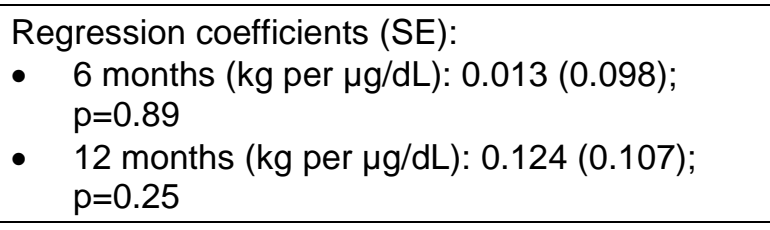 \\
\hline
\end{tabular}

\section{Schell et al. 2009}

Longitudinal cohort study; $\mathrm{n}=244$ children (aged 312 months)

\section{Maternal PbB mean: 2.8} $\mathrm{p}=0.25$ 
Table 2-43. Summary of Epidemiological Studies Evaluating Anthropometric Measurements in Infants and Children with Mean Blood Lead Concentration $(\mathrm{PbB}) \leq 10 \mu \mathrm{g} / \mathrm{dL}^{\mathrm{a}}$

\begin{tabular}{|c|c|c|c|}
\hline $\begin{array}{l}\text { Reference and study } \\
\text { population }^{b}\end{array}$ & $\mathrm{PbB}(\mu \mathrm{g} / \mathrm{dL})^{\mathrm{c}}$ & Outcome evaluated & Result $^{d}$ \\
\hline & & Weight for length & $\begin{array}{l}\text { Regression coefficients (SE): } \\
\text { - } \quad 6 \text { months }(\text { per } \mu \mathrm{g} / \mathrm{dL}):-0.158(0.111) ; \mathrm{p}=0.16 \\
\text { - } 12 \text { months (per } \mu \mathrm{g} / \mathrm{dL}): 0.084(0.111) ; \mathrm{p}=0.45\end{array}$ \\
\hline & & Head circumference & $\begin{array}{l}\text { Regression coefficients (SE): } \\
\begin{array}{l}6 \text { months }(\mathrm{cm} \text { per } \mu \mathrm{g} / \mathrm{dL}):-0.242(0.094) ; \\
p=0.01^{*} \\
12 \text { months }(\mathrm{cm} \text { per } \mu \mathrm{g} / \mathrm{dL}):-0.220(0.109) \\
p=0.05^{*}\end{array}\end{array}$ \\
\hline & & $\begin{array}{l}\text { Upper arm } \\
\text { circumference }\end{array}$ & $\begin{array}{l}\text { Regression coefficients (SE): } \\
\quad 12 \text { months }(\mathrm{cm} \text { per } \mu \mathrm{g} / \mathrm{dL}):-0.132(0.114) \\
\mathrm{p}=0.25\end{array}$ \\
\hline Yang et al. 2013a & Child PbB mean: 7.30 & Height & $\beta$ coefficient(cm per $\mu \mathrm{g} / \mathrm{dL}):-0.10 ; p=0.02^{*}$ \\
\hline \multirow{2}{*}{$\begin{array}{l}\text { Cross sectional study; } \\
n=246 \text { children (aged } 3-8 \text { years) }\end{array}$} & & Weight & $\beta$ coefficient (kg per $\mu \mathrm{g} / \mathrm{dL}):-0.14 ; p=0.01^{*}$ \\
\hline & & BMI & $\beta$ coefficient $\left(\mathrm{kg} / \mathrm{m}^{2}\right.$ per $\left.\mu \mathrm{g} / \mathrm{dL}\right):-0.08 ; p=0.24$ \\
\hline
\end{tabular}

aSee the Supporting Document for Epidemiological Studies for Lead, Table 13 for more detailed descriptions of studies.

bParticipants had no known occupational exposure to $\mathrm{Pb}$.

'Values are for maternal $\mathrm{PbB}$, unless otherwise specified.

${ }^{\mathrm{d}}$ Asterisk and bold indicate association with $\mathrm{Pb}$; unless otherwise specified, values in parenthesis are $95 \% \mathrm{Cls}$; $\mathrm{p}$-values $<0.05$ unless otherwise noted in the table.

eStunting (defined as length-for-age z score <-2), wasting (defined as weight-for-length z-score <-2), and underweight (defined as weight-for-age z-score <-2)

$\mathrm{BMI}=$ body mass index; $\mathrm{Cl}=$ confidence interval; Gmean = geometric mean; $\mathrm{NS}=$ not statistically significant; $\mathrm{OR}=$ odds ratio; $\mathrm{Pb}=$ lead; $\mathrm{SD}=\mathrm{standard}$ deviation; SE = standard error 


\section{Table 2-44. Summary of Epidemiological Studies Evaluating the Onset of Puberty in Children with Mean Blood Lead Concentration $(\mathrm{PbB}) \leq 10 \mu \mathrm{g} / \mathrm{dL}^{\mathrm{a}}$}

\begin{tabular}{|c|c|c|c|}
\hline $\begin{array}{l}\text { Reference and study } \\
\text { population }^{\text {b }}\end{array}$ & $\mathrm{PbB}(\mu \mathrm{g} / \mathrm{dL})^{\mathrm{c}}$ & Outcome evaluated & Result $^{d}$ \\
\hline \multicolumn{4}{|l|}{ Onset of puberty in females } \\
\hline Den Hond et al. 2011 & Median: 1.81 & Pubic hair development & OR: $0.65(0.45,0.93) ; p=0.020^{*}$ \\
\hline \multicolumn{4}{|l|}{$\begin{array}{l}\text { Cross-sectional study; } n=792 \text { girls } \\
\text { (aged } 14-15 \text { years) }\end{array}$} \\
\hline $\begin{array}{l}\text { Denham et al. } 2005 \\
\text { Cross-sectional study; } n=138 \text { girls } \\
\text { (aged 10-16.9 years) }\end{array}$ & Mean: 0.49 & Attainment of menarche & $\begin{array}{l}\beta \text { coefficient (SE) predicting likelihood of } \\
\text { attaining menarche (per In } \\
\mu g / d L):-1.29(0.494) ; p=0.01^{*}\end{array}$ \\
\hline $\begin{array}{l}\text { Gollenberg et al. } 2010 \\
\text { Cross-sectional study; n=705 girls } \\
\text { (aged 6-11 years) }\end{array}$ & $\begin{array}{l}\text { Median: } 2.5 \\
\text { Tertiles } \\
\text { - } \quad \text { T1: }<1.0 \\
\text { - } \quad \text { T2: } 1-4.99 \\
\text { - } \quad \text { T3: } \geq 5.00\end{array}$ & $\begin{array}{l}\text { Inhibin B pubertal } \\
\text { cutoff value }\end{array}$ & $\begin{array}{l}\text { OR for exceeding pubertal cutoff value: } \\
\begin{array}{l}\text { - T2 (OR): } 0.38(0.12,1.15)^{*} \\
\text { - T3 (OR): } 0.26(0.11,0.60)^{*}\end{array}\end{array}$ \\
\hline \multirow{3}{*}{$\begin{array}{l}\text { Naicker et al. } 2010 \\
\text { Cross-sectional, longitudinal } \\
\text { study; } n=682 \text { girls (aged } 13 \text { years) }\end{array}$} & \multirow[t]{3}{*}{ Mean: 4.9} & Breast development & $\begin{array}{l}\text { Trend analysis over ages } 8-16 \text { years: } \\
\mathrm{p}<0.001^{*}\end{array}$ \\
\hline & & Pubic hair development & $\begin{array}{l}\text { Trend analysis over ages } 8-16 \text { years: } \\
p<0.001^{*}\end{array}$ \\
\hline & & Attainment of menarche & $\begin{array}{l}\text { Trend analysis over ages } 8-16 \text { years: } \\
p<0.001^{*}\end{array}$ \\
\hline
\end{tabular}




\section{Table 2-44. Summary of Epidemiological Studies Evaluating the Onset of Puberty in Children with Mean Blood Lead Concentration $(\mathrm{PbB}) \leq 10 \mu \mathrm{g} / \mathrm{dL}^{\mathrm{a}}$}

\begin{tabular}{ll}
\hline $\begin{array}{l}\text { Reference and study } \\
\text { population }^{\mathrm{b}}\end{array}$ & $\mathrm{PbB}(\mu \mathrm{g} / \mathrm{dL})^{\mathrm{c}}$ \\
\hline Selevan et al. 2003 & Gmean \\
& - NHW: 1.4 \\
$\begin{array}{l}\text { Cross-sectional study; } \\
\mathrm{n}=2,186 \text { girls (aged 8-18 years) }\end{array}$ & - NHAA: 2.1 \\
\end{tabular}

-
- MA: 1.7

Outcome evaluated Result ${ }^{d}$

Breast development $\quad$ NHW OR: $0.82(0.47,1.42)$

- NHAA OR: $0.64(0.42,0.97)$; $p<0.05 *$

- MA OR: $0.76(0.63,0.91) ; p<0.05^{*}$

Pubic hair development $\bullet \quad$ NHW OR: $0.75(0.37,1.51)$

- NHAA OR: $0.62(0.41,0.96) ; P<0.05$

- MA OR: $0.70(0.54,0.91) ; p<0.05$

Age of menarche $\quad$ NHW HR: $0.74(0.55,1.002)$

- NHAA HR: $0.78(0.63,0.98)$; $p<0.05$ (age at menarche delayed 3.6 months)*

- MA HR: $0.90(0.73,1.11)$

Wolff et al. 2008
Cross-sectional study; n=192 girls
(aged 9 years)

Median: 2.4

Breast development $\quad$ PR for breast stage $\geq 2$ versus stage 1: $1.01(0.79,1.30)$

Pubic hair development $P R$ for pubic hair stage $\geq 2$ versus stage 1: $1.25(0.83,1.88)$

\begin{tabular}{|c|c|c|c|}
\hline \multirow{3}{*}{$\begin{array}{l}\text { Wu et al. 2003b } \\
\text { Cross-sectional study; } \\
n=1,706 \text { girls (aged 8-16 years) }\end{array}$} & \multirow{3}{*}{$\begin{array}{l}\text { Mean: } 2.5 \\
\text { Tertiles: } \\
\text { - } \quad \text { T1: } 0.7-2.0 \text { (reference) } \\
\text { - } \quad \text { T2: } 2.1-4.9 \\
\text { - } \quad \text { T3: } 5.0-21.7\end{array}$} & Breast development & $\begin{array}{ll}- & \text { OR for T2: } 1.51(0.90,2.53) \\
-\quad & \text { OR for T3: } 1.20(0.51,2.85)\end{array}$ \\
\hline & & Pubic hair development & $\begin{array}{ll}- & \text { OR for T2: } 0.48(0.25,0.92)^{*} \\
- & \text { OR for T3: } 0.27(0.08,0.93)^{*}\end{array}$ \\
\hline & & Attainment of menarche & $\begin{array}{ll}- & \text { OR for T2: } 0.42(0.18,0.97)^{*} \\
- & \text { OR for T3: } 0.19(0.08,0.43)^{*}\end{array}$ \\
\hline
\end{tabular}


Table 2-44. Summary of Epidemiological Studies Evaluating the Onset of Puberty in Children with Mean Blood Lead Concentration $(\mathrm{PbB}) \leq 10 \mu \mathrm{g} / \mathrm{dL}^{\mathrm{a}}$

\begin{tabular}{|c|c|c|c|}
\hline $\begin{array}{l}\text { Reference and study } \\
\text { population }^{b}\end{array}$ & $\mathrm{PbB}(\mu \mathrm{g} / \mathrm{dL})^{\mathrm{c}}$ & Outcome evaluated & Result $^{d}$ \\
\hline \multicolumn{4}{|l|}{ Onset of puberty in males } \\
\hline \multicolumn{4}{|l|}{$\begin{array}{l}\text { Cross-sectional study; } \mathrm{n}=887 \text { boys } \\
\text { (aged } 12-15 \text { years) }\end{array}$} \\
\hline $\begin{array}{l}\text { Hauser et al. } 2008 \\
\text { Cross-sectional study; } \\
\text { n=489 peripubertal boys (aged 8- } \\
9 \text { years) }\end{array}$ & Median: 3 & Genitalia development & $\begin{array}{l}\text { OR for having entered genitalia stage } \mathrm{G} 2 \text { for } \\
\mathrm{PbB} \geq 5 \text { compared to } \mathrm{PbB}<5: 0.57(0.34 \\
0.95) ; \mathrm{p}=0.03^{*}\end{array}$ \\
\hline \multirow{3}{*}{$\begin{array}{l}\text { Williams et al. } 2010 \\
\text { Longitudinal cohort; } \\
\text { n=489 peripubertal boys (aged 8- } \\
9 \text { years) }\end{array}$} & \multirow[t]{3}{*}{ Median: 3} & Testicular volume & $\begin{array}{l}\text { HR for testicular volume }<3 \mathrm{~mL} \text { for } \mathrm{PbB} \\
\geq 5 \mu \mathrm{g} / \mathrm{dL} \text { compared to } \mathrm{PbB}<5 \mu \mathrm{g} / \mathrm{dL}: \\
0.73(0.55,0.97) ; \mathrm{p}=0.03^{*}\end{array}$ \\
\hline & & Genitalia stage & $\begin{array}{l}\text { HR for having entered genitalia stage } \mathrm{G} 2 \text { for } \\
\mathrm{PbB} \geq 5 \mu \mathrm{g} / \mathrm{dL} \text { compared to } \mathrm{PbB}<5 \mu \mathrm{g} / \mathrm{dL} \text { : } \\
0.76(0.59,0.98) ; \mathrm{p}=0.04^{*}\end{array}$ \\
\hline & & Pubic hair stage & $\begin{array}{l}\text { HR for having entered pubic hair stage } \mathrm{G} 2 \text { for } \\
\mathrm{PbB} \geq 5 \mu \mathrm{g} / \mathrm{dL} \text { compared to } \mathrm{PbB}<5 \mu \mathrm{g} / \mathrm{dL} \text { : } \\
0.69(0.44,1.07) ; \mathrm{p}=0.10\end{array}$ \\
\hline
\end{tabular}


Table 2-44. Summary of Epidemiological Studies Evaluating the Onset of Puberty in Children with Mean Blood Lead Concentration $(\mathrm{PbB}) \leq 10 \mu \mathrm{g} / \mathrm{dL}^{\mathrm{a}}$

\section{Reference and study \\ population $^{\mathrm{b}}$}

Williams et al. 2019

$\mathrm{PbB}(\mu \mathrm{g} / \mathrm{dL})^{\mathrm{c}}$

Median: 3

Outcome evaluated

Result $^{d}$

Longitudinal cohort; $n=481$ boys

(enrolled at ages 8-9 years)

\section{Onset of puberty}

Difference in age (shift in mean age in months) $(95 \% \mathrm{Cl})$ for $\mathrm{PbB} \geq 5 \mu \mathrm{g} / \mathrm{dL}$ compared to $\mathrm{PbB}$, based on:

- Genitalia: 8.40 (3.70, 13.10); $p<0.001^{*}$

- $\quad$ Pubic hair: 8.12(3.46, 12.78); $p<0.001^{*}$

- Testicular volume: 7.68 (3.46, 11.90); $p<0.001 *$

Onset of sexual

maturity
Difference in age (shift in mean age in months) (95\% Cl) for $\mathrm{PbB} \geq 5 \mu \mathrm{g} / \mathrm{dL}$ compared to $\mathrm{PbB}$, based on:

- Genitalia: $4.20(0.56,7.84) ; p=0.024^{*}$

- $\quad$ Pubic hair: $4.23(-0.31,8.77) ; p=0.068$

- Testicular volume: 5.14 (1.70, 8.58); $\mathrm{p}=0.003^{*}$

aSee the Supporting Document for Epidemiological Studies for Lead, Table 13 for more detailed descriptions of studies.

bParticipants had no known occupational exposure to $\mathrm{Pb}$.

cValues are for maternal $\mathrm{PbB}$, unless otherwise specified.

${ }^{\mathrm{d}}$ Asterisk and bold indicate association with $\mathrm{Pb}$; unless otherwise specified, values in parenthesis are $95 \%$ Cls; $\mathrm{p}$-values $<0.05$ unless otherwise noted in the table

$\mathrm{Cl}=$ confidence interval; Gmean = geometric mean; HR = hazard ratio; $\mathrm{MA}=$ Mexican Americans; NHW = Non-Hispanic whites; NHAA = Non-Hispanic African Americans; $\mathrm{OR}=$ odds ratio; $\mathrm{Pb}=$ lead; $\mathrm{PR}=$ prevalence ratio; $\mathrm{SE}=$ standard error 
Associations Between Bone Pb and Birth Outcome and Postnatal Growth. Studies evaluating associations between maternal bone $\mathrm{Pb}$ and birth outcome (birth weight and length, head circumference) and postnatal growth (infant and child weight gain) are summarized in Table 2-45. Studies were conducted in mother-infant/child pairs residing in Mexico City. Maternal tibia $\mathrm{Pb}$ was inversely associated with birth weight (Cantonwine et al. 2010; González-Cossío; Kordas et al. 2009), birth length (Hernandez-Avila et al. 2002), and head circumference (Hernandez-Avila et al. 2002; Kordas et al. 2009). Maternal patella $\mathrm{Pb}$ was associated with decreased head circumference (Hernandez-Avila et al. 2002), but not birth weight (Afeiche et al. 2011; González-Cossío) or birth length (Hernandez-Avila et al. 2002).

Infant weight gain measured at 1 month of age was inversely associated with maternal patella $\mathrm{Pb}$, but not maternal tibia $\mathrm{Pb}$ (Sanin et al. 2001); no associations between maternal tibia or patella $\mathrm{Pb}$ were observed from birth to 12 months of age (Afeiche et al. 2011). Maternal patella $\mathrm{Pb}$ was inversely associated with weight gain in girls, but not boys, at 5 years of age; however, no associations were observed for maternal tibia $\mathrm{Pb}$ for boys or girls. In contrast, no associations were observed in a prospective study examining the relationships between maternal patella or tibia $\mathrm{Pb}$ (measured 1 month postpartum) and BMI, percent body fat, weight-for-age score, or height-for-age score in children ages 4-6 years (Renzetti et al. 2017). Taken together, results of these studies provide evidence that long-term maternal $\mathrm{Pb}$ exposure is inversely associated with infant size and post-natal growth.

\section{Table 2-45. Associations Between Maternal Bone $\mathrm{Pb}$ and Birth Outcome and Postnatal Growth}

\begin{tabular}{|c|c|c|c|c|c|c|}
\hline \multirow[b]{2}{*}{ Reference } & \multirow[b]{2}{*}{ Population ${ }^{a}$} & \multicolumn{5}{|c|}{ Effect } \\
\hline & & $\begin{array}{l}\text { Birth } \\
\text { weight }\end{array}$ & $\begin{array}{l}\text { Birth } \\
\text { length }\end{array}$ & $\begin{array}{l}\text { Head } \\
\text { circumference }\end{array}$ & $\begin{array}{l}\text { Infant weight } \\
\text { gain }\end{array}$ & $\begin{array}{l}\text { Child } \\
\text { weight } \\
\text { gain }\end{array}$ \\
\hline $\begin{array}{l}\text { Afeiche et al. } \\
2011\end{array}$ & $\begin{array}{l}\text { Mother-infant pairs } \\
\text { (522 boys; } 477 \text { girls) }\end{array}$ & $\begin{array}{l}\text { O T (M, F) } \\
\text { O P }(M, F)\end{array}$ & - & - & $\begin{array}{l}0 \mathrm{~T}(\mathrm{M}, \mathrm{F})^{\mathrm{b}} \\
0 \mathrm{P}(\mathrm{M}, \mathrm{F})^{\mathrm{b}}\end{array}$ & $\begin{array}{c}0 \mathrm{~T}(\mathrm{M}, \mathrm{F}) \\
0 \mathrm{P}(\mathrm{M}) \\
\downarrow \mathrm{P}(\mathrm{F})^{\mathrm{C}}\end{array}$ \\
\hline $\begin{array}{l}\text { Cantonwine et } \\
\text { al. } 2010\end{array}$ & $\begin{array}{l}538 \text { mother-infant } \\
\text { pairs }\end{array}$ & $\downarrow T$ & - & - & - & - \\
\hline $\begin{array}{l}\text { Gonzalez- } \\
\text { Cossio et al. } \\
1997\end{array}$ & $\begin{array}{l}272 \text { mother-infant } \\
\text { pairs }\end{array}$ & $\begin{array}{l}\downarrow T \\
0 \mathrm{P}\end{array}$ & - & - & - & - \\
\hline $\begin{array}{l}\text { Hernandez- } \\
\text { Avila et al. } \\
2002\end{array}$ & $\begin{array}{l}223 \text { mother-infant } \\
\text { pairs }\end{array}$ & - & $\begin{array}{l}\downarrow T \\
O P\end{array}$ & $\begin{array}{l}\downarrow T \\
\downarrow P\end{array}$ & - & - \\
\hline $\begin{array}{l}\text { Kordas et al. } \\
2009\end{array}$ & $\begin{array}{l}474 \text { mother-infant } \\
\text { pairs }\end{array}$ & $\downarrow T$ & $0 \mathrm{~T}$ & $\downarrow T$ & - & - \\
\hline
\end{tabular}




\section{Table 2-45. Associations Between Maternal Bone Pb and Birth Outcome and Postnatal Growth}

\begin{tabular}{|c|c|c|c|c|c|c|}
\hline \multirow[b]{2}{*}{ Reference } & \multirow[b]{2}{*}{ Population $^{a}$} & \multicolumn{5}{|c|}{ Effect } \\
\hline & & $\begin{array}{l}\text { Birth } \\
\text { weight }\end{array}$ & $\begin{array}{l}\text { Birth } \\
\text { length }\end{array}$ & $\begin{array}{l}\text { Head } \\
\text { circumference }\end{array}$ & $\begin{array}{l}\text { Infant weight } \\
\text { gain }\end{array}$ & $\begin{array}{l}\text { Child } \\
\text { weight } \\
\text { gain }\end{array}$ \\
\hline $\begin{array}{l}\text { Renzetti et al. } \\
2017\end{array}$ & $\begin{array}{l}424(P) \text { and } 430(T) \\
\text { mother-child pairs }\end{array}$ & - & - & - & - & $\begin{array}{l}0 \mathrm{~T}^{\mathrm{d}} \\
0 \mathrm{P}^{\mathrm{d}}\end{array}$ \\
\hline $\begin{array}{l}\text { Sanin et al. } \\
2001\end{array}$ & $\begin{array}{l}329 \text { mother-infant } \\
\text { pairs }\end{array}$ & - & - & - & $\begin{array}{l}0 \mathrm{~T}^{\mathrm{e}} \\
\downarrow \mathrm{Pe}\end{array}$ & - \\
\hline
\end{tabular}

aFrom Mexico City.

${ }^{b}$ Measured from birth to 12 months of age.

cMeasured at 5 years of age.

dMeasured at 4-6 years; assessments included BMI, percentage body fat, weight-for-age, and height-for-age. No associations between maternal $\mathrm{Pb}$ and any of the assessments were observed.

eMeasured at 1 month of age.

$\downarrow=$ inverse association; $0=$ no association; $-=$ not reported; $\mathrm{F}=$ female; $\mathrm{M}=$ male; $\mathrm{P}=$ patella; $\mathrm{Pb}=\mathrm{lead} ; \mathrm{T}=$ tibia

Mechanisms of Action. General mechanisms of toxicity of $\mathrm{Pb}$ (reviewed in Section 2.21) are likely involved in adverse development effects. EPA (2014c) specifically noted that delayed puberty may result from alterations in pulsatile release of sex hormones and that insulin-like growth factor 1 (IGF-1) may play a role in this effect. Pb is distributed to the fetus and has been measured in umbilical cord blood, placenta, and follicular fluid (See Section 3.1.2, Toxicokinetics, Distribution), providing a toxicokinetic mechanism for direct exposure of the fetus.

\subsection{CANCER}

Overview. Numerous epidemiological studies have investigated associations between $\mathrm{Pb}$ exposure and cancer. Studies include exposure of workers and general populations, with many studies reporting PbB. In most studies, mean PbBs in these studies are $<10 \mu \mathrm{g} / \mathrm{dL}$. Although studies provide limited evidence of carcinogenicity of $\mathrm{Pb}$ in humans, results are inconsistent and interpretation may be limited due to confounding factors.

Many studies of occupational cohorts and cancer risks do not report PbB data. These studies have reported associations between occupational exposure to $\mathrm{Pb}$ and cancer, including overall cancer mortality and cancers of the lung, brain, stomach, kidney, and bladder. However, results are inconsistent and interpretation may be limited due to confounding factors. 
The following cancers have been associated with PbB:

- $\quad \leq 10 \mu \mathrm{g} / \mathrm{dL}$ :

o Increased risk of all cancer; evaluated in multiple studies with mixed results.

o Increased risk of lung cancer; evaluated in multiple studies with mixed results.

- $\quad>10 \mu \mathrm{g} / \mathrm{dL}:$

o Increased risk of all cancer; evaluated in multiple studies with mixed results.

o Increased risk of respiratory tract cancers (bronchus, trachea, lung); evaluated in multiple studies with mixed results.

o Increased risk of stomach cancer; evaluated in multiple studies with mixed results.

o Increased risk of intestinal cancer.

o Increased risk of cancer of the larynx.

o Increased risk of glioma.

Carcinogenicity Classifications of $\mathbf{P b}$ and $\mathbf{P b}$ Compounds. IARC has classified inorganic $\mathrm{Pb}$ compounds as probably carcinogenic to humans (Group 2A) based on sufficient evidence in animals and limited evidence in humans; evidence for organic $\mathrm{Pb}$ compounds was considered to be inadequate in humans and animals (IARC 2006). The National Toxicology Program $14^{\text {th }}$ Report on Carcinogens classified $\mathrm{Pb}$ and $\mathrm{Pb}$ compounds as reasonably anticipated to be human carcinogens (NTP 2016). As the basis of the Group 2A classification for inorganic Pb compounds, IARC (2006) cited multiple animal studies showing kidney cancer following chronic oral and parenteral exposure (Azar et al. 1973; Balo et al. 1965; Fears et al. 1989; Kasprzak et al. 1985; Koller et al. 1985; Van Esch and Kroes 1969; Zawirska 1981; Zollinger 1953), renal tubular adenoma in offspring of mice exposed during gestation and lactation (Waalkes et al. 1995), and brain gliomas following oral exposure of rats (Zawirska 1981; Zawirska and Medras 1972). For epidemiological studies of occupational cohorts, IARC (2006) noted limited evidence of carcinogenicity of the lung, stomach, kidney, and brain/nervous system, although studies yielded inconsistent results, and interpretation of results was compromised due to potential confounding factors (e.g., smoking, occupational exposure to other carcinogens such as arsenic).

Confounding Factors and Effect Modifiers. Numerous factors can influence results of epidemiological studies evaluating associations between $\mathrm{Pb}$ exposure and cancer, including smoking status, family history of cancer, and co-exposure to other carcinogens. Failure to account for these factors may attenuate or strengthen the apparent associations between $\mathrm{Pb}$ exposure and the outcome, depending on the direction of 
the effect of the variable on the outcome. For example, many occupational studies include smelters where exposure to arsenic and other carcinogenic metals (e.g., cadmium) can be correlated with exposure to $\mathrm{Pb}$. Exposures to $\mathrm{Pb}$ occur throughout the lifetime and a cross-sectional evaluation of $\mathrm{PbB}$ may not adequately represent the exposure history of the individual.

Measures of Exposure. Numerous studies evaluating cancer in general populations and Pb-exposed workers report $\mathrm{PbB}$ as a measure of exposure. A few studies measured exposure by bone $\mathrm{Pb}$ concentrations, cumulative blood $\mathrm{Pb}$ index, or cumulative exposure (Bhatti et al. 2009; Englyst et al. 2001; Ionescu et al. 2007; Rajaraman et al. 2006); however, these studies did not report PbB.

Characterization of Effects. Numerous epidemiological studies have assessed associations between $\mathrm{PbB}$ and cancer. Studies of general populations and workers are briefly summarized in Table 2-46. Studies of general populations include large cross-sectional studies $(n=5,482-13,946)$ of NHANES participants (Cheung et al. 2013; Jemal et al. 2002; Menke et al. 2013; Schober et al. 2006). Mean PbBs in most studies are $<10 \mu \mathrm{g} / \mathrm{dL}$, although in some studies that stratify by $\mathrm{PbB}$, the highest exposure categories are $>10 \mu \mathrm{g} / \mathrm{dL}$ (Jemal et al. 2002; Kelly et al. 2013; Schober et al. 2006). Results of two studies with PbB $<10 \mu \mathrm{g} / \mathrm{dL}$ show increased risks of all cancer and of lung cancer (Cheung et al. 2013; Schober et al. 2006), although other studies show no increases in cancer risk (Jemal et al. 2002; Khalil et al. 2009; Kelly et al. 2013; Menke et al. 2013; Santibanez et al. 2008; Wiesskopf et al. 2009). Results of occupational exposure studies are mixed and do not establish a pattern of effects of exposure-response relationships. PbBs in these studies generally are $>40 \mu \mathrm{g} / \mathrm{dL}$. Studies have reported associations between $\mathrm{PbB}$ and all cancers (Anttila et al. 1995; Lundstrom et al. 1997; Lustberg and Silbergeld 2002; McElvenny et al. 2015; Wong and Harris et al. 2000), cancers of the bronchus, trachea, and lung (Anttila et al. 1995; Barry and Steenland 2019; Chowdhury et al. 2014; Kim et al. 2015; Lundstrom et al. 1997; McElvenny et al. 2015; Steenland and Boffetta 2000; Steenland et al. 2017, 2019), cancer of the larynx (Barry and Steenland 2019; Chowdhury et al. 2014; Steenland et al. 2019), esophageal cancer (Steenland et al. 2019), stomach cancer (Cooper et al. 1985; Steenland and Boffetta 2000; Steenland et al. 2017, 2019; Wong and Harris et al. 2000), intestinal or rectal cancer (Kim et al. 2015; Steenland et al. 2019), bladder cancer (Steenland et al. 2017), and gliomas (Anttila et al. 1996).

Many studies of occupational cohorts with high exposure to $\mathrm{Pb}$ and cancer risks do not report $\mathrm{PbB}$ data (Bertazzi and Zocchetti 1980; Bhatti et al. 2009; Cocco et al. 1994, 1997, 1998a, 1998b, 1999a, 1999b; Davies 1984a, 1984b; Dingwall-Fordyce and Lane 1963; Fayerweather et al. 1997; Hu et al. 1999; Jones et al. 2007; Kauppinen et al. 1992; Lin et al. 2009; McElroy et al. 2008; Michaels et al. 1991; Pan et al. 
Table 2-46. Summary of Epidemiological Studies Evaluating Cancer Endpoints and Blood Lead Concentration (PbB)

\begin{tabular}{|c|c|c|c|}
\hline Reference and study population & $\mathrm{PbB}(\mu \mathrm{g} / \mathrm{dL})$ & Cancer outcomes & Effects $^{a}$ \\
\hline \multicolumn{4}{|l|}{ General population } \\
\hline Cheung et al. 2013 & Mean (SE): 4.44 (0.14) & All cancer & OR: $1.071(1.036,1.106)^{*}$ \\
\hline \multicolumn{2}{|l|}{ Cross-sectional study; n=3,482 (NHANES III) } & Lung cancer & OR: $1.090(1.054,1.127)^{*}$ \\
\hline $\begin{array}{l}\text { Jemal et al. } 2002 \\
\text { Cross-sectional study; } n=3,592 \text { (NHANES II, } \\
\text { age } 6 \text { months-74 years) }\end{array}$ & $\begin{array}{ll}\text { Quartiles: } \\
\text { - } \quad \text { Q1: } \leq 9.8 \\
\text { - } \quad \text { Q2: } 9.9-12.9 \\
\text { - } \quad \text { Q3: } 13.0-16.9 \\
\text { - } \quad \text { Q4: } \geq 17.0 \\
\end{array}$ & All cancer & Adjusted RR Q4: $1.50(0.75,3.01)$ \\
\hline $\begin{array}{l}\text { Khalil et al. } 2009 \\
\text { Prospective cohort study; } \mathrm{n}=532 \text { women } \\
\text { (age 65-87 years) }\end{array}$ & $\begin{array}{l}\text { Mean: } 5.3 \\
<8(n=453) \\
\geq 8(n=79)\end{array}$ & All cancer & $\begin{array}{l}\text { Adjusted } \mathrm{HR} \mathrm{PbB} \geq 8 \text { (versus <8): } 1.64 \\
(0.73,3.71)\end{array}$ \\
\hline & \multirow{2}{*}{$\begin{array}{l}\text { Mean (range) } \\
\text { - } \quad \text { Males: } 6.18(1.54,67.2) \\
\text { - } \quad \text { Females: } 5.27(1.1,40.1) \\
\text { Quartiles } \\
\text { - } \quad \text { Q1: } 1.5423-3.986 \\
\text { - } \quad \text { 2: } 3.9504-5.8763 \\
\text { - } \quad \text { Q3: } 5.8832-8.7218 \\
\text { - } \quad \text { 4: } 8.7531-40.0843 \\
\end{array}$} & $\mathrm{NHL}$ & $\begin{array}{l}\text { OR Q4: } 0.93(0.43,2.02) \\
\text { p-trend }=0.849\end{array}$ \\
\hline $\begin{array}{l}\text { Nested case-control study; } \mathrm{n}=194 \text { cases } \\
\text { NHL; } 76 \text { cases MM; and } 270 \text { controls (mean } \\
\text { age } 53.08 \text { years) }\end{array}$ & & MM & $\begin{array}{l}\text { OR Q4: } 1.63(0.45,5.94) \\
p \text {-trend }=0.533\end{array}$ \\
\hline $\begin{array}{l}\text { Menke et al. } 2006 \\
\text { Cross-sectional study; } n=13,946 \text { (NHANES } \\
\text { 1988-1994; mean age } 44.4 \text { years) }\end{array}$ & $\begin{array}{l}\text { Mean: } 2.58 \\
\text { Tertiles: } \\
\text { - T1: }<1.93 \\
\text { - T2: } 1.94-3.62 \\
-\quad \text { T3: } \geq 3.62 \\
\end{array}$ & All cancer & $\begin{array}{l}\text { Adjusted OR } \\
\text { - T2: } 0.72(0.46,1.12) ; p \text {-trend }=0.130 \\
\text { - T3: } 1.10(0.82,1.47) ; p \text {-trend }=0.101\end{array}$ \\
\hline $\begin{array}{l}\text { Santibanez et al. } 2008 \\
\text { Case-control study; } n=185 \text { esophageal } \\
\text { cancer patients; } 285 \text { controls (age 30- } \\
80 \text { years) }\end{array}$ & $\begin{array}{l}\text { Low: } \leq 4.9 \\
\text { High: }>4.9\end{array}$ & Esophageal & $\begin{array}{l}\text { Adjusted OR } \\
\text { - } \quad \text { Low: } 0.79(0.43,1.46) \\
\text { - } \quad \text { High: } 1.69(0.57,5.03)\end{array}$ \\
\hline
\end{tabular}


Table 2-46. Summary of Epidemiological Studies Evaluating Cancer Endpoints and Blood Lead Concentration (PbB)

\begin{tabular}{|c|c|c|c|}
\hline Reference and study population & $\mathrm{PbB}(\mu \mathrm{g} / \mathrm{dL})$ & Cancer outcomes & Effects $^{a}$ \\
\hline $\begin{array}{l}\text { Schober et al. } 2006 \\
\text { Cross-sectional study; } n=9,757 \text { (NHANES III; } \\
\text { age } \geq 40 \text { years) }\end{array}$ & $\begin{array}{l}\text { Tertiles } \\
\text { - T1: }<5(\text { mean } 2.6) \\
\text { - T2: } 5-9(\text { mean } 6.3) \\
-\quad \text { T3: }>10(\text { mean } 11.8) \\
\end{array}$ & All cancer & $\begin{array}{l}\text { Adjusted RR } \\
\text { - T2: } 1.44(1.12,1.86)^{*} \\
\text { - T3: } 1.69(1.14,2.52)^{*} \\
\text { - } \quad \text {-trend<0.01* }\end{array}$ \\
\hline $\begin{array}{l}\text { Weisskopf et al. } 2009 \\
\text { Prospective study; } n=868 \text { men (Normative } \\
\text { Aging Study; age } 21-80 \text { years) } \\
\end{array}$ & $\begin{array}{l}\text { Mean (SD): } 5.6(3.4) \\
\text { Tertiles: } \\
\text { - T1: }<4 \\
\text { - T2: } 4-6 \\
\text { - T3: }>6 \\
\end{array}$ & All cancer & $\begin{array}{l}\text { Adjusted HR T3: } 0.48(0.25-0.91)^{*} ; \\
p \text {-trend }=0.02\end{array}$ \\
\hline \multicolumn{4}{|l|}{ Workers } \\
\hline Anttila et al. 1995 & \multirow{2}{*}{$\begin{array}{l}\text { Tertiles: } \\
\text { - T1: } 0-18.6 \\
\text { - T2: } 20.7-39.4 \\
\text { - T3: } 41.1-161.6\end{array}$} & All cancer & $\begin{array}{l}\text { SMR T2: } 1.4(1.1,1.8)^{*} \\
\text { SMR T3: } 1.2(0.9,1.8)\end{array}$ \\
\hline $\begin{array}{l}\text { Cross-sectional study; } n=20,700 \text { workers } \\
\text { (age } 30-74 \text { years) }\end{array}$ & & Lung, trachea & $\begin{array}{l}\text { SMR T2: } 2.0(1.2,3.2)^{*} \\
\text { SMR T3: } 1.5(0.8,2.1)\end{array}$ \\
\hline Anttila et al. 1996 & \multirow{2}{*}{$\begin{array}{l}\text { Tertiles: } \\
-\quad \text { T1: } 2.1-14.5 \\
\text { - } \quad \text { T2: } 16.6-26.9 \\
\text { - } \quad \text { T3: } 29.0-89.1\end{array}$} & $\begin{array}{l}\text { All nervous system } \\
\text { cancers }\end{array}$ & $\begin{array}{l}\text { Adjusted OR T3: } 2.2(0.7,6.6) \\
\text { p-trend=0.17 }\end{array}$ \\
\hline $\begin{array}{l}\text { Cross-sectional study; } n=20,741 \text { workers } \\
\text { (age } 18-74 \text { years) }\end{array}$ & & Glioma & $\begin{array}{l}\text { Adjusted OR T3: } 11(1.0,626)^{*} \\
\text { p-trend: } 0.037\end{array}$ \\
\hline
\end{tabular}


Table 2-46. Summary of Epidemiological Studies Evaluating Cancer Endpoints and Blood Lead Concentration (PbB)

\begin{tabular}{|c|c|}
\hline Reference and study population & $\mathrm{PbB}(\mu \mathrm{g} / \mathrm{dL})$ \\
\hline Barry and Steenland 2019 & Quartiles \\
\hline $\begin{array}{l}\text { Retrospective study; } n=58,368 \text { male workers } \\
\text { (follow-up of Chowdhury et al. 2014) }\end{array}$ & $\begin{array}{ll}- & \text { Q1: } 0-<5 \\
- & \text { Q2: } 5-<25 \\
- & \text { Q3: } 25-<40 \\
- & \text { Q4: } \geq 40\end{array}$ \\
\hline
\end{tabular}

\begin{tabular}{l} 
Cancer outcomes \\
Colon \\
\hline Kidney \\
\hline Liver
\end{tabular}

Effects $^{\mathrm{a}}$

Lung HR Q4: $1.19(0.54,2.61)$ HR Q4: $0.97(0.43,2.20)$ HR Q4: $0.92(0.32,2.58)$ HR Q4: $1.53(0.79,2.99)$

HR Q2: $1.61(1.04,2.48)^{*}$ HR Q3: $2.03(1.34,3.10)^{*}$ HR Q4: $2.92(1.91,4.46)^{*}$ p-trend: $<0.01^{*}$

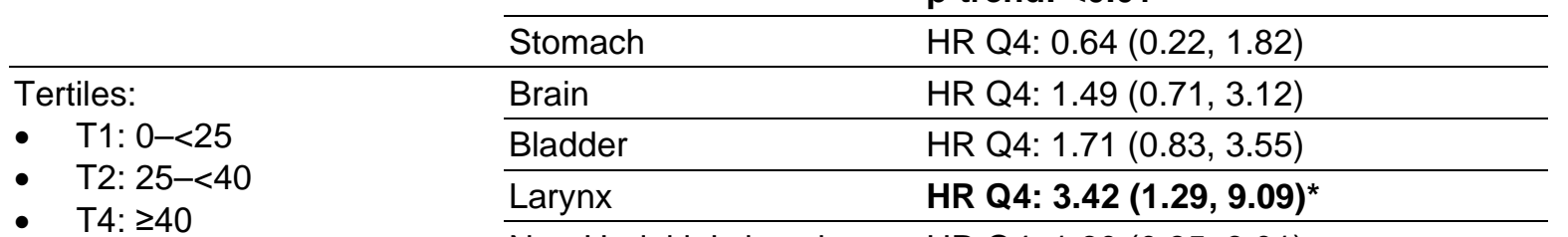

- $\mathrm{T} 4: \geq 40$

\section{Chowdhury et al. 2014}

Survey study/cross-sectional study; $\mathrm{n}=58,368$ male workers (mean age 38.9 years)

\section{Quartiles}

- Q1: 0-<5

- Q2: 5-<25

- Q3: $25-<40$

- $\mathrm{Q} 4: \geq 40$

\section{Non-Hodgkin's lymphoma}

Pancreas HR Q4: $1.15(0.72,1.85)$

Rectal HR Q4: $2.06(0.87,4.84)$

\begin{tabular}{ll}
\hline Lung & SMR Q4: 1.20 $(\mathbf{1 . 0 3}, \mathbf{1 . 3 9})^{\star}$ \\
\hline Brain & SMR Q4: 0.83 $(0.41,1.49)$ \\
\hline Kidney & SMR Q4: 0.72 $(0.33,1.37)$ \\
\hline Stomach & SMR Q4: 0.92 $(0.44,1.69)$ \\
\hline Esophagus & SMR Q4: 0.65 $(0.32,1.16)$ \\
\hline Larynx & SMR Q4: 2.11 (1.05, 3.77)* \\
\hline Bladder & SMR Q4: 0.70 $(0.28,1.45)$ \\
\hline All cancer & Battery PMR: 1.06 $(0.96,1.16)$ \\
& Smelters PMR: $1.02(0.87,1.19)$ \\
\hline Stomach & Battery PMR: 1.54 (1.11, 2.15)* \\
& Smelters PMR: $1.03(0.75,1.42)$ \\
\hline Large intestine & Battery PMR: 0.98 $(0.69,1.40)$ \\
& Smelters PMR: $1.19(0.62,2.28)$ \\
\hline
\end{tabular}

\section{Cooper et al. 1985}

Cohort study; $n=4,519$ battery workers; 2,300 smelters

\section{Mean}

- Battery $(n=1,326): 62.7$

- Smelters $(n=537): 79.7$ 
Table 2-46. Summary of Epidemiological Studies Evaluating Cancer Endpoints and Blood Lead Concentration (PbB)

\begin{tabular}{|c|c|c|c|}
\hline Reference and study population & $\mathrm{PbB}(\mu \mathrm{g} / \mathrm{dL})$ & Cancer outcomes & Effects $^{a}$ \\
\hline & & Larynx & $\begin{array}{l}\text { Battery PMR: } 1.19(0.54,2.65) \\
\text { Smelters PMR }(95 \% \mathrm{Cl}): 1.06(0.27 \\
4.21)\end{array}$ \\
\hline & & Bronchus, trachea, lung & $\begin{array}{l}\text { Battery PMR: } 1.16(0.97,1.39) \\
\text { Smelters PMR: } 1.13(0.84,1.51)\end{array}$ \\
\hline & & Brain and other CNS & $\begin{array}{l}\text { Battery PMR: } 1.09(0.55,2.18) \\
\text { Smelters PMR: } 0.97(0.32,3.01)\end{array}$ \\
\hline \multirow{5}{*}{$\begin{array}{l}\text { Kim et al. } 2015 \\
\text { Cross-sectional study; } n=81,067 \text { inorganic } \\
\text { Pb workers ( } 54,788 \text { males; } 26,279 \text { females; } \\
\text { age } 20-\leq 50 \text { years) }\end{array}$} & \multirow{5}{*}{$\begin{array}{l}\text { Mean (SD) } \\
\text { - } \quad \text { Males: } 8.8(8.5) \\
\text { - } \quad \text { Females } 5.8(5.4) \\
\text { Tertiles: } \\
\text { - } \quad \text { T1: }<10 \\
\text { - T2: } 10-20 \\
\text { - } \quad \text { T3: }>20\end{array}$} & All cancer & $\begin{array}{l}\text { Males: RR T3: } 0.95(0.56,1.61) \\
\text { Females RR T3: } 1.68(0.40,7.13)\end{array}$ \\
\hline & & Stomach & $\begin{array}{l}\text { Males: RR T3: } 0.80(0.23,2.71) \\
\text { Females RR T2: } 1.82(0.20,16.36) \\
\text { Females T3: no cases }\end{array}$ \\
\hline & & Colo-rectal & $\begin{array}{l}\text { Males: RR T3: } 1.86(0.35,9.79) \\
\text { Females RR T2: } \mathbf{1 3 . 4 2}\left(\mathbf{1 . 2 1}, \mathbf{1 4 9 . 4 ) ^ { * }} \text {; }\right. \\
\text { p<0.05 } \\
\text { Females T3: no cases }\end{array}$ \\
\hline & & Liver & $\begin{array}{l}\text { Males: RR T3: } 1.72(0.72,4.14) \\
\text { Females T2 RR: } 0.83(0.10,6.56) \\
\text { Females T3: no cases }\end{array}$ \\
\hline & & Bronchus, lung & 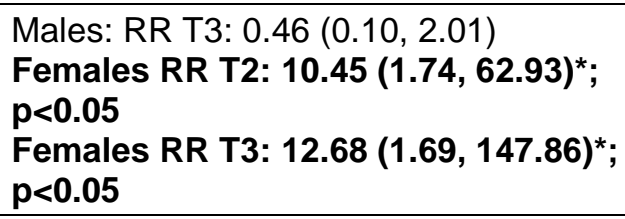 \\
\hline \multirow[b]{2}{*}{ Cross-sectional study; n=3979 workers } & \multirow{2}{*}{$\begin{array}{l}\text { Mean: } \\
\text { - } \quad \text { In 1950: } 62.2 \\
\text { - } \quad \text { In 1987: } 33.2\end{array}$} & All cancer & SMR: $1.2(1.0,1.5)^{*}$ \\
\hline & & Lung & SMR: $2.8(2.0,3.8)^{*}$ \\
\hline Lundstrom et al. 2006 & $\begin{array}{l}\text { Peak: } \\
\text { Cases }(n=40): 49.7\end{array}$ & Lung & OR: $0.93(0.60,1.44)$ \\
\hline $\begin{array}{l}\text { Nested case-referent study; 3,979 smelter } \\
\text { workers }\end{array}$ & Referents ( $n=114): 55.9$ & & \\
\hline
\end{tabular}


Table 2-46. Summary of Epidemiological Studies Evaluating Cancer Endpoints and Blood Lead Concentration (PbB)

\section{Reference and study population}

Lustberg and Silbergeld 2002

Cross-sectional study; $n=4,292$; age 3074 years (NHANES II)

\section{McElvenny et al. 2015}

Cohort study; $n=9,122$ workers; mean age 29.2 years

$\mathrm{PbB}(\mu \mathrm{g} / \mathrm{dL})$

Tertiles:

- $T 1(n=818):<10$

- T2 $(n=2,735): 10-19$

- T3 (n=637): 20-29

Mean (SD): 44.3 (22.7)

Range: $2.3-321.5$

\begin{tabular}{|c|c|c|c|}
\hline & & \multirow{3}{*}{$\frac{\text { Colon }}{\text { Kidney }}$} & \multirow[b]{2}{*}{ SMR: $0.98(0.77,1.26)$} \\
\hline & & & \\
\hline & & & SMR: $1.30(0.91,1.86)$ \\
\hline & & Bladder & SMR: $0.95(0.67,1.35)$ \\
\hline & & Bronchus, trachea, lung & SMR: $1.42(1.29,1.57)^{*}$ \\
\hline & & Brain & SMR: $0.92(0.61,1.38)$ \\
\hline \multirow{5}{*}{$\begin{array}{l}\text { Selevan et al. } 1985 \\
\text { Retrospective cohort study; } n=1,987 \text { male } \\
\text { workers }\end{array}$} & \multirow[t]{5}{*}{ Mean: 56.3} & All cancer & SMR: $0.95(0.78,1.14)$ \\
\hline & & Digestive organs & SMR: $0.77(0.52,1.10)$ \\
\hline & & Respiratory system & SMR: $1.11(0.80,1.51)$ \\
\hline & & Kidney & SMR: $2.04(0.75,4.44)$ \\
\hline & & Bladder & SMR: $1.44(0.53,3.14)$ \\
\hline Steenland and Boffetta 2000 & \multirow[t]{3}{*}{ Range of study means: $26-80$} & Lung & RR: $1.14(1.04,1.25)^{\star}$ \\
\hline \multirow{2}{*}{$\begin{array}{l}\text { Meta-analysis; data from eight studies on } \mathrm{Pb} \\
\text { workers; } n=36,027 \text { workers }\end{array}$} & & Stomach & RR: $1.34(1.14,1.57)^{*}$ \\
\hline & & Brain & RR: $1.06(0.81,1.40)$ \\
\hline Steenland et al. 1992 & \multirow[t]{4}{*}{ Mean: 56.3} & All Cancer & SMR: $0.98(0.84,1.12)$ \\
\hline \multirow{3}{*}{$\begin{array}{l}\text { Cohort study (same cohort as Selevan et al. } \\
\text { 1985); } n=1,990 \text { male smelter workers }\end{array}$} & & Colon & SMR: $0.48(0.22,0.90)$ \\
\hline & & Lung & SMR: $1.18(0.92,1.48)$ \\
\hline & & Kidney & SMR: $1.93(0.88,3.67)$ \\
\hline
\end{tabular}


Table 2-46. Summary of Epidemiological Studies Evaluating Cancer Endpoints and Blood Lead Concentration (PbB)

\section{Reference and study population}

Steenland et al. 2017

Cohort study; $\mathrm{n}=88,000 \mathrm{~Pb}$ workers

Steenland et al. 2019
Cohort study; $n=29,874 \mathrm{~Pb}$ workers

Cohort study; $n=29,874$ Pb workers

Median: 29

$\mathrm{PbB}(\mu \mathrm{g} / \mathrm{dL})$
Median: 26

(n)

\begin{tabular}{l} 
Cancer outcomes \\
Bladder $(>40 \mu \mathrm{g} / \mathrm{dL})$ \\
\hline Kidney $(>40 \mu \mathrm{g} / \mathrm{dL})$ \\
\hline Larynx $(>40 \mu \mathrm{g} / \mathrm{dL})$ \\
\hline Lung $(20-<30 \mu \mathrm{g} / \mathrm{dL})$
\end{tabular}

Effects $^{\mathrm{a}}$

Stomach $(20-<40 \mu \mathrm{g} / \mathrm{dL})$ HR: $1.62(1.13,2.32)^{*}$

HR: $1.86(1.04,3.33)^{*}$

HR: $2.69(1.07,6.76)^{*}$

Brain $(>40 \mu \mathrm{g} / \mathrm{dL}) \quad$ HR: $1.71(0.94,3.12)$

\begin{tabular}{ll} 
Brain $(>40 \mu \mathrm{g} / \mathrm{dL})$ & HR: $1.71(0.94,3.12)$ \\
\hline Bladder $(>40 \mu \mathrm{g} / \mathrm{dL})$ & HR: $1.24(0.87,1,75)$
\end{tabular}

Esophagus (30-39 $\mu \mathrm{g} / \mathrm{dL})$ HR: $2.00(1.08,3.71)^{*}$

\begin{tabular}{ll}
\hline Kidney $(>40 \mu \mathrm{g} / \mathrm{dL})$ & HR: $1.00(0.66,1.51)$ \\
\hline Larynx $(>40 \mu \mathrm{g} / \mathrm{dL})$ & HR: $1.92(0.94,3.91)$ \\
\hline Lung $(20-29 \mu \mathrm{g} / \mathrm{dL})$ & HR: $1.39(\mathbf{1 . 1 7}, \mathbf{1 . 6 5})^{*}$ \\
\hline Rectum $(>40 \mu \mathrm{g} / \mathrm{dL})$ & HR: $1.49(\mathbf{1 . 0 3}, \mathbf{2 . 1 7})^{*}$ \\
\hline Stomach $(20-29 \mu \mathrm{g} / \mathrm{dL})$ & HR: $1.55\left(\mathbf{1 . 1 0 , 2 . 1 8 ) ^ { * }}\right.$ \\
\hline All cancer & SMR: $1.045(1.012,1.080)^{*}$ \\
\hline Stomach & SMR: $1.474(1.125,1.898)^{*}$ \\
\hline Large intestine & SMR: $0.994(0.789,1.235)$ \\
\hline Bronchus, trachea, lung & SMR: $1.164(1.039,1.299)$ \\
\hline Kidney & SMR: $0.636(0.339,1.087)$ \\
\hline CNS & SMR: $0.748(0.419,1.234)$
\end{tabular}

aAsterisk and bold indicate association with $\mathrm{Pb}$; unless otherwise specified, values in parenthesis are $95 \% \mathrm{Cls}$; $\mathrm{p}$-values <0.05 unless otherwise noted in the table.

$\mathrm{Cl}=$ confidence interval; CNS = central nervous system; HR = hazard ratio; $\mathrm{MM}=$ multiple myeloma; NHANES = National Health and Nutrition Examination Survey; NHL = non-Hodgkin's lymphoma; $\mathrm{OR}=$ odds ratio; $\mathrm{Pb}=$ lead; $\mathrm{PMR}=$ proportionate mortality ratio; $\mathrm{RR}=$ rate ratio or relative ratio; $\mathrm{SD}=\mathrm{standard}$

deviation; SE = standard error; SMR = standard mortality ratio

Cohort study; $n=4,519$ battery workers;

2,300 smelters (same cohort as Cooper et al. 1985)
Mean:

- All workers: 64.0

- Battery workers: 62.7

- $\quad$ Smelters: 79.7 
2011; Partanen et al. 1991; Pesch et al. 2000; Rajaraman et al. 2006; Risch et al. 1988; Rousseau et al. 2007; Sankila et al. 1990; Sheffet et al. 1982; Siemiatycki 1991; Sweeney et al. 1986; van Wijngaarden and Dosemeci 2006; Wingren and Englander 1990). Although results of these studies are mixed and interpretation may be limited due to confounding factors, associations have been reported between occupational exposure to $\mathrm{Pb}$ and cancer, including overall cancer mortality and cancers of the lung, brain, stomach, kidney, and bladder.

Mechanisms of Action. Numerous mechanisms for Pb-induced carcinogenicity have been proposed (EPA 2014c); however, it is likely that a combination of mechanisms, rather than a single mechanism, is involved. Although $\mathrm{Pb}$ is considered to be only weakly mutagenic, it has been shown to produce DNA damage (single and double strand breaks), sister chromatid exchanges (SCEs), chromosome aberrations, micronuclei (MN) formation, and cytogenetic damage. Epigenetic mechanisms (e.g., changes in gene expression in the absence of changes to DNA), post-translational alterations to protein structure, and immune modulation of tumorigenesis in response to $\mathrm{Pb}$-induced ROS oxidative damage and inflammation have also been proposed as possible mechanisms involved in Pb-induced carcinogenesis.

\subsection{GENOTOXICITY}

The genotoxicity of $\mathrm{Pb}$ has been studied in $\mathrm{Pb}$ workers and the general population, in in vivo animal models, and in vitro cultures of microorganisms and mammalian cells. For the following discussions, data from epidemiological studies on genotoxicity were obtained from the primary literature. Information on in vitro studies and in vivo animal studies was taken from comprehensive reviews of $\mathrm{Pb}$ genotoxicity (EPA 2014c; Garcia-Leston et al. 2010; IARC 2006; NTP 2003).

\section{Epidemiological Studies}

Overview. Epidemiological studies have examined genotoxic effects associated with $\mathrm{Pb}$ exposure in adults (general populations and workers) and children. Most studies were conducted in small populations of workers. Numerous studies with $\mathrm{PbB} \geq 10 \mu \mathrm{g} / \mathrm{dL}$ report associations for exposure to $\mathrm{Pb}$ and genotoxic endpoints (gene mutation, DNA damage, SCE, MN formation, and DNA methylation), although some inverse associations have been reported. Few epidemiology studies have evaluated genotoxicity at $\mathrm{PbB}$ $\leq 10 \mu \mathrm{g} / \mathrm{dL}$. 
The following genotoxic effects have been associated with PbB:

- $\quad \leq 10 \mu \mathrm{g} / \mathrm{dL}$ :

o Gene mutation.

o DNA damage; evaluated in a few studies with mixed results.

o DNA methylation; positive results, corroborated in a few studies.

- $\quad>10 \mu \mathrm{g} / \mathrm{dL}$ :

o DNA damage; corroborated in numerous studies.

o Decreased telomere length.

o Chromosomal aberrations; evaluated in numerous studies with mainly positive results.

o Sister chromatid exchange; evaluated in numerous studies with mainly positive results.

o Micronuclei formation; evaluated in numerous studies with mainly positive results.

o DNA methylation.

Measures of Exposure. Studies evaluating the association between genotoxic effects and $\mathrm{Pb}$ exposure typically evaluate exposure by measurement of PbB.

Confounding Factors and Effect Modifiers. Most epidemiological studies evaluating genotoxic effects were conducted in worker populations. Therefore, potential co-exposure to other genotoxic compounds (such as arsenic) could occur, complicating interpretation of results. In addition, many studies were conducted in small populations $(\mathrm{n}<100)$. Variable outcomes of genotoxicity studies in human populations may derive from the influence of experimental variables that may act as confounders, such as duration and route of $\mathrm{Pb}$ exposure, cell culturing time following the exposure, smoking habits, and simultaneous exposure to other toxic agents that could act by modifying the genotoxic response of the cells to $\mathrm{Pb}$ exposure and similarly, modifying the results of the studies (García-Lestón et al. 2010).

Characterization of Effects. General trends for studies demonstrating associations between $\mathrm{PbB}$ and genotoxic effects are shown in Table 2-47. Additional study details are provided in the Supporting Document for Epidemiological Studies for Lead, Table 14. Although few studies have evaluated genotoxic effects at $\mathrm{PbB} \leq 10 \mu \mathrm{g} / \mathrm{dL}$ (see discussion below), numerous studies in adult workers with mean PbBs ranging from 20 to $>50 \mu \mathrm{g} / \mathrm{dL}$ provide evidence of increased DNA damage, chromosomal aberrations, SCEs, and MN. One study reported decreased telomere length in workers (Pawlas et al. 2016). A few studies in workers reported negative findings for chromosomal aberrations (Anwar and Kamal 1988; Bulsma and DeFrance 1976; Mäki-Paakkanen et al. 1981; Schwanitz et al. 1975) and SCEs 
(Grandjean et al. 1983; Mäki-Paakkanen et al. 1981); however, positive results for these endpoints were reported in other studies at similar PbBs.

\section{Table 2-47. Overview of Epidemiology Studies Evaluating Genotoxicity Associated with Chronic Exposure to Lead (Pb)}

\begin{tabular}{lll}
\hline $\begin{array}{l}\text { Mean PbB } \\
(\mu \mathrm{g} / \mathrm{dL})\end{array}$ & $\begin{array}{l}\text { Effects associated with Pb } \\
\text { exposure }\end{array}$ & References \\
\hline$\leq 10$ & Gene mutation & Van Larebeke et al. 2004 \\
& DNA damage/repair & Akram et al. 2019; Jasso-Pineda et al. 2012; \\
& Decreased telomere length & Pawlas et al. 2015 \\
& MN & Mielzynska et al. 2006; Wu et al. 2017 \\
& DNA methylation & Hanna et al. 2012; Li et al. 2016b; Pilsner et al. 2009 \\
\hline$>10-30$ & DNA damage/repair & Chinde et al. 2014; Danadevi et al. 2003; Dobrakowski et al. \\
& & 2017; Jannuzzi and Alpertunga 2016; Kašuba et al. 2012; \\
& & Kayaalti et al. 2015b; Méndez-Gómez et al. 2008; Shaik and \\
& Jamil 2009 \\
& Chromosomal aberrations & Pinto et al. 2000 \\
& SCE & Anwar and Kamal 1988; Pinto et al. 2000 \\
& MN & Chinde et al. 2014; Khan et al. 2010b; Kašuba et al. \\
& 2012;Nordenson et al. 1978; Pinto et al. 2000 \\
\hline$>30-50$ & DNA damage/repair & Dobrakowski et al. 2017; Fracasso et al. 2002; Grover et al. \\
& 2010; Pawlas et al. 2017 \\
& Decreased telomere length & Pawlas et al. 2016 \\
& Chromosomal aberrations & Forni et al. 1976; Grover et al. 2010; Schwanitz et al. 1970 \\
& SCE & Duydu et al. 2001, 2005; Wiwanitkit et al. 2008; Wu et al. \\
& 2002 \\
& MN & Grover et al. 2010; Hamurcu et al. 2001; Minozzo et al. 2004 \\
& DNA methylation & Devoz et al. 2017 \\
\hline DNA damage/repair & de Restrepo et al. 2000 \\
Chromosomal aberrations & Al-Hakkak et al. 1986; Forni et al. 1976; Huang et al. 1988; \\
& Nordenson et al. 1978; Schwanitz et al. 1970 \\
& SCE & Huang et al. 1988 \\
MN & Shaik and Jamil 2009; Singh et al. 2013; Vaglenov et al. \\
& 1998, 2001 \\
\hline &
\end{tabular}

DNA = deoxyribonucleic acid; $\mathrm{MN}=$ micronuclei; $\mathrm{PbB}=$ blood lead concentration; $\mathrm{SCE}=$ sister chromatid exchange

Results of genotoxicity studies conducted in small populations of children ( $\mathrm{n}=12-103)$ are inconsistent; for study details, see the Supporting Document for Epidemiological Studies for Lead, Table 14. Mixed results were observed for studies on DNA damage, with positive associations at mean PbBs of 7.3 and $28.5 \mu \mathrm{g} / \mathrm{dL}$ (Méndez-Gómez et al. 2008; Jasso-Pineda et al. 2012) and no associations at a mean PbB of $19.5 \mu \mathrm{g} / \mathrm{dL}$ (Méndez-Gómez et al. 2008). No associations were observed for chromosome aberrations at 
a PbB range of 12-33 $\mu \mathrm{g} / \mathrm{dL}$ (Bauchinger et al. 1977) and for SCE at mean PbBs of 7.69 and $62.7 \mu \mathrm{g} / \mathrm{dL}$ (Dalpra et al. 1983; Mielzynska et al. 2006). MN formation was positively associated with a mean $\mathrm{PbB}$ of $7.69 \mu \mathrm{g} / \mathrm{dL}$ (Mielzynska et al. 2006), and altered DNA methylation was found in newborns at mean umbilical cord PbB of $6.6 \mu \mathrm{g} / \mathrm{dL}$ (Pilsner et al. 2009) and mean prenatal maternal RBC Pb of $1.2 \mu \mathrm{g} / \mathrm{dL}$ (Wu et al. 2017).

Effect at Blood Pb Levels $\leq 10 \mu \mathrm{g} / \mathrm{dL}$. Results of studies evaluating genotoxic effects of $\mathrm{PbB} \leq 10 \mu \mathrm{g} / \mathrm{dL}$ are summarized in Table 2-48, with study details provided in the Supporting Document for Epidemiological Studies for Lead, Table 14. Few studies have evaluated genotoxicity at $\mathrm{PbB} \leq 10 \mu \mathrm{g} / \mathrm{dL}$. Some endpoints were only evaluated in a single study; therefore, it is difficult to draw conclusions. With the exception of a large study conducted in NHANES participants (Zota et al. 2015), genotoxic effects were evaluated in small study populations ( $\mathrm{n}=12-103)$. Gene mutations were observed in a single study of Finnish women at a PbB range of 1.6-5.2 $\mu \mathrm{g} / \mathrm{dL}$ (Van Larebeke et al. 2004). Results of studies on DNA damage are mixed, with no associations in adult workers at PbB means of 2.1-4.4 $\mu \mathrm{g} / \mathrm{dL}$ (Al Bakheet et al. 2013; Hengstler et al. 2003), and positive associations in a small study of children with a mean PbB of $7.3 \mu \mathrm{g} / \mathrm{dL}$ (Jasso-Pineda et al. 2012). No effect on telomere length was observed in a large NHANES study of adults with a mean $\mathrm{PbB}$ of $1.67 \mu \mathrm{g} / \mathrm{dL}$ (Zota et al. 2015). No associations were observed for SCE in a single study in workers with a mean PbB of $9.3 \mu \mathrm{g} / \mathrm{dL}$ and for MN in children with a mean PbB of 7.69 g/dL (Mielzyńska et al. 2006; Wu et al. 2002). Studies on DNA methylation showed positive associations in adult women undergoing in vitro fertilization (median $\mathrm{PbB} 2.88 \mu \mathrm{g} / \mathrm{dL}$ ), in children (mean PbB: $1.36 \mu \mathrm{g} / \mathrm{dL}$ ), and in newborns (mean umbilical cord PbB $6.6 \mu \mathrm{g} / \mathrm{dL}$ or prenatal maternal RBC Pb 1.2 g/dL) (Hanna et al. 2012; Li et al. 2016b; Pilsner et al. 2009; Wu et al. 2017).

In Vivo Animal Models and In Vitro Cultures of Mammalian Cells and Microorganisms. Numerous studies have investigated the genotoxicity of $\mathrm{Pb}$ using in vivo animal models and cultured mammalian cells and microorganisms. Rather than reviewing these numerous studies, an overview of findings is summarized below. This information was taken from the following reviews: EPA 2006, 2014c; IARC 2006; NTP 2016.

In vivo studies in animals. DNA damage has been observed in several in vivo exposure studies in rodents. DNA damage (single strand breaks), as measured in comet assays, was observed in various organ systems, bone marrow, leukocytes, and spermatozoa of mice and rats following repeated inhalation or oral exposures to $\mathrm{Pb}$ or $\mathrm{Pb}$ acetate. Many of these studies administered $\mathrm{Pb}$ by parenteral routes (intravenous, intraperitoneal). Narayana and Al-Bader (2011) and Narayana and Raghupathy (2012) did 
2. HEALTH EFFECTS

Table 2-48. Results of Genotoxicity Studies at Blood Lead Concentration (PbB) $\leq 10 \mu \mathrm{g} / \mathrm{dL}$

\begin{tabular}{|c|c|c|c|c|c|c|c|c|}
\hline $\begin{array}{l}\text { PbB or range } \\
(\mu g / d L)\end{array}$ & Population (n) & $\begin{array}{l}\text { Gene } \\
\text { mutation }\end{array}$ & $\begin{array}{l}\text { DNA } \\
\text { damage }\end{array}$ & $\begin{array}{l}\text { Telomere } \\
\text { length }\end{array}$ & SCE & $\mathrm{MN}$ & $\begin{array}{l}\text { DNA } \\
\text { methylation }\end{array}$ & Reference \\
\hline $1.6-5.2$ & Women (99) & $\uparrow$ & NA & NA & NA & NA & NA & Van Larebeke et al. 2004 \\
\hline 2.1 & Men (40) & NA & 0 & NA & NA & NA & NA & Al Bakheet et al. 2013 \\
\hline 3.28 & Children (99) & NA & NA & $\downarrow$ & NA & NA & NA & Pawlas et al. 2015 \\
\hline 4.4 & Workers (78) & NA & 0 & NA & NA & NA & NA & Hengstler et al. 2003 \\
\hline 7.3 & Children (12) & NA & $\uparrow$ & NA & NA & NA & NA & Jasso-Pineda et al. 2012 \\
\hline 1.67 & Adults $(6,796)^{a}$ & NA & NA & 0 & NA & NA & NA & Zota et al. 2015 \\
\hline 9.3 & Workers (34) & NA & NA & NA & 0 & NA & NA & Wu et al. 2002 \\
\hline 7.69 & Children & NA & NA & NA & NA & 0 & NA & Mielzyńska et al. 2006 \\
\hline$>0.73$ & Women (43) & NA & NA & NA & NA & NA & $\downarrow$ & Hanna et al. 2012 \\
\hline 1.45 & Adults $(78)^{b}$ & NA & NA & NA & NA & NA & $\downarrow$ & Li et al. 2016b \\
\hline $6.6^{c}$ & Newborns (103) & NA & NA & NA & NA & NA & $\uparrow \downarrow$ & Pilsner et al. 2009 \\
\hline 8 & Workers (100) & NA & $\uparrow$ & NA & NA & NA & NA & Akram et al. 2019 \\
\hline $1.2^{\mathrm{d}}$ & Newborns (268) & NA & NA & NA & NA & & $\downarrow$ & Wu et al. 2017 \\
\hline
\end{tabular}

aNHANES participants.

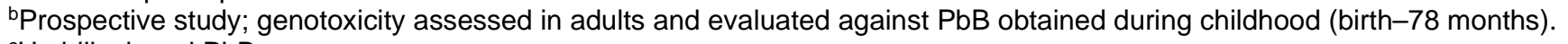

umbilical cord PbB.

dMaternal RBC lead at gestation week 28.

$\uparrow=$ increase observed for specific effect; $\downarrow=$ decrease observed for specific effect; $\uparrow \downarrow=$ decreased DNA methylations at some differentially methylated regions, and increased DNA methylation at other regions; $0=$ no effect observed; DNA = deoxyribonucleic acid; $M N=$ micronuclei; $N A=$ not assessed;

NHANES = National Health and Nutrition Examination Survey; RBC = red blood cell; SCE = sister chromatid exchange 
not find DNA damage in rats that received oral doses of lead nitrate at levels that produced necrotic changes in the liver. Global hypomethylation in hepatic DNA of rats was observed following single intravenous injection of $\mathrm{Pb}$ nitrate; hypomethylation was associated with an increase in cell proliferation. Exposure to $\mathrm{Pb}$ compounds is correlated with increased DNA synthesis and cell proliferation in the mammalian liver following intravenous injection. Numerous studies have assessed $\mathrm{Pb}$ compounds for chromosomal damage. Chromosomal aberrations were observed in bone marrow cells and spermatocytes of mice and rats following single or repeated exposure (intraperitoneal, gavage, dietary); however, the increase in aberrations did not consistently demonstrate dose-dependence.

Exposure to $\mathrm{Pb}$ compounds has been associated with SCEs in bone marrow of mice and rats following intravenous exposure. Studies assessing $\mathrm{Pb}$ compounds for $\mathrm{MN}$ formation in bone marrow erythrocytes of rats and mice were positive for multiple exposure routes (gavage, drinking water, intraperitoneal).

In vitro studies in human cell lines. In vitro studies in human cells lines have yielded mixed results. $\mathrm{Pb}$ acetate was weakly mutagenic in keratinocytes in the presence of 6-thioguanine, but not mutagenic in human foreskin, fibroblasts, or lung carcinoma cells. Results of assays assessing Pb compounds for DNA damage in human cell cultures were inconsistent. Double or single DNA strand breaks have been observed in peripheral blood lymphocytes, endothelial cells, hTERT-immortalized human skin fibroblasts, and HepG2 cells, but not in HeLa cells. DNA-protein crosslinks were observed in lymphoma cells exposed to $100 \mu \mathrm{M} \mathrm{Pb}$ acetate, although cross-links were not observed for $\mathrm{Pb}$ nitrate at concentrations up to $10,000 \mu \mathrm{M}$. Studies investigating SCEs and MN formation in human lymphocytes were positive following exposure to $\mathrm{Pb}$ nitrate and $\mathrm{Pb}$ chloride; however, no SCEs were observed in human lung cells or primary lymphocytes exposed to $\mathrm{Pb}$. Interpretation of in vitro studies is challenging because concentrations used in these studies typically are very high and are not relevant to environmental or occupational exposures. As discussed in Section 3.1.2 (Toxicokinetics, Distribution), $>99 \%$ of $\mathrm{Pb}$ in blood is bound to erythrocytes, leaving $<1 \%$ available in plasma. Thus, plasma levels of $\mathrm{Pb}$ are far lower (at least two orders of magnitude) than the concentrations examined in in vitro studies in human cell lines. This leads to the introduction of considerable bias when interpreting study results (Bannon and Williams 2017).

In vitro studies in prokaryotic and mammalian cells. Mutagenicity tests of $\mathrm{Pb}$ compounds in prokaryotic organisms have mostly yielded negative results. Studies assessed gene mutation and DNA damage in Salmonella typhimurium, Escherichia coli, and Bacillus subtilis and gene conversion and mitotic recombination in Saccharomyces cerevisiae in the presence or absence of metabolic activation. The only 
$\mathrm{Pb}$ compound that yielded positive results for gene mutation in S. typhimurium and E. coli was $\mathrm{Pb}$ bromide. Results of in vitro studies in mammalian cells for $\mathrm{Pb}$ compounds are mixed. Mutagenicity assays (hypoxanthine phosphorybosyl transferase [HPRT] and glutamate pyruvate transaminase [gpt] assays) were mutagenic in Chinese hamster ovary (CHO) and CHV79 cells at higher concentrations $(>100 \mu \mathrm{M})$ and negative at lower concentrations $(<100 \mu \mathrm{M})$. Pb chloride was the only $\mathrm{Pb}$ compound that was consistently mutagenic (gpt assay) in CHO cells at low concentrations (0.1-1.1 $\mu \mathrm{M}$; equivalent to 2.3-23 $\mu \mathrm{g} / \mathrm{dL}$ ). Comet assays assessing $\mathrm{Pb}$ acetate for DNA damage (single strand breaks) in undifferentiated PC12 cells and mouse bone marrow mesenchymal stem cells were positive. Concentration-dependent increases in DNA-protein crosslinks were observed in hepatoma cells exposed to $\mathrm{Pb}$ nitrate, although $\mathrm{Pb}$ acetate did not induce single or double DNA strand breaks or DNA crosslinks in CHV79 cells. Exposure to $\mathrm{Pb}$ nitrate or $\mathrm{Pb}$ glutamate did not induce chromosomal aberrations in $\mathrm{CHO}$ cells. Assays assessing Pb compounds for SCEs in CHV79 cells were negative when fewer cells per concentrations were utilized (25-30 cells), but were positive when the number of cells per concentration was increased (100 cells). Conflicting results were reported for MN formation in Chinese hamster cells.

Mechanisms of Action. Several mechanisms of action are likely involved in the genotoxic effects of $\mathrm{Pb}$ (EPA 2014c; IARC 2006; NTP 2016). Studies in occupationally exposed populations have found significant correlations between DNA breaks, decreased glutathione levels in the lymphocytes, and increased production of ROS, which may indicate oxidative stress as a possible mechanism for this response. The production of ROS after $\mathrm{Pb}$ exposure is a multi-pathway process, which results from oxidation of ALA, membrane and lipid oxidation, $\mathrm{NAD}(\mathrm{P}) \mathrm{H}$ oxidase activation, and antioxidant enzyme depletion. Disruption of functional metal ions that form enzymes (superoxide dismutase [SOD], catalase [CAT], and glutathione peroxidase [GPx]) may occur as part of this process.

\subsection{GENERAL CELLULAR MECHANISMS OF ACTION}

\subsubsection{Perturbation of Ion Homeostasis}

$\mathrm{Pb}$ exerts many of its adverse effects by perturbing ion homeostasis. This perturbation occurs when $\mathrm{Pb}$ displaces other metal ions such as iron, calcium, zinc, magnesium, selenium, and manganese, interfering with the critical biological processes mediated by the ions themselves or by enzymes and proteins that require these ions (reviewed by EPA 2014c; Flora et al. 2012). Among the biological processes that Pb has been shown to affect via its impact on ion homeostasis are: calcium homeostasis; transportation of 
ions across cell membranes; cellular energetics; and the functioning of numerous proteins involved in cell signaling, growth and differentiation, gene expression, energy metabolism, and biosynthetic pathways.

Calcium Homeostasis. Many of Pb’s adverse effects can be traced back to its ability to displace calcium, leading to perturbations of numerous calcium-dependent cellular functions, including energy metabolism, apoptosis, cellular motility, signal transduction, and hormonal regulation (reviewed by EPA 2014c). In addition, intracellular migration of $\mathrm{Pb}$ has been shown in several cell lines (HEK293, HeLa, and PC12) to occur via calcium channels; higher $\mathrm{Pb}$ permeation correlated with lower calcium concentrations, suggesting that $\mathrm{Pb}$ competed with calcium for the channel binding sites.

Ion Transport. $\mathrm{Pb}$ has been shown to disrupt the transportation of critical cations across the cell membrane by decreasing the activity of ATPases (including $\mathrm{Na}+\mathrm{K}+-, \mathrm{Ca} 2+$, and Mg2+-ATPases; reviewed by EPA 2014c). Pb-induced inhibition of ATPase activities has been shown in the kidneys, livers, erythrocytes, and brain synaptosomes of rats exposed to $\mathrm{Pb}$ in drinking water; in testes of rat pups exposed during lactation and postweaning; in primary cerebellar granule neuronal cultures of rat pups exposed pre- and postnatally; in rabbit kidney membranes and sarcoplasmic reticulum exposed in vitro; and in human erythrocyte ghosts. Furthermore, blood or hair Pb levels were inversely correlated with ATPase activities in erythrocytes in several human epidemiological studies.

In addition to ATPases, Pb's action on ion transport includes competitive inhibition of voltage-gated calcium channels (reviewed by EPA 2014c). A number of in vitro studies have demonstrated inhibition of calcium transport via voltage gated channels in cultured neurons and neuroblastoma cells, bovine adrenal chromaffin cells, and human embryonic kidney cells. Inhibition of calcium transportation via voltage-gated channels can disrupt release of neurotransmitters, and impaired neurotransmitter release has, in fact, been shown with $\mathrm{Pb}$ exposure at low in vitro levels. In addition to inhibiting calciumdependent neurotransmitter release, $\mathrm{Pb}$ may mimic calcium, thereby increasing neurotransmitter release in some circumstances. For example, $\mathrm{Pb}$ exposure in vitro has been shown to induce the spontaneous release of norepinephrine from bovine adrenal chromaffin cells and increase the release of catecholamine from PC12 cells. It has been suggested that Pb may trigger spontaneous neurotransmitter release via activation of calcium/calmodulin-dependent protein kinase II-dependent phosphorylation of synapsin I, or by directly activating synaptotagmin I (a calcium-sensing protein that regulates neurotransmitter release). Intracellular migration of $\mathrm{Pb}$ has been shown to occur via calcium channels; higher $\mathrm{Pb}$ permeation in several cell lines (HEK293, HeLa, and PC12) correlated with lower calcium concentrations, suggesting that $\mathrm{Pb}$ competed with calcium for the channel binding sites. 
$\mathrm{Pb}$ also disrupts the activity of calcium-dependent potassium channels, as shown by increased efflux of potassium from inverted erythrocyte vesicles, and alterations in potassium channel activation in erythrocytes exposed to $\mathrm{Pb}$ (reviewed by EPA 2014c). The nature of the effect on potassium channels is dose-dependent; at low $\mathrm{Pb}$ concentrations $(<10 \mu \mathrm{M})$, potassium channels are activated, while inhibition of the channels is seen at higher $\mathrm{Pb}$ concentrations. As with calcium channels, alterations in potassium channel activity may also disrupt neurotransmitter release. In rats exposed to $\mathrm{Pb}$ in utero and postnatally, potassium-stimulated release of hippocampal GABA was decreased at low exposure levels, but enhanced GABA release was observed at higher exposures (in the absence of calcium).

Cellular Energetics. Evidence indicating that $\mathrm{Pb}$ exposure perturbs mitochondrial function and cellular energy metabolism is abundant (as reviewed by EPA 2014c). In rats exposed to $\mathrm{Pb}$ via diet or drinking water, renal tubular and epididymal mitochondria exhibited swelling, rupture of the outer membrane, distorted cristae or loss of cristae, vacuolization, inclusion bodies, and fusion with nearby mitochondria. As discussed further in Section 2.21.6, Apoptosis, $\mathrm{Pb}$ exposure has been shown to open the mitochondrial transmembrane pore, initiating the apoptotic caspase cascade. Evidence for Pb’s effect on energy metabolism includes decreased ATP levels and/or adenylate energy charge (AEC) (along with increased ADP, AMP, and/or adenosine levels) in forebrain synaptosomes from rats exposed via drinking water, in cerebellar granule neuronal cultures from rats exposed by drinking water, in PC-12 cells exposed in vitro, and in isolated mitochondria exposed in vitro. In osteoblasts exposed in vitro, $\mathrm{Pb}$ inhibited both coupled and uncoupled respiratory oxygen use in mitochondria. Pb has been proposed to behave as a classic chemical uncoupler of respiration, abolishing the proton gradient necessary for oxidative phosphorylation. In the muscles of rats exposed to $\mathrm{Pb}$ in drinking water, decreased activities of the enzymes of complex I and IV of the respiratory chain were observed. However, in forebrain synaptosomes from rats exposed to $\mathrm{Pb}$ in vivo, oxidative phosphorylation was not inhibited, despite the fact that ATP levels were decreased.

$\mathrm{Pb}$ may affect cellular energetics via perturbation of the glycolysis pathway. Decreased glycolysis was observed in osteoblasts and erythrocytes exposed to Pb in vitro (reviewed by EPA 2014c). However, increased levels of glycolytic enzymes were noted in workers with higher blood $\mathrm{Pb}$ levels, when compared with workers with lower blood $\mathrm{Pb}$, suggesting that $\mathrm{Pb}$ may activate anaerobic glycolysis.

Depletion of cellular nucleotide pools required for ATP synthesis has also been observed after Pb exposure of human erythrocytes in vitro and in rats exposed via drinking water (reviewed by EPA 2014c). This effect may be mediated by Pb-induced inhibition of enzymes involved in nucleotide biosynthesis in 
erythrocytes, including adenine phosphoribosyltransferase (see Impaired Protein Function below) and NAD synthetase (which depends on magnesium for activity). In support of the latter mechanism, in humans exposed to $\mathrm{Pb}, \mathrm{PbB}$ levels were inversely correlated with NAD synthetase activity.

Impaired Protein Function. Pb impairs the functions of numerous proteins, with concomitant effects on signaling, growth and differentiation, gene expression, energy metabolism, and biosynthetic pathways. The mechanisms by which $\mathrm{Pb}$ alters protein activity are by displacing metal cofactors or binding to sulfhydryl groups (reviewed by EPA 2014c). Table 2-49 shows proteins known to be bound to or otherwise altered by $\mathrm{Pb}$, along with their functions and brief summaries of the evidence for $\mathrm{Pb}$-induced alterations. As the table suggests, Pb-induced alterations in proteins may play a role in its adverse effects on the neurological, hematological, cardiovascular, and skeletal systems.

Through its displacement of calcium, Pb perturbs the function of several calcium-dependent proteins, including protein kinase $\mathrm{C}$, calmodulin, osteocalcin, the mitochondrial transmembrane pore, and NAD(P)H oxidase (reviewed by EPA 2014c). The protein kinase $\mathrm{C}$ family of enzymes is important to cell signaling, growth, and differentiation. Pb exposure has been shown to activate PKC in a number of cell types tested in vitro (see table), and to decrease its activity in mouse macrophages and rat brain cortex. Pb stimulates calmodulin activity, as shown by increased activity of several calmodulindependent enzymes, and increased binding of calmodulin to brain membranes. In experiments testing the affinity of metal cations to bind calmodulin, $\mathrm{Pb}$ was more potent than mercury, cadmium, iron, and even calcium. Pb binding to calmodulin has been postulated as a mechanism for its stimulatory effect on $\mathrm{Ca}^{2+} / \mathrm{Mg}^{2+}$ ATPase. Calmodulin plays an essential role in maintaining calcium homeostasis and regulating calcium-dependent cell signaling important to structural integrity, gene expression, and maintaining membrane potential (reviewed by EPA 2014c).

Skeletal effects of $\mathrm{Pb}$ may be mediated in part by Pb's interference with another calcium-dependent protein: osteocalcin (reviewed by EPA 2014c). The binding of Pb to osteocalcin is much stronger than binding of calcium, and $\mathrm{Pb}$ binding alters the structure of osteocalcin. The conformational change in osteocalcin induced by $\mathrm{Pb}$ has been postulated as the mechanism by which $\mathrm{Pb}$ exposure diminishes the adsorption of osteocalcin to hydroxyapatite. 


\section{Table 2-49. Effects of Lead (Pb) on Function of Various Proteins}

\begin{tabular}{|c|c|c|}
\hline Protein & General function & Effect of Pb; summary of evidence \\
\hline \multicolumn{3}{|c|}{ Calcium-dependent proteins } \\
\hline $\begin{array}{l}\text { Calcium binding proteins } \\
\text { (CABPs I and II) }\end{array}$ & $\begin{array}{l}\text { Regulation of calcium } \\
\text { signaling, especially in } \\
\text { neuronal cells }\end{array}$ & $\begin{array}{l}\text { No data } \\
-\mathrm{Ca}^{2+} \text { displacement shown in vitro. }\end{array}$ \\
\hline $\begin{array}{l}\mathrm{Ca}^{2+} \text {-dependent } \mathrm{K}^{+} \\
\text {channel }\end{array}$ & $\begin{array}{l}\text { Ion transport; activation of } \\
\text { channels regulates neuron } \\
\text { firing and neurotransmitter } \\
\text { release }\end{array}$ & $\begin{array}{l}\text { Activates or inhibits channel } \\
\text {-Pb promoted efflux of } \mathrm{K}^{+} \text {from inverted red blood cell vesicles. } \\
\text { - } \mathrm{Pb} \text { induced activation of } \mathrm{K}^{+} \text {channel in erythrocytes at low } \mathrm{Pb} \text { concentrations and } \\
\text { inhibited activity at high concentrations. }\end{array}$ \\
\hline Calmodulin & $\begin{array}{l}\text { Cell signaling, including } \\
\text { structural integrity, gene } \\
\text { expression, and maintenance } \\
\text { of membrane potential }\end{array}$ & $\begin{array}{l}\text { Amplifies calmodulin activity } \\
\text {-Pb activated calmodulin-dependent phosphodiesterase and cyclic nucleotide } \\
\text { phosphodiesterase activities. } \\
\text {-Pb stimulated brain membrane phosphorylation. } \\
\text {-Pb increased binding of calmodulin to brain membranes. }\end{array}$ \\
\hline $\begin{array}{l}\text { Mitochondrial } \\
\text { transmembrane pore } \\
(\mathrm{MTMP})\end{array}$ & $\begin{array}{l}\text { Triggers mitochondrial } \\
\text { apoptosis cascade when open }\end{array}$ & $\begin{array}{l}\text { Opens MTMP, triggering apoptosis } \\
\text {-Pb increased mitochondria-regulated apoptotic indicators (cytochrome c, caspases) } \\
\text { in rat retinal rod cells and hepatic oval cells in vitro. }\end{array}$ \\
\hline $\mathrm{NAD}(\mathrm{P}) \mathrm{H}$ oxidase & $\begin{array}{l}\text { Inflammatory mediator; triggers } \\
\text { oxidative burst (via production } \\
\text { of superoxide) in response to } \\
\text { infection }\end{array}$ & $\begin{array}{l}\text { Increases activity, leading to ROS generation } \\
-\mathrm{Pb} \text { increased protein levels of glycosylated subunit of } \mathrm{NAD}(\mathrm{P}) \mathrm{H} \text { oxidase in brain, } \\
\text { heart, and renal cortex of rats exposed via drinking water and in human coronary } \\
\text { artery endothelial cells in vitro. }\end{array}$ \\
\hline Osteocalcin & $\begin{array}{l}\text { Bone resorption, osteoclast } \\
\text { differentiation, and bone } \\
\text { growth }\end{array}$ & $\begin{array}{l}\text { Alters binding of osteocalcin to hydroxyapatite } \\
\text {-Pb exposure has been shown to both increase and decrease binding of osteocalcin } \\
\text { to hydroxyapatite. }\end{array}$ \\
\hline Parvalbumin & $\begin{array}{l}\text { Unclear; may buffer } \mathrm{Ca}^{2+} \\
\text { levels; expressed at high levels } \\
\text { in interneurons }\end{array}$ & $\begin{array}{l}\text { No data } \\
-\mathrm{Ca}^{2+} \text { displacement shown in vitro. }\end{array}$ \\
\hline Phospholipase $\mathrm{A}_{2}$ & $\begin{array}{l}\text { Hydrolyze fatty acids from } \\
\text { membrane phospholipids; } \\
\text { released fatty acids are } \\
\text { metabolized to bioactive lipid } \\
\text { mediators }\end{array}$ & $\begin{array}{l}\text { No data } \\
-\mathrm{Ca}^{2+} \text { displacement shown in vitro. }\end{array}$ \\
\hline
\end{tabular}




\section{Table 2-49. Effects of Lead (Pb) on Function of Various Proteins}

\begin{tabular}{|c|c|c|}
\hline Protein & General function & Effect of Pb; summary of evidence \\
\hline Protein kinase $\mathrm{C}(\mathrm{PKC})$ & $\begin{array}{l}\text { Cell signaling, especially } \\
\text { growth and differentiation }\end{array}$ & $\begin{array}{l}\text { Increases or decreases activity } \\
\text {-Pb shown to activate PKC in vitro in bovine adrenal chromaffin cells, rat brain } \\
\text { microvessels, human erythrocytes, and rabbit mesenteric arteries. } \\
\text {-Pb decreased PKC activity in mouse macrophages and rat brain cortex. }\end{array}$ \\
\hline Synaptotagmin I & $\begin{array}{l}\mathrm{Ca}^{2+} \text { sensor regulating } \\
\text { neurotransmitter release }\end{array}$ & $\begin{array}{l}\text { No data } \\
-\mathrm{Ca}^{2+} \text { displacement shown in vitro. }\end{array}$ \\
\hline Troponin C & $\begin{array}{l}\mathrm{Ca}^{2+} \text { sensor regulating muscle } \\
\text { contraction }\end{array}$ & $\begin{array}{l}\text { No data } \\
-\mathrm{Ca}^{2+} \text { displacement shown in vitro. }\end{array}$ \\
\hline \multicolumn{3}{|l|}{ Heme-dependent proteins } \\
\hline Catalase & $\begin{array}{l}\text { Antioxidant; scavenger of } \\
\text { hydrogen peroxide }\end{array}$ & $\begin{array}{l}\text { Increases or decreases activity } \\
\text {-Pb shown to increase activity in some studies and decrease activity in others, } \\
\text { possibly due to differences in species, exposure duration, dose, or other study design } \\
\text { variations. }\end{array}$ \\
\hline Guanylate cyclase & $\begin{array}{l}\text { Catalyzes synthesis of cGMP, } \\
\text { which stimulates } \\
\text { vasorelaxation in vascular } \\
\text { tissues }\end{array}$ & $\begin{array}{l}\text { Impairs production of cGMP } \\
\text {-Pb reduced cGMP in plasma and urine of rats exposed by drinking water. } \\
\text {-Pb decreased protein levels of soluble guanylate cyclase in vascular tissue. }\end{array}$ \\
\hline Hemoglobin & Oxygen transportation & $\begin{array}{l}\text { Impairs heme production needed for synthesis of hemoglobin } \\
\text {-Pb binding to hemoglobin demonstrated in human blood. }\end{array}$ \\
\hline \multicolumn{3}{|c|}{ Magnesium-dependent proteins } \\
\hline $\begin{array}{l}\text { Adenine and } \\
\text { hypoxanthine/guanine } \\
\text { phosphoribosyltransferases }\end{array}$ & Recycling of nucleotides & $\begin{array}{l}\text { Inhibits activity } \\
\text {-Pb inhibited phosphoribosyltransferase activities in erythrocytes of rats exposed via } \\
\text { drinking water and in human erythrocytes in vitro. }\end{array}$ \\
\hline NAD synthetase (Mg) & Nucleotide biosynthesis & $\begin{array}{l}\text { Decreases activity } \\
\text {-Blood Pb was inversely correlated with NAD synthetase activity in humans. }\end{array}$ \\
\hline Pyrimidine 5'-nucleotidase & $\begin{array}{l}\text { Dephosphorylates pyrimidine } \\
\text { nucleotides in erythrocytes, } \\
\text { preserving purine nucleotides } \\
\text { (e.g., ATP, ADP) necessary for } \\
\text { energy }\end{array}$ & $\begin{array}{l}\text { Alters protein conformation and amino acid positioning at active site, possibly by } \\
\text { occupying active site } \\
\text {-Pb binding and protein conformation changes observed in vitro. } \\
\text {-Pyrimidine nucleotide accumulation in erythrocytes is seen in Pb poisoning. }\end{array}$ \\
\hline
\end{tabular}


2. HEALTH EFFECTS

\section{Table 2-49. Effects of Lead (Pb) on Function of Various Proteins}

\begin{tabular}{|c|c|c|}
\hline Protein & General function & Effect of Pb; summary of evidence \\
\hline \multicolumn{3}{|l|}{ Zinc-dependent proteins } \\
\hline $\begin{array}{l}\text {-ALA ( } \delta \text {-ALAD or } \\
\text { porphobilinogen synthase) }\end{array}$ & $\begin{array}{l}\text { Heme biosynthesis (converts } \\
\delta-A L A \text { to porphobilinogen) }\end{array}$ & $\begin{array}{l}\text { Depletes } \delta \text {-ALAD, preventing heme biosynthesis and leading to accumulation of } \\
\delta \text {-ALA. } \\
\text { - } \delta \text {-ALAD shown to be major binding target of } \mathrm{Pb} \text { in erythrocytes. }\end{array}$ \\
\hline GATA zinc finger proteins & $\begin{array}{l}\text { Activation/suppression of DNA } \\
\text { transcription }\end{array}$ & $\begin{array}{l}\text { Decreases ability of GATA proteins to bind to DNA and regulate transcription } \\
\text {-Pb binding to cysteine residues and displacement of } \mathrm{Zn} \text { from GATA proteins } \\
\text { observed in vitro. } \\
\text {-Pb-bound GATA proteins exhibited reduced DNA binding. }\end{array}$ \\
\hline $\begin{array}{l}\text { Transcription factors } \\
\text { TFIIIA, Sp1, and Erg-1 }\end{array}$ & $\begin{array}{l}\text { Activation/suppression of DNA } \\
\text { transcription }\end{array}$ & $\begin{array}{l}\text { Decreases ability of TFIIIA, Sp1, and Erg-1 to bind to DNA and regulate transcription } \\
-\mathrm{Pb} \text { exposure caused dissociation of TFIIIA-DNA adducts. } \\
\text {-Pb exposure altered DNA binding profile of Sp-1 and Erg-1 in rat pups exposed via } \\
\text { lactation, leading to changes in gene expression. }\end{array}$ \\
\hline \multicolumn{3}{|c|}{ Proteins altered by lead interaction with other cations or sulfhydryl groups } \\
\hline $\begin{array}{l}\text { ATPases }\left(\mathrm{Ca}^{2+}-, \mathrm{Mg}^{2+}-\right. \\
\left.\text { and } \mathrm{Na}^{+} / \mathrm{K}^{+-}\right)\end{array}$ & Ion transport & $\begin{array}{l}\text { Decreases activity } \\
\text {-Pb decreased ATPase activities in brain, kidneys, liver, testes, and erythrocytes } \\
\text { (cells or tissues). }\end{array}$ \\
\hline $\begin{array}{l}\text { cGMP phosphodiesterase } \\
(\mathrm{Zn}, \mathrm{Mg})\end{array}$ & Hydrolysis of cGMP & $\begin{array}{l}\text { Inhibits activity } \\
\text {-Decreased activity observed in homogenized bovine retinas exposed to } \mathrm{Pb} \text { in vitro. }\end{array}$ \\
\hline Ferrochelatase (Fe) & $\begin{array}{l}\text { Heme biosynthesis; } \\
\text { incorporates } \mathrm{Fe}^{2+} \text { into } \\
\text { protoporphyrin IX to form heme }\end{array}$ & $\begin{array}{l}\text { Inhibits insertion of Fe into protoporphyrin ring, leading to substitution by } \mathrm{Zn} \\
\text {-Zn-protoporphyrin levels correlated with blood Pb levels in humans. }\end{array}$ \\
\hline $\begin{array}{l}\text { Glutathione peroxidase } \\
\text { and glutathione } \\
\text { S-transferase (Se) }\end{array}$ & Antioxidants & $\begin{array}{l}\text { Reduces uptake of Se and depletes cellular GSH and protein thiols, resulting in } \\
\text { altered GST and GPx enzyme activities } \\
\text {-Decreased activity, often with compensatory upregulation of the enzymes, seen in } \\
\text { Pb-exposed animals and humans. }\end{array}$ \\
\hline
\end{tabular}


Table 2-49. Effects of Lead (Pb) on Function of Various Proteins

\begin{tabular}{ll}
\hline Protein & General function \\
\hline Metallothionein $(\mathrm{Zn}, \mathrm{Cu})$ & $\begin{array}{c}\text { Trace element homeostasis; } \\
\text { free radical scavenging }\end{array}$
\end{tabular}

Effect of Pb; summary of evidence

Sequestered by metallothionein, providing protective effect

- $\mathrm{Pb}$ toxicity is seen at lower blood $\mathrm{Pb}$ levels in humans with low expression of metallothionein or low $\mathrm{Pb}$ binding to metallothionein.

-Pb induced production of metallothionein in mice exposed via intraperitoneal or intravenous injection and in rats exposed via intraperitoneal injection, but not in rats exposed via drinking water.

-Presence of zinc metallothionein reduced effect of $\mathrm{Pb}$ on membrane integrity in hepatocytes exposed in vitro.

- $\mathrm{Pb}$ nephrotoxicity and preneoplastic and neoplastic lesions in the testes, bladder, and kidneys were more severe or seen at increased incidences in metallothionein-null mice compared with wild-type.

\begin{tabular}{lll}
\hline Superoxide dismutase & $\begin{array}{l}\text { Antioxidant; catalyzes } \\
\text { conversion of superoxide to } \\
\text { hydrogen peroxide; inhibits } \\
\text { oxidative inactivation of nitric } \\
\text { oxide }\end{array}$ & $\begin{array}{l}\text { Increased or decreased activity } \\
\text {-Pb shown to increase activity in several studies and decrease activity in others, } \\
\text { possibly due to differences in species, exposure duration, dose, or other study design } \\
\text { variations. }\end{array}$ \\
\hline Thymosin $\beta-4$ & $\begin{array}{l}\text { Actin regulation; exerts } \\
\text { angiogenic, anti-inflammatory, } \\
\text { and cardioprotective effects on } \\
\text { the heart }\end{array}$ \\
\hline
\end{tabular}

ADP = adenosine diphosphate; $\delta$-ALA = aminolevulinic acid $; \delta-A L A D=$ aminolevulinic acid dehydratase; ATP = adenosine triphosphate; $A T P a s e=$ family of phosphatase enzymes that breakdown ATP and ADP; cGMP = cyclic guanosine monophosphate; DNA = deoxyribonucleic acid; Erg-1 = early growth response protein 1; GST = glutathione S-transferase; GSH = glutathione; GPX = glutathione peroxidase; $N A D=$ nicotinamide adenine dinucleotide; $N A D(P) H=$ the reduced form of nicotinamide adenine dinucleotide phosphate; ROS = reactive oxygen species; Sp1 = Transcription factor specificity protein 1; TFIIIA = transcription factor IIIA

Sources: EPA 2014c; Ahamed and Siddiqui 2007; Flora et al. 2012; Gonick 2011 
Other calcium-dependent proteins bound to or impaired by Pb include parvalbumin, phospholipase A2, synaptotagmin I (see Ion Transport above), troponin C, the mitochondrial transmembrane pore (see Section 2.21.6, Apoptosis), and NAD(P)H oxidase (see Section 2.21.3, Oxidative Stress) (reviewed by EPA 2014c).

$\mathrm{Pb}$ also displaces zinc in a number of critical proteins, including ALAD, GATA proteins, and several zinc-binding transcription factors (TFIIIA, Sp1, and Erg-1) (reviewed by EPA 2014c). Section 2.8 provides a detailed discussion of Pb's effects on ALAD and heme biosynthesis. Binding of Pb to zincbinding domains in GATA proteins and transcription factors inhibits their binding to DNA and impairs their ability to regulate gene expression (see Section 2.21.5, Epigenetic Effects, below for further detail).

Through competitive inhibition of magnesium-dependent proteins, $\mathrm{Pb}$ also affects the activities of adenine and hypoxanthine/guanine phosphoribosyltransferases, cyclic guanosine monophosphate (cGMP) phosphodiesterase, and pyrimidine 5'-nucleotidase (reviewed by EPA 2014c). In erythrocytes, adenine phosphoribosyltransferase catalyzes the synthesis of nucleotides via the adenine salvage pathway; $\mathrm{Pb}$ exposure has been shown to decrease nucleotide pools in human erythrocytes in vitro and in erythrocytes from rats exposed via drinking water. Inhibition of cGMP phosphodiesterase, a magnesium-dependent enzyme regulating cGMP signaling in smooth muscle contraction and relaxation, has been observed in homogenized bovine retinas cultured with $\mathrm{Pb}$. $\mathrm{Pb}$ inhibits magnesium binding in pyrimidine 5'-nucleotidase, inhibiting its activity by changing its active site conformation. Pyrimidine 5'-nucleotidase occurs at high levels in erythrocytes, where it dephosphorylates pyrimidine nucleotides while leaving purine nucleotides (used as an energy source in erythrocytes, as they lack mitochondria), intact. Basophilic stippling of erythrocytes, a common feature of $\mathrm{Pb}$ poisoning, is also seen in individuals with inherited pyrimidine-5'-nucleotidase deficiency (Rees et al. 2003), providing supporting evidence that $\mathrm{Pb}$ inactivates the enzyme.

\subsubsection{Protein Binding/Sequestration}

A number of low molecular-weight proteins, including metallothionein, have been shown to bind (through thiol residues) to $\mathrm{Pb}$, forming inclusion bodies in the kidney, liver, lung, and glial cells (reviewed by EPA 2014c; Gonick 2011). In the case of metallothionein, the effect of the binding is to sequester $\mathrm{Pb}$, protecting the exposed cells and tissues. The strongest evidence for the protective effect of metallothionein comes from studies of metallothionein-null mice, which exhibit more severe Pb-induced renal toxicity, as well as increased incidences of neoplastic and nonneoplastic lesions in the testes, 
bladder, and kidneys, compared with wild-type mice. Supporting this finding is the observation that higher blood $\mathrm{Pb}$ levels, as well as more pronounced $\mathrm{Pb}$-induced effects on systolic blood pressure and kidney function, were observed in exposed workers with a metallothionein mutation (compared with those exhibiting a normal metallothionein genotype). Metallothionein levels have been shown to be induced by $\mathrm{Pb}$ exposure in mice and in rats pretreated with zinc.

In erythrocytes, the major Pb-binding protein is ALAD; hemoglobin also binds $\mathrm{Pb}$ (reviewed by EPA 2014c; Gonick 2011). In exposed humans, polymorphisms in the ALAD gene that increase the $\mathrm{Pb}$-binding capacity of its protein product (e.g., ALAD-2) were observed to decrease blood $\mathrm{Pb}$ levels and biomarkers for $\mathrm{Pb}$ toxicity, including plasma levulinic acid, zinc protoporphyrin, cortical bone $\mathrm{Pb}$ levels, and dimercaptosuccinic acid-chelatable $\mathrm{Pb}$ levels. Other proteins that bind $\mathrm{Pb}$ in erythrocytes include pyrimidine 5'-nucleotidase and acyl-coenzyme A binding protein.

In rat kidneys, inclusion bodies consisting of Pb-bound proteins have been observed in a number of studies (reviewed by EPA 2014c; Gonick 2011). These inclusion bodies are initially observed in the cytosol, but appear to translocate to the nucleus, as they disappear concomitantly with the appearance of intranuclear inclusion bodies. The primary Pb-bound protein in the kidney (a $32 \mathrm{kDa}$ protein with an isoelectric point of 6.3, named p32/6.3) has not been identified, but has been shown to be enriched in the brain and is highly conserved across species (rats, mice, dogs, chickens, and humans). Studies in rats exposed by food or drinking water showed that p32/6.3 is not found in the kidneys of untreated rats but rather is induced by $\mathrm{Pb}$ exposure. Other Pb-binding proteins identified in the kidneys of rats or humans include acyl-CoA binding protein and thymosin $\beta-4$ (the latter is involved in actin regulation).

\subsubsection{Oxidative Stress}

$\mathrm{Pb}$ exposure has resulted in oxidative damage in several tissues in humans and rats, including the brain, kidneys, reproductive organs, heart, and erythrocytes (reviewed by EPA 2014c; Ahamed and Siddiqui 2007). Oxidative damage may play a role in Pb-induced toxicity in these tissues, including neurological effects, hypertension and other cardiovascular effects, and diminished fertility. Pb induces oxidative stress through several mechanisms, including increased production of ROS via inhibition of heme biosynthesis and activation of NAD(P)H oxidase; stimulation of lipid peroxidation and alteration of lipids enhancing their susceptibility to lipid peroxidation; and inactivation and/or depletion of antioxidant enzymes. Through the increased production of ROS, which sequesters nitric oxide, $\mathrm{Pb}$ exposure also leads to perturbation of nitric oxide signaling that is critical to vasodilation. 
Exposure to $\mathrm{Pb}$ triggers increased production of ROS via its effects on heme biosynthesis. In erythrocytes, $\mathrm{Pb}$ has been shown to bind to $\delta$-ALAD as well as to inhibit its activity by interfering with the zinc ions the enzyme requires for heme biosynthesis; in fact, inhibition of $\delta$-ALAD activity is inversely correlated with PbB levels in humans (reviewed by EPA 2014c; Ahamed and Siddiqui 2007). $\delta$-ALAD catalyzes the conversion of $\delta$-ALA to porphobilinogen; thus, its inhibition results in accumulation of $\delta$-ALA in blood and in urine. In these environments, $\delta$-ALA undergoes autoxidation, yielding superoxide and hydroxyl radicals, as well as hydrogen peroxide and an ALA radical. In addition, through subsequent reduction of ferricytochrome c and transfer of electrons from oxyhemoglobin, methemoglobin, and ferric and ferrous iron complexes, oxidized $\delta$-ALA also produces ROS.

$\mathrm{Pb}$ may also increase intracellular ROS by upregulating expression of NAD(P)H oxidase, an enzyme that produces superoxide anion via reaction of $\mathrm{NAD}(\mathrm{P}) \mathrm{H}$ and molecular oxygen, but data are limited (reviewed by EPA 2014c). Increased protein expression of the glycosylated subunit of NAD(P)H oxidase was observed in tissues of rats exposed to $\mathrm{Pb}$ in drinking water, and in human endothelial cells in vitro.

ROS produced via $\mathrm{Pb}$ effects on $\delta$-ALA and/or NAD(P)H oxidase can damage membrane lipids through peroxidation. In addition, however, $\mathrm{Pb}$ has been shown to catalyze ferrous ion-initiated lipid peroxidation (reviewed by EPA 2014c). Furthermore, there is evidence that Pb exerts effects on membrane lipids that render them more vulnerable to peroxidation (reviewed by EPA 2014c; Ahamed and Siddiqui 2007). For example, $\mathrm{Pb}$ has been shown to alter the composition of fatty acids in chicks exposed by drinking water, such that a higher fraction of longer fatty acids (such as arachidonic acid) and lower fraction of shorter fatty acids (compared with controls) were observed. Oxidative potential of fatty acids is correlated with both length and desaturation (i.e., the number of double bonds; the hydrogen on a double bond is easier to remove). It has been proposed that $\mathrm{Pb}$ may stimulate both elongation and desaturation of fatty acids, increasing their susceptibility to peroxidation. Alterations in lipid composition may also affect membrane permeability and functions, including the activity of membrane-associated enzymes, solute transport functions, endo- and exocytosis, and signal transduction.

Increased circulating ROS (specifically, superoxide anion) can inactivate nitric oxide, an endogenously produced molecule that plays an important role in vasodilation (reviewed by EPA 2014c). Depletion of nitric oxide has been observed in animals exposed to $\mathrm{Pb}$, as well as in human and animal immune cells treated in vitro. In addition, nitric oxide depletion is believed to be the mechanism behind $\mathrm{Pb}$-induced upregulation of nitric oxide synthases seen in vascular tissues after Pb exposure. Nitric oxide depletion 
occurs when it reacts with superoxide anion to form the highly reactive peroxynitrite anion, which itself damages DNA and proteins. Levels of nitrotyrosine, which results from peroxynitrite-induced nitration of tyrosine residues in proteins, were increased in plasma and other tissues after in vivo exposure to $\mathrm{Pb}$. In vascular tissues, nitric oxide induces vasorelaxation via cGMP signaling (reviewed by EPA 2014c). Exposure of rats to $\mathrm{Pb}$ in drinking water for 1-3 months markedly reduced cGMP levels in both blood and urine. Synthesis of cGMP is catalyzed by soluble guanylate cyclase, a heme-dependent enzyme. $\mathrm{Pb}$ exposure has been shown to reduce protein levels of soluble guanylate cyclase in vascular tissues; alleviation of this effect by antioxidant treatment (ascorbic acid) demonstrated that this finding was mediated, at least in part, by increased oxidative stress.

In human epidemiological studies, the ratio of oxidized glutathione (glutathione disulfide or GSSG) to reduced glutathione (GSH), a measure of oxidative stress, was positively correlated with blood Pb levels (reviewed by EPA 2014c; Ahamed and Siddiqui 2007; Flora et al. 2012). The effects of Pb on oxidative stress levels may occur through depletion of antioxidant levels in addition to stimulation of ROS, as oxidative stress occurs when the antioxidant capacity of the body is exceeded. $\mathrm{Pb}$ forms covalent bonds with sulfhydryl groups in antioxidant enzymes such as GSH, glutathione reductase (GR), and glutathione S-transferase (GST) (reviewed by EPA 2014c; Ahamed and Siddiqui 2007; Flora et al. 2012). In humans, animals, and in vitro studies, decreased GSH in blood and organs has been associated with $\mathrm{Pb}$ exposure. After long-term exposure to $\mathrm{Pb}$, increased GSH levels, attributed to compensatory upregulation of GSH biosynthesis, have been reported. Like GSH, GR (which reduces GSSG back to GSH) and GST also have disulfides at their active site that could be bound by $\mathrm{Pb}$. Studies examining GR and GST activity after Pb exposure used varying study designs and showed both increases and decreases; it is not clear whether the differences in results reflect species, strain, dose, or duration differences.

Pb's capacity to compete with cations and its interference with heme biosynthesis have also been suggested as potential mechanisms for its ability to alter levels of SOD, CAT, GPx, and GST (reviewed by EPA 2014c; Flora et al. 2012; Ahamed and Siddiqui 2007). SOD forms require copper, zinc, or manganese, cations that $\mathrm{Pb}$ may displace, while catalase is a heme-dependent enzyme. Several studies in humans and animals have shown alterations in SOD and CAT activity, with some evidence for a nonlinear dose-response relationship. EPA (2014c) suggested that increased SOD and CAT may occur at low doses as a result of ROS generation by $\mathrm{Pb}$, while at higher doses, $\mathrm{Pb}$ may inactivate the enzymes. $\mathrm{Pb}$ exposure also alters activities of GPx and GST, potentially by reducing the uptake of selenium (required by GPx) and/or disrupting protein thiols (necessary for GST function). Decreased GPx and GST 
activities have been observed, along with compensatory upregulation of these enzymes, in Pb-exposed humans and animals.

\subsubsection{Inflammation}

Increasing oxidative stress through ROS generation and depletion of antioxidant enzymes may be one mechanism by which $\mathrm{Pb}$ induces an inflammatory response (reviewed by EPA 2014c). Inflammation, considered a hallmark of Pb exposure (EPA 2014c), may also be triggered by pro-inflammatory signaling and cytokine production. Inflammation has been seen after $\mathrm{Pb}$ exposure in many different cell types, as well as in the kidneys of rats exposed to $\mathrm{Pb}$ in drinking water.

Oxidative stress is known to activate the pro-inflammatory nuclear transcription factor kappa B (NFkB). In the rat kidney, $\mathrm{Pb}$-induced inflammation was accompanied by activation of $\mathrm{NF} \kappa \mathrm{B}$ as well as lymphocyte and macrophage infiltration (reviewed by EPA 2014c). Pb has been shown to stimulate the expression of pro-inflammatory signal mediators including NFkB, activator protein-1 (AP-1), and c-Jun, and to stimulate phosphorylation of the Erk/MAPK pathway. In addition, exposure to $\mathrm{Pb}$ is associated with increased production of prostaglandins, which also mediate pro-inflammatory messaging. Increases in arachidonic acid production, leading to increases in prostaglandins E2 and F2 and thromboxane levels, have been seen in $\mathrm{Pb}$-exposed workers as well as in animals and in cultured cells systems exposed to $\mathrm{Pb}$. In vascular smooth muscle cells, $\mathrm{Pb}$ has been shown to activate phospholipase A2, which may explain its ability to stimulate the release of arachidonic acid.

In both human epidemiological and laboratory animal studies, $\mathrm{Pb}$ exposure has been demonstrated to increase cytokine production (reviewed by EPA 2014c). In these studies, a fairly consistent picture of decreasing Th-1 cytokines and increasing Th-2 cytokines has emerged. EPA (2014c) outlined three modes by which $\mathrm{Pb}$ influences cytokine production: (1) direct action on macrophages to increase proinflammatory cytokines such as TNF- $\alpha$ and interleukin 6 (IL-6); (2) skew the ratio of IL-12 to IL-10, leading to suppression of Th-1 cell responses and stimulation of Th-2 cell responses; and (3) during acquired immune response occurring after $\mathrm{Pb}$ exposure, production of cytokines by Th-1 lymphocytes is suppressed, and Th-2 cytokines are increased. The net result of these changes is consistent with the proinflammatory picture seen with $\mathrm{Pb}$ exposure.

Human epidemiological studies have provided evidence that $\mathrm{Pb}$ exposure skews immune responses toward Th-2 pro-inflammatory responses (reviewed by EPA 2014c). Higher blood Pb levels in children 
were associated with increased serum levels of Il-4 (which induces differentiation of Th0 cells to the Th-2 phenotype) and lower levels of interferon gamma (IFN- $\gamma$ ). In adult students in Korea, higher blood $\mathrm{Pb}$ levels were positively associated with increased TNF- $\alpha$ and IL-6; a $1 \mu \mathrm{g} / \mathrm{dL}$ increase in blood Pb was associated with a $23 \%$ increase in log TNF- $\alpha$ and a $26 \%$ increase in IL-6. Finally, in occupationallyexposed workers, higher blood Pb levels were associated with increases in IL-2, IL-10, IL-6, TNF- $\alpha$, and granulocyte colony stimulating factor (G-CSF), and, in one study, lower levels of Th- 1 cytokines IL-1 $\beta$ and IFN- $\gamma$. Similar effects were seen in mice exposed to $\mathrm{Pb}$ in feed; blood levels of Th-1 cytokines (IL-2 and IFN- $\gamma$ ) were decreased at low dietary doses, while increases in IL-4 were seen as the Pb dose increased. Based on these data, EPA (2014c) suggested that the immune system response to $\mathrm{Pb}$ may exhibit nonlinearities at low doses. In rats exposed to $\mathrm{Pb}$ via intraperitoneal injection, increased levels of TNF- $\alpha$ were seen in the hippocampus, and increased IL-6 was noted in the forebrain. In vitro data have also shown alterations in cytokine production after exposure to $\mathrm{Pb}$.

\subsubsection{Epigenetic Effects}

In a small number of studies, $\mathrm{Pb}$ has been shown to induce epigenetic effects, including perturbations in DNA methylation as well as alterations in mitogenesis (reviewed by EPA 2014c; Bakulski et al. 2012). In human studies, maternal blood $\mathrm{Pb}$ was correlated with decreased DNA methylation of Alu retrotransposable elements in umbilical cord blood, and bone Pb levels were correlated with decreased DNA methylation of LINE-1 retrotransposons in elderly men, while higher blood $\mathrm{Pb}$ was associated with increased methylation of p16 tumor suppressor gene promoters in occupationally exposed individuals. Other evidence for effects of $\mathrm{Pb}$ on DNA methylation include a study in primates in which the activity of DNA methyltransferase 1 was decreased by early life $\mathrm{Pb}$ exposure, and in vitro data showing decreased global DNA methylation in rat pheochromocytoma cells. Hypomethylation of DNA has been shown to trigger changes in gene expression that may lead to alterations in tissue differentiation.

$\mathrm{Pb}$ exposure also induces effects on mitogenesis, including both increases in cell proliferation and decreases in some systems (reviewed by EPA 2014c). Increased cell proliferation and/or DNA synthesis have been reported in workers exposed to $\mathrm{Pb}$, in hepatocytes of rats exposed by intravenous injection of $\mathrm{Pb}$ nitrate, and in mouse lung after exposure to $\mathrm{Pb}$ acetate via inhalation. In in vitro studies, results were mixed: in some cases cell proliferation was decreased, as Pb exposure resulted in cell cycle arrest. Effects of $\mathrm{Pb}$ exposure on gene expression have been demonstrated in several studies (reviewed by EPA 2014c). Although the exact mechanisms by which $\mathrm{Pb}$ alters gene expression have not been elucidated, $\mathrm{Pb}$ is known to interfere with GATA proteins and several transcription factors (TFIIIA, Sp1, and Erg-1) 
through its interaction with zinc-binding domains, reducing the ability of these proteins to bind to DNA and exert their transcriptional regulation functions. In vivo and in vitro studies have shown that $\mathrm{Pb}$ alters the transcription of genes for metabolic enzymes including GST-P and GST-Ya, CYPs 1A1 and 1A2, and $\mathrm{NAD}(\mathrm{P}) \mathrm{H}$ :quinone oxidoreductase, as well as genes involved in the pentose phosphate pathway and amino acid metabolism.

\subsubsection{Apoptosis}

As discussed earlier, $\mathrm{Pb}$ is capable of opening the mitochondrial transmembrane pore (MTMP, the first step in the mitochondrial apoptosis cascade), possibly by displacing calcium on the matrix side of the pore (reviewed by EPA 2014c). Evidence for this effect includes observations of mitochondrial swelling and decreased membrane potential in rat primary cerebellar granule neuronal cultures, astroglia, proximal tubule cells, and retinal rod photoreceptor cells. In addition, release of cytochrome c and activation of caspases 3 and 9 were observed in rat retinal rod cells and hepatic oval cells exposed to $\mathrm{Pb}$ in vitro. In lymphocytes of Pb-exposed humans, increased apoptosis, karyorrhexis, and karyolysis (early indicators of apoptosis) were observed. Other tissues have also exhibited increased apoptosis after $\mathrm{Pb}$ exposure, including liver, fibroblasts, and alveolar macrophages. 


\section{CHAPTER 3. TOXICOKINETICS, SUSCEPTIBLE POPULATIONS, BIOMARKERS, CHEMICAL INTERACTIONS}

\subsection{TOXICOKINETICS}

Overview. The toxicokinetics of $\mathrm{Pb}$ in humans has been extensively studied and several models have been published that simulate the absorption and complex distribution and elimination of $\mathrm{Pb}$ from blood, soft tissues, and bone.

- Absorption:

o Respiratory tract: Inorganic $\mathrm{Pb}$ in submicron size particles can be almost completely absorbed through the respiratory tract, whereas larger particles may be moved after deposition in the respiratory tract by mucociliary clearance toward the oropharynx and swallowed.

o Gastrointestinal tract: The fraction of ingested $\mathrm{Pb}$ absorbed from the gastrointestinal tract depends on many factors, including age, diet, nutrition, and physiological characteristics of $\mathrm{Pb}$ in the medium ingested.

o Children can absorb $40-50 \%$ of an oral dose of water-soluble $\mathrm{Pb}$ compared to $3-10 \%$ for adults.

o Gastrointestinal absorption of inorganic Pb occurs primarily in the duodenum by saturable mechanisms.

o Dermal: Inorganic Pb can be absorbed following inhalation, oral, and dermal exposure, but the latter route is much less efficient than the former two, with the exception of handto-mouth behavior. Studies in animals have shown that organic $\mathrm{Pb}$ is absorbed through the skin.

- Distribution:

o The distribution of $\mathrm{Pb}$ in the body is route-independent and, in adults, approximately $94 \%$ of the total body burden of $\mathrm{Pb}$ is in the bones compared to approximately $73 \%$ in children.

o $\mathrm{Pb}$ in blood is primarily in red blood cells. Conditions such as pregnancy, lactation, menopause, and osteoporosis increase bone resorption and consequently also increase $\mathrm{Pb}$ in blood. 
o $\mathrm{Pb}$ can be transferred from the mother to the fetus and also from the mother to infants via maternal milk.

- Metabolism:

o Metabolism of inorganic $\mathrm{Pb}$ consists of formation of complexes with a variety of protein and nonprotein ligands.

o Organic Pb compounds are actively metabolized in the liver by oxidative dealkylation by P-450 enzymes.

- Excretion:

o $\mathrm{Pb}$ is excreted primarily in urine and feces regardless of the route of exposure. Minor routes of excretion include sweat, saliva, hair, nails, breast milk, and seminal fluid.

o Elimination of $\mathrm{Pb}$ is multiphasic, reflecting pools of $\mathrm{Pb}$ in the body that have varying retention times. The apparent elimination half-time in blood varies with age and exposure history and ranges from 1 week to 2 years. Elimination of $\mathrm{Pb}$ from bone occurs with an apparent half-time of 1-2 decades.

- $\quad$ Toxicokinetics models:

o Several models of $\mathrm{Pb}$ pharmacokinetics have been proposed to characterize such parameters as intercompartmental $\mathrm{Pb}$ exchange rates, retention of $\mathrm{Pb}$ in various tissues, and relative rates of distribution among the tissue groups.

o Some models are currently being used or are being considered for broad application in $\mathrm{Pb}$ risk assessment.

\subsubsection{Absorption}

\section{Inhalation Exposure}

Inorganic $\boldsymbol{P b}$. Inorganic $\mathrm{Pb}$ in ambient air consists of aerosols of particulates that can be deposited in the respiratory tract when the aerosols are inhaled. Amounts and patterns of deposition of particulate aerosols in the respiratory tract are affected by the size of the inhaled particles, age-related factors that determine breathing patterns (e.g., nose versus mouth breathing), airway geometry, and air-stream velocity within the respiratory tract (James et al. 1994). Absorption of deposited $\mathrm{Pb}$ is influenced by particle size and solubility as well as the pattern of regional deposition within the respiratory tract. Larger particles 
$(>2.5 \mu \mathrm{m})$ that are deposited in the ciliated airways (nasopharyngeal and tracheobronchial regions) can be transferred by mucociliary transport into the esophagus and swallowed. Smaller particles ( 2.5 to $<1 \mu \mathrm{m})$, which can be deposited in the alveolar region, can be absorbed after extracellular dissolution or ingestion by phagocytic cells (Bailey and Roy 1994).

Deposition in, and clearance from, the respiratory tract have been measured in adult humans (Chamberlain et al. 1978; Hursh and Mercer 1970; Hursh et al. 1969; Morrow et al. 1980; Wells et al. 1975). In these studies, exposures were to Pb-bearing particles having mass median aerodynamic diameters (MMADs) below $1 \mu \mathrm{m}$ and, therefore, deposition of the inhaled Pb particles can be assumed to have been primarily in the bronchiolar and alveolar regions of the respiratory tract (James et al. 1994) where transport of deposited $\mathrm{Pb}$ to the gastrointestinal tract is likely to have been only a minor component of particle clearance (Hursh et al. 1969). Approximately 25\% of inhaled $\mathrm{Pb}$ chloride or Pb hydroxide (MMAD 0.26 and $0.24 \mu \mathrm{m}$, respectively) was deposited in the respiratory tract in adult subjects who inhaled an inorganic $\mathrm{Pb}$ aerosol through a standard respiratory mouthpiece for 5 minutes (Morrow et al. 1980). Approximately 95\% of deposited inorganic $\mathrm{Pb}$ that was inhaled as submicron particles was absorbed (Hursh et al. 1969; Wells et al. 1975). Rates of clearance from the respiratory tract of inorganic $\mathrm{Pb}$ inhaled as submicron particles of $\mathrm{Pb}$ oxide, or $\mathrm{Pb}$ nitrate, were described with half-times $\left(\mathrm{t}_{1 / 2}\right)$ of 0.8 hours (22\%), 2.5 hours (34\%), 9 hours (33\%), and 44 hours (12\%) (Chamberlain et al. 1978). These rates are thought to represent, primarily, absorption from the bronchiolar and alveolar regions of the respiratory tract. Absorption half-times have been estimated in adults who inhaled aerosols of $\mathrm{Pb}$ and bismuth isotopes generated from decay of ${ }^{220} \mathrm{Rn}$ or ${ }^{222} \mathrm{Rn}$ (Butterweck et al. 2002; Marsh and Birchall 1999). The absorption half-time was approximately 10 hours in subjects who inhaled aerosols having an activity median particle diameter of approximately $160 \mathrm{~nm}$ (range 50-500 nm), and approximately 68 minutes for aerosols having diameters of approximately $0.3-3 \mathrm{~nm}$.

Rates and amounts of absorption of inhaled $\mathrm{Pb}$ particles $>2.5 \mu \mathrm{m}$ will be determined, primarily by rates of transport to and absorption from the gastrointestinal tract. Absorption of $\mathrm{Pb}$ from the gastrointestinal tract varies with the chemical form ingested, age, meal status (e.g., fed versus fasted), and nutritional factors (see Section 3.1.1 Oral Exposure).

Organic Pb. Clinical studies of subjects who inhaled tetraethyl or tetramethyl $\mathrm{Pb}$ found that $60-80 \%$ of the $\mathrm{Pb}$ deposited in the respiratory tract was absorbed (Heard et al. 1979). Following a single exposure to vapors of radioactive $\left({ }^{203} \mathrm{~Pb}\right.$ ) tetraethyl $\mathrm{Pb}$ (approximately $1 \mathrm{mg} / \mathrm{m}^{3}$ breathed through a mouthpiece for 12 minutes) in four male subjects, $37 \%$ of inhaled ${ }^{203} \mathrm{~Pb}$ was initially deposited in the respiratory tract, of 
which approximately 20\% was exhaled in the subsequent 48 hours (Heard et al. 1979). One hour after the exposure, approximately $50 \%$ of the ${ }^{203} \mathrm{~Pb}$ burden was associated with liver, $5 \%$ was associated with kidney, and the remaining burden was widely distributed throughout the body (determined by external gamma counting), suggesting near complete absorption of the $\mathrm{Pb}$ that was not exhaled. In a similar experiment conducted with $\left({ }^{203} \mathrm{~Pb}\right)$ tetramethyl $\mathrm{Pb}, 51 \%$ of the inhaled ${ }^{203} \mathrm{~Pb}$ dose was initially deposited in the respiratory tract, of which approximately $40 \%$ was exhaled in 48 hours. The distribution of ${ }^{203} \mathrm{~Pb}$ 1 hour after the exposure was similar to that observed following exposure to tetraethyl $\mathrm{Pb}$.

The relatively rapid and near complete absorption of tetraalkyl $\mathrm{Pb}$ that is inhaled and deposited in the respiratory tract is also supported by studies conducted in animal models (Boudene et al. 1977; Morgan and Holmes 1978).

\section{Oral Exposure}

Inorganic Pb. The extent and rate of gastrointestinal absorption of ingested inorganic $\mathrm{Pb}$ are influenced by physiology (e.g., age, fasting, nutritional calcium and iron status, pregnancy), physicochemical characteristics of the medium ingested (e.g., particle size, mineralogy, solubility, and $\mathrm{Pb}$ species) and the ingested $\mathrm{Pb}$ dose.

Mechanisms of Absorption. Gastrointestinal absorption of inorganic $\mathrm{Pb}$ occurs primarily in the duodenum (Mushak 1991). The exact mechanisms of absorption are unknown and may involve active transport and/or diffusion through intestinal epithelial cells (transcellular) or between cells (paracellular), and may involve ionized $\mathrm{Pb}\left(\mathrm{Pb}^{+2}\right)$ and/or inorganic or organic complexes of $\mathrm{Pb}$. In vitro studies of $\mathrm{Pb}$ speciation in simulated human intestinal chyme indicate that the concentration of ionized $\mathrm{Pb}$ is negligible at $\mathrm{Pb}$ concentrations below $10^{-3} \mathrm{M}(207 \mathrm{mg} / \mathrm{L})$ and that $\mathrm{Pb}$ phosphate and bile acid complexes are the dominant forms when inorganic $\mathrm{Pb}$ salts (e.g., $\mathrm{Pb}$ nitrate) are added to chyme (Oomen et al. 2003a). However, these complexes may be sufficiently labile to provide ionized $\mathrm{Pb}$ for transport across cell membranes (Oomen et al. 2003b). Saturable mechanisms of absorption have been inferred from measurements of net flux kinetics of $\mathrm{Pb}$ in in situ perfused mouse intestine, in situ ligated chicken intestine, and in vitro isolated segments of rat intestine (Aungst and Fung 1981; Barton 1984; Flanagan et al. 1979; Mykkänen and Wasserman 1981). By analogy to other divalent cations, saturable transport mechanisms for $\mathrm{Pb}^{+2}$ may exist within the mucosal and serosal membranes and within the intestinal epithelial cell. For calcium and iron, these are thought to represent membrane carriers (e.g., $\mathrm{Ca}^{2+}-\mathrm{Mg}^{2+}$-ATPase, $\mathrm{Ca}^{2+} / \mathrm{Na}^{+}$exchange, DMT1) or facilitated diffusion pathways (e.g., $\mathrm{Ca}^{2+}$ channel) and 
intracellular binding proteins for $\mathrm{Ca}^{2+}$ (Bronner et al. 1986; Fleming et al. 1998b; Gross and Kumar 1990; Teichmann and Stremmel 1990).

Effect of Age. Gastrointestinal absorption of water-soluble $\mathrm{Pb}$ appears to be higher in children than in adults. Estimates derived from dietary balance studies conducted in infants and children (ages 2 weeks to 8 years) indicate that approximately $40-50 \%$ of ingested $\mathrm{Pb}$ is absorbed (Alexander et al. 1974; Ziegler et al. 1978). In adults, estimates of absorption of ingested water-soluble $\mathrm{Pb}$ compounds (e.g., Pb chloride, $\mathrm{Pb}$ nitrate, $\mathrm{Pb}$ acetate) ranged from 3 to 10\% in fed subjects (Heard and Chamberlain 1982; James et al. 1985; Rabinowitz et al. 1980; Watson et al. 1986). Data available on Pb absorption between childhood and adulthood ages are very limited. While no absorption studies have been conducted on subjects in this age range, the kinetics of the change in stable isotope signatures of blood $\mathrm{Pb}$ in mothers and their children, as both come into equilibrium with a novel environmental $\mathrm{Pb}$ isotope profile, suggest that children ages 6-11 years and their mothers may absorb a similar percentage of ingested $\mathrm{Pb}$ (Gulson et al. 1997b).

Studies in experimental animals provide additional evidence for an age-dependency of gastrointestinal absorption of $\mathrm{Pb}$. Absorption of $\mathrm{Pb}$, administered as $\mathrm{Pb}$ acetate (6.37 $\mathrm{mg} \mathrm{Pb} / \mathrm{kg}$, gavage), was higher in juvenile Rhesus monkeys (38\% of dose) compared to adult female monkeys (26\% of the dose) (Pounds et al. 1978). Rat pups absorb approximately 40-50 times more $\mathrm{Pb}$ from the diet than do adult rats (Aungst et al. 1981; Forbes and Reina 1972; Kostial et al. 1978). This age difference in absorption may be due, in part, to the shift from the neonatal to adult diet, and to postnatal physiological development (enzymes, transporters, gastric pH) of the gastrointestinal tract (Weis and LaVelle 1991).

Effect of Fasting. The presence of food in the gastrointestinal tract decreases absorption of water-soluble Pb (Blake and Mann 1983; Blake et al. 1983; Heard and Chamberlain 1982; James et al. 1985; Maddaloni et al. 1998; Rabinowitz et al. 1980). In adults, absorption of a tracer dose of $\mathrm{Pb}$ acetate in water was approximately 63\% when ingested by fasted subjects and 3\% when ingested with a meal (James et al. 1985). Heard and Chamberlain (1982) reported nearly identical results. The arithmetic mean of reported estimates of absorption in fasted adults was 57\% (calculated by ATSDR based on Blake et al. 1983; Heard and Chamberlain 1982; James et al. 1985; Rabinowitz et al. 1980). Reported fed/fasted ratios for absorption in adults range from 0.04 to 0.2 (Blake et al. 1983; Heard and Chamberlain 1983; James et al. 1985; Rabinowitz et al. 1980). Mineral content is one contributing factor to the lower absorption of $\mathrm{Pb}$ when $\mathrm{Pb}$ is ingested with a meal; in particular, the presence of calcium and phosphate in a meal will depress the absorption of ingested Pb (Blake and Mann 1983; Blake et al. 1983; Heard and Chamberlain 
1982). Suppression of absorption by meals may explain the observation of lower PbB in children (age 35 years) who ate breakfast compared to children who went without breakfast, after controlling for nutritional variables (Liu et al. 2011).

Effect of Nutrition. Pb absorption in children is affected by nutritional iron status. Children who are iron deficient have higher PbBs than similarly exposed children who are iron replete, which would suggest that iron deficiency may result in higher absorption of $\mathrm{Pb}$ or, possibly, other changes in $\mathrm{Pb}$ biokinetics that would contribute to higher PbBs (Mahaffey and Annest 1986; Marcus and Schwartz 1987). Genetic variation in genes involved in iron metabolism appear to affect PbBs; however, it is not certain if these associations are caused by changes in $\mathrm{Pb}$ absorption. These include variants in the hemochromatosis (HFE) and transferrin genes, which have been associated with higher PbBs in children (Hopkins et al. 2008), and with lower PbBs and bone Pb levels in elderly men (Wright et al. 2004).

Evidence for the effect for iron deficiency on $\mathrm{Pb}$ absorption has been provided from animal studies. In rats, iron deficiency increases the gastrointestinal absorption of $\mathrm{Pb}$, possibly by enhancing binding of $\mathrm{Pb}$ to iron binding proteins in the intestine (Bannon et al. 2003; Barton et al. 1978b; Morrison and Quaterman 1987). Interactions between iron and $\mathrm{Pb}$ appear to involve either intracellular transfer or basolateral transfer mechanisms. Iron $\left(\mathrm{FeCl}_{2}\right)$ added to the mucosal fluid of the everted rat duodenal sac decreases serosal transfer, but not mucosal uptake of Pb (Barton 1984). When mRNA for DMT1, a mucosal membrane carrier for iron (which also transports other divalent metal cations), was suppressed in Caco 2 cells (a human gastrointestinal cell line), the rate of iron and cadmium uptake decreased by 50\% compared to cells in which DMT1 mRNA was not suppressed; however, DMT1 mRNA suppression did not alter the rate of $\mathrm{Pb}$ uptake by Caco 2 cells, indicating that $\mathrm{Pb}$ may enter Caco 2 cells through a mechanism that is independent of DMT1 (Bannon et al. 2003). The above observations suggest that ratelimiting saturable mechanisms for $\mathrm{Pb}$ absorption are associated with transfer of $\mathrm{Pb}$ from cell to blood rather than with mucosal transfer. Similar mechanisms may contribute to Pb-iron and Pb-calcium absorption interactions in humans, and possibly interactions between $\mathrm{Pb}$ and other divalent cations such as cadmium, copper, magnesium, and zinc.

Dietary calcium intake affects $\mathrm{Pb}$ absorption. An inverse relationship has been observed between dietary calcium intake and $\mathrm{PbBs}$ in children, suggesting that children who are calcium-deficient may absorb more $\mathrm{Pb}$ than calcium-replete children (Elias et al. 2007; Mahaffey et al. 1986; Schell et al. 2004; Ziegler et al. 1978). An effect of calcium on $\mathrm{Pb}$ absorption is also evident in adults. In experimental studies of adults, absorption of a single dose of $\mathrm{Pb}(100-300 \mu \mathrm{g} \mathrm{Pb}$ chloride) was lower when the $\mathrm{Pb}$ was ingested together 
with calcium carbonate (0.2-1 g calcium carbonate) than when the $\mathrm{Pb}$ was ingested without additional calcium (Blake and Mann 1983; Heard and Chamberlain 1982). A similar effect of calcium occurs in rats (Barton et al. 1978a). Complexation with calcium (and phosphate) in the gastrointestinal tract and competition for a common transport protein have been proposed as possible mechanisms for this interaction (Barton et al. 1978a; Heard and Chamberlain 1982). Absorption of $\mathrm{Pb}$ from the gastrointestinal tract is enhanced by dietary calcium depletion or administration of cholecalciferol (Mykkänen and Wasserman 1981, 1982). This "cholecalciferol-dependent" component of Pb absorption appears to involve a stimulation of the serosal transfer of $\mathrm{Pb}$ from the epithelium, not stimulation of mucosal uptake of Pb (Mykkänen and Wasserman 1981, 1982). This is similar to the effects of cholecalciferol on calcium absorption (Bronner et al. 1986; Fullmer and Rosen 1990).

In a study of young children (ages 6-12 months), PbBs increased in association with lower dietary Zn levels (Schell et al. 2004); however, it is not certain if these associations were caused by changes in $\mathrm{Pb}$ absorption.

Effect of Pregnancy. Absorption of Pb may increase during pregnancy. Although there is no direct evidence for this in humans, an increase in $\mathrm{Pb}$ absorption may contribute, along with other mechanisms (e.g., increased mobilization of bone $\mathrm{Pb}$ ), to the increase in PbBs that has been observed during the latter half of pregnancy (see Section 3.1.2, Pb Distribution during Pregnancy and Maternal-Fetal-Infant Transfer).

Effect of Dose. Pb absorption in humans may be a capacity-limited process, in which case, the percentage of ingested $\mathrm{Pb}$ that is absorbed may decrease with increasing rate of $\mathrm{Pb}$ intake. Studies, to date, do not provide a firm basis for discerning if the gastrointestinal absorption of $\mathrm{Pb}$ is limited by dose. Numerous observations of nonlinear relationships between $\mathrm{PbB}$ and $\mathrm{Pb}$ intake in humans provide support for the existence of a saturable absorption mechanism or some other capacity-limited process in the distribution of Pb in humans (Pocock et al. 1983; Sherlock and Quinn 1986; Sherlock et al. 1984) (see Section 3.1.2, $\mathrm{Pb}$ in Blood and $\mathrm{Pb}$ in Plasma for discussion of saturable uptake of $\mathrm{Pb}$ in red blood cells). However, in immature swine that received oral doses of $\mathrm{Pb}$ in soil, $\mathrm{Pb}$ dose-blood $\mathrm{Pb}$ relationships were curvilinear, whereas dose-tissue $\mathrm{Pb}$ relationships for bone, kidney, and liver were linear. The same pattern (nonlinearity for $\mathrm{PbB}$ and linearity for tissues) was observed in swine administered $\mathrm{Pb}$ acetate intravenously (Casteel et al. 1997, 2006). These results suggest that the nonlinearity in the Pb dose-blood $\mathrm{Pb}$ relationship may derive from an effect of $\mathrm{Pb}$ dose on some aspect of the biokinetics of $\mathrm{Pb}$ other than absorption. In fasted rats, absorption was estimated at 42 and $2 \%$ following single oral administration of 
1 and $100 \mathrm{mg} \mathrm{Pb} / \mathrm{kg}$, respectively, as $\mathrm{Pb}$ acetate, suggesting a limitation on absorption imposed by dose (Aungst et al. 1981). Evidence for capacity-limited processes at the level of the intestinal epithelium (Aungst and Fung 1981; Barton 1984; Flanagan et al. 1979; Mykkänen and Wasserman 1981) suggests that the intake-uptake relationship for $\mathrm{Pb}$ is likely to be nonlinear; however, the dose at which absorption becomes appreciably limited in humans is not known.

Effect of Particle Size. Particle size influences the degree of gastrointestinal absorption (Ruby et al. 1999). In rats, an inverse relationship was found between absorption and particle size of $\mathrm{Pb}$ in diets containing metallic $\mathrm{Pb}$ particles that were $\leq 250 \mu \mathrm{m}$ in diameter (Barltrop and Meek 1979). Tissue $\mathrm{Pb}$ concentration was a 2.3-fold higher when rats ingested an acute dose (37.5 mg Pb/kg) of Pb particles that were $<38 \mu \mathrm{m}$ in diameter than when rats ingested particles having diameters in the range of $150-250 \mu \mathrm{m}$ (Barltrop and Meek 1979). Dissolution kinetics experiments with Pb-bearing mine waste soil suggest that surface area effects control dissolution rates for particles sizes of $<90 \mu \mathrm{m}$ diameter; however, dissolution of $90-250 \mu \mathrm{m}$ particle size fractions appeared to be controlled more by surface morphology (Davis et al. 1994). Similarly, Healy et al. (1982) found that the solubility of Pb sulfide in gastric acid in vitro was much greater for particles that were $30 \mu \mathrm{m}$ in diameter than for particles that were $100 \mu \mathrm{m}$ in diameter.

Absorption from Soil. Absorption of $\mathrm{Pb}$ from the gastrointestinal tract involves absorptive transport of soluble $\mathrm{Pb}$ species (e.g., $\mathrm{Pb}^{2+}$ ) across the gastrointestinal tract epithelium. In order for $\mathrm{Pb}$ to be absorbed from soil, it must first be made bioaccessible in the gastrointestinal tract. The process of rendering soil $\mathrm{Pb}$ bioaccessible may involve: (1) physical and/or chemical digestion of the soil particles to expose $\mathrm{Pb}$ deposits to gastrointestinal tract fluids; (2) transfer of $\mathrm{Pb}$ minerals from exposed surfaces on soil particles to the aqueous environment of the gastrointestinal tract; and (3) chemical transformation of $\mathrm{Pb}$ minerals to soluble $\mathrm{Pb}$ species (e.g., $\mathrm{Pb}^{2+}$ ) that are substrates for absorptive transport. Although absorptive transport of $\mathrm{Pb}$ occurs predominantly, if not solely, in the upper small intestine, bioaccessibility processes occurring in the stomach appear to be major determinants of $\mathrm{Pb}$ absorption.

Adult subjects who ingested soil (particle size $<250 \mu \mathrm{m}$ ) collected from the Bunker Hill National Priorities List (NPL) site absorbed 26\% of the resulting $250 \mu \mathrm{g} / 70 \mathrm{~kg}$ body weight Pb dose when the soil was ingested in the fasted state, and $2.5 \%$ when the same soil $\mathrm{Pb}$ dose was ingested with a meal (Maddaloni et al. 1998). The value reported for fasted subjects (26\%) was approximately half that reported for soluble $\mathrm{Pb}$ ingested by fasting adults, or approximately 60\% (Blake et al. 1983; Heard and Chamberlain 1983; James et al. 1985; Rabinowitz et al. 1980). Measurements of the absorption of soil Pb in infants or children have not been reported. 
Absorption of $\mathrm{Pb}$ from ingested soils and surface dust has been studied more extensively in animals (Bannon et al. 2009; Barltrop and Meek 1979; Bradham et al. 2016, 2019; Brown et al. 2004; Casteel et al. 1997, 2006; Freeman et al. 1992, 1994, 1996; Healy et al. 1982; Hettiearachchi et al. 2003; Juhasz et al. 2009; Ryan et al. 2004; Weis and Lavelle 1991). These studies have shown that absorption of soil Pb varies depending upon the $\mathrm{Pb}$ mineralogy and physical characteristics of the $\mathrm{Pb}$ in the soil (e.g., encapsulated or exposed, particle size). Studies conducted in swine and other animal models have provided estimates of relative bioavailability (RBA) of $\mathrm{Pb}$ in soils collected from sites impacted by a variety of sources of $\mathrm{Pb}$ contamination including ore and ore processing, shooting of $\mathrm{Pb}$ munitions, and Pb-based paint (Bannon et al. 2009; Barltrop and Meek 1979; Bradham et al. 2016, 2019; Brown et al. 2004; Casteel et al. 1997, 2006; Freeman et al. 1992, 1994, 1996; Healy et al. 1982; Hettiearachchi et al. 2003; Juhasz et al. 2009; Ryan et al. 2004; Weis and Lavelle 1991). RBA is the ratio of the absolute bioavailability (or absorption fraction) of $\mathrm{Pb}$ in soil to that of a water-soluble reference (Pb acetate). $\mathrm{RBA}$ has been measured in animal models using various approaches, including measurement of blood and tissue $\mathrm{Pb}$ in animals following dosing with soil or $\mathrm{Pb}$ acetate. RBA estimates from these studies ranged from 1 to $100 \%$ (mean 60\%, $n=33$, calculated by ATSDR). RBAs for soils (sieved to $<250 \mu \mathrm{m}$ ) from firing ranges where the predominant form of $\mathrm{Pb}$ was $\mathrm{Pb}$ carbonate were approximately $100 \%$ (Bannon et al. 2009). A soil amended with NIST paint standard (a mixture of $\mathrm{Pb}$ carbonate and $\mathrm{Pb}$ oxide) had an RBA of 92\%. Smelter slag and soils in which the dominant source of $\mathrm{Pb}$ was smelter slag had relatively low RBA (14-40\%). Galena (lead sulfide) in soil also had relatively low RBA (1-6\%).

Casteel et al. (2006) estimated Pb RBA of 19 soils in swine and categorized the RBA according to $\mathrm{Pb}$ mineral associations. Electron microprobe analyses of Pb-bearing grains in the various soils revealed that the grains ranged from as small as 1-2 $\mu \mathrm{m}$ up to a maximum of $250 \mu \mathrm{m}$ (the sieve size used in preparation of the samples) and that $\mathrm{Pb}$ was present in a wide range of different mineral associations (phases), including various oxides, sulfides, sulfates, and phosphates. These variations in size and mineral content of the Pb-bearing grains are the suspected cause of variations in the gastrointestinal absorption of $\mathrm{Pb}$ from different samples of soil. Based on these very limited data, the RBA of Pb mineral phases were rankordered (Table 3-1). 
Table 3-1. Ranking of Relative Bioavailability of Lead (Pb) Mineral Phases in Soil ${ }^{\mathrm{a}}$

\begin{tabular}{lll}
\hline $\begin{array}{l}\text { Low bioavailability } \\
\text { (RBA<0.25) }\end{array}$ & $\begin{array}{l}\text { Medium bioavailability } \\
(\mathrm{RBA}=0.25-0.75)\end{array}$ & $\begin{array}{l}\text { High bioavailability } \\
(\mathrm{RBA}>0.75)\end{array}$ \\
\hline Angelsite & Pb oxide & Cerussite \\
$\mathrm{Fe}(\mathrm{M})$ oxide & Pb phosphate & Mn(M) oxide \\
$\mathrm{Fe}(\mathrm{M})$ sulfate & & \\
Galena & & \\
$\mathrm{Pb}(\mathrm{M})$ oxide & & \\
\hline
\end{tabular}

aEstimates are based on studies of immature swine.

$\mathrm{Fe}=$ iron; $\mathrm{M}=$ metal; $\mathrm{Mn}$ = manganese RBA = relative bioavailability (compared to $\mathrm{Pb}$ acetate)

Source: Casteel et al. 2006

Several studies have shown that elevating the phosphate concentration of soil can decrease soil Pb RBA (Brown et al. 2004; Hettiarachichi et al. 2003; Ryan et al. 2004). The mechanism for the effect is thought to be the formation of a relatively insoluble form of $\mathrm{Pb}$ in soil, pyromorphite, which has a low RBA (Scheckel et al. 2013).

Bioaccessibility in Soil and its Relationship to Relative Bioavailability. Empirical evidence supporting the importance of gastric bioaccessibility in $\mathrm{Pb}$ absorption comes from studies of relationships between extractability of $\mathrm{Pb}$ from soil measured in vitro and $\mathrm{Pb}$ RBA measured in animals. In vitro extractability of $\mathrm{Pb}$ from soil (in vitro bioaccessibility, IVBA) strongly correlates with RBA measured swine assays when the extraction is performed at gastric $\mathrm{pH}\left(\mathrm{r}^{2}=0.92, \mathrm{n}=18\right.$; Drexler and Brattin 2007).

Bioaccessibility estimates obtained from IVBA assays are sensitive to assay conditions such as $\mathrm{pH}$, liquid:soil ratios, inclusion or absence of food material, and differences in methods used to separate dissolved and particle-bound $\mathrm{Pb}$ (e.g., centrifugation versus filtration); as a result, different assays can yield different results when applied to the same soils or surface dusts (Dong et al. 2016; Juhasz et al. 2011; Lu et al. 2011; Roussel et al. 2010; Saikat et al. 2007; Smith et al. 2011; Van de Wiele et al. 2007). For this reason, application of IVBA assays for predicting RBA must be supported by demonstration of a strong correlation between IVBA and RBA (Drexler and Brattin 2007). Even in the absence of validation of RBA predictions, IVBA assays may be useful for predicting relative differences in RBA between soils. For example, the relative change in Pb RBA resulting from treatment of soils with phosphate amendments was predicted from IVBA measurements even though the IVBA assay performed poorly at predicting the actual RBA of the soils (Juhasz et al. 2016). Bioaccessibility measured with IVBA assays has been shown to increase with decreasing particle size (varied from $<2,000$ to $<50 \mu \mathrm{m}$ ) (Juhasz et al. 2011) and increase with increasing soil acidity and organic matter content (Jin et al. 2005). 


\section{Dermal Exposure}

Inorganic $\mathbf{P b}$. Dermal absorption of inorganic $\mathrm{Pb}$ compounds is generally considered to be much less than absorption by inhalation or oral routes of exposure; however, few studies have provided quantitative estimates of dermal absorption of inorganic $\mathrm{Pb}$ in humans, and the quantitative significance of the dermal absorption pathway as a contributor to $\mathrm{Pb}$ body burden in humans remains an uncertainty. $\mathrm{Pb}$ was detected in the upper layers of the stratum corneum of Pb-battery workers, prior to their shifts and after cleaning of the skin surface (Sun et al. 2002), suggesting adherence and/or possible dermal penetration of $\mathrm{Pb}$. Following skin application of ${ }^{203} \mathrm{~Pb}$-labeled $\mathrm{Pb}$ acetate in cosmetic preparations $(0.12 \mathrm{mg} \mathrm{Pb}$ in $0.1 \mathrm{~mL}$ or $0.18 \mathrm{mg} \mathrm{Pb}$ in $0.1 \mathrm{~g}$ of a cream) to eight male volunteers for 12 hours, absorption was $\leq 0.3 \%$, based on whole-body, urine, and blood ${ }^{203} \mathrm{~Pb}$ measurements, and was predicted to be $0.06 \%$ during normal use of such preparations (Moore et al. 1980). Most of the absorption took place within 12 hours of exposure. $\mathrm{Pb}$ also appears to be absorbed across human skin when applied to the skin as $\mathrm{Pb}$ nitrate; however, quantitative estimates of absorption have not been reported. $\mathrm{Pb}$ (4.4 mg, as $\mathrm{Pb}$ nitrate) was applied (vehicle or solvent not reported) to an occluded filter placed on the forearm of an adult subject for 24 hours, after which, the patch was removed, the site cover and the forearm were rinsed with water, and total $\mathrm{Pb}$ was quantified in the cover material and rinse (Stauber et al. 1994). The amount of Pb recovered from the cover material and rinse was $3.1 \mathrm{mg}$ (70\% of the applied dose). Based on this recovery measurement, $1.3 \mathrm{mg}$ (30\%) of the applied dose remained either in the skin or had been absorbed in 24 hours; the amount that remained in or on the skin and the fate of this $\mathrm{Pb}$ (e.g., exfoliation) was not determined. Exfoliation has been implicated as an important pathway of elimination of other metals from skin (e.g., inorganic mercury; Hursh et al. 1989). Pb concentrations in sweat collected from the right arm increased 4-fold following the application of $\mathrm{Pb}$ to the left arm, indicating that some $\mathrm{Pb}$ had been absorbed (amounts of sweat collected or total Pb recovered in sweat were not reported; Stauber et al. 1994). In similar experiments with three subjects, measurements of ${ }^{203} \mathrm{~Pb}$ in blood, sweat, and urine, made over a 24-hour period following dermal exposures to $5 \mathrm{mg} \mathrm{Pb}$ as ${ }^{203} \mathrm{~Pb}$ nitrate or acetate, accounted for $<1 \%$ of the applied (or adsorbed) dose (Stauber et al. 1994). This study also reported that absorption of $\mathrm{Pb}$ could not be detected from measurements of $\mathrm{Pb}$ in sweat following dermal exposure to $\mathrm{Pb}$ as $\mathrm{Pb}$ carbonate.

Information on relative dermal permeability of inorganic and organic $\mathrm{Pb}$ salts of $\mathrm{Pb}$ comes from studies of in vitro preparations of excised skin; the rank ordering of penetration rates through excised human skin 
was: $\mathrm{Pb}$ nuolate $(\mathrm{Pb}$ linoleic and oleic acid complex $)>\mathrm{Pb}$ naphthanate $>\mathrm{Pb}$ acetate $>\mathrm{Pb}$ oxide (nondetectable) (Bress and Bidanset 1991).

Studies conducted in animals provide additional evidence that dermal absorption of inorganic $\mathrm{Pb}$ is substantially lower than absorption from the inhalation or oral route. In a comparative study of dermal absorption of inorganic and organic salts of $\mathrm{Pb}$ conducted in rats, approximately $100 \mathrm{mg}$ of $\mathrm{Pb}$ was applied in an occluded patch to the shaved backs of rats. Based on urinary $\mathrm{Pb}$ measurements made prior to and for 12 days following exposure, $\mathrm{Pb}$ compounds could be ranked according to the relative amounts absorbed (i.e., percent of dose recovered in urine; calculated by ATSDR): $\mathrm{Pb}$ naphthalene (0.17\%), $\mathrm{Pb}$ nitrate (0.03\%), $\mathrm{Pb}$ stearate (0.006\%), Pb sulfate (0.006\%), $\mathrm{Pb}$ oxide $(0.005 \%)$, and metal $\mathrm{Pb}$ powder (0.002\%). This rank order (i.e., $\mathrm{Pb}$ naphthalene $>\mathrm{Pb}$ oxide) is consistent with a rank ordering of penetration rates of inorganic and organic $\mathrm{Pb}$ salts through excised skin from humans and guinea pigs: $\mathrm{Pb}$ nuolate $(\mathrm{Pb}$ linoleic and oleic acid complex $)>\mathrm{Pb}$ naphthanate $>\mathrm{Pb}$ acetate $>\mathrm{Pb}$ oxide (nondetectable) (Bress and Bidanset 1991). The estimates for percent of dose excreted underestimate actual absorption as these estimates do not account for the $\mathrm{Pb}$ retained in bone and other tissues.

Following application of $\mathrm{Pb}$ acetate to the shaved clipped skin of rats, the concentration of $\mathrm{Pb}$ in the kidneys was found to be higher relative to controls, suggesting that absorption of $\mathrm{Pb}$ had occurred (Laug and Kunze 1948). This study also observed that dermal absorption of $\mathrm{Pb}$ from $\mathrm{Pb}$ arsenate was significantly less than from $\mathrm{Pb}$ acetate, and that mechanical injury to the skin significantly increased the dermal penetration of $\mathrm{Pb}$.

Organic Pb. Relative to inorganic $\mathrm{Pb}$ and organic $\mathrm{Pb}$ salts, tetraalkyl $\mathrm{Pb}$ compounds have been shown to be rapidly and extensively absorbed through the skin of rabbits and rats (Kehoe and Thamann 1931; Laug and Kunze 1948). A $0.75-\mathrm{mL}$ amount of tetraethyl Pb, which was allowed to spread uniformly over an area of $25 \mathrm{~cm}^{2}$ on the abdominal skin of rabbits, resulted in $10.6 \mathrm{mg}$ of $\mathrm{Pb}$ in the carcass at 0.5 hours and $4.41 \mathrm{mg}$ at 6 hours (Kehoe and Thamann 1931). Tetraethyl Pb was reported to be absorbed by the skin of rats to a much greater extent than $\mathrm{Pb}$ acetate, $\mathrm{Pb}$ oleate, and $\mathrm{Pb}$ arsenate (Laug and Kunze 1948).

Evidence for higher dermal permeability of organic $\mathrm{Pb}$ compounds compared to inorganic organic salts of $\mathrm{Pb}$ also comes from in vitro studies conducted with excised skin. The rank order of absorption rates through excised skin from humans and guinea pigs was as follows: tetrabutyl $\mathrm{Pb}>\mathrm{Pb}$ nuolate $(\mathrm{Pb}$ linoleic and oleic acid complex) $>\mathrm{Pb}$ naphthanate $>\mathrm{Pb}$ acetate $>\mathrm{Pb}$ oxide (nondetectable) (Bress and Bidanset 1991). 


\subsubsection{Distribution}

Inorganic $\boldsymbol{P} \boldsymbol{b}$. Absorbed inorganic $\mathrm{Pb}$ appears to be distributed in essentially the same manner regardless of the route of absorption (Chamberlain et al. 1978; Kehoe 1987); therefore, the distribution of absorbed $\mathrm{Pb}$ (i.e., by any route) is discussed in this section, rather than in separate sections devoted to specific routes of exposure. The expression "body burden" is used here to refer to the total amount of $\mathrm{Pb}$ in the body. Most of the available information about the distribution of $\mathrm{Pb}$ to major organ systems (e.g., bone, soft tissues) derives from autopsy studies conducted in the 1960s and 1970s and reflect body burdens accrued during periods when ambient and occupational exposure levels were much higher than current levels (Barry 1975, 1981; Gross et al. 1975; Schroeder and Tipton 1968). A more recent autopsy study found lower Pb concentrations in autopsies performed during the period 2004-2013 (Mari et al. 2014). In general, these studies indicate that the distribution of $\mathrm{Pb}$ appears to be similar in children and adults, although a larger fraction of the $\mathrm{Pb}$ body burden of adults resides in bone. Several models of $\mathrm{Pb}$ pharmacokinetics have been proposed to characterize such parameters as intercompartmental $\mathrm{Pb}$ exchange rates, retention of $\mathrm{Pb}$ in various tissues, and relative rates of distribution among the tissue groups (see Section 3.1.5 for further discussion of models).

$\boldsymbol{P b}$ in Blood. Concentrations of $\mathrm{Pb}$ in blood vary considerably with age, physiology/life stage (e.g., pregnancy, lactation, menopause), and numerous factors that affect exposure to $\mathrm{Pb}$. $\mathrm{PbBs}$ in various demographic strata of the U.S. population are periodically estimated from the NHANES. Based on data from NHANES (2015-2016, CDC 2018a), the geometric mean PbB of U.S. adults, age $\geq 20$ years, was $0.920 \mu \mathrm{g} / \mathrm{dL}$ (95\% CI 0.862, 0.982). The geometric mean PbB of U.S. children, age 1-5 years, was 0.758 (95\% CI 0.675, 0.850). PbBs in the United States have decreased considerably in the last several decades as a result of removal of $\mathrm{Pb}$ from gasoline and restrictions placed on the use of $\mathrm{Pb}$ in residential paints (Brody et al. 1994; CDC 2011, 2018a; Pirkle et al. 1994, 1998; Schwartz and Pitcher 1989). While historically, the geometric mean PbB in U.S. children has been higher than that of the adult population, recent estimates indicate that geometric means in children have fallen below that of adults.

Pb in Red Blood Cells. Pb in blood is primarily in the red blood cells (99\%) (Bergdahl et al. 1997a, 1998, 1999; Hernandez-Avila et al. 1998; Manton et al. 2001; Schutz et al. 1996; Smith et al. 2002). Although the mechanisms by which Pb crosses cell membranes have not been fully elucidated, results of studies in intact red blood cells and red blood cell ghosts indicate that there are two, and possibly three, pathways for facilitated transfer of $\mathrm{Pb}$ across the red cell membrane. The major proposed pathway is an anion exchanger that is dependent upon $\mathrm{HCO}_{3}{ }^{-}$and is blocked by anion exchange inhibitors (Bannon et al. 
2000, Simons 1985, 1986a, 1986b, 1993). A second minor pathway, which does not exhibit $\mathrm{HCO}_{3}{ }^{-}$ dependence and is not sensitive to anion exchange inhibitors, may also exist (Simons 1986b). Pb and calcium may also share a permeability pathway, which may be a $\mathrm{Ca}^{2+}$-channel (Calderon-Salinas et al. 1999). $\mathrm{Pb}$ is transferred out of the erythrocyte by an active transport pathway, most likely a $\left(\mathrm{Ca}^{2+}, \mathrm{Mg}^{2+}\right)$ ATPase (Simons 1988).

$\mathrm{Pb}$ in erythrocytes binds to several intracellular proteins. ALAD is the primary binding ligand for $\mathrm{Pb}$ in erythrocytes (Bergdahl et al. 1997a, 1998; Sakai et al. 1982; Xie et al. 1998). Pb binding to ALAD is saturable; the binding capacity has been estimated to be approximately $85 \mu \mathrm{g} / \mathrm{dL}$ red blood cells (or approximately $40 \mu \mathrm{g} / \mathrm{dL}$ whole blood) and the apparent dissociation constant has been estimated to be approximately $1.5 \mu \mathrm{g} / \mathrm{L}$ (Bergdahl et al. 1998). Two other Pb-binding proteins have been identified in erythrocytes, a $45 \mathrm{kDa}$ protein $(\mathrm{Kd} 5.5 \mu \mathrm{g} / \mathrm{L})$ and a smaller protein(s) having a molecular weight $<10 \mathrm{kDa}$ (Bergdahl et al. 1996, 1997a, 1998). Of the three principal Pb-binding proteins identified in erythrocytes, ALAD has the strongest affinity for $\mathrm{Pb}$ (Bergdahl et al. 1998) and appears to dominate the ligand distribution of $\mathrm{Pb}$ (35-84\% of total erythrocyte $\mathrm{Pb}$ ) at blood $\mathrm{Pb}$ levels below $40 \mu \mathrm{g} / \mathrm{dL}$ (Bergdahl et al. 1996, 1998; Sakai et al. 1982). The decrease in hematocrit that occurs in early infancy (51\% at birth to $35 \%$ at 6 months) may decrease the total binding capacity of blood and PbBs over the first postnatal 6 months (Simon et al. 2007).

$\mathrm{Pb}$ binds to and inhibits the activity of ALAD (Gercken and Barnes 1991; Gibbs et al. 1985; Jaffe et al. 2000; Sakai et al. 1982, 1983). Binding of zinc is essential for ALAD activity, and Pb inhibits activity of ALAD by displacing zinc (Jaffe et al. 2000). Synthesis of ALAD appears to be induced in response to inhibition of ALAD and, therefore, in response to binding of Pb to ALAD (Boudene et al. 1984; Fujita et al. 1982). Several mechanisms may participate in the induction of ALAD, including (1) inhibition of ALAD directly by $\mathrm{Pb}$; (2) inhibition by protoporphyrin, secondary to accumulation of protoporphyrin as a result of $\mathrm{Pb}$ inhibition of ferrochelatase; and (3) accumulation of ALA (a substrate of ASAD), secondary to inhibition of ALAD, which may stimulate ALAD synthesis in bone marrow cells (Boudene et al. 1984; Fujita et al. 1982).

ALAD is a polymorphic enzyme with two alleles (ALAD 1 and ALAD 2) and three genotypes (ALAD 1,1, ALAD 1,2, and ALAD 2,2) (Battistuzzi et al. 1981, Scinicariello et al. 2007). Numerous studies have examined the relationship between ALAD genotype and PbBs and the results of these studies are mixed with some studies finding higher PbBs in association with the ALAD 2 allele and other studies finding no associations or lower PbBs associated with the ALAD 2 allele (see Section 3.2). One possible 
mechanism by which ALAD polymorphism could affect $\mathrm{PbBs}$ is by allelic variation in $\mathrm{Pb}$ binding to ALAD (Bergdahl et al. 1997b). However, competitive displacement studies with recombinant human ALAD 1 and ALAD 2 did not indicate differences in affinity for Pb relative to zinc (Jaffe et al. 2000).

$\boldsymbol{P b}$ in Blood Plasma. $\mathrm{Pb}$ binds to several constituents in plasma and it has been proposed that $\mathrm{Pb}$ in plasma exists in four states: loosely bound to serum albumin or other proteins with relatively low affinity for $\mathrm{Pb}$, complexed to low molecular weight ligands such as amino acids and carboxylic acids, tightly bound to a circulating metalloprotein, and as free $\mathrm{Pb}^{2+}$ (Al-Modhefer et al. 1991). Free ionized $\mathrm{Pb}$ (i.e., $\mathrm{Pb}^{2+}$ ) in plasma represents an extremely small percentage of total plasma $\mathrm{Pb}$. The concentration of $\mathrm{Pb}^{2+}$ in fresh serum, as measured by an ion-selective $\mathrm{Pb}$ electrode, was reported to be 1/5,000 of the total serum $\mathrm{Pb}$ (Al-Modhefer et al. 1991). Approximately 40-75\% of $\mathrm{Pb}$ in the plasma is bound to plasma proteins, of which albumin appears to be the dominant ligand (Al-Modhefer et al. 1991; Ong and Lee 1980). Pb also binds to transferrins and $\gamma$-globulins (Guo et al. 2014; Ong and Lee 1980). Pb in serum that is not bound to protein exists largely as complexes with low molecular weight sulfhydryl compounds (e.g., cysteine, homocysteine). Other potential low molecular weight Pb-binding ligands in serum may include citrate, cysteamine, ergothioneine, glutathione, histidine, and oxylate (Al-Modhefer et al. 1991).

Saturable binding to red blood cell proteins contributes to curvature to the blood Pb-plasma $\mathrm{Pb}$ relationship with an increase in the plasma/blood Pb ratio with increasing PbB (Barbosa et al. 2006a; Bergdahl et al. 1997b, 1998, 1999; DeSilva 1981; Jin et al. 2008; Kang et al. 2009; Manton et al. 2001; Rentschler et al. 2012; Smith et al. 2002; Tian et al. 2013). The curvature becomes evident at PbBs well above $10 \mu \mathrm{g} / \mathrm{dL}$. As binding sites for $\mathrm{Pb}$ in red blood cells become saturated, a larger fraction of the blood $\mathrm{Pb}$ is available in plasma to distribute to brain and other $\mathrm{Pb}$-responsive tissues. This contributes to a curvature in the relationship between $\mathrm{Pb}$ intake and $\mathrm{PbB}$, with the blood $\mathrm{Pb} /$ intake slope decreasing with increasing $\mathrm{Pb}$ intake, which has been observed in children (Sherlock and Quinn 1986) and immature swine (Casteel et al. 2006). Saturable binding of $\mathrm{Pb}$ to red blood cell proteins also contributes to a curvilinear relationship between blood $\mathrm{Pb}$ and urinary $\mathrm{Pb}$, whereas the relationship between plasma $\mathrm{Pb}$ concentration and urine $\mathrm{Pb}$ is linear (Bergdahl et al. 1997b).

$\boldsymbol{P b}$ in Bone. In human adults, approximately $>90 \%$ of the total body burden of $\mathrm{Pb}$ is found in the bones. Based on analyses of post-mortem tissues, bone accounted for $94 \%$ of the total $\mathrm{Pb}$ body burden of adults and $73 \%$ of the body burden in children (Barry 1975). Pb concentrations in bone increase with age, indicative of a relatively slow turnover of $\mathrm{Pb}$ in adult bone (Barry 1975, 1981; Gross et al. 1975; Schroeder and Tipton 1968; Wilker et al. 2011). A portion of Pb in bone readily exchanges with the 
plasma $\mathrm{Pb}$ pool and, as a result, bone $\mathrm{Pb}$ is a reservoir for replenishment of $\mathrm{Pb}$ eliminated from blood by excretion (Alessio 1988; Behinaein et al. 2012, 2014; Chettle et al. 1991; Hryhorczuk et al. 1985; Nie et al. 2005; Nilsson et al. 1991; Rabinowitz et al. 1976). Pb in adult bone can serve to maintain blood $\mathrm{Pb}$ levels long after exposure has ended (Fleming et al. 1997; Inskip et al. 1996; Kehoe 1987; O'Flaherty et al. 1982; Smith et al. 1996). It can also serve as a source of $\mathrm{Pb}$ transfer to the fetus when maternal bone is resorbed for the production of the fetal skeleton (Franklin et al. 1997; Gulson et al. 1997b, 1999b, 2003).

$\mathrm{Pb}$ forms highly stable complexes with phosphate and can replace calcium in the calcium-phosphate salt, hydroxyapatite, which comprises the primary crystalline matrix of bone (Bres et al. 1986; Lloyd et al. 1975; Meirer et al. 2011; Miyake 1986; Verbeeck et al. 1981). As a result, Pb deposits in bone during the normal mineralization process that occurs during bone growth and remodeling and is released to the blood during the process of bone resorption (Aufderheide and Wittmers 1992; O'Flaherty 1991b, 1993). During infancy and childhood, bone calcification is most active in trabecular bone, whereas in adulthood, calcification occurs at sites of remodeling in cortical and trabecular bone. This suggests that $\mathrm{Pb}$ accumulation will occur predominantly in trabecular bone during childhood, and in both cortical and trabecular bone in adulthood (Aufderheide and Wittmers 1992). The association of Pb uptake and release from bone with the normal physiological processes of bone formation and resorption renders $\mathrm{Pb}$ biokinetics sensitive to these processes. Physiological states (e.g., pregnancy, menopause, advanced age) or disease-related states (e.g., osteoporosis, prolonged immobilization) that are associated with increased bone resorption will tend to promote the release of $\mathrm{Pb}$ from bone, which, in turn, may contribute to an increase in the concentration of Pb in blood (Berkowtiz et al. 2004; Bonithon-Kopp et al. 1985; Garrido Latorre et al. 2003; Hernandez-Avila et al. 2000; Jackson et al. 2010; Markowitz and Weinberger 1990; Mendola et al. 2013; Nash et al. 2004; Nie et al. 2009; Popovic et al. 2005; Silbergeld et al. 1988; Symanski and Hertz-Picciotto 1995; Thompson et al. 1985).

Two physiological compartments appear to exist for $\mathrm{Pb}$ in cortical and trabecular bone, to varying degrees. In one compartment, bone $\mathrm{Pb}$ is essentially inert, having an elimination half-time of several decades. A labile compartment exists as well that allows for maintenance of an equilibrium of $\mathrm{Pb}$ between bone and soft tissue or blood (Rabinowitz et al. 1976). Although a high bone formation rate in early childhood results in the rapid uptake of circulating $\mathrm{Pb}$ into mineralizing bone, bone $\mathrm{Pb}$ is also recycled to other tissue compartments or excreted in accordance with a high bone resorption rate (O'Flaherty 1995a). Thus, most of the Pb acquired early in life is not permanently fixed in the bone (O'Flaherty 1995a). In general, bone turnover rates decrease as a function of age, resulting in slowly increasing bone Pb levels among adults (Barry 1975; Gross et al. 1975; Schroeder and Tipton 1968). 
Bone $\mathrm{Pb}$ burdens in adults are slowly lost by diffusion (heteroionic exchange) as well as by resorption (O'Flaherty 1995a, 1995b). An XRF study of tibia Pb concentrations in individuals $>10$ years old showed a gradual increase in bone $\mathrm{Pb}$ after age 20 (Kosnett et al. 1994). In 60-70-year-old men, the total bone $\mathrm{Pb}$ burden may be $\geq 200 \mathrm{mg}$, while children $<16$ years old have been shown to have a total bone $\mathrm{Pb}$ burden of $8 \mathrm{mg}$ (Barry 1975). However, in some bones (i.e., mid femur and pelvic bone), the increase in Pb content plateaus at middle age and then decreases at higher ages; the decrease with age was more pronounced in females (Drasch et al. 1987). Osteoporosis and release of $\mathrm{Pb}$ from resorbed bone to blood may contribute to decreasing bone $\mathrm{Pb}$ content in females (Gulson et al. 2002).

Evidence for the exchange of bone $\mathrm{Pb}$ and soft tissue $\mathrm{Pb}$ stores comes from analyses of stable $\mathrm{Pb}$ isotope signatures of $\mathrm{Pb}$ in bone and blood. A comparison of blood and bone $\mathrm{Pb}$ stable isotope signatures in five adults indicated that bone $\mathrm{Pb}$ stores contributed to approximately $40-70 \%$ of the $\mathrm{Pb}$ in blood (Smith et al. 1996). During pregnancy, the mobilization of bone Pb increases, as the bone is resorbed to produce the fetal skeleton. Analysis for kinetics of changes in the stable isotope signatures of blood $\mathrm{Pb}$ in pregnant women as they came into equilibrium with a novel environmental $\mathrm{Pb}$ isotope signature indicated that 10 $88 \%$ of the $\mathrm{Pb}$ in blood may derive from the mobilization of bone $\mathrm{Pb}$ store and approximately $80 \%$ of cord blood may be contributed from maternal bone Pb (Gulson 2000; Gulson et al. 1997b, 1999c, 2003). The mobilization of bone $\mathrm{Pb}$ during pregnancy may contribute, along with other mechanisms (e.g., increased absorption), to the increase in $\mathrm{Pb}$ concentration that has been observed during the later stages of pregnancy (Gulson et al. 1997b, 2016; Lagerkvist et al. 1996; Schuhmacher et al. 1996). Bone resorption during pregnancy can be reduced by ingestion of calcium supplements (Janakiraman et al. 2003). Additional evidence for increased mobilization of bone $\mathrm{Pb}$ into blood during pregnancy is provided from studies in nonhuman primates and rats (Franklin et al. 1997; Maldonado-Vega et al. 1996). Direct evidence for transfer of maternal bone $\mathrm{Pb}$ to the fetus has been provided from stable $\mathrm{Pb}$ isotope studies in Cynomolgus monkeys (Macaca fascicularis) that were dosed with $\mathrm{Pb}$ having a different stable isotope ratio than the $\mathrm{Pb}$ to which the monkeys were exposed at an earlier age; approximately $7-39 \%$ of the maternal $\mathrm{Pb}$ burden that was transferred to the fetus appeared to have been derived from the maternal skeleton (Franklin et al. 1997).

In addition to pregnancy, other states of increased bone resorption appear to result in release of bone $\mathrm{Pb}$ to blood; these include lactation, osteoporosis, and severe weight loss. Analysis of kinetics of changes in the stable isotope signatures of blood $\mathrm{Pb}$ in postpartum women as they came into equilibrium with a novel environmental $\mathrm{Pb}$ isotope signature indicated that the release of maternal bone $\mathrm{Pb}$ to blood appears to accelerate during lactation (Gulson et al. 2002, 2003, 2004). This is consistent with declines in patella 
bone $\mathrm{Pb}$ (measured by XRF) during lactation without calcium supplementation (Hernandez-Avila et al. 1996). Similar approaches have detected increased release of bone $\mathrm{Pb}$ to blood in women, in association with menopause (Gulson et al. 2002). These observations are consistent with epidemiological studies that have shown increases in $\mathrm{PbB}$ after menopause and in association with decreasing bone density in postmenopausal women (Berkowitz et al. 2004; Garrido Latorre et al. 2003; Hernandez-Avila et al. 2000; Korrick et al. 2002; Nash et al. 2004; Popovic et al. 2005; Symanski and Hertz-Picciotto 1995). In a prospective study of women who were scheduled to undergo bilateral oophorectomy for benign conditions, blood and tibia bone $\mathrm{Pb}$ (measured by XRF and adjusted for bone mineral density) did not change 6-18 months post-surgery, regardless of whether patients were given estrogen replacement therapy (Berkowitz et al. 2004). Severe weight loss (28\% of BMI in 6 months) in women, which increased bone turnover, increased PbB (Riedt et al. 2009).

$\boldsymbol{P b}$ in Soft Tissues. Several studies have compared soft tissue concentrations of $\mathrm{Pb}$ in autopsy samples of soft tissues (Barry 1975, 1981; Gross et al. 1975; Schroeder and Tipton 1968). These studies were conducted in the 1960s and 1970s and, therefore, reflect burdens accrued during periods when ambient and occupational exposure levels were much higher than current levels. A more recent autopsy study found lower Pb concentrations in autopsies performed during the period 2004-2013 (Mari et al. 2014). Average PbBs reported in the adult subjects were approximately $20 \mu \mathrm{g} / \mathrm{dL}$ in the Barry (1975) and Gross et al. (1975) studies, whereas more current estimates of the average for adults in the United States are $<5 \mu \mathrm{g} / \mathrm{dL}$ (CDC 2018a). Levels in other soft tissues also appear to have decreased substantially since these studies were reported (Barregård et al. 1999; Mari et al. 2014). For example, average $\mathrm{Pb}$ concentrations in kidney cortex of male adults were $0.78 \mu \mathrm{g} / \mathrm{g}$ wet tissue and $0.79 \mu \mathrm{g} / \mathrm{g}$, as reported by Barry (1975) and Gross et al. (1975), respectively (samples in the Barry study were from subjects who had no known occupational exposures). An analysis of kidney biopsy samples collected in Sweden found that the mean level of $\mathrm{Pb}$ in kidney cortex among subjects not occupationally exposed to $\mathrm{Pb}$ was $0.18 \mu \mathrm{g} / \mathrm{g}$ (maximum, $0.56 \mu \mathrm{g} / \mathrm{g}$ ) (Barregård et al. 1999). Mari et al. (2014) reported a value of $0.18 \mu \mathrm{g} / \mathrm{g}$ for mean kidney $\mathrm{Pb}$ concentration in 20 autopsies performed in Spain. In spite of the downward trends in soft tissue Pb levels, the autopsy studies provide a basis for describing the relative soft tissue distribution of $\mathrm{Pb}$ in adults and children. Most of the $\mathrm{Pb}$ in soft tissue is in liver. Relative amounts of $\mathrm{Pb}$ in soft tissues as reported by Schroeder and Tipton (1968), expressed as percent of total soft tissue Pb, were: liver, 33\%; skeletal muscle, 18\%; skin, 16\%; dense connective tissue, 11\%; fat, $6.4 \%$; kidney, 4\%; lung, 4\%; aorta, $2 \%$; and brain, $2 \%$ (other tissues were $<1 \%$ ). The highest soft tissue concentrations in adults also occur in liver and kidney cortex (Barry 1975; Gerhardsson et al. 1986, 1995b; Gross et al. 1975; Mari et al. 2014; Oldereid et al. 1993). The relative distribution of $\mathrm{Pb}$ in soft tissues, in males and females, 
expressed in terms of tissue:liver concentration ratios, were: liver, 1.0 (approximately $1 \mu \mathrm{g} / \mathrm{g}$ wet weight); kidney cortex, 0.8; kidney medulla, 0.5; pancreas, 0.4; ovary, 0.4; spleen, 0.3; prostate, 0.2; adrenal gland, 0.2; brain, 0.1; fat, 0.1; testis, 0.08; heart, 0.07; and skeletal muscle, 0.05 (Barry 1975; Gross et al. 1975). In contrast to $\mathrm{Pb}$ in bone, which accumulates $\mathrm{Pb}$ with continued exposure in adulthood, concentrations in soft tissues (e.g., liver and kidney) are relatively constant in adults (Barry 1975; Treble and Thompson 1997), reflecting a faster turnover of Pb in soft tissue, relative to bone.

Mechanisms by which $\mathrm{Pb}$ enters soft tissues have not been fully characterized (Bressler et al. 2005). Studies conducted in preparations of mammalian small intestine support the existence of saturable and nonsaturable pathways of $\mathrm{Pb}$ transfer and suggest that $\mathrm{Pb}$ can interact with transport mechanisms for calcium and iron (see Section 3.1.1). Pb can enter cells through voltage-gated L-type $\mathrm{Ca}^{2+}$ channels in bovine adrenal medullary cells (Legare et al. 1998; Simons and Pocock 1987; Tomsig and Suszkiw 1991) and through store-operated $\mathrm{Ca}^{2+}$ channels in pituitary GH3, glial C3, human embryonic kidney, and bovine brain capillary endothelial cells (Kerper and Hinkle 1997a, 1997b). Anion exchangers may also participate in Pb transport in astrocytes (Bressler et al. 2005). In addition to the small intestine, DMT1 is expressed in the kidney (Canonne-Hergaux et al. 1999); however, little information is available regarding the transport of $\mathrm{Pb}$ across the renal tubular epithelium. In Madin-Darby canine kidney cells (MDCK), $\mathrm{Pb}$ has been shown to undergo transepithelial transport by a mechanism distinct from the anion exchanger that has been identified in red blood cells (Bannon et al. 2000). The uptake of Pb into MDCK cells was both time and temperature dependent. Overexpression of DMT1 in the human embryonic kidney fibroblast cells (HEK293) resulted in increased Pb uptake compared to HEK293 cells in which DMT1 was not overexpressed (Bannon et al. 2002). Based on this limited information, it appears that DMT1 may play a role in the renal transport of $\mathrm{Pb}$.

$\mathrm{Pb}$ in other soft tissues such as kidney, liver, and brain exists predominantly bound to protein. High affinity cytosolic $\mathrm{Pb}$ binding proteins have been identified in rat kidney and brain (DuVal and Fowler 1989; Gonick et al. 2011). The $\mathrm{Pb}$ binding proteins of rat are cleavage products of $\alpha 2 \mu$-globulin, a member of the protein superfamily known as retinol-binding proteins (Fowler and DuVal 1991). $\alpha 2 \mu$-Globulin is synthesized in the liver under androgen control and has been implicated in the mechanism of male rat hyaline droplet nephropathy produced by certain hydrocarbons (EPA 1991; Swenberg et al. 1989); however, there is no evidence that $\mathrm{Pb}$ induces male-specific nephropathy or hyaline droplet nephropathy. The precise role for $\mathrm{Pb}$ binding proteins in the toxicokinetics and toxicity of $\mathrm{Pb}$ has not been firmly established; however, it has been proposed that binding proteins may serve as a cytosolic Pb "receptor" that, when transported into the nucleus, binds to chromatin and modulates gene 
expression (Fowler and DuVal 1991; Mistry et al. 1985, 1986). Other high-affinity Pb binding proteins (Kd approximately $14 \mathrm{nM}$ ) have been isolated in human kidney, two of which have been identified as a $5 \mathrm{kD}$ peptide, thymosin 4, and a $9 \mathrm{kD}$ peptide, acyl-CoA binding protein (Smith et al. 1998b). $\mathrm{Pb}$ also binds to metallothionein, but does not appear to be a significant inducer of the protein in comparison with the inducers of cadmium and zinc (Eaton et al. 1980; Waalkes and Klaassen 1985). In vivo, only a small fraction of the $\mathrm{Pb}$ in the kidney is bound to metallothionein, and appears to have a binding affinity that is less than $\mathrm{Cd}^{2+}$, but higher than $\mathrm{Zn}^{2+}$ (Ulmer and Vallee 1969); thus, $\mathrm{Pb}$ will more readily displace zinc from metallothionein than cadmium (Goering and Fowler 1987; Nielson et al. 1985; Waalkes et al. 1984).

Pb Distribution during Pregnancy and Maternal-Fetal-Infant Transfer. PbBs tend to be lower in pregnant women compared to non-pregnant women of similar age, BMI, iron status, and smoking status (Jain 2013a; Liu et al. 2013). This difference may reflect increased elimination of $\mathrm{Pb}$ from the maternal system (Jain 2013b). Maternal PbB changes during and following pregnancy. A U-shaped temporal pattern has been observed in which maternal PbBs decrease during the second trimester and increase during the third trimester and postpartum period (Gulson et al. 2004, 1997b, 2016; Hertz-Picciotto et al. 2000; Lagerkvist et al. 1996; Lamadrid-Figueroa et al. 2006; Rothenberg et al. 1994). Several factors appear to contribute to these changes. During the second trimester, increased plasma volume contributes to hemodilution of maternal blood $\mathrm{Pb}$ and a lowering in the $\mathrm{PbB}$ (Hytten 1985). During the third trimester, growth of the fetal skeleton accelerates, which results in increased mobilization of calcium and $\mathrm{Pb}$ from the maternal skeleton, increasing maternal PbB (Gulson et al. 1998b, 2003). Postpartum calcium demand increases further during lactation and breastfeeding, which promotes further mobilization of calcium and $\mathrm{Pb}$ from bone and sustains or increases maternal PbBs (Gulson et al. 1998b; Hansen 2011; Tellez-Rojo et al. 2002). Increased demand for calcium in the third trimester and postpartum (to supply calcium for breast milk) is also evident from studies of the effects of dietary calcium supplementation during pregnancy. Calcium supplementation of the maternal diet decreased or delayed the onset of the increase in maternal PbB during the third trimester and postpartum period and delayed mobilization of maternal bone Pb in the third trimester (Ettinger et al. 2009; Gulson et al. 2004, 2016; Manton et al. 2003). The increase in PbB associated with late pregnancy was greater in older women who had a longer history of Pb exposure and, presumably, higher bone Pb levels (Miranda et al. 2010). Pb has been detected in follicular fluid at concentrations similar to that in blood plasma (Silberstein et al. 2006).

A portion of the maternal $\mathrm{Pb}$ burden is transferred to the placenta and fetus during pregnancy (EstebanVasaloo et al. 2012; Franklin et al. 1997; Gulson et al. 2003, 2016; Irwinda et al. 2019; Kayaalti et al. 
2016; Kazi et al. 2014; O’Flaherty 1998; Reddy et al. 2014). Measurements of stable Pb isotope ratios in pregnant women and cord blood, as they came into equilibrium with a novel environmental $\mathrm{Pb}$ isotope signature, indicated that approximately $80 \%$ of $\mathrm{Pb}$ in fetal cord blood appears to derive from maternal bone stores (Gulson et al. 1997b, 1999c, 2000, 2003, 2016). Stable isotope studies have also demonstrated transfer of $\mathrm{Pb}$ from the maternal skeleton to fetus in nonhuman primates (Franklin et al. 1997; O'Flaherty 1998). Transplacental transfer of $\mathrm{Pb}$ may be facilitated by an increase in the plasma/PbB ratio during pregnancy (Lamadrid-Figueroa et al. 2006; Montenegro et al. 2008).

Fetal and maternal PbBs and placental Pb concentrations are correlated (Amaral et al. 2010; Baeyens et al. 2014; Baranowska-Boisiacka et al. 2016; Carbone et al. 1998; Chen et al. 2014; Goyer 1990; Graziano et al. 1990; Gulson et al. 2016; Kayaalti et al. 2015b; Kazi et al. 2014; Kim et al. 2015; Kordas et al. 2009; Patel and Prabhu 2009; Reddy et al. 2014). Estimates of the maternal/fetal PbB ratio, based on cord blood $\mathrm{Pb}$ measurements at the time of delivery, range from 0.7 to 1.0 at mean maternal $\mathrm{PbBs}$ ranging from 1 to $9 \mu \mathrm{g} / \mathrm{dL}$. In one of the larger studies of fetal $\mathrm{PbB}$, maternal and cord $\mathrm{PbB}$ were measured at delivery in 888 mother-infant pairs; the cord/maternal ratio was relatively constant, 0.93, over a blood $\mathrm{Pb}$ range of approximately 3-40 $\mu \mathrm{g} / \mathrm{dL}$ (Graziano et al. 1990). An analysis of data from 159 mother-infant pairs revealed that higher blood pressure and alcohol consumption late in pregnancy were associated with higher concentrations of $\mathrm{Pb}$ in cord blood relative to maternal blood, while higher hemoglobin and sickle cell trait were associated with lower cord blood $\mathrm{Pb}$ relative to maternal blood $\mathrm{Pb}$ (Harville et al. 2005). No associations were found for calcium intake, physical activity, or smoking. Placental Pb concentrations were found to correlate with ALAD polymorphisms, with higher concentrations observed in association with ALAD2 (Kayaalti et al. 2015b).

Maternal $\mathrm{Pb}$ is transferred to infants during breastfeeding. Stable $\mathrm{Pb}$ isotope dilution studies suggested that $\mathrm{Pb}$ in breast milk can contribute substantially to the isotope profile of infant blood (approximately 40-80\%; Gulson et al. 1998b). Numerous studies have reported Pb concentrations in maternal blood and breast milk. In general, these studies indicate that $\mathrm{Pb}$ concentrations in breast milk are correlated with $\mathrm{Pb}$ concentrations in maternal blood or plasma. Milk/maternal concentration ratios are $<0.1$, although values of 0.9 have been reported (Baranowska-Boisiacka et al. 2016; Counter et al. 2014; Ettinger et al. 2006, 2014; Gulson et al. 1998a; Koyashiki et al. 2010). Ettinger et al. (2004, 2006) assessed factors influencing breast milk $\mathrm{Pb}$ concentration in a group of 367 women and found that $\mathrm{PbB}$ (mean 8-9 $\mu \mathrm{g} / \mathrm{dL}$; range 2-30) was a stronger predictor of breast milk $\mathrm{Pb}$ (mean 0.9-1.4 $\mu \mathrm{g} / \mathrm{dL}$; range $0.2-8 \mu \mathrm{g} / \mathrm{dL}$ ) than bone $\mathrm{Pb}$, and that tibia $\mathrm{Pb}$ (mean $9.5 \mu \mathrm{g} / \mathrm{g}$; range $<1-76.5 \mu \mathrm{g} / \mathrm{dL}$ ) was a stronger predictor of breast milk $\mathrm{Pb}$ than patella bone $\mathrm{Pb}$ (mean $14.6 \mu \mathrm{g} / \mathrm{dL}$; range $<1-67.2 \mu \mathrm{g} / \mathrm{dL}$ ). Dietary intake of polyunsaturated fatty 
acids (PUFA) may decrease transfer of $\mathrm{Pb}$ from bone to breast milk (Arora et al. 2008). $\mathrm{Pb}$ concentrations in maternal blood and breast milk have been shown to correlate with $\mathrm{PbBs}$ in breastfeeding infants (Ettinger et al. 2014; Farhat et al. 2013). Breast milk Pb concentrations explained 37\% of the variation in infant blood $\mathrm{Pb}$ of breastfeeding infants (Ettinger et al. 2014).

Organic Pb. Information on the distribution of $\mathrm{Pb}$ in humans following exposures to organic $\mathrm{Pb}$ is extremely limited. One hour following 1-2-minute inhalation exposures to ${ }^{203} \mathrm{~Pb}$ tetraethyl or tetramethyl $\mathrm{Pb}\left(1 \mathrm{mg} / \mathrm{m}^{3}\right)$, approximately $50 \%$ of the ${ }^{203} \mathrm{~Pb}$ body burden was associated with liver and $5 \%$ was associated with kidney; the remaining ${ }^{203} \mathrm{~Pb}$ was widely distributed throughout the body (Heard et al. 1979). The kinetics of ${ }^{203} \mathrm{~Pb}$ in blood of these subjects showed an initial declining phase during the first 4 hours (tetramethyl $\mathrm{Pb}$ ) or 10 hours (tetraethyl $\mathrm{Pb}$ ) after the exposure, followed by a phase of gradual increase in $\mathrm{PbB}$ that lasted for up to 500 hours after the exposure. Radioactive $\mathrm{Pb}$ in blood was highly volatile immediately after the exposure and transitioned to a nonvolatile state thereafter. These observations may reflect an early distribution of organic $\mathrm{Pb}$ from the respiratory tract, followed by a redistribution of de-alkylated $\mathrm{Pb}$ compounds (see Section 3.1.3 for further discussion of alkyl $\mathrm{Pb}$ metabolism).

In a man and woman who accidentally inhaled a solvent containing $31 \%$ tetraethyl $\mathrm{Pb}(17.6 \% \mathrm{~Pb}$ by weight), $\mathrm{Pb}$ concentrations in the tissues, from highest to lowest, were liver, kidney, brain, pancreas, muscle, and heart (Bolanowska et al. 1967). In another incident, a man ingested a chemical containing 59\% tetraethyl $\mathrm{Pb}$ (38\% $\mathrm{Pb}$ w/w); $\mathrm{Pb}$ concentration was highest in the liver followed by kidney, pancreas, brain, and heart (Bolanowska et al. 1967).

\subsubsection{Metabolism}

Inorganic Pb. Metabolism of inorganic $\mathrm{Pb}$ consists of formation of complexes with a variety of protein and nonprotein ligands (see Section 3.1.2 for further discussion). Major extracellular ligands include albumen and nonprotein sulfhydryls. The major intracellular ligand in red blood cells is ALAD. Pb also forms complexes with proteins in the cell nucleus and cytosol.

Organic Pb. Alkyl Pb compounds are actively metabolized in the liver by oxidative dealkylation catalyzed by cytochrome P-450. Relatively few studies that address the metabolism of alkyl Pb compounds in humans have been reported. Studies of workers who were exposed to tetraethyl $\mathrm{Pb}$ have shown that tetraethyl $\mathrm{Pb}$ is excreted in the urine as diethyl $\mathrm{Pb}$, ethyl $\mathrm{Pb}$, and inorganic $\mathrm{Pb}$ (Turlakiewicz 
and Chmielnicka 1985; Vural and Duydu 1995; Zhang et al. 1994). Trialkyl Pb metabolites were found in the liver, kidney, and brain following exposure to the tetraalkyl compounds in workers; these metabolites have also been detected in brain tissue of nonoccupational subjects (Bolanowska et al. 1967; Nielsen et al. 1978). In volunteers exposed by inhalation to 0.64 and $0.78 \mathrm{mg} \mathrm{Pb} / \mathrm{m}^{3}$ of ${ }^{203} \mathrm{~Pb}$-labeled tetraethyl and tetramethyl $\mathrm{Pb}$, respectively, $\mathrm{Pb}$ was cleared from the blood within 10 hours, followed by a re-appearance of radioactivity back into the blood after approximately 20 hours (Heard et al. 1979). The high level of radioactivity initially in the plasma indicates the presence of tetraalkyl/trialkyl $\mathrm{Pb}$. The subsequent rise in blood radioactivity, however, probably represents water-soluble inorganic $\mathrm{Pb}$ and trialkyl and dialkyl $\mathrm{Pb}$ compounds that were formed from the metabolic conversion of the volatile parent compounds (Heard et al. 1979).

\subsubsection{Excretion}

Independent of the route of exposure, absorbed $\mathrm{Pb}$ is excreted primarily in urine and feces; sweat, saliva, hair and nails, breast milk, and seminal fluids are minor routes of excretion (Chamberlain et al. 1978; Griffin et al. 1975; Hernandez-Ochoa et al. 2005; Hursh and Suomela 1968; Hursh et al. 1969; Kehoe 1987; Rabinowitz et al. 1976; Sears et al. 2012; Stauber et al. 1994). Fecal excretion accounts for approximately one-third of total excretion of absorbed $\mathrm{Pb}$ (fecal/urinary excretion ratio of approximately 0.5), based on intravenous injection studies conducted in humans (Chamberlain et al. 1978). A similar value for fecal/urinary excretion ratio, approximately 0.5 , has been observed following inhalation of submicron Pb particles (Chamberlain et al. 1978; Hursh et al. 1969). Contributors to fecal excretion may include secretion into the bile, gastric fluid, and saliva (Rabinowitz et al. 1976). Biliary excretion of $\mathrm{Pb}$ has been observed in the dog, rat, and rabbit (Klaassen and Shoeman 1974; O'Flaherty 1993).

Mechanisms by which inorganic $\mathrm{Pb}$ is excreted in urine have not been fully characterized. Such studies have been hampered by the difficulties associated with measuring ultrafilterable $\mathrm{Pb}$ in plasma and thereby in measuring the GFR of Pb. Renal plasma clearance was approximately $20-30 \mathrm{~mL} /$ minute in a subject who received a single intravenous injection of a ${ }^{203} \mathrm{~Pb}$ chloride tracer (Chamberlain et al. 1978). Urinary $\mathrm{Pb}$ excretion is strongly correlated with the GFR of $\mathrm{Pb}$ (Araki et al. 1986) and plasma $\mathrm{Pb}$ concentration (Bergdahl et al. 1997b; Rentschler et al. 2012) (i.e., urinary excretion is proportional to GFR x plasma Pb concentration). Estimates of plasma-to-urine clearance of $\mathrm{Pb}$ range from 13 to $22 \mathrm{~L} /$ day, with a mean of 18 L/day (Araki et al. 1986; Manton and Cook 1984; Manton and Malloy 1983; Chamberlain et al. 1978). The rate of urinary excretion of $\mathrm{Pb}$ was less than the GFR of ultrafilterable $\mathrm{Pb}$, suggesting renal tubular reabsorption of $\mathrm{Pb}$ from the glomerular filtrate (Araki et al. 1986, 1990). Measurement of the renal 
clearance of ultrafilterable $\mathrm{Pb}$ in plasma indicates that in dogs, $\mathrm{Pb}$ undergoes glomerular filtration and net tubular reabsorption (Araki et al. 1986, 1990; Vander et al. 1977; Victery et al. 1979). Net tubular secretion of $\mathrm{Pb}$ has been demonstrated in dogs made alkalotic by infusions of bicarbonate (Victery et al. 1979). Renal clearance of blood $\mathrm{Pb}$ increases with increasing $\mathrm{PbBs}>25 \mu \mathrm{g} / \mathrm{dL}$ (Chamberlain 1983). The mechanism for this has not been elucidated and could involve a shift in the distribution of $\mathrm{Pb}$ in blood towards a fraction having a higher GFR (e.g., lower molecular weight complex), a capacity-limited mechanism in the tubular reabsorption of $\mathrm{Pb}$, or the effects of $\mathrm{Pb}$-induced nephrotoxicity on $\mathrm{Pb}$ reabsorption. Renal clearance of blood $\mathrm{Pb}$ has been estimated in approximately 7,600 subjects who participated in the NHANES 2009-2016 (Diamond et al. 2019). Blood Pb concentrations ranged from 0.05 to $34 \mu \mathrm{g} / \mathrm{dL}$, with medians of $0.54 \mu \mathrm{g} / \mathrm{dL}$ in adolescents (12-<20 years old) and $1.08 \mu \mathrm{g} / \mathrm{dL}$ in adults ( $\geq 20$ years old). The median blood $\mathrm{Pb}$ clearance was $0.043 \mathrm{~L} /$ day in adolescents and $0.040 \mathrm{~L} /$ day in adults. Blood Pb clearance was approximately 3\% of GFR, estimated from creatinine clearance.

\section{Excretion and Routes of Exposure}

\section{Inhalation Exposure}

Inorganic $\mathrm{Pb}$. Inorganic $\mathrm{Pb}$ inhaled as submicron particles is deposited primarily in the bronchiolar and alveolar regions of the respiratory tract, from where it is absorbed and excreted primarily in urine and feces (Chamberlain et al. 1978; Hursh et al. 1969; Kehoe 1987). Fecal/urinary excretion ratios were approximately 0.5 following inhalation of submicron Pb-bearing particles (Chamberlain et al. 1978; Hursh et al. 1969). Higher fecal-urinary ratios would be expected following inhalation of larger particle sizes (e.g., $>1 \mu \mathrm{m}$ ) as these particles would be cleared to the gastrointestinal tract from where a smaller percentage would be absorbed (Kehoe 1987; see Section 3.1.1).

Organic $\mathrm{Pb}$. $\mathrm{Pb}$ derived from inhaled tetraethyl and tetramethyl $\mathrm{Pb}$ is excreted in exhaled air, urine, and feces (Heard et al. 1979). Following 1-2-minute inhalation exposures to ${ }^{203} \mathrm{~Pb}$ tetraethyl $\left(1 \mathrm{mg} / \mathrm{m}^{3}\right)$, in four male subjects, $37 \%$ of inhaled ${ }^{203} \mathrm{~Pb}$ was initially deposited in the respiratory tract, of which approximately 20\% was exhaled in the subsequent 48 hours (Heard et al. 1979). In a similar experiment conducted with $\left({ }^{203} \mathrm{~Pb}\right)$ tetramethyl $\mathrm{Pb}, 51 \%$ of the inhaled ${ }^{203} \mathrm{~Pb}$ dose was initially deposited in the respiratory tract, of which approximately $40 \%$ was exhaled in 48 hours. Pb that was not exhaled was excreted in urine and feces. Fecal/urinary excretion ratios were 1.8 following exposure to tetraethyl $\mathrm{Pb}$ and 1.0 following exposure to tetramethyl $\mathrm{Pb}$ (Heard et al. 1979). Occupational monitoring studies of workers who were exposed to tetraethyl $\mathrm{Pb}$ have shown that tetraethyl $\mathrm{Pb}$ is excreted in the urine as 
diethyl Pb, ethyl Pb, and inorganic Pb (Turlakiewicz and Chmielnicka 1985; Vural and Duydu 1995; Zhang et al. 1994).

\section{Oral Exposure}

Inorganic $\mathrm{Pb}$. Much of the available information on the excretion of ingested $\mathrm{Pb}$ in adults derives from studies conducted on five male adults who received daily doses of ${ }^{207} \mathrm{~Pb}$ nitrate for periods up to 210 days (Rabinowitz et al. 1976). The dietary intakes of the subjects were reduced to accommodate the tracer doses of ${ }^{207} \mathrm{~Pb}$ without increasing daily intake, thus preserving a steady state with respect to total $\mathrm{Pb}$ intake and excretion. Total $\mathrm{Pb}$ intakes (diet plus tracer) ranged from approximately 210 to $360 \mu \mathrm{g} /$ day. Urinary excretion accounted for approximately $12 \%$ of the daily intake (range for five subjects: $7-17 \%$ ) and fecal excretion, approximately 90\% of the daily intake (range, 87-94\%). Based on measurements of tracer and total $\mathrm{Pb}$ in saliva, gastric secretions, bile, and pancreatic secretions (samples collected from three subjects by intubation), gastrointestinal secretion of Pb was estimated to be approximately $2.4 \%$ of intake (range, 1.9-3.3\%). In studies conducted at higher ingestion intakes, 1-3 mg/day for up to 208 weeks, urinary $\mathrm{Pb}$ excretion accounted for approximately 5\% of the ingested dose (Kehoe 1987). Elimination of $\mathrm{Pb}$ is multiphasic, reflecting pools of $\mathrm{Pb}$ in the body that have varying retention times. Elimination from blood and soft tissues is faster than bone (Nilsson et al. 1991; Rabinowitz et al. 1976). As a result, after an abrupt decrease in exposure, $\mathrm{PbB}$ declines at an apparent rate that reflects excretion of $\mathrm{Pb}$ from blood and replenishment of $\mathrm{Pb}$ in blood from bone stores. The elimination half-time of $\mathrm{Pb}$ in blood in retired $\mathrm{Pb}$ workers was tri-exponential, with approximately $22 \%$ of elimination occurring at a half-time of 34 days (95\% CI: 29, 41), 28\% at a half-time of 1.2 years (95\% CI: 0.85, 1.8), and 50\% at a half-time of 13 years (95\% CI: 10, 18) (Nilsson et al. 1991). The corresponding mono-exponential halftime for finger bone (XRF) in these same subjects was 16 years (85\% CL 12, 23). Apparent elimination half-times for blood $\mathrm{Pb}$ in children also vary considerably, dependent in part on age and exposure history of the child that establishes levels of Pb in bone (Manton et al. 2000; Specht et al. 2018). Manton et al. (2000) estimated apparent elimination half-times for PbB in children (ages 2-3 years at time of exposure) that ranged from 8 to 38 months. However, these estimates reflect both excretion of $\mathrm{Pb}$ from blood as well as transfer of $\mathrm{Pb}$ from bone to blood; the latter would tend to increase the apparent blood elimination half-time. Specht et al. (2018) estimated blood $\mathrm{Pb}$ elimination half-times for $\mathrm{Pb}$ transferred from bone to blood (estimated with XRF measurements and biokinetics modeling). Estimated blood Pb half-times were $6.9 \pm 4$ (SD) days in children 1-3 years old and 19.3 \pm 14.1 days in children $>3$ years old (Specht et al. 2018). 
Dermal Exposure. Inorganic $\mathrm{Pb}$ is excreted in sweat and urine following dermal exposure to $\mathrm{Pb}$ nitrate or $\mathrm{Pb}$ acetate (Moore et al. 1980; Stauber et al. 1994).

\subsubsection{Physiologically Based Pharmacokinetic (PBPK)/Pharmacodynamic (PD) Models}

PBPK models use mathematical descriptions of the uptake and disposition of chemical substances to quantitatively describe the relationships among critical biological processes (Krishnan et al. 1994). PBPK models are also called biologically based tissue dosimetry models. PBPK models are increasingly used in risk assessments, primarily to predict the concentration of potentially toxic moieties of a chemical that will be delivered to any given target tissue following various combinations of route, dose level, and test species (Clewell and Andersen 1985). Physiologically based pharmacodynamic (PBPD) models use mathematical descriptions of the dose-response function to quantitatively describe the relationship between target tissue dose and toxic endpoints.

Early Pb modeling applications relied on classical pharmacokinetics. Compartments representing individual organs or groups of organs that share a common characteristic were defined as volumes, or pools, that are kinetically homogeneous. For example, the body could be represented by a central compartment (e.g., blood plasma), and one or two peripheral compartments, which might be "shallow" or “deep” (i.e., they may exchange relatively rapidly or relatively slowly with blood plasma) (O'Flaherty 1987). One of the first of such models was proposed by Rabinowitz et al. (1976) based on a study of the kinetics of ingested stable $\mathrm{Pb}$ isotope tracers and $\mathrm{Pb}$ balance data in five healthy adult males. The Rabinowitz model included three compartments: a central compartment representing blood and other tissues and spaces in rapid equilibrium with blood (e.g., interstitial fluid); a shallow tissue compartment, representing soft tissues and rapidly exchanging pools within the skeleton; and a deep tissue compartment, representing, primarily, slowly exchanging pools of $\mathrm{Pb}$ within bone. Excretion pathways represented in the model included urinary, from the central compartment, and bile, sweat, hair, and nails, from the shallow tissue compartment. The model predicted pseudo-first-order half-times for $\mathrm{Pb}$ of approximately 25,28 , and $10^{4}$ days in the central, shallow tissue, and deep compartments, respectively. The slow kinetics of the deep tissue compartment led to the prediction that it would contain most of the $\mathrm{Pb}$ burden after lengthy exposures (e.g., years), consistent with $\mathrm{Pb}$ measurements made in human autopsy samples (see Section 3.1.2 Distribution). Note that this model did not simulate the distribution of $\mathrm{Pb}$ within blood (e.g., erythrocytes and plasma), nor did it simulate subcompartments within bone or physiological processes of bone turnover that might affect kinetics of the deep tissue compartment. 
Marcus (1985b) reanalyzed the data from stable isotope tracer studies of Rabinowitz et al. (1976) and derived an expanded multicompartment kinetic model for $\mathrm{Pb}$ that included separate compartments for cortical (slow, $\mathrm{t}_{1 / 2} 1.2 \times 10^{4}-3.5 \times 10^{4}$ days) and trabecular (fast, $\mathrm{t}_{1 / 2}$ 100-700 days), an approach subsequently adopted in several models (Bert et al. 1989; EPA 1994a, 1994b; Leggett 1993; O’Flaherty 1993, 1995a). A more complex representation of the $\mathrm{Pb}$ disposition in bone included explicit simulation of diffusion of $\mathrm{Pb}$ within the bone volume of the osteon and exchange with blood at the canaliculus (Marcus 1985a). The bone diffusion model was based on Pb kinetics data from studies conducted in dogs. Marcus (1985c) also introduced nonlinear kinetics of exchange of Pb between plasma and erythrocytes. The blood model included four blood subcompartments: diffusible $\mathrm{Pb}$ in plasma, proteinbound Pb in plasma, a "shallow" erythrocyte pool, and a "deep" erythrocyte pool. This model predicted the curvilinear relationship between plasma and PbBs observed in humans (see Section 3.1.2 Distribution for further discussion of plasma-erythrocyte Pb concentrations).

Additional information on $\mathrm{Pb}$ biokinetics, bone mineral metabolism, and $\mathrm{Pb}$ exposures has led to further refinements and expansions of these earlier modeling efforts. Four pharmacokinetic models, in particular, are currently being used or are being considered for broad application in Pb risk assessment: (1) the O'Flaherty Model, which simulates Pb kinetics from birth through adulthood (O'Flaherty 1993, 1995a); (2) the EPA Integrated Exposure Uptake BioKinetic (IEUBK) Model for Lead in Children developed by EPA (1994a, 1994b); (3) the Leggett Model, which simulates Pb kinetics from birth through adulthood (Leggett 1993); and (4) the EPA All Ages Lead Model (AALM, EPA 2014a). The AALM is currently under review by EPA; a version of the model is available at https://cfpub.epa.gov/ncea/risk/ recordisplay.cfm?deid=343670 (January 10, 2020). The structure and parameterization of the O'Flaherty Model is distinct from both the IEUBK Model and Leggett Model. The AALM is an update of the O’Flaherty and Leggett models, extended to include a multi-media exposure model.

The IEUBK Model simulates multimedia exposures, uptake, and kinetics of $\mathrm{Pb}$ in children ages 0-7 years for predicting pseudo-steady state relationships between $\mathrm{Pb}$ exposure and $\mathrm{PbB}$; the model is not intended for use in predicting short-term kinetics of blood $\mathrm{Pb}$ or $\mathrm{Pb}$ concentrations in tissues other than whole blood. The O'Flaherty Model, Leggett Model, and AALM are lifetime models, and include parameters that simulate uptake and kinetics of Pb during infancy, childhood, adolescence, and adulthood. $\mathrm{Pb}$ exposure (e.g., residence-specific environmental $\mathrm{Pb}$ concentrations, childhood activity patterns) is not readily described by current versions of the O'Flaherty and Leggett models. The IEUBK Model and AALM include parameters for simulating exposures and uptake to estimate average daily uptake of $\mathrm{Pb}$ 
( $\mu \mathrm{g} /$ day) among populations potentially exposed via soil and dust ingestion, air inhalation, tap water ingestion, diet, and miscellaneous (other) intakes. All four models have been calibrated, to varying degrees, against empirical physiological data on animals and humans, and data on PbBs in individuals and/or populations (Beck et al. 2001; Bowers and Mattuck 2001; Cal EPA 2013; EPA 1994a, 1994c, 2014a, 2014b, 2016; Griffin et al. 1999; Hogan et al. 1998; Leggett 1993; Li et al. 2016a; MacMillan et al. 2015; O'Flaherty 1993, 1995a, 1998, 2000; Pounds and Leggett 1998; White et al. 1998; Von Lindern et al. 2003, 2016).

The focus on relying on PbBs for model evaluation and calibration derives from several concerns. The empirical basis for a relationship between low levels of $\mathrm{Pb}$ exposure and behavioral dysfunction largely consists of prospective epidemiological studies relating various indices of dysfunction with $\mathrm{PbB}$ (see Section 3.3). In this context, $\mathrm{PbB}$ has been related to health effects of $\mathrm{Pb}$, and this is the main reason that the focus of interest in the models has been on estimating PbBs. Also, the most available data with which to calibrate and validate the models have been data relating exposure and/or $\mathrm{Pb}$ intake to blood concentration. Thus, there is greater confidence in the validity of the models for estimating blood concentrations, rather than $\mathrm{Pb}$ levels in other physiologic compartments. Although the principal adverse health effects of $\mathrm{Pb}$ have been related to concentrations of $\mathrm{Pb}$ in blood, other biomarkers of $\mathrm{Pb}$ exposure, such as bone $\mathrm{Pb}$ concentrations, are also of value in assessing associations between $\mathrm{Pb}$ exposure and health; hence, there is a need for models that predict concentrations of $\mathrm{Pb}$ in tissues other than blood (see Section 3.3).

The following four pharmacokinetic models are discussed in great detail below: (1) the O'Flaherty Model (O'Flaherty 1993, 1995a); (2) the IEUBK Model for Lead in Children (EPA 1994a, 1994b); (3) the Leggett Model (Leggett 1993); and (4) AALM (EPA 2014a).

\subsubsection{O'Flaherty Model}

The O'Flaherty Model simulates Pb exposure, uptake, and disposition in humans, from birth through adulthood (O'Flaherty 1993, 1995a). Figure 3-1 shows a conceptualized representation of the O'Flaherty Model, including the movement of $\mathrm{Pb}$ from exposure media (i.e., intake via inhalation or ingestion) to the lungs and gastrointestinal tract, followed by the subsequent exchanges between blood plasma, liver, kidney, richly-perfused tissues, poorly-perfused tissues, bone compartments, and excretion from liver and/or kidney. The model simulates both age- and media-specific absorption. Because many of the pharmacokinetic functions are based on body weight and age, the model can be used to estimate PbBs 


\section{Figure 3-1. Compartments and Pathways of Lead (Pb) Exchange in the O'Flaherty Model* $^{*}$}

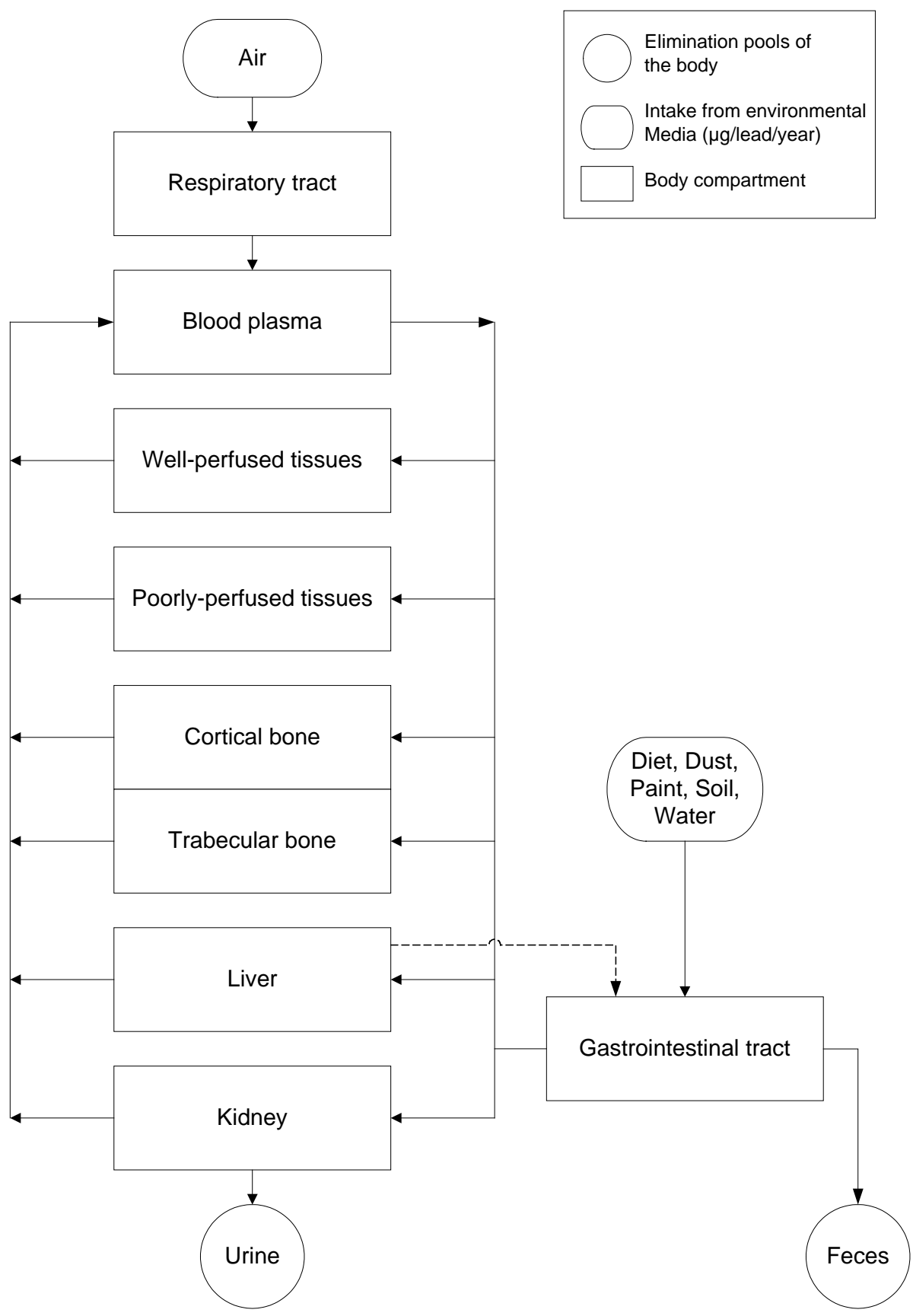

*Schematic model for $\mathrm{Pb}$ kinetics in which $\mathrm{Pb}$ distribution is represented by flows from blood plasma to liver, kidney, richly-perfused tissues, poorly-perfused tissues, and cortical and trabecular bone. The model simulates tissue growth with age, including growth and resorption of bone mineral.

Sources: O'Flaherty 1991b, 1993, 1995a 
across a broad age range, including infants, children, adolescents, and adults. The model uses physiologically based parameters to describe the volume, composition, and metabolic activity of blood, soft tissues, and bone that determine the disposition of $\mathrm{Pb}$ in the human body.

A central feature of the model is the growth curve, a logistic expression relating body weight to age. The full expression relating weight to age has five parameters (constants), so that it can readily be adapted to fit a range of standardized growth curves for men and women. Tissue growth and volumes are linked to body weight; this provides explicit modeling of concentrations of $\mathrm{Pb}$ in tissues. Other physiologic functions (e.g., bone formation) are linked to body weight, age, or both.

$\mathrm{Pb}$ exchange between blood plasma and bone is simulated as parallel processes occurring in cortical (80\% of bone volume) and trabecular bone (20\% of bone volume). Uptake and release of $\mathrm{Pb}$ from trabecular bone and metabolically active cortical bone are functions of bone formation and resorption rates, respectively. Rates of bone formation and resorption are simulated as age-dependent functions, which gives rise to an age-dependence of $\mathrm{Pb}$ kinetics in bone. The model simulates an age-related transition from immature bone, in which bone turnover (formation and resorption) rates are relatively high, to mature bone, in which turnover is relatively slow. Changes in bone mineral turnover associated with senescence (e.g., postmenopausal osteoporosis) are not represented in the model. In addition to metabolically active regions of bone, in which $\mathrm{Pb}$ uptake and loss is dominated by bone formation and loss, a region of slow kinetics in mature cortical bone is also simulated, in which Pb uptake and release to blood occur by heteroionic exchange with other minerals (e.g., calcium). Heteroionic exchange is simulated as a radial diffusion in bone volume of the osteon. All three processes are linked to body weight, or the rate of change of weight with age. This approach allows for explicit simulation of the effects of bone formation (e.g., growth) and loss, changes in bone volume, and bone maturation on $\mathrm{Pb}$ uptake and release from bone. Exchanges of Pb between blood plasma and soft tissues (e.g., kidney and liver) are represented as flow-limited processes. The model simulates saturable binding of $\mathrm{Pb}$ in erythrocytes; this replicates the curvilinear relationship between plasma and erythrocyte $\mathrm{Pb}$ concentrations observed in humans (see Section 3.1.2). Excretory routes include kidney to urine and liver to bile. Total excretion (clearance from plasma attributable to bile and urine) is simulated as a function of GFR. Biliary and urinary excretory rates are proportioned as 70 and $30 \%$ of the total plasma clearance, respectively.

The O'Flaherty Model simulates $\mathrm{Pb}$ intake from inhalation and ingestion. Inhalation rates are agedependent. Absorption of inhaled $\mathrm{Pb}$ is simulated as a fraction (0.5) of the amount inhaled, and is 
independent of age. The model simulates ingestion exposures from infant formula, soil and dust ingestion, and drinking water ingestion. Rates of soil and dust ingestion are age-dependent, increasing to approximately $130 \mathrm{mg} /$ day at age 2 years, and declining to $<1 \mathrm{mg}$ /day after age 10 years. Gastrointestinal absorption of $\mathrm{Pb}$ in diet and drinking water is simulated as an age-dependent fraction, declining from 0.58 of the ingestion rate at birth to 0.08 after age 8 years. These values can be factored to account for relative bioavailability when applied to absorption of $\mathrm{Pb}$ ingested in dust or soil.

The O'Flaherty Model, as described in O’Flaherty (1993, 1995a), utilizes point estimates for parameter values and yields point estimates as output; however, a subsequent elaboration of the model has been developed that utilizes a Monte Carlo approach to simulate variability in exposure, absorption, and erythrocyte $\mathrm{Pb}$ binding capacity (Beck et al. 2001). This extension of the model can be used to predict the probability that children exposed to $\mathrm{Pb}$ in environmental media will have PbBs exceeding a health-based reference value (e.g., $5 \mu \mathrm{g} / \mathrm{dL}$ ).

The model was designed to operate with an exposure time step on 1 year (the smallest time interval for a single exposure event). However, the implementation code allows constructions of simulations with an exposure time step as small as 1 day, which would allow simulation of rapidly changing intermittent exposures (e.g., an acute exposure event).

The O'Flaherty Model was initially calibrated to predict blood, bone, and tissue Pb concentrations in rats (O'Flaherty 1991a), and subsequently modified to reflect anatomical and physiological characteristics in children (O'Flaherty 1995a), adults (O'Flaherty 1993), and Cynomolgus monkeys (M. fasicularis) (O’Flaherty et al. 1998). Model parameters were modified to correspond with available information on species- and age-specific anatomy and physiological processes described above. Comparisons of predicted and observed PbB in children and adults are reported in O'Flaherty (1993, 1995a). MacMillan et al. (2015) evaluated performance of the model for predicting population blood and bone $\mathrm{Pb}$ levels in a convenience sample of 263 individuals (age range 1-83 years) who experienced low chronic exposure. Based on this evaluation, model performance for predicting general trends in population PbBs and cortical bone $\mathrm{Pb}$ concentrations was improved by revising parameters that determine binding of $\mathrm{Pb}$ in red blood cells. Revisions included decreasing the maximum and affinity constants (BIND and KBIND, respectively) and increasing clearance of $\mathrm{Pb}$ from blood to bone by increasing the permeability constant for $\mathrm{Pb}$ diffusion across the canaliculi-bone interface from canaliculi to bone $\left(P_{0}\right)$. 


\subsubsection{EPA IEUBK Model}

The EPA IEUBK Model for Lead in Children simulates Pb exposure, uptake, and disposition in human children from birth to age 7 years (EPA 1994a, 1994b, 2002a; White et al. 1998). Figure 3-2 shows a conceptualized representation of the IEUBK Model. The model has four major submodels: (1) exposure model, in which average daily intakes of $\mathrm{Pb}(\mu \mathrm{g} /$ day) are calculated for each inputted exposure concentration (or rates) of Pb in air, diet, dust, soil, and water; (2) uptake model, which converts environmental media-specific $\mathrm{Pb}$ intake rates calculated from the exposure model into a media-specific time-averaged uptake rate ( $\mu \mathrm{g} /$ day) of $\mathrm{Pb}$ to the central compartment (blood plasma); (3) biokinetic model, which simulates the transfer of absorbed $\mathrm{Pb}$ between blood and other body tissues, elimination of $\mathrm{Pb}$ from the body (via urine, feces, skin, hair, and nails), and predicts an average $\mathrm{PbB}$ for the exposure time period of interest; and (4) blood $\mathrm{Pb}$ probability model, which applies a log-normal distribution (using geometric mean and geometric standard deviation for parameters) to predict probabilities for the occurrence of a specified given $\mathrm{PbB}$ in a population of similarly exposed children.

Exposure Model. The exposure model simulates intake of $\mathrm{Pb}(\mu \mathrm{g} /$ day) for inputted exposures to $\mathrm{Pb}$ in air $\left(\mu \mathrm{g} / \mathrm{m}^{3}\right)$, drinking water $(\mu \mathrm{g} / \mathrm{L})$, soil-derived dust $(\mu \mathrm{g} / \mathrm{g})$, or diet $(\mu \mathrm{g} /$ day $)$. The exposure model operates on a 1-year time step, the smallest time interval for a single exposure event. The model accepts inputs for media intake rates (e.g., air volumes, breathing rates, drinking water consumption rate, soil and dust ingestion rate). The air exposure pathway is partitioned in exposures to outdoor air and indoor air, with age-dependent values for time spent outdoors and indoors (hours/day). Exposure to $\mathrm{Pb}$ to soil-derived dust is also partitioned into outdoor and indoor contributions. The intakes from all ingested exposure media (diet, drinking water, soil-derived dust) are summed to calculate a total intake to the gastrointestinal tract, for estimating capacity-limited absorption (see description of the uptake model).

Uptake Model. The uptake model simulates $\mathrm{Pb}$ absorption for the gastrointestinal tract as the sum of capacity-limited (represented by a Michaelis-Menten type relationship) and unlimited processes (represented by a first-order, linear relationship). These two terms are intended to represent two different mechanisms of $\mathrm{Pb}$ absorption, an approach that is in accord with limited available data in humans and animals that suggest a capacity limitation to $\mathrm{Pb}$ absorption (see Section 3.2.1). One of the parameters for the capacity-limited absorption process (that represents that maximum rate of absorption) is agedependent. The above representation gives rise to a decrease in the fractional absorption of ingested $\mathrm{Pb}$ as a function of total $\mathrm{Pb}$ intake as well as an age-dependence of fractional $\mathrm{Pb}$ absorption. Absorption 
Figure 3-2. Structure of the IEUBK Model for Lead (Pb) in Children*
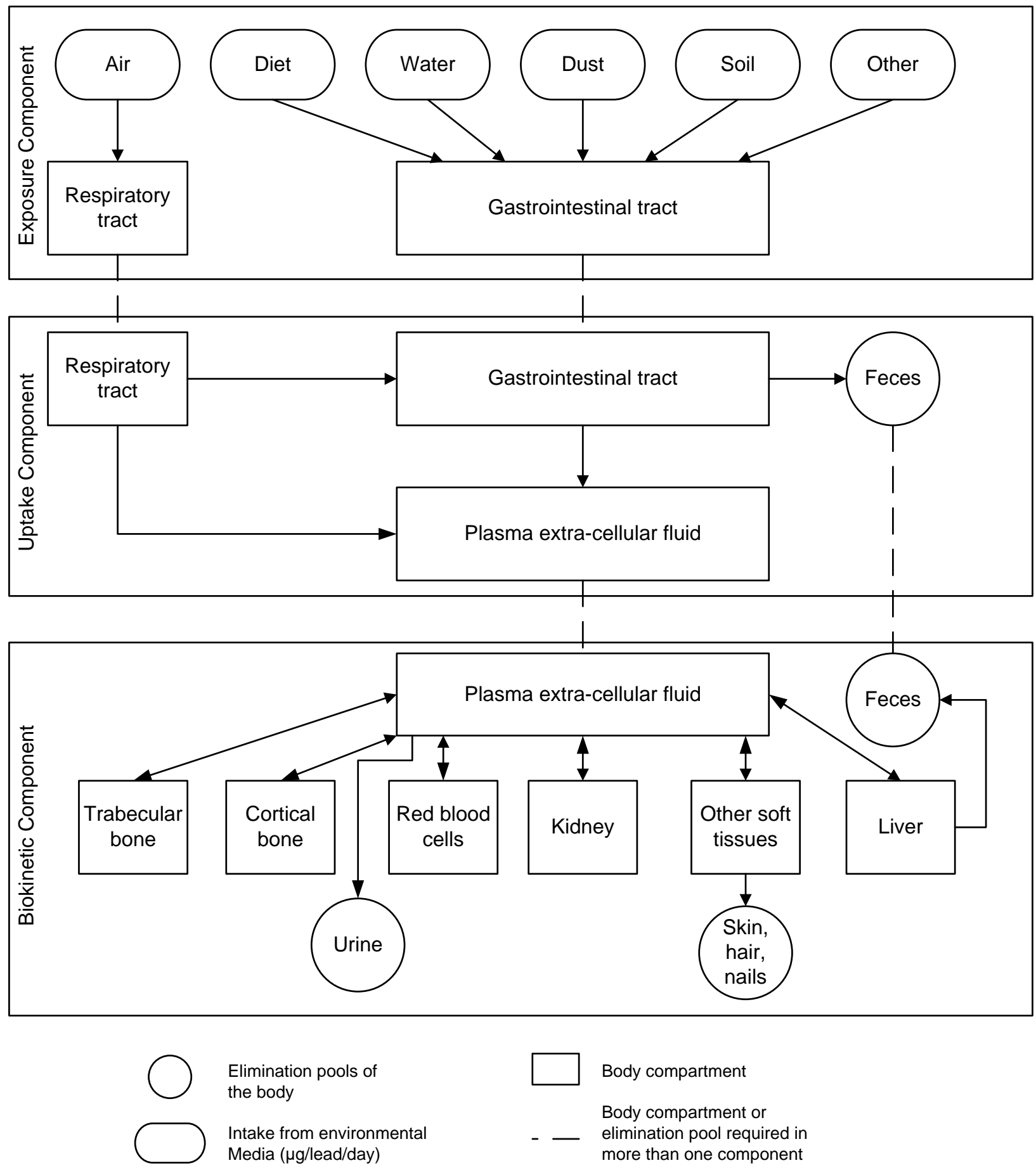

Elimination pools of the body

Intake from environmental Media ( $\mu \mathrm{g} / \mathrm{lead} / \mathrm{day})$

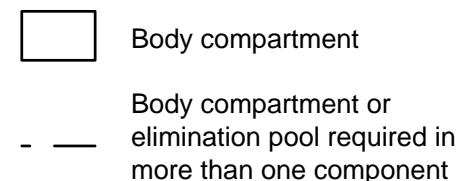

*Schematic for integrated $\mathrm{Pb}$ exposure-kinetics model in which simulated multi-media exposures are linked to simulations of lead uptake (i.e., absorption into the plasma-extracellular fluid), tissue distribution, and excretion).

Sources: EPA 1994a, 1994b 
fractions are also medium-specific. At 30 months of age, at low intakes ( $<200 \mu \mathrm{g} / \mathrm{day}$ ), below the rates at which capacity-limitation has a significant impact on absorption, the fraction of ingested $\mathrm{Pb}$ in food or drinking water that is absorbed is 0.5 and decreases to approximately 0.11 (intake, $>5,000 \mu \mathrm{g} /$ day). For $\mathrm{Pb}$ ingested in soil or dust, fractional absorption is 0.35 at low intakes ( $<200 \mu \mathrm{g} / \mathrm{day})$ and decreases to 0.09 (intake, $>5,000 \mu \mathrm{g} /$ day).

The uptake model assumes that $32 \%$ of inhaled $\mathrm{Pb}$ is absorbed. This value was originally assigned based on a scenario of exposure to active smelter emissions, which assumed the particle size distribution in the vicinity of an active $\mathrm{Pb}$ smelter ( $<1 \mu \mathrm{m}, 12.5 \%$; $1-2.5 \mu \mathrm{m}, 12.5 \%$; 2-15 $\mu \mathrm{m}, 20 \%$; $15-30 \mu \mathrm{m}, 40 \%$; $>30 \mu \mathrm{m}, 15 \%$ ); size-specific deposition fractions for the nasopharyngeal, tracheobronchial, and alveolar regions of the respiratory tract; and region-specific absorption fractions. $\mathrm{Pb}$ deposited in the alveolar region is assumed to be completely absorbed from the respiratory tract, whereas $\mathrm{Pb}$ deposited in the nasopharyngeal and tracheobronchial regions (30-80\% of the $\mathrm{Pb}$ particles in the size range $1-15 \mu \mathrm{m}$ ) is assumed to be transported to the gastrointestinal tract.

Biokinetics Model. The biokinetics model includes a central compartment, six peripheral body compartments, and three elimination pools (urine, feces, lumped pool representing skin, hair, and nails). The body compartments include plasma and extracellular fluid (central compartment), red blood cells, kidney, liver, trabecular bone, cortical bone, and other soft tissue (EPA 1994a). The model simulates growth of the body and tissues, compartment volumes, and $\mathrm{Pb}$ masses and concentrations in each compartment. $\mathrm{PbB}$ at birth (neonatal) is assumed to be 0.85 of the maternal blood $\mathrm{Pb}$. Neonatal $\mathrm{Pb}$ masses and concentrations are assigned to other compartments based on a weighted distribution of the neonatal PbB. Exchanges between the central compartment and tissue compartments are simulated as first-order processes, which are parameterized with unidirectional, first-order rate constants. Bone is simulated as two compartments: a relatively fast trabecular bone compartment (representing $20 \%$ of bone volume) and a relatively slow cortical bone compartment (representing $80 \%$ of the bone volume). Saturable uptake of $\mathrm{Pb}$ into erythrocytes is simulated, with a maximum erythrocyte $\mathrm{Pb}$ concentration of $12 \mu \mathrm{g} / \mathrm{dL}$. Excretory routes simulated include urine, from the central compartment; bile-feces, from the liver; and a lumped excretory pathway representing losses from skin, hair and nail, from the other soft tissue compartment.

Blood Pb Probability Model. Inputs to the IEUBK Model are exposure point estimates that are intended to represent time-averaged central tendency exposures. The output of the model is a central tendency estimate of $\mathrm{PbB}$ for children who might experience the inputted exposures. However, within a group of 
similarly exposed children, PbBs would be expected to vary among children as a result of inter-individual variability in media intakes, absorption, and biokinetics. The model simulates the combined impact of these sources of variability as a lognormal distribution of $\mathrm{PbB}$ for which the geometric mean is given by the central tendency PbB outputted from the biokinetics model and the GSD is an input parameter. The resulting lognormal distribution also provides the basis for predicting the probability of occurrence of given $\mathrm{PbB}$ within a population of similarly exposed children. The model can be iterated for varying exposure concentrations (e.g., a series of increasing soil Pb concentrations) to predict the media concentration that would be associated with a probability of 0.05 for the occurrence of a PbB exceeding $10 \mu \mathrm{g} / \mathrm{dL}$. A subsequent elaboration of the model has been developed that utilizes a Monte Carlo approach to simulate variability and uncertainty in exposure and absorption (Goodrum et al. 1996; Griffin et al. 1999). This extension of the model provides an alternative to the blood $\mathrm{Pb}$ probability model for incorporating, explicitly, estimates of variability (and uncertainty in variability) in exposure and absorption into predictions of an expected probability distribution of PbBs. More recently, Zartarian et al. (2017) provided an analysis coupling the IEUBK model with EPA's Stochastic Human Exposure and Dose Simulation (SHEDS)-Multimedia Model that considered general U.S. childhood exposures probabilistically and assessed primary sources of $\mathrm{Pb}$ exposure across the distribution of $\mathrm{PbB}$.

Performance of the IEUBK Model has been evaluated for predicting observed PbBs in children (Hogan et al. 1998; Li et al. 2016a; Von Lindern et al. 2003, 2016). The largest evaluation utilized longitudinal exposure and blood $\mathrm{Pb}$ data for approximately 2,200 children who resided near a former smelter in northern Idaho (Bunker Hill site) during a 14-year period of remediation activities (Von Lindern et al. 2003, 2016). The observed annual blood Pb geometric means ranged from 2.5 to $10.6 \mu \mathrm{g} / \mathrm{dL}$. The model predicted the time course of the observed $\mathrm{PbBs}$ as the remediation progressed when the gastrointestinal absorption fraction was calibrated to agree with blood Pb observations (Von Lindern et al. 2003). A similar outcome was obtained in a subsequent analysis in which the gastrointestinal absorption fraction was adjusted to agree with site measurements of soil Pb RBA, and soil and dust ingestion rates were calibrated to the blood $\mathrm{Pb}$ observations (Von Lindern et al. 2016). The mean difference between predicted and observed annual geometric mean PbBs (predicted - observed) was - $0.31 \mu \mathrm{g} / \mathrm{dL}$ (range: $-1.07,1.93$ ) and the mean relative percent difference was -8.4\% (range: -23-21\%). Applications of the IEUBK Model to the Bunker Hill site were reviewed by the National Research Council (NRC 2005). Hogan et al. (1998) evaluated the IEUBK Model performance based on residential exposure and blood data for approximately 478 children who resided near three $\mathrm{Pb}$ mining and smelting sites. The observed geometric means for the three sites ranged from 5.2 to $6.8 \mu \mathrm{g} / \mathrm{dL}$. The IEUBK Model predictions agreed reasonably well with observations for children whose exposures were predominantly 
from their residence (e.g., who spent no more than 10 hours/week away from home). The mean difference between predicted and observed site geometric mean PbBs (predicted-observed) was $0.03 \mu \mathrm{g} / \mathrm{dL}$ (range -0.6-0.7) and the mean relative percent difference was $-0.4 \%$ (range $-12-10 \%$ ). The predicted geometric mean PbBs were within $0.7 \mu \mathrm{g} / \mathrm{dL}$ of the observed geometric means at each site. The prediction of the percentage of children expected to have PbBs exceeding $10 \mu \mathrm{g} / \mathrm{dL}$ were within $4 \%$ of the observed percentage at each site. Li et al. (2016a) compared predictions of PbB to observations in a cohort of 760 children in Central China. The observed residence area geometric means ranged from 5 to $14 \mu \mathrm{g} / \mathrm{dL}$. When exposure parameters were set to the study population (e.g., exposure media $\mathrm{Pb}$ concentration and intakes), predicted and observed PbBs were not significantly different. The mean difference between predicted and observed geometric mean PbBs for 21 residence areas (predictedobserved) was $0.55 \mu \mathrm{g} / \mathrm{dL}$ (range -2.0-3.2) and the mean relative percent difference was 3.5\% (range -3228\%). These evaluations provide support for the validity of the IEUBK Model for estimating PbBs in children at sites where their exposures can be adequately characterized. Similar empirical comparisons of the IEUBK Model have shown that agreement between model predictions and observed PbBs at specific locations is influenced by numerous factors, including the extent to which the exposure and blood $\mathrm{Pb}$ measurements are adequately matched, and site-specific factors (e.g., soil characteristics, behavior patterns, bioavailability) that may affect $\mathrm{Pb}$ intake or uptake in children (Bowers and Mattuck 2001; Von Lindern et al. 2003, 2016). In addition to the above empirical comparisons, the computer code used to implement the IEUBK Model (IEUBK version 0.99d) has undergone an independent validation and verification and has been shown to accurately implement the conceptual IEUBK Model (Zaragoza and Hogan 1998).

\subsubsection{Leggett Model}

The Leggett Model simulates $\mathrm{Pb}$ intake, absorption, and disposition in humans, from birth through adulthood (Leggett 1993). Figure 3-3 shows a conceptualized representation of the model, including the movement of $\mathrm{Pb}$ from exposure media (i.e., intake via inhalation or ingestion) to the lungs and gastrointestinal tract, followed by the subsequent exchanges between diffusible blood plasma, soft tissues, bone compartments, and excretion from liver, kidneys, and sweat. A detailed exposure module is not linked to the Leggett Model; rather, Pb exposure estimates are incorporated into the model as age-specific point estimates of average daily intake ( $\mu \mathrm{g} / \mathrm{day})$ from inhalation and ingestion. A description of the model and its potential application to risk assessment are provided below. 
Figure 3-3. Compartments and Pathways of Lead (Pb) Exchange in the Leggett Model*

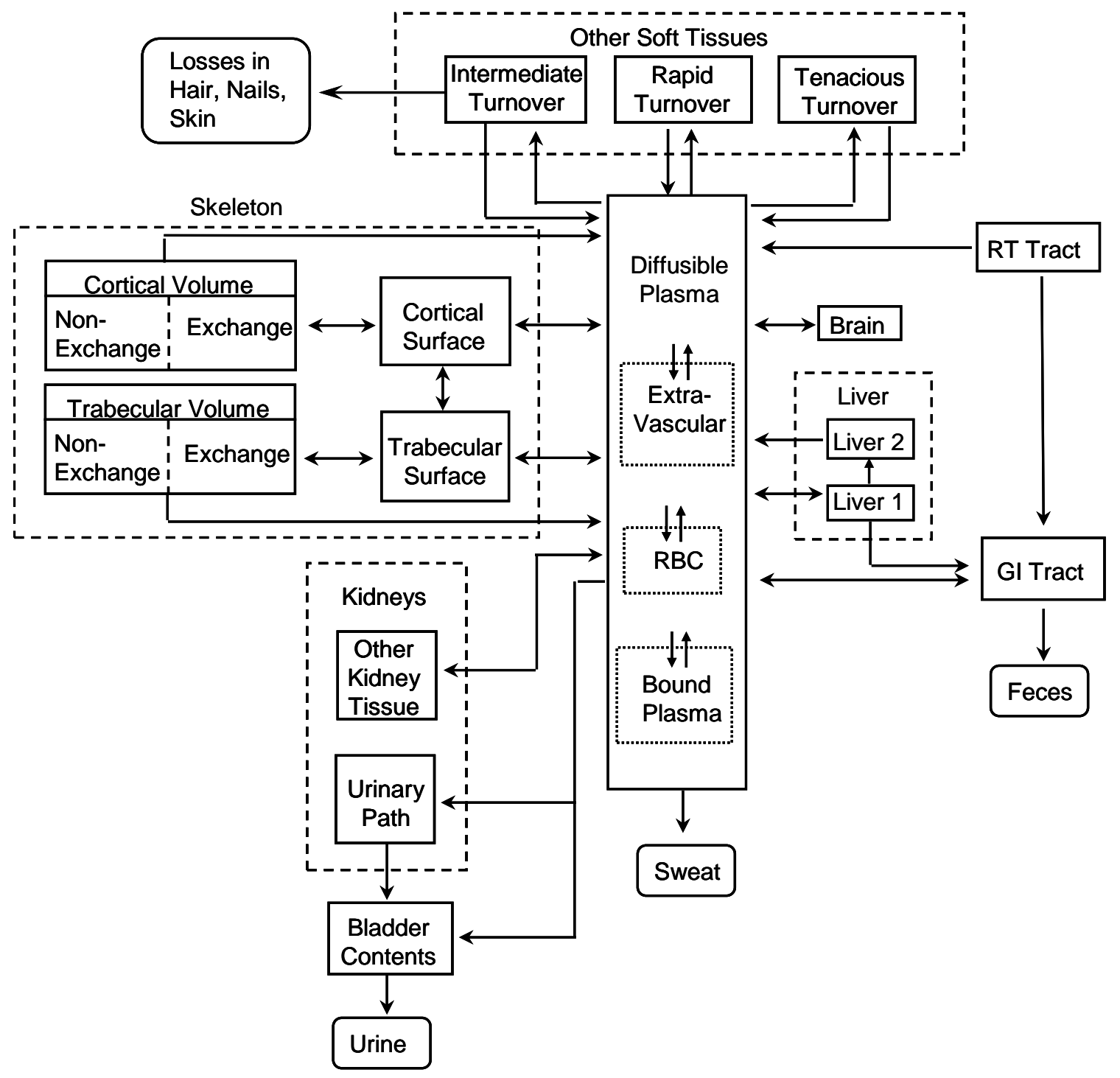

*Schematic model for $\mathrm{Pb}$ kinetics in which $\mathrm{Pb}$ distribution is represented by exchanges between the central plasmaextracellular fluid and tissue compartments. Bone is represented as having surface (which rapidly exchanges with plasma-extracellular fluid) and volume compartments; the latter simulates slow exchange with the surface and slow return of $\mathrm{Pb}$ to the plasma-extracellular fluid from bone resorption.

$\mathrm{GI}$ = gastrointestinal; RBC = red blood cell; RT = respiratory

Source: Leggett 1993 
The Leggett Model includes a central compartment, 15 peripheral body compartments, and 4 elimination pools (urine, feces, sweat, and lumped pool representing skin, hair, and nails), as illustrated in Figure 3-3. Transport of $\mathrm{Pb}$ from blood plasma to tissues is assumed to follow first-order kinetics. Transfer rate constants vary with age and PbB. Above a nonlinear threshold concentration in red blood cells (assumed to be $60 \mu \mathrm{g} / \mathrm{dL}$ ), the rate constant for transfer to red blood cells declines and constants to all other tissues increase proportionally (Leggett 1993). This replicates the nonlinear relationship between plasma and red blood cells observed in humans (see Section 3.1.2). The model simulates blood volume as an agedependent function, which allows simulation of plasma and PbBs. Pb masses are simulated in all other tissues (tissue volumes are not simulated).

Unidirectional, first-order transfer rates $\left(\mathrm{day}^{-1}\right)$ between compartments were developed for six age groups, and intermediate age-specific values are obtained by linear interpolation. The total transfer rate from diffusible plasma to all destinations combined is assumed to be 2,000 day ${ }^{-1}$, based on isotope tracer studies in humans receiving $\mathrm{Pb}$ via injection or inhalation. Values for transfer rates in various tissues and tissue compartments are based on measured deposition fractions or instantaneous fractional outflows of $\mathrm{Pb}$ between tissue compartments (Leggett 1993).

The Leggett Model was developed from a biokinetic model originally developed for the International Commission on Radiological Protection (ICRP) for calculating radiation doses from environmentally important radionuclides, including radioisotopes of $\mathrm{Pb}$ (Leggett 1993). The Leggett Model simulates agedependent bone physiology using a model structure developed for application to the alkaline earth elements, but parameterized using data specific to $\mathrm{Pb}$ where possible. The model simulates both rapid exchange of $\mathrm{Pb}$ with plasma via bone surface and slow loss by bone resorption. Cortical bone volume ( $80 \%$ of bone volume) and trabecular bone volume (20\% of bone volume) are simulated as bone surface compartments, which rapidly exchange $\mathrm{Pb}$ with the blood plasma, and bone volume, within which are exchangeable and nonexchangeable pools. Pb enters the exchangeable pool of bone volume via the bone surface and can return to the bone surface, or move to the nonexchangeable pool, from where it can return to the blood only when bone is resorbed. Rate constants for transfer of $\mathrm{Pb}$ from the nonexchangeable pools and blood plasma vary with age to reflect the age-dependence of bone turnover.

The liver is simulated as two compartments: one compartment has a relatively rapid uptake of $\mathrm{Pb}$ from plasma and a relatively short removal half-time (days) for transfers to plasma and to the small intestine by biliary secretion, and a second compartment simulates a more gradual transfer to plasma of approximately $10 \%$ of $\mathrm{Pb}$ uptake in liver. The kidney is simulated as two compartments: one that exchanges slowly with 
blood plasma and accounts for $\mathrm{Pb}$ accumulation in kidney tissue, and a second compartment that receives $\mathrm{Pb}$ from blood plasma and rapidly transfers $\mathrm{Pb}$ to urine, with essentially no accumulation (urinary pathway). Other soft tissues are simulated as three compartments representing rapid, intermediate, and slow turnover rates (without specific physiologic correlates). Other excretory pathways (hair, nails, and skin) are represented as a lumped pathway from the intermediate turnover rate soft tissue compartment.

The Leggett Model simulates $\mathrm{Pb}$ intakes from inhalation, ingestion, or intravenous injection. The latter was included to accommodate model evaluations based on intravenous injection studies in humans and animal models. The respiratory tract is simulated as four compartments into which inhaled $\mathrm{Pb}$ is deposited and absorbed with half-times of 1, 3, 10, and 48 hours. Four percent of the inhaled $\mathrm{Pb}$ is assumed to be transferred to the gastrointestinal tract. These parameter values reflect the data on which the model was based, which were derived from studies in which human subjects inhaled submicron Pb-bearing particles (Chamberlain et al. 1978; Hursh and Mercer 1970; Hursh et al. 1969; Morrow et al. 1980; Wells et al. 1975). These assumptions would not necessarily apply to exposures to large airborne particles (see Section 3.1.1). Absorption of ingested $\mathrm{Pb}$ is simulated as an age-dependent fraction of the ingestion rate, declining from 0.45 at birth to 0.3 at age 1 year (to age 15 years), and to 0.15 after age 25 years.

Output from the Leggett Model has been compared with data in children and adult subjects exposed to $\mathrm{Pb}$ in order to calibrate model parameters (Leggett et al. 1993; Pounds and Leggett 1998). Nie et al. (2005) evaluated performance of the Leggett Model for predicting bone Pb concentrations in $539 \mathrm{~Pb}$ workers. The data included periodic monitoring of PbBs and XRF bone Pb measurements made in 1994 and 1999. $\mathrm{Pb}$ intakes of each individual were calibrated to agree with measured PbBs. The Leggett Model underpredicted observed cortical bone $\mathrm{Pb}$ concentrations by a factor of 3-4, and underpredicted trabecular bone $\mathrm{Pb}$ concentration by a factor of 12-18. EPA (2014a) evaluated performance of the Leggett Model for predicting $\mathrm{PbBs}$ in children and blood and bone $\mathrm{Pb}$ concentrations in adults. The evaluation of predictions for children used data on PbBs reported in the NHANES for the years 2007-2008, and required making assumptions about $\mathrm{Pb}$ exposures in this population. The Leggett Model overpredicted observed PbBs in children 1-7 years of age by a factor of 2-3. Cal EPA (2013) evaluated the Leggett Model for predicting PbBs in smelter workers whose occupational exposures were interrupted during a workers strike. Pre-hire background $\mathrm{Pb}$ intakes and pre-strike intakes were calibrated to agree with measured $\mathrm{PbBs}$ and the predicted rate of decline in blood $\mathrm{Pb}$ that occurred during the strike period was compared to observations. Cal EPA (2013) reported "the average difference between the measured and predicted post-strike BLL was unacceptably large and indicated significant under-prediction of BLLs”. 
The average difference was $>4 \mu \mathrm{g} / \mathrm{dL}$ in a cohort that had a mean post-strike PbB of $31 \mu \mathrm{g} / \mathrm{dL}$ (no further details were provided). Performance was substantially improved when various parameters were calibrated to the observations. These included parameters that control transfers between plasma and bone and red blood cell saturation (see Cal EPA [2013] for details of parameter value changes). The mean difference between predicted and observed annual geometric mean PbBs (predicted-observed) was -0.9 $\mu \mathrm{g} / \mathrm{dL}$ (range -26-32) and the mean relative percent difference was $-8.8 \%$ (range: -55-320\%). Cal EPA (2013) reported several other evaluations of their recalibrated model, including observed and predicted relationships between plasma and whole $\mathrm{PbBs}$ in adults, and predicted distribution of $\mathrm{Pb}$ in bone and soft tissues compared to estimates from human autopsy studies.

\subsubsection{EPA All Ages Lead Model (AALM)}

The AALM simulates blood and tissue $\mathrm{Pb}$ masses $(\mu \mathrm{g})$ and concentrations $(\mu \mathrm{g} / \mathrm{g})$ resulting from exposures to $\mathrm{Pb}$ in air, drinking water, surface dust (e.g., indoor dust, soil dust), food, or miscellaneous $\mathrm{Pb}$ ingestion pathways. The AALM exposure module allows the user to simulate multi-pathway exposures that are constant or that vary in time increments as small as 1 day and that occur at any age from birth to 90 years. The user can select to run a systemic biokinetics simulation based on either the Leggett (AALM-LG) or O’Flaherty (AALM-OF) biokinetics models. Parameters in both systemic models were re-calibrated with observations of blood, bone, and soft tissue $\mathrm{Pb}$ concentrations in children and adults (EPA 2014a). The version of the AALM described in EPA (2014a) was implemented in Advanced Continuous Simulation Language (acslX, ver. 3.1.4.2). The ICRP Human Respiratory Tract Model (HRTM) deposition and absorption parameters are used in both the AALM-LG and AALM-OF, which allows simulation of inhaled $\mathrm{Pb}$ particles of specified size ranges and absorption kinetics (ICRP 1994). The gastrointestinal tract model includes age-dependent absorption fractions and parameters for RBA of $\mathrm{Pb}$ from all ingestion pathways.

The structures of the two systemic biokinetics models in AALM-OF and AALM-LG are based on the O’Flaherty and Leggett models, respectively, with the following modifications. Growth parameters from the O’Flaherty Model are used in both models to simulate age-dependent body weight tissue weights. This provides a means for calculating tissue concentrations as the Pb mass ( $\mu g$ ) divided by the tissue weight (g). Concentrations of $\mathrm{Pb}$ in bone wet weight are converted to concentration per g bone mineral by dividing the wet weight concentration by the ash fraction of bone. This conversion provides a means for comparing model predictions of bone $\mathrm{Pb}$ concentration with bone XRF data, which is typically reported in units of $\mathrm{Pb}$ per g bone mineral. Parameters for $\mathrm{RBA}$ of $\mathrm{Pb}$ in each intake medium include the 
gastrointestinal tract model. This provides a means for independently adjusting the absorption fraction for each of the intake pathways (including respiratory tract-to-gastrointestinal tract) and maintains mass balance for fecal excretion of unabsorbed $\mathrm{Pb}$. Inhalation, deposition, mucociliary clearance, and absorptive clearance of airborne $\mathrm{Pb}$ is simulated with a simplified implementation of the ICRP HRTM.

The AALM systemic biokinetic models were recalibrated from the original Leggett and O’Flaherty Models (EPA 2014b). The sequential recalibration utilized several sources of data on blood and bone $\mathrm{Pb}$ concentrations in humans. Parameters that control the uptake and retention of $\mathrm{Pb}$ in red blood cells were recalibrated using paired data on whole blood and plasma $\mathrm{Pb}$ concentrations in children and adults (Bergdahl et al. 1997c, 1998, 1999; Hernández-Avila et al. 1998; Manton et al. 2001; Schütz et al. 1996; Smith et al. 2002). Parameters that control plasma-to-urine clearance were recalibrated based on clearance estimates from studies that measured paired plasma concentration and urinary $\mathrm{Pb}$ excretion in adults (Araki et al. 1986; Chamberlain et al. 1978; Manton and Cook 1984; Manton and Malloy 1983). Autopsy data from children and adults were used to evaluate parameters that control the relationship between of tissue $\mathrm{Pb}$ concentrations and bone Pb concentrations (Barry 1975). The relationship between bone and plasma $\mathrm{Pb}$ concentrations was evaluated with paired data for plasma $\mathrm{Pb}$ concentration and XRF bone $\mathrm{Pb}$ in adults (Cake et al. 1996; Hernández-Avila et al. 1998). The long-term rate elimination of $\mathrm{Pb}$ from blood and bone was evaluated with data on blood and XRF bone $\mathrm{Pb}$ in retired $\mathrm{Pb}$ workers (Nilsson et al. 1991).

The calibrated AALM was evaluated with data on PbBs measured in infants (Ryu et al. 1983; Sherlock and Quinn 1986) or adults (Rabinowitz et al. 1976) who consumed known quantities of Pb. In the Ryu et al. (1983) study, PbBs were monitored in formula-fed infants who were fed measured quantiles of formula. PbBs predicted from the AALM-LG were within 1 SD of the group means and the $\mathrm{r}^{2}$ for predictions was 0.85 . Predictions from the AALM-OF were uniformly higher than observations and the $\mathrm{r}^{2}$ for predictions was 0.76. Sherlock and Quinn (1986) measured PbB in infants at age 13 weeks and estimated dietary intake of $\mathrm{Pb}$ for each infant based on $\mathrm{Pb}$ measurements made in duplicate diet samples collected daily during week 13. The observed dose-blood $\mathrm{Pb}$ relationship was predicted with $\mathrm{r}^{2}$ values of 0.95 for AALM-LG and 0.98 for AALM-OF. Rabinowitz et al. (1976) conducted a pharmacokinetics study in which four adults ingested daily doses of $\left[{ }^{207} \mathrm{~Pb}\right]$ nitrate for periods up to 124 days. Concentrations of ${ }^{207} \mathrm{~Pb}$ in blood, urine, and feces were then monitored during and following cessation of exposure, and data on daily intakes and blood concentrations for each subject were reported. Absorption fractions for $\mathrm{Pb}$ were estimated for each individual based on mass balance in feces. AALM-LG 
predictions are closer to the observations; $\mathrm{r}^{2}$ values ranged from 0.92 to 0.98 for four subjects in the study. The AALM-OF predicted a slower accrual and decline of blood $\mathrm{Pb}$, and lower peak $\mathrm{PbBs}\left(\mathrm{r}^{2}<0.25\right)$.

\subsubsection{Model Comparisons}

The O’Flaherty, IEUBK, and Leggett Model differ considerably in the way each represents tissues, exchanges of $\mathrm{Pb}$ between tissues, and $\mathrm{Pb}$ exposure. The AALM includes biokinetics models based on, but updated from, the O’Flaherty and Leggett models.

Figure 3-4 compares the PbBs predicted by each model for a hypothetical child who ingests $100 \mu \mathrm{g}$ $\mathrm{Pb} /$ day in soil for a period of 1 year beginning at the age of 2 years (e.g., equivalent to ingestion of $100 \mu \mathrm{g}$ soil/day at a soil $\mathrm{Pb}$ concentration of $1,000 \mathrm{mg} \mathrm{Pb} / \mathrm{g}$ soil). The $100-\mu \mathrm{g} /$ day exposure is superimposed on a baseline exposure that yields a PbB of approximately $2 \mu \mathrm{g} / \mathrm{dL}$ at 2 years of age. All five models predict an increase in $\mathrm{PbB}$ towards a quasi-steady state during the exposure period, followed by a decline towards the pre-exposure baseline $\mathrm{PbB}$ with an apparent half-time of approximately 1 month. Predicted $\mathrm{PbBs}$ at the end of the 12-month soil exposure period were 10, 23, 5.9, 9.4, and 10.4 $\mu \mathrm{g} / \mathrm{dL}$ for the IEUBK Model, Leggett Model, O’Flaherty Model, AALM-LG, and AALM-OF, respectively. Differences in the magnitude of the predicted impact of the soil exposure on $\mathrm{PbB}$ reflect differences in assumptions about $\mathrm{Pb}$ biokinetics and cannot be attributed solely to different assumptions about $\mathrm{Pb}$ bioavailability. Bioavailability assumptions in the models for the age range 2-3 years are: O’Flaherty Model, 45\% (50\% at age 2 years, decreasing to $40 \%$ at age 3 years); IEUBK Model, 30\% (soil Pb at low intakes); Leggett Model, 30\%; and AALM-LG and AALM-OF 34\% (38\% at age 2 years and decreasing to 30\% at age 3 years). A comparison of model predictions for a similar exposure during adulthood (100 $\mu \mathrm{g} \mathrm{Pb} / \mathrm{day}$ for 1 year, beginning at age 25) is shown in Figure 3-5. Predicted PbBs at the end of the 12-month soil exposure period were 8.4, 3.3, 4.0, and $4.8 \mu \mathrm{g} / \mathrm{dL}$ for the Leggett Model, O'Flaherty Model, AALM-LG, and AALM-OF, respectively. All four models predict a smaller change in PbB in adults, compared to children, for a similar increment in exposure. This is attributed, in part, to assumptions of lower $\mathrm{Pb}$ bioavailability in adults (i.e., O’Flaherty, 8\%; Leggett, 15\%; AALM-LG and AALM-OF, 8\%). 


\section{Figure 3-4. Blood Lead Concentrations (PbBs) in Children Predicted by the IEUBK, Leggett, and O'Flaherty Models and AALM*}

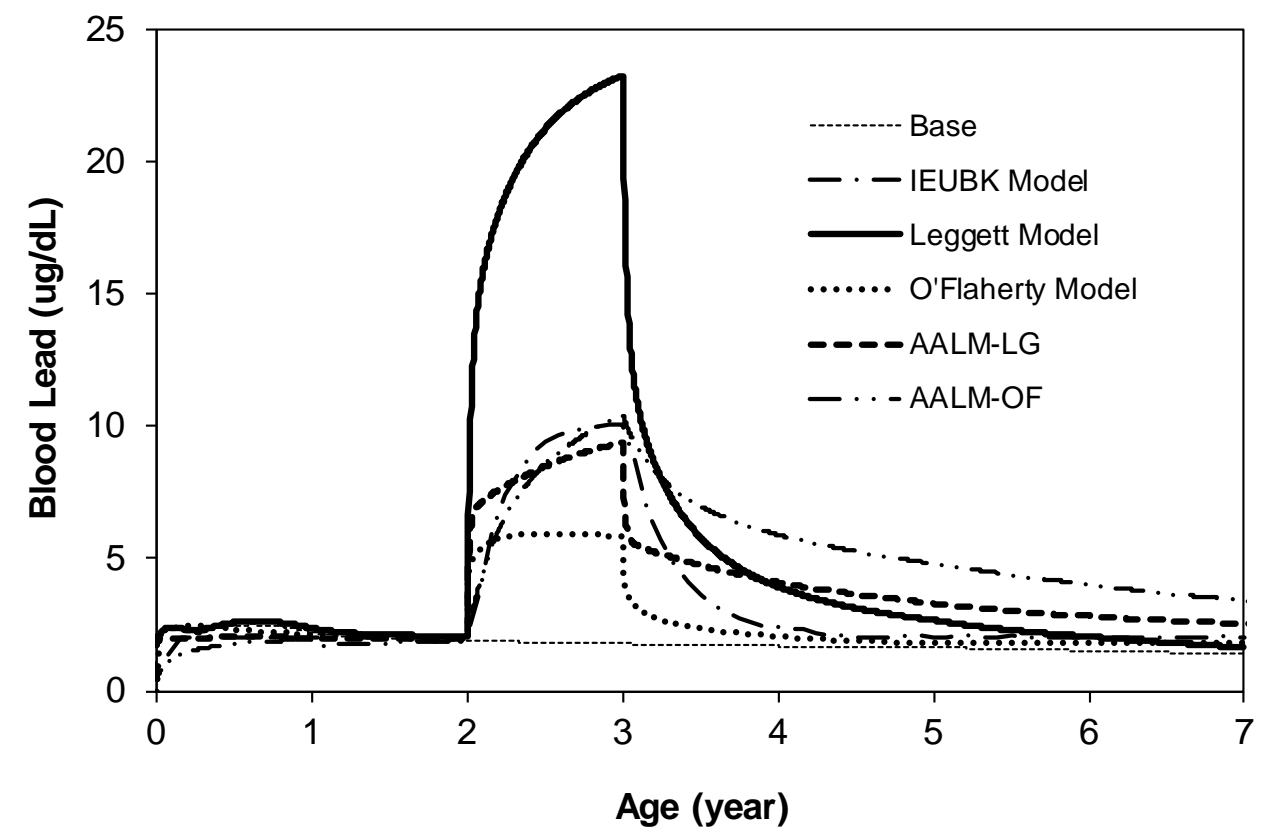

*The simulations are of a hypothetical child who has a PbB of $2 \mu \mathrm{g} / \mathrm{dL}$ at age 2 years, and then experiences a 1-year exposure to $100 \mu \mathrm{g} \mathrm{Pb} /$ day. The $100 \mu \mathrm{g} /$ day exposure was simulated as an exposure to lead in soil in the IEUBK Model. Default bioavailability assumptions were applied in all three models.

Figure 3-5. Blood Lead Concentrations (PbBs) in Adults Predicted by the Leggett and O'Flaherty Models and AALM*

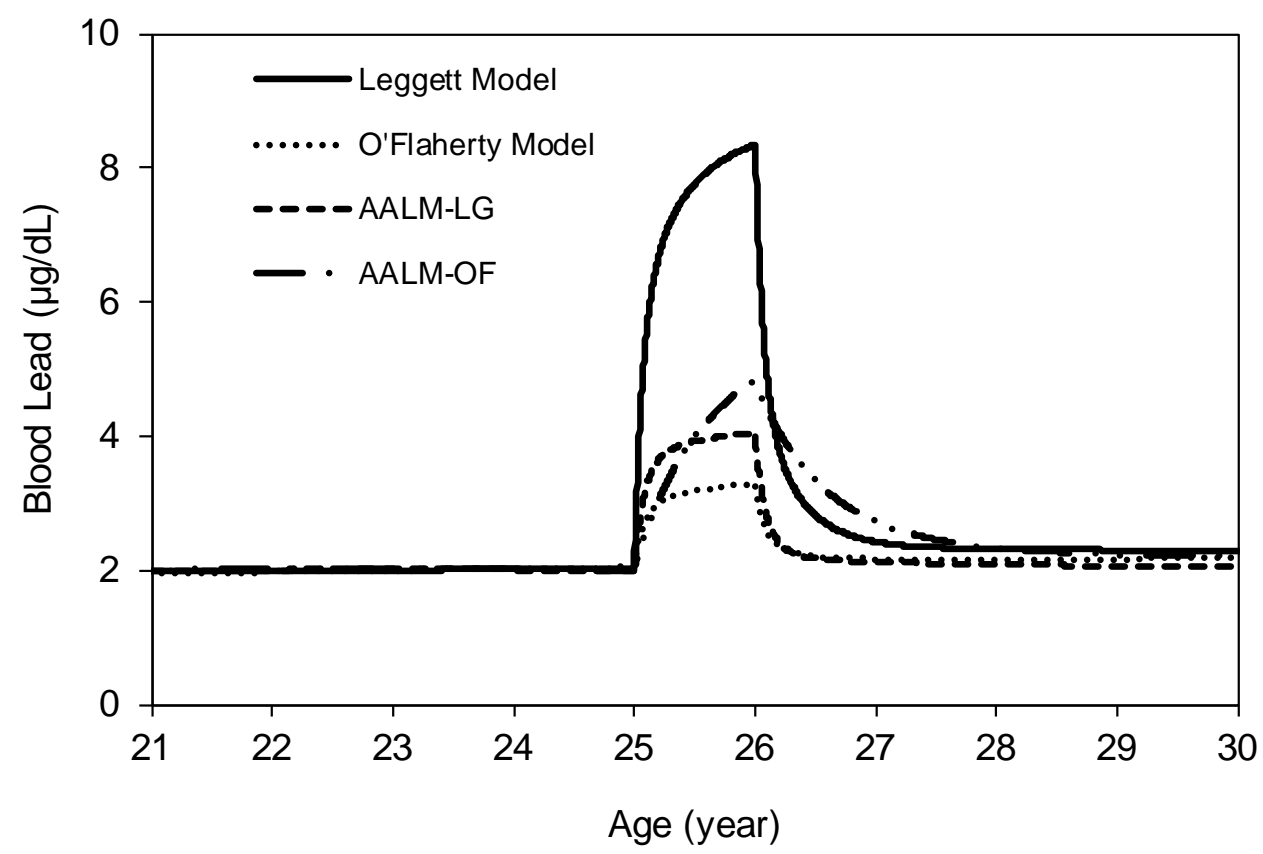

*The simulations are of a hypothetical adult who has a PbB of $2 \mu \mathrm{g} / \mathrm{dL}$ at age 25 years, and then experiences a 1-year exposure to $100 \mu \mathrm{g}$ Pb/day. Default bioavailability assumptions were applied in all three models. 


\subsubsection{Slope Factor Models}

Slope factor models have been used as simpler alternatives to compartmental models for predicting PbBs, or the change in $\mathrm{PbB}$, associated with a given exposure (Abadin et al. 1997; Bowers et al. 1994; Carlisle and Wade 1992; EPA 2017d; Maddaloni et al. 2005; Stern 1994, 1996). In slope factor models, Pb biokinetics is represented with a simple linear relationship between the $\mathrm{PbB}$ and either $\mathrm{Pb}$ uptake (biokinetic slope factor, BSF) or Pb intake (intake slope factor, ISF). The models take the general mathematical forms:

$$
\begin{gathered}
P b B=E \cdot I S F \\
P b B=E \cdot A F \cdot B S F
\end{gathered}
$$

where $\mathrm{E}$ is an expression for exposure (e.g., soil intake $\mathrm{x}$ soil $\mathrm{Pb}$ concentration) and AF is the absorption fraction for $\mathrm{Pb}$ in the specific exposure medium of interest. Intake slope factors are based on ingested $\mathrm{Pb}$, rather than absorbed $\mathrm{Pb}$ and, therefore, integrate both absorption and biokinetics into a single slope factor, whereas models that utilize a biokinetic slope factor (BSF) to account for absorption in the relationship include an absorption parameter. Slope factors used in various models are presented in Table 3-2. Of the various models presented in Table 3-2, the Bowers et al. (1994) and EPA (2017b) models implement BSFs. The slope factors used in both models (approximately $0.4 \mu \mathrm{g} / \mathrm{dL}$ per $\mu \mathrm{g} \mathrm{Pb} /$ day) are similar to BSFs predicted from the O'Flaherty Model $(0.65 \mu \mathrm{g} / \mathrm{dL}$ per $\mu \mathrm{g} \mathrm{Pb}$ uptake/day) and Leggett Model ( $0.43 \mu \mathrm{g} / \mathrm{dL}$ per $\mu \mathrm{g} \mathrm{Pb}$ uptake/day) for simulations of adult exposures (Maddaloni et al. 2005).

\begin{tabular}{|c|c|c|c|c|c|}
\hline \multirow[b]{2}{*}{ Model } & \multirow[b]{2}{*}{ Receptor } & \multirow[b]{2}{*}{ Intake route } & \multicolumn{2}{|c|}{ Slope factor } & \multirow{2}{*}{$\begin{array}{l}\text { Absorption } \\
\text { fraction }\end{array}$} \\
\hline & & & Intake & Biokinetics & \\
\hline Bowers et al. 1994 & Adult & Ingestion of soil/dust & ND & 0.375 & 0.08 \\
\hline $\begin{array}{l}\text { Carlisle and Wade } \\
1992\end{array}$ & Child & $\begin{array}{l}\text { Ingestion of soil/dust } \\
\text { Ingestion of water }\end{array}$ & $\begin{array}{l}0.07 \\
0.04\end{array}$ & ND & ND \\
\hline $\begin{array}{l}\text { Carlisle and Wade } \\
1992\end{array}$ & Adult & $\begin{array}{l}\text { Ingestion of soil/dust } \\
\text { Ingestion of water }\end{array}$ & $\begin{array}{l}0.018 \\
0.04\end{array}$ & ND & ND \\
\hline Cal EPA 2017 & Child & $\begin{array}{l}\text { Ingestion of soil/dust } \\
\text { Inhalation of } \\
\text { respirable dust } \\
\text { Dermal contact }\end{array}$ & $\begin{array}{l}\text { ND } \\
0.192 \\
0.0001\end{array}$ & $\begin{array}{l}0.16 \\
\text { ND } \\
\text { ND }\end{array}$ & $\begin{array}{l}0.44 \\
\text { ND } \\
\text { ND }\end{array}$ \\
\hline $\begin{array}{l}\text { EPA 2017d; } \\
\text { Maddaloni et al. } \\
2005\end{array}$ & Adult & Ingestion of soil/dust & ND & 0.4 & 0.12 \\
\hline
\end{tabular}

Table 3-2. Comparison of Slope Factors in Selected Slope Factor Models 
Table 3-2. Comparison of Slope Factors in Selected Slope Factor Models

\begin{tabular}{llllll}
\hline & & & \multicolumn{2}{c}{ Slope factor } & Absorption \\
\cline { 3 - 5 } Model & Receptor & Intake route & Intake & Biokinetics & fraction \\
\hline Stern 1994 & Child & Ingestion of soil/dust & $T(0.056,0.16,0.18)$ & ND & ND \\
\hline Stern 1996 & Adult & Ingestion of soil dust & $\cup(0.014,0.034)$ & ND & ND \\
\hline
\end{tabular}

$\mathrm{ND}=$ no data; $\mathrm{T}=$ triangular probability distribution function (PDF); $\mathrm{U}=$ uniform PDF

\subsection{CHILDREN AND OTHER POPULATIONS THAT ARE UNUSUALLY SUSCEPTIBLE}

This section discusses potential health effects from exposures during the period from conception to maturity at 18 years of age in humans. Potential effects on offspring resulting from exposures of parental germ cells are considered, as well as any indirect effects on the fetus and neonate resulting from maternal exposure during gestation and lactation. Children may be more or less susceptible than adults to health effects from exposure to hazardous substances and the relationship may change with developmental age.

This section also discusses unusually susceptible populations. A susceptible population may exhibit different or enhanced responses to certain chemicals than most persons exposed to the same level of these chemicals in the environment. Factors involved with increased susceptibility may include genetic makeup, age, health and nutritional status, and exposure to other toxic substances (e.g., cigarette smoke). These parameters can reduce detoxification or excretion or compromise organ function.

Populations at greater exposure risk to unusually high exposure levels to $\mathrm{Pb}$ are discussed in Section 5.7, Populations with Potentially High Exposures.

Age. Children and the elderly are likely to have increased susceptibility to Pb compared to non-elderly adults. As reviewed in Section 3.1.2 (Distribution), Pb crosses the placenta and is distributed to the fetus; neonates are also exposed to $\mathrm{Pb}$ in breast milk. Epidemiological studies show that umbilical cord $\mathrm{PbB}$ (reflective of neonatal $\mathrm{PbB}$ ) and $\mathrm{PbB}$ in infants are associated with adverse health outcomes during childhood, including decrements in neurological function (reviewed in Chapter 2). Results of a few studies that have followed children to early adulthood show an association between child $\mathrm{PbB}$ and behavioral and neuroanatomical changes in adults, suggesting a possible role of exposures in childhood to adult outcomes. Children are likely to be more susceptible than adults to $\mathrm{Pb}$ for the following reasons:

(1) it is generally accepted that developing systems are more susceptible than mature systems;

(2) absorption of $\mathrm{Pb}$ is higher in children compared to adults (see Section 3.1.1, Absorption); and 
(3) children exhibit behaviors that increase ingestion of Pb surface dusts (e.g., hand-to-mouth activity, pica behavior [the compulsive, habitual consumption of nonfood items]), proximity of breathing zone to entrained surface dust).

Regarding the elderly, it is well-established that physiological functions (e.g., renal, neurological, cardiovascular) decline with age. Thus, populations with age-related compromises in physiological function would be anticipated to be more susceptible to $\mathrm{Pb}$ than younger populations. Furthermore, because aging is associated with bone loss, $\mathrm{Pb}$ is mobilized into blood, resulting in potential increases in $\mathrm{PbB}$.

Sex. As reviewed in Chapter 2, some epidemiological studies examined health outcomes in populations stratified by sex. However, studies have not demonstrated clear sex-related susceptibilities to Pb-induced toxicity for any health effect outcome. In women, pregnancy, lactation, and post-menopausal status may increase bone demineralization, mobilizing bone $\mathrm{Pb}$ into the blood and potentially redistributing $\mathrm{Pb}$ to other tissues.

Nutritional Status. As discussed in Sections 3.1 (Toxicokinetics) and 3.4 (Interactions with other Chemicals), dietary calcium and nutritional status of iron and zinc can affect absorption of $\mathrm{Pb}$, potentially leading to alterations in PbB and health effects. See Sections 3.1 and 3.4 for additional details.

Pre-existing Conditions, Diseases, and Exposure to Other Substances. Because health effects associated with $\mathrm{Pb}$ are observed in every organ system, it is assumed that any condition or disease that compromises physiological functions could cause increased susceptibility to $\mathrm{Pb}$. Examples of underlying conditions include diseases of the kidney (e.g., glomerular nephritis), neurological system (e.g., autism), hematological system (e.g., anemia, thalassemia), and cardiovascular system (e.g., hypertension, cardiac conduction disorders). Similarly, increased susceptibility to $\mathrm{Pb}$ would be anticipated due to use of alcohol, tobacco, or any other substance that causes deficits in physiological function.

Genetic Polymorphisms. Numerous genetic polymorphisms that may alter susceptibility to $\mathrm{Pb}$ through altered toxicokinetics (i.e., absorption, distribution, and retention of $\mathrm{Pb}$ ) or toxicodynamics (e.g., effects) have been identified. The most well-studied polymorphisms are $\delta$-ALAD and the VDR. Several other polymorphisms that may alter susceptibility to $\mathrm{Pb}$ have been identified, although little data are available. In addition to the references listed below, information also was obtained from a recent review by Broberg et al. 2015. 
$A L A D$. As reviewed in Section 2.8 (Heath Effects, Hematological), $\mathrm{Pb}$ binds to and inhibits $\delta$-ALAD, causing decreased hemoglobin formation, measurable decreases in blood hemoglobin concentration, and anemia. $\delta$-ALAD is the major binding site for $\mathrm{Pb}$ in the blood (see Section 3.1.2). As such, polymorphisms of ALAD have the potential to alter $\mathrm{Pb}$ toxicokinetics, and thereby alter health effects. Many studies have evaluated the potential effects of ALAD polymorphisms on $\mathrm{Pb}$ distribution and toxicity. Information reviewed below was obtained from the following publications: Åkesson et al. (2000); Alexander et al. (1998b); Astrin et al. (1987); Battistuzzi et al. (1981); Bellinger et al. (1994); Bergdahl et al. (1997a, 1997b); Chia et al. (2005); Chiu et al. (2013); Fang et al. (2010); Fleming et al. (1998a); Gao et al. (2010); Hsieh et al. (2000); Hu et al. (2001); Huo et al. (2014); Jaffe et al. (2000, 2001); Kim et al. (2004); Krieg et al. (2009); Lee et al. (2001); Mitra et al. (2017); Ong et al. (1990); Pagliuca et al. (1990); Pawlas et al. (2012); Petrucci et al. (1982); Sakai et al. (2000); Schwartz (1995); Schwartz et al. (1995, 1997a, 1997b, 2000a, 2000b); Scinicariello et al. (2007, 2010); Shen et al. (2001); Sithisarankul et al. (1997); Smith (1995); Suzen et al. (2003); Szymanska-Chaowska et al. (2015); Tasmin et al. (2015); Warrington et al. (2015); Weaver et al. (2008); Wetmur et al. (1991a, 1991b); Wu et al. (2003a); and Zheng et al. (2011).

The ALAD gene encodes for the heme metabolism enzyme $\delta$-ALAD. ALAD is a polymorphic enzyme with two alleles (ALAD-1 and ALAD-2) and three genotypes (ALAD 1,1; ALAD 1,2; and ALAD 2,2). The ALAD 2,2 genotype is rare, and is found in 1\% of Caucasians; in contrast, the ALAD 1,1 and ALAD 1,2 genotypes occur in 80 and 19\%, respectively, of Caucasians. The ALAD 2,2 genotype occurs in $<1 \%$ of Asian and African populations. A study using NHANES III data (1988-1994) reported that 15.6\% of non-Hispanic whites, 2.6\% non-Hispanic blacks, and 8.8\% Mexican Americans carried the ALAD-2 allele (Scinicariello et al. 2010). The ALAD-2 protein has a higher binding affinity than the ALAD-1 protein for $\mathrm{Pb}$. Due to this higher binding affinity, it has been proposed that ALAD-2 sequesters $\mathrm{Pb}$ in erythrocytes, limiting distribution of $\mathrm{Pb}$ to other tissues. Numerous studies have shown that ALAD-2 carriers have higher PbB than ALAD-1 carriers. Although it has been demonstrated that ALAD genotype affects the toxicokinetics of $\mathrm{Pb}$, the association between adverse effects of $\mathrm{Pb}$ and ALAD genotype have not been definitively established.

$V D R$. Several studies have evaluated the potential effects of VDR polymorphisms on $\mathrm{Pb}$ uptake and distribution. Information reviewed below was obtained from the following publications: Ames et al. (1999); Cooper and Umbach (1996); Gundacker et al. (2009, 2010); Haynes et al. (2003); Krieg et al. (2010); Mitra et al. (2017); Morrison et al. (1992); Onalaja and Claudio (2000); Rezende et al. (2008); 
Schwartz et al. (2000a, 2000b); Szymanska-Chaowska et al. (2015); Theppeang et al. (2004); and Weaver et al. (2003b).

The VDR is located in the nucleus of intestinal, renal, and bone cells. It is involved in maintaining calcium and phosphate homeostasis and regulating bone metabolism. Binding of vitamin D3 (the active form of vitamin D) to the VDR activates genes that encode for various calcium-binding proteins involved in intestinal absorption and accumulation of calcium in bone. The VDR regulates the production of calcium-binding proteins, and accounts for up to $75 \%$ of the total genetic effect on bone density. Because $\mathrm{Pb}$ can replace and mimic calcium, the VDR plays a critical role in the accumulation of $\mathrm{Pb}$ in bone. The VDR has several polymorphic forms that are defined based on restriction enzyme digestion; these include FokI with three genotypes (FF, Ff, and ff) and BsmI with three genotypes (BB, Bb, bb). The FF genotype has been associated with higher $\mathrm{PbB}$ and increased bone mineral density and calcium uptake. The BB genotype has been associated with higher $\mathrm{PbB}$ and bone $\mathrm{Pb}$. However, the role of VDR polymorphisms in the $\mathrm{Pb}$ uptake into bone remains to be fully elucidated.

Hemochromatosis gene (HFE). Information on HFE polymorphisms was taken from the following publications: Åkesson et al. (2000); Barton et al. (1994); Fan et al. (2014); Hopkins et al. (2008); Mitra et al. (2017); Onalaja and Claudio (2000); Park et al. (2009a); Wang et al. (2007); Wright et al. (2004); and Zhang et al. (2010).

Hemochromatosis is an autosomal, recessive disease characterized by the excessive accumulation of iron in the body. In individuals with hemochromatosis, excess iron accumulates in various organs of the body and causes damage to the liver and compromises cardiovascular function. Hemochromatosis is caused by mutations of the HFE gene, which result in defects to the HFE protein. In individuals with normal HFE, HFE binds to transferrin, decreasing the gastrointestinal absorption of iron; however, in individuals with hemochromatosis, the HFE protein is not functional, leading to an increased accumulation of iron. The absorption of $\mathrm{Pb}$ is linked to iron status such that $\mathrm{Pb}$ absorption increases when iron is limited. HFE polymorphisms have been shown to enhance Pb-induced cognitive impairment (Wang et al. 2007) and the HFE H63D polymorphism appears to enhance positive associations between bone $\mathrm{Pb}$ and pulse pressure (Zhang et al. 2010). However, the influence of HFE variants on absorption and health effects of $\mathrm{Pb}$ is still being defined.

Other polymorphisms. Several other polymorphisms have been examined to evaluate potential alterations in susceptibility to adverse effects of $\mathrm{Pb}$; however, little data are available. These include: 
- Apoprotein E (APOE). APOE is an intracellular transporter of cholesterol and fatty acids that is synthesized by astrocytes in the brain and plays a key role in the structure of cell membranes and myelin. There are three alleles of the APOE gene: E2, E3, and E4. It has been proposed that APOE gene variants may alter susceptibility to $\mathrm{Pb}$-induced changes in neurodevelopment and neurological deficits (Stewart et al. 2002; Wright et al. 2003a).

- Dopamine receptor D4 (DRD4), Dopamine Receptor D2 (DRD2), and Dopamine Transporter (DAT1). $\mathrm{Pb}$ is associated with alterations in the dopaminergic system, which is involved in cognition and behavior. Thus, polymorphisms of DRD4, DRD2, and DAT1 may alter susceptibility to Pb-induced neurocognitive impairment (Froehlich et al. 2007; Kordas et al. 2011; Roy et al. 2011).

- $\quad$-Methyl-D-aspartate receptors (NMDAR subunits GRIN2A and GRIN2B). NMDARs mediate excitatory actions of glutamate in the central nervous system, which affect learning and memory. Polymorphisms in GRIN2A and GRIN2B have been shown to be interacting factors with PbB in association between $\mathrm{PbB}$ and cognitive performance in children (Rooney et al. 2018).

- Glutathione S-transferase mu 1 (GSTM1). Glutathione is an intracellular scavenger of oxidants and electrophiles. It is encoded by the polymorphic gene GSTM1. Genetic alterations causing a decrease in functional glutathione could result in increased oxidative damage or inflammation (Kim et al. 2007).

- Endothelial nitric oxide synthase (eNOS). Nitric oxide, an endogenous signaling molecule involved in vasodilation, is produced by a family of nitric oxide synthase enzymes, including eNOS. Polymorphisms of eNOS could increase susceptibility to Pb (Barbosa et al. 2006b).

- Metallothionein (MT). MT binds to and sequesters Pb. It has been proposed that polymorphisms of MT (MT1 and MT2) may affect binding of Pb to MT and lead to an increased PbB (Chen et al. 2010; Fernandes et al. 2016; Mitra et al. 2017; Yang et al. 2013b).

- Peptide transporter 2 (PEPT2). Polymorphisms of PEPT2 have been associated with increased PbB in children (Sobin et al. 2009).

- Tumor necrosis factor-alpha (TNF- $\alpha)$ : TNF- $\alpha$ is a cell signaling protein involved in the development of inflammation. Genetic variants in TNF- $\alpha$ have the potential to alter susceptibility to $\mathrm{Pb}$ (Kim et al. 2007). 


\subsection{BIOMARKERS OF EXPOSURE AND EFFECT}

Biomarkers are broadly defined as indicators signaling events in biologic systems or samples. They have been classified as biomarkers of exposure, biomarkers of effect, and biomarkers of susceptibility (NAS/NRC 1989).

A biomarker of exposure is a xenobiotic substance or its metabolite(s) or the product of an interaction between a xenobiotic agent and some target molecule(s) or cell(s) that is measured within a compartment of an organism (NAS/NRC 1989). The preferred biomarkers of exposure are generally the substance itself, substance-specific metabolites in readily obtainable body fluid(s), or excreta. Biomarkers of exposure to $\mathrm{Pb}$ are discussed in Section 3.3.1. The National Report on Human Exposure to Environmental Chemicals provides an ongoing assessment of the exposure of a generalizable sample of the U.S. population to environmental chemicals using biomonitoring (see http://www.cdc.gov/exposurereport/). If available, biomonitoring data for Pb from this report are discussed in Section 5.6, General Population Exposure.

Biomarkers of effect are defined as any measurable biochemical, physiologic, or other alteration within an organism that (depending on magnitude) can be recognized as an established or potential health impairment or disease (NAS/NRC 1989). This definition encompasses biochemical or cellular signals of tissue dysfunction (e.g., increased liver enzyme activity or pathologic changes in female genital epithelial cells), as well as physiologic signs of dysfunction such as increased blood pressure or decreased lung capacity. Note that these markers are not often substance specific. They also may not be directly adverse, but can indicate potential health impairment (e.g., DNA adducts). Biomarkers of effect caused by $\mathrm{Pb}$ are discussed in Section 3.3.2.

A biomarker of susceptibility is an indicator of an inherent or acquired limitation of an organism's ability to respond to the challenge of exposure to a specific xenobiotic substance. It can be an intrinsic genetic or other characteristic or a preexisting disease that results in an increase in absorbed dose, a decrease in the biologically effective dose, or a target tissue response. If biomarkers of susceptibility exist, they are discussed in Section 3.2, Children and Other Populations that are Unusually Susceptible. 


\subsubsection{Biomarkers of Exposure}

Biomarkers of exposure in practical use today are measurements of total $\mathrm{Pb}$ levels in body fluids or tissues, such as blood, bone, or urine. Tetraalkyl Pb compounds may also be measured in the breath. Of these, $\mathrm{PbB}$ is the most widely used and is considered to be the most reliable biomarker for general clinical use and public health surveillance. Currently, $\mathrm{PbB}$ measurement is the screening test of choice to identify children with elevated PbBs (CDC 2012d). Venous sampling of blood is preferable to finger prick sampling, which has a considerable risk of surface $\mathrm{Pb}$ contamination from the finger if proper finger cleaning is not carried out. In children, PbBs greater than the blood lead reference value identify highrisk childhood populations and geographic areas most in need of primary prevention (CDC 2012d). In 2012, the blood lead reference value was defined as $5 \mu \mathrm{g} / \mathrm{dL}$ (CDC 2012d). Based on an analyses of NHANES data, geometric mean PbB decreased in the United States population during the period 20092014 (Tsoi et al. 2016) and the percentage of children (<6 years of age) in the United States who had PbB $\geq 5 \mu \mathrm{g} / \mathrm{dL}$ in the survey period 2014-2015 decreased compared to the survey period 2009-2010 (Baertkein and Yendell 2017; McClure et al. 2016).

$\boldsymbol{P b B}$. Measurement of $\mathrm{PbB}$ is the most widely used biomarker of $\mathrm{Pb}$ exposure. $\mathrm{CDC}$ considers $\mathrm{PbB}$ to be elevated in children when it exceeds a reference value defined as the $97.5^{\text {th }}$ percentile for the U.S. population. The blood lead reference value was set at $5 \mu \mathrm{g} / \mathrm{dL}$ in 2012, based on data from NHANES 2007-2008 and 2009-2010, is $5 \mu \mathrm{g} / \mathrm{dL}$ (CDC 2012d). Elevated PbB (e.g., $>5 \mu \mathrm{g} / \mathrm{dL}$ ) is an indication of excessive exposure in infants and children. The biological exposure index (BEI) for $\mathrm{Pb}$ in blood of exposed workers is $20 \mu \mathrm{g} / \mathrm{dL}$ (ACGIH 2018). The BEI also notes to advise "female workers of childbearing age about the risk of delivering a child with a PbB over the current CDC reference value.” The Occupational Safety and Health Administration's (OSHA) permissible exposure limit (PEL) for Pb (50 $\mu \mathrm{g} / \mathrm{m}^{3}$ air, 8-hour time-weighted average [TWA]) was established to keep a majority of worker PbBs below $40 \mu \mathrm{g} / \mathrm{dL}$ (OSHA 2016a). The National Institute for Occupational Safety and Health (NIOSH) recommended exposure limit (REL) for workers (50 $\mu \mathrm{g} / \mathrm{m}^{3}$ air, 8-hour TWA) is established to ensure that the PbB does not exceed $60 \mu \mathrm{g} / \mathrm{dL}$ (NIOSH 2016b).

The extensive use of $\mathrm{PbB}$ as a dose metric reflects mainly the greater feasibility of incorporating $\mathrm{PbB}$ measurements into clinical or epidemiological studies, compared to other potential dose indicators, such as $\mathrm{Pb}$ in kidney, plasma, or bone. $\mathrm{PbB}$ measurements have several limitations as measures of total $\mathrm{Pb}$ body burden. Blood comprises $<2 \%$ of the total $\mathrm{Pb}$ burden; most of the $\mathrm{Pb}$ burden resides in bone (Barry 1975). Pb is eliminated from blood more rapidly than from bone (Behinaein et al. 2014; Brito et al. 2005; 
Chamberlain et al. 1978; Griffin et al. 1975; Manton et al. 2001; Nie et al. 2005; Nilsson et al. 1991; Rabinowitz et al. 1976; Rentschler et al. 2012); therefore, the Pb concentration in blood reflects mainly the exposure history of the previous few months and does not necessarily reflect the larger burden and much slower elimination kinetics of $\mathrm{Pb}$ in bone (Graziano 1994; Lyngbye et al. 1990). Slow release of $\mathrm{Pb}$ from bone can contribute to blood Pb levels long after external exposure has ceased (Fleming et al. 1997; Inskip et al. 1996; Kehoe 1987; McNeill et al. 2000; O'Flaherty et al. 1982; Smith et al. 1996). The relationship between $\mathrm{Pb}$ intake and $\mathrm{PbB}$ is curvilinear; the increment in $\mathrm{PbB}$ per unit of intake decreases with increasing PbB (Ryu et al. 1983; Sherlock and Quinn 1986; Sherlock et al. 1982, 1984). Pb intake$\mathrm{PbB}$ relationships also vary with age as a result of age-dependency of gastrointestinal absorption of $\mathrm{Pb}$, and vary with diet and nutritional status (Mushak 1991). A practical outcome of the above characteristics of $\mathrm{PbB}$ is that $\mathrm{PbB}$ can change relatively rapidly (e.g., days to weeks) in response to changes in exposure; thus, $\mathrm{PbB}$ can be influenced by short-term variability in exposure that may have only minor effects on total $\mathrm{Pb}$ body burden. A single $\mathrm{PbB}$ determination cannot distinguish between lower-level intermediate or chronic exposure and higher-level acute exposure. Similarly, a single measurement may fail to detect a higher exposure that occurred (or ended) several months earlier. Time-integrated measurements of $\mathrm{PbB}$ (CBLI) may provide a means for accounting for some of these factors and thereby provide a better measure of long-term exposure (Armstrong et al. 1992; Behinaein et al. 2014; Chuang et al. 2000; Fleming et al. 1997; Gerhardsson et al. 1993; Healey et al. 2008; Hu et al. 2007; McNeill et al. 2000; Nie et al. 2011a; Roels et al. 1995). The correlation observed between CBLI and tibia bone Pb concentrations provides supporting evidence for this (Hu et al. 2007).

Bone and Tooth Pb Measurements. The development of noninvasive XRF techniques for measuring $\mathrm{Pb}$ concentrations in bone has enabled the exploration of bone $\mathrm{Pb}$ as a biomarker of $\mathrm{Pb}$ exposure in children and in adults (Behinaein et al. 2011; Chettle et al. 2003; Hu et al. 2007; Ji et al. 2014; Nie et al. 2011b; Specht et al. 2016; Todd et al. 2000). Pb in bone is considered a biomarker of cumulative exposure to $\mathrm{Pb}$ because $\mathrm{Pb}$ accumulates in bone over the lifetime and most of the $\mathrm{Pb}$ body burden resides in bone. $\mathrm{Pb}$ is not distributed uniformly in bone. $\mathrm{Pb}$ will accumulate in those regions of bone undergoing the most active calcification at the time of exposure. During infancy and childhood, bone calcification is most active in trabecular bone, whereas in adulthood, calcification occurs at sites of remodeling in both cortical and trabecular bone. This suggests that $\mathrm{Pb}$ accumulation will occur predominantly in trabecular bone during childhood, and in both cortical and trabecular bone in adulthood (Aufderheide and Wittmers 1992). Patella, calcaneus, and sternum XRF measurements primarily reflect $\mathrm{Pb}$ in trabecular bone, whereas XRF measurements of midtibia, phalanx, or ulna primarily reflect primarily $\mathrm{Pb}$ in cortical bone. $\mathrm{Pb}$ levels in cortical bone may be a better indicator of long-term cumulative exposure than $\mathrm{Pb}$ in 
trabecular bone, possibly because $\mathrm{Pb}$ in trabecular bone may exchange more actively with $\mathrm{Pb}$ in blood than does cortical bone. This is consistent with estimates of a longer elimination half-time of $\mathrm{Pb}$ in cortical bone, compared to trabecular bone (Behinaein et al. 2014; Borjesson et al. 1997; Brito et al. 2005; Nie et al. 2005; Nilsson et al. 1991; Schutz et al. 1987). Longitudinal studies that have repeatedly measured bone $\mathrm{Pb}$ (by XRF) over many years have shown more rapid declines in trabecular bone compared to cortical bone (Kim et al. 1997; Wilker et al. 2011). Estimates of cortical bone Pb elimination half-times (5-50 years) show a dependence on $\mathrm{Pb}$ burden, with longer half-times in people who have higher total body burdens (estimated from CBLI) and bone Pb burdens (Behinaein et al. 2014; Brito et al. 2005; Nie et al. 2005). Further evidence that cortical bone Pb measurements may provide a better reflection of long-term exposure than do measurements of trabecular bone comes from studies in which cortical and trabecular bone $\mathrm{Pb}$ measurements have been compared to $\mathrm{PbB}$. Pb levels in trabecular bone (in adults) correlate more highly with contemporary $\mathrm{PbB}$ than do levels of $\mathrm{Pb}$ in cortical bone (Erkkila et al. 1992; Hernandez-Avila et al. 1996; Hu et al. 1996b, 1998; Watanabe et al. 1994). Cortical bone $\mathrm{Pb}$ measurements correlate well with time-integrated $\mathrm{PbB}$ measurements, which would be expected to be a better reflection of cumulative exposure than contemporary PbB measurements (Behinaein et al. 2012; Borjesson et al. 1997; Hu et al. 2007; Roels et al. 1994). Bone Pb levels tend to increase with age (Hu et al. 1996b; Kosnett et al. 1994; Roy et al. 1997), although the relationship between age and bone $\mathrm{Pb}$ may be stronger after adolescence (Hoppin et al. 1997). These observations are consistent with cortical bone reflecting cumulative exposures over the lifetime.

Standard methods for bone $\mathrm{Pb}$ XRF measurements have not been universally accepted, in part, because the technology continues to be improved, and this needs to be considered in comparisons of measurements reported by different laboratories and at different times in development of the methodology used. Historically, two XRF methods have seen the most use in bone Pb epidemiology: K-shell and L-shell methods. The K-shell method is the more widely used, although, improvements in L-shell technology continue to be reported (Nie et al. 2011a). One study reported a correlation of 0.65 between bone $\mathrm{Pb}$ measurements made with a portable L-shell device and a K-shell method (Nie et al. 2011a). In general, recent advances in K-shell technology have yielded higher sensitivities (approximately $3 \mu \mathrm{g} / \mathrm{g}$ tibia mineral; Behinaein et al. 2011) than L-shell technology (approximately $8 \mu \mathrm{g} / \mathrm{g}$ tibia bone mineral; Nie et al. 2011a). Precision of K-shell XRF bone Pb measurements have been extensively discussed (Aro et al. 2000; Behinaein et al. 2014; Todd et al. 2000, 2001, 2002). Methodological factors can contribute substantially to observed variability in bone $\mathrm{Pb}$ measurements in populations (Behinaein et al. 2014). These factors include bone $\mathrm{Pb}$ target, radioactive source, measurement time, and data reduction methods (e.g., approach to handling negative values). Measurement uncertainty also appears to contribute by 
biological factors, such as BMI and bone mineral content (Behinaein et al. 2014; Berkowitz et al. 2004; Hu et al. 2007; Theppeang et al. 2008). The association between BMI and measurement uncertainty may reflect the effect attenuation of the XRF signal by tissue overlaying the target bone site (Behinaein et al. 2014). Bone mineral can be a factor because XRF measures bone Pb fluorescence in relation to fluorescence from bone calcium and the result is expressed in units of $\mu \mathrm{g} \mathrm{Pb}$ per g bone mineral. As a result, variability in bone mineral content can contribute to variability in measured bone $\mathrm{Pb}$. Typically, potential associations between bone density and bone $\mathrm{Pb}$ concentration are not evaluated in epidemiologic studies (Berkowitz et al. 2004; Hu et al. 2007; Theppeang et al. 2008). An important consequence of expressing bone $\mathrm{Pb}$ measures relative to bone mineral content is that lower bone mineral density is associated with greater measurement uncertainty in bone $\mathrm{Pb}$. This uncertainty can have important implications for studies in older women for whom low bone mineral density is more common than in other populations including men and younger adults.

Tooth $\mathrm{Pb}$ has been considered a potential biomarker for measuring long-term exposure to $\mathrm{Pb}$ (e.g., years) because $\mathrm{Pb}$ that accumulates in tooth dentin and enamel appears to be retained until the tooth is shed or extracted (Costa de Almeida et al. 2007; Ericson 2001; Fosse et al. 1995; Gomes et al. 2004; Gulson and Wilson 1994: Gulson et al. 1996; Omar et al. 2001; Rabinowitz 1995; Rabinowitz et al. 1989, 1993; Robbins et al. 2010; Steenhout and Pourtois 1987; Tvinnereim et al. 1997). Formation of enamel and primary dentin of deciduous teeth begins in utero and is complete prior to the time children begin to crawl. Formation of secondary dentin begins after completion of the tooth root and continues through childhood until the tooth is lost, or otherwise loses vitality. $\mathrm{Pb}$ in shed deciduous teeth is not uniformly distributed. Differences in $\mathrm{Pb}$ levels and stable isotope signatures of the enamel and dentin suggest that $\mathrm{Pb}$ uptake occurs differentially in enamel and dentin (Gulson 1996; Gulson and Wilson 1994). Pb in enamel is thought to reflect primarily $\mathrm{Pb}$ exposure that occurs in utero and early infancy, prior to tooth eruption. Dentin appears to continue to accumulate $\mathrm{Pb}$ after eruption of the tooth; therefore, dentin $\mathrm{Pb}$ is thought to reflect exposure that occurs up to the time the teeth are shed or extracted (Gulson 1996; Gulson and Wilson 1994; Rabinowitz 1995; Rabinowitz et al. 1993). The technique of laser ablation-inductively coupled plasma-mass spectrometry (LA-ICP-MS) allows measurement of $\mathrm{Pb}$ levels in regions of dentin formed at various times during deciduous tooth formation in utero and after birth (Arora et al. 2014; Shepherd et al. 2016). Accumulation of $\mathrm{Pb}$ in dentin of permanent teeth may continue for the life of the tooth (Steenhout 1982; Steenhout and Pourtois 1981). Because enamel is in direct contact with the external environment, enamel $\mathrm{Pb}$ levels may be more influenced than dentin $\mathrm{Pb}$ by external $\mathrm{Pb}$ levels and tooth wear (Purchase and Fergusson 1986). 
An analysis of eight cross-sectional and/or prospective studies that reported tooth $\mathrm{Pb}$ and $\mathrm{PbBs}$ of the same children found considerable consistency among the studies (Rabinowitz 1995). The mean tooth Pb levels ranged from $<3$ to $>12 \mu \mathrm{g} / \mathrm{g}$. Dentin $\mathrm{Pb}$ was found to be predictive of $\mathrm{Pb}$ in tibia, patella, and mean bone $\mathrm{Pb}$ in 32 of 63 subjects at follow-up of $\leq 13$ years (Kim et al. 1996b). The authors estimated that a $10 \mu \mathrm{g} / \mathrm{g}$ increase in dentin $\mathrm{Pb}$ levels in childhood was predictive of a $1 \mu \mathrm{g} / \mathrm{g}$ increase in tibia $\mathrm{Pb}$ levels, a $5 \mu \mathrm{g} / \mathrm{g}$ in patella $\mathrm{Pb}$ levels, and a $3 \mu \mathrm{g} / \mathrm{g}$ increase in mean bone $\mathrm{Pb}$ among the young adults. Arora et al. (2014) found that Pb levels in primary (prenatal) dentin were more strongly correlated with PbBs at birth (correlation coefficient, $\mathrm{r}=0.69, \mathrm{n}=27$ ), whereas Pb levels in secondary (postnatal) dentin were more strongly correlated with CBLI ( $\mathrm{r}=0.38, \mathrm{n}=75)$. Shepherd et al. (2016) combined LA-ICP-MS with histological determinations of dentin age to reconstruct the history of incorporation of environmental $\mathrm{Pb}$ from various sources.

Plasma Pb Concentration. The concentration of $\mathrm{Pb}$ in plasma (e.g., approximately $0.04 \mu \mathrm{g} / \mathrm{dL}$ at $\mathrm{PbB}$ of $10 \mu \mathrm{g} / \mathrm{dL}$ ) is extremely difficult to measure accurately because levels in plasma are near the quantitation limits of most analytical techniques (Bergdahl and Skerfving 1997; Bergdahl et al. 1997a) and because hemolysis that occurs with typical analytical practices can contribute to substantial measurement error (Bergdahl et al. 1998, 2006; Cavalleri et al. 1978; Smith et al. 1998a). ICP-MS offers sensitivity sufficient for measurements of $\mathrm{Pb}$ in plasma (Schütz et al. 1996). The technique has been applied to assessing $\mathrm{Pb}$ exposures in adults (Barbosa et al. 2006a; Cake et al. 1996; Hernandez-Avila et al. 1998; Manton et al. 2001; Smith et al. 2002; Tellez-Rojo et al. 2004; Tian et al. 2013). A direct comparison of $\mathrm{Pb}$ concentrations in plasma and serum yielded similar results (Bergdahl et al. 2006); however, the interchangeability of plasma and serum $\mathrm{Pb}$ measurements for biomonitoring of $\mathrm{Pb}$ exposure or body burden had not been thoroughly evaluated in large numbers of subjects (Bergdahl et al. 2006; Manton et al. 2001; Smith et al. 2002).

Urinary Pb. Measurements of urinary $\mathrm{Pb}$ levels have been used to assess $\mathrm{Pb}$ exposure (e.g., Chiang et al. 2008; Fels et al. 1998; Fukui et al. 1999; Gerhardsson et al. 1992; Lilis et al. 1968; Lin et al. 2001; Mendy et al. 2012; Mortada et al. 2001; Navas-Acien et al. 2005; Rentschler et al. 2012; Roels et al. 1994; Sun et al. 2008b). However, like PbB, urinary Pb excretion mainly reflects recent exposure and thus shares many of the same limitations for assessing Pb body burden or long-term exposure (Sakai 2000; Skerfving 1988). Although collection of urine is noninvasive, urine $\mathrm{Pb}$ levels exhibit variability with $\mathrm{PbB}$, and interpretation of urine $\mathrm{Pb}$ levels requires estimates of GFR and measurement of urine volume (NTP 2012). A significant, but relatively weak correlation between urinary $\mathrm{Pb}$ levels ( $\mu \mathrm{g} / \mathrm{g}$ creatinine) and individual $\mathrm{Pb}$ intakes $(\mu \mathrm{g} /$ day) was observed in a study of $10-12$-year-old children $(\beta: 0.053, \mathrm{R}=0.320$, 
$\mathrm{p}=0.02, \mathrm{~N}=57$; Chiang et al. 2008). In this study, urine sampling and measurements used to estimate intake were separated by as long as 6 months for some children, which may have contributed to the relatively weak correlation. The measurement is further complicated by variability in urine volume, which can affect concentrations independent of excretion rate (Diamond 1988) and the potential effects of decrements in kidney function on excretion, in association with high, nephrotoxic Pb exposures or kidney disease (Lilis et al. 1968; Wedeen et al. 1975). Urinary Pb concentration increases exponentially with $\mathrm{PbB}$ and can exhibit relatively high intra-individual variability, even at similar PbBs (Gulson et al. 1998a; Skerfving et al. 1985). However, the relationship between plasma $\mathrm{Pb}$ and urinary $\mathrm{Pb}$ ( $\mu \mathrm{g} \mathrm{Pb} / \mathrm{g}$ creatinine) was linear in a small group of children (Rentschler et al. 2012). The linear relationship between plasma and urinary $\mathrm{Pb}$ may reflect the importance of plasma $\mathrm{Pb}$ in determining the rate of glomerular filtration and renal tubular transport of $\mathrm{Pb}$ (see Section 3.1.4). Urinary diethyl $\mathrm{Pb}$ has been proposed as a qualitative marker of exposure to tetraethyl Pb (Turlakiewicz and Chmielnicka 1985; Vural and Duydu 1995; Zhang et al. 1994).

The measurement of $\mathrm{Pb}$ excreted in urine following an injection (intravenous or intramuscular) of the chelating agent, calcium disodium EDTA (EDTA provocation), or oral dosing with dimercaptosuccinic acid (DMSA) has been used to detect elevated body burden of $\mathrm{Pb}$ in adults (Biagini et al. 1977; Lee et al. 2009; Lilis et al. 1968; Lin et al. 2003, 2006a, 2006b; Schwartz et al. 2000a, 2000c; Wedeen 1992; Wedeen et al. 1975)and children (Chisolm et al. 1976; Markowitz and Rosen 1981). However, the American College of Medical Toxicology (ACMT 2010) position statement on post-chelator challenge urinary metal testing states that "post-challenge urinary metal testing has not been scientifically validated, has no demonstrated benefit, and may be harmful when applied in the assessment and treatment of patients in whom there is concern for metal poisoning.” The assay is not a substitute for $\mathrm{PbB}$ measurements in the clinical setting. Note that children whose $\mathrm{PbBs}$ are $\geq 45 \mu \mathrm{g} / \mathrm{dL}$ should not receive a provocative chelation test; they should be immediately referred for appropriate chelation therapy (CDC 2002a, 2012e). For additional information on recommended actions based on PbB level in children and adults, see Section 3.5 (Methods for Reducing Toxic Effects). Further limitations for routine use of the test are that EDTA must be given parenterally and requires timed urine collections. A study conducted in rats found that intraperitoneal administration of a single dose of EDTA following 3-4-month exposures to $\mathrm{Pb}$ in drinking water increased levels of $\mathrm{Pb}$ in the liver and brain (Cory-Slechta et al. 1987) raising concern for similar effects in humans who undergo the EDTA provocation test. The use of EDTA to assess bone stores of $\mathrm{Pb}$ (Wedeen 1992) is largely being supplanted by more direct, noninvasive procedures for measuring $\mathrm{Pb}$ in bone. DMSA is a $\mathrm{Pb}$ chelating agent that can be administered orally. 
DMSA-chelatable Pb has been used as marker of Pb body burden in adults (Schwartz et al. 1997b, 2000a, 2000c; Scinicariello et al. 2007; Weaver et al. 2003a, 2003b).

$\boldsymbol{P b}$ in Saliva and Sweat. Pb is excreted in human saliva and sweat (Genuis et al. 2011; Lilley et al. 1988; Omokhodion and Crockford 1991; Rabinowitz et al. 1976; Stauber and Florence 1988; Sears et al. 2012; Stauber et al. 1994). Sweat has not been widely adopted for monitoring Pb exposures. Lilley et al. (1988) found that $\mathrm{Pb}$ concentrations in sweat were elevated in Pb workers; however, sweat and PbBs were poorly correlated. This may reflect excretion of $\mathrm{Pb}$ in or on the skin that had not been absorbed into blood. Studies conducted in rats have found relatively strong correlations between $\mathrm{Pb}$ concentrations in plasma and saliva (e.g., $\mathrm{r}^{2}>0.9$ ), compared to blood $\mathrm{Pb}$ and saliva; therefore, saliva may serve as a better predictor of plasma $\mathrm{Pb}$ than $\mathrm{PbB}$ (Timchalk et al. 2006). However, studies of saliva $\mathrm{Pb}$ conducted in humans have had mixed results, with some studies showing relatively strong correlations between salivary Pb concentration and PbB (Brodeur et al. 1983; Omokhodion and Crockford 1991; P'an 1981), and other studies showing weak or inconsistent relationships (Barbosa et al. 2006c; Costa de Almeida et al. 2009, 2010, 2011; Nriagu et al. 2006). Variable outcomes from these studies may reflect differences in $\mathrm{PbBs}$, exposure history and/or dental health (i.e., transfer of $\mathrm{Pb}$ between dentin and saliva), and methods used for determining $\mathrm{Pb}$ in saliva. Other complicating factors reported in the literature include uncontrolled variation in salivary flow rates (Barbosa et al. 2005; Esteban and Castano 2009) and potential blood contamination of saliva (Koh and Koh 2007).

Hair and Nail Pb. Pb is incorporated into human hair and hair roots (Bos et al. 1985; Rabinowitz et al. 1976) and has been explored as a possibly noninvasive approach for estimating $\mathrm{Pb}$ body burden (Gerhardsson et al. 1995b; Wilhelm et al. 1989). The method is subject to error from contamination of the surface with environmental $\mathrm{Pb}$ and contaminants in artificial hair treatments (i.e., dyeing, bleaching, permanents) and is a relatively poor predictor of $\mathrm{PbB}$, particularly at low concentrations $(<12 \mu \mathrm{g} / \mathrm{dL})$ (Campbell and Toribara 2001; Drasch et al. 1997; Esteban et al. 1999; Rodrigues et al. 2008). Nevertheless, levels of $\mathrm{Pb}$ in hair were positively correlated with children's classroom attention deficit behavior in a study (Tuthill 1996). Pb in hair was correlated with liver and kidney $\mathrm{Pb}$ in a study of deceased smelter workers (Gerhardsson et al. 1995b). Correlations between maternal and infant hair $\mathrm{Pb}$ concentrations have been observed (Kordas et al. 2010). Although hair Pb measurements have been used in some epidemiologic studies (Bao et al. 2009; Huel et al. 2008; Marcus et al. 2010; Shah et al. 2011), an empirical basis for interpreting hair $\mathrm{Pb}$ measurements in terms of body burden or exposure has not been firmly established. Nail $\mathrm{Pb}$ has also been utilized as a marker of $\mathrm{Pb}$ exposure, although nails may be contaminated with Pb from external sources (Barbosa et al. 2005; Gerhardsson et al. 1995b). 
Semen $\mathbf{P b}$. Pb concentrations in semen have been explored as an internal exposure biomarker for adverse effects of Pb on the testes (Hernandez-Ochoa et al. 2005; Kasperczyk et al. 2015; Slivkova et al. 2009; Taha et al. 2013; Wu et al. 2012). Correlations between concentrations of Pb in semen and blood have been reported and vary in strength across studies (Alexander et al. 1998a, 1998b; Farias et al. 2005; Hernandez-Ochoa et al. 2005; Mendiola et al. 2011; Telisman et al. 2000). This variation may relate, in part, to analytical challenges in the measurement of the relatively low concentrations of $\mathrm{Pb}$ in semen. Using ICP-MS and rigorous collection methods to avoid contamination, Farias et al. (2005) reported a detection limit of $0.2 \mu \mathrm{g} / \mathrm{L}$ semen. Mean semen $\mathrm{Pb}$ concentration in a group of 160 men (age range 1948 years) who were not exposed to $\mathrm{Pb}$ occupationally was $2.66 \mu \mathrm{g} / \mathrm{L}$ (range $0.08-19.42$ ) and was significantly correlated with $\mathrm{PbB}$ (mean $10.8 \mu \mathrm{g} / \mathrm{dL}$, range 4.5-40.2) and tibia bone $\mathrm{Pb}$ (mean $14.51 \mu \mathrm{g} / \mathrm{g}$, range not-detected-44.71 $\mu \mathrm{g} / \mathrm{g})$.

Stable Pb Isotopes. Analysis of the relative abundance of stable isotopes of $\mathrm{Pb}$ in blood and other accessible body fluids (e.g., breast milk, urine) has been used to differentiate exposures from multiple sources (Flegal and Smith 1995). Relative abundances of stable isotopes of $\mathrm{Pb}\left({ }^{204} \mathrm{~Pb},{ }^{206} \mathrm{~Pb}\right.$, ${ }^{207} \mathrm{~Pb}$, and ${ }^{208} \mathrm{~Pb}$ ) in $\mathrm{Pb}$ ores vary with the age of the ore (which determines the extent to which the parent isotopes have undergone radioactive decay to stable $\mathrm{Pb}$ ). Humans have $\mathrm{Pb}$ isotope abundance profiles that reflect the profiles of $\mathrm{Pb}$ deposits to which they have been exposed. $\mathrm{Pb}$ isotope studies can be used to exclude sources of $\mathrm{Pb}$ contributing to exposure. Similarly, if exposure abruptly changes to a $\mathrm{Pb}$ source having a different isotope abundance profile, the kinetics of the change in profile in the person can be measured, reflecting the kinetics of uptake and distribution of $\mathrm{Pb}$ from the new source (Gulson et al. 2003; Maddaloni et al. 1998; Manton et al. 2003). Numerous examples of the application of stable isotope abundance measurements for studying sources of $\mathrm{Pb}$ exposures have been reported (Angle et al. 1995; Graziano et al. 1996; Gulson and Wilson 1994; Gulson et al. 1996, 1997b, 1999c, 2016; Manton 1977, 1998).

Effect Biomarkers Used to Assess Exposure to Pb. Certain physiological changes that are associated with $\mathrm{Pb}$ exposure have been used as biomarkers of exposure (see Section 3.3.2). These include measurements of biomarkers of impaired heme biosynthesis (blood zinc protoporphyrin, urinary coproporphyrin, erythrocyte ALAD activity, serum ALA). These types of measurements have largely been supplanted with measurement of $\mathrm{PbB}$ for the purpose of assessing $\mathrm{Pb}$ exposure due to the higher sensitivity of $\mathrm{PbB}$ measurements in quantifying lower level $\mathrm{Pb}$ exposures. 


\subsubsection{Biomarkers of Effect}

Certain effects of $\mathrm{Pb}$ have been used in diagnosing $\mathrm{Pb}$ poisoning to support measurements of $\mathrm{PbB}$; however, none of these diagnostic aids are considered preferable to measurement of $\mathrm{PbB}$. A multisite study of populations living near four NPL sites was conducted to assess the relationship between exposure ( $\mathrm{PbB}$ and area of residence) and biomarkers of four organ systems: immune system dysfunction, kidney dysfunction, liver dysfunction, and hematopoietic dysfunction (ATSDR 1995). The geometric mean $\mathrm{PbB}$ in those living in the target areas was $4.26 \mu \mathrm{g} / \mathrm{dL}(\mathrm{n}=1,645)$ compared with $3.45 \mu \mathrm{g} / \mathrm{dL}$ for a group living in comparison areas $(\mathrm{n}=493)$. In children $<6$ years old, the corresponding means were 5.37 versus $3.96 \mu \mathrm{g} / \mathrm{dL}$. In subjects $\geq 15$ years old, the target and comparison values were 3.06 and $3.63 \mu \mathrm{g} / \mathrm{dL}$, respectively. Ninety percent of target and $93 \%$ of comparison area participants had $\mathrm{PbBs}<10 \mu \mathrm{g} / \mathrm{dL} . \mathrm{Pb}$ in soil and water was found to be higher in comparison areas than in the target areas, but $\mathrm{Pb}$ in house dust and in interior paint was higher in the target areas. $\mathrm{PbB}$ correlated with $\mathrm{Pb}$ in soil and dust, but not with $\mathrm{Pb}$ in paint and water. Multivariate regression analyses showed that of all the biomarkers analyzed, $\mathrm{PbB}$ was significantly associated with, and predictive of, hematocrit in adults $\geq 15$ years of age and with increased mean serum IgA in children 6-71 months of age. The biological significance of these associations is unclear since both hematocrit and IgA levels were well within normal ranges and were hardly different than levels in subjects from the comparison areas.

$\mathrm{Pb}$ inhibits heme biosynthesis, which is necessary for production of red blood cells. Hematologic tests such as hemoglobin concentration may suggest toxicity, but this is not specific for $\mathrm{Pb}$ (Bernard and Becker 1988). However, inhibition of ferrochelatase in the heme pathway causes accumulation of protoporphyrin in erythrocytes (CDC 1985). Most protoporphyrin in erythrocytes (about 90\%) exists as zinc-protoporphyrin (ZPP). This fraction is preferentially measured by hematofluorometers. Extraction methods measure all of the protoporphyrin present, but strip the zinc from the ZPP during the extraction process. For this reason, extraction results are sometimes referred to as (zinc) free erythrocyte protoporphyrin (FEP). Although the chemical forms measured by the two methods differ slightly, on a weight basis, they are roughly equivalent; thus, results reported as EP, ZPP, or FEP all reflect essentially the same analyte. An elevated EP level is one of the earliest and most reliable indicators of impairment of heme biosynthesis and reflects average $\mathrm{Pb}$ levels at the site of erythropoiesis over the previous 4 months (Janin et al. 1985). The concentration of EP rises above background at PbBs of 25-30 $\mu \mathrm{g} / \mathrm{dL}$, above which, there is a positive correlation between PbB and EP (CDC 1985; Gennart et al. 1992a; Roels and Lauwerys 1987; Soldin et al. 2003; Wildt et al. 1987). Pb toxicity is generally considered to be present when a $\mathrm{PbB} \geq 10 \mu \mathrm{g} / \mathrm{dL}$ is associated with an EP level $\geq 35 \mu \mathrm{g} / \mathrm{dL}$ (CDC 1991; Somashekaraiah et al. 
1990). This effect is detectable in circulating erythrocytes only after a lag time reflecting maturation in which the entire population of red blood cells has turned over (i.e., 120 days) (EPA 1986a; Moore and Goldberg 1985). Similarly, elevated EP can reflect iron deficiency, sickle cell anemia, and hyperbilirubinemia (jaundice). Therefore, reliance on EP levels alone for initial screening could result in an appreciable number of false positive cases (CDC 1985; Mahaffey and Annest 1986; Marcus and Schwartz 1987). Conversely, since EP does not go up until the PbB exceeds $25 \mu \mathrm{g} / \mathrm{dL}$, and the blood lead reference value was set at $5 \mu \mathrm{g} / \mathrm{dL}$ in 2012, relying on EP measures would result in many false negative cases. Some have estimated that relying only on ZPP screening to predict future Pb toxicity would miss approximately three cases with toxic PbBs in every 200 workers at risk (Froom et al. 1998). A limitation of measuring porphyrin accumulation is that porphyrin is labile because of photochemical decomposition; thus, assay samples must be protected from light. However, other diseases or conditions such as porphyria, liver cirrhosis, iron deficiency, age, and alcoholism may also produce similar effects on heme synthesis (Somashekaraiah et al. 1990).

$\mathrm{ALAD}$, an enzyme occurring early in the heme pathway, is also considered a sensitive indicator of $\mathrm{Pb}$ effect (Graziano 1994; Hernberg et al. 1970; Morris et al. 1988; Somashekaraiah et al. 1990; Tola et al. 1973). ALAD activity is negatively correlated with PbBs of 5-95 $\mu \mathrm{g} / \mathrm{dL}$, with $>50 \%$ inhibition occurring at PbBs $>20 \mu \mathrm{g} / \mathrm{dL}$ (Hernberg et al. 1970; Morita et al. 1997; Roels and Lauwerys 1987). However, ALAD activity may also be decreased with other diseases or conditions such as porphyria, liver cirrhosis, and alcoholism (Somashekaraiah et al. 1990). ALAD was found to be a more sensitive biomarker than urinary ALA and ZPP at PbBs between 21 and $30 \mu \mathrm{g} / \mathrm{dL}$ (Schuhmacher et al. 1997). A marked increase in urinary excretion of ALA, the intermediate that accumulates from decreased ALAD, can be detected when PbB exceeds $35 \mu \mathrm{g} / \mathrm{dL}$ in adults and 25-75 $\mu \mathrm{g} / \mathrm{dL}$ in children (NAS 1972; Roels and Lauwerys 1987; Sakai and Morita 1996; Schuhmacher et al. 1997).

Another potential biomarker for hematologic effects of $\mathrm{Pb}$ is the observation of basophilic stippling and premature erythrocyte hemolysis (Paglia et al. 1975, 1977). Pb can impair the activity of pyrimidine 5'-nucleotidase, resulting in a corresponding increase in pyrimidine nucleotides in red blood cells, which leads to a deficiency in maturing erythroid elements and thus, decreased red blood cells. However, this effect is nonspecific; it is encountered with benzene and arsenic poisoning (Smith et al. 1938) and in a genetically-induced enzyme-deficiency syndrome (Paglia et al. 1975, 1977). Furthermore, since basophilic stippling is not universally found in chronic $\mathrm{Pb}$ poisoning, it is relatively insensitive to lesser degrees of Pb toxicity (CDC 1985). The activity of adenine dinucleotide synthetase (NADS) in 
erythrocytes has also been explored as a biomarker for predicting PbBs $>40 \mu \mathrm{g} / \mathrm{dL}$; NADS activity is negatively correlated with PbB over the range 5-80 $\mu \mathrm{g} / \mathrm{dL}$ (Morita et al. 1997).

Reduction in the serum 1,25-dihydroxyvitamin D concentration has been reported as an indicator of increased $\mathrm{Pb}$ absorption or $\mathrm{Pb}$ concentrations in the blood (Rosen et al. 1980). Pb inhibits the formation of this active metabolite of vitamin D, which occurs in bone mineral metabolism (EPA 1986a; Landrigan 1989). Children with PbBs of 12-120 $\mu \mathrm{g} / \mathrm{dL}$ showed decreased serum 1,25-dihydroxyvitamin D concentrations comparable to those found in patients with hypoparathyroidism, uremia, and metabolic bone disease (Mahaffey et al. 1982; Rosen et al. 1980). This biomarker is clearly not specific for $\mathrm{Pb}$ exposure and several diseases can influence this measurement.

One of the most sensitive systems affected by $\mathrm{Pb}$ exposure is the nervous system. Encephalopathy is characterized by symptoms such as coma, seizures, ataxia, apathy, bizarre behavior, and incoordination (CDC 1985). Children are more sensitive to neurological changes than adults. In children, encephalopathy has been associated with PbBs as low as $70 \mu \mathrm{g} / \mathrm{dL}$ (CDC 1985). An early sign of peripheral manifestations of neurotoxicity is gastrointestinal colic, which can occur with PbBs above $50 \mu \mathrm{g} / \mathrm{dL}$. The most sensitive peripheral index of neurotoxicity of $\mathrm{Pb}$ is reported to be slowed conduction velocity in small motor fibers of the ulnar nerve in workers with PbBs of 30-40 $\mu \mathrm{g} / \mathrm{dL}$ (Landrigan 1989). Other potential biomarkers of $\mathrm{Pb}$ suggested for neurotoxicity in workers are neurological and behavioral tests, as well as cognitive and visual sensory function tests (Williamson and Teo 1986). However, these tests are not specific to elevated $\mathrm{Pb}$ exposure.

Functional deficits associated with Pb-induced nephrotoxicity increase in severity with increasing PbB. Effects include decreased glomerular filtration, enzymuria and proteinuria, and impaired transport function. Biomarkers for these changes include elevation of serum creatinine, urinary enzymes (e.g., NAG), or protein (albumin, $\beta 2 \mu$-globulin, $\alpha 1 \mu$-globulin, retinol binding protein). However, none of these markers are specific for $\mathrm{Pb}$-induced nephrotoxicity. A characteristic histologic feature of $\mathrm{Pb}$ nephrotoxicity is the formation of intranuclear inclusion bodies in the renal proximal tubule (Choie and Richter 1972; Goyer et al. 1970a, 1970b). 


\subsection{INTERACTIONS WITH OTHER CHEMICALS}

Interactions between $\mathrm{Pb}$ and other chemicals can be classified into two categories: interactions with contaminants that are commonly found together with $\mathrm{Pb}$ at hazardous waste sites, and interactions with essential elements (ATSDR 2004a, 2004b, 2006; EPA 2014c).

Interactions with Other Contaminants. Several metals and metalloids frequently are found together with $\mathrm{Pb}$ at hazardous waste sites, including arsenic (As), cadmium (Cd), manganese (Mn), zinc (Zn), copper (Cu), and inorganic mercury (Hg). ATSDR (2004a, 2004b, 2006) has conducted assessments to predict interactions of these chemicals with $\mathrm{Pb}$; conclusions are presented in Table 3-3. For each co-contaminant, interactions were classified as less than additive (indicating an antagonistic effect with $\mathrm{Pb}$ ), additive (indicating no effect of combined exposure), or greater than additive (indicating a synergistic effect with $\mathrm{Pb})$. Greater-than-additive effects were observed for neurological effects for As and Cd, male reproductive effects for $\mathrm{Cd}$, and renal effects for $\mathrm{Hg}$. Interactions for other metals were either less than additive or additive for cardiovascular (Cd, Zn), developmental (Zn), hematological (As, Cd, Mn, Zn, $\mathrm{Cu}$ ), immunological (Cd), neurological effects ( $\mathrm{Zn}$ ), renal (As, $\mathrm{Cd}, \mathrm{Mn}, \mathrm{Zn}, \mathrm{Cu}$ ), and male reproductive (Zn) effects. Other metals that may interact with $\mathrm{Pb}$ include selenium and chromium(VI) (Nordberg et al. 2015). Observed interactions of metals and metalloids with $\mathrm{Pb}$ could be the results of alterations to $\mathrm{Pb}$ toxicokinetics, toxicodynamics, or a combination of both.

\section{Table 3-3. Influence of Other Metals and Metalloids on Lead (Pb) Toxicity}

\begin{tabular}{lllllll}
\hline & \multicolumn{7}{c}{ Metal } \\
\cline { 2 - 7 } Organ system & Arsenic $^{\mathrm{a}}$ & Cadmium $^{\mathrm{a}}$ & Manganese $^{\mathrm{b}}$ & Zinc $^{\mathrm{b}}$ & Copper $^{\mathrm{b}}$ & $\begin{array}{l}\text { Inorganic } \\
\text { mercury }^{\mathrm{c}}\end{array}$ \\
\hline Cardiovascular & - & $<$ or 0 & - & $<$ & - & - \\
\hline Developmental & - & - & - & $<$ & - & - \\
\hline Hematological & $<$ or 0 & $<$ or 0 & 0 & $<$ or 0 & $<$ & - \\
\hline Immunological & - & $<$ & - & - & - & - \\
\hline Neurological & $>$ & $>$ & - & $<$ or 0 & $<$ & - \\
\hline Renal & 0 & $<$ or 0 & 0 & $<$ & - & $>$ \\
\hline Male reproductive & - & $>$ & - & $<$ & - & - \\
\hline
\end{tabular}

aATSDR 2004a.

${ }^{\mathrm{b} A T S D R} 2004 \mathrm{~b}$.

'ATSDR 2006.

$<=$ less than additive; $0=$ additive (no effect) $>=$ greater than additive; $-=$ not assessed 
Interactions with Essential Elements. In physiological systems, Pb mimics divalent cations (calcium, iron, zinc). Substitution of $\mathrm{Pb}$ for essential elements in membrane transport systems is the mechanism by which $\mathrm{Pb}$ is absorbed from the intestine and crosses cell membranes throughout the body. Thus, numerous interactions between $\mathrm{Pb}$ and essential elements have been observed, including the following (additional details on these finding are provided in Section 3.1, Toxicokinetics):

- Dietary calcium intake appears to affect $\mathrm{Pb}$ absorption. An inverse relationship has been observed between dietary calcium intake and PbBs in children (Elias et al. 2007; Mahaffey et al. 1986; Schell et al. 2004; Ziegler et al. 1978).

- Nutritional iron status may affect Pb absorption in children. Higher PbBs have been observed in iron-deficient children compared to children who are iron replete. This observation suggests that iron deficiency may result in higher absorption of $\mathrm{Pb}$ or, possibly, other changes in $\mathrm{Pb}$ biokinetics that would contribute to higher PbBs (Mahaffey and Annest 1986; Marcus and Schwartz 1987).

- In young children (ages 6-12 months), PbB increased in association with lower dietary Zn levels (Schell et al. 2004). It is not clear, however, if these associations were caused by changes in $\mathrm{Pb}$ absorption.

\subsection{METHODS FOR REDUCING TOXIC EFFECTS}

This section will describe clinical practice and research concerning methods for reducing toxic effects of exposure to $\mathrm{Pb}$. However, because some of the treatments discussed may be experimental and unproven, this section should not be used as a guide for treatment of exposures to $\mathrm{Pb}$. When specific exposures have occurred, poison control centers, medical toxicologists, or other clinicians with expertise and experience treating and managing Pb-exposed adults and/or children should be consulted. The following resources provide specific information about treatment and management of patients following exposure to $\mathrm{Pb}$ :

AAP. 2005. Lead exposure in children: Prevention, detection, and management. Pediatrics 116(4):1036-1046. 10.1542/peds.2005-1947.

AAP. 2016. Council on Environmental Health. Prevention of childhood lead toxicity. Pediatrics 38(1):e20161493

ATSDR. 2017. Case studies in environmental medicine (CSEM). Lead toxicity. https://www.atsdr.cdc.gov/csem/lead/docs/csem-lead_toxicity_508.pdf. August 30, 2018.

Calello DP, Henretig FM. 2014. Lead. In: Goldfrank's toxicologic emergencies. Tenth ed. New York, NY: McGraw-Hill, 1219-1234.

Holland MG, Cawthon D. 2016. ACOEM Position Statement. Workplace lead exposure. J Occup Environ Med 58(12):e371-e374. 
Leikin JB, Paloucek FP. 2008. Lead. In: Poisoning and toxicology handbook. Fourth ed. Boca Raton, FL: CRC Press, 807-811.

CDC. 2002a. Managing elevated blood levels among young children. Recommendations from the Advisory Committee on Childhood Lead Poisoning. Centers for Disease Control and Prevention. https://www.cdc.gov/nceh/lead/casemanagement/managingEBLLs.pdf. July 18, 2018.

Kosnett MJ. 2001. Lead. In: Ford M, Delaney KA, Ling L, et al., eds. Clinical toxicology. St. Louis: WB Saunders, 723-736.

Kosnett MJ. 2005. Lead. In: Brent J, Wallace KL, Burkhart KK, et al., eds. Critical care toxicology. Philadelphia, PA: Elsevier Mosby, 821-836.

PEHSU. 2013. Recommendations on medical management of childhood lead exposure and poisoning. Pediatric Environmental Health Specialty Units.

Additional publicly available clinical resources for the health care professional can be found in Appendix D.

\subsubsection{Reducing Absorption Following Exposure}

No treatment modalities to reduce $\mathrm{Pb}$ absorption have been developed. Therefore, the most important intervention is to identify and remove the source of exposure (AAP 2005, 2016; ATSDR 2017; CDC 2012e). Pb absorption from the gastrointestinal tract is influenced by nutrition, especially calcium, iron, and vitamin C (AAP 2005; CDC 2012e). It is recommended that a child's diet contain ample amounts of iron and calcium to reduce the likelihood of increased absorption of $\mathrm{Pb}$ and that children eat regular meals since more $\mathrm{Pb}$ is absorbed on an empty stomach (AAP 2005; CDC 2002a, 2012e). Good sources of iron include liver, fortified cereal, cooked legumes, and spinach, whereas milk, yogurt, cheese, and cooked greens are good sources of calcium (CDC 1991).

General recommendations to reduce absorption of $\mathrm{Pb}$ following acute exposure include the following (AAP 2016; ATSDR 2017; Calello and Henretig 2014; Kosnett et al. 2007):

- remove the individual from the source of exposure;

- mitigate source of exposure;

- if suspected that elevated $\mathrm{PbB}$ is due to ingestion of a foreign object (e.g., Pb paint chips, toys or jewelry containing $\mathrm{Pb}, \mathrm{Pb}$ ammunition), radiographic imaging is suggested;

- if elevated $\mathrm{PbB}$ is due to ingestion of a foreign object, decontamination of the bowel (surgical or gastric lavage) is indicated; and

- ensure that diet is adequate in calcium, iron, and vitamin C. 
For children, specific recommended actions based on PbB levels are summarized in Table 3-4. CDC considers $\mathrm{PbB}$ to be elevated in children when it exceeds a reference value defined as the $97.5^{\text {th }}$ percentile for the U.S. population. In 2012, CDC adopted a blood lead reference value, based on data from NHANES 2007-2008 and 2009-2010, of 5 g/dL (CDC 2016).

Table 3-4. Recommended Actions Based on Child Blood Lead Level (PbB)

\begin{tabular}{|c|c|}
\hline $\mathrm{PbB}(\mu \mathrm{g} / \mathrm{dL})$ & Recommended actions \\
\hline$<$ Reference value ${ }^{a}$ & $\begin{array}{l}\text { - Routine assessment of nutritional and developmental milestones } \\
\text { - Education on common sources of } \mathrm{Pb} \text { exposure } \\
\text { - Follow-up PbB monitoring }\end{array}$ \\
\hline $5-19$ & $\begin{array}{ll}\text { - } & \text { Follow recommendations for }<\text { Reference value } \\
\text { - } & \text { Nutritional counseling for calcium and iron intake }\end{array}$ \\
\hline $20-44$ & $\begin{array}{l}\text { - } \quad \text { Complete history and physical examination with neurodevelopmental } \\
\text { - } \quad \text { Environmental assessment of home and lead hazard reduction } \\
\text { - } \quad \text { Follow-up PbB monitoring } \\
\text { - } \text { Assess iron status, hemoglobin, and hematocrit } \\
\text { - Abdominal x-ray and bowel decontamination if indicated }\end{array}$ \\
\hline$\geq 45$ and $\leq 69$ & $\begin{array}{l}\text { - Follow recommendations for } 45-69 \mu \mathrm{g} / \mathrm{dL} \\
\text { - Complete neurological examination } \\
\text { - } \quad \text { Consider oral chelation therapy with consultation with a medical } \\
\text { - } \quad \text { Coxicologist or pediatric environmental health expert or unit } \\
\text { - Consider hospitalization if cannot assure mitigation of } \mathrm{Pb} \text { source }\end{array}$ \\
\hline$\geq 70$ & $\begin{array}{l}\text { - Hospitalize } \\
\text { - Initiate chelation therapy with consultation with a medical toxicologist or } \\
\text { pediatric environmental health expert or unit } \\
\text { - } \text { Follow recommendations for } \geq 45 \text { and } \leq 69 \mu \mathrm{g} / \mathrm{dL} \\
\text { - Environmental investigation of the home and lead hazard reduction; child } \\
\text { receiving chelation therapy should not return to home until lead hazard } \\
\text { remediation is completed }\end{array}$ \\
\hline
\end{tabular}

${ }^{2} 5 \mu \mathrm{g} / \mathrm{dL}$ (CDC 2012d).

Source: CDC $2012 f$

For occupational exposures, OSHA and NIOSH have developed recommendations to reduce $\mathrm{Pb}$ exposure through procedures and surveillance. In 1987, NIOSH created the Adult Blood Lead Epidemiology and Surveillance (ABLES) program to monitor adult PbBs through coordinated efforts with state agencies (NIOSH 2017a). This program was designed to decrease the rate of adults with $\mathrm{PbBs} \geq 10 \mu \mathrm{g} / \mathrm{dL}$ as a result of work-related $\mathrm{Pb}$ exposure. In 2015, NIOSH designated PbB of $5 \mu \mathrm{g} / \mathrm{dL}$ as the PbB reference value and defined elevated $\mathrm{PbB}$ as $\mathrm{PbB} \geq 5 \mu \mathrm{g} / \mathrm{dL}$ (NIOSH 2017a). Several federal and state agencies work together to reduce the rate of elevated PbBs among workers. The OSHA (1995) mandated rule on 
$\mathrm{Pb}$ provides recommendations to reduce occupational $\mathrm{Pb}$ exposure for general industry, shipyard employment, and construction through use of respirators, protective clothing, routine biological monitoring of $\mathrm{PbB}$ and zinc protoporphyrin, and medical assessments for workers with elevated $\mathrm{PbB}$. More recently, Holland and Cawthon (2016) suggested the actions based on PbB levels, with a baseline $\mathrm{PbB}<5 \mu \mathrm{g} / \mathrm{dL}$ (Table 3-5).

Table 3-5. Recommended Actions for Workers Based on Blood Lead Level (PbB)

\begin{tabular}{|c|c|}
\hline $\mathrm{PbB}(\mu \mathrm{g} / \mathrm{dL})$ & Recommended actions \\
\hline All workers & $\begin{array}{l}\text { - } \quad \text { PbB monitoring at initial employment } \\
\text { - } \quad \text { Monitor PbB every } 6 \text { months after initial employment monitoring } \\
\text { - } \quad \text { PbB goal is }<5 \mu \mathrm{g} / \mathrm{dL} \text { for pregnant workers }\end{array}$ \\
\hline$\geq 5-9$ & $\begin{array}{l}\text { - Increase monitoring if indicated } \\
\text { - Recommend removal for pregnant workers or workers who are trying to become } \\
\text { pregnant; return to work may be considered if two consecutive PbB measurements } \\
\text { are }<5 \mu \mathrm{g} / \mathrm{dL} \\
\text { - Continue } \mathrm{PbB} \text { monitoring as noted above }\end{array}$ \\
\hline 10-19 & $\begin{array}{l}\text { - Monitor PbB every } 2 \text { months until two consecutive } \mathrm{PbB} \text { measurements are } \\
\quad<10 \mu \mathrm{g} / \mathrm{dL} \\
\text { - Mandatory medical removal for pregnant workers or workers who are trying to } \\
\text { become pregnant; return to work may be considered if two consecutive } \mathrm{PbB} \\
\text { measurements are }<5 \mu \mathrm{g} / \mathrm{dL} \\
\text { - Continue } \mathrm{PbB} \text { monitoring as noted above } \\
\text { - Evaluate exposure, controls, and work practices }\end{array}$ \\
\hline$\geq 20$ & $\begin{array}{l}\text { - Remove from work if repeat } \mathrm{PbB} \text { measurement in } 4 \text { weeks is } \geq 20 \mu \mathrm{g} / \mathrm{dL} \text { or if single } \\
\mathrm{PbB} \text { measurement is } \geq 30 \mu \mathrm{g} / \mathrm{dL} \\
\text { - } \text { Monitor } \mathrm{PbB} \text { monthly; return to work after two consecutive monthly } \mathrm{PbB} \\
\text { measurements are }<15 \mu \mathrm{g} / \mathrm{dL} \\
\text { - Continue } \mathrm{PbB} \text { monitoring as noted above } \\
\text { - Evaluate exposure, controls, and work practices }\end{array}$ \\
\hline$\geq 30$ & $\begin{array}{l}\text { - } \text { Removed from exposure immediately } \\
\text { - } \text { Monitor PbB monthly; return to work after two consecutive monthly PbB } \\
\text { measurements are }<15 \mu \mathrm{g} / \mathrm{dL} \\
\text { - } \quad \text { Continue PbB monitoring as noted above } \\
\text { - Evaluate exposure, controls, and work practices }\end{array}$ \\
\hline
\end{tabular}

Source: Holland and Cawthon (2016)

\subsubsection{Reducing Body Burden}

$\mathrm{Pb}$ is initially distributed throughout the body and then redistributed to soft tissues and bone. In human adults and children, approximately 94 and $73 \%$ of the total body burden of $\mathrm{Pb}$ is found in bones, respectively. $\mathrm{Pb}$ may be stored in bone for long periods of time, but may be mobilized, thus achieving a steady state of intercompartmental distribution (see Section 3.3.2). 
Currently available methods to obviate the toxic effects of $\mathrm{Pb}$ are based on their ability to reduce the body burden of $\mathrm{Pb}$ by chelation. All of the chelating agents bind inorganic $\mathrm{Pb}$, enhance its excretion, and facilitate the transfer of $\mathrm{Pb}$ from soft tissues to the circulation where it can be excreted. Since the success of chelation therapy depends on excretion of chelated $\mathrm{Pb}$ via the kidney, caution should be used when treating a patient with renal failure. For all cases where chelation therapy is considered or implemented, medical providers should consult with a medical toxicologist or an expert in the medical management of Pb toxicity (CDC 2002a, 2012e). Chelation treatment should be administered in conjunction with meticulous supportive therapy (Calello and Henretig 2014). Most of the information below regarding chelators was obtained from Calello and Henretig (2014) and Kosnett (2005, 2007).

Several pharmacological substances are available for chelation therapy for $\mathrm{Pb}$ intoxication. Chelating agents currently in use are dimercaprol (British Anti-Lewisite, or BAL), CaNa2-EDTA (or EDTA), and 2,3-dimercaptosuccinic acid (DMSA; Succimer ${ }^{\circledR}$ ). Dosages and administration protocols for these agents vary with patient age, $\mathrm{PbB}$ level, and symptom types and severity. Specific treatment protocols should be developed in consultation with clinical experts in the management of $\mathrm{Pb}$ toxicity for the most current chelation therapy procedures for children and adults (CDC 2002a, 2012e).

Dimercaprol (BAL). The mechanism of action of BAL is through formation of stable chelate-metal compounds intra- and extracellularly. BAL is administered parenterally. The onset of action for BAL is 30 minutes. BAL increases fecal excretion of $\mathrm{Pb}$ as chelated $\mathrm{Pb}$ is excreted predominantly in bile within 4-6 hours; BAL also increases urinary excretion of chelated $\mathrm{Pb}$. A number of adverse reactions have been associated with BAL, including nausea, vomiting, hypertension, tachycardia, headache, increased secretions, anxiety, abdominal pain, and fever.

$\boldsymbol{C a N a}_{2}$-EDTA (or EDTA). EDTA works by forming a stable metal-chelate complex that is excreted by the kidney. It increases renal excretion of $\mathrm{Pb} 20-50$ times. EDTA is administered parenterally. Numerous adverse effects have been described due to treatment with EDTA including rash, fever, fatigue, thirst, myalgias, chills, and cardiac dysrhythmias. Since EDTA chelates zinc, patients with low zinc stores may be adversely affected by EDTA. Since EDTA also chelates other metals, administration of EDTA (or BAL) to persons occupationally exposed to cadmium may result in increased renal excretion of cadmium and renal damage. 
2,3-Dimercaptosuccinic acid (DMSA; Succimer $^{\circledR}$ ). The mechanism of action of DMSA is similar to BAL. DMSA is administered orally. DMSA has been shown to be as effective as EDTA in increasing the urinary excretion of $\mathrm{Pb}$. Minimal adverse effects that have been reported include anorexia, nausea, vomiting, and rashes. DMSA increases the excretion of zinc, but to a much lesser extent than other chelators, and has minimal effects on calcium, iron, magnesium, and copper. 


\section{CHAPTER 4. CHEMICAL AND PHYSICAL INFORMATION}

\subsection{CHEMICAL IDENTITY}

$\mathrm{Pb}$ is a naturally occurring element with an abundance of $0.0016 \%$ in the earth's crust (Davidson et al. 2014). It is a member of Group 14 (IVA) of the periodic table. Natural Pb is a mixture of four stable isotopes: ${ }^{204} \mathrm{~Pb}(1.4 \%),{ }^{206} \mathrm{~Pb}(24.1 \%),{ }^{207} \mathrm{~Pb}(22.1 \%)$, and ${ }^{208} \mathrm{~Pb}(52.4 \%)$. The $\mathrm{Pb}$ isotopes ${ }^{206} \mathrm{~Pb}$, ${ }^{207} \mathrm{~Pb}$, and ${ }^{208} \mathrm{~Pb}$ are the stable decay product of the naturally occurring decay series of uranium, actinium, and thorium, respectively (Haynes 2014).

$\mathrm{Pb}$ is found in concentrated and easily accessible $\mathrm{Pb}$ ore deposits that are widely distributed throughout the world (King et al. 2014). Its properties, such as corrosion resistance, density, and low melting point, make it a familiar metal in pipes, solder, weights, and storage batteries. The chemical identities of $\mathrm{Pb}$ and several of its compounds are provided in Table 4-1.

Table 4-1. Chemical Identity of Lead and Compounds

\begin{tabular}{|c|c|c|c|c|}
\hline Characteristic & Lead & Lead(II) acetate & Lead(II) azide & Lead(II) bromide \\
\hline $\begin{array}{l}\text { Synonym(s) and } \\
\text { registered trade } \\
\text { name(s) }\end{array}$ & $\begin{array}{l}\text { C.I. 77575; C.I. } \\
\text { Pigment metal 4; } \\
\text { Glover; Lead flake; } \\
\text { Lead S2; Omaha; } \\
\text { Omaha \& Grant; SI; } \\
\text { SO }^{\text {a }}\end{array}$ & $\begin{array}{l}\text { Acetic acid } \\
\text { lead(2+) salt }(2: 1) ; \\
\text { neutral lead } \\
\text { acetate; plumbous } \\
\text { acetate; normal } \\
\text { lead acetate; sugar } \\
\text { of lead; salt of } \\
\text { Saturn }\end{array}$ & Lead azide $^{\mathrm{b}}$ & $\begin{array}{l}\text { Lead bromide } \\
\left(\mathrm{PbBr}_{2}\right) ; \text { plumbous } \\
\text { bromide }^{\mathrm{b}}\end{array}$ \\
\hline Chemical formula & $\mathrm{Pb}^{\mathrm{b}}$ & $\mathrm{Pb}\left(\mathrm{CH}_{3} \mathrm{CO}_{2}\right)_{2}{ }^{\mathrm{b}}$ & $\mathrm{Pb}\left(\mathrm{N}_{3}\right)_{2}{ }^{\mathrm{b}}$ & $\mathrm{PbBr}_{2}{ }^{\mathrm{b}}$ \\
\hline Chemical structure & Not applicable & Not applicable & Not applicable & Not applicable \\
\hline $\begin{array}{l}\text { CAS Registry } \\
\text { Number }\end{array}$ & $7439-92-1^{b}$ & $301-04-2^{b}$ & $13424-46-9^{b}$ & $10031-22-8^{b}$ \\
\hline
\end{tabular}


Table 4-1. Chemical Identity of Lead and Compounds

\begin{tabular}{|c|c|c|c|c|}
\hline Characteristic & Lead(II) chloride & Lead(II) chromate & $\begin{array}{l}\text { Lead(II) } \\
\text { tetrafluoroborate }^{c}\end{array}$ & Lead(II) iodide \\
\hline $\begin{array}{l}\text { Synonym(s) and } \\
\text { registered trade } \\
\text { name(s) }\end{array}$ & $\begin{array}{l}\text { Lead chloride } \\
\left(\mathrm{PbCl}_{2}\right) ; \text { Lead(2+) } \\
\text { chloride; Plumbous } \\
\text { chloride }^{\text {b }}\end{array}$ & $\begin{array}{l}\text { Chromic acid } \\
\left(\mathrm{H}_{2} \mathrm{CrO}_{4} \text { lead }(2+)\right. \\
\text { salt (1:1); Chrome } \\
\text { yellow; Cologne } \\
\text { yellow; King's } \\
\text { yellow; Leipzig } \\
\text { yellow; Paris } \\
\text { yellow; C.I. } \\
\text { Pigment Yellow 34; } \\
\text { lead chromium } \\
\text { oxide (PbCrO } 4) ; \\
\text { plumbous } \\
\text { chromate; C.I. } \\
77600^{b}\end{array}$ & $\begin{array}{l}\text { Tetrafluoro } \\
\text { borate(1-) } \\
\text { Lead(2+) }\end{array}$ & $\begin{array}{l}\text { Lead iodide }\left(\mathrm{Pbl}_{2}\right) \\
\text { Plumbous iodide }\end{array}$ \\
\hline Chemical formula & $\mathrm{PbCl}_{2}{ }^{\mathrm{b}}$ & $\mathrm{PbCrO}_{4}^{\mathrm{b}}$ & $\mathrm{Pb}\left(\mathrm{BF}_{4}\right)_{2}{ }^{\mathrm{a}}$ & $\mathrm{Pbl}_{2}{ }^{\mathrm{b}}$ \\
\hline Chemical structure & Not applicable & Not applicable & Not applicable & Not applicable \\
\hline $\begin{array}{l}\text { CAS Registry } \\
\text { Number }\end{array}$ & $7758-95-4^{b}$ & $7758-97-6^{b}$ & $13814-96-5^{a}$ & $10101-63-0^{b}$ \\
\hline
\end{tabular}


Table 4-1. Chemical Identity of Lead and Compounds

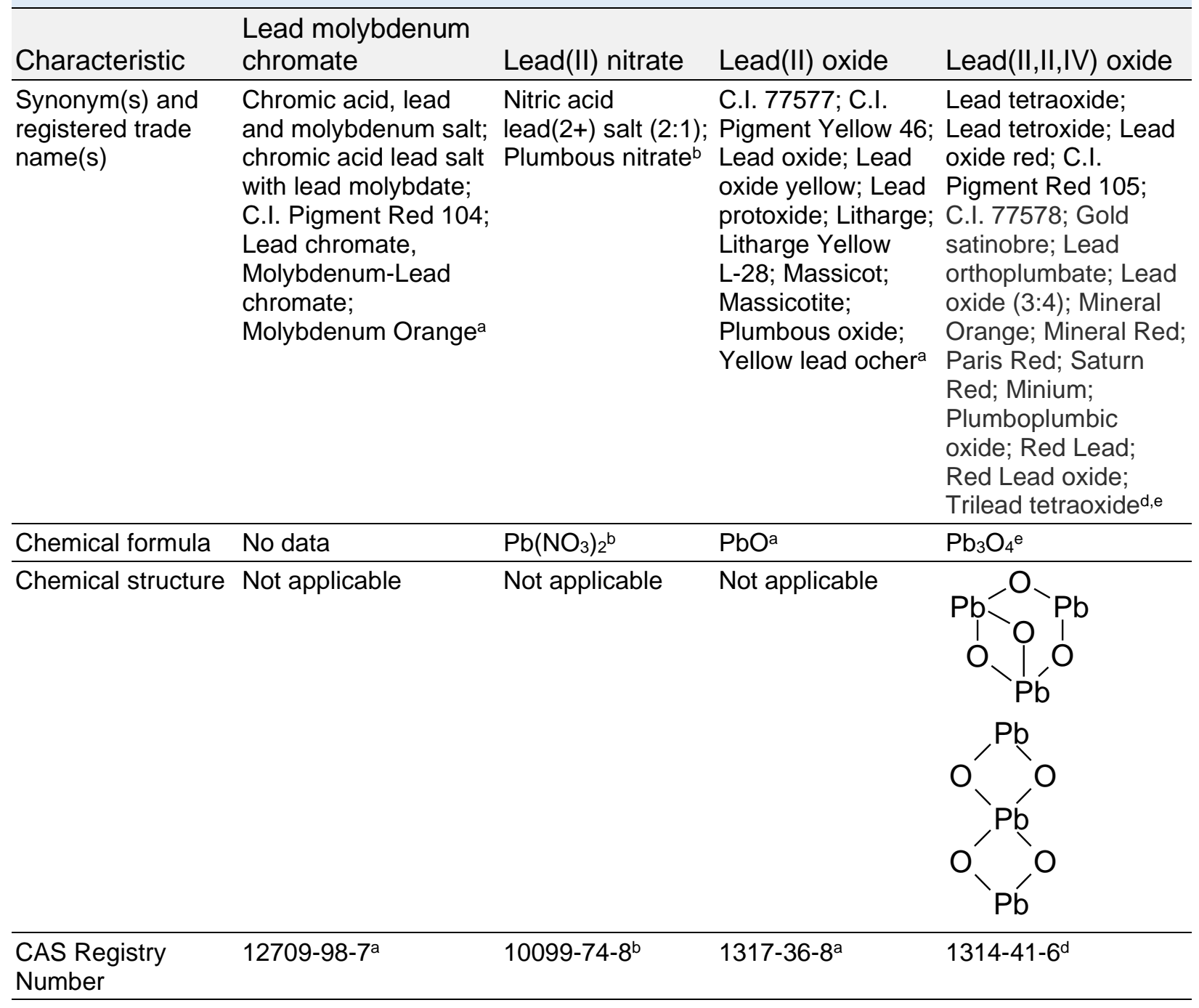


Table 4-1. Chemical Identity of Lead and Compounds

\begin{tabular}{|c|c|c|c|}
\hline Characteristic & Lead(II) phosphate & Lead(II) styphnate & Lead(II) sulfate \\
\hline $\begin{array}{l}\text { Synonym(s) and } \\
\text { registered trade } \\
\text { name(s) }\end{array}$ & $\begin{array}{l}\text { C.I. 77622; Lead } \\
\text { orthophosphate; Lead } \\
\text { phosphate (3:2); } \\
\text { Lead(2+) phosphate; } \\
\text { normal lead } \\
\text { orthophosphate; } \\
\text { Phosphoric acid, } \\
\text { lead(2+) salt (2:3); } \\
\text { Plumbous phosphate; } \\
\text { Trilead phosphate }\end{array}$ & Lead trinitroresorcinate & $\begin{array}{l}\text { Anglesite; C.I. 77630; } \\
\text { C.I. Pigment White 3; } \\
\text { Fast White; } \\
\text { Freemans White } \\
\text { Lead; Lead bottoms; } \\
\text { Milk white; Mulhouse } \\
\text { White; Sulfuric acid, } \\
\text { lead(2+) salt }(1: 1)^{\mathrm{a}}\end{array}$ \\
\hline Chemical formula & $\mathrm{Pb}_{3}\left(\mathrm{PO}_{4}\right)_{2}^{\mathrm{a}}$ & $\mathrm{Pb}\left(\mathrm{C}_{6} \mathrm{HN}_{3} \mathrm{O}_{8}\right)_{2}{ }^{\dagger}$ & $\mathrm{PbSO}_{4}{ }^{\mathrm{b}}$ \\
\hline Chemical structure & Not applicable & Not applicable & Not applicable \\
\hline $\begin{array}{l}\text { CAS Registry } \\
\text { Number }\end{array}$ & $7446-27-7^{a}$ & $15245-44-0^{f}$ & $7446-14-2^{b}$ \\
\hline Characteristic & Lead(II) sulfide & Tetraethyl lead & Lead(II) carbonate \\
\hline $\begin{array}{l}\text { Synonym(s) and } \\
\text { registered trade } \\
\text { name(s) }\end{array}$ & $\begin{array}{l}\text { C.I. 77640; Galena; } \\
\text { Natural lead sulfide; } \\
\text { Plumbous sulfide }\end{array}$ & $\begin{array}{l}\text { Tetraethylplumbane; Lead tetraethyl; } \\
\text { TEL }^{\text {b }}\end{array}$ & $\begin{array}{l}\text { Carbonic acid, } \\
\text { lead( } 2+) \text { salt }(1: 1) ; \\
\text { Cerussite; Dibasic } \\
\text { lead carbonate; } \\
\text { Lead }(2+) \text { carbonate; } \\
\text { White lead }{ }^{\mathrm{a}}\end{array}$ \\
\hline Chemical formula & $\mathrm{PbS}^{\mathrm{a}}$ & $\mathrm{Pb}\left(\mathrm{C}_{2} \mathrm{H}_{5}\right)_{4}^{\mathrm{a}}$ & $\mathrm{PbCO}_{3}{ }^{\mathrm{a}}$ \\
\hline Chemical structure & Not applicable & & Not applicable \\
\hline $\begin{array}{l}\text { CAS Registry } \\
\text { Number }\end{array}$ & $1314-87-0^{a}$ & $78-00-2^{b}$ & $598-63-0^{a}$ \\
\hline
\end{tabular}

aLewis 2012.

bO'Neil et al. 2013.

'Stable only in aqueous solution (Haynes 2014).

dNLM 2020.

eHaynes 2014.

fBoileau et al. 2012.

CAS $=$ Chemical Abstracts Services

\subsection{PHYSICAL AND CHEMICAL PROPERTIES}

$\mathrm{Pb}$, a blueish-white metal with bright luster, is very soft, highly malleable, ductile, a poor conductor of electricity, and is very resistant to corrosion (Haynes 2014). A clean Pb surface will not be attacked by dry air; however, in moist air, the surface will react and become coated with a layer of lead(II) oxide (PbO). This coating may be hydrated and combine with carbon dioxide to form lead(II) carbonate $\left(\mathrm{PbCO}_{3}\right)$ (Carr et al. 2004). This protective coating of insoluble $\mathrm{Pb}$ compounds slows or halts corrosion of the underlying metal. $\mathrm{Pb}$ is rarely found in its metallic form in nature and commonly occurs as a 
mineral with sulfur or oxygen. The most important $\mathrm{Pb}$ mineral is galena ( $\mathrm{PbS})$. Other common $\mathrm{Pb}$-containing minerals include anglesite $\left(\mathrm{PbSO}_{4}\right)$, cerussite $\left(\mathrm{PbCO}_{3}\right)$, and minium $\left(\mathrm{Pb}_{3} \mathrm{O}_{4}\right)(\mathrm{Carr}$ et al. 2004; Davidson et al. 2014; Haynes 2014).

$\mathrm{Pb}$ can exist in the 0 oxidation state in metallic $\mathrm{Pb}$ and in compounds as the +2 or +4 oxidation states. In the environment, $\mathrm{Pb}$ is primarily found in the +2 state in inorganic compounds. The chemistry of inorganic $\mathrm{Pb}$ compounds is generally similar to that of the Group 2(II) or alkaline earth metals. There are three common oxides of $\mathrm{Pb}$ : lead(II) oxide (PbO); lead(II,IV) oxide or lead tetroxide $\left(\mathrm{Pb}_{3} \mathrm{O}_{4}\right)$; and lead(IV) oxide or lead dioxide $\left(\mathrm{PbO}_{2}\right)$. The +4 state is only formed under strongly oxidizing conditions. Inorganic $\mathrm{Pb}(+4)$ compounds are relatively unstable and would not be expected to be found under ordinary environmental conditions. $\mathrm{Pb}$ is amphoteric, meaning that it can react with acids and bases. In acid, $\mathrm{Pb}$ forms $\mathrm{Pb}(+2)$ (plumbous) and $\mathrm{Pb}(+4)$ (plumbic) salts and in basic solution, it forms plumbites $\left(\mathrm{PbO}_{2}{ }^{2-}\right)$ and plumbates $\left(\mathrm{Pb}(\mathrm{OH})_{6}{ }^{2-}\right)$ (Carr et al. 2004). In organolead compounds, $\mathrm{Pb}$ is typically in the tetravalent (+4) oxidation state (Carr et al. 2004; Haynes 2014).

Data on the physical and chemical properties of $\mathrm{Pb}$ and several of its compounds are provided in Table 4-2. 
Table 4-2. Physical and Chemical Properties of Lead and Compounds

\begin{tabular}{|c|c|c|c|c|}
\hline Property & Lead & Lead(II) acetate & Lead(II) azide & Lead(II) bromide \\
\hline Molecular weight & $207.2^{\mathrm{a}}$ & $325.3^{\mathrm{b}}$ & $291.24^{a}$ & $367.0^{\mathrm{b}}$ \\
\hline Color & $\begin{array}{l}\text { Bluish-white, } \\
\text { silvery, gray metala }\end{array}$ & White crystals ${ }^{b}$ & $\begin{array}{l}\text { Needles or white } \\
\text { powder a }\end{array}$ & $\begin{array}{l}\text { White orthorhombic } \\
\text { crystals }^{\mathrm{b}}\end{array}$ \\
\hline Physical state & Solid & Solid & Solid & Solid \\
\hline Melting point & $327.4^{\circ} \mathrm{C}^{\mathrm{a}}$ & $280^{\circ} \mathrm{C}^{\mathrm{b}}$ & $\begin{array}{l}\text { Decomposes at } \\
190^{\circ} \mathrm{C}^{\mathrm{c}}\end{array}$ & $371^{\circ} \mathrm{C}^{\mathrm{b}}$ \\
\hline Boiling point & $1,740^{\circ} \mathrm{C}^{\mathrm{a}}$ & Decomposes $^{b}$ & No data & $892^{\circ} \mathrm{C}^{\mathrm{b}}$ \\
\hline Density & $\begin{array}{l}11.34 \mathrm{~g} / \mathrm{cm}^{3} \text { at } \\
20^{\circ} \mathrm{C}^{\mathrm{a}}\end{array}$ & $3.25 \mathrm{~g} / \mathrm{cm}^{3 \mathrm{~b}}$ & $\begin{array}{l}4.17 \mathrm{~g} / \mathrm{cm}^{3} \text { at } \\
20^{\circ} \mathrm{C}^{\mathrm{c}}\end{array}$ & $6.69 \mathrm{~g} / \mathrm{cm}^{3 \mathrm{~b}}$ \\
\hline Odor & No data & $\begin{array}{l}\text { Slightly acetic odor } \\
\text { (trihydrate) }^{\mathrm{a}}\end{array}$ & No data & No data \\
\hline \multicolumn{5}{|l|}{ Odor threshold: } \\
\hline Water & No data & No data & No data & No data \\
\hline Air & No data & No data & No data & No data \\
\hline \multicolumn{5}{|l|}{ Solubility: } \\
\hline Water & Insoluble ${ }^{d}$ & $\begin{array}{l}443,000 \mathrm{mg} / \mathrm{L} \text { at } \\
20^{\circ} \mathrm{C}^{\mathrm{b}}\end{array}$ & $230 \mathrm{mg} / \mathrm{L}$ at $18^{\circ} \mathrm{C}^{\mathrm{a}}$ & $9,750 \mathrm{mg} / \mathrm{L}$ at $25^{\circ} \mathrm{C}^{\mathrm{b}}$ \\
\hline Acids & $\begin{array}{l}\text { Soluble in dilute } \\
\text { nitric acidd; reacts } \\
\text { with sulfuric acid }\end{array}$ & Soluble in acide & $\begin{array}{l}\text { Freely soluble in } \\
\text { acetic acida }\end{array}$ & No data \\
\hline Bases & No data & Soluble in alkalie & No data & No data \\
\hline Organic solvents & $\begin{array}{l}\text { Soluble in glycerin; } \\
\text { slightly soluble in } \\
\text { alcohole }\end{array}$ & $\begin{array}{l}\text { Slightly soluble in } \\
\text { alcohol; freely } \\
\text { soluble in glycerol }\end{array}$ & No data & Insoluble in alcohol ${ }^{b}$ \\
\hline \multicolumn{5}{|c|}{ Partition coefficients: } \\
\hline Log $K_{o w}$ & No data & No data & No data & No data \\
\hline Log $K_{o c}$ & No data & No data & No data & No data \\
\hline Vapor pressure & $\begin{array}{l}1.77 \mathrm{mmHg} \text { at } \\
1,000^{\circ} \mathrm{C}^{\mathrm{a}}\end{array}$ & No data & No data & $0.0075 \mathrm{mmHg}$ at $374^{\circ} \mathrm{C}^{\mathrm{b}}$ \\
\hline $\begin{array}{l}\text { Henry's law } \\
\text { constant }\end{array}$ & No data & No data & No data & No data \\
\hline $\begin{array}{l}\text { Autoignition } \\
\text { temperature }\end{array}$ & No data & No data & No data & No data \\
\hline Flashpoint & No data & No data & No data & No data \\
\hline Flammability limits & No data & No data & No data & No data \\
\hline Conversion factors & Not relevant ${ }^{f}$ & Not relevant ${ }^{f}$ & Not relevant ${ }^{f}$ & Not relevant ${ }^{f}$ \\
\hline Explosive limits & No data & No data & $\begin{array}{l}\text { Explodes at } \\
350^{\circ} \mathrm{C}^{\mathrm{a}}\end{array}$ & No data \\
\hline Valence state & 0 & +2 & +2 & +2 \\
\hline
\end{tabular}


Table 4-2. Physical and Chemical Properties of Lead and Compounds

\begin{tabular}{|c|c|c|c|c|}
\hline Property & Lead(II) chloride & Lead(II) chromate & $\begin{array}{l}\text { Lead(II) } \\
\text { tetrafluoroborate }\end{array}$ & Lead iodide \\
\hline Molecular weight & $278.1^{9}$ & $323.19^{a}$ & $380.8^{\mathrm{b}}$ & $461.05^{9}$ \\
\hline Color & $\begin{array}{l}\text { White, orthorhombic } \\
\text { needles }{ }^{g}\end{array}$ & $\begin{array}{l}\text { Yellow or orange- } \\
\text { yellow powdera }\end{array}$ & No data & $\begin{array}{l}\text { Yellow } \\
\text { hexagonal } \\
\text { crystalsg }\end{array}$ \\
\hline Physical state & Solid & Solid & $\begin{array}{l}\text { Stable only in } \\
\text { aqueous solution }\end{array}$ & Solid \\
\hline Melting point & $501^{\circ} \mathrm{C}^{g}$ & $844^{\circ} \mathrm{C}^{\mathrm{a}}$ & No data & $402^{\circ} \mathrm{C}^{g}$ \\
\hline Boiling point & $950^{\circ} \mathrm{C}^{\mathrm{g}}$ & No data & No data & $954^{\circ} \mathrm{C}^{\mathrm{g}}$ \\
\hline Density & $5.85 \mathrm{~g} / \mathrm{cm}^{3 \mathrm{~g}}$ & $6.12 \mathrm{~g} / \mathrm{cm}^{3 \mathrm{~b}}$ & No data & $6.16 \mathrm{~g} / \mathrm{cm}^{3 \mathrm{~g}}$ \\
\hline Odor & No data & No data & No data & No data \\
\hline Odor threshold & No data & No data & No data & No data \\
\hline \multicolumn{5}{|l|}{ Solubility: } \\
\hline Water & $9,900 \mathrm{mg} / \mathrm{L}$ at $20^{\circ} \mathrm{Cg}$ & $0.2 \mathrm{mg} / \mathrm{L}^{\mathrm{a}}$ & Soluble ${ }^{b}$ & $\begin{array}{l}630 \mathrm{mg} / \mathrm{L} \text { at } \\
20^{\circ} \mathrm{C}^{\mathrm{g}}\end{array}$ \\
\hline Acids & $\begin{array}{l}\text { Slightly soluble in } \\
\text { dilute hydrochloric } \\
\text { acidg }\end{array}$ & $\begin{array}{l}\text { Soluble in dilute } \\
\text { nitric acid; insoluble } \\
\text { in acetic acida }\end{array}$ & No data & No data \\
\hline Bases & $\begin{array}{l}\text { Slightly soluble in } \\
\text { dilute ammoniag }\end{array}$ & No data & No data & No data \\
\hline Organic solvents & Insoluble in alcoholg & No data & No data & $\begin{array}{l}\text { Insoluble in } \\
\text { alcoholg }\end{array}$ \\
\hline \multicolumn{5}{|l|}{ Partition coefficients: } \\
\hline Log $K_{\text {ow }}$ & No data & No data & No data & No data \\
\hline Log Koc & No data & No data & No data & No data \\
\hline Vapor pressure & $7.5 \mathrm{mmHg}$ at $637^{\circ} \mathrm{C}^{b}$ & No data & No data & $\begin{array}{l}0.75 \mathrm{mmHg} \text { at } \\
470^{\circ} \mathrm{C}^{\mathrm{b}}\end{array}$ \\
\hline $\begin{array}{l}\text { Henry's law } \\
\text { constant }\end{array}$ & No data & No data & No data & No data \\
\hline $\begin{array}{l}\text { Autoignition } \\
\text { temperature }\end{array}$ & No data & No data & No data & No data \\
\hline Flashpoint & No data & No data & No data & No data \\
\hline Flammability limits & No data & No data & No data & No data \\
\hline Conversion factors & Not relevant ${ }^{f}$ & Not relevant ${ }^{f}$ & Not relevant ${ }^{f}$ & Not relevant ${ }^{f}$ \\
\hline Explosive limits & No data & No data & No data & No data \\
\hline Valence state & +2 & +2 & +2 & +2 \\
\hline
\end{tabular}


Table 4-2. Physical and Chemical Properties of Lead and Compounds

\begin{tabular}{|c|c|c|c|c|}
\hline Property & $\begin{array}{l}\text { Lead molybdenum } \\
\text { chromate }\end{array}$ & Lead(II) nitrate & Lead(II) oxide & Lead(II,II,IV) oxide \\
\hline Molecular weight & No data & 331.239 & 223.219 & $685.57 \mathrm{e}$ \\
\hline Color & No data & $\begin{array}{l}\text { Cubic or monoclinic } \\
\text { colorless crystalsg }\end{array}$ & $\begin{array}{l}\text { Reddish-yellow; } \\
\text { yellow (above } \\
\left.489^{\circ} \mathrm{C}\right)^{\mathrm{g}}\end{array}$ & $\begin{array}{l}\text { Bright red heavy } \\
\text { powdera; red } \\
\text { tetrahedral crystalsb }\end{array}$ \\
\hline Physical state & No data & Solid & Solid & Solid \\
\hline Melting point & No data & $\begin{array}{l}\text { Begins to } \\
\text { decompose above } \\
205^{\circ} \mathrm{Cg}\end{array}$ & $\begin{array}{l}897^{\circ} \mathrm{C} \text { (begins to } \\
\text { sublime before } \\
\text { melting) }\end{array}$ & $830^{\circ} \mathrm{C}^{\mathrm{b}} ; 500^{\circ} \mathrm{C}^{\mathrm{e}}$ \\
\hline Boiling point & No data & No data & $\begin{array}{l}\text { Decomposes at } \\
1,472^{\circ} \mathrm{Cg}\end{array}$ & $\begin{array}{l}\text { Decomposes between } \\
500-530^{\circ} \mathrm{C}^{d}\end{array}$ \\
\hline Density & No data & $4.53 \mathrm{~g} / \mathrm{cm}^{3 \mathrm{~g}}$ & $\begin{array}{l}9.53 \mathrm{~g} / \mathrm{cm}^{3} \\
(\text { Litharge })^{\mathrm{g}} \\
9.6 \\
\mathrm{~g} / \mathrm{cm}^{3} \text { (Massicot) }{ }^{\mathrm{g}}\end{array}$ & $\begin{array}{l}8.92 \mathrm{~g} / \mathrm{cm}^{3 \mathrm{~b}} \\
9.1 \mathrm{~g} / \mathrm{cm}^{3 \mathrm{e}}\end{array}$ \\
\hline Odor & No data & No data & No data & No data \\
\hline Odor threshold: & No data & No data & No data & No data \\
\hline \multicolumn{5}{|l|}{ Solubility: } \\
\hline Water & No data & $\begin{array}{l}56: 5 \mathrm{~g} / 100 \mathrm{~mL} \text { at } \\
20^{\circ} \mathrm{C}^{\mathrm{g}}\end{array}$ & $\begin{array}{l}50.4 \mathrm{mg} / \mathrm{L} \text { at } 25^{\circ} \mathrm{C} \\
\left(\text { Litharge) }{ }^{\mathrm{g}} ;\right. \\
106.5 \mathrm{mg} / \mathrm{L} \text { at } \\
25^{\circ} \mathrm{C} \text { (Massicot) }{ }^{\mathrm{g}}\end{array}$ & Insoluble in water ${ }^{d}$ \\
\hline Acid & No data & $\begin{array}{l}\text { Insoluble in } \\
\text { concentrated nitric } \\
\text { acid }^{\mathrm{a}}\end{array}$ & Solubleg & $\begin{array}{l}\text { Dissolves in acetic } \\
\text { acid or hot } \\
\text { hydrochloric acid }{ }^{b, g}\end{array}$ \\
\hline Base & No data & $\begin{array}{l}\text { Soluble in alkali and } \\
\text { ammoniag }\end{array}$ & Solubleg & No data \\
\hline Organic solvents & No data & $\begin{array}{l}87.7 \mathrm{mg} / \mathrm{L}(43 \% \\
\text { aqueous ethanol) at } \\
22^{\circ} \mathrm{C}^{g}\end{array}$ & $\begin{array}{l}\text { Insoluble in } \\
\text { alcohola }^{\text {a }}\end{array}$ & Insoluble in alcoholg \\
\hline \multicolumn{5}{|c|}{ Partition coefficients: } \\
\hline Log Kow & No data & No data & No data & No data \\
\hline Log $K_{o c}$ & No data & No data & No data & No data \\
\hline Vapor pressure & No data & No data & $\begin{array}{l}0.0075 \mathrm{mmHg} \text { at } \\
724^{\circ} \mathrm{C}^{\mathrm{b}}\end{array}$ & No data \\
\hline $\begin{array}{l}\text { Henry's law } \\
\text { constant }\end{array}$ & No data & No data & No data & No data \\
\hline $\begin{array}{l}\text { Autoignition } \\
\text { temperature }\end{array}$ & No data & No data & No data & No data \\
\hline Flashpoint & No data & No data & No data & No data \\
\hline Flammability limits & No data & No data & No data & No data \\
\hline Conversion factors & Not relevant ${ }^{f}$ & Not relevant ${ }^{f}$ & Not relevant ${ }^{f}$ & Not relevant ${ }^{f}$ \\
\hline Explosive limits & No data & No data & No data & No data \\
\hline Valence state & +2 & +2 & +2 & $+2,+2,+4$ \\
\hline
\end{tabular}


Table 4-2. Physical and Chemical Properties of Lead and Compounds

\begin{tabular}{|c|c|c|c|}
\hline Property & Lead(II) phosphate & Lead(II) styphnate & Lead(II) sulfate \\
\hline Molecular weight & $811.54^{\mathrm{a}}$ & $450.29^{\mathrm{h}}$ & $303.25^{g}$ \\
\hline Color & White powdera & $\begin{array}{l}\text { Monoclinic orange-yellow } \\
\text { crystal (monohydrate) }\end{array}$ & $\begin{array}{l}\text { White, heavy, crystalline } \\
\text { powdera }^{2}\end{array}$ \\
\hline Physical state & Solid & Solid & Solid \\
\hline Melting point & $1,014^{\circ} \mathrm{C}^{\mathrm{a}}$ & No data & $1,170^{\circ} \mathrm{C}^{\mathrm{g}}$ \\
\hline Boiling point & No data & No data & No data \\
\hline Density & $6.9 \mathrm{~g} / \mathrm{cm}^{3 a}$ & $\begin{array}{l}3.1 \mathrm{~g} / \mathrm{cm}^{3} \text { (monohydrate); } \\
2.9 \mathrm{~g} / \mathrm{cm}^{3} \text { (anhydrous) }\end{array}$ & $6.2 \mathrm{~g} / \mathrm{cm}^{3 \mathrm{~g}}$ \\
\hline Odor & No data & No data & No data \\
\hline Odor threshold: & No data & No data & No data \\
\hline \multicolumn{4}{|l|}{ Solubility: } \\
\hline Water & Insoluble & Insoluble & $42.5 \mathrm{mg} / \mathrm{L}$ at $25^{\circ} \mathrm{Cg}$ \\
\hline Acid & Soluble in nitric acida & No data & Soluble in concentrated acids 9 \\
\hline Base & $\begin{array}{l}\text { Soluble in fixed alkali } \\
\text { hydroxides }\end{array}$ & No data & Soluble in alkalies ${ }^{g}$ \\
\hline Organic solvents & Insoluble in alcohola & No data & Insoluble in alcohola \\
\hline \multicolumn{4}{|c|}{ Partition coefficients: } \\
\hline Log Kow & No data & No data & No data \\
\hline $\log K_{o c}$ & No data & No data & No data \\
\hline Vapor pressure & No data & No data & No data \\
\hline $\begin{array}{l}\text { Henry's law } \\
\text { constant }\end{array}$ & No data & No data & No data \\
\hline $\begin{array}{l}\text { Autoignition } \\
\text { temperature }\end{array}$ & No data & No data & No data \\
\hline Flashpoint & No data & No data & No data \\
\hline Flammability limits & No data & No data & No data \\
\hline Conversion factors & Not relevant $^{f}$ & Not relevant $^{f}$ & Not relevant $^{f}$ \\
\hline Explosive limits & No data & Detonates at $260^{\circ} \mathrm{C}^{b}$ & No data \\
\hline Valence state & +2 & +2 & +2 \\
\hline
\end{tabular}


Table 4-2. Physical and Chemical Properties of Lead and Compounds

\begin{tabular}{|c|c|c|c|}
\hline Property & Lead(II) sulfide & Tetraethyl lead & Lead(II) carbonate \\
\hline Molecular weight & $239.25^{9}$ & $323.45^{\mathrm{a}}$ & $267.22^{\mathrm{g}}$ \\
\hline Color & $\begin{array}{l}\text { Metallic black cubic } \\
\text { crystals }^{\mathrm{g}}\end{array}$ & Colorless $^{a}$ & Colorless rhombic crystalsg \\
\hline Physical state & Solid & Liquida $^{a}$ & Solid \\
\hline Melting point & $1,114^{\circ} \mathrm{C}^{\mathrm{d}}$ & No data & $315^{\circ} \mathrm{C}$ (decomposes) ${ }^{g}$ \\
\hline Boiling point & Sublimes at $1,281^{\circ} \mathrm{C}^{\mathrm{d}}$ & $\begin{array}{l}200^{\circ} \mathrm{C} ; 227.7^{\circ} \mathrm{C} \text { (with } \\
\text { decomposition) }^{\mathrm{a}}\end{array}$ & No data \\
\hline Density & $7.57-7.59 \mathrm{~g} / \mathrm{cm}^{3 \mathrm{~g}}$ & $1.653 \mathrm{~g} / \mathrm{cm}^{3 a}$ & $6.6 \mathrm{~g} / \mathrm{cm}^{3 \mathrm{~g}}$ \\
\hline Odor & No data & No data & No data \\
\hline Odor threshold: & No data & No data & No data \\
\hline \multicolumn{4}{|l|}{ Solubility: } \\
\hline Water & $124.4 \mathrm{mg} / \mathrm{L} 20^{\circ} \mathrm{Cg}$ & $0.29 \mathrm{mg} / \mathrm{L}^{\mathrm{i}}$ & $1.1 \mathrm{mg} / \mathrm{L}$ at $20^{\circ} \mathrm{C}^{g}$ \\
\hline Acid & Soluble in nitric acidg & No data & Soluble ${ }^{g}$ \\
\hline Base & Insoluble in alkalies ${ }^{d}$ & No data & $\begin{array}{l}\text { Soluble in alkalies; insoluble in } \\
\text { ammoniag }^{9}\end{array}$ \\
\hline Organic solvents & Insoluble in alcohola & $\begin{array}{l}\text { Soluble in benzene, } \\
\text { petroleum ether, gasoline; } \\
\text { slightly soluble in } \text { alcohola }^{a}\end{array}$ & Insoluble in alcoholg \\
\hline \multicolumn{4}{|l|}{ Partition coefficients: } \\
\hline Log Kow & No data & $4.15^{j}$ & No data \\
\hline $\log K_{o c}$ & No data & No data & No data \\
\hline Vapor pressure & $\begin{array}{l}0.0075 \mathrm{mmHg} \text { at } \\
705^{\circ} \mathrm{C}^{\mathrm{b}}\end{array}$ & $0.26 \mathrm{mmHg}$ at $25^{\circ} \mathrm{C}^{\mathrm{j}}$ & No data \\
\hline $\begin{array}{l}\text { Henry's law } \\
\text { constant }\end{array}$ & No data & No data & No data \\
\hline $\begin{array}{l}\text { Autoignition } \\
\text { temperature }\end{array}$ & No data & No data & No data \\
\hline Flashpoint & No data & $200^{\circ} \mathrm{F}\left(93^{\circ} \mathrm{C}\right)(\text { closed cup })^{\mathrm{k}}$ & No data \\
\hline Flammability limits & No data & $\begin{array}{l}\text { Lower flammable limit: } \\
1.8 \% \text { by volumek }\end{array}$ & No data \\
\hline Conversion factors & Not relevant ${ }^{f}$ & No data & Not relevant ${ }^{f}$ \\
\hline Explosive limits & No data & No data & No data \\
\hline Valence state & +2 & +4 & +2 \\
\hline
\end{tabular}

aO'Neil et al. 2013.

bHaynes 2014.

cAkhavan 2004.

dLarrañaga et al. 2016.

e Jacob 2012.

fSince these compounds exist in the atmosphere in the particulate state, their concentrations are expressed as $\mu \mathrm{g} / \mathrm{m}^{3}$ only.

${ }^{\mathrm{g}}$ Carr et al. 2004.

hMolecular weight calculated from atomic weights.

'Feldhake and Stevens 1963.

jWang et al. 1996.

kNFPA 2002. 


\section{CHAPTER 5. POTENTIAL FOR HUMAN EXPOSURE}

\subsection{OVERVIEW}

$\mathrm{Pb}$ and $\mathrm{Pb}$ compounds have been identified in at least 1,287 and 46 sites, respectively, of the 1,867 hazardous waste sites that have been proposed for inclusion on the EPA National Priorities List (NPL) (ATSDR 2019). However, the number of sites evaluated for $\mathrm{Pb}$ is not known. The number of sites in each state is shown in Figures 5-1 and 5-2, respectively. Of these 1,287 sites for $\mathrm{Pb}, 1,273$ are located within the United States, 2 are located in the Virgin Islands, 2 are located in Guam, and 10 are located in Puerto Rico (not shown). All the sites for Pb compounds are only in the United States.

Figure 5-1. Number of NPL Sites with Lead Contamination

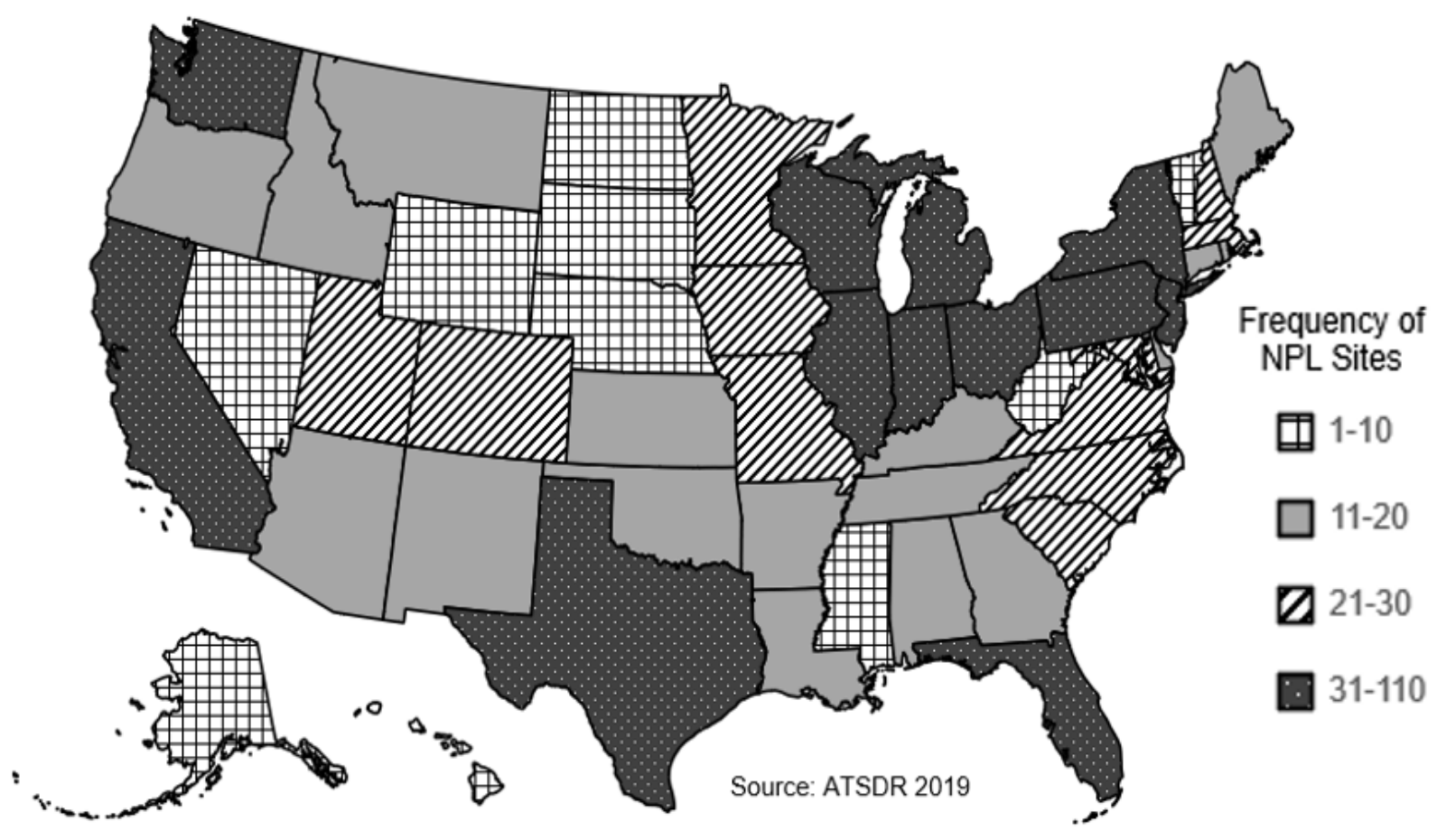


Figure 5-2. Number of NPL Sites with Lead Compound Contamination

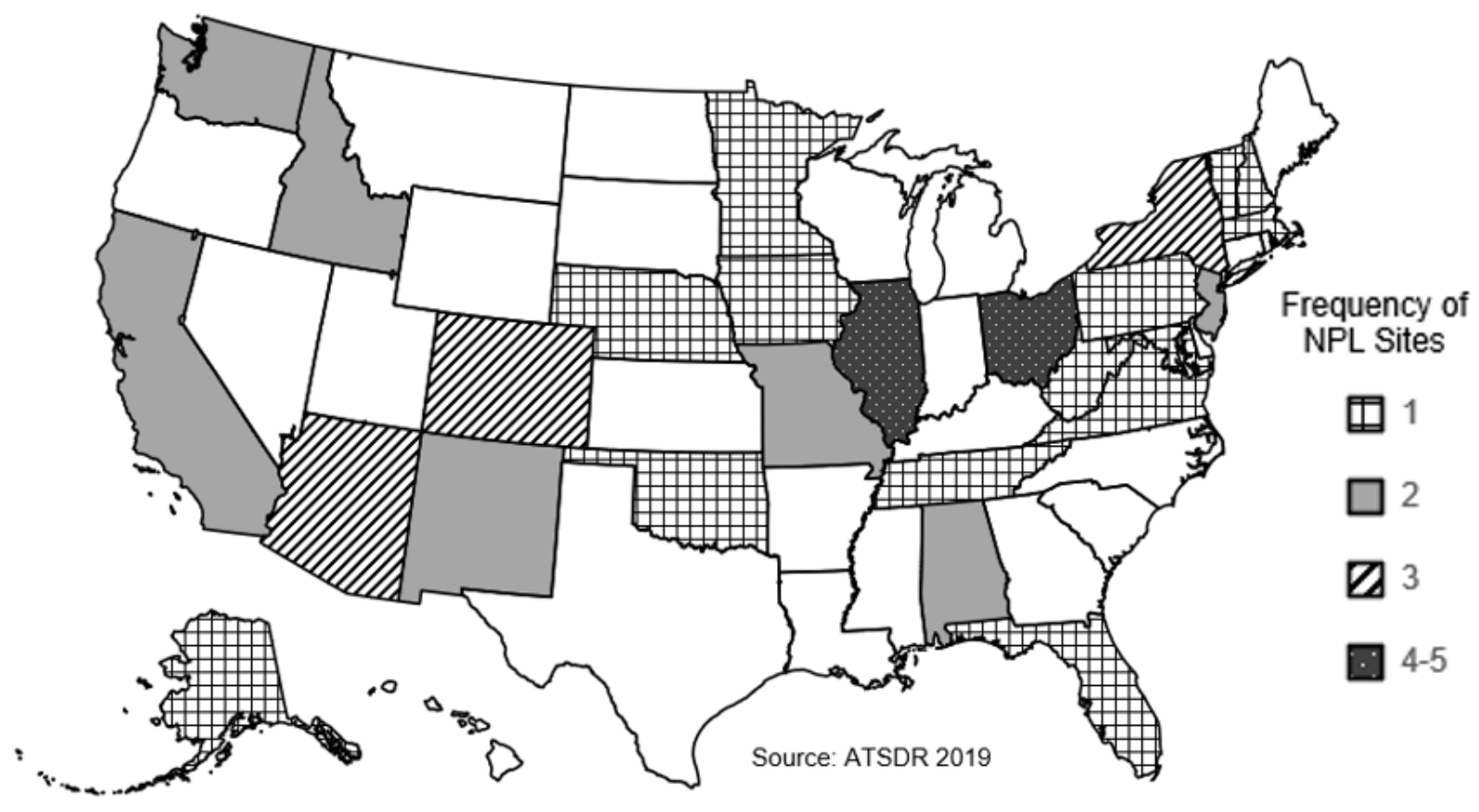

- $\quad \mathrm{Pb}$ is an element found in concentrated and easily accessible $\mathrm{Pb}$ ore deposits that are widely distributed throughout the world.

- The general population may be exposed to $\mathrm{Pb}$ in ambient air, foods, drinking water, soil, and dust. For adults, exposure to levels of $\mathrm{Pb}$ beyond background are usually associated with occupational exposures.

- For children, exposure to high levels of $\mathrm{Pb}$ are associated with living in areas contaminated by $\mathrm{Pb}$ (e.g., soil or indoor dust in older homes with Pb paint). Exposure usually occurs by hand-tomouth activities.

- As an element, $\mathrm{Pb}$ does not degrade. However, particulate matter contaminated with $\mathrm{Pb}$ can move through air, water, and soil.

- Atmospheric deposition is the largest source of $\mathrm{Pb}$ found in soils. $\mathrm{Pb}$ is transferred continuously between air, water, and soil by natural chemical and physical processes such as weathering, runoff, precipitation, dry deposition of dust, and stream/river flow; however, soil and sediments appear to be important sinks for $\mathrm{Pb}$.

- $\quad \mathrm{Pb}$ adsorbs strongly to most soils, which limits the rate of leaching of $\mathrm{Pb}$ from soil. Soil acidity $(\mathrm{pH})$ is the most important factor affecting solubility, mobility, and phytoavailability of $\mathrm{Pb}$ in soil. 
Other conditions that increase $\mathrm{Pb}$ mobility in soils are reducing conditions (low redox potential; for example, anoxia) and high chloride content.

$\mathrm{Pb}$ is dispersed throughout the environment primarily as the result of anthropogenic activities. In the air, $\mathrm{Pb}$ is in the form of particles and is removed by rain or gravitational settling. The solubility of $\mathrm{Pb}$ compounds in water is a function of $\mathrm{pH}$, ionic strength, and the presence of humic material. Solubility is highest in acidic water. Soil and sediment are an important sink for $\mathrm{Pb}$. Because $\mathrm{Pb}$ is strongly adsorbed to soil, very little is transported through runoff to surface water or leached to groundwater except under acidic conditions. Anthropogenic sources of $\mathrm{Pb}$ include the mining and smelting of ore, manufacture and use of $\mathrm{Pb}$-containing products, combustion of coal and oil, and waste incineration. Many anthropogenic sources of $\mathrm{Pb}$, most notably leaded gasoline, $\mathrm{Pb}$-based paint, $\mathrm{Pb}$ solder in food cans, $\mathrm{Pb}$-arsenate pesticides, and shot and sinkers, have been eliminated or are regulated. $\mathrm{Pb}$ compounds released to the environment may be transformed to other $\mathrm{Pb}$ compounds; however, $\mathrm{Pb}$ is an element and cannot be destroyed or degraded. Because $\mathrm{Pb}$ does not degrade over time, deposits of $\mathrm{Pb}$ in the environment by current and former uses leave their legacy as higher concentrations of $\mathrm{Pb}$ in the environment. These deposits can continue to be a source for potential $\mathrm{Pb}$ exposure (e.g., soil particles containing $\mathrm{Pb}$ also may be resuspended and redeposited). Plants and animals may bioconcentrate $\mathrm{Pb}$, but $\mathrm{Pb}$ is not biomagnified in the aquatic or terrestrial food chain.

The general population may be exposed to $\mathrm{Pb}$ in ambient air, foods, drinking water, soil, and dust. Segments of the general population at highest risk of health effects from $\mathrm{Pb}$ exposure are preschool-age children and pregnant women and their fetuses. Other segments of the general population with an increased exposure include individuals living near sites where $\mathrm{Pb}$ was produced or disposed. Some of the more important $\mathrm{Pb}$ exposures have occurred as a result of living in urban environments, particularly in areas near stationary emission sources (e.g., smelters); renovation of homes containing Pb-based paint; pica (the compulsive, habitual consumption of nonfood items); contact with interior $\mathrm{Pb}$ paint dust; occupational exposure; and secondary occupational exposure (e.g., families of workers in $\mathrm{Pb}$ industries). Higher exposures may also occur to residents living in close proximity to NPL sites that contain elevated levels of $\mathrm{Pb}$.

The primary source of $\mathrm{Pb}$ in the environment has historically been anthropogenic emissions to the atmosphere. In 1984, combustion of leaded gasoline was responsible for approximately $90 \%$ of all anthropogenic $\mathrm{Pb}$ emissions. The United States gradually phased out the use of $\mathrm{Pb}$ alkyls in gasoline, and by 1990, auto emissions accounted for only 33\% of the annual Pb emissions (EPA 1996b). Use of $\mathrm{Pb}$ 
additives in most motor fuels was totally banned after December 31, 1995 (EPA 1996a). The ban went into effect on February 2, 1996. The ban did not include off-road vehicles, including aircraft, racing cars, farm equipment, and marine engines. $\mathrm{Pb}$ additives are still used in fuels for piston driven airplane engines and it continues to be commercially available for other off-road uses. Atmospheric deposition is the largest source of $\mathrm{Pb}$ found in soils. $\mathrm{Pb}$ is transferred continuously between air, water, and soil by natural chemical and physical processes such as weathering, runoff, precipitation, dry deposition of dust, and stream/river flow; however, soil and sediments appear to be important sinks for $\mathrm{Pb}$. $\mathrm{Pb}$ particles are removed from the atmosphere primarily by wet and dry deposition. The average residence time in the atmosphere is 10 days. Over this time, long-distance transport, up to thousands of kilometers, may take place. The speciation of $\mathrm{Pb}$ in these media varies widely depending upon such factors as temperature, $\mathrm{pH}$, and the presence of humic materials. $\mathrm{Pb}$ is largely associated with suspended solids and sediments in aquatic systems, and it occurs in relatively immobile forms in soil.

\subsection{PRODUCTION, IMPORT/EXPORT, USE, AND DISPOSAL}

\subsubsection{Production}

The most important mineable $\mathrm{Pb}$ ore is galena (PbS), which is commonly associated with other minerals, typically zinc ores. Anglesite $\left(\mathrm{PbSO}_{4}\right)$ and cerussite $\left(\mathrm{PbCO}_{3}\right)$, formed by the weathering of galena, are two other important $\mathrm{Pb}$ minerals. $\mathrm{Pb}$ is processed from ore to refined metal in four steps: ore dressing; smelting; drossing; and refining. Ore dressing involves crushing, grinding, and beneficiation (concentration) (King et al. 2014).

Since 1998, U.S. production of Pb has shifted to the domestic secondary Pb industry (USGS 2014). Since 2014, primary Pb metal has not been produced in the United States (USGS 2016). The Doe Run Resources Corporation operated the last domestic primary Pb smelter-refinery facility in the United States at Herculaneum, Missouri and it was closed at the end of 2013. Pb-acid batteries are the dominant source of recoverable $\mathrm{Pb}$ scrap, accounting for nearly 100\% of all secondary Pb (USGS 2016, 2019).

Domestic mines produced 368,000 metric tons of recoverable $\mathrm{Pb}$ in 2014, a more than $11 \%$ increase from 2013. Nearly all of the secondary Pb produced in 2014 was by 7 companies operating 12 plants in Alabama, California, Florida, Indiana, Minnesota, Missouri, New York, Pennsylvania, Tennessee, and Texas (USGS 2016). Secondary (recycled) Pb, derived from mainly scrapped Pb-acid batteries, accounted for all of the domestic refined Pb production in 2014. Due to plant closings, U.S. production 
of secondary refined $\mathrm{Pb}$ decreased in 2014 by $11 \%$ to 1.02 metric tons, from 1.5 metric tons in 2013 (USGS 2016).

World mine production of $\mathrm{Pb}$ was 4.91 million metric tons in 2014, a decrease of 9\% from 2013. The United States accounted for approximately 8\% of global mine production in 2014. The United States ranked third in global mine production behind China and Australia, which accounted for 49 and 15\%, respectively. World production of refined $\mathrm{Pb}$ (primary and secondary) was 10.6 million metric tons in 2014. China produced about $45 \%$ of global refined $\mathrm{Pb}$ in 2014 with the United States as the second leading world producer of refined Pb, accounting for 10\% (USGS 2016). In 2017 and 2018 worldwide mine production of $\mathrm{Pb}$ was reported as 4.58 and 4.40 million metric tons, respectively (USGS 2019). As in previous years, China was the dominant producer accounting for nearly half of the world production.

Manufacturers and importers of $\mathrm{Pb}$ metal and selected $\mathrm{Pb}$ compounds are listed in Table 5-1. These data are from EPA's Chemical Data Access Tool (now called Chemical Data Reporting [CDR]), which provides information on chemicals submitted to the EPA under the Toxic Substance Control Act that are manufactured or imported into the United States. Manufacturing volumes for more recent years are not available in the CDR as most manufacturers have withheld these data as confidential business information; however, as in previous years, the U.S. Geological Survey (USGS) reported total Pb mined in the United States in its Minerals Commodity summaries and these data for 2015-2018 are provided in Table 5-2. According to the USGS, five Pb mines located in the state of Missouri along with five mines in Alaska, Idaho, and the state of Washington accounted for all domestic Pb mine production (USGS 2019).

\section{Table 5-1. U.S. Manufacturers of Lead Metal and Selected Lead Compounds}

\begin{tabular}{l} 
Company \\
\hline Lead \\
$5 n$ Plus Inc. \\
Colfin Specialty Steel Corp. \\
Compliance Administrators \& Project Services Inc. \\
Concorde/Interspace Battery \\
Doe Run Co. \\
East Penn Manufacturing Co. Inc. \\
Exide Technologies
\end{tabular}

Domestic manufacturing Location (pounds/year)

$\begin{array}{lr}\text { Fairfield, Connecticut } & 36,671 \\ \text { New Brighton, Pennsylvania } & 2,552 \\ \text { Bloomington, California } & 848,008 \\ \text { West Covina, California } & 348,998 \\ \text { Herculaneum, Missouri } & 280,000,000 \\ \text { Lyon Station, Pennsylvania } & 194,537,569 \\ \text { Bristol, Tennessee } & 150,000\end{array}$


Table 5-1. U.S. Manufacturers of Lead Metal and Selected Lead Compounds

\begin{tabular}{|c|c|c|}
\hline Company & Location & $\begin{array}{l}\text { Domestic } \\
\text { manufacturing } \\
\text { (pounds/year) }\end{array}$ \\
\hline & Columbus, Georgia & $4,200,000$ \\
\hline & Forest City, Missouri & $84,000,000$ \\
\hline & Fort Smith, Arkansas & $3,600,000$ \\
\hline & Frisco, Texas & $140,000,000$ \\
\hline & Kansas City, Kansas & $9,100,000$ \\
\hline & Los Angeles, California & $230,000,000$ \\
\hline & Manchester, lowa & $16,000,000$ \\
\hline & Muncie, Indiana & $160,000,000$ \\
\hline & Reading, Pennsylvania & $130,000,000$ \\
\hline & Salina, Kansas & 990,000 \\
\hline \multirow[t]{2}{*}{ Gopher Resource } & Eagan, Minnesota & $310,000,000$ \\
\hline & Tampa, Florida & $38,000,000$ \\
\hline \multirow[t]{4}{*}{ Horsehead Holding Corp. } & Chicago, Illinois & $2,444,492$ \\
\hline & Palmerton, Pennsylvania & $3,867,016$ \\
\hline & Rockwood, Tennessee & $1,872,054$ \\
\hline & Snelling, South Carolina & $2,012,236$ \\
\hline \multirow[t]{7}{*}{ Johnson Controls } & Canby, Oregon & $36,832,250$ \\
\hline & Geneva, Illinois & $47,025,828$ \\
\hline & Holland, Ohio & $82,721,150$ \\
\hline & Kernersville, North Carolina & $204,679,893$ \\
\hline & Middletown, Delaware & $86,732,852$ \\
\hline & Tampa, Florida & $3,069,380$ \\
\hline & Yuma, Arizona & $359,977,380$ \\
\hline \multirow[t]{2}{*}{ Johnson Controls Distribution Center } & Saint Joseph, Missouri & $2,550,177$ \\
\hline & St. Joseph, Missouri & $266,151,342$ \\
\hline Renco Group Inc. & Boss, Missouri & $310,000,000$ \\
\hline Sanders Lead Co., Inc. & Troy, Alabama & $471,954,520$ \\
\hline Stemar Investments Inc. & Butler, Pennsylvania & 40,506 \\
\hline Yuasa Battery Inc. & Laureldale, Pennsylvania & $1,492,754$ \\
\hline \multicolumn{3}{|l|}{ Lead(II) nitrate } \\
\hline American Pacific Corp. & Cedar City, Utah & 42,500 \\
\hline \multicolumn{3}{|l|}{ Lead(II) oxide } \\
\hline \multirow[t]{3}{*}{ C\&D Technologies Inc. } & Attica, Indiana & $18,657,255$ \\
\hline & Leola, Pennsylvania & $1,348,311$ \\
\hline & Milwaukee, Wisconsin & $48,491,557$ \\
\hline Crown Battery Manufacturing Co. & Fremont, Ohio & $25,600,000$ \\
\hline
\end{tabular}


Table 5-1. U.S. Manufacturers of Lead Metal and Selected Lead Compounds

\begin{tabular}{|c|c|c|}
\hline Company & Location & $\begin{array}{l}\text { Domestic } \\
\text { manufacturing } \\
\text { (pounds/year) }\end{array}$ \\
\hline Fiamm Energy LLC & Waynesboro, Georgia & $4,700,000$ \\
\hline \multirow[t]{2}{*}{ Hammond Group Inc. } & Hammond, Indiana & $3,585,529$ \\
\hline & Pottstown, Pennsylvania & $8,287,521$ \\
\hline Renco Group Inc. & Boss, Missouri & $7,700,000$ \\
\hline Steel Dust Recycling & Millport, Alabama & $2,000,000$ \\
\hline Superior Battery Manufacturing & Russell Springs, Kentucky & $16,866,793$ \\
\hline \multirow[t]{2}{*}{ Trojan Battery Co. } & Lithonia, Georgia & $38,540,700$ \\
\hline & Santa Fe Springs, California & $35,241,500$ \\
\hline \multicolumn{3}{|l|}{ Lead(II) styphnate } \\
\hline Alliant Techsystems Inc. & Lewiston, Idaho & 78,767 \\
\hline Alliant Techsystems Operations LLC & Independence, Missouri & 43,489 \\
\hline \multicolumn{3}{|l|}{ Lead(II) sulfate } \\
\hline Crown Battery Manufacturing Co. & Fremont, Ohio & 768,000 \\
\hline \multirow[t]{2}{*}{ East Penn Manufacturing Co., Inc. } & Corydon, lowa & $17,006,710$ \\
\hline & Lyon Station, Pennsylvania & $220,436,420$ \\
\hline \multirow[t]{6}{*}{ Johnson Controls } & Canby, Oregon & $6,098,880$ \\
\hline & Geneva, Illinois & $11,340,306$ \\
\hline & Holland, Ohio & $10,714,048$ \\
\hline & Middletown, Delaware & $5,749,910$ \\
\hline & Tampa, Florida & $5,506,240$ \\
\hline & Yuma, Arizona & 86,756 \\
\hline \multirow[t]{2}{*}{ Johnson Controls Distribution Center } & Saint Joseph, Missouri & 306,021 \\
\hline & St. Joseph, Missouri & $29,577,055$ \\
\hline Palos Verdes Bldg Corp. & Augusta, Georgia & $6,904,629$ \\
\hline Superior Battery Manufacturing & Russell Springs, Kentucky & $22,905,105$ \\
\hline \multirow[t]{2}{*}{ Trojan Battery Co. } & Lithonia, Georgia & $58,127,100$ \\
\hline & Santa Fe Springs, California & $53,083,500$ \\
\hline \multicolumn{3}{|l|}{ Lead(II) chloride } \\
\hline \multirow[t]{2}{*}{ Horsehead Holding Corp. } & Monaca, Pennsylvania & $1,891,700$ \\
\hline & Palmerton, Pennsylvania & $11,484,955$ \\
\hline
\end{tabular}


Table 5-2. U.S. Lead Production 2015-2018

\begin{tabular}{lcccr}
\hline & \multicolumn{4}{c}{ Production volumes in metric tons } \\
\hline & 2015 & 2016 & 2017 & \multicolumn{1}{c}{2018} \\
\hline Mine, lead in concentrates & 370,000 & 346,000 & 310,000 & 260,000 \\
Secondary refinery, old scrap & $1,050,000$ & 986,000 & $1,130,000$ & $1,300,000$ \\
\hline
\end{tabular}

Source: USGS 2019

Tables 5-3 (Pb) and 5-4 (Pb compounds) list the facilities in each state that manufacture or process $\mathrm{Pb}$ or $\mathrm{Pb}$ compounds, the intended use, and the range of maximum amounts of $\mathrm{Pb}$ that are stored on site. The data listed in Tables 5-3 and 5-4 are derived from the Toxics Release Inventory (TRI) (TRI18 2020). The data presented in Table 5-3 are for Pb metal and the data from Table 5-4 are for all Pb compounds. Facilities with $\geq 10$ full-time employees in certain TRI-covered industry sectors (e.g., manufacturing) must submit data on releases and other waste management for TRI-listed chemicals $(\mathrm{Pb}$ and $\mathrm{Pb}$ compounds are TRI listed). Therefore, there are sources for $\mathrm{Pb}$ and $\mathrm{Pb}$ compounds not contained in the TRI database. In comparing TRI data with that of previous years, it is important to note that starting in 2001, the threshold for reporting $\mathrm{Pb}$ and all $\mathrm{Pb}$ compounds was reduced to 100 pounds, except for $\mathrm{Pb}$ contained in a stainless steel, brass, or bronze alloy. Previously, reporting was only required of facilities that manufactured or processed $>25,000$ pounds annually or that used $>10,000$ pounds annually. Beginning in 1998, additional industries were required to report, including metal mining, coal mining, electrical utilities, and Resource Conservation and Recovery Act (RCRA)/Solvent Recovery. Table 5-3 lists the producers of primary $\mathrm{Pb}$ metal and selected $\mathrm{Pb}$ compounds. Companies listed are those producing $\mathrm{Pb}$ compounds in commercial quantities $>5,000$ pounds or $\$ 10,000$ in value annually. Table 5-4 shows the U.S. production volumes for $\mathrm{Pb}$ for 2010 through 2013. During this time, the primary $\mathrm{Pb}$ production declined, while secondary $\mathrm{Pb}$ production was relatively constant.

\section{Table 5-3. Facilities that Produce, Process, or Use Lead}

\begin{tabular}{|c|c|c|c|c|}
\hline State $^{a}$ & $\begin{array}{l}\text { Number of } \\
\text { facilities }\end{array}$ & $\begin{array}{l}\text { Minimum } \\
\text { amount on site } \\
\text { in pounds }{ }^{b}\end{array}$ & $\begin{array}{l}\text { Maximum } \\
\text { amount on site } \\
\text { in pounds }{ }^{b}\end{array}$ & Activities and uses ${ }^{c}$ \\
\hline AK & 4 & 100 & 99,999 & 12 \\
\hline $\mathrm{AL}$ & 105 & 0 & $999,999,999$ & $1,4,5,6,7,8,9,10,11,12,13,14$ \\
\hline AR & 67 & 0 & $49,999,999$ & $1,2,5,6,7,8,9,11,12,13,14$ \\
\hline $\mathrm{AZ}$ & 52 & 0 & $9,999,999$ & $1,3,5,7,8,9,10,11,12,14$ \\
\hline $\mathrm{CA}$ & 188 & 0 & $9,999,999$ & $1,2,3,5,6,7,8,9,10,11,12,13,14$ \\
\hline $\mathrm{CO}$ & 33 & 0 & 99,999 & $1,5,6,7,8,10,11,12,14$ \\
\hline
\end{tabular}


5. POTENTIAL FOR HUMAN EXPOSURE

Table 5-3. Facilities that Produce, Process, or Use Lead

\begin{tabular}{|c|c|c|c|c|}
\hline State $^{a}$ & $\begin{array}{l}\text { Number of } \\
\text { facilities }\end{array}$ & $\begin{array}{l}\text { Minimum } \\
\text { amount on site } \\
\text { in pounds }{ }^{b}\end{array}$ & $\begin{array}{l}\text { Maximum } \\
\text { amount on site } \\
\text { in pounds }{ }^{b}\end{array}$ & 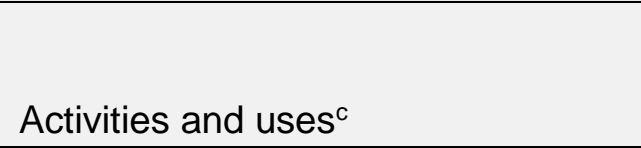 \\
\hline CT & 42 & 0 & 99,999 & $1,3,4,5,6,7,8,9,10,11,12,13,14$ \\
\hline $\mathrm{DC}$ & 6 & 0 & 9,999 & $1,7,8,11,12,13,14$ \\
\hline $\mathrm{DE}$ & 6 & 0 & 99,999 & $7,12,14$ \\
\hline $\mathrm{FL}$ & 223 & 0 & 999,999 & $1,2,3,5,6,7,8,9,10,11,12,13,14$ \\
\hline $\mathrm{GA}$ & 108 & 0 & 999,999 & $1,2,3,5,6,7,8,9,10,11,12,13,14$ \\
\hline GU & 1 & 0 & 99 & 1,5 \\
\hline $\mathrm{HI}$ & 2 & 0 & 99,999 & 8,12 \\
\hline IA & 114 & 0 & $9,999,999$ & $1,2,5,6,7,8,9,11,12,13,14$ \\
\hline ID & 27 & 0 & $49,999,999$ & $1,2,3,5,8,9,11,12,13,14$ \\
\hline IL & 228 & 0 & $49,999,999$ & $1,2,3,4,5,7,8,9,10,11,12,13,14$ \\
\hline IN & 161 & 0 & 999,999 & $1,2,3,4,5,6,7,8,9,10,11,12,13,14$ \\
\hline KS & 70 & 0 & $9,999,999$ & $1,3,5,7,8,9,10,12,13,14$ \\
\hline KY & 79 & 0 & 999,999 & $1,2,3,5,6,7,8,9,10,11,12,13,14$ \\
\hline LA & 46 & 0 & 999,999 & $1,2,3,5,8,9,10,11,12,13,14$ \\
\hline$\overline{M A}$ & 55 & 0 & 999,999 & $1,2,4,5,6,7,8,9,10,11,12,13,14$ \\
\hline $\mathrm{MD}$ & 35 & 0 & 99,999 & $1,5,7,8,9,10,11,12,14$ \\
\hline $\mathrm{ME}$ & 20 & 0 & 99,999 & $1,2,3,4,5,8,9,11,12,13,14$ \\
\hline $\mathrm{Ml}$ & 148 & 0 & 999,999 & $1,2,3,4,5,7,8,9,10,11,12,13,14$ \\
\hline $\mathrm{MN}$ & 123 & 0 & 99,999 & $1,2,3,5,6,7,8,9,10,11,12,13,14$ \\
\hline $\mathrm{MO}$ & 92 & 0 & $9,999,999$ & $1,3,5,6,7,8,9,10,11,12,13,14$ \\
\hline MS & 62 & 0 & 999,999 & $1,5,7,8,9,10,12,14$ \\
\hline MT & 15 & 0 & 99,999 & $1,2,5,11,12,13,14$ \\
\hline NC & 162 & 0 & $9,999,999$ & $1,2,3,5,6,7,8,9,11,12,13,14$ \\
\hline ND & 14 & 0 & 9,999 & $1,5,8,9,12,14$ \\
\hline $\mathrm{NE}$ & 62 & 0 & 999,999 & $1,2,3,5,7,8,9,11,12,13,14$ \\
\hline $\mathrm{NH}$ & 30 & 0 & 99,999 & $1,2,3,4,5,7,8,9,10,11,12,14$ \\
\hline $\mathrm{NJ}$ & 43 & 0 & 99,999 & $1,2,5,7,8,9,11,12,13,14$ \\
\hline NM & 17 & 0 & 999,999 & $1,5,6,8,9,10,11,12,14$ \\
\hline$\overline{N V}$ & 28 & 0 & 999,999 & $1,2,4,5,8,9,12,13,14$ \\
\hline NY & 140 & 0 & 999,999 & $1,2,3,5,7,8,9,10,11,12,13,14$ \\
\hline $\mathrm{OH}$ & 237 & 0 & $9,999,999$ & $1,2,3,4,5,6,7,8,9,10,11,12,13,14$ \\
\hline OK & 54 & 0 & 999,999 & $1,2,3,5,7,8,11,12,14$ \\
\hline OR & 48 & 0 & $9,999,999$ & $1,2,3,5,7,8,10,11,12,13,14$ \\
\hline PA & 182 & 0 & $99,999,999$ & $1,2,3,4,5,6,7,8,9,10,11,12,13,14$ \\
\hline$\overline{P R}$ & 5 & 100 & 9,999 & $2,3,8,9,12$ \\
\hline $\mathrm{RI}$ & 15 & 0 & 999,999 & $1,5,7,8,9,10,11,12,13,14$ \\
\hline SC & 81 & 0 & 999,999 & $1,2,3,4,5,6,7,8,9,11,12,13,14$ \\
\hline SD & 14 & 0 & 99,999 & $1,2,5,7,8,9,13,14$ \\
\hline
\end{tabular}


Table 5-3. Facilities that Produce, Process, or Use Lead

\begin{tabular}{|c|c|c|c|c|}
\hline State $^{a}$ & $\begin{array}{l}\text { Number of } \\
\text { facilities }\end{array}$ & $\begin{array}{l}\text { Minimum } \\
\text { amount on site } \\
\text { in pounds }{ }^{b}\end{array}$ & $\begin{array}{l}\text { Maximum } \\
\text { amount on site } \\
\text { in pounds }\end{array}$ & Activities and uses ${ }^{c}$ \\
\hline TN & 110 & 0 & $99,999,999$ & $1,2,3,4,5,6,7,8,9,10,11,12,13,14$ \\
\hline $\mathrm{TX}$ & 326 & 0 & $49,999,999$ & $1,2,4,5,6,7,8,9,10,11,12,13,14$ \\
\hline UT & 50 & 0 & 999,999 & $1,5,6,8,9,10,11,12,13,14$ \\
\hline VA & 98 & 0 & 999,999 & $1,2,3,5,7,8,9,10,11,12,13,14$ \\
\hline $\mathrm{VI}$ & 3 & 0 & 99 & $7,9,14$ \\
\hline VT & 11 & 0 & 99,999 & $1,2,3,5,8,11,12,13,14$ \\
\hline$\overline{W A}$ & 57 & 0 & 999,999 & $1,2,3,4,5,7,8,9,11,12,13,14$ \\
\hline WI & 165 & 0 & $9,999,999$ & $1,2,3,5,6,7,8,9,10,11,12,13,14$ \\
\hline WV & 31 & 0 & 999,999 & $1,2,5,8,9,10,11,12,13,14$ \\
\hline WY & 12 & 0 & 99,999 & $1,5,6,8,9,10,11,12,13,14$ \\
\hline
\end{tabular}

apost office state abbreviations used.

${ }^{b}$ Amounts on site reported by facilities in each state.

'Activities/Uses:
1. Produce
6. Reactant
2. Import
7. Formulation Component
11. Manufacture Aid
3. Used Processing
4. Sale/Distribution
8. Article Component
12. Ancillary
13. Manufacture Impurity
5. Byproduct
10. Chemical Processing Aid
14. Process Impurity

Source: TRI18 2020 (Data are from 2018)

Table 5-4. Facilities that Produce, Process, or Use Lead Compounds

\begin{tabular}{|c|c|c|c|c|}
\hline State $^{a}$ & $\begin{array}{l}\text { Number of } \\
\text { facilities }\end{array}$ & $\begin{array}{l}\text { Minimum } \\
\text { amount on site } \\
\text { in pounds }{ }^{b}\end{array}$ & $\begin{array}{l}\text { Maximum } \\
\text { amount on site } \\
\text { in pounds }{ }^{b}\end{array}$ & Activities and uses ${ }^{c}$ \\
\hline$A K$ & 16 & 0 & $499,999,999$ & $1,5,9,12,13,14$ \\
\hline $\mathrm{AL}$ & 130 & 0 & $49,999,999$ & $1,2,3,4,5,6,7,8,9,10,11,12,13,14$ \\
\hline AR & 68 & 0 & $49,999,999$ & $1,2,3,4,5,6,7,8,9,10,11,12,13,14$ \\
\hline$A Z$ & 65 & 0 & $49,999,999$ & $1,2,3,4,5,7,8,9,10,11,12,13,14$ \\
\hline $\mathrm{CA}$ & 315 & 0 & $9,999,999$ & $1,2,3,4,5,6,7,8,9,10,11,12,13,14$ \\
\hline $\mathrm{CO}$ & 76 & 0 & $49,999,999$ & $1,2,3,4,5,7,8,9,11,12,13,14$ \\
\hline CT & 39 & 0 & $49,999,999$ & $1,2,3,4,5,6,7,8,9,10,11,12,13,14$ \\
\hline $\mathrm{DC}$ & 1 & 100 & 999 & 14 \\
\hline $\mathrm{DE}$ & 5 & 100 & $49,999,999$ & $1,2,3,4,5,7,8,9,12,13,14$ \\
\hline FL & 150 & 0 & $49,999,999$ & $1,2,3,4,5,7,8,9,11,12,13,14$ \\
\hline GA & 108 & 0 & $9,999,999$ & $1,2,3,4,5,6,7,8,9,11,12,13,14$ \\
\hline GU & 3 & 0 & 99 & $1,5,7,9,12,13,14$ \\
\hline $\mathrm{HI}$ & 14 & 0 & 99,999 & $1,2,5,7,9,12,13,14$ \\
\hline IA & 66 & 0 & $9,999,999$ & $1,2,3,4,5,7,8,9,10,11,12,13,14$ \\
\hline
\end{tabular}


Table 5-4. Facilities that Produce, Process, or Use Lead Compounds

\begin{tabular}{|c|c|c|c|c|}
\hline State $^{\mathrm{a}}$ & $\begin{array}{l}\text { Number of } \\
\text { facilities }\end{array}$ & $\begin{array}{l}\text { Minimum } \\
\text { amount on site } \\
\text { in pounds }{ }^{b}\end{array}$ & $\begin{array}{l}\text { Maximum } \\
\text { amount on site } \\
\text { in pounds }{ }^{b}\end{array}$ & 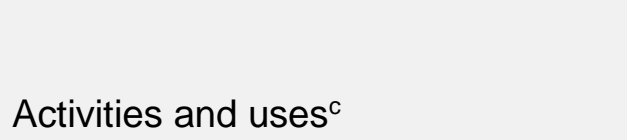 \\
\hline ID & 31 & 0 & $9,999,999$ & $1,2,3,4,5,7,8,11,12,13,14$ \\
\hline IL & 166 & 0 & $49,999,999$ & $1,2,3,4,5,6,7,8,9,10,11,12,13,14$ \\
\hline IN & 151 & 0 & $99,999,999$ & $1,2,3,4,5,6,7,8,9,10,11,12,13,14$ \\
\hline KS & 40 & 0 & 9,999,999 & $1,2,3,4,5,6,7,8,9,10,11,12,13,14$ \\
\hline KY & 64 & 0 & $9,999,999$ & $1,2,3,4,5,6,7,8,9,10,11,12,13,14$ \\
\hline LA & 85 & 0 & $9,999,999$ & $1,2,3,4,5,6,7,8,9,10,11,12,13,14$ \\
\hline MA & 53 & 0 & $49,999,999$ & $1,2,3,4,5,6,7,8,9,10,11,12,13,14$ \\
\hline MD & 35 & 0 & $99,999,999$ & $1,2,3,4,5,6,7,8,9,12,13,14$ \\
\hline ME & 13 & 0 & 999,999 & $1,2,4,5,8,9,12,13,14$ \\
\hline $\mathrm{Ml}$ & 102 & 0 & $9,999,999$ & $1,2,3,4,5,6,7,8,9,10,11,12,13,14$ \\
\hline MN & 50 & 0 & 49,999,999 & $1,2,3,4,5,6,7,8,9,10,11,12,13,14$ \\
\hline MO & 74 & 0 & $999,999,999$ & $1,2,3,4,5,6,7,8,9,11,12,13,14$ \\
\hline MP & 1 & 0 & 99 & $1,5,12,13,14$ \\
\hline MS & 50 & 0 & $99,999,999$ & $1,2,3,4,5,6,7,8,11,12,13,14$ \\
\hline MT & 20 & 0 & $9,999,999$ & $1,2,3,4,5,6,7,9,12,13,14$ \\
\hline NC & 149 & 0 & 49,999,999 & $1,2,3,4,5,6,7,8,9,10,11,12,13,14$ \\
\hline ND & 20 & 0 & 99,999 & $1,2,3,4,5,7,8,9,12,13,14$ \\
\hline NE & 24 & 0 & $9,999,999$ & $1,3,4,5,6,7,8,9,10,12,13,14$ \\
\hline $\mathrm{NH}$ & 14 & 0 & 999,999 & $1,2,5,7,8,9,11,12,14$ \\
\hline $\mathrm{NJ}$ & 55 & 0 & 999,999 & $1,2,3,4,5,7,8,9,10,11,12,13,14$ \\
\hline NM & 18 & 0 & 49,999,999 & $1,2,3,4,5,7,8,9,11,12,13,14$ \\
\hline NV & 51 & 0 & 49,999,999 & $1,2,3,5,6,7,8,9,10,11,12,13,14$ \\
\hline NY & 82 & 0 & 49,999,999 & $1,2,3,4,5,6,7,8,9,10,11,12,13,14$ \\
\hline $\mathrm{OH}$ & 175 & 0 & $49,999,999$ & $1,2,3,4,5,6,7,8,9,10,11,12,13,14$ \\
\hline OK & 81 & 0 & $49,999,999$ & $1,2,3,4,5,6,7,8,9,10,11,12,13,14$ \\
\hline OR & 55 & 0 & 9,999,999 & $1,2,3,4,5,6,7,8,9,10,11,12,13,14$ \\
\hline PA & 195 & 0 & $99,999,999$ & $1,2,3,4,5,6,7,8,9,10,11,12,13,14$ \\
\hline PR & 11 & 0 & 99,999 & $1,2,3,4,5,6,7,8,9,12,14$ \\
\hline RI & 19 & 0 & 99,999 & $1,2,3,4,5,7,8,9,10,11,12,13,14$ \\
\hline SC & 101 & 0 & 49,999,999 & $1,2,3,4,5,6,7,8,9,11,12,13,14$ \\
\hline SD & 11 & 0 & $9,999,999$ & $1,3,4,5,6,7,8,9,12,13,14$ \\
\hline TN & 89 & 0 & $9,999,999$ & $1,2,3,4,5,6,7,8,9,11,12,13,14$ \\
\hline $\mathrm{TX}$ & 330 & 0 & $499,999,999$ & $1,2,3,4,5,6,7,8,9,10,11,12,13,14$ \\
\hline UT & 41 & 0 & $499,999,999$ & $1,2,3,4,5,6,7,8,9,10,11,12,13,14$ \\
\hline VA & 84 & 0 & $49,999,999$ & $1,3,4,5,6,7,8,9,10,11,12,13,14$ \\
\hline VI & 2 & 0 & 99 & $1,5,12,0$ \\
\hline VT & 5 & 0 & 9,999 & $7,8,14$ \\
\hline WA & 76 & 0 & $9,999,999$ & $1,2,3,4,5,6,7,8,9,11,12,13,14$ \\
\hline
\end{tabular}


Table 5-4. Facilities that Produce, Process, or Use Lead Compounds

\begin{tabular}{|c|c|c|c|c|}
\hline State $^{a}$ & $\begin{array}{l}\text { Number of } \\
\text { facilities }\end{array}$ & $\begin{array}{l}\text { Minimum } \\
\text { amount on site } \\
\text { in pounds }{ }^{b}\end{array}$ & $\begin{array}{l}\text { Maximum } \\
\text { amount on site } \\
\text { in pounds }\end{array}$ & Activities and uses ${ }^{c}$ \\
\hline WI & 100 & 0 & 999,999 & $1,2,3,4,5,6,7,8,9,10,11,12,13,14$ \\
\hline WV & 44 & 0 & $49,999,999$ & $1,3,4,5,7,8,9,10,11,12,13,14$ \\
\hline$\overline{W Y}$ & 16 & 0 & 999,999 & $1,2,3,4,5,8,9,12,13,14$ \\
\hline
\end{tabular}

aPost office state abbreviations used.

${ }^{\mathrm{b}}$ Amounts on site reported by facilities in each state.

'Activities/Uses:
1. Produce
6. Reactant
2. Import
7. Formulation Component
11. Manufacture Aid
3. Used Processing
4. Sale/Distribution
8. Article Component
9. Repackaging
12. Ancillary
5. Byproduct
10. Chemical Processing Aid
13. Manufacture Impurity
14. Process Impurity

Source: TRI18 2020 (Data are from 2018)

\subsubsection{Import/Export}

In 2014, 1,080 and 593,000 metric tons of $\mathrm{Pb}$ as base bullion and pigs and bars, respectively, were imported into the United States. Imports have increased since 2010 when 602 and 271,000 metric tons of $\mathrm{Pb}$ as base bullion and pigs and bars, respectively, were imported. In 2014, 65,100 metric tons, $\mathrm{Pb}$ content of Pb pigments and compounds were imported in the United States (USGS 2016). In 2015, 2016, 2017, and 2018 imports of Pb refined metal (unwrought) were reported as 521,000, 533,000, 658,000, and 580,000 metric tons, respectively (USGS 2019).

Exports of $\mathrm{Pb}$ in ore and concentrates and $\mathrm{Pb}$ materials, excluding scrap were 299,000 and 83,500 metric tons, respectively, in 2010 as compared to 365,000 and 61,300 metric tons, respectively, in 2014. In 2013 and 2014, 34,900 and 36,400 metric tons of Pb scrap were exported, respectively (USGS 2016). Total exports of $\mathrm{Pb}(\mathrm{Pb}$ in concentrates and refined metal, unwrought gross weight) were reported as 406,000, 384,000, 293,000, and 324,000 metric tons in 2015, 2016, 2017, and 2018, respectively (USGS 2019).

\subsubsection{Use}

$\mathrm{Pb}$ may be used in the form of metal, either pure or alloyed with other metals, or as chemical compounds. The main uses of $\mathrm{Pb}$ and its compounds are in $\mathrm{Pb}$-acid batteries, with most other applications using $\mathrm{Pb}$ alloys. The commercial importance of $\mathrm{Pb}$ is based on its physical properties, including its low melting 
point, ease of casting, high density, softness, malleability, low strength, ease of fabrication, acid resistance, electrochemical reaction with sulfuric acid, and chemical stability in air, water, and soil (King et al. 2014).

In the United States in 2014, Pb was consumed by over 70 companies to manufacture products such as ammunition; building-construction materials; covering for power and communication cable; $\mathrm{Pb}$-acid storage batteries; $\mathrm{Pb}$ oxides for ceramics, chemicals, glass, and pigments; $\mathrm{Pb}$ sheet; and solder for construction, electronic components and accessories, metal containers, and motor vehicles (USGS 2016). In 2018, it was estimated that the Pb-acid battery industry accounted for $>85 \%$ of the domestic consumption of $\mathrm{Pb}$ in the United States (USGS 2019). Pb-acid batteries were primarily used as startinglighting-ignition (SLI) batteries for automobiles and trucks and as industrial-type batteries for standby power for computer and telecommunications networks and for motive power. Global consumption of refined Pb was 11.71 million metric tons in 2018, (USGS 2019).

Prior to the EPA beginning to regulate the Pb content in gasoline during the early 1970s, approximately 250,000 tons of organic $\mathrm{Pb}$ (e.g., tetraethyl $\mathrm{Pb}$ ) were added to gasoline on an annual basis in the United States (Giddings 1973). These Pb-based "anti-knock” additives increased the octane rating of the gasoline and, as a result, increased engine efficiency (Giddings 1973). In 1971, the average Pb content for a gallon of gasoline purchased in the United States was $2.2 \mathrm{~g} /$ gallon (Giddings 1973). After determining that $\mathrm{Pb}$ additives would impair the performance of emission control systems installed on motor vehicles, and that $\mathrm{Pb}$ particle emission from motor vehicles presented a significant health risk to urban populations, EPA, in 1973, initiated a phase-down program designed to minimize the amount of $\mathrm{Pb}$ in gasoline over time. By 1988, the phase-down program had reduced the total $\mathrm{Pb}$ usage in gasoline to $<1 \%$ of the amount of $\mathrm{Pb}$ used in the peak year of 1970 (EPA 1996a).

In 1990, a Congressional amendment to the Clean Air Act (CAA) banned the use of gasoline containing $\mathrm{Pb}$ or $\mathrm{Pb}$ additives as fuel in most motor vehicles. On February 2, 1996, the EPA incorporated the statutory ban in a direct final rule, which defined unleaded gasoline as gasoline containing trace amounts of $\mathrm{Pb}$ up to $0.05 \mathrm{~g}$ /gallon (EPA 1996a). The definition still allowed trace amounts of $\mathrm{Pb}$, but expressly prohibited the use of any $\mathrm{Pb}$ additive in the production of unleaded gasoline. The term "lead additive" was defined to include pure $\mathrm{Pb}$ as well as $\mathrm{Pb}$ compounds (EPA 1996a). Although the regulatory action of Congress banned the use of leaded gasoline as fuel in motor vehicles, it did not restrict other potential uses of gasoline containing $\mathrm{Pb}$ or $\mathrm{Pb}$ additives (EPA 1996a). Gasoline produced with $\mathrm{Pb}$ additives continues to be made and marketed for use as fuels in aircraft, race cars, and non-road engines such as 
farm equipment engines and marine engines to the extent allowed by law (EPA 1996a), but tetraethyl Pb has not been produced in the United States since March 1991. All gasoline sold for motor vehicle use since January 1, 1996 has been unleaded (EPA 2020a).

Table 5-5 lists the uses of the specific Pb compounds identified in Chapter 4.

Table 5-5. Current and Former Uses of Selected Lead Compounds

\begin{tabular}{ll}
\hline Compound & Uses \\
\hline Lead(II) acetate & $\begin{array}{l}\text { Dyeing of textiles, waterproofing, varnishes, lead driers, chrome pigments, } \\
\text { gold cyanidation process, insecticide, anti-fouling paints, analytical reagent, } \\
\text { hair dye }\end{array}$ \\
\hline Lead(II) azide & $\begin{array}{l}\text { Primary detonating compound for high explosives, firing of Pb-based } \\
\text { ammunition }\end{array}$ \\
\hline Lead(II) bromide & $\begin{array}{l}\text { Photopolymerization catalyst, inorganic filler in fire-retardant plastics, } \\
\text { general purpose welding flux }\end{array}$ \\
\hline Lead(II) carbonate & $\begin{array}{l}\text { Polymerization catalyst, component of high pressure lubricating greases, } \\
\text { coating on vinyl chloride polymers }\end{array}$ \\
\hline Lead(II) chloride & Preparation of lead salts, lead chromate pigments, analytical reagent \\
\hline Lead(II) chromate & $\begin{array}{l}\text { Pigment in industrial paints, rubber, plastics, ceramic coatings; organic } \\
\text { analysis }\end{array}$ \\
\hline Lead(II) tetrafluoroborate & $\begin{array}{l}\text { Salt for electroplating lead; can be mixed with stannous fluoborate to } \\
\text { electroplate any composition of tin and lead as an alloy }\end{array}$ \\
\hline Lead(II) iodide & Bronzing, printing, photography, cloud seeding \\
\hline Lead molybdenum chromate & Analytical chemistry, pigments \\
\hline Lead(II) nitrate & $\begin{array}{l}\text { Lead salts, mordant in dyeing and printing calico, matches, mordant for } \\
\text { staining mother of pearl, oxidizer in the dye industry, sensitizer in } \\
\text { photography, explosives, tanning, process engraving, and lithography }\end{array}$ \\
\hline Lead(II) oxide & $\begin{array}{l}\text { Storage batteries, ceramic cements and fluxes, pottery and glazes, glass, } \\
\text { chromium pigments, oil refining, varnishes, paints, enamels, assay of } \\
\text { precious metal ores, manufacture of red lead, cement (with glycerol), acid- } \\
\text { resisting compositions, match-head compositions, other lead compounds, } \\
\text { rubber accelerator }\end{array}$ \\
\hline Tetraethyl lead & Stabilizing agent in plastics \\
\hline Lead(II) phosphate & Primary explosive, firing of Pb-based ammunition \\
\hline Lead(II) styphnate & $\begin{array}{l}\text { Storage batteries, paints, ceramics, pigments, electrical and other vinyl } \\
\text { compounds requiring high heat stability }\end{array}$ \\
\hline Lead(II) sulfate & $\begin{array}{l}\text { Ceramics, infrared radiation detector, semi-conductor, ceramic glaze, } \\
\text { source of lead }\end{array}$ \\
\hline Anti-knock agent in aviation gasoline \\
\hline
\end{tabular}

Sources: Boileau et al. 1987; Carr 1995; Carr et al. 2004; Davidson et al. 2014

$\mathrm{Pb}$ arsenate, basic $\mathrm{Pb}$ arsenate, and $\mathrm{Pb}$ arsenite were formerly used as herbicides, insecticides, or rodenticides. Until the 1960s, they were widely used to control pests in fruit orchards, especially apple 
orchards (EPA 2002c; PAN Pesticides Database 2004; Peryea 1998; Wisconsin DHS 2002). All insecticidal use of $\mathrm{Pb}$ arsenate was officially banned on August 1, 1988. However, all registrations for its insecticidal use had lapsed before that time.

\subsubsection{Disposal}

Secondary (recycled) $\mathrm{Pb}$, derived mainly from scrapped $\mathrm{Pb}$-acid batteries, accounted for $100 \%$ of refined $\mathrm{Pb}$ production in the United States in 2014. Almost all of the Pb recycled in 2014 was recovered by 7 companies operating 12 plants in Alabama, California, Florida, Indiana, Minnesota, Missouri, New York, Pennsylvania, Tennessee, and Texas (USGS 2016). More than 99\% of all battery Pb is recycled and new batteries contain between 60 and 80\% recycled $\mathrm{Pb}$ and plastic, respectively (BCI 2019). Scrap $\mathrm{Pb}$ is also recovered from dross, dust, residue, and sludge generated by smelting of metals, $\mathrm{Pb}$ pipe and sheet, printing materials, sheaths from power and telephone cable, and vehicle wheel weights (USGS 2014).

Disposal of wastes containing $\mathrm{Pb}$ or $\mathrm{Pb}$ compounds is controlled by several federal regulations (see Chapter 7). Pb is listed as a toxic substance under Section 313 of the Emergency Planning and Community Right to Know Act (EPCRA) under Title III of the Superfund Amendments and Reauthorization Act (SARA) (EPA 1988). Pb-containing waste products include storage batteries, ammunition waste, ordnance, sheet $\mathrm{Pb}$, solder, pipes, traps, and other metal products; solid waste and tailings from $\mathrm{Pb}$ mining; items covered with Pb-based paint; and solid wastes created by mineral ore processing, iron and steel production, copper and zinc smelting, and the production and use of various Pb-containing products (EPA 1982a).

In the United States., federal laws require, used nickel cadmium (Ni-Cd) and lead (Pb) batteries to be managed as Universal Waste and recycled or disposed of in accordance under Title 40 Parts 266 and 273 of the Code of Federal Regulations (EPA 2020b). The Mercury-Containing and Rechargeable Battery Management Act (the Battery Act) of 1996 removed certain barriers to the recycling of batteries including small, sealed lead acid (SSLA) batteries (EPA 2002b). The intent was to provide the efficient and costeffective collection and recycling or proper disposal of batteries to keep them out of the waste stream. The Act established uniform national labeling requirements, mandated that batteries under the Act be “easily removable” from consumer products where possible, made the Universal Waste Rule effective in all 50 states for the collection, storage, and transportation of batteries covered by the Battery Act, and 
required EPA to establish a public education program on battery recycling and the proper handling and disposal of used batteries (EPA 1997a).

According to data from the TRI, total disposal of $\mathrm{Pb}$ and $\mathrm{Pb}$ compounds varied during the period of 20052015 from 387 million pounds in 2009 to 832 million pounds in 2013, with an overall increase of 20\% during this time period. The metal mining sector contributes most to the disposal of $\mathrm{Pb}$ and $\mathrm{Pb}$ compounds, with metal mines reporting 85\% of total $\mathrm{Pb}$ and $\mathrm{Pb}$ compound releases in 2015.

\subsection{RELEASES TO THE ENVIRONMENT}

Facilities with $\geq 10$ full-time employees in certain industry sectors (e.g., manufacturing) covered by the TRI (e.g., manufacturing) must submit data to TRI on releases and other waste management for TRIlisted chemicals ( $\mathrm{Pb}$ and $\mathrm{Pb}$ compounds are TRI listed). Therefore, TRI data do not reflect all sources of $\mathrm{Pb}$ releases (EPA 2005a). TRI-covered facilities are required to report information to the TRI only if they employ the equivalent of $\geq 10$ full-time employees; if their facility is included in Standard Industrial Classification (SIC) Codes 10 (except 1011, 1081, and 1094), 12 (except 1241), 20-39, 4911 (limited to facilities that combust coal and/or oil for the purpose of generating electricity for distribution in commerce), 4931 (limited to facilities that combust coal and/or oil for the purpose of generating electricity for distribution in commerce), 4939 (limited to facilities that combust coal and/or oil for the purpose of generating electricity for distribution in commerce), 4953 (limited to facilities regulated under RCRA Subtitle C, 42 U.S.C. section 6921 et seq.), 5169, 5171, and 7389 (limited S.C. section 6921 et seq.), 5169, 5171, and 7389 (limited to facilities primarily engaged in solvents recovery services on a contract or fee basis); and if their facility produces, imports, or processes $>25,000$ pounds of any TRI chemical or otherwise uses $>10,000$ pounds of a TRI chemical in a calendar year (EPA 2005a).

Starting in 2001, the threshold to trigger reporting of $\mathrm{Pb}$ in most $\mathrm{Pb}$ compounds was reduced to 100 pounds. The higher threshold still applies to $\mathrm{Pb}$ contained in stainless steel, brass, or bronze alloys. The threshold for $\mathrm{Pb}$ is determined using the weight of the metal, whereas the threshold for $\mathrm{Pb}$ compounds is determined by the weight of the entire compound. Prior to 1998, only facilities classified within the SIC codes 20-39 (Manufacturing Industries) were required to report. After 1998, the industries required to report were enlarged to include other industrial sectors, such as metal mining, coal mining, electrical utilities, and hazardous waste treatment (EPA 2001). 
$\mathrm{Pb}$ is a naturally-occurring element that is typically found combined in various minerals. It occurs in the Earth's crust primarily as the mineral galena ( $\mathrm{PbS})$, and to a lesser extent as anglesite $\left(\mathrm{PbSO}_{4}\right)$ and cerussite $\left(\mathrm{PbCO}_{3}\right.$ ) (Carr et al. 2004; Davidson et al. 2014; Haynes 2014). Pb minerals are found in association with zinc, copper, and iron sulfides as well as gold, silver, bismuth, and antimony minerals. It also occurs as a trace element in coal, oil, and wood. Typical Pb concentrations in some ores and fuels are: copper ores, 11,000 ppm; Pb and zinc ores, 24,000 ppm; gold ores, 6.60 ppm; bituminous coal, 3111 ppm; crude oil, 0.31 ppm; No. 6 fuel oil, 1 ppm; and wood, 20 ppm (EPA 2001).

Leaded gasoline remains commercially available for off-road uses, including aircraft, racing cars, farm equipment, and marine engines. Currently, the largest contributor to atmospheric $\mathrm{Pb}$ emissions in the United States is piston-engine aircraft emissions (EPA 2016c). Industrial sources of Pb can result from the mining and smelting of $\mathrm{Pb}$ ores, as well as other ores in which $\mathrm{Pb}$ is a byproduct or contaminant. Fuel combustion also contributes to releases of $\mathrm{Pb}$ to the environment. As a result of these processes, $\mathrm{Pb}$ may be released to land, water, and air. Many of the anthropogenic sources of $\mathrm{Pb}$ have been eliminated or phased out because of Pb’s persistence, bioaccumulative nature, and toxicity. These include $\mathrm{Pb}$-based paint in 1978, Pb-containing pesticides in 1988, and $\mathrm{Pb}$ in gasoline for use in on-road vehicles in 1996. In early 2017, the use of Pb ammunition and Pb sinkers was banned on most federal lands; however, this ban was temporarily halted soon after. Because $\mathrm{Pb}$ does not degrade and remains in the environment long after its release, these former uses continue to be a potential source for $\mathrm{Pb}$ exposure.

\subsubsection{Air}

According to the TRI, in 2018, a total of 57,240 pounds of Pb were released to air from 4,064 reporting facilities (TRI18 2020). In addition, a total of 343,142 pounds of $\mathrm{Pb}$ compounds were released to air from 3,789 reporting facilities (TRI18 2020). Tables 5-6 and 5-7 list amounts of $\mathrm{Pb}$ and $\mathrm{Pb}$ compounds released from these facilities grouped by state, respectively. 
Table 5-6. Releases to the Environment from Facilities that Produce, Process, or Use Lead ${ }^{\mathrm{a}}$

\begin{tabular}{|c|c|c|c|c|c|c|c|c|c|}
\hline \multirow[b]{3}{*}{ State $^{c}$} & \multirow[b]{3}{*}{$\mathrm{RF}^{\mathrm{d}}$} & \multicolumn{8}{|c|}{ Reported amounts released in pounds per yearb } \\
\hline & & \multirow[b]{2}{*}{ Air } & \multirow[b]{2}{*}{ Water $^{f}$} & \multirow[b]{2}{*}{$U^{g}$} & \multirow[b]{2}{*}{ Land $^{\mathrm{h}}$} & \multirow[b]{2}{*}{ Other ${ }^{\mathrm{i}}$} & \multicolumn{3}{|c|}{ Total release } \\
\hline & & & & & & & On-site & Off-site $^{k}$ & $\begin{array}{l}\text { On- and } \\
\text { off-site }\end{array}$ \\
\hline AK & 4 & 1 & 0 & 0 & 22,766 & 0 & 22,167 & 600 & 22,767 \\
\hline $\mathrm{AL}$ & 105 & 2,944 & 1,133 & 11 & $1,481,278$ & 7,384 & $1,483,641$ & 9,109 & $1,492,750$ \\
\hline AR & 66 & 2,318 & 107 & 0 & 176,475 & 730 & 177,674 & 1,955 & 179,629 \\
\hline$A Z$ & 52 & 103 & 129 & 0 & 42,201 & 331 & 40,794 & 1,970 & 42,765 \\
\hline $\mathrm{CA}$ & 179 & 662 & 1,313 & 0 & 585,305 & 58,212 & 572,870 & 72,623 & 645,493 \\
\hline $\mathrm{CO}$ & 34 & 296 & 20 & 0 & 108,291 & 51 & 108,414 & 244 & 108,658 \\
\hline $\mathrm{CT}$ & 42 & 52 & 541 & 0 & 2 & 5,360 & 62 & 5,893 & 5,955 \\
\hline DC & 6 & 1 & 2 & 0 & 6,493 & 1,000 & 6,464 & 1,031 & 7,495 \\
\hline $\mathrm{DE}$ & 6 & 9 & 3 & 89 & 7,852 & 0 & 7,864 & 89 & 7,954 \\
\hline $\mathrm{FL}$ & 221 & 693 & 89 & 1 & 98,375 & 15,249 & 84,880 & 29,527 & 114,408 \\
\hline $\mathrm{GA}$ & 107 & 3,397 & 1,000 & 0 & 18,503 & 8,671 & 20,456 & 11,114 & 31,571 \\
\hline GU & 1 & 28 & 0 & 0 & 0 & 0 & 0 & 0 & 28 \\
\hline $\mathrm{HI}$ & 2 & 0 & 0 & 0 & 10,359 & 0 & 10,358 & 1 & 10,359 \\
\hline IA & 114 & 10,241 & 80 & 849 & 6,205 & 12,559 & 14,396 & 15,538 & 29,934 \\
\hline ID & 27 & 268 & 45 & 0 & 367,560 & 29,707 & 367,635 & 29,945 & 397,579 \\
\hline $\mathrm{IL}$ & 228 & 3,147 & 2,286 & 27 & 649,364 & 43,310 & 613,450 & 84,683 & 698,133 \\
\hline IN & 160 & 1,056 & 272 & 22 & 454,088 & 557,867 & 7,434 & $1,005,871$ & $1,013,305$ \\
\hline $\mathrm{KS}$ & 70 & 536 & 26 & 0 & 24,749 & 729 & 13,856 & 12,183 & 26,039 \\
\hline $\mathrm{KY}$ & 79 & 891 & 509 & 0 & 32,991 & 5,941 & 30,618 & 9,715 & 40,333 \\
\hline LA & 45 & 511 & 967 & 13 & 22,715 & 462 & 22,763 & 1,905 & 24,669 \\
\hline MA & 55 & 70 & 10,245 & 28 & 36,566 & 3,555 & 15,467 & 34,998 & 50,465 \\
\hline MD & 34 & 25 & 59 & 0 & 13,986 & 86 & 13,467 & 689 & 14,156 \\
\hline $\mathrm{ME}$ & 20 & 3 & 23 & 0 & 219 & 123 & 39 & 329 & 368 \\
\hline $\mathrm{MI}$ & 147 & 2,095 & 396 & 11 & 41,090 & 5,359 & 19,114 & 29,837 & 48,951 \\
\hline $\mathrm{MN}$ & 123 & 1,199 & 232 & 5 & 4,017 & 624 & 1,971 & 4,106 & 6,077 \\
\hline $\mathrm{MO}$ & 92 & 483 & 225 & 1 & 20,478 & 729 & 12,844 & 9,072 & 21,915 \\
\hline MS & 62 & 1,102 & 35 & 1 & 63,143 & 1,327 & 63,240 & 2,368 & 65,608 \\
\hline MT & 13 & 16 & 2 & 0 & 36,873 & 0 & 36,872 & 20 & 36,892 \\
\hline $\mathrm{NC}$ & 162 & 567 & 708 & 248 & 122,679 & 1,295 & 16,540 & 108,957 & 125,497 \\
\hline ND & 13 & 1 & 229 & 0 & 6,038 & 7 & 6,130 & 144 & 6,275 \\
\hline $\mathrm{NE}$ & 61 & 4,505 & 1,379 & 0 & 79,160 & 9,063 & 83,397 & 10,709 & 94,107 \\
\hline $\mathrm{NH}$ & 29 & 17 & 6 & 0 & 726 & 26,933 & 743 & 26,939 & 27,682 \\
\hline $\mathrm{NJ}$ & 42 & 198 & 27 & 17 & 3,286 & 4,976 & 1,419 & 7,086 & 8,505 \\
\hline $\mathrm{NM}$ & 16 & 12 & 0 & 0 & 6,472 & 61 & 5,582 & 963 & 6,545 \\
\hline NV & 27 & 502 & 1 & 1 & $2,554,095$ & 150 & $2,554,538$ & 210 & $2,554,748$ \\
\hline
\end{tabular}




\begin{tabular}{|c|c|c|c|c|c|c|c|c|c|}
\hline \multirow[b]{3}{*}{ State $^{c}$} & \multirow[b]{3}{*}{$\mathrm{RF}^{\mathrm{d}}$} & \multicolumn{8}{|c|}{ Reported amounts released in pounds per year ${ }^{b}$} \\
\hline & & \multirow[b]{2}{*}{ Air ${ }^{\mathrm{e}}$} & \multirow[b]{2}{*}{ Water $^{f}$} & \multirow[b]{2}{*}{$\mathrm{UI}^{\mathrm{g}}$} & \multirow[b]{2}{*}{ Land $^{\mathrm{h}}$} & \multirow[b]{2}{*}{ Other ${ }^{\mathrm{i}}$} & \multicolumn{3}{|c|}{ Total release } \\
\hline & & & & & & & On-site & Off-site & $\begin{array}{l}\text { On- and } \\
\text { off-site }\end{array}$ \\
\hline NY & 140 & 499 & 89 & 0 & 21,001 & 9,770 & 14,560 & 16,799 & 31,359 \\
\hline $\mathrm{OH}$ & 236 & 4,321 & 540 & 232 & 68,683 & 33,151 & 34,929 & 71,997 & 106,926 \\
\hline OK & 54 & 451 & 11 & 14 & 81,644 & 3,422 & 81,961 & 3,581 & 85,542 \\
\hline OR & 48 & 77 & 45 & 0 & $1,495,571$ & 742 & $1,494,474$ & 1,961 & $1,496,435$ \\
\hline PA & 183 & 6,456 & 1,908 & 425 & 167,238 & 486,904 & 12,164 & 650,767 & 662,931 \\
\hline PR & 4 & 4 & 0 & 0 & 3,777 & 47 & 3,780 & 48 & 3,828 \\
\hline $\mathrm{RI}$ & 14 & 10 & 1 & 0 & 3 & 5,086 & 10 & 5,091 & 5,101 \\
\hline $\mathrm{SC}$ & 80 & 517 & 212 & 0 & 43,446 & 947 & 35,803 & 9,319 & 45,123 \\
\hline SD & 14 & 100 & 0 & 0 & 0 & 25 & 100 & 25 & 126 \\
\hline $\mathrm{TN}$ & 109 & 756 & 222 & 1 & 33,156 & 8,866 & 21,918 & 21,083 & 43,001 \\
\hline$T X$ & 325 & 1,223 & 1,027 & 2,833 & 468,642 & 45,651 & 468,737 & 50,638 & 519,376 \\
\hline UT & 49 & 835 & 24 & 0 & 95,346 & 80 & 89,493 & 6,793 & 96,286 \\
\hline $\mathrm{VA}$ & 93 & 1,176 & 574 & 17 & 184,110 & 26,197 & 83,245 & 128,828 & 212,074 \\
\hline $\mathrm{VI}$ & 3 & 2 & 0 & 0 & 31 & 0 & 24 & 8 & 32 \\
\hline $\mathrm{VT}$ & 11 & 1 & 6 & 0 & 16,304 & 397 & 16,289 & 419 & 16,708 \\
\hline WA & 54 & 115 & 184 & 0 & 382,285 & 212,687 & 371,196 & 224,075 & 595,272 \\
\hline $\mathrm{WI}$ & 163 & 1,921 & 638 & 0 & 35,520 & 13,924 & 5,242 & 46,762 & 52,004 \\
\hline WV & 30 & 410 & 190 & 52 & 144,313 & 38 & 144,201 & 803 & 145,004 \\
\hline$W Y$ & 10 & 416 & 1 & 0 & 47,579 & 1 & 47,733 & 263 & 47,996 \\
\hline Total & 4,064 & 57,240 & 27,764 & 4,901 & $10,393,047$ & $1,649,785$ & $9,363,048$ & $2,769,688$ & $12,132,737$ \\
\hline
\end{tabular}

aThe TRI data should be used with caution since only certain types of facilities are required to report. This is not an exhaustive list. Data are rounded to nearest whole number.

bData in TRI are maximum amounts released by each facility.

cPost office state abbreviations are used.

dNumber of reporting facilities.

eThe sum of fugitive and point source releases are included in releases to air by a given facility.

fSurface water discharges, waste water treatment-(metals only), and publicly owned treatment works (POTWs) (metal and metal compounds).

gClass I wells, Class II-V wells, and underground injection.

hesource Conservation and Recovery Act (RCRA) subtitle C landfills; other onsite landfills, land treatment, surface impoundments, other land disposal, other landfills.

iStorage only, solidification/stabilization (metals only), other off-site management, transfers to waste broker for disposal, unknown

iThe sum of all releases of the chemical to air, land, water, and underground injection wells.

kTotal amount of chemical transferred offOsite, including to POTWs.

$\mathrm{RF}=$ reporting facilities; $\mathrm{UI}=$ underground injection

Source: TRI18 2020 (Data are from 2018) 
Table 5-7. Releases to the Environment from Facilities that Produce, Process, or Use Lead Compounds ${ }^{a}$

\begin{tabular}{|c|c|c|c|c|c|c|c|c|c|}
\hline \multirow[b]{3}{*}{ State $^{\mathrm{c}}$} & \multirow[b]{3}{*}{$\mathrm{RF}^{\mathrm{d}}$} & \multicolumn{8}{|c|}{ Reported amounts released in pounds per year ${ }^{b}$} \\
\hline & & \multirow[b]{2}{*}{ Aire } & \multirow[b]{2}{*}{ Water $^{f}$} & \multirow[b]{2}{*}{ UI ${ }^{g}$} & \multirow[b]{2}{*}{ Land $^{\mathrm{h}}$} & \multirow[b]{2}{*}{ Other $^{i}$} & \multicolumn{3}{|c|}{ Total release } \\
\hline & & & & & & & On-site & Off-site ${ }^{k}$ & $\begin{array}{l}\text { On- and } \\
\text { off-site }\end{array}$ \\
\hline AK & 15 & 19,345 & 139 & 0 & $499,679,935$ & 1,006 & $499,696,960$ & 3,465 & $499,700,425$ \\
\hline $\mathrm{AL}$ & 130 & 6,801 & 4,859 & 0 & $1,254,651$ & 4,503 & $1,167,133$ & 103,682 & $1,270,814$ \\
\hline$\overline{\mathrm{AR}}$ & 67 & 5,268 & 1,098 & 0 & 481,839 & 53,191 & 239,131 & 302,264 & 541,396 \\
\hline$A Z$ & 64 & 64,290 & 103 & 0 & $27,547,626$ & 7,110 & $27,600,302$ & 18,827 & $27,619,128$ \\
\hline CA & 305 & 5,816 & 435 & 0 & $2,656,973$ & 27,145 & $1,127,596$ & $1,562,773$ & $2,690,369$ \\
\hline $\mathrm{CO}$ & 76 & 2,931 & 244 & 1 & $10,195,005$ & 239 & $10,180,490$ & 17,930 & $10,198,420$ \\
\hline CT & 39 & 445 & 565 & 0 & 32,116 & 14,510 & 708 & 46,929 & 47,637 \\
\hline$\overline{D C}$ & 1 & 0 & 0 & 0 & 24 & 0 & 24 & 0 & 24 \\
\hline $\mathrm{DE}$ & 5 & 127 & 17 & 0 & 489 & 1,070 & 599 & 1,103 & 1,703 \\
\hline $\mathrm{FL}$ & 150 & 5,429 & 1,691 & 1 & 972,024 & 2,470 & 946,498 & 35,116 & 981,615 \\
\hline $\mathrm{GA}$ & 107 & 6,011 & 4,228 & 0 & 573,267 & 242 & 361,887 & 221,860 & 583,747 \\
\hline GU & 3 & 30 & 0 & 0 & 1,510 & 0 & 1,540 & 0 & 1,540 \\
\hline $\mathrm{HI}$ & 14 & 2,406 & 35 & 18 & 57,605 & 4 & 57,568 & 2,501 & 60,068 \\
\hline IA & 66 & 3,101 & 568 & 4 & 98,007 & 47,899 & 35,369 & 114,209 & 149,578 \\
\hline ID & 31 & 1,337 & 105 & 0 & 587,617 & 1,071 & 588,601 & 1,529 & 590,130 \\
\hline $\mathrm{IL}$ & 163 & 10,458 & 3,316 & 158 & $1,044,749$ & 382,696 & 219,957 & $1,221,420$ & $1,441,377$ \\
\hline IN & 147 & 26,832 & 60,668 & 86 & $3,554,799$ & $1,702,496$ & $1,345,912$ & $3,998,968$ & $5,344,880$ \\
\hline $\mathrm{KS}$ & 40 & 2,171 & 126 & 35 & 33,471 & 7,290 & 31,594 & 11,499 & 43,092 \\
\hline $\mathrm{KY}$ & 64 & 15,233 & 719 & 272 & 758,794 & 116,393 & 739,954 & 151,456 & 891,411 \\
\hline LA & 82 & 8,193 & 8,065 & 303 & 694,825 & 3,582 & 504,890 & 210,078 & 714,968 \\
\hline $\mathrm{MA}$ & 53 & 888 & 357 & 0 & 20,874 & 2,919 & 2,655 & 22,382 & 25,037 \\
\hline $\mathrm{MD}$ & 35 & 209 & 203 & 0 & 4,611 & 55,261 & 1,443 & 58,842 & 60,285 \\
\hline $\mathrm{ME}$ & 13 & 750 & 510 & 0 & 23,485 & 8,099 & 4,729 & 28,116 & 32,844 \\
\hline $\mathrm{MI}$ & 100 & 4,220 & 979 & 13 & $2,091,026$ & 18,914 & $1,514,558$ & 600,594 & $2,115,152$ \\
\hline $\mathrm{MN}$ & 50 & 2,961 & 291 & 0 & 980,965 & 2,228 & 97,635 & 888,810 & 986,445 \\
\hline $\mathrm{MO}$ & 73 & 12,990 & 1,919 & 206 & $18,739,390$ & 243,396 & $18,118,610$ & 879,290 & $18,997,900$ \\
\hline $\mathrm{MP}$ & 1 & 2 & 0 & 0 & 1 & 0 & 3 & 0 & 3 \\
\hline $\mathrm{MS}$ & 50 & 2,260 & 1,770 & 197,869 & 261,610 & 3,066 & 235,034 & 231,540 & 466,575 \\
\hline MT & 20 & 3,438 & 20 & 4,503 & $6,091,825$ & 581 & $6,092,803$ & 7,565 & $6,100,367$ \\
\hline $\mathrm{NC}$ & 145 & 10,843 & 973 & 0 & 728,520 & 35,993 & 551,282 & 225,047 & 776,329 \\
\hline ND & 19 & 4,103 & 19 & 0 & 90,847 & 5,984 & 76,746 & 24,207 & 100,953 \\
\hline $\mathrm{NE}$ & 24 & 1,473 & 125 & 0 & 50,473 & 529 & 46,977 & 5,623 & 52,600 \\
\hline $\mathrm{NH}$ & 14 & 20 & 23 & 0 & 1,509 & 6,243 & 94 & 7,702 & 7,796 \\
\hline $\mathrm{NJ}$ & 54 & 871 & 12,266 & 0 & 410,073 & 234,474 & 62,349 & 595,336 & 657,685 \\
\hline NM & 17 & 902 & 283 & 1 & $2,978,296$ & 31,276 & $2,893,375$ & 117,383 & $3,010,758$ \\
\hline $\mathrm{NV}$ & 49 & 9,161 & 1 & 0 & $49,276,056$ & 40 & $49,282,572$ & 2,685 & $49,285,258$ \\
\hline$\overline{N Y}$ & 82 & 2,294 & 1,167 & 0 & 209,644 & 26,579 & 132,597 & 107,087 & 239,684 \\
\hline
\end{tabular}


Table 5-7. Releases to the Environment from Facilities that Produce, Process, or Use Lead Compounds ${ }^{a}$

\begin{tabular}{|c|c|c|c|c|c|c|c|c|c|}
\hline \multirow[b]{3}{*}{ State $^{c}$} & \multirow[b]{3}{*}{$\mathrm{RF}^{\mathrm{d}}$} & \multicolumn{8}{|c|}{ Reported amounts released in pounds per yearb } \\
\hline & & \multirow[b]{2}{*}{ Air } & \multirow[b]{2}{*}{ Water $^{f}$} & \multirow[b]{2}{*}{$\mathrm{UI}^{\mathrm{g}}$} & \multirow[b]{2}{*}{ Land $^{\mathrm{h}}$} & \multirow[b]{2}{*}{ Other ${ }^{i}$} & \multicolumn{3}{|c|}{ Total release } \\
\hline & & & & & & & On-site & Off-site ${ }^{k}$ & $\begin{array}{l}\text { On- and } \\
\text { off-site }\end{array}$ \\
\hline $\mathrm{OH}$ & 175 & 9,346 & 17,583 & 5,952 & $1,483,307$ & 263,386 & $1,093,350$ & 686,224 & $1,779,574$ \\
\hline OK & 80 & 11,075 & 378 & 58 & 307,485 & 945 & 307,785 & 12,157 & 319,941 \\
\hline OR & 55 & 1,591 & 2,127 & 0 & 5,176 & 1,827 & 6,272 & 4,449 & 10,721 \\
\hline $\mathrm{PA}$ & 195 & 13,623 & 15,874 & 0 & $3,488,454$ & 229,953 & $3,176,873$ & 571,031 & $3,747,904$ \\
\hline $\mathrm{PR}$ & 11 & 759 & 13 & 0 & 236 & 1,300 & 772 & 1,536 & 2,308 \\
\hline $\mathrm{RI}$ & 19 & 23 & 15 & 0 & 1,018 & 124 & 24 & 1,155 & 1,179 \\
\hline $\mathrm{SC}$ & 100 & 4,198 & 1,783 & 0 & 459,973 & 38,536 & 101,830 & 402,660 & 504,490 \\
\hline SD & 11 & 149 & 0 & 0 & $1,757,845$ & 9 & $1,757,955$ & 48 & $1,758,003$ \\
\hline $\mathrm{TN}$ & 89 & 4,151 & 2,653 & 184 & $3,375,608$ & 11,693 & $2,953,656$ & 440,633 & $3,394,289$ \\
\hline$T X$ & 323 & 14,625 & 2,732 & 2,309 & $1,365,901$ & 3,582 & $1,170,161$ & 218,988 & $1,389,149$ \\
\hline UT & 40 & 17,378 & 657 & 0 & $185,430,965$ & 41,774 & $185,290,906$ & 199,868 & $185,490,774$ \\
\hline $\mathrm{VA}$ & 82 & 10,922 & 8,300 & 0 & 201,670 & 12,410 & 206,084 & 27,217 & 233,301 \\
\hline $\mathrm{VI}$ & 2 & 13 & 0 & 0 & 0 & 0 & 13 & 0 & 13 \\
\hline $\mathrm{VT}$ & 5 & 10 & 0 & 0 & 117 & 0 & 10 & 117 & 127 \\
\hline WA & 76 & 4,205 & 843 & 0 & $2,976,519$ & 10,118 & $2,925,078$ & 66,607 & $2,991,685$ \\
\hline WI & 98 & 3,448 & 1,598 & 0 & 279,692 & 27,606 & 74,486 & 237,858 & 312,344 \\
\hline $\mathrm{WV}$ & 44 & 3,235 & 920 & 0 & 362,089 & 1,142 & 240,520 & 126,866 & 367,385 \\
\hline$\overline{W Y}$ & 16 & 783 & 2 & 0 & 79,050 & 284 & 67,659 & 12,461 & 80,119 \\
\hline Total & 3,789 & 343,142 & 163,361 & 211,975 & $833,759,635$ & $3,693,189$ & $823,333,608$ & $14,837,694$ & $838,171,302$ \\
\hline
\end{tabular}

aThe TRI data should be used with caution since only certain types of facilities are required to report. This is not an exhaustive list. Data are rounded to nearest whole number.

${ }^{b}$ Data in TRI are maximum amounts released by each facility.

'Post office state abbreviations are used.

${ }^{\mathrm{d}}$ Number of reporting facilities.

eThe sum of fugitive and point source releases are included in releases to air by a given facility.

fSurface water discharges, waste water treatment (metals only), and publicly owned treatment works (POTWs) (metal and metal compounds).

${ }^{9}$ Class I wells, Class II-V wells, and underground injection.

${ }^{h}$ Resource Conservation and Recovery Act (RCRA) subtitle C landfills; other onsite landfills, land treatment, surface impoundments, other land disposal, other landfills.

iStorage only, solidification/stabilization (metals only), other offsite management, transfers to waste broker for disposal, unknown

iThe sum of all releases of the chemical to air, land, water, and underground injection wells.

kTotal amount of chemical transferred offsite, including to POTWs.

$\mathrm{RF}=$ reporting facilities; $\mathrm{UI}=$ underground injection

Source: TRI18 2020 (Data are from 2018) 
The decrease in national Pb emissions between 1970 to 2011 is estimated to be $99.6 \%$ (220,000 tons), which is mostly attributed to the elimination of leaded gasoline for on-road vehicles. Since 2000, nonroad engines and metals industrial processing have accounted for most of the anthropogenic $\mathrm{Pb}$ emissions in the United States (EPA 2015). Based on data from the National Emissions Inventory (NEI 2014), the following sectors contribute the largest portions of total $\mathrm{Pb}$ emissions in the United States: mobile-aircraft (63\%), industrial processes-not elsewhere classified (6.8\%), industrial processes-ferrous metals (6.8\%), fuel combustion-electric generation-coal (5.5\%), and industrial processes-non-ferrous metals (4.1\%) (EPA 2016c). Historical trends of Pb emissions in the United States are provided in Table 5-8 (EPA 2015).

\section{Table 5-8. Historic Levels of Lead Emissions to the Atmosphere in the United States (in Thousand Metric Tons)}

\begin{tabular}{lccccccccccc}
\hline & 1970 & 1975 & 1980 & 1985 & 1990 & 1995 & 1999 & 2002 & 2005 & 2008 & 2011 \\
\hline On-road vehicles & 172 & 130.2 & 60.5 & 18.1 & 2.17 & 2.05 & 1 & 0 & 0 & 0 & 0 \\
\hline $\begin{array}{l}\text { Metals industrial } \\
\text { processing }\end{array}$ & 24.22 & 9.923 & 3.03 & 2.1 & 0.5 & 0.49 & 0.96 & 0.4 & 0.3 & 0.16 & 0.14 \\
\hline Fuel combustion & 10.62 & 10.35 & 4.3 & 0.52 & 0.42 & 0.02 & 0 & 0.39 & 0.14 & 0.12 & 0.09 \\
\hline Nonroad engines & 9.737 & 6.13 & 4.2 & 0.92 & 0.78 & 0.54 & 0.55 & 0.45 & 0.66 & 0.56 & 0.49 \\
\hline Other sources & 4.331 & 3.053 & 2.12 & 1.31 & 1.11 & 0.83 & 0.84 & 0.43 & 0.25 & 0.11 & 0.1 \\
\hline
\end{tabular}

Source: EPA 2015

According to the data from the NEI, the largest portions of total Pb emissions are in the U.S. mobileaircraft sector. Murphy et al. (2008) studied weekly patterns of metals and other aerosol components using data collected from 2000 to 2006 at Interagency Monitoring of Protected Visual Environments (IMPROVE) sites, and these data suggested that $\mathrm{Pb}$ concentrations were impacted by piston aircraft emissions.

As indicated in Table 5-6, by the early 2000s, transportation (i.e., automotive) emissions were no longer the dominant source of $\mathrm{Pb}$ emitted to the atmosphere. When such emissions were prevalent, $>90 \%$ (mass basis) of automotive $\mathrm{Pb}$ emissions from leaded gasoline were in the form of inorganic particulate matter (e.g., $\mathrm{Pb}$ bromochloride $[\mathrm{PbBrCl}]$ ) and $<10 \%$ (mass basis) were in the form of organolead vapors (e.g., $\mathrm{Pb}$ alkyls). In 1984, the average $\mathrm{Pb}$ content of gasoline was $0.44 \mathrm{~g}$ Pb/gallon (EPA 1986b); however, as of January 1986, the allowable $\mathrm{Pb}$ content of leaded gasoline dropped to $0.1 \mathrm{~g}$ Pb/gallon (EPA 1985d).

Between January and June of 1990, the actual average $\mathrm{Pb}$ concentration in leaded gasoline was $0.085 \mathrm{~g}$ $\mathrm{Pb}$ /gallon, indicating consumption of approximately 230,000 $\mathrm{kg}$ of $\mathrm{Pb}$ for the production of 2.74 billion 
gallons of leaded gasoline. In the early 1980s, EPA allowed up to $0.05 \mathrm{~g}$ of $\mathrm{Pb}$ in a gallon of unleaded gasoline (EPA 1982b).

According to data from TRI, on-site air releases of $\mathrm{Pb}$ and $\mathrm{Pb}$ compounds varied over the same period from 431,311 pounds in 2014 to 1,037,265 pounds in 2006, with an overall decrease of 40\%. In 2018, 91,028 pounds of $\mathrm{Pb}$ and $\mathrm{Pb}$ compounds were released to air. The electric utility and primary metals industry sectors contributed to this overall decrease; both sectors have decreased air $\mathrm{Pb}$ and $\mathrm{Pb}$ compounds releases by approximately $70 \%$ from 2005 to 2015. The primary metal sector, which includes iron and steel manufacturers and smelting operations, contributes the greatest quantity of $\mathrm{Pb}$ and $\mathrm{Pb}$ compounds to air releases (EPA 2017a, 2017b).

While Pb levels in paints for interior use have been restricted since the 1950s, older houses and furniture may still be covered with leaded paint. Releases from Pb-based paints are frequently confined to the area in the immediate vicinity of painted surfaces, and deterioration or removal of the paint by sanding or sandblasting can result in high localized concentrations of $\mathrm{Pb}$ dust in both indoor and outdoor air.

The largest volume of organolead vapors released to the atmosphere results from industrial processes; prior to its phaseout and ban, leaded gasoline containing tetraethyl $\mathrm{Pb}$ as an anti-knock additive was also a major contributor. Tetraalkyl $\mathrm{Pb}$ vapors are photoreactive, and their presence in local atmospheres is transitory. Halogenated $\mathrm{Pb}$ compounds are formed during combustion by reaction of the tetraalkyl $\mathrm{Pb}$ compounds with halogenated $\mathrm{Pb}$ scavenger compounds. These halogenated $\mathrm{Pb}$ compounds ultimately give rise to $\mathrm{Pb}$ oxides and carbonates in the environment (EPA 1985b). Tetraalkyl Pb compounds once contributed $5-10 \%$ of the total particulate $\mathrm{Pb}$ present in the atmosphere. Organolead vapors were most likely to occur in occupational settings (e.g., gasoline transport and handling operations, gas stations, and parking garages) and high-traffic areas (Nielsen 1984).

\subsubsection{Water}

According to the TRI, in 2018, a total of 27,764 pounds of Pb were released to water from 4,064 reporting facilities (TRI18 2020). In addition, a total of 163,361 pounds of $\mathrm{Pb}$ compounds were released to water from 3,789 reporting facilities (TRI18 2020). Tables 5-6 and 5-7 list amounts of $\mathrm{Pb}$ and $\mathrm{Pb}$ compounds released from these facilities grouped by state, respectively. 
The following industry sectors accounted for the majority of release of $\mathrm{Pb}$ to surface water in 2018: chemicals (14\%); paper (12\%); primary metals (10\%); transportation equipment (5\%); and fabricated metals (2\%). The following industry sectors accounted for the majority of release of $\mathrm{Pb}$ compounds to surface water in 2018: paper (20\%); electric utilities (8\%); primary metals (8\%); metal mining (3\%) (TRI18 2020). The trends in discharges of $\mathrm{Pb}$ and $\mathrm{Pb}$ compounds to surface water from 2001 to 2018 are presented in Table 5-9.

Table 5-9. U.S. Surface Water Discharges of Lead and Lead Compounds
(Pounds/Year)

Source: EPA 2017c; TRI18 2020

Data reported by Environment and Climate Change Canada (2016) show that other industries, which include the iron and steel industry, oil and gas industry, and cement and concrete industry, contributed 136.9 tonnes of the total $\mathrm{Pb}$ released to water in 2014. This release includes 134.1 tonnes of $\mathrm{Pb}$ that were released when a dam securing a tailings pond from the Mount Polley mine in central British Columbia breached on August 4, 2014, spilling mining waste into Polley Lake and surrounding waters. Waste, pulp, paper, and paperboard industry, and non-ferrous smelting and refining were the next largest contributors (Table 5-10). In 2013, Pb releases to water were similar for other industries and waste. 
Table 5-10. Canada Surface Water Discharges of Lead and Lead Compounds (Tonnes)

\begin{tabular}{llllll}
\hline Year & $\begin{array}{l}\text { Other } \\
\text { industries }\end{array}$ & Waste & $\begin{array}{l}\text { Pulp, paper, and } \\
\text { paperboard industry }\end{array}$ & $\begin{array}{l}\text { Non-ferrous smelting } \\
\text { and refining }\end{array}$ & Other sources \\
\hline 2003 & 4.38 & 15.49 & 2.55 & 1.74 & 0.18 \\
\hline 2004 & 3.97 & 11.53 & 2.84 & 2.26 & 0.26 \\
\hline 2005 & 6.11 & 9.47 & 3.29 & 1.82 & 0.58 \\
\hline 2006 & 5 & 9.9 & 2.35 & 1.65 & 0.24 \\
\hline 2007 & 3.63 & 6.42 & 2.37 & 1.64 & 0.19 \\
\hline 2008 & 4.76 & 11.58 & 2.42 & 2.04 & 0.16 \\
\hline 2009 & 3.39 & 8.49 & 2.25 & 2.13 & 0.19 \\
\hline 2010 & 3.21 & 11.97 & 2.12 & 1.45 & 0.14 \\
\hline 2011 & 3.65 & 8.97 & 2.91 & 1.5 & 0.16 \\
\hline 2012 & 4.66 & 4.69 & 2.8 & 1.75 & 0.12 \\
\hline 2013 & 4.17 & 4.66 & 2.42 & 1.48 & 0.13 \\
\hline 2014 & 136.92 & 5.11 & 1.85 & 1.77 & 0.13 \\
\hline
\end{tabular}

Source: Environment and Climate Change Canada (2016)

Urban runoff and atmospheric deposition are significant indirect sources of $\mathrm{Pb}$ found in the aquatic environment. Pb reaching surface waters is sorbed to suspended solids and sediments (EPA 1982a; EPA 2006, 2014c).

$\mathrm{Pb}$ is released into surface water from $\mathrm{Pb}$ shot and $\mathrm{Pb}$ sinkers. A study of a shooting range in Southwestern Virginia found that the dissolved $\mathrm{Pb}$ content of surface water ranged up to $473 \mathrm{ppb}$, with the highest concentrations closest to the backstop (Craig et al. 1999). Upstream from the site, the $\mathrm{Pb}$ concentration was 0.5 ppb. In 1991, the U.S. Fish and Wildlife Service banned the use of Pb shot when hunting waterfowl, such as geese or ducks, in order to avoid releasing $\mathrm{Pb}$ directly to surface water.

\subsubsection{Soil}

According to the TRI, in 2018, a total of 10,393,047 pounds of $\mathrm{Pb}$ were released to the land, both on-site and off-site, by 4,064 reporting facilities (TRI18 2020). Table 5-6 lists amounts of Pb released from these facilities grouped by state. In addition, a total of 833,759,635 pounds of $\mathrm{Pb}$ compounds were released to land, both on-site and off-site, by 3,789 reporting facilities (TRI18 2020). Table 5-7 lists amounts of Pb compounds released from these facilities grouped by state. In addition, 27,764 and 211,975 pounds of $\mathrm{Pb}$ and $\mathrm{Pb}$ compounds, respectively, were injected underground. 
$\mathrm{Pb}$-containing material from home and commercial use may be sent to municipal landfills. It is important to note that land is the ultimate repository for $\mathrm{Pb}$, and $\mathrm{Pb}$ released to air and water ultimately is deposited in soil or sediment. For example, $\mathrm{Pb}$ released to the air from leaded gasoline or in stack gas from smelters and power plants will settle on soil, sediment, foliage, or other surfaces. The heaviest contamination occurs near the highway, in the case of leaded gasoline, or near the facility, in the case of a power plant or smelter. Road dust contributes to $\mathrm{Pb}$ in soil. Pb concentrations were higher in surface soils within $1,000 \mathrm{~m}$ of roadways (134 kg/ha) as compared to outside the 1,000-m region (38.7 kg/ha) (Yesilonis et al. 2008). Wheel weights can contribute to releases of $\mathrm{Pb}$ along roadways. Aucott and Caldarelli (2012) estimated that approximately 12 tons of $\mathrm{Pb}$ as wheel weights are deposited on New Jersey roadways; however, they estimated that only a small amount enters the environment as small particulate from grinding. Root (2000) also estimated a rate of Pb deposition in Albuquerque, New Mexico as 50$70 \mathrm{~kg} / \mathrm{km} /$ year. However, use of $\mathrm{Pb}$ wheel weights are on the decline due to legislation, voluntary phaseout, and new wheel technology (Aucott and Caldarelli 2012).

\subsubsection{Paint}

Although the sale of residential Pb-based paint was banned in the United States in 1978, flaking paint, paint chips, and weathered powdered paint, which are most commonly associated with deteriorated housing stock in urban areas, remain major sources of $\mathrm{Pb}$ exposure for young children residing in these houses, particularly for children afflicted with pica (the compulsive, habitual consumption of nonfood items) (Bornschein et al. 1986; EPA 1986b). Pb concentrations of $1-5 \mathrm{mg} / \mathrm{cm}^{2}$ have been found in chips of Pb-based paint (Billick and Gray 1978), suggesting that consumption of a single chip of paint would provide greater short-term exposure than any other source of Pb (EPA 1986b). An estimated 40-50\% of occupied housing in the United States may contain Pb-based paint on exposed surfaces (Chisolm 1986).

In the late 1980s, the U.S. Department of Housing and Urban Development (HUD) conducted a national survey of Pb-based paint in housing. The EPA subsequently sponsored a comprehensive technical report on the HUD-sponsored survey to provide estimates of the extent of Pb-based paint in housing. In the EPA report, a home is considered to have Pb-based paint if the measured $\mathrm{Pb}$ concentration on any painted surface is $\geq 1.0 \mathrm{mg} / \mathrm{cm}^{2}$. The EPA report estimates that 64 million ( \pm 7 million) homes, or $83 \%$ ( $\pm 9 \%$ ) of privately-owned housing units built before 1980, have Pb-based paint somewhere in the building. Approximately 12 million ( \pm 5 million) of these homes are occupied by families with children under the age of 7 years. Approximately 49 million ( \pm 7 million) privately owned homes have Pb-based paint in 
their interiors. By contrast, approximately 86\% ( $\pm 8 \%$ ) of all pre-1980 public housing family units have Pb-based paint somewhere in the building (EPA 1995b).

Damaged Pb-based paint is associated with excessive dust $\mathrm{Pb}$ levels. Approximately 14 million homes (19\% of pre-1980 housing) have $>5$ square feet of damaged Pb-based paint, and nearly half (47\%) of those homes have excessive dust Pb levels (EPA 1995b).

In the Cincinnati prospective $\mathrm{Pb}$ study of public and private low- and moderate-income housing, the $\mathrm{Pb}$ concentration ranges were: painted interior walls, $0.1-35 \mathrm{mg} / \mathrm{cm}^{2}$; interior home surface dust, 0.04$39 \mathrm{mg} / \mathrm{m}^{2}$ and $72-16,200 \mu \mathrm{g} / \mathrm{g}$; interior home dustfall, 0.0040-60 mg/m²/30 days; exterior dust scrapings, 20-108,000 $\mu \mathrm{g} / \mathrm{g}$; and dust on children's hands, 1-191 $\mu \mathrm{g}$. The Pb levels in older private deteriorating or dilapidated housing were higher than the levels in newer public and rehabilitated housing (Clark et al. 1985).

Releases from Pb-based paints are frequently confined to the area in the immediate vicinity of painted surfaces, and deterioration or removal of the paint can result in high localized concentrations of $\mathrm{Pb}$ in dust in air (from sanding and sandblasting) and on exposed surfaces. A study was conducted in New Orleans where power sanding is a common practice during repainting old houses; median, $90^{\text {th }}$ percentile, and maximum $\mathrm{Pb}$ concentrations in 31 study houses were 35, 126, and $257 \mathrm{mg} / \mathrm{g}$, respectively (Mielke et al. 2001). Pb concentrations in dust and soil samples from one study of a house where the paint chips contained about $90 \mathrm{mg} \mathrm{Pb} / \mathrm{g}$ were very high. If the house had been sanded down to bare wood, $7.4 \mathrm{~kg}$ of $\mathrm{Pb}$ would have been released to the environment. Disturbance of older structures containing Pb-based paints is now a significant contributor to total $\mathrm{Pb}$ releases.

The authors of a report of findings from NHANES III, conducted in 1988-1991, commented that of the multiple sources of exposure, $\mathrm{Pb}$-based paint is the principal high-dose source of $\mathrm{Pb}$. Exposure occurs not only through the direct ingestion of flaking and chalking paint, but also through the inhalation of dust and soil contaminated with paint (Brody et al. 1994). According to a study by the New York State Department of Health, renovation and remodeling activities that disturb Pb-based paints in homes can produce significant amounts of $\mathrm{Pb}$ dust, which can be inhaled or ingested (CDC 1997a). 


\subsection{ENVIRONMENTAL FATE}

The atmosphere is the main environmental transport media for $\mathrm{Pb}$ that is deposited onto surface water and soils (EPA 2006, 2014c). Upon release to the atmosphere, Pb particles are dispersed and ultimately removed from the atmosphere by wet or dry deposition. $\mathrm{Pb}$ deposition is typically greatest closer to $\mathrm{Pb}$ emission sources. An important factor in determining the atmospheric transport of $\mathrm{Pb}$ is particle size distribution. Large particles settle out of the atmosphere more rapidly and are deposited relatively close to emission sources and smaller particles may be transported much farther distances. After deposition, particles may be resuspended and redeposited. The cycling of $\mathrm{Pb}$ in aquatic environments is governed by chemical, biological, and mechanical processes. The exchange between sediment and surface water will be affected by $\mathrm{pH}$, ionic strength, formation of organic complexes with $\mathrm{Pb}$ ions, and oxidation-reduction potential of the environment (EPA 2006, 2014c).

\subsubsection{Transport and Partitioning}

Transport and partitioning of $\mathrm{Pb}$ in the environment is an interplay of various processes (EPA 2014c). Global atmospheric deposition of $\mathrm{Pb}$ peaked in the 1970s and has declined since then; however, these deposits are still in the environment and can be transported and partitioned between environmental compartments. Past and current releases of $\mathrm{Pb}$ to the air result in the deposition of $\mathrm{Pb}$ on land and in surface water. While soil is a repository for $\mathrm{Pb}$, it is not a passive repository, and resuspension of $\mathrm{Pb}$ contaminated soil-derived dust particulates can contribute to $\mathrm{Pb}$ exposure (Laidlaw and Filippelli 2008; Laidlaw et al. 2012). Pb in soil can be washed off surfaces into waters, and within water, it can partition between water and sediments (EPA 2006, 2014c).

Air. EPA (2006) summarized that the major pathway for the transport of $\mathrm{Pb}$ in the environment is the atmosphere and that airborne $\mathrm{Pb}$ tends to be in the form of submicron aerosols, which can travel large distances. After release to the atmosphere, $\mathrm{Pb}$ particles are dispersed and ultimately removed from the atmosphere by wet or dry deposition. Dry deposition was the major removal process for $\mathrm{Pb}$ in coarse particulate matter and wet deposition was the most important removal process for fine particulate matter. Soil-bound $\mathrm{Pb}$ and contaminated road dust can be resuspended and can be a significant source of airborne $\mathrm{Pb}$ in areas near major sources of Pb emissions (EPA 2006, 2014c).

In the atmosphere, non-organic compounds of $\mathrm{Pb}$ exist primarily in the particulate form. The median particle distribution for $\mathrm{Pb}$ emissions from smelters is $1.5 \mu \mathrm{m}$, with $86 \%$ of the particle sizes under $10 \mu \mathrm{m}$ 
(Corrin and Natusch 1977). The smallest Pb-containing particulate matter $(<1 \mu \mathrm{m})$ is associated with high-temperature combustion processes. Upon release to the atmosphere, $\mathrm{Pb}$ particles are dispersed and ultimately removed from the atmosphere by wet or dry deposition. Approximately $40-70 \%$ of the deposition of $\mathrm{Pb}$ is by wet fallout; $20-60 \%$ of particulate $\mathrm{Pb}$ once emitted from automobiles is deposited near the source. An important factor in determining the atmospheric transport of $\mathrm{Pb}$ is particle size distribution. Large particles, particularly those with aerodynamic diameters of $>2 \mu \mathrm{m}$, settle out of the atmosphere more rapidly and are deposited relatively close to emission sources (e.g., $25 \mathrm{~m}$ from the roadway for those size particles emitted in motor vehicle exhaust in the past); smaller particles may be transported thousands of kilometers away from the emission source.

The amount of $\mathrm{Pb}$ scavenged from the atmosphere by wet deposition varies widely; wet deposition can account for $40-70 \%$ of $\mathrm{Pb}$ deposition depending on such factors as geographic location and amount of emissions in the area (Nielsen 1984). An annual scavenging ratio (concentration in precipitation, mg/L, to concentration in air, $\mu \mathrm{g} / \mathrm{m}^{3}$ ) of $0.18 \times 10^{-6}$ has been calculated for $\mathrm{Pb}$, making it the lowest value among seven trace metals studied (iron, aluminum, manganese, copper, zinc, cadmium); this indicates that $\mathrm{Pb}$ (which initially exists as fine particles in the atmosphere) is removed from the atmosphere by wet deposition relatively inefficiently.

While $\mathrm{Pb}$ particles from automobile emissions are quite relatively small $(<0.1 \mu \mathrm{m}$ in diameter), they may coagulate to form larger particulates (Chamberlain et al. 1979). Pb has been found in sediment cores of lakes in Ontario and Quebec, Canada far from any point sources of Pb releases, suggesting that longrange atmospheric transport was occurring (Evans and Rigler 1985). Sabin and Schiff (2008) reported that median dry deposition fluxes along a coastal transect in southern California ranged from 0.52 to $14 \mu \mathrm{g} / \mathrm{m}^{2}$-day in 2006. Pb fluxes ranged from 20 to $330 \mu \mathrm{g} / \mathrm{m}^{2}$-day in 1975. Osterberg et al. (2008) reported elevated concentrations of $\mathrm{Pb}$ in a 1970-1998 ice core from the summit of Mt. Logan, Canada, and indicated that elevated levels correspond to increased industrial activity in Asia over the same time period. Mean $\mathrm{Pb}$ concentrations in the 1970-1998 portion were $68.9 \mathrm{ng} / \mathrm{L}$, more than 10 -fold above the natural background (5.6 ng/L).

$\mathrm{Pb}$ in soil in urban areas of older cities may be a source of airborne $\mathrm{Pb}$ (Laidlaw and Filippelli 2008). Studies of the $\mathrm{Pb}$ species found in airborne particulate matter collected in El Paso, Texas found that $\mathrm{Pb}$ humate was the dominant form of $\mathrm{Pb}$ in air samples. Pb-humate, a stable, sorbed complex formed in the humus fraction of $\mathrm{Pb}$ contaminated soil, is the major Pb species in soils in El Paso (Pingitore et al. 2009). In a review, Cho et al. (2011) noted that, over the past 40 years, lead-bound air particulates have shifted to 
larger air particulate sizes as concentrations of $\mathrm{Pb}$ in urban areas have decreased. They note that this shift has occurred as the use of leaded gasoline was phased-out and that industrial emissions and resuspension of road dust became more important sources of $\mathrm{Pb}$. In addition to soil-derived dust, re-entrainment of dusts near highways and deteriorating $\mathrm{Pb}$-based paint from elevated steel structures can contribute to airborne $\mathrm{Pb}$ (Sabin et al. 2006; Weiss et al. 2006). Studies suggest that there is long-range transport of $\mathrm{Pb}$ bound to particulate matter from industrial emissions. Dust samples from surface glaciers and in dust traps in remote areas on the west coast of New Zealand's South Island were identified as being both Australian and New Zealand in origin. Samples were enriched in metals, including $\mathrm{Pb}$, and the degree of metal enrichment indicted that they were transported from eastern Australia (Marx et al. 2008).

Water. The amount of soluble $\mathrm{Pb}$ in surface waters depends upon the $\mathrm{pH}$ and the ionic strength of the water. Equilibrium calculations show that at $\mathrm{pH}>5.4$, the total solubility of $\mathrm{Pb}$ is approximately $30 \mu \mathrm{g} / \mathrm{L}$ in hard water and approximately $500 \mu \mathrm{g} / \mathrm{L}$ in soft water. Sulfate ions, if present in soft water, limit the $\mathrm{Pb}$ concentration in solution through the formation of $\mathrm{Pb}$ sulfate. Above $\mathrm{pH}$ 5.4, the $\mathrm{Pb}$ carbonates, $\mathrm{PbCO}_{3}$ and $\mathrm{Pb}_{2}(\mathrm{OH})_{2} \mathrm{CO}_{3}$, limit the amount of soluble $\mathrm{Pb}$. The carbonate concentration is in turn dependent upon the partial pressure of carbon dioxide, $\mathrm{pH}$, and temperature (EPA 1986b).

A significant fraction of $\mathrm{Pb}$ carried by river water is expected to be in an undissolved form, which can consist of colloidal particles or larger undissolved particles of $\mathrm{Pb}$ carbonate, $\mathrm{Pb}$ oxide, $\mathrm{Pb}$ hydroxide, or other $\mathrm{Pb}$ compounds incorporated in other components of surface particulate matter from runoff. $\mathrm{Pb}$ may occur either as sorbed ions or surface coatings on sediment mineral particles, or it may be carried as a part of suspended living or nonliving organic matter in water.

Sediment and Soil. EPA (2006, 2014c) reviewed and summarized the factors affecting the behavior of $\mathrm{Pb}$ in soil. While $\mathrm{Pb}$ is relatively immobile in soil and has a long retention time in most soils, it has some capacity to leach through the soil column and potentially contaminate groundwater. $\mathrm{Pb}$ sorbs strongly to soil components and is only weakly soluble in pore water, making the leaching of $\mathrm{Pb}$ in soil a slower process as compared to other contaminants. Various soil conditions and characteristics affect the sorbing capacity of the soil and the solubility of contaminants including hydraulic conductivity of the soils, composition of the soil solution, organic matter, clay mineral content of the soil, $\mathrm{pH}$, and microbial activity (EPA 2006). In soil, Pb can be partitioned between the soil water, precipitate forms, secondary iron and manganese oxides, carbonates, organic matter, sulfides, or the surfaces of clay, humus, or silicate particles. Pb adsorbed to the surfaces of colloid soil particles (e.g., organic matter, clay, oxides, and carbonates) are the most labile fraction. High chloride content in soil also enhances Pb solubility. At low 
$\mathrm{pH}$, metal species bound to carbonates, hydroxides, and other soil components are more likely to dissolve into solution, increasing rates of $\mathrm{Pb}$ migration through the soil. EPA (2014c) reported that soil $\mathrm{pH}$ is the most important factor affecting solubility, mobility, and phytoavailability of $\mathrm{Pb}$ in soil; however, reducing conditions (e.g., anoxia) in soil also increase $\mathrm{Pb}$ mobility. In addition, dissolved organic matter is more important than iron oxyhydroxides in Pb mobility in soil.

The fate of $\mathrm{Pb}$ in soil is affected by the adsorption at mineral interfaces, precipitation of sparingly soluble solid forms of the compound, and formation of relatively stable organic-metal complexes or chelates with soil organic matter. These processes are dependent on such factors as soil $\mathrm{pH}$, soil type, particle size, organic matter content of soil, presence of inorganic colloids and iron oxides, cation exchange capacity (CEC), and amount of Pb in soil (Getz et al. 1977; Reddy et al. 1995). Soil samples were extracted from the Powder River Basin in Wyoming to determine the relative distribution and speciation of $\mathrm{Pb}$ and other metals in acidic environments (Reddy et al. 1995). At near neutral $\mathrm{pH}$, organic carbon-Pb complexes were the predominant species in the soil water extracts. At low $\mathrm{pH}$, dissolved $\mathrm{Pb}$ in ionic form $\left(\mathrm{Pb}^{2+}\right)$ and ion pairs (e.g., $\mathrm{PbSO}_{4}$ ) were the predominant species. It was concluded that the mobility of $\mathrm{Pb}$ will increase in environments having low $\mathrm{pH}$ due to the enhanced solubility of $\mathrm{Pb}$ under acidic conditions. The accumulation of $\mathrm{Pb}$ in most soils is primarily a function of the rate of deposition from the atmosphere. Most $\mathrm{Pb}$ is retained strongly in soil, and very little is transported through runoff to surface water or leached to groundwater except under acidic conditions (EPA 1986b; Getz et al. 1977). Clays, silts, iron and manganese oxides, and soil organic matter can bind metals electrostatically (cation exchange) as well as chemically (specific adsorption) (Reed et al. 1995). Although sorption to organic matter in soil limits the rate and extent of leaching, $\mathrm{Pb}$ may enter surface waters as a result of erosion of $\mathrm{Pb}$-containing soil particulates. Pb bromochloride, the primary form of $\mathrm{Pb}$ emitted from motor vehicles, which once burned leaded gasoline in the presence of organohalogen scavenger compounds, is converted to the less-soluble $\mathrm{Pb}$ sulfate either by reactions in the atmosphere or by reactions at the soil surface, thus limiting its mobility in soil. It has been determined that $\mathrm{Pb}$ oxides, carbonates, oxycarbonates, sulfates, and oxysulfates become the most prominent constituents of aged automobile exhaust particles (i.e., those collected at locations more remote from traffic sources) (Ter Haar and Bayard 1971). Pb may also be immobilized by ion exchange with hydrous oxides or clays or by chelation with humic or fulvic acids in the soil (Olson and Skogerboe 1975). In soils with $\mathrm{pH} \geq 5$ and with at least $5 \%$ organic matter content, atmospheric $\mathrm{Pb}$ is retained in the upper $2-5 \mathrm{~cm}$ of undisturbed soil. Inorganic $\mathrm{Pb}$ may be bound into crystalline matrices of rocks and remain essentially immobile; it can also occur in water entrapped in soil macro- and micropores (Reed et al. 1995). In soil with high organic matter content and a $\mathrm{pH}$ of 6-8, $\mathrm{Pb}$ may form insoluble organic $\mathrm{Pb}$ complexes; if the soil has less organic matter at the same $\mathrm{pH}$, hydrous $\mathrm{Pb}$ 
oxide complexes may form or $\mathrm{Pb}$ may precipitate out with carbonate or phosphate ions. At a pH of 4-6, the organic Pb complexes become soluble and leach out or may be taken up by plants (EPA 1986b). Entrainment or suspension of soil particles in moving air is another route of Pb transport (EPA 1982c). This process may be important in contributing to the atmospheric burden of $\mathrm{Pb}$ around some $\mathrm{Pb}$ smelting facilities and NPL sites that contain elevated levels of Pb in soil.

The downward movement of elemental $\mathrm{Pb}$ and inorganic $\mathrm{Pb}$ compounds from soil to groundwater by leaching is very slow under most natural conditions except for highly acidic situations (Getz et al. 1977). The conditions that induce leaching are the presence of $\mathrm{Pb}$ in soil at concentrations that either approach or exceed the CEC of the soil, the presence of materials in soil that are capable of forming soluble chelates with $\mathrm{Pb}$, and a decrease in the $\mathrm{pH}$ of the leaching solution (e.g., acid rain) (Getz et al. 1977). Favorable conditions for leaching may be present in some soils near Pb smelting and NPL sites. Tetraalkyl Pb compounds, such as tetraethyl $\mathrm{Pb}$, are insoluble in water and would not be expected to leach in soil. However, they can be transported through a soil column when it is present in a migrating plume of gasoline (USAF 1995). In aqueous media, tetraalkyl Pb compounds are first degraded to their respective ionic trialkyl $\mathrm{Pb}$ species and are eventually mineralized to inorganic $\mathrm{Pb}\left(\mathrm{Pb}^{2+}\right)$ by biological and chemical degradation processes (Ou et al. 1995).

In a study of Pb migration in forest soils in Vermont, Miller and Friedland (1994) used Pb deposition time series and measurements of organic soil horizon Pb content made in 1966, 1980, and 1990 to compute dynamic response times for $\mathrm{Pb}$ storage in several types of soil. The authors concluded that maximum $\mathrm{Pb}$ concentrations in organic soil occurred around 1980, with concentrations of about $85 \mu \mathrm{g} / \mathrm{g}$ in soils of the northern hardwood forests of the study area and about $200 \mu \mathrm{g} / \mathrm{g}$ in soils of the spruce-fir forests. The large surge of atmospheric $\mathrm{Pb}$ deposited in these forests during the time when leaded gasoline was routinely used in motor vehicles is being redistributed in the soil profile rather than being retained in the organic horizon. Based on an analysis of $\mathrm{Pb}$ transit times through mineral soil horizons, the pulse of $\mathrm{Pb}$ may begin to be released to upland streams sometime in the middle of the next century (Miller and Friedland 1994). However, Wang et al. (1995) observed that Pb migration in forest soils is slowed considerably due to a decrease in solubility when $\mathrm{Pb}$ moves from the soil surface horizon to streams. Their results suggest that $\mathrm{Pb}$ is effectively trapped in the subsurface soil horizons, which may greatly reduce its release to streams.

Lewis et al. (2010) studied the distribution, chemical speciation, and mobility of $\mathrm{Pb}$ and antimony from small arms ammunition in a coarse-grained surface sand and reported that the transport of $\mathrm{Pb}$ was small in 
this soil type. Ninety-three percent of the mass of the bullets was found in the top $30 \mathrm{~cm}$ of the sand. $\mathrm{Pb}$ was mostly associated with the following grain sizes in decreasing order $>5.0 \mathrm{~mm}(\sim 3.3 \mathrm{~g} / \mathrm{kg}), 1.2-$ $5.0 \mathrm{~mm}(\sim 1.5 \mathrm{~g} / \mathrm{kg})$, and $<0.06 \mathrm{~mm}(\sim 0.25 \mathrm{~m} / \mathrm{kg})$. In the 0.06-0.6 mm fractions, Pb concentrations were just above background levels $(0.0004 \mathrm{~g} / \mathrm{kg})$. Declining concentrations with depth has also been observed in clay/loam shooting range soils (Vantelon et al. 2005). $\mathrm{Pb}$ in the fine fraction ( $<2 \mathrm{~mm}$ ) shooting range soils also showed a depth distribution, with the highest concentrations in the top $10 \mathrm{~cm}$ (Cao et al. 2003a, 2003b; Hui et al. 2002; Lin et al. 1995; Perroy et al. 2014; Selonen et al. 2012). In a study of various contaminant levels in soil at a major training facility used for testing military tanks and munitions, $\mathrm{Pb}$ concentrations in the $0-15 \mathrm{~cm}$ soil depth ranged from 249.2 to $1,963.7 \mathrm{mg} / \mathrm{kg}$ (Berthelot et al. 2008).

Flooding events can change the spatial distribution of $\mathrm{Pb}$ in soil and sediments (EPA 2014c). Zahran et al. (2010) and Presley et al. (2010) reported variations in Pb concentrations in soil samples from schoolyards in New Orleans, Louisiana before and after Hurricanes Katrina and Rita in 2005, with some sites increasing and others decreasing in Pb concentrations. Forty-six census tracts in New Orleans were sampled before and after Hurricanes Katrina and Rita; 29 of these showed a decline in Pb concentrations, with 6 samples $>400 \mathrm{mg} / \mathrm{kg}$. Prior to these hurricanes, 15 of 46 samples had $\mathrm{Pb}$ concentrations $>400 \mathrm{mg} / \mathrm{kg}$. Across the tracts, the average median $\mathrm{Pb}$ concentration decreased from 328.5 to $203.33 \mathrm{mg} / \mathrm{kg}$ (Zahran et al. 2010). Presley et al. (2010) reported similar trends. Of the 17 schoolyard sites that were sampled, 7 sites had concentrations exceeding Pb concentrations of $400 \mathrm{mg} / \mathrm{kg}$ in June 2005, and in January 2006, Pb concentrations at 3 sites exceeded this concentration. The geometric mean concentration of the sites decreased from 290.0 to $207.4 \mathrm{mg} / \mathrm{kg}$; however, at two sites, $\mathrm{Pb}$ concentrations increased from 804.0 to $1,740.0 \mathrm{mg} / \mathrm{kg}$ and from $1,090.0$ to 2,500.0 mg/kg. During a 4-day storm event, 2,400 tonnes of suspended particulate matter were transported in a historical mining, ore processing, and smelting region in the Czech Republic that contained various metals including 2,954 kg of $\mathrm{Pb}$ (Žak et al. 2009).

Other Media. Plants and animals may bioconcentrate Pb, but biomagnification is not expected. In general, the highest $\mathrm{Pb}$ concentrations are found in aquatic and terrestrial organisms with habitats near $\mathrm{Pb}$ mining, smelting, and refining facilities; storage battery recycling plants; areas affected by high automobile and truck traffic; sewage sludge and spoil disposal areas; sites where dredging has occurred; areas of heavy hunting and fishing ( $\mathrm{Pb}$ from spent shot or sinkers); and urban and industrialized areas. $\mathrm{Pb}$ may be present on plant surfaces as a result of atmospheric deposition; its presence in internal plant tissues indicates biological uptake from the soil and leaf surfaces. Although the bioavailability of $\mathrm{Pb}$ in soil to plants is limited because of the strong adsorption of $\mathrm{Pb}$ to soil organic matter, bioavailability 
increases with increased soil organic matter content and with decreased soil $\mathrm{pH}$ (more acidic). Plants grown in $\mathrm{Pb}$-contaminated soils were shown to accumulate low levels of $\mathrm{Pb}$ in the edible portions of the plant from adherence of dusts and translocation into the tissues (Finster et al. 2004). Thirty-two different types of fruits or vegetables were grown in urban gardens with soils containing high $\mathrm{Pb}$ levels (27$4,580 \mathrm{mg} / \mathrm{kg}$ ). Samples were harvested and washed with either water or detergents and analyzed for $\mathrm{Pb}$ content. Only one fruiting vegetable among 52 samples contained $\mathrm{Pb}$ levels greater than the detection limit of $10 \mu \mathrm{g} / \mathrm{g}$ in the edible portion. However, $39 \%$ of the leafy vegetables and herbs had $\mathrm{Pb}$ levels $>10 \mu \mathrm{g} / \mathrm{g}$ in the edible shoot portion following washing of the vegetables with detergent and water (Finster et al. 2004).

$\mathrm{Pb}$ may be taken up in edible plants from the soil via the root system, by direct foliar uptake and translocation within the plant, and by surface deposition of particulate matter. The amount of $\mathrm{Pb}$ in soil that is bioavailable to a vegetable plant depends on factors such as cation exchange capacity, $\mathrm{pH}$, amount of organic matter present, soil moisture content, and type of amendments added to the soil. Background agricultural soil $\mathrm{Pb}$ concentrations for major growing areas of the United States have been determined (Holmgren et al. 1993).

The influence of various combinations of soil amendments on $\mathrm{Pb}$ uptake by soybeans was studied for a metal-contaminated alluvial soil (Pierzynski and Schwab 1993). Addition of limestone was found to be most effective in reducing the bioavailability of metals (including $\mathrm{Pb}$ ) as indicated by the reduction in labile soil metals, increased yields, and decreased soybean tissue metal content. Uptake of metals by lettuce and radishes grown in a loam soil spiked with cadmium chloride and $\mathrm{Pb}$ nitrate (from 100 to $1,000 \mathrm{mg} / \mathrm{kg}$ ) was also studied (Nwosu et al. 1995). Results indicated that the mean uptake of $\mathrm{Pb}$ by lettuce increased as the concentration of $\mathrm{Pb}$ rose in the soil mixture. However, the uptake was low and this finding is inconsistent with other reports. $\mathrm{Pb}$ was not bioaccumulated by either plant regardless of soil $\mathrm{Pb}$ concentrations. The response of kidney bean growth to the concentration and chemical form of $\mathrm{Pb}$ in soils obtained near a zinc smelter in Japan has been studied (Xian 1989). It was found that the amount of $\mathrm{Pb}$ in the total plant (approximately 35-80 $\mu \mathrm{g}$ ) correlated strongly with the concentration of $\mathrm{Pb}$ in the soil ( $0-240 \mathrm{mg} / \mathrm{kg})$. The best relationship was found between the amount of metal uptake and the concentration of exchangeable and carbonate forms of $\mathrm{Pb}$ in the soil.

Uptake of $\mathrm{Pb}$ in animals may occur as a result of inhalation of contaminated ambient air or ingestion of contaminated plants. However, $\mathrm{Pb}$ is not biomagnified in aquatic or terrestrial food chains. Older organisms tend to contain the greatest body burdens of $\mathrm{Pb}$. In aquatic organisms, $\mathrm{Pb}$ concentrations are 
usually highest in benthic organisms and algae, and lowest in upper trophic level predators (e.g., carnivorous fish). Exposure of a fresh water fish to several sublethal concentrations of $\mathrm{Pb}$ for a period of 30 days showed significant accumulation of $\mathrm{Pb}$ in the blood and tissues. The $\mathrm{Pb}$ accumulation in tissues was found to increase with $\mathrm{Pb}$ in water up to a concentration of $5 \mathrm{mg} / \mathrm{L}(\mu \mathrm{g} / \mathrm{mL})$; at concentrations of 10 and $20 \mathrm{mg} / \mathrm{L}$, the $\mathrm{Pb}$ accumulation in the tissues, although indicating an increase, was not proportional to the $\mathrm{Pb}$ concentration in water (Tulasi et al. 1992). High bioconcentration factors (BCFs) were determined in studies using oysters (6,600 for Crassostrea virginica), fresh water algae (92,000 for Senenastrum capricornutum), and rainbow trout (726 for Salmo gairdneri). However, most median BCF values for aquatic biota were significantly lower: 42 for fish, 536 for oysters, 500 for insects, 725 for algae, and 2,570 for mussels (Eisler 1988). Pb is toxic to all aquatic biota, and organisms higher up in the food chain may experience $\mathrm{Pb}$ poisoning as a result of eating $\mathrm{Pb}$-contaminated food. Organolead compounds, such as trialkyl and tetraalkyl $\mathrm{Pb}$ compounds, are more toxic than inorganic forms and have been shown to bioconcentrate in aquatic organisms.

Biomagnification of organolead compounds has not been found to occur. Depuration is relatively rapid, with half-life values of 30-45 hours for rainbow trout exposed to tetramethyl $\mathrm{Pb}$. Tetraalkyl $\mathrm{Pb}$ compounds are more toxic than trialkyl Pb compounds, and ethyl forms are more toxic than methyl forms (Eisler 1988). Isolation of a Pseudomonas aeruginosa strain designated CHL004, which is able to remove $\mathrm{Pb}$ from solidified media and soil, has been reported (Vesper et al. 1996). The rate of uptake of $\mathrm{Pb}$ nitrate by CHL004 was very rapid initially and then decreased greatly.

\subsubsection{Transformation and Degradation}

As an element, $\mathrm{Pb}$ cannot be degraded in the environment, but may undergo various precipitation or ligand exchange reactions. Pb will typically be found in compounds with oxygen and sulfur, and may undergo oxidation-reduction reactions under different environmental conditions. Under most environmental conditions, $\mathrm{Pb}$ will most likely exist in its $\mathrm{Pb}(\mathrm{II})$ oxidation state. $\mathrm{Pb}$ can be complexed by various ligands present in the environment (e.g., fulvic and humic acids). Despite forming complexes with organic matter, it is unlikely that it would be incorporated into organic compounds under environmental conditions. Transformations of $\mathrm{Pb}$ compounds that occur during their movement through the environment will be between various inorganic compounds.

Air. According to EPA (2014c), Pb accumulated on airborne mineral dusts can be transformed into different compounds during transport. It was also noted that $\mathrm{Pb}$ can accumulate on coarse particulate 
matter during transport in air and undergo chemical transformations. For example, $\mathrm{Pb}$ sulfate $\left(\mathrm{PbSO}_{4}\right)$, one of the main components of Pb-containing aerosols from coal combustion, can react with calcite $\left(\mathrm{CaCO}_{3}\right)$ in particulate matter to form various $\mathrm{Pb}$ carbonate compounds on the calcite surface. Another study included in the discussion noted that $\mathrm{Pb}$ levels in the $\mathrm{PM}_{10}$ fraction from dust storms collected in Israel were enriched with $\mathrm{Pb}$ at levels higher than those found in their source in the Sahara desert, suggesting that the dust samples accumulated $\mathrm{Pb}$ during transit between the Sahara desert and Israel (EPA 2014c).

Before the ban on sales of leaded gasoline, $\mathrm{Pb}$ particles were emitted to the atmosphere from automobile exhaust as $\mathrm{Pb}$ halides (mostly $\mathrm{PbBrCl}$ ) and as double salts with ammonium halides (e.g., $2 \mathrm{PbBrCl} \cdot \mathrm{NH}_{4} \mathrm{Cl}$, $\mathrm{Pb}_{3}\left[\mathrm{PO}_{4}\right]_{2}$, and $\mathrm{PbSO}_{4}$ ) (Biggins and Harrison 1979; Ter Haar and Bayard 1971). After 18 hours, approximately $75 \%$ of the bromine and $30-40 \%$ of the chlorine was released, and $\mathrm{Pb}$ carbonates, oxycarbonates, and oxides were produced. These $\mathrm{Pb}$ oxides are subject to further weathering to form additional carbonates and sulfates (Olson and Skogerboe 1975). Pb particles are emitted from mines and smelters primarily in the form of elemental $\mathrm{Pb}$ and $\mathrm{Pb}$-sulfur compounds, $\mathrm{PbSO}_{4}, \mathrm{PbO} \cdot \mathrm{PbSO}_{4}$, and $\mathrm{PbS}$ (Corrin and Natusch 1977; EPA 1986b; Spear et al. 1998). The Pb emitted from the combustion of waste oil was found to be in the form of $\mathrm{PbCl}_{2}, \mathrm{PbO}$, and elemental $\mathrm{Pb}\left(\mathrm{Pb}^{0}\right)$ (Nerin et al. 1999). In the atmosphere, $\mathrm{Pb}$ exists primarily in the form of $\mathrm{PbSO}_{4}$ and $\mathrm{PbCO}_{3}$ (EPA 1986b).

While $\mathrm{Pb}$ is no longer added to gasoline for on-road use, the inorganic $\mathrm{Pb}$ degradation products of these organolead compounds may still be present in the environment. Based on the vapor pressure of tetraethyl $\mathrm{Pb}\left(0.26 \mathrm{mmHg}\right.$ at $\left.25^{\circ} \mathrm{C}\right)$ and tetramethyl $\mathrm{Pb}\left(26.0 \mathrm{mmHg}\right.$ at $\left.20^{\circ} \mathrm{C}\right)$, these two compounds exist almost entirely in the vapor phase in the atmosphere (Eisenreich et al. 1981). When exposed to sunlight, they decompose rapidly to trialkyl and dialkyl $\mathrm{Pb}$ compounds, and eventually to inorganic $\mathrm{Pb}$ oxides by a combination of direct photolysis, reaction with hydroxyl radicals, and reaction with ozone. The half-life of tetraethyl $\mathrm{Pb}$ in reactions with hydroxyl radicals during summer is approximately 5.7 hours, based on a rate constant of $6.8 \times 10^{-11} \mathrm{~cm}^{3} /$ molecule-second (Nielsen et al. 1991). The half-life for tetramethyl $\mathrm{Pb}$ is about 65 hours, based on a rate constant of $5.9 \times 10^{-12} \mathrm{~cm}^{3} /$ molecule-second. In the winter, both compounds have half-lives of up to several days since the concentration of atmospheric hydroxyl radicals is lower than in summer months (DeJonghe and Adams 1986).

Water. The fate of $\mathrm{Pb}$ in water will be determined by the conditions of the water, including acidity $(\mathrm{pH})$, ionic strength, oxidation-reduction potential, flow rate, and amount and composition of suspended materials (EPA 2014c). The $\mathrm{pH}$ of water is an important factor in determining the fate of $\mathrm{Pb}$ in water. At 
neutral to more basic $\mathrm{pH}, \mathrm{Pb}$ will tend to be complexed, precipitated, or sorbed to suspended sediments in water (EPA 2014c). Pb will form compounds of low solubility with the major anions found in natural waters. The maximum solubility of $\mathrm{Pb}$ in hard water is about $30 \mu \mathrm{g} / \mathrm{L}$ at $\mathrm{pH}>5.4$ and the maximum solubility of $\mathrm{Pb}$ in soft water is approximately $500 \mu \mathrm{g} / \mathrm{L}$ at $\mathrm{pH}>5.4$ (EPA 1977). In the environment, the divalent form $\left(\mathrm{Pb}^{2+}\right)$ is the stable ionic species of $\mathrm{Pb}$. Hydroxide, carbonate, sulfide, and, more rarely, sulfate may act as solubility controls in precipitating $\mathrm{Pb}$ from water. At $\mathrm{pH}<5.4$, the formation of $\mathrm{Pb}$ sulfate limits the concentration of soluble $\mathrm{Pb}$ in water, while at $\mathrm{pH}>5.4$, the formation of $\mathrm{Pb}$ carbonates limits the amount of soluble $\mathrm{Pb}$ (EPA 1979). The relatively volatile organolead compound, tetramethyl $\mathrm{Pb}$, may form as a result of biological alkylation of organic and inorganic $\mathrm{Pb}$ compounds by microorganisms in anaerobic lake sediments; however, if the water over the sediments is aerobic, volatilization of tetramethyl $\mathrm{Pb}$ from the sediments is not considered to be important because the tetramethyl $\mathrm{Pb}$ will be oxidized (EPA 1979).

The speciation of $\mathrm{Pb}$ was found to differ in fresh water and seawater. In fresh water, $\mathrm{Pb}$ may partially exist as the divalent cation $\left(\mathrm{Pb}^{2+}\right)$ at $\mathrm{pHs}$ below 7.5, but complexes with dissolved carbonate to form insoluble $\mathrm{PbCO}_{3}$ under alkaline conditions (Long and Angino 1977). Even small amounts of carbonate ions formed in the dissolution of atmospheric $\mathrm{CO}_{2}$ are sufficient to keep $\mathrm{Pb}$ concentrations in rivers at the $500 \mu \mathrm{g} / \mathrm{L}$ solubility limit (EPA 1979). Pb chloride and $\mathrm{Pb}$ carbonate are the primary compounds formed in seawater (Long and Angino 1977). The speciation of $\mathrm{Pb}$ in water is also dependent on the presence of other ligands in water. $\mathrm{Pb}$ is known to form strong complexes with humic acid and other organic matter (Denaix et al. 2001; Gao et al. 1999; Guibaud et al. 2003). Pb-organic matter complexes are stable to a $\mathrm{pH}$ of 3 with the affinity increasing with increasing $\mathrm{pH}$, but decreasing with increased water hardness (EPA 1979). In seawater, there is the presence of $\mathrm{Pb}$ complexed to Fe-Mn oxides, which is due to the content of these oxides in seawater (Elbaz-Poulichet et al. 1984). Sorption of $\mathrm{Pb}$ to polar particulate matter in fresh water and estuarine environments is an important process for the removal of $\mathrm{Pb}$ from these surface waters. The adsorption of $\mathrm{Pb}$ to organic matter, clay, and mineral surfaces, and coprecipitation and/or sorption by hydrous iron and manganese oxides increases with increasing pH (EPA 1979).

Sediment and Soil. Pb in its naturally-occurring mineral forms is a component of many soils in the United States. The speciation of $\mathrm{Pb}$ in soils is dependent upon the properties of the soil. In a calcareous soil, $\mathrm{PbSO}_{4}$ and $\mathrm{PbCO}_{3}$ were shown to account for $<5 \%$ of the total $\mathrm{Pb}$ content, whereas in roadside dust, $\mathrm{PbSO}_{4}$, elemental $\mathrm{Pb}, \mathrm{Pb}_{3} \mathrm{O}_{4}, \mathrm{PbO} \cdot \mathrm{PbSO}_{4}$, and $2 \mathrm{PbCO}_{3} \cdot \mathrm{Pb}(\mathrm{OH})_{2}$ were present in significant quantities (Chaney et al. 1988). It was also reported that after adding 3,000-4,000 mg/kg of $\mathrm{Pb}$ in the form of 
$\mathrm{PbSO}_{4}$, subsequent extractions revealed that the $\mathrm{Pb}$ sulfate was rapidly transformed to other $\mathrm{Pb}$ compounds in the soil (Chaney et al. 1988).

Nearly all forms of $\mathrm{Pb}$ that are released to soil from anthropogenic sources, such as elemental $\mathrm{Pb}, \mathrm{PbSO}_{4}$, $\mathrm{PbCO}_{3}, \mathrm{PbS}, \mathrm{Pb}(\mathrm{OH})_{2}, \mathrm{PbCrO}_{4}$, and $\mathrm{PbClBr}$, are transformed by chemical and biotic processes to adsorbed forms in soil (Chaney et al. 1988). The transformation process involves the formation of $\mathrm{Pb}$ complexes with binding sites on clay minerals, humic acid and other organic matter, and hydrous iron oxides (Chaney et al. 1988; Chuan et al. 1996; Sauve et al. 1997). The ability of soils to bind $\mathrm{Pb}$ is dependent on soil pH and the cation exchange capacity of the soil components (e.g., hydrous iron oxides on clay and organic matter) (Chaney et al. 1988; EPA 1986b). Only a small fraction (0.1-1\%) of Pb appears to remain water-soluble in soil (Khan and Frankland 1983). The solubility of $\mathrm{Pb}$ in soil is dependent on $\mathrm{pH}$, being sparingly soluble at $\mathrm{pH} 8$ and becoming more soluble as the $\mathrm{pH}$ approaches 5 (Chuan et al. 1996). Between pH 5 and 3.3, large increases in Pb solubility in soil are observed. These changes in $\mathrm{Pb}$ solubility appear to correlate with the $\mathrm{pH}$-dependent adsorption and dissolution of Fe-Mn oxyhydroxides. In addition to $\mathrm{pH}$, other factors that influence $\mathrm{Pb}$ solubility in soil are total $\mathrm{Pb}$ content and the concentrations of phosphate and carbonate in soils (Bradley and Cox 1988; Ge et al. 2000; Pardo et al. 1990; Sauve et al. 1997).

Large particles of elemental $\mathrm{Pb}$ (e.g., shot and bullet fragments) degrade from weathering processes (Cao et al. 2003a, 2003b). Weathering includes physical transformation of larger particles to smaller particles (particle dissolution), as well as oxidation of the particle surface (coating) to $\mathrm{PbO}_{2}$, with subsequent further oxidation to carbonates, phosphates, and sulfates (Cao et al. 2003a, 2003b; Hardison et al. 2004; Hashimoto 2013; Lewis et al. 2010; Lin et al. 1995; Rooney et al. 2007; Vantenlon et al. 2005). Particle dissolution rates for shotgun pellets in soils have been estimated to range from 1 to $20 \mathrm{mg} / \mathrm{g}$ pellet/year, depending on soil type, precipitation, and vegetation cover (Jorgenson and Willems 1987; Takamatsu et al. 2010).

Since the ban on the use of leaded gasoline, atmospheric $\mathrm{Pb}$ deposition to soil has decreased considerably. However, the deposited organolead compounds and their transformation products remain in the soil. Limited data indicate that tetraethyl and tetramethyl $\mathrm{Pb}$ are converted into water-soluble $\mathrm{Pb}$ compounds in soil through microbial metabolism (Ou et al. 1994). Using an Arredondo fine sand from Florida (92\% sand, $7 \%$ silt, $1 \%$ clay, $11.8 \mathrm{~g} / \mathrm{kg}$ organic carbon, $\mathrm{pH}$ 5.5), tetraethyl $\mathrm{Pb}$ was shown to degrade sequentially to monoionic triethyl $\mathrm{Pb}$, diionic diethyl $\mathrm{Pb}$, and eventually $\mathrm{Pb}^{+2}$ (Ou et al. 1994). Experiments were conducted using non-sterilized and autoclaved soil samples. The presence of 
monoionic triethyl $\mathrm{Pb}$ and diionic diethyl $\mathrm{Pb}$ was generally lower in the autoclaved samples, suggesting that both abiotic and biotic mechanisms are responsible for the degradation of tetraethyl $\mathrm{Pb}$. At the end of a 28-day incubation period, no tetraethyl $\mathrm{Pb}$ was present in the soil; however, there were significant quantities of monoionic triethyl $\mathrm{Pb}$ and diionic diethyl $\mathrm{Pb}$, which suggest that the degradation products are more persistent than the original species. Although tetraethyl and tetramethyl $\mathrm{Pb}$ are not expected to leach significantly through soil, their more water-soluble metabolites may be subject to leaching (EPA 1985a).

$\mathrm{Pb}$ content in plants is largely the result of atmospheric deposition. This is due to the strong retention of particulate matter on plant surfaces that is difficult to remove through washing (EPA 1977). Uptake of Pb into plant tissue appears to involve a combination of uptake from the leaf surface and uptake from roots, with the relative contribution of each pathway dependent on species and soil characteristics (Angelova et al. 2010; Bindler et al. 2008; Chrastny et al. 2010; Cui et al. 2007; Guyette et al. 1991; Hu and Ding 2009; Nwosu et al. 1995). Pb taken up by the root systems remains largely associated with root tissues (Comino et al. 2011; Businelli et al. 2011; Deng et al. 2004; Mellem et al. 2009; Murray et al. 2009; Nan and Cheng 2001; Sonmez et al. 2008; Wang et al. 2011). Translocation from roots to stem and leaf tissue has been shown to occur in some species (Peralta-Videa et al. 2009; Shaheen and Tsadilas 2009; Tamura et al. 2005; Wang et al. 2006; Zaprjanova et al. 2010). Eventually, the Pb will be returned to soil when these plants decay unless they are harvested (to possibly enter the food chain) or removed.

\subsection{LEVELS IN THE ENVIRONMENT}

Reliable evaluation of the potential for human exposure to $\mathrm{Pb}$ depends, in part, on the reliability of supporting analytical data from environmental samples and biological specimens. Concentrations of $\mathrm{Pb}$ in unpolluted atmospheres and in pristine surface waters are often so low as to be near the limits of current analytical methods. In reviewing data on $\mathrm{Pb}$ levels monitored or estimated in the environment, it should also be noted that the amount of chemical identified analytically is not necessarily equivalent to the amount that is bioavailable.

Table 5-11 shows the lowest limit of detections that are achieved by analytical analysis in environmental media. An overview summary of the range of concentrations detected in environmental media is presented in Table 5-12. 
Table 5-11. Lowest Limit of Detection Based on Standards ${ }^{a}$

\begin{tabular}{|c|c|c|}
\hline Media & Detection limit & Reference \\
\hline \multirow[t]{13}{*}{ Air } & $1.5 \mathrm{ng} / \mathrm{cm}^{2}(\mathrm{XRF})$ & EPA, 1999, Method IO-3.3 \\
\hline & $2.6 \mu \mathrm{g} / \mathrm{sample}$ & NIOSH 2017b, Method 7082 \\
\hline & $6 \mu \mathrm{g} / \mathrm{sample}$ & NIOSH 1998, Method 7702 \\
\hline & $0.02 \mu \mathrm{g} / \mathrm{sample}$ & NIOSH 1994c, Method 7105 \\
\hline & $0.05 \mu \mathrm{g} / \mathrm{sample}$ & NIOSH 2016a, Method 7701 \\
\hline & $0.062 \mu \mathrm{g} / \mathrm{filter}$ & NIOSH 2003c, Method 7300 \\
\hline & $0.062 \mu \mathrm{g} /$ filter & NIOSH 2003a, Method 7301 \\
\hline & $0.023 \mu \mathrm{g} / \mathrm{mL}$ & NIOSH 2003b, Method 7303 \\
\hline & $0.6 \mu \mathrm{g} / \mathrm{sample}$ & NIOSH 2014a, Method 7302 \\
\hline & $1 \mu \mathrm{g} /$ sample & NIOSH 2014b, Method 7304 \\
\hline & $0.062 \mu \mathrm{g} / \mathrm{sample}$ & NIOSH 2015, Method 7306 \\
\hline & $0.03 \mu \mathrm{g} / \mathrm{mL}$ & OSHA 2002, Method ID-121 \\
\hline & $2.1 \mu \mathrm{g} / \mathrm{sample}$ & OSHA 2002, Method ID-125G \\
\hline $\begin{array}{l}\text { Drinking } \\
\text { water }\end{array}$ & $\begin{array}{l}1.1 \mu \mathrm{g} / \mathrm{L} \text { (ICP-AES) } \\
0.02 \mu \mathrm{g} / \mathrm{L} \text { (ICP-MS) }\end{array}$ & $\begin{array}{l}\text { EPA } 2003 \text { Method } 200.5 \\
\text { EPA } 1994 f \text { Method } 200.8\end{array}$ \\
\hline \multirow{6}{*}{$\begin{array}{l}\text { Surface water } \\
\text { and } \\
\text { groundwater }\end{array}$} & $\mathrm{r} 0.07 \mu \mathrm{g} / \mathrm{L}$ & EPA 1997b \\
\hline & $\begin{array}{l}2.4 \mu \mathrm{g} / \mathrm{L} \text { (GFAA) } \\
0.28 \mu \mathrm{g} / \mathrm{L} \text { (GFAA with preconcentration) } \\
0.07 \mu \mathrm{g} / \mathrm{L} \text { (ICP-MS) }\end{array}$ & EPA 1997b \\
\hline & $\begin{array}{l}0.05 \mu \mathrm{g} / \mathrm{L}(\mathrm{ICP}-\mathrm{MS}) \\
60 \mu \mathrm{g} / \mathrm{L}(\mathrm{ICP}-\mathrm{OES})\end{array}$ & USGS 1989 \\
\hline & $\begin{array}{l}1 \mu \mathrm{g} / \mathrm{L}(\mathrm{GFAA}) \\
1.1 \mu \mathrm{g} / \mathrm{L}(\mathrm{AVICP}-\mathrm{AES})\end{array}$ & USGS 1993 \\
\hline & $\begin{array}{l}10 \mu \mathrm{g} / \mathrm{L}(\text { ICP) } \\
100 \mu \mathrm{g} / \mathrm{L} \text { (total recoverable, FLAA) } \\
1 \mu \mathrm{g} / \mathrm{L} \text { (whole water recoverable, GFAA) } \\
0.5 \mu \mathrm{g} / \mathrm{L} \text { (dissolved in water by GFAA) } \\
100 \mu \mathrm{g} / \mathrm{L} \text { (suspended recoverable, FLAA) } \\
100 \mu \mathrm{g} / \mathrm{L} \text { (dissolved, FLAA) }\end{array}$ & USGS 1989 \\
\hline & $\begin{array}{l}0.6 \mu \mathrm{g} / \mathrm{L}(\mathrm{ICP}-\mathrm{MS}) \\
0.7 \mu \mathrm{g} / \mathrm{L}(\mathrm{GFAA}) \\
10 \mu \mathrm{g} / \mathrm{L}(\mathrm{ICP}-\mathrm{AES})\end{array}$ & EPA 1994d \\
\hline \multirow[t]{2}{*}{ Soil/sediment } & $\begin{array}{l}0.15 \mu \mathrm{g} / \mathrm{g} \text { (ICP-MS) } \\
0.2 \mu \mathrm{g} / \mathrm{g} \text { (XRF) } \\
0.2 \mu \mathrm{g} / \mathrm{g} \text { (GF-AAS) }\end{array}$ & NOAA 1998 \\
\hline & $10 \mu \mathrm{g} / \mathrm{g}(\mathrm{FLAA})$ & USGS 1989 \\
\hline
\end{tabular}


Table 5-11. Lowest Limit of Detection Based on Standards ${ }^{a}$

\begin{tabular}{lll}
\hline Media & Detection limit & Reference \\
\hline Wipes & $0.042 \mu \mathrm{g} /$ wipe & NIOSH 2003d, Method 9102 \\
\cline { 2 - 3 } & $0.02 \mu \mathrm{g} / \mathrm{cm}^{2}$ for $100-\mathrm{cm}^{2}$ area (FLAA or & NIOSH 1996a, Method 9100 \\
& ICP); & \\
\cline { 2 - 3 } $0.001 \mu \mathrm{g} / \mathrm{cm}^{2}$ for $100-\mathrm{cm}^{2}$ area (GF-AAS) & \\
\cline { 2 - 3 } & Range: $5-15 \mu \mathrm{g} /$ wipe sample & NIOSH 2003e, Method 9105 \\
\hline
\end{tabular}

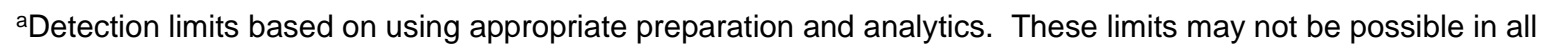
situations.

AES = atomic emission spectroscopy; AVICP = axially viewed inductively coupled plasma; FLAA = flame atomic absorption; GFAA = graphite furnace atomic absorption; GF-AAS = graphite furnace-atomic absorption spectrometer; GRAV = gravimetry; ICP = inductively coupled plasma; MS = mass spectrometry; OES = optical emission spectrometry; $\mathrm{Pb}=$ lead; $\mathrm{XRF}=\mathrm{x}$-ray fluorescence

\section{Table 5-12. Lead Levels in Water, Soil, and Air of National Priorities List (NPL)} Sites

\begin{tabular}{|c|c|c|c|c|c|}
\hline Medium & Median $^{a}$ & $\begin{array}{l}\text { Geometric } \\
\text { mean }^{\mathrm{a}}\end{array}$ & $\begin{array}{l}\text { Geometric } \\
\text { standard } \\
\text { deviation }^{\mathrm{a}}\end{array}$ & $\begin{array}{l}\text { Number of } \\
\text { quantitative } \\
\text { measurements }\end{array}$ & NPL sites \\
\hline Water (ppb) & 75 & 118 & 13.8 & 1,452 & 659 \\
\hline Soil (ppb) & $1,110,000$ & 885,000 & 19.7 & 1,453 & 661 \\
\hline Air (ppbv) & 0.194 & 0.286 & 32.3 & 85 & 51 \\
\hline
\end{tabular}

aConcentrations found in ATSDR site documents from 1981 to 2019 for 1,867 NPL sites (ATSDR 2019). Maximum concentrations were abstracted for types of environmental media for which exposure is likely. Pathways do not necessarily involve exposure or levels of concern.

\subsubsection{Air}

Four national monitoring networks collect data on $\mathrm{Pb}$ concentrations in ambient air to report to the Air Quality System (AQS). State and local agencies carry out monitoring at state and local monitoring stations (SLAMS). These data are primarily used to evaluate compliance with the National Ambient Air Quality Standard (NAAQS) for Pb. Pb levels are also monitored in the Chemical Speciation Network (CSN), Interagency Monitoring of Protected Visual Environments (IMPROVE), and National Air Toxics Trends Station (NATTS) networks. Pb concentrations in air are measured in three particulate matter (PM) size fractions: total suspended particles (TSP), $\mathrm{PM}_{10}$, and $\mathrm{PM}_{2.5}$. The CSN and IMPROVE networks monitor $\mathrm{Pb}$ in $\mathrm{PM}_{2.5}$ and the NATTS network monitors $\mathrm{Pb}$ in $\mathrm{PM}_{10}$. These networks are designed to meet different objectives than those of the Pb NAAQS monitoring network (EPA 2006, 2014c). EPA (2014c) analyzed data from these monitoring systems and presented data summaries for 
source-oriented (defined as near point sources and exceeded a defined emission threshold) and nonsource-oriented Pb monitors across the United States for 2008-2010 (EPA 2014c). Maximum 3-month daily average $\mathrm{Pb}$ concentrations were calculated for non-source-oriented Pb-TSP monitors for 47 counties across the United States (1.5\% of U.S. counties) and for source-oriented Pb-TSP monitors for 50 counties across the United States (1.6\% of U.S. counties) during the period 2008-2010. Summaries of these analyses are presented in Table 5-13.

\section{Table 5-13. Summary Data for Lead Monitors Across the United States, 2008- $2010\left(\mu \mathrm{g} / \mathrm{m}^{3}\right)$}

\begin{tabular}{lccccc}
\hline & Mean & Median & $95^{\text {th }} \%$ & $99^{\text {th }} \%$ & Maximum \\
\hline Monthly (source-oriented) & 0.20 & 0.063 & 0.86 & 1.6 & 4.4 \\
\hline Monthly (nonsource-oriented) & 0.012 & 0.010 & 0.040 & 0.052 & 0.14 \\
\hline
\end{tabular}

Source: EPA 2014c

$\mathrm{Pb}$ levels have been declining in the ambient air of the United States for several decades and according to the EPA, there has been approximately a 94\% decrease since 2000 (EPA 2018a). Figure 5-3 shows the annual maximum 3-month average Pb level in the United States based upon data at 24 monitoring sites.

\section{Figure 5-3. Annual Maximum 3-Month Average Representing the National Trend}

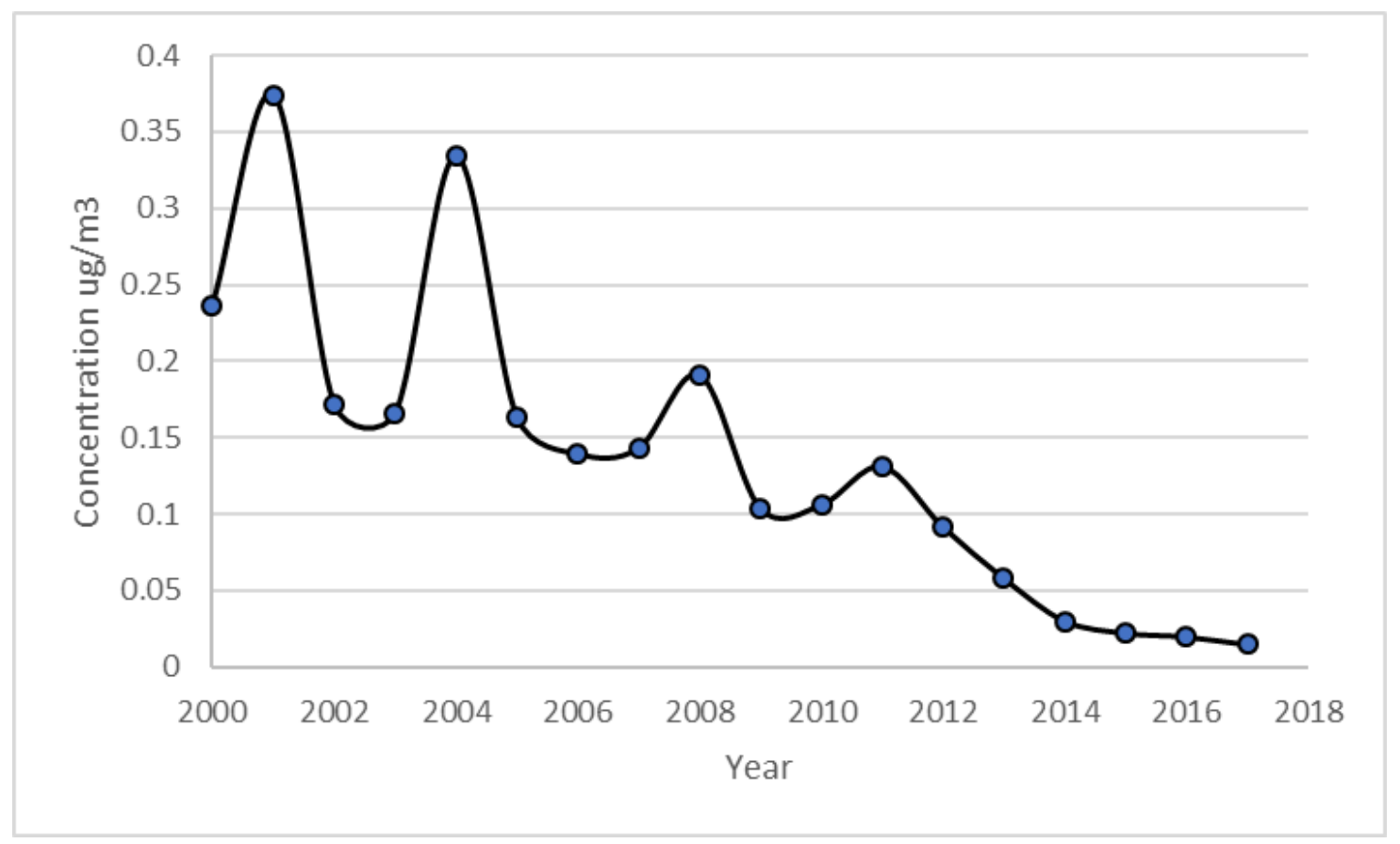

Source: EPA 2018a 
Data compiled from the EPA AQS database from 2015 to 2018 were used to calculate the percentile distribution of arithmetic mean 3-month averages at locations across the United States. These data are summarized in Table 5-14.

\section{Table 5-14. Percentile Distribution of Mean Lead (TSP) Concentrations $\left(\mu \mathrm{g} / \mathrm{m}^{3}\right)$ Measured in Ambient Air at Locations Across the United States}

\begin{tabular}{llllll}
\hline & \multicolumn{5}{c}{ Percentile } \\
\cline { 2 - 5 } Year & 25th & 50th & 75th & 95th & Maximum \\
\hline 2015 & 0.0036 & 0.0090 & 0.0216 & 0.0753 & 0.1942 \\
2016 & 0.0038 & 0.0093 & 0.0220 & 0.0782 & 0.1466 \\
2017 & 0.0039 & 0.0080 & 0.0190 & 0.0756 & 0.2087 \\
2018 & 0.0035 & 0.0090 & 0.0313 & 0.1248 & 0.5574 \\
\hline
\end{tabular}

TSP $=$ total suspended particles

Source: EPA 2018b

$\mathrm{Pb}$ in indoor air is related to $\mathrm{Pb}$ in housedust, and predominant sources are outdoor air and degraded Pb-based paint (EPA 2006). Smoking can also contribute to higher concentrations of $\mathrm{Pb}$ in indoor air. $\mathrm{Pb}$ concentrations in air and dust in the indoor environment were measured in residential homes as part of the National Human Exposure Assessment Survey (NHEXAS) in EPA Region V (Indiana, Illinois, Michigan, Minnesota, Ohio, and Wisconsin). Mean ( \pm 1 SD) and median concentrations of $\mathrm{Pb}$ in indoor air from 213 residences were $15.2 \mathrm{ng} / \mathrm{m}^{3}\left(37.6 \mathrm{ng} / \mathrm{m}^{3}\right)$ and $6.17 \mathrm{ng} / \mathrm{m}^{3}$, respectively, with a maximum value of $293.5 \mathrm{ng} / \mathrm{m}^{3}$ (Bonanno et al. 2001). The median $\mathrm{Pb}$ concentration in outdoor air was $8.84 \mathrm{ng} / \mathrm{m}^{3}$ (Clayton et al. 2002). Pb concentrations were higher in households where one or more residents smoked indoors (mean concentration of $21.8 \mathrm{ng} / \mathrm{m}^{3}$ ) as compared to households with nonsmoking residents (mean concentration of $7.79 \mathrm{ng} / \mathrm{m}^{3}$ ) (Bonanno et al. 2001). In dust collected from the living areas of 238 residences, the mean ( \pm 1 SD) and median Pb concentrations were $467.4 \mu \mathrm{g} / \mathrm{g}(2,100 \mu \mathrm{g} / \mathrm{g})$ and $131.6 \mu \mathrm{g} / \mathrm{g}$, respectively, with a maximum value of $30,578 \mu \mathrm{g} / \mathrm{g}$. Dust samples collected from window sills had mean ( \pm 1 SD) and median Pb concentrations of $987 \mu \mathrm{g} / \mathrm{g}(2,723 \mu \mathrm{g} / \mathrm{g})$ and $207.5 \mu \mathrm{g} / \mathrm{g}$, respectively, with a maximum value of $21,120 \mu \mathrm{g} / \mathrm{g}$. For both indoor air and dust measurements, higher concentrations of Pb were correlated with dilapidated and suburban homes. Dixon et al. (2009) analyzed children's exposures to residential dust Pb using data from the NHANES survey and associated demographics as well as smoking status to exposure levels. Children who resided in homes in which smoking occurred indoors had significantly $(\mathrm{p}=0.015)$ higher PbB levels than children who lived in homes of nonsmokers. 
In another analysis of the NHEXAS EPA Region V data, Pellizzari et al. (1999) looked at potential differences in $\mathrm{Pb}$ concentrations in indoor air and personal air exposures between minorities (e.g., Hispanics and African-Americans) and nonminorities (e.g., Caucasian). Some differences were noted in the mean ( \pm 1 SD) Pb concentrations between minorities of $57 \mathrm{ng} / \mathrm{m}^{3}\left( \pm 24 \mathrm{ng} / \mathrm{m}^{3}\right)$ and nonminorities of $22 \mathrm{ng} / \mathrm{m}^{3}\left( \pm 3.4 \mathrm{ng} / \mathrm{m}^{3}\right)$ in personal air exposures, although the differences were not significant $(\mathrm{p}=0.147)$. Similarly, differences were noted between minorities $\left(26 \pm 12 \mathrm{ng} / \mathrm{m}^{3}\right)$ and nonminorities $\left(13 \pm 2.6 \mathrm{ng} / \mathrm{m}^{3}\right)$ in indoor air, although these were also not significantly different $(p=0.266)$. When the age of the home was considered in the analysis, it was found that $\mathrm{Pb}$ concentrations were significantly ( $\mathrm{p}=0.036$ ) higher in homes built before 1940 than in homes built between 1960 and 1979, with mean ( \pm 1 SD) values of $46 \mathrm{ng} / \mathrm{m}^{3}\left( \pm 1.6 \mathrm{ng} / \mathrm{m}^{3}\right)$ and $13 \mathrm{ng} / \mathrm{m}^{3}\left( \pm 2.1 \mathrm{ng} / \mathrm{m}^{3}\right)$, respectively. The $\mathrm{Pb}$ concentrations measured in indoor air in homes built before 1940 were not significantly different from mean ( $\pm 1 \mathrm{SD}) \mathrm{Pb}$ concentrations of $22 \mathrm{ng} / \mathrm{m}^{3}\left( \pm 5.1 \mathrm{ng} / \mathrm{m}^{3}\right)$ and $23 \mathrm{ng} / \mathrm{m}^{3}\left( \pm 5.1 \mathrm{ng} / \mathrm{m}^{3}\right)$ measured in indoor air in homes built between 1940 and 1959 and between 1980 and 1995, respectively.

\subsubsection{Water}

$\mathrm{Pb}$ has been monitored in surface water, groundwater, and drinking water throughout the United States and other countries. The concentration of $\mathrm{Pb}$ in surface water is highly variable depending upon sources of pollution, $\mathrm{Pb}$ content of sediments, and characteristics of the system ( $\mathrm{pH}$, temperature, etc.). $\mathrm{Pb}$ concentrations in surface water are generally higher in urban areas than in rural areas (EPA 1982c), and $\mathrm{Pb}$ measured in natural or "pristine” surface waters may be due to anthropogenic input. Western Airborne Contaminants Assessment Project (WACAP) data collected at five U.S. National Parks showed median $\mathrm{Pb}$ levels in surface waters ranging from 0.006 to $0.075 \mu \mathrm{g} / \mathrm{L}$ (EPA 2014c). The median Pb level in natural river water was $5 \mu \mathrm{g} / \mathrm{L}$, with a range of 0.6-120 $\mu \mathrm{g} / \mathrm{L}$; however, lower Pb levels are to be expected after leaded gasoline was banned in 1985, which resulted in decreased rates of atmospheric deposition (Bowen et al. 1966; King et al. 2014). The National Academies of Science reported Pb concentration levels in surface water and groundwater (EPA 1986b). The mean Pb concentration level in surface water was $4 \mu \mathrm{g} / \mathrm{L}$ with a range from below the detection limit to $890 \mu \mathrm{g} / \mathrm{L}$ (EPA 2014c); concentrations $>100 \mu \mathrm{g} / \mathrm{L}$ were observed near sources of urban runoff or industrial discharge. Mean levels of $\mathrm{Pb}$ in surface water measured at 50,000 surface water stations throughout the United States were $3.9 \mu \mathrm{g} / \mathrm{L}$ (based on 39,490 occurrences) (Eckel and Jacob 1988). Using the EPA Storage and Retrieval (STORET) database, from January 1, 2005 to May 16, 2005, Pb had been detected in surface water in Washington, Utah at concentrations of 20.5 and $142 \mu \mathrm{g} / \mathrm{L}$ and surface water from Salt Lake City, Utah at $7.75 \mu \mathrm{g} / \mathrm{L}$ (EPA 2005b). Pb was not detected above the detection limits in 224 other surface water samples obtained 
from various locations in Utah and Iowa over the sampling period (EPA 2005b). Pb content in groundwater is driven largely by the surrounding bedrock geochemistry; $\mathrm{Pb}$ concentrations are generally low in groundwater and natural springs ranging from below the detection limit to $100 \mu \mathrm{g} / \mathrm{L}$ (EPA 2014c). A USGS study of groundwater in the United States from 2000 to 2016 concluded that $<1 \%$ of measured $\mathrm{Pb}$ concentrations are $>15 \mu \mathrm{g} / \mathrm{L}$, but when high levels are detected, they are typically associated with geographic locations where the $\mathrm{Pb}$ solubility potentials (the amount of $\mathrm{Pb}$ that could dissolve before $\mathrm{a} \mathrm{Pb}$ mineral precipitates out of solution) are naturally high (Jurgens et al. 2019). Pb levels in seawater are typically in the range of $0.001-0.036 \mu \mathrm{g} / \mathrm{L}$ in the open ocean and about $0.050-0.30 \mu \mathrm{g} / \mathrm{L}$ in coastal waters influenced by anthropogenic activity (Angel et al. 2016).

Urban storm water runoff is an important source of $\mathrm{Pb}$ entering receiving waterways. Sources of $\mathrm{Pb}$ in runoff can be contributed to substantial direct atmospheric deposition, as well as indirect release from building materials, soil, and road dust, and industrial discharge. $\mathrm{Pb}$ is found in building material (brick, concrete, painted and unpainted wood, roofing, and vinyl), and automotive sources (brakes, used oil), which contribute to runoff (Davis et al. 2001). The largest contributing sources were siding and roofing. Soto-Jiménez and Flegal (2009) evaluated the sources of Pb pollution in the Gulf of California, northwest Mexico by sampling urban and rural areas for Pb levels and isotope ratios. Urban street dust (157 $\mu \mathrm{g} / \mathrm{g})$, agricultural soils $(29.0 \mu \mathrm{g} / \mathrm{g})$, and surface estuary sediments $(35.6 \mu \mathrm{g} / \mathrm{g})$ were all higher than natural bedrock $(16.0 \mu \mathrm{g} / \mathrm{g})$. Isotopic ratios in rural and soil runoff samples were comparable to natural $\mathrm{Pb}$ containing bedrock. $\mathrm{Pb}$ concentrations in the suspended particulate matter were measured in sewage effluent (132 $\mu \mathrm{g} / \mathrm{g})$, agricultural effluent $(29.3 \mu \mathrm{g} / \mathrm{g})$, river runoff $(7.3 \mu \mathrm{g} / \mathrm{g})$, and estuary water $(68.3 \mu \mathrm{g} / \mathrm{g})$. Urban, street dust, and sewage showed contributions from automotive emissions from past leaded gasoline combustion.

$\mathrm{Pb}$ in drinking water can derive from source water contamination as described above, but the more common source of $\mathrm{Pb}$ in drinking water is from internal corrosion of water distribution system piping and plumbing. Internal corrosion of $\mathrm{Pb}$ service lines, $\mathrm{Pb}$-based pipe solder, brass meters and plumbing fixtures, and dissolution of existing protective scales contribute directly to Pb levels in drinking water. The Lead and Copper Rule (LCR) was promulgated in 1991 with the purpose of protecting public health by minimizing $\mathrm{Pb}$ and copper levels in drinking water, primarily by reducing water corrosivity (EPA 2004). The LCR established a $\mathrm{Pb}$ action level (AL) of $15 \mu \mathrm{g} / \mathrm{L}$ and a maximum contaminant level goal (MCLG) of zero. The $\mathrm{Pb}$ action level is based on feasibility of public water systems to control corrosion in their distribution systems and is not a health benchmark for $\mathrm{Pb}$ in drinking water. The $\mathrm{Pb}$ action level is exceeded if the concentration of the $90^{\text {th }}$ percentile first draw tap sample (collected after a minimum 
stagnation period of 6 hours from high risk sites) exceeds $15 \mu \mathrm{g} / \mathrm{L}$ (EPA 2016a). If the Pb AL is exceeded, the LCR can require public water systems to take steps to minimize the risk of Pb exposure that may include source water monitoring/treatment, public education, water quality monitoring, implementing corrosion control treatment, and Pb service line replacement. In October 2019, EPA proposed significant changes to the LCR (EPA 2019a). These changes include: (1) identify areas most in need of remediation of $\mathrm{Pb}$ service lines; (2) establish a trigger level of $10 \mu \mathrm{g} / \mathrm{L}$ for requiring corrosion control in drinking water systems that do not currently treat for corrosion; (3) require water system to replace $\mathrm{Pb}$ service lines; (4) increase sampling reliability by prohibiting pre-stagnation flushing and other methods; (5) require systems to notify customers of action level exceedance within 24 hours; and (6) protect children in schools by expanding testing at drinking water outlets.

Analyses done in support of the short-term revisions to the LCR at the beginning of the $21^{\text {st }}$ century suggest that in $2003,<2 \%$ of public water systems serving $>3,300$ people exceeded the $\mathrm{Pb}$ action level of $15 \mu \mathrm{g} / \mathrm{L}$ (EPA 2007a). Additionally, a 2004 study conducted by the EPA on LCR compliance monitoring for public water systems serving $>3,300$ people indicated that $<4 \%$ of those systems exceeded the $\mathrm{Pb}$ action level (Hill 2011). It is important to note that states were not required until 2002 to report $90^{\text {th }}$ percentile $\mathrm{Pb}$ concentrations to the EPA unless those samples exceeded the $\mathrm{Pb} \mathrm{AL}$; therefore, it is difficult to accurately compare differences between tap water Pb levels prior to LCR implementation and immediately following LCR implementation with current nationwide Pb concentration levels (Hill 2011). Nevertheless, the EPA evaluated water sample data from 166 large public water systems (systems serving >50,000 people) that exceeded the Pb AL in 1992 and 1993 (Hill 2011). Of the large systems that exceeded the Pb AL in 1992-1993, only 15 of those systems continued to exceed the $\mathrm{Pb}$ AL between 2000 and 2004, and their associated average $90^{\text {th }}$ percentile $\mathrm{Pb}$ concentration levels significantly decreased from 32 to $8.2 \mu \mathrm{g} / \mathrm{L}$.

The amount of $\mathrm{Pb}$ contained in pipes and plumbing fittings has been strictly regulated since 1986. Section 1417 of the Safe Drinking Water Act (SDWA) was amended to ban the use of service lines, pipe fittings, pipe solder, and fixtures that are not " $\mathrm{Pb}$ free" (not more than $0.2 \% \mathrm{~Pb}$ for pipe solder and flux, and not more than $8 \% \mathrm{~Pb}$ for pipe fittings and service lines) and are connected to a public water system and intended to provide water for human consumption. The 1996 Amendment broadened this ban by limiting the amount of leaching of $\mathrm{Pb}$ from new plumbing, and an industry standard was established. In 2011, the Reduction of Lead in Drinking Water Act amended Section 1417, revising the existing SDWA definition of " $\mathrm{Pb}$ free" and getting rid of the leaching certification requirement. Implemented in 2014, the 
act reduced the allowable level of $\mathrm{Pb}$ by "not more than a weighted average of 0.25 percent $\mathrm{Pb}$ when used with respect to the wetted surfaces of pipes, pipe fittings, plumbing fittings and fixtures.”

According to EPA's National Public Water Systems Compliance Report for calendar year 2013 (EPA 2013), $73 \%$ of public water systems in the United States, serving approximately $77 \%$ of the population, had no significant reported violations of any type. Significant violations include all violations of healthbased standards, including violations of the maximum contaminant levels, treatment technique requirements, and significant monitoring and reporting requirements. In 2013, 7\% of public water systems had no reported violations of health-based standards, and 5\% of all health-based standard violations were LCR violations.

In the spring of 2014, the source of drinking water in the city of Flint, Michigan was switched from treated water obtained from Lake Huron to the Flint River. However, the treated water from the Flint River was more corrosive and did not contain corrosion inhibitors, which resulted in $\mathrm{Pb}$ leaching from the city’s aging service lines. Sampling data conducted in August of 2015 showed that the $90^{\text {th }}$ percentile concentration of $\mathrm{Pb}$ in first-draw drinking water was $26.8 \mu \mathrm{g} / \mathrm{L}$ for 268 samples of tap water, which far exceeded the EPA AL of $15 \mu \mathrm{g} / \mathrm{L}$ (Pieper et al. 2018). In response to the high Pb levels in Flint drinking water, the city reconnected to the DWSD in October of 2015. By August of 2017, the $90^{\text {th }}$ percentile concentration of $\mathrm{Pb}$ in first-draw tap water was $7.9 \mu \mathrm{g} / \mathrm{L}$ (Pieper et al. 2018).

\subsubsection{Sediment and Soil}

$\mathrm{Pb}$ is a naturally occurring metal found in the earth's crust at about 15-20 mg/kg (Goyer 2001). However, the concentration of $\mathrm{Pb}$ in the top layers of soil varies widely due to deposition and accumulation of atmospheric particulates from anthropogenic sources. The concentration of soil $\mathrm{Pb}$ generally decreases as distance from contaminating sources increases. The estimated $\mathrm{Pb}$ levels in the upper layer of soil beside roadways are typically 30-2,000 $\mu \mathrm{g} / \mathrm{g}$ higher than natural levels, although these levels drop exponentially up to $25 \mathrm{~m}$ from the roadway (EPA 1986b). Soil adjacent to a smelter in Missouri had Pb levels in excess of 60,000 $\mu \mathrm{g} / \mathrm{g}$ (Palmer and Kucera 1980). Soils adjacent to houses with exterior Pb-based paints have reported $\mathrm{Pb}$ levels $>10,000 \mu \mathrm{g} / \mathrm{g}$ (EPA 1986b). As a result of $\mathrm{Pb}$ reactions with the soil, extractable $\mathrm{Pb}$ in surface soil samples (0-5 cm depth) from an agricultural area near a car battery manufacturing plant (taken at $0.3 \mathrm{~km}$ from the source) decreased from 117 to $1 \mu \mathrm{g} / \mathrm{g}$ within 1 year after the plant stopped operating (Schalscha et al. 1987). Soil collected by scraping the top $2.5 \mathrm{~cm}$ of soil surface near homes and streetside in Louisiana and Minnesota contained median Pb concentrations of $>840 \mu \mathrm{g} / \mathrm{g}$ in New 
Orleans and $265 \mu \mathrm{g} / \mathrm{g}$ in Minneapolis. In contrast, the small towns of Natchitoches, Louisiana, and Rochester, Minnesota had soil Pb concentrations of $<50$ and $58 \mu \mathrm{g} / \mathrm{g}$, respectively. These data suggest that $\mathrm{Pb}$-contaminated soil is a major source of $\mathrm{Pb}$ exposure in urban areas (Mielke 1993). As would be expected, soils in elementary school properties were also found to have the same pattern of Pb levels as the soils in the surrounding residences. Pb concentrations in soils collected from inner-city schools in New Orleans were higher (median concentration of $96.5 \mu \mathrm{g} / \mathrm{g}$ ) than soils collected from mid-city $(30.0 \mu \mathrm{g} / \mathrm{g})$ and outer-city $(16.4 \mu \mathrm{g} / \mathrm{g})$ elementary schools (Higgs et al. 1999).

The former use of $\mathrm{Pb}$ in paints, particularly in older structures, is also a source of $\mathrm{Pb}$ in soil and within homes. Mielke and Gonzales (2008) reported median Pb concentrations of 76,603 mg/kg (464$317,151 \mathrm{mg} / \mathrm{kg}$ ) and $416 \mathrm{mg} / \mathrm{kg}$ (24-63,313 mg/kg) for exterior and interior paints, respectively, in 40 paint chip samples collected from homes in metropolitan New Orleans. The authors noted that the age of the house is often used as a surrogate for the amount of $\mathrm{Pb}$ in paints; the mid-1920s being the peak use of leaded paint with declines until 1978. Demolition and renovation of buildings where leaded paint was used can result in transport of $\mathrm{Pb}$ to soil surrounding the building as well as indoor dust that contains $\mathrm{Pb}$.

$\mathrm{Pb}$ concentrations were measured in residential transects through Lubbock, Texas. Pb concentrations through the city showed a trend of decreasing $\mathrm{Pb}$ concentrations with increasing distance from the city center, which also paralleled a decrease in the property age. The highest $\mathrm{Pb}$ concentrations in the city center were $90.0-174.0 \mathrm{mg} / \mathrm{kg}$, with a median of $35.4 \mathrm{mg} / \mathrm{kg}$, and decreased out to the farther part of the residential transect to $6.0-9.0 \mathrm{mg} / \mathrm{kg}$. The highest concentrations outside city development were $4.9 \mathrm{mg} / \mathrm{kg}$ (Brown et al. 2008).

Studies conducted in Maryland and Minnesota indicate that within large, light-industrial, urban settings such as Baltimore, the highest soil Pb levels generally occur near inner-city areas, especially where high traffic flows have long prevailed (Mielke et al. 1983, 1984, 1989) and that the amount of $\mathrm{Pb}$ in the soil is correlated with the size of the city (Mielke 1991). In 1981, soil Pb levels in the Minneapolis/St. Paul inner-city area were 60 times higher $(423 \mu \mathrm{g} / \mathrm{g})$ than levels found in rural Minnesota $(6.7 \mu \mathrm{g} / \mathrm{g})$, with almost all the increase (95\%) resulting from the combustion of leaded gasoline. A study conducted in Minneapolis, Minnesota, after the $\mathrm{Pb}$ content of gasoline had been significantly reduced, found that median soil Pb levels taken from the foundations of homes, in yards, and adjacent to the street were 700, 210, and $160 \mu \mathrm{g} / \mathrm{g}$, respectively; median soil $\mathrm{Pb}$ concentrations in comparable samples from the smaller city of Rochester, Minnesota, did not exceed $100 \mu \mathrm{g} / \mathrm{g}$ at any location tested (Mielke et al. 1989). The Minneapolis data suggested that average $\mathrm{Pb}$ levels were elevated in soil samples taken from the 
foundations of homes, but that $\mathrm{Pb}$ levels were low $(<50 \mu \mathrm{g} / \mathrm{g})$ in areas where children could be expected to play, such as parks that were located away from traffic, but were higher in play areas around private residences. Soil samples taken from around the foundations of homes with painted exteriors had the highest $\mathrm{Pb}$ levels (mean concentrations of $522 \mu \mathrm{g} / \mathrm{g}$ ), but levels around homes composed of brick or stucco were significantly lower (mean concentration $158 \mu \mathrm{g} / \mathrm{g}$ ) (Schmitt et al. 1988). Severely contaminated soils (levels as high as $20,136 \mu \mathrm{g} / \mathrm{g}$ ) were located near house foundations adjacent to private dwellings with exterior $\mathrm{Pb}$-based paint. Elevated soil $\mathrm{Pb}$ concentrations were found in larger urban areas, with 27, 26, 32, and 42\% of the soil samples exceeding $300 \mu \mathrm{g} / \mathrm{g} \mathrm{Pb}$ in Duluth, inner-city North Minneapolis, inner-city St. Paul, and inner-city South Minneapolis, respectively. Only 5\% of the soil samples taken from the smaller urban areas of Rochester and St. Cloud, Minnesota, had Pb levels $>150 \mu \mathrm{g} / \mathrm{g}$. It has been suggested that the higher $\mathrm{Pb}$ levels associated with soils taken from around painted homes in the inner city are the result of greater atmospheric $\mathrm{Pb}$ content, resulting from the burning of leaded gasoline in cars and the washdown of building surfaces to which the small $\mathrm{Pb}$ particles adhere by rain (Mielke et al. 1989). A state-wide Minnesota study concluded that exterior Pb-based paint was the major source of contamination in severely contaminated soils located near the foundations of private residences and that aerosol $\mathrm{Pb}$ accounted for virtually all of the contamination found in soils removed from the influence of Pb-based paint. Contamination due to Pb-based paint was found to be "highly concentrated over a limited area, while contamination due to aerosol $\mathrm{Pb}$ was found to be less concentrated, but more widespread” (Schmitt et al. 1988).

$\mathrm{Pb}$ was analyzed in dust wipes and soil samples from 67 public housing projects containing 487 dwelling units across the United States (Succop et al. 2001). A total of 5,906 dust wipes and 1,222 soil samples were included in the data set. The median soil levels were $194 \mathrm{ppm}$ near the foundation, $177 \mathrm{ppm}$ near the walkways, and 145 ppm elsewhere in the yard. The maximum level, 3,900 ppm, was found in a foundation sample. Median dust $\mathrm{Pb}$ loading $\left(\mu \mathrm{g} \mathrm{m}^{-2}\right)$ from kitchens, living rooms, and two children's bedrooms were 151 ( $5^{\text {th }}-95^{\text {th }}$ percentile range: 22,674$), 936(86,10,190)$, and $8,560(818,313,000)$ for floor window sills and window troughs, respectively. Thirteen percent of the floor samples and $30 \%$ of the window sill samples from the rooms exceeded the HUD Interim Dust Lead Standards of 431 and $2,690 \mu \mathrm{g} \mathrm{m}^{-2}$ for floor and window sill samples, respectively.

\subsubsection{Paint}

Weathering and deterioration of Pb-based paint can contribute to the Pb content of dust and soil (Clark et al. 2004; Hunt et al. 1993; Jaeger et al. 1998; Lucas et al. 2014; Marcus and Elias 1995). A soil Pb study 
in Minneapolis, Minnesota, found that soil samples taken from around the foundations of homes with painted exteriors had a mean concentration of $522 \mu \mathrm{g} / \mathrm{g}$, while soil samples taken from around the foundations of brick or stucco had a mean concentration of $158 \mu \mathrm{g} / \mathrm{g}$ (Schmitt et al. 1988). Pb-based paint, removed from surfaces by burning (gas torch or hot air gun), scraping, or sanding have been found to result, at least temporarily, in higher levels of exposure for families residing in these homes. A 2006 survey of U.S. housing stock estimated that 35\% of 106 million housing units contained Pb-based paint and that approximately 20\% of houses contained deteriorating Pb-based paint (HUD 2011).

\subsubsection{Other Media}

$\mathrm{Pb}$ has been detected in a variety of foods and spices (Lin et al. 2010). Pb may be introduced into food through uptake from soil into plants or atmospheric deposition onto plant surfaces, during transport to market, processing, and kitchen preparation (EPA 1986b). The ban on leaded gasoline as well as the use of welded (non-soldered) food cans during the 1980s are largely responsible for the decreases in levels of $\mathrm{Pb}$ in the U.S. diet beginning in the 1980s (FDA 2006). The FDA analyzed samples of foods commonly eaten by toddlers and infants for $\mathrm{Pb}$ and noted that levels of $\mathrm{Pb}$ in infant and toddler foods, on average, are relatively low (FDA 2016a). These results are summarized in Table 5-15. Selected data from the 20062011 FDA Total Diet Study Market Baskets are presented in Table 5-16 (FDA 2016b). Mean Pb levels in dairy products (e.g., milk, cheese, ice cream, cream, yogurt) were generally low or below the detection limit. The dairy product category with the highest $\mathrm{Pb}$ level was for low-fat fruit-flavored yogurt, with a mean concentration of $0.002 \mathrm{mg} / \mathrm{kg}$ for 24 analyses. Mean concentrations of $\mathrm{Pb}$ in fruits and vegetable were also generally low, with the highest concentrations in raisins $(0.005 \mathrm{mg} / \mathrm{kg})$, spinach $(0.004 \mathrm{mg} / \mathrm{kg})$, and lettuce $(0.004 \mathrm{mg} / \mathrm{kg})$. Mean concentration of $\mathrm{Pb}$ in baby foods ranged from not detected to $0.013 \mathrm{mg} / \mathrm{kg}$. The highest levels reported were found in sweet potatoes $(0.013 \mathrm{mg} / \mathrm{kg})$, arrowroot cookies (0.012 mg/kg), grape juice (0.011 mg/kg), teething biscuits $(0.008 \mathrm{mg} / \mathrm{kg})$, and apple-cherry juice $(0.008 \mathrm{mg} / \mathrm{kg})$. Based on a multimedia $\mathrm{Pb}$ exposure modeling analysis for children $1-5$ years old, below the $70^{\text {th }}$ percentile of $\mathrm{PbB}$ in the general U.S. population, dietary intake was a major background exposure pathway (Zartarian et al. 2017)

Table 5-15. Lead Levels in Foods Commonly Eaten by Toddlers and Infants

\begin{tabular}{lll}
\hline Product category & Average $^{\mathrm{a}}(\mathrm{range})(\mu \mathrm{g} / \mathrm{kg})$ & Number of samples \\
\hline Cereal, infant/toddler (rice) & $15.6(5.0-82.0)$ & 76 \\
\hline Cereal, infant/toddler (multigrain) & $7.2(6.0-8.0)$ & 6 \\
\hline Cereal, infant/toddler (non-rice) & $4.8(0.4-17.0)$ & 30 \\
\hline
\end{tabular}


Table 5-15. Lead Levels in Foods Commonly Eaten by Toddlers and Infants

\begin{tabular}{lll}
\hline Product category $^{\mathrm{A}}$ & Average $^{\mathrm{a}}(\mathrm{range})(\mu \mathrm{g} / \mathrm{kg})$ & Number of samples \\
\hline Apples $^{\mathrm{b}}$ & 3.3 & 10 \\
\hline Cereal, oat ring & $7.8(3.3-16.4)$ & 30 \\
\hline Grapes & $3.7(3.3-7.6)$ & 10 \\
\hline Juice, grape & $5.6(0.3-41.3)$ & 30 \\
\hline Juice boxes and pouches & $3.3(0.3-17.0)$ & 40 \\
\hline Peanut butter & $5.3(3.3-45.2)$ & 29 \\
\hline Quinoa & $22.2(0.4-98.0)$ & 30 \\
\hline Raisins & $18.1(1.8-151)$ & 23 \\
\hline Stage 2 toddler foods & $5.2(1.0-22.2)$ & 35 \\
\hline Teething biscuits & $12.0(2.0-131)$ & 27 \\
\hline Toddler puffs & $19.1(3.3--91.0)$ & 31 \\
\hline
\end{tabular}

aThe average concentration reported for each product category was calculated using all values. For those samples with results below the detection limit, half of the detection limit was used to calculate the average.

${ }^{\mathrm{b}}$ All of the apple samples were below the limit of detection.

Source: FDA 2016a

Table 5-16. Selected Mean Lead Concentrations in Food from the FDA Total Diet Study

\begin{tabular}{lllll}
\hline Food & Mean (range) $(\mathrm{mg} / \mathrm{kg})^{\mathrm{a}}$ & $\begin{array}{l}\text { Number of } \\
\text { analyses }\end{array}$ & $\begin{array}{l}\text { Number } \\
<\text { LOD }\end{array}$ & $\begin{array}{l}\text { LOD } \\
(\mathrm{mg} / \mathrm{kg})\end{array}$ \\
\hline Syrup, chocolate & $0.016(0-0.027)$ & 24 & 1 & 0.007 \\
\hline Apricots, canned in heavy/light syrup & $0.015(0-0.036)$ & 24 & 1 & 0.007 \\
\hline Baby food, sweet potatoes & $0.013(0-0.034)$ & 24 & 5 & 0.007 \\
\hline Peach, canned in light/medium syrup & $0.013(0-0.038)$ & 24 & 2 & 0.007 \\
\hline Candy bar, milk chocolate, plain & $0.013(0-0.027)$ & 24 & 5 & 0.01 \\
\hline Baby food, arrowroot cookies & $0.012(0-0.031)$ & 24 & 9 & 0.01 \\
\hline Sweet potatoes, canned & $0.012(0-0.018)$ & 24 & 2 & 0.007 \\
\hline Shrimp, boiled & $0.012(0-0.18)$ & 24 & 18 & 0.01 \\
\hline Baby food, juice, grape & $0.011(0-0.02)$ & 24 & 1 & 0.004 \\
\hline Fruit cocktail, canned in light syrup & $0.011(0-0.025)$ & 24 & 4 & 0.007 \\
\hline Brownie & $0.01(0-0.032)$ & 24 & 5 & 0.007 \\
\hline
\end{tabular}

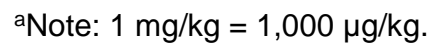

FDA $=$ U.S. Food and Drug Administration; LOD = limit of detection

Source: FDA 2016b 
The U.S. Fish and Wildlife Service reported the concentrations of metals in a total of 315 composite samples of whole fish sampled from 109 stations nationwide from late 1994 to early 1995 . For $\mathrm{Pb}$, the geometric mean, maximum, and $85^{\text {th }}$ percentile concentrations ( $\mu \mathrm{g} / \mathrm{g}$ wet weight) were $0.11,4.88$, and 0.22, respectively. The mean concentration of $\mathrm{Pb}$ was significantly lower than in the 1980-1981 survey. $\mathrm{Pb}$ concentrations in fish have declined steadily from 1976 to 1984, suggesting that reductions of leaded gasoline and controls on mining and industrial discharges have reduced $\mathrm{Pb}$ in the aquatic environment (Schmitt and Brumbaugh 1990).

In order to reduce $\mathrm{Pb}$ exposure from consumption of $\mathrm{Pb}$-contaminated fish and shellfish, consumption advisories are issued by states recommending that individuals restrict their consumption of specific fish and shellfish species from certain water bodies where $\mathrm{Pb}$ concentrations in fish and shellfish tissues exceed the human health level of concern. This level of concern is set by individual state agencies and used to issue advisories recommending no consumption, or restricted consumption, of contaminated fish and shellfish from certain waterbody types (e.g., lakes and/or rivers). In 1995, the EPA Office of Water issued guidance to states on sampling and analysis procedures to use in assessing the health risks from consuming locally caught fish and shellfish. The risk assessment method proposed by EPA was specifically designed to assist states in developing fish consumption advisories for recreational and subsistence fishers (EPA 1995a). These two groups within the general population consume larger quantities of fish and shellfish than the general population and frequently fish the same water bodies routinely. Because of this, these populations are at greater risk of exposure to $\mathrm{Pb}$ and other chemical contaminants if the waters they fish are contaminated. In 2007, eight advisories restricting the consumption of Pb-contaminated fish and shellfish were in effect in five states (Hawaii, Idaho, Washington, Kansas, and Missouri) and one territory (American Samoa) (EPA 2007b).

Elevated levels of $\mathrm{Pb}$ in the blood of cattle grazing near a $\mathrm{Pb}$ smelter have been reported, although no implications regarding $\mathrm{Pb}$ in beef were made. The mean $\mathrm{Pb}$ levels for the herd were highest near the smelter and decreased with distance. Ingestion of soil along with the forage was thought to be a large source of additional metal (Neuman and Dollhopf 1992). Evidence has also been shown for transfer of $\mathrm{Pb}$ to milk and edible tissue in cattle poisoned by licking the remains of storage batteries burned and left in a pasture (Oskarsson et al. 1992). Levels of $\mathrm{Pb}$ in muscle of acutely sick cows that were slaughtered ranged from 0.23 to $0.5 \mathrm{mg} / \mathrm{kg}$ (wet weight basis). Normal $\mathrm{Pb}$ levels in bovine meat from Swedish farms are $<0.005 \mathrm{mg} / \mathrm{kg}$. For eight cows that were less exposed, levels of $\mathrm{Pb}$ in milk taken 2 weeks after the exposure were $0.08 \pm 0.04 \mathrm{mg} / \mathrm{kg}$. The highest $\mathrm{Pb}$ level found in the milk of eight cows studied for 18 weeks was $0.22 \mathrm{mg} / \mathrm{kg}$. Pb in most milk samples decreased to values $<0.03 \mathrm{mg} / \mathrm{kg} 6$ weeks after 
exposure. Two affected cows delivered a calf at 35 and 38 weeks after the exposure. There was a high $\mathrm{Pb}$ level in the blood of the cows at the time of delivery, which suggests mobilization of $\mathrm{Pb}$ in connection with the latter stages of gestation and delivery. Pb levels in colostrum were increased as compared to mature milk samples taken 18 weeks after exposure. The concentration of $\mathrm{Pb}$ in milk produced after delivery decreased rapidly with time and was almost down to the limit of detection in mature milk.

In a survey, 324 multivitamin-mineral products were analyzed for $\mathrm{Pb}$ content (Mindak et al. 2008). Estimates of $\mathrm{Pb}$ exposure from these products were derived for four groups summarized in Table 5-17. The overall median value for $\mathrm{Pb}$ exposure was $0.576 \mu \mathrm{g} / \mathrm{day}$. Five samples would have provided exposures that exceeded $4 \mu \mathrm{g} / \mathrm{day}$. The authors reported that the estimates of $\mathrm{Pb}$ exposures were below the provisional total tolerable intake levels for the four population groups (Mindak et al. 2008). Twentyone elements, including $\mathrm{Pb}$, were analyzed in various botanical and dietary supplements; $\mathrm{Pb}$ concentrations ranged from not detected to $4.21 \mu \mathrm{g} / \mathrm{g}$. None of the products analyzed would result in a maximum exposure that exceeds a tolerable level of exposure (Avula et al. 2010).

\section{Table 5-17. Estimated Median and Maximum Lead Exposures}

\begin{tabular}{lll}
\hline Population group & Median $(\mu \mathrm{g} /$ day $)$ & Maximum $(\mu \mathrm{g} /$ day $)$ \\
\hline Young children $(0-6$ years $)$ & 0.123 & 2.88 \\
\hline Older children $(7+$ years $)$ & 0.356 & 1.78 \\
\hline Pregnant or lactating women & 0.845 & 8.97 \\
\hline Adult women & 0.842 & 4.92 \\
\hline
\end{tabular}

Source: Adapted with permission from Mindak et al. (2008), American Chemical Society.

Many non-Western folk remedies used to treat diarrhea or other ailments may contain substantial amounts of Pb. Examples of these include: Alarcon, Ghasard, Alkohl, Greta, Azarcon, Liga, Bali Goli, Pay-loo-ah, Coral, and Rueda. In addition, an adult case of $\mathrm{Pb}$ poisoning was attributed to an Asian remedy for menstrual cramps known as Koo Sar. The pills contained $\mathrm{Pb}$ at levels as high as $12 \mathrm{ppm}$ (CDC 1998). The source of the $\mathrm{Pb}$ was thought to be in the red dye used to color the pills. $\mathrm{Pb}$ was the most common heavy metal contaminant/adulterant found in samples $(\mathrm{n}=54)$ of Asian traditional remedies available at health food stores and Asian groceries in Florida, New York, and New Jersey (Garvey et al. 2001). Sixty percent of the remedies tested would give a daily dose of $\mathrm{Pb}$ in excess of $300 \mathrm{mg}$ when taken according to labeling instructions. Pb poisoning has been caused by ingestion of a Chinese herbal medicine to which metallic $\mathrm{Pb}$ was added to increase its weight and sales price (Wu et al. 1996). Ayurveda is a traditional form of medicine practiced in India and other South Asian countries; the medications used often contain herbs, minerals, metals, or animal products and are made in standardized 
and nonstandardized formulations (CDC 2004b). CDC (1998, 2002b) reported cases of elevated PbBs in children after consuming candy from Mexico or using various folk remedies. Elevated PbBs were reported in two 7-year-old children in Rhode Island. A sample of litargirio, which was used as an antiperspirant/deodorant, found in the home contained 79\% Pb (CDC 2005).

During 2011-2012, six cases of Pb poisoning were associated with the use of 10 oral Ayurvedic medications made in India. Pb concentrations in these medications were as high as $2.4 \%$. Blood $\mathrm{Pb}$ levels of these women ranged from 16 to $64 \mu \mathrm{g} / \mathrm{dL}$ (CDC 2012c). In 2004-2012, the New York City Department of Health and Mental Hygiene identified 22 oral medications, supplements, or remedies containing high levels of heavy metals, including $\mathrm{Pb}$ (Table 5-18).

Table 5-18. Lead Content in Ayurvedic Medications and Other Health Remedies

\begin{tabular}{lllc}
\hline Product & $\begin{array}{l}\text { Country where } \\
\text { manufactured }\end{array}$ & $\begin{array}{l}\text { Country where } \\
\text { purchased }\end{array}$ & $\begin{array}{l}\text { Lead content } \\
\text { (ppm) }\end{array}$ \\
\hline Calabash Chalk (Nzu) & Unknown & United States & 6.6 \\
\hline Emperor's Tea Pill (concentrated) & China & United States & 5,400 \\
\hline Garbha Chintamani Ras (Vrihat) (Swarna Yukt) & India & India & 120 \\
\hline Garbha Dharak Yog & India & India & 110 \\
\hline Garbhapal Ras & India & India & 22,000 \\
\hline Garbhapal Ras & India & United States & 15,000 \\
\hline Hepatico Extract (concentrated) & China & United States & 5,900 \\
\hline Jambrulin & India & United States & 243,000 \\
\hline Kankayan Bati (Gulma) & India & United States & 12 \\
\hline Lakshmivilash Ras (Nardiya) & India & United States & 260 \\
\hline Laxmana Louh & India & India & 180 \\
\hline Maha Sudarshan & India & United States & 41 \\
\hline Mahashakti Rasayan & India & India & 9,400 \\
\hline Mahayogaraj Guggulu (enriched with silver) & India & United States & 47,000 \\
\hline Ovarin & India & India & 24,000 \\
\hline Pigmento & India & India & 7.3 \\
\hline Pregnita & India & India & 12,000 \\
\hline Sorin & India & India & 46,707 \\
\hline Tierra Santa & Mexico & United States & 13 \\
\hline Vasant Kusumakar Ras (with Gold and Pearl) & India & India & 29 \\
\hline Vatvidhwansan Ras & India & United States & 20,000 \\
\hline Vita Breath & United States & United States & 1,100 \\
\hline & & & \\
\hline
\end{tabular}

Source: CDC 2012c 
A study was conducted in an urban neighborhood in Chicago in order to gauge the levels of $\mathrm{Pb}$ in an array of fruits, vegetables, and herbs (Finster et al. 2004). The soil Pb concentrations where the plants were sampled varied from 27 to 4,580 ppm (median 800 ppm, geometric mean 639 ppm). Detectable Pb levels in the edible fruit, vegetables, and herbs sampled ranged from 11 to $81 \mathrm{ppm}$. Only one fruiting vegetable (cucumber 81 ppm) among the 52 sampled had detectable levels of $\mathrm{Pb}$ in the edible portion. However, 12 of the 31 leafy vegetables and herbs sampled contained $\mathrm{Pb}$ in the edible shoot part of the plant (range, 11-60 ppm). The Pb concentrations in the four samples of root vegetables ranged from 10 to $21 \mathrm{ppm}$. No significant correlation was found between the $\mathrm{Pb}$ concentrations in the edible portion of plant and the soil Pb level.

$\mathrm{Pb}$ may leach from $\mathrm{Pb}$ crystal decanters and glasses into the liquids they contain. Port wine that contained an initial concentration of $89 \mu \mathrm{g} / \mathrm{L} \mathrm{Pb}$ was stored for 4 months in crystal decanters containing up to 32\% $\mathrm{Pb}$ oxide. At the end of 4 months, Pb concentrations in the port were 5,331, 3,061, and 2,162 $\mu \mathrm{g} / \mathrm{L}$ in decanters containing 32, 32, and $24 \% \mathrm{~Pb}$ oxide, respectively. $\mathrm{Pb}$ was also found to elute from $\mathrm{Pb}$ crystal wine glasses within minutes. Mean $\mathrm{Pb}$ concentrations in wine contained in 12 glasses rose from $33 \mu \mathrm{g} / \mathrm{L}$ initially to 68, 81, 92, and $99 \mu \mathrm{g} / \mathrm{L}$ after 1, 2, 3, and 4 hours, respectively (Graziano and Blum 1991).

Hair dyes and some cosmetics may contain Pb compounds (Cohen and Roe 1991). Hair dyes formulated with $\mathrm{Pb}$ acetate may have $\mathrm{Pb}$ concentrations 3-10 times the allowable concentration in paint. Measured $\mathrm{Pb}$ concentrations of 2,300-6,000 $\mu \mathrm{g}$ of $\mathrm{Pb} /$ gram of product have been reported (Mielke et al. 1997). $\mathrm{Pb}$ acetate is soluble in water and easily transferred to hands and other surfaces during and following application of a hair dye product. Measurements of 150-700 $\mu \mathrm{g}$ of $\mathrm{Pb}$ on each hand following application have been reported (Mielke et al. 1997). In addition to transfer of $\mathrm{Pb}$ to the hand-to-mouth pathway of the person applying the product, $\mathrm{Pb}$ can be transferred to any other surface (comb, hair dryer, outside of product container, counter top, etc.) that comes into contact with the product. It is also on the hair that it is applied to and the hands applying it. Objects coming into contact with hair dyed with a Pb-containing product also become contaminated. A dry hand passed through dry hair dyed with a Pb-containing product in cream form was been shown to pick up about $786 \mu \mathrm{g}$ of $\mathrm{Pb}$. A dry hand passed through dry hair dyed using foam or liquid Pb-containing hair dye products picked up less $\mathrm{Pb}: 69 \mu \mathrm{g} / \mathrm{hand}$ for foam products and $73 \mu \mathrm{g} / \mathrm{hand}$ for liquid products (Mielke et al. 1997). An elevated PbB (12 $\mu \mathrm{g} / \mathrm{dL}$ ) in an infant was observed after the use of tiro, a Nigerian eye cosmetic applied to the infant's eyes (CDC 2012a). Elevated PbBs (27.0 and $33.5 \mu \mathrm{g} / \mathrm{dL}$ ) were reported in two young children in New Mexico after the use of kajal, a cosmetic imported from Afghanistan, that was applied to the children's eyelids. The 
kajal was reported to contain 54\% Pb (CDC 2013). Sindoor, a cosmetic and cultural/religious powder used in Hindu cultures, has been found to contain very high amounts of Pb (Lin et al. 2010).

Cases of $\mathrm{Pb}$ poisoning have been related to less common sources of exposure. Illicit "moonshine" whiskey made in stills composed of Pb-soldered parts (e.g., truck radiators) may contain high levels of $\mathrm{Pb}$. Detectable levels of $\mathrm{Pb}$ with a maximum concentration of $5.3 \mathrm{mg} / \mathrm{L}$ were found in 7 of 12 samples of Georgia moonshine whiskey (Gerhardt et al. 1980). Of the 115 suspected moonshine samples seized by local law enforcement between 1995 and 2001 and analyzed by the Bureau of Alcohol, Tobacco, and Firearms, 33 samples (28.7\%) contained $\mathrm{Pb}$ levels $>300 \mu \mathrm{g} / \mathrm{dL}$. The median and maximum levels were 44.0 and 53,200 $\mu \mathrm{g} / \mathrm{dL}$, respectively (Parramore et al. 2001).

Firing of $\mathrm{Pb}$ ammunition may result in exposure to $\mathrm{Pb}$ aerosols and dusts generated during gun or rifle discharge at levels up to $1,000 \mu \mathrm{g} / \mathrm{m}^{3}$ (EPA 1985c), from Pb pellets ingested by or imbedded in animals that are used as food sources, and from $\mathrm{Pb}$ pellets or fragments imbedded in humans from shooting incidents (see Appendix C, Ingestion of Lead Debris). Exposures to airborne $\mathrm{Pb}$ dust from firearm discharge in indoor shooting ranges has been shown to result in increases in PbBs that are 1.5-2 times higher than preexposure concentrations (Greenberg and Hamilton 1999; Gulson et al. 2002). However, the use of copper-jacketed bullets, nonlead primers, and well-ventilated indoor firing ranges lessen the impact of airborne $\mathrm{Pb}$ on blood $\mathrm{Pb}$ levels (Gulson et al. 2002).

$\mathrm{A} \mathrm{Pb}$ poisoning hazard for young children exists in imported vinyl miniblinds that had $\mathrm{Pb}$ added to stabilize the plastic. Over time, the plastic deteriorates to produce $\mathrm{Pb}$ dust that can be ingested when the blinds are touched by children, who then put their hands in their mouths (CPSC 1996). The U.S. Consumer Product Safety Commission (CPSC) has requested that manufacturers change the manufacturing process to eliminate the $\mathrm{Pb}$. As a consequence, vinyl miniblinds should now be $\mathrm{Pb}-\mathrm{free}$. The CPSC recommends that consumers with young children remove old vinyl miniblinds from their homes and replace them with new miniblinds made without added $\mathrm{Pb}$ or with alternative window coverings.

Inexpensive metallic jewelry items specifically intended for children and teenagers have been shown to contain varying levels of $\mathrm{Pb}$ (Maas et al. 2005). A total of 311 chemical assays conducted using 285 jewelry items purchased in 20 different stores in California revealed that a considerable amount of $\mathrm{Pb}$ was added to the items, presumably to increase their weight or to impart some type of metallic coating to the surface of the item. The mean weight percentage of $\mathrm{Pb}$ for all 311 assays was $30.6 \%$. Of the 
311 samples tested, 169 contained at least $3 \% \mathrm{~Pb}$ by weight in at least one portion of the jewelry piece and 123 of the samples were found to contain $>50 \% \mathrm{~Pb}$ by weight (Maas et al. 2005). In addition, 62 pieces of the purchased jewelry were tested for surface levels of $\mathrm{Pb}$ that could potentially be transferred dermally through the routine handling of these pieces. Using standard laboratory wipes, the surface of the jewelry pieces were wiped for a total of 20 seconds and subsequently analyzed for $\mathrm{Pb}$ content. Mean Pb levels in the wipes ranged from 0.06 to $541.97 \mu \mathrm{g}$. The authors characterized the potential $\mathrm{Pb}$ exposure from these dermal transfer experiments as either low exposure $(<1 \mu \mathrm{g}$ of $\mathrm{Pb}$ transferred to the laboratory wipe), moderate exposure (1-10 $\mu \mathrm{g}$ of $\mathrm{Pb}$ transferred to the laboratory wipe), high exposure (10-50 $\mu \mathrm{g}$ of $\mathrm{Pb}$ transferred to the laboratory wipe), and very high exposure ( $>50 \mu \mathrm{g}$ of $\mathrm{Pb}$ transferred to the laboratory wipe). Approximately 35\% of the 62 pieces tested were characterized as having low exposure, $48 \%$ were characterized as moderate exposure, $11 \%$ were characterized as high exposure, and 5\% were characterized as very high exposure (Maas et al. 2005).

\subsection{GENERAL POPULATION EXPOSURE}

Measurements of $\mathrm{Pb}$ in blood, urine, and tissues (postmortem) have been used to assess exposures of individuals to $\mathrm{Pb}$. Table 5-19 shows the lowest limit of detections that are achieved by analytical analysis of blood, urine and tissues.

Table 5-19. Lowest Limit of Detection Based on Standards ${ }^{a}$

\begin{tabular}{lll}
\hline Media & Detection limit & Reference \\
\hline Whole blood/urine/tissue & $0.05 \mu \mathrm{g} \mathrm{Pb} / \mathrm{g}$ blood or $\mathrm{mL}$ urine & NIOSH 1994b, Method 8003 \\
\cline { 2 - 3 } & $1 \mu \mathrm{g} / 100 \mathrm{~g}$ blood; $0.2 \mu \mathrm{g} / \mathrm{g}$ tissue & NIOSH 1994a, Method 8005 \\
\hline Animal tissue & $0.1 \mu \mathrm{g} / \mathrm{g}$ (ICP-MS or GFAA) & NOAA 1998 \\
\hline
\end{tabular}

${ }^{a}$ Detection limits based on using appropriate preparation and analytics. These limits may not be possible in all situations.

GFAA = graphite furnace atomic absorption; ICP-MS = inductively coupled plasma-mass spectrometry

Prior to the 1980s, aerolized $\mathrm{Pb}$ emissions from the use of leaded gasoline was the main source of $\mathrm{Pb}$ exposure for the general U.S. population. Aerolized $\mathrm{Pb}$ can be either inhaled or ingested after deposition on surfaces and food crops. Adult $\mathrm{Pb}$ exposures tend to be limited to occupational or recreational sources. For children, the primary source of $\mathrm{Pb}$ exposure is from surface dusts (on the ground or entrained) that contain $\mathrm{Pb}$ from a variety of sources including deteriorated Pb-based paint (Bornschein et al. 1986; CDC 2009; Dixon et al. 2009; Egeghy et al. 2005; EPA 1996c; Garavan et al. 2008; Gulson et al. 2009; 
Lanphear and Roghmann 1997; Lanphear et al. 1998a; Lewin et al. 1999; Malcoe et al. 2002; Mielke et al. 2007; Succop et al. 1998; Von Lindern et al. 2003, 2016; Zahran et al. 2013). Young children are particularly vulnerable to $\mathrm{Pb}$ exposure because of hand-to-mouth activity, which contributes to ingestion of $\mathrm{Pb}$ in surface dusts. $\mathrm{Pb}$ in the fine particle fraction of surface dusts $(<150 \mu \mathrm{m})$ readily adheres to the skin surface, from which it can be inadvertently ingested from hand-to-mouth activity (Choate et al. 2006a, 2006b; Clausing et al. 1987; Davis and Mirick 2006; Davis et al. 1990; Siciliano et al. 2009; Yamamoto et al. 2006). Several studies have attempted to quantify soil and dust ingestion in children (Chien et al. 2017; Ozkaynak et al. 2011; Sedman et al. 1994; Stanek et al. 2012; Von Lindern et al. 2016; Wilson et al. 2013) and adults (Calabrese et al. 1990; Doyle et al. 2012; Irvine et al. 2014; Stanek et al. 1997).

Although air $\mathrm{Pb}$ can be a direct pathway of exposure in children, it can also be an indirect pathway from its effect on Pb concentration in surface dusts (Brunekreef 1984; Hayes et al. 1994; Hilts 2003; Rabinowitz et al. 1985; Schnaas et al. 2004; Schwartz and Pitcher 1989; Tripathi et al. 2001). Secondhand smoke may also contribute to increased Pb exposure (Apostolou et al. 2012; Mannino et al. 2003; Richter et al. 2013). Dietary sources of $\mathrm{Pb}$ can originate from direct or indirect transfer of atmospheric $\mathrm{Pb}$ emissions to secondary media such as water, food crops, game, and fish. Pb in the maternal system can also be transferred to the fetus during gestation and to the nursing infant (EPA 2014c).

Several studies provided data on $\mathrm{Pb}$ levels in food, with which dietary intakes of $\mathrm{Pb}$ for the general population in the United States have been estimated (FDA 2016a, 2016b). An analysis of individual food intakes and PbB from NHANES (2006-2008) estimated that diet explained approximately $2.9 \%$ of the variations of $\mathrm{PbB}$ in children and 1.6\% in adults (Davis et al. 2014). A randomized survey of 250 individuals (adults and children) from the Midwest United States conducted over the period 19951997 estimated average dietary Pb intake to be approximately $10 \mu \mathrm{g} /$ day (Clayton et al. 1999). The EPA has estimated mean dietary $\mathrm{Pb}$ intakes in children ages 6-84 months to be approximately $2 \mu \mathrm{g} /$ day (EPA 2014c). The ban on the use of welded (non-soldered) food cans during the 1980s has resulted in a decrease in $\mathrm{Pb}$ exposure from foods (FDA 2006). In recent surveys, the mean Pb levels in dairy products (e.g., milk, cheese, ice cream, cream, yogurt) were generally low or below the detection limit. Mean concentrations of $\mathrm{Pb}$ in fruits and vegetables were also generally low. Mean concentration of $\mathrm{Pb}$ in baby foods ranged from not detected to $0.013 \mathrm{mg} / \mathrm{kg}$. Possible sources of $\mathrm{Pb}$ in food samples include introduction during processing or preparation with drinking water contaminated with $\mathrm{Pb}$, deposition of $\mathrm{Pb}$ onto raw materials for each food, and $\mathrm{Pb}$ exposure in livestock that produce dairy or meat ingredients (EPA 2014c). Pb has also been reported in home-prepared reconstituted infant formula. Although, at one 
time, use of $\mathrm{Pb}$ solder in formula containers contributed to PbB from formula consumption (Ryu et al. 1983), this practice was phased out after 1970 in the United States and subsequently banned (FDA 1995). However, tap water remains a potential source of $\mathrm{Pb}$ in home-prepared formula at locations where tap water $\mathrm{Pb}$ concentrations are elevated. In a study conducted in the Boston area in 1997, 2 of 40 samples of home-prepared formula had $\mathrm{Pb}$ concentrations $>15 \mu \mathrm{g} / \mathrm{L}$. In both cases, the reconstituted formula had been prepared using cold tap water run for 5-30 seconds, drawn from the plumbing of houses $>20$ years old. Pb-containing ceramic ware used in food preparation has also been associated with childhood $\mathrm{Pb}$ exposure in children of Hispanic ethnicity in San Diego County, California. One study (Gersberg et al. 1997) used the IEUBK Model to determine that dietary Pb exposure from beans prepared in Mexican ceramic bean pots may account for a major fraction of blood $\mathrm{Pb}$ burden in children whose families use such ceramic ware.

The main source of $\mathrm{Pb}$ in drinking water is from the corrosion of $\mathrm{Pb}$ service lines, which are pipes constructed of pure $\mathrm{Pb}$ that connect the water distribution main to a building's internal plumbing. Other common sources of $\mathrm{Pb}$ in drinking water are exposed leaded solder or corroded fixtures containing $\mathrm{Pb}$ (EPA 2016a). While Pb was restricted to no more than 8\% in plumbing materials in 1986, older homes and neighborhoods may still contain $\mathrm{Pb}$ service lines, $\mathrm{Pb}$ connections, $\mathrm{Pb}$ solder, or other $\mathrm{Pb}$-based plumbing materials that may contaminate drinking water during its delivery from its source to homes. Corrosion of these older plumbing materials can result in leaching of Pb into drinking water (CDC 2012b; Hanna-Attisha et al. 2016). Flint, Michigan is an example of how a water system with Pb sources in drinking water infrastructure resulted in elevated $\mathrm{Pb}$ levels in drinking water. For decades, the drinking water for the City of Flint was purchased from the Detroit Water and Sewer Department (DWSD). This water had optimized corrosion control and was treated with orthophosphate, a corrosion inhibitor that reduces $\mathrm{Pb}$ solubility and leaching from leaded plumbing materials by the formation of protective scales on the pipe's interior surface. When the water source was changed to the Flint River in 2014, corrosion control was not implemented, which allowed $\mathrm{Pb}$ to leach into the drinking water (EPA 2017e). $\mathrm{Pb}$ concentration in first-draw tap water tends to be higher than after the plumbing system has been flushed, although with $\mathrm{Pb}$ service lines, it is possible to see higher $\mathrm{Pb}$ concentrations in flushed water, if flushing is sufficient to draw stagnant water from the service line to the tap. Gulson et al. (1997a) measured Pb in household water throughout the day when the plumbing system of an unoccupied test house was not flushed. Water concentration data ranged from $119 \mu \mathrm{g} / \mathrm{L}$ for the initial (first-draw) sample to 35-52 $\mu \mathrm{g} / \mathrm{L}$ for hourly samples to $1.7 \mu \mathrm{g} / \mathrm{L}$ for a fully flushed sample. The $1991 \mathrm{LCR}$ was implemented to protect public health by minimizing $\mathrm{Pb}$ and copper levels in drinking water, by primarily reducing water corrosivity (EPA 2010). The rule set a Pb action level of $15 \mu \mathrm{g} / \mathrm{L}$ based on $90^{\text {th }}$ percentile levels of tap 
water samples. The LCR established tap sampling monitoring requirements for public water systems. One-liter samples are taken at the tap where water has stood in the pipes for at least 6 hours (first-draw) in homes and buildings that are considered high-risk of $\mathrm{Pb}$ and copper contamination, and the number of samples are based on the system size. Pb action level exceedances can trigger a number of steps that a water system can take to reduce $\mathrm{Pb}$ exposure. These requirements include implementing a corrosion control treatment program, monitoring and/or treating source water, public education, and $\mathrm{Pb}$ service line replacement (EPA 2004). As discussed in Section 5.5.2, EPA has proposed major changes in the LCR as of October 2019.

Other less common sources of $\mathrm{Pb}$ exposure also exist. Exposure may also result from engaging in hobbies that use $\mathrm{Pb}$ (e.g., leaded solder is used in making stained glass, molten $\mathrm{Pb}$ used in casting, leaded glazes and frits are used in making pottery, and $\mathrm{Pb}$ compounds as coloring agents in glassblowing) (Grabo 1997). The use of inadequately glazed or heavily worn earthenware vessels for food storage and cooking may result in Pb exposure (CDC 1985; EPA 1986b). Various folk remedies and Ayurvedic medication (CDC 1998, 2004b, 2012c; Garvey et al. 2001; Wu et al. 1996) and some cosmetics (Mielke et al. 1997) may also be sources of $\mathrm{Pb}$ exposure. Moonshine consumption was strongly associated with elevated PbBs (Morgan and Parramore 2001). A 2000 study found a median PbB of $11 \mu \mathrm{g} / \mathrm{dL}$ among 35 moonshine consumers versus $2.5 \mu \mathrm{g} / \mathrm{dL}$ in 68 randomly-selected nonmoonshine consumers (Parramore et al. 2001). Exposure to infants and children can occur from mouthing of leaded jewelry and toys containing $\mathrm{Pb}$ or painted with leaded paint (CDC 2018c).

Plastic food wrappers may be printed with pigments that contain $\mathrm{Pb}$ chromates. Plastic wrappers used for 14 different national brands of bread collected in New Jersey contained a mean concentration of $26 \mathrm{mg}$ of $\mathrm{Pb}$ for a bag size of 2,000 $\mathrm{cm}^{2}$. A survey of 106 homemakers who buy such breads indicated that $39 \%$ of them reused the bags and $16 \%$ of the respondents turned the bags inside out to reuse them, suggesting that the potential exists for $\mathrm{Pb}$ leaching from the paint into the stored food (Weisel et al. 1991).

Blood Pb levels measured as a part of the NHANES revealed that between 1976 and 1991, the mean PbBs of the U.S. population aged 1-74 years old dropped 78\%, from 12.8 to $2.8 \mu \mathrm{g} / \mathrm{dL}$. The prevalence of $\mathrm{PbBs} \geq 10 \mu \mathrm{g} / \mathrm{dL}$ also decreased sharply from 77.8 to $4.3 \%$. The major cause of the observed decline in $\mathrm{PbBs}$ is most likely the removal of $99.8 \%$ of $\mathrm{Pb}$ from gasoline and the removal of $\mathrm{Pb}$ from soldered cans (Pirkle et al. 1994). Data from the Fourth National Report on Human Exposure to Environmental Chemical are summarized in Tables 5-20 and 5-21, which provide geometric means of Pb levels in the blood and urine in segments of the U.S. population. 
Table 5-20. Geometric Mean Blood Lead Levels $(\mu \mathrm{g} / \mathrm{dL})$ and the $95^{\text {th }}$ Percentile Confidence Interval, by Race/Ethnicity, Sex, and Age for the Years for 2011-2016

\begin{tabular}{|c|c|c|c|}
\hline & Survey years & Geometric mean (95\% confidence interval) & Sample size \\
\hline \multirow[t]{3}{*}{ Total } & $11-12$ & $0.973(0.916-1.04)$ & 7,920 \\
\hline & $13-14$ & $0.858(0.813-0.906)$ & 5,215 \\
\hline & $15-16$ & $0.820(0.772-0.872)$ & 4,988 \\
\hline \multicolumn{4}{|l|}{ Age group } \\
\hline \multirow[t]{3}{*}{$1-5$ years } & $11-12$ & $0.970(0.877-1.07)$ & 713 \\
\hline & $13-14$ & $0.782(0.705-0.869)$ & 818 \\
\hline & $15-16$ & $0.758(0.675-0.850$ & 790 \\
\hline \multirow[t]{3}{*}{$6-11$ years } & $11-12$ & $0.681(0.623-0.744)$ & 1,048 \\
\hline & $13-14$ & $0.567(0.529-0.607)$ & 1,075 \\
\hline & $15-16$ & $0.571(0.523-0.623)$ & 565 \\
\hline \multirow[t]{3}{*}{$12-19$ years } & $11-12$ & $0.554(0.511-0.601)$ & 1,129 \\
\hline & $13-14$ & $0.506(0.464-0.551)$ & 627 \\
\hline & $15-16$ & $0.467(0.433-0.504$ & 1,023 \\
\hline \multirow[t]{3}{*}{20 years and older } & $11-12$ & $1.09(1.03-1.16)$ & 5,030 \\
\hline & $13-14$ & $0.967(0.921-1.02)$ & 2,695 \\
\hline & $15-16$ & $0.920(0.862-0.982)$ & 2,610 \\
\hline \multicolumn{4}{|l|}{ Gender } \\
\hline \multirow[t]{3}{*}{ Males } & $11-12$ & $1.13(1.06-1.21)$ & 3,968 \\
\hline & $13-14$ & $0.994(0.919-1.08)$ & 2,587 \\
\hline & $15-16$ & $1.13(1.06-1.21)$ & 3,968 \\
\hline \multirow[t]{3}{*}{ Females } & $11-12$ & $0.842(0.796-0.890)$ & 3,952 \\
\hline & $13-14$ & $0.746(0.715-0.777)$ & 2,628 \\
\hline & $15-16$ & $0.735(0.679-0.795)$ & 2,500 \\
\hline \multicolumn{4}{|l|}{ Race/ethnicity } \\
\hline \multirow[t]{3}{*}{ Mexican Americans } & $11-12$ & $0.838(0.767-0.916)$ & 1,077 \\
\hline & $13-14$ & $0.746(0.685-0.813)$ & 969 \\
\hline & $15-16$ & $0.704(0.659-0.759)$ & 994 \\
\hline \multirow[t]{3}{*}{ Non-Hispanic blacks } & $11-12$ & $0.998(0.947-1.05)$ & 2,195 \\
\hline & $13-14$ & $0.871(0.787-0.963)$ & 1,119 \\
\hline & $15-16$ & $0.856(0.763-0.962$ & 1,070 \\
\hline \multirow[t]{3}{*}{ Non-Hispanic whites } & $11-12$ & $0.993(0.914-1.08)$ & 2,493 \\
\hline & $13-14$ & $0.882(0.820-0.950)$ & 1,848 \\
\hline & $15-16$ & $0.835(0.774-0.900)$ & 1,511 \\
\hline \multirow[t]{3}{*}{ All Hispanics } & $11-12$ & $0.855(0.793-0.922)$ & 1,931 \\
\hline & $13-14$ & $0.742(0.695-0.793)$ & 1,481 \\
\hline & $15-16$ & $0.703(0.658-0.750)$ & 1,664 \\
\hline \multirow[t]{2}{*}{ Asians } & $11-12$ & $1.15(1.06-1.24)$ & 1,005 \\
\hline & $13-14$ & $1.01(0.923-1.11)$ & 510 \\
\hline
\end{tabular}


Table 5-20. Geometric Mean Blood Lead Levels $(\mu \mathrm{g} / \mathrm{dL})$ and the $95^{\text {th }}$ Percentile Confidence Interval, by Race/Ethnicity, Sex, and Age for the Years for 2011-2016

Survey years Geometric mean (95\% confidence interval) Sample size

$15-16 \quad 1.07(0.976-1.18) \quad 479$

Source: CDC 2018a

Table 5-21. Geometric Mean Urine Lead Levels $(\mu \mathrm{g} / \mathrm{dL})$ and the $95^{\text {th }}$ Percentile Confidence Interval, by Race/Ethnicity, Sex, and Age

\begin{tabular}{|c|c|c|c|}
\hline & Surve & Geometric mean (95\% confidence interval) & Sample size \\
\hline \multirow[t]{3}{*}{ Total } & $11-12$ & $0.360(0.328-0.396)$ & 2,504 \\
\hline & $13-14$ & $0.277(0.257-0.298)$ & 2,664 \\
\hline & $15-16$ & & 3,061 \\
\hline \multicolumn{4}{|l|}{ Age group } \\
\hline $3-5$ years & $15-16$ & $0.257(0.225-0.292)$ & 486 \\
\hline \multirow[t]{3}{*}{$6-11$ years } & $11-12$ & $0.346(0.292-0.410)$ & 399 \\
\hline & $13-14$ & $0.222(0.192-0.258)$ & 402 \\
\hline & $15-16$ & $0.346(0.292-0.410)$ & 399 \\
\hline \multirow[t]{3}{*}{$12-19$ years } & $11-12$ & $0.259(0.219-0.305)$ & 390 \\
\hline & $13-14$ & $0.201(0.166-0.245)$ & 451 \\
\hline & $15-16$ & $0.196(0.183-0.211)$ & 402 \\
\hline \multirow[t]{3}{*}{20 years and older } & $11-12$ & $0.381(0.348-0.416)$ & 1,715 \\
\hline & $13-14$ & $0.297(0.280-0.315)$ & 1,811 \\
\hline & $15-16$ & $0.304(0.276-0.334)$ & 1,794 \\
\hline \multicolumn{4}{|l|}{ Gender } \\
\hline \multirow[t]{3}{*}{ Males } & $11-12$ & $0.414(0.367-0.466)$ & 1,262 \\
\hline & $13-14$ & $0.315(0.295-0.337)$ & 1,318 \\
\hline & $15-16$ & $0.313(0.285-0.343)$ & 1,524 \\
\hline \multirow[t]{3}{*}{ Females } & $11-12$ & $0.316(0.282-0.355)$ & 1,242 \\
\hline & $13-14$ & $0.245(0.222-0.269)$ & 1,346 \\
\hline & $15-16$ & $0.259(0.233-0.288)$ & 1,537 \\
\hline \multicolumn{4}{|l|}{ Race/ethnicity } \\
\hline \multirow[t]{3}{*}{ Mexican Americans } & $11-12$ & $0.372(0.320-0.431)$ & 317 \\
\hline & $13-14$ & $0.277(0.240-0.319)$ & 453 \\
\hline & $15-16$ & $0.259(0.233-0.288)$ & 585 \\
\hline \multirow{3}{*}{$\begin{array}{l}\text { Non-Hispanic } \\
\text { blacks }\end{array}$} & $11-12$ & $0.431(0.385-0.483)$ & 669 \\
\hline & $13-14$ & $0.371(0.320-0.429)$ & 581 \\
\hline & & $0.340(0.298-0.388)$ & 671 \\
\hline \multirow{3}{*}{$\begin{array}{l}\text { Non-Hispanic } \\
\text { whites }\end{array}$} & $11-12$ & $0.346(0.311-0.385)$ & 820 \\
\hline & $13-14$ & $0.267(0.245-0.290)$ & 985 \\
\hline & $15-16$ & $0.275(0.247-0.305)$ & 924 \\
\hline All Hispanics & $11-12$ & $0.372(0.327-0.423)$ & 573 \\
\hline
\end{tabular}




\begin{tabular}{|c|c|c|c|}
\hline & \multicolumn{2}{|c|}{ Survey years Geometric mean (95\% confidence interval) } & \multirow{2}{*}{$\frac{\text { Sample size }}{701}$} \\
\hline & $13-14$ & $0.270(0.239-0.305)$ & \\
\hline & $15-16$ & $0.284(0.258-0.312)$ & 982 \\
\hline \multirow[t]{3}{*}{ Asians } & $11-12$ & $0.383(0.341-0.429)$ & 353 \\
\hline & $13-14$ & $0.257(0.230-0.287)$ & 292 \\
\hline & $15-16$ & $0.292(0.264-0.324)$ & 332 \\
\hline
\end{tabular}

Source: CDC 2019

The Adult Blood Lead Epidemiology and Surveillance (ABLES) program tracks adult (aged $\geq 16$ years) cases with elevated $\mathrm{PbBs}$ from workplace exposure. In 2016, 26 states submitted $\mathrm{PbB}$ data on 18,093 adults with $\mathrm{PbBs} \geq 10 \mu \mathrm{g} / \mathrm{dL}$. $\mathrm{PbBs} \geq 10 \mu \mathrm{g} / \mathrm{dL}$ declined from 26.6 adults per 100,000 employed in 2010 to 15.8 per 100,000 employed in 2016 (results for data submitted as of December 2018). In 2016, among adults with known exposures, $90.3 \%$ had occupational exposure. The majority of these adults were employed in manufacturing, construction, mining, and services. Table 5-22 presents industries within each sector with the most workers with occupational exposures resulting in $\mathrm{PbB} \geq 25 \mu \mathrm{g} / \mathrm{dL}$ during 2010-2016 (NIOSH 2017a).

\section{Table 5-22. Industries by Sector with Most Workers having Blood Lead Concentrations (PbBs) $\geq 25 \mu \mathrm{g} / \mathrm{dL}, 2010-2016$}

\begin{tabular}{ll}
\hline NORA Sector & Industry NAICS Code \\
\hline Manufacturing & Storage battery manufacturing (33591) \\
\hline Nonferrous metal (except copper and aluminum) rolling, drawing, \\
extruding, and alloying (33149)
\end{tabular}

Services (except public safety) All other amusement and recreation industries (71399)

Remediation services (56291) 
Table 5-22. Industries by Sector with Most Workers having Blood Lead Concentrations (PbBs) $\geq 25 \mu \mathrm{g} / \mathrm{dL}, 2010-2016$

\begin{tabular}{ll}
\hline NORA Sector & Industry NAICS Code \\
\hline & Automotive mechanical and electrical repair and maintenance (81111) \\
\cline { 2 - 2 } & Other services (except public safety industries) (71394) \\
\hline Mining & Copper, nickel, lead, and zinc mining (21223) \\
\hline
\end{tabular}

NAICS = North American Industry Classification System; NORA = National Occupational Research Agenda

Source: NIOSH 2017a

Raymond and Brown (2015a, 2015b, 2017) and analyzed the 2007-2012 and 2009-2014 datasets from the Childhood Blood Lead Surveillance (CBLS) system. In 2007, a total of 38 states identified and reported 37,289 children ( $<6$ years) with $\mathrm{PbB} \geq 10 \mu \mathrm{g} / \mathrm{dL}$. In 2012, a total of 30 jurisdictions identified and reported approximately 138,000 children ( $<6$ years) with $\mathrm{PbB} \geq 5 \mu \mathrm{g} / \mathrm{dL}$. In 2012, federal funding ended and several states lost their state-wide $\mathrm{Pb}$ poisoning prevention programs and in 2013, the number of states reporting data declined, as did the number of children reported to the CDC with $\mathrm{PbB} \geq 5 \mu \mathrm{g} / \mathrm{dL}$. In October 2013, federal funding resumed and in 2013, 27 states, the District of Columbia, and New York City reported data. In 2014, 30 states, the District of Columbia, and New York City reported data.

Table 5-23 summarizes the number and rate per 100,000 children aged $<5$ years with blood Pb levels 5$9 \mu \mathrm{g} / \mathrm{dL}$ reported in the 2010-2014 CBLS system. PbBs $\geq 10 \mu \mathrm{g} / \mathrm{dL}$ continue to be more prevalent among children with known risk factors, such as minority race or ethnicity, urban residence, residing in homes built prior to the 1950s, and low family income (CDC 2009).

\section{Table 5-23. Number and Rate per 100,000 Children Aged $<5$ Years with Blood Lead Levels 5-9 $\mu \mathrm{g} / \mathrm{dL}$ in the Childhood Blood Lead Surveillance System, United States, 2010-2014}

\begin{tabular}{lcccc}
\hline & \multicolumn{2}{c}{$<1$ Year } & 1-4 Years \\
\cline { 2 - 5 } Year & Number & Rate & Number & Rate \\
\hline $2010^{\mathrm{a}}$ & 18,598 & 448.48 & 137,887 & 805.62 \\
\hline $2011^{\mathrm{b}}$ & 13,981 & 352.69 & 130,838 & 810.56 \\
\hline $2012^{\mathrm{c}}$ & 7,876 & 199.74 & 95,854 & 596.58 \\
\hline $2013^{\mathrm{d}}$ & 5,494 & 138.26 & 57,293 & 360.46 \\
\hline $2014^{\mathrm{e}}$ & 5,904 & 148.51 & 70,680 & 444.49 \\
\hline
\end{tabular}

a37 jurisdictions reporting.

b36 jurisdictions reporting.

c30 jurisdictions reporting.

d29 jurisdictions reporting.

e32 jurisdictions reporting. 


\section{Table 5-23. Number and Rate per 100,000 Children Aged $<5$ Years with Blood Lead Levels 5-9 $\mu \mathrm{g} / \mathrm{dL}$ in the Childhood Blood Lead Surveillance System, United States, 2010-2014}

\begin{tabular}{lrrrr}
\hline & \multicolumn{3}{c}{$<$ Year } & $1-4$ Years \\
\cline { 2 - 5 } Year & Number & Rate & Number & Rate \\
\hline
\end{tabular}

Source: Raymond and Brown 2017

Various studies suggest that ingestion of game hunted with $\mathrm{Pb}$ shot is associated with increased PbBs. Johansen et al. (2006) collected blood samples from 50 men in Nuuk, Greenland to study the relationship between the consumption of birds hunted with $\mathrm{Pb}$ shot and $\mathrm{PbBs}$. Men who regularly ate hunted birds killed with $\mathrm{Pb}$ shot had mean $\mathrm{PbB}$ ranging from $6.2 \mu \mathrm{g} / \mathrm{dL}$ in the group eating 0.1-5 bird equivalents per month to $12.8 \mu \mathrm{g} / \mathrm{dL}$ in those eating >30 bird equivalents per month. In addition, levels were highest in mid-winter when consumption of hunted birds was highest. Those who did not consume hunted birds had a mean $\mathrm{PbB}$ of $1.5 \mu \mathrm{g} / \mathrm{dL}$. These results are consistent with earlier surveys of Arctic hunting communities. A 1992 survey of 492 Inuit adults from the Arctic region of Quebec, Canada showed that consumption of waterfowl, along with age and smoking, were associated with elevated $\mathrm{PbB}$ (Dewailly et al. 2001). The geometric mean $\mathrm{PbB}$ was $0.42 \mu \mathrm{mol} / \mathrm{L}(8.7 \mu \mathrm{g} / \mathrm{dL})$, with a range of $0.04-2.28 \mu \mathrm{mol} / \mathrm{L}$ $(0.8-47 \mu \mathrm{g} / \mathrm{dL})$. In a cohort of Inuit newborns from northern Quebec, where the population consumed game killed with $\mathrm{Pb}$ shot, the geometric umbilical cord $\mathrm{PbB}$ was $3.9 \mu \mathrm{g} / \mathrm{dL}$ (range $0.2-27 \mu \mathrm{g} / \mathrm{dL}$ ); $7 \%$ of Inuit newborns had cord PbBs $>10 \mu \mathrm{g} / \mathrm{dL}$ as compared to $0.16 \%$ of the non-Inuit population in southern Quebec (Lévesque et al. 2003).

Second-hand smoke may also contribute to increased Pb exposure (Apostolou et al. 2012; Mannino et al. 2003; Richter et al. 2013). Pb is a component of tobacco and tobacco smoke, and smokers often have higher Pb blood levels than nonsmokers (Bonanno et al. 2001; Mannino et al. 2003). Using data from the NHEXAS EPA Region V study, PbB levels in smokers and nonsmokers were analyzed and a correlation between tobacco smoke and exposure levels was observed (Bonanno et al. 2001). The mean PbBs in smokers, nonsmokers exposed to environmental tobacco smoke (ETS), and nonsmokers without ETS were 2.85, 2.06, and $1.81 \mu \mathrm{g} / \mathrm{dL}$, respectively (Bonanno et al. 2001). Recent $\mathrm{Pb}$ urine concentrations for the U.S. adult population from the NHANES by smoking status are presented in Table 5-24. 
Table 5-24. Geometric Mean Urine Lead Levels $(\mu \mathrm{g} / \mathrm{dL})$ and the 95th Percentile Confidence Interval by Smoking Status

\begin{tabular}{|c|c|c|c|}
\hline & Survey years & $\begin{array}{l}\text { Geometric mean (95\% } \\
\text { confidence interval) }\end{array}$ & Sample size \\
\hline \multicolumn{4}{|c|}{ Cigarette smokers } \\
\hline \multirow[t]{2}{*}{ Total } & $11-12$ & $2.36(1.71-4.62)$ & 876 \\
\hline & $13-14$ & $1.51(1.30-1.91)$ & 957 \\
\hline \multicolumn{4}{|l|}{ Age group } \\
\hline 20-49 years & $11-12$ & $1.78(1.41-3.07)$ & 522 \\
\hline 18-49 years & $13-14$ & 1.34 (1.13-1.92) & 583 \\
\hline \multirow[t]{2}{*}{50 years and older } & $11-12$ & 3.35 (1.62-6.83) & 354 \\
\hline & $13-14$ & $1.72(1.40-2.03)$ & 374 \\
\hline \multicolumn{4}{|l|}{ Gender } \\
\hline \multirow[t]{2}{*}{ Males } & $11-12$ & $3.07(1.73-5.03)$ & 527 \\
\hline & $13-14$ & $1.91(1.48-2.14)$ & 512 \\
\hline \multirow[t]{2}{*}{ Females } & $11-12$ & $1.58(1.14-3.45)$ & 349 \\
\hline & $13-14$ & $1.30(1.12-1.41)$ & 445 \\
\hline \multicolumn{4}{|c|}{ Nonsmokers $^{\mathrm{a}}$} \\
\hline \multirow[t]{2}{*}{ Total } & $11-12$ & $1.38(1.25-1.58)$ & 1,343 \\
\hline & $13-14$ & $1.16(0.950-1.51)$ & 1,487 \\
\hline \multicolumn{4}{|l|}{ Age group } \\
\hline $20-49$ years & $11-12$ & $1.26(1.02-1.38)$ & 671 \\
\hline 18-49 years & $13-14$ & $0.880(0.720-1.04)$ & 778 \\
\hline \multirow[t]{2}{*}{50 years and older } & $11-12$ & $1.63(1.29-2.16)$ & 672 \\
\hline & $13-14$ & $1.48(1.12-2.52)$ & 709 \\
\hline \multicolumn{4}{|l|}{ Gender } \\
\hline \multirow[t]{2}{*}{ Males } & $11-12$ & $1.61(1.18-2.13)$ & 635 \\
\hline & $13-14$ & $1.51(1.04-2.68)$ & 663 \\
\hline \multirow[t]{2}{*}{ Females } & $11-12$ & 1.32 (1.06-1.38) & 708 \\
\hline & $13-14$ & $0.238(0.219-0.258)$ & 824 \\
\hline
\end{tabular}

${ }^{\mathrm{a} C}$ igarette nonsmokers who used other tobacco products were excluded.

Source: CDC 2018a

Studies have been conducted to determine exposure of firearm instructors to $\mathrm{Pb}$ at outdoor firing ranges when either nonjacketed (pure $\mathrm{Pb}$ ) or jacketed (copper-coated) bullets were used. Instructors are likely to have higher exposure than shooters because they spend more time at the range. In studies at an outdoor range in Virginia, the mean breathing zone $\mathrm{Pb}$ level when nonjacketed bullets were fired was $67.1 \mu \mathrm{g} / \mathrm{m}^{3}$ for one instructor and $211.1 \mu \mathrm{g} / \mathrm{m}^{3}$ for another (Tripathi and Llewellyn 1990). When jacketed bullets were used, breathing zone levels decreased to $\leq 8.7 \mu \mathrm{g} / \mathrm{m}^{3}$. PbBs of the instructors did not exceed the OSHA Pb standard's medical removal level of $2.4 \mu \mathrm{mol} / \mathrm{L}(60 \mu \mathrm{g} / \mathrm{dL})$ in either case (OSHA 2016a). 
When shooters fired conventional $\mathrm{Pb}$ bullets, their mean exposures to airborne $\mathrm{Pb}$ were $128 \mu \mathrm{g} / \mathrm{m}^{3}$ in the personal breathing zone and $68 \mu \mathrm{g} / \mathrm{m}^{3}$ in the general area. When totally copper-jacketed $\mathrm{Pb}$ bullets were fired, the mean breathing zone and general area air sample concentrations were 9.53 and $5.80 \mu \mathrm{g} / \mathrm{m}^{3}$, respectively (Tripathi and Llewellyn 1990). At an outdoor uncovered range in Los Angeles, instructors who spent an average of 15-20 hours/week behind the firing line were found to be exposed to breathing zone $\mathrm{Pb}$ concentrations of 460 and $510 \mu \mathrm{g} / \mathrm{m}^{3}$ measured as 3-hour, time-weighted averages. The $\mathrm{PbB}$ of one instructor reached $3.38 \mu \mathrm{mol} / \mathrm{L}(70 \mu \mathrm{g} / \mathrm{dL})$. After reassignment to other duties, repeat testing indicated his PbB had dropped to $1.35 \mu \mathrm{mol} / \mathrm{L}$ (28 $\mu \mathrm{g} / \mathrm{dL})$ (Goldberg et al. 1991).

In 1991, NIOSH conducted a survey of the Federal Bureau of Investigations (FBI) Firearms Training Unit firing ranges and related facilities to determine occupational $\mathrm{Pb}$ exposures among FBI and Drug Enforcement Agency (DEA) firing range personnel (NIOSH 1996b). Sixty-one personal breathing-zone and 30 area samples for airborne $\mathrm{Pb}$ were collected. Exposures ranged up to $51.7 \mu \mathrm{g} / \mathrm{m}^{3}$ (mean, $12.4 \mu \mathrm{g} / \mathrm{m}^{3}$ ), $2.7 \mu \mathrm{g} / \mathrm{m}^{3}$ (mean, $0.6 \mu \mathrm{g} / \mathrm{m}^{3}$ ), and $4.5 \mu \mathrm{g} / \mathrm{m}^{3}$ (mean, $0.6 \mu \mathrm{g} / \mathrm{m}^{3}$ ) for range instructors, technicians, and gunsmiths, respectively. Exposure of custodians ranged from nondetectable to $220 \mu \mathrm{g} / \mathrm{m}^{3}$ during short-term cleaning of a large indoor range. Carpet dust sampling of dormitory rooms of students who practiced at the firing ranges revealed higher $(\mathrm{p}<0.0005)$ dust- $\mathrm{Pb}$ concentrations when compared to nonstudent dormitories (dust-Pb concentration range of 116-546 $\mu \mathrm{g} / \mathrm{g}$ with a geometric mean of $214 \mu \mathrm{g} / \mathrm{g}$ in the student's rooms versus a dust- $\mathrm{Pb}$ concentration range of 50-188 $\mu \mathrm{g} / \mathrm{g}$ with a geometric mean of $65 \mu \mathrm{g} / \mathrm{g}$ for the nonstudent rooms). This suggested that the students were contaminating their living quarters with $\mathrm{Pb}$.

The American Academy of Pediatrics (AAP) $(1998,2005)$ concluded that although monitoring data demonstrate a decline in $\mathrm{PbBs}, \mathrm{Pb}$ remains a common, preventable, environmental health threat. Most $\mathrm{Pb}$ poisoning in children is the result of dust and chips from deteriorating $\mathrm{Pb}$ paint on interior surfaces (AAP 2005, 2016; ATSDR 2017). The AAP supported the CDC guidelines endorsing universal screening in certain areas and targeted screening for children at high risk (CDC 1997b, 2005). Many children continue to be at risk for ingestion of Pb-based paint and of soil and dust contaminated through the deterioration of Pb-based paint and the residues from combustion of leaded gasoline. A 1974 study indicated that elevated PbBs in children were most likely a result of ingesting Pb-contaminated soil, and that the most likely source was Pb-based paint rather than Pb from automotive exhaust (Ter Haar and Aronow 1974). However, more recent studies have shown that children with the highest PbBs live in areas with high traffic flow where $\mathrm{Pb}$ particles in the air may fall directly to the soil or adhere to the outer surfaces of building and wash to the soil with rain (Mielke et al. 1989, 2008, 2010). The CDC concluded that a 
common source of $\mathrm{Pb}$ exposure for children who have elevated $\mathrm{PbB}$ is $\mathrm{Pb}$-based paint that has deteriorated into paint chips and Pb dusts (CDC 1997b, 2012d).

$\mathrm{Pb}$ can readily cross the placenta; therefore, exposure of women to $\mathrm{Pb}$ during pregnancy results in uptake by the fetus. Furthermore, since the physiological stress of pregnancy may result in mobilization of $\mathrm{Pb}$ from maternal bone, fetal uptake of $\mathrm{Pb}$ can occur from a mother who was exposed to $\mathrm{Pb}$ before pregnancy, even if no $\mathrm{Pb}$ exposure occurs during pregnancy. Maternal $\mathrm{Pb}$ can also be transferred to breastfeeding infants.

Malcoe et al. (2002) assessed Pb sources and their effect on blood Pb in rural Native American and white children living in a former mining region. Blood samples, residential environmental samples (soil, dust, paint, water), and caregiver interviews (hand-mouth behaviors, socioeconomic conditions) were obtained from a representative sample of 245 children ages 1-6 years. There were no ethnic differences in the results. However, poor children were especially vulnerable. Regression analysis showed that mean floor dust $\mathrm{Pb}$ loading $>10.1 \mu \mathrm{g} / \mathrm{ft}^{2}$ and yard soil $\mathrm{Pb}>165.3 \mathrm{mg} / \mathrm{kg}$ were independently associated with blood $\mathrm{Pb}$ levels $\geq 10 \mu \mathrm{g} / \mathrm{dL}$.

The $\mathrm{Pb}$ content of dusts can be a significant source of exposure, especially for young children. Baseline estimates of potential human exposure to dusts, including intake due to normal hand-to-mouth activity, are $0.2 \mathrm{~g}$ /day for children 1-6 years old versus $0.1 \mathrm{~g} /$ day for adults when both indoor and outdoor ingestion of soil including dust is considered (EPA 1989a). For children who engage in pica behavior (the compulsive, habitual consumption of nonfood items), the ingestion rate of soil can be as high as $5 \mathrm{~g} /$ day. Although ingestion of Pb-containing paint may lead to elevated $\mathrm{PbBs}$ in young children, a major source of elevated PbBs $(>10 \mu \mathrm{g} / \mathrm{dL})$ in children is often contaminated household dust and subsequent hand contamination and repetitive mouthing (Bornschein et al. 1986; Charney et al. 1980; Dixon et al. 2009; Lanphear and Roghmann 1997; Lanphear et al. 1998a; Succop et al. 1998). Weathering of Pb-based paint can contribute to the Pb content of dust and soil. Pb levels of indoor dust and outdoor soil were found to be strongly predictive of PbBs in over 200 urban and suburban infants followed from birth to 2 years of age; however, PbBs were not correlated with indoor air or tap water Pb levels, nor the size of nearby roadways. Indoor dust $\mathrm{Pb}$ levels and soil $\mathrm{Pb}$ levels in the homes of children with high $\mathrm{PbBs}$ $(>8.8 \mu \mathrm{g} / \mathrm{dL}$ ) were $72 \mu \mathrm{g} /$ wipe (window sill dust) and $1,011 \mu \mathrm{g} / \mathrm{g}$, respectively; children with low PbBs $(<3.7 \mu \mathrm{g} / \mathrm{dL})$ were exposed to $22 \mu \mathrm{g} /$ wipe and $380 \mu \mathrm{g} / \mathrm{g}$, respectively. In addition, $79 \%$ of the homes of children with high PbBs had been renovated, while only 56\% of the homes of children with low PbBs had been renovated, suggesting that renovating the interior of homes previously painted with leaded paint may 
increase, at least temporarily, a child's exposure to Pb dust (Rabinowitz et al. 1985). Regular use of dust control methods (e.g., wet mopping of floors, damp-sponging of horizontal surfaces, high-efficiency vacuum cleaner) has been shown in some, although not all, cases to reduce indoor dust, $\mathrm{Pb}$ dust, and blood $\mathrm{Pb}$ levels in some, although not all, older homes containing leaded paints (Lanphear et al. 2000b; Rhoads et al. 1999). Decreases of between 17 and 43\% in blood Pb concentrations were observed in children where regular dust control methods had been used to reduce indoor levels of $\mathrm{Pb}$ (Rhoads et al. 1999). EPA (2014c) summarized concentrations of Pb in house dust in the United Stated from 2006 to 2011; these data are presented in Table 5-25.

PbB samples from 1,473 children $<5$ years old were analyzed prior to and after the change in drinking water source in the city of Flint, Michigan (Hanna-Attisha et al. 2016). Prior to the change, 2.4\% of the children had PbB levels exceeding $5 \mu \mathrm{g} / \mathrm{dL}$ ( $\mathrm{n}=736$ ). Following the change in water source, $4.9 \%$ of children's PbB levels exceeded 5 gg/dL for samples obtained from January 1 to September 15, 2015 ( $n=737$ ). The study also found that in areas where $\geq 25 \%$ of the drinking water samples exceeded $15 \mu \mathrm{g} / \mathrm{L}$, the percentage of children with PbB levels $>5 \mu \mathrm{g} / \mathrm{dL}$ increased from 4.0 to $10.6 \%$. Gomez et al. (2018) analyzed PbB levels for children $<5$ years old in Flint, Michigan over an 11-year time span from 2006 to 2016. The percentage of children with PbB levels $>5.0 \mu \mathrm{g} / \mathrm{dL}$ declined from $11.8 \%$ in 2006 to 3.2\% by 2016. The study authors noted an uptick in the geometric mean PbB level during the height of the Flint water crisis from $1.19 \pm 0.02$ to $1.30 \pm 0.02 \mu \mathrm{g} / \mathrm{dL}$ in $2014-2015$, but it declined to $1.15 \pm 0.02 \mu \mathrm{g} / \mathrm{dL}$ in 2016 after the water source was switched back to the DWSD. The authors concluded that while there was a slight increase in $\mathrm{PbB}$ levels during the time at which the source of drinking water was changed for residents of Flint, the overall trend for the 11-year time span was decreasing PbB levels with a nearly $73 \%$ reduction in the percentage of children having levels $>5 \mu \mathrm{g} / \mathrm{dL}$. A second study analyzed PbB levels for females aged 12-50 years prior to (April 25, 2012-October 15, 2013), during (April 25, 2014-October 15, 2015), and immediately after (April 25, 2016-October 15, 2017) the Flint water crisis (Gomez et al. 2019). The authors found that blood levels did not increase for females of child-bearing age residing in Flint during the period when the water supply was changed from the DWSD to the Flint River. The geometric means reported were $0.69 \mu \mathrm{g} / \mathrm{dL}$ (April 25, 2012-October 15, 2013), $0.65 \mu \mathrm{g} / \mathrm{dL}$ (April 25, 2014-October 15, 2015), and $0.55 \mu \mathrm{g} / \mathrm{dL}$, (April 25, 2016-October 15, 2017). 
Table 5-25. Measurements of Lead in Indoor Dust in the United States from 2006 to 2011

\begin{tabular}{|c|c|c|}
\hline Location & Sample site & Value reported \\
\hline New York City, New York & $\begin{array}{l}\text { Glass plate next to open } \\
\text { window of academic } \\
\text { building }\end{array}$ & Median weekly dust loading: 52 $\mu \mathrm{g} / \mathrm{m}^{2}$ \\
\hline $\begin{array}{l}\text { Eureka, Utah near Eureka Mills } \\
\text { Superfund Site }\end{array}$ & $\begin{array}{l}\text { Indoor home site (not } \\
\text { specified) }\end{array}$ & $\begin{array}{l}\text { Dust concentrations, range: } 160- \\
2,000 \mathrm{mg} / \mathrm{kg}\end{array}$ \\
\hline $\begin{array}{l}\text { Denver, Colorado, near } \\
\text { Vasquez Blvd and I-70 } \\
\text { Superfund Site }\end{array}$ & $\begin{array}{l}\text { Indoor home site (not } \\
\text { specified) }\end{array}$ & Dust concentrations, range: $11-660 \mathrm{mg} / \mathrm{kg}$ \\
\hline $\begin{array}{l}\text { East Helena, Montana, near } \\
\text { East Helena Superfund Site }\end{array}$ & $\begin{array}{l}\text { Indoor home site (not } \\
\text { specified) }\end{array}$ & $\begin{array}{l}\text { Dust concentrations, range: } 68- \\
1,000 \mathrm{mg} / \mathrm{kg}\end{array}$ \\
\hline Syracuse, New York & Floor & $\begin{array}{l}\text { Dust concentrations, range: } 209- \\
1,770 \mathrm{mg} / \mathrm{kg}\end{array}$ \\
\hline \multirow[t]{4}{*}{ United States (nationwide) } & Smooth floor & $\begin{array}{l}\text { Median dust loading: } 1.7 \mu \mathrm{g} / \mathrm{m}^{2} \\
\text { Average dust loading: } 4.4 \mu \mathrm{g} / \mathrm{m}^{2}\end{array}$ \\
\hline & Rough floor & $\begin{array}{l}\text { Median dust loading: } 5.6 \mu \mathrm{g} / \mathrm{m}^{2} \\
\text { Average dust loading: } 16 \mu \mathrm{g} / \mathrm{m}^{2}\end{array}$ \\
\hline & Smooth windowsill & $\begin{array}{l}\text { Median dust loading: } 2.5 \mu \mathrm{g} / \mathrm{m}^{2} \\
\text { Average dust loading: } 190 \mu \mathrm{g} / \mathrm{m}^{2}\end{array}$ \\
\hline & Rough windowsill & $\begin{array}{l}\text { Median dust loading: } 55 \mu \mathrm{g} / \mathrm{m}^{2} \\
\text { Average dust loading: } 480 \mu \mathrm{g} / \mathrm{m}^{2}\end{array}$ \\
\hline \multirow[t]{3}{*}{ Milwaukee, Wisconsin } & Central perimeter & Average dust concentration: $107 \mu \mathrm{g} / \mathrm{m}^{2}$ \\
\hline & Entry & Average dust concentration: $140 \mu \mathrm{g} / \mathrm{m}^{2}$ \\
\hline & Window & Average dust concentration: $151 \mu \mathrm{g} / \mathrm{m}^{2}$ \\
\hline \multirow[t]{2}{*}{ Rural towns, Idaho } & Vacuum & $\begin{array}{l}\text { Dust concentration } \\
\text { Median: } 120 \mathrm{mg} / \mathrm{kg} \\
\text { Maximum: } 830 \mathrm{mg} / \mathrm{kg}\end{array}$ \\
\hline & Floor & $\begin{array}{l}\text { Median dust concentration: } 95 \mathrm{mg} / \mathrm{kg} \\
\text { Maximum dust concentration: } 1,300 \mathrm{mg} / \mathrm{kg}\end{array}$ \\
\hline \multirow[t]{2}{*}{$\begin{array}{l}\text { Bunker Hill, Idaho Superfund } \\
\text { Site }\end{array}$} & Vacuum & $\begin{array}{l}\text { Median dust concentration: } 470 \mathrm{mg} / \mathrm{kg} \\
\text { Maximum dust concentration: } 2,000 \mathrm{mg} / \mathrm{kg}\end{array}$ \\
\hline & Floor & $\begin{array}{l}\text { Median dust concentration: } 290 \mathrm{mg} / \mathrm{kg} \\
\text { Maximum dust concentration: } 4,600 \mathrm{mg} / \mathrm{kg}\end{array}$ \\
\hline
\end{tabular}

Source: EPA 2014c

Lanphear and Roghmann (1997) and Lanphear et al. (1996a, 1996b, 1998b) studied factors affecting $\mathrm{PbBs}$ in urban children and found the following independent predictors of children's PbBs: dust $\mathrm{Pb}$ loading in homes (carpets, uncarpeted floors, window sills, and troughs), African-American race/ethnicity, foundation perimeter soil $\mathrm{Pb}$ levels, ingestion of soil or dirt, $\mathrm{Pb}$ content and condition of interior painted surfaces, and first-flush kitchen drinking water Pb levels (Lanphear et al. 1996a, 1996b). Differences in housing conditions and exposures to $\mathrm{Pb}$-containing house dust appear to contribute to the 
racial differences in urban children's PbBs. In addition, white children were more likely to put soil in their mouths (outdoor exposure) and suck their fingers, and African-American children were more likely to put their mouths on window sills (indoor exposure) and to use a bottle. Interior $\mathrm{Pb}$ exposures were more significant for African American children and exterior Pb exposures were more significant for white children (Lanphear et al. 1996a, 1996b). Mouthing behaviors are an important mechanism of Pb exposure among urban children (Lanphear and Roghmann 1997). Community characteristics such as residence within a city, proportion of African Americans, lower housing value, housing built before 1950, higher population density, higher rates of poverty, lower percent of high school graduates, and lower rates of owner-occupied housing have been used to identify children with elevated blood levels (Lanphear et al. 1998b). An analysis of children's PbBs and multiple measures of $\mathrm{Pb}$ concentrations in household dust, tap water, foundation perimeter soil, and interior house paint has been used to predict the effect of changing concentrations of $\mathrm{Pb}$ in environmental media on children's PbBs. An increase in dust $\mathrm{Pb}$ loading from background to $200 \mu \mathrm{g} / \mathrm{ft}^{2}$ was estimated to produce an increase of $23.3 \%$ in the percentage of children estimated to have a $\mathrm{PbB}>10 \mu \mathrm{g} / \mathrm{dL}$; an increase in tap water $\mathrm{Pb}$ concentration from background to $15 \mu \mathrm{g} / \mathrm{L}$ was estimated to produce an increase of $13.7 \%$ in the percentage of children estimated to have a $\mathrm{PbB}$ level $>10 \mu \mathrm{g} / \mathrm{dL}$; and an increase in soil $\mathrm{Pb}$ concentration from background to $400 \mu \mathrm{g} / \mathrm{g}$ was estimated to produce an increase of $11.6 \%$ in the percentage of children estimated to have a PbB level $>10 \mu \mathrm{g} / \mathrm{dL}$ (Lanphear et al. 1998a).

Outdoor $\mathrm{Pb}$ dust was found to be a more potent contaminant of children's hands than indoor dust at daycare centers in New Orleans; boys, in general, had higher hand Pb levels than girls. The conclusions were based on $\mathrm{Pb}$ analysis of hand wipe samples taken before and after children played outdoors at four different daycare centers (a private inner-city site, a private outer-city site, a public inner-city site, and a public outer-city site). The private inner-city site had a severely contaminated outdoor play area with measured soil $\mathrm{Pb}$ concentrations ranging from 287 to 1,878 $\mathrm{mg} / \mathrm{kg}$. The outdoor play area at the public inner-city site, where children exhibited the lowest hand $\mathrm{Pb}$ measurements of any site in the study, had been completely paved over with concrete or rubberized asphalt and had well-maintained equipment (Viverette et al. 1996).

EPA conducted the Urban Soil Lead Abatement Demonstration Project (USLADP), also known as the “Three City Lead Study,” in Boston, Baltimore, and Cincinnati (EPA 1996c). The purpose was to determine whether abatement of $\mathrm{Pb}$ in soil could reduce $\mathrm{PbBs}$ of inner-city children. No significant evidence was found that soil abatement had any direct impact on children's PbBs in either the Baltimore or Cincinnati studies. In the Boston study, however, a mean soil Pb reduction of 1,856 ppm resulted in a 
mean decline of $1.28 \mu \mathrm{g} / \mathrm{dL} \mathrm{PbB}$ at 11 months postabatement (Weitzman et al. 1993). Phase II extended the study to 2 years and included soil abatement of the two comparison areas from Phase I (Aschengrau et al. 1994). Combined results from Phase I and II suggested a higher impact of soil remediation on PbBs $(2.2-2.7 \mu \mathrm{g} / \mathrm{dL})$. EPA reanalyzed the data from the USLADP in an integrated report (EPA 1996c). They concluded that when soil is a significant source of $\mathrm{Pb}$ in the child's environment, under certain conditions, the abatement of that soil will result in a reduction in exposure and consequently, $\mathrm{PbB}$ level. The results of the USLADP suggest that a number of factors are important in determining the influence of soil remediation on PbBs in children. These include the site-specific exposure scenario, the magnitude of the remediation, and the magnitude of additional sources of $\mathrm{Pb}$ exposure.

Authors of a study of PbBs in children in Toronto, Canada, before and after abatement of $\mathrm{Pb}$-contaminated soil and house dust found that they could neither strongly support nor refute beneficial effects of abatement. The failure to reach a definite conclusion from the results of the study, which included data from 12 cross-sectional blood-screening surveys that were conducted over an 8-year period, was due, in part, to a low response rate (32-75\%) to questionnaires used to determine behavioral, household, lifestyle, neighborhood, and environmental factors relating to study participants (Langlois et al. 1996).

Seasonal variations in PbBs in children have been observed in a number of studies (Gulson et al. 2008; Haley and Talbot 2004; Havlena et al. 2009; Kemp et al. 2007; Johnson and Bretsch 2002; Johnson et al. 1996; Laidlaw et al. 2005; Yiin et al. 2000). These studies suggest a general trend of increasing PbB during late summer and early fall. In addition to seasonal patterns in behavior (e.g., outdoor activities), seasonal patterns in weather (humidity and wind velocity) that promote re-entrainment and transport of dust $\mathrm{Pb}$ may contribute to the observed seasonal patterns in PbB (Laidlaw et al. 2005, 2012).

In addition to the ingestion of hand soil/dust through normal hand-to-mouth activity, some children engage in pica behavior (consumption of nonfood items), which can put them at increased risk through ingestion of large amounts of soil contaminated with $\mathrm{Pb}$. It has been estimated that an average child may ingest between 20 and $50 \mathrm{mg}$ of soil/day and that a pica child may ingest $\geq 5,000 \mathrm{mg}$ of soil/day (LaGoy 1987; Mielke et al. 1989). If the soil contains $100 \mu \mathrm{g} / \mathrm{g}$ of $\mathrm{Pb}$, an average child may be exposed to $5 \mu \mathrm{g}$ $\mathrm{Pb}$ /day from this source alone (Mielke et al. 1989), and a pica child may be exposed to $>100$ times that amount. 
Improper removal of $\mathrm{Pb}$ from housing known to contain $\mathrm{Pb}$-based paint can significantly increase $\mathrm{Pb}$ levels in dust, thus causing $\mathrm{Pb}$ toxicity in children living in the home during the Pb-removal process. Four such cases have been documented (Amitai et al. 1987). In January 1995, the New York State Department of Health identified 320 children in 258 households in New York State (excluding New York City) with $\mathrm{PbBs} \geq 20 \mu \mathrm{g} / \mathrm{dL}$ that were considered to be attributable to residential renovation and remodeling (CDC 1997a).

Workers occupationally exposed to $\mathrm{Pb}$ can carry $\mathrm{Pb}$ home on clothing, bodies, or tools (take home exposure). PbBs of children in households of occupationally exposed workers were almost twice those of children in neighboring homes whose parents were not occupationally exposed to $\mathrm{Pb}$ (median ranges were 10-14 and 5-8 $\mu \mathrm{g} / \mathrm{dL}$, respectively) (Grandjean and Bach 1986). Young children ( $<6$ years old) of workers exposed to high levels of $\mathrm{Pb}$ in workplace air at an electronic components plant (61-1,700 $\mu \mathrm{g}$ $\mathrm{Pb} / \mathrm{m}^{3}$ ambient concentrations) had significantly elevated PbBs $(13.4 \mu \mathrm{g} / \mathrm{dL})$ compared with children from the same locale whose parents did not work in the electronics plant (7.1 $\mu \mathrm{g} / \mathrm{dL})$ (Kaye et al. 1987). Based upon data collected from 1987 to 1994, children aged $1-5$ years $(n=139)$ of workers whose occupation resulted in $\mathrm{Pb}$ exposure had a geometric mean $\mathrm{PbB}$ of $9.3 \mu \mathrm{g} / \mathrm{dL}$ as compared to a U.S. population geometric mean of $3.6 \mu \mathrm{g} / \mathrm{dL}$ (Roscoe et al. 1999). Of this group, $52 \%$ of the children had $\mathrm{PbBs}$ $\geq 10 \mu \mathrm{g} / \mathrm{dL}$ compared to $8.9 \%$ of the U.S. population and $21 \%$ had $\mathrm{PbBs} \geq 20 \mu \mathrm{g} / \mathrm{dL}$ compared to $1.1 \%$ of the U.S. population (Roscoe et al. 1999). However, improved industrial hygiene procedures are likely to have decreased worker take-home exposures. Exposures of Pb workers' families have been identified in nearly 30 different industries and occupations. Industries in which exposure of family members has been reported most often include $\mathrm{Pb}$ smelting, battery manufacturing and recycling, radiator repair, electrical components manufacturing, pottery and ceramics, and stained glass making (NIOSH 1995). Children of Pb-exposed construction workers may also be at increased risk (Whelan et al. 1997).

Children may be exposed to $\mathrm{Pb}$ because of activities associated with certain hobbies and artistic activities practiced by adults in the home. Some of the more obvious hobbies and activities involving use of Pb-containing materials include casting, stained glass, pottery, painting, glassblowing, and screenprinting. Activities involving use of Pb-containing materials should always be done in an area well-ventilated with outdoor air and should never be done with children in the same room or in close proximity. Maas et al. (2005) indicated that high levels of $\mathrm{Pb}$ are prevalent in inexpensive cosmetic jewelry that is sold to the general public at retail stores. 
Accidental or intentional ingestion of folk remedies (e.g., Chinese herbal medicines and Ayurvedic medicines containing $\mathrm{Pb}$ ) or use of the $\mathrm{Pb}$ containing eye cosmetic tiro in children (discussed in Section 5.5.5) represents another source for potential Pb-poisoning in children. Sindoor, a cosmetic and cultural/religious powder used in Hindu cultures, has been found to contain very high amounts of $\mathrm{Pb}$ (Lin et al. 2010). Hair dyes formulated with $\mathrm{Pb}$ acetate represent a potential source for $\mathrm{Pb}$-poisoning both by accidental ingestion and by hand-to-mouth activity following contact with Pb-contaminated surfaces, including dyed hair of adults (Mielke et al. 1997).

Children may be exposed to Pb through the inhalation of second-hand smoke. Mannino et al. (2003) employed data from the NHANES III and analyzed PbBs of children aged 4-16 years who were exposed to high, low, and intermediate levels of second-hand smoke. Serum levels of the nicotine biomarker cotinine were used to classify the children into one of the three second-hand smoke exposure categories. The geometric mean PbBs were 1.5, 1.9, and $2.6 \mu \mathrm{g} / \mathrm{dL}$ for children with low $(\leq 0.050-0.104 \mathrm{ng} / \mathrm{mL})$, intermediate (0.105-0.562 ng/mL), and high (0.563-14.9 ng/mL) serum cotinine levels, respectively (Mannino et al. 2003).

\subsection{POPULATIONS WITH POTENTIALLY HIGH EXPOSURES}

In addition to workers exposed to $\mathrm{Pb}$ in the workplace and family members of workers exposed via take home exposure, other population groups are at risk for potential exposure to high levels of $\mathrm{Pb}$. These include populations residing in older housing or buildings that contain deteriorating leaded paint or that have galvanized pipes, $\mathrm{Pb}$ service lines, or scales that contain $\mathrm{Pb}$ within a distribution public water system; in high-traffic areas with legacies from leaded gasoline; near sites where $\mathrm{Pb}$ was produced or disposed; or near one of the NPL hazardous waste sites where $\mathrm{Pb}$ has been detected in some environmental media (ATSDR 2017b; EPA 2014c, 2016a). Since Pb is often detected in tobacco and tobacco smoke, persons who use chewing tobacco or smoke or are exposed to second-hand smoke, may have higher PbB levels than persons that do not use these products (Apostolou et al. 2012; Bonanno et al. 2001; Richter et al. 2013). Recent studies have also found e-cigarettes to be a potential source of $\mathrm{Pb}$ exposure (Olmedo et al. 2018). Other $\mathrm{Pb}$ sources that can contribute to elevated exposures to individual children or adults include mouthing or ingestion of toys containing $\mathrm{Pb}$ and consumption of candy and folk remedies and illicitly manufactured drugs that contain Pb (CDC 2002b, 2018c).

General population exposure is most likely to occur through the ingestion of food and water contaminated with $\mathrm{Pb}$. Based on a multimedia $\mathrm{Pb}$ exposure modeling analysis for children 1-5 years old at upper 
percentiles of $\mathrm{PbB}$ in the U.S. population, soil and dust ingestion are dominant exposure pathways, but for lower percentiles, other age groups (e.g., younger children), or specific local U.S. locations, the main exposure source/pathway could be different (Zartarian et al. 2017). However, some individuals and families may be exposed to additional sources of $\mathrm{Pb}$ in their homes. This is particularly true of older homes that may contain Pb-based paint. In an attempt to reduce the amount of exposure due to deteriorating leaded paint, the paint is commonly removed from homes by burning (gas torch or hot air gun), scraping, or sanding. These activities have been found to result, at least temporarily, in higher levels of exposure for families residing in these homes. In addition, those individuals involved in the paint removal process (i.e., do-it-yourself renovators and professionals who remove $\mathrm{Pb}$ ) can be exposed to such excessive levels that $\mathrm{Pb}$ poisoning may occur (Chisolm 1986; Fischbein et al. 1981; Rabinowitz et al. 1985). Special populations at risk of high exposure to tetraethyl $\mathrm{Pb}$ include workers at hazardous waste sites and those involved in the manufacture and dispensing of tetraethyl $\mathrm{Pb}$ (Bress and Bidanset 1991). 


\section{CHAPTER 6. ADEQUACY OF THE DATABASE}

Section 104(i)(5) of CERCLA, as amended, directs the Administrator of ATSDR (in consultation with the Administrator of EPA and agencies and programs of the Public Health Service) to assess whether adequate information on the health effects of $\mathrm{Pb}$ is available. Where adequate information is not available, ATSDR, in conjunction with NTP, is required to assure the initiation of a program of research designed to determine the adverse health effects (and techniques for developing methods to determine such health effects) of $\mathrm{Pb}$.

Data needs are defined as substance-specific informational needs that, if met, would reduce the uncertainties of human health risk assessment. This definition should not be interpreted to mean that all data needs discussed in this section must be filled. In the future, the identified data needs will be evaluated and prioritized, and a substance-specific research agenda will be proposed.

\subsection{INFORMATION ON HEALTH EFFECTS}

Studies evaluating the health effects of exposure of humans $\mathrm{Pb}$ that are discussed in Chapter 2 are summarized in Figure 2-1. The purpose of this figure is to illustrate the information concerning the health effects of $\mathrm{Pb}$. The number of human studies included in the profile for each endpoint is indicated regardless of whether an effect was found.

The health effects of $\mathrm{Pb}$ have been extensively studied in humans, including numerous studies in children. Due to the extent of the database in humans, a comprehensive review of the complete epidemiological database is not feasible. Epidemiological studies included in Chapter 2 were selected to identify the major lines of evidence regarding health effects in humans. Because the database of epidemiological studies is so large, animal studies were not included in the profile. Due to the increasing awareness that low-level environmental exposure resulting in blood $\mathrm{Pb}$ concentrations $(\mathrm{PbB})<10 \mu \mathrm{g} / \mathrm{dL}$ is associated with adverse effects, particularly in children, the primary objective of current research is focused on health effects associated with $\mathrm{PbB} \leq 10 \mu \mathrm{g} / \mathrm{dL}$. Additional details on studies with $\mathrm{PbB} \leq 10 \mu \mathrm{g} / \mathrm{dL}$, including statistical analyses and assessment of confounding factors, are provided in the Supporting Document for Epidemiological Studies for Lead.

Health effects of $\mathrm{Pb}$ in humans are not defined in terms of route or duration of exposure. Epidemiological studies on $\mathrm{Pb}$ toxicity rely on internal exposure metrics (e.g., $\mathrm{PbB}$ ), rather than measurements of external 
exposures (e.g., concentration of $\mathrm{Pb}$ in water or air) or ingested dose. Furthermore, once absorbed into the body, the health effects of $\mathrm{Pb}$ are the same, regardless of the route of exposure. Environmental exposure to $\mathrm{Pb}$ occurs continuously over a lifetime and $\mathrm{Pb}$ can be retained in the body for decades; therefore, health effects of $\mathrm{Pb}$ in humans are considered to be associated with chronic exposure, rather than to shorter exposures.

\subsection{IDENTIFICATION OF DATA NEEDS}

A data need, as defined in ATSDR's Decision Guide for Identifying Substance-Specific Data Needs Related to Toxicological Profiles (ATSDR 1989), is substance-specific information necessary to conduct comprehensive public health assessments. Generally, ATSDR defines a data gap more broadly as any substance-specific information missing from the scientific literature.

Increased awareness of the potential adverse consequences of low environmental exposures to $\mathrm{Pb}$ has led to changes in U.S. public health policy, with a focus on lowering PbB levels to well below $10 \mu \mathrm{g} / \mathrm{dL}$ (CDC 2012d; EPA 2016b). In 2012, the CDC concluded that the $97.5^{\text {th }}$ percentile of the U.S. PbB distribution (based on NHANES data) should be considered a reference value for identifying children who have “elevated” $\mathrm{PbB}$ (CDC 2012d). At that time, the $97.5^{\text {th }}$ percentile was approximately $5 \mu \mathrm{g} / \mathrm{dL}$. Therefore, additional epidemiological studies for all health outcomes are needed. The objective of these additional studies would be to define the low end of the dose-response curve (e.g., at $\mathrm{PbB} \leq 5 \mu \mathrm{g} / \mathrm{dL}$ ) and to identify threshold levels for health outcomes.

MRLs. Epidemiological studies have identified health effects of $\mathrm{Pb}$ in all organ systems. However, exposure thresholds for effects have not been identified, and it is not possible to determine from the epidemiological data which organ system are the most sensitive (i.e., primary) targets for Pb toxicity. Because clear thresholds for these effects have not been identified, MRLs for Pb have not been derived. Additional epidemiological studies would provide more data to further characterize effects; however, as PbBs continue to decline and effects are observed at the lowest PbB examined, identification of control groups has become increasingly difficult. Thus, it is not anticipated that additional epidemiological studies would identify threshold values for Pb-induced toxicity endpoints.

Health Effects. As noted above, epidemiological studies have identified health effects of $\mathrm{Pb}$ in every organ system at the lowest PbB evaluated. Additional prospective studies on all health outcomes would provide important information to further characterize the effects of $\mathrm{Pb}$ and evaluate potential implications 
for long-term effects. However, as noted above, it is not anticipated that additional epidemiological studies would identify threshold values for health effects.

Epidemiology and Human Dosimetry Studies. Several models of the Pb exposure-biokinetics toxicokinetics in humans have been developed and used in dosimetry studies. Additional studies would be helpful for addressing major uncertainties in these models, including: (1) absence of calibration data for the kinetics of $\mathrm{Pb}$ in blood and bone in children in association with exposures that have been quantified with high certainty; (2) absence of calibration data on bone Pb concentrations in adolescents and adults in association with exposures that have been quantified with high certainty; (3) absence of data on the absolute bioavailability of ingested $\mathrm{Pb}$ in older children and adolescents; (4) incomplete understanding of $\mathrm{Pb}$ kinetics during periods of changing bone metabolism, including adolescence, pregnancy, and menopause; and (5) incomplete understanding of inter- and intra-individual variability in model parameter values in humans. In addition, there is a need for studies that can evaluate or validate model predictions of concentrations of $\mathrm{Pb}$ in blood and other tissues in populations in which $\mathrm{PbBs}$ are typical of the U.S. population $(\leq 5 \mu \mathrm{g} / \mathrm{dL})$.

Biomarkers of Exposure and Effect. Measurement of blood Pb concentration is the most widely used biomarker of $\mathrm{Pb}$ exposure and is used to identity children who have elevated exposures. Measurement of bone $\mathrm{Pb}$ by XRF has been used to estimate $\mathrm{Pb}$ body burden in adults, which is a more accurate biomarker of long-term exposure than PbB. Additional studies that could improve and evaluate the validity of non-invasive biomarkers (e.g., hair, saliva, sweat, deciduous teeth, urine) for quantifying exposure would be helpful for population monitoring of $\mathrm{Pb}$ exposures and for epidemiology of $\mathrm{Pb}$ health effects.

Absorption, Distribution, Metabolism, and Excretion. Studies of $\mathrm{Pb}$ absorption are limited to studies in infants and adults. No data are available on the absorption of $\mathrm{Pb}$ in older children and adolescents. Additional studies of $\mathrm{Pb}$ absorption in this age category would be useful for improving exposure-biokinetic models.

A variety of factors are known to influence the absorption of ingested $\mathrm{Pb}$, including the chemical form of the ingested $\mathrm{Pb}$, the presence of food in the gastrointestinal tract, diet, and nutritional status with respect to calcium, vitamin D, and iron; however, for the most part, the mechanisms by which these interactions occur are not fully understood. This reflects, in part, a lack of understanding of the mechanisms by which $\mathrm{Pb}$ is absorbed in the gastrointestinal tract, and studies aimed at elucidating such mechanisms would be 
helpful for developing PBPK models that accurately simulate relationships between $\mathrm{Pb}$ exposure and $\mathrm{Pb}$ in blood and other target and biomarker tissue.

The quantitative significance of the dermal absorption pathway as a contributor to $\mathrm{Pb}$ body burden remains an uncertainty. Few studies are available on $\mathrm{Pb}$ absorption after dermal exposure of inorganic $\mathrm{Pb}$ compounds in humans. Children may experience extensive dermal contact with $\mathrm{Pb}$ in soil, sand, or surface water and suspended sediment (e.g., beach or shoreline exposure scenario), even a low percent absorption across the skin may represent a significant internal dose. Therefore, additional studies designed to quantify dermal absorption of inorganic $\mathrm{Pb}$ compounds from both aqueous media and from soil would be helpful for improving PBPK models, in particular, studies that enable measurements to be extrapolated to children.

Comparative Toxicokinetics. Animal models (e.g., swine, mouse) have been used extensively as a model for assessing relative bioavailability of $\mathrm{Pb}$ in ingested soil in humans and for evaluating in vitro approaches to assessing bioaccessibility of $\mathrm{Pb}$. However, no studies are available in which the absolute or relative bioavailability of ingested $\mathrm{Pb}$ has been quantitatively compared in animal models and humans. Such studies would be useful for validating both the in vivo swine model and the in vitro bioaccessibility model.

Children's Susceptibility. Children are likely to have increased susceptibility to Pb compared to adults for several reasons: increased susceptibility of developing physiological systems compared to mature systems; increased absorption of $\mathrm{Pb}$ in children compared to adults; and common childhood behaviors (e.g., hand-to-mouth activity, pica behavior [the compulsive, habitual consumption of nonfood items], proximity of breathing zone to entrained surface dust). In addition, several other factors may affect children's susceptibility to $\mathrm{Pb}$, including (but not limited to) family socio-economic status, parent education, parent alcohol, tobacco, and drug use, allergen exposure, and family history of disease, although these factors may not be unique to children. Additional studies evaluating these factors would provide an increased understanding of relative contributions of these factors to child $\mathrm{PbB}$ and associated health effects.

Physical and Chemical Properties. No data needs were identified regarding physical and chemical properties of $\mathrm{Pb}$. 
Production, Import/Export, Use, Release, and Disposal. Continued monitoring of $\mathrm{Pb}$ production, import/export, use, release, and disposal would be helpful for identifying sources of potential human exposure. In particular, additional data on releases of $\mathrm{Pb}$ from leaded gasoline used in pistondriven engines would be helpful for determining potential contributions of this source to human exposure. Industrial wastes, as well as consumer products, containing $\mathrm{Pb}$ are disposed of in municipal and hazardous waste landfills. Current information on the amounts being disposed would be helpful for evaluating potential for exposures to $\mathrm{Pb}$ from these sources.

Environmental Fate. Additional information on the atmospheric transformations of organic and inorganic $\mathrm{Pb}$ compounds would be helpful for identifying $\mathrm{Pb}$ compounds to which humans are most likely to be exposed by inhalation. Additional data regarding the chemical speciation and the transformation pathways of $\mathrm{Pb}$ in soils and water with varying properties such as $\mathrm{pH}$, oxygen content, and salinity would be helpful for improved understanding of the environmental fate of $\mathrm{Pb}$ in soils and water.

Bioavailability from Environmental Media. Studies conducted in animal models show that oral $\mathrm{RBA}$ of soil $\mathrm{Pb}$ varies depending upon the $\mathrm{Pb}$ mineralogy and physical characteristics of the $\mathrm{Pb}$ in the soil. There is only one published study that assessed the bioavailability of $\mathrm{Pb}$ in humans (adults) who ingested hazardous waste site soil. Additional studies of this type would provide an improved basis for estimating $\mathrm{Pb}$ uptake in people who are exposed to $\mathrm{Pb}$ in soil. No studies have measured oral $\mathrm{RBA}$ of surface dusts. Since this is an important exposure pathway, especially in urban environments, studies of oral Pb RBA of surface dusts collected from various types of indoor and outdoor surfaces, including those impacted by paint $\mathrm{Pb}$, would be helpful.

Recent interest in the use of soil-amending agents (e.g., phosphate) to reduce soil Pb bioavailability, would be served by additional studies directed at developing methods for monitoring the magnitude and persistence of the effect of amending agents on $\mathrm{Pb}$ bioavailability and for predicting the magnitude of the effect for improved design of amending projects.

Food Chain Bioaccumulation. No data needs were identified regarding food chain bioaccumulation.

Exposure Levels in Environmental Media. Reliable monitoring data for the levels of $\mathrm{Pb}$ in contaminated media at hazardous waste sites are needed so that the information obtained on levels of $\mathrm{Pb}$ in the environment can be used in combination with the known body burden of $\mathrm{Pb}$ to assess the potential risk of adverse health effects in populations living in the vicinity of hazardous waste sites. Continued 
monitoring of $\mathrm{Pb}$ levels in air, drinking water, and diet (e.g., food and bottled water) would be helpful for evaluating potential for exposures to $\mathrm{Pb}$ from these sources. Continued testing of consumer products would be helpful for identifying potential localized sources of human exposure (e.g., ceramics, cosmetics, jewelry, toys).

Exposure Levels in Humans. Continued updating of national (e.g., NHANES) and regional surveys of $\mathrm{Pb}$ biomarkers (e.g., $\mathrm{PbB}$ ) would be helpful for assessing temporal and demographic trends in $\mathrm{Pb}$ exposure in the U.S. population as well as for evaluating associations between $\mathrm{Pb}$ exposure and health metrics (e.g., those included in the NHANES), and for evaluating models that relate exposure to PbB.

Exposures of Children. Since an important variable in estimating $\mathrm{Pb}$ intakes from measurements of surface dust $\mathrm{Pb}$ levels is the rate of surface dust ingestion, improved estimates of soil ingestion would increase confidence in predictions of $\mathrm{Pb}$ intakes associated with exposures to $\mathrm{Pb}$ in surface dusts. In some contexts, exposure to surface dust $\mathrm{Pb}$ is measured in terms of $\mathrm{Pb}$ loading $\left(\mu \mathrm{g} / \mathrm{Pb} / \mathrm{cm}^{2}\right.$ of surface area available for contact); however, $\mathrm{Pb}$ loading measurements do not provide a direct way of estimating $\mathrm{Pb}$ ingestion without corresponding estimates of dust loading and surface dust ingestion rates. Improved methods for translating measurements of $\mathrm{Pb}$ loading into estimates of surface dust $\mathrm{Pb}$ concentration or surface dust $\mathrm{Pb}$ intake would be helpful for improving models for predicting exposure-Pb relationships in children.

\subsection{ONGOING STUDIES}

Ongoing studies on $\mathrm{Pb}$ are outlined in Table 6-1. Note that the studies listed below are funded by the National Institute of Health (NIH) and do not include ongoing studies that are funded by other sources.

\section{Table 6-1. Ongoing Studies on Lead (Pb)}

\begin{tabular}{llll}
\hline Investigator & Affiliation & Research description & Sponsor \\
\hline Bhattacharya, A & $\begin{array}{l}\text { University of } \\
\text { Cincinnati }\end{array}$ & $\begin{array}{l}\text { Epidemiological study evaluating the effects of } \\
\text { childhood Pb exposure on bone and musculature in } \\
\text { African-American women }\end{array}$ & NIEHS \\
\hline Kordas, K & $\begin{array}{l}\text { State University } \\
\text { of New York at } \\
\text { Buffalo }\end{array}$ & $\begin{array}{l}\text { Epidemiological study on the interaction between } \\
\text { metals and neurobehavioral outcomes in children } \\
\text { and adolescents }\end{array}$ & $\mathrm{NIEHS}$ \\
\hline Lamas, G & $\begin{array}{l}\text { Mt. Sinai Medical } \\
\text { Center }\end{array}$ & $\begin{array}{l}\text { Investigation effects of chelation-reduced PbB on } \\
\text { myocardial infarction }\end{array}$ & $\mathrm{NCCIH}$ \\
\hline
\end{tabular}




\section{Table 6-1. Ongoing Studies on Lead (Pb)}

\begin{tabular}{|c|c|c|c|}
\hline Investigator & Affiliation & Research description & Sponsor \\
\hline Lu, Q & $\begin{array}{l}\text { Harvard School of } \\
\text { Public Health }\end{array}$ & $\begin{array}{l}\text { Study in children to evaluate SPP1 upregulation as } \\
\text { a critical mechanism linking Pb exposure with } \\
\text { neural stem cell function and neurodevelopment in } \\
\text { children }\end{array}$ & NIEHS \\
\hline Papautsky, I & $\begin{array}{l}\text { University of } \\
\text { Illinois at Chicago }\end{array}$ & $\begin{array}{l}\text { Longitudinal study to evaluate the relationship } \\
\text { between } \mathrm{PbB} \text { and functional gait and static and } \\
\text { dynamic balance in an adolescent cohort }\end{array}$ & NIEHS \\
\hline Reuben, A & Duke University & $\begin{array}{l}\text { Longitudinal birth cohort study of neuroimaging } \\
\text { data to determine whether childhood } \mathrm{Pb} \text { exposure } \\
\text { relates to degenerative alterations in neural } \\
\text { structure or function by late midlife }\end{array}$ & NIEHS \\
\hline Upson, $\mathrm{K}$ & $\begin{array}{l}\text { Michigan State } \\
\text { University }\end{array}$ & $\begin{array}{l}\text { Prospective cohort study to evaluate the } \\
\text { association between } \mathrm{PbB} \text { and uterine fibroid tumors }\end{array}$ & NINR \\
\hline Wang, G & $\begin{array}{l}\text { Johns Hopkins } \\
\text { University }\end{array}$ & $\begin{array}{l}\text { Prospective birth cohort study to evaluate the } \\
\text { relationship between PbB and placental pathology } \\
\text { and cardiometabolic outcomes in childhood }\end{array}$ & NIEHS \\
\hline Weuve, J & $\begin{array}{l}\text { Boston University } \\
\text { Medical Campus }\end{array}$ & $\begin{array}{l}\text { Pilot study to evaluate XRF energy-dispersed X-ray } \\
\text { fluorescence measurement of } \mathrm{Pb} \text { in bone and } \\
\text { toenails }\end{array}$ & NIEHS \\
\hline
\end{tabular}

$\mathrm{NCCIH}=$ National Center for Complementary and Integrative Health; NIEHS = National Institute of Environmental Health Sciences; NINR = National Institute of Nursing Research; PbB = blood lead concentration; SSP1 = secreted phosphoprotein 1; XRF = X-ray fluorescence

Source: NIH Reporter 2020 (https://projectreporter.nih.gov/reporter.cfm) 


\section{CHAPTER 7. REGULATIONS AND GUIDELINES}

Pertinent international and national regulations, advisories, and guidelines regarding lead in air, water, and other media are summarized in Table 7-1. This table is not an exhaustive list, and current regulations should be verified by the appropriate regulatory agency.

ATSDR develops MRLs, which are substance-specific guidelines intended to serve as screening levels by ATSDR health assessors and other responders to identify contaminants and potential health effects that may be of concern at hazardous waste sites. See Section 1.3 and Appendix A for detailed information on the MRLs for $\mathrm{Pb}$. As discussed in Appendix A, no MRLs were derived for $\mathrm{Pb}$.

Table 7-1. Regulations and Guidelines Applicable to Lead (Pb)

\begin{tabular}{|c|c|c|c|}
\hline Agency & Description & Information & Reference \\
\hline \multicolumn{4}{|c|}{ Air } \\
\hline EPA & RfC & Not evaluated & IRIS $\underline{2002}, \underline{2004}$ \\
\hline EPA & NAAQS & $0.15 \mu \mathrm{g} / \mathrm{m}^{3}$ a & EPA 2019b \\
\hline $\mathrm{WHO}$ & Air quality guidelines & Not listed & WHO 2010 \\
\hline \multicolumn{4}{|c|}{ Water \& Food } \\
\hline \multirow[t]{9}{*}{ EPA } & $\begin{array}{l}\text { Drinking water standards and health } \\
\text { advisories }\end{array}$ & No data & EPA 2018c \\
\hline & $\begin{array}{l}\text { National primary drinking water } \\
\text { regulations for inorganic lead }\end{array}$ & & EPA 2009 \\
\hline & MCL or TT & $\mathrm{TT}^{\mathrm{b}}$ & \\
\hline & Action level & $0.015 \mathrm{mg} / \mathrm{L}$ & \\
\hline & Public health goal & zero & \\
\hline & Lead and copper rule proposal & & EPA 2019a \\
\hline & Trigger level (proposed) & $10 \mu \mathrm{g} / \mathrm{L}^{\mathrm{c}}$ & \\
\hline & RfD & & \\
\hline & Tetraethyl lead & $1 \times 10^{-7} \mathrm{mg} / \mathrm{kg} / \mathrm{day}$ & IRIS 2002 \\
\hline \multirow[t]{2}{*}{ WHO } & Drinking water quality guidelines & & WHO 2017 \\
\hline & Provisional guideline value, lead & $0.01 \mathrm{mg} / \mathrm{L}(10 \mu \mathrm{g} / \mathrm{L})^{\mathrm{d}}$ & \\
\hline \multirow[t]{2}{*}{ FDA } & Substances Added to Foode & Not listed & FDA 2019a \\
\hline & Allowable level of lead in bottled water & $0.005 \mathrm{mg} / \mathrm{L}$ & FDA 2019b \\
\hline
\end{tabular}


Table 7-1. Regulations and Guidelines Applicable to Lead (Pb)

\begin{tabular}{|c|c|c|c|}
\hline Agency & Description & Information & Reference \\
\hline \multicolumn{4}{|c|}{ Cancer } \\
\hline \multirow[t]{2}{*}{ HHS } & Carcinogenicity classification & & NTP 2016 \\
\hline & Lead and lead compounds & $\begin{array}{l}\text { Reasonably anticipated to } \\
\text { be human carcinogens }\end{array}$ & \\
\hline \multirow[t]{2}{*}{ EPA } & Carcinogenicity classification & & $\underline{\text { IRIS } 2004}$ \\
\hline & Lead and compounds (inorganic) & $\mathrm{B} 2^{\mathrm{f}}$ & \\
\hline \multirow[t]{4}{*}{ IARC } & Carcinogenicity classification & & \\
\hline & Lead & Group 2Bg & IARC 1987, $\underline{2019}$ \\
\hline & Lead compounds, inorganic & Group $2 A^{\mathrm{h}}$ & IARC $\underline{2006}, \underline{2019}$ \\
\hline & Lead compounds, organic & Group $3^{\mathrm{i}}$ & IARC $\underline{2006}, \underline{2019}$ \\
\hline \multicolumn{4}{|c|}{ Occupational } \\
\hline \multirow[t]{15}{*}{ OSHA } & PEL (8-hour TWA) for general indust & & \\
\hline & $\begin{array}{l}\text { Lead (elemental, inorganic and } \\
\text { organic soaps) }\end{array}$ & $50 \mu \mathrm{g} / \mathrm{m}^{3}$ & $\underline{\text { OSHA 2019a }}$ \\
\hline & Tetraethyl lead and tetramethyl lea & $0.075 \mathrm{mg} / \mathrm{m}^{3 j}$ & $\underline{\text { OSHA 2019b }}$ \\
\hline & $\begin{array}{l}\text { PEL (8-hour TWA) for construction } \\
\text { and shipyards }\end{array}$ & & \\
\hline & $\begin{array}{l}\text { Lead (elemental, inorganic and } \\
\text { organic soaps) }\end{array}$ & $50 \mu \mathrm{g} / \mathrm{m}^{3}$ & OSHA $\underline{2019 c}, \underline{2019 a}$ \\
\hline & Tetraethyl lead & $0.1 \mathrm{mg} / \mathrm{m}^{3 \mathrm{j}}$ & OSHA 2019d, 2019e \\
\hline & Tetramethyl lead & $0.15 \mathrm{mg} / \mathrm{m}^{3 \mathrm{j}}$ & OSHA 2019d, $\underline{2019 f}$ \\
\hline & $\begin{array}{l}\text { Action level (8-hour TWA) for genera } \\
\text { industry, construction }\end{array}$ & & \\
\hline & $\begin{array}{l}\text { Lead (elemental, inorganic and } \\
\text { organic soaps) }\end{array}$ & $30 \mu \mathrm{g} / \mathrm{m}^{3}$ & OSHA 2019a, $\underline{2019 \mathrm{c}}$ \\
\hline & $\begin{array}{l}\text { Medical removal protection for gener } \\
\text { industry }\end{array}$ & & $\underline{\text { OSHA 2019a }}$ \\
\hline & Temporary removal blood lead lev & $\geq 60 \mu \mathrm{g} / 100 \mathrm{~g}$ & \\
\hline & Return to work blood lead level & $<40 \mu \mathrm{g} / 100 \mathrm{~g}$ & \\
\hline & $\begin{array}{l}\text { Medical removal protection for } \\
\text { construction and shipyards }\end{array}$ & & $\underline{\text { OSHA 2019c }}$ \\
\hline & Temporary removal blood lead lev & $\geq 50 \mu \mathrm{g} / \mathrm{dL}$ & \\
\hline & Return to work blood lead level & $<40 \mu \mathrm{g} / \mathrm{dL}$ & \\
\hline \multirow[t]{6}{*}{$\mathrm{NIOSH}$} & REL (8-hour TWA) & & \\
\hline & Lead and compounds (as Pb) & $0.05 \mathrm{mg} / \mathrm{m}^{3}$ & NIOSH 2019a \\
\hline & $\begin{array}{l}\text { Tetraethyl lead (as } \mathrm{Pb} \text { ) and } \\
\text { tetramethyl lead (as } \mathrm{Pb})\end{array}$ & $0.075 \mathrm{mg} / \mathrm{m}^{3 \mathrm{j}}$ & $\mathrm{NIOSH} \underline{2019 b}, \underline{2019 \mathrm{c}}$ \\
\hline & IDLH & & \\
\hline & Lead and compounds (as Pb) & $100 \mathrm{mg} / \mathrm{m}^{3}$ & NIOSH 2019a \\
\hline & $\begin{array}{l}\text { Tetraethyl lead (as Pb) and } \\
\text { tetramethyl lead (as Pb) }\end{array}$ & $40 \mathrm{mg} / \mathrm{m}^{3}$ & $\mathrm{NIOSH} \underline{2019 b}, \underline{2019 \mathrm{c}}$ \\
\hline
\end{tabular}


Table 7-1. Regulations and Guidelines Applicable to Lead (Pb)

\begin{tabular}{|c|c|c|c|}
\hline Agency & Description & Information & Reference \\
\hline \multicolumn{4}{|c|}{ Emergency Criteria } \\
\hline EPA & AEGLs-air & No data & EPA 2018c \\
\hline$\overline{\mathrm{DOE}}$ & PACs-airk & & DOE 2018a \\
\hline \multicolumn{4}{|c|}{ Lead } \\
\hline & PAC-1 & $0.15 \mathrm{mg} / \mathrm{m}^{3}$ & \\
\hline & PAC-2 & $120 \mathrm{mg} / \mathrm{m}^{3}$ & \\
\hline & PAC-3 & $700 \mathrm{mg} / \mathrm{m}^{3}$ & \\
\hline \multicolumn{4}{|c|}{ Tetraethyl lead } \\
\hline & PAC-1 & $0.3 \mathrm{mg} / \mathrm{m}^{3}$ & \\
\hline & PAC-2 & $4 \mathrm{mg} / \mathrm{m}^{3}$ & \\
\hline & PAC-3 & $40 \mathrm{mg} / \mathrm{m}^{3}$ & \\
\hline \multicolumn{4}{|c|}{ Tetramethyl lead } \\
\hline & PAC-1 & $0.45 \mathrm{mg} / \mathrm{m}^{3}$ & \\
\hline & PAC-2 & $4 \mathrm{mg} / \mathrm{m}^{3}$ & \\
\hline & PAC-3 & $40 \mathrm{mg} / \mathrm{m}^{3}$ & \\
\hline \multicolumn{4}{|c|}{ Lead acetate } \\
\hline & PAC-1 & $5 \mathrm{mg} / \mathrm{m}^{3}$ & \\
\hline & PAC-2 & $55 \mathrm{mg} / \mathrm{m}^{3}$ & \\
\hline & PAC-3 & $330 \mathrm{mg} / \mathrm{m}^{3}$ & \\
\hline \multicolumn{4}{|c|}{ Lead carbonate } \\
\hline & PAC-1 & $0.19 \mathrm{mg} / \mathrm{m}^{3}$ & \\
\hline & PAC-2 & $24 \mathrm{mg} / \mathrm{m}^{3}$ & \\
\hline & PAC-3 & $900 \mathrm{mg} / \mathrm{m}^{3}$ & \\
\hline \multicolumn{4}{|c|}{ Lead dioxide and lead sulfide } \\
\hline & PAC-1 & $0.17 \mathrm{mg} / \mathrm{m}^{3}$ & \\
\hline & PAC-2 & $140 \mathrm{mg} / \mathrm{m}^{3}$ & \\
\hline & PAC-3 & $810 \mathrm{mg} / \mathrm{m}^{3}$ & \\
\hline \multicolumn{4}{|c|}{ Lead tetroxide } \\
\hline & PAC-1 & $0.17 \mathrm{mg} / \mathrm{m}^{3}$ & \\
\hline & PAC-2 & $130 \mathrm{mg} / \mathrm{m}^{3}$ & \\
\hline & PAC-3 & $770 \mathrm{mg} / \mathrm{m}^{3}$ & \\
\hline \multicolumn{4}{|c|}{ Lead sulfide } \\
\hline & PAC-1 & $0.17 \mathrm{mg} / \mathrm{m}^{3}$ & \\
\hline & PAC-2 & $140 \mathrm{mg} / \mathrm{m}^{3}$ & \\
\hline & PAC-3 & $810 \mathrm{mg} / \mathrm{m}^{3}$ & \\
\hline \multicolumn{4}{|c|}{ Lead oxide } \\
\hline & PAC-1 & $0.16 \mathrm{mg} / \mathrm{m}^{3}$ & \\
\hline & PAC-2 & $130 \mathrm{mg} / \mathrm{m}^{3}$ & \\
\hline & PAC-3 & $750 \mathrm{mg} / \mathrm{m}^{3}$ & \\
\hline
\end{tabular}




\section{Table 7-1. Regulations and Guidelines Applicable to Lead (Pb)}

\begin{tabular}{|c|c|c|c|}
\hline Agency & Description & Information & Reference \\
\hline \multicolumn{4}{|c|}{ Lead sulfate } \\
\hline & PAC-1 & $0.22 \mathrm{mg} / \mathrm{m}^{3}$ & \\
\hline & PAC-2 & $170 \mathrm{mg} / \mathrm{m}^{3}$ & \\
\hline & PAC-3 & $1,000 \mathrm{mg} / \mathrm{m}^{3}$ & \\
\hline \multicolumn{4}{|c|}{ Lead phosphate } \\
\hline & PAC-1 & $0.2 \mathrm{mg} / \mathrm{m}^{3}$ & \\
\hline & PAC-2 & $150 \mathrm{mg} / \mathrm{m}^{3}$ & \\
\hline & PAC-3 & $910 \mathrm{mg} / \mathrm{m}^{3}$ & \\
\hline \multicolumn{4}{|c|}{ Lead chloride } \\
\hline & PAC-1 & $0.2 \mathrm{mg} / \mathrm{m}^{3}$ & \\
\hline & PAC-2 & $160 \mathrm{mg} / \mathrm{m}^{3}$ & \\
\hline & PAC-3 & $940 \mathrm{mg} / \mathrm{m}^{3}$ & \\
\hline \multicolumn{4}{|c|}{ Lead chromate } \\
\hline & PAC-1 & $0.036 \mathrm{mg} / \mathrm{m}^{3}$ & \\
\hline & PAC-2 & $16 \mathrm{mg} / \mathrm{m}^{3}$ & \\
\hline & PAC-3 & $97 \mathrm{mg} / \mathrm{m}^{3}$ & \\
\hline \multicolumn{4}{|c|}{ Lead bromide } \\
\hline & PAC-1 & $0.27 \mathrm{mg} / \mathrm{m}^{3}$ & \\
\hline & PAC-2 & $200 \mathrm{mg} / \mathrm{m}^{3}$ & \\
\hline & PAC-3 & $1,200 \mathrm{mg} / \mathrm{m}^{3}$ & \\
\hline \multicolumn{4}{|c|}{ Lead nitrate } \\
\hline & PAC-1 & $0.24 \mathrm{mg} / \mathrm{m}^{3}$ & \\
\hline & PAC-2 & $180 \mathrm{mg} / \mathrm{m}^{3}$ & \\
\hline & PAC-3 & $1,100 \mathrm{mg} / \mathrm{m}^{3}$ & \\
\hline \multicolumn{4}{|c|}{ Lead iodide } \\
\hline & PAC-1 & $0.33 \mathrm{mg} / \mathrm{m}^{3}$ & \\
\hline & PAC-2 & $270 \mathrm{mg} / \mathrm{m}^{3}$ & \\
\hline & PAC-3 & $1,600 \mathrm{mg} / \mathrm{m}^{3}$ & \\
\hline \multicolumn{4}{|c|}{ Lead fluoroborate } \\
\hline & PAC-1 & $0.28 \mathrm{mg} / \mathrm{m}^{3}$ & \\
\hline & PAC-2 & $220 \mathrm{mg} / \mathrm{m}^{3}$ & \\
\hline & PAC-3 & $1,300 \mathrm{mg} / \mathrm{m}^{3}$ & \\
\hline \multicolumn{4}{|c|}{$\begin{array}{l}\text { Miscellaneous Federal Guidance } \\
\end{array}$} \\
\hline $\mathrm{CDC}$ & PbB reference value & $5 \mu \mathrm{g} / \mathrm{dL}$ & CDC 2012d, 2012e \\
\hline$\overline{E P A}$ & $\begin{array}{l}\text { Dust-lead hazard standards } \\
\text { Floors } \\
\text { Window sills }\end{array}$ & $\begin{array}{l}10 \mu \mathrm{g} / \mathrm{ft}^{2} \\
100 \mu \mathrm{g} / \mathrm{ft}^{2}\end{array}$ & EPA 2019c \\
\hline
\end{tabular}


Table 7-1. Regulations and Guidelines Applicable to Lead (Pb)

\begin{tabular}{llll}
\hline Agency & Description & Information & Reference \\
\hline EPA & Soil screening level & $400 \mathrm{ppm}$ & $\begin{array}{l}\text { EPA 1994e, 1998; } \\
\text { 2016d }\end{array}$ \\
\hline HUD & Dust lead hazard action levels & $\underline{\text { HUD 2017 }}$ \\
& Floors & $\geq 10 \mu \mathrm{g} / \mathrm{ft}^{2}$ & \\
Window sills & $\geq 100 \mu \mathrm{g} / \mathrm{ft}^{2}$ & \\
& Dust lead clearance action levels & & \\
& Interior floors & $<10 \mu \mathrm{g} / \mathrm{ft}^{2}$ & \\
& Porch floors & $<40 \mu \mathrm{g} / \mathrm{ft}^{2}$ & \\
& Window sills & $<100 \mu \mathrm{g} / \mathrm{ft}^{2}$ & \\
& Window troughs & $<100 \mu \mathrm{g} / \mathrm{ft}^{2}$ & \\
&
\end{tabular}

aNot-to-exceed air $\mathrm{Pb}$ concentration of $0.15 \mu \mathrm{g} / \mathrm{m}^{3}$ in total suspended solids for a 3-month rolling average, evaluated over a 3-year period (i.e., the 3-month rolling average cannot exceed $0.15 \mu \mathrm{g} / \mathrm{m}^{3}$ over a 3-year period). blf $>10 \%$ of tap water samples exceed the action level, a water system must take additional steps to control the corrosiveness of its water.

${ }^{c}$ Exceedance would trigger additional planning, monitoring, and treatment requirements, which vary depending on the characteristics of the water system.

${ }^{\mathrm{d}}$ The guideline value is designated as provisional on the basis of treatment performance and analytical achievability because it is extremely difficult to achieve a lower concentration by central conditioning, such as phosphate dosing. eThe Substances Added to Food inventory replaces EAFUS and contains the following types of ingredients: food and color additives listed in FDA regulations, flavoring substances evaluated by FEMA or JECFA, GRAS substances listed in FDA regulations, substances approved for specific uses in food prior to September 6, 1958, substances that are listed in FDA regulations as prohibited in food, delisted color additives, and some substances "no longer FEMA GRAS."

fGroup B2: probable human carcinogen.

gGroup 2B: possibly carcinogenic to humans.

${ }^{\mathrm{h}} \mathrm{Group} 2 \mathrm{~A}$ : probably carcinogenic to humans.

'Group 3: not classifiable as to carcinogenicity to humans.

iskin designation.

kDefinitions of PAC terminology are available from U.S. Department of Energy (DOE 2018b).

AEGL = acute exposure guideline levels; $C D C=$ Centers for Disease Control and Prevention; $D O E=$ Department of Energy; EAFUS = Everything Added to Food in the United States; EPA = Environmental Protection Agency;

FDA = Food and Drug Administration; FEMA = Flavor and Extract Manufacturers Association of the United States; GRAS = generally recognized as safe; HHS = Department of Health and Human Services; HUD = Housing and Urban Development; IARC = International Agency for Research on Cancer; IDLH = immediately dangerous to life or health concentration; IRIS = Integrated Risk Information System; JECFA = Joint FAO/WHO Expert Committee on Food Additives; $\mathrm{MCL}=$ maximum contaminant level; NAAQS = National Ambient Air Quality Standard; $\mathrm{NIOSH}=$ National Institute for Occupational Safety and Health; NTP = National Toxicology Program; $\mathrm{OSHA}=$ Occupational Safety and Health Administration; $\mathrm{PAC}=$ Protective Action Criteria; $\mathrm{PbB}=$ blood lead concentration; $\mathrm{PEL}=$ permissible exposure limit; $\mathrm{REL}=$ recommended exposure limit; $\mathrm{RfC}=$ inhalation reference concentration; $\mathrm{RfD}=$ oral reference dose; $\mathrm{TT}$ = treatment technique; TWA = time-weighted average; $\mathrm{WHO}=$ World Health Organization 


\section{CHAPTER 8. REFERENCES}

AAP. 1998. Screening for elevated blood lead levels. American Academy of Pediatrics. Pediatrics 101(6):1072-1078.

AAP. 2005. Lead exposure in children: Prevention, detection, and management. American Academy of Pediatrics. Pediatrics 116(4):1036-1046.

AAP. 2016. Prevention of childhood lead toxicity. Council on Environmental Health. (Erratum in: Pediatrics 140(2):e20171490). American Academy of Pediatrics. Pediatrics 138(1):e20161493. http://doi.org/10.1542/peds.2016-1493.

Abadin HG, Wheeler JS, Jones DE, et al. 1997. A framework to guide public health assessment decisions at lead sites. J Clean Technol Environ Toxicol Occup Med 6(3):225-237.

Abam E, Okediran BS, Odukoya OO, et al. 2008. Reversal of ionoregulatory disruptions in occupational lead exposure by vitamin C. Environ Toxicol Pharmacol 26(3):297-304. http://doi.org/10.1016/j.etap.2008.05.008.

Abdelouahab N, Mergler D, Takser L, et al. 2008. Gender differences in the effects of organochlorines, mercury, and lead on thyroid hormone levels in lakeside communities of Quebec (Canada). Environ Res 107(3):380-392. http://doi.org/10.1016/j.envres.2008.01.006.

ACGIH. 2018. TLVs and BEIs based on the documentation of the threshold limit values for chemical substances and physical agents and biological exposure indices. Cincinnati, OH: American Conference of Governmental Industrial Hygienists.

ACMT. 2010. American College of Medical Toxicology position statement on post-chelator challenge urinary metal testing. American College of Medical Toxicology. J Med Toxicol 6:74-75.

Adebonojo FO. 1974. Hematologic status of urban black children in Philadelphia. Clin Pediatr (Phila) 13(10):874-888.

Ademuyiwa O, Ugbaja RN, Idumebor F, et al. 2005. Plasma lipid profiles and risk of cardiovascular disease in occupational lead exposure in Abeokuta, Nigeria. Lipids Health Dis 4:19. http://doi.org/10.1186/1476-511X-4-19.

Afeiche M, Peterson KE, Sánchez BN, et al. 2011. Prenatal lead exposure and weight of 0- to 5 year-old children in Mexico City. Environ Health Perspect 119(10):1436-1441. http://doi.org/10.1289/ehp.1003184.

Ahamed M, Siddiqui MK. 2007. Low level lead exposure and oxidative stress: Current opinions. Clin Chim Acta 383(1-2):57-64. http://doi.org/10.1016/j.cca.2007.04.024.

Ahamed M, Verma S, Kumar A, et al. 2005. Environmental exposure to lead and its correlation with biochemical indices in children. Sci Total Environ 346(1-3):48-55. http://doi.org/10.1016/j.scitotenv.2004.12.019.

Ahamed M, Verma S, Kumar A, et al. 2006. Delta-aminolevulinic acid dehydratase inhibition and oxidative stress in relation to blood lead among urban adolescents. Hum Exp Toxicol 25(9):547553. http://doi.org/10.1191/0960327106het657oa.

Ahamed M, Singh S, Behari JR, et al. 2007. Interaction of lead with some essential trace metals in the blood of anemic children from Lucknow, India. Clin Chim Acta 377(1-2):92-97. http://doi.org/10.1016/j.cca.2006.08.032.

Ahn J, Kim NS, Lee BK, et al. 2018. Association of blood pressure with blood lead and cadmium levels in Korean adolescents: Analysis of data from the 2010-2016 Korean National Health and Nutrition Examination Survey. J Korean Med Sci 33(44):e278. http://doi.org/10.3346/jkms.2018.33.e278.

Aiba Y, Ohshiba S, Horiguchi S, et al. 1999. Peripheral hemodynamics evaluated by acceleration plethysmography in workers exposed to lead. Ind Health 37:3-8.

Åkesson A, Stal P, Vahter M. 2000. Phlebotomy increases cadmium uptake in hemochromatosis. Environ Health Perspect 108:289-291.

Åkesson A, Lundh T, Vahter M, et al. 2005. Tubular and glomerular kidney effects in Swedish women with low environmental cadmium exposure. Environ Health Perspect 113(11):1627-1631. http://doi.org/10.1289/ehp.8033. 
Akhavan J. 2004. Explosives and propellants. In: Kirk-Othmer encyclopedia of chemical technology. Vol. 10. New York: John Wiley \& Sons, 719-744. http://onlinelibrary.wiley.com/doi/10.1002/0471238961.0524161212091404.a01.pub2/pdf. March 30, 2017.

Akram Z, Riaz S, Kayani MA, et al. 2019. Lead induces DNA damage and alteration of ALAD and antioxidant genes mRNA expression in construction site workers. Arch Environ Occup Health 74(4):171-178. http://doi.org/10.1080/19338244.2018.1428523.

Al Bakheet SA, Attafi IM, Maayah ZH, et al. 2013. Effect of long-term human exposure to environmental heavy metals on the expression of detoxification and DNA repair genes. Environ Pollut 181:226-232.

Alessio L. 1988. Relationship between "chelatable lead" and the indicators of exposure and effect in current and past occupational life. Sci Total Environ 71:293-299.

Alessio L, Bertazzi PA, Monelli O, et al. 1976. Free erythrocyte protoporphyrin as an indicator of the biological effect of lead in adult males. Int Arch Occup Environ Health 37:89-105.

Alexander FW, Clayton BE, Delves HT. 1974. Mineral and trace-metal balances in children receiving normal and synthetic diets. Q J Med 169:89-111.

Alexander BH, Checkoway H, Faustman EM, et al. 1998a. Contrasting associations of blood and semen lead concentrations with semen quality among lead smelter workers. Am J Ind Med 34:464-469.

Alexander BH, Checkoway H, Costa-Mallen P, et al. 1998b. Interaction of blood lead and deltaaminolevulinic acid dehydratase genotype on markers of heme synthesis and sperm production in lead smelter workers. Environ Health Perspect 106:213-216.

Al-Hakkak ZS, Hamamy HA, Murad AM, et al. 1986. Chromosome aberrations in workers at a storage battery plant in Iraq. Mutat Res Genet Toxicol 171(1):53-60.

Ali Z, Ulrik CS. 2013. Obesity and asthma: A coincidence or a causal relationship? A systematic review. Respir Med 107:1287-1300.

Aligne CA, Auinger P, Byrd RS, et al. 2000. Risk factors for pediatric asthma: Contributions of poverty, race, and urban residence. Am J Respir Crit Care Med 162(3 Pt. 1):873-877.

Almeida Lopes ACB, Silbergeld EK, Navas-Acien A, et al. 2017. Association between blood lead and blood pressure: A population-based study in Brazilian adults. Environ Health 16(1):27. http://doi.org/10.1186/s12940-017-0233-5.

Al-Modhefer AJ, Bradbury MWB, Simons TJB. 1991. Observations on the chemical nature of lead in human blood serum. Clin Sci 81:823-829.

Al-Neamy FR, Almehdi AM, Alwash R, et al. 2001. Occupational lead exposure and amino acid profiles and liver function tests in industrial workers. Int J Environ Health Res 11(2):181-188.

Alomran AH, Shleamoon MN. 1988. The influence of chronic lead exposure on lymphocyte proliferative response and immunoglobulin levels in storage battery workers. J Biol Sci 19(3):575585.

Al-Saleh I, Shinwari N, Mashhour A, et al. 2005. Is lead considered as a risk factor for high blood pressure during menopause period among Saudi women? Int J Hyg Environ Health 208(5):341-356.

Al-Saleh I, Shinwari N, Mashhour A, et al. 2014. Birth outcome measures and maternal exposure to heavy metals (lead, cadmium and mercury) in Saudi Arabian population. Int J Hyg Environ Health 217(2-3):205-218. http://doi.org/10.1016/j.ijheh.2013.04.009.

Alvarez-Ortega N, Caballero-Gallardo K, Olivero-Verbel J. 2019. Toxicological effects in children exposed to lead: A cross-sectional study at the Colombian Caribbean coast. Environ Int 130:104809. http://doi.org/10.1016/j.envint.2019.05.003.

Amaral JH, Rezende VB, Quintana SM, et al. 2010. The relationship between blood and serum lead levels in peripartum women and their respective umbilical cords. Basic Clin Pharmacol Toxicol 107(6):971-975. http://doi.org/10.1111/j.1742-7843.2010.00616.x.

Amato MS, Magzamen S, Imm P, et al. 2013. Early lead exposure ( $<3$ years old) prospectively predicts fourth grade school suspension in Milwaukee, Wisconsin (USA). Environ Res 126:60-65. http://doi.org/10.1016/j.envres.2013.07.008. 


\section{REFERENCES}

Ames SK, Ellis KJ, Gunn SK, et al. 1999. Vitamin D receptor gene Fok1 polymorphism predicts calcium absorption and bone mineral density in children. J Bone Miner Res 14(5):740-746.

Amitai Y, Graef JW, Brown MJ, et al. 1987. Hazards of 'deleading' homes of children with lead poisoning. Am J Dis Child 141:758-760.

An HC, Sung JH, Lee J, et al. 2017. The association between cadmium and lead exposure and blood pressure among workers of a smelting industry: A cross-sectional study. Ann Occup Environ Med 29:47. http://doi.org/10.1186/s40557-017-0202-z.

Anetor J, Adeniyi F. 1997. Decreased immune status in Nigerian workers occupationally exposed to lead. Afr J Med Sci 27(3-4):169-172.

Angel BM, Apte SC, Batley GE, et al. 2016. Lead solubility in seawater: An experimental study. Huanjing Huaxue 13(3):489. http://doi.org/10.1071/en15150.

Angelova VR, Ivanova RV, Todorov JM, et al. 2010. Lead, cadmium, zinc, and copper bioavailability in the soil-plant-animal system in a polluted area. ScientificWorldJournal 10:273-285. http://doi.org/10.1100/tsw.2010.33.

Angle CR, Manton WI, Stanek KL. 1995. Stable isotope identification of lead sources in preschool children - The Omaha Study. Clin Toxicol 33(6):657-662.

Angle CR, McIntire MS, Swanson MS, et al. 1982. Erythrocyte nucleotides in children -- Increased blood lead and cytidine triphosphate. Pediatr Res 16:331-334.

Anttila A, Heikkila P, Pukkala E, et al. 1995. Excess lung cancer among workers exposed to lead. Scand J Work Environ Health 21:460-469.

Anttila A, Heikkila P, Nykyri E, et al. 1996. Risk of nervous system cancer among workers exposed to lead. J Occup Environ Med 38(2):131-136.

Anwar WA, Kamal AA. 1988. Cytogenetic effects in a group of traffic policemen in Cairo. Mutat Res 208(3-4):225-231.

Aoki Y, Brody DJ, Flegal KM, et al. 2016. Blood lead and other metal biomarkers as risk factors for cardiovascular disease mortality. Medicine 95(1):e2223. http://doi.org/10.1097/md.0000000000002223.

Apostoli P, Maranelli G, Cas LD, et al. 1990. Blood lead and blood pressure: A cross sectional study in a general population group. Cardiologia 35(7):597-603.

Apostolou A, Garcia-Esquinas E, Fadrowski JJ, et al. 2012. Secondhand tobacco smoke: A source of lead exposure in US children and adolescents. Am J Public Health 102(20):12. http://doi.org/10.2105/AJPH.2011.300161.

Araki S, Murata K, Aono H. 1987. Central and peripheral nervous system dysfunction in workers exposed to lead, zinc and copper. Int Arch Occup Environ Health 59:177-187.

Araki S, Sata F, Murata K. 1990. Adjustment for urinary flow rate: An improved approach to biological monitoring. Int Arch Occup Environ Health 62(6):471-477.

Araki S, Honma T, Yanagihara S, et al. 1980. Recovery of slowed nerve conduction velocity in leadexposed workers. Int Arch Occup Environ Health 46:151-157.

Araki S, Aono H, Yokoyama K, et al. 1986. Filterable plasma concentration, glomerular filtration, tubular balance, and renal clearance of heavy metals and organic substances in metal workers. Arch Environ Health 41(4; 4):216-221.

Araki S, Sato H, Yokoyama K, et al. 2000. Subclinical neurophysiological effects of lead: A review on peripheral, central, and autonomic nervous system effects in lead workers. Am J Ind Med 37:193204.

Arbuckle TE, Davis K, Boylan K, et al. 2016. Processed data for CHMS 2007-2009: Bisphenol A, phthalates and lead and learning and behavioral problems in Canadian children 6-19 years of age. Data Brief 8:784-802. http://doi.org/10.1016/j.dib.2016.06.017.

Ari E, Kaya Y, Demir H, et al. 2011. The correlation of serum trace elements and heavy metals with carotid artery atherosclerosis in maintenance hemodialysis patients. Biol Trace Elem Res 144(13):351-359. http://doi.org/10.1007/s12011-011-9103-0. 
Armstrong R, Chettle DR, Scott MC, et al. 1992. Repeated measurements of tibia lead concentrations by in vivo X-ray fluorescence in occupational exposure. Br J Ind Med 49(1):14-16.

Arnvig E, Grandjean P, Beckmann J. 1980. Neurotoxic effects of heavy lead exposure determined with psychological tests. Toxicol Lett 5:399-404.

Aro A, Amarasiriwardena C, Lee ML, et al. 2000. Validation of K x-ray fluorescence bone lead measurements by inductively coupled plasma mass spectrometry in cadaver legs. Med Phys 27(1):119-123

Arora M, Ettinger AS, Peterson KE, et al. 2008. Maternal dietary intake of polyunsaturated fatty acids modifies the relationship between lead levels in bone and breast milk. J Nutr 138:73-79.

Arora M, Weuve J, Weisskopf MG, et al. 2009. Cumulative lead exposure and tooth loss in men: The normative aging study. Environ Health Perspect 117(10):1531-1534.

Arora M, Austin C, Sarrafpour B, et al. 2014. Determining prenatal, early childhood and cumulative long-term lead exposure using micro-spatial deciduous dentine levels. PLoS ONE 9(5):e97805. http://doi.org/10.1371/journal.pone.0097805.

Aschengrau A, Beiser A, Bellinger D, et al. 1994. The impact of soil lead abatement on urban children's blood lead levels: Phase II results from Boston Lead-in-Soil Demonstration project. Environ Res 67:125-148.

Assennato G, Paci C, Baser ME, et al. 1987. Sperm count suppression without endocrine dysfunction in lead-exposed men. Arch Environ Health 42:124-127.

Astrin KH, Bishop DF, Wetmur JG, et al. 1987. delta-Aminolevulinic acid dehydratase isozymes and lead toxicity. Ann N Y Acad Sci 514:23-39.

ATSDR. 1989. Decision guide for identifying substance-specific data needs related to toxicological profiles; Notice. Agency for Toxic Substances and Disease Registry. Fed Regist 54(174):3761837634.

ATSDR. 1995. Multisite lead and cadmium exposure study with biological markers incorporated. Atlanta, GA: Agency for Toxic Substances and Disease Registry, U.S. Department of Health and Human Services.

ATSDR. 2004a. Interaction profile for arsenic, cadmium, chromium, and lead. Atlanta, GA: Agency for Toxic Substances and Disease Registry. http://www.atsdr.cdc.gov/interactionprofiles/ip04.html. July 3, 2017.

ATSDR. 2004b. Interaction profile for lead, manganese, zinc, and copper. Atlanta, GA: Agency for Toxic Substances and Disease Registry. http://www.atsdr.cdc.gov/interactionprofiles/ip06.html. July 3, 2017.

ATSDR. 2006. Interaction profile for chlorpyrifos, lead, mercury, and methylmercury. Atlanta, GA: Agency for Toxic Substances and Disease Registry. http://www.atsdr.cdc.gov/interactionprofiles/IP11/ip11.pdf. July 3, 2017.

ATSDR. 2007. Toxicological profile for lead. Agency for Toxic Substances and Disease Registry. https://www.atsdr.cdc.gov/toxprofiles/tp13.pdf. June 20, 2017.

ATSDR. 2017. Case studies in environmental medicine (CSEM). Lead toxicity. Agency for Toxic Substances and Disease Registry. https://www.atsdr.cdc.gov/csem/lead/docs/csemlead_toxicity_508.pdf. August 30, 2018.

ATSDR. 2019. Lead. Full SPL data. Substance priority list (SPL) resource page. Agency for Toxic Substances and Disease Registry.

Aucott M, Caldarelli A. 2012. Quantity of lead released to the environment in New Jersey in the form of motor vehicle wheel weights. Water Air Soil Pollut 223(4):1743-1752. http://doi.org/10.1007/s11270-011-0979-2.

Aufderheide AC, Wittmers LE. 1992. Selected aspects of the spatial distribution of lead in bone. Neurotoxicology 13:809-820.

Aungst BJ, Fung HL. 1981. Kinetic characterization of in vitro lead transport across the rat small intestine. Toxicol Appl Pharmacol 61:39-47. 
Aungst BJ, Dolce JA, Fung HL. 1981. The effect of dose on the disposition of lead in rats after intravenous and oral administration. Toxicol Appl Pharmacol 61:48-57.

Avula B, Wang YH, Smillie TJ, et al. 2010. Quantitative determination of multiple elements in botanicals and dietary supplements using ICP-MS. J Agric Food Chem 58:8887-8894.

Awad el Karim MA, Hamed AS, Elhaimi YA, et al. 1986. Effects of exposure to lead among lead-acid battery factory workers in Sudan. Arch Environ Health 41(4):261-265. http://doi.org/10.1080/00039896.1986.9938342.

Azar A, Trochimowicz HJ, Maxfield ME. 1973. Review of lead studies in animals carried out at Haskell Laboratory: Two year feeding study and response to hemorrhage study In: Barth D, Berlin A, Engel R, et al., eds. Environmental health aspects of lead: Proceedings of an international symposium. Vol. 5004. Luxembourg: Commission of the European Communities, 199-210.

Baertlein L, Yendell S. 2017. Selection bias in estimates of elevated blood lead levels in children. J Pediatr 181:330. http://doi.org/10.1016/j.jpeds.2016.09.044.

Baeyens W, Vrjens J, Gao Y, et al. 2014. Trace metals in blood and urine of newborn/mother pairs, adolescents and adults of the Flemish population (2007-2011). Int J Hyg Environ Health 217(8):878-890.

Bagci C, Bozkurt A, Cakmak E, et al. 2004. Blood lead levels of the battery and exhaust workers and their pulmonary function tests. Int J Clin Pract 58(6):568-572.

Baghurst PA, Robertson EF, McMichael AJ, et al. 1987. The Port Pirie cohort study: Lead effects on pregnancy outcome and early childhood development. Neurotoxicology 8(3):395-402.

Baghurst PA, McMichael AJ, Wigg NR, et al. 1992. Environmental exposure to lead and children's intelligence at the age of seven years. N Engl J Med 327(18):1279-1284.

Baghurst PA, McMichael AJ, Tong S, et al. 1995. Exposure to environmental lead and visual-motor integration at age 7 years: The Port Pirie cohort study. Epidemiology 6(2):104-109.

Bailey MR, Roy M. 1994. Clearance of particles from the respiratory tract. In: Human respiratory tract model for radiological protection: A report of a task group of the International Commission on Radiological Protection. Oxford, UK: Pergamon Press, 301-413.

Baker EL, Landrigan PJ, Barbour AG, et al. 1979. Occupational lead poisoning in the United States: Clinical and biochemical findings related to blood lead levels. Br J Ind Med 36:314-322.

Baker EL, Feldman RG, White RF, et al. 1983. The role of occupational lead exposure in the genesis of psychiatric and behavioral disturbances. Acta Psychiatr Scand Suppl 303:38-48.

Bakulski KM, Rozek LS, Dolinoy DC, et al. 2012. Alzheimer's disease and environmental exposure to lead: The epidemiologic evidence and potential role of epigenetics. Curr Alzheimer Res 9(5):563573.

Balo J, Attila B, Bela S. 1965. [Experimental adenomas of the kidney produced by chronic administration of lead phosphate]. Magy Onkol 9:141-151. (Hungarian)

Bandeen-Roche K, Glass TA, Bolla KI, et al. 2009. Cumulative lead dose and cognitive function in older adults. Epidemiology 20(6):831-839. http://doi.org/10.1097/EDE.0b013e3181b5f100.

Bannon DI, Williams MA. 2017. Matching target dose to target organ. F1000 Research 5:2785. https://f1000research.com/articles/5-2785/v2. February 18, 2020.

Bannon DI, Olivi L, Bressler J. 2000. The role of anion exchange in the uptake of Pb by human erythrocytes and Madin-Darby canine kidney cells. Toxicology 147:101-107.

Bannon DI, Portnoy ME, Olivi L, et al. 2002. Uptake of lead and iron by divalent metal transporter 1 in yeast and mammalian cells. Biochem Biophys Res Commun 295(4):978-984.

Bannon DI, Abounader R, Lees PS, et al. 2003. Effect of DMT1 knockdown on iron, cadmium, and lead uptake in Caco-2 cells. Am J Physiol Cell Physiol 284(1):C44-50. http://doi.org/10.1152/ajpcell.00184.2002.

Bannon DI, Drexler JW, Fent GM, et al. 2009. Evaluation of small arms range soils for metal contamination and lead bioavailability. Environ Sci Technol 43(24):9071-9076. http://doi.org/10.1021/es901834h. 
Bao QS, Lu CY, Song H, et al. 2009. Behavioural development of school-aged children who live around a multi-metal sulphide mine in Guangdong province, China: A cross-sectional study. BMC Public Health 9(1):217. http://doi.org/10.1186/1471-2458-9-217.

Baranowska-Bosiacka I, Kosinska I, Jamiol D, et al. 2016. Environmental lead (Pb) exposure versus fatty acid content in blood and milk of the mother and in the blood of newborn children. Biol Trace Elem Res 170:279-287. http://doi.org/10.1007/s12011-015-0482-5.

Barbosa F, Tanus-Santos JE, Gerlach RQ, et al. 2005. A critical review of biomarkers used for monitoring human exposure to lead: Advantages, limitations, and future needs. Environ Health Perspect 113(12):1669-1674.

Barbosa F, Ramires I, Rodrigues MHC, et al. 2006a. Contrasting effects of age on the plasma/whole blood lead ratio in men and women with a history of lead exposure. Environ Res 102(1):90-95. http://doi.org/10.1016/j.envres.2006.03.007.

Barbosa F, Sandrim VC, Uzuelli JA, et al. 2006b. eNOS genotype-dependent correlation between whole blood lead and plasma nitric oxide products concentrations. Nitric Oxide 14(1):58-64. http://doi.org/10.1016/j.niox.2005.09.007.

Barbosa F, Correa Rodrigues MH, Buzalaf MR, et al. 2006c. Evaluation of the use of salivary lead levels as a surrogate of blood lead or plasma lead levels in lead exposed subjects. Arch Toxicol 80(10):633-637. http://doi.org/10.1007/s00204-006-0096-y.

Barltrop D, Meek F. 1979. Effect of particle size on lead absorption from the gut. Arch Environ Health 34(4):280-285. http://doi.org/10.1080/00039896.1979.10667414.

Barman T, Kalahasthi R, Rajmohan HR. 2014. Effects of lead exposure on the status of platelet indices in workers involved in a lead-acid battery manufacturing plant. J Expo Sci Environ Epidemiol 24(6):629-633. http://doi.org/10.1038/jes.2014.4.

Barnes DG, Dourson M. 1988. Reference dose (RfD): Description and use in health risk assessments. Regul Toxicol Pharmacol 8(4):471-486.

Barregård L, Svalander C, Schütz A, et al. 1999. Cadmium, mercury, and lead in kidney cortex of the general Swedish population: A study of biopsies from living kidney donors. Environ Health Perspect 107(11):867-871.

Barry PSI. 1975. A comparison of concentrations of lead in human tissues. Br J Ind Med 32:119-139.

Barry PSI. 1981. Concentrations of lead in the tissues of children. Br J Ind Med 38:61-71.

Barry V, Steenland K. 2019. Lead exposure and mortality among U.S. workers in a surveillance program: Results from 10 additional years of follow-up. Environ Res 177:108625. http://doi.org/10.1016/j.envres.2019.108625.

Barry V, Todd AC, Steenland K. 2019. Bone lead associations with blood lead, kidney function and blood pressure among US, lead-exposed workers in a surveillance programme. Occup Environ Med 76(5):349-354. http://doi.org/10.1136/oemed-2018-105505.

Barth A, Schaffer AW, Osterode W, et al. 2002. Reduced cognitive abilities in lead-exposed men. Int Arch Occup Environ Health 75:394-398.

Barton JC. 1984. Active transport of lead-210 by everted segments of rat duodenum. Am J Physiol 247:G193-G198.

Barton JC, Conrad ME, Harrison L, et al. 1978a. Effects of calcium on the absorption and retention of lead. J Lab Clin Med 91(3):366-376.

Barton JC, Conrad ME, Nuby S, et al. 1978b. Effects of iron on the absorption and retention of lead. J Lab Clin Med 92(4):536-547.

Barton JC, Patton MA, Edwards CQ, et al. 1994. Blood lead concentrations in hereditary hemochromatosis. J Lab Clin Med 124(2):193-198.

Basaran N, Undeger U. 2000. Effects of lead on immune parameters in occupationally exposed workers. Am J Ind Med 38:349-354.

Battistuzzi G, Petrucci R, Silvagni L, et al. 1981. $\delta$-Aminolevulinate dehydrase: A new genetic polymorphism in man. Ann Hum Genet 45(3):223-229. 
Bauchinger M, Dresp J, Schmid E, et al. 1977. Chromosome analyses of children after ecological lead exposure. Mutat Res 56:75-80.

BCI. 2019. National recycling rate study. Battery Council International.

Beck BD, Mattuck RL, Bowers TS, et al. 2001. The development of a stochastic physiologically-based pharmacokinetic model for lead. Sci Total Environ 274:15-19.

Beckley AL, Caspi A, Broadbent J, et al. 2018. Association of childhood blood lead levels with criminal offending. JAMA Pediatrics 172(2):166-173. http://doi.org/10.1001/jamapediatrics.2017.4005.

Beers MH, Berkow R, eds. 1999. Injuries, poisoning, and cardiopulmonary resuscitation. In: The Merck manual of diagnosis and therapy Whitehouse Station, NJ: Merck Research Laboratories, 2254-2300.

Behinaein S, Chettle DR, Atanackovic J, et al. 2011. In vivo measurement of lead in the bones of smelter workers using the four-element 'clover-leaf' geometry detector system. Phys Med Biol 56(3):653-665. http://doi.org/10.1088/0031-9155/56/3/008.

Behinaein S, Chettle DR, Egden LM, et al. 2012. Nonlinearity in the relationship between bone lead concentrations and CBLI for lead smelter employees. J Environ Monit 14(12):3267-3275. http://doi.org/10.1039/c2em30652b.

Behinaein S, Chettle DR, Egden LM, et al. 2014. The estimation of the rates of lead exchange between body compartments of smelter employees. Environ Sci Process Impacts 16(7):1705-1715. http://doi.org/10.1039/c4em00032c.

Bellinger DC. 2004. Assessing environmental neurotoxicant exposures and child neurobehavior: Confounded by confounding? Epidemiology 15(4):383-384.

Bellinger DC. 2011. The protean toxicities of lead: New chapters in a familiar story. Int J Environ Res Public Health 8(7):2593-2628. http://doi.org/10.3390/ijerph8072593.

Bellinger D, Needleman HL. 2003. Intellectual impairment and blood lead levels. N Engl J Med 349(5):500. http://doi.org/10.1056/NEJM200307313490515.

Bellinger D, Leviton A, Sloman J. 1990. Antecedents and correlates of improved cognitive performance of children exposed in utero to low levels of lead. Environ Health Perspect 89:5-11.

Bellinger DC, Stiles KM, Needleman HL. 1992. Low-level lead exposure, intelligence and academic achievement: A long-term follow-up study. Pediatrics 90(6):855-861.

Bellinger DC, Leviton A, Waternaux C, et al. 1987. Longitudinal analyses of prenatal and postnatal lead exposure and early cognitive development. N Engl J Med 316:1037-1043.

Bellinger D, Sloman J, Leviton A, et al. 1991. Low-level lead exposure and children's cognitive function in the preschool years. Pediatrics 87(2):219-227.

Bellinger D, Hu H, Titlebaum L, et al. 1994. Attentional correlates of dentin and bone lead levels in adolescents. Arch Environ Health 49(2):98-105. http://doi.org/10.1080/00039896.1994.9937461.

Bener A, Almehdi AM, Alwash R, et al. 2001. A pilot survey of blood lead levels in various types of workers in the United Arab Emirates. Environ Int 27(4):311-314. http://doi.org/10.1016/S01604120(01)00061-7.

Bergdahl IA, Skerfving S. 1997. Partition of circulating lead between plasma and red cells does not seem to be different for internal and external sources of lead. Letter to the editor. Am J Ind Med 32:317-318.

Bergdahl IA, Schutz A, Grubb A. 1996. Application of liquid chromatography-inductively coupled plasma mass spectrometry to the study of protein-bond lead in human erythrocytes. J Anal Atom Spectrom 11:735-738.

Bergdahl IA, Grubb A, Schütz A, et al. 1997a. Lead binding to $\delta$-aminolevulinic acid dehydratase (ALAD) in human erythrocytes. Basic Clin Pharmacol Toxicol 81(4):153-158.

Bergdahl IA, Gerhardsson L, Schütz A, et al. 1997b. delta-Aminolevulinic acid dehydratase polymorphism: Influence on lead levels and kidney function in humans. Arch Environ Health 52(2):91-96.

Bergdahl IA, Schutz A, Gerhardsson L, et al. 1997c. Lead concentrations in human plasma, urine and whole blood. Scand J Work Environ Health 23(5):359-363. 
Bergdahl IA, Sheveleva M, Schutz A, et al. 1998. Plasma and blood lead in humans: Capacity-limited binding to delta-aminolevulinic acid dehydratase and other lead-binding components. Toxicol Sci 46:247-253.

Bergdahl IA, Vahter M, Counter SA, et al. 1999. Lead in plasma and whole blood from lead-exposed children. Environ Res 80:25-33. http://doi.org/10.1006/enrs.1998.3880.

Bergdahl IA, Gerhardsson L, Liljelind IE, et al. 2006. Plasma-lead concentration: Investigations into its usefulness for biological monitoring of occupational lead exposure. Am J Ind Med 49(2):93-101.

Bergeret A, Pouget E, Tedone R, et al. 1990. Neutrophil functions in lead-exposed workers. Hum Exp Toxicol 9(4):231-233. http://doi.org/10.1177/096032719000900405.

Berkowitz GS, Wolff MS, Lapinski RH, et al. 2004. Prospective study of blood and tibia lead in women undergoing surgical menopause. Environ Health Perspect 112(17):1673-1678.

Bernard BP, Becker CE. 1988. Environmental lead exposure and the kidney. Clin Toxicol 26(1\&2):134.

Bernard AM, Vyskocil A, Roels H, et al. 1995. Renal effects in children living in the vicinity of a lead smelter. Environ Res 68:91-95.

Bert JL, Van DLJ, Grace JR. 1989. A generalized model for the prediction of lead body burdens. Environ Res 48:117-127.

Bertazzi PA, Zocchetti C. 1980. A mortality study of newspaper printing workers. Am J Ind Med 1:8597.

Berthelot Y, Valton E, Auroy A, et al. 2008. Integration of toxicological and chemical tools to assess the bioavailability of metals and energetic compounds in contaminated soils. Chemosphere 74:166-177.

Bhattacharya A, Shukla R, Dietrich KN, et al. 2006. Effect of early lead exposure on the maturation of children's postural balance: A longitudinal study. Neurotoxicol Teratol 28(3):376-385. http://doi.org/10.1016/j.ntt.2006.02.003.

Bhatti P, Stewart PA, Hutchinson A, et al. 2009. Lead exposure, polymorphisms in genes related to oxidative stress, and risk of adult brain tumors. Cancer Epidemiol Biomarkers Prev 18(6):18411848. http://doi.org/10.1158/1055-9965.EPI-09-0197.

Biagini G, Caudarella R, Vangelista A. 1977. Renal morphological and functional modification in chronic lead poisoning. In: Brown SS, ed. Clinical chemistry and chemical toxicology of metals. Elsevier/North-Holland Biomedical Press, 123-126.

Biggins PDE, Harrison RM. 1979. Atmospheric chemistry of automotive lead. Environ Sci Technol 13:558-565.

Billick IH, Gray VE. 1978. Executive summary. In: Lead based paint poisoning research: Review and evaluation, 1971-1977. U.S. Deptartment of Housing and Urban Development, vi-vii.

Bindler R, Renberg I, Klaminder J. 2008. Bridging the gap between ancient metal pollution and contemporary biogeochemistry. J Paleolimnol 40(3):755-770.

Blackowicz MJ, Hryhorczuk DO, Rankin KM, et al. 2016. The impact of low-level lead toxicity on school performance among Hispanic subgroups in the Chicago public schools. Int J Environ Res Public Health 13(8):774. http://doi.org/10.3390/ijerph13080774.

Blake K, Mann M. 1983. Effect of calcium and phosphorus on the gastrointestinal absorption of 203Pb in man. Environ Res 30(1):188-194.

Blake KC, Barbezat H, Mann M. 1983. Effect of dietary constituents on the gastrointestinal absorption of 203Pb in man. Environ Res 30:182-187.

Bloom MS, Parsons PJ, Steuerwald AJ, et al. 2010. Toxic trace metals and human oocytes during in vitro fertilization (IVF). Reprod Toxicol 29(3):298-305. http://doi.org/10.1016/j.reprotox.2010.01.003.

Bloom MS, Louis GMB, Sundaram R, et al. 2011. Associations between blood metals and fecundity among women residing in New York State. Reprod Toxicol 31(2):158-163. http://doi.org/10.1016/j.reprotox.2010.09.013. 
Bloom MS, Buck Louis GM, Sundaram R, et al. 2015. Birth outcomes and background exposures to select elements, the Longitudinal Investigation of Fertility and the Environment (LIFE). Environ Res 138:118-129. http://doi.org/10.1016/j.envres.2015.01.008.

Bockelmann I, Pfister EA, McGauran N, et al. 2002. Assessing the suitability of cross-sectional and longitudinal cardiac rhythm tests with regard to identifying effects of occupational chronic lead exposure. J Occup Environ Med 44:59-65.

Boileau J, Fauquignon C, Napoly C. 1987. Explosives. In: Ullmann's encyclopedia of industrial chemistry. Vol. A10. Weinheim, Germany: VCH Verlag GmbH \& Co., 143-172.

Boileau J, Fauguigson C, Hlueber B, et al. 2012. Explosives. In: Ullmann's encyclopedia of industrial chemistry. Weinheim, Germany: Wiley-VCH Verlag GmbH \& Co., 621-654. http://onlinelibrary.wiley.com/doi/10.1002/14356007.a10_143.pub2/pdf. March 30, 2017.

Bolanowska W, Piotrowski J, Garczynski H. 1967. Triethyl lead in the biological material in cases of acute tetraethyl lead poisoning. Arch Toxikol 22:278-282.

Bonanno LJ, Freeman NCG, Greenburg M, et al. 2001. Multivariate analysis on levels of selected metals, particulate matter, VOC, and household characteristics and activities from the midwestern states NHEXAS. Appl Occup Environ Hyg 16(9):859-874.

Bonde JP, Joffe M, Apostoli P, et al. 2002. Sperm count and chromatin structure in men exposed to inorganic lead: Lowest adverse effect levels. Occup Environ Med 59(4):234-242.

Bonithon-Kopp C, Huel G, Grasmick C, et al. 1986. Effects of pregnancy on the inter-individual variations in blood levels of lead, cadmium and mercury. Biol Res Pregnancy Perinatol 7(1):37-42.

Borja-Aburto VH, Hertz-Picciotto I, Lopez MR, et al. 1999. Blood lead levels measured prospectively and risk of spontaneous abortion. Am J Epidemiol 150:590-597.

Borjesson J, Gerhardsson L, Schutz A, et al. 1997. In vivo measurements of lead in fingerbone in active and retired lead smelters. Int Arch Occup Environ Health 69:97-105.

Bornschein RL, Succop PA, Krafft KM, et al. 1986. Exterior surface dust lead, interior house dust lead and childhood lead exposure in an urban environment. Trace Subst Environ Health 20:322-332.

Bornschein RL, Grote J, Mitchell T, et al. 1989. Effects of prenatal lead exposure on infant size at birth. In: Smith MA, Grant LD, Sors AI, eds. Lead exposure and child development. Dordrecht: Springer, 307-319. http://doi.org/10.1007/978-94-009-0847-5_18.

Bos AJJ, van der Stap CCAH, Valkovic V, et al. 1985. Incorporation routes of elements into human hair: Implications for hair analysis used for monitoring. Sci Total Environ 42:157-169.

Boscolo P, Di Gioacchino M, Sabbioni E, et al. 1999. Expression of lymphocyte subpopulations, cytokine serum levels, and blood and urinary trace elements in asymptomatic atopic men exposed to an urban environment. Int Arch Occup Environ Health 72(1):26-32.

http://doi.org/10.1007/s004200050330.

Boscolo P, Di Gioacchino M, Sabbioni E, et al. 2000. Lymphocyte subpopulations, cytokines and trace elements in asymptomatic atopic women exposed to an urban environment. Life Sci 67(10):11191126. http://doi.org/10.1016/S0024-3205(00)00712-8.

Bost L, Primatesta P, Dong W, et al. 1999. Blood lead and blood pressure: Evidence from the health survey for England 1995. J Hum Hypertens 13(2; 2):123-128.

Bouchard MF, Bellinger DC, Weuve J, et al. 2009. Blood lead levels and major depressive disorder, panic disorder, and generalized anxiety disorder in US young adults. Arch Gen Psychiatry 66(12):1313-1319. http://doi.org/10.1001/archgenpsychiatry.2009.164.

Boucher O, Jacobson SW, Plusquellec P, et al. 2012. Prenatal methylmercury, postnatal lead exposure, and evidence of attention deficit/hyperactivity disorder among Inuit children in Arctic Quebec. Environ Health Perspect 120(10):1456-1461. http://doi.org/10.1289/ehp.1204976.

Boucher O, Muckle G, Jacobson JL, et al. 2014. Domain-specific effects of prenatal exposure to PCBs, mercury, and lead on infant cognition: Results from the Environmental Contaminants and Child Development Study in Nunavik. Environ Health Perspect 122(3):310-316. http://doi.org/10.1289/ehp.1206323.

Boudene C, Malet D, Masse R. 1977. Fate of Pb inhaled by rats. Toxicol Appl Pharmacol 41:271-276. 
Boudene C, Despaux-Pages N, Comoy E, et al. 1984. Immunological and enzymatic studies of erythrocytic $\delta$-aminolevulinate dehydratase. Int Arch Occup Environ Health 55(1):87-96.

Bound JP, Harvey PW, Francis BJ, et al. 1997. Involvement of deprivation and environmental lead in neural tube defects: A matched case-control study. Arch Dis Child 76(2):107-112.

Bouton C, Pevsner J. 2000. Effects of lead on gene expression. Neurotoxicology 21(6):1045-1056.

Bowen HJM. 1966. Trace elements in biochemistry. New York, NY: Academic Press. 31-32.

Bowers TS, Mattuck RL. 2001. Further comparisons of empirical and epidemiological data with predictions of the Integrated Exposure Uptake Biokinetic Model for lead in children. Hum Ecol Risk Assess 7:1699-1713.

Bowers TS, Beck BD. 2006. What is the meaning of non-linear dose-response relationships between blood lead concentrations and IQ? Neurotoxicology 27(4):520-524. http://doi.org/10.1016/j.neuro.2006.02.001. http://www.ncbi.nlm.nih.gov/pubmed/16551479.

Bowers TS, Beck BD, Karam HS. 1994. Assessing the relationship between environmental lead concentrations and adult blood lead levels. Risk Anal 14(2):183-189.

Bradham KD, Green W, Hayes H, et al. 2016. Estimating relative bioavailability of soil lead in the mouse. J Toxicol Environ Health A 79(24):1179-1182. http://doi.org/10.1080/15287394.2016.1221789.

Bradham KD, Nelson CM, Diamond GL, et al. 2019. Dietary lead and phosphate interactions affect oral bioavailability of soil lead in the mouse. Environ Sci Technol 53(21):12556-12564. http://doi.org/10.1021/acs.est.9b02803.

Bradley SB, Cox JJ. 1988. The potential availability of cadmium, copper, iron, lead, manganese, nickel and zinc in standard river sediment (NBS 1645). Environ Technol Lett 9:733-739.

Braun JM, Kahn RS, Froehlich T, et al. 2006. Exposures to environmental toxicants and attention deficit hyperactivity disorder in U.S. children. Environ Health Perspect 114(12):1904-1909. http://doi.org/10.1289/ehp.9478.

Braun JM, Froehlich TE, Daniels JL, et al. 2008. Association of environmental toxicants and conduct disorder in U.S. children: NHANES 2001-2004. Environ Health Perspect 116(7):956-962. http://doi.org/10.1289/ehp.11177.

Braun JM, Hoffman E, Schwartz J, et al. 2012. Assessing windows of susceptibility to lead-induced cognitive deficits in Mexican children. Neurotoxicology 33(5):1040-1047. http://doi.org/10.1016/j.neuro.2012.04.022.

Braun JM, Wright RJ, Just AC, et al. 2014. Relationships between lead biomarkers and diurnal salivary cortisol indices in pregnant women from Mexico City: A cross-sectional study. Environ Health 13(1):50. http://doi.org/10.1186/1476-069x-13-50.

Braunstein GD, Dahlgren J, Loriaux DL. 1978. Hypogonadism in chronically lead-poisoned men. Infertility 1(1):33-51.

Brender JD, Suarez L, Felkner M, et al. 2006. Maternal exposure to arsenic, cadmium, lead, and mercury and neural tube defects in offspring. Environ Res 101(1):132-139. http://doi.org/10.1016/j.envres.2005.08.003.

Bres EF, Voegel JC, Barry JC, et al. 1986. Feasibility study for the detection of lead substitution sites in the hydroxyapatite crystal structure using high resolution electron microscopy (HREM) at optimum focus. J Appl Cryst 19:168-173.

Bress WC, Bidanset JH. 1991. Percutaneous in vivo and in vitro absorption of lead. Vet Hum Toxicol 33(3):212-214.

Bressler J, Kim K, Chakraborti T, et al. 1999. Molecular mechanisms of lead neurotoxicity. Neurochem Res 24(4):595-600.

Bressler JP, Olivi L, Kim Y, et al. 2005. Plasma membrane transporters for lead and cadmium. J Appl Pharmacol 13(1):1-6.

Brito JAA, McNeill FE, Webber CE, et al. 2005. Grid search: An innovative method for the estimation of the rates of lead exchange between body compartments. J Environ Monit 7(3):241-247. 
Broberg K, Engstrom K, Ameer S. 2015. Gene-environment interactions for metals. In: Nordberg GF, Fowler BA, Nordberg M, eds. Handbook on the toxicology of metals. 4th ed. Elsevier, 239-264. http://doi.org/10.1016/B978-0-444-59453-2.00012-3.

Brodeur J, Lacasse Y, Talbot D. 1983. Influence of removal from occupational lead exposure on blood and saliva lead concentrations. Toxicol Lett 19(1-2):195-199.

Brody DJ, Pirkle JL, Kramer RA, et al. 1994. Blood lead levels in the US population: Phase I of the third National Health and Nutrition Examination Survey (NHANES III, 1968-1991). J Am Med Assoc 272(4):277-283.

Bronner F, Pansu D, Stein WD. 1986. An analysis of intestinal calcium transport across the rat intestine. Am J Physiol 250(13):G561-G569.

Brown S, Chaney RL, Hallfrisch J, et al. 2004. In situ soil treatments to reduce the phyto- and bioavailability of lead, zinc and cadmium. J Environ Qual 33:522-531.

Brown RW, Gonzales C, Hooper MJ, et al. 2008. Soil lead (Pb) in residential transects through Lubbock, Texas: A preliminary assessment. Environ Geochem Health 30(6):541-547. http://doi.org/10.1007/s10653-008-9180-y.

Brubaker CJ, Dietrich KN, Lanphear BP, et al. 2010. The influence of age of lead exposure on adult gray matter volume. Neurotoxicology 31(3):259-266. http://doi.org/10.1016/j.neuro.2010.03.004.

Brunekreef B. 1984. The relationship between air lead and blood lead in children: A critical review. Sci Total Environ 38:79-123.

Buc H, Kaplan J. 1978. Red-cell pyrimidine 5'-nucleotidase and lead poisoning. Clin Chim Acta 87(1):49-55.

Buchet JP, Roels H, Bernard A, et al. 1980. Assessment of renal function of workers exposed to inorganic lead, cadmium or mercury vapor. J Occup Environ Med 22(11):741-750.

Budtz-Jorgensen E, Bellinger D, Lanphear B, et al. 2013. An international pooled analysis for obtaining a benchmark dose for environmental lead exposure in children. Risk Anal 33(3):450-461. http://doi.org/10.1111/j.1539-6924.2012.01882.x.

Bulsma JB, de France HF. 1976. Cytogenetic investigations in volunteers ingesting inorganic lead. Int Arch Occup Environ Health 38:145-148.

Burns JS, Williams PL, Lee ML, et al. 2017. Peripubertal blood lead levels and growth among Russian boys. Environ Int 106:53-59. http://doi.org/10.1016/j.envint.2017.05.023.

Buser MC, Scinicariello F. 2017. Cadmium, lead, and depressive symptoms: Analysis of National Health and Nutrition Examination Survey 2011-2012. J Clin Psychiatry 78(5):e515-e521. http://doi.org/10.4088/JCP.15m10383.

Bushnik T, Levallois P, D'Amour M, et al. 2014. Association between blood lead and blood pressure: Results from the Canadian Health Measures Survey (2007 to 2011). Health Rep 25(7):12-22.

Businelli D, Onofri A, Massaccesi L. 2011. Factors involved in uptake of lead by some edible crops grown in agricultural soils of Central Italy. Soil Sci 176(9):472-478.

Butterweck G, Schuler C, Vezzù G, et al. 2002. Experimental determination of the absorption rate of unattached radon progeny from respiratory tract to blood. Radiat Prot Dosimetry 102(4):343- 348.

Cabral M, Dieme D, Verdin A, et al. 2012. Low-level environmental exposure to lead and renal adverse effects: A cross-sectional study in the population of children bordering the Mbeubeuss landfill near Dakar, Senegal. Hum Exp Toxicol 31(12):1280-1291. http://doi.org/10.1177/0960327112446815.

Cake K, Bowins R, Vaillancourt C, et al. 1996. Partition of circulating lead between serum and red cells is different for internal and external sources of lead. Am J Ind Med 29(5):440-445.

Cal EPA. 2013. Estimating workplace air and worker blood lead concentration using an updated physiologically-based pharmacokinetic (PBPB) model. California Environmental Protection Agency. https://oehha.ca.gov/media/downloads/air/document/pbpk2013.pdf. April 03, 2014.

Cal EPA. 2017. User's guide to LeadSpread 8 and recommendations for evaluation of lead exposures in adults. California Environmental Protection Agency. http://www.dtsc.ca.gov/AssessingRisk/upload/LeadSpread8_UserGuide.pdf. September 1, 2017. 


\section{REFERENCES}

Calabrese EJ, Stanek EJ, Gilbert CE, et al. 1990. Preliminary adult soil ingestion estimates: Results of a pilot study. Regul Toxicol Pharmacol 12(1):88-95.

Calderon-Salinas JV, Quintanar-Escorcia MA, Gonzalez-Martinez MT, et al. 1999. Lead and calcium transport in human erythrocyte. Hum Exp Toxicol 18:327-332.

Calello DP, Henretig FM. 2014. Lead. In: Goldfrank's toxicologic emergencies. Tenth ed. New York, NY: McGraw-Hill, 1219-1234.

Campara P, D'Andrea F, Micciolo R, et al. 1984. Psychological performance of workers with blood-lead concentration below the current threshold limit value. Int Arch Occup Environ Health 53:233-246.

Campbell JR, Toribara TY. 2001. Hair-root lead to screen for lead toxicity. J Trace Elem Exp Med 14:69-72.

Campbell JR, Auinger P. 2007. The association between blood lead levels and osteoporosis among adults - Results from the third National Health and Nutrition Examination Survey (NHANES III). Environ Health Perspect 115(7):1018-1022. http://doi.org/10.1289/ehp.9716.

Campbell JR, Moss ME, Raubertas RF. 2000a. The association between caries and childhood lead exposure. Environ Health Perspect 108(11):1099-1102.

Campbell TF, Needleman HL, Riess JA, et al. 2000b. Bone lead levels and language processing performance. Dev Neuropsychol 18(2):171-186.

Campbell JR, Rosier RN, Novotny L, et al. 2004. The association between environmental lead exposure and bone density in children. Environ Health Perspect 112:1200-1203.

Can S, Bagci C, Ozaslan M, et al. 2008. Occupational lead exposure effect on liver functions and biochemical parameters. Acta Physiol Hung 95(4):395-403.

http://doi.org/10.1556/APhysiol.95.2008.4.6.

Canfield RL, Henderson CR, Cory-Slechta DA, et al. 2003. Intellectual impairment in children with blood lead concentrations below 10 micrograms per deciliter. N Engl J Med 348(16):1517-1526. http://doi.org/10.1056/NEJMoa022848.

Canonne-Hergaux F, Gruenheid S, Ponka P, et al. 1999. Cellular and subcellular localization of the nramp2 iron transporter in the intestinal brush border and regulation by dietary iron. Blood 93(12):4406-4417.

Cantonwine D, Hu H, Tellez-Rojo MM, et al. 2010. HFE gene variants modify the association between maternal lead burden and infant birthweight: A prospective birth cohort study in Mexico City, Mexico. Environmental Health: A Global Access Science Source 9:43. http://doi.org/10.1186/1476069X-9-43.

Cao X, Ma LQ, Chen M, et al. 2003a. Lead transformation and distribution in the soils of shooting ranges in Florida, USA. Sci Total Environ 307:179-189.

Cao X, Ma LQ, Chen M, et al. 2003b. Weathering of lead bullets and their environmental effects at outdoor shooting ranges. J Environ Qual 32:526-534.

Carbone R, Laforgia N, Crollo E, et al. 1998. Maternal and neonatal lead exposure in southern Italy. Biol Neonate 73:362-366.

Cardenas A, Roels H, Bernard A, et al. 1993. Markers of early renal changes induced by industrial pollutants. II. Application to workers exposed to lead. Br J Ind Med 50(1):28-36.

Cardozo dos Santos A, Colacciopo S, Dal Bó CM, et al. 1994. Occupational exposure to lead, kidney function tests, and blood pressure. Am J Ind Med 26(5):635-643.

Carlisle JC, Wade MJ. 1992. Predicting blood lead concentrations from environmental concentrations. Regul Toxicol Pharmacol 16(3):280-289.

Carr DS. 1995. Lead compounds: Lead salts. In: Kirk-Othmer encyclopedia of chemical technology. 4th edition ed. New York: John Wiley \& Sons, 132-152.

Carr DS, Spangenberg WC, Chronley K, et al. 2004. Lead compounds. In: Kirk-Othmer encyclopedia of chemical technology. New York: John Wiley \& Sons, 1-26.

Casjens S, Pesch B, van Thriel C, et al. 2018. Associations between blood lead, olfaction and fine-motor skills in elderly men: Results from the Heinz Nixdorf Recall Study. Neurotoxicology 68:66-72. http://doi.org/10.1016/j.neuro.2018.06.013. 
Cassidy-Bushrow AE, Havstad S, Basu N, et al. 2016. Detectable blood lead level and body size in early childhood. Biol Trace Elem Res 171(1):41-47. http://doi.org/10.1007/s12011-015-0500-7.

Casteel SW, Cowart RP, Weis CP, et al. 1997. Bioavailability of lead to juvenile swine dosed with soil from the Smuggler Mountain NPL site of Aspen, Colorado. Toxicol Sci 36(2):177-187.

Casteel SW, Weis CP, Henningsen GM, et al. 2006. Estimation of relative bioavailability of lead in soil and soil-like materials using young swine. Environ Health Perspect 114(8):1162-1171.

Cavalleri A, Minoia C, Pozzoli L, et al. 1978. Determination of plasma lead levels in normal subjects and lead-exposed workers. Br J Ind Med 35:21-26.

CDC. 1985. Preventing lead poisoning in young children. Atlanta, GA: Centers for Disease Control and Prevention. Publication No. 99-2230.

CDC. 1991. Preventing lead poisoning in young children. Atlanta, GA: Centers for Disease Control and Prevention.

CDC. 1997a. Children with elevated blood lead levels attributed to home renovation and remodeling activities - New York, 1993-1994. Centers for Disease Control and Prevention. MMWR Morb Mortal Wkly Rep 45:1120-1123. https://www.cdc.gov/mmwr/preview/mmwrhtml/00045033.htm. February 18, 2020.

CDC. 1997b. Update: Blood lead levels - United States, 1991-1994. Centers for Disease Control and Prevention. MMWR Morb Mortal Wkly Rep 46(7):141-146. http://www.cdc.gov/epo/mmwr/preview/mmwrhtml/00048339.htm. February 18, 2020.

CDC. 1998. Lead poisoning associated with imported candy and powdered food coloring - California and Michigan. Centers for Disease Control and Prevention. MMWR Morb Mortal Wkly Rep 47(48):1041-1043. http://www.cdc.gov/mmwr/preview/mmwrhtml/00055939.htm. February 18, 2020.

CDC. 2002a. Managing elevated blood levels among young children. Recommendations from the Advisory Committee on Childhood Lead Poisoning. Centers for Disease Control and Prevention. http://www.cdc.gov/nceh/lead/casemanagement/managing EBLLs.pdf. July 18, 2018.

CDC. 2002b. Childhood lead poisoning associated with tamarind candy and fold remedies ---California, 1999-2000. Centers for Disease Control and Prevention. MMWR Morb Mortal Wkly Rep 51(31):684-686. https://www.cdc.gov/mmwr/preview/mmwrhtml/mm5131a3.htm. June 28, 2017.

CDC. 2004b. Lead poisoning associated with ayurvedic medications - five states, 2000-2003. MMWR Morb Mortal Wkly Rep 53(26):582-584.

https://www.cdc.gov/mmwr/preview/mmwrhtml/mm5326a3.htm.

CDC. 2005. Lead poisoning associated with use of litargirio-Rhode Island, 2003. Centers for Disease Control and Prevention. MMWR Morb Mortal Wkly Rep 54(09):227-229. https://www.cdc.gov/mmwr/preview/mmwrhtml/mm5409a5.htm. June 28, 2017.

CDC. 2009. Fourth national report on human exposure to environmental chemicals. Atlanta, GA: Centers for Disease Control and Prevention. http://www.cdc.gov/exposurereport. April 20, 2014.

CDC. 2011. State-based prevalence data of ADHD diagnosis. Atlanta, GA: Centers for Disease Control and Prevention. http://www.cdc.gov/ncbddd/adhd/prevalence.html. February 18, 2020.

CDC. 2012a. Infant lead poisoning associated with use of tiro, an eye cosmetic from Nigeria - Boston, Massachusetts, 2011. Centers for Disease Control and Prevention. MMWR Morb Mortal Wkly Rep 61(30):574-576.

https://www.cdc.gov/mmwr/preview/mmwrhtml/mm6130a3.htm?s_cid=mm6130a3_w. June $29,2017$.

CDC. 2012b. Lead in drinking water and human blood lead levels in the United States. Centers for Disease Control and Prevention. MMWR Morb Mortal Wkly Rep 61(Supplement: August 10, 2012):1-9. https://www.cdc.gov/mmwr/pdf/other/su6104.pdf. June 29,2017.

CDC. 2012c. Lead poisoning in pregnant women who used Ayurvedic medications from India-New York City, 2011-2012. Centers for Disease Control and Prevention. MMWR Morb Mortal Wkly Rep 61(33):641-646. 
https://www.cdc.gov/mmwr/preview/mmwrhtml/mm6133a1.htm?s_cid=mm6133a1_w. June 28, 2017.

CDC. 2012d. CDC response to Advisory Committee on Childhood Lead Poisoning Prevention.

Recommendations in "Low level lead exposure harms children: A renewed call for primary prevention.”. Atlanta, GA: Centers for Disease Control and Prevention. https://www.cdc.gov/nceh/lead/acclpp/cdc_response_lead_exposure_recs.pdf. December 8, 2017.

CDC. 2012e. Low level lead exposure harms children: A renewed call for primary prevention. Atlanta, GA: Centers for Disease Control and Prevention. https://www.cdc.gov/nceh/lead/acclpp/final_document_030712.pdf. August 25, 2017.

CDC. 2012f. Recommended actions based on blood lead level. Centers for Disease Control and Prevention. https://www.cdc.gov/nceh/lead/advisory/acclpp/actions-blls.htm. June 16, 2020.

CDC. 2013. Childhood lead exposure associated with the use of kajal, an eye cosmetic from Afghanistan - Albuquerque, New Mexico, 2013. Centers for Disease Control and Prevention. MMWR Morb Mortal Wkly Rep 62(46):917-919. https:/www.cdc.gov/mmwr/pdf/wk/mm6246.pdf. June 29,2017.

CDC. 2016. Standard surveillance definitions and classifications. Atlanta, GA: Centers for Disease Control and Prevention. https://www.cdc.gov/nceh/lead/data/case-definitions-classifications.htm. February 18, 2020.

CDC. 2018a. Fourth national report on human exposure to environmental chemicals. Updated tables, March 2018, Volumes 1 \& 2. Atlanta, GA: Centers for Disease Control and Prevention. https://www.cdc.gov/exposurereport/. May 01, 2018.

CDC. 2018b. Lead. Atlanta, GA: Centers for Disease Control and Prevention. https://www.cdc.gov/nceh/lead/default.htm. February 5, 2019.

CDC. 2018c. Lead hazards in some holiday toys and toy jewelry. Atlanta, GA: Centers for Disease Control and Prevention. https://www.cdc.gov/features/leadintoys/index.html. March 08, 2018.

CDC. 2019. Fourth report on human exposure to environmental chemicals, updated tables (January 2019). Atlanta, GA: Centers for Disease Control and Prevention. https://www.cdc.gov/exposurereport/. January 23, 2020.

Cecil KM, Brubaker CJ, Adler CM, et al. 2008. Decreased brain volume in adults with childhood lead exposure. PLoS Med 5(5):e112. http://doi.org/10.1371/journal.pmed.0050112.

Cecil KM, Dietrich KN, Altaye M, et al. 2011. Proton magnetic resonance spectroscopy in adults with childhood lead exposure. Environ Health Perspect 119(3):403-408. http://doi.org/10.1289/ehp.1002176.

Chamberlain AC. 1983. Effect of airborne lead on blood lead. Atmos Environ 17:677-692.

Chamberlain AC, Heard MJ, Little P, et al. 1978. Investigations into lead from motor vehicles. Harwell: Atomic Energy Research Establishment. United Kingdom Atomic Energy Authority. International Lead Zinc Research Organization. AERE-R9198.

Chamberlain AC, Heard MJ, Little P, et al. 1979. The dispersion of lead from motor exhausts. Phil Trans R Soc London A 290:577-589.

Chandramouli K, Steer CD, Ellis M, et al. 2009. Effects of early childhood lead exposure on academic performance and behaviour of school age children. Arch Dis Child 94(11):844-848. http://doi.org/10.1136/adc.2008.149955.

Chaney RL, Mielke HW, Sterrett SB. 1988. Speciation, mobility and bioavailability of soil lead. In: Davies BE, Wixson BG, eds. Lead in soil: Issues and guidelines. Northwood: Science Reviews Ltd., 105-130.

Chang SH, Cheng BH, Lee SL, et al. 2006. Low blood lead concentration in association with infertility in women. Environ Res 101(3):380-386. http://doi.org/10.1016/j.envres.2005.10.004.

Chao K, Hutton H, Levin A. 2015. Laboratory assessment of kidney disease. Glomerular filtration rate, urinalysis and proteinuria. In: Shorecki K, Chertow GM, Mardsen PA, et al., eds. Brenner and Rector's the kidney. Elsevier, 780-803.

Charney E, Sayre J, Coulter M. 1980. Increased lead absorption in inner city children: Where does the lead come from? Pediatrics 65(2):226-231. 
Chen PC, Pan IJ, Wang JD. 2006. Parental exposure to lead and small for gestational age births. Am J Ind Med 49(6):417-422. http://doi.org/10.1002/ajim.20313.

Chen A, Dietrich KN, Ware JH, et al. 2005. IQ and blood lead from 2 to 7 years of age: Are the effects in older children the residual of high blood lead concentrations in 2-year-olds? Environ Health Perspect 113(5):597-601. http://doi.org/10.1289/ehp.7625.

Chen A, Cai B, Dietrich KN, et al. 2007. Lead exposure, IQ, and behavior in urban 5- to 7-year-olds: Does lead affect behavior only by lowering IQ? Pediatrics 119(3):e650-e658. http://doi.org/10.1542/peds.2006-1973.

Chen HI, Chiu YW, Hsu YK, et al. 2010. The association of metallothionein-4 gene polymorphism and renal function in long-term lead-exposed workers. Biol Trace Elem Res 137:55-62.

Chen CC, Yen HW, Lo YH, et al. 2013. The association of prolonged QT interval on electrocardiography and chronic lead exposure. J Occup Environ Med 55(6):614-619. http://doi.org/10.1097/JOM.0b013e318291787a.

Chen Z, Myers R, Wei T, et al. 2014. Placental transfer and concentrations of cadmium, mercury, lead, and selenium in mothers, newborns, and young children. J Expo Sci Environ Epidemiol 24(5):537544. http://doi.org/10.1038/jes.2014.26.

Chen C, Wang N, Zhai H, et al. 2016. Associations of blood lead levels with reproductive hormone levels in men and postmenopausal women: Results from the SPECT-China Study. Sci Rep 6:37809. http://doi.org/10.1038/srep37809.

Chen C, Li Q, Nie X, et al. 2017. Association of lead exposure with cardiovascular risk factors and diseases in Chinese adults. Environ Sci Pollut Res Int 24(28):22275-22283. http://doi.org/10.1007/s11356-017-9884-6.

Chen Y, Xu X, Zeng Z, et al. 2019. Blood lead and cadmium levels associated with hematological and hepatic functions in patients from an e-waste-polluted area. Chemosphere 220:531-538. http://doi.org/10.1016/j.chemosphere.2018.12.129.

Cheng Y, Schwartz J, Vokonas PS, et al. 1998. Electrocardiographic conduction disturbances in association with low-level lead exposure (the Normative Aging Study). Am J Cardiol 82:594-599.

Cheng Y, Schwartz J, Sparrow D, et al. 2001. Bone lead and blood lead levels in relation to baseline blood pressure and the prospective development of hypertension: The Normative Aging Study. Am J Epidemiol 153(2):164-171. http://doi.org/10.1093/aje/153.2.164.

Chettle DR, Scott MC, Somervaille LJ. 1991. Lead in bone: Sampling and quantitation using K X-rays excited by 109Cd. Environ Health Perspect 91:49-55.

Chettle DR, Arnold ML, Aro ACA, et al. 2003. An agreed statement on calculating lead concentration and uncertainty in XRF in vivo bone lead analysis. Appl Radiat Isot 58:603-605.

Cheung MR. 2013. Blood lead concentration correlates with all cause, all cancer and lung cancer mortality in adults: A population based study. Asian Pac J Cancer Prev 14(5):3105-3108. http://doi.org/10.7314/apjcp.2013.14.5.3105.

Chia KS, Mutti A, Tan C, et al. 1994. Urinary N-acetyl-beta-D-glucosaminidase activity in workers exposed to inorganic lead. Occup Environ Med 51:125-129.

Chia KS, Jeyaratnam J, Tan C, et al. 1995a. Glomerular function of lead-exposed workers. Toxicol Lett 77(1-3):319-328.

Chia KS, Jeyaratnam J, Lee J, et al. 1995b. Lead-induced nephropathy: Relationship between various biological exposure indices and early markers of nephrotoxicity. Am J Ind Med 27(6):883-895.

Chia SE, Chia HP, Ong CN, et al. 1996. Cumulative concentrations of blood lead and postural stability. Occup Environ Med 53:264-268.

Chia SE, Zhou H, Tham MT, et al. 2005. Possible influence of "delta"-aminolevulinic acid dehydratase polymorphism and susceptibility to renal toxicity of lead: A study of a Vietnamese population. Environ Health Perspect 113(10):1313-1317. http://doi.org/10.1289/ehp.7904.

Chiang WF, Yang HJ, Lung SC, et al. 2008. A comparison of elementary schoolchildren's exposure to arsenic and lead. J Environ Sci Health C Environ Carcinog Ecotoxicol Rev 26(3):237-255. http://doi.org/10.1080/10590500802343958. 
Chien LC, Tsou MC, Hsi HC, et al. 2017. Soil ingestion rates for children under 3 years old in Taiwan. J Expo Sci Environ Epidemiol 27(1):33-40. http://doi.org/10.1038/jes.2015.61.

Chinde S, Kumari M, Devi KR, et al. 2014. Assessment of genotoxic effects of lead in occupationally exposed workers. Environ Sci Pollut Res Int 21(19):11469-11480. http://doi.org/10.1007/s11356014-3128-9.

Chiodo LM, Jacobson SW, Jacobson JL. 2004. Neurodevelopmental effects of postnatal lead exposure at very low levels. Neurotoxicol Teratol 26(3):359-371. http://doi.org/10.1016/j.ntt.2004.01.010.

Chisolm JJ. 1977. Lead poisoning. In: Rudolph AM, ed. Pediatrics. New York, NY: Prentice-Hall, Inc., 797-806.

Chisolm JJ. 1986. Editorial. Removal of lead paint from old housing: The need for a new approach. Am J Public Health 76(3):236-237.

Chisolm JJ, Mellits ED, Barrett MB. 1976. Interrelationships among blood lead concentration, quantitative daily ALA-U and urinary lead output following calcium EDTA. In: Nordberg GF, ed. Effects and dose relationships of toxic metals. Amsterdam, The Netherlands: Elsevier Scientific Publishing Company, 416-433.

Chiu Y, Liu T, Chuang H. 2013. The effects of lead exposure on the activities of $\delta$-aminolevulinic acid dehydratase with the modification of the relative genotypes. E3S Web Conf 1:26005. http://doi.org/10.1051/e3sconf/20130126005.

Cho SH, Richmond-Bryant J, Thornburg J, et al. 2011. A literature review of concentrations and size distributions of ambient airborne Pb-containing particulate matter. Atmos Environ 45(28):50055015. http://doi.org/10.1016/j.atmosenv.2011.05.009.

Choate LM, Ranville JF, Bunge AL, et al. 2006a. Dermally adhered soil: 1. Amount and particle-size distribution. Integr Environ Assess Manag 2(4):375-384.

Choate LM, Ranville JF, Bunge AL, et al. 2006b. Dermally adhered soil: 2. Reconstruction of drysieve particle-size distributions from wet-sieve data. Integr Environ Assess Manag 2(4):385-390.

Choi YH, Hu H, Mukherjee B, et al. 2012. Environmental cadmium and lead exposures and hearing loss in U.S. adults: The National Health and Nutrition Examination Survey, 1999 to 2004. Environ Health Perspect 120(11):1544-1550. http://doi.org/10.1289/ehp.1104863.

Choi WJ, Kwon HJ, Lim MH, et al. 2016. Blood lead, parental marital status and the risk of attentiondeficit/hyperactivity disorder in elementary school children: A longitudinal study. Psychiatry Res 236:42-46. http://doi.org/10.1016/j.psychres.2016.01.002.

Choie DD, Richter GW. 1972. Lead poisoning: Rapid formation of intranuclear inclusions. Science 177:1194-1195.

Chowdhury R, Sarnat SE, Darrow L, et al. 2014. Mortality among participants in a lead surveillance program. Environ Res 132:100-104. http://doi.org/10.1016/j.envres.2014.03.008.

Chrastny V, Komarek M, Hajek T. 2010. Lead contamination of an agricultural soil in the vicinity of a shooting range. Environ Monit Assess 162:37-46. http://doi.org/10.1007/s10661-009-0774-3.

Chu NF, Liou SH, Wu TN, et al. 1999. Reappraisal of the relation between blood lead concentration and blood pressure among the general population in Taiwan. Occup Environ Med 56:30-33.

Chuan MC, Shu GY, Liu JC. 1996. Solubility of heavy metals in a contaminated soil: Effects of redox potential and pH. Water Air Soil Pollut 90:543-556.

Chuang HY, Schwartz J, Tsai SY, et al. 2000. Vibration perception thresholds in workers with long term exposure to lead. Occup Environ Med 57(9):588-594.

Chuang HY, Kuo CH, Chiu YW, et al. 2007. A case-control study on the relationship of hearing function and blood concentrations of lead, manganese, arsenic, and selenium. Sci Total Environ 387(1-3):79-85. http://doi.org/10.1016/j.scitotenv.2007.07.032.

Chung HK, Chang YS, Ahn CW. 2015. Effects of blood lead levels on airflow limitations in Korean adults: Findings from the 5th KNHNES 2011. Environ Res 136:274-279.

http://doi.org/10.1016/j.envres.2014.10.027. 
Chwalba A, Maksym B, Dobrakowski M, et al. 2018. The effect of occupational chronic lead exposure on the complete blood count and the levels of selected hematopoietic cytokines. Toxicol Appl Pharmacol 355:174-179. http://doi.org/10.1016/j.taap.2018.05.034.

Clark C, Bornschein R, Succop P, et al. 1985. Condition and type of housing as an indicator of potential environmental lead exposure and pediatric blood lead levels. Environ Res 38(1):46-53.

Clark S, Menrath W, Chen M, et al. 2004. The influence of exterior dust and soil lead on interior dust lead levels in housing that had undergone lead-based paint hazard control. J Occup Environ Hyg 1(5):273-282. http://doi.org/10.1080/15459620490439036.

Clausing P, Brunekreef B, van Wijnen JH. 1987. A method for estimating soil ingestion by children. Int Arch Occup Environ Health 59(1):73-82.

Clayton CA, Pellizzari ED, Quackenboss JJ. 2002. National Human Exposure Assessment Survey: Analysis of exposure pathways and routes for arsenic and lead in EPA Region 5. J Expo Anal Environ Epidemiol 12:29-43.

Clayton CA, Pellizzari ED, Whitmore RW, et al. 1999. National Human Exposure Assessment Survey (NHEXAS): Distributions and associations of lead, arsenic and volatile organic compounds in EPA region 5. J Expo Anal Environ Epidemiol 9(5):381-392.

Clewell HJ, Andersen ME. 1985. Risk assessment extrapolations and physiological modeling. Toxicol Ind Health 1(4):111-131.

Coate D, Fowles R. 1989. Is there statistical evidence for a blood lead-blood pressure relationship? J Health Econ 8:173-184.

Cocco P, Dosemeci M, Heineman EF. 1998a. Brain cancer and occupational exposure to lead. J Occup Environ Med 40(11):937-942.

Cocco P, Ward MH, Dosemeci M. 1998b. Occupational risk factors for cancer of the gastric cardia. J Occup Environ Med 40(10):855-861.

Cocco P, Heineman EF, Dosemeci M. 1999a. Occupational risk factors for cancer of the central nervous system (CNS) among US women. Am J Ind Med 36:70-74.

Cocco P, Ward MH, Dosemeci M. 1999b. Risk of stomach cancer associated with 12 workplace hazards: Analysis of death certificates from 24 states of the United States with the aid of job exposure matrices. Occup Environ Med 56:781-787.

Cocco PL, Carta P, Belli S, et al. 1994. Mortality of Sardinian lead and zinc miners: 1960-1968. Occup Environ Med 51:674-682.

Cocco P, Hua F, Boffetta P, et al. 1997. Mortality of Italian lead smelter workers. Scand J Work Environ Health 23:15-23.

Cockcroft DW, Gault MH. 1976. Prediction of creatinine clearance from serum creatinine. Nephron 16:31-41.

Cohen AJ, Roe FJC. 1991. Review of lead toxicology relevant to the safety assessment of lead acetate as a hair colouring. Food Chem Toxicol 29(7):485-507.

Cohen N, Modai D, Golik A, et al. 1989. Increased concanavalin A-induced suppressor cell activity in humans with occupational lead exposure. Environ Res 48(1):1-6. http://doi.org/10.1016/S00139351(89)80079-9.

Comino E, Menegatti S, Fiorucci A, et al. 2011. Accumulation and translocation capacity of As, Co, $\mathrm{Cr}$ and $\mathrm{Pb}$ by forage plants. Agrochimica 55(EPFL-ARTICLE-171227):105-115.

Conterato GM, Bulcao RP, Sobieski R, et al. 2013. Blood thioredoxin reductase activity, oxidative stress and hematological parameters in painters and battery workers: Relationship with lead and cadmium levels in blood. J Appl Toxicol 33(2):142-150. http://doi.org/10.1002/jat.1731.

Cools A, Salle HJA, Verberk MM, et al. 1976. Short communications. Biochemical response of male volunteers ingesting inorganic lead for 49 days. Int Arch Occup Environ Health 38:129-139.

Coon S, Stark A, Peterson E, et al. 2006. Whole-body lifetime occupational lead exposure and risk of Parkinson's disease. Environ Health Perspect 114(12):1872-1876. http://doi.org/10.1289/ehp.9102.

Cooper WC. 1988. Deaths from chronic renal disease in US battery and lead production workers. Environ Health Perspect 78:61-63. 
Cooper GS, Umbach DM. 1996. Are vitamin D receptor polymorphisms associated with bone mineral density? A meta-analysis. J Bone Miner Res 11(12):1841-1849.

Cooper WC, Wong O, Kheifets L. 1985. Mortality among employees of lead battery plants and leadproducing plants, 1947-1980. Scand J Work Environ Health 11:331-345.

Corrin ML, Natusch DF. 1977. Physical and chemical characteristics of environmental lead. In: Lead in the environment. Washington, DC: National Science Foundation, 7-31.

Cory-Slechta DA. 1995. Relationships between lead-induced learning impairments and changes in dopaminergic, cholinergic, and glutamatergic neurotransmitter system functions. Annu Rev Pharmacol Toxicol 35:391-415.

Cory-Slechta DA. 2003. Lead-induced impairments in complex cognitive function: Offerings from experimental studies. Child Neuropsychol 9(1):54-75.

Cory-Slechta DA, Weiss B, Cox C. 1987. Mobilization and redistribution of lead over the course of calcium disodium ethylenediamine tetraacetate chelation therapy. J Pharmacol Exp Ther 243(3):804-813.

Costa de Almeida GR, Pereira Saraiva Mda C, Barbosa F, et al. 2007. Lead contents in the surface enamel of deciduous teeth sampled in vivo from children in uncontaminated and in leadcontaminated areas. Environ Res 104(3):337-345. http://doi.org/10.1016/j.envres.2007.03.007.

Costa de Almeida GR, Umbelino de Freitas C, Barbosa F, et al. 2009. Lead in saliva from lead-exposed and unexposed children. Sci Total Environ 407(5):1547-1550. http://doi.org/10.1016/j.scitotenv.2008.10.058.

Costa de Almeida GR, de Freitas Tavares CF, de Souza AM, et al. 2010. Whole blood, serum, and saliva lead concentrations in 6- to 8-year-old children. Sci Total Environ 408(7):1551-1556. http://doi.org/10.1016/j.scitotenv.2009.12.034.

Costa de Almeida GR, de Sousa Guerra C, de Angelo Souza Leite G, et al. 2011. Lead contents in the surface enamel of primary and permanent teeth, whole blood, serum, and saliva of 6- to 8-year-old children. Sci Total Environ 409(10):1799-1805. http://doi.org/10.1016/j.scitotenv.2011.01.004.

Coste J, Mandereau L, Pessione F, et al. 1991. Lead-exposed workmen and fertility: A cohort study on 354 subjects. Eur J Epidemiol 7(2):154-158.

Counter SA, Buchanan LH, Ortega F, et al. 2014. Lead levels in the breast milk of nursing Andean mothers living in a lead-contaminated environment. J Toxicol Environ Health A 77(17):993-1003. http://doi.org/10.1080/15287394.2014.897281.

Cox WM, Pesola GR. 2005. Buckshot ingestion. N Engl J Med 353(26):e23.

CPSC. 1996. CPSC finds lead poisoning hazard for young children in imported vinyl miniblinds. U.S. Consumer Product Safety Commission. https://groups.google.com/forum/\#!topic/misc.kids/Actbkpo1E_Q. July 12, 2017.

Craig JR, Rimstidt JD, Bonnaffon CA, et al. 1999. Surface water transport of lead at shooting range. Bull Environ Contam Toxicol 63:312-319.

Cramer K, Goyer RA, Jagenburg R, et al. 1974. Renal ultrastructure, renal function, and parameters of lead toxicity in workers with different periods of lead exposure. Br J Ind Med 31:113-127.

Crump KS, Van Landingham C, Bowers TS, et al. 2013. A statistical reevaluation of the data used in the Lanphear et al. (2005) pooled-analysis that related low levels of blood lead to intellectual deficits in children. Crit Rev Toxicol 43(9):785-799. http://doi.org/10.3109/10408444.2013.832726.

Cui S, Zhou Q, Chao L. 2007. Potential hyperaccumulation of Pb, Zn, Cu and Cd in endurant plants distributed in an old smeltery, northeast China. Environ Geol 51(6):1043-1048.

Cullen MR, Kayne RD, Robins JM. 1984. Endocrine and reproductive dysfunction in men associated with occupational inorganic lead intoxication. Arch Environ Health 39(6):431-440.

Dallaire R, Dewailly E, Ayotte P, et al. 2014. Growth in Inuit children exposed to polychlorinated biphenyls and lead during fetal development and childhood. Environ Res 134:17-23. http://doi.org/10.1016/j.envres.2014.06.023.

Dalpra L, Tibiletti MG, Nocera G, et al. 1983. SCE analysis in children exposed to lead emission from a smelting plant. Mutat Res 120:249-256. 
Danadevi K, Rozati R, Banu BS, et al. 2003. DNA damage in workers exposed to lead using comet assay. Toxicology 187(2):183-193.

Davidson A, Ryman J, Sutherland CA, et al. 2014. Lead. In: Ullmann's encyclopedia of industrial chemistry. Weinheim, Germany: Wiley-VCH Verlag GmbH \& Co. http://doi.org/10.1002/14356007.a15_193.pub3.

Davies JM. 1984a. Lung cancer mortality among workers making lead chromate and zinc chromate pigments at three English factories. Br J Ind Med 41:158-169.

Davies JM. 1984b. Long term mortality study of chromate pigment workers who suffered lead poisoning. Br J Ind Med 41:170-178.

Davis JM, Svendsgaard DJ. 1990. Nerve conduction velocity and lead: A critical review and metaanalysis. In: Advances in neurobehavioral toxicology: Applications in environmental and occupational health. Chelsea, MI: Lewis Publishers, 353-376.

Davis S, Mirick DK. 2006. Soil ingestion in children and adults in the same family. J Expo Sci Environ Epidemiol 16(1):63-75. http://doi.org/10.1038/sj.jea.7500438.

Davis A, Ruby MV, Bergstrom PD. 1994. Factors controlling lead bioavailability in the Butte mining district, Montana, USA. Environ Geochem Health 16(3/4; 3/4):147-157.

Davis AP, Shokouhian M, Ni S. 2001. Loading estimates of lead, copper, cadmium, and zinc in urban runoff from specific sources. Chemosphere 44:997-1009.

Davis MA, Gilbert-Diamond D, Karagas MR, et al. 2014. A dietary-wide association study (DWAS) of environmental metal exposure in US children and adults. PLoS ONE 9(9):e104768. http://doi.org/10.1371/journal.pone.0104768.

de Burbure C, Buchet JP, Leroyer A, et al. 2006. Renal and neurologic effects of cadmium, lead, mercury, and arsenic in children: Evidence of early effects and multiple interactions at environmental exposure levels. Environ Health Perspect 114(4):584-590. http://doi.org/10.1289/ehp.8202.

De Kort WL, Zwennis WC. 1988. Blood lead and blood pressure: Some implications for the situation in The Netherlands. Environ Health Perspect 78:67-70.

De Kort WLAM, Verschoor MA, Wibowo AAE, et al. 1987. Occupational exposure to lead and blood pressure: A study in 105 workers. Am J Ind Med 11:145-156.

de Restrepo HG, Sicard D, Torres MM. 2000. DNA damage and repair in cells of lead exposed people. Am J Ind Med 38(3):330-334.

Deierlein AL, Teitelbaum SL, Windham GC, et al. 2019. Lead exposure during childhood and subsequent anthropometry through adolescence in girls. Environ Int 122:310-315. http://doi.org/10.1016/j.envint.2018.11.031.

DeJonghe W, Adams F. 1986. Biogeochemical cycling of organic lead compounds. Adv Environ Sci Technol 17:561-594.

Den Hond E, Nawrot T, Staessen JA. 2001. Relationship between blood pressure and blood lead in NHANES III. J Hypertens 19(2):S57.

Den Hond E, Nawrot T, Staessen JA. 2002. The relationship between blood pressure and blood lead in NHANES III. J Hum Hypertens 16:563-568.

Den Hond E, Dhooge W, Bruckers L, et al. 2011. Internal exposure to pollutants and sexual maturation in Flemish adolescents. J Expo Sci Environ Epidemiol 21(3):224-233. http://doi.org/10.1038/jes.2010.2.

Denaix L, Semlali RM, Douay F. 2001. Dissolved and colloidal transport of Cd, Pb, and Zn in a silt loam soil affected by atmospheric industrial deposition. Environ Pollut 113:29-38.

Deng H, Ye Z, Wong M. 2004. Accumulation of lead, zinc, copper and cadmium by 12 wetland plant species thriving in metal-contaminated sites in China. Environ Pollut 132(1):29-40.

Denham M, Schell LM, Deane G, et al. 2005. Relationship of lead, mercury, mirex, dichlorodiphenyldichloroethylene, hexachlorobenzene, and polychlorinated biphenyls to timing of menarche among Akwesasne Mohawk girls. Pediatrics 115(2):e127-e134.

http://doi.org/10.1542/peds.2004-1161. 
Desilva PE. 1981. Determination of lead in plasma and studies on its relationship to lead in erythrocytes. Br J Ind Med 38:209-217.

Desrochers-Couture M, Oulhote Y, Arbuckle TE, et al. 2018. Prenatal, concurrent, and sex-specific associations between blood lead concentrations and IQ in preschool Canadian children. Environ Int 121(Pt 2):1235-1242. http://doi.org/10.1016/j.envint.2018.10.043.

Desrochers-Couture M, Courtemanche Y, Forget-Dubois N, et al. 2019. Association between early lead exposure and externalizing behaviors in adolescence: A developmental cascade. Environ Res 178:108679. http://doi.org/10.1016/j.envres.2019.108679.

Devoz PP, Gomes WR, De Araujo ML, et al. 2017. Lead (Pb) exposure induces disturbances in epigenetic status in workers exposed to this metal. J Toxicol Environ Health A 80(19-21):10981105. http://doi.org/10.1080/15287394.2017.1357364.

Dewailly E, Ayotte P, Bruneau S, et al. 2001. Exposure of the Inuit population of Nunavik (Arctic Quebec) to lead and mercury. Arch Environ Health 56(4):350-357.

Di Lorenzo L, Silvestroni A, Martino MG, et al. 2006. Evaluation of peripheral blood neutrophil leucocytes in lead-exposed workers. Int Arch Occup Environ Health 79(6):491-498. http://doi.org/10.1007/s00420-005-0073-4.

Diamond GL. 1988. Biological monitoring of urine for exposure to toxic metals. In: Clarkson TW, Friberg L, Nordberg GF, et al., eds. Biological monitoring of toxic metals. New York, NY: Plenum Publishing Corporation, 515-529.

Diamond GL. 2005. Risk assessment of nephrotoxic metals. In: Tarloff J, Lash L, eds. The toxicology of the kidney. London, England: CRC Press, 1099-1132.

Diamond GL, Thayer WC, Brown JS, et al. 2019. Estimates of urinary blood lead clearance and its relationship to glomerular filtration rate based on a large population survey. J Toxicol Environ Health A 82(5):379-382. http://doi.org/10.1080/15287394.2019.1603280.

Dietrich KN, Berger OG, Succop PA. 1993b. Lead exposure and the motor developmental status of urban six-year-old children in the Cincinnati prospective study. Pediatrics 91(2):301-307.

Dietrich KN, Krafft KM, Bier M, et al. 1986. Early effects of fetal lead exposure: Neurobehavioral findings at 6 months. Int J Biosoc Res 8(2):151-168.

Dietrich KN, Krafft KM, Shukla R, et al. 1987. The neurobehavioral effects of early lead exposure. In: Schroeder SR, ed. Toxic substances and mental retardation: Neurobehavioral toxicology and teratology. Washington, DC: American Association on Mental Deficiency, 71-95.

Dietrich KN, Krafft KM, Bier M, et al. 1989. Neurobehavioral effects of foetal lead exposure: The first year of life. In: Lead exposure and child development. Dordrecht: Springer, 320-331. http://doi.org/10.1007/978-94-009-0847-5_19.

Dietrich KN, Succop PA, Berger OG, et al. 1991. Lead exposure and the cognitive development of urban preschool children: The Cincinnati Lead Study cohort at age 4 years. Neurotoxicol Teratol 13(2):203-211.

Dietrich KN, Succop PA, Berger OG, et al. 1992. Lead exposure and the central auditory processing abilities and cognitive development of urban children: The Cincinnati Lead Study cohort at age 5 years. Neurotoxicol Teratol 14(1):51-56.

Dietrich KN, Berger OG, Succop PA, et al. 1993a. The developmental consequences of low to moderate prenatal and postnatal lead exposure: Intellectual attainment in the Cincinnati Lead Study Cohort following school entry. Neurotoxicol Teratol 15(1):37-44.

Dietrich KN, Douglas RM, Succop PA, et al. 2001. Early exposure to lead and juvenile delinquency. Neurotoxicol Teratol 23(6):511-518.

Ding N, Wang X, Weisskopf MG, et al. 2016. Lead-related genetic loci, cumulative lead exposure and incident coronary heart disease: The normative aging study. PLoS ONE 11(9):e0161472. http://doi.org/10.1371/journal.pone.0161472.

Ding N, Wang X, Tucker KL, et al. 2019. Dietary patterns, bone lead and incident coronary heart disease among middle-aged to elderly men. Environ Res 168:222-229. http://doi.org/10.1016/j.envres.2018.09.035. 
Dingwall-Fordyce I, Lane RE. 1963. A follow-up study of lead workers. Br J Ind Med 20:313-315.

Disha, Sharma S, Goyal M, et al. 2019. Association of raised blood lead levels in pregnant women with preeclampsia: A study at tertiary centre. Taiwan J Obstet Gynecol 58(1):60-63. http://doi.org/10.1016/j.tjog.2018.11.011.

Dixon SL, Gaitens JM, Jacobs DE, et al. 2009. Exposure of U.S. children to residential dust lead, 19992004: II. The contribution of lead-contaminated dust to children's blood lead levels. Environ Health Perspect 117(3):468-474. http://doi.org/10.1289/ehp.11918.

do Nascimento SN, Charao MF, Moro AM, et al. 2014. Evaluation of toxic metals and essential elements in children with learning disabilities from a rural area of southern Brazil. Int J Environ Res Public Health 11(10):10806-10823. http://doi.org/10.3390/ijerph111010806.

Dobrakowski M, Boron M, Czuba ZP, et al. 2016. Blood morphology and the levels of selected cytokines related to hematopoiesis in occupational short-term exposure to lead. Toxicol Appl Pharmacol 305:111-117. http://doi.org/10.1016/j.taap.2016.06.015.

Dobrakowski M, Pawlas N, Kasperczyk A, et al. 2017. Oxidative DNA damage and oxidative stress in lead-exposed workers. Hum Exp Toxicol 36(7):744-754. http://doi.org/10.1177/0960327116665674.

DOE. 2018a. Table 3: Protective Action Criteria (PAC) Rev. 29a based on applicable 60-minute AEGLs, ERPGs, or TEELs. The chemicals are listed by CASRN. June 2018. Oak Ridge, TN: U.S. Department of Energy. https://sp.eota.energy.gov/pac/docs/Revision_29A_Table3.pdf. July 26, 2018.

DOE. 2018b. Protective Action Criteria (PAC) with AEGLs, ERPGs, \& TEELs: Rev. 29A, June 2018. Oak Ridge, TN: U.S. Department of Energy. https://sp.eota.energy.gov/pac/. July 26, 2018.

Dong Z, Yan K, Liu Y, et al. 2016. A meta-analysis to correlate lead bioavailability and bioaccessibility and predict lead bioavailability. Environ Int 92-93:139-145. http://doi.org/10.1016/j.envint.2016.04.009.

Dongre NN, Suryakar AN, Patil AJ, et al. 2013. Biochemical effects of lead exposure on battery manufacture workers with reference to blood pressure, calcium metabolism and bone mineral density. Indian J Clin Biochem 28(1):65-70. http://doi.org/10.1007/s12291-012-0241-8.

Dorsey CD, Lee B, Bolla KI, et al. 2006. Comparison of patella lead with blood lead and tibia lead and their associations with neurobehavioral test scores. J Occup Environ Med 48(5):489-496.

Doyle JR, Blais JM, Holmes RD, et al. 2012. A soil ingestion pilot study of a population following a traditional lifestyle typical of rural or wilderness areas. Sci Total Environ 424:110-120. http://doi.org/10.1016/j.scitotenv.2012.02.043.

Drasch GA, Bohm J, Baur C. 1987. Lead in human bones. Investigations on an occupationally nonexposed population in southern Bavaria (F.R.G.). I. Adults. Sci Total Environ 64:303-315.

Drasch G, Wanghofer E, Roider G. 1997. Are blood, urine, hair, and muscle valid biomonitors for the internal burden of men with the heavy metals mercury, lead and cadmium: An investigation on 150 deceased. Trace Elem Electrolytes 14(3):116-123.

Drexler JW, Brattin WJ. 2007. An in vitro procedure for estimation of lead relative bioavailability: With validation. Hum Ecol Risk Assess 13(2):383-401. http://doi.org/10.1080/10807030701226350.

Dundar B, Öktem F, Arslan MK, et al. 2006. The effect of long-term low-dose lead exposure on thyroid function in adolescents. Environ Res 101(1):140-145. http://doi.org/10.1016/j.envres.2005.10.002.

DuVal GE, Fowler BA. 1989. Preliminary purification and characterization studies of a low molecular weight, high affinity cytosolic lead-binding protein in rat brain. Biochem Biophys Res Commun 159:177-184.

Duydu Y, Dur A, Süzen HS. 2005. Evaluation of increased proportion of cells with unusually high sister chromatid exchange counts as a cytogenetic biomarker for lead exposure. Biol Trace Elem Res 104(2):121-129. http://doi.org/10.1385/BTER:104:2:121.

Duydu Y, Süzen H, Aydin A, et al. 2001. Correlation between lead exposure indicators and sister chromatid exchange (SCE) frequencies in lymphocytes from inorganic lead exposed workers. Arch Environ Contam Toxicol 41(2):241-246. 
Dye BA, Hirsch R, Brody DJ. 2002. The relationship between blood lead levels and periodontal bone loss in the United States, 1988-1994. Environ Health Perspect 110(10):997-1002.

Eaton DL, Stacey NH, Wong KL, et al. 1980. Dose response effects of various metal ions on rat liver metallothionein, glutathione, heme oxygenase, and cytochrome P-450. Toxicol Appl Pharmacol 55:393-402.

Eckel WP, Jacob TA. 1988. Ambient levels of 24 dissolved metals in U.S. surface and ground waters. Prepr Pap Natl Meet Am Chem Soc Div Environ Chem 28:371-372.

Egeghy PP, Quackenboss JJ, Catlin S, et al. 2005. Determinants of temporal variability in NHEXASMaryland environmental concentrations, exposures, and biomarkers. J Expo Anal Environ Epidemiol 15(5):388-397. http://doi.org/10.1038/sj.jea.7500415.

Ehrlich R, Robins T, Jordaan E, et al. 1998. Lead absorption and renal dysfunction in a South African battery factory. Occup Environ Med 55:453-460.

Eisenreich SJ, Looney BB, Thornton JD. 1981. Airborne organic contaminants in the Great Lakes ecosystem. Environ Sci Technol 15(1):30-38.

Eisler R. 1988. Lead hazards to fish, wildlife, and invertebrates: A synoptic review. Fish and Wildlife Service. U.S. Department of the Interior. Biological Report 85(1.14).

Elbaz-Poulichet F, Holliger P, Huang WW, et al. 1984. Lead cycling in estuaries, illustrated by the Gironde estuary, France. Nature 308:409-414.

Elias SM, Hashim Z, Marjan ZM, et al. 2007. Relationship between blood lead concentration and nutritional status among Malay primary school children in Kuala Lumpur, Malaysia. Asia Pac J Public Health 19(3):29-37. http://doi.org/10.1177/101053950701900306.

Elmarsafawy SF, Jain NB, Schwartz J, et al. 2006. Dietary calcium as a potential modifier of the relationship of lead burden to blood pressure. Epidemiology 17(5):531-537.

Elwood PC, Davey-Smith G, Oldham PD, et al. 1988a. Two Welsh surveys of blood lead and blood pressure. Environ Health Perspect 78:119-121.

Elwood PC, Yarnell JWG, Oldham PD, et al. 1988b. Blood pressure and blood lead in surveys in Wales. Am J Epidemiol 127(5):942-945.

Emory E, Ansari Z, Pattillo R, et al. 2003. Maternal blood lead effects on infant intelligence at age 7 months. Am J Obstet Gynecol 188(4):S26-S32.

Englyst V, Lundstrom NG, Gerhardsson L, et al. 2001. Lung cancer risks among lead smelter workers also exposed to arsenic. Sci Total Environ 273:77-82.

Environment and Climate Change Canada. 2016. Canadian environmental sustainability indicators: Releases of harmful substances to the environment. Minister of Environment and Climate Change. http://www.ec.gc.ca/indicateurs-indicators/3C4C1124-63E6-40BB-941A-

EB87E1A23387/Releases\%20of\%20Harmful\%20Substances_EN.pdf. June 26, 2017.

EPA. 1977. Standards of performance for secondary lead smelters. U.S. Environmental Protection Agency. Code of Federal Regulations. 40 CFR 60; Subpart L.

EPA. 1979. Lead. Water-related environmental fate of 129 priority pollutants. U.S. Environmental Protection Agency. 13-11 - 13-19. EPA440479029a.

EPA. 1982a. Standards of performance for lead-acid battery manufacturing plants. U.S. Environmental Protection Agency. Code of Federal Regulations. 40 CFR 60; Subpart KK.

EPA. 1982b. Test methods. U.S. Environmental Protection Agency. Code of Federal Regulations. 40 CFR 80.3.

EPA. 1982c. An exposure and risk assessment for lead. Washington, DC: U.S. Environmental Protection Agency. EPA440485010. PB85220606.

EPA. 1985a. Controls applicable to gasoline refiners and importers. U.S. Environmental Protection Agency. Code of Federal Regulations. 40 CFR 80.20.

EPA. 1985b. Determination of reportable quantities. U.S. Environmental Protection Agency. Code of Federal Regulations. 40 CFR 117.3.

EPA. 1985c. Lead exposures in the human environment. Research Triangle Park, NC: U.S. Environmental Protection Agency. EPA600D86185. PB86241007. 
EPA. 1985d. Regulation of fuels and fuel additives; gasoline lead content. Fed Regist 50(45):93869399.

EPA. 1986a. Air quality criteria for lead. Research Triangle Park, NC: U.S. Environmental Protection Agency. EPA600883028F.

EPA. 1986b. Superfund record of decision (EPA Region 5): Forest Waste Disposal Site, Genesee County, Michigan, June 1986. Washington, DC: U.S. Environmental Protection Agency. EPARODR0586034. PB87189890.

EPA. 1988. Specific toxic chemical listings. U.S. Environmental Protection Agency. Code of Federal Regulations. 40 CFR 372.65.

EPA. 1989a. Exposure factors handbook. Washington, DC: U.S. Environmental Protection Agency. EPA600889043.

EPA. 1989b. National primary drinking water regulations. U.S. Environmental Protection Agency. Code of Federal Regulations. 40 CFR 141,142.

EPA. 1991. Reference air concentrations. Health based limits for exclusion of waste-derived residues. U.S. Environmental Protection Agency. Code of Federal Regulations. 40 CFR 266, Appendices IV and VII.

EPA. 1994a. Guidance manual for the integrated exposure uptake biokinetic model for lead in children. U.S. Environmental Protection Agency. EPA540R93081. PB93963510.

EPA. 1994b. Technical support document: Parameters and equations used in integrated exposure uptake biokinetic model for lead in children (v0.99d). Washington, DC: U.S. Environmental Protection Agency. EPA540R94040. PB94963505.

EPA. 1994c. Validation strategy for the integrated exposure uptake biokinetic model for lead in children. Washington, DC: U.S. Environmental Protection Agency. EPA540R94039. PB94963504.

EPA. 1994d. Methods for the determination of metals in environmental samples. Supplement 1. Cincinnati, OH: U.S. Environmental Protection Agency. EPA600R94111. https://nepis.epa.gov/Exe/ZyPURL.cgi?Dockey=300036HL.txt. March 30, 2017.

EPA. 1994e. Revised interim soil lead guidance for CERCLA sites and RCRA corrective action facilities. Washington, DC: U.S. Environmental Protection Agency. OSWER Directive \#9355.412.

EPA. 1994f. Method 200.8, revision 5.4: Determination of trace elements in waters and wastes by inductively coupled plasma-mass spectrometry. Cincinnati, OH: U.S. Environmental Protection Agency. https://www.epa.gov/sites/production/files/2015-08/documents/method_200-8_rev_54_1994.pdf. December 8, 2017.

EPA. 1995a. Guidance for assessing chemical contaminant data for use in fish advisories. Volume 1: Fish sampling and analysis. U.S. Environmental Protection Agency. EPA823R95007.

EPA. 1995b. Report on the national survey of lead based paint in housing - base report. U.S. Environmental Protection Agency.

EPA. 1996a. Prohibition on gasoline containing lead or lead additives for highway use. U.S. Environmental Protection Agency. Fed Regist 61(23):3832.

EPA. 1996b. National air quality and emissions trends report 1995. U.S. Environmental Protection Agency. EPA454R96005.

EPA. 1996c. Urban soil lead abatement demonstration project. Volume I: EPA integrated report. U.S. Environmental Protection Agency. EPA600P93001aF.

EPA. 1997a. Implementation of the mercury-containing and rechargeable battery management act. U.S. Environmental Protection Agency. https://nepis.epa.gov/Exe/ZyPURL.cgi?Dockey=10000MXZ.txt. February 18, 2020.

EPA. 1997b. Methods for the determination of chemical substances in marine and estuarine environmental matrices- 2nd edition. Washington, DC: U.S. Environmental Protection Agency. EPA600R97072. https://cfpub.epa.gov/si/si_public_record_report.cfm?dirEntryId=309412. March 30, 2017. 


\section{REFERENCES}

EPA. 1998. Clarification to the 1994 revised interim soil lead guidance for CERCLA sites and RCRA corrective action facilities. U.S. Environmental Protection Agency. EPA540F98030. https://www.epa.gov/superfund/lead-superfund-sites-guidance. August 25, 2017.

EPA. 1999. Determination of metals in ambient particulate matter using X-ray fluorescence (XRF) spectroscopy. Compendium Method 10-3.3. Cincinnati, OH: U.S. Environmental Protection Agency. EPA625R96010a. https://www3.epa.gov/ttnamti1/files/ambient/inorganic/mthd-3-3.pdf. March 30, 2017.

EPA. 2001. Guidance for reporting releases and other waste management quantities of toxic chemicals: Lead and lead compounds. Washington, DC: U.S. Environmental Protection Agency. https://ofmpub.epa.gov/apex/guideme_ext/guideme_ext/guideme/file/lead\%20and\%20lead\%20comp ounds.pdf. February 25, 2020.

EPA. 2002a. Reference manual for the Integrated Exposure Uptake Biokinetic Model for lead in children (IEUBK) Windows 32 bit version. U.S. Environmental Protection Agency. EPA9285.7-44 https://www.epa.gov/superfund/lead-superfund-sites-software-and-users-manuals\#technical. February 18, 2020.

EPA. 2002b. The "battery act". Enforcement alert. U.S. Environmental Protection Agency. EPA300N02002.

EPA. 2002c. National primary drinking water regulations. Washington, DC: U.S. Environmental Protection Agency. EPA816F02013. http://www.epa.gov/safewater/mcl.html. February 18, 2020.

EPA. 2003. Method 200.5: Determination of trace elements in drinking water by axially viewed inductively coupled plasma- atomic emission spectrometry. Cincinnati, OH: U.S. Environmental Protection Agency. EPA600R06115. https://www.epa.gov/sites/production/files/201508/documents/method_200-5_rev_4-2_2003.pdf. March 30, 2017.

EPA. 2004. Lead and copper rule: A quick reference guide. U.S. Environmental Protection Agency. EPA816F04009. https://nepis.epa.gov/Exe/ZyPURL.cgi?Dockey=30006646.txt. February 5, 2019.

EPA. 2005a. Toxic chemical release inventory reporting forms and instructions: Revised 2004 version. Section 313 of the Emergency Planning and Community Right-to-Know Act (Title III of the Superfund Amendments and Reauthorization Act of 1986). U.S. Environmental Protection Agency. EPA260B05001.

EPA. 2005b. STORET database access. U.S. Environmental Protection Agency. http://www.epa.gov/storet/dbtop.html. May 20, 2005.

EPA. 2006. Air quality criteria for lead. Volume 1 of II. U.S. Environmental Protection Agency. EPA600R5144aF. http://ofmpub.epa.gov/eims/eimscomm.getfile?p_download_id=459555. June 27, 2017.

EPA. 2007a. National primary drinking water regulations for lead and copper: Short-term regulatory revisions and clarifications. U.S. Environmental Protection Agency. Fed Regist 72(195):5778257820. https://www.gpo.gov/fdsys/pkg/FR-2007-10-10/pdf/E7-19432.pdf. June 28, 2017.

EPA. 2007b. The national listing of fish advisories. Advisory report output. U.S. Environmental Protection Agency.

EPA. 2009. National primary drinking water regulations. Washington, DC: U.S. Environmental Protection Agency. EPA816F090004. https://www.epa.gov/sites/production/files/201606/documents/npwdr_complete_table.pdf. February 28, 2017.

EPA. 2010. Lead and copper rule monitoring and reporting guidance for public water systems. U.S. Environmental Protection Agency. EPA816R10004. https://nepis.epa.gov/Exe/ZyPURL.cgi?Dockey=P100DP2P.txt. February 5, 2019.

EPA. 2013. Providing safe drinking water in America: 2013 National Public Water Systems Compliance Report. U.S. Environmental Protection Agency. https://www.epa.gov/sites/production/files/201506/documents/sdwacom2013.pdf. February 18, 2020.

EPA. 2014a. Development and evaluation of the all ages lead model (AALM). U.S. Environmental Protection Agency. 
EPA. 2014b. Approach for estimating exposures and incremental health effects from lead due to renovation, repair, and painting activities in public and commercial buildings. U.S. Environmental Protection Agency. https://www.epa.gov/sites/production/files/201408/documents/approachdocument_0.pdf. April 03, 2017.

EPA. 2014c. Integrated science assessment for lead. Contains errata sheet created 5/12/2014. U.S. Environmental Protection Agency. EPA600R10075F. https://cfpub.epa.gov/ncea/isa/recordisplay.cfm?deid=255721. October 21, 2016.

EPA. 2014d. Lead and lead compounds. Chemical data access tool (CDAT). [2012 Chemical Data Reporting (CDR)]. U.S. Environmental Protection Agency. https://java.epa.gov/oppt_chemical_search/. June 26, 2017.

EPA. 2014e. Identification and consideration of errors in Lanphear et al. (2005), "Low-level environmental lead exposure and children's intellectual function: An international pooled analysis". Memorandum to Integrated Science Assessment for lead docket (EPA-HQ-ORD-2011-0051). Research Triangle Park, NC: U.S. Environmental Protection Agency. https://ofmpub.epa.gov/eims/eimscomm.getfile?p_download_id=518543. February 5, 2019.

EPA. 2015. Report on the environment. Lead emissions. U.S. Environmental Protection Agency. https://cfpub.epa.gov/roe/. June 27, 2017.

EPA. 2016a. Optimal corrosion control treatment evaluation technical recommendations for primacy agencies and public water systems. U.S. Environmental Protection Agency. EPA816B16003. https://www.epa.gov/dwreginfo/optimal-corrosion-control-treatment-evaluation-technicalrecommendations. February 18, 2020.

EPA. 2016b. Memorandum. Updated scientific considerations for lead in soil cleanups. U.S. Environmental Protection Agency. https://assets.documentcloud.org/documents/3525442/EPAMemo-Updated-Scientific-Considerations-for.pdf. June 28, 2017.

EPA. 2016c. 2014 National emissions inventory, version 1. Technical support document. Research Triangle Park, NC: U.S. Environmental Protection Agency. https://www.epa.gov/sites/production/files/2016-12/documents/nei2014v1_tsd.pdf. February 18, 2020.

EPA. 2016d. Updated scientific considerations for lead in soil cleanups. U.S. Environmental Protection Agency, Office of Land and Emergency Management. https://quicksilver.epa.gov/work/08/1884174.pdf. August 25, 2017.

EPA. 2017a. Lead air releases trend in the 2015 TRI national analysis. U.S. Environmental Protection Agency.

EPA. 2017b. Supporting data files for the 2015 TRI national analysis: 2015 Toxics Release Inventory national analysis: Releases of chemicals: Lead air releases trend. U.S. Environmental Protection Agency. https://www.epa.gov/trinationalanalysis/supporting-data-files-2015-tri-national-analysis.

EPA. 2017c. TRI basic data files: Calendar years 1987 - 2015 [US data from 2015]. U.S. Environmental Protection Agency.

EPA. 2017d. Transmittal of update to the adult lead methodology's default baseline blood lead concentration and geometric standard deviation parameters. OLEM Directive 9285-56. Washington, DC: U.S. Environmental Protection Agency. https://semspub.epa.gov/work/HQ/196766.pdf. August 25, 2017.

EPA. 2017e. Review of Michigan's lead and copper rule. Review of the Michigan Department of Environmental Quality Drinking Water Program 2016. U.S. Environmental Protection Agency. 1629.

EPA. 2018a. Lead trends: National trends in lead levels. U.S. Environmental Protection Agency. https://www.epa.gov/air-trends/lead-trends. February 18, 2020.

EPA. 2018b. Air quality system: Lead. U.S. Environmental Protection Agency. https://www.epa.gov/aqs. February 18, 2020. 
EPA. 2018c. 2018 Edition of the drinking water standards and health advisories tables. Washington, DC: U.S. Environmental Protection Agency. EPA822F18001. https://www.epa.gov/sites/production/files/2018-03/documents/dwtable2018.pdf. May 4, 2017.

EPA. 2018d. Acute Exposure Guideline Levels (AEGLs) values. U.S. Environmental Protection Agency. https://www.epa.gov/sites/production/files/201808/documents/compiled_aegls_update_27jul2018.pdf. June 5, 2019.

EPA. 2019a. National primary drinking water regulations: Proposed lead and copper rule revisions. U.S. Environmental Protection Agency. Fed Regist 84(219):61684-61774. https://www.regulations.gov/contentStreamer?documentId=EPA-HQ-OW-2017-03000001\&contentType=pdf. January 14, 2020.

EPA. 2019b. National primary and secondary ambient air quality standards for lead. U.S. Environmental Protection Agency. Code of Federal Regulations. 40 CFR 50.16. https://www.govinfo.gov/content/pkg/CFR-2019-title40-vol2/pdf/CFR-2019-title40-vol2-sec5016.pdf. February 18, 2020.

EPA. 2019c. Review of the dust-lead hazard standards and the definition of lead based paints. U. S. Environmental Protection Agency. Fed Regist 84(131):32632-32648. https://www.regulations.gov/document?D=EPA-HQ-OPPT-2018-0166-0360. October 25, 2019.

EPA. 2020a. Regulation of fuels and fuel additives. U.S. Environmental Protection Agency. Code of Federal Regulations. 40 CFR 80.

EPA. 2020b. Standards for the management of specific hazardous wastes and specific types of hazardous waste management facilities. U.S. Environmental Protection Agency. Code of Federal Regulations. 40 CFR 266.

Erenberg G, Rinsler SS, Fish BG. 1974. Lead neuropathy and sickle cell disease. Pediatrics 54:438-441.

Erfurth EM, Gerhardsson L, Nilsson A, et al. 2001. Effects of lead on the endocrine system in lead smelter workers. Arch Environ Health 56(5):449-455.

Ergurhan-Ilhan I, Cadir B, Koyuncu-Arslan M, et al. 2008. Level of oxidative stress and damage in erythrocytes in apprentices indirectly exposed to lead. Pediatr Int 50(1):45-50. http://doi.org/10.1111/j.1442-200X.2007.02442.x.

Ericson JE. 2001. Enamel lead biomarker for prenatal exposure assessment. Environ Res 87(3):136140. http://doi.org/10.1006/enrs.2001.4283.

Erie JC, Good JA, Butz JA. 2009. Excess lead in the neural retina in age-related macular degeneration. Am J Ophthalmol 148(6):890-894. http://doi.org/10.1016/j.ajo.2009.07.001.

Erkkila J, Armstrong R, Riihimaki V, et al. 1992. In vivo measurements of lead in bone at four anatomical sites: Long term occupational and consequent endogenous exposure. Br J Ind Med 49:631-644.

Ernhart CB, Morrow-Tlucak M, Wolf AW, et al. 1989. Low level lead exposure in the prenatal and early preschool periods: Intelligence prior to school entry. Neurotoxicol Teratol 11(2):161-170.

Esteban M, Castano A. 2009. Non-invasive matrices in human biomonitoring: A review. Environ Int 35:438-449.

Esteban E, Rubin CH, Jones RL, et al. 1999. Hair and blood as substrates for screening children for lead poisoning. Arch Environ Health 54(6):436-440.

Esteban-Vasallo MD, Aragones N, Pollan M, et al. 2012. Mercury, cadmium, and lead levels in human placenta: A systematic review. Environ Health Perspect 120(10):1369-1377. http://doi.org/10.1289/ehp.1204952.

Ethier AA, Muckle G, Bastien C, et al. 2012. Effects of environmental contaminant exposure on visual brain development: A prospective electrophysiological study in school-aged children.

Neurotoxicology 33(5):1075-1085. http://doi.org/10.1016/j.neuro.2012.05.010.

Ettinger AS, Tellez-Rojo MM, Amarasiriwardena C, et al. 2004. Levels of lead in breast milk and their relation to maternal blood and bone lead levels at one month postpartum. Environ Health Perspect 112:926-931 
Ettinger AS, Tellez-Rojo MM, Amarasiriwardena C, et al. 2006. Influence of maternal bone lead burden and calcium intake on levels of lead in breast milk over the course of lactation. Am J Epidemiol 163(1):48-56.

Ettinger AS, Lamadrid-Figueroa H, Tellez-Rojo MM, et al. 2009. Effect of calcium supplementation on blood lead levels in pregnancy: A randomized placebo-controlled trial. Environ Health Perspect 117(1):26-31.

Ettinger AS, Roy A, Amarasiriwardena CJ, et al. 2014. Maternal blood, plasma, and breast milk lead: Lactational transfer and contribution to infant exposure. Environ Health Perspect 122(1):87-92. http://doi.org/10.1289/ehp.1307187.

Eum KD, Nie LH, Schwartz J, et al. 2011. Prospective cohort study of lead exposure and electrocardiographic conduction disturbances in the Department of Veterans Affairs Normative Aging Study. Environ Health Perspect 119(7):490-494. http://doi.org/10.1289/ehp.1003279.

Eum KD, Wang FT, Schwartz J, et al. 2013. Modifying roles of glutathione S-transferase polymorphisms on the association between cumulative lead exposure and cognitive function. Neurotoxicology 39:65-71. http://doi.org/10.1016/j.neuro.2013.08.002.

Eum KD, Weisskopf MG, Nie LH, et al. 2014. Cumulative lead exposure and age at menopause in the Nurses' Health Study cohort. Environ Health Perspect 122(3):229-234. http://doi.org/10.1289/ehp.1206399.

Eum KD, Seals RM, Taylor KM, et al. 2015. Modification of the association between lead exposure and amyotrophic lateral sclerosis by iron and oxidative stress related gene polymorphisms. Amyotroph Lateral Scler Frontotemporal Degener 16(1-2):72-79. http://doi.org/10.3109/21678421.2014.964259.

Evans RD, Rigler FH. 1985. Long distance transport of anthropogenic lead as measured by lake sediments. Water Air Soil Pollut 24:141-151.

Evans M, Elinder CG. 2011. Chronic renal failure from lead: Myth or evidence-based fact? Kidney Int 79(3):272-279. http://doi.org/10.1038/ki.2010.394.

Evens A, Hryhorczuk D, Lanphear BP, et al. 2015. The impact of low-level lead toxicity on school performance among children in the Chicago public schools: A population-based retrospective cohort study. Environ Health 14:21. http://doi.org/10.1186/s12940-015-0008-9.

Ewers U, Stiller-Winkler R, Idel H. 1982. Serum immunoglobulin, complement C3, and salivary IgA levels in lead workers. Environ Res 29(2):351-357. http://doi.org/10.1016/0013-9351(82)90036-6.

Factor-Litvak P, Graziano JH, Kline JK, et al. 1991. A prospective study of birthweight and length of gestation in a population surrounding a lead smelter in Kosovo, Yugoslavia. Int J Epidemiol 20:722728.

Factor-Litvak P, Kline JK, Popovac D, et al. 1996. Blood lead and blood pressure in young children. Epidemiology 7(6):633-637. http://doi.org/10.1097/00001648-199611000-00012.

Factor-Litvak P, Slavkovich V, Liu X, et al. 1998. Hyperproduction of erythropoietin in nonanemic lead-exposed children. Environ Health Perspect 106(6):361-364.

Factor-Litvak P, Wasserman G, Kline JK, et al. 1999. The Yugoslavia prospective study of environmental lead exposure. Environ Health Perspect 107(1):9-15.

Fadrowski JJ, Navas-Acien A, Tellez-Plaza M, et al. 2010. Blood lead level and kidney function in US adolescents: The Third National Health and Nutrition Examination Survey. Arch Intern Med 170(1):75-82. http://doi.org/10.1001/archinternmed.2009.417.

Famurewa AC, Ugwuja EI. 2017. Association of blood and seminal plasma cadmium and lead levels with semen quality in non-occupationally exposed infertile men in Abakaliki, South East Nigeria. J Family Reprod Health 11(2):97-103.

Fan G, Du G, Li H, et al. 2014. The effect of the hemochromatosis (HFE) genotype on lead load and iron metabolism among lead smelter workers. PLoS ONE 9(7):e101537. http://doi.org/10.1371/journal.pone.0101537. 
Fan Y, Sheng J, Liang C, et al. 2020. Association of blood lead levels with the risk of depressive symptoms in the elderly Chinese population: Baseline data of a cohort study. Biol Trace Elem Res 194(1):76-83. http://doi.org/10.1007/s12011-019-01755-x.

Fang F, Kwee LC, Allen KD, et al. 2010. Association between blood lead and the risk of amyotrophic lateral sclerosis. Am J Epidemiol 171(10):1126-1133. http://doi.org/10.1093/aje/kwq06.

Fanning D. 1988. A mortality study of lead workers, 1926-1985. Arch Environ Health 43(3):247-251.

Faramawi MF, Delongchamp R, Lin YS, et al. 2015. Environmental lead exposure is associated with visit-to-visit systolic blood pressure variability in the US adults. Int Arch Occup Environ Health 88(3):381-388. http://doi.org/10.1007/s00420-014-0970-5.

Farhat A, Mohammadzadeh A, Balali-Mood M, et al. 2013. Correlation of blood lead level in mothers and exclusively breastfed infants: A study on infants aged less than six months. Asia Pac J Med Toxicol 2:150-152.

Farias P, Echavarria M, Hernandez-Avila M, et al. 2005. Bone, blood and semen lead in men with environmental and moderate occupational exposure. Int J Environ Health Res 15(1):21-31.

Farooqui Z, Bakulski KM, Power MC, et al. 2017. Associations of cumulative Pb exposure and longitudinal changes in Mini-Mental Status Exam scores, global cognition and domains of cognition: The VA Normative Aging Study. Environ Res 152:102-108. http://doi.org/10.1016/j.envres.2016.10.007.

Fayerweather WE, Karns ME, Nuwayhid IA, et al. 1997. Case-control study of cancer risk in tetraethyl lead manufacturing. Am J Ind Med 31:28-35.

Fazli D, Malekirad AA, Mirzaee M, et al. 2014. Study on the link between lead exposure and hematological, psychological, and memorial parameters in automobile repair workers. Sci Res 6:712-719.

FDA. 1995. Substances prohibited from use in human food. Substances prohibited from indirect addition to human food through food-contact surfaces. U.S. Food and Drug Administration. Code of Federal Regulations. 21 CFR 189.240.

FDA. 2006. Supporting document for recommended maximum level for lead in candy likely to be consumed frequently by small children. U.S. Food and Drug Administration. https://www.fda.gov/food/foodborneillnesscontaminants/metals/ucm172050.htm. June 28, 2017.

FDA. 2016a. Lead and cadmium in foods. Combination metals testing. U.S. Food and Drug Administration. https://www.fda.gov/Food/FoodborneIllnessContaminants/Metals/ucm521427.htm. March 23, 2017.

FDA. 2016b. Total diet study elements results summary statistics. Market baskets 2006 through 2011. U.S. Food and Drug Administration. March 23, 2017.

FDA. 2019a. Substances added to food. Washington, DC: U.S. Food and Drug Administration. https://www.accessdata.fda.gov/scripts/fdcc/?set=FoodSubstances. September 2, 2019.

FDA. 2019b. Subpart B - Requirements for specific standardized beverages. Bottled water. U.S. Food and Drug Administration. Code of Federal Regulations. 21 CFR 165.110. https://www.govinfo.gov/content/pkg/CFR-2019-title21-vol2/pdf/CFR-2019-title21-vol2part165.pdf. December 5, 2019.

Fears TR, Elashoff RM, Schneiderman MA. 1989. The statistical analysis of a carcinogen mixture experiment. III. Carcinogens with different target systems, aflatoxin B1, n-butyl-N(4hydroxybutyl)nitrosamine, lead acetate, and thiouracil. Toxicol Ind Health 5(1):1-23.

Feldhake CJ, Stevens CD. 1963. The solubility of tetraethyllead in water. J Chem Eng Data 8(2):196197. http://doi.org/10.1021/je60017a016.

Fels LM, Bundschuh I, Gwinner W, et al. 1994. Early urinary markers of target nephron segments as studied in cadmium toxicity. Kidney Int Suppl 47:S81-88.

Fels LM, Wunsch M, Baranowski J, et al. 1998. Adverse effects of chronic low level lead exposure on kidney function - A risk group study in children. Nephrol Dial Transplant 13:2248-2256.

Fenga C, Gangemi S, Alibrandi A, et al. 2016. Relationship between lead exposure and mild cognitive impairment. J Prev Med Hyg 57(4):E205-E210. 
Fergusson DM, Horwood LJ, Lynskey MT. 1993. Early dentine lead levels and subsequent cognitive and behavioural development. J Child Psychol Psychiatry 34(2):215-227. http://doi.org/10.1111/j.1469-7610.1993.tb00980.x.

Fernandes KC, Martins AC, Oliveira AA, et al. 2016. Polymorphism of metallothionein 2A modifies lead body burden in workers chronically exposed to the metal. Public health genomics 19(1):47-52. http://doi.org/10.1159/000441713.

Finster ME, Gray KA, Binns HJ. 2004. Lead levels of edibles grown in contaminated residential soils: A field survey. Sci Total Environ 320(2):245-257.

Fischbein A, Anderson KE, Sassa S, et al. 1981. Lead poisoning from "Do-It-Yourself" heat guns for removing lead-based paint: Report of two cases. Environ Res 24:425-431.

Fischbein A, Tsang P, Luo JCJ, et al. 1993. Phenotypic aberrations of CD3+ and CD4+ cells and functional impairments of lymphocytes at low-level occupational exposure to lead. Clin Immunol Immunopathol 66(2):163-168.

Flanagan PR, Hamilton DL, Haist J, et al. 1979. Interrelationships between iron and lead absorption in iron-deficient mice. Gastroenterology 77:1074-1081.

Flegal AR, Smith DR. 1995. Measurements of environmental lead contamination and human exposure. Rev Environ Contam Toxicol 143:1-45.

Fleisch AF, Burns JS, Williams PL, et al. 2013. Blood lead levels and serum insulin-like growth factor 1 concentrations in peripubertal boys. Environ Health Perspect 121(7):854-858. http://doi.org/10.1289/ehp.1206105.

Fleming D, Boulay D, Richard NS, et al. 1997. Accumulated body burden and endogenous release of lead in employees of a lead smelter. Environ Health Perspect 105(2):224-233.

Fleming MD, Romano MA, Su MA, et al. 1998a. Nramp2 is mutated in the anemic Belgrade (b) rat: Evidence of a role for Nramp2 in endosomal iron transport. Proc Natl Acad Sci 95(3):1148-1153.

Fleming DEB, Chettle DR, Wetmur JG, et al. 1998b. Effect of the delta-aminolevulinate dehydratase polymorphism on the accumulation of lead in bone and blood in lead smelter workers. Environ Res 77:49-61.

Flora G, Gupta D, Tiwari A. 2012. Toxicity of lead: A review with recent updates. Interdiscip Toxicol 5(2):47-58. http://doi.org/10.2478/v10102-012-0009-2.

Forbes GB, Reina JC. 1972. Effect of age on gastrointestinal absorption (Fe, Sr, Pb) in the rat. J Nutr 102:647-652.

Forni A, Cambiaghi G, Secchi GC. 1976. Initial occupational exposure to lead. Chromosome and biochemical findings. Arch Environ Health 31:73-78.

Fosse G, Wesenberg GR, Tvinnereim HM, et al. 1995. Lead in deciduous teeth from larger cities of some countries. Int J Environ Stud 47:203-210.

Foster P, Gray LE. 2008. Toxic responses of the reproductive system. In: Klaassen CD, ed. Casarett and Doull's toxicology. The basic science of poisons. New York, NY: The McGraw-Hill Companies, Inc, 761-806.

Fowler BA, DuVal G. 1991. Effects of lead on the kidney: Roles of high-affinity lead-binding proteins. Environ Health Perspect 91:77-80.

Fracasso ME, Perbellini L, Soldà S, et al. 2002. Lead induced DNA strand breaks in lymphocytes of exposed workers: Role of reactive oxygen species and protein kinase C. Mutat Res Genet Toxicol Environ Mutagen 515(1):159-169.

Franklin CA, Inskip MJ, Baccanale CL, et al. 1997. Use of sequentially administered stable lead isotopes to investigate changes in blood lead during pregnancy in a nonhuman primate (Macaca fascicularis). Fundam Appl Toxicol 39:109-119.

Fraser S, Muckle G, Despres C. 2006. The relationship between lead exposure, motor function and behaviour in Inuit preschool children. Neurotoxicol Teratol 28(1):18-27. http://doi.org/10.1016/j.ntt.2005.10.008.

Freeman GB, Johnson JD, Killinger JM, et al. 1992. Relative bioavailability of lead from mining waste soil in rats. Fundam Appl Toxicol 19(3):388-398. 
Freeman GB, Johnson JD, Liao SC, et al. 1994. Absolute bioavailability of lead acetate and mining waste lead in rats. Toxicology 91:151-163.

Freeman GB, Dill JA, Johnson JD, et al. 1996. Comparative absorption of lead from contaminated soil and lead salts by weanling Fischer 344 rats. Fundam Appl Toxicol 33:109-119.

Frisancho AR, Ryan AS. 1991. Decreased stature associated with moderate blood lead concentrations in Mexican-American children. Am J Clin Nutr 54:516-519.

Froehlich TE, Lanphear BP, Dietrich KN, et al. 2007. Interactive effects of a DRD4 polymorphism, lead and sex on executive functions in children. Biol Psychiatry 62(3):243-249. http://doi.org/10.1016/j.biopsych.2006.09.039.

Froehlich TE, Lanphear BP, Auinger P, et al. 2009. Association of tobacco and lead exposures with attention-deficit/hyperactivity disorder. Pediatrics 124(6):E1054-E1063. http://doi.org/10.1542/peds.2009-0738.

Froom P, Kristal-Boneh E, Benbassat J, et al. 1998. Predictive value of determinations of zinc protoporphyrin for increased blood lead concentrations. Clin Chem 44(6):1283-1288.

Fruh V, Rifas-Shiman SL, Amarasiriwardena C, et al. 2019. Prenatal lead exposure and childhood executive function and behavioral difficulties in project viva. Neurotoxicology 75:105-115. http://doi.org/10.1016/j.neuro.2019.09.006.

Fujita H, Sato K, Sano S. 1982. Increase in the amount of erythrocyte delta-aminolevulinic acid dehydratase in workers with moderate lead exposure. Int Arch Occup Environ Health 50:287-297.

Fukui Y, Miki M, Ukai H, et al. 1999. Urinary lead as a possible surrogate of blood lead among workers occupationally exposed to lead. Int Arch Occup Environ Health 72(8):516-520.

Fukumoto K, Karai I, Horiguchi S. 1983. Effect of lead on erythrocyte membranes. Br J Ind Med 40:220-223.

Fullmer CS, Rosen JF. 1990. Effect of dietary calcium and lead status on intestinal calcium absorption. Environ Res 51:91-99.

Gambelunghe A, Sallsten G, Borne Y, et al. 2016. Low-level exposure to lead, blood pressure, and hypertension in a population-based cohort. Environ Res 149:157-163. http://doi.org/10.1016/j.envres.2016.05.015.

Gao K, Pearce J, Jones J, et al. 1999. Interaction between peat, humic acid and aqueous metal ions. Environ Geochem Health 21(1):13-26.

Gao A, Lu XT, Li QY, et al. 2010. Effect of the delta-aminolevulinic acid dehydratase gene polymorphism on renal and neurobehavioral function in workers exposed to lead in China. Sci Total Environ 408(19):4052-4055. http://doi.org/10.1016/j.scitotenv.2010.04.024.

Garavan C, Breen J, Moles R, et al. 2008. A case study of the health impacts in an abandoned lead mining area, using children's blood lead levels. Int J Min Reclam Environ 22(4):265-284.

Garcia-Esquinas E, Aragones N, Fernandez MA, et al. 2014. Newborns and low to moderate prenatal environmental lead exposure: Might fathers be the key? Environ Sci Pollut Res Int 21(13):78867898. http://doi.org/10.1007/s11356-014-2738-6.

Garcia-Leston J, Mendez J, Pasaro E, et al. 2010. Genotoxic effects of lead: An updated review. Environ Int 36(6):623-636. http://doi.org/10.1016/j.envint.2010.04.011.

Garcia-Leston J, Roma-Torres J, Mayan O, et al. 2012. Assessment of immunotoxicity parameters in individuals occupationally exposed to lead. J Toxicol Environ Health A 75(13-15):807-818. http://doi.org/10.1080/15287394.2012.690327.

García-Lestón J, Roma-Torres J, Vilares M, et al. 2011. Biomonitoring of a population of Portuguese workers exposed to lead. Mutat Res 721(1):81-88. http://doi.org/10.1016/j.mrgentox.2011.01.001.

Garcon G, Leleu B, Marez T, et al. 2007. Biomonitoring of the adverse effects induced by the chronic exposure to lead and cadmium on kidney function: Usefulness of alpha-glutathione S-transferase. Sci Total Environ 377(2-3):165-172. http://doi.org/10.1016/j.scitotenv.2007.02.002.

Garrido Latorre F, Hernandez-Avila M, Orozco JT, et al. 2003. Relationship of blood and bone lead to menopause and bone mineral density among middle-age women in Mexico City. Environ Health Perspect 111(4):631-636. 
Gartside PS. 1988. The relationship of blood lead levels and blood pressure in NHANES II: Additional calculations. Environ Health Perspect 78:31-34.

Garvey GJ, Hahn G, Lee RV, et al. 2001. Heavy metal hazards of Asian traditional remedies. Int J Environ Health Res 11(1):63-71.

Ge Y, Murray P, Hendershot W. 2000. Trace metal speciation and bioavailability in urban soils. Environ Pollut 107(1):137-144.

Geier DA, Kern JK, Geier MR. 2017. Blood lead levels and learning disabilities: A cross-sectional study of the 2003-2004 National Health and Nutrition Examination Survey (NHANES). Int J Environ Res Public Health 14(10):1202. http://doi.org/10.3390/ijerph14101202.

Geier DA, Kern JK, Geier MR. 2018. A cross-sectional study of the relationship between blood lead levels and reported attention deficit disorder: An assessment of the economic impact on the United States. Metab Brain Dis 33(1):201-208. http://doi.org/10.1007/s11011-017-0146-6.

Gemmel A, Tavares M, Alperin S, et al. 2002. Blood lead level and dental caries in school-age children. Environ Health Perspect 110(10):A625-A630.

Gennart JP, Bernard A, Lauwerys R. 1992. Assessment of thyroid, testes, kidney and autonomic nervous system function in lead-exposed workers. Int Arch Occup Environ Health 64:49-57.

Genuis SJ, Birkholz D, Rodushkin I, et al. 2011. Blood, urine, and sweat (BUS) study: Monitoring and elimination of bioaccumulated toxic elements. Arch Environ Contam Toxicol 61(2):344-357. http://doi.org/10.1007/s00244-010-9611-5.

Gercken B, Barnes RM. 1991. Determination of lead and other trace element species in blood by size exclusion chromatography and inductively coupled plasma/mass spectrometry. Anal Chem 63:283287.

Gerhardsson L, Brune D, Nordberg GF, et al. 1986. Distribution of cadmium, lead and zinc in lung, liver and kidney in long-term exposed smelter workers. Sci Total Environ 50:65-85.

Gerhardsson L, Chettle D, Englyst V, et al. 1992. Kidney effects in long term exposed lead smelter workers. Br J Ind Med 49(3):186-192.

Gerhardsson L, Attewell R, Chettle DR, et al. 1993. In vivo measurements of lead in bone in long-term exposed lead smelter workers. Arch Environ Health 48(3):147-156. http://doi.org/10.1080/00039896.1993.9940813.

Gerhardsson L, Hagmar L, Rylander L, et al. 1995a. Mortality and cancer incidence among secondary lead smelter workers. Occup Environ Med 52:667-672.

Gerhardsson L, Englyst V, Lundstrom NG, et al. 1995b. Lead in tissues of deceased lead smelter worker. J Trace Elem Med Biol 9:136-143.

Gerhardt RE, Crecelius EA, Hudson JB. 1980. Trace element content of moonshine. Arch Environ Health 35:332-334.

Gerr F, Letz R, Stokes L, et al. 2002. Association between bone lead concentration and blood pressure among young adults. Am J Ind Med 42:98-106.

Gersberg RM, Gaynor K, Tenczar D, et al. 1997. Quantitative modeling of lead exposure from glazed ceramic pottery in childhood lead poisoning cases. Int J Environ Health Res 7:193-202.

Getz LL, Rolfe GL, Haney AW, et al. 1977. Transport and distribution in a watershed ecosystem. In: Boggess WR, ed. Lead in the environment. Washington, DC: National Science Foundation, 105134.

Ghiasvand M, Aghakhani K, Salimi A, et al. 2013. Ischemic heart disease risk factors in lead exposed workers: Research study. J Occup Med Toxicol 8:11. http://doi.org/10.1186/1745-6673-8-11.

Ghiasvand M, Mohammadi S, Roth B, et al. 2016. The relationship between occupational exposure to lead and hearing loss in a cross-sectional survey of Iranian workers. Front Public Health 4:19. http://doi.org/10.3389/fpubh.2016.00019.

Gibbs PNB, Gore MG, Jordan PM. 1985. Investigation of the effect of metal ions on the reactivity of thiol groups in human 5-aminolaevulinate dehydratase. Biochem J 225:573-580.

Giddings JC. 1973. Lead in gasoline. In: Chemistry, man, and environmental change. An integrated approach. New York, NY: Canfield Press, 351-353. 


\section{REFERENCES}

Gilbert M, Lasley SM. 2002. Long-term consequences of developmental exposure to lead or polychlorinated biphenyls: Synaptic transmission and plasticity in the rodent CNS. Environ Toxicol Pharmacol 12(2):105-117.

Glass TA, Bandeen-Roche K, McAtee M, et al. 2009. Neighborhood psychosocial hazards and the association of cumulative lead dose with cognitive function in older adults. Am J Epidemiol 169(6):683-692. http://doi.org/10.1093/aje/kwn390.

Glenn BS, Stewart WF, Links JM, et al. 2003. The longitudinal association of lead with blood pressure. Epidemiology 14(1):30-36.

Glenn BS, Bandeen-Roche K, Lee BK, et al. 2006. Changes in systolic blood pressure associated with lead in blood and bone. Epidemiology 17(5):538-544. http://doi.org/10.1097/01.ede.0000231284.19078.4b.

Goering PL, Fowler BA. 1987. Metal constitution of metallothionein influences inhibition of $\delta$ aminolaevulinic acid dehydratase (porphobilinogen synthase) by lead. Biochem J 245(2):339-345.

Goldberg RL, Hicks AM, O'Leary LM, et al. 1991. Lead exposure at uncovered outdoor firing ranges. J Occup Med 33(6):718-719.

Gollenberg AL, Hediger ML, Lee PA, et al. 2010. Association between lead and cadmium and reproductive hormones in peripubertal U.S. girls. Environ Health Perspect 118(12):1782-1787. http://doi.org/10.1289/ehp.1001943.

Golub NI, Winters PC, van Wijngaarden E. 2010. A population-based study of blood lead levels in relation to depression in the United States. Int Arch Occup Environ Health 83(7):771-777.

Gomaa A, Howard H, Bellinger D, et al. 2002. Maternal bone lead as an independent risk factor for fetal neurotoxicity: A prospective study. Pediatrics 110(1):110-118.

Gomes VE, Rosario de Sousa ML, Barbosa F, et al. 2004. In vivo studies on lead content of deciduous teeth superficial enamel of preschool children. Sci Total Environ 320(1):25-35. http://doi.org/10.1016/j.scitotenv.2003.08.013.

Gomez HF, Borgialli DA, Sharman M, et al. 2018. Blood lead levels of children in Flint, Michigan: 2006-2016. J Pediatr 197:158-164. http://doi.org/10.1016/j.jpeds.2017.12.063.

Gomez HF, Borgialli DA, Sharman M, et al. 2019. Blood lead levels in females of childbearing age in Flint, Michigan, and the water crisis. Obstet Gynecol 134(3):628-635. http://doi.org/10.1097/aog.0000000000003416.

Gonick HC. 2011. Lead-binding proteins: A review. Journal of toxicology 2011:686050. http://doi.org/10.1155/2011/686050.

González-Cossío T, Peterson KE, Sanin L, et al. 1997. Decrease in birth weight in relation to maternal bone-lead burden. Pediatrics 100(5):856-862.

Goodman M, LaVerda N, Clarke C, et al. 2002. Neurobehavioural testing in workers occupationally exposed to lead: Systematic review and meta-analysis of publications. Occup Environ Med 59(4):217-223.

Goodrum PE, Diamond GL, Hassett JM, et al. 1996. Monte Carlo modeling of childhood lead exposure: Development of a probabilistic methodology for use with the USEPA IEUBK model for lead in children. Hum Ecol Risk Assess 2(4):681-708.

Goyer RA. 1989. Mechanisms of lead and cadmium nephrotoxicity. Toxicol Lett 46:153-162.

Goyer RA. 1990. Transplacental transport of lead. Environ Health Perspect 89:101-105.

Goyer RA. 2001. Lead. In: Bingham E, Cohrssen B, Powell CH, eds. Patty's toxicology. Vol. 2. 5th ed. New York, NY: John Wiley \& Sons, Inc., 611-675.

Goyer RA, Leonard DL, Moore JF, et al. 1970a. Lead dosage and the role of the intranuclear inclusion body: An experimental study. Arch Environ Health 20:705-711.

Goyer RA, May P, Cates MM, et al. 1970b. Lead and protein content of isolated intranuclear inclusion bodies from kidneys of lead-poisoned rats. Lab Invest 22(3):245-251.

Grabo TN. 1997. Unknown toxic exposures: Arts and crafts materials. AAOHN J 45(3):124-130.

Grandjean P. 1979. Occupational lead exposure in Denmark: Screening with the haematofluorometer. Br J Ind Med 36:52-58. 
Grandjean P, Lintrup J. 1978. Erythrocyte-Zn-protoporphyrin as an indicator of lead exposure. Scand J Clin Lab Invest 38:669-675.

Grandjean P, Bach E. 1986. Indirect exposures: The significance of bystanders at work and at home. Am Ind Hyg Assoc J 47(12):819-824.

Grandjean P, Wulf HC, Niebuhr E. 1983. Sister chromatid exchange in response to variations in occupational lead exposure. Environ Res 32(1):199-204.

Grandjean P, Jorgensen PJ, Viskum S. 1991. Temporal and interindividual variation in erythrocyte zincprotoporphyrin in lead exposed workers. Br J Ind Med 48:254-257.

Grandjean P, Hollnagel H, Hedegaard L, et al. 1989. Blood lead-blood pressure relations: Alcohol intake and hemoglobin as confounders. Am J Epidemiol 129(4):732-739.

Grashow R, Miller MW, McKinney A, et al. 2013a. Lead exposure and fear-potentiated startle in the VA Normative Aging Study: A pilot study of a novel physiological approach to investigating neurotoxicant effects. Neurotoxicol Teratol 38:21-28. http://doi.org/10.1016/j.ntt.2013.04.003.

Grashow R, Spiro A, Taylor KM, et al. 2013b. Cumulative lead exposure in community-dwelling adults and fine motor function: Comparing standard and novel tasks in the VA normative aging study. Neurotoxicology 35:154-161. http://doi.org/10.1016/j.neuro.2013.01.005.

Grashow R, Sparrow D, Hu H, et al. 2015. Cumulative lead exposure is associated with reduced olfactory recognition performance in elderly men: The Normative Aging Study. Neurotoxicology 49:158-164. http://doi.org/10.1016/j.neuro.2015.06.006.

Graziano JH. 1994. Validity of lead exposure markers in diagnosis and surveillance. Clin Chem 40(7):1387-1390.

Graziano JH, Blum C. 1991. Lead exposure from lead crystal. Lancet 337:141-142.

Graziano JH, Popovac D, Factor-Litvak P, et al. 1990. Determinants of elevated blood lead during pregnancy in a population surrounding a lead smelter in Kosovo, Yugoslavia. Environ Health Perspect 89:95-100.

Graziano JH, Blum CB, Lolacono NJ, et al. 1996. A human in vivo model for the determination of lead bioavailability using stable isotope dilution. Environ Health Perspect 104(2):176-179. http://doi.org/10.1289/ehp.96104176.

Graziano J, Slavkovich V, Liu X, et al. 2004. A prospective study of prenatal and childhood lead exposure and erythropoietin production. J Occup Environ Med 46(9):924-929.

Greenberg M, Hamilton R. 1999. Lack of blood lead elevations in police officers following small arms qualification on an indoor range. J Toxicol Clin Toxicol 37(5):627.

Griffin TB, Coulston F, Wills H. 1975. Biological and clinical effects of continuous exposure to airborne particulate lead. Arh Hig Rada Toksikol 26:191-208.

Griffin S, Goodrum PE, Diamond GL, et al. 1999. Application of a probabilistic risk assessment methodology to a lead smelter site. Hum Ecol Risk Assess 5(4):845-868.

Gross M, Kumar R. 1990. Physiology and biochemistry of vitamin D-dependent calcium binding proteins. Am J Physiol 259:F195-F209.

Gross SB, Pfitzer EA, Yeager DW, et al. 1975. Lead in human tissues. Toxicol Appl Pharmacol 32:638651.

Grover P, Rekhadevi PV, Danadevi K, et al. 2010. Genotoxicity evaluation in workers occupationally exposed to lead. Int J Hyg Environ Health 213(2):99-106. http://doi.org/10.1016/j.ijheh.2010.01.005.

Guibaud G, Tixier N, Bouju A, et al. 2003. Relation between extracellular polymers' composition and its ability to complex $\mathrm{Cd}, \mathrm{Cu}$ and $\mathrm{Pb}$. Chemosphere 52(10):1701-1710.

Gulson BL. 1996. Tooth analyses of sources and intensity of lead exposure in children. Environ Health Perspect 104(3):306-312. http://doi.org/10.1289/ehp.96104306.

Gulson BL. 2000. Revision of estimates of skeletal contribution to blood during pregnancy and postpartum period. J Lab Clin Med 136(3):250-251. http://doi.org/10.1067/mlc.2000.108752.

Gulson B, Wilson D. 1994. History of lead exposure in children revealed from isotopic analyses of teeth. Arch Environ Health 49(4):279-283. http://doi.org/10.1080/00039896.1994.9937480. 
Gulson BL, Palmer JM, Bryce A. 2002. Changes in blood lead of a recreational shooter. Sci Total Environ 293(1):143-150.

Gulson BL, Mizon KJ, Korsch MJ, et al. 1996. Impact on blood lead in children and adults following relocation from their source of exposure and contribution of skeletal tissue to blood lead. Bull Environ Contam Toxicol 56(4):543-550. http://doi.org/10.1007/s001289900078.

Gulson BL, James M, Giblin AM, et al. 1997a. Maintenance of elevated lead levels in drinking water from occasional use and potential impact on blood leads in children. Sci Total Environ 205:271-275.

Gulson BL, Jameson CW, Mahaffey KR, et al. 1997b. Pregnancy increases mobilization of lead from maternal skeleton. J Lab Clin Med 130:51-62.

Gulson BL, Jameson CW, Mahaffey KR, et al. 1998a. Relationships of lead in breast milk to lead in blood, urine, and diet of the infant and mother. Environ Health Perspect 106(10):667-674.

Gulson BL, Mahaffey KR, Jameson CW, et al. 1998b. Mobilization of lead from the skeleton during the postnatal period is larger than during pregnancy. J Lab Clin Med 131:324-329.

Gulson BL, Gray B, Mahaffey KR, et al. 1999a. Comparison of the rates of exchange of lead in the blood of newly born infants and their mothers with lead from their current environment. J Lab Clin Med 133(2):171-178.

Gulson BL, Pounds JG, Mushak P, et al. 1999b. Estimation of cumulative lead releases (lead flux) from the maternal skeleton during pregnancy and lactation. J Lab Clin Med 134(6):631-640.

Gulson BL, Mahaffey KR, Jameson CW, et al. 1999c. Impact of diet on lead in blood and urine in female adults and relevance to mobilization of lead from bone stores. Environ Health Perspect 107(4):257-263.

Gulson BL, Mizon KJ, Korsch MJ, et al. 2003. Mobilization of lead from human bone tissue during pregnancy and lactation - A summary of long-term research. Sci Total Environ 303:79-104.

Gulson BL, Mizon KJ, Palmer JM, et al. 2004. Blood lead changes during pregnancy and postpartum with calcium supplementation. Environ Health Perspect 12(15):1499-1507.

Gulson B, Mizon K, Taylor A, et al. 2008. Longitudinal monitoring of selected elements in blood of healthy young children. J Trace Elem Med Biol 22(3):206-214. http://doi.org/10.1016/j.jtemb.2008.04.001.

Gulson B, Korsch M, Matisons M, et al. 2009. Windblown lead carbonate as the main source of lead in blood of children from a seaside community: An example of local birds as "canaries in the mine". Environ Health Perspect 117(1):148-154. http://doi.org/10.1289/ehp.11577.

Gulson B, Mizon K, Korsch M, et al. 2016. Revisiting mobilisation of skeletal lead during pregnancy based on monthly sampling and cord/maternal blood lead relationships confirm placental transfer of lead. Arch Toxicol 90(4):805-816. http://doi.org/10.1007/s00204-015-1515-8.

Gump BB, Stewart P, Reihman J, et al. 2005. Prenatal and early childhood blood lead levels and cardiovascular functioning in 9 1/2 year old children. Neurotoxicol Teratol 27(4):655-665. http://doi.org/10.1016/j.ntt.2005.04.002.

Gump BB, Stewart P, Reihman J, et al. 2008. Low-level prenatal and postnatal blood lead exposure and adrenocortical responses to acute stress in children. Environ Health Perspect 116(2):249-255. http://doi.org/10.1289/ehp.10391.

Gump BB, Mackenzie JA, Bendinskas K, et al. 2011. Low-level Pb and cardiovascular responses to acute stress in children: The role of cardiac autonomic regulation. Neurotoxicol Teratol 33(2):212219. http://doi.org/10.1016/j.ntt.2010.10.001.

Gundacker C, Wittmann KJ, Kukuckova M, et al. 2009. Genetic background of lead and mercury metabolism in a group of medical students in Austria. Environ Res 109:786-796.

Gundacker C, Fröhlich S, Graf-Rohrmeister K, et al. 2010. Perinatal lead and mercury exposure in Austria. Sci Total Environ 408(23):5744-5749. http://doi.org/10.1016/j.scitotenv.2010.07.079.

Guo M, He L, Strong PJ, et al. 2014. Binding between lead ions and the high-abundance serum proteins. Chemosphere 112:472-480. http://doi.org/10.1016/j.chemosphere.2014.05.018.

Gurer-Orhan H, Sabır HU, Özgüneş H. 2004. Correlation between clinical indicators of lead poisoning and oxidative stress parameters in controls and lead-exposed workers. Toxicology 195(2):147-154. 
Gustafson A, Hedner P, Schutz A, et al. 1989. Occupational lead exposure and pituitary function. Int Arch Occup Environ Health 61:277-281.

Guyette RP, Cutter BE, Henderson GS. 1991. Long-term correlations between mining activity and levels of lead and cadmium in tree-rings of eastern red-cedar. J Environ Qual 20(1):146-150

Haenninen H, Hernberg S, Mantere P, et al. 1978. Psychological performance of subjects with low exposure to lead. J Occup Med 20(10):683-689.

Haley VB, Talbot TO. 2004. Seasonality and trend in blood lead levels of New York State children. BMC Pediatr 4(1):8. http://doi.org/10.1186/1471-2431-4-8.

Hamurcu Z, Donmez H, Saraymen R, et al. 2001. Micronucleus frequency in human lymphocyte exposed to occupational lead, zinc, and cadmium. Biol Trace Elem Res 83(2):97-102.

Han L, Wang X, Han R, et al. 2018. Association between blood lead level and blood pressure: An occupational population-based study in Jiangsu province, China. PLoS ONE 13(7):e0200289. http://doi.org/10.1371/journal.pone.0200289.

Hanna CW, Bloom MS, Robinson WP, et al. 2012. DNA methylation changes in whole blood is associated with exposure to the environmental contaminants, mercury, lead, cadmium and bisphenol A, in women undergoing ovarian stimulation for IVF. Hum Reprod 27(5):1401-1410. http://doi.org/10.1093/humrep/des038.

Hanna-Attisha M, LaChance J, Sadler RC, et al. 2016. Elevated blood lead levels in children associated with the Flint drinking water crisis: A spatial analysis of risk and public health response. Am J Public Health 106(2):283-290. http://doi.org/10.2105/ajph.2015.303003.

Hanninen H, Aitio A, Kovala T, et al. 1998. Occupational exposure to lead and neuropsychological dysfunction. Occup Environ Med 55:202-209.

Hansen S, Nieboer E, Sandanger TM, et al. 2011. Changes in maternal blood concentrations of selected essential and toxic elements during and after pregnancy. J Environ Monit 13(8):2143-2152. http://doi.org/10.1039/c1em10051c.

Harari F, Sallsten G, Christensson A, et al. 2018. Blood lead levels and decreased kidney function in a population-based cohort. Am J Kidney Dis 72(3):381-389. http://doi.org/10.1053/j.ajkd.2018.02.358.

Hardison DW, Ma LQ, Luongo T, et al. 2004. Lead contamination in shooting range soils from abrasion of lead bullets and subsequent weathering. Sci Total Environ 328:175-183. http://doi.org/10.1016/j.scitotenv.2003.12.013.

Harlan WR. 1988. The relationship of blood lead levels to blood pressure in the U.S. population. Environ Health Perspect 78:9-13.

Harlan WR, Landis JR, Schmouder RL, et al. 1985. Blood lead and blood pressure. Relationship in the adolescent and adult US population. J Am Med Assoc 253:530-534.

Harville EW, Hertz-Picciotto I, Schramm M, et al. 2005. Factors influencing the difference between maternal and cord blood lead. Occup Environ Med 62(4):263-269.

Hauser R, Sergeyev O, Korrick S, et al. 2008. Association of blood lead levels with onset of puberty in Russian boys. Environ Health Perspect 116(7):976-980. http://doi.org/10.1289/ehp.10516.

Havlena J, Kanarek MS, Coons M. 2009. Factors associated with the seasonality of blood lead levels among preschool Wisconsin children. WMJ 108(3):151-155.

Hayes EB, McElvaine MD, Orbach HG, et al. 1994. Long-term trends in blood lead levels among children in Chicago: Relationship to air lead levels. Pediatrics 93(2):195-200.

Haynes WM. 2014. Lead. In: CRC handbook of chemistry and physics. 95th ed. Boca Raton, FL: CRC Press, 4-20.

Haynes EN, Kalkwarf HJ, Hornung R, et al. 2003. Vitamin D receptor Fok1 polymorphism and blood lead concentration in children. Environ Health Perspect 111:1665-1669.

He J, Ning H, Huang R. 2019. Low blood lead levels and attention-deficit hyperactivity disorder in children: A systematic review and meta-analysis. Environ Sci Pollut Res Int 26(18):17875-17884. http://doi.org/10.1007/s11356-017-9799-2. 
Healey N, Chettle DR, McNeill FE, et al. 2008. Uncertainties in the relationship between tibia lead and cumulative blood lead index. Environ Health Perspect 116(3):A109-110. http://doi.org/10.1289/ehp.10778.

Healy MA, Harrison PG, Aslam M, et al. 1982. Lead sulphide and traditional preparations: Routes for ingestion, and solubility and reactions in gastric fluid. J Clin Hosp Pharm 7:169-173.

Heard MJ, Chamberlain AC. 1982. Effect of minerals and food on uptake of lead from the gastrointestinal tract in humans. Hum Toxicol 1:411-415.

Heard MJ, Chamberlain AC, Sherlock JC. 1983. Uptake of lead by humans and effect of minerals and food. Sci Total Environ 30:245-253. http://doi.org/10.1016/0048-9697(83)90016-5.

Heard MJ, Wells AC, Newton D, et al. 1979. Human uptake and metabolism of tetra ethyl and tetra methyl lead vapour labelled with 203Pb. In: International conference on management and control of heavy metals in the environment. Edinburgh, United Kingdom CEP Consultants, Ltd., 103-108.

Hengstler JG, Bolm-Audorff U, Faldum A, et al. 2003. Occupational exposure to heavy metals: DNA damage induction and DNA repair inhibition prove co-exposures to cadmium, cobalt and lead as more dangerous than hitherto expected. Carcinogenesis 24(1):63-73.

Hense HW, Filipiak B, Keil U. 1993. The association of blood lead and blood pressure in population surveys. Epidemiology 4:173-179.

Heo Y, Lee BK, Ahn KD, et al. 2004. Serum IgE elevation correlates with blood lead levels in battery manufacturing workers. Hum Exp Toxicol 23(5):209-213. http://doi.org/10.1191/0960327104ht442oa.

Hernandez-Avila M, Gonzalez-Cossio T, Palazuelos E, et al. 1996. Dietary and environmental determinants of blood and bone lead levels in lactating postpartum women living in Mexico City. Environ Health Perspect 104(10):1076-1082.

Hernandez-Avila M, Smith D, Meneses F, et al. 1998. The influence of bone and blood lead on plasma lead levels in environmentally exposed adults. Environ Health Perspect 106(8):473-477.

Hernandez-Avila M, Villalpano CG, Palazuelos E, et al. 2000. Determinants of blood lead levels across the menopausal transition. Arch Environ Health 53:355-360.

Hernandez-Avila M, Peterson KE, Gonzalez-Cossio T, et al. 2002. Effect of maternal bone lead on length and head circumference of newborns and 1-month-old infants. Arch Environ Health 57(5):482-488.

Hernández-Ochoa I, García-Vargas G, López-Carrillo L, et al. 2005. Low lead environmental exposure alters semen quality and sperm chromatin condensation in northern Mexico. Reprod Toxicol 20(2):221-228. http://doi.org/10.1016/j.reprotox.2005.01.007.

Hernberg S, Nikkanen J, Mellin G, et al. 1970. $\delta$-Aminolevulinic acid dehydrase as a measure of lead exposure. Arch Environ Health 21:140-145.

Hertz-Picciotto I, Croft J. 1993. Review of the relation between blood lead and blood pressure. Epidemiol Rev 15(2):352-373.

Hertz-Picciotto I, Schramm M, Watt-Morse M, et al. 2000. Patterns and determinants of blood lead during pregnancy. Am J Epidemiol 152:829-837.

Hettiarachchi GM, Pierzynski GM, Oehme FW, et al. 2003. Treatment of contaminated soil with phosphorus and manganese oxide reduces lead absorption by Sprague-Dawley rats. J Environ Qual 32:1335-1345.

Higgs FJ, Mielke HW, Brisco M. 1999. Soil lead at elementary public schools: Comparison between school properties and residential neighbourhoods of New Orleans. Environ Geochem Health 21(1):27-36.

Hill CP. 2011. Overview of internal corrosion impacts in drinking water distribution systems. In: Internal corrosion control in water distribution systems. American Water Works Association, 1-11.

Hilts SR. 2003. Effect of smelter emission reductions on children's blood lead levels. Sci Total Environ 303(1-2):51-58.

Hirata M, Kosaka H. 1993. Effects of lead exposure on neurophysiological parameters. Environ Res 63:60-69. 
Hogan K, Marcus A, Smith R, et al. 1998. Integrated exposure uptake biokinetic model for lead in children: Empirical comparisons with epidemiologic data. Environ Health Perspect 106(Suppl 6):1557-1567.

Hogstedt C, Hane M, Agrell A, et al. 1983. Neuropsychological test results and symptoms among workers with well-defined long-term exposure to lead. Br J Ind Med 40:99-105.

Holland MG, Cawthon D. 2016. ACOEM position statement. Workplace lead exposure. J Occup Environ Med 58(12):e371-e374.

Holmgren GGS, Meyer MW, Chaney RL, et al. 1993. Cadmium, lead, zinc, copper, and nickel in agricultural soils of the United States of America. J Environ Qual 22:335-348.

Hon KL, Ching GK, Hung EC, et al. 2009. Serum lead levels in childhood eczema. Clin Exp Dermatol 34(7):e508-e509. http://doi.org/10.1111/j.1365-2230.2009.03596.x.

Hon KLE, Wang SS, Hung ECW, et al. 2010. Serum levels of heavy metals in childhood eczema and skin diseases: Friends or foes. Pediatric Allergy and Immunology 21(5):831-836. http://doi.org/10.1111/j.1399-3038.2010.01022.x.

Hong CD, Hanenson IB, Lerner S, et al. 1980. Occupational exposure to lead: Effects on renal function. Kidney Int 18:489-494.

Hong YC, Kulkarni SS, Lim YH, et al. 2014. Postnatal growth following prenatal lead exposure and calcium intake. Pediatrics 134(6):1151-1159. http://doi.org/10.1542/peds.2014-1658.

Hong SB, Im MH, Kim JW, et al. 2015. Environmental lead exposure and attention deficit/hyperactivity disorder symptom domains in a community sample of South Korean school-age children. Environ Health Perspect 123(3):271-276. http://doi.org/10.1289/ehp.1307420.

Hopkins MR, Ettinger AS, Hernández-Avila M, et al. 2008. Variants in iron metabolism genes predict higher blood lead levels in young children. Environ Health Perspect 116(9):1261-1266. http://doi.org/10.1289/ehp.11233.

Hoppin JA, Aro A, Hu H, et al. 1997. In vivo bone lead measurement in suburban teenagers. Pediatrics 100(3):365-370. http://doi.org/10.1542/peds.100.3.365.

Hornung RW, Lanphear BP. 2014. The supralinear dose-response for environmental toxicants: A statistical artifact? Clin Toxicol (Phila) 52(2):88-90. http://doi.org/10.3109/15563650.2013.878946.

Hornung RW, Lanphear BP, Dietrich KN. 2009. Age of greatest susceptibility to childhood lead exposure: A new statistical approach. Environ Health Perspect 117(8):1309-1312. http://doi.org/10.1289/ehp.0800426.

Hryhorczuk DO, Rabinowitz MB, Hessl SM, et al. 1985. Elimination kinetics of blood lead in workers with chronic lead intoxication. Am J Ind Med 8(1):33-42. http://doi.org/10.1002/ajim.4700080105.

Hsiao CL, Wu KH, Wan KS. 2011. Effects of environmental lead exposure on T-helper cell-specific cytokines in children. J Immunotoxicol 8(4):284-287. http://doi.org/10.3109/1547691X.2011.592162.

Hsiao CY, Wu HI, Lai JS, et al. 2001. A longitudinal study of the effects of long-term exposure to lead among lead battery factory workers in Taiwan (1989-1999). Sci Total Environ 279(1):151-158.

Hsieh LL, Liou SH, Chen YH, et al. 2000. Association between aminolevulinate dehydrogenase genotype and blood lead levels in Taiwan. J Occup Environ Med 42(2):151-155.

Hsieh TJ, Chen YC, Li CW, et al. 2009. A proton magnetic resonance spectroscopy study of the chronic lead effect on the basal ganglion and frontal and occipital lobes in middle-age adults. Environ Health Perspect 117(6):941-945. http://doi.org/10.1289/ehp.0800187.

Hsu PC, Chang HY, Guo YL, et al. 2009. Effect of smoking on blood lead levels in workers and role of reactive oxygen species in lead-induced sperm chromatin DNA damage. Fertil Steril 91(4):10961103. http://doi.org/10.1016/j.fertnstert.2008.01.005.

Hu X, Ding Z. 2009. Lead/cadmium contamination and lead isotopic ratios in vegetables grown in periurban and mining/smelting contaminated sites in Nanjing, China. Bull Environ Contam Toxicol 82(1):80-84. http://doi.org/10.1007/s00128-008-9562-y.

$\mathrm{Hu} \mathrm{H}$, Hashimoto D, Besser M. 1996b. Levels of lead in blood and bone of women giving birth in a Boston Hospital. Arch Environ Health 51(1):52-58. 
Hu H, Rabinowitz M, Smith D. 1998. Bone lead as a biological marker in epidemiologic studies of chronic toxicity: Conceptual paradigms. Environ Health Perspect 106(1):1-8. http://doi.org/10.1289/ehp.981061.

Hu H, Aro A, Payton M, et al. 1996a. The relationship of bone and blood lead to hypertension. The normative aging study. J Am Med Assoc 275(15):1171-1176.

Hu J, Little J, Xu T, et al. 1999. Risk factors for meningioma in adults: A case-control study in northeast China. Int J Cancer 83:299-304.

$\mathrm{Hu} \mathrm{H}$, Wu MT, Cheng Y, et al. 2001. The a-aminolevulinic acid dehydratase (ALAD) polymorphism and bone and blood lead levels in community-exposed men: The Normative Aging Study. Environ Health Perspect 109:827-832.

Hu H, Tellez-Rojo MM, Bellinger D, et al. 2006. Fetal lead exposure at each stage of pregnancy as a predictor of infant mental development. Environ Health Perspect 114(11):1730-1735. http://doi.org/10.1289/ehp.9067.

Hu H, Shih R, Rothenberg S, et al. 2007. The epidemiology of lead toxicity in adults: Measuring dose and consideration of other methodologic issues. Environ Health Perspect 115(3):455-462. http://doi.org/10.1289/ehp.9783.

Huang XP, Feng ZY, Zhai WL, et al. 1988. Chromosomal aberrations and sister chromatid exchanges in workers exposed to lead. Biomed Environ Sci 1:382-387.

Huang J, Wu J, Li T, et al. 2011. Effect of exposure to trace elements in the soil on the prevalence of neural tube defects in a high-risk area of China. Biomed Environ Sci 24(2):94-101. http://doi.org/10.3967/0895-3988.2011.02.002.

Huang S, Hu H, Sanchez BN, et al. 2016. Childhood blood lead levels and symptoms of Attention Deficit Hyperactivity Disorder (ADHD): A cross-sectional study of Mexican children. Environ Health Perspect 124(6):868-874. http://doi.org/10.1289/ehp.1510067.

HUD. 2011. American health homes survey. Lead and arsenic findings. U.S. Department of Housing and Urban Development. https://portal.hud.gov/hudportal/documents/huddoc\%3Fid=AHHS_Report.pdf. July 12, 2017.

HUD. 2017. Revised dust-lead action levels for risk assessment and clearance; clearance of porch floors. Washington, DC: U.S. Department of Housing and Urban Development. https://portal.hud.gov/hudportal/documents/huddoc?id=leaddustclearance.pdf. August 25, 2017.

Huel G, Sahuquillo J, Debotte G, et al. 2008. Hair mercury negatively correlates with calcium pump activity in human term newborns and their mothers at delivery. Environ Health Perspect 116(2):263267. http://doi.org/10.1289/ehp.10381.

Huh DA, Choi YH, Ji MS, et al. 2018. Comparison of pure-tone average methods for estimation of hearing loss caused by environmental exposure to lead and cadmium: Does the pure-tone average method which uses low-frequency ranges underestimate the actual hearing loss caused by environmental lead and cadmium exposure? Audiol Neurotol 23(5):259-269. http://doi.org/10.1159/000494049.

Hui CA. 2002. Lead distribution throughout soil, flora, and an invertebrate at a wetland skeet range. J Toxicol Environ Health A 65:1093-1107. http://doi.org/10.1080/0098410029007128 9.

Hunt A, Johnson DL, Thornton I, et al. 1993. Apportioning the sources of lead in house dusts in the London borough of Richmond, England. Sci Total Environ 138(1-3):183-206.

Huo X, Peng L, Qiu B, et al. 2014. ALAD genotypes and blood lead levels of neonates and children from e-waste exposure in Guiyu, China. Environ Sci Pollut Res Int 21(10):6744-6750. http://doi.org/10.1007/s11356-014-2596-2.

Hursh JB, Suomela J. 1968. Absorption of $212 \mathrm{~Pb}$ from the gastrointestinal tract of man. Acta Radiol Ther Phys Biol 7(2):108-120. http://doi.org/10.3109/02841866809133184.

Hursh J, Mercer T. 1970. Measurement of 212Pb loss rate from human lungs. J Appl Physiol 28(3):268274.

Hursh JB, Schraub A, Sattler EL, et al. 1969. Fate of 212Pb inhaled by human subjects. Health Phys 16:257-267. 


\section{REFERENCES}

Hursh JB, Clarkson TW, Miles EF, et al. 1989. Percutaneous absorption of mercury vapor by man. Arch Environ Health 44(2):120-127.

Hwang YH, Chiang HY, Yen-Jean MC, et al. 2009. The association between low levels of lead in blood and occupational noise-induced hearing loss in steel workers. Sci Total Environ 408(1):43-49. http://doi.org/10.1016/j.scitotenv.2009.09.016.

Hytten F. 1985. Blood volume changes in normal pregnancy. Clin Haematol 14(3):601-612.

IARC. 1987. Lead and lead compounds. In: IARC monographs on the evaluation of carcinogenic risks to humans. Supplement 7. Overall evaluations of carcinogenicity: An updating of IARC monographs volumes 1 to 42. Lyon, France: International Agency for Research on Cancer, 230232. http://monographs.iarc.fr/ENG/Monographs/suppl7/Suppl7-95.pdf. May 4, 2017.

IARC. 2006. IARC Monographs on the evaluation of carcinogenic risks to humans. Volume 87. Inorganic and organic lead compounds. Lyon, France: International Agency for Research on Cancer. http://monographs.iarc.fr/ENG/Monographs/vol87/mono87.pdf. May 4, 2017.

IARC. 2019. Agents classified by the IARC Monographs, Volumes 1-124. Lyon, France: International Agency for Research on Cancer. https://monographs.iarc.fr/list-of-classifications. September 2, 2019.

ICRP. 1994. Human respiratory tract model for radiological protection. International Commission on Radiological Protection. 36-53; 72-77; Annex F 415-432.

Ignasiak Z, Slawinska T, Rozek K, et al. 2006. Lead and growth status of schoolchildren living in the copper basin of south-western Poland: Differential effects on bone growth. Ann Hum Biol 33(4):401-414. http://doi.org/10.1080/03014460600730752.

Iijima K, Otake T, Yoshinaga J, et al. 2007. Cadmium, lead, and selenium in cord blood and thyroid hormone status of newborns. Biol Trace Elem Res 119(1):10-18. http://doi.org/10.1007/s12011007-0057-1.

Inskip MJ, Franklin CA, Baccanale CL, et al. 1996. Measurement of the flux of lead from bone to blood in a nonhuman primate (Macaca fascicularis) by sequential administration of stable lead isotopes. Fundam Appl Toxicol 33:235-245.

Ionescu JG, Novotny J, Stejskal V, et al. 2007. Breast tumours strongly accumulate transition metals. Maedica 2(1):5-9.

Irgens A, Kruger K, Skorve AH, et al. 1998. Reproductive outcome in offspring of parents occupationally exposed to lead in Norway. Am J Ind Med 34(5):431-437. http://doi.org/10.1002/(sici)1097-0274(199811)34:5<431::aid-ajim3>3.0.co;2-t.

IRIS. 2002. Tetraethyl lead; CASRN 78-00-2. Integrated Risk Information System. Washington, DC: U.S. Environmental Protection Agency. https://cfpub.epa.gov/ncea/iris/iris_documents/documents/subst/0109_summary.pdf. May 4, 2017.

IRIS. 2004. Lead and compounds (inorganic); CASRN 7439-92-1. Integrated Risk Information System. Washington, DC: U.S. Environmental Protection Agency. https://cfpub.epa.gov/ncea/iris/iris_documents/documents/subst/0277_summary.pdf. May 4, 2017.

Irvine G, Doyle JR, White PA, et al. 2014. Soil ingestion rate determination in a rural population of Alberta, Canada practicing a wilderness lifestyle. Sci Total Environ 470-471:138-146. http://doi.org/10.1016/j.scitotenv.2013.09.037.

Irwinda R, Wibowo N, Putri AS. 2019. The concentration of micronutrients and heavy metals in maternal serum, placenta, and cord blood: A cross-sectional study in preterm birth. J Pregnancy 2019:5062365. http://doi.org/10.1155/2019/5062365.

Iwata T, Yano E, Karita K, et al. 2005. Critical dose of lead affecting postural balance in workers. Am J Ind Med 48(5):319-325. http://doi.org/10.1002/ajim.20220.

Jackson LW, Cromer BA, Panneerrselvamm A. 2010. Association between bone turnover, micronutrient intake, and blood lead levels in pre- and postmenopausal women, NHANES 1999-2002. Environ Health Perspect 118(11):1590-1596. 
Jackson LW, Howards PP, Wactawski-Wende J, et al. 2011. The association between cadmium, lead and mercury blood levels and reproductive hormones among healthy, premenopausal women. Hum Reprod 26(10):2887-2895. http://doi.org/10.1093/humrep/der250.

Jacobs DE. 2012. Lead. In: Patty's toxicology. John Wiley \& Sons, Inc., 381-426. http://doi.org/10.1002/0471435139.tox034.pub2.

Jaeger RJ, Weiss AL, Manton WI. 1998. Isotopic ratio analysis in residential lead-based paint and associated surficial dust. J Toxicol Clin Toxicol 36(7):691-703.

Jaffe EK, Volin M, Bronson-Mullins CR, et al. 2000. An artificial gene for human porphobilinogen synthase allows comparison of an allelic variation implicated in susceptibility to lead poisoning. J Biol Chem 275(4):2619-2626.

Jaffe EK, Martins J, Li J, et al. 2001. The molecular mechanism of lead inhibition of human porphobilinogen synthase. J Biol Chem 276(2):1531-1537. http://doi.org/10.1074/jbc.M007663200.

Jain RB. 2013a. Effect of pregnancy on the levels of blood cadmium, lead, and mercury for females aged 17-39 years old: Data from National Health and Nutrition Examination Survey 2003-2010. J Toxicol Environ Health A 76(1):58-69. http://doi.org/10.1080/15287394.2012.722524.

Jain RB. 2013b. Effect of pregnancy on the levels of urinary metals for females aged 17-39 years old: Data from National Health and Nutrition Examination Survey 2003-2010. J Toxicol Environ Health A 76(2):86-97.

Jain NB, Potula V, Schwartz J, et al. 2007. Lead levels and ischemic heart disease in a prospective study of middle-aged and elderly men: The VA Normative Aging Study. Environ Health Perspect 115(6):871-875. http://doi.org/10.1289/ehp.9629.

James HM, Hilburn ME, Blair JA. 1985. Effects of meals and meal times on uptake of lead from the gastrointestinal tract in humans. Hum Toxicol 4:401-407.

James AC, Stahlhofen W, Rudolf G, et al. 1994. Deposition of inhaled particles. Ann ICRP 24(13):231-299.

Janakiraman V, Ettinger A, Mercado-Garcia A, et al. 2003. Calcium supplements and bone resorption in pregnancy: A randomized crossover trial. Am J Prev Med 24(3):260-264.

Janin Y, Couinaud C, Stone A, et al. 1985. The "Lead-induced colic" syndrome in lead intoxication. Surg Annu 17:287-307.

Jannuzzi AT, Alpertunga B. 2016. Evaluation of DNA damage and DNA repair capacity in occupationally lead-exposed workers. Toxicol Ind Health 32(11):1859-1865. http://doi.org/10.1177/0748233715590919.

Jasso-Pineda Y, Diaz-Barriga F, Calderon J, et al. 2012. DNA damage and decreased DNA repair in individuals exposed to arsenic and lead in a mining site. Biol Trace Elem Res 146(2):141-149.

Jedrychowski W, Perera FP, Jankowski J, et al. 2009. Very low prenatal exposure to lead and mental development of children in infancy and early childhood: Krakow prospective cohort study. Neuroepidemiology 32(4):270-278. http://doi.org/10.1159/000203075.

Jedrychowski W, Perera F, Maugeri U, et al. 2011. Intrauterine exposure to lead may enhance sensitization to common inhalant allergens in early childhood: A prospective prebirth cohort study. Environ Res 111(1):119-124. http://doi.org/10.1016/j.envres.2010.11.002.

Jelliffe-Pawlowski LL, Miles SQ, Courtney JG, et al. 2006. Effect of magnitude and timing of maternal pregnancy blood lead $(\mathrm{Pb})$ levels on birth outcomes. J Perinatol 26(3):154-162. http://doi.org/10.1038/sj.jp.7211453.

Jemal A, Graubard BI, Devesa SS, et al. 2002. The association of blood lead level and cancer mortality among whites in the United States. Environ Health Perspect 110(4):325-329.

Jhun MA, Hu H, Schwartz J, et al. 2015. Effect modification by vitamin D receptor genetic polymorphisms in the association between cumulative lead exposure and pulse pressure: A longitudinal study. Environ Health 14:5. http://doi.org/10.1186/1476-069x-14-5.

Ji JS, Elbaz A, Weisskopf MG. 2013. Association between blood lead and walking speed in the National Health and Nutrition Examination Survey (NHANES 1999-2002). Environ Health Perspect 121(6):711-716. http://doi.org/10.1289/ehp.1205918. 


\section{REFERENCES}

Ji JS, Schwartz J, Sparrow D, et al. 2014. Occupational determinants of cumulative lead exposure: Analysis of bone lead among men in the VA normative aging study. J Occup Environ Med 56(4):435-440. http://doi.org/10.1097/jom.0000000000000127.

Ji JS, Power MC, Sparrow D, et al. 2015. Lead exposure and tremor among older men: The VA normative aging study. Environ Health Perspect 123(5):445-450. http://doi.org/10.1289/ehp.1408535.

Ji Y, Hong X, Wang G, et al. 2018. A prospective birth cohort study on early childhood lead levels and attention deficit hyperactivity disorder: New insight on sex differences. J Pediatr 199:124-131.e128. http://doi.org/10.1016/j.jpeds.2018.03.076.

Jiang YM, Long LL, Zhu XY, et al. 2008. Evidence for altered hippocampal volume and brain metabolites in workers occupationally exposed to lead: A study by magnetic resonance imaging and 1H magnetic resonance spectroscopy. Toxicol Lett 181(2):118-125. http://doi.org/10.1016/j.toxlet.2008.07.009.

Jin CW, Zhang SJ, He YF, et al. 2005. Lead contamination in tea garden soils and factors affecting its bioavailability. Chemosphere 59:1151-1159. http://doi.org/10.1016/j.chemosphere.2004.11.058.

Jin YP, Liao YJ, Lu CW, et al. 2006. Health effects in children aged 3-6 years induced by environmental lead exposure. Ecotoxicol Environ Saf 63(2):313-317. http://doi.org/10.1016/j.ecoenv.2005.05.011.

Jin C, Li Y, Li YL, et al. 2008. Blood lead: Its effect on trace element levels and iron structure in hemoglobin. Nucl Instrum Methods Phys Res B 266(16):3607-3613. http://doi.org/10.1016/j.nimb.2008.05.087.

Jing J, Thapa S, Delhey L, et al. 2019. The relation of blood lead and QRS-T angle in American adults. Arch Environ Occup Health 74(5):287-291. http://doi.org/10.1080/19338244.2018.1488674.

Johansen P, Pedersen HS, Asmund G, et al. 2006. Lead shot from hunting as a source of lead in human blood. Environ Pollut 142:93-97.

Johnson DL, Bretsch JK. 2002. Soil lead and children's blood lead levels in Syracuse, NY, USA. Environ Geochem Health 24:375-385.

Johnson DL, McDade K, Griffith D. 1996. Seasonal variation in paediatric blood lead levels in Syracuse, NY, USA. Environ Geochem Health 18(2):81-88. http://doi.org/10.1007/bf01771136.

Jones SR, Atkin P, Holroyd C, et al. 2007. Lung cancer mortality at a UK tin smelter. Occup Med (Lond) 57(4):238-245. http://doi.org/10.1093/occmed/kql153.

Joo H, Lim MH, Ha M, et al. 2017. Secondhand smoke exposure and low blood lead levels in association with attention-deficit hyperactivity disorder and its symptom domain in children: A community-based case-control study. Nicotine Tob Res 19(1):94-101. http://doi.org/10.1093/ntr/ntw152.

Joo H, Choi JH, Burm E, et al. 2018. Gender difference in the effects of lead exposure at different time windows on neurobehavioral development in 5-year-old children. Sci Total Environ 615:1086-1092. http://doi.org/10.1016/j.scitotenv.2017.10.007.

Jorgensen S, Willems M. 1987. The fate of lead of soil: The transformation of lead pellets in shootingrange soils. Ambio 16(1):11-15.

Jorgensen TH, De Backer O, Gerds TA, et al. 2018. Immediate post-procedural 12-lead electrocardiography as predictor of late conduction defects after transcatheter aortic valve replacement. JACC Cardiovasc Interv 11(15):1509-1518. http://doi.org/10.1016/j.jcin.2018.04.011.

Joseph CLM, Havstad S, Ownby DR, et al. 2005. Blood lead level and risk of asthma. Environ Health Perspect 113(7):900-904. http://doi.org/10.1289/ehp.7453.

Juhasz AL, Weber J, Smith E. 2011. Impact of soil particle size and bioaccessibility on children and adult lead exposure in peri-urban contaminated soil. J Hazard Mater 186(2-3):1870-1879. http://doi.org/10.1016/j.jhazmat.2010.12.095.

Juhasz AL, Weber J, Smith E, et al. 2009. Evaluation of SBRC-gastric and SBRC-intestinal methods for the prediction of in vivo relative lead bioavailability in contaminated soils. Environ Sci Technol 43(12):4503-4509. http://doi.org/10.1021/es803238u. 


\section{REFERENCES}

Juhasz AL, Scheckel KG, Betts AR, et al. 2016. Predictive capabilities of in vitro assays for estimating $\mathrm{Pb}$ relative bioavailability in phosphate amended soils. Environ Sci Technol 50(23):13086-13094. http://doi.org/10.1021/acs.est.6b04059.

Jurgens BC, Parkhurst DL, Belitz K. 2019. Assessing the lead solubility potential of untreated groundwater of the United States. Environ Sci Technol 53(6):3095-3103. http://doi.org/10.1021/acs.est.8b04475.

Jusko TA, Lockhart DW, Sampson PD, et al. 2006. Response to: "What is the meaning of non-linear dose-response relationships between blood lead concentrations and IQ?". Neurotoxicology 27(6):1123-1125. http://doi.org/10.1016/j.neuro.2006.09.004.

Jusko TA, Henderson CR, Lanphear BP, et al. 2008. Blood lead concentrations $<10 \mu \mathrm{g} / \mathrm{dL}$ and child intelligence at 6 years of age. Environ Health Perspect 116(2):243-248. http://doi.org/10.1289/ehp.10424.

Kahn LG, Liu X, Rajovic B, et al. 2014. Blood lead concentration and thyroid function during pregnancy: Results from the Yugoslavia prospective study of environmental lead exposure. Environ Health Perspect 122(10):1134-1140. http://doi.org/10.1289/ehp.1307669.

Kalahasthi R, Barman T. 2016. Effect of lead exposure on the status of reticulocyte count indices among workers from lead battery manufacturing plant. Toxicol Res 32(4):281-287. http://doi.org/10.5487/tr.2016.32.4.281.

Kamel F, Umbach DM, Munsat TL, et al. 2002. Lead exposure and amyotrophic lateral sclerosis. Epidemiology 13:311-319.

Kang HG, Jeong SH, Cho MR, et al. 2009. Time-dependent changes in lead and delta-aminolevulinic acid after subchronic lead exposure in rats. Hum Exp Toxicol 28(10):647-654. http://doi.org/10.1177/0960327109107046.

Kang GH, Uhm JY, Choi YG, et al. 2018. Environmental exposure of heavy metal (lead and cadmium) and hearing loss: Data from the Korea National Health and Nutrition Examination Survey (KNHANES 2010-2013). Ann Occup Environ Med 30:22. http://doi.org/10.1186/s40557-0180237-9.

Kapuku GK, Harshfield GA, Davis HC, et al. 2006. Early markers of cardiovascular disease. Vascul Pharmacol 45(5):277-280. http://doi.org/10.1016/j.vph.2006.08.009.

Karakulak UN, Yilmaz OH, Tutkun E, et al. 2017. Evaluation of the ambulatory arterial stiffness index in lead-exposed workers. Anatol J Cardiol 18(1):10-14. http://doi.org/10.14744/AnatolJCardiol.2017.7170.

Karakulak UN, Okutucu S, Lokman U, et al. 2019. Evaluation of erectile dysfunction and left ventricular diastolic parameters in lead exposed workers. Acta Cardiol Sin 35(1):75-84. http://doi.org/10.6515/acs.201901_35(1).20180716a.

Karimooy HN, Mood MB, Hosseini M, et al. 2010. Effects of occupational lead exposure on renal and nervous system of workers of traditional tile factories in Mashhad (northeast of Iran). Toxicol Ind Health 26(9):633-638. http://doi.org/10.1177/0748233710377774.

Karita K, Yano E, Dakeishi M, et al. 2005. Benchmark dose of lead inducing anemia at the workplace. Risk Anal 25(4):957-962. http://doi.org/10.1111/j.1539-6924.2005.00652.x.

Karmaus W, Brooks KR, Nebe T, et al. 2005. Immune function biomarkers in children exposed to lead and organochlorine compounds: A cross-sectional study. Environ Health 4(1):5. http://doi.org/10.1186/1476-069X-4-5.

Kasperczyk A, Kasperczyk S, Horak S, et al. 2008. Assessment of semen function and lipid peroxidation among lead exposed men. Toxicol Appl Pharmacol 228(3):378-384. http://doi.org/10.1016/j.taap.2007.12.024.

Kasperczyk S, Blaszczyk I, Dobrakowski M, et al. 2013. Exposure to lead affects male biothiols metabolism. Ann Agric Environ Med 20(4):721-725.

Kasperczyk A, Dobrakowski M, Czuba ZP, et al. 2015. Environmental exposure to lead induces oxidative stress and modulates the function of the antioxidant defense system and the immune 


\section{REFERENCES}

system in the semen of males with normal semen profile. Toxicol Appl Pharmacol 284(3):339-344. http://doi.org/10.1016/j.taap.2015.03.001.

Kasprzak KS, Hoover KL, Poirier LA. 1985. Effects of dietary calcium acetate on lead subacetate carcinogenicity in kidneys of male Sprague-Dawley rats. Carcinogenesis 6(2):279-282.

Kasuba V, Rozgaj R, Milic M, et al. 2012. Evaluation of genotoxic effects of lead in pottery-glaze workers using micronucleus assay, alkaline comet assay and DNA diffusion assay. Int Arch Occup Environ Health 85(7):807-818. http://doi.org/10.1007/s00420-011-0726-4.

Kauppinen T, Riala R, Seitsamo J, et al. 1992. Primary liver cancer and occupational exposure. Scand J Work Environ Health 18(1):18-25.

Kayaalti Z, Yavuz I, Soylemez E, et al. 2015a. Evaluation of DNA damage using 3 comet assay parameters in workers occupationally exposed to lead. Arch Environ Occup Health 70(3):120-125. http://doi.org/10.1080/19338244.2013.787964.

Kayaalti Z, Kaya-Akyuzlu D, Soylemez E, et al. 2015b. Maternal hemochromatosis gene H63D singlenucleotide polymorphism and placental lead levels. Environ Res 140:456-461.

Kayaalti Z, Sert S, Kaya-Akyuzlu D, et al. 2016. Association between delta-aminolevulinic acid dehydratase polymorphism and placental lead levels. Environ Toxicol Pharmacol 41:147-151. http://doi.org/10.1016/j.etap.2015.11.017.

Kaye WE, Novotny TE, Tucker M. 1987. New ceramics-related industry implicated in elevated blood lead levels in children. Arch Environ Health 42(2):161-164.

Kazi TG, Shah F, Shaikh HR, et al. 2014. Exposure of lead to mothers and their new born infants, residents of industrial and domestic areas of Pakistan. Environ Sci Pollut Res Int 21(4):3021-3030. http://doi.org/10.1007/s11356-013-2223-7.

Kehoe RA. 1987. Studies of lead administration and elimination in adult volunteers under natural and experimentally induced condition over extended periods of time. Food Chem Toxicol 25(6):425494.

Kehoe RA, Thamann F. 1931. The behavior of lead in the animal organism, II. Tetraethyl lead. Am J Hyg 13:478-498.

Kelly RS, Lundh T, Porta M, et al. 2013. Blood erythrocyte concentrations of cadmium and lead and the risk of B-cell non-Hodgkin's lymphoma and multiple myeloma: A nested case-control study. PLoS ONE 8(11):e81892. http://doi.org/10.1371/journal.pone.0081892.

Kemp FW, Neti PVSV, Howell RW, et al. 2007. Elevated blood lead concentrations and vitamin D deficiency in winter and summer in young urban children. Environ Health Perspect 115(4):630-635. http://doi.org/10.1289/ehp.9389.

Kerper LE, Hinkle PM. 1997a. Lead uptake in brain capillary endothelial cells: Activation by calcium store depletion. Toxicol Appl Pharmacol 146(1):127-133.

Kerper LE, Hinkle PM. 1997b. Cellular uptake of lead is activated by depletion of intracellular calcium stores. J Biol Chem 272(13):8346-8352.

Kerr BT, Ochs-Balcom HM, Lopez P, et al. 2019. Effects of ALAD genotype on the relationship between lead exposure and anthropometry in a Cohort of Mexican children. Environ Res 170:65-72. http://doi.org/10.1016/j.envres.2018.12.003.

Khalil N. 2010. Re: Blood lead mortality study: Khalil et al., 2009. Integrated science assessment for lead: Supporting documents: Supplemental data from Dr. Khalil. Docket ID: EPA-HQ-ORD-20110051. http://www.regulations.gov/\#!documentDetail;D=EPA-HQ-ORD-2011-0051-0006. February 25, 2020.

Khalil N, Cauley JA, Wilson JW, et al. 2008. Relationship of blood lead levels to incident nonspine fractures and falls in older women: The Study of Osteoporotic Fractures. J Bone Miner Res 23(9):1417-1425. http://doi.org/10.1359/jbmr.080404.

Khalil N, Wilson JW, Talbott EO, et al. 2009. Association of blood lead concentrations with mortality in older women: A prospective cohort study. Environmental Health: A Global Access Science Source 8:15. http://doi.org/10.1186/1476-069x-8-15. 
Khan DH, Frankland B. 1983. Chemical forms of cadmium and lead in some contaminated soils. Environ Pollut Ser B 6:15-31.

Khan DA, Qayyum S, Saleem S, et al. 2008. Lead-induced oxidative stress adversely affects health of the occupational workers. Toxicol Ind Health 24(9):611-618. http://doi.org/10.1177/0748233708098127.

Khan D, Qayyum S, Saleem S, et al. 2010a. Lead exposure and its adverse health effects among occupational worker's children. Toxicol Ind Health 26(8):497. http://doi.org/10.1177/0748233710373085.

Khan MI, Ahmad I, Mahdi AA, et al. 2010b. Elevated blood lead levels and cytogenetic markers in buccal epithelial cells of painters in India: Genotoxicity in painters exposed to lead containing paints. Environ Sci Pollut Res Int 17(7):1347-1354. http://doi.org/10.1007/s11356-010-0319-x.

Kieltucki J, Dobrakowski M, Pawlas N, et al. 2017. The analysis of QT interval and repolarization morphology of the heart in chronic exposure to lead. Hum Exp Toxicol 36(10):1081-1086. http://doi.org/10.1177/0960327116680277.

Kim KN, Kwon HJ, Hong YC. 2016. Low-level lead exposure and autistic behaviors in school-age children. Neurotoxicology 53:193-200. http://doi.org/10.1016/j.neuro.2016.02.004.

Kim R, Hu H, Rotnitzky A, et al. 1995. A longitudinal study of chronic lead exposure and physical growth in Boston children. Environ Health Perspect 103(10):952-957.

Kim R, Rotnitzky A, Sparrow D, et al. 1996a. A longitudinal study of low-level lead exposure and impairment of renal function: The normative aging study. J Am Med Assoc 275(15):1177-1181.

Kim R, Hu H, Rotnitzky A, et al. 1996b. Longitudinal relationship between dentin lead levels in childhood and bone lead levels in young adulthood. Arch Environ Health 51(5):375-382.

Kim R, Landrigan C, Mossmann P, et al. 1997. Age and secular trends in bone lead levels in middleaged and elderly men: Three-year longitudinal follow-up in the normative aging study. Am J Epidemiol 146(4):586-591.

Kim HS, Lee SS, Lee GS, et al. 2004. The protective effect of delta-aminolevulinic acid dehydratase 1-2 and 2-2 isozymes against blood lead with higher hematologic parameters. Environ Health Perspect 112:538-541.

Kim JH, Lee KH, Yoo DH, et al. 2007. GSTM1 and TNF-alpha gene polymorphisms and relations between blood lead and inflammatory markers in a non-occupational population. Mutat Res 629(1):32-39. http://doi.org/10.1016/j.mrgentox.2007.01.004.

Kim S, Arora M, Fernandez C, et al. 2013a. Lead, mercury, and cadmium exposure and attention deficit hyperactivity disorder in children. Environ Res 126:105-110.

Kim Y, Ha EH, Park H, et al. 2013b. Prenatal lead and cadmium co-exposure and infant neurodevelopment at 6 months of age: The Mothers and Children's Environmental Health (MOCEH) study. Neurotoxicology 35:15-22. http://doi.org/10.1016/j.neuro.2012.11.006.

Kim MG, Ryoo JH, Chang SJ, et al. 2015. Blood Lead levels and cause-specific mortality of inorganic lead-exposed workers in South Korea. PLoS ONE 10(10):e0140360. http://doi.org/10.1371/journal.pone.0140360.

Kim YS, Ha M, Kwon HJ, et al. 2017a. Association between low blood lead levels and increased risk of dental caries in children: A cross-sectional study. BMC Oral Health 17(1):42. http://doi.org/10.1186/s12903-017-0335-z.

Kim JH, Park Y, Kim SK, et al. 2017b. Timing of an accelerated body mass increase in children exposed to lead in early life: A longitudinal study. Sci Total Environ 584-585:72-77. http://doi.org/10.1016/j.scitotenv.2017.01.122.

Kimber I, Stonard MD, Gidlow DA, et al. 1986. Influence of chronic low-level exposure to lead on plasma immunoglobulin concentration and cellular immune function in man. Int Arch Occup Environ Health 57:117-125.

King M, Ramachandran V, Prengaman RD, et al. 2014. Lead and lead alloys. In: Kirk-Othmer encyclopedia of chemical technology. Hoboken, NJ: Wiley-Blackwell. http://doi.org/10.1002/0471238961.1205010411091407.a01.pub3. 
Kirkby H, Gyntelberg F. 1985. Blood pressure and other cardiovascular risk factors of long-term exposure to lead. Scand J Work Environ Health 11(1):15-19. http://doi.org/10.5271/sjweh.2259.

Klaassen C. 2001. Heavy metals and heavy metal antagonists. In: Hardman JG, Limbard LE, eds. Goodman and Gilman's The pharmacological basis of therapeutics. 10th ed. New York, NY: McGraw-Hill Companies, 1851-1875.

Klaassen CD, Shoeman DW. 1974. Biliary excretion of lead in rats, rabbits and dogs. Toxicol Appl Pharmacol 29:434-446.

Koh DS, Koh GC. 2007. The use of salivary biomarkers in occupational and environmental medicine. Occup Environ Med 64:202-210. http://doi.org/10.1136/oem.2006.026567.

Koller LD, Kerkvliet NI, Exon JH. 1985. Neoplasia induced in male rats fed lead acetate, ethyl urea, and sodium nitrite. Toxicol Pathol 13(1):50-57.

Kordas K, Canfield RL, Lopez P, et al. 2006. Deficits in cognitive function and achievement in Mexican first-graders with low blood lead concentrations. Environ Res 100(3):371-386. http://doi.org/10.1016/j.envres.2005.07.007.

Kordas K, Ettinger AS, Lamadrid-Figueroa H, et al. 2009. Methylenetetrahydrofolate reductase (MTHFR) C677T, A1298C and G1793A genotypes, and the relationship between maternal folate intake, tibia lead and infant size at birth. Br J Nutr 102(6):907-914. http://doi.org/10.1017/s0007114509318280.

Kordas K, Queirolo EL, Ettinger AS, et al. 2010. Prevalence and predictors of exposure to multiple metals in preschool children from Monetvideo, Uraguay. Sci Total Environ 408:4488-4494.

Kordas K, Ettinger AS, Bellinger DC, et al. 2011. A dopamine receptor (DRD2) but not dopamine transporter (DAT1) gene polymorphism is associated with neurocognitive development of Mexican preschool children with lead exposure. J Pediatr 159(4):638-643. http://doi.org/10.1016/j.jpeds.2011.03.043.

Korrick SA, Hunter DJ, Rotnitzky A, et al. 1999. Lead and hypertension in a sample of middle-aged women. Am J Public Health 89(3):330-335.

Korrick SA, Schwartz J, Tsaih SW, et al. 2002. Correlates of bone and blood lead levels among middleaged and elderly women. Am J Epidemiol 156(4):335-343.

Kosnett MJ. 2001. Lead. In: Ford M, Delaney KA, Ling L, et al., eds. Clinical toxicology. St. Louis, MO: WB Saunders, 723-736.

Kosnett MJ. 2005. Lead. In: Brent J, Wallace KL, Burkhart KK, et al., eds. Critical care toxicology: Diagnosis and management of the critically poisoned patient. Philadelphia, PA: Elsevier Mosby, 821-836.

Kosnett MJ, Becker CE, Osterloh JD, et al. 1994. Factors influencing bone lead concentration in a suburban community assessed by noninvasive K x-ray fluorescence. J Am Med Assoc 271(3):197203.

Kosnett MJ, Wedeen RP, Rothenberg SJ, et al. 2007. Recommendations for medical management of adult lead exposure. Environ Health Perspect 115(3):463-471. http://doi.org/10.1289/ehp.9784.

Kostial K, Kello D, Jugo S, et al. 1978. Influence of age on metal metabolism and toxicity. Environ Health Perspect 25:81-86.

Koyashiki GAK, Paoliello MMB, Matsuo T, et al. 2010. Lead levels in milk and blood from donors to the breast milk bank in southern Brazil. Environ Res 110:265-271.

Kresovich JK, Argos M, Turyk ME. 2015. Associations of lead and cadmium with sex hormones in adult males. Environ Res 142:25-33. http://doi.org/10.1016/j.envres.2015.05.026.

Krieg EF. 2007. The relationships between blood lead levels and serum follicle stimulating hormone and luteinizing hormone in the third national health and nutrition examination survey. Environ Res 104(3):374-382. http://doi.org/10.1016/j.envres.2006.09.009.

Krieg EF, Chrislip DW, Crespo CJ, et al. 2005. The relationship between blood lead levels and neurobehavioral test performance in NHANES III and related occupational studies. Public Health Rep 120(3):240-251. 


\section{REFERENCES}

Krieg EF, Butler MA, Chang M, et al. 2009. Lead and cognitive function in ALAD genotypes in the Third National Health and Nutrition Examination Survey. Neurotoxicol Teratol 31(6):364-371. http://doi.org/10.1016/j.ntt.2009.08.003.

Krieg EF, Butler MA, Chang M, et al. 2010. Lead and cognitive function in VDR genotypes in the Third National Health and Nutrition Examination Survey. Neurotoxicol Teratol 32(2):262-272. http://doi.org/10.1016/j.ntt.2009.12.004.

Krishnan K, Anderson ME, Clewell HJ, et al. 1994. Physiologically based pharmacokinetic modeling of chemical mixtures. In: Yang RSH, ed. Toxicology of chemical mixtures. Case studies, mechanisms, and novel approaches. San Diego, CA: Academic Press, 399-437.

Kristal-Boneh E, Coller D, Froom P, et al. 1999. The association between occupational lead exposure and serum cholesterol and lipoprotein levels. Am J Public Health 89(7):1083-1087.

Kromhout D. 1988. Blood lead and coronary heart disease risk among elderly men in Zutphen, The Netherlands. Environ Health Perspect 78:43-46.

Kromhout D, Wibowo A, Herber R, et al. 1985. Trace metals and coronary heart disease risk indicators in 152 elderly men (the Zutphen Study). Am J Epidemiol 122(3):378-385.

Krueger WS, Wade TJ. 2016. Elevated blood lead and cadmium levels associated with chronic infections among non-smokers in a cross-sectional analysis of NHANES data. Environ Health 15(1):16. http://doi.org/10.1186/s12940-016-0113-4.

Kumar BD, Krishnaswamy K. 1995. Detection of occupational lead nephropathy using early renal markers. J Toxicol Clin Toxicol 33(4):331-335.

Kuruvilla A, Pillay VV, Adhikari P, et al. 2006. Clinical manifestations of lead workers of Mangalore, India. Toxicol Ind Health 22(9):405-413.

Lagerkvist BJ, Ekesrydh S, Englyst V, et al. 1996. Increased blood lead and decreased calcium levels during pregnancy: A prospective study of Swedish women living near a smelter. Am J Public Health 86(9):1247-1252.

LaGoy PK. 1987. Estimated soil ingestion rates for use in risk assessment. Risk Anal 7(3):355-359.

Laidlaw MAS, Filippelli GM. 2008. Resuspension of urban soils as a persistent source of lead poisoning in children: A review and new directions. Appl Geochem 23(8):2021-2039. http://doi.org/10.1016/j.apgeochem.2008.05.009.

Laidlaw MA, Mielke HW, Filippelli GM, et al. 2005. Seasonality and children's blood lead levels: Developing a predictive model using climatic variables and blood lead data from Indianapolis, Indiana, Syracuse, New York, and New Orleans, Louisiana (USA). Environ Health Perspect 113(6):793-800.

Laidlaw MAS, Zahran S, Mielke HW, et al. 2012. Re-suspension of lead contaminated urban soil as a dominant source of atmospheric lead in Birmingham, Chicago, Detroit and Pittsburgh, USA. Atmos Environ 49:302-310. http://doi.org/10.1016/j.atmosenv.2011.11.030.

La-Llave-Leon O, Mendez-Hernandez EM, Castellanos-Juarez FX, et al. 2017. Association between blood lead levels and delta-aminolevulinic acid dehydratase in pregnant women. Int J Environ Res Public Health 14(4):432. http://doi.org/10.3390/ijerph14040432.

Lamadrid-Figueroa H, Téllez-Rojo MM, Hernández-Cadena L, et al. 2006. Biological markers of fetal lead exposure at each stage of pregnancy. J Toxicol Environ Health A 69(19):1781-1796. http://doi.org/10.1080/15287390600630195.

Lamadrid-Figueroa H, Téllez-Rojo MM, Hernández-Avila M, et al. 2007. Association between the plasma/whole blood lead ratio and history of spontaneous abortion: A nested cross-sectional study. BMC Pregnancy Childbirth 7:22. http://doi.org/10.1186/1471-2393-7-22.

Lamb MR, Janevic T, Liu X, et al. 2008. Environmental lead exposure, maternal thyroid function, and childhood growth. Environ Res 106(2):195-202. http://doi.org/10.1016/j.envres.2007.09.012.

Lancranjan I, Popescu HI, Gavanescu O, et al. 1975. Reproductive ability of workmen occupationally exposed to lead. Arch Environ Health 30:396-401.

Landrigan PJ. 1989. Toxicity of lead at low dose - Editorial. Br J Ind Med 46:593-596. 
Landrigan PJ, Baker EL, Feldman RG, et al. 1976. Increased lead absorption with anemia and slowed nerve conduction in children near a lead smelter. J Pediatr 89(6; 6):904-910.

Langlois P, Smith L, Fleming S, et al. 1996. Blood lead levels in Toronto children and abatement of lead-contaminated soil and house dust. Arch Environ Health 51(1):59-67.

Lanphear BP, Roghmann KJ. 1997. Pathways of lead exposure in urban children. Environ Res 74:6773.

Lanphear BP, Weitzman M, Eberly S. 1996a. Racial differences in urban children's environmental exposures to lead. Am J Public Health 86:1460-1463.

Lanphear BP, Eberly S, Howard CR. 2000b. Long-term effect of dust control on blood lead concentrations. Pediatrics 106(4):1-4.

Lanphear BP, Weitzman M, Winter NL, et al. 1996b. Lead-contaminated house dust and urban children's blood lead levels. Am J Public Health 86:1416-1421.

Lanphear BP, Burgoon DA, Rust SW, et al. 1998a. Environmental exposures to lead and urban children's blood lead levels. Environ Res Section A 76:120-130.

Lanphear BP, Byrd RS, Auinger P, et al. 1998b. Community characteristics associated with elevated blood lead levels in children. Pediatrics 101(2):264-271.

Lanphear BP, Dietrich K, Auinger P, et al. 2000a. Cognitive deficits associated with blood lead concentrations $<10$ microg/dL in US children and adolescents. Public Health Rep 115(6):521-529.

Lanphear BP, Hornung R, Khoury J, et al. 2005. Low-level environmental lead exposure and children's intellectual function: An international pooled analysis. Environ Health Perspect 113(7):894-899.

Lanphear BP, Rauch S, Auinger P, et al. 2018. Low-level lead exposure and mortality in US adults: A population-based cohort study. Lancet Public Health 3(4):e177-e184. http://doi.org/10.1016/S24682667(18)30025-2.

Lanphear BP, Hornung R, Khoury J, et al. 2019. Erratum: "Low-level environmental lead exposure and children's intellectual function: An international pooled analysis". Environ Health Perspect 127(9):99001. http://doi.org/10.1289/ehp5685.

Larrañaga MD, Lewis RJ, Sr., Lewis RA, eds. 2016. Lead and lead compounds. In: Hawley's condensed chemical dictionary. Sixteenth ed. Hoboken, NJ: John Wiley \& Sons, Inc., 817-824.

Lasley SM, Gilbert ME. 2000. Glutamatergic components underlying lead-induced impairments in hippocampal synaptic plasticity. Neurotoxicology 21(6):1057-1068.

Laug EP, Kunze FM. 1948. The penetration of lead through the skin. J Ind Hyg Toxicol 30(4; 4):256259.

Lauwerys R, Buchet JP, Roels H, et al. 1978. Placental transfer of lead, mercury, cadmium, and carbon monoxide in women. I. Comparison of the frequency distributions of the biological indices in maternal and umbilical cord blood. Environ Res 15:278-289.

Lee HS, Park T. 2018. Nuclear receptor and VEGF pathways for gene-blood lead interactions, on bone mineral density, in Korean smokers. PLoS ONE 13(3):e0193323. http://doi.org/10.1371/journal.pone.0193323.

Lee BK, Lee GS, Stewart WF, et al. 2001. Associations of blood pressure and hypertension with lead dose measures and polymorphisms in the vitamin D receptor and delta-aminolevulinic acid dehydratase genes. Environ Health Perspect 109(4):383-389.

Lee TH, Tseng MC, Chen CJ, et al. 2009. Association of high body lead store with severe intracranial carotid atherosclerosis. Neurotoxicology 30(6):876-880. http://doi.org/10.1016/j.neuro.2009.07.004.

Lee BK, Ahn J, Kim NS, et al. 2016a. Association of blood pressure with exposure to lead and cadmium: Analysis of data from the 2008-2013 Korean National Health and Nutrition Examination Survey. Biol Trace Elem Res 174(1):40-51. http://doi.org/10.1007/s12011-016-0699-y.

Lee W, Yoon JH, Roh J, et al. 2016b. The association between low blood lead levels and the prevalence of prehypertension among nonhypertensive adults in Korea. Am J Hum Biol 28(5):729-735. http://doi.org/10.1002/ajhb.22857. 
Leem AY, Kim SK, Chang J, et al. 2015. Relationship between blood levels of heavy metals and lung function based on the Korean National Health and Nutrition Examination Survey IV-V. Int J Chron Obstruct Pulmon Dis 10:1559-1570. http://doi.org/10.2147/copd.s86182.

Legare ME, Barhoumi R, Hebert E, et al. 1998. Analysis of Pb2+ entry into cultured astroglia. Toxicol Sci 46:90-100.

Leggett RW. 1993. An age-specific kinetic model of lead metabolism in humans. Environ Health Perspect 101(7):598-616.

Leikin JB, Paloucek FP. 2008. Lead. In: Poisoning and toxicology handbook. Fourth ed. Boca Raton, FL: CRC Press, 807-811.

Lerda D. 1992. Study of sperm characteristics in persons occupationally exposed to lead. Am J Ind Med 22:567-571.

Lévesque B, Duchesne JF, Gariepy C, et al. 2003. Monitoring of umbilical cord blood lead levels and sources assessment among the Inuit. Occup Environ Med 60(9):693-695.

Levey AS, Bosch JP, Lewis JB, et al. 1999. A more accurate method to estimate glomerular filtration rate from serum creatinine: A new prediction equation. Ann Intern Med 130(6):461-479.

Levey AS, Stevens LA, Schmid CH, et al. 2009. A new equation to estimate glomerular filtration rate. Ann Intern Med 150:604-612.

Lewin MD, Sarasua S, Jones PA. 1999. A multivariate linear regression model for predicting children's blood lead levels based on soil lead levels: A study at four Superfund sites. Environ Res 81(Section A):52-61.

Lewis RJ. 2012. Lead and lead compounds. In: Sax's dangerous properties of industrial materials. Vol. 4. 12th ed. John Wiley \& Sons, Inc., 2711-2725.

Lewis RC, Meeker JD. 2015. Biomarkers of exposure to molybdenum and other metals in relations to testosterone among men from the United States National Health and Nutrition Examination Survey 2011-2012. Fertil Steril 103:172-178.

Lewis J, Sjostrom J, Skyllberg U, et al. 2010. Distribution, chemical speciation, and mobility of lead and antimony originating from small arms ammunition in a coarse-grained unsaturated surface sand. $\mathrm{J}$ Environ Qual 39:863-870.

Li CJ, Yeh CY, Chen RY, et al. 2015. Biomonitoring of blood heavy metals and reproductive hormone level related to low semen quality. J Hazard Mater 300:815-822. http://doi.org/10.1016/j.hazmat.2015.08.027.

Li Y, Hu J, Wu W, et al. 2016a. Application of IEUBK model in lead risk assessment of children aged 61-84 months old in central China. Sci Total Environ 541:673-682. http://doi.org/10.1016/j.scitotenv.2015.09.103.

Li Y, Xie C, Murphy SK, et al. 2016b. Lead exposure during early human development and DNA methylation of imprinted gene regulatory elements in adulthood. Environ Health Perspect 124(5):666-673. http://doi.org/10.1289/ehp.1408577.

Li S, Xu J, Liu Z, et al. 2017a. The non-linear association between low-level lead exposure and maternal stress among pregnant women. Neurotoxicology 59:191-196. http://doi.org/10.1016/j.neuro.2016.07.005.

Li J, Wang H, Hao JH, et al. 2017b. Maternal serum lead level during pregnancy is positively correlated with risk of preterm birth in a Chinese population. Environ Pollut 227:484-489. http://doi.org/10.1016/j.envpol.2017.05.009.

Li C, Ni ZM, Ye LX, et al. 2018. Dose-response relationship between blood lead levels and hematological parameters in children from central China. Environ Res 164:501-506. http://doi.org/10.1016/j.envres.2018.03.018.

Liebelt EL, Schonfeld DJ, Gallagher P. 1999. Elevated blood lead levels in children are associated with lower erythropoietin concentrations. J Pediatr 134:107-109.

Lilis R, Gavrilescu N, Nestorescu B, et al. 1968. Nephropathy in chronic lead poisoning. Br J Ind Med 25:196-202. 


\section{REFERENCES}

Lilis R, Eisinger J, Blumberg W, et al. 1978. Hemoglobin, serum iron, and zinc protoporphyrin in leadexposed workers. Environ Health Perspect 25:97-102.

Lilis R, Fischbein A, Valciukas JA, et al. 1980. Kidney function and lead: Relationships in several occupational groups with different levels of exposure. Am J Ind Med 1(3-4):405-412.

Lilley SG, Florence TM, Stauber JL. 1988. The use of sweat to monitor lead absorption through the skin. Sci Total Environ 76:267-278.

Lin TA, Tai-Yi J. 2007. Benchmark dose approach for renal dysfunction in workers exposed to lead. Environ Toxicol 22(3):229-233. http://doi.org/10.1002/tox.20260.

Lin CN, Wang LH, Shen KH. 2009. Determining urinary trace elements ( $\mathrm{Cu}, \mathrm{Zn}, \mathrm{Pb}, \mathrm{As}$, and Se) in patients with bladder cancer. J Clin Lab Anal 23(3):192-195. http://doi.org/10.1002/jcla.20318.

Lin Z, Comet B, Qvarfort U, et al. 1995. The chemical and mineralogical behaviour of PB in shooting range soils from central Sweden. Environ Pollut 89(3):303-309.

Lin JL, Tan DT, Hsu KH, et al. 2001. Environmental lead exposure and progressive renal insufficiency. Arch Intern Med 161:264-271.

Lin J, Lin-Tan D, Hsu K, et al. 2003. Environmental lead exposure and progression of chronic renal diseases in patients without diabetes. N Engl J Med 348(4):277-286.

Lin JL, Lin-Tan DT, Li YJ, et al. 2006a. Low-level environmental exposure to lead and progressive chronic kidney diseases. Am J Med 119(8):1-9. http://doi.org/10.1016/j.amjmed.2006.01.005.

Lin JL, Lin-Tan DT, Yu CC, et al. 2006b. Environmental exposure to lead and progressive diabetic nephropathy in patients with type II diabetes. Kidney Int 69(11):2049-2056.

Lin CG, Schaider LA, Brabander DJ, et al. 2010. Pediatric lead exposure from imported Indian spice and cultural powders. Pediatrics 125(4):e828-e835. http://doi.org/10.1542/peds.2009-1396.

Lin CC, Chen YC, Su FC, et al. 2013. In utero exposure to environmental lead and manganese and neurodevelopment at 2 years of age. Environ Res 123:52-57. http://doi.org/10.1016/j.envres.2013.03.003.

Lin Y, Huang L, Xu J, et al. 2019. Blood lead, bone lead and child attention-deficit-hyperactivitydisorder-like behavior. Sci Total Environ 659:161-167. http://doi.org/10.1016/j.scitotenv.2018.12.219.

Lin-Tan DT, Lin JL, Yen TH, et al. 2007. Long-term outcome of repeated lead chelation therapy in progressive non-diabetic chronic kidney diseases. Nephrol Dial Transplant 22(10):2924-2931. http://doi.org/10.1093/ndt/gfm342.

Little BB, Spalding S, Walsh B, et al. 2009. Blood lead levels and growth status among AfricanAmerican and Hispanic children in Dallas, Texas-1980 and 2002: Dallas Lead Project II. Ann Hum Biol 36(3):331-341. http://doi.org/10.1080/03014460902806615.

Little BB, Ignasiak Z, Slawinska T, et al. 2017. Blood lead levels, pulmonary function and agility in Polish schoolchildren. Ann Hum Biol 44(8):723-728. http://doi.org/10.1080/03014460.2017.1387284.

Liu J, McCauley L, Compher C, et al. 2011. Regular breakfast and blood lead levels among preschool children. Environ Health 10:28. http://doi.org/10.1186/1476-069X-10-28.

Liu R, Gress J, Gao J, et al. 2013. Impacts of two best management practices on $\mathrm{Pb}$ weathering and leachability in shooting range soils. Environ Monit Assess 185:6477-6484. http://doi.org/10.1007/s10661-012-3039-5.

Liu J, Gao D, Chen Y, et al. 2014a. Lead exposure at each stage of pregnancy and neurobehavioral development of neonates. Neurotoxicology 44:1-7. http://doi.org/10.1016/j.neuro.2014.03.003.

Liu J, Chen Y, Gao D, et al. 2014b. Prenatal and postnatal lead exposure and cognitive development of infants followed over the first three years of life: A prospective birth study in the Pearl River Delta region, China. Neurotoxicology 44:326-334. http://doi.org/10.1016/j.neuro.2014.07.001.

Liu C, Huo X, Lin P, et al. 2015a. Association between blood erythrocyte lead concentrations and hemoglobin levels in preschool children. Environ Sci Pollut Res Int 22(12):9233-9240. http://doi.org/10.1007/s11356-014-3992-3. 


\section{REFERENCES}

Liu J, Liu X, Pak V, et al. 2015b. Early blood lead levels and sleep disturbance in preadolescence. Sleep 38(12):1869-1874. http://doi.org/10.5665/sleep.5230.

Liu Z, He C, Chen M, et al. 2018a. The effects of lead and aluminum exposure on congenital heart disease and the mechanism of oxidative stress. Reprod Toxicol 81:93-98. http://doi.org/10.1016/j.reprotox.2018.07.081.

Liu Y, Huo X, Xu L, et al. 2018b. Hearing loss in children with e-waste lead and cadmium exposure. Sci Total Environ 624:621-627. http://doi.org/10.1016/j.scitotenv.2017.12.091.

Liu Y, Peterson KE, Montgomery K, et al. 2019a. Early lead exposure and childhood adiposity in Mexico city. Int J Hyg Environ Health 222(6):965-970. http://doi.org/10.1016/j.ijheh.2019.06.003.

Liu Y, Tellez-Rojo MM, Sanchez BN, et al. 2019b. Early lead exposure and pubertal development in a Mexico City population. Environ Int 125:445-451. http://doi.org/10.1016/j.envint.2019.02.021.

Lloyd RD, Mays CW, Atherton DR, et al. 1975. 210Pb studies in beagles. Health Phys 28:575-583.

Lockett CJ, Arbuckle D. 1987. Lead, ferritin, zinc, and hypertension. Bull Environ Contam Toxicol 38:975-980.

Loghman-Adham M. 1997. Renal effects of environmental and occupational lead exposure. Environ Health Perspect 105(9):928-939.

Long DT, Angino EE. 1977. Chemical speciation of $\mathrm{Cd}, \mathrm{Cu}, \mathrm{Pb}$, and $\mathrm{Zn}$ in mixed freshwater, seawater, and brine solutions. Geochim Cosmochim Acta 41:1183-1191.

Lopez CM, Pineiro AE, Nunez N, et al. 2000. Thyroid hormone changes in males exposed to lead in the Buenos Aires area (Argentina). Pharmacol Res Commun 42(6):599-602.

Lu Y, Yin W, Huang L, et al. 2011. Assessment of bioaccessibility and exposure risk of arsenic and lead in urban soils of Guangzhou City, China. Environ Geochem Health 33(2):93-102. http://doi.org/10.1007/s10653-010-9324-8.

Lucas JP, Bellanger L, Le Strat Y, et al. 2014. Source contributions of lead in residential floor dust and within-home variability of dust lead loading. Sci Total Environ 470-471:768-779. http://doi.org/10.1016/j.scitotenv.2013.10.028.

Lucchini R, Albini E, Cortesi I, et al. 2000. Assessment of neurobehavioral performance as a function of current and cumulative occupational lead exposure. Neurotoxicology 21(5):805-812.

Lundstrom NG, Nordberg G, Englyst V, et al. 1997. Cumulative lead exposure in relation to mortality and lung cancer morbidity in a cohort of primary smelter workers. Scand J Work Environ Health 23:24-30.

Lundstrom NG, Englyst V, Gerhardsson L, et al. 2006. Lung cancer development in primary smelter workers: A nested case-referent study. J Occup Environ Med 48(4):376-380. http://doi.org/10.1097/01.jom.0000201556.95982.95.

Luo J, Hendryx M. 2014. Relationship between blood cadmium, lead, and serum thyroid measures in US adults - The National Health and Nutrition Examination Survey (NHANES) 2007-2010. Int J Environ Health Res 24(2):125-136. http://doi.org/10.1080/09603123.2013.800962.

Lustberg M, Silbergeld E. 2002. Blood lead levels and mortality. Arch Intern Med 162(21):2443-2449.

Lutz PM, Wilson TJ, Ireland J, et al. 1999. Elevated immunoglobulin E (IgE) levels in children with exposure to environmental lead. Toxicology 134(1):63-78. http://doi.org/10.1016/S0300483X(99)00036-0.

Lyngbye T, Jorgensen J, Grandjean P, et al. 1990. Validity and interpretation of blood lead levels: A study on Danish school children. Scand J Clin Lab Invest 50:441-449.

Maas RP, Patch SC, Pandolfo TJ, et al. 2005. Lead content and exposure from children's and adult's jewelry products. Bull Environ Contam Toxicol 74:437-444.

Machida M, Sun SJ, Oguma E, et al. 2009. High bone matrix turnover predicts blood levels of lead among perimenopausal women. Environ Res 109(7):880-886. http://doi.org/10.1016/j.envres.2009.06.005.

MacMillan JW, Behinaein S, Chettle DR, et al. 2015. Physiologically based modeling of lead kinetics: A pilot study using data from a Canadian population. Environ Sci Process Impacts 17(12):21222133. http://doi.org/10.1039/c5em00517e. 
Maddaloni M, Lolacono N, Manton W, et al. 1998. Bioavailability of soilborne lead in adults, by stable isotope dilution. Environ Health Perspect 106(Suppl 6):1589-1594.

Maddaloni M, Ballew M, Diamond G, et al. 2005. Assessing lead risks at non-residential hazardous waste sites. Hum Ecol Risk Assess 11:967-1003.

Magzamen S, Imm P, Amato MS, et al. 2013. Moderate lead exposure and elementary school end-ofgrade examination performance. Ann Epidemiol 23(11):700-707. http://doi.org/10.1016/j.annepidem.2013.08.007.

Magzamen S, Amato MS, Imm P, et al. 2015. Quantile regression in environmental health: Early life lead exposure and end-of-grade exams. Environ Res 137:108-119. http://doi.org/10.1016/j.envres.2014.12.004.

Mahaffey KR, Annest JL. 1986. Association of erythrocyte protoporphyrin with blood lead level and iron status in the second National Health and Nutrition Examination Survey, 1976-1980. Environ Res 41(1):327-338.

Mahaffey KR, Gartside PS, Glueck CJ. 1986. Blood lead levels and dietary calcium intake in 1-to 11year-old children: The Second National Health and Nutrition Examination Survey, 1976 to 1980. Pediatrics 78(2):257-262.

Mahaffey KR, Rosen JF, Chesney RW, et al. 1982. Association between age, blood lead concentration, and serum 1,25-dihydroxycholecalciferol levels in children. Am J Clin Nutr 35:1327-1331.

Maheswaran R, Gill JS, Beevers DG. 1993. Blood pressure and industrial lead exposure. Am J Epidemiol 137(6):645-653.

Maizlish NA, Parra G, Feo O. 1995. Neurobehavioural evaluation of Venezuelan workers exposed to inorganic lead. Occup Environ Med 52:408-414.

Maki-Paakkanen J, Sorsa M, Vainio H. 1981. Chromosome aberrations and sister chromatid exchanges in lead-exposed workers. Hereditas 94:269-275.

Malcoe LH, Lynch RA, Keger MC, et al. 2002. Lead sources, behaviors, and socioeconomic factors in relation to blood lead of native and white children: A community-based assessment of a former mining area. Environ Health Perspect 110(Suppl 2):221-231.

Malcolm D, Barnett HAR. 1982. A mortality study of lead workers 1925-76. Br J Ind Med 39:404-410. Maldonado-Vega M, Cerbon-Solorzano J, Albores-Medina A, et al. 1996. Lead: Intestinal absorption and bone mobilization during lactation. Hum Exp Toxicol 15(11):872-877.

Malekirad AA, Kalantari-Dehaghi R, Abdollahi M. 2013. Clinical, haematological, and neurocognitive findings in lead-exposed workers of a battery plant in Iran. Arh Hig Rada Toksikol 64(4):497-503. http://doi.org/10.2478/10004-1254-64-2013-2385.

Mannino DM, Albalak R, Grosse S, et al. 2003. Second-hand smoke exposure and blood lead levels in U.S. children. Epidemiology 14(6):719-727.

Mantere P, Hänninen H, Hernberg S. 1982. Subclinical neurotoxic lead effects: Two-year follow-up studies with psychological test methods. Neurobehav Toxicol Teratol 4:725-727.

Manton WI. 1977. Sources of lead in blood. Identification by stable isotopes. Arch Environ Health 32(4):149-159. http://doi.org/10.1080/00039896.1977.10667273.

Manton WI. 1998. Isotope ratios and the source of lead in lead poisoning. J Toxicol Clin Toxicol 36(7):705-706. http://doi.org/10.3109/15563659809162618.

Manton WI, Malloy CR. 1983. Distribution of lead in body fluids after ingestion of soft solder. Br J Ind Med 40(1):51-57.

Manton WI, Cook JD. 1984. High accuracy (stable isotope dilution) measurements of lead in serum and cerebrospinal fluid. Br J Ind Med 41:313-319.

Manton WI, Rothenberg SJ, Manalo M. 2001. The lead content of blood serum. Environ Res 86(Section A):263-273.

Manton WI, Angle CR, Stanek KL, et al. 2000. Acquisition and retention of lead by young children. Environ Res 82:60-80.

Manton W, Angle C, Stanek K, et al. 2003. Release of lead from bone in pregnancy and lactation. Environ Res 92(2):139-151. 
Marcus AH. 1985a. Multicompartment kinetic model for lead. I. Bone diffusion models for long-term retention. Environ Res 36:441-458.

Marcus AH. 1985b. Multicompartment kinetic model for lead. II. Linear kinetics and variable absorption in humans without excessive lead exposure. Environ Res 36:459-472.

Marcus AH. 1985c. Multicompartment kinetic model for lead. III. Lead in blood plasma and erythrocytes. Environ Res 36:473-489.

Marcus AH, Schwartz J. 1987. Dose-response curves for erythrocyte protoporphyrin vs blood lead: Effects of iron status. Environ Res 44(2):221-227.

Marcus DK, Fulton JJ, Clarke EJ. 2010. Lead and conduct problems: A meta-analysis. J Clin Child Psychol 39(2):234-241. http://doi.org/10.1080/15374411003591455.

Mari M, Nadal M, Schuhmacher M, et al. 2014. Human exposure to metals: Levels in autopsy tissues of individuals living near a hazardous waste incinerator. Biol Trace Elem Res 159(1-3):15-21.

Markowitz ME, Rosen JF. 1981. Zinc (Zn) and copper (Cu) metabolism in CaNa-2 EDTA-treated children with plumbism. Pediatr Res 15:635.

Markowitz ME, Weinberger HL. 1990. Immobilization-related lead toxicity in previously lead-poisoned children. Pediatrics 86:455-457.

Marques RC, Bernardi JV, Dorea JG, et al. 2014. Perinatal multiple exposure to neurotoxic (lead, methylmercury, ethylmercury, and aluminum) substances and neurodevelopment at six and 24 months of age. Environ Pollut 187:130-135. http://doi.org/10.1016/j.envpol.2014.01.004.

Marsden PA. 2003. Increased body lead burden - Cause or consequence of chronic renal insufficiency? N Engl J Med 348(4):345-347. http://doi.org/10.1056/NEJMe020164.

Marsh J, Birchall A. 1999. Determination of lung-to-blood absorption rates for lead and bismuth which are appropriate for radon progeny. Radiat Prot Dosimetry 83(4):331- 337.

Martin D, Glass TA, Bandeen-Roche K, et al. 2006. Association of blood lead and tibia lead with blood pressure and hypertension in a community sample of older adults. Am J Epidemiol 163(5):467-478. http://doi.org/10.1093/aje/kwj060.

Marx SK, Kamber BS, McGowan HA. 2008. Scavenging of atmospheric trace metal pollutants by mineral dusts: Inter-regional transport of Australian trace metal pollution to New Zealand. Atmos Environ 42(10):2460-2478. http://doi.org/10.1016/j.atmosenv.2007.12.014.

Matte TD, Figueroa JP, Burr G, et al. 1989. Lead exposure among lead-acid battery workers in Jamaica. Am J Ind Med 16:167-177.

Mazumdar M, Bellinger DC, Gregas M, et al. 2011. Low-level environmental lead exposure in childhood and adult intellectual function: A follow-up study. Environ Health 10(1):24. http://doi.org/10.1186/1476-069X-10-24.

McClure LF, Niles JK, Kaufman HW. 2016. Blood lead levels in young children: US, 2009-2015. J Pediatr 175:173-181. http://doi.org/10.1016/j.jpeds.2016.05.005.

McDonald JA, Potter NU. 1996. Lead's legacy? Early and late mortality of 454 lead-poisoned children. Arch Environ Health 51(2):116-121.

McElroy JA, Shafer MM, Gangnon RE, et al. 2008. Urinary lead exposure and breast cancer risk in a population-based case-control study. Cancer Epidemiol Biomarkers Prev 17(9):2311-2317. http://doi.org/10.1158/1055-9965.epi-08-0263.

McElvenny DM, Miller BG, MacCalman LA, et al. 2015. Mortality of a cohort of workers in Great Britain with blood lead measurements. Occup Environ Med 72(9):625-632. http://doi.org/10.1136/oemed-2014-102637.

McFarlane AC, Searle AK, Van Hooff M, et al. 2013. Prospective associations between childhood lowlevel lead exposure and adult mental health problems: The Port Pirie cohort study. Neurotoxicology 39:11-17. http://doi.org/10.1016/j.neuro.2013.08.003.

McGregor AJ, Mason HJ. 1990. Chronic occupational lead exposure and testicular endocrine function. Hum Exp Toxicol 9:371-376.

McLaine P, Navas-Acien A, Lee R, et al. 2013. Elevated blood lead levels and reading readiness at the start of kindergarten. Pediatrics 131(6):1081-1089. http://doi.org/10.1542/peds.2012-2277. 
McMichael AJ, Vimpani GV, Robertson EF, et al. 1986. The Port Pirie cohort study: Maternal blood lead and pregnancy outcome. J Epidemiol Community Health 40(1):18-25.

McMichael AJ, Baghurst PA, Wigg NR, et al. 1988. Port Pirie cohort study: Environmental exposure to lead and children's abilities at the age of four years. N Engl J Med 319(8):468-475.

McNeill FE, Stokes L, Brito JA, et al. 2000. 109Cd K x-ray fluorescence measurements of tibial lead content in young adults exposed to lead in early childhood. Occup Environ Med 57(7):465-471. http://doi.org/10.1136/oem.57.7.465.

Meeker JD, Rossano MG, Protas B, et al. 2008. Cadmium, lead, and other metals in relation to semen quality: Human evidence for molybdenum as a male reproductive toxicant. Environ Health Perspect 116(11):1473-1479. http://doi.org/10.1289/ehp.11490.

Meeker JD, Rossano MG, Protas B, et al. 2010. Environmental exposure to metals and male reproductive hormones: Circulating testosterone is inversely associated with blood molybdenum. Fertil Steril 93(1):130-140. http://doi.org/10.1016/j.fertnstert.2008.09.044.

Meirer F, Pemmer B, Pepponi G, et al. 2011. Assessment of chemical species of lead accumulated in tidemarks of human articular cartilage by X-ray absorption near-edge structure analysis. J Synchrotron Radiat 18(Pt 2):238-244. http://doi.org/10.1107/S0909049510052040.

Mellem JJ, Baijnath H, Odhav B. 2009. Translocation and accumulation of Cr, Hg, As, Pb, Cu and Ni by Amaranthus dubius (Amaranthaceae) from contaminated sites. J Environ Sci Health A Tox Hazard Subst Environ Eng 44(6):568-575. http://doi.org/10.1080/10934520902784583.

Méndez-Gómez J, García-Vargas GG, López-Carrillo L, et al. 2008. Genotoxic effects of environmental exposure to arsenic and lead on children in region Lagunera, Mexico. Ann N Y Acad Sci 1140:358367. http://doi.org/10.1196/annals.1454.027.

Mendiola J, Moreno JM, Roca M, et al. 2011. Relationships between heavy metal concentrations in three different body fluids and male reproductive parameters: A pilot study. Environ Health 10(1):6. http://doi.org/10.1186/1476-069X-10-6.

Menditto A, Morisi G, Spagnolo A, et al. 1994. Association of blood lead to blood pressure in men aged 55 to 75 years: Effect of selected social and biochemical confounders. NFR Study Group. Environ Health Perspect 102 Suppl 9:107-111.

Mendola P, Brett K, Dibari JN, et al. 2013. Menopause and lead body burden among US women aged 45-55, NHANES 1999-2010. Environ Res 121:110-113. http://doi.org/10.1016/j.envres.2012.12.009.

Mendy A, Gasana J, Vieira ER. 2012. Urinary heavy metals and associated medical conditions in the US adult population. Int J Environ Health Res 22(2):105-118. http://doi.org/10.1080/09603123.2011.605877.

Mendy A, Gasana J, Vieira ER. 2013. Low blood lead concentrations and thyroid function of American adults. Int J Environ Health Res 23(6):461-473. http://doi.org/10.1080/09603123.2012.755155.

Menke A, Muntner P, Batuman V, et al. 2006. Blood lead below 0.48 micromol/L (10 microg/dL) and mortality among US adults. Circulation 114(13):1388-1394. http://doi.org/10.1161/circulationaha.106.628321.

Meredith PA, Moore MR, Campbell BC, et al. 1978. delta-Aminolaevulinic acid metabolism in normal and lead-exposed humans. Toxicology 9:1-9.

Meyer-Baron M, Seeber A. 2000. A meta-analysis for neurobehavioural results due to occupational lead exposure with blood lead concentrations $<70 \mathrm{ug} / 100 \mathrm{ml}$. Arch Toxicol 73:510-518.

Michaels D, Zoloth SR, Stern FB. 1991. Does low-level lead exposure increase risk of death? A mortality study of newspaper printers. Int J Epidemiol 20(4):978-983.

Mielke HW. 1991. Lead in residential soils: Background and preliminary results of New Orleans. Water Air Soil Pollut 57(1):111-119.

Mielke HW. 1993. Lead dust contaminated U.S.A. communities: Comparison of Louisiana and Minnesota. Appl Geochem Suppl(2):257-261.

Mielke HW, Gonzales C. 2008. Mercury (Hg) and lead (Pb) in interior and exterior New Orleans house paint films. Chemosphere 72(6):882-885. http://doi.org/10.1016/j.chemosphere.2008.03.061. 
Mielke HW, Laidlaw MA, Gonzales C. 2010. Lead (Pb) legacy from vehicle traffic in eight California urbanized areas: Continuing influence of lead dust on children's health. Sci Total Environ 408(19):3965-3975. http://doi.org/10.1016/j.scitotenv.2010.05.017.

Mielke HW, Anderson JC, Berry KJ, et al. 1983. Lead concentrations in inner-city soils as a factor in the child lead problem. Am J Public Health 73(12; 12):1366-1369.

Mielke HW, Burroughs S, Wade R, et al. 1984. Urban lead in Minnesota: Soil transect results of four cities. J Minn Acad Sci 50(1):19-24.

Mielke HW, Adams JL, Reagan PL, et al. 1989. Soil-dust lead and childhood lead exposure as a function of city size and community traffic flow: The case for lead abatement in Minnesota. In: Lead in soil: Issues and guidelines. CRC Press, 253-271.

Mielke HW, Dugas D, Mielke PW, et al. 1997. Associations between soil lead and childhood blood lead in urban New Orleans and rural Lafourche Parish of Louisiana. Environ Health Perspect 105:950954.

Mielke HW, Powell ET, Shah A, et al. 2001. Multiple metal contamination from house paints: Consequences of power sanding and paint scraping in New Orleans. Environ Health Perspect 109(9):973-978.

Mielke HW, Gonzales CR, Powell E, et al. 2007. Nonlinear association between soil lead and blood lead of children in metropolitan New Orleans, Louisiana: 2000-2005. Sci Total Environ 388(1-3):43-53. http://doi.org/10.1016/j.scitotenv.2007.08.012.

Mielke HW, Gonzales C, Powell E, et al. 2008. Urban soil-lead (Pb) footprint: Retrospective comparison of public and private properties in New Orleans. Environ Geochem Health 30(3):231242. http://doi.org/10.1007/s10653-007-9111-3.

Mielzyńska D, Siwińska E, Kapka L, et al. 2006. The influence of environmental exposure to complex mixtures including PAHs and lead on genotoxic effects in children living in Upper Silesia, Poland. Mutagenesis 21(5):295-304. http://doi.org/10.1093/mutage/gel037.

Miller EK, Friedland AJ. 1994. Lead migration in forest soils: Response to changing atmospheric inputs. Environ Sci Technol 28(4):662-669.

Min KB, Min JY. 2015. Environmental lead exposure and increased risk for total and allergen-specific IgE in US adults. J Allergy Clin Immunol 135(1):275-277. http://doi.org/10.1016/j.jaci.2014.08.052.

Min YS, Ahn YS. 2017. The association between blood lead levels and cardiovascular diseases among lead-exposed male workers. Scand J Work Environ Health 43(4):385-390. http://doi.org/10.5271/sjweh.3631.

Min JY, Min KB, Kim R, et al. 2008a. Blood lead levels and increased bronchial responsiveness. Biol Trace Elem Res 123(1-3):41-46. http://doi.org/10.1007/s12011-008-8099-6.

Min KB, Min JY, Cho SI, et al. 2008b. Relationship between low blood lead levels and growth in children of white-collar civil servants in Korea. Int J Hyg Environ Health 211(1-2):82-87. http://doi.org/10.1016/j.ijheh.2007.03.003.

Min MYO, Singer LT, Kirchner HL, et al. 2009. Cognitive development and low-level lead exposure in poly-drug exposed children. Neurotoxicol Teratol 31(4):225-231. http://doi.org/10.1016/j.ntt.2009.03.002.

Mindak WR, Cheng J, Canas BJ, et al. 2008. Lead in women's and children's vitamins. J Agric Food Chem 56:6892-6896.

Minozzo R, Deimling LI, Gigante LP, et al. 2004. Micronuclei in peripheral blood lymphocytes of workers exposed to lead. Mutat Res 565(1):53-60. http://doi.org/10.1016/j.mrgentox.2004.09.003.

Miranda ML, Kim D, Reiter J, et al. 2009. Environmental contributors to the achievement gap. Neurotoxicology 30(6):1019-1024. http://doi.org/10.1016/j.neuro.2009.07.012.

Miranda ML, Edwards SE, Swamy GK, et al. 2010. Blood lead levels among pregnant women: Historical versus contemporaneous exposures. Int J Environ Res Public Health 7(4):1508-1519. http://doi.org/10.3390/ijerph7041508. 
Mishra KP, Singh VK, Rani R, et al. 2003. Effect of lead exposure on the immune response of some occupationally exposed individuals. Toxicology 188(2-3):251-259. http://doi.org/10.1016/s0300483x(03)00091-X.

Mishra KP, Rani R, Yadav VS, et al. 2010. Effect of lead exposure on lymphocyte subsets and activation markers. Immunopharmacol Immunotoxicol 32(3):446-449. http://doi.org/10.3109/08923970903503668.

Mistry P, Lucier GW, Fowler BA. 1985. High affinity lead binding proteins from rat kidney cytosol mediate cell-free nuclear translocation of lead. J Pharmacol Exp Ther 232:462-469.

Mistry P, Mastri C, Fowler BA. 1986. Influence of metal ions on renal cytosolic lead-binding proteins and nuclear uptake of lead in the kidney. Biochem Pharmacol 35:711-713.

Mitra P, Sharma S, Purohit P, et al. 2017. Clinical and molecular aspects of lead toxicity: An update. Crit Rev Clin Lab Sci 54(7-8):506-528. http://doi.org/10.1080/10408363.2017.1408562.

Miyake M. 1986. Structure refinements of $\mathrm{Pb2}+$ ion-exchanged apatites by x-ray powder pattern-fitting. Journal of Solid State Chemistry 61(2):230-235. http://doi.org/10.1016/0022-4596(86)90026-5.

Mohammad IK, Mahdi AA, Raviraja A, et al. 2008. Oxidative stress in painters exposed to low lead levels. Arh Hig Rada Toksikol 59(3):161-169. http://doi.org/10.2478/10004-1254-59-2008-1883.

Møller L, Kristensen TS. 1992. Blood lead as a cardiovascular risk factor. Am J Epidemiol 136(9):1091-1100.

Montenegro MF, Barbosa F, Tanus-Santos JE. 2008. Assessment of how pregnancy modifies plasma lead and plasma/whole blood lead ratio in ALAD 1-1 genotype women. Basic Clin Pharmacol Toxicol 102(4):347-351. http://doi.org/10.1111/j.1742-7843.2007.00205.x.

Moon SS. 2013. Association of lead, mercury and cadmium with diabetes in the Korean population: The Korea National Health and Nutrition Examination Survey (KNHANES) 2009-2010. Diabet Med 30(4):e143-148. http://doi.org/10.1111/dme.12103.

Moore MR, Goldberg A. 1985. Health implications of the hematopoietic effects of lead. In: Dietary and environmental lead: Human health effects. Elsevier, 261-314.

Moore MR, Meredith PA, Watson WS, et al. 1980. The percutaneous absorption of lead-203 in humans from cosmetic preparations containing lead acetate, as assessed by whole-body counting and other techniques. Food Chem Toxicol 18:399-405.

Moran-Martinez J, Carranza-Rosales P, Morales-Vallarta M, et al. 2013. Chronic environmental exposure to lead affects semen quality in a Mexican men population. Iran J Reprod Med 11(4):267274.

Moreau T, Orssaud G, Juguet B, et al. 1982. [Blood lead levels and arterial pressure. Initial results of a cross sectional study of 431 male subjects]. Rev Epidemiol Sante Publique 30(3):395-397. (French)

Moreau T, Hannaert P, Orssaud G, et al. 1988. Influence of membrane sodium transport upon the relation between blood lead and blood pressure in a general male population. Environ Health Perspect 78:47-51.

Morgan A, Holmes A. 1978. The fate of lead in petrol-engine exhaust particulates inhaled by the rat. Environ Res 15:44-56.

Morgan B, Parramore C. 2001. Elevated blood lead levels associated with the consumption of illicitly distilled moonshine. J Toxicol Clin Toxicol 39(5):551.

Morita Y, Sakai T, Araki S, et al. 1997. Nicotinamide adenine dinucleotide synthetase activity in erythrocytes as a tool for the biological monitoring of lead exposure. Int Arch Occup Environ Health 70:195-198.

Morris V, Markowitz ME, Rosen JF. 1988. Serial measurements of aminolevulinic acid dehydratase in children with lead toxicity. J Pediatr 112(6):916-919.

Morris C, McCarron DA, Bennett WM. 1990. Low-level lead exposure, blood pressure, and calcium metabolism. Am J Kidney Dis 15:568-574.

Morrison JN, Quarterman J. 1987. The relationship between iron status and lead absorption in rats. Biol Trace Elem Res 14(1-2):115-126. http://doi.org/10.1007/bf02795602. 
Morrison NA, Yeoman R, Kelly PJ, et al. 1992. Contribution of trans-acting factor alleles to normal physiological variability: Vitamin D receptor gene polymorphism and circulating osteocalcin. Proc Natl Acad Sci U S A 89(15):6665-6669.

Morrow P, Beiter H, Amato F, et al. 1980. Pulmonary retention of lead: an experimental study in man. Environ Res 21(2):373-384.

Mortada WI, Sobh MA, El-Defrawy MM, et al. 2001. Study of lead exposure from automobile exhaust as a risk for nephrotoxicity among traffic policemen. Am J Nephrol 21:274-279.

Moss ME, Lanphear BP, Auinger P. 1999. Association of dental caries and blood lead levels. JAMA 281(24):2294-2298.

Mujaj B, Yang WY, Zhang ZY, et al. 2019. Renal function in relation to low-level environmental lead exposure. Nephrol Dial Transplant 34(6):941-946. http://doi.org/10.1093/ndt/gfy279.

Muldoon SB, Cauley JA, Kuller LH, et al. 1996. Effects of blood lead levels on cognitive function of older women. Neuroepidemiology 15:62-72.

Muntner P, He J, Vupputuri S, et al. 2003. Blood lead and chronic kidney disease in the general United States population: Results from NHANES III. Kidney Int 63(3):1044-1050.

Muntner P, Menke A, DeSalvo KB, et al. 2005. Continued decline in blood lead levels among adults in the United States - The National Health and Nutrition Examination Surveys. Arch Intern Med 165(18):2155-2161. http://doi.org/10.1001/archinte.165.18.2155.

Muntner P, Menke A, Batuman V, et al. 2007. Association of tibia lead and blood lead with end-stage renal disease: A pilot study of African-Americans. Environ Res 104(3):396-401. http://doi.org/10.1016/j.envres.2007.04.001.

Murata K, Iwata T, Dakeishi M, et al. 2009. Lead toxicity: Does the critical level of lead resulting in adverse effects differ between adults and children? J Occup Health 51(1):1-12. http://doi.org/10.1539/joh.K8003.

Murphy MJ, Graziano JH, Popovac D, et al. 1990. Past pregnancy outcomes among women living in the vicinity of a lead smelter in Kosova, Yugoslavia. Am J Public Health 80:33-35.

Murphy DM, Capps SL, Daniel JS, et al. 2008. Weekly patterns of aerosol in the United States. Atmos Chem Phys 8(10):2729-2739. http://doi.org/10.5194/acp-8-2729-2008.

Murray H, Thompson K, Macfie SM. 2009. Site- and species-specific patterns of metal bioavailability in edible plants. Botany 87(7):702-711.

Mushak P. 1991. Gastro-intestinal absorption of lead in children and adults: Overview of biological and biophysico-chemical aspects. Chem Spec Bioavailab 3(3-4):87-104.

Mykkanen HM, Wasserman RH. 1981. Gastrointestinal absorption of lead (203Pb) in chicks: Influence of lead, calcium, and age. J Nutr 111:1757-1765.

Mykkanen HM, Wasserman RH. 1982. Effect of vitamin D on the intestinal absorption of 203Pb and 47Ca in chicks. J Nutr 112:520-527.

Naha N, Chowdhury AR. 2006. Inorganic lead exposure in battery and paint factory: Effect on human sperm structure and functional activity. J UOEH 28(2):157-171.

Naicker N, Norris SA, Mathee A, et al. 2010. Lead exposure is associated with a delay in the onset of puberty in South African adolescent females: Findings from the birth to twenty cohort. Sci Total Environ 408(21):4949-4954. http://doi.org/10.1016/j.scitotenv.2010.07.037.

Nan Z, Cheng G. 2001. Accumulation of $\mathrm{Cd}$ and $\mathrm{Pb}$ in spring wheat (Triticum aestivum L.) grown in calcareous soil irrigated with wastewater. Bull Environ Contam Toxicol 66(6):748-754.

Narayana K, Al-Bader M. 2011. Ultrastructural and DNA damaging effects of lead nitrate in the liver. Exp Toxicol Pathol 63(1-2):43-51. http://doi.org/10.1016/j.etp.2009.09.007.

Narayana K, Raghupathy R. 2012. DNA damage in lead-exposed hepatocytes: Coexistence of apoptosis and necrosis? Drug Chem Toxicol 35(2):208-217. http://doi.org/10.3109/01480545.2011.589849.

NAS. 1972. Lead: Airborne lead in perspective. Washington, DC: National Academy of Sciences, 71177, 281-313. 


\section{REFERENCES}

NAS. 2013. Potential health risks to DOD firing-range personnel from recurrent lead exposure.

Washington, DC: National Academy of Sciences, National Research Council. http://nap.edu/18249. February 19, 2020.

NAS/NRC. 1989. Report of the oversight committee. In: Biologic markers in reproductive toxicology. Washington, DC: National Academy of Sciences, 15-35.

Nash D, Magder L, Lustberg M, et al. 2003. Blood lead, blood pressure, and hypertension in perimenopausal and postmenopausal women. JAMA 289(12):1523-1532.

Nash D, Magder LS, Sherwin R, et al. 2004. Bone density-related predictors of blood lead level among peri-and postmenopausal women in the United States the Third National Health and Nutrition Examination Survey, 1988-1994. Am J Epidemiol 160(9):901-911.

Navas-Acien A, Selvin E, Sharrett AR, et al. 2004. Lead, cadmium, smoking, and increased risk of peripheral arterial disease. Circulation 109:3196-3201.

Navas-Acien A, Silbergeld EK, Sharrett AR, et al. 2005. Metals in urine and peripheral arterial disease. Environ Health Perspect 113(2):164-169. http://doi.org/10.1289/ehp.7329.

Navas-Acien A, Guallar E, Silbergeld EK, et al. 2007. Lead exposure and cardiovascular disease - A systematic review. Environ Health Perspect 115:472-482. http://doi.org/10.1289/ehp.9785.

Navas-Acien A, Schwartz BS, Rothenberg SJ, et al. 2008. Bone lead levels and blood pressure endpoints: A meta-analysis. Epidemiology 19(3):496-504. http://doi.org/10.1097/EDE.0b013e31816a2400.

Navas-Acien A, Tellez-Plaza M, Guallar E, et al. 2009. Blood cadmium and lead and chronic kidney disease in US adults: A joint analysis. Am J Epidemiol 170(9):1156-1164. http://doi.org/10.1093/aje/kwp248.

Nawrot TS, Thijs L, Hond EMD, et al. 2002. An epidemiological re-appraisal of the association between blood pressure and blood lead: A meta-analysis. J Hum Hypertens 16:123-131.

Needleman H. 2004. Lead poisoning. Annu Rev Med 55:209-222.

Needleman HL, Gunnoe C, Leviton A, et al. 1979. Deficits in psychologic and classroom performance of children with elevated dentine lead levels. N Engl J Med 300(13):689-695.

Needleman HL, Rabinowitz M, Leviton A, et al. 1984. The relationship between prenatal exposure to lead and congenital anomalies. J Am Med Assoc 251(22):2956-2959.

Needleman HL, Schell A, Bellinger D, et al. 1990. The long-term effects of exposure to low doses of lead in childhood: An 11-year follow-up report. N Engl J Med 322(2):83-88.

Needleman HL, Riess JA, Tobin MJ, et al. 1996. Bone lead levels and delinquent behavior. J Am Med Assoc 275:363-369.

Needleman HL, McFarland C, Ness RB, et al. 2002. Bone lead levels in adjudicated delinquents. A case control study. Neurotoxicol Teratol 24:711-717.

Nelson AE, Chaudhary S, Kraus VB, et al. 2011. Whole blood lead levels are associated with biomarkers of joint tissue metabolism in African American and white men and women: The Johnston County Osteoarthritis Project. Environ Res 111(8):1208-1214. http://doi.org/10.1016/j.envres.2011.08.002.

Neri LC, Hewitt D, Orser B. 1988. Blood lead and blood pressure: Analysis of cross-sectional and longitudinal data from Canada. Environ Health Perspect 78:123-126.

Nerin C, Domeno C, Garcia JI, et al. 1999. Distribution of Pb, V, Cr, Ni, Cd, Cu and Fe in particles formed from the combustion of waste oils. Chemosphere 38(7):1533-1540.

Neuberger JS, Hu SC, Drake KD, et al. 2009. Potential health impacts of heavy-metal exposure at the Tar Creek Superfund site, Ottawa County, Oklahoma. Environ Geochem Health 31(1):47-59. http://doi.org/10.1007/s10653-008-9154-0.

Neugebauer J, Wittsiepe J, Kasper-Sonnenberg M, et al. 2015. The influence of low level pre- and perinatal exposure to PCDD/Fs, PCBs, and lead on attention performance and attention-related behavior among German school-aged children: Results from the Duisburg Birth Cohort Study. Int J Hyg Environ Health 218(1):153-162. http://doi.org/10.1016/j.ijheh.2014.09.005. 
Neuman DR, Dollhopf DJ. 1992. Lead levels in blood from cattle residing near a lead smelter. J Environ Qual 21:181-184.

NFPA. 2002. Tetraethyl lead. In: Spencer AB, Colonna GR, eds. Fire protection guide to hazardous materials. Quincy, MA: National Fire Protection Association, 325-106.

$\mathrm{Ng} \mathrm{TP}$, Goh HH, Ng YL, et al. 1991. Male endocrine functions in workers with moderate exposure to lead. Br J Ind Med 48:485-491.

Ngueta G, Verner MA, Fiocco AJ, et al. 2018. Blood lead levels and hypothalamic-pituitary-adrenal function in middle-aged individuals. Environ Res 160:554-561. http://doi.org/10.1016/j.envres.2017.10.032.

Nie H, Chettle DR, Webber CE, et al. 2005. The study of age influence on human bone lead metabolism by using a simplified model and X-ray fluorescence data. J Environ Monit 7(11):1069-1073. http://doi.org/10.1039/b507749d.

Nie H, Sanchez BN, Wilker E, et al. 2009. Bone lead and endogenous exposure in an environmentally exposed elderly population: The Normative Aging Study. J Occup Environ Med 51:848-857.

Nie LH, Wright RO, Bellinger DC, et al. 2011a. Blood lead levels and cumulative blood lead index (CBLI) as predictors of late neurodevelopment in lead poisoned children. Biomarkers 16(6):517524. http://doi.org/10.3109/1354750x.2011.604133.

Nie LH, Sanchez S, Newton K, et al. 2011b. In vivo quantification of lead in bone with a portable x-ray fluorescence system - Methodology and feasibility. Phys Med Biol 56(3):N39-51. http://doi.org/10.1088/0031-9155/56/3/n01.

Nie X, Chen Y, Chen Y, et al. 2017. Lead and cadmium exposure, higher thyroid antibodies and thyroid dysfunction in Chinese women. Environ Pollut 230:320-328. http://doi.org/10.1016/j.envpol.2017.06.052.

Nielsen T. 1984. Atmospheric occurrence of organolead compounds. In: Grandjean P, ed. Biological effects of organolead compounds. Boca Raton, FL: CRC Press, 44-62.

Nielsen T, Jensen KA, Grandjean P. 1978. Organic lead in normal human brains. Nature 274:602-603.

Nielsen OJ, O'Farrell DJ, Treacy JJ, et al. 1991. Rate constants for the gas-phase reactions of hydroxyl radicals with tetramethyllead and tetraethyllead. Environ Sci Technol 25(6):1098-1103.

Nielson KB, Atkin CL, Winge DR. 1985. Distinct metal-binding configurations in metallothionein. J Biol Chem 260:5342-5350.

Nihei MK, Guilarte TR. 2002. Molecular mechanisms of low-level Pb2 neurotoxicity. In: Massaro EJ, ed. Handbook of neurotoxicology. Totowa, NJ: Humana Press, 107-133.

Nilsson U, Attewell R, Christoffersson JO, et al. 1991. Kinetics of lead in bone and blood after end of occupational exposure. Pharmacol Toxicol 69:477-484.

NIOSH. 1994a. Method 8005, Issue 2: Elements in blood or tissue. NIOSH manual of analytical methods (NMAM). National Institute for Occupational Safety and Health. https://www.cdc.gov/niosh/docs/2003-154/pdfs/8005.pdf. March 30, 2017.

NIOSH. 1994b. Method 8003: Lead in blood and urine. NIOSH manual of analytical methods (NMAM). Cincinnati, OH: National Institute for Occupational Safety and Health.

NIOSH. 1994c. Method 7105, Issue 2: Lead by GFAAS. NIOSH manual of analytical methods (NMAM). National Institute for Occupational Safety and Health. https://www.cdc.gov/niosh/docs/2003-154/pdfs/7105. March 29, 2017.

NIOSH. 1995. Report to congress on workers' home contamination study conducted under the Worker's Family Protection Act (29 U.S.C. 671a). Cincinnati, OH: National Institute for Occupational Safety and Health. DHHS (NIOSH) Publication No. 95-123. PB96192000HEF.

NIOSH. 1996a. Method 9100, Issue 2: Lead in surface wipe samples. NIOSH manual of analytical methods (NMAM). National Institute for Occupational Safety and Health. https://www.cdc.gov/niosh/docs/2003-154/pdfs/9100.pdf. March 30, 2017.

NIOSH. 1996b. NIOSH health hazard evaluation report: HETA 91-0346-2572 FBI academy Quantico, Virginia. Cincinnati, OH: National Institute for Occupational Safety and Health. 
NIOSH. 1998. Method 7702, Issue 1: Lead by field portable XRF. NIOSH manual of analytical methods (NMAM). National Institute for Occupational Safety and Health. http://www.cdc.gov/niosh/docs/2003-154/pdfs/7702.pdf. March 29, 2017.

NIOSH. 2003a. Method 7301, Issue 1: Elements by ICP (Aqua Regia Ashing). NIOSH manual of analytical methods (NMAM). National Institute for Occupational Safety and Health. https://www.cdc.gov/niosh/docs/2003-154/pdfs/7301.pdf. March 29, 2017.

NIOSH. 2003b. Method 7303, Issue 1: Elements by ICP (Hot Block/HCl/HNO3 Ashing). NIOSH manual of analytical methods (NMAM). National Institute for Occupational Safety and Health. https://www.cdc.gov/niosh/docs/2003-154/pdfs/7303.pdf. February 18, 2020.

NIOSH. 2003c. Method 7300, Issue 3: Elements by (ICP). NIOSH manual of analytical methods (NMAM). National Institute for Occupational Safety and Health. https://www.cdc.gov/niosh/docs/2003-154/pdfs/7300.pdf. March 29, 2017.

NIOSH. 2003d. Method 9102, Issue 1: Elements on wipes methods. NIOSH manual of analytical methods (NMAM). National Institute for Occupational Safety and Health. https://www.cdc.gov/niosh/docs/2003-154/pdfs/9102.pdf. March 30, 2017.

NIOSH. 2003e. Method 9105, Issue 1: Lead in dust wipes. NIOSH manual of analytical methods (NMAM). National Institute for Occupational Safety and Health. https://www.cdc.gov/niosh/docs/2003-154/pdfs/9105.pdf. March 30, 2017.

NIOSH. 2014a. Method 7302, Issue 1: Elements by ICP (microwave digestion). NIOSH manual of analytical methods (NMAM). National Institute for Occupational Safety and Health. https://www.cdc.gov/niosh/docs/2014-151/pdfs/methods/7302.pdf. March 29, 2017.

NIOSH. 2014b. Method 7304, Issue 1: Elements by ICP (microwave digestion). NIOSH manual of analytical methods (NMAM). National Institute for Occupational Safety and Health. https://www.cdc.gov/niosh/docs/2014-151/pdfs/methods/7304.pdf. March 29, 2017.

NIOSH. 2015. Method 7306, Issue 1: Elements by cellulosic internal capsule sampler. NIOSH manual of analytical methods (NMAM). National Institute for Occupational Safety and Health. https://www.cdc.gov/niosh/docs/2014-151/pdfs/methods/7306.pdf. March 29, 2017.

NIOSH. 2016a. Method 7701, Issue 3: Lead by portable ultrasonic extraction/ASV. NIOSH manual of analytical methods (NMAM). National Institute for Occupational Safety and Health. https://www.cdc.gov/niosh/docs/2014-151/pdfs/methods/7701.pdf. March 29, 2017.

NIOSH. 2016b. Lead. NIOSH pocket guide to chemical hazards. Atlanta, GA: National Institute for Occupational Safety and Health. https://www.cdc.gov/niosh/npg/npgd0368.html. May 10, 2017.

NIOSH. 2017a. Elevated blood lead levels among employed adults - United States, 2014 Adult blood lead epidemiology and surveillance (ABLES). National Institute for Occupational Safety and Health.

NIOSH. 2017b. Method 7082, Issue 2: Lead by flame AAS NIOSH manual of analytical methods (NMAM). National Institute for Occupational Safety and Health. https://www.cdc.gov/niosh/docs/2014-151/pdfs/methods/7082.pdf. March 13, 2019.

NIOSH. 2019a. Lead. NIOSH pocket guide to chemical hazards. Atlanta, GA: National Institute for Occupational Safety and Health. https://www.cdc.gov/niosh/npg/npgd0368.html. October 25, 2019.

NIOSH. 2019b. Tetraethyl lead (as Pb). NIOSH pocket guide to chemical hazards. Atlanta, GA: National Institute for Occupational Safety and Health. https://www.cdc.gov/niosh/npg/npgd0601.html. October 25, 2019.

NIOSH. 2019c. Tetramethyl lead (as Pb). NIOSH pocket guide to chemical hazards. Atlanta, GA: National Institute for Occupational Safety and Health. https://www.cdc.gov/niosh/npg/npgd0603.html. October 25, 2019.

Nishioka E, Yokoyama K, Matsukawa T, et al. 2014. Evidence that birth weight is decreased by maternal lead levels below 5 ug/dl in male newborns. Reprod Toxicol 47:21-26. http://doi.org/10.1016/j.reprotox.2014.05.007. 


\section{REFERENCES}

Niu Y, Yu W, Fang S, et al. 2015. Lead poisoning influences TCR-related gene expression patterns in peripheral blood T-lymphocytes of exposed workers. J Immunotoxicol 12(1):92-97. http://doi.org/10.3109/1547691x.2014.899412.

Nkomo P, Mathee A, Naicker N, et al. 2017. The association between elevated blood lead levels and violent behavior during late adolescence: The South African Birth to Twenty Plus cohort. Environ Int 109:136-145. http://doi.org/10.1016/j.envint.2017.09.004.

NLM. 2020. ChemIDplus: Lead tetroxide (CASRN: 1314-41-6). U.S. National Library of Medicine. https://chem.nlm.nih.gov/chemidplus/name/lead\%20tetraoxide. January 23, 2020.

NOAA. 1998. Sampling and analytical methods of the National Status and Trends Program Mussel Watch Project: 1993-1996 Update. Silver Spring, MD: National Oceanic and Atmospheric Administration. NOS ORCA 130. http://aquaticcommons.org/2201/. March 30, 2017.

Nordberg GF, Gerhardsson L, Mumtaz MM, et al. 2015. Interactions and mixtures in metal toxicology. In: Nordberg GF, Fowler BA, Nordberg M, eds. Handbook on the toxicology of metals. 4th ed. Elsevier, 213-238.

Nordenson I, Beckman G, Beckman L, et al. 1978. Occupational and environmental risks in and around a smelter in northern Sweden. IV. Chromosomal aberrations in workers exposed to lead. Hereditas 88:263-267.

NRC. 2005. Superfund and mining megasites. Lessons from Coeur d'Alene River Basin. National Research Council. http://dels.nas.edu/Report/Superfund-Mining-Megasites-Lessons-from/11359. February 18, 2020.

Nriagu J, Burt B, Linder A, et al. 2006. Lead levels in blood and saliva in a low-income population of Detroit, Michigan. Int J Hyg Environ Health 209(2):109-121. http://doi.org/10.1016/j.ijheh.2005.11.005.

NTP. 2003. Report on carcinogens background document for lead and lead compounds Research Triangle Park, NC: National Toxicology Program. https://ntp.niehs.nih.gov/ntp/newhomeroc/roc11/lead-public_508.pdf. January 23, 2020.

NTP. 2012. NTP monograph on health effects of low-level lead. National Toxicology Program. xiii, xv148.

https://ntp.niehs.nih.gov/ntp/ohat/lead/final/monographhealtheffectslowlevellead_newissn_508.pdf. May 31, 2017.

NTP. 2016. Lead and lead compounds, CAS No. 7439-92-1 (lead). Report on carcinogens. Research Triangle Park, NC: National Toxicology Program. https://ntp.niehs.nih.gov/ntp/roc/content/profiles/lead.pdf. May 4, 2017.

Nwosu JU, Harding AK, Linder G. 1995. Cadmium and lead uptake by edible crops grown in a silt loam soil. Bull Environ Contam Toxicol 54:570-578.

Obeng-Gyasi E, Obeng-Gyasi B. 2018. Blood pressure and oxidative stress among U.S. adults exposed to lead in military environments - A preliminary study. Diseases 6(4):97. http://doi.org/10.3390/diseases6040097.

Odland JO, Nieboer E, Romanova N, et al. 1999. Blood lead and cadmium and birth weight among subarctic and arctic populations of Norway and Russia. Acta Obstet Gynecol Scand 78:852-860.

O'Flaherty EJ. 1987. Modeling: An introduction. In: Pharmacokinetics in risk assessment: Drinking water and health. Vol. 8. Washington, DC: National Academy Press, National Academy of Sciences, 27-33.

O'Flaherty EJ. 1991a. Physiologically based models for bone-seeking elements. II. Kinetics of lead disposition in rats. Toxicol Appl Pharmacol 111:313-331.

O'Flaherty EJ. 1991b. Physiologically based models for bone-seeking elements: III. Human skeletal and bone growth. Toxicol Appl Pharmacol 111(2):332-341.

O'Flaherty EJ. 1993. Physiologically based models for bone-seeking elements. IV. Kinetics of lead disposition in humans. Toxicol Appl Pharmacol 118(1):16-29.

O'Flaherty EJ. 1995a. Physiologically based models for bone-seeking elements. V. Lead absorption and disposition in childhood. Toxicol Appl Pharmacol 131:297-308. 


\section{REFERENCES}

O'Flaherty EJ. 1995b. PBK modeling for metals. Examples with lead, uranium, and chromium. Toxicol Lett 82/83:367-372.

O'Flaherty EJ. 1998. A physiologically based kinetic model for lead in children and adults. Environ Health Perspect 106(Suppl 6):1495-1503.

O'Flaherty EJ. 2000. Modeling normal aging bone loss, with consideration of bone loss in osteoporosis. Toxicol Sci 55:171-188.

O'Flaherty EJ, Hammond PB, Lerner SI. 1982. Dependence of apparent blood lead half-life on the length of previous lead exposure in humans. Fundam Appl Toxicol 2:49-55.

Oldereid NB, Thomassen Y, Attramadal A, et al. 1993. Concentrations of lead, cadmium and zinc in the tissues of reproductive organs of men. J Reprod Fertil 99:421-425.

Olivero-Verbel J, Duarte D, Echenique M, et al. 2007. Blood lead levels in children aged 5-9 years living in Cartagena, Colombia. Sci Total Environ 372(2-3):707-716. http://doi.org/10.1016/j.scitotenv.2006.10.025.

Olmedo P, Goessler W, Tanda S, et al. 2018. Metal concentrations in e-cigarette liquid and aerosol samples: The contribution of metallic coils. Environ Health Perspect 126(2):027010. http://doi.org/10.1289/EHP2175.

Olson KW, Skogerboe RK. 1975. Identification of soil lead compounds from automobile sources. Environ Sci Technol 9:227-230.

Omae K, Sakurai H, Higashi T, et al. 1990. No adverse effects of lead on renal function in lead-exposed workers. Ind Health 28(2):77-83.

Omar M, Ibrahim M, Assem H, et al. 2001. Teeth and blood lead levels in Egyptian schoolchildren: Relationship to health effects. J Appl Toxicol 21(4):349-352. http://doi.org/10.1002/jat.771.

Omokhodion FO, Crockford GW. 1991. Lead in sweat and its relationship to salivary and urinary levels in normal healthy subjects. Sci Total Environ 103(2-3):113-122.

Onalaja AO, Claudio L. 2000. Genetic susceptibility to lead poisoning. Environ Health Perspect 108(Suppl 1):23-28.

O'Neil MJ, Heckelman PE, Dobbelaar PH, et al. 2013. Lead and lead compounds. In: The Merck index. London, England: The Royal Society of Chemistry, 1004-1007, 1706.

Ong CN, Lee WR. 1980. Distribution of lead-203 in human peripheral blood in vitro. Br J Ind Med 37:78-84.

Ong C, Kong Y, Ong H, et al. 1990. The in vitro and in vivo effects of lead on $\delta$-aminolevulinic acid dehydratase and pyrimidine 5'-nucleotidase. Pharmacol Toxicol 66(1):23-26.

Onuegbu AJ, Olisekodiaka MJ, Nwaba EI, et al. 2011. Assessment of some renal indices in people occupationally exposed to lead. Toxicol Ind Health 27(5):475-479. http://doi.org/10.1177/0748233710390020.

Oomen AG, Tolls J, Sips AJAM, et al. 2003a. Lead speciation in artificial human digestive fluid. Arch Environ Contam Toxicol 44:107-115.

Oomen AG, Rompelberg CJ, Bruil MA, et al. 2003b. Development of an in vitro digestion model for estimating the bioaccessibility of soil contaminants. Arch Environ Contam Toxicol 44(3):281-287.

Opler MGA, Brown AS, Graziano J, et al. 2004. Prenatal lead exposure, delta-aminolevulinic acid, and schizophrenia. Environ Health Perspect 112(5):548-552. http://doi.org/10.1289/ehp.10464.

Opler MGA, Buka SL, Groeger J, et al. 2008. Prenatal exposure to lead, delta-aminolevulinic acid, and schizophrenia: Further evidence. Environ Health Perspect 116(11):1586-1590. http://doi.org/10.1289/ehp.10464.

Orisakwe OE, Nwachukwu E, Osadolor HB, et al. 2007. Liver and kidney function tests amongst paint factory workers in Nkpor, Nigeria. Toxicol Ind Health 23(3):161-165.

Orssaud G, Claude JR, Moreau T, et al. 1985. Blood lead concentration and blood pressure. Br Med J 290:244.

OSHA. 1995. Subpart Z - Toxic and hazardous substances. Lead-general. Occupational Safety and Health Administration. Code of Federal Regulations. 29 CFR 1910.1025. https://www.osha.gov/laws-regs/regulations/standardnumber/1910/1910.1025. February 19, 2020. 
OSHA. 2002a. Metal \& metalloid particulates in workplace atmospheres (atomic absorption). Occupational Safety and Health Administration. https://www.osha.gov/dts/sltc/methods/inorganic/id121/id121.pdf. January 13, 2020.

OSHA. 2002b. Metal and metalloid particulates in workplace atmospheres (ICP analysis). Occupational Safety and Health Administration. https://www.osha.gov/dts/sltc/methods/inorganic/id125g/id125g.pdf. January 13, 2020.

OSHA. 2016a. Subpart Z - Toxic and hazardous substances. Lead - general. Occupational Safety and Health Administration. Code of Federal Regulations. 29 CFR 1910.1025. https:/www.gpo.gov/fdsys/pkg/CFR-2016-title29-vol6/pdf/CFR-2016-title29-vol6-sec19101025.pdf. May 4, 2017.

OSHA. 2016b. Subpart Z - Toxic and hazardous substances. Air contaminants - general industry. Occupational Safety and Health Administration. Code of Federal Regulations. 29 CFR 1910.1000. https:/www.gpo.gov/fdsys/pkg/CFR-2016-title29-vol6/pdf/CFR-2016-title29-vol6-sec19101000.pdf. March 6, 2017.

OSHA. 2019a. Occupational safety and health standards for shipyard employment. Subpart Z - Toxic and hazardous substances. Lead. Occupational Safety and Health Administration. Code of Federal Regulations. 29 CFR 1915.1025. https://www.osha.gov/lawsregs/regulations/standardnumber/1915/1915.1025. October 25, 2019.

OSHA. 2019b. Occupational safety and health standards. Subpart Z - Toxic and hazardous substances. Air contaminants. Table Z-1: Limits for air contaminants. Occupational Safety and Health Administration. Code of Federal Regulations. 29 CFR 1910.1000. https://www.osha.gov/lawsregs/regulations/standardnumber/1910/1910.1000TABLEZ1. October 25, 2019.

OSHA. 2019c. Safety and health regulations for construction. Lead. Occupational Safety and Health Administration. Code of Federal Regulations. 29 CFR 1926.62. https://www.osha.gov/lawsregs/regulations/standardnumber/1926/1926.62. October 25, 2019.

OSHA. 2019d. Safety and health regulations for construction. Subpart D - Occupational health and environment controls. Gases, vapors, fumes, dusts, and mists. Occupational Safety and Health Administration. Code of Federal Regulations. 29 CFR 1926.55 Appendix A. https://www.osha.gov/laws-regs/regulations/standardnumber/1926/1926.55AppA. October 25, 2019.

OSHA. 2019e. Occupational safety and health standards for shipyard employment. Subpart Z - Toxic and hazardous substances. Air contaminants. Occupational Safety and Health Administration. Code of Federal Regulations. 29 CFR 1915.1000. https://www.osha.gov/lawsregs/regulations/standardnumber/1915/1915.1000. October 25, 2019.

OSHA. 2019f. Occupational safety and health standards. Subpart Z - Toxic and hazardous substances. Lead. Occupational Safety and Health Administration. Code of Federal Regulations. 29 CFR 1910.1025. https://www.osha.gov/laws-regs/regulations/standardnumber/1910/1910.1025. October 25, 2019.

Oskarsson A, Jorhem L, Sundberg J, et al. 1992. Lead poisoning in cattle - Transfer of lead to milk. Sci Total Environ 111:83-94.

Osman K, Pawlas K, Schutz A, et al. 1999. Lead exposure and hearing effects in children in Katowice, Poland. Environ Res 80 Section A:1-8.

Osterberg E, Mayewski P, Kreutz K, et al. 2008. Ice core record of rising lead pollution in the North Pacific atmosphere. Geophys Res Lett 35(5):L05810. http://doi.org/10.1029/2007gl032680.

Osterode W, Barnas U, Geissler K. 1999. Dose dependent reduction of erythroid progenitor cells and inappropriate erythropoietin response in exposure to lead: New aspects of anaemia induced by lead. Occup Environ Med 56:106-109.

Otto D, Robinson G, Baumann S, et al. 1985. 5-Year follow-up study of children with low-to-moderate lead absorption: Electrophysiological evaluation. Environ Res 38(1):168-186.

Ou LT, Thomas JE, Jing W. 1994. Biological and chemical degradation of tetraethyl lead in soil. Bull Environ Contam Toxicol 52:238-245. 
Ou LT, Jing W, Thomas JE. 1995. Biological and chemical degradation of ionic ethyllead compounds in soil. Environ Toxicol Chem 14(4):545-551.

Ozkaynak H, Xue J, Zartarian VG, et al. 2011. Modeled estimates of soil and dust ingestion rates for children. Risk Anal 31(4):592-608. http://doi.org/10.1111/j.1539-6924.2010.01524.x.

Padilla MA, Elobeid M, Ruden DM, et al. 2010. An examination of the association of selected toxic metals with total and central obesity indices: NHANES 99-02. Int J Environ Res Public Health 7:3332-3347. http://doi.org/10.3390/ijerph7093332.

Paglia DE, N VW, Dahlgren JG. 1975. Effects of low-level lead exposure on pyrimidine 5'-nucleotidase and other erythrocyte enzymes. Possible role of pyrimidine 5'-nucleotidase in the pathogenesis of lead-induced anemia. J Clin Invest 56:1164-1169.

Paglia D, Valentine W, Fink K. 1977. Lead poisoning. Further observations on erythrocyte pyrimidinenucleotidase deficiency and intracellular accumulation of pyrimidine nucleotides. J Clin Invest 60(6):1362-1366.

Pagliuca A, Mufti GJ, Baldwin D, et al. 1990. Lead poisoning: Clinical, biochemical, and haematological aspects of a recent outbreak. J Clin Pathol 43:277-281.

Palaniappan K, Roy A, Balakrishnan K, et al. 2011. Lead exposure and visual-motor abilities in children from Chennai, India. Neurotoxicology 32(4):465-470. http://doi.org/10.1016/j.neuro.2011.03.011.

Palmer KT, Kucera CL. 1980. Lead contamination of sycamore and soil from lead mining and smelting operations in eastern Missouri. J Environ Qual 9(1):106-111.

Pan SY, Morrison H, Gibbons L, et al. 2011. Breast cancer risk associated with residential proximity to industrial plants in Canada. J Occup Environ Med 53(5):522-529. http://doi.org/10.1097/JOM.0b013e318216d0b3.

P'An AYS. 1981. Lead levels in saliva and in blood. J Toxicol Environ Health 7(2):273-280. http://doi.org/10.1080/15287398109529978.

PAN Pesticides Database. 2004. Chemical names that begin with: Lead. PAN Pesticides Databasechemicals. Pesticide Action Network. http://www.pesticideinfo.org/. February 22, 2005.

Parajuli RP, Fujiwara T, Umezaki M, et al. 2013. Association of cord blood levels of lead, arsenic, and zinc with neurodevelopmental indicators in newborns: A birth cohort study in Chitwan Valley, Nepal. Environ Res 121:45-51. http://doi.org/10.1016/j.envres.2012.10.010.

Pardo R, Barrado E, Perez L, et al. 1990. Determination and speciation of heavy metals in sediments of the Pisuerga River. Water Res 24(3):373-379.

Park SK, Schwartz J, Weisskopf M, et al. 2006. Low-level lead exposure, metabolic syndrome, and heart rate variability: The VA normative aging study. Environ Health Perspect 114:1718-1724.

Park SK, Hu H, Wright RO, et al. 2009a. Iron metabolism genes, low-level lead exposure, and QT interval. Environ Health Perspect 117(1):80-85.

Park SK, Mukherjee B, Xia X, et al. 2009b. Bone lead level prediction models and their application to examine the relationship of lead exposure and hypertension in the third National Health and Nutrition Examination Survey. J Occup Environ Med 51(12):1422-1436. http://doi.org/10.1097/JOM.0b013e3181bf6c8d.

Park SK, Elmarsafawy S, Mukherjee B, et al. 2010. Cumulative lead exposure and age-related hearing loss: The VA Normative Aging Study. Hear Res 269(1-2):48-55. http://doi.org/10.1016/j.heares.2010.07.004.

Park SJ, Lee JH, Woo SJ, et al. 2015. Five heavy metallic elements and age-related macular degeneration: Korean National Health and Nutrition Examination Survey, 2008-2011. Ophthalmology 122(1):129-137. http://doi.org/10.1016/j.ophtha.2014.07.039.

Park JH, Seo JH, Hong YS, et al. 2016. Blood lead concentrations and attention deficit hyperactivity disorder in Korean children: A hospital-based case control study. BMC pediatrics 16(1):156. http://doi.org/10.1186/s12887-016-0696-5.

Park WJ, Kim SH, Kang W, et al. 2019. Blood lead level and Helicobacter pylori infection in a healthy population: A cross-sectional study. Arch Environ Occup Health 74:1-6. http://doi.org/10.1080/19338244.2019.1654969. 
Parkinson DK, Ryan C, Bromet EJ, et al. 1986. A psychiatric epidemiologic study of occupational lead exposure. Am J Epidemiol 123(2; 2):261-269.

Parkinson DK, Hodgson MJ, Bromet EJ, et al. 1987. Occupational lead exposure and blood pressure. $\mathrm{Br}$ J Ind Med 44(11):744-748.

Parramore CS, Morgan BW, Ethridge MW. 2001. Lead contaminated moonshine: A report of ATF analyzed samples. J Toxicol Clin Toxicol 39(5):520.

Partanen T, Heikkila P, Hernberg S, et al. 1991. Renal cell cancer and occupational exposure to chemical agents. Scand J Work Environ Health 17(4):231-239.

Pasternak MD, Becker CE, Lash A, et al. 1989. Cross-sectional neurotoxicology study of lead-exposed cohort. Clin Toxicol 27(1 and 2):37-51.

Patel AB, Prabhu AS. 2009. Determinants of lead level in umbilical cord blood. Indian Pediatr 46(9):791-793.

Patil AJ, Bhagwat VR, Patil JA, et al. 2006. Effect of lead (Pb) exposure on the activity of superoxide dismutase and catalase in battery manufacturing workers (BMW) of Western Maharashtra (India) with reference to heme biosynthesis. Int J Environ Res Public Health 3(4):329-337.

Patil AJ, Bhagwat VR, Patil JA, et al. 2007. Occupational lead exposure in battery manufacturing workers, silver jewelry workers, and spray painters in western Maharashtra (India): Effect on liver and kidney function. J Basic Clin Physiol Pharmacol 18(2):87-100. http://doi.org/10.1515/JBCPP.2007.18.2.87.

Pawlas N, Broberg K, Olewinska E, et al. 2012. Modification by the genes ALAD and VDR of leadinduced cognitive effects in children. Neurotoxicology 33:37-43.

Pawlas N, Broberg K, Olewinska E, et al. 2015. Genetic modification of ALAD and VDR on leadinduced impairment of hearing in children. Environ Toxicol Pharmacol 39(3):1091-1098. http://doi.org/10.1016/j.etap.2015.03.008.

Pawlas N, Plachetka A, Kozlowska A, et al. 2016. Telomere length, telomerase expression, and oxidative stress in lead smelters. Toxicol Ind Health 32(12):1961-1970. http://doi.org/10.1177/0748233715601758.

Pawlas N, Olewinska E, Markiewicz-Gorka I, et al. 2017. Oxidative damage of DNA in subjects occupationally exposed to lead. Adv Clin Exp Med 26(6):939-945. http://doi.org/10.17219/acem/64682.

Payton M, Hu H, Sparrow D, et al. 1994. Low-level lead exposure and renal function in the normative aging study. Am J Epidemiol 140(9):821-829.

Payton M, Riggs KM, Sprio A, et al. 1998. Relations of bone and blood lead to cognitive function: The VA normative aging study. Neurotoxicol Teratol 20(1):19-27.

PEHSU. 2013. Recommendations on medical management of childhood lead exposure and poisoning. Pediatric Environmental Health Specialty Units. American Academy of Pediatrics. https://www.pehsu.net/_Library/facts/medical-mgmnt-childhood-lead-exposure-June-2013.pdf. February 19, 2020.

Pekcici R, Kavlakoğlu B, Yilmaz S, et al. 2010. Effects of lead on thyroid functions in lead-exposed workers. Cent Eur J Med 5(2):215-218. http://doi.org/10.2478/s11536-009-0092-8.

Pellizzari ED, Perritt RL, Clayton CA. 1999. National human exposure assessment survey (NHEXAS): Exploratory survey of exposure among population subgroups in EPA Region V. J Expo Anal Environ Epidemiol 9:49-55.

Peralta-Videa JR, Lopez ML, Narayan M, et al. 2009. The biochemistry of environmental heavy metal uptake by plants: Implications for the food chain. Int J Biochem Cell Biol 41(8-9):1665-1677. http://doi.org/10.1016/j.biocel.2009.03.005.

Pergande M, Jung K, Precht S, et al. 1994. Changed excretion or urinary proteins and enzymes by chronic exposure to lead. Nephrol Dial Transplant 9:613-618.

Perkins M, Wright RO, Amarasiriwardena CJ, et al. 2014. Very low maternal lead level in pregnancy and birth outcomes in an eastern Massachusetts population. Ann Epidemiol 24(12):915-919. http://doi.org/10.1016/j.annepidem.2014.09.007. 
Perlstein T, Weuve J, Schwartz J, et al. 2007. Cumulative community-level lead exposure and pulse pressure: The Normative Aging Study. Environ Health Perspect 115(12):1696-1700. http://doi.org/10.1289/ehp.10350.

Perneger TV, Nieto FJ, Whelton PK, et al. 1993. A prospective study of blood pressure and serum creatinine: Results from the 'Clue' Study and the ARIC Study. J Am Med Assoc 269:488-493.

Perroy RL, Belby CS, Mertens CJ. 2014. Mapping and modeling three dimensional lead contamination in the wetland sediments of a former trap-shooting range. Sci Total Environ 487:72-81. http://doi.org/10.1016/j.scitotenv.2014.03.102.

Peryea FJ. 1998. Historical use of lead arsenate insecticides, resulting soil contamination and implications for soil remediation. In: Proceedings. 16th World Congress of Soil Science (CD rom), Montpellier, France, 20-26 Aug. 1998. Vol. Scientific registration no: 274. Washington State University. http://soils.tfrec.wsu.edu/leadhistory.htm. March 4, 2005.

Pesch B, Haerting J, Ranft U, et al. 2000. Occupational risk factors for renal cell carcinoma: Agentspecific results from a case-control study in Germany. Int J Epidemiol 29:1014-1024.

Peters JL, Kubzansky L, McNeely E, et al. 2007. Stress as a potential modifier of the impact of lead levels on blood pressure: The Normative Aging Study. Environ Health Perspect 115(8):1154-1159. http://doi.org/10.1289/ehp.10002.

Petrucci R, Leonardi A, Battistuzzi G. 1982. The genetic polymorphism of delta-aminolevulinate dehydrase in Italy. Hum Genet 60(3):289-290.

Pieper KJ, Martin R, Tang M, et al. 2018. Evaluating water lead levels during the Flint water crisis. Environ Sci Technol 52(15):8124-8132. http://doi.org/10.1021/acs.est.8b00791.

Pierzynski GM, Schwab AP. 1993. Heavy metals in the environment. Bioavailability of zinc, cadmium, and lead in a metal-contaminated alluvial soil. J Environ Qual 22:247-254.

Pilsner JR, Hu H, Ettinger A, et al. 2009. Influence of prenatal lead exposure on genomic methylation of cord blood DNA. Environ Health Perspect 117(9):1466-1471. http://doi.org/10.1289/ehp.0800497.

Pineda-Zavaleta AP, García-Vargas G, Borja-Aburto VH, et al. 2004. Nitric oxide and superoxide anion production in monocytes from children exposed to arsenic and lead in region Lagunera, Mexico. Toxicol Appl Pharmacol 198(3):283-290. http://doi.org/10.1016/j.taap.2003.10.034.

Pingitore NE, Clague JW, Amaya MA, et al. 2009. Urban airborne lead: X-ray absorption spectroscopy establishes soil as dominant source. PLoS ONE 4(4):e5019. http://doi.org/10.1371/journal.pone.0005019.

Pinkerton LE, Biagini RE, Ward EM, et al. 1998. Immunologic findings among lead-exposed workers. Am J Ind Med 33(4):400-408. http://doi.org/10.1002/(SICI)1097-0274(199804)33:4<400::AIDAJIM11>3.0.CO;2-2.

Pinto D, Ceballos JM, García G, et al. 2000. Increased cytogenetic damage in outdoor painters. Mutat Res Genet Toxicol Environ Mutagen 467(2):105-111. http://doi.org/10.1016/S1383-5718(00)000243.

Pinto de Almeida AR, Carvalho FM, Spinola AG, et al. 1987. Renal dysfunction in Brazilian lead workers. Am J Nephrol 7(6):455-458. http://doi.org/10.1159/000167519.

Pirkle JL, Schwartz J, Landis JR, et al. 1985. The relationship between blood lead levels and blood pressure and its cardiovascular risk implications. Am J Epidemiol 121(2):246-258.

Pirkle JL, Brody DJ, Gunter EW, et al. 1994. The decline in blood lead levels in the United States. The National Health and Nutrition Examination Surveys (NHANES). J Am Med Assoc 272(4):284-291.

Pirkle JL, Kaufmann RB, Brody DJ, et al. 1998. Exposure of the U.S. population to lead, 1991-1994. Environ Health Perspect 106(11):745-750.

Pizent A, Macan J, Jurasović J, et al. 2008. Association of toxic and essential metals with atopy markers and ventilatory lung function in women and men. Sci Total Environ 390(2-3):369-376. http://doi.org/10.1016/j.scitotenv.2007.10.049.

Pocock SJ, Shaper AG, Walker M, et al. 1983. Effects of tap water lead, water hardness, alcohol, and cigarettes on blood lead concentrations. J Epidemiol Community Health 37:1-7. 
Pocock SJ, Shaper AG, Ashby D, et al. 1984. Blood lead concentration, blood pressure, and renal function. Br Med J 289:872-874.

Pocock SJ, Shaper AG, Ashby D, et al. 1988. The relationship between blood lead, blood pressure, stroke, and heart attacks in middle-aged British men. Environ Health Perspect 78:23-30.

Polanska K, Hanke W, Pawlas N, et al. 2018. Sex-dependent impact of low-level lead exposure during prenatal period on child psychomotor functions. Int J Environ Res Public Health 15(10):2263. http://doi.org/10.3390/ijerph15102263.

Pollack AZ, Schisterman EF, Goldman LR, et al. 2011. Cadmium, lead, and mercury in relation to reproductive hormones and anovulation in premenopausal women. Environ Health Perspect 119(8):1156-1161. http://doi.org/10.1289/ehp.1003284.

Pollack AZ, Mumford SL, Wactawski-Wende J, et al. 2013. Bone mineral density and blood metals in premenopausal women. Environ Res 120:76-81.

Pollack AZ, Mumford SL, Mendola P, et al. 2015. Kidney biomarkers associated with blood lead, mercury, and cadmium in premenopausal women: A prospective cohort study. J Toxicol Environ Health A 78(2):119-131. http://doi.org/10.1080/15287394.2014.944680.

Popovic M, McNeill FE, Chettle DR, et al. 2005. Impact of occupational exposure on lead levels in women. Environ Health Perspect 113(4):478-484.

Poreba R, Poreba M, Gać P, et al. 2011. Ambulatory blood pressure monitoring and structural changes in carotid arteries in normotensive workers occupationally exposed to lead. Hum Exp Toxicol 30(9):1174-1180. http://doi.org/10.1177/0960327110391383.

Poreba R, Gac P, Poreba M, et al. 2012. Assessment of cardiovascular risk in workers occupationally exposed to lead without clinical presentation of cardiac involvement. Environ Toxicol Pharmacol 34(2):351-357. http://doi.org/10.1016/j.etap.2012.05.008.

Poreba R, Gac P, Poreba M, et al. 2013. Echocardiographic assessment of myocardial function in workers occupationally exposed to lead without clinically evident heart disease. Environ Toxicol Pharmacol 36(2):522-528. http://doi.org/10.1016/j.etap.2013.05.010.

Pounds JG, Leggett RW. 1998. The ICRP age-specific biokinetic model for lead: Validations, empirical comparisons, and explorations. Environ Health Perspect 106(Suppl 6):1505-1511.

Pounds JG, Marlar RJ, Allen JR. 1978. Metabolism of lead-210 in juvenile and adult rhesus monkeys (Macaca mulatta). Bull Environ Contam Toxicol 19(1):684-691.

Power MC, Korrick S, Tchetgen Tchetgen EJ, et al. 2014. Lead exposure and rate of change in cognitive function in older women. Environ Res 129:69-75. http://doi.org/10.1016/j.envres.2013.12.010.

Presley SM, Abel MT, Austin GP, et al. 2010. Metal concentrations in schoolyard soils from New Orleans, Louisiana before and after hurricanes Katrina and Rita. Chemosphere 80(1):67-73. http://doi.org/10.1016/j.chemosphere.2010.03.031.

Proctor SP, Rotnitzky A, Sparrow D, et al. 1996. The relationship of blood lead and dietary calcium to blood pressure in the normative aging study. Int J Epidemiol 25(3):528-536.

Przybyla J, Houseman EA, Smit E, et al. 2017. A path analysis of multiple neurotoxic chemicals and cognitive functioning in older US adults (NHANES 1999-2002). Environ Health 16(1):19. http://doi.org/10.1186/s12940-017-0227-3.

Pugh Smith P, Nriagu JO. 2011. Lead poisoning and asthma among low-income and African American children in Saginaw, Michigan. Environ Res 111(1):81-86. http://doi.org/10.1016/j.envres.2010.11.007.

Purchase NG, Fergusson JE. 1986. Lead in teeth: The influence of the tooth type and the sample within a tooth on lead levels. Sci Total Environ 52(3):239-250. http://doi.org/10.1016/00489697(86)90124-5.

Queirolo EI, Ettinger AS, Stoltzfus RJ, et al. 2010. Association of anemia, child and family characteristics with elevated blood lead concentrations in preschool children from Montevideo, Uruguay. Arch Environ Occup Health 65(2):94-100. http://doi.org/10.1080/19338240903390313. 


\section{REFERENCES}

Queiroz MLS, Almeida M, Gallão MI, et al. 1993. Defective neutrophil function in workers occupationally exposed to lead. Pharmacol Toxicol 72(2):73-77. http://doi.org/10.1111/j.16000773.1993.tb00293.x.

Queiroz MLS, Costa FF, Bincoletto C, et al. 1994a. Engulfment and killing capabilities of neutrophils and phagocytic splenic function in persons occupationally exposed to lead. Int J Immunopharmacol 16(3):239-244. http://doi.org/10.1016/0192-0561(94)90018-3.

Queiroz MLS, Perlingeiro RCR, Bincoletto C, et al. 1994b. Immunoglobulin levels and cellular immune function in lead exposed workers. Immunopharmacol Immunotoxicol 16(1):115-128. http://doi.org/10.3109/08923979409029904.

Rabinowitz MB. 1995. Relating tooth and blood lead levels in children. Bull Environ Contam Toxicol 55:853-857.

Rabinowitz MB, Wetherill GW, Kopple JD. 1976. Kinetic analysis of lead metabolism in healthy humans. J Clin Invest 58:260-270.

Rabinowitz MB, Kopple JD, Wetherill GW. 1980. Effect of food intake and fasting on gastrointestinal lead absorption in humans. Am J Clin Nutr 33:1784-1788.

Rabinowitz M, Leviton A, Bellinger D. 1985. Home refinishing, lead paint and infant blood lead levels. Am J Public Health 75(4):403-404.

Rabinowitz MB, Leviton A, Bellinger DC. 1989. Blood lead--tooth lead relationship among Boston children. Bull Environ Contam Toxicol 43(4):485-492. http://doi.org/10.1007/bf01701924.

Rabinowitz MB, Leviton A, Bellinger D. 1993. Relationships between serial blood lead levels and exfoliated tooth dentin lead levels: Models of tooth lead kinetics. Calcif Tissue Int 53(5):338-341. http://doi.org/10.1007/bf01351840.

Rabinowitz M, Bellinger D, Leviton A, et al. 1987. Pregnancy hypertension, blood pressure during labor, and blood lead levels. Hypertension 10:447-451.

Rabinowitz MB, Allred EN, Bellinger DC, et al. 1990. Lead and childhood propensity to infectious and allergic disorders: Is there an association? Bull Environ Contam Toxicol 44(5):657-660. http://doi.org/10.1007/BF01701784.

Rabito FA, Kocak M, Werthmann DW, et al. 2014. Changes in low levels of lead over the course of pregnancy and the association with birth outcomes. Reprod Toxicol 50:138-144. http://doi.org/10.1016/j.reprotox.2014.10.006.

Raihan MJ, Briskin E, Mahfuz M, et al. 2018. Examining the relationship between blood lead level and stunting, wasting and underweight - A cross-sectional study of children under 2 years-of-age in a Bangladeshi slum. PLoS ONE 13(5):e0197856. http://doi.org/10.1371/journal.pone.0197856.

Rajan P, Kelsey KT, Schwartz JD, et al. 2007. Lead burden and psychiatric symptoms and the modifying influence of the delta-aminolevulinic acid dehydratase (ALAD) polymorphism: The VA Normative Aging Study. Am J Epidemiol 166(12):1400-1408. http://doi.org/10.1093/aje/kwm220.

Rajan P, Kelsey KT, Schwartz JD, et al. 2008. Interaction of the delta-aminolevulinic acid dehydratase polymorphism and lead burden on cognitive function: The VA Normative Aging Study. J Occup Environ Med 50(9):1053-1061. http://doi.org/10.1097/JOM.0b013e3181792463.

Rajaraman P, Stewart PA, Samet JM, et al. 2006. Lead, genetic susceptibility, and risk of adult brain tumors. Cancer Epidemiol Biomarkers Prev 15(12):2514-2520. http://doi.org/10.1158/10559965.EPI-06-0482.

Rapisarda V, Ledda C, Ferrante M, et al. 2016. Blood pressure and occupational exposure to noise and lead (Pb): A cross-sectional study. Toxicol Ind Health 32(10):1729-1736. http://doi.org/10.1177/0748233715576616.

Ravnskov U. 1992. Cholesterol lowering trials in coronary heart disease: Frequency of citation and outcome. Br Med J 305:15-19.

Raymond J, Brown MJ. 2015a. Childhood blood lead levels - United States, 2007-2012 [Erratum: MMWR 64(45): 1277]. MMWR Morb Mortal Wkly Rep 62(54):76-80. http://doi.org/10.15585/mmwr.mm6254a5. 
Raymond J, Brown MJ. 2015b. Childhood blood lead levels - United States, 2007-2012 [Erratum to: MMWR 62(54): 76-80]. MMWR Morb Mortal Wkly Rep 64(45):1277.

https://www.cdc.gov/mmwr/pdf/wk/mm6445.pdf. June 29, 2017.

Raymond J, Brown MJ. 2017. Childhood blood lead levels in children aged $<5$ years - United States, 2009-2014. MMWR Morb Mortal Wkly Rep 66(3):1-7.

https://www.cdc.gov/mmwr/volumes/66/ss/pdfs/ss6603.pdf. June 29, 2017.

Reddy KJ, Wang L, Gloss SP. 1995. Solubility and mobility of copper, zinc, and lead in acidic environments. Plant Soil 171:53-58.

Reddy YS, Aparna Y, Ramalaksmi BA, et al. 2014. Lead and trace element levels in placenta, maternal and cord blood: A cross-sectional pilot study. J Obstet Gynaecol Res 40(12):2184-2190. http://doi.org/10.1111/jog.12469.

Reed BE, Moore RE, Cline SR. 1995. Soil flushing of a sandy loam contaminated with $\mathrm{Pb}(\mathrm{II}), \mathrm{PbSO} 4$ (s), PbCo3 (3) or Pb-naphthalene: Column results. J Soil Contam 4(3):243-267.

Rees DC, Duley JA, Marinaki AM. 2003. Pyrimidine 5' nucleotidase deficiency. Br J Haematol 120(3):375-383.

Reimer W, Tittelbach U. 1989. [Verhalten von herzfrequenz, blutdruck und systolischen zeitintervallen in ruhe und wahrend einhandarbeit bei bleiexponierten und kontrollpersonen]. Z Gesamte Hyg 35:491-492. (German)

Rentschler G, Broberg K, Lundh T, et al. 2012. Long-term lead elimination from plasma and whole blood after poisoning. Int Arch Occup Environ Health 85(3):311-316. http://doi.org/10.1007/s00420-011-0673-0.

Renzetti S, Just AC, Burris HH, et al. 2017. The association of lead exposure during pregnancy and childhood anthropometry in the Mexican PROGRESS cohort. Environ Res 152:226-232. http://doi.org/10.1016/j.envres.2016.10.014.

RePORTER. 2020. Lead. National Institutes of Health, Research Portfolio Online Reporting Tools. http://projectreporter.nih.gov/reporter.cfm. January 7, 2020.

Reuben A, Caspi A, Belsky DW, et al. 2017. Association of childhood blood lead levels with cognitive function and socioeconomic status at age 38 years and with IQ change and socioeconomic mobility between childhood and adulthood. JAMA 317(12):1244-1251. http://doi.org/10.1001/jama.2017.1712.

Rezende VB, Barbosa F, Montenegro MF, et al. 2008. Haplotypes of vitamin D receptor modulate the circulating levels of lead in exposed subjects. Arch Toxicol 82:29-36.

Rhoads GG, Ettinger AS, Weisel CP, et al. 1999. The effect of dust lead control on blood lead in toddlers: A randomized trial. Pediatrics 103(3):551-555.

Rhodes D, Spiro III A, Aro A, et al. 2003. Relationship of bone and blood lead levels to psychiatric symptoms: The normative aging study. J Occup Environ Med 45(11):1144-1151.

Richter PA, Bishop EE, Wang J, et al. 2013. Trends in tobacco smoke exposure and blood lead levels among youths and adults in the United States: The National Health and Nutrition Examination Survey, 1999-2008. Preventing chronic disease 10:E213. http://doi.org/10.5888/pcd10.130056.

Riddell TJ, Solon O, Quimbo SA, et al. 2007. Elevated blood-lead levels among children living in the rural Philippines. Bull World Health Organ 85(9):674-680.

Riedt CS, Buckley BT, Brolin RE, et al. 2009. Blood lead levels and bone turnover with weight reduction in women. J Expo Sci Environ Epidemiol 19:90-96.

Ris MD, Dietrich KN, Succop PA, et al. 2004. Early exposure to lead and neuropsychological outcome in adolescence. J Int Neuropsychol Soc 10(2):261-270. http://doi.org/10.1017/S1355617704102154.

Risch HA, Burch JD, Miller AB, et al. 1988. Occupational factors and the incidence of cancer of the bladder in Canada. Br J Ind Med 45:361-367.

Robbins N, Zhang ZF, Sun J, et al. 2010. Childhood lead exposure and uptake in teeth in the Cleveland area during the era of leaded gasoline. Sci Total Environ 408(19):4118-4127. http://doi.org/10.1016/j.scitotenv.2010.04.060. 


\section{REFERENCES}

Robins JM, Cullen MR, Connors BB, et al. 1983. Depressed thyroid indexes associated with occupational exposure to inorganic lead. Arch Intern Med 143:220-224.

Robinson G, Baumann S, Kleinbaum D, et al. 1985. Effects of low to moderate lead exposure on brainstem auditory evoked potentials in children. In: Environmental health, Document 3:

Neurobehavioral methods in occupational and environmental health. Copenhagen, Denmark: World Health Organization, 177-182.

Rodamilans M, Osaba MJ, To-Figueras J, et al. 1988. Lead toxicity on endocrine testicular function in a occupationally exposed population. Hum Toxicol 7:125-128.

Rodosthenous RS, Burris HH, Svensson K, et al. 2017. Prenatal lead exposure and fetal growth: Smaller infants have heightened susceptibility. Environ Int 99:228-233.

http://doi.org/10.1016/j.envint.2016.11.023.

Rodrigues JL, Batista BL, Nunes JA, et al. 2008. Evaluation of the use of human hair for biomonitoring the deficiency of essential and exposure to toxic elements. Sci Total Environ 405(1-3):370-376. http://doi.org/10.1016/j.scitotenv.2008.06.002.

Rodrigues EG, Bellinger DC, Valeri L, et al. 2016. Neurodevelopmental outcomes among 2- to 3-yearold children in Bangladesh with elevated blood lead and exposure to arsenic and manganese in drinking water. Environ Health 15:44. http://doi.org/10.1186/s12940-016-0127-y.

Roels H, Lauwerys R. 1987. Evaluation of dose-effect and dose-response relationships for lead exposure in different Belgian population groups (fetus, child, adult men and women). Trace Elem Med 4(2):80-87.

Roels H, Lauwerys R, Buchet J, et al. 1975. Response of free erythrocyte porphyrin and urinary $\delta$ aminolevulinic acid in men and women moderately exposed to lead. Int Arch Occup Environ Health 34(2):97-108.

Roels H, Buchet JP, Lauwerys R, et al. 1976. Impact of air pollution by lead on the heme biosynthetic pathway in school-age children. Arch Environ Health Nov/Dec:310-315.

Roels HA, Balis-Jacques MN, Buchet J-P, et al. 1979. The influence of sex and of chelation therapy on erythrocyte protoporphyrin and urinary [delta]-aminolevulinic acid in lead-exposed workers. J Occup Environ Med 21(8):527-539.

Roels H, Lauwerys R, Konings J, et al. 1994. Renal function and hyperfiltration capacity in lead smelter workers with high bone lead. Occup Environ Med 51:505-512.

Roels H, Konings J, Green S, et al. 1995. Time-integrated blood lead concentration is a valid surrogate for estimating the cumulative lead dose assessed by tibial lead measurement. Environ Res 69(2):7582.

Rokadia H, Agarwal S. 2013. Serum heavy metals and obstructive lung disease: Results from the National Health and Nutrition Examination Survey. Chest 143(2):388-397. http://doi.org/10.1378/chest.12-0595.

Romeo R, Aprea C, Boccalon P, et al. 1996. Serum erythropoietin and blood lead concentrations. Int Arch Occup Environ Health 69:73-75.

Ronco AM, Gutierrez Y, Gras N, et al. 2010. Lead and arsenic levels in women with different body mass composition. Biol Trace Elem Res 136:269-278. http://doi.org/10.1007/s12011-009-8546-z.

Rooney CP, McLaren RG, Condron LM. 2007. Control of lead solubility in soil contaminated with lead shot: Effect of soil pH. Environ Pollut 149:149-157. http://doi.org/10.1016/j.envpol.2007.01.009.

Rooney JPK, Woods NF, Martin MD, et al. 2018. Genetic polymorphisms of GRIN2A and GRIN2B modify the neurobehavioral effects of low-level lead exposure in children. Environ Res 165:1-10. http://doi.org/10.1016/j.envres.2018.04.001.

Root RA. 2000. Lead loading of urban streets by motor vehicle wheel weights. Environ Health Perspect 108(10):937-940.

Roscoe RJ, Gittleman JL, Deddens JA, et al. 1999. Blood lead levels among children of lead-exposed workers: A meta-analysis. Am J Med 36(4):475-481.

Rosen JF, Chesney RW, Hamstra A, et al. 1980. Reduction in 1,25-dihydroxyvitamin D in children with increased lead absorption. N Engl J Med 302(20):1128-1131. 
Rosenman KD, Sims A, Luo Z, et al. 2003. Occurrence of lead-related symptoms below the current occupational safety and health act allowable blood lead levels. J Occup Environ Med 45(5):546-555.

Rothenberg SJ, Schnaas L, Cansino-Ortiz S, et al. 1989. Neurobehavioral deficits after low level lead exposure in neonates: The Mexico City pilot study. Neurotoxicol Teratol 11:85-93.

Rothenberg SJ, Karchmer S, Schnaas L, et al. 1994a. Changes in serial blood lead levels during pregnancy. Environ Health Perspect 102(10):876-880. http://doi.org/10.1289/ehp.94102876.

Rothenberg SJ, Manalo M, Jiang J, et al. 1999a. Blood lead level and blood pressure during pregnancy in South Central Los Angeles. Arch Environ Health 54(6):382-389. http://doi.org/10.1080/00039899909603369.

Rothenberg SJ, Manalo M, Jiang J, et al. 1999b. Maternal blood lead level during pregnancy in South Central Los Angeles. Arch Environ Health 54(3):151-157. http://doi.org/10.1080/00039899909602253.

Rothenberg SJ, Kondrashov V, Manalo M, et al. 2002. Increases in hypertension and blood pressure during pregnancy with increased bone lead levels. Am J Epidemiol 156(12):1079-1087.

Rousseau MC, Parent ME, Nadon L, et al. 2007. Occupational exposure to lead compounds and risk of cancer among men: A population-based case-control study. Am J Epidemiol 166(9):1005-1014. http://doi.org/10.1093/aje/kwm183.

Roussel H, Waterlot C, Pelfrene A, et al. 2010. Cd, Pb and Zn oral bioaccessibility of urban soils contaminated in the past by atmospheric emissions from two lead and zinc smelters. Arch Environ Contam Toxicol 58(4):945-954. http://doi.org/10.1007/s00244-009-9425-5.

Roy MM, Gordon CL, Beaumont LF, et al. 1997. Further experience with bone lead content measurements in residents of southern Ontario. Appl Radiat Isot 48(3):391-396. http://doi.org/10.1016/s0969-8043(96)00223-0.

Roy A, Bellinger D, Hu H, et al. 2009. Lead exposure and behavior among young children in Chennai, India. Environ Health Perspect 117(10):1607-1611. http://doi.org/10.1289/ehp.0900625.

Roy A, Hu H, Bellinger DC, et al. 2011. Hemoglobin, lead exposure, and intelligence quotient: Effect modification by the DRD2 Taq IA polymorphism. Environ Health Perspect 119(1):144-149. http://doi.org/10.1289/ehp.0901878.

Ruby MV, Schoof R, Brattin W, et al. 1999. Advances in evaluating the oral bioavailability of inorganics in soil for use in human health risk assessment. Environ Sci Technol 33(21):3697-3705.

Ruebner RL, Hooper SR, Parrish C, et al. 2019. Environmental lead exposure is associated with neurocognitive dysfunction in children with chronic kidney disease. Pediatr Nephrol 34(11):23712379. http://doi.org/10.1007/s00467-019-04306-7.

Ryan JA, Scheckel KG, Berti WR, et al. 2004. Reducing children's risk from lead in soil. Environ Sci Technol 38:19A-24A.

Ryu JE, Ziegler EE, Nelson SE, et al. 1983. Dietary intake of lead and blood lead concentration in early infancy. Am J Dis Child 137:886-891.

Sabin LD, Schiff KC. 2008. Dry atmospheric deposition rates of metals along a coastal transect in southern California. Atmos Environ 42(27):6606-6613. http://doi.org/10.1016/j.atmosenv.2008.04.042.

Sabin LD, Lim JH, Stolzenbach KD, et al. 2006. Atmospheric dry deposition of trace metals in the coastal region of Los Angeles, California, USA. Environ Toxicol Chem 25(9):2334-2341. http://doi.org/10.1897/05-300r.1.

Sadeghniiat-Haghighi K, Saraie M, Ghasemi M, et al. 2013. Assessment of peripheral neuropathy in male hospitalized patients with lead toxicity in Iran. J Res Med Sci 18(1):6-9.

Saikat S, Barnes B, Westwood D. 2007. A review of laboratory results for bioaccessibility values of arsenic, lead and nickel in contaminated UK soils. J Environ Sci Health A Tox Hazard Subst Environ En 42(9):1213-1221. http://doi.org/10.1080/10934520701435486.

Sakai T. 2000. Biomarkers of lead exposure. Ind Health 38:127-142. 


\section{REFERENCES}

Sakai T, Morita Y. 1996. delta-Aminolevulinic acid in plasma or whole blood as a sensitive indicator of lead effects, and its relation to the other heme-related parameters. Int Arch Occup Environ Health 68:126-132.

Sakai T, Yanagihara S, Kunugi Y, et al. 1982. Relationships between distribution of lead in erythrocytes in vivo and in vitro and inhibition of ALA-D. Br J Ind Med 39:382-387.

Sakai T, Yanagihara S, Kunugi Y, et al. 1983. Mechanisms of ALA-D inhibition by lead and of its restoration by zinc and dithiothreitol. Br J Ind Med 40:61-66.

Sakai T, Morita Y, Araki T, et al. 2000. Relationship between delta-aminolevulinic acid dehydratase genotypes and heme precursors in lead workers. Am J Ind Med 38:355-360.

Sakata S, Shimizu S, Ogoshi K, et al. 2007. Inverse relationship between serum erythropoietin and blood lead concentrations in Kathmandu tricycle taxi drivers. Int Arch Occup Environ Health 80(4):342345. http://doi.org/10.1007/s00420-006-0125-4.

Sallmén M, Lindbohm M-L, Nurminen M. 2000. Paternal exposure to lead and infertility. Epidemiology 11(2):148-152.

Sallmén M, Anttila A, Lindbohm ML, et al. 1995. Time to pregnancy among women occupationally exposed to lead. J Occup Environ Med 37(8):931-934.

Sanin LH, Gonzalez-Cossio T, Romieu I, et al. 2001. Effect of maternal lead burden on infant weight and weight gain at one month of age among breastfed infants. Pediatrics 107(5):1016-1023.

Sankila R, Karjalainen A, Pukkala E, et al. 1990. Cancer risk among glass factory workers: An excess of lung cancer? Br J Ind Med 47:815-818.

Sanna E, Vallascas E. 2011. Hair lead levels to evaluate the subclinical impact of lead on growth in Sardinian children (Italy). Am J Hum Biol 23(6):740-746. http://doi.org/10.1002/ajhb.21203.

Santibanez M, Vioque J, Alguacil J, et al. 2008. Occupational exposures and risk of oesophageal cancer by histological type: A case-control study in eastern Spain. Occup Environ Med 65(11):774-781. http://doi.org/10.1136/oem.2007.037929.

Sarasua SM, Vogt RF, Henderson LO, et al. 2000. Serum immunoglobulins and lymphocyte subset distributions in children and adults living in communities assessed for lead and cadmium exposure. J Toxicol Environ Health A 60(1):1-15. http://doi.org/10.1080/009841000156556.

Sata F, Araki S, Tanigawa T, et al. 1998. Changes in T cell subpopulations in lead workers. Environ Res 76(1):61-64.

Sauve S, McBride MB, Hendershot WH. 1997. Speciation of lead in contaminated soils. Environ Pollut 98(2):149-155.

Schalscha EB, Morales M, Pratt PF. 1987. Lead and molybdenum in soils and forage near an atmospheric source. J Environ Qual 16(4):313-315.

Schaumberg DA, Mendes F, Balaram M, et al. 2004. Accumulated lead exposure and risk of age-related cataract in men. JAMA 292(22):2750-2754.

Scheckel KG, Diamond GL, Burgess MF, et al. 2013. Amending soils with phosphate as means to mitigate soil lead hazard: A critical review of the state of the science. J Toxicol Environ Health B Crit Rev 16(6):337-380. http://doi.org/10.1080/10937404.2013.825216.

Schell LM, Denham M, Stark AD, et al. 2004. Relationship between blood lead concentration and dietary intakes of infants from 3 to 12 months of age. Environ Res 96(3):264-273. http://doi.org/10.1016/j.envres.2004.02.008.

Schell LM, Denham M, Stark AD, et al. 2009. Growth of infants' length, weight, head and arm circumferences in relation to low levels of blood lead measured serially. Am J Hum Biol 21(2):180187. http://doi.org/10.1002/ajhb.20842.

Schmitt MDC, Trippler DJ. 1988. Soil lead concentrations in residential Minnesota as measured by ICPAES. Water Air Soil Pollut 39:157-168.

Schmitt CJ, Brumbaugh WG. 1990. National contaminant biomonitoring program: Concentrations of arsenic, cadmium, copper, lead, mercury, selenium, and zinc in U.S. freshwater fish, 1976-1984. Arch Environ Contam Toxicol 19:731-747. 
Schnaas L, Rothenberg SJ, Perroni E, et al. 2000. Temporal pattern in the effect of postnatal blood lead level on intellectual development of young children. Neurotoxicol Teratol 22:805-810.

Schnaas L, Rothenberg SJ, Flores MF, et al. 2004. Blood lead secular trend in a cohort of children in Mexico City (1987-2002). Environ Health Perspect 112(10):1110-1115.

Schnaas L, Rothenberg SJ, Flores MF, et al. 2006. Reduced intellectual development in children with prenatal lead exposure. Environ Health Perspect 114(5):791-797. http://doi.org/10.1289/ehp.8552.

Schober SE, Mirel LB, Graubard BI, et al. 2006. Blood lead levels and death from all causes, cardiovascular disease, and cancer: Results from the NHANES III Mortality Study. Environ Health Perspect 114(10):1538-1541.

Schroeder HA, Tipton IH. 1968. The human body burden of lead. Arch Environ Occup Health 17(6):965-978.

Schuhmacher M, Hernandez M, Domingo JL, et al. 1996. A longitudinal study of lead mobilization during pregnancy: Concentration in maternal and umbilical cord blood. Trace Elem Electrolytes 13:177-181.

Schuhmacher M, Paternain JL, Domingo JL, et al. 1997. An assessment of some biomonitors indicative of occupational exposure to lead. Trace Elem Electrolytes 14(3):145-149.

Schumacher C, Brodkin CA, Alexander B, et al. 1998. Thyroid function in lead smelter workers: Absence of subacute or cumulative effects with moderate lead burdens. Int Arch Occup Environ Health 71(7):453-458.

Schütz A, Skerfving S, Ranstam J, et al. 1987. Kinetics of lead in blood after the end of occupational exposure. Scand J Work Environ Health 13:221-231.

Schütz A, Bergdahl IA, Ekholm A, et al. 1996. Measurement by ICP-MS of lead in plasma and whole blood of lead workers and controls. Occup Environ Med 53:736-740.

Schwanitz G, Lehnert G, Gebhart E. 1970. Chromosome damage after occupational exposure to lead. Dtsch Med Wochenschr 95(32):1636-1641.

Schwanitz G, Gebhart E, Rott H-D, et al. 1975. [Chromosomenuntersuchungen bei Personen mit beruflicher Bleiexposition]. Dtsch Med Wochenschr 100(18):1007-1011. (German)

Schwartz J. 1995. Lead, blood pressure, and cardiovascular disease in men. Arch Environ Health 50(1):31-37.

Schwartz J, Otto D. 1987. Blood lead, hearing threshold, and neurobehavioral development in children and youth. Arch Environ Health 42(2; 2):153-160.

Schwartz J, Pitcher H. 1989. The relationship between gasoline lead and blood lead in the United States. J off Stat 5:421-431.

Schwartz J, Otto D. 1991. Lead and minor hearing impairment. Arch Environ Health 46(5):300-305.

Schwartz J, Landrigan PJ, Feldman RG, et al. 1988. Threshold effect in lead-induced peripheral neuropathy. J Pediatr 112:12-17.

Schwartz J, Landrigan PJ, Baker EL, et al. 1990. Lead-induced anemia: Dose-response relationships and evidence for a threshold. Am J Public Health 80(2):165-168.

Schwartz BS, Lee BK, Stewart W, et al. 1995. Associations of delta-aminolevulinic acid dehydratase genotype with plant, exposure duration, and blood lead and zinc protoporphyrin levels in Korean lead workers. Am J Epidemiol 142(7):738-745.

Schwartz BS, Lee BK, Stewart W, et al. 1997a. Associations of subtypes of hemoglobin with deltaaminolevulinic acid dehydratase genotype and dimercaptosuccinic acid-chelatable lead levels. Arch Environ Health 52(2):97-103.

Schwartz BS, Lee B-K, Stewart W, et al. 1997b. delta-Aminolevulinic acid dehydratase genotype modifies four hour urinary lead excretion after oral administration of dimercaptosuccinic acid. Occup Environ Med 54(4):241-246.

Schwartz BS, Lee B-K, Lee G-S, et al. 2000a. Associations of blood lead, dimercaptosuccinic acidchelatable lead, and tibia lead with polymorphisms in the vitamin D receptor and [delta]aminolevulinic acid dehydratase genes. Environ Health Perspect 108(10):949-954. 
Schwartz BS, Stewart W, Bolla K, et al. 2000b. Past adult lead exposure is associated with longitudinal decline in cognitive function. Neurology 55(8):1144-1150.

Schwartz BS, Stewart WF, Todd AC, et al. 2000c. Different associations of blood lead, meso 2,3dimercaptosuccinic acid (DMSA)-chelatable lead, and tibial lead levels with blood pressure in 543 organolead manufacturing workers. Arch Environ Health 55:85-92.

Schwartz BS, Lee B, Lee G, et al. 2001. Associations of blood lead, dimercaptosuccinic acid-chelatable lead, and tibia lead with neurobehavioral test scores in South Korean lead workers. Am J Epidemiol 153(5):453-464.

Schwartz BS, Lee BK, Bandeen-Roche K, et al. 2005. Occupational lead exposure and longitudinal decline in neurobehavioral test scores. Epidemiology 16(1):106-113. http://doi.org/10.1097/01.ede.0000147109.62324.51.

Scinicariello F, Buser MC. 2015. Blood cadmium and depressive symptoms in young adults (aged 20-39 years). Psychol Med 45(4):807-815.

Scinicariello F, Abadin HG, Murray HE. 2011. Association of blood lead and blood pressure in the NHANES 1999-2006. Environ Res 111(8):1249-1257.

Scinicariello F, Murray HE, Moffett DB, et al. 2007. Lead and delta-aminolevulinic acid dehydratase polymorphism: Where does it lead? A meta-analysis. Environ Health Perspect 115(1):35-41. http://doi.org/10.1289/ehp.9448.

Scinicariello F, Yesupriya A, Chang MH, et al. 2010. Modification by ALAD of the association between blood lead and blood pressure in the U.S. population: Results from the Third National Health and Nutrition Examination Survey. Environ Health Perspect 118(2):259-264. http://doi.org/10.1289/ehp.0900866.

Scinicariello F, Buser MC, Mevissen M, et al. 2013. Blood lead level association with lower body weight in NHANES 1999-2006. Toxicol Appl Pharmacol 273(3):516-523. http://doi.org/10.1016/j.taap.2013.09.022.

Sears ME, Kerr KJ, Bray RI. 2012. Arsenic, cadmium, lead, and mercury in sweat: A systematic review. J Environ Public Health 2012:184745. http://doi.org/10.1155/2012/184745.

Secchi GC, Erba L, Cambiaghi G. 1974. delta-Aminolevulinic dehydratase activity of erythrocytes and liver tissue in man. Relationship to lead exposure. Arch Environ Health 28:130-132.

Sedman RM, Mahmood RJ. 1994. Soil ingestion by children and adults reconsidered using the results of recent tracer studies. Air Waste 44(2):141-144. http://doi.org/10.1080/1073161x.1994.10467242.

Seegal RF, Fitzgerald EF, McCaffrey RJ, et al. 2013. Tibial bone lead, but not serum polychlorinated biphenyl, concentrations are associated with neurocognitive deficits in former capacitor workers. J Occup Environ Med 55(5):552-562. http://doi.org/10.1097/JOM.0b013e318285f3fd.

Selevan SG, Landrigan PJ, Stern FB, et al. 1985. Mortality of lead smelter workers. Am J Epidemiol 122:673-683.

Selevan SG, Rice DC, Hogan KA, et al. 2003. Blood lead concentration and delayed puberty in girls. N Engl J Med 348(16):1527-1536. http://doi.org/10.1056/NEJMoa020880.

Selonen S, Liiri M, Strommer R, et al. 2012. The fate of lead at abandoned and active shooting ranges in a boreal pine forest. Environ Toxicol Chem 31(12):2771-2779. http://doi.org/10.1002/etc.1998.

Seo J, Lee BK, Jin SU, et al. 2014. Lead-induced impairments in the neural processes related to working memory function. PLoS ONE 9(8):e105308. http://doi.org/10.1371/journal.pone.0105308.

Seto DSY, Freeman JM. 1964. Lead neuropathy in childhood. Am J Dis Child 107:337-342.

Shadbegian R, Guignet D, Klemick H, et al. 2019. Early childhood lead exposure and the persistence of educational consequences into adolescence. Environ Res 178:108643. http://doi.org/10.1016/j.envres.2019.108643.

Shah F, Kazi TG, Afridi HI, et al. 2010. Environmental exposure of lead and iron deficit anemia in children age ranged 1-5 years: A cross sectional study. Sci Total Environ 408(22):5325-5330. http://doi.org/10.1016/j.scitotenv.2010.07.091. 
Shah F, Kazi TG, Afridi HI, et al. 2011. The influence of environmental exposure on lead concentrations in scalp hair of children in Pakistan. Ecotoxicol Environ Saf 74(4):727-732. http://doi.org/10.1016/j.ecoenv.2010.10.036.

Shaheen SM, Tsadilas CD. 2009. Concentration of lead in soils and some vegetable plants in north Nile Delta as affected by soil type and irrigation water. Commun Soil Sci Plant Anal 40(1-6):327-344.

Shaik AP, Jamil K. 2009. Individual susceptibility and genotoxicity in workers exposed to hazardous materials like lead. J Hazard Mater 168(2-3):918-924. http://doi.org/10.1016/j.jhazmat.2009.02.129.

Shao W, Liu Q, He X, et al. 2017. Association between level of urinary trace heavy metals and obesity among children aged 6-19 years: NHANES 1999-2011. Environ Sci Pollut Res Int 24:1157311581. http://doi.org/10.1007/s11356-017-8803-1.

Shaper AG, Pocock SJ, Walker M, et al. 1981. British regional heart study: Cardiovascular risk factors in middle-aged men in 24 towns. Br Med J 283:179-186.

Sharp DS, Osterloh J, Becker CE, et al. 1988. Blood pressure and blood lead concentration in bus drivers. Environ Health Perspect 78:131-137.

Sharp DS, Smith AH, Holman BL, et al. 1989. Elevated blood pressure in treated hypertensives with low-level lead accumulation. Arch Environ Health 44:18-22.

Sharp DS, Benowitz NL, Osterloh JD, et al. 1990. Influence of race, tobacco use, and caffeine use on the relation between blood pressure and blood lead concentration. Am J Epidemiol 131(5):845-854.

Sheffet A, Thind I, Miller AM, et al. 1982. Cancer mortality in a pigment plant utilizing lead and zinc chromates. Arch Environ Health 37(1):44-52.

Shen XM, Yan CH, Guo D, et al. 1998. Low-level prenatal lead exposure and neurobehavioral development of children in the first year of life: A prospective study in Shanghai. Environ Res 79:1-8.

Shen X, Wu S, Yan C, et al. 2001. delta-Aminolevulinate dehydratase polymorphism and blood lead levels in Chinese children. Environ Res 85(3):185-190.

Shepherd TJ, Dirks W, Roberts NM, et al. 2016. Tracing fetal and childhood exposure to lead using isotope analysis of deciduous teeth. Environ Res 146:145-153. http://doi.org/10.1016/j.envres.2015.12.017.

Sherlock J, Quinn M. 1986. Relationship between blood lead concentrations and dietary lead intake in infants: The Glasgow Duplicate Diet Study 1979-1980. Food Addit Contam 3(2):167-176.

Sherlock J, Smart G, Forbes GI, et al. 1982. Assessment of lead intakes and dose-response for a population in Ayr exposed to a plumbosolvent water supply. Hum Toxicol 1:115-122.

Sherlock JC, Ashby D, Delves HT, et al. 1984. Reduction in exposure to lead from drinking water and its effect on blood lead concentrations. Hum Toxicol 3:383-392.

Shiau C, Wang J, Chen P. 2004. Decreased fecundity among male lead workers. Occup Environ Med 61(11):915-923.

Shih RA, Glass TA, Bandeen-Roche K, et al. 2006. Environmental lead exposure and cognitive function in community-dwelling older adults. Neurology 67(9):1556-1562. http://doi.org/10.1212/01.wnl.0000239836.26142.c5.

Shih RA, Hu H, Weisskopf MG, et al. 2007. Cumulative lead dose and cognitive function in adults: A review of studies that measured both blood lead and bone lead. Environ Health Perspect 115:483492. http://doi.org/10.1289/ehp.9786.

Shiue I. 2014. Higher urinary heavy metal, phthalate, and arsenic but not parabens concentrations in people with high blood pressure, U.S. NHANES, 2011-2012. Int J Environ Res Public Health 11:5989-5999. http://doi.org/10.3390/ijerph110605989.

Siciliano SD, James K, Zhang G, et al. 2009. Adhesion and enrichment of metals on human hands from contaminated soil at an Arctic urban brownfield. Environ Sci Technol 43(16):6385-6390.

Siegel M, Forsyth B, Siegel L, et al. 1989. The effect of lead on thyroid function in children. Environ Res 49:190-196. 


\section{REFERENCES}

Siemiatycki J. 1991. Exposure assessment. In: Risk factors for cancer in the workplace. Boca Raton, FL: CRC Press, 45-49, 84, 141-145, 198-199, 210, 215.

Silbergeld EK, Schwartz J, Mahaffey K. 1988. Lead and osteoporosis: Mobilization of lead from bone in postmenopausal women. Environ Res 47(1):79-94.

Silberstein T, Saphier O, Paz-Tal O, et al. 2006. Lead concentrates in ovarian follicle compromises pregnancy. J Trace Elem Med Biol 20(3):205-207. http://doi.org/10.1016/j.jtemb.2006.05.001.

Silver MK, Li X, Liu Y, et al. 2016. Low-level prenatal lead exposure and infant sensory function. Environ Health 15(1):65. http://doi.org/10.1186/s12940-016-0148-6.

Simon DL, Maynard EJ, Thomas KD. 2007. Living in a sea of lead - Changes in blood- and hand-lead of infants living near a smelter. J Expo Sci Environ Epidemiol 17(3):248-259. http://doi.org/10.1038/sj.jes.7500512.

Simons TJB. 1985. Influence of lead ions on cation permeability in human red cell ghosts. J Membr Biol 84:61-71.

Simons TJB. 1986a. The role of anion transport in the passive movement of lead across the human red cell membrane. J Physiol 378:287-312.

Simons TJB. 1986b. Passive transport and binding of lead by human red blood cells. J Physiol 378:267286.

Simons TJB. 1988. Active transport of lead by the calcium pump in human red cell ghosts. J Physiol 405:105-113.

Simons TJ. 1993. Lead transport and binding by human erythrocytes in vitro. Toxicol Lett 423:307-313.

Simons TJB, Pocock G. 1987. Lead enters bovine adrenal medullary cells through calcium channels. J Neurochem 48:383-389.

Singh Z, Chadha P, Sharma S. 2013. Evaluation of oxidative stress and genotoxicity in battery manufacturing workers occupationally exposed to lead. Toxicol Int 20(1):95-100. http://doi.org/10.4103/0971-6580.111550.

Singh B, Chandran V, Bandhu HK, et al. 2000. Impact of lead exposure on pituitary-thyroid axis in humans. Biometals 13:187-192.

Sioen I, Den Hond E, Nelen V, et al. 2013. Prenatal exposure to environmental contaminants and behavioural problems at age 7-8 years. Environ Int 59:225-231. http://doi.org/10.1016/j.envint.2013.06.014.

Sirivarasai J, Wananukul W, Kaojarern S, et al. 2013. Association between inflammatory marker, environmental lead exposure, and glutathione S-transferase gene. Biomed Res Int 2013:474963. http://doi.org/10.1155/2013/474963.

Sithisarankul P, Schwartz BS, Lee BK, et al. 1997. Aminolevulinic acid dehydratase genotype mediates plasma levels of the neurotoxin, 5-aminolevulinic acid, in lead-exposed workers. Am J Ind Med 32(1):15-20. http://doi.org/10.1002/(sici)1097-0274(199707)32:1<15::aid-ajim2>3.0.co;2-q.

Skerfving S. 1988. Biological monitoring of exposure to inorganic lead. In: Clarkson TW, Friberg L, Nordberg G, et al., eds. Biological monitoring of toxic metals. New York, NY: Plenum Press, 169197.

Skerfving S, Bergdahl IA. 2015. Lead. In: Nordberg GF, Fowler BA, eds. Handbook of toxicology of metals. Specific metals, Volume II. 4th ed. Boston, MA: Academic Press, 911-967.

Skerfving S, Ahlgren L, Christoffersson JO, et al. 1985. Metabolism of inorganic lead in man. Nutr Res Suppl 1:601-607.

Slivkova J, Popelkova M, Massanyi P, et al. 2009. Concentration of trace elements in human semen and relation to spermatozoa quality. J Environ Sci Health A Tox Hazard Subst Environ Eng 44(4):370375. http://doi.org/10.1080/10934520802659729.

Smith GR. 1995. Lead. Minerals yearbook: Volume I. Metals and minerals. U.S. Geological Survey. http://minerals.usgs.gov/minerals/pubs/commodity/lead/380495.pdf. October 5, 2007.

Smith FL, Rathmell TK, Marcil GE. 1938. The early diagnosis of acute and latent plumbism. Am J Clin Pathol 8:471-508. 
Smith DR, Osterloh JD, Flegal AR. 1996. Use of endogenous, stable lead isotopes to determine release of lead from the skeleton. Environ Health Perspect 104(1):60-66.

Smith DR, Ilustre RP, Osterloh JD. 1998a. Methodological considerations for the accurate determination of lead in human plasma and serum. Am J Ind Med 33:430-438.

Smith DR, Kahng MW, Quintanilla-Vega B, et al. 1998b. High-affinity renal lead-binding proteins in environmentally-exposed humans. Toxicol Appl Pharmacol 115:39-52.

Smith D, Hernandez-Avila M, Tellez-Rojo MM, et al. 2002. The relationship between lead in plasma and whole blood in women. Environ Health Perspect 110(3):263-268.

Smith E, Weber J, Naidu R, et al. 2011. Assessment of lead bioaccessibility in peri-urban contaminated soil. J Hazard Mater 186(1):300-305. http://doi.org/10.1016/j.jhazmat.2010.10.111.

Sobin C, Gutierrez M, Alterio H. 2009. Polymorphisms of delta-aminolevulinic acid dehydratase (ALAD) and peptide transporter 2 (PEPT2) genes in children with low-level lead exposure. Neurotoxicology 30(6):881-887. http://doi.org/10.1016/j.neuro.2009.08.006.

Sobin C, Flores-Montoya MG, Gutierrez M, et al. 2015. delta-Aminolevulinic acid dehydratase single nucleotide polymorphism 2 (ALAD2) and peptide transporter $2 * 2$ haplotype (hPEPT2*2) differently influence neurobehavior in low-level lead exposed children. Neurotoxicol Teratol 47:137-145. http://doi.org/10.1016/j.ntt.2014.12.001.

Sokas RK, Simmens S, Sophar K, et al. 1997. Lead levels in Maryland construction workers. Am J Ind Med 31:188-194.

Soldin OP, Pezzullo JC, Hanak B, et al. 2003. Changing trends in the epidemiology of pediatric lead exposure: Interrelationship of blood lead and ZPP concentrations and a comparison to the US population. Ther Drug Monit 25(4):415-420.

Solliway BM, Schaffer A, Pratt H, et al. 1996. Effects of exposure to lead on selected biochemical and haematological variables. Pharmacol Toxicol 78:18-22.

Somashekaraiah BV, Venkaiah B, Prasad ARK. 1990. Biochemical diagnosis of occupational exposure to lead toxicity. Bull Environ Contam Toxicol 44:268-275.

Songdej N, Winters PC, McCabe MJ, et al. 2010. A population-based assessment of blood lead levels in relation to inflammation. Environ Res 110(3):272-277. http://doi.org/10.1016/j.envres.2009.12.008.

Sonmez O, Bukun B, Kaya C, et al. 2008. The assessment of tolerance to heavy metals (Cd, $\mathrm{Pb}$ and $\mathrm{Zn}$ ) and their accumulation in three weed species. Pak J Bot 40(2):747-754.

Sönmez F, Dönmez O, Keskınoğlu A, et al. 2002. Lead exposure and urinary N-acetyl $\beta$ D glucosaminidase activity in adolescent workers in auto repair workshops. J Adolesc Health 30(3):213-216.

Soto-Jiménez MF, Flegal AR. 2009. Origin of lead in the Gulf of California Ecoregion using stable isotope analysis. J Geochem Explor 101(3):209-217. http://doi.org/10.1016/j.gexplo.2008.07.003.

Spear TM, Svee W, Vincent JH, et al. 1998. Chemical speciation of lead dust associated with primary lead smelting. Environ Health Perspect 106(9):565-571.

Specht AJ, Weisskopf M, Nie LH. 2018. Childhood lead biokinetics and associations with age among a group of lead-poisoned children in China. J Expo Sci Environ Epidemiol 29(3):416-423. http://doi.org/10.1038/s41370-018-0036-y.

Specht AJ, Lin Y, Weisskopf M, et al. 2016. XRF-measured bone lead (Pb) as a biomarker for $\mathrm{Pb}$ exposure and toxicity among children diagnosed with $\mathrm{Pb}$ poisoning. Biomarkers 21(4):347-352. http://doi.org/10.3109/1354750x.2016.1139183.

Spector JT, Navas-Acien A, Fadrowski J, et al. 2011. Associations of blood lead with estimated glomerular filtration rate using MDRD, CKD-EPI and serum cystatin C-based equations. Nephrol Dial Transplant 26(9):2786-2792. http://doi.org/10.1093/ndt/gfq773.

Staessen JA, Roels H, Fagard R. 1996b. Lead exposure and conventional and ambulatory blood pressure. A prospective population study. J Am Med Assoc 275(20):1563-1570.

Staessen J, Bulpitt C, Roels H, et al. 1984. Urinary cadmium and lead concentrations and their relation to blood pressure in a population with low exposure. Br J Ind Med 41(2):241-248. 


\section{REFERENCES}

Staessen J, Yeoman WB, Fletcher AE, et al. 1990. Blood lead concentration, renal function, and blood pressure in London civil servants. Br J Ind Med 47:442-447.

Staessen JA, Lauwerys RR, Buchet JP, et al. 1992. Impairment of renal function with increasing blood lead concentrations in the general population. N Engl J Med 327:151-156.

Staessen JA, Lauwerys RR, Bulpitt CJ, et al. 1994. Is a positive association between lead exposure and blood pressure supported by animal experiments? Curr Opin Nephrol Hypertens 3(3):257-263.

Staessen JA, Buchet J-P, Ginucchio G, et al. 1996a. Public health implications of environmental exposure to cadmium and lead: An overview of epidemiological studies in Belgium. J Cardiovasc Risk 3(1):26-41.

Staessen JA, T O'Brien E, Thijs L, et al. 2000. Modern approaches to blood pressure measurement. Occup Environ Med 57(8):510-520.

Staessen JA, Nawrot T, Den Hond E, et al. 2001. Renal function, cytogenetic measurements, and sexual development in adolescents in relation to environmental pollutants: A feasibility study of biomarkers. Lancet 357(9269):1660-1669.

Stanek EJ, Calabrese EJ, Xu B. 2012. Meta-analysis of mass-balance studies of soil ingestion in children. Risk Anal 32(3):433-447. http://doi.org/10.1111/j.1539-6924.2011.01673.x.

Stanek EJ, Calabrese EJ, Barnes R, et al. 1997. Soil ingestion in adults - Results of a second pilot study. Ecotoxicol Environ Saf 36(3):249-257. http://doi.org/10.1006/eesa.1996.1510.

Stauber JL, Florence TM. 1988. A comparative study of copper, lead, cadmium and zinc in human sweat and blood. Sci Total Environ 74:235-247.

Stauber JL, Florence TM, Gulson BL, et al. 1994. Percutaneous absorption of inorganic lead compounds. Sci Total Environ 145:55-70.

Steenhout A. 1982. Kinetics of lead storage in teeth and bones: An epidemiologic approach. Arch Environ Health 37(4):224-231. http://doi.org/10.1080/00039896.1982.10667569.

Steenhout A, Pourtois M. 1981. Lead accumulation in teeth as a function of age with different exposures. Br J Ind Med 38(3):297-303. http://doi.org/10.1136/oem.38.3.297.

Steenhout A, Pourtois M. 1987. Age-related lead kinetics in children. In: Trace elements in human health and disease. Second Nordic symposium, Odense, Denmark. Copenhagen, Denmark: World Health Organization, 144-147.

Steenland K, Boffetta P. 2000. Lead and cancer in humans: Where are we now? Am J Ind Med 38:295299.

Steenland K, Selevan S, Landrigan P. 1992. The mortality of lead smelter workers: An update. Am J Public Health 82(12):1641-1644.

Steenland K, Barry V, Anttila A, et al. 2017. A cohort mortality study of lead-exposed workers in the USA, Finland and the UK. Occup Environ Med 74(11):785-791. http://doi.org/10.1136/oemed2017-104311.

Steenland K, Barry V, Anttila A, et al. 2019. Cancer incidence among workers with blood lead measurements in two countries. Occup Environ Med 76(9):603-610. http://doi.org/10.1136/oemed2019-105786.

Stern AH. 1994. Derivation of a target level of lead in soil at residential sites corresponding to a de minimis contribution to blood lead concentration. Risk Anal 14(6):1049-1056.

Stern AH. 1996. Derivation of a target concentration of $\mathrm{Pb}$ in soil based on elevation of adult blood pressure. Risk Anal 16(2):201-210.

Stewart WF, Schwartz BS, Simon D, et al. 2002. ApoE genotype, past adult lead exposure, and neurobehavioral function. Environ Health Perspect 110(5):501-505.

Stollery BT, Banks HA, Broadbent DE, et al. 1989. Cognitive functioning in lead workers. Br J Ind Med 46:698-707.

Stollery BT, Broadbent DE, Banks HA, et al. 1991. Short term prospective study of cognitive functioning in lead workers. Br J Ind Med 48:739-749.

Stroustrup A, Hsu HH, Svensson K, et al. 2016. Toddler temperament and prenatal exposure to lead and maternal depression. Environ Health 15(1):71. http://doi.org/10.1186/s12940-016-0147-7. 
Stuik EJ. 1974. Biological response of male and female volunteers to inorganic lead. Int Arch Arbeitsmed 33:83-97.

Succop P, Bornschein R, Brown K, et al. 1998. An empirical comparison of lead exposure pathway models. Environ Health Perspect 106 Suppl 6:1577-1583.

Succop P, Clark S, Tseng CY, et al. 2001. Evaluation of public housing lead risk assessment data. Environ Geochem Health 23:1-15.

Sun CC, Wong TT, Hwang YH, et al. 2002. Percutaneous absorption of inorganic lead compounds. AIHA J (Fairfax, Va) 63(5):641-646. http://doi.org/10.1080/15428110208984751.

Sun L, Hu J, Zhao Z, et al. 2003. Influence of exposure to environmental lead on serum immunoglobulin in preschool children. Environ Res 92(2):124-128. http://doi.org/10.1016/S0013-9351(02)00090-7.

Sun Y, Sun D, Zhou Z, et al. 2008a. Osteoporosis in a Chinese population due to occupational exposure to lead. Am J Ind Med 51(6):436-442. http://doi.org/10.1002/ajim.20567.

Sun Y, Sun DH, Zhou ZJ, et al. 2008b. Estimation of benchmark dose for bone damage and renal dysfunction in a Chinese male population occupationally exposed to lead. Ann Occup Hyg 52(6):527-533. http://doi.org/10.1093/annhyg/men031.

Suszkiw JB. 2004. Presynaptic disruption of transmitter release by lead. Neurotoxicology 25(4):599604.

Süzen HS, Duydu Y, Aydın A, et al. 2003. Influence of the delta-aminolevulinic acid dehydratase (ALAD) polymorphism on biomarkers of lead exposure in Turkish storage battery manufacturing workers. Am J Ind Med 43(2):165-171.

Sweeney MH, Beaumont JJ, Waxweiler RJ, et al. 1986. An investigation of mortality from cancer and other causes of death among workers employed at an east Texas chemical plant. Arch Environ Health 41(1):23-28.

Swenberg JA, Short B, Borghoff S, et al. 1989. The comparative pathobiology of $\alpha 2 \mathrm{u}$-globulin nephropathy. Toxicol Appl Pharmacol 97(1):35-46.

Symanski E, Hertz-Picciotto I. 1995. Blood lead levels in relation to menopause, smoking, and pregnancy history. Am J Epidemiol 141(11):1047-1058.

Szymanska-Chabowska A, Laczmanski L, Jedrychowska I, et al. 2015. The relationship between selected VDR, HFE and ALAD gene polymorphisms and several basic toxicological parameters among persons occupationally exposed to lead. Toxicology 334:12-21. http://doi.org/10.1016/j.tox.2015.05.002.

Taha EA, Sayed SK, Ghandour NM, et al. 2013. Correlation between seminal lead and cadmium and seminal parameters in idiopathic oligoasthenozoospermic males. Cent European J Urol 66(1):84-92. http://doi.org/10.5173/ceju.2013.01.art28.

Takamatsu T, Murata T, Koshikawa MK, et al. 2010. Weathering and dissolution rates among Pb shot pellets at differing elemental compositions exposed to various aqueous and soil conditions. Arch Environ Contam Toxicol 59:91-99. http://doi.org/10.1007/s00244-009-9449-x.

Tamura H, Honda M, Sato T, et al. 2005. Pb hyperaccumulation and tolerance in common buckwheat (Fagopyrum esculentum Moench). J Plant Res 118(5):355-359. http://doi.org/10.1007/s10265-0050229-z.

Tasmin S, Furusawa H, Ahmad SA, et al. 2015. delta-Aminolevulinic acid dehydratase (ALAD) polymorphism in lead exposed Bangladeshi children and its effect on urinary aminolevulinic acid (ALA). Environ Res 136:318-323. http://doi.org/10.1016/j.envres.2014.08.045.

Taylor CM, Golding J, Emond AM. 2015. Adverse effects of maternal lead levels on birth outcomes in the ALSPAC study: A prospective birth cohort study. BJOG 122(3):322-328. http://doi.org/10.1111/1471-0528.12756.

Taylor CM, Golding J, Hibbeln J, et al. 2013. Environmental factors predicting blood lead levels in pregnant women in the UK: The ALSPAC study. PLoS ONE 8(9):e72371. http://doi.org/10.1371/journal.pone.0072371. 


\section{REFERENCES}

Taylor CM, Kordas K, Golding J, et al. 2017. Effects of low-level prenatal lead exposure on child IQ at 4 and 8 years in a UK birth cohort study. Neurotoxicology 62:162-169. http://doi.org/10.1016/j.neuro.2017.07.003.

Taylor CM, Emond AM, Lingam R, et al. 2018. Prenatal lead, cadmium and mercury exposure and associations with motor skills at age 7 years in a UK observational birth cohort. Environ Int 117:4047. http://doi.org/10.1016/j.envint.2018.04.032.

Teichmann R, Stremmel W. 1990. Iron uptake by human upper small intestine microvillous membrane vesicles. Indication for a facilitated transport mechanism mediated by a membrane iron-binding protein. J Clin Invest 86(6):2145-2153.

Telisman S, Cvitkovic P, Jurasovic J, et al. 2000. Semen quality and reproductive endocrine function in relation to biomarkers of lead, cadmium, zinc, and copper in men. Environ Health Perspect 108(1):45-53.

Telisman S, Colak B, Pizent A, et al. 2007. Reproductive toxicity of low-level lead exposure in men. Environ Res 105(2):256-266. http://doi.org/10.1016/j.envres.2007.05.011.

Tellez-Rojo MM, Hernandez-Avila M, Gonzalez-Cossio T, et al. 2002. Impact of breastfeeding on the mobilization of lead from bone. Am J Epidemiol 155(5):420-428.

Tellez-Rojo MM, Hernandez-Avila M, Lamadrid-Figueroa H, et al. 2004. Impact of bone lead and bone resorption on plasma and whole blood lead levels during pregnancy. Am J Epidemiol 160(7):668678.

Téllez-Rojo MM, Bellinger DC, Arroyo-Quiroz C, et al. 2006. Longitudinal associations between blood lead concentrations lower than $10 \mathrm{microg} / \mathrm{dL}$ and neurobehavioral development in environmentally exposed children in Mexico City. Pediatrics 118(2):e323-e330. http://doi.org/10.1542/peds.20053123.

Ter Haar GL, Bayard MA. 1971. Composition of airborne lead particles. Nature 232:553-554.

Ter Haar G, Aronow R. 1974. New information on lead in dirt and dust as related to the childhood lead problem. Environ Health Perspect 7:83-89.

Theppeang K, Schwartz BS, Lee B-K, et al. 2004. Associations of patella lead with polymorphisms in the vitamin $\mathrm{D}$ receptor, $\delta$-aminolevulinic acid dehydratase and endothelial nitric oxide synthase genes. J Occup Environ Med 46(6):528-537.

Theppeang K, Glass TA, Bandeen-Roche K, et al. 2008. Associations of bone mineral density and lead levels in blood, tibia, and patella in urban-dwelling women. Environ Health Perspect 116(6):784790. http://doi.org/10.1289/ehp.10977.

Thomas S, Arbuckle TE, Fisher M, et al. 2015. Metals exposure and risk of small-for-gestational age birth in a Canadian birth cohort: The MIREC study. Environ Res 140:430-439. http://doi.org/10.1016/envres.2015.04.018.

Thompson GN, Robertson EF, Fitzgerald S. 1985. Lead mobilization during pregnancy. Med J Aust 143:131.

Tian L, Zheng G, Sommar JN, et al. 2013. Lead concentration in plasma as a biomarker of exposure and risk, and modification of toxicity by delta-aminolevulinic acid dehydratase gene polymorphism. Toxicol Lett 221(2):102-109. http://doi.org/10.1016/j.toxlet.2013.06.214.

Timchalk C, Lin Y, Weitz KK, et al. 2006. Disposition of lead (Pb) in saliva and blood of SpragueDawley rats following a single or repeated oral exposure to Pb-acetate. Toxicology 222(1):86-94.

Todd AC, Carroll S, Godbold JH, et al. 2000. Variability in XRF-measured tibia lead levels. Phys Med Biol 45(12):3737-3748.

Todd AC, Buchanan R, Carroll S, et al. 2001. Tibia lead levels and methodological uncertainty in 12year-old children. Environ Res 86:60-65.

Todd AC, Carroll S, Geraghty C, et al. 2002. L-shell x-ray fluorescence measurements of lead in bone: Accuracy and precision. Phys Med Biol 47:1399-1419.

Tola S, Hernberg S, Asp S, et al. 1973. Parameters indicative of absorption and biological effect in new lead exposure: A prospective study. Br J Ind Med 30(2):134-141. 
Tomoum HY, Mostafa GA, Ismail NA, et al. 2010. Lead exposure and its association with pubertal development in school-age Egyptian children: Pilot study. Pediatr Int 52(1):89-93. http://doi.org/10.1111/j.1442-200X.2009.02893.x.

Tomsig J, Suszkiw JB. 1991. Permeation of Pb2+ through calcium channels: Fura-2 measurements of voltage-and dihydropyridine-sensitive $\mathrm{Pb} 2+$ entry in isolated bovine chromaffin cells. Biochim Biophys Acta Biomembr 1069(2):197-200.

Tong S, McMichael AJ, Baghurst PA. 2000. Interactions between environmental lead exposure and sociodemographic factors on cognitive development. Arch Environ Health 55(5):330-335. http://doi.org/10.1080/00039890009604025.

Toscano CD, Guilarte TR. 2005. Lead neurotoxicity: From exposure to molecular effects. Brain Res Brain Res Rev 49(3):529-554. http://doi.org/10.1016/j.brainresrev.2005.02.004.

Treble RG, Thompson RS. 1997. Preliminary results of a survey of lead levels in human liver tissue. Bull Environ Contam Toxicol 59:688-695.

TRI18. 2020. TRI explorer: Providing access to EPA's toxics release inventory data. Washington, DC: Toxics Release Inventory. U.S. Environmental Protection Agency. http://www.epa.gov/triexplorer/. February 18, 2020.

Triebig G, Weltle D, Valentin H. 1984. Investigations on neurotoxicity of chemical substances at the workplace. Int Arch Occup Environ Health 53:189-204.

Tripathi RK, Llewellyn GC. 1990. Deterioration of air quality in firing ranges: A review of airborne lead exposures. In: Llewellyn GC, O'Rear CE, eds. Biodeterioration research 3: Mycotoxins, biotoxins, wood decay, air quality, cultural properties, general biodeterioration, and degradation. New York, NY: Plenum Press, 445-457.

Tripathi R, Raghunath R, Kumar AV, et al. 2001. Atmospheric and children's blood lead as indicators of vehicular traffic and other emission sources in Mumbai, India. Sci Total Environ 267(1):101-108.

Tsaih SW, Korrick S, Schwartz J, et al. 2004. Lead, diabetes, hypertension, and renal function: The normative aging study. Environ Health Perspect 112(11):1178-1182. http://doi.org/10.1289/ehp.7024.

Tsoi MF, Cheung CL, Cheung TT, et al. 2016. Continual decrease in blood lead level in Americans: United States National Health Nutrition and Examination Survey 1999-2014. Am J Med 129(11):1213-1218. http://doi.org/10.1016/j.amjmed.2016.05.042.

Tulasi SJ, Reddy PUM, Rao JVR. 1992. Accumulation of lead and effects on total lipids and lipid derivatives in the freshwater fish Anabas testudineus (Bloch). Ecotoxicol Environ Saf 23(1):33-38.

Tuppurainen M, Wagar G, Kurppa K, et al. 1988. Thyroid function as assessed by routine laboratory tests of workers with long-term lead exposure. Scand J Work Environ Health 14:175-180.

Turlakiewicz Z, Chmielnicka J. 1985. Diethyllead as a specific indicator of occupational exposure to tetraethyllead. Br J Ind Med 42:682-685.

Tuthill RW. 1996. Hair lead levels related to children's classroom attention-deficit behavior. Arch Environ Health 51(3):214-220.

Tvinnereim HM, Eide R, Riise T, et al. 1997. Lead in primary teeth from Norway: Changes in lead levels from the 1970s to the 1990s. Sci Total Environ 207(2-3):165-177. http://doi.org/10.1016/s0048-9697(97)00263-5.

Ukaejiofo EO, Thomas N, Ike SO. 2009. Haematological assessment of occupational exposure to lead handlers in Enugu urban, Enugu State, Nigeria. Niger J Clin Pract 12(1):58-64.

Ulmer DD, Vallee BL. 1969. Effects of lead on biochemical systems. In: Hemphill DD, ed. Trace substances in environmental health. Columbia, MO: University of Missouri Press, 7-27.

Undeger U, Basaran N, Canpinar H, et al. 1996. Immune alterations in lead-exposed workers. Toxicology 109:167-172.

USAF. 1995. The fate and behavior of lead alkyls in the subsurface environment. Tyndall Air Force Base, FL: U.S. Air Force. AL/EQ-TR-1994-0026. 


\section{REFERENCES}

USGS. 1989. Techniques of Water-Resources Investigations of the United States Geological Survey: Chapter A1: Methods for the determination of inorganic substances in water and fluvial sediments. U.S. Geological Survey. https://pubs.er.usgs.gov/publication/twri05A1. March 30, 2017.

USGS. 1993. Methods of analysis by the U.S. Geological Survey National Water Quality Laboratory. Determination of inorganic and organic constituents in water and fluvial sediments. Denver, CO: U.S. Geological Survey. Open-File Report 93-125. https://pubs.usgs.gov/of/1993/0125/report.pdfUSGS. March 30, 2017.

USGS. 2014. Comparison of the U.S. lead recycling industry in 1998 and 2011. U.S. Geological Survey. Scientific Investigations Report 2014-5086. https://pubs.usgs.gov/sir/2014/5086/pdf/sir2014-5086.pdf. June 26, 2017.

USGS. 2016. 2014 Mineral yearbook. Lead [advance release]. U.S. Geological Survey. https://minerals.usgs.gov/minerals/pubs/commodity/lead/myb1-2014-lead.pdf. June 26, 2017.

USGS. 2019. Mineral commodity summaries 2019. Reston, VA: U.S. Geological Survey. http://doi.org/10.3133/70202434.

Vaglenov A, Carbonell E, Marcos R. 1998. Biomonitoring of workers exposed to lead. Genotoxic effects, its modulation by polyvitamin treatment and evaluation of induced radioresistance. Mutat Res 418:79-92.

Vaglenov A, Creus A, Laltchev S, et al. 2001. Occupational exposure to lead and induction of genetic damage. Environ Health Perspect 109(3):295-298.

Valciukas JA, Lilis R, Eisinger J, et al. 1978. Behavioral indicators of lead neurotoxicity: Results of a clinical field survey. Int Arch Occup Environ Health 41(4):217-236.

Valentino M, Governa M, Marchiseppe I, et al. 1991. Effects of lead on polymorphonuclear leukocyte (PMN) functions in occupationally exposed workers. Arch Toxicol 65(8):685-688.

Valentino M, Rapisarda V, Santarelli L, et al. 2007. Effect of lead on the levels of some immunoregulatory cytokines in occupationally exposed workers. Hum Exp Toxicol 26(7):551-556. http://doi.org/10.1177/0960327107073817.

Van de Wiele TR, Oomen AG, Wragg J, et al. 2007. Comparison of five in vitro digestion models to in vivo experimental results: Lead bioaccessibility in the human gastrointestinal tract. J Environ Sci Health A Tox Hazard Subst Environ Eng 42(9):1203-1211. http://doi.org/10.1080/10934520701434919.

Van Esch GJ, Kroes R. 1969. The induction of renal tumours by feeding basic lead acetate to mice and hamsters. Br J Cancer 23(4):765-771.

Van Larebeke N, Koppen G, Nelen V, et al. 2004. Differences in HPRT mutant frequency among middle-aged Flemish women in association with area of residence and blood lead levels. Biomarkers 9(1):71-84. http://doi.org/10.1080/13547500310001652160.

van Wijngaarden E, Dosemeci M. 2006. Brain cancer mortality and potential occupational exposure to lead: Findings from the National Longitudinal Mortality Study, 1979-1989. Int J Cancer 119(5):1136-1144. http://doi.org/10.1002/ijc.21947.

van Wijngaarden E, Campbell JR, Cory-Slechta DA. 2009. Bone lead levels are associated with measures of memory impairment in older adults. Neurotoxicology 30(4):572-580. http://doi.org/10.1016/j.neuro.2009.05.007.

Vander AJ, Taylor DL, Kalitis K, et al. 1977. Renal handling of lead in dogs: Clearance studies. Am J Physiol 233(6):F532-F538.

Vantelon D, Lanzirotti A, Scheinost AC, et al. 2005. Spatial distribution and speciation of lead around corroding bullets in a shooting range soil studies by micro-X-ray fluorescence and absorption spectroscopy. Environ Sci Technol 39(13):4808-4815.

Verbeeck RMH, Lassuyt CJ, Heijligers HJM, et al. 1981. Lattice parameters and cation distribution of solid solutions of calcium and lead hydroxyapatite. Calcif Tissue Int 33(1):243-247. http://doi.org/10.1007/BF02409444.

Verberk MM, Willems TEP, Verplanke AJW, et al. 1996. Environmental lead and renal effects in children. Arch Environ Health 51(1):83-87. 
Verschoor M, Wibowo A, Herber R, et al. 1987. Influence of occupational low-level lead exposure on renal parameters. Am J Ind Med 12(4):341-351.

Vesper SJ, Donovan-Brand R, Paris KP, et al. 1996. Microbial removal of lead from solid media and soil. Water Air Soil Pollut 86:207-219.

Victery W, Vander AJ, Mouw DR. 1979. Effect of acid-base status on renal excretion and accumulation of lead in dogs and rats. Am J Physiol 237(5):F398-F407.

Vigeh M, Yokoyama K, Kitamura F, et al. 2010. Early pregnancy blood lead and spontaneous abortion. Women Health 50(8):756-766.

Vigeh M, Yokoyama K, Seyedaghamiri Z, et al. 2011. Blood lead at currently acceptable levels may cause preterm labour. Occup Environ Med 68(3):231-234. http://doi.org/10.1136/oem.2009.050419.

Vigeh M, Yokoyama K, Matsukawa T, et al. 2014. Low level prenatal blood lead adversely affects early childhood mental development. J Child Neurol 29(10):1305-1311. http://doi.org/10.1177/0883073813516999.

Viverette L, Mielke HW, Brisco M, et al. 1996. Environmental health in minority and other underserved populations: Benign methods for identifying lead hazards at day care centres of New Orleans. Environ Geochem Health 18(1):41-45. http://doi.org/10.1007/bf01757218.

Vlasak T, Jordakieva G, Gnambs T, et al. 2019. Blood lead levels and cognitive functioning: A metaanalysis. Sci Total Environ 668:678-684. http://doi.org/10.1016/j.scitotenv.2019.03.052.

von Lindern I, Spalinger S, Petroysan V, et al. 2003. Assessing remedial effectiveness through the blood lead:soil/dust lead relationship at the Bunker Hill Superfund Site in the Silver Valley of Idaho. Sci Total Environ 303(1-2):139-170.

von Lindern I, Spalinger S, Stifelman ML, et al. 2016. Estimating children's soil/dust ingestion rates through retrospective analyses of blood lead biomonitoring from the Bunker Hill Superfund Site in Idaho. Environ Health Perspect 124:1462-1470. http://doi.org/10.1289/ehp.1510144.

Vupputuri S, He J, Muntner P, et al. 2003. Blood lead level is associated with elevated blood pressure in blacks. Hypertension 41(3):463-468.

Vural N, Duydu Y. 1995. Biological monitoring of lead in workers exposed to tetraethyllead. Sci Total Environ 171:183-187.

Waalkes MP, Klaassen CD. 1985. Concentration of metallothionein in major organs of rats after administration of various metals. Fundam Appl Toxicol 5:473-477.

Waalkes MP, Harvey MJ, Klaassen CD. 1984. Relative in vitro affinity of hepatic metallothionein for metals. Toxicol Lett 20:33-39.

Waalkes MP, Diwan BA, Ward JM, et al. 1995. Renal tubular tumors and atypical hyperplasias in B6C3F1. Cancer Res 55:5265-5271.

Wang EX, Bormann FH, Benoit G. 1995. Evidence of complete retention of atmospheric lead in the soils of northern hardwood forested ecosystems. Environ Sci Technol 29:735-739.

Wang Y, Allen AG, Harrison RM. 1996. Determination of octanol-water partition coefficients, water solubility and vapour pressures of alkyl-lead compounds. Appl Organomet Chem 10(10):773-778.

Wang IJ, Karmaus WJJ, Yang CC. 2017a. Lead exposure, IgE, and the risk of asthma in children. J Expo Sci Environ Epidemiol 27(5):478-483. http://doi.org/10.1038/jes.2017.5.

Wang G, Su MY, Chen YH, et al. 2006. Transfer characteristics of cadmium and lead from soil to the edible parts of six vegetable species in southeastern China. Environ Pollut 144(1):127-135. http://doi.org/10.1016/j.envpol.2005.12.023.

Wang FT, Hu H, Schwartz J, et al. 2007. Modifying effects of the HFE polymorphisms on the association between lead burden and cognitive decline. Environ Health Perspect 115(8):1210-1215. http://doi.org/10.1289/ehp.9855.

Wang HL, Chen XT, Yang B, et al. 2008. Case-control study of blood lead levels and attention deficit hyperactivity disorder in Chinese children. Environ Health Perspect 116(10):1401-1406. http://doi.org/10.1289/ehp.11400. 


\section{REFERENCES}

Wang Q, Zhao HH, Chen JW, et al. 2010. delta-Aminolevulinic acid dehydratase activity, urinary deltaaminolevulinic acid concentration and zinc protoporphyrin level among people with low level of lead exposure. Int J Hyg Environ Health 213(1):52-58. http://doi.org/10.1016/j.ijheh.2009.08.003.

Wang ZW, Nan ZR, Wang SL, et al. 2011. Accumulation and distribution of cadmium and lead in wheat (Triticum aestivum L.) grown in contaminated soils from the oasis, north-west China. J Sci Food Agric 91(2):377-384. http://doi.org/10.1002/jsfa.4196.

Wang N, Chen C, Nie X, et al. 2015. Blood lead level and its association with body mass index and obesity in China - Results from SPECT-China study. Scientific reports 5:18299. http://doi.org/10.1038/srep18299.

Wang H, Li J, Hao JH, et al. 2017b. High serum lead concentration in the first trimester is associated with an elevated risk of small-for-gestational-age infants. Toxicol Appl Pharmacol 332:75-80. http://doi.org/10.1016/j.taap.2017.07.020.

Wang J, Gao ZY, Yan J, et al. 2017c. Sex differences in the effects of prenatal lead exposure on birth outcomes. Environ Pollut 225:193-200. http://doi.org/10.1016/j.envpol.2017.03.031.

Wang W, Moroi S, Bakulski K, et al. 2018. Bone lead levels and risk of incident primary open-angle glaucoma: The VA Normative Aging Study. Environ Health Perspect 126(8):087002. http://doi.org/10.1289/ehp3442.

Wang M, Hossain F, Sulaiman R, et al. 2019. Exposure to inorganic arsenic and lead and autism spectrum disorder in children: A systematic review and meta-analysis. Chem Res Toxicol 32(10):1904-1919. http://doi.org/10.1021/acs.chemrestox.9b00134.

Warrington NM, Zhu G, Dy V, et al. 2015. Genome-wide association study of blood lead shows multiple associations near ALAD. Hum Mol Genet 24(13):3871-3879. http://doi.org/10.1093/hmg/ddv112.

Wasserman GA, Graziano JH, Factor-Litvak P, et al. 1994. Consequences of lead exposure and iron supplementation on childhood development at age 4 years. Neurotoxicol Teratol 16(3):233-240.

Wasserman GA, Liu X, Lolacono NJ, et al. 1997. Lead exposure and intelligence in 7-year-old children: The Yugoslavia Prospective Study. Environ Health Perspect 105(9):956-962.

Wasserman GA, Liu X, Popovac D, et al. 2000. The Yugoslavia prospective lead study: Contributions of prenatal and postnatal lead exposure to early intelligence. Neurotoxicol Teratol 22:811-818.

Wasserman GA, Factor-Litvak P, Liu X, et al. 2003. The relationship between blood lead, bone lead and child intelligence. Child Neuropsychol 9(1):22-34.

Watanabe H, Hu H, Rotnitzky A. 1994. Correlates of bone and blood lead levels in carpenters. Am J Ind Med 26(2):255-264. http://doi.org/10.1002/ajim.4700260211.

Watson WS, Morrison J, Bethel MIF, et al. 1986. Food iron and lead absorption in humans. Am J Clin Nutr 44:248-256.

Weaver V, Lee B, Ahn K, et al. 2003a. Associations of lead biomarkers with renal function in Korean lead workers. Occup Environ Med 60(8):551-562.

Weaver VM, Schwartz BS, Ahn KD, et al. 2003b. Associations of renal function with polymorphisms in the delta-aminolevulinic acid dehydratase, vitamin $\mathrm{D}$ receptor, and nitric oxide synthase genes in Korean lead workers. Environ Health Perspect 111(13):1613-1619.

Weaver VM, Jaar BG, Schwartz BS, et al. 2005a. Associations among lead dose biomarkers, uric acid, and renal function in Korean lead workers. Environ Health Perspect 113(1):36-42.

Weaver VM, Schwartz BS, Jaar BG, et al. 2005b. Associations of uric acid with polymorphisms in the "delta"-aminolevulinic acid dehydratase, vitamin D receptor, and nitric oxide synthase genes in Korean lead workers. Environ Health Perspect 113(11):1509-1515. http://doi.org/10.1289/ehp.7927.

Weaver VM, Lee BK, Todd AC, et al. 2006. Effect modification by delta-aminolevulinic acid dehydratase, vitamin $\mathrm{D}$ receptor, and nitric oxide synthase gene polymorphisms on associations between patella lead and renal function in lead workers. Environ Res 102(1):61-69. http://doi.org/10.1016/j.envres.2006.01.001. 


\section{REFERENCES}

Weaver VM, Ellis LR, Lee BK, et al. 2008. Associations between patella lead and blood pressure in lead workers. Am J Ind Med 51(5):336-343. http://doi.org/10.1002/ajim.20573.

Weaver VM, Griswold M, Todd AC, et al. 2009. Longitudinal associations between lead dose and renal function in lead workers. Environ Res 109(1):101-107. http://doi.org/10.1016/j.envres.2008.09.005.

Wedeen RP. 1992. Removing lead from bone: Clinical implications of bone lead stores. Neurotoxicology 13:843-852.

Wedeen RP, Mallik DK, Batuman V. 1979. Detection and treatment of occupational lead nephropathy. Arch Intern Med 139:53-57.

Wedeen RP, Maesaka JK, Weiner B, et al. 1975. Occupational lead nephropathy. Am J Med 59:630641.

Weis CP, LaVelle JM. 1991. Characteristics to consider when choosing an animal model for the study of lead bioavailability. Chem Spec Bioavailab 3(3-4):113-119.

Weisel C, Demak M, Marcus S, et al. 1991. Soft plastic bread packaging: Lead content and reuse by families. Am J Public Health 81(6):756-758.

Weiss ST, Munoz A, Stein A, et al. 1986. The relationship of blood lead to blood pressure in a longitudinal study of working men. Am J Epidemiol 123(5):800-808.

Weiss ST, Munoz A, Stein A, et al. 1988. The relationship of blood lead to systolic blood pressure in a longitudinal study of policemen. Environ Health Perspect 78:53-56.

Weiss AL, Caravanos J, Blaise MJ, et al. 2006. Distribution of lead in urban roadway grit and its association with elevated steel structures. Chemosphere 65(10):1762-1771. http://doi.org/10.1016/j.chemosphere.2006.04.079.

Weisskopf MG, Wright RO, Schwartz J, et al. 2004. Cumulative lead exposure and prospective change in cognition among elderly men. The VA Normative Aging Study. Am J Epidemiol 160(12):11841193. http://doi.org/10.1093/aje/kwh333.

Weisskopf MG, Proctor SP, Wright RO, et al. 2007. Cumulative lead exposure and cognitive performance among elderly men. Epidemiology 18(1):59-66. http://doi.org/10.1097/01.ede.0000248237.35363.29.

Weisskopf MG, Nitin J, Nie H, et al. 2009. A prospective study of bone lead concentration and death from all causes, cardiovascular diseases, and cancer in the department of veterans affairs normative aging study. Circulation 120(12):1056-1064. http://doi.org/10.1161/circulationaha.108.827121.

Weitzman M, Aschengrau A, Bellinger D, et al. 1993. Lead-contaminated soil abatement and urban children's blood lead levels. J Am Med Assoc 269(13):1647-1654.

Wells A, Venn J, Heard M. 1975. Deposition in the lung and uptake to blood of motor exhaust labelled with 203Pb. Inhaled Part 4:175-189.

Wells EM, Navas-Acien A, Herbstman JB, et al. 2011. Low-level lead exposure and elevations in blood pressure during pregnancy. Environ Health Perspect 119:664-669.

Wells EM, Bonfield TL, Dearborn DG, et al. 2014. The relationship of blood lead with immunoglobulin E, eosinophils, and asthma among children: NHANES 2005-2006. Int J Hyg Environ Health 217(23):196-204. http://doi.org/10.1016/j.ijheh.2013.04.010.

Were FH, Moturi MC, Gottesfeld P, et al. 2014. Lead exposure and blood pressure among workers in diverse industrial plants in Kenya. J Occup Environ Hyg 11(11):706-715. http://doi.org/10.1080/15459624.2014.908258.

Wetmur JG, Lehnert G, Desnick RJ. 1991b. The delta-aminolevulinate dehydratase polymorphism: Higher blood lead levels in lead workers and environmentally exposed children with 1-2 and 2-2 isozymes. Environ Res 56:109-119.

Wetmur JG, Kaya AH, Plewinska M, et al. 1991a. Molecular characterization of the human deltaaminolevulinate dehydratase 2 (ALAD2) allele: Implications for molecular screening of individuals for genetic susceptibility to lead poisoning. Am J Hum Genet 49(4):757-763.

Weuve J, Kelsey KT, Schwartz J, et al. 2006. delta-Aminolevulinic acid dehydratase polymorphism and the relation between low level lead exposure and the Mini-Mental Status Examination in older men: 


\section{REFERENCES}

The Normative Aging Study. Occup Environ Med 63(11):746-753.

http://doi.org/10.1136/oem.2006.027417.

Weuve J, Korrick SA, Weisskopf MA, et al. 2009. Cumulative exposure to lead in relation to cognitive function in older women. Environ Health Perspect 117(4):574-580. http://doi.org/10.1289/ehp.11846.

Weuve J, Press DZ, Grodstein F, et al. 2013. Cumulative exposure to lead and cognition in persons with Parkinson's disease. Mov Disord 28(2):176-182. http://doi.org/10.1002/mds.25247.

Whelan EA, Piacitelli GM, Gerwel B, et al. 1997. Elevated blood lead levels in children of construction workers. Am J Public Health 87(8):1352-1355.

White PD, Van LP, Davis BD, et al. 1998. The conceptual structure of the integrated exposure uptake biokinetic model for lead in children. Environ Health Perspect 106(Suppl 6):1513-1530.

WHO. 2010. Guidelines for indoor air quality: Selected pollutants. Geneva, Switzerland: World Health Organization. http://www.euro.who.int/_data/assets/pdf_file/0009/128169/e94535.pdf. January 08, 2014.

WHO. 2017. Guidelines for drinking-water quality. Fourth edition incorporating the first addendum. Geneva, Switzerland: World Health Organization. http://apps.who.int/iris/bitstream/10665/254637/1/9789241549950-eng.pdf?ua=1. February 28, 2017.

Wildt K, Eliasson R, Berlin M. 1983. Effects of occupational exposure to lead on sperm and semen. In: Clarkson TW, Nordberg GF, Sager PR, eds. Reproductive and developmental toxicity of metals. Boston, MA: Springer-Verlag, 279-300. http://doi.org/10.1007/978-1-4615-9346-11.

Wildt K, Berlin M, Isberg PE. 1987. Monitoring of zinc protoporphyrin levels in blood following occupational lead exposure. Am J Ind Med 12(4):385-398.

Wilhelm M, Lombeck I, Hafner D, et al. 1989. Hair lead levels in young children from the FRG. J Trace Elem Electrolytes Health Dis 3:165-170.

Wilker E, Korrick S, Nie LH, et al. 2011. Longitudinal changes in bone lead levels: The VA Normative Aging Study. J Occup Environ Med 53(8):850-855. http://doi.org/10.1097/JOM.0b013e31822589a9.

Williams PL, Sergeyev O, Lee MM, et al. 2010. Blood lead levels and delayed onset of puberty in a longitudinal study of Russian boys. Pediatrics 125(5):1088-1096. http://doi.org/10.1542/peds.20092575.

Williams PL, Bellavia A, Korrick SA, et al. 2019. Blood lead levels and timing of male sexual maturity: A longitudinal study of Russian boys. Environ Int 125:470-477. http://doi.org/10.1016/j.envint.2019.01.070.

Williamson AM, Teo RKC. 1986. Neurobehavioral and memory of occupational lead workers. Br J Ind Med 43:373-380.

Wilson R, Jones-Otazo H, Petrovic S, et al. 2013. Revisiting dust and soil ingestion rates based on handto-mouth transfer. Hum Ecol Risk Assess 19(1):158-188.

Wingren G, Englander V. 1990. Mortality and cancer morbidity in a cohort of Swedish glassworkers. Int Arch Occup Environ Health 62:253-257.

Winter AS, Sampson RJ. 2017. From lead exposure in early childhood to adolescent health: A Chicago birth cohort. Am J Public Health 107(9):1496-1501. http://doi.org/10.2105/ajph.2017.303903.

Wisconsin DHS. 2002. Lead arsenate pesticides. Madison, WI: Wisconsin Department of Health Services. P-45007.

Wiwanitkit V, Suwansaksri J, Soogarun S. 2008. White blood cell sister chromatid exchange among a sample of Thai subjects exposed to lead: Lead-induced genotoxicity. Toxicol Environ Chem 90(4):765-768. http://doi.org/10.1080/02772240701712758.

Wolf C, Wallnofer A, Waldhor T, et al. 1995. Effect of lead on blood pressure in occupationally nonexposed men. Am J Ind Med 27(6):897-903.

Wolff MS, Britton JA, Boguski L, et al. 2008. Environmental exposures and puberty in inner-city girls. Environ Res 107(3):393-400. http://doi.org/10.1016/j.envres.2008.03.006. 


\section{REFERENCES}

Wong O, Harris F. 2000. Cancer mortality study of employees at lead battery plants and lead smelters, 1947-1995. Am J Ind Med 38:255-270.

Wright RO, Hu H, Silverman EK, et al. 2003a. Apolipoprotein E genotype predicts 24-month Bayley scales infant development score. Pediatr Res 54(6):819-825.

Wright RO, Tsaih SW, Schwartz J, et al. 2003b. Lead exposure biomarkers and mini-mental status exam scores in older men. Epidemiology 14(6):713-718.

Wright RO, Silverman EK, Schwartz J, et al. 2004. Association between hemochromatosis genotype and lead exposure among elderly men: The Normative Aging Study. Environ Health Perspect 112(6):746-750.

Wu T, Buck GM, Mendola P. 2003b. Blood lead levels and sexual maturation in U.S. girls: The Third National Health and Nutrition Examination Survey, 1988-1994. Environ Health Perspect 111(5):737-741. http://doi.org/10.1289/ehp.6008.

Wu TN, Yang KC, Wang CM, et al. 1996. Lead poisoning caused by contaminated Cordyceps, a Chinese herbal medicine: Two case reports. Sci Total Environ 182:193-195.

Wu F, Chang P, Wu C, et al. 2002. Correlations of blood lead with DNA-protein cross-links and sister chromatid exchanges in lead workers. Cancer Epidemiol Biomarkers Prev 11(3):287-290.

Wu M, Kelsey K, Schwartz J, et al. 2003a. A delta-aminolevulinic acid dehydratase (ALAD) polymorphism may modify the relationship of low-level lead exposure to uricemia and renal function: The normative aging study. Environ Health Perspect 111(3):335-340.

Wu HM, Lin-Tan DT, Wang ML, et al. 2012. Lead level in seminal plasma may affect semen quality for men without occupational exposure to lead. Reprod Biol Endocrinol 10:91.

Wu S, Hivert MF, Cardenas A, et al. 2017. Exposure to low levels of lead in utero and umbilical cord blood DNA methylation in project viva: An epigenome-wide association study. Environ Health Perspect 125(8):087019. http://doi.org/10.1289/ehp1246.

Wu Y, Sun J, Wang M, et al. 2018. The relationship of children's intelligence quotient and blood lead and zinc levels: A meta-analysis and system review. Biol Trace Elem Res 182(2):185-195. http://doi.org/10.1007/s12011-017-1093-0.

Xian X. 1989. Response of kidney bean to concentration and chemical form of cadmium, zinc, and lead in polluted soils. Environ Pollut 57:127-137.

Xie Y, Chiba M, Shinohara A, et al. 1998. Studies on lead-binding protein and interaction between lead and selenium in the human erythrocytes. Ind Health 36:234-239.

Xie X, Ding G, Cui C, et al. 2013. The effects of low-level prenatal lead exposure on birth outcomes. Environ Pollut 175:30-34. http://doi.org/10.1016/j.envpol.2012.12.013.

Xu X, Chen X, Zhang J, et al. 2015. Decreased blood hepatitis B surface antibody levels linked to ewaste lead exposure in preschool children. J Hazard Mater 298:122-128. http://doi.org/10.1016/j.jhazmat.2015.05.020.

Yamamoto N, Takahashi Y, Yoshinaga J, et al. 2006. Size distributions of soil particles adhered to children's hands. Arch Environ Contam Toxicol 51(2):157-163. http://doi.org/10.1007/s00244-0057012-y.

Yang H, Huo X, Yekeen TA, et al. 2013a. Effects of lead and cadmium exposure from electronic waste on child physical growth. Environ Sci Pollut Res Int 20(7):4441-4447. http://doi.org/10.1007/s11356-012-1366-2.

Yang CC, Chen HI, Chiu YW, et al. 2013b. Metallothionein 1A polymorphisms may influence urine uric acid and $\mathrm{N}$-acetyl-beta-D-glucosaminidase (NAG) excretion in chronic lead-exposed workers. Toxicology 306:68-73. http://doi.org/10.1016/j.tox.2013.02.007.

Yang WY, Zhang ZY, Thijs L, et al. 2017. Left ventricular structure and function in relation to environmental exposure to lead and cadmium. Journal of the American Heart Association 6(2):e004692. http://doi.org/10.1161/jaha.116.004692.

Yang WY, Efremov L, Mujaj B, et al. 2018. Association of office and ambulatory blood pressure with blood lead in workers before occupational exposure. J Am Soc Hypertens 12(1):14-24. http://doi.org/10.1016/j.jash.2017.10.010. 


\section{REFERENCES}

Yazbeck C, Thiebaugeorges O, Moreau T, et al. 2009. Maternal blood lead levels and the risk of pregnancy-induced hypertension: The EDEN cohort study. Environ Health Perspect 117(10):15261530. http://doi.org/10.1289/ehp.0800488.

Yesilonis ID, Pouyat RV, Neerchal NK. 2008. Spatial distribution of metals in soils in Baltimore, Maryland: Role of native parent material, proximity to major roads, housing age and screening guidelines. Environ Pollut 156(3):723-731. http://doi.org/10.1016/j.envpol.2008.06.010.

Yiin LM, Rhoads GG, Lioy PJ. 2000. Seasonal influences on childhood lead exposure. Environ Health Perspect 108(2):177-182.

Yin Y, Zhang T, Dai Y, et al. 2008. The effect of plasma lead on anembryonic pregnancy. Ann N Y Acad Sci 1140:184-189. http://doi.org/10.1196/annals.1454.042.

Yokoyama K, Araki S, Murata K, et al. 1997. Subclinical vestibulo-cerebellar, anterior cerebellar lobe and spinocerebellar effects in lead workers in relation to concurrent and past exposure. Neurotoxicology 18(2):371-380.

Yoon JH, Ahn YS. 2016. The association between blood lead level and clinical mental disorders in fifty thousand lead-exposed male workers. J Affect Disord 190:41-46. http://doi.org/10.1016/j.jad.2015.09.030.

Yorita Christensen KL. 2013. Metals in blood and urine, and thyroid function among adults in the United States 2007-2008. Int J Hyg Environ Health 216(6):624-632.

Youravong N, Teanpaisan R. 2015. The periodontal health of lead-exposed children living in a shipyard industrial area. Toxicol Ind Health 31(5):459-466. http://doi.org/10.1177/0748233712472529.

Yu C, Lin J, Lin-Tan D. 2004. Environmental exposure to lead and progression of chronic renal diseases: A four-year prospective longitudinal study. J Am Soc Nephrol 15(4):1016-1022.

Yu CJ, Du JC, Chiou HC, et al. 2016. Attention deficit/hyperactivity disorder and urinary nonylphenol levels: A case-control study in Taiwanese children. PLoS ONE 11(2):e0149558. http://doi.org/10.1371/journal.pone.0149558.

Yu CG, Wei FF, Yang WY, et al. 2019a. Heart rate variability and peripheral nerve conduction velocity in relation to blood lead in newly hired lead workers. Occup Environ Med 76(6):382-388. http://doi.org/10.1136/oemed-2018-105379.

Yu CG, Yang WY, Saenen N, et al. 2019b. Neurocognitive function in relation to blood lead among young men prior to chronic occupational exposure. Scand J Work Environ Health 45(3):298-307. http://doi.org/10.5271/sjweh.3798.

Yücesoy B, Turhan A, Üre M, et al. 1997. Simultaneous effects of lead and cadmium on NK cell activity and some phenotypic parameters. Immunopharmacol Immunotoxicol 19(3):339-348. http://doi.org/10.3109/08923979709046980.

Zahran S, Mielke HW, Gonzales CR, et al. 2010. New Orleans before and after hurricanes Katrina/Rita: A quasi-experiment of the association between soil lead and children's blood lead. Environ Sci Technol 44(12):4433-4440. http://doi.org/10.1021/es100572s.

Zak K, Rohovec J, Navratil T. 2009. Fluxes of heavy metals from a highly polluted watershed during flood events: A case study of the Litavka River, Czech Republic. Water Air Soil Pollut 203(14):343-358. http://doi.org/10.1007/s11270-009-0017-9.

Zaprjanova P, Dospatliev L, Angelova V, et al. 2010. Correlation between soil characteristics and lead and cadmium content in the aboveground biomass of Virginia tobacco. Environ Monit Assess 163(1-4):253-261. http://doi.org/10.1007/s10661-009-0831-y.

Zaragoza L, Hogan K. 1998. The integrated exposure uptake biokinetic model for lead in children: Independent validation and verification. Environ Health Perspect 106(Suppl 6):1551-1556.

Zartarian V, Xue J, Tornero-Velez R, et al. 2017. Children's lead exposure: A multimedia modeling analysis to guide public health decision-making. Environ Health Perspect 125(9):e97009. http://doi.org/10.1289/EHP1605.

Zawia NH, Crumpton T, Brydie M, et al. 2000. Disruption of the zinc finger domain: A common target that underlies many of the effects of lead. Neurotoxicology 21(6):1069-1080. 


\section{REFERENCES}

Zawirska B. 1981. The role of the kidneys in disorders of porphyrin metabolism during carcinogenesis induced with lead acetate. Environ Res 24:391-408.

Zawirska B, Medraś K. 1972. The role of the kidneys in disorders of porphyrin metabolism during carcinogenesis induced with lead acetate. Arch Immunol Ther Exp (Warsz) 20(2):257-272.

Zeng X, Xu X, Zheng X, et al. 2016. Heavy metals in PM2.5 and in blood, and children's respiratory symptoms and asthma from an e-waste recycling area. Environ Pollut 210:346-353. http://doi.org/10.1016/j.envpol.2016.01.025.

Zeng X, Xu X, Boezen HM, et al. 2017. Decreased lung function with mediation of blood parameters linked to e-waste lead and cadmium exposure in preschool children. Environ Pollut 230:838-848. http://doi.org/10.1016/j.envpol.2017.07.014.

Zentner LE, Rondó PH, Mastroeni SS. 2006. Lead contamination and anthropometry of the newborn baby. J Trop Pediatr 52(5):369-371. http://doi.org/10.1093/tropej/fml009.

Zeyrek D, Soran M, Cakmak A, et al. 2009. Serum copper and zinc levels in mothers and cord blood of their newborn infants with neural tube defects: A case-control study. Indian Pediatr 46(8):675-680.

Zhai H, Chen C, Wang N, et al. 2017. Blood lead level is associated with non-alcoholic fatty liver disease in the Yangtze River Delta region of China in the context of rapid urbanization. Environ Health 16(1):93. http://doi.org/10.1186/s12940-017-0304-7.

Zhang W, Zhang GG, He HZ, et al. 1994. Early health effects and biological monitoring in persons occupationally exposed to tetraethyl lead. Int Arch Occup Environ Health 65:395-399.

Zhang N, Baker HW, Tufts M, et al. 2013. Early childhood lead exposure and academic achievement: Evidence from Detroit public schools, 2008-2010. Am J Public Health 103(3):e72-77. http://doi.org/10.2105/ajph.2012.301164.

Zhang XL, Guariglia SR, McGothan JL, et al. 2015. Presynaptic mechanisms of lead neurotoxicity: Effects on vesicular release, vesicle clustering and mitochondria number. PLoS ONE 10(5):e0127461.

Zhang A, Park SK, Wright RO, et al. 2010. HFE H63D polymorphism as a modifier of the effect of cumulative lead exposure on pulse pressure: The Normative Aging Study. Environ Health Perspect 118(9):1261-1266. http://doi.org/10.1289/ehp.1002251.

Zhang A, Hu H, Sánchez BN, et al. 2011. Association between prenatal lead exposure and blood pressure in female offspring. Environ Health Perspect 120(3):445-450. http://doi.org/10.1289/ehp.1103736.

Zhao ZY, Li R, Sun L, et al. 2004. Effect of lead exposure on the immune function of lymphocytes and erythrocytes in preschool children. J Zhejiang Univ Sci 5(8):1001-1004. http://doi.org/10.1007/BF02947614.

Zheng G, Tian L, Liang Y, et al. 2011. delta-Aminolevulinic acid dehydratase genotype predicts toxic effects of lead on workers' peripheral nervous system. Neurotoxicology 32:374-382.

Zhou L, Xu J, Zhang J, et al. 2017. Prenatal maternal stress in relation to the effects of prenatal lead exposure on toddler cognitive development. Neurotoxicology 59:71-78. http://doi.org/10.1016/j.neuro.2017.01.008.

Zhu M, Fitzgerald EF, Gelberg KH, et al. 2010. Maternal low-level lead exposure and fetal growth. Environ Health Perspect 118(10):1471-1475. http://doi.org/10.1289/ehp.0901561.

Ziegler EE, Edwards BB, Jensen RL, et al. 1978. Absorption and retention of lead by infants. Pediatr Res 12(1):29-34.

Zimmermann-Tansella C, Campara P, Andrea FD, et al. 1983. Psychological and physical complaints of subjects with low exposure to lead. Hum Toxicol 2:615-623.

Zollinger HU. 1953. Durch chronische bleivergiftung erzeugte nierenadenome und-carcinome bei ratten und ihre beziechungen zu den entsprehenden neubildungen menschen. Virchows Arch 323:694-710.

Zota AR, Shenassa ED, Morello-Frosch R. 2013. Allostatic load amplifies the effect of blood lead levels on elevated blood pressure among middle-aged U.S. adults: A cross-sectional study. Environ Health 12(1):64. http://doi.org/10.1186/1476-069x-12-64. 


\section{REFERENCES}

Zota AR, Needham BL, Blackburn EH, et al. 2015. Associations of cadmium and lead exposure with leukocyte telomere length: Findings from National Health and Nutrition Examination Survey, 19992002. Am J Epidemiol 181(2):127-136. http://doi.org/10.1093/aje/kwu293. 


\section{APPENDIX A. ATSDR MINIMAL RISK LEVEL WORKSHEETS}

MRLs are derived when reliable and sufficient data exist to identify the target organ(s) of effect or the most sensitive health effect(s) for a specific duration for a given route of exposure. An MRL is an estimate of the daily human exposure to a hazardous substance that is likely to be without appreciable risk of adverse noncancer health effects over a specified route and duration of exposure. MRLs are based on noncancer health effects only; cancer effects are not considered. These substance-specific estimates, which are intended to serve as screening levels, are used by ATSDR health assessors to identify contaminants and potential health effects that may be of concern at hazardous waste sites. It is important to note that MRLs are not intended to define clean-up or action levels.

MRLs are derived for hazardous substances using the NOAEL/uncertainty factor approach. They are below levels that might cause adverse health effects in the people most sensitive to such chemicalinduced effects. MRLs are derived for acute (1-14 days), intermediate (15-364 days), and chronic ( $\geq 365$ days) durations and for the oral and inhalation routes of exposure. Currently, MRLs for the dermal route of exposure are not derived because ATSDR has not yet identified a method suitable for this route of exposure. MRLs are generally based on the most sensitive substance-induced endpoint considered to be of relevance to humans. Serious health effects (such as irreparable damage to the liver or kidneys, or birth defects) are not used as a basis for establishing MRLs. Exposure to a level above the MRL does not mean that adverse health effects will occur.

MRLs are intended only to serve as a screening tool to help public health professionals decide where to look more closely. They may also be viewed as a mechanism to identify those hazardous waste sites that are not expected to cause adverse health effects. Most MRLs contain a degree of uncertainty because of the lack of precise toxicological information on the people who might be most sensitive (e.g., infants, elderly, nutritionally or immunologically compromised) to the effects of hazardous substances. ATSDR uses a conservative (i.e., protective) approach to address this uncertainty consistent with the public health principle of prevention. Although human data are preferred, MRLs often must be based on animal studies because relevant human studies are lacking. In the absence of evidence to the contrary, ATSDR assumes that humans are more sensitive to the effects of hazardous substance than animals and that certain persons may be particularly sensitive. Thus, the resulting MRL may be as much as 100 -fold below levels that have been shown to be nontoxic in laboratory animals. 
Proposed MRLs undergo a rigorous review process: Health Effects/MRL Workgroup reviews within the Division of Toxicology and Human Health Sciences, expert panel peer reviews, and agency-wide MRL Workgroup reviews, with participation from other federal agencies and comments from the public. They are subject to change as new information becomes available concomitant with updating the toxicological profiles. Thus, MRLs in the most recent toxicological profiles supersede previously published MRLs. For additional information regarding MRLs, please contact the Division of Toxicology and Human Health Sciences, Agency for Toxic Substances and Disease Registry, 1600 Clifton Road NE, Mailstop S102-1, Atlanta, Georgia 30329-4027.

The literature evaluating the health effects of $\mathrm{Pb}$ is enormous, and includes an extensive database in humans, including children. Effects are diverse and exposure to $\mathrm{Pb}$ is associated with toxicity to every organ system. For the most studied endpoints (neurological, renal, cardiovascular, hematological, immunological, reproductive, and developmental), effects occur at the lowest PbBs studied $(\leq 5 \mu \mathrm{g} / \mathrm{dL})$. Exposure thresholds for effects on specific organ systems have not been identified (i.e., no safe level has been identified). Cognitive deficits in children occurring at the lowest $\mathrm{PbBs}(\leq 5 \mu \mathrm{g} / \mathrm{dL}$ ) are the best substantiated effects. However, because the lowest $\mathrm{PbBs}$ are associated with serious adverse effects (e.g., declining cognitive function in children), MRLs for Pb have not been derived. 


\title{
APPENDIX B. LITERATURE SEARCH FRAMEWORK FOR LEAD
}

The objective of the toxicological profile is to evaluate the potential for human exposure and the potential health hazards associated with inhalation, oral, or dermal/ocular exposure to $\mathrm{Pb}$.

\section{B.1 LITERATURE SEARCH AND SCREEN}

A literature search and screen were conducted to identify studies examining health effects, toxicokinetics, mechanisms of action, susceptible populations, biomarkers, chemical interactions, physical and chemical properties, production, use, environmental fate, environmental releases, and environmental and biological monitoring data for $\mathrm{Pb}$. ATSDR primarily focused on peer-reviewed articles without publication date or language restrictions. Non-peer-reviewed studies that were considered relevant to the assessment of the health effects of $\mathrm{Pb}$ have undergone peer review by at least three ATSDR-selected experts who have been screened for conflict of interest. The inclusion criteria used to identify relevant studies examining the health effects of $\mathrm{Pb}$ are presented in Table B-1.

\section{Table B-1. Inclusion Criteria for the Literature Search and Screen}

\author{
Health Effects \\ Species \\ Human \\ Laboratory mammals \\ Route of exposure \\ Inhalation \\ Oral \\ Dermal (or ocular) \\ Parenteral (these studies will be considered supporting data) \\ Health outcome \\ Death \\ Systemic effects \\ Body weight effects \\ Respiratory effects \\ Cardiovascular effects \\ Gastrointestinal effects \\ Hematological effects \\ Musculoskeletal effects \\ Hepatic effects \\ Renal effects \\ Dermal effects \\ Ocular effects \\ Endocrine effects \\ Immunological effects \\ Neurological effects \\ Reproductive effects \\ Developmental effects \\ Other noncancer effects
}


Table B-1. Inclusion Criteria for the Literature Search and Screen

\begin{tabular}{l}
\hline Cancer \\
\hline Toxicokinetics \\
Absorption \\
Distribution \\
Metabolism \\
Excretion \\
PBPK models \\
Biomarkers \\
Biomarkers of exposure \\
Biomarkers of effect \\
Interactions with other chemicals \\
Potential for human exposure \\
Releases to the environment \\
Air \\
Water \\
Soil \\
Environmental fate \\
Transport and partitioning \\
Transformation and degradation \\
Environmental monitoring \\
Air \\
Water \\
Sediment and soil \\
Other media \\
General populations \\
Occupation populations \\
\hline
\end{tabular}

\section{B.1.1 Literature Search}

The current literature search was intended to update the draft toxicological profile for $\mathrm{Pb}$ released for public comment in 2019; thus, the literature search was restricted to studies published between February 2015 and September 2019. The following main databases were searched in September 2019:

- PubMed

- National Library of Medicine’s TOXLINE

- $\quad$ Scientific and Technical Information Network's TOXCENTER

The search strategy used the chemical names, Chemical Abstracts Service (CAS) numbers, synonyms, Medical Subject Headings (MeSH) headings, and keywords for Pb. The query strings used for the literature search are presented in Table B-2.

The search was augmented by searching the Toxic Substances Control Act Test Submissions (TSCATS), NTP website, and National Institute of Health Research Portfolio Online Reporting Tools Expenditures 
and Results (NIH RePORTER) databases using the queries presented in Table B-3. Additional databases were searched in the creation of various tables and figures, such as the TRI Explorer, the Substance Priority List (SPL) resource page, and other items as needed. Regulations applicable to $\mathrm{Pb}$ were identified by searching international and U.S. agency websites and documents.

Review articles were identified and used for the purpose of providing background information and identifying additional references. ATSDR also identified reports from the grey literature, which included unpublished research reports, technical reports from government agencies, conference proceedings and abstracts, and theses and dissertations.

\section{Table B-2. Database Query Strings}

\section{Database \\ search date Query string}

\section{PubMed}

09/2019

((((10031-22-8[rn] OR 10099-74-8[rn] OR 10101-63-0[rn] OR 11119-70-3[rn] OR 1270998-7[rn] OR 1309-60-0[rn] OR 1314-41-6[rn] OR 1314-87-0[rn] OR 1317-36-8[rn] [ 1342446-9[rn] OR 13814-96-5[rn] OR 15245-44-0[rn] OR 16040-38-3[rn] OR 39377-56-5[rn] OR 598-63-0[rn] OR 7439-92-1[rn] OR 7446-14-2[rn] OR 7446-27-7[rn] OR 7758-95-4[rn] OR 7758-97-6[rn] OR 78-00-2[rn] OR 301-04-2[rn] ) AND ((("Lead/toxicity"[mh] OR "Lead/adverse effects"[mh] OR "Lead/poisoning"[mh] OR "Lead/pharmacokinetics"[mh]) OR ("Lead"[mh] AND ("environmental exposure"[mh] OR ci[sh])) OR ("Lead"[mh] AND toxicokinetics[mh:noexp]) OR ("Lead/blood"[mh] OR "Lead/cerebrospinal fluid"[mh] OR "Lead/urine"[mh]) OR ("Lead"[mh] AND ("endocrine system"[mh] OR "hormones, hormone substitutes, and hormone antagonists"[mh] OR "endocrine disruptors"[mh])) OR ("Lead"[mh] AND ("computational biology"[mh] OR "medical informatics"[mh] OR genomics[mh] OR genome[mh] OR proteomics[mh] OR proteome[mh] OR metabolomics[mh] OR metabolome[mh] OR genes[mh] OR "gene expression"[mh] OR phenotype[mh] OR genetics[mh] OR genotype[mh] OR transcriptome[mh] OR ("systems biology"[mh] AND ("environmental exposure"[mh] OR "epidemiological monitoring"[mh] OR analysis[sh])) OR "transcription, genetic "[mh] OR "reverse transcription"[mh] OR "transcriptional activation"[mh] OR "transcription factors"[mh] OR ("biosynthesis"[sh] AND (RNA[mh] OR DNA[mh])) OR "RNA, messenger"[mh] OR "RNA, transfer"[mh] OR "peptide biosynthesis"[mh] OR "protein biosynthesis"[mh] OR "reverse transcriptase polymerase chain reaction"[mh] OR "base sequence"[mh] OR "trans-activators"[mh] OR "gene expression profiling"[mh])) OR ("Lead/antagonists and inhibitors"[mh]) OR ("Lead/metabolism"[mh] AND ("humans"[mh] OR "animals"[mh])) OR ("Lead"[majr] AND cancer[sb]) OR ("Lead/pharmacology"[majr])) AND (2016/02/01 : 3000[mhda] OR 2016/02/01 : 3000[crdt] OR 2016/02/01 : 3000[edat] OR 2015/02/01 : 3000[dp]))) OR ("lead poisoning"[mh] AND (2016/02/01 : 3000[mhda] OR 2016/02/01 : 3000[crdt] OR 2016/02/01 : 3000[edat] OR 2015/02/01 : 3000[dp])) OR ((("Tetraethyl Lead/toxicity"[mh] OR "Tetraethyl Lead/adverse effects"[mh] OR "Tetraethyl Lead/poisoning"[mh] OR "Tetraethyl Lead/pharmacokinetics"[mh]) OR ("Tetraethyl Lead"[mh] AND ("environmental exposure"[mh] OR ci[sh])) OR ("Tetraethyl Lead"[mh] AND toxicokinetics[mh:noexp]) OR ("Tetraethyl Lead/blood"[mh] OR "Tetraethyl Lead/cerebrospinal fluid"[mh] OR "Tetraethyl Lead/urine"[mh]) OR ("Tetraethyl Lead"[mh] AND ("endocrine system"[mh] OR "hormones, hormone substitutes, and hormone antagonists"[mh] OR "endocrine disruptors"[mh])) OR ("Tetraethyl Lead"[mh] AND ("computational biology"[mh] OR "medical informatics"[mh] OR genomics[mh] OR genome[mh] OR proteomics[mh] OR proteome[mh] OR metabolomics[mh] OR metabolome[mh] OR genes[mh] OR "gene expression"[mh] OR phenotype[mh] OR genetics[mh] OR genotype[mh] OR transcriptome[mh] OR ("systems biology"[mh] AND ("environmental exposure"[mh] OR "epidemiological monitoring"[mh] OR analysis[sh])) OR "transcription, genetic "[mh] OR "reverse transcription"[mh] OR 
Table B-2. Database Query Strings

\section{Database} search date Query string

"transcriptional activation"[mh] OR "transcription factors"[mh] OR ("biosynthesis"[sh] AND (RNA[mh] OR DNA[mh])) OR "RNA, messenger"[mh] OR "RNA, transfer"[mh] OR "peptide biosynthesis"[mh] OR "protein biosynthesis"[mh] OR "reverse transcriptase polymerase chain reaction"[mh] OR "base sequence"[mh] OR "trans-activators"[mh] OR "gene expression profiling"[mh])) OR ("Tetraethyl Lead/antagonists and inhibitors"[mh]) OR ("Tetraethyl Lead/metabolism"[mh] AND ("humans"[mh] OR "animals"[mh])) OR ("Tetraethyl Lead"[majr] AND cancer[sb]) OR ("Tetraethyl Lead/pharmacology"[majr])) AND (2016/02/01 : 3000[mhda] OR 2016/02/01 : 3000[crdt] OR 2016/02/01 : 3000[edat] OR 2015/02/01 : 3000[dp])) OR ((301-04-2[rn] AND (("Organometallic

Compounds/toxicity"[mh] OR "Organometallic Compounds/adverse effects"[mh] OR "Organometallic Compounds/poisoning"[mh] OR "Organometallic Compounds/pharmacokinetics"[mh]) OR ("Organometallic Compounds"[mh] AND ("environmental exposure"[mh] OR ci[sh])) OR ("Organometallic Compounds"[mh] AND toxicokinetics[mh:noexp]) OR ("Organometallic Compounds/blood"[mh] OR "Organometallic Compounds/cerebrospinal fluid"[mh] OR "Organometallic Compounds/urine"[mh]) OR ("Organometallic Compounds"[mh] AND ("endocrine system"[mh] OR "hormones, hormone substitutes, and hormone antagonists"[mh] OR "endocrine disruptors"[mh])) OR ("Organometallic Compounds"[mh] AND ("computational biology"[mh] OR "medical informatics"[mh] OR genomics[mh] OR genome[mh] OR proteomics[mh] OR proteome[mh] OR metabolomics[mh] OR metabolome[mh] OR genes[mh] OR "gene expression"[mh] OR phenotype[mh] OR genetics[mh] OR genotype[mh] OR transcriptome[mh] OR ("systems biology"[mh] AND ("environmental exposure"[mh] OR "epidemiological monitoring"[mh] OR analysis[sh])) OR "transcription, genetic "[mh] OR "reverse transcription"[mh] OR "transcriptional activation"[mh] OR "transcription factors"[mh] OR ("biosynthesis"[sh] AND (RNA[mh] OR DNA[mh])) OR "RNA, messenger"[mh] OR "RNA, transfer"[mh] OR "peptide biosynthesis"[mh] OR "protein biosynthesis"[mh] OR "reverse transcriptase polymerase chain reaction"[mh] OR "base sequence"[mh] OR "trans-activators"[mh] OR "gene expression profiling"[mh])) OR ("Organometallic Compounds/antagonists and inhibitors"[mh]) OR ("Organometallic Compounds/metabolism"[mh] AND ("humans"[mh] OR "animals"[mh])) OR ("Organometallic Compounds"[majr] AND cancer[sb]) OR ("Organometallic Compounds/pharmacology"[majr]))) AND (2016/02/01 : 3000[mhda] OR 2016/02/01 : 3000[crdt] OR 2016/02/01 : 3000[edat] OR 2015/02/01 : 3000[dp])) OR ((("1,3-

Benzenediol, 2,4,6-trinitro-, lead(2+) salt"[tw] OR "Borate(1-), tetrafluoro-, lead (2+)"[tw] OR "Borate(1-), tetrafluoro-, lead(2+)"[tw] OR "Chromic acid lead salt with lead molybdate"[tw] OR "Chromic acid, lead and molybdenum salt"[tw] OR "Chromium lead molybdenum oxide"[tw] OR "Lead (II) iodide"[tw] OR "Lead 2,4,6-trinitro-m-phenylene dioxide"[tw] OR "Lead bis(tetrafluoroborate)"[tw] OR "Lead borofluoride"[tw] OR "Lead boron fluoride"[tw] OR "Lead Brown"[tw] OR "Lead chromate molybdate"[tw] OR "lead diiodide"[tw] OR "Lead dioxide"[tw] OR "Lead fluoborate"[tw] OR "Lead fluoroborate"[tw] OR "Lead iodide"[tw] OR "Lead molybdate chromate"[tw] OR "Lead molybdenum chromate"[tw] OR "Lead oxide"[tw] OR "Lead peroxide"[tw] OR "Lead styphnate"[tw] OR "Lead superoxide"[tw] OR "Lead tetrafluoroborate"[tw] OR "Lead tricinate"[tw] OR "Lead trinitroresorcinate"[tw] OR "Lead(II) iodide"[tw] OR "Lead(II) styphnate"[tw] OR "Lead(II) tetrafluoroborate"[tw] OR "Lead(IV) oxide"[tw] OR "Lead-molybdenum chromate"[tw] OR "Molybdenum-lead chromate"[tw] OR "Plumbic oxide"[tw] OR "Plumbous iodide"[tw] OR "Plumbum jodatum"[tw] OR "Resorcinol, 2,4,6-trinitro-, lead(2+) salt"[tw] OR "Thiolead A"[tw] OR "Tricinat"[tw]) AND (to[sh] OR po[sh] OR ae[sh] OR pk[sh] OR ai[sh] OR ci[sh] OR bl[sh] OR cf[sh] OR ur[sh] OR "pharmacology"[sh:noexp] OR "environmental exposure"[mh] OR "endocrine system"[mh] OR "hormones, hormone substitutes, and hormone antagonists"[mh] OR "endocrine 
Table B-2. Database Query Strings

Database

search date Query string

disruptors"[mh] OR "Computational biology"[mh] OR "Medical Informatics"[mh] OR

Genomics[mh] OR Genome[mh] OR Proteomics[mh] OR Proteome[mh] OR

Metabolomics[mh] OR Metabolome[mh] OR Genes[mh] OR "Gene expression"[mh] OR

Phenotype[mh] OR genetics[mh] OR genotype[mh] OR Transcriptome[mh] OR ("Systems

Biology"[mh] AND ("Environmental Exposure"[mh] OR "Epidemiological Monitoring"[mh]

OR analysis[sh])) OR "Transcription, Genetic "[mh] OR "Reverse transcription"[mh] OR

"Transcriptional activation"[mh] OR "Transcription factors"[mh] OR ("biosynthesis"[sh] AND

(RNA[mh] OR DNA[mh])) OR "RNA, Messenger"[mh] OR "RNA, Transfer"[mh] OR

"peptide biosynthesis"[mh] OR "protein biosynthesis"[mh] OR "Reverse Transcriptase

Polymerase Chain Reaction"[mh] OR "Base Sequence"[mh] OR "Trans-activators"[mh] OR

"Gene Expression Profiling"[mh] OR cancer[sb] OR (me[sh] AND ("humans"[mh] OR

"animals"[mh])))) AND (2016/02/01 : 3000[mhda] OR 2016/02/01 : 3000[crdt] OR

2016/02/01 : 3000[edat] OR 2015/02/01 : 3000[dp])) OR (("Plumbism"[tw] OR

"saturnism"[tw] OR "colica pictorum"[tw] OR "Devon colic"[tw] OR "painter's colic"[tw]) NOT

"lead poisoning"[mh]) OR ((("1,3-Benzenediol, 2,4,6-trinitro-, lead(2+) salt (1:1)"[tw] OR

"Acetic acid lead(2+) salt"[tw] OR "Acetic acid, lead salt"[tw] OR "Acetic acid, lead(2+)

salt"[tw] OR "Acetic acid, lead(2+) salt"[tw] OR "Anglislite"[tw] OR "Azarcon"[tw] OR

"Borate(1-), tetrafluoro-, lead (2+)"[tw] OR "Borate(1-), tetrafluoro-, lead(2+) (2:1)"[tw] OR

"C.I. Pigment Metal 4"[tw] OR "C.I. Pigment Yellow 46"[tw] OR "CARBONIC ACID, LEAD

SALT (1:1)"[tw] OR "Carbonic acid, lead salt (2+) (1:1) "[tw] OR "Carbonic acid, lead(2+)

salt"[tw] OR "Cerussete"[tw] OR "Cerussite"[tw] OR "Chrome Orange"[tw] OR "Chrome

Yellow"[tw] OR "Chromic acid (H2CrO4), lead(2+) salt (1:1)"[tw] OR "Chromic acid lead salt "[tw] OR "CHROMIC ACID, LEAD (2+) SALT (1:1)"[tw] OR "Chromic Acid, Lead (II) Salt (1:1)"[tw] OR "Chromic acid, lead and molybdenum salt"[tw] OR "Chromic acid, lead salt"[tw] OR "Chromic acid, lead(2+) salt (1:1)"[tw] OR "Chromium lead molybdenum oxide"[tw] OR "Chromium lead oxide"[tw] OR "Cl pigment metal 4"[tw] OR "Cl Pigment Yellow 46"[tw] OR "Collodial lead phosphate"[tw] OR "Dibasic lead acetate"[tw] OR "Dibasic lead carbonate"[tw] OR "Dibasic lead sulfate"[tw] OR "Entan"[tw] OR "Fast White"[tw] OR "Flowsperse R 12"[tw] OR "Freemans White Lead"[tw] OR "Galena"[tw] OR "Glover"[tw] OR "Gold Satinobre"[tw] OR "Heuconin 5"[tw] OR "Lead (II) carbonate"[tw] OR "Lead (II) chloride"[tw] OR "Lead (II) chromate"[tw] OR "Lead (II) iodide"[tw] OR "Lead (II) nitrate"[tw] OR "Lead (II) oxide"[tw] OR "Lead (II) sulfate"[tw] OR "Lead (II) sulfide"[tw] OR "Lead (II, IV) oxide"[tw] OR "Lead (IV) oxide "[tw] OR "Lead 2,4,6-trinitro-m-phenylene dioxide"[tw] OR "Lead acetate"[tw] OR "Lead azide"[tw] OR "Lead

bis(tetrafluoroborate)"[tw] OR "Lead borofluoride"[tw] OR "Lead boron fluoride"[tw] OR "Lead Bottoms"[tw] OR "Lead bromide"[tw] OR "Lead brown"[tw] OR "Lead carbonate"[tw] OR "Lead chloride"[tw] OR "Lead chromate"[tw] OR "Lead chromate(VI)"[tw] OR "Lead chromium oxide (PbCrO4)"[tw] OR "Lead di(acetate)"[tw] OR "Lead diacetate"[tw] OR "Lead diazide"[tw] OR "Lead dibasic acetate"[tw] OR "Lead dibromide"[tw] OR "Lead dichloride"[tw] OR "Lead diiodide"[tw] OR "Lead dinitrate"[tw] OR "Lead dioxide"[tw] OR "Lead element"[tw] OR "Lead flake"[tw] OR "Lead fluoborate"[tw] OR "Lead fluoroborate"[tw] OR "Lead iodide"[tw] OR "Lead metal"[tw] OR "Lead molybdate chromate"[tw] OR "Lead molybdenum chromate"[tw] OR "Lead monooxide"[tw] OR "Lead monosulfate"[tw] OR "Lead monosulfide"[tw] OR "Lead monoxide"[tw] OR "Lead nitrate"[tw] OR "Lead orthophosphate"[tw] OR "Lead orthoplumbate"[tw] OR "Lead oxide"[tw] OR "Lead peroxide"[tw] OR "Lead phosphate"[tw] OR "Lead protoxide"[tw] OR "Lead S 2"[tw] OR "Lead S2"[tw] OR "Lead styphnate"[tw] OR "Lead sulfate"[tw] OR "Lead sulfide"[tw] OR "Lead sulphate"[tw] OR "Lead sulphide"[tw] OR "Lead superoxide"[tw] OR "Lead tetraethide"[tw] OR "Lead tetraethyl"[tw] OR "Lead tetrafluoroborate"[tw] OR "Lead tetraoxide"[tw] OR "Lead tetroxide"[tw] OR "Lead tricinate"[tw] OR "Lead 
APPENDIX B

Table B-2. Database Query Strings

Database

search date Query string

trinitroresorcinate"[tw] OR "Lead(+2) sulfate"[tw] OR "Lead(2+) acetate"[tw] OR "Lead(2+)

azide"[tw] OR "Lead(2+) bis(nitrate)"[tw] OR "Lead(2+) bromide"[tw] OR "Lead(2+)

carbonate"[tw] OR "Lead(2+) chloride"[tw] OR "Lead(2+) nitrate"[tw] OR "Lead(2+)

oxide"[tw] OR "Lead(2+) phosphate"[tw] OR "Lead(2+) phosphate (Pb3(PO4)2)"[tw] OR

"Lead(2+) salt carbamic acid (1:1) "[tw] OR "Lead(2+) sulfate"[tw] OR "Lead(2+)

sulfide"[tw] OR "Lead(II) acetate"[tw] OR "Lead(II) azide"[tw] OR "Lead(II) bromide"[tw] OR

"Lead(II) carbonate"[tw] OR "Lead(II) chloride"[tw] OR "Lead(II) chromate"[tw] OR "Lead(II) dinitrate"[tw] OR "Lead(II) iodide"[tw] OR "Lead(II) nitrate"[tw] OR "Lead(II) oxide"[tw] OR "Lead(II) phosphate"[tw] OR "Lead(II) phosphate (3:2)"[tw] OR "Lead(II) styphnate"[tw] OR "Lead(II) sulfate"[tw] OR "Lead(II) sulfide"[tw] OR "Lead(II) tetrafluoroborate"[tw] OR "Lead(IV) oxide"[tw] OR "Lead, elemental"[tw] OR "Lead, inorganic"[tw] OR "Lead, tetraethyl"[tw] OR "Lead, tetraethyl-"[tw] OR "Lead-molybdenum chromate"[tw] OR "Litharge"[tw] OR "Massicot"[tw] OR "Massicotite"[tw] OR "Mennige"[tw] OR "Milk White"[tw] OR "mine orange"[tw] OR "Mineral Orange"[tw] OR "Mineral red"[tw] OR "minio anaranjado"[tw] OR "Minium"[tw] OR "Molybdenum-lead chromate"[tw] OR "Mulhouse White"[tw] OR "Nitric acid lead(2+) salt"[tw] OR "Nitric acid, lead(2+) salt"[tw] OR "Orange lead"[tw] OR "Orangemennige"[tw] OR "Paris Red"[tw] OR "PbSO4"[tw] OR "Perlex paste 500"[tw] OR "Perlex paste 600A"[tw] OR "Phoenicochroite"[tw] OR "Phosphoric acid, lead salt"[tw] OR "Phosphoric acid, lead(2+) salt (2:3)"[tw] OR "Pigment Red 105"[tw] OR "Pigment White 3"[tw] OR "Pigment Yellow 34"[tw] OR "Pigment Yellow 46"[tw] OR "Plumbane"[tw] OR "Plumbi"[tw] OR "Plumbic oxide"[tw] OR "Plumboplumbic oxide"[tw] OR "Plumbous"[tw] OR "Plumbum"[tw] OR "Red lead"[tw] OR "Resorcinol, 2,4,6-trinitro-, lead(2+) salt (1:1)"[tw] OR "Rough lead bullion"[tw] OR "Royal Yellow 6000"[tw] OR "Salt of saturn"[tw] OR "Sandix"[tw] OR "Saturn red"[tw] OR "Sugar of lead"[tw] OR "Sulfuric acid, lead(2+) salt"[tw] OR "Tetra Ethylene Lead"[tw] OR "Tetra(methylethyl)lead"[tw] OR "Tetraethyl lead"[tw] OR "Tetraethyl plumbane"[tw] OR "Tetraethyllead"[tw] OR "Tetraethyllead, liquid"[tw] OR "tetraethylplomb"[tw] OR "Tetraethylplombane"[tw] OR "Tetraethylplumbane"[tw] OR "tetraetilplomo"[tw] OR "Thiolead A"[tw] OR "Tricinat"[tw] OR "Trilead bis(orthophosphate)"[tw] OR "Trilead phosphate"[tw] OR "Trilead tetraoxide"[tw] OR "Trilead tetroxide"[tw] OR "Unichem PBA"[tw] OR "Yellow lead ocher"[tw]) NOT medline[sb]) AND (2016/02/01 : 3000[crdt] OR 2016/02/01 : 3000[edat] OR 2015/02/01 : $3000[\mathrm{dp}]))))$ OR (((("Lead"[ti] NOT "lead to"[ti]) OR "Pb"[ti] OR "PbS"[ti] OR "PbO"[ti]) NOT medline[sb]) AND (2016/02/01 : 3000[crdt] OR 2016/02/01 : 3000[edat] OR 2015/02/01 : $3000[\mathrm{dp}]))$

\section{Toxline}

09/2019 Date limit: 2015 to present:

(10031-22-8[rn] OR 10099-74-8[rn] OR 10101-63-0[rn] OR 11119-70-3[rn] OR 12709-987[rn] OR 1309-60-0[rn] OR 1314-41-6[rn] OR 1314-87-0[rn] OR 1317-36-8[rn] OR 1342446-9[rn] OR 13814-96-5[rn] OR 15245-44-0[rn] OR 16040-38-3[rn] OR 39377-56-5[rn] OR 598-63-0[rn] OR 7439-92-1[rn] OR 7446-14-2[rn] OR 7446-27-7[rn] OR 7758-95-4[rn] OR 7758-97-6[rn] OR 78-00-2[rn]) AND ( ANEUPL [org] OR BIOSIS [org] OR CIS [org] OR DART [org] OR EMIC [org] OR EPIDEM [org] OR HEEP [org] OR HMTC [org] OR IPA [org] OR RISKLINE [org] OR MTGABS [org] OR NIOSH [org] OR NTIS [org] OR PESTAB [org] OR PPBIB [org] )

("1,3-Benzenediol, 2,4,6-trinitro-, lead(2+) salt (1:1)" OR "Acetic acid lead(2+) salt" OR "Acetic acid, lead salt" OR "Acetic acid, lead(2+) salt" OR "Acetic acid, lead(2+) salt" OR "Anglislite" OR "Azarcon" OR "Borate(1-), tetrafluoro-, lead (2+)" OR "Borate(1-), tetrafluoro-, lead(2+) (2:1)" OR "C.I. Pigment Metal 4" OR "C.I. Pigment Yellow 46" OR "CARBONIC ACID, LEAD SALT (1:1)" OR "Carbonic acid, lead salt (2+) (1:1)") AND ( ANEUPL [org] OR BIOSIS [org] OR CIS [org] OR DART [org] OR EMIC [org] OR EPIDEM 
Table B-2. Database Query Strings

Database

search date Query string

[org] OR HEEP [org] OR HMTC [org] OR IPA [org] OR RISKLINE [org] OR MTGABS [org] OR NIOSH [org] OR NTIS [org] OR PESTAB [org] OR PPBIB [org] )

("Carbonic acid, lead(2+) salt" OR "Cerussete" OR "Cerussite" OR "Chrome Orange" OR "Chrome Yellow" OR "Chromic acid (H2CrO4), lead(2+) salt (1:1)" OR "Chromic acid lead salt " OR "CHROMIC ACID, LEAD (2+) SALT (1:1)" OR "Chromic Acid, Lead (II) Salt (1:1)" OR "Chromic acid, lead and molybdenum salt" OR "Chromic acid, lead salt" OR "Chromic acid, lead(2+) salt (1:1)" OR "Chromium lead molybdenum oxide") AND ( ANEUPL [org] OR BIOSIS [org] OR CIS [org] OR DART [org] OR EMIC [org] OR EPIDEM [org] OR HEEP [org] OR HMTC [org] OR IPA [org] OR RISKLINE [org] OR MTGABS [org] OR NIOSH [org] OR NTIS [org] OR PESTAB [org] OR PPBIB [org] )

("Chromium lead oxide" OR "Cl pigment metal 4" OR "Cl Pigment Yellow 46" OR "Collodial lead phosphate" OR "Dibasic lead acetate" OR "Dibasic lead carbonate" OR "Dibasic lead sulfate" OR "Entan" OR "Fast White" OR "Flowsperse R 12" OR "Freemans White Lead" OR "Galena" OR "Glover" OR "Gold Satinobre" OR "Heuconin 5" OR "Lead (II) carbonate" OR "Lead (II) chloride" OR "Lead (II) chromate" OR "Lead (II) iodide" OR "Lead (II) nitrate" OR "Lead (II) oxide" OR "Lead (II) sulfate" OR "Lead (II) sulfide") AND ( ANEUPL [org] OR BIOSIS [org] OR CIS [org] OR DART [org] OR EMIC [org] OR EPIDEM [org] OR HEEP [org] OR HMTC [org] OR IPA [org] OR RISKLINE [org] OR MTGABS [org] OR NIOSH [org] OR NTIS [org] OR PESTAB [org] OR PPBIB [org] )

("Lead (II,IV) oxide" OR "Lead (IV) oxide " OR "Lead 2,4,6-trinitro-m-phenylene dioxide" OR "Lead acetate" OR "Lead azide" OR "Lead bis(tetrafluoroborate)" OR "Lead borofluoride" OR "Lead boron fluoride" OR "Lead Bottoms" OR "Lead bromide" OR "Lead brown" OR "Lead carbonate" OR "Lead chloride" OR "Lead chromate" OR "Lead chromate(VI)" OR "Lead chromium oxide (PbCrO4)" OR "Lead di(acetate)") AND ( ANEUPL [org] OR BIOSIS [org] OR CIS [org] OR DART [org] OR EMIC [org] OR EPIDEM [org] OR HEEP [org] OR HMTC [org] OR IPA [org] OR RISKLINE [org] OR MTGABS [org] OR NIOSH [org] OR NTIS [org] OR PESTAB [org] OR PPBIB [org] )

("Lead diacetate" OR "Lead diazide" OR "Lead dibasic acetate" OR "Lead dibromide" OR "Lead dichloride" OR "Lead diiodide" OR "Lead dinitrate" OR "Lead dioxide" OR "Lead element" OR "Lead flake" OR "Lead fluoborate" OR "Lead fluoroborate" OR "Lead iodide" OR "Lead metal" OR "Lead molybdate chromate" OR "Lead molybdenum chromate" OR "Lead monooxide" OR "Lead monosulfate" OR "Lead monosulfide") AND ( ANEUPL [org] OR BIOSIS [org] OR CIS [org] OR DART [org] OR EMIC [org] OR EPIDEM [org] OR HEEP [org] OR HMTC [org] OR IPA [org] OR RISKLINE [org] OR MTGABS [org] OR NIOSH [org] OR NTIS [org] OR PESTAB [org] OR PPBIB [org] )

("Lead monoxide" OR "Lead nitrate" OR "Lead orthophosphate" OR "Lead orthoplumbate" OR "Lead oxide" OR "Lead peroxide" OR "Lead phosphate" OR "Lead protoxide" OR "Lead S 2" OR "Lead S2" OR "Lead styphnate" OR "Lead sulfate" OR "Lead sulfide" OR "Lead sulphate" OR "Lead sulphide" OR "Lead superoxide" OR "Lead tetraethide" OR "Lead tetraethyl" OR "Lead tetrafluoroborate" OR "Lead tetraoxide") AND ( ANEUPL [org] OR BIOSIS [org] OR CIS [org] OR DART [org] OR EMIC [org] OR EPIDEM [org] OR HEEP [org] OR HMTC [org] OR IPA [org] OR RISKLINE [org] OR MTGABS [org] OR NIOSH [org] OR NTIS [org] OR PESTAB [org] OR PPBIB [org] )

("Lead tetroxide" OR "Lead tricinate" OR "Lead trinitroresorcinate" OR "Lead(+2) sulfate" OR "Lead(2+) acetate" OR "Lead(2+) azide" OR "Lead(2+) bis(nitrate)" OR "Lead(2+) bromide" OR "Lead(2+) carbonate" OR "Lead(2+) chloride" OR "Lead(2+) nitrate" OR "Lead(2+) oxide" OR "Lead(2+) phosphate" OR "Lead(2+) phosphate (Pb3(PO4)2)" OR "Lead(2+) salt carbamic acid (1:1) " OR "Lead(2+) sulfate") AND ( ANEUPL [org] OR BIOSIS [org] OR CIS [org] OR DART [org] OR EMIC [org] OR EPIDEM [org] OR HEEP 
Table B-2. Database Query Strings

\section{Database}

search date Query string

[org] OR HMTC [org] OR IPA [org] OR RISKLINE [org] OR MTGABS [org] OR NIOSH [org] OR NTIS [org] OR PESTAB [org] OR PPBIB [org] )

("Lead(2+) sulfide" OR "Lead(II) acetate" OR "Lead(II) azide" OR "Lead(II) bromide" OR "Lead(II) carbonate" OR "Lead(II) chloride" OR "Lead(II) chromate" OR "Lead(II) dinitrate" OR "Lead(II) iodide" OR "Lead(II) nitrate" OR "Lead(II) oxide" OR "Lead(II) phosphate" OR "Lead(II) phosphate (3:2)" OR "Lead(II) styphnate" OR "Lead(II) sulfate" OR "Lead(II) sulfide" OR "Lead(II) tetrafluoroborate" OR "Lead(IV) oxide") AND ( ANEUPL [org] OR BIOSIS [org] OR CIS [org] OR DART [org] OR EMIC [org] OR EPIDEM [org] OR HEEP [org] OR HMTC [org] OR IPA [org] OR RISKLINE [org] OR MTGABS [org] OR NIOSH [org] OR NTIS [org] OR PESTAB [org] OR PPBIB [org] )

("Lead, elemental" OR "Lead, inorganic" OR "Lead, tetraethyl" OR "Lead, tetraethyl-" OR "Lead-molybdenum chromate" OR "Litharge" OR "Massicot" OR "Massicotite" OR "Mennige" OR "Milk White" OR "mine orange" OR "Mineral Orange" OR "Mineral red" OR "minio anaranjado" OR "Minium" OR "Molybdenum-lead chromate" OR "Mulhouse White" OR "Nitric acid lead(2+) salt" OR "Nitric acid, lead(2+) salt") AND ( ANEUPL [org] OR BIOSIS [org] OR CIS [org] OR DART [org] OR EMIC [org] OR EPIDEM [org] OR HEEP [org] OR HMTC [org] OR IPA [org] OR RISKLINE [org] OR MTGABS [org] OR NIOSH [org] OR NTIS [org] OR PESTAB [org] OR PPBIB [org] )

("Orange lead" OR "Orangemennige" OR "Paris Red" OR "PbSO4" OR "Perlex paste 500" OR "Perlex paste 600A" OR "Phoenicochroite" OR "Phosphoric acid, lead salt" OR "Phosphoric acid, lead(2+) salt (2:3)" OR "Pigment Red 105" OR "Pigment White 3" OR "Pigment Yellow 34" OR "Pigment Yellow 46" OR "Plumbane" OR "Plumbi" OR "Plumbic oxide" OR "Plumboplumbic oxide" OR "Plumbous" OR "Plumbum") AND ( ANEUPL [org] OR BIOSIS [org] OR CIS [org] OR DART [org] OR EMIC [org] OR EPIDEM [org] OR HEEP [org] OR HMTC [org] OR IPA [org] OR RISKLINE [org] OR MTGABS [org] OR NIOSH [org] OR NTIS [org] OR PESTAB [org] OR PPBIB [org] )

("Red lead" OR "Resorcinol, 2,4,6-trinitro-, lead(2+) salt (1:1)" OR "Rough lead bullion" OR "Royal Yellow 6000" OR "Salt of saturn" OR "Sandix" OR "Saturn red" OR "Sugar of lead" OR "Sulfuric acid, lead(2+) salt" OR "Tetra Ethylene Lead" OR "Tetra(methylethyl)lead" OR "Tetraethyl lead" OR "Tetraethyl plumbane" OR "Tetraethyllead" OR "Tetraethyllead, liquid" OR "tetraethylplomb") AND ( ANEUPL [org] OR BIOSIS [org] OR CIS [org] OR DART [org] OR EMIC [org] OR EPIDEM [org] OR HEEP [org] OR HMTC [org] OR IPA [org] OR RISKLINE [org] OR MTGABS [org] OR NIOSH [org] OR NTIS [org] OR PESTAB [org] OR PPBIB [org] )

("Tetraethylplombane" OR "Tetraethylplumbane" OR "tetraetilplomo" OR "Thiolead A" OR "Tricinat" OR "Trilead bis(orthophosphate)" OR "Trilead phosphate" OR "Trilead tetraoxide" OR "Trilead tetroxide" OR "Unichem PBA" OR "Yellow lead ocher") AND ( ANEUPL [org] OR BIOSIS [org] OR CIS [org] OR DART [org] OR EMIC [org] OR EPIDEM [org] OR HEEP [org] OR HMTC [org] OR IPA [org] OR RISKLINE [org] OR MTGABS [org] OR NIOSH [org] OR NTIS [org] OR PESTAB [org] OR PPBIB [org] )

Term searched as exact words:

"lead" AND ( ANEUPL [org] OR BIOSIS [org] OR CIS [org] OR DART [org] OR EMIC [org] OR EPIDEM [org] OR HEEP [org] OR HMTC [org] OR IPA [org] OR RISKLINE [org] OR MTGABS [org] OR NIOSH [org] OR NTIS [org] OR PESTAB [org] OR PPBIB [org] )

Date limit: 2013 to present:

"Plumbism" OR "saturnism" OR "colica pictorum" OR "Devon colic" OR "painter's colic" 
Table B-2. Database Query Strings

\begin{tabular}{|c|c|c|}
\hline $\begin{array}{l}\text { Database } \\
\text { search date }\end{array}$ & Que & y string \\
\hline & & 12709-98-7 OR 1309-60-0 OR 1314-41-6 OR 1314-87-0 OR 1317-36-8 \\
\hline & & OR 13424-46-9 \\
\hline & L2 & 239150 SEA 13814-96-5 OR 15245-44-0 OR 16040-38-3 OR 301-04-2 OR \\
\hline & & 39377-56-5 OR 598-63-0 OR 7439-92-1 OR 7446-14-2 OR 7446-27-7 \\
\hline & & OR 7758-95-4 OR 7758-97-6 OR 78-00-2 \\
\hline & L3 & 244612 SEA L1 OR L2 \\
\hline & L4 & 244408 SEA L3 NOT TSCATS/FS \\
\hline & L5 & 225905 SEA L4 NOT PATENT/DT \\
\hline & L6 & 26519 SEA L5 AND ED>=20160201 \\
\hline & & ACT TOXQUERY/Q \\
\hline & L7 & QUE (CHRONIC OR IMMUNOTOX? OR NEUROTOX? OR TOXICOKIN? OR \\
\hline & & BIOMARKER? OR NEUROLOG?) \\
\hline & L8 & QUE (PHARMACOKIN? OR SUBCHRONIC OR PBPK OR EPIDEMIOLOGYIST,CT, \\
\hline & & \\
\hline & L9 & QUUE (ACUTE OR SUBACUTE OR LD50\# OR LD(W)50 OR LC50\# OR \\
\hline & & LC(W)50) \\
\hline & L10 & QUE (TOXICITY OR ADVERSE OR POISONING)/ST,CT,IT \\
\hline & L11 & QUE (INHAL? OR PULMON? OR NASAL? OR LÚNG? OR RESPIR?) \\
\hline & L12 & QUE ((OCCUPATION? OR WORKPLACE? OR WORKER?) AND EXPOS?) \\
\hline & L13 & QUE (ORAL OR ORALLY OR INGEST? OR GAVAGE? OR DIET OR DIETS OR \\
\hline & & DIETARY OR DRINKING(W)WATER?) \\
\hline & L14 & QUE (MAXIMUM AND CONCENTRÁTION? AND (ALLOWABLE OR PERMISSIBLE)) \\
\hline & L15 & QUE (ABORT? OR ABNORMALIT? OR EMBRYO? OR CLEFT? OR FETUS?) \\
\hline & L16 & QUE (FOETUS? OR FETAL? OR FOETAL? OR FERTIL? OR MALFORM? OR \\
\hline & & OVUM?) \\
\hline & L17 & QUE (OVA OR OVARY OR PLACENTA? OR PREGNAN? OR PRENATAL?) \\
\hline & L18 & QUE (PERINATAL? OR POSTNATAL? OR REPRODUC? OR STERIL? OR \\
\hline & L19 & QUE (SPERM OR SPERMAC? OR SPERMAG? OR SPERMATI? OR SPERMAS? OR \\
\hline & & SPERMATOB? OR SPERMATOC? OR SPERMATOG?) \\
\hline & L20 & QUE (SPERMATOI? OR SPERMATOL? OR SPERMATOR? OR SPERMATOX? OR \\
\hline & & SPERMATOZ? OR SPERMATU? OR SPERMI? OR SPERMO?) \\
\hline & L21 & QUE (NEONAT? OR NEWBORN? OR DEVELOPMENT OR DEVELOPMENTAL?) \\
\hline & L22 & QUE (ENDOCRIN? AND DISRUPT?) \\
\hline & L23 & QUE (ZYGOTE? OR CHILD OR CHILDREN OR ADOLESCEN? OR INFANT?) \\
\hline & L24 & QUE (WEAN? OR OFFSPRING OR AGE(W)FACTOR?) \\
\hline & L25 & QUE (DERMAL? OR DERMIS OR SKIN OR EPIDERM? OR CUTANEOUS?) \\
\hline & L26 & QUE (CARCINOG? OR COCARCINOG? OR CANCER? OR PRECANCER? OR \\
\hline & & NEOPLAS?) \\
\hline & L27 & QUE (TUMOR? OR TUMOUR? OR ONCOGEN? OR LYMPHOMA? OR CARCINOM?) \\
\hline & L28 & QUE (GENETOX? OR GENOTOX? OR MUTAGEN? OR GENETIC(W)TOXIC?) \\
\hline & L29 & QUE (NEPHROTOX? OR HEPATOTOX?) \\
\hline & L30 & QUE (ENDOCRIN? OR ESTROGEN? OR ANDROGEN? OR HORMON?) \\
\hline & L31 & QUE (OCCUPATION? OR WORKER? OR WORKPLACE? OR EPIDEM?) \\
\hline & L32 & QUE L7 OR L8 OR L9 OR L10 OR L11 OR L12 OR L13 OR L14 OR L15 \\
\hline & & OR L16 OR L17 OR L18 OR L19 OR L20 OR L21 OR L22 OR L23 OR L24 \\
\hline & & OR L25 OR L26 OR L27 OR L28 OR L29 OR L30 OR L31 \\
\hline & L33 & QUE (RAT OR RATS OR MOUSE OR MICE OR GUINEA(W)PIG? OR MURIDAE \\
\hline & & OR DOG OR DOGS OR RABBIT? OR HAMSTER? OR PIG OR PIGS OR SWINE \\
\hline & & OR PORCINE OR MONKEY? OR MACAQUE?) \\
\hline & L34 & QUE (MARMOSET? OR FERRET? OR GERBIL? OR RODENT? OR LAGOMORPHA \\
\hline & & OR BABOON? OR CANINE OR CAT OR CATS OR FELINE OR MURINE) \\
\hline & L35 & QUE L32 OR L33 OR L34 \\
\hline & L36 & QUE (HUMAN OR HUMANS OR HOMINIDAE OR MAMMALS OR MAMMAL? OR \\
\hline
\end{tabular}


Table B-2. Database Query Strings

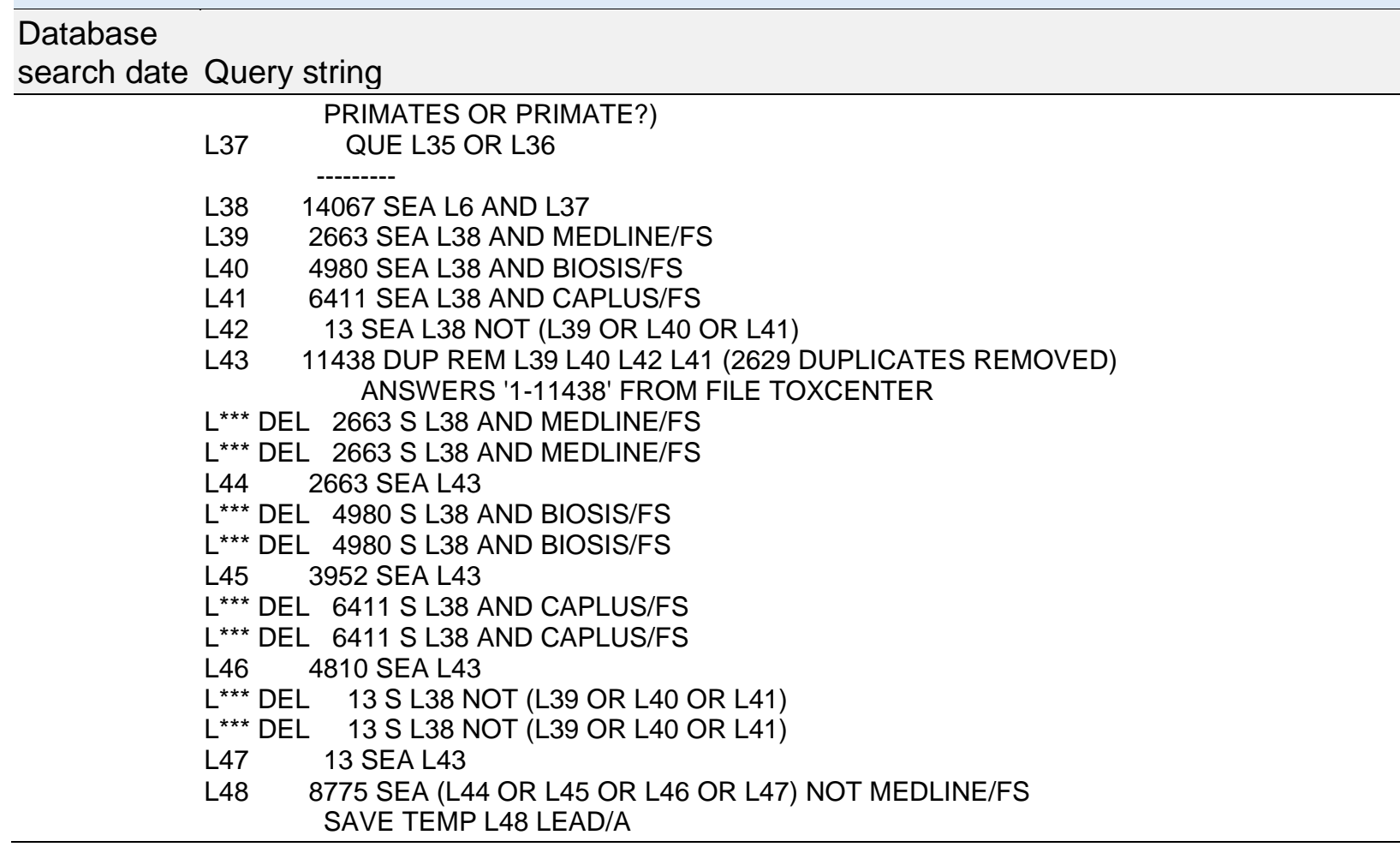

\section{Table B-3. Strategies to Augment the Literature Search}

\section{Source ChemView}

09/2019
Query and number screened when available

Compounds searched: 10031-22-8; 10099-74-8; 10101-63-0; 11119-70-3; 12709-98-7; 1309-60-0; 1314-41-6; 1314-87-0; 1317-36-8; 13424-46-9; 1381496-5; 15245-44-0; 16040-38-3; 301-04-2; 39377-56-5; 598-63-0; 7439-92-1; 7446-14-2; 7446-27-7; 7758-95-4; 7758-97-6; 78-00-2

\section{NTP}

09/2019
NTP Site Search (http://ntpsearch.niehs.nih.gov/home), date limit 2015 to present:

"10031-22-8" "10099-74-8" "10101-63-0" "11119-70-3"

"12709-98-7" "1309-60-0" "1314-41-6" "1314-87-0"

"1317-36-8" "13424-46-9" "13814-96-5" "15245-44-0"

"16040-38-3" "301-04-2" "39377-56-5" "598-63-0"

"7439-92-1" "7446-14-2" "7446-27-7" "7758-95-4"

"7758-97-6" "78-00-2"

Limited to content types reports \& publications; systematic reviews; ROC profiles, reviews, or candidates; or testing status, date limit 2015 to present: "lead"

\section{Regulations.gov}

10/2019
Compounds searched: 10031-22-8; 10099-74-8; 10101-63-0; 11119-70-3; 12709-98-7; 1309-60-0; 1314-41-6; 1314-87-0; 1317-36-8; 13424-46-9; 13814- 
Table B-3. Strategies to Augment the Literature Search

\begin{tabular}{ll}
\hline Source & Query and number screened when available \\
\hline $96-5 ; 15245-44-0 ; 16040-38-3 ; 301-04-2 ; 39377-56-5 ;$ 598-63-0; 7439-92-1; \\
7446-14-2; 7446-27-7; 7758-95-4; 7758-97-6; 78-00-2
\end{tabular}

NIH RePORTER

01/2020
Search in: Projects AdminIC: All, Fiscal Year: Active Projects Text Search (Advanced):

"1,3-Benzenediol, 2,4,6-trinitro-, lead" OR "Acetic acid lead " OR "Acetic acid, lead salt" OR "Acetic acid, lead " OR "Acetic acid, lead " OR "Anglislite" OR "Azarcon" OR "Borate(1-), tetrafluoro-, lead" OR "Borate(1-), tetrafluoro-, lead" OR "C.I. Pigment Metal 4" OR "C.I. Pigment Yellow 46" OR "CARBONIC ACID, LEAD SALT" OR "Carbonic acid, lead salt" OR "Carbonic acid, lead" OR "Cerussete" OR "Cerussite" OR "Chrome Orange" OR "Chrome Yellow" OR "Chromic acid (H2CrO4), lead" OR "Chromic acid lead salt " OR "CHROMIC ACID, LEAD" OR "Chromic Acid, Lead (II) Salt" OR "Chromic acid, lead and molybdenum salt" OR "Chromic acid, lead salt" OR "Chromic acid, lead" OR "Chromium lead molybdenum oxide" OR "Chromium lead oxide" OR "Cl pigment metal 4" OR "CI Pigment Yellow 46" OR "Collodial lead phosphate" OR "Dibasic lead acetate" OR "Dibasic lead carbonate" OR "Dibasic lead sulfate" OR "Entan" OR "Fast White" OR "Flowsperse R 12" OR "Freemans White Lead" OR "Galena" OR "Glover" OR "Gold Satinobre" OR "Heuconin 5" OR "Lead (II) carbonate" OR "Lead (II) chloride" OR "Lead (II) chromate" OR "Lead (II) iodide" OR "Lead (II) nitrate" OR "Lead (II) oxide" OR "Lead (II) sulfate" OR "Lead (II) sulfide" OR "Lead (II, IV) oxide" OR "Lead (IV) oxide " OR "Lead 2,4,6-trinitro-m-phenylene dioxide" OR "Lead acetate" OR "Lead azide" OR "Lead bis(tetrafluoroborate)" OR "Lead borofluoride" OR "Lead boron fluoride" OR "Lead Bottoms" OR "Lead bromide" OR "Lead brown" OR "Lead carbonate" OR "Lead chloride" OR "Lead chromate" OR "Lead chromate(VI)" OR "Lead chromium oxide (PbCrO4)" OR "Lead di(acetate)" OR "Lead diacetate" OR "Lead diazide" OR "Lead dibasic acetate" OR "Lead dibromide" OR "Lead dichloride" OR "Lead diiodide" OR "Lead dinitrate" OR "Lead dioxide" OR "Lead element" OR "Lead flake" OR "Lead fluoborate" OR "Lead fluoroborate" OR "Lead iodide" OR "Lead metal" OR "Lead molybdate chromate" OR "Lead molybdenum chromate" OR "Lead monooxide" OR "Lead monosulfate" OR "Lead monosulfide" OR "Lead monoxide" OR "Lead nitrate" OR "Lead orthophosphate" OR "Lead orthoplumbate" OR "Lead oxide" OR "Lead peroxide" OR "Lead phosphate" OR "Lead protoxide" OR "Lead S 2" OR "Lead S2" OR "Lead styphnate" OR "Lead sulfate" OR "Lead sulfide" OR "Lead sulphate" OR "Lead sulphide" OR "Lead superoxide" OR "Lead tetraethide" OR "Lead tetraethyl"

"Lead tetrafluoroborate" OR "Lead tetraoxide" OR "Lead tetroxide" OR "Lead tricinate" OR "Lead trinitroresorcinate" OR "Lead(II) acetate" OR "Lead(II) azide" OR "Lead(II) bromide" OR "Lead(II) carbonate" OR "Lead(II) chloride" OR "Lead(II) chromate" OR "Lead(II) dinitrate" OR "Lead(II) iodide" OR "Lead(II) nitrate" OR "Lead(II) oxide" OR "Lead(II) phosphate" OR "Lead(II) phosphate" OR "Lead(II) styphnate" OR "Lead(II) sulfate" OR "Lead(II) sulfide" OR "Lead(II) tetrafluoroborate" OR "Lead(IV) oxide" OR "Lead, elemental" OR "Lead, inorganic" OR "Lead, tetraethyl" OR "Lead, tetraethyl-" OR "Leadmolybdenum chromate" OR "Litharge" OR "Massicot" OR "Massicotite" OR "Mennige" OR "Milk White" OR "mine orange" OR "Mineral Orange" OR "Mineral red" OR "minio anaranjado" OR "Minium" OR "Molybdenum-lead chromate" OR "Mulhouse White" OR "Nitric acid lead" OR "Nitric acid, lead" OR "Orange lead" OR "Orangemennige" OR "Paris Red" OR "PbSO4" OR "Perlex 
Table B-3. Strategies to Augment the Literature Search

\begin{tabular}{ll}
\hline Source & Query and number screened when available \\
\hline paste 500" OR "Perlex paste 600A" OR "Phoenicochroite" OR "Phosphoric \\
acid, lead salt" OR "Phosphoric acid, lead" OR "Pigment Red 105" OR "Pigment \\
White 3" OR "Pigment Yellow 34" OR "Pigment Yellow 46" OR "Plumbane" OR \\
"Plumbi" OR "Plumbic oxide" OR "Plumboplumbic oxide" OR "Plumbous" OR \\
"Plumbum" OR "Red lead" OR "Resorcinol, 2,4,6-trinitro-, lead" OR "Rough \\
lead bullion" OR "Royal Yellow 6000" OR "Salt of saturn" OR "Sandix" OR \\
"Saturn red" OR "Sugar of lead" OR "Sulfuric acid, lead" OR "Tetra Ethylene \\
Lead" OR "Tetra(methylethyl)lead" OR "Tetraethyl lead" OR "Tetraethyl \\
plumbane" OR "Tetraethyllead" OR "Tetraethyllead, liquid" OR \\
"tetraethylplomb" OR "Tetraethylplombane" OR "Tetraethylplumbane" OR \\
"tetraetilplomo" OR "Thiolead A" OR "Tricinat" OR "Trilead bis(orthophosphate)" \\
OR "Trilead phosphate" OR "Trilead tetraoxide" OR "Trilead tetroxide" OR \\
"Unichem PBA" OR "Yellow lead ocher" OR "Lead(2) sulfate" OR "Lead(2) \\
acetate" OR "Lead(2) azide" OR "Lead(2) bis(nitrate)" OR "Lead(2) bromide" \\
OR "Lead(2) carbonate" OR "Lead(2) chloride" OR "Lead(2) nitrate" OR \\
"Lead(2) oxide" OR "Lead(2) phosphate" OR "Lead(2) phosphate (Pb3(PO4)2)" \\
OR "Lead(2) salt carbamic acid" OR "Lead(2) sulfate" OR "Lead(2) sulfide" \\
"lead poisoning" OR "Plumbism" OR "saturnism" OR "colica pictorum" OR \\
"Devon colic" OR "painter's colic" \\
"blood lead" \\
Search in: Projects Limit to: Project Title, $\quad$ AdminlC: All, Fiscal Year: Active \\
Projects Text Search (Advanced): \\
"lead" not ("lead academic" or "lead optimization") \\
Identified throughout the assessment process
\end{tabular}

The 2019 results were:

- Number of records identified from PubMed, TOXLINE, and TOXCENTER (after duplicate removal): 15,240

- Number of records identified from other strategies: 107

- Total number of records to undergo literature screening: 15,347

\section{B.1.2 Literature Screening}

A two-step process was used to screen the literature search to identify relevant studies on $\mathrm{Pb}$ :

- Title and abstract screen

- Full text screen

Title and Abstract Screen. Within the reference library, titles and abstracts were screened manually for relevance. Studies that were considered relevant (see Table B-1 for inclusion criteria) were moved to the second step of the literature screening process. Studies were excluded when the title and abstract clearly indicated that the study was not relevant to the toxicological profile.

- $\quad$ Number of titles and abstracts screened: 388

- $\quad$ Number of studies considered relevant and moved to the next step: 388 
Full Text Screen. The second step in the literature screening process was a full text review of individual studies considered relevant in the title and abstract screen step. Each study was reviewed to determine whether it was relevant for inclusion in the toxicological profile.

- $\quad$ Number of studies undergoing full text review: 388

- Number of studies cited in the pre-public draft of the toxicological profile: 1,589

- Total number of studies cited in the profile: 1,803

A summary of the results of the literature search and screening is presented in Figure B-1.

\section{Figure B-1. September 2019 Literature Search Results and Screen for Lead (Pb)}
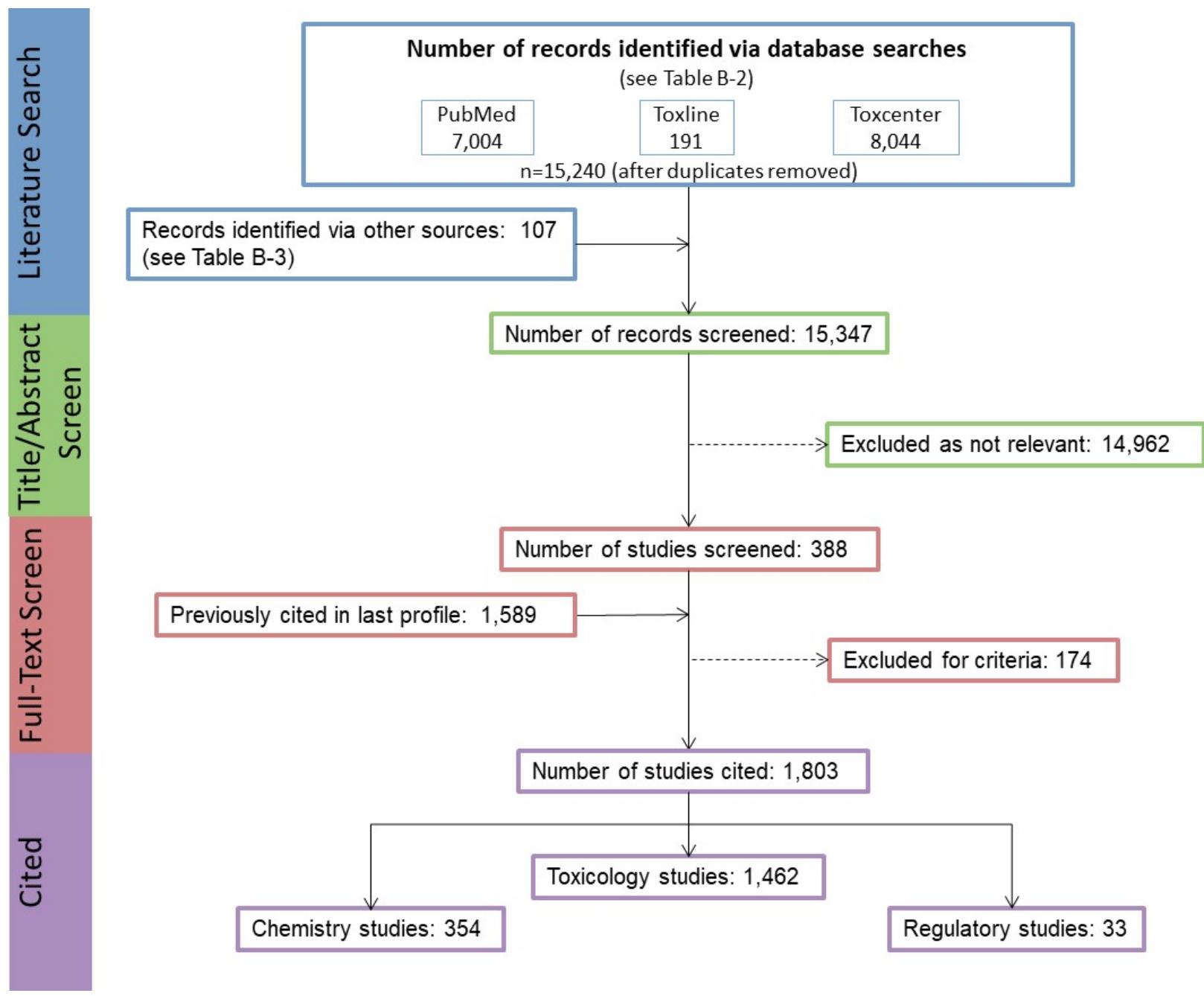


\section{APPENDIX C. INGESTION OF LEAD DEBRIS}

The main focus of this ATSDR Toxicological Profile for Lead is on health effects of chronic low-level environmental exposures. The profile also provides information on the clinical presentation of acute $\mathrm{Pb}$ toxicity, which occurs when large amounts of $\mathrm{Pb}$ are ingested. In children, this often occurs through ingestion of paint chips containing $\mathrm{Pb}, \mathrm{Pb}$-contaminated soils, or other non-solid forms of $\mathrm{Pb}$. Ingestion of solid forms of $\mathrm{Pb}$ ( $\mathrm{Pb}$ debris) is a unique exposure scenario in which there is accidental or purposeful ingestion of visible debris containing $\mathrm{Pb}$. This exposure may be acute (debris is expelled or removed from the body soon after ingestion) or chronic ( $\mathrm{Pb}$ debris is retained within the body, leading to continued elevation in $\mathrm{PbB}$ ). There are several sources of $\mathrm{Pb}$ debris, including $\mathrm{Pb}$ shot or other debris found at firing or artillery ranges, or $\mathrm{Pb}$ shot found in wild game meats. The information presented below reviews toxicokinetics and adverse health effects of ingested $\mathrm{Pb}$ debris. Information regarding the chemistry, fate, and transport of $\mathrm{Pb}$ debris is reviewed in Chapter 5. It should also be noted that in addition to ingestion of $\mathrm{Pb}$ debris, retained $\mathrm{Pb}$ shot or shrapnel, especially in military personnel, could contribute to elevated $\mathrm{PbB}$ (Gerhardsson et al. 2002; McQuirter et al. 2004); this possibility should be considered in individuals as appropriate.

Overview. No controlled studies in humans have evaluated bioavailability or toxicity of ingested $\mathrm{Pb}$ debris (e.g., Pb shot and other Pb-containing debris from artillery or shooting ranges). Available information is anecdotal, obtained from case reports. Thus, data are not sufficient to determine the bioavailability of ingested $\mathrm{Pb}$ debris or to develop dose-response relationships for toxicity. Case reports of acute exposures from ingestion of $\mathrm{Pb}$ debris are summarized in Table $\mathrm{C}-1$; these reports demonstrate the following:

- $\mathrm{PbB}$ rises rapidly (within hours to a few days) following ingestion of $\mathrm{Pb}$ debris.

- The clinical presentation of toxicity following ingestion of $\mathrm{Pb}$ debris is the same as that observed for acute $\mathrm{Pb}$ poisoning from ingestion of other forms of $\mathrm{Pb}$ (see Section 2.2).

- Severity of toxicity of ingested $\mathrm{Pb}$ debris will depend upon how much $\mathrm{Pb}$ is absorbed (e.g., toxicity is related to $\mathrm{PbB}$; see Section 2.2).

- The onset of toxicity can be rapid (within hours to a few days).

- Following removal of $\mathrm{Pb}$ debris from the body, $\mathrm{PbBs}$ decrease; however, applying clinical protocols for chelation therapy results in a more rapid decrease in $\mathrm{PbB}$.

- Ingested $\mathrm{Pb}$ debris can be retained in the appendix of some individuals and continue to contribute to elevated $\mathrm{PbB}$. 
Table C-1. Selected Case Studies of Ingestion of Solid Lead $(\mathrm{Pb})$ Debris or Pb Retained in Gunshot Wounds

\begin{tabular}{|c|c|c|c|}
\hline Reference and exposure & $\begin{array}{l}\text { Blood lead concentration (PbB) } \\
(\mu \mathrm{g} / \mathrm{dL})\end{array}$ & Effects & Treatment \\
\hline $\begin{array}{l}\text { Banner et al. } 2012 \\
\text { A 15-year-old boy ingested a "handful" of } \\
\text { Pb shot. He was admitted to the hospital } \\
\text { for treatment } 14 \text { days after exposure. }\end{array}$ & $\begin{array}{l}\text { Post-ingestion } \\
\text { o } 8 \text { days: } 54 \\
\text { o } 14 \text { days: } 41 \\
\text { Post-treatment (2 weeks): }<5\end{array}$ & $\begin{array}{l}\text { - } \quad \text { Most Pb was located in the } \\
\text { appendix (14 days post- } \\
\text { ingestion) } \\
\text { - } \quad \text { Abdominal pain } \\
\text { - } \quad \text { Elevated free erythrocyte } \\
\text { protoporphyrin }\end{array}$ & $\begin{array}{ll}\text { - } & \text { Whole bowel irrigation } \\
\text { - } & \text { Appendectomy } \\
\text { - } & \text { Chelation }\end{array}$ \\
\hline $\begin{array}{l}\text { CDC } 2006 \\
\text { A 4-year-old boy with previously diagnosed } \\
\text { microcephaly and mental delays ingested } \\
\text { a metallic charm containing Pb. Time from } \\
\text { exposure to first medical visit was not } \\
\text { reported. }\end{array}$ & At death: 180 & $\begin{array}{ll}\text { - } & \text { Charm was retained in the } \\
\text { - } & \text { Intramach (was not removed) } \\
\text { - } & \text { Cerebral edema } \\
\text { - } & \text { Seizures } \\
\text { - } & \text { Death }\end{array}$ & Supportive therapy \\
\hline $\begin{array}{l}\text { Clifton et al. } 2002 \\
\text { A 21-month-old girl ingested Pb BB pellets. } \\
\text { She was taken to the hospital } \\
\text { approximately } 6 \text { hours post-ingestion. }\end{array}$ & $\begin{array}{ll}\text { - } & \text { Pre-ingestion (routine): } 12 \\
\text { - } & \text { Post-ingestion (6 hours): } 47 \\
\text { - } & \text { Post-removal of pellets: } 25 \\
\text { - } & \text { Post-treatment (10 days): } 16\end{array}$ & $\begin{array}{ll}\text { - } & \text { Hyperactivity } \\
\text { - } & \text { No signs of neurological or } \\
& \text { gastrointestinal toxicity }\end{array}$ & $\begin{array}{l}\text { - } \quad \text { Bowel irrigation } \\
\text { - Colonoscopy for } \\
\text { removal of pellets } \\
\text { - Chelation }\end{array}$ \\
\hline $\begin{array}{l}\text { Cox and Pesola } 2005 \\
\text { A 73-year-old woman ingested Pb shot in } \\
\text { game over decades. }\end{array}$ & Not reported & $\begin{array}{l}\text { - Pb shot accumulated in the } \\
\text { appendix } \\
\text { - No information on adverse } \\
\text { health effects was reported }\end{array}$ & Not reported \\
\hline $\begin{array}{l}\text { Durlach et al. } 1986 \\
\text { A 30-year-old man ingested Pb shot in } \\
\text { game regularly over an unspecified period } \\
\text { of time. }\end{array}$ & $\begin{array}{l}\text { - At initial examination: } 67.4 \\
\text { Post-treatment } \\
\text { o } 10 \text { days: } 52.2 \\
\text { o } 13 \text { days: } 24.5 \\
\text { o } 1 \text { month: } 36.8 \\
\text { o } 1.5 \text { months: } 31.6\end{array}$ & $\begin{array}{l}\text { - } \quad \text { Pb shot accumulated in the } \\
\text { appendix } \\
\text { - } \quad \text { Acute abdominal pain }\end{array}$ & $\begin{array}{ll}\text { - } & \text { Bowel irritation } \\
\text { - } & \text { Chelation } \\
\text { - } & \text { Appendectomy }\end{array}$ \\
\hline
\end{tabular}


Table C-1. Selected Case Studies of Ingestion of Solid Lead (Pb) Debris or Pb Retained in Gunshot Wounds

\begin{tabular}{llll}
\hline Reference and exposure & $\begin{array}{l}\text { Blood lead concentration }(\mathrm{PbB}) \\
(\mu \mathrm{g} / \mathrm{dL})\end{array}$ & Effects & Treatment \\
\hline Fergusson et al. 1997 & $\bullet \quad$ Day of ingestion: 4 & No signs of toxicity observed & Endoscopy \\
A 4-year-old girl ingested a Pb fishing & - Day after ingestion: 16 & &
\end{tabular}

sinker. She was evaluated in the emergency room within 1 hour of ingestion.

\begin{tabular}{|c|c|c|c|}
\hline $\begin{array}{l}\text { Gerhardsson et al. } 2002 \\
\text { A man in his "late } 40 \text { s" had retained Pb } \\
\text { shot following a gunshot wound to the } \\
\text { shoulder. Reconstructive surgery occurred } \\
54 \text { days post-accident. Some, but not all, } \\
\text { of the Pb shot was removed during } \\
\text { surgery. }\end{array}$ & $\begin{array}{l}\text { Approximate (data presented } \\
\text { graphically), time after accident: } \\
\text { - } \quad 25 \text { days: } 28 \\
\text { - } \quad 50 \text { days: } 41 \\
\text { - } \quad 54 \text { days (day of surgery): } 55 \\
\text { - } \quad \text { 60 days: } 31 \\
\text { - } \quad 75 \text { days: } 48 \\
\text { - } \quad 200 \text { days: } 36 \\
\text { - } \quad 375 \text { days: } 30\end{array}$ & $\begin{array}{l}\text { - } \quad \text { No signs of toxicity observed } \\
\text { - Not all of the Pb shot could be } \\
\text { removed during surgery }\end{array}$ & $\begin{array}{l}\text { Surgical removal of } \mathrm{Pb} \\
\text { pellets }\end{array}$ \\
\hline $\begin{array}{l}\text { Guillard et al. } 2006 \\
\text { A 2-year-old boy ingested toy money made } \\
\text { from pure metallic } \mathrm{Pb} \text {. }\end{array}$ & $\begin{array}{l}\text { Time post-ingestion } \\
\text { - } 1 \text { day: } 31.3 \\
\text { - } \quad 8 \text { days: } 61.1 \\
\text { - } 1 \text { month: } 30.0 \\
\text { - } \quad 4 \text { months: } 24.9 \\
\text { - } 10 \text { months: } 9.9\end{array}$ & $\begin{array}{l}\text { Development of microcytic } \\
\text { anemia and increased blood } \\
\text { zinc protoporphyrin } \\
\text { - } \quad \text { No signs of toxicity observed }\end{array}$ & $\begin{array}{ll}\text { - } & \text { Removal of object } \\
\text { - } & \text { Chelation (8 days } \\
\text { post-ingestion) }\end{array}$ \\
\hline $\begin{array}{l}\text { Gustavsson and Gerhardsson } 2005 \\
\text { A 45-year-old woman with elevated PbB } \\
\text { was found to have Pb shot in her intestine } \\
\text { from ingestion of game. The Pb shot was } \\
\text { spontaneously eliminated. Time from } \\
\text { ingestion was estimated to be sometime } \\
\text { between } 1993 \text { and } 2001 \text {. }\end{array}$ & $\begin{array}{l}\text { Time of assessment: } \\
\text { - } \quad \text { January } 2002: 55.0 \\
\text { - } \quad \text { April } 2003 \text { (2 months post- } \\
\text { - } \quad \text { elimination): } 34.5 \\
\quad \text { November 2003: } 7.2\end{array}$ & $\begin{array}{l}\text { - Malaise and fatigue } \\
\text { - "Diffuse gastrointestinal } \\
\text { symptoms" }\end{array}$ & $\begin{array}{l}\text { No treatment (object was } \\
\text { spontaneously eliminated) }\end{array}$ \\
\hline
\end{tabular}


Table C-1. Selected Case Studies of Ingestion of Solid Lead (Pb) Debris or Pb Retained in Gunshot Wounds

\begin{tabular}{|c|c|c|c|}
\hline Reference and exposure & $\begin{array}{l}\text { Blood lead concentration (PbB) } \\
(\mu \mathrm{g} / \mathrm{dL})\end{array}$ & Effects & Treatment \\
\hline $\begin{array}{l}\text { Hatten et al. } 2013 \\
\text { Case 1: A 15-year-old boy ingested } \\
\text { rifle cartridges approximately } 1 \text { month } \\
\text { prior to evaluation. }\end{array}$ & $\begin{array}{l}\text { Case } 1 \\
\text { - } \quad \text { Initial assessment: } 146 \\
\text { - } \quad 19 \text { days post-treatment: } 53 \\
\text { - } \quad 3 \text { months post-treatment: } 38\end{array}$ & $\begin{array}{ll}\text { Case } 1 \\
-\quad \text { Decreased activity level } \\
\text { - } \quad \text { Vomiting, diarrhea, anorexia } \\
\text { - } \quad \text { Hyperactive patellar and } \\
\quad \text { brachioradialis reflexes }\end{array}$ & $\begin{array}{l}\text { Case } 1 \\
-\quad \text { Cartridges removed } \\
\text { by endoscopy } \\
\text { - } \quad \text { Chelation }\end{array}$ \\
\hline $\begin{array}{l}\text { - Case 2: A 65-year-old woman } \\
\text { ingested several handfuls of bullets. }\end{array}$ & $\begin{array}{l}\text { Case 2: Days after ingestion } \\
\text { - } \quad \text { Day 1: } 9.7 \\
\text { - } \quad \text { Day 2: } 25.7 \\
\text { - } \quad \text { Day 3: } 40.5 \\
\text { - } \quad \text { Day } 60: 17.2\end{array}$ & $\begin{array}{l}\text { Case } 2 \\
\text { - No signs of toxicity were } \\
\text { observed }\end{array}$ & $\begin{array}{l}\text { Case } 2 \\
-\quad \text { Endoscopy }\end{array}$ \\
\hline Larsen and Blanton 2000 & Not reported & - Abdominal pain and anorexia & Appendectomy \\
\hline
\end{tabular}

A 9-year-old boy ingested $\mathrm{Pb}$ shot in game; the $\mathrm{Pb}$ shot was retained in the appendix.

\begin{tabular}{|c|c|c|c|}
\hline $\begin{array}{l}\text { Lyons and Filston } 1994 \\
\text { A 4-year-old boy ingested Pb shot, which } \\
\text { was lodged in his appendix. }\end{array}$ & $\begin{array}{l}\text { - Peak (time of assessment not } \\
\text { reported): } 23 \\
\text { - Prior to surgery (1.5 months after } \\
\text { ingestion): } 12\end{array}$ & $\begin{array}{l}\text { - Abdominal discomfort, nausea, } \\
\text { vomiting, diarrhea } \\
\text { - Headache }\end{array}$ & Appendectomy \\
\hline $\begin{array}{l}\text { Madsen et al. } 1988 \\
\text { Seven patients with } \mathrm{Pb} \text { shot retained in the } \\
\text { appendix. }\end{array}$ & Range: $4.6-18.2$ & Not reported & Not reported \\
\hline $\begin{array}{l}\text { McKinney and McKinney } 2000 \\
\text { A 5.5-year-old girl ingested several Pb } \\
\text { pellets. }\end{array}$ & $\begin{array}{l}\text { Time after ingestion } \\
-\quad 13 \text { hours: } 57 \\
\text { - } \quad 36 \text { hours: } 79 \\
\text { After treatment: } \\
\text { - } 14 \text { days: } 14.3 \\
-\quad 6 \text { months: } 25\end{array}$ & $\begin{array}{l}\text { - Vomiting and abdominal pain } \\
\text { - Decreased blood hemoglobin } \\
\text { and hematocrit } \\
\text { - Mild" speech and language } \\
\text { delays noted post-treatment }\end{array}$ & $\begin{array}{l}\text { - Whole bowel irrigation } \\
\text { - Chelation }\end{array}$ \\
\hline
\end{tabular}


Table C-1. Selected Case Studies of Ingestion of Solid Lead (Pb) Debris or Pb Retained in Gunshot Wounds

\begin{tabular}{|c|c|c|c|}
\hline Reference and exposure & $\begin{array}{l}\text { Blood lead concentration (PbB) } \\
(\mu \mathrm{g} / \mathrm{dL})\end{array}$ & Effects & Treatment \\
\hline $\begin{array}{l}\text { McNutt et al. } 2001 \\
\text { A } 45 \text {-year-old male ingested } 206 \mathrm{~Pb} \\
\text { bullets. Medical evaluation occurred } \\
5 \text { days after ingestion. Bullets were } \\
\text { spontaneously eliminated over } 4-47 \text { days } \\
\text { after first medical evaluation. }\end{array}$ & $\begin{array}{l}\text { Time after ingestion: } \\
\text { - } \quad 5 \text { days: } 391 \\
\text { - } \quad 10 \text { days: } 171 \\
\text { - } \quad 25 \text { days: } 41 \\
\text { - } \quad 6 \text { weeks: } 24\end{array}$ & $\begin{array}{l}\text { - Abdominal pain and } \\
\text { gastrointestinal bleeding } \\
\text { - Anemia }\end{array}$ & $\begin{array}{l}\text { Chelation started at initial } \\
\text { medical visit }\end{array}$ \\
\hline
\end{tabular}

after first medical evaluation.

McQuirter et al. 2004

Subjects $(n=451)$ 1-year following gunshot

- $\quad$ PbB at time after injury: $1.9 \quad$ Not reported

Not reported

wound with retained bullets.
0 days: 2.1
o 3 days: 7.6
o 18 days: 25.1
o 3 months: 38.1
6 months: 28.5
o 12 months: 15.8

CDC 2004a

- 2-3 weeks after ingestion: 123

- After treatment: 57

A 4-year-old boy ingested a Pb medallion.

- Abdominal pain, vomiting, diarrhea

- Normocytic anemia, elevated protoporphyrin

\begin{tabular}{|c|c|c|c|}
\hline $\begin{array}{l}\text { Mowad et al. } 1998 \\
\text { An 8-year-old boy ingested several Pb } \\
\text { fishing sinkers. Medical assessment was } \\
\text { within } 1 \text { days of ingestion. }\end{array}$ & $\begin{array}{l}\text { Time after ingestion: } \\
\text { - } 1 \text { day: } 53 \\
\text { - } \quad 6 \text { days: } 45 \text { (start of chelation) } \\
\text { - } \quad 1 \text { month: } 3\end{array}$ & No signs of toxicity observed & $\begin{array}{ll}\text { - } & \text { Bowel irrigation } \\
\text { - } & \text { Colonoscopy } \\
\text { - } & \text { Chelation }\end{array}$ \\
\hline $\begin{array}{l}\text { Rosenberg and Haynes } 2019 \\
\text { A 3-year-old ingested Pb pellets. }\end{array}$ & $\begin{array}{l}\text { Time after ingestion: } \\
\text { - } 7 \text { days: } 27 \\
\text { - } \quad \text { Post-surgical removal: } 14\end{array}$ & Not reported & $\begin{array}{l}\text { Laproscopic removal of } \\
\text { pellets }\end{array}$ \\
\hline
\end{tabular}


Table C-1. Selected Case Studies of Ingestion of Solid Lead (Pb) Debris or Pb Retained in Gunshot Wounds

\begin{tabular}{|c|c|c|c|}
\hline Reference and exposure & $\begin{array}{l}\text { Blood lead concentration (PbB) } \\
(\mu \mathrm{g} / \mathrm{dL})\end{array}$ & Effects & Treatment \\
\hline \multirow{3}{*}{$\begin{array}{l}\text { Rozier and Liebelt } 2019 \\
\text { A 2-year-old boy, a 10-year-old boy, and a } \\
\text { 16-year old girl ingested Pb pellets. }\end{array}$} & $\begin{array}{l}\text { 2-year-old boy, PbB measurement: } \\
\text { - } \quad \text { Day 0: } 65 \\
\text { - } 5 \text { days post-chelation: } 25.2\end{array}$ & 2-year-old boy: asymptomatic & $\begin{array}{l}\text { 2-year-old boy } \\
\text { - } \quad \text { Bowel irrigation } \\
\text { - } \quad \text { Chelation }\end{array}$ \\
\hline & $\begin{array}{l}\text { 10-year-old boy, PbB measurement: } \\
\text { - } 3 \text { days post-ingestion: } 70 \\
\text { - } 7 \text { months post-chelation: } 9.5\end{array}$ & 10-year-old boy: not reported & $\begin{array}{l}\text { 10-year-old boy } \\
\text { - } \quad \text { Bowel irrigation } \\
\text { - } \quad \text { Chelation }\end{array}$ \\
\hline & $\begin{array}{l}\text { 16-year old girl, } \mathrm{PbB} \text { measurement: } \\
\text { - } 9 \text { days post-ingestion: } 53 \\
\text { - } 13 \text { days post-treatment: } 3 \\
\text { - } 5 \text { weeks post-treatment: } 13\end{array}$ & $\begin{array}{l}\text { 16-year old girl: } \\
-\quad \text { abdominal pain } \\
\text { - } \quad \text { shortness of breath }\end{array}$ & $\begin{array}{l}\text { 16-year old girl } \\
\text { - } \quad \text { Bowel irrigation } \\
\text { - } \quad \text { Chelation } \\
\text { - } \quad \text { Colonoscopy }\end{array}$ \\
\hline $\begin{array}{l}\text { Treble and Thompson } 2002 \\
\text { A 2.5-year-old girl ingested } \mathrm{Pb} \text { pellets. }\end{array}$ & $\begin{array}{l}\text { Time after ingestion } \\
-\quad 1.5 \text { hours: } 56 \\
-\quad 29 \text { hours: } 35 \\
-\quad 94 \text { hours: } 35\end{array}$ & No signs of toxicity observed & Laxatives \\
\hline $\begin{array}{l}\text { Zardawi and Siriweera } 2013 \\
\text { An 8-year-old boy ingested } \mathrm{Pb} \text { pellets in } \\
\text { game over a 2-year period. }\end{array}$ & $\begin{array}{l}\text { Elevated PbB (17.4-27.4) over } \\
2 \text { years } \\
\text { Pellets observed in appendix }\end{array}$ & Hyperactivity & $\begin{array}{l}\text { - } \text { Bowel irrigation } \\
\text { - }\end{array}$ \\
\hline
\end{tabular}


Confounding Factors. There are several uncertainties from case reports on ingestion of $\mathrm{Pb}$ debris. Therefore, it is not possible to determine dose, bioavailability, or accurate plasma-time concentration curves. Uncertainties include:

- Baseline PbB data are rarely available. Thus, it is difficult to determine the contribution of ingested $\mathrm{Pb}$ debris to measured $\mathrm{PbB}$ following ingestion.

- Time from ingestion of $\mathrm{Pb}$ debris to first clinical evaluation and $\mathrm{PbB}$ assessment is often unknown.

- $\quad$ No quantitative data on the dose of $\mathrm{Pb}$ ingested in debris are reported.

- No quantitative data on fecal excretion of ingested $\mathrm{Pb}$ are reported.

- Information on the chemical composition of $\mathrm{Pb}$ debris often is not reported.

- No information on potential differences in the bioavailability of different types of $\mathrm{Pb}$ debris is available

Bioavailability of $\mathbf{P b}$ Debris. No quantitative estimates on the bioavailability of $\mathrm{Pb}$ debris in humans are available. Several case reports show increased $\mathrm{PbB}$ following ingestion of $\mathrm{Pb}$ debris, demonstrating that ingested Pb is absorbed (CDC 2006; Clifton et al. 2002; Durlach et al. 1986; Fergusson et al. 1997; Greensher et al. 1974; Guillard et al. 2006; Hatten et al. 2013; McKinney and McKinney 2000; McNutt et al. 2001; CDC 2004a; Mowad et al. 1998; Treble and Thompson 2002); see Table C-1 for details. However, due to lack of information on ingested dose, quantitative estimates of absorption cannot be determined. No information on bioavailability of $\mathrm{Pb}$ debris in animals was identified. Lead debris retained within the body will continue to contribute to elevated $\mathrm{PbB}$ until it is removed from the body, either spontaneously or by medical intervention (Banner et al. 2012; Clifton et al. 2002; Durlach et al. 1986; Gerhardsson et al. 2002; Guillard et al. 2006; McQuirter et al. 2004).

Lead debris must become bioaccessible (i.e., soluble) in the gastrointestinal tract in order for it to be absorbed. It is likely that processes thought to contribute to rendering ingested soil $\mathrm{Pb}$ bioaccessible also are important in rendering ingested $\mathrm{Pb}$ debris bioaccessible (see Section 3.1.1). IVBA assays that measure extractable $\mathrm{Pb}$ have not been evaluated for predicting bioavailability or RBA of ingested $\mathrm{Pb}$ debris, although one study found that IVBA measured at gastric $\mathrm{pH}$ predicted the relatively high in vivo RBA (100\%) of firing range soils (Bannon et al. 2009; see Section 3.1.1). 
Although dose-PbB relationships and bioavailability cannot be reliably established from the published case history of $\mathrm{Pb}$ debris ingestion, it is possible to use exposure-biokinetics models to reconstruct the time course of $\mathrm{PbB}$ expected for a given acute dose of soluble $\mathrm{Pb}$ and, from this, estimate the relative bioavailability of $\mathrm{Pb}$ from ingested $\mathrm{Pb}$ shot that would result in a given peak $\mathrm{PbB}$. This scenario assumes that $\mathrm{Pb}$ debris is not retained in the body. The AALM-LG (EPA 2014a) can simulate the internal biokinetics of $\mathrm{Pb}$ associated with daily doses of $\mathrm{Pb}$. This model predicts that a child 30 months of age who has a baseline $\mathrm{PbB}$ of $1 \mu \mathrm{g} / \mathrm{dL}$ would experience a $10 \mu \mathrm{g} / \mathrm{dL}$ increase in $\mathrm{PbB}$ in response to ingestion of approximately $1 \mathrm{mg}$ of soluble $\mathrm{Pb}$ (Figure C-1). The peak PbB would occur during the day of ingestion and $\mathrm{PbB}$ would return to approximately 120\% of baseline in approximately 35 days following the dose. If this prediction is extrapolated to the ingestion of $\mathrm{Pb}$ shot or other debris, the $1 \mathrm{mg}$ dose of soluble $\mathrm{Pb}$ could occur in association with a dose of $100 \mathrm{mg}$ of debris having an RBA of $1 \%$, or $1 \mathrm{~g}$ of debris having an RBA of 0.1\% (see Section 3.1.5.4 EPA All Ages Lead Model [AALM] for more information). Figure C-1 also shows the predicted $\mathrm{PbB}$ pattern for six repeated, weekly events in which the child ingested $1 \mathrm{mg}$ of soluble $\mathrm{PbB}$. This would result in periodic increases in $\mathrm{PbB}$, with the maximum following each exposure event increasing until a pseudo-steady-state $\mathrm{PbB}$ was reached at approximately $14.5 \mu \mathrm{g} / \mathrm{dL}$ (13.5 $\mu \mathrm{g} / \mathrm{dL}$ above baseline). The PbB would return to approximately $120 \%$ of baseline in approximately 570 days after the last exposure event. This longer time to baseline following multiple exposures reflects the accrual of $\mathrm{Pb}$ in bone with multiple dosing and the relatively slow transfer of $\mathrm{Pb}$ from bone to blood after exposure ceases (see Section 3.1).

Ingestion of soil from firing ranges may also contribute to $\mathrm{PbB}$. A study in juvenile swine of eight soils (sieved to $<250 \mu \mathrm{m}$ ) from small arms firing ranges showed a relative bioavailability range of 77-140\%, with a mean of $108 \%$ (SD or SE [not specified]: 18\%). Soil from this site largely consisted of highly bioavailable $\mathrm{Pb}$ carbonate. However, this study did not provide information on bioavailability of $\mathrm{Pb}$ debris. 


\section{Figure C-1. PbB Predicted from AALM-LG for a $0.9 \mathrm{mg}$ Dose of Soluble $\mathrm{Pb}$ Ingested by a Child 30 Months of Age}

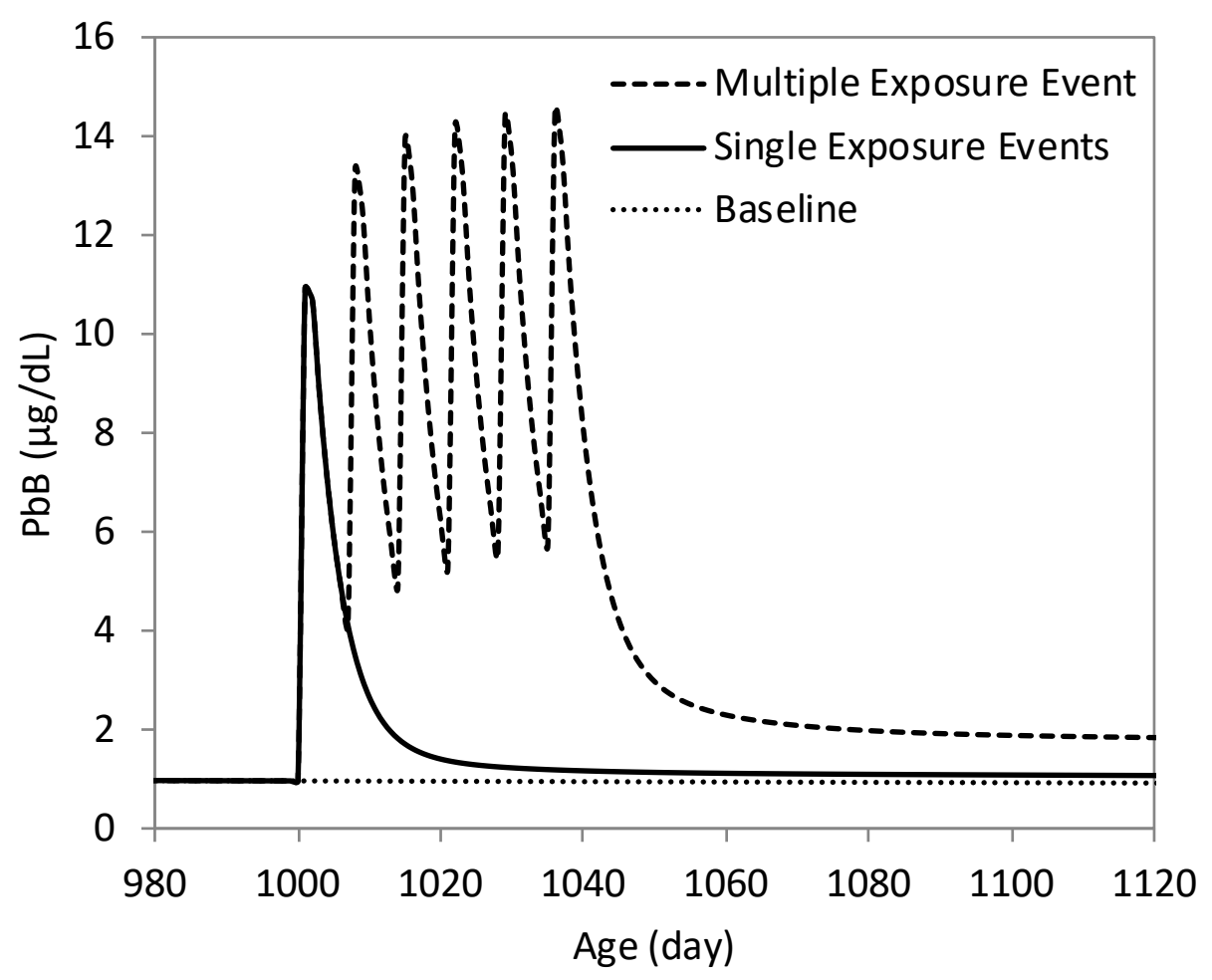

Retention of $\mathbf{P b}$ Debris in the Appendix. Case reports show that $\mathrm{Pb}$ debris can be retained within the appendix (Banner et al. 2012; Cox and Pesola 2005; Durlach et al. 1986; Larsen and Blanton 2000; Lyons and Filston 1994; Madsen et al. 1988; Reddy 1985; Zardawi and Siriweera 2013); see Table C-1 for details. For this to occur, the appendix must be oriented with respect to the cecum in such a way to allow objects to pass through the appendiceal-cecal orifice; approximately $45 \%$ of the population have appendices with this orientation. However, approximately 65\% of the population have appendices that might hinder foreign body access into the appendiceal lumen due to atypical anatomic position, adhesions, or kinks (Klingler et al. 1998). In addition to orientation of the appendix, the physical size and shape of the debris likely contribute to retention. Although it is not possible to determine the incidence of $\mathrm{Pb}$ debris lodged in the gastrointestinal tract or the appendix because not all cases of ingestion of $\mathrm{Pb}$ debris are reported in the published literature, approximately $45 \%$ of the population is predisposed to retention of $\mathrm{Pb}$ debris on orientation of the appendix.

Toxicity of Ingested $\mathbf{P b}$ Debris. Regardless of the source of $\mathrm{Pb}$ (e.g., ingested $\mathrm{Pb}$ debris, $\mathrm{Pb}$ paint, $\mathrm{Pb}-$ contaminated soil, occupational exposure), once $\mathrm{Pb}$ is absorbed into the body, toxicity will be related to $\mathrm{PbB}$; thus, bioavailability and duration of elevated $\mathrm{PbB}$, rather than the form of $\mathrm{Pb}$ ingested, will determine adverse health outcomes. If ingested $\mathrm{Pb}$ debris is not retained by the body, toxicity of $\mathrm{PbB}$ 
would be consistent with that described for acute $\mathrm{Pb}$ toxicity. A summary of peak PbBs and associated toxicity following exposure of ingested $\mathrm{Pb}$ debris is shown in Table C-2. Severity of toxicity increases with $\mathrm{PbB}$. At $\mathrm{PbB} \leq 47 \mu \mathrm{g} / \mathrm{dL}$, the only adverse health effect observed was a single report of headache at a $\mathrm{PbB}$ of $12 \mu \mathrm{g} / \mathrm{dL}$. With increased $\mathrm{PbB}$, effects were observed in several organ systems and severity of effects increased. At a PbB range of 54-146 $\mu \mathrm{g} / \mathrm{dL}$, abdominal colic, vomiting, hematological effects, and neurological effects were observed, and at a PbB of $180 \mu \mathrm{g} / \mathrm{dL}$, severe effects (seizure and cerebral edema) leading to death were observed. In most cases, the onset of toxicity occurs within hours or days of ingestion. If $\mathrm{PbB}$ remains elevated, either due to inadequate medical intervention or $\mathrm{Pb}$ that is retained within the body (i.e., appendix, gastrointestinal tract, etc.) adverse health effects associated with chronically elevated PbB would be expected to occur (see Chapter 2, Health Effects). As reviewed in Chapter 2, PbBs $\leq 10 \mu \mathrm{g} / \mathrm{dL}$ are associated with adverse health effects to numerous organ systems, including developmental and neurological effects, with severity exhibiting dose-dependence. Given the many factors that can affect development of $\mathrm{Pb}$-induced toxicity, case reports of individuals cannot provide generalizations of exposure-response relationships.

\begin{tabular}{|c|c|c|}
\hline Peak PbB $(\mu \mathrm{g} / \mathrm{dL})^{\mathrm{a}}$ & Effects associated with $\mathrm{Pb}$ exposure & References \\
\hline \multirow[t]{2}{*}{$12-16$} & No effects observed & Fergusson et al. 1997 \\
\hline & Headache & Lyons and Filston 1994 \\
\hline $40.5-47$ & No effects observed & Clifton et al. 2002; Hatten et al. 2013 \\
\hline \multirow[t]{3}{*}{$54-61$} & No effects observed & $\begin{array}{l}\text { Mowad et al. 1998; Treble and } \\
\text { Thompson } 2002\end{array}$ \\
\hline & Abdominal colic & Banner et al. 2012 \\
\hline & Hematological effects ${ }^{b}$ & \\
\hline \multirow[t]{3}{*}{79} & Abdominal colic and vomiting & McKinney and McKinney 2000 \\
\hline & Hematological effects ${ }^{c}$ & \\
\hline & Neurological effects ${ }^{d}$ & \\
\hline 123 & $\begin{array}{l}\text { Abdominal colic, vomiting, diarrhea } \\
\text { Hematological effects }\end{array}$ & CDC 2004a \\
\hline \multirow[t]{2}{*}{146} & Vomiting & Hatten et al. 2013 \\
\hline & Neurological signs ${ }^{f}$ & \\
\hline \multirow[t]{4}{*}{180} & Vomiting & CDC 2006 \\
\hline & Seizures & \\
\hline & Cerebral edema & \\
\hline & Death & \\
\hline
\end{tabular}


Table C-2. Peak Blood Lead Concentration (PbB) and Acute Toxicity Associated with Ingestion of Lead $(\mathrm{Pb})$ Debris

\begin{tabular}{lll}
\hline Peak PbB $(\mu \mathrm{g} / \mathrm{dL})^{\mathrm{a}}$ & Effects associated with $\mathrm{Pb}$ exposure & References \\
\hline 391 & $\begin{array}{l}\text { Abdominal colic, gastrointestinal bleeding } \\
\text { Anemia }\end{array}$ & McNutt et al. 2001 \\
&
\end{tabular}

aPeak blood $\mathrm{Pb}$ reported.

${ }^{b}$ Elevated free erythrocyte protoporphyrin or microcytic anemia and increased blood zinc protoporphyrin.

'Decreased blood hemoglobin and hematocrit.

d"Mild" speech and language delays.

eNormocytic anemia, elevated protoporphyrin

fDecreased activity level and hyperactive patellar and brachioradialis reflexes.

\section{REFERENCES}

Banner B, Schaeffer S, Badillo RB, et al. 2012. Multiple lead appendoliths following ingestion of lead shot: Time course and removal by laporoscopic appendectomy. Clin Toxicol 50(4):266-267. http://doi.org/10.3109/15563650.2012.658473.

Bannon DI, Drexler JW, Fent GM, et al. 2009. Evaluation of small arms range soils for metal contamination and lead bioavailability. Environ Sci Technol 43(24):9071-9076. http://doi.org/10.1021/es901834h.

CDC. 2004a. Brief report: Lead poisoning from ingestion of a toy necklace- Oregon, 2003. Centers for Disease Control and Prevention. MMWR Morb Mortal Wkly Rep 53(23):509-511. https://www.cdc.gov/mmwr/preview/mmwrhtml/mm5323a5.htm.

CDC. 2006. Death of a child after ingestion of a metallic charm -- Minnesota, 2006. MMWR Morb Mortal Wkly Rep 55(12):340-341. https://www.cdc.gov/mmwr/pdf/wk/mm5512.pdf. May 23, 2017.

Clifton JC, Sigg T, Burda AM, et al. 2002. Acute pediatric lead poisoning: Combined whole bowel irrigation, succimer therapy, and endoscopic removal of ingested lead pellets. Pediatr Emerg Care 18(3):200-202. http://doi.org/10.1097/01.pec.0000019226.25165.86.

Cox WM, Pesola GR. 2005. Buckshot ingestion. N Engl J Med 353(26):e23.

Durlach V, Lisovoski F, Gross A, et al. 1986. Appendicectomy in an unusual case of lead poisoning. Lancet 1(8482):687-688.

EPA. 2014a. Development and evaluation of the all ages lead model (AALM). U.S. Environmental Protection Agency.

Fergusson J, Malecky G, Simpson E. 1997. Lead foreign body ingestion in children. J Paediatr Child Health 33:542-544.

Gerhardsson L, Dahlin L, Knebel R, et al. 2002. Blood lead concentration after a shotgun accident. Environ Health Perspect 110:115-117.

Greensher J, Mofenson HC, Balakrishnan C, et al. 1974. Leading poisoning from ingestion of lead shot. Pediatrics 54(5):641-643.

Guillard O, Flamen P, Fauconneau B. 2006. A case of acute lead poisoning in a 2-year-old child. Br J Clin Pharmacol 62(2):247-247. http://doi.org/10.1111/j.1365-2125.2006.02603.x.

Gustavsson P, Gerhardsson L. 2005. Intoxication from an accidentally ingested lead shot retained in the gastrointestinal tract. Environ Health Perspect 113:491-493.

Hatten BW, Bueso A, Craven P, et al. 2013. Lead toxicity and endoscopic removal of ingested firearm cartridges. Clin Toxicol (Phila) 51(5):448-450. http://doi.org/10.3109/15563650.2013.792114.

Klingler PJ, Seelig MH, DeVault KR, et al. 1998. Ingested foreign bodies within the appendix: A 100year review of the literature. Dig Dis 16(5):308-314. 
Larsen AR, Blanton RH. 2000. Appendicitis due to bird shot ingestion: A case study. Am Surg 66(6):589-591.

Lyons JD, Filston HC. 1994. Lead intoxication from a pellet entrapped in the appendix of a child: Treatment considerations. J Pediatr Surg 29(12):1618-1620.

Madsen HH, Skjodt T, Jorgensen PJ, et al. 1988. Blood lead levels in patients with lead shot retained in the appendix. Acta Radiol 29(6):745-746. http://doi.org/10.1080/02841858809171977.

McNutt TK, Chambers-Emerson J, Dethlefsen M, et al. 2001. Bite the bullet: Lead poisoning after ingestion of 206 lead bullets. Vet Hum Toxicol 43(5):288-289.

McQuirter JL, Rothenberg SJ, Dinkins GA, et al. 2004. Change in blood lead concentration up to 1 year after a gunshot wound with a retained bullet. Am J Epidemiol 159(7):683-692. http://doi.org/10.1093/aje/kwh074.

Mowad E, Haddad I, Gemmel DJ. 1998. Management of lead poisoning from ingested fishing sinkers. Arch Pediatr Adolesc Med 152(5):485-488.

Reddy ER. 1985. Retained lead shot in the appendix. J Can Assoc Radiol 36(1):47-48.

Rosenberg A, Haynes J. 2019. Management of ingested lead pellets with elevated blood lead levels in a pediatric patient. J Pediatr Gastroenterol Nutr 69(6):e159-e160. http://doi.org/10.1097/mpg.0000000000002438.

Rozier B, Liebelt E. 2019. Lead pellet ingestion in 3 children: Another source for lead toxicity. Pediatr Emerg Care 35(5):385-388. http://doi.org/10.1097/pec.0000000000001469.

Treble RG, Thompson TS. 2002. Elevated blood lead levels resulting from the ingestion of air rifle pellets. J Anal Toxicol 26:370-373.

Zardawi I, Siriweera E. 2013. Images in clinical medicine. Pellets in the appendix. N Engl J Med 369(6):e7. http://doi.org/10.1056/NEJMicm1214754. 


\section{APPENDIX D. QUICK REFERENCE FOR HEALTH CARE PROVIDERS}

Toxicological Profiles are a unique compilation of toxicological information on a given hazardous substance. Each profile reflects a comprehensive and extensive evaluation, summary, and interpretation of available toxicologic and epidemiologic information on a substance. Health care providers treating patients potentially exposed to hazardous substances may find the following information helpful for fast answers to often-asked questions.

\section{Primary Chapters/Sections of Interest}

Chapter 1: Relevance to Public Health: The Relevance to Public Health Section provides an overview of exposure and health effects and evaluates, interprets, and assesses the significance of toxicity data to human health. A table listing minimal risk levels (MRLs) is also included in this chapter.

Chapter 2: Health Effects: Specific health effects identified in both human and animal studies are reported by type of health effect (e.g., death, hepatic, renal, immune, reproductive), route of exposure (e.g., inhalation, oral, dermal), and length of exposure (e.g., acute, intermediate, and chronic).

NOTE: Not all health effects reported in this section are necessarily observed in the clinical setting.

\section{Pediatrics:}

Section 3.2 Children and Other Populations that are Unusually Susceptible Section 3.3 Biomarkers of Exposure and Effect

\section{ATSDR Information Center}

Phone: 1-800-CDC-INFO (800-232-4636) or 1-888-232-6348 (TTY)

Internet: http://www.atsdr.cdc.gov

The following additional materials are available online:

Case Studies in Environmental Medicine are self-instructional publications designed to increase primary health care providers' knowledge of a hazardous substance in the environment and to aid in the evaluation of potentially exposed patients (see https://www.atsdr.cdc.gov/csem/csem.html).

Managing Hazardous Materials Incidents is a three-volume set of recommendations for on-scene (prehospital) and hospital medical management of patients exposed during a hazardous materials incident (see https://www.atsdr.cdc.gov/MHMI/index.asp). Volumes I and II are planning guides to assist first responders and hospital emergency department personnel in planning for incidents that involve hazardous materials. Volume III-Medical Management Guidelines for Acute Chemical Exposures - is a guide for health care professionals treating patients exposed to hazardous materials.

Fact Sheets (ToxFAQs ${ }^{\mathrm{TM}}$ ) provide answers to frequently asked questions about toxic substances (see https://www.atsdr.cdc.gov/toxfaqs/Index.asp). 


\section{Other Agencies and Organizations}

The National Center for Environmental Health (NCEH) focuses on preventing or controlling disease, injury, and disability related to the interactions between people and their environment outside the workplace. Contact: NCEH, Mailstop F-29, 4770 Buford Highway, NE, Atlanta, GA 30341-3724 • Phone: 770-488-7000 • FAX: 770-488-7015 • Web Page: https://www.cdc.gov/nceh/.

The National Institute for Occupational Safety and Health (NIOSH) conducts research on occupational diseases and injuries, responds to requests for assistance by investigating problems of health and safety in the workplace, recommends standards to the Occupational Safety and Health Administration (OSHA) and the Mine Safety and Health Administration (MSHA), and trains professionals in occupational safety and health. Contact: NIOSH, 395 E Street, S.W., Suite 9200, Patriots Plaza Building, Washington, DC 20201 • Phone: 202-245-0625 or 1-800-CDC-INFO (800-232-4636) • Web Page: https://www.cdc.gov/niosh/.

The National Institute of Environmental Health Sciences (NIEHS) is the principal federal agency for biomedical research on the effects of chemical, physical, and biologic environmental agents on human health and well-being. Contact: NIEHS, PO Box 12233, 104 T.W. Alexander Drive, Research Triangle Park, NC 27709 • Phone: 919-541-3212 • Web Page: https://www.niehs.nih.gov/.

\section{Clinical Resources (Publicly Available Information)}

The Association of Occupational and Environmental Clinics (AOEC) has developed a network of clinics in the United States to provide expertise in occupational and environmental issues. Contact: AOEC, 1010 Vermont Avenue, NW, \#513, Washington, DC 20005 • Phone: 202-347-4976 • FAX: 202-347-4950 • e-mail: AOEC@AOEC.ORG • Web Page: http://www.aoec.org/.

The American College of Occupational and Environmental Medicine (ACOEM) is an association of physicians and other health care providers specializing in the field of occupational and environmental medicine. Contact: ACOEM, 25 Northwest Point Boulevard, Suite 700, Elk Grove Village, IL 60007-1030 • Phone: 847-818-1800 • FAX: 847-818-9266 • Web Page: http://www.acoem.org/.

The American College of Medical Toxicology (ACMT) is a nonprofit association of physicians with recognized expertise in medical toxicology. Contact: ACMT, 10645 North Tatum Boulevard, Suite 200-111, Phoenix AZ 85028 • Phone: 844-226-8333 • FAX: 844-226-8333 • Web Page: http://www.acmt.net.

The Pediatric Environmental Health Specialty Units (PEHSUs) is an interconnected system of specialists who respond to questions from public health professionals, clinicians, policy makers, and the public about the impact of environmental factors on the health of children and reproductive-aged adults. Contact information for regional centers can be found at http://pehsu.net/findhelp.html.

The American Association of Poison Control Centers (AAPCC) provide support on the prevention and treatment of poison exposures. Contact: AAPCC, 515 King Street, Suite 510, Alexandria VA 22314 • Phone: 701-894-1858 • Poison Help Line: 1-800-222-1222 • Web Page: http://www.aapcc.org/. 


\section{APPENDIX E. GLOSSARY}

Absorption-The process by which a substance crosses biological membranes and enters systemic circulation. Absorption can also refer to the taking up of liquids by solids, or of gases by solids or liquids.

Acute Exposure-Exposure to a chemical for a duration of $\leq 14$ days, as specified in the Toxicological Profiles.

Adsorption-The adhesion in an extremely thin layer of molecules (as of gases, solutes, or liquids) to the surfaces of solid bodies or liquids with which they are in contact.

Adsorption Coefficient $\left(\mathbf{K}_{\mathbf{o c}}\right)$ - The ratio of the amount of a chemical adsorbed per unit weight of organic carbon in the soil or sediment to the concentration of the chemical in solution at equilibrium.

Adsorption Ratio (Kd) — The amount of a chemical adsorbed by sediment or soil (i.e., the solid phase) divided by the amount of chemical in the solution phase, which is in equilibrium with the solid phase, at a fixed solid/solution ratio. It is generally expressed in micrograms of chemical sorbed per gram of soil or sediment.

Benchmark Dose (BMD) or Benchmark Concentration (BMC)—is the dose/concentration corresponding to a specific response level estimate using a statistical dose-response model applied to either experimental toxicology or epidemiology data. For example, a $\mathrm{BMD}_{10}$ would be the dose corresponding to a $10 \%$ benchmark response (BMR). The BMD is determined by modeling the doseresponse curve in the region of the dose-response relationship where biologically observable data are feasible. The BMDL or BMCL is the 95\% lower confidence limit on the BMD or BMC.

Bioconcentration Factor (BCF) - The quotient of the concentration of a chemical in aquatic organisms at a specific time or during a discrete time period of exposure divided by the concentration in the surrounding water at the same time or during the same period.

Biomarkers-Indicators signaling events in biologic systems or samples, typically classified as markers of exposure, effect, and susceptibility.

Cancer Effect Level (CEL) - The lowest dose of a chemical in a study, or group of studies, that produces significant increases in the incidence of cancer (or tumors) between the exposed population and its appropriate control.

Carcinogen-A chemical capable of inducing cancer.

Case-Control Study-A type of epidemiological study that examines the relationship between a particular outcome (disease or condition) and a variety of potential causative agents (such as toxic chemicals). In a case-control study, a group of people with a specified and well-defined outcome is identified and compared to a similar group of people without the outcome.

Case Report - A report that describes a single individual with a particular disease or exposure. These reports may suggest some potential topics for scientific research, but are not actual research studies.

Case Series-Reports that describe the experience of a small number of individuals with the same disease or exposure. These reports may suggest potential topics for scientific research, but are not actual research studies. 
Ceiling Value-A concentration that must not be exceeded.

Chronic Exposure-Exposure to a chemical for $\geq 365$ days, as specified in the Toxicological Profiles.

Clastogen-A substance that causes breaks in chromosomes resulting in addition, deletion, or rearrangement of parts of the chromosome.

Cohort Study-A type of epidemiological study of a specific group or groups of people who have had a common insult (e.g., exposure to an agent suspected of causing disease or a common disease) and are followed forward from exposure to outcome, and who are disease-free at start of follow-up. Often, at least one exposed group is compared to one unexposed group, while in other cohorts, exposure is a continuous variable and analyses are directed towards analyzing an exposure-response coefficient.

Cross-sectional Study - A type of epidemiological study of a group or groups of people that examines the relationship between exposure and outcome to a chemical or to chemicals at a specific point in time.

Data Needs—Substance-specific informational needs that, if met, would reduce the uncertainties of human health risk assessment.

Developmental Toxicity-The occurrence of adverse effects on the developing organism that may result from exposure to a chemical prior to conception (either parent), during prenatal development, or postnatally to the time of sexual maturation. Adverse developmental effects may be detected at any point in the life span of the organism.

Dose-Response Relationship-The quantitative relationship between the amount of exposure to a toxicant and the incidence of the response or amount of the response.

Embryotoxicity and Fetotoxicity-Any toxic effect on the conceptus as a result of prenatal exposure to a chemical; the distinguishing feature between the two terms is the stage of development during which the effect occurs. Effects include malformations and variations, altered growth, and in utero death.

Epidemiology - The investigation of factors that determine the frequency and distribution of disease or other health-related conditions within a defined human population during a specified period.

Excretion-The process by which metabolic waste products are removed from the body.

Genotoxicity-A specific adverse effect on the genome of living cells that, upon the duplication of affected cells, can be expressed as a mutagenic, clastogenic, or carcinogenic event because of specific alteration of the molecular structure of the genome.

Half-life-A measure of rate for the time required to eliminate one-half of a quantity of a chemical from the body or environmental media.

Health Advisory-An estimate of acceptable drinking water levels for a chemical substance derived by EPA and based on health effects information. A health advisory is not a legally enforceable federal standard, but serves as technical guidance to assist federal, state, and local officials.

Immediately Dangerous to Life or Health (IDLH) — A condition that poses a threat of life or health, or conditions that pose an immediate threat of severe exposure to contaminants that are likely to have adverse cumulative or delayed effects on health. 
Immunotoxicity-Adverse effect on the functioning of the immune system that may result from exposure to chemical substances.

Incidence-The ratio of new cases of individuals in a population who develop a specified condition to the total number of individuals in that population who could have developed that condition in a specified time period.

Intermediate Exposure-Exposure to a chemical for a duration of 15-364 days, as specified in the Toxicological Profiles.

In Vitro-Isolated from the living organism and artificially maintained, as in a test tube.

In Vivo-Occurring within the living organism.

Lethal Concentration $(\mathbf{L O})\left(\mathbf{L C}_{\mathbf{L O}}\right)$ - The lowest concentration of a chemical in air that has been reported to have caused death in humans or animals.

Lethal Concentration ${ }_{(50)}\left(\mathbf{L C}_{50}\right)$ - A calculated concentration of a chemical in air to which exposure for a specific length of time is expected to cause death in $50 \%$ of a defined experimental animal population.

Lethal Dose $(\mathbf{L O})\left(\mathbf{L D}_{\mathbf{L o}}\right)$ - The lowest dose of a chemical introduced by a route other than inhalation that has been reported to have caused death in humans or animals.

Lethal Dose $\mathbf{( 5 0}_{(\mathbf{5 0}}\left(\mathbf{L D}_{\mathbf{5 0}}\right)$ - The dose of a chemical that has been calculated to cause death in $50 \%$ of a defined experimental animal population.

Lethal Time $\left(\mathbf{5 0}_{\mathbf{5}} \mathbf{( L T}_{\mathbf{5 0}}\right)$ - A calculated period of time within which a specific concentration of a chemical is expected to cause death in $50 \%$ of a defined experimental animal population.

Lowest-Observed-Adverse-Effect Level (LOAEL) — The lowest exposure level of chemical in a study, or group of studies, that produces statistically or biologically significant increases in frequency or severity of adverse effects between the exposed population and its appropriate control.

Lymphoreticular Effects-Represent morphological effects involving lymphatic tissues such as the lymph nodes, spleen, and thymus.

Malformations-Permanent structural changes that may adversely affect survival, development, or function.

Metabolism-Process in which chemical substances are biotransformed in the body that could result in less toxic and/or readily excreted compounds or produce a biologically active intermediate.

Minimal Risk Level (MRL)—An estimate of daily human exposure to a hazardous substance that is likely to be without an appreciable risk of adverse noncancer health effects over a specified route and duration of exposure.

Modifying Factor (MF) —A value (greater than zero) that is applied to the derivation of a Minimal Risk Level (MRL) to reflect additional concerns about the database that are not covered by the uncertainty factors. The default value for a MF is 1. 
Morbidity - The state of being diseased; the morbidity rate is the incidence or prevalence of a disease in a specific population.

Mortality-Death; the mortality rate is a measure of the number of deaths in a population during a specified interval of time.

Mutagen-A substance that causes mutations, which are changes in the DNA sequence of a cell's DNA. Mutations can lead to birth defects, miscarriages, or cancer.

Necropsy-The gross examination of the organs and tissues of a dead body to determine the cause of death or pathological conditions.

Neurotoxicity-The occurrence of adverse effects on the nervous system following exposure to a hazardous substance.

No-Observed-Adverse-Effect Level (NOAEL) - The dose of a chemical at which there were no statistically or biologically significant increases in frequency or severity of adverse effects seen between the exposed population and its appropriate control. Although effects may be produced at this dose, they are not considered to be adverse.

Octanol-Water Partition Coefficient $\left(\mathbf{K}_{\mathbf{o w}}\right)$ - The equilibrium ratio of the concentrations of a chemical in $n$-octanol and water, in dilute solution.

Odds Ratio (OR) - A means of measuring the association between an exposure (such as toxic substances and a disease or condition) that represents the best estimate of relative risk (risk as a ratio of the incidence among subjects exposed to a particular risk factor divided by the incidence among subjects who were not exposed to the risk factor). An odds ratio that is greater than 1 is considered to indicate greater risk of disease in the exposed group compared to the unexposed group.

Permissible Exposure Limit (PEL)—An Occupational Safety and Health Administration (OSHA) regulatory limit on the amount or concentration of a substance not to be exceeded in workplace air averaged over any 8-hour work shift of a 40-hour workweek.

Pesticide-General classification of chemicals specifically developed and produced for use in the control of agricultural and public health pests (insects or other organisms harmful to cultivated plants or animals).

Pharmacokinetics - The dynamic behavior of a material in the body, used to predict the fate (disposition) of an exogenous substance in an organism. Utilizing computational techniques, it provides the means of studying the absorption, distribution, metabolism, and excretion of chemicals by the body.

Pharmacokinetic Model-A set of equations that can be used to describe the time course of a parent chemical or metabolite in an animal system. There are two types of pharmacokinetic models: data-based and physiologically-based. A data-based model divides the animal system into a series of compartments, which, in general, do not represent real, identifiable anatomic regions of the body, whereas the physiologically-based model compartments represent real anatomic regions of the body.

Physiologically Based Pharmacodynamic (PBPD) Model-A type of physiologically based doseresponse model that quantitatively describes the relationship between target tissue dose and toxic endpoints. These models advance the importance of physiologically based models in that they clearly describe the biological effect (response) produced by the system following exposure to an exogenous substance. 
Physiologically Based Pharmacokinetic (PBPK) Model—A type of physiologically based doseresponse model that is comprised of a series of compartments representing organs or tissue groups with realistic weights and blood flows. These models require a variety of physiological information, including tissue volumes, blood flow rates to tissues, cardiac output, alveolar ventilation rates, and possibly membrane permeabilities. The models also utilize biochemical information, such as blood:air partition coefficients, and metabolic parameters. PBPK models are also called biologically based tissue dosimetry models.

Prevalence-The number of cases of a disease or condition in a population at one point in time.

Prospective Study - A type of cohort study in which a group is followed over time and the pertinent observations are made on events occurring after the start of the study.

Recommended Exposure Limit (REL) — A National Institute for Occupational Safety and Health (NIOSH) time-weighted average (TWA) concentration for up to a 10-hour workday during a 40-hour workweek.

Reference Concentration (RfC)—An estimate (with uncertainty spanning perhaps an order of magnitude) of a continuous inhalation exposure to the human population (including sensitive subgroups) that is likely to be without an appreciable risk of deleterious noncancer health effects during a lifetime. The inhalation RfC is expressed in units of $\mathrm{mg} / \mathrm{m}^{3}$ or $\mathrm{ppm}$.

Reference Dose (RfD) — An estimate (with uncertainty spanning perhaps an order of magnitude) of the daily oral exposure of the human population to a potential hazard that is likely to be without risk of deleterious noncancer health effects during a lifetime. The oral RfD is expressed in units of mg/kg/day.

Reportable Quantity (RQ) - The quantity of a hazardous substance that is considered reportable under the Comprehensive Environmental Response, Compensation, and Liability Act (CERCLA). RQs are (1) $\geq 1$ pound or (2) for selected substances, an amount established by regulation either under CERCLA or under Section 311 of the Clean Water Act. Quantities are measured over a 24-hour period.

Reproductive Toxicity-The occurrence of adverse effects on the reproductive system that may result from exposure to a hazardous substance. The toxicity may be directed to the reproductive organs and/or the related endocrine system. The manifestation of such toxicity may be noted as alterations in sexual behavior, fertility, pregnancy outcomes, or modifications in other functions that are dependent on the integrity of this system.

Retrospective Study - A type of cohort study based on a group of persons known to have been exposed at some time in the past. Data are collected from routinely recorded events, up to the time the study is undertaken. Retrospective studies are limited to causal factors that can be ascertained from existing records and/or examining survivors of the cohort.

Risk-The possibility or chance that some adverse effect will result from a given exposure to a hazardous substance.

Risk Factor-An aspect of personal behavior or lifestyle, an environmental exposure, existing health condition, or an inborn or inherited characteristic that is associated with an increased occurrence of disease or other health-related event or condition. 
Risk Ratio/Relative Risk-The ratio of the risk among persons with specific risk factors compared to the risk among persons without risk factors. A risk ratio that is greater than 1 indicates greater risk of disease in the exposed group compared to the unexposed group.

Short-Term Exposure Limit (STEL)—A STEL is a 15-minute TWA exposure that should not be exceeded at any time during a workday.

Standardized Mortality Ratio (SMR) — A ratio of the observed number of deaths and the expected number of deaths in a specific standard population.

Target Organ Toxicity-This term covers a broad range of adverse effects on target organs or physiological systems (e.g., renal, cardiovascular) extending from those arising through a single limited exposure to those assumed over a lifetime of exposure to a chemical.

Teratogen-A chemical that causes structural defects that affect the development of an organism.

Threshold Limit Value (TLV)_An American Conference of Governmental Industrial Hygienists (ACGIH) concentration of a substance to which it is believed that nearly all workers may be repeatedly exposed, day after day, for a working lifetime without adverse effect. The TLV may be expressed as a Time-Weighted Average (TLV-TWA), as a Short-Term Exposure Limit (TLV-STEL), or as a ceiling limit (TLV-C).

Time-Weighted Average (TWA)—An average exposure within a given time period.

Toxicokinetic - The absorption, distribution, metabolism, and elimination of toxic compounds in the living organism.

Toxics Release Inventory (TRI) - The TRI is an EPA program that tracks toxic chemical releases and pollution prevention activities reported by industrial and federal facilities.

Uncertainty Factor (UF)—A factor used in operationally deriving the Minimal Risk Level (MRL), Reference Dose (RfD), or Reference Concentration (RfC) from experimental data. UFs are intended to account for (1) the variation in sensitivity among the members of the human population, (2) the uncertainty in extrapolating animal data to the case of human, (3) the uncertainty in extrapolating from data obtained in a study that is of less than lifetime exposure, and (4) the uncertainty in using lowestobserved-adverse-effect level (LOAEL) data rather than no-observed-adverse-effect level (NOAEL) data. A default for each individual UF is 10; if complete certainty in data exists, a value of 1 can be used; however, a reduced UF of 3 may be used on a case-by-case basis ( 3 being the approximate logarithmic average of 10 and 1$)$.

Xenobiotic_-Any substance that is foreign to the biological system. 


\section{APPENDIX F. ACRONYMS, ABBREVIATIONS, AND SYMBOLS}

\begin{tabular}{|c|c|}
\hline AAPCC & American Association of Poison Control Centers \\
\hline ACGIH & American Conference of Governmental Industrial Hygienists \\
\hline ACOEM & American College of Occupational and Environmental Medicine \\
\hline ACMT & American College of Medical Toxicology \\
\hline ADI & acceptable daily intake \\
\hline ADME & absorption, distribution, metabolism, and excretion \\
\hline AEGL & Acute Exposure Guideline Level \\
\hline AIC & Akaike’s information criterion \\
\hline AIHA & American Industrial Hygiene Association \\
\hline ALT & alanine aminotransferase \\
\hline AOEC & Association of Occupational and Environmental Clinics \\
\hline AP & alkaline phosphatase \\
\hline AST & aspartate aminotransferase \\
\hline atm & atmosphere \\
\hline ATSDR & Agency for Toxic Substances and Disease Registry \\
\hline AWQC & Ambient Water Quality Criteria \\
\hline $\mathrm{BCF}$ & bioconcentration factor \\
\hline $\mathrm{BMD} / \mathrm{C}$ & benchmark dose or benchmark concentration \\
\hline $\mathrm{BMD}_{\mathrm{X}}$ & dose that produces a X\% change in response rate of an adverse effect \\
\hline BMDL $_{X}$ & 95\% lower confidence limit on the $\mathrm{BMD}_{\mathrm{X}}$ \\
\hline BMDS & Benchmark Dose Software \\
\hline BMR & benchmark response \\
\hline BUN & blood urea nitrogen \\
\hline $\mathrm{C}$ & centigrade \\
\hline CAA & Clean Air Act \\
\hline CAS & Chemical Abstract Services \\
\hline CDC & Centers for Disease Control and Prevention \\
\hline CEL & cancer effect level \\
\hline CERCLA & Comprehensive Environmental Response, Compensation, and Liability Act \\
\hline CFR & Code of Federal Regulations \\
\hline $\mathrm{Ci}$ & curie \\
\hline CI & confidence interval \\
\hline $\mathrm{cm}$ & centimeter \\
\hline CPSC & Consumer Products Safety Commission \\
\hline CWA & Clean Water Act \\
\hline DNA & deoxyribonucleic acid \\
\hline DOD & Department of Defense \\
\hline DOE & Department of Energy \\
\hline DWEL & drinking water exposure level \\
\hline EAFUS & Everything Added to Food in the United States \\
\hline ECG/EKG & electrocardiogram \\
\hline EEG & electroencephalogram \\
\hline EPA & Environmental Protection Agency \\
\hline ERPG & emergency response planning guidelines \\
\hline $\mathrm{F}$ & Fahrenheit \\
\hline F1 & first-filial generation \\
\hline FDA & Food and Drug Administration \\
\hline FIFRA & Federal Insecticide, Fungicide, and Rodenticide Act \\
\hline FR & Federal Register \\
\hline
\end{tabular}


FSH follicle stimulating hormone

g gram

GC

gd

gas chromatography

GGT

GRAS

gestational day

HEC

$\gamma$-glutamyl transferase

HED

generally recognized as safe

HHS

human equivalent concentration

HPLC

human equivalent dose

HSDB

Department of Health and Human Services

IARC

high-performance liquid chromatography

IDLH

Hazardous Substance Data Bank

IRIS

$\mathrm{Kd}$

$\mathrm{kg}$

kkg

International Agency for Research on Cancer

immediately dangerous to life and health

$\mathrm{K}_{\mathrm{oc}}$

$\mathrm{K}_{\mathrm{ow}}$

$\mathrm{L}$

LC

$\mathrm{LC}_{50}$

$\mathrm{LC}_{\mathrm{Lo}}$

$\mathrm{LD}_{50}$

LD Lo

Integrated Risk Information System

adsorption ratio

kilogram

kilokilogram; 1 kilokilogram is equivalent to 1,000 kilograms and 1 metric ton

organic carbon partition coefficient

octanol-water partition coefficient

liter

liquid chromatography

lethal concentration, 50\% kill

lethal concentration, low

lethal dose, $50 \%$ kill

lethal dose, low

$\mathrm{LDH} \quad$ lactic dehydrogenase

LH luteinizing hormone

LOAEL lowest-observed-adverse-effect level

LSE

$\mathrm{LT}_{50}$

$\mathrm{m}$

Level of Significant Exposure

lethal time, 50\% kill

$\mathrm{mCi}$

MCL

MCLG

MF

$\mathrm{mg}$

$\mathrm{mL}$

meter

millicurie

maximum contaminant level

maximum contaminant level goal

modifying factor

milligram

milliliter

$\mathrm{mm}$

millimeter

$\mathrm{mmHg}$

mmol

MRL

MS

millimeters of mercury

millimole

Minimal Risk Level

MSHA

$\mathrm{Mt}$

mass spectrometry

NAAQS

Mine Safety and Health Administration

metric ton

NAS

NCEH

National Ambient Air Quality Standard

National Academy of Science

ND

National Center for Environmental Health

not detected

ng nanogram

NHANES National Health and Nutrition Examination Survey

NIEHS

National Institute of Environmental Health Sciences 
NIOSH National Institute for Occupational Safety and Health

NLM

$\mathrm{nm}$ National Library of Medicine

nmol nanometer

NOAEL NPL

NR nanomole

NRC

NS no-observed-adverse-effect level

NTP

OR National Priorities List not reported

OSHA

PAC National Research Council

not specified

National Toxicology Program

odds ratio

PAH

Occupational Safety and Health Administration

PBPD

PBPK

PEHSU

PEL Protective Action Criteria

polycyclic aromatic hydrocarbon physiologically based pharmacodynamic physiologically based pharmacokinetic

PEL-C permissible exposure limit-ceiling value

pg

PND

POD

$\mathrm{ppb}$

ppbv

ppm

ppt

REL

REL-C

RfC

RfD

RNA

SARA

SCE

SD

SE

SGOT

SGPT

SIC

SMR

SRBC

STEL

TLV

TLV-C

TRI

TSCA

TWA

UF

U.S. picogram

postnatal day

point of departure

parts per billion

parts per billion by volume

parts per million

parts per trillion

recommended exposure level/limit

recommended exposure level-ceiling value

reference concentration

reference dose

ribonucleic acid

Superfund Amendments and Reauthorization Act

sister chromatid exchange

standard deviation

standard error

serum glutamic oxaloacetic transaminase (same as aspartate aminotransferase or AST)

serum glutamic pyruvic transaminase (same as alanine aminotransferase or ALT)

standard industrial classification

standardized mortality ratio

sheep red blood cell

short term exposure limit

threshold limit value

threshold limit value-ceiling value

Toxics Release Inventory

Toxic Substances Control Act

time-weighted average

uncertainty factor

USDA United States Department of Agriculture

USGS United States Geological Survey

USNRC U.S. Nuclear Regulatory Commission 
VOC volatile organic compound

WBC white blood cell

WHO World Health Organization

$>\quad$ greater than

$\geq \quad$ greater than or equal to

$=$ equal to

$<\quad$ less than

$\leq \quad$ less than or equal to

$\%$ percent

$\alpha \quad$ alpha

$\beta \quad$ beta

$\gamma \quad$ gamma

$\delta \quad$ delta

$\mu \mathrm{m} \quad$ micrometer

$\mu \mathrm{g} \quad$ microgram

$\mathrm{q}_{1}{ }^{*} \quad$ cancer slope factor

$-\quad$ negative

$+\quad$ positive

(+) weakly positive result

$(-) \quad$ weakly negative result 